Page 1 of

\begin{tabular}{|c|c|c|c|c|}
\hline \multirow{3}{*}{ 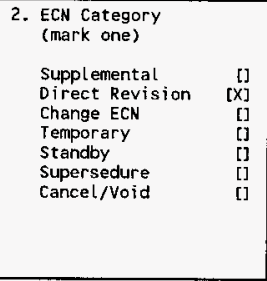 } & \multicolumn{2}{|c|}{$\begin{array}{l}\text { 3. Originator's Name, Organization, MSIN, } \\
\text { and Telephone No. } \\
\text { T. M. Brown, R2-12, 373-4437 }\end{array}$} & $\begin{array}{l}\text { 4. USQ Required? } \\
{[] \text { Yes }[X] \text { No }}\end{array}$ & \\
\hline & \multicolumn{2}{|c|}{$\begin{array}{l}\text { 6. Project Iitle/No./Work Order No. } \\
\text { Supporting Document for the } \\
\text { Historical Tank Content } \\
\text { Estimate for S-Tank Farm }\end{array}$} & $\begin{array}{l}\text { 7. Bldg./Sys./Fac. No. } \\
2750 / 200 \mathrm{E}\end{array}$ & \\
\hline & \multicolumn{2}{|c|}{$\begin{array}{l}\text { 9. Document Numbers Changed by this ECN } \\
\text { (includes sheet no. and rev.) } \\
\text { WHC-SD-WM-ER-323, Rev. } 0\end{array}$} & $\begin{array}{l}\text { 10. Related ECN No(s). } \\
N / A\end{array}$ & \\
\hline \multirow{2}{*}{$\begin{array}{l}\text { 12a. Modification Work } \\
\text { [] Yes (fill out Blk. } \\
12 b \text { ) } \\
{[X] \text { No (NA Blks. 12b, }} \\
12 c, 12 d)\end{array}$} & \multirow[t]{2}{*}{$\begin{array}{l}\text { 12b. Work Package } \\
\text { No. } \\
\text { N/A }\end{array}$} & \multirow{2}{*}{$\begin{array}{l}\begin{array}{l}\text { 12c. Modification Work Complete } \\
N / A\end{array} \\
\text { Design Authority/Cog. Engineer } \\
\text { Signature \& Date }\end{array}$} & \multicolumn{2}{|c|}{$\begin{array}{l}\text { 12d. Restored to Original Cor } \\
t \text { ion (Temp. or Standby ECN or } \\
N / A\end{array}$} \\
\hline & & & \begin{tabular}{l|l} 
Engineer \\
te
\end{tabular} & \\
\hline \multicolumn{5}{|c|}{$\begin{array}{l}\text { 13a. Description of change } \\
\text { 1. Complete rewrite and update of } \\
\text { 2. Added HTCE and Supporting Docu } \\
\text { 3. Added SW quadrant tank farms t } \\
\text { 4. Updated and restructured waste } \\
\text { 5. Removed temperature data table } \\
\text { 6. Removed drywell data, plots an } \\
\text { 7. Removed chemical and radionucl } \\
\text { 8. Removed unknown tank transfer } \\
\text { 9. Added the Hanford Tank Chemica }\end{array}$} \\
\hline
\end{tabular}

14a. Justification (mark one)

Criteria Change

[] Design Improvement [X]

Environmental

[]

Facility Deactivation

As-Found

[] Facilitate Const

Const. Error/Omission

[] Design Error/Omission

14b. Justification Details

To update the supporting document with more complete historical and tank inventory information.

15. Distribution (include name, MSIN, and no. of copies)

See attached distribution sheet. 
THIS PAGE INTENTIONALLY

LEFT BLANK 


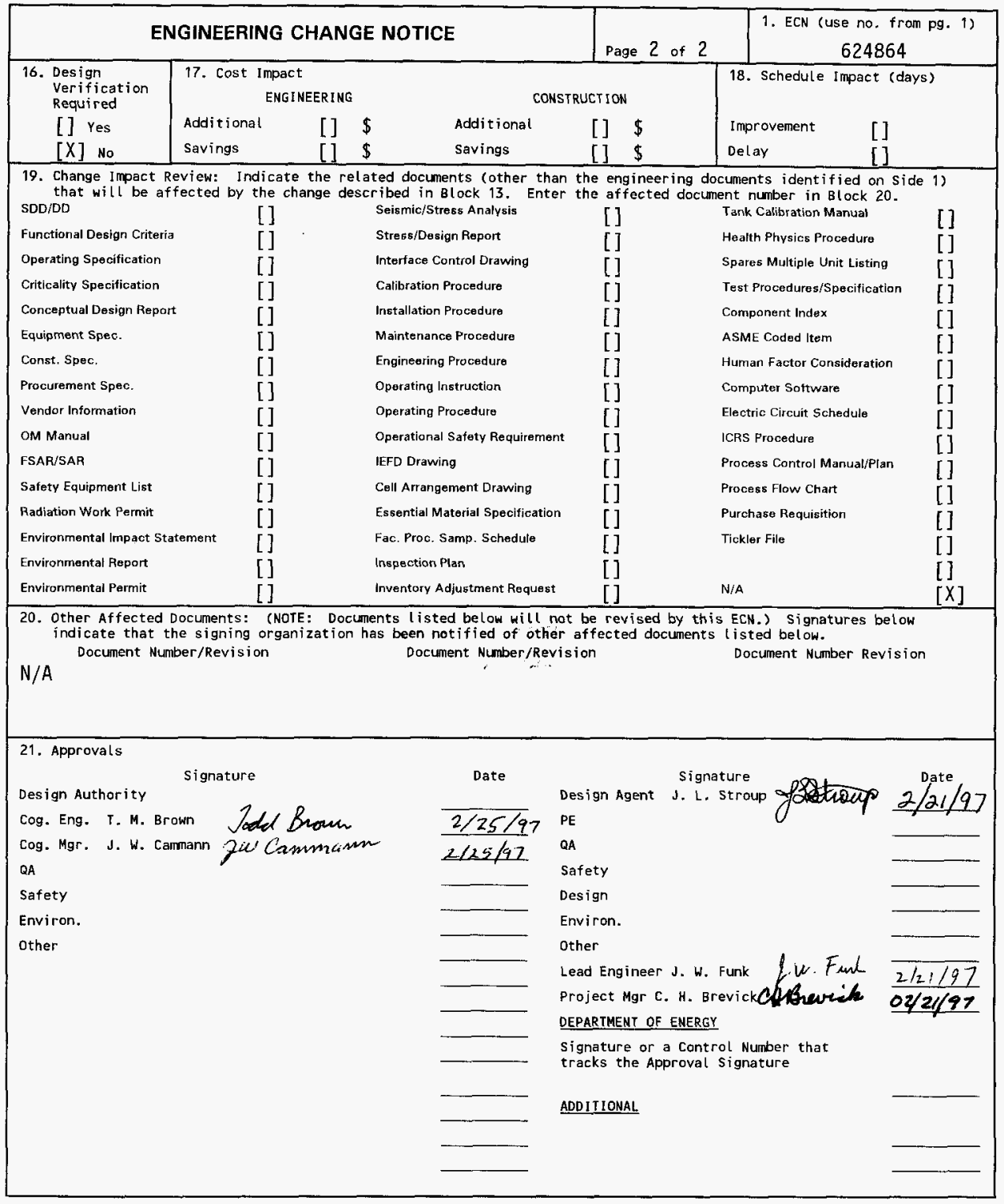




\section{TH: $\cdots$ INTENTIONALLY LLFT BLANK}




\title{
Supporting Document for the Historical Tank Content Estimate for S-Tank Farm
}

\author{
C. H. Brevick, J. L. Stroup, J. W. Funk
}

Fluor Daniel Northwest Inc., Richland, WA 99352

U.S. Department of Energy Contract DE-AC06-96RL13200
EDT/ECN: $\quad 624864$
Org Code: 408
UC: 2070
B\&R Code: EW3120074
Charge Code: $E 18675$
Total Pages: 430

Key Words: Southwest quadrant, Historical Tank Content Estimate, tank farms, tank level, tank temperture, aerial photos, in-tank montages, TLM, SMM, waste temperature plots, inventory estimates, riser locations

Abstract: This Supporting Document provides historical in-depth characterization information on S-Tank Farm, such as historical waste transfer and level data, tank physical information, temperature plots, 1 iquid observation well plots, chemical analyte and radionuclide inventories for the Historical Tank Content Estimate Report for the Southwest Quadrant of the Hanford 200 West Area

\footnotetext{
TRADEMARK DISCLAIMER. Reference herein to any specific comercial product, process, or service by trade name, trademark, manufacturer, or otherwise, does not necessarily constitute or imply its endorsement, recommendation, or favoring by the United States Government or any agency thereof or its contractors or subcontractors.

Printed in the United States of America. To obtain copies of this document, contact: Document Control Services, P.O. Box 950, Mailstop H6-08, Richland WA 99352, Phone (509) 372-2420;

Fax (509) 376-4989.
}
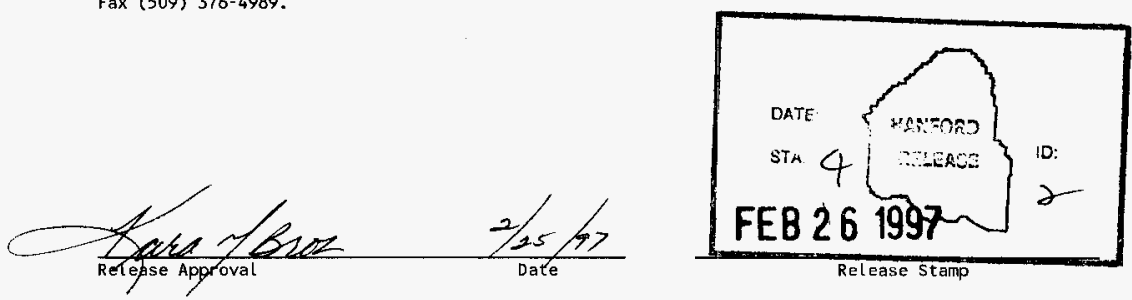

Approved for Public Release 
THIS PAGE INTENTIONALLY

LEFT BLANK 
(2) Title

Supporting Document for the Historical Tank Content Estimate for S-Tank Farm CHANGE CONTROL RECORD

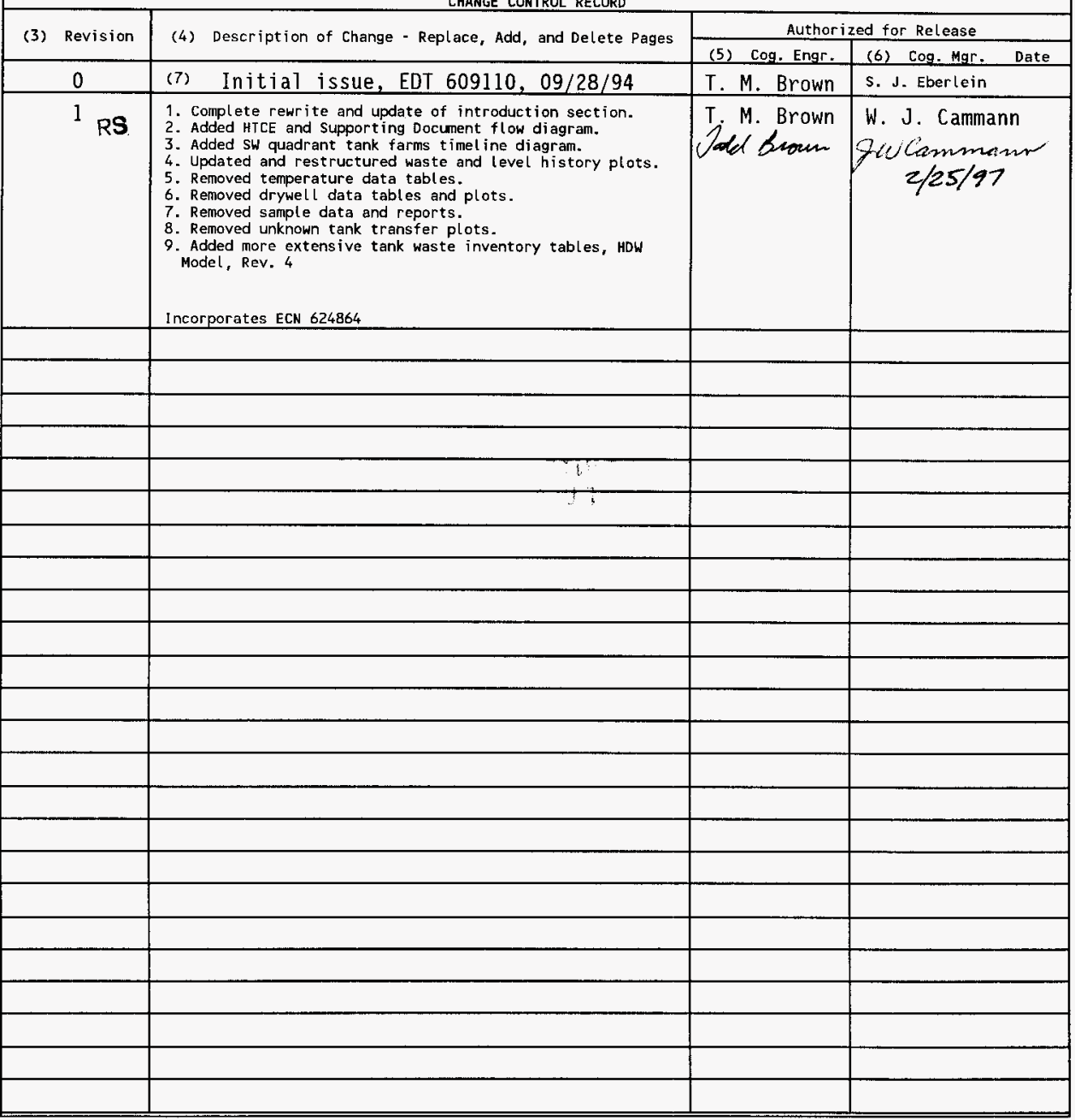


THIS PAGE INTENTIONALLY LEFT BLANK 


\title{
SUPPORTING DOCUMENT FOR THE \\ HISTORICAL TANK CONTENT ESTIMATE FOR
}

\section{S TANK FARM}

\author{
Prepared for \\ Lockheed Martin Hanford Corporation \\ January 1997
}

Prepared by

K. L. Ewer

J. W. Funk

R. G. Hale

G. A. Lisle

C. V. Salois

M. R. Umphrey

Fluor Daniel Northwest, Inc.

Richland, Washington 
THIS PACE INTENTIONALLY LEFT BLANK 


\section{SUPPORTING DOCUMENT FOR THE HISTORICAL TANK CONTENT ESTIMATE FOR}

\section{S TANK FARM}

WORK ORDER E18675

\section{APPROVED:}

Fluor Daniel Northwest, Inc. Qee Zimmerman

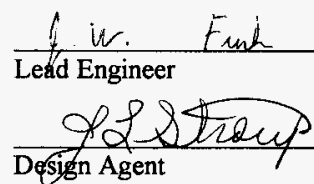

Chas It Brarich

Project Manager

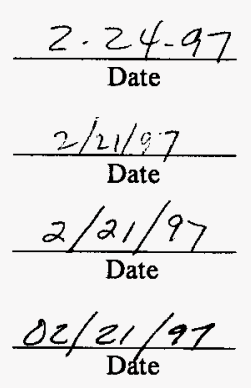

Lockheed Martin Hanford Corporation

$\frac{211}{\text { Technical Lead, TWRS DQO, Models and Inventory }}$ 
THIS PACT: INTENTIONALLY LEFT BLANK 


\section{ACKNOWLEDGMENTS}

A project of the this magnitude would not be possible without the help of a significant number of persons and organizations. Fluor Daniel Northwest, Inc., would like to acknowledge the contributions made by our Los Alamos National Laboratory counterparts: Stephen F. Agnew, Kenneth A. Jurgensen, Robert A. Corbin, Tomasita B. Duran, Bonnie L. Young, Theodore P. Ortiz, John FitzPatrick, and James Boyer. Also, Todd Brown, Brett Simpson and Jerry Cammann of Lockheed Martin Hanford Corporation are recognized for their contributions. 
THIS PAGE INTENTIONALLY LEFT BLANK 


\section{INFORMATION FEEDBACK CARD}

\section{SUPPORTING DOCUMENT FOR THE HISTORICAL TANK CONTENT ESTIMATE FOR S TANK FARM}

\section{COMMENTS AND CONTRIBUTIONS}

The reader is requested to use this card to comment on this document, report any discrepancies, or contribute new information to improve the accuracy and content of the document. Please use the space provided, add additional pages if necessary, and return the comments to the person noted at the address listed below.

Send Comments to: Mr. Jerry W. Cammann

Lockheed Martin Hanford Corporation

TWRS DQO, Models and Inventory

P.O. Box 1970, MSIN R2-12

Richland, WA 99352 
THIS F GE INTENTIONALLY LEFT BLANK 


\section{TABLE OF CONTENTS}

1.0 Introduction $\ldots \ldots \ldots \ldots \ldots \ldots \ldots \ldots \ldots \ldots \ldots \ldots \ldots \ldots \ldots \ldots$

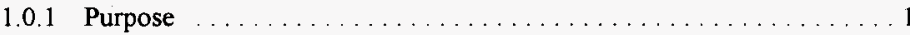

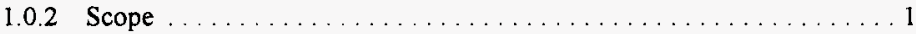

1.0 .3 Approach . . . . . . . . . . . . . . . . . . . 1

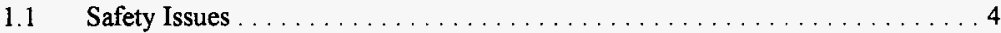

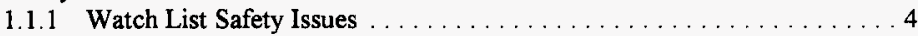

1.1 .2 Non-Watch List Safety Issues $\ldots \ldots \ldots \ldots \ldots \ldots \ldots \ldots \ldots$

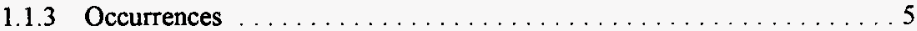

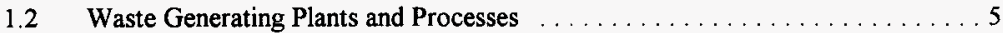

1.2.1 Plants Processes . . . . . . . . . . . . . . . . . . . 5

1.2 .2 Waste Management Operations . . . . . . . . . . . . . . . . 9

1.2.3 Miscellaneous Waste Sources and Equipment $\ldots \ldots \ldots \ldots \ldots \ldots$

1.2 .4 Time Lines . . . . . . . . . . . . . . . . . . . . . . . 11

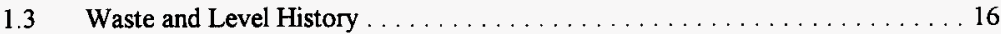

1.3.1 Source of Data . . . . . . . . . . . . . . . . . . . 16

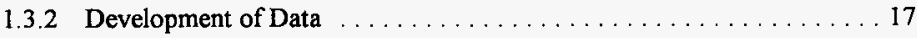

1.3 .3 Assumptions . . . . . . . . . . . . . . . . . . . . . 19

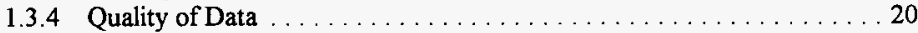

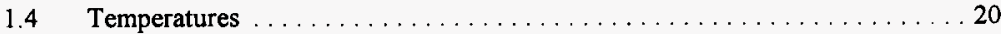

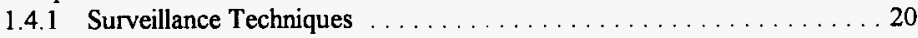

1.4 .2 Source of Data . . . . . . . . . . . . . . . . . . 20

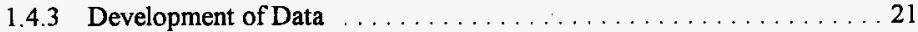

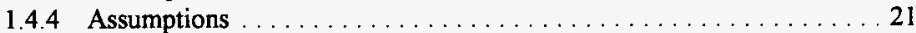

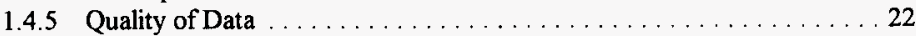

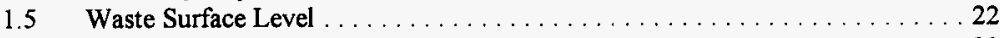

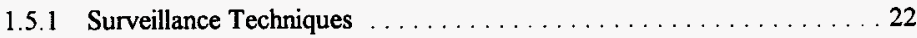

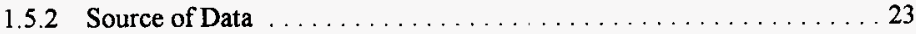

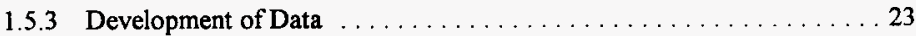

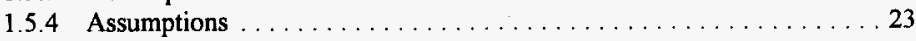

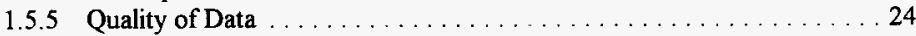

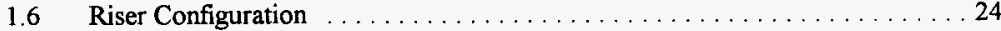

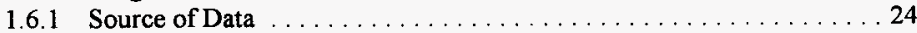

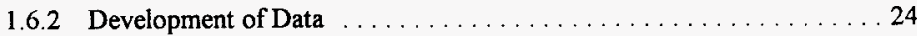

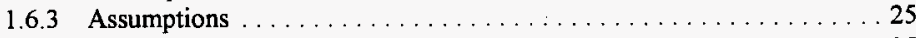

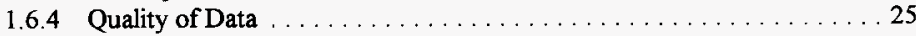

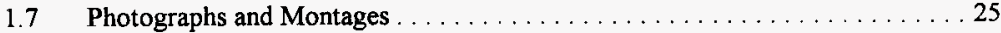

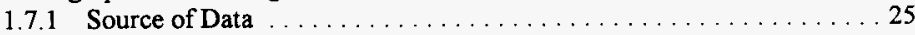

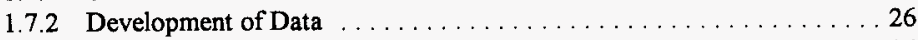

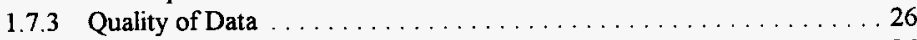

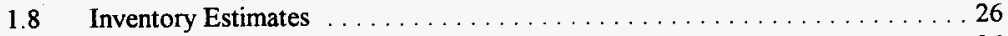

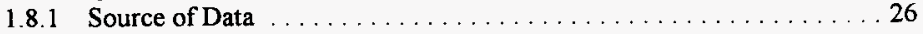

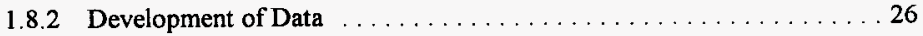




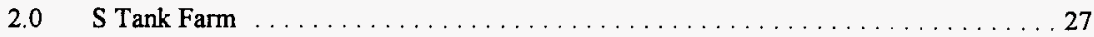

2.0 .1 S Tank Farm Information $\ldots \ldots \ldots \ldots \ldots \ldots \ldots \ldots \ldots, 27$

2.0 .2 S Tank Farm Waste and Level History $\ldots \ldots \ldots \ldots \ldots \ldots \ldots \ldots$

2.0 .3 S Tank Farm Temperature History $\ldots \ldots \ldots \ldots \ldots \ldots \ldots \ldots \ldots$

2.0.4 S Tank Farm Occurrences . . . . . . . . . . . . . . . . . . . . 27

2.0 .5 S Tank Farm Current Status . . . . . . . . . . . . . . . 27

2.0.6 S Tank Farm Photograph and Montages ............... 28

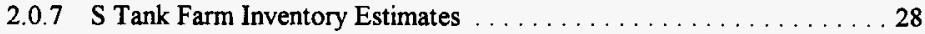

2.1 Tank 241-S-101 ... . . . . . . . . . . . . . . . . . . . . 29

2.1.1 Waste and Level History of Tank $241-\mathrm{S}-101 \ldots \ldots \ldots \ldots$

2.1.2 Temperature History of Tank $241-\mathrm{S}-101 \ldots \ldots \ldots \ldots \ldots$

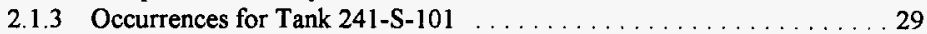

2.1.4 Current Status of Tank 241-S-101 . . . . . . . . . . . . . . . . . 29

2.1.5 Interior Montage of Tank $241-\mathrm{S}-101 \ldots \ldots \ldots \ldots \ldots \ldots$

2.1.6 Inventory Estimate of Tank $241-\mathrm{S}-101 \ldots \ldots \ldots \ldots \ldots \ldots \ldots$

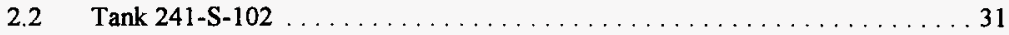

2.2.1 Waste and Level History of Tank $241-S-102 \ldots \ldots \ldots \ldots \ldots \ldots \ldots 31$

2.2.2 Temperature History of Tank $241-\mathrm{S}-102 \ldots \ldots \ldots \ldots \ldots \ldots \ldots \ldots$

2.2.3 Occurrences for Tank $241-S-102 \ldots \ldots \ldots \ldots \ldots \ldots \ldots \ldots \ldots \ldots$

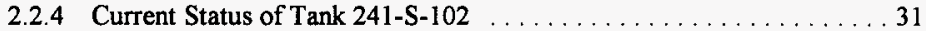

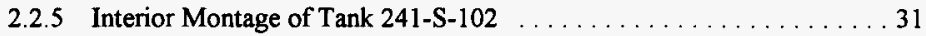

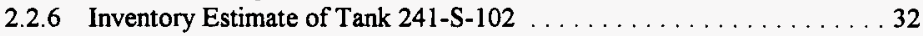

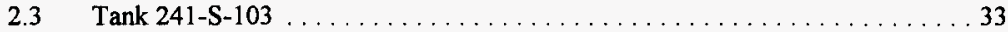

2.3.1 Waste and Level History of Tank $241-\mathrm{S}-103 \ldots \ldots \ldots \ldots \ldots \ldots$

2.3.2 Temperature History of Tank $241-\mathrm{S}-103 \ldots \ldots \ldots \ldots \ldots \ldots \ldots$

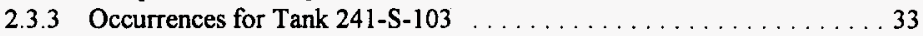

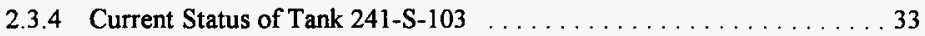

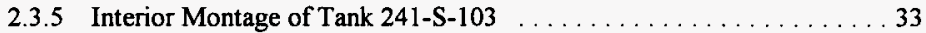

2.3.6 Inventory Estimate of Tank $241-S-103 \ldots \ldots \ldots \ldots \ldots \ldots$

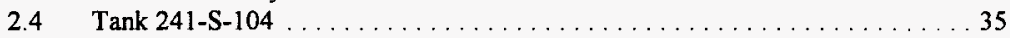

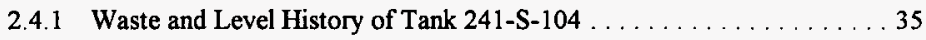

2.4.2 Temperature History of Tank $241-\mathrm{S}-104 \ldots \ldots \ldots \ldots \ldots \ldots \ldots \ldots$

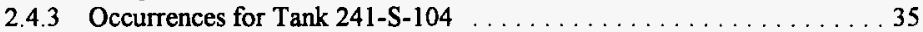

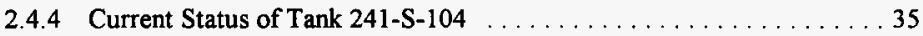

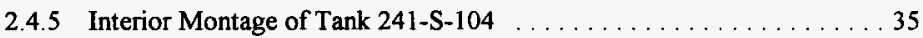

2.4 .6 Inventory Estimate of Tank $241-S-104 \ldots \ldots \ldots \ldots \ldots \ldots \ldots \ldots$

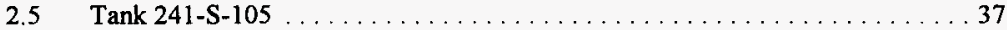

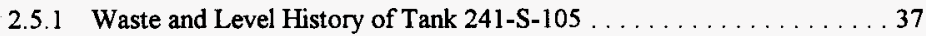

2.5.2 Temperature History of Tank $241-\mathrm{S}-105 \ldots \ldots \ldots \ldots \ldots \ldots \ldots \ldots$

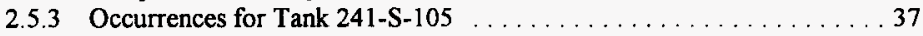

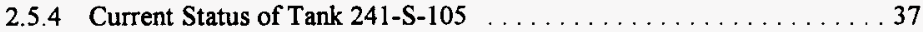

2.5.5 Interior Montage of Tank $241-\mathrm{S}-105 \ldots \ldots \ldots \ldots \ldots \ldots \ldots \ldots$

2.5.6 Inventory Estimate of Tank $241-\mathrm{S}-105 \ldots \ldots \ldots \ldots \ldots \ldots$

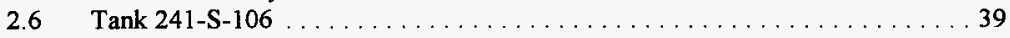

2.6.1 Waste and Level History of Tank $241-\mathrm{S}-106 \ldots \ldots \ldots \ldots \ldots \ldots$

2.6.2 Temperature History of Tank $241-\mathrm{S}-106 \ldots \ldots \ldots \ldots \ldots \ldots$

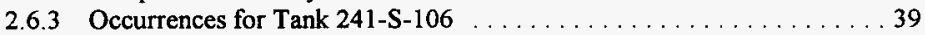




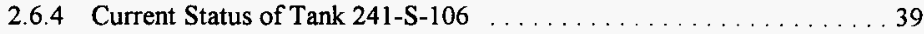

2.6 .5 Interior Montage of Tank $241-\mathrm{S}-106 \ldots \ldots \ldots \ldots$

2.6.6 Inventory Estimate of Tank $241-S-106 \ldots \ldots \ldots \ldots \ldots \ldots \ldots$

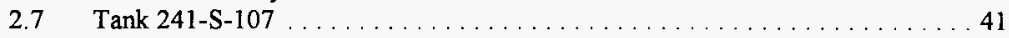

2.7.1 Waste and Level History of Tank $241-\mathrm{S}-107 \ldots \ldots \ldots \ldots \ldots . . .41$

2.7.2 Temperature History of Tank $241-\mathrm{S}-107 \ldots \ldots \ldots \ldots \ldots . . . . .41$

2.7.3 Occurrences for Tank $241-\mathrm{S}-107 \ldots \ldots \ldots \ldots \ldots \ldots \ldots \ldots \ldots$

2.7.4 Current Status of Tank $241-S-107 \ldots \ldots \ldots \ldots \ldots \ldots \ldots \ldots \ldots$

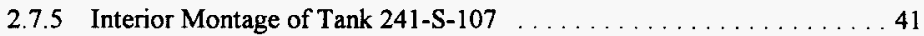

2.7.6 Inventory Estimate of Tank $241-\$-107 \ldots \ldots \ldots \ldots \ldots \ldots \ldots 2$

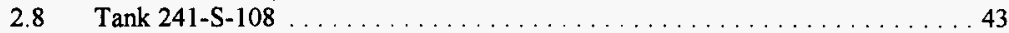

2.8.1 Waste and Level History of Tank $241-\mathrm{S}-108 \ldots \ldots \ldots \ldots \ldots \ldots . . . .43$

2.8.2 Temperature History of Tank $241-\mathrm{S}-108 \ldots \ldots \ldots \ldots \ldots \ldots \ldots$

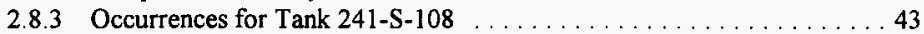

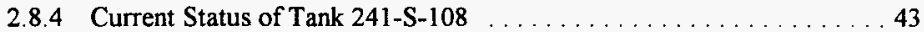

2.8.5 Interior Montage of Tank $241-\mathrm{S}-108 \ldots \ldots \ldots \ldots \ldots \ldots \ldots$

2.8.6 Inventory Estimate of Tank $241-\mathrm{S}-108 \ldots \ldots \ldots \ldots \ldots \ldots . \ldots 4$

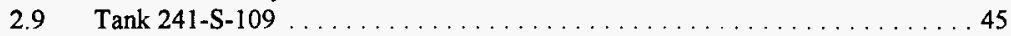

2.9.1 Waste and Level History of Tank $241-\mathrm{S}-109 \ldots \ldots \ldots \ldots \ldots .45$

2.9.2 Temperature History of Tank $241-\mathrm{S}-109 \ldots \ldots \ldots \ldots \ldots \ldots \ldots . \ldots \ldots$

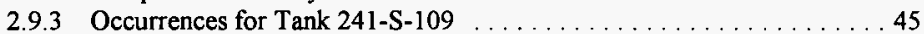

2.9.4 Current Status of Tank 241-S-109 . . . . . . . . . . . . . . . . . . . 45

2.9 .5 Interior Montage of Tank $241-\mathrm{S}-109 \ldots \ldots \ldots \ldots \ldots \ldots \ldots$

2.9.6 Inventory Estimate of Tank $241-\mathrm{S}-109 \ldots \ldots \ldots \ldots \ldots \ldots \ldots$

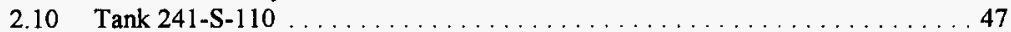

2.10.1 Waste and Level History of Tank $241-S-110 \ldots \ldots \ldots \ldots \ldots .47$

2.10.2 Temperature History of Tank $241-\mathrm{S}-110 \ldots \ldots \ldots \ldots \ldots \ldots \ldots 4$

2.10 .3 Occurrences for Tank $241-\mathrm{S}-110 \ldots \ldots \ldots \ldots \ldots \ldots \ldots \ldots$

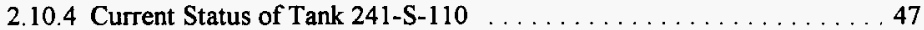

2.10.5 Interior Montage of Tank $241-\mathrm{S}-110 \ldots \ldots \ldots \ldots \ldots \ldots \ldots, 47$

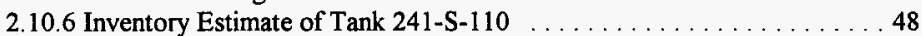

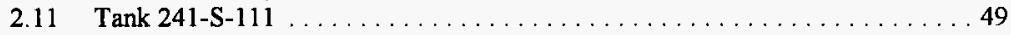

2.11.1 Waste and Level History of Tank $241-S-111 \ldots \ldots \ldots \ldots \ldots .49$

2.11.2 Temperature History of Tank $241-\mathrm{S}-111 \ldots \ldots \ldots \ldots \ldots \ldots$

2.11.3 Occurrences for Tank $241-\mathrm{S}-111 \ldots \ldots \ldots \ldots \ldots$

2.11.4 Current Status of Tank 241-S-111 . . . . . . . . . . . . . . 49

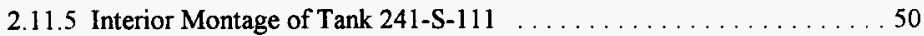

2.11.6 Inventory Estimate of Tank $241-\mathrm{S}-111 \ldots \ldots \ldots \ldots \ldots \ldots \ldots$

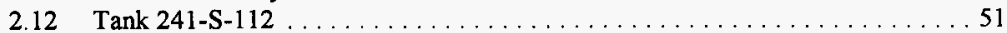

2.12.1 Waste and Level History of Tank $241-S-112 \ldots \ldots \ldots \ldots \ldots \ldots . \ldots 1$

2.12 .2 Temperature History of Tank $241-\mathrm{S}-112 \ldots \ldots \ldots \ldots \ldots \ldots \ldots .51$

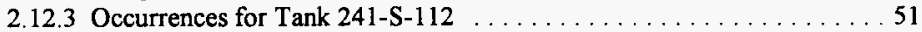

2.12.4 Current Status of Tank $241-S-112 \ldots \ldots \ldots \ldots \ldots \ldots \ldots \ldots \ldots$

2.12.5 Interior Montage of Tank $241-\mathrm{S}-112 \ldots \ldots \ldots \ldots \ldots \ldots \ldots \ldots \ldots$

2.12.6 Inventory Estimate of Tank $241-\mathrm{S}-112 \ldots \ldots \ldots \ldots \ldots \ldots \ldots \ldots \ldots \ldots$ 


\section{APPENDICES}

Appendix A Glossary

Appendix B References

Appendix C Waste and Level History Sketches and Data

Appendix D Temperature Graphs

Appendix E Waste Surface Level Graphs

Appendix F Riser Configuration Sketches and Tables

Appendix $G$ Tank Farm Photograph and Tank Montages

Appendix $\mathrm{H}$ Inventory Estimates 


\section{TRADEMARKS}

Microsoft Excel is a registered trademark of Microsoft Corporation.

ENRAF is a registered trademark of Delft Instruments.

AutoCAD is a registered trademark of Autodesk, Inc. 
THIS PA P ' MITT' 'TIONALLY LEH I bLAiNK 
1 
T!" P PAGE INTENTIONALLY LEFT BLANK 


\subsection{Introduction}

\subsubsection{Purpose}

The purpose of this historical characterization document is to present the synthesized summaries of the historical records concerning the physical characteristics, and radiological, and chemical composition of mixed wastes stored in underground single-shell tanks and the physical conditions of these tanks. The single-shell tanks are located on the United States Department of Energy's Hanford Site, approximately 25 miles northwest of Richland, Washington. The document will be used to assist in characterizing the waste in the tanks in conjunction with the current program of sampling and analyzing the tank wastes. Los Alamos National Laboratory (LANL) developed computer models that used the historical data to attempt to characterize the wastes and to generate estimates of each tank's inventory. A historical review of the tanks may reveal anomalies or unusual contents that could be critical to characterization and post characterization activities.

This document was developed by reviewing the operating plant process histories, waste transfer data, and available physical and chemical data from numerous resources. These resources were generated by numerous contractors from 1945 to the present.

Waste characterization, the process of describing the character or quality of a waste, is required by Federal law (Resource Conservation and Recovery Act [RCRA]) and state law (Washington Administrative Code [WAC] 173-303, Dangerous Waste Regulations). Characterizing the waste is necessary to determine methods to safely retrieve, transport, and/or treat the wastes.

This document is not intended for use as a total design basis document. Further investigations of the information may be required before using this data for design purposes or safety analysis.

\subsubsection{Scope}

The scope of this document covers available information about the wastes contained in the single-shell tanks in the S Tank Farm. Waste transfer and level data, tank physical information, and surveillance data of tanks and wastes have been compiled for this document. The inventory estimates of waste types and volumes generated by the computer modeling programs developed by LANL are included also. A summary of this information is contained in the Historical Tank Content Estimate (HTCE) for the Southwest Quadrant of the Hanford 200 West Area (Brevick et al., 1997c). The Southwest Quadrant (SW Quadrant) document covers three single-shell tank farms. These three tank farms, S, SX, and U, are located in the 200 West Area and are shown on the map in Figure 1 . A flow diagram showing the relationships between the sources of data, the HTCE, and the supporting documents is in Figure 2.

This document also includes information on the safety issues affecting the tanks and the plants and processes that produced the waste in the underground waste storage tanks.

\subsubsection{Approach}

This document was compiled from work performed by Fluor Daniel Northwest, Inc. (FDNW), LANL, and Lockheed Martin Hanford Corporation (LMHC), as well as work performed by 


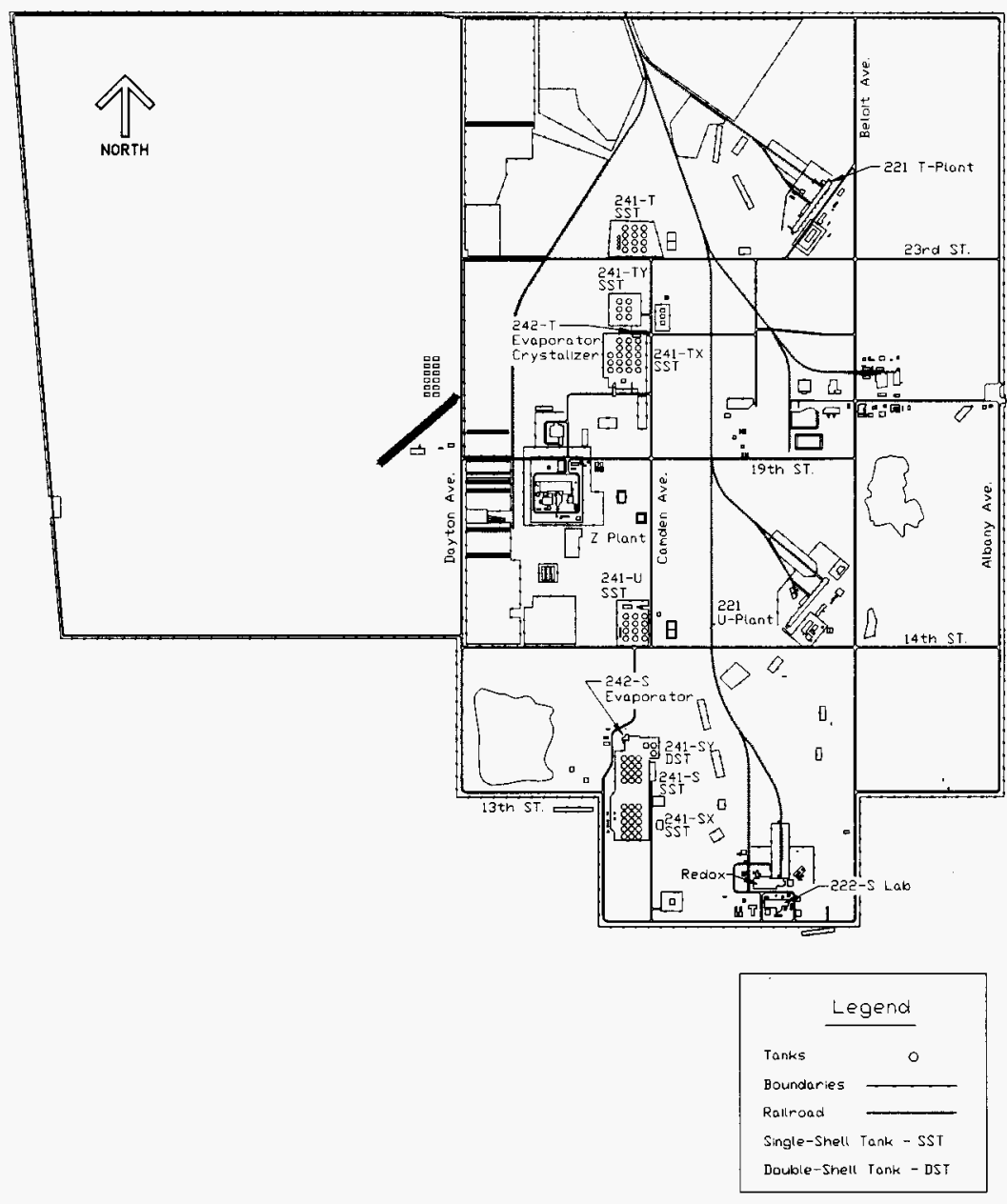

Figure 1. 200 West Area. 


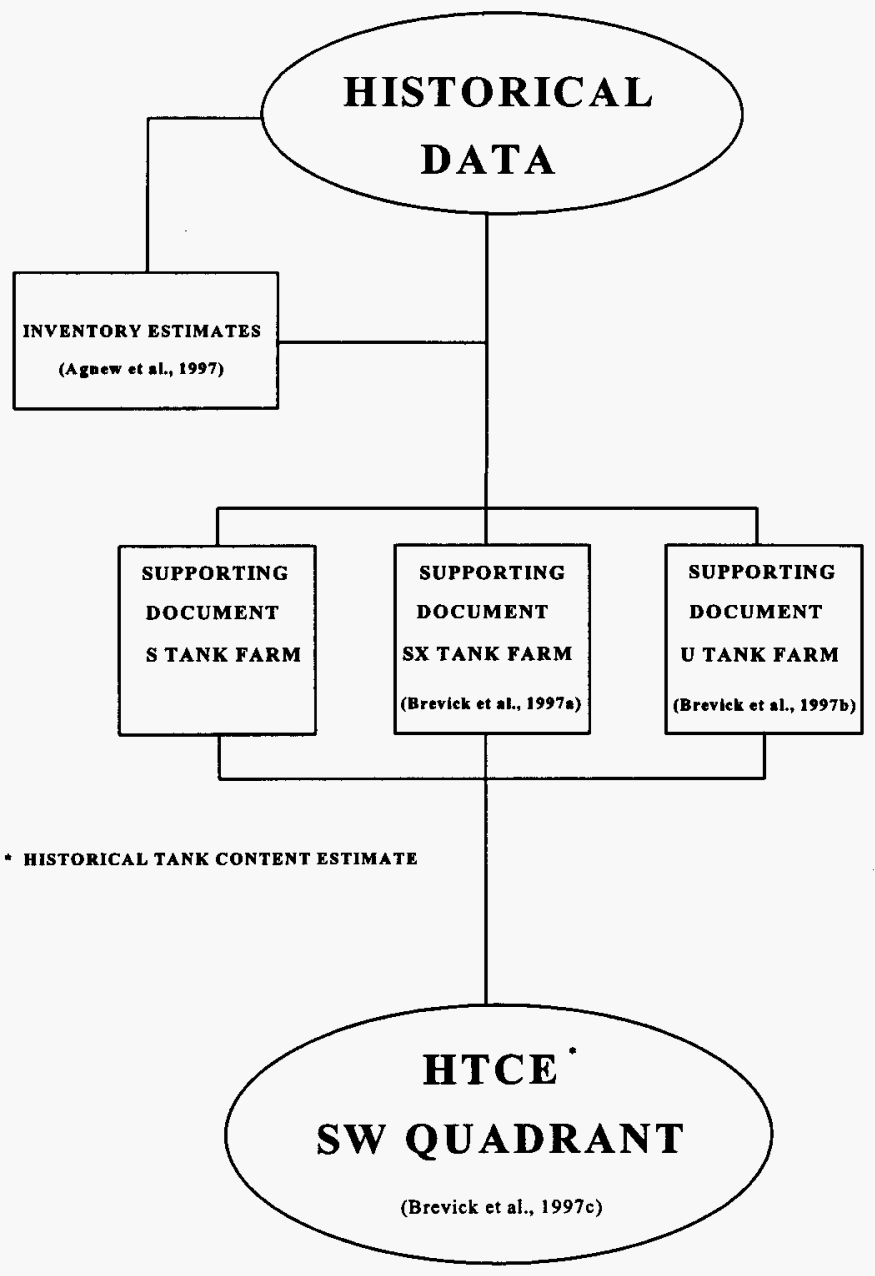

Figure 2. HTCE and Supporting Document Flow Diagram. 
Westinghouse Hanford. FDNW reviewed the historical records of the tanks and incorporated the inventory estimates and models of waste layers in the tanks being developed by LANL into this document.

\subsection{Safety Issues}

The safety issues that affect the tanks can be divided into two groups: watch list and non-watch list. The watch lists are listings of tanks believed to pose potential safety hazards to workers, the environment, and the public. Non-watch list issues are of concern because of their possible effect on workers and the environment. Occurrences are unusual events on the Hanford Site that sometimes are related to safety issues.

\subsubsection{Watch List Safety Issues}

Watch list safety issues for these tanks were identified as "issues/situations that contain most of the necessary conditions that could lead to worker (onsite) or offsite radiation exposure through an uncontrolled release of fission products" under Public Law 101-510, Section 3137, of the National Defense Authorization Act of Fiscal Year 1991 (i.e., the Wyden Amendment). As of June 30, 1996, 44 single-shell tanks and 6 double-shell tanks are on watch lists (Hanlon, 1996i). See the Approach for Tank Safety Characterization of Hanford Site Waste (Eberlein et al., 1995) for more information on the watch list issues.

\subsubsection{Non-Watch List Safety Issues}

Non-watch list issues include safety hazards such as leaking tanks. Tank leaks are a safety hazard because of their potential to release chemicals and radioactive liquids into the ground. Corrosion is the main cause of tank leaks. Three other safety issues that do not require a watch list and continual monitoring under the Wyden Amendment include criticality, tank bumps, and toxic vapor releases. The following sections provide a general description of the different non-watch list safety issues. See the Hanford Site Tank Farm Facilities Interim Safety Basis (Leach and Stahl, 1993) for more information.

\section{- Corrosion}

Corrosion is the most probable degradation mechanism of the steel tank liners resulting from contact with liquid, liquid-vapor, vapor, and solid phases of the wastes. The corrosion mechanisms that reduce the thickness of the carbon steel liners can be divided into two categories: localized and general or uniform. Localized corrosion occurs on a localized area of the liner surface. Some of the localized corrosion mechanisms include pitting corrosion, stress corrosion cracking, and crevice corrosion. General or uniform corrosion occurs over the entire liner surface. Corrosion of the steel tank liners may involve more than one of these mentioned mechanisms. Corrosion is a safety issue because it has the potential to degrade the tank liner to the point of causing a leak or, more seriously, structural failure of the tank. Either condition could release contamination to the environment.

\section{Criticality}

Criticality is a self-sustained, nuclear chain reaction that can occur when a sufficient mass of fissile material is present in the proper configuration along with a neutron source to start the nuclear reaction. Criticality in the tank farms has been declared an unreviewed safety question, even though 
the Hanford Site Tank Farm Facilities Interim Safety Basis (Leach and Stahl, 1993) indicates that a "nuclear criticality accident in the tank farms is probably not an imminent risk." The unreviewed safety question on criticality in the tank farms remains because the inventory of fissile material and its distribution within the tanks cannot be confirmed as being within the approved safety envelope defined in the current safety analysis reports. Criticality is a safety issue because of the potential to release contamination to the environment.

\section{- Tank Bumps}

A tank bump is the sudden pressurization of the tank. This phenomenon occurs when solids overheat in the lower portion of the tank followed by uncontrolled mixing of these solids. The stirred hot solids rapidly transfer heat to the liquid in the tank, some of which quickly vaporizes. The rapid vapor generation causes a sudden internal tank pressurization that causes a bump. Uncontrolled mixing of heated solids can occur when an airlift circulator fails allowing the solids to heat up followed by rapid startup of the airlift circulator which causes rapid mixing. Uncontrolled mixing can also occur when a natural "rollover" of waste occurs in the tank. Tank bumps are a safety issue because of their potential to release contamination to the environment.

\section{- Toxic Vapor Releases}

Toxic vapor releases are a recently analyzed safety concern at the Hanford Site. The entire issue of toxic gas releases at the tank farms is being investigated (Leach and Stahl, 1993).

\subsubsection{Occurrences}

Over the years, unusual events (occurrences) have occurred at the S Tank Farm. An occurrence is an event that falls outside the normal operating, maintenance and/or construction procedures of the tank farm. Occurrences have been documented by various reporting methods including unusual occurrences reports, off-normal reports, event fact sheets, and occurrence reports. Before it was included in this document, the occurrence documentation that could be located was evaluated for its significance in determining the waste content of the tanks. The types of significant occurrences included are those involving surface level changes, temperature changes, and radioactivity changes (activity in the drywells). This document does not contain complete information from the reports, only summaries of the events. For more information on occurrences, refer to the Occurrences Section for the specific tank.

\subsection{Waste Generating Plants and Processes}

\subsubsection{Plants Processes}

Brief descriptions and histories of the plants and processes that generated waste now contained in the single-shell and double-shell tanks are presented in alphabetical order. Typically, the name of the plant and the process are synonymous. The dates and events described in the following brief histories are presented on time lines in Figures 3 and 4. Although not all of the processes listed below contributed waste directly to tanks in the Southwest Quadrant, the waste they generated could have been transferred indirectly from tank to tank. 


\section{- A Plant (PUREX)}

The Plutonium Uranium Extraction (PUREX) plant (i.e., A Plant) began operating in January 1956 (Gerber, 1993a). "The PUREX process is an advanced solvent extraction process that uses a tributyl phosphate in kerosene solvent for recovering uranium and plutonium from nitric acid solutions of irradiated uranium. Nitric acid is used instead of metallic nitrates to promote the extraction of uranium and plutonium from aqueous phase to an organic phase." (Wilson and Reep, 1991, p. B-4). Two campaigns of the Thorex process were conducted in 1966 and 1971 (Jungfleisch, 1984). The Thorex process recovered ${ }^{233} \mathrm{U}$ from thorium irradiated in the Hanford Site reactors (Wilson and Reep, 1991). PUREX reprocessed aluminum-clad fuel elements and zirconium alloy-clad fuel elements, and provided plutonium for research reactor development, safety programs, and defense. Also, PUREX recovered slightly enriched uranium to be recycled as fuel in reactors generating electricity and plutonium (Rockwell, 1985). PUREX was put on standby in 1972 (Gerber, 1993a).

The PUREX plant was restarted in November 1983 but was shut down in December 1988 (see Figure 3). The plant was shut down due to the lack of steam pressure needed to operate the support backup safety equipment. There was a brief stabilization run in early 1990. In October 1990, PUREX was placed on standby by Secretary of Energy James Watkins. DOE issued the final closure order in December 1992 (Gerber, 1993b).

\section{- B Plant}

B Plant used the bismuth phosphate process at first, and later changed its processing capabilities to strontium and cesium fractionation. The bismuth phosphate process "separated plutonium from uranium and the bulk of fission products in irradiated fuel by co-precipitation with bismuth phosphate from a uranium nitrate solution. The plutonium was then separated from fission products by successive precipitation cycles using bismuth phosphate and lanthanum fluoride. The plutonium was isolated as a peroxide and, after dissolving in nitric acid, was concentrated as plutonium nitrate. The waste containing the uranium from which the plutonium had been separated, was made alkaline (neutralized) and stored in underground single-shell tanks. Other acid waste (which included most of the fission products) generated by this process was neutralized and stored in other single-shell tanks" (Wilson and Reep, 1991, p. B-3). "Some of the strontium and cesium fission products were removed (fractionated) from the waste and separately isolated to reduce the heat generation in the tanks. B Plant . . was modified in 1968 to permit removal of these fission products by a combination of precipitation, solvent extraction, and ion-exchange steps. The residual acid waste from the processing was neutralized and stored in single-shell tanks" (Wilson and Reep, 1991, pp. B-4 and B-5).

B Plant began its first batch run on April 13, 1945 (Anderson, 1990), and was shutdown in 1952 (Gerber, 1993b) (see Figure 3). Shortly after the renovations to B Plant were completed in December 1955, the 4X Program was abandoned. The 4X Program "planned to utilize the capabilities of all four Hanford processing plants (B, T, REDOX, and PUREX)" (Gerber, 1993b, p. 12); however, the large production and economic efficiency of the PUREX plant caused the $4 X$ Program to be abandoned (Gerber, 1993b). B Plant restarted in 1968 to recover cesium and strontium from stored liquid waste. Cesium and strontium recovery was completed in September 1983 and February 1985, respectively (Rockwell, 1985).

\section{5-B (WESF)}

The Waste Encapsulation and Storage Facility (WESF) converted solutions of cesium and strontium nitrates recovered in B Plant to strontium fluoride and cesium chloride solids that are doubly 
encapsulated in metal (Ballinger and Hall, 1991). "Strontium and cesium capsules have been used in applications of fission byproducts for gamma and heat sources" (Wilson and Reep, 1991, p. B-5).

WESF was constructed in 1974 (see Figure 3). The process optimization for cesium and strontium was completed in 1978 and 1981, respectively (Rockwell, 1985). The cesium processing ended in 1983 and strontium encapsulation in 1985. The capsule return program started in 1988 and ended in 1995 (Gerber, 1996).

\section{- C Plant (Strontium Semiworks)}

The Strontium or Hot Semiworks Facility (i.e., C Plant) began operating in 1952 as a hot pilot plant for the REDOX process (see Figure 3). In 1954, the plant was converted to a pilot plant for the PUREX process and continued operating until 1956 (Ballinger and Hall, 1991). "The process building (201-C) contains three hot cells equipped only for contact maintenance and is supported by an aqueous makeup and control building (271-C) and a solvent handling building (276-C). The facility also includes a fiberglass exhaust filter and a 200-ft stack." (PNL, 1991, Vol. 1, p. 3.6). In 1960, the plant was reactivated as a pilot plant used to recover ${ }^{90} \mathrm{Sr},{ }^{147} \mathrm{Pm}$, and ${ }^{144} \mathrm{Cs}$ from PUREX waste. The plant was shut down in 1967 and the building and the site have been decontaminated and decommissioned (PNL, 1991).

\section{S Plant (REDOX)}

The Reduction and Oxidation extraction (REDOX) plant (i.e., S Plant) began processing on January 9, 1952 (Anderson, 1990) (see Figure 3). "The REDOX extraction process was a secondgeneration recovery process and the first process to recover both plutonium and uranium. It used a continuous solvent extraction process to extract plutonium and uranium from dissolved fuel into a methyl isobutyl ketone (hexone) solvent. The slightly acidic wastestream contained the fission products and large quantities of aluminum nitrate that were used to promote the extraction of plutonium and uranium. This waste was neutralized and stored in single-shell tanks. The volume of high-level waste from this process was much smaller than that from the bismuth phosphate process, but larger than that from the PUREX process" (Wilson and Reep, 1991, pp. B-3 and B-4). REDOX operated until 1967 (Rockwell, 1985).

\section{- T Plant}

T Plant was the first full-scale separations plant at the Hanford Site. T Plant used the bismuth phosphate process to separate plutonium from uranium and the bulk of fission products in irradiated fuel (B Plant used the same process). "The waste containing the uranium from which the plutonium had been separated was made alkaline (neutralized) and stored in underground single-shell tanks. Other acid waste (which included most of the fission products) generated by this process was neutralized and stored in other single-shell tanks" (Wilson and Reep, 1991, p. B-3).

T Plant began operating in 1944 (Rockwell, 1985) as a separations plant and continued until March 1956 (Gerber, 1994) (see Figure 4). T Plant's mission was changed in 1957 to the repair and high-level decontamination of equipment (Rockwell, 1985). T Plant was converted to a "central decontamination facility for the site. As such, failed and contaminated equipment was assessed and either repaired or discarded there for over three decades" (Gerber, 1994, p. 1). Early decontamination operations used steam, sand, chemicals, and detergents. "Smaller equipment pieces were immersed in decontamination solutions in 'thimble tanks,' and larger pieces were flushed with water, chemical solutions, sand-blasted, steam-blasted, high-pressure sprayed (using pressures up to 10,000 pounds per 
square inch), and/or scrubbed with detergents. During the initial years, a strong nitric acid flush (approximately 60\%) usually began the decontamination process, followed by a caustic wash with sodium hydroxide combined with sodium phosphate, boric acid, versene, sodium dichromate, sodium tartrate, or sodium citrate. However, it was learned that versene and tartrate, in particular, adversely affected the ability of soil cribs to absorb the rinsate materials. High-pressure sprays often used $1,1,1$ trichloroethane or perchloroethylene, and detergents generally were chloride-based. By the mid-1960s, commercially prepared and trademarked chemical mixtures had replaced most of the simpler chemicals used in the early years. Many commercial products were based on oxalic acid, phosphates, nitric acidferrous ammonium sulfate combinations, potassium permanganate, and sodium bisulfate, with some unknown additives" (Gerber, 1994, pp. 40-42). The facility was modified in 1978 to store pressurized water reactor (PWR) core II fuel assemblies (Rockwell, 1985).

\section{- U Plant}

U Plant (221-U) was built as one of three original bismuth phosphate process facilities, but it was not used for that purpose. U Plant was modified extensively and used for the uranium recovery process, operating from 1952 to 1958 (see Figure 4). Uranium in waste from the bismuth phosphate process initially was stored in the single-shell tanks. Later, the waste was sluiced, dissolved in nitric acid, and processed through a solvent extraction process using tributyl phosphate in kerosene to recover the uranium. The process was similar to that used later in the plutonium-uranium extraction (PUREX) process except that plutonium was not recovered. The acid waste from the uranium recovery process was made alkaline and returned to single-shell tanks. The tributyl phosphate waste was treated with potassium ferrocyanide as a cesium and strontium scavenger. The recovery process resulted in an increase in nonradioactive salts and a small increase in waste volume (Wilson and Reep, 1991).

\section{4-U ( $\mathrm{UO}_{3}$, Uranium Trioxide Plant)}

The 224-U Building was converted to a uranium trioxide $\left(\mathrm{UO}_{3}\right)$ plant that began operating in 1952 (see Figure 4). The $\mathrm{UO}_{3}$ plant was capable of handling the uranyl nitrate hexahydrate (UNH) stream from REDOX, U Plant, and PUREX. "The basic $\mathrm{UO}_{3}$ process, calcining, consisted of concentrating and then heating liquid UNH until it converted to a stable, orange-yellow powder. The nitric acid in the UNH solution could be recovered in the same process. The $\mathrm{UO}_{3}$ powder was the base material needed for the manufacture of uranium hexafluoride $\left(\mathrm{UF}_{6}\right.$ ), the primary feed material for the United States' gaseous diffusion plants. Because the largest of these plants was located in Ohio and Tennessee, it was considered safer to ship the material across the country in powder rather than in liquid form" (Gerber, 1993b, pp. 33-34). The $\mathrm{UO}_{3}$ plant was shut down in 1972, but restarted in 1984. Since 1984, there have been 17 campaigns at the plant averaging 8 days each. Final deactivation of the plant was ordered in 1992. In April 1993, the $\mathrm{UO}_{3}$ plant resumed operations to convert 200,000 gallons of remaining $\mathrm{UNH}$ to $\mathrm{UO}_{3}$ powder. A final deactivation plan was written in the summer of 1993 (Gerber, 1993b).

\section{- Z Plant (PFP, Plutonium Finishing Plant)}

The Plutonium Finishing Plant (PFP) or Z Plant, previously called Plutonium Recovery and Finishing Operations, processed plutonium and prepared plutonium products. "Waste from this plant contained only minor amounts of fission products but did contain low concentration of plutonium and other transuranic elements and was high in metallic nitrates. Initially, this waste was discharged via cribs to soil columns, which absorbed the transuranic elements and retained them close to the point of discharge. Beginning in 1973, waste from PFP was stored with other waste in underground tanks" (Wilson and Reep, 1991, p. B-4). "Three types of feed materials are processed at the PFP to produce 
plutonium metal. Feed material types are handled differently in different process lines Historically, the main feed for the PFP was purified plutonium nitrate solution that was produced elsewhere in a fuel reprocessing plant. This feed was charged directly to one of the main process lines, which was initially a glovebox line. The glovebox line was replaced by remote mechanical lines, which were upgraded over the years. In time, processes were added to handle rework and scrap plutonium. These processes were used to convert the rework and scrap materials into a purified plutonium nitrate solution that could be handled by the main process" (Duncan and Mayancsik, 1993, pp. 2-1-2-2).

In July 1949, PFP began operations with a glovebox line (see Figure 4). The remote mechanical A line replaced the glovebox line in May 1953. Installment of the Recuplex Facility at PFP was completed in April 1955. The remote mechanical C line was installed in July 1960. In September 1961, the 232-Z Building had an incinerator and leaching equipment installed. In June 1964, the Plutonium Reclamation Facility (PRF) replaced the functions of the Recuplex Facility. Fabrication of plutonium metal nuclear weapon components ceased at the PFP in December 1965. In April 1973, the 232-Z Incinerator was shut down and the remote mechanical $\mathrm{C}$ line was placed on standby. The PRF was placed on standby in February 1979, and the remote mechanical A line was shutdown in December 1979. In January 1984, the PRF was restarted for a series of campaigns. The remote mechanical C line was restarted in June 1985 for a series of campaigns. In September 1986, operations at PFP were halted for nine months. This partial listing of the process history in the PFP is from Duncan et al. (1993).

\subsubsection{Waste Management Operations}

This section describes the different methods used to concentrate waste in the 200 Areas. Evaporating, and in-tank solidification are methods used to reduce the volumes of supernate. Brief descriptions and histories of the operations are presented in alphabetical order. The events and dates described in the brief histories are presented on a time line (Figure 5).

\section{- 242-A Evaporator-Crystallizer}

"The program objective was to reduce the volume of tanked waste liquors through the boiloff of water. This was accomplished by boiling the liquor in an enclosed vessel at reduced pressure. The evaporation was carried out until a slurry containing about $30 \mathrm{wt} \%$ solids was formed. The slurry was returned to underground waste tanks for cooling, crystallization, and settling. The principal products of waste solidification have been large volumes of sodium nitrate salt cakes and waste liquors that are rich in sodium hydroxide and sodium aluminate" (Wilson and Reep, 1991, p. B-5).

The 242-A Evaporator-Crystallizer began operating on March 18, 1977 (Anderson, 1990) (see Figure 5). In 1981, the evaporator was shut down for ten months to tie AW Tank Farm into the process (Rockwell, 1985). The evaporator was shut down in 1989 because of regulatory issues, but was restarted in 1994 after extensive modifications (Gerber, 1996).

\section{2-B Evaporator}

"The first type of waste solidification facility, the 242-B and 242-T Concentrators, was originally used for concentration of bismuth phosphate process waste. In 1951, they began to concentrate cladding/first cycle waste. These concentrators were steam-heated pot evaporators operated outside the waste tanks and at atmospheric pressure. The liquors were partially boiled down 
and cycled to underground waste storage tanks" (Jungfleisch, 1984, p. 1-5). This evaporator ran for approximately four years (Anderson, 1990) (see Figure 5)

\section{2-S Evaporator-Crystallizer}

The 242-S Evaporator-Crystallizer was designed to boil off water from the waste in an enclosed vessel at reduced pressure, similar to the 242-A Evaporator-Crystallizer. "The evaporation was carried out until a slurry containing about $30 \mathrm{wt} \%$ solids was formed. The slurry was returned to underground waste tanks for cooling, crystallization, and settling. The principal products of waste solidification have been large volumes of sodium nitrate salt cakes and waste liquors that are rich in sodium hydroxide and sodium aluminate" (Wilson and Reep, 1991, p. B-5). The evaporator began operating on November 1, 1973 (Anderson, 1990) and was shut down in 1981 (Gerber, 1996) (see Figure 5).

242-T Evaporator

The 242-T Evaporator, like the 242-B Evaporator, began operating in 1951 (Gerber, 1992) to reclaim nonboiling waste storage capacity in existing tanks (see Figure 5). The evaporator was shut down in the summer of 1955 and modified for tributyl phosphate scavenging (Godfrey, 1965), although scavenging was never performed in this evaporator. The evaporator was restarted on December 3, 1965, and operated until April 15, 1976 (Anderson, 1990).

\section{In-Tank Solidification}

The in-tank solidification systems immobilized high level wastes, that were not self-boiling, by concentrating the waste directly inside the tanks to form radionuclide-bearing salt cakes (Shefcik, 1964). The first in-tank solidification unit (ITS-1) and the second in-tank solidification unit (ITS-2) operated in tanks in the BY Tank Farm (Caudill, 1965 and 1967). "...[O]ne used a hot air sparge (ITS-1) and the other used an immersed electrical heater (ITS-2). The ITS-1 operations were conducted in individual tanks. The ITS- 2 concentrations were performed by heating the contents of one tank and moving the heated liquor through a series of other tanks" (Wilson and Reep, 1991, p. B-5).

ITS units 1 and 2 began operating on March 19, 1965, and February 17, 1968, respectively (see Figure 5). ITS-1 was converted to a cooler for ITS-2 on August 24, 1971. Both units were shut down on June 30, 1974 (Anderson, 1990).

\subsubsection{Miscellaneous Waste Sources and Equipment}

Wastes from various other sources on the Hanford Site have been added to the tanks. Some wastes are from the 300 Area, the 100 Area production reactors, various laboratories, and catch tanks.

\section{- Critical Mass Laboratories}

The critical mass laboratories were used to study the physics of plutonium solutions and solids to avoid accidently creating a criticality or self-sustained nuclear reaction. The first facility began operating in the 120 Building near $100 \mathrm{~F}$ in April 1950 and closed in December 1951. The second facility, the 209-E Building, was located next to the Strontium Semiworks and began operating in July 1961 (Ballinger and Hall, 1991). The plutonium used in the lab was reprocessed in PUREX.

\section{4-AR, -BXR, and -CR Process Vaults}

Three of the process vaults are the 244-AR Vault, the 244-BXR Vault, and the 244-CR Vault. These vaults were composed of several process vessels or tanks used to prepare waste for treatment or 
storage. Specific wastes from tanks can be pumped temporarily to the vaults and later sent directly to desired tanks or processing facilities.

The AR Vault, located north and west of the A Tank Farm, was constructed in 1966. The vault facilities include a canyon building with process cells containing tanks. The AR Vault has been on standby since 1978 (Leach and Stahl, 1993).

The 244-BXR Vault, located south of the BX Tank Farm began, operating in 1952 (Rodenhizer, 1987) and became inactive in 1956. The waste in the vault was difficult to handle so the vault was jetted with high-pressure steam in 1976. The 244-BXR Vault was used to process sludge in the recovery of uranium from bismuth phosphate metal waste in the tanks (Rodenhizer, 1987).

The 244-CR Vault, constructed in 1952, is located south of the C Tank Farm (Leach and Stahl, 1993). Salt-well waste from the C Tank Farm is interimly stored in the CR Vault. The 244-CR Vault was used to process sludge in the recovery of uranium from bismuth phosphate metal waste in the tanks (Rodenhizer, 1987).

\section{4-AR and 204-S Railroad Car Facilities}

The 204-AR rail car unloading facility built in 1981 (Leach and Stahl, 1993), replaced the 204-S rail car unloading facility. The facilities were built for pumping liquid radioactive waste from tank cars and sending the waste to 200 East Area tank farms (Leach and Stahl, 1993).

\subsubsection{Time Lines}

Time lines presented on the following pages represent many of the events that occurred during the history of the major plants and waste management operations on the Hanford Site. These are the same events as those described in the description of each facility. The plants, associated processes, and methods for managing waste are the main sources of the wastes stored in the tanks. Abbreviations are defined in the preceding text and in the glossary in Appendix A.

One time line represents the history of each of the tank farms in the Southwest Quadrant of the 200 West Area (Figure 6). The events represented include the dates of construction, the individual tank's entry into service and removal from service, and the deactivation of each tank farm. 
THIS F.' 'TTITIONALLY

LL-1 i BLANK 


\section{PLANTS / PROCESS - TIME LINE}

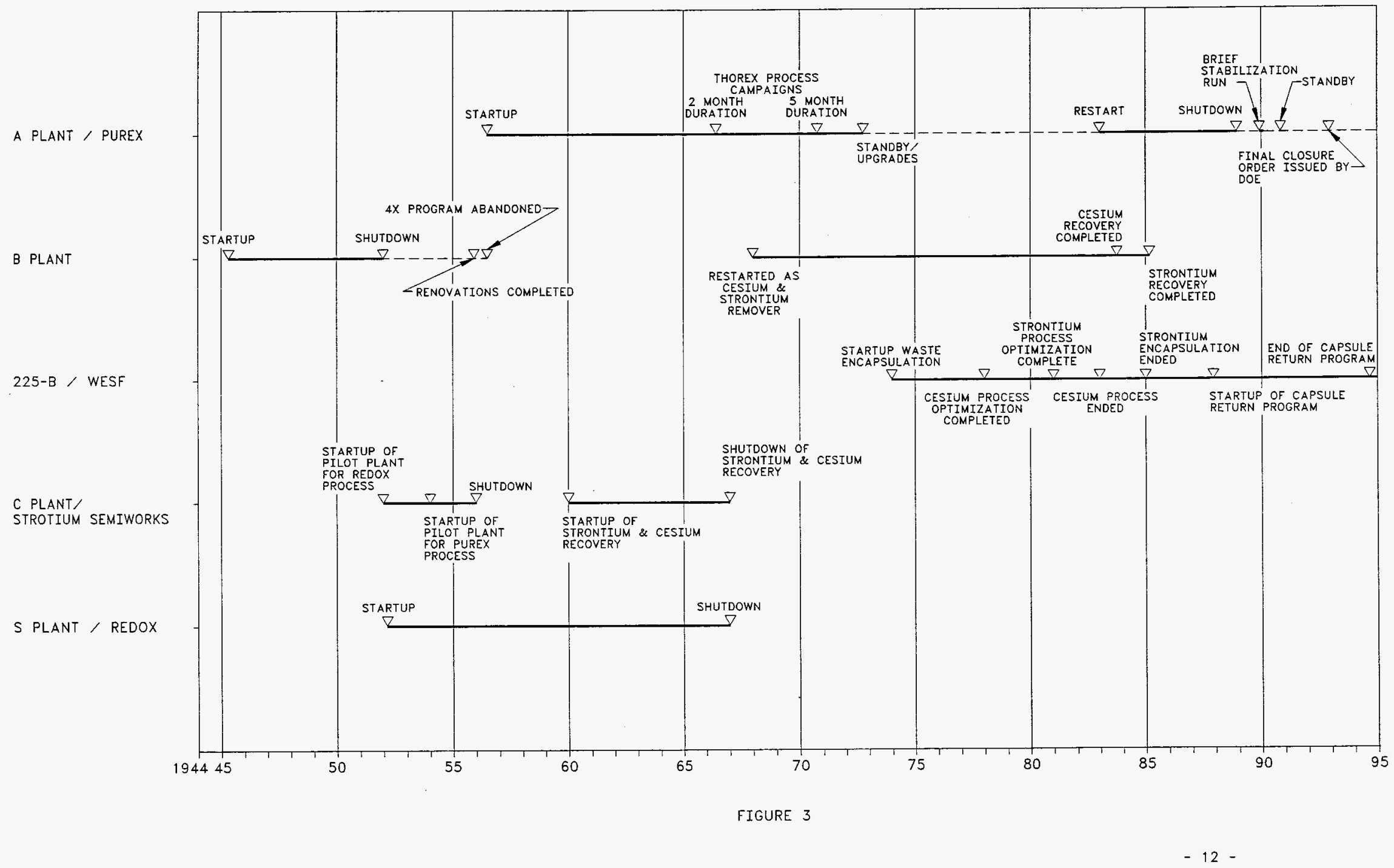


THIS PAGE INTENTIONALLY LEFT BLANK 


\section{PLANTS / PROCESS - TIME LINE}

T PLANT
U PLANT
224-U
(U0, URANIUM
TRIOXIDE PLANT)
Z PLANT /
(PFP PLUTONIUM
FINISHING PLANT)

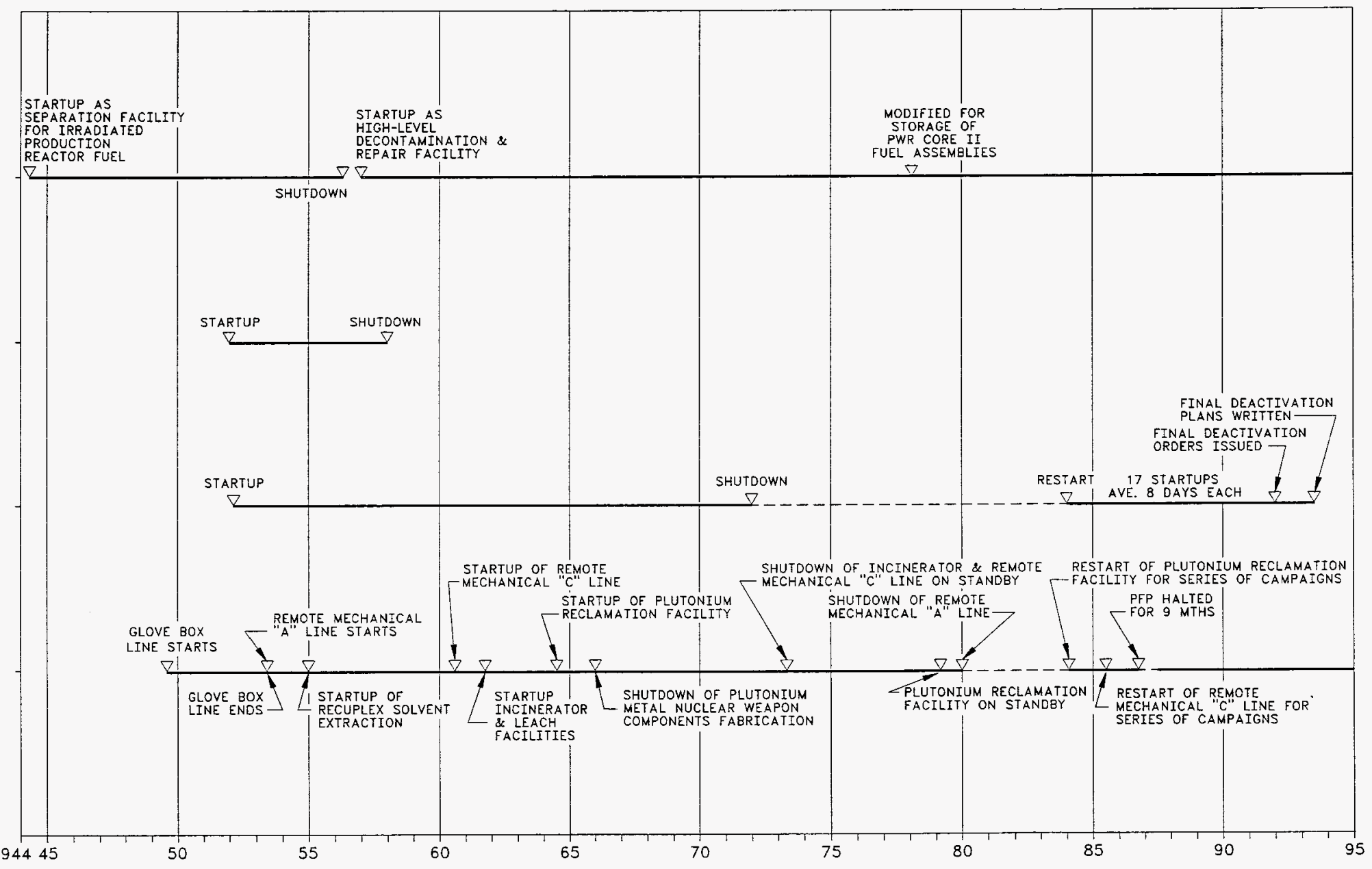

FIGURE 4 
THIS PAGE INTENTIONALLY

LEFT BLANK 


\section{WASTE MANAGEMENT - TIME LINE}

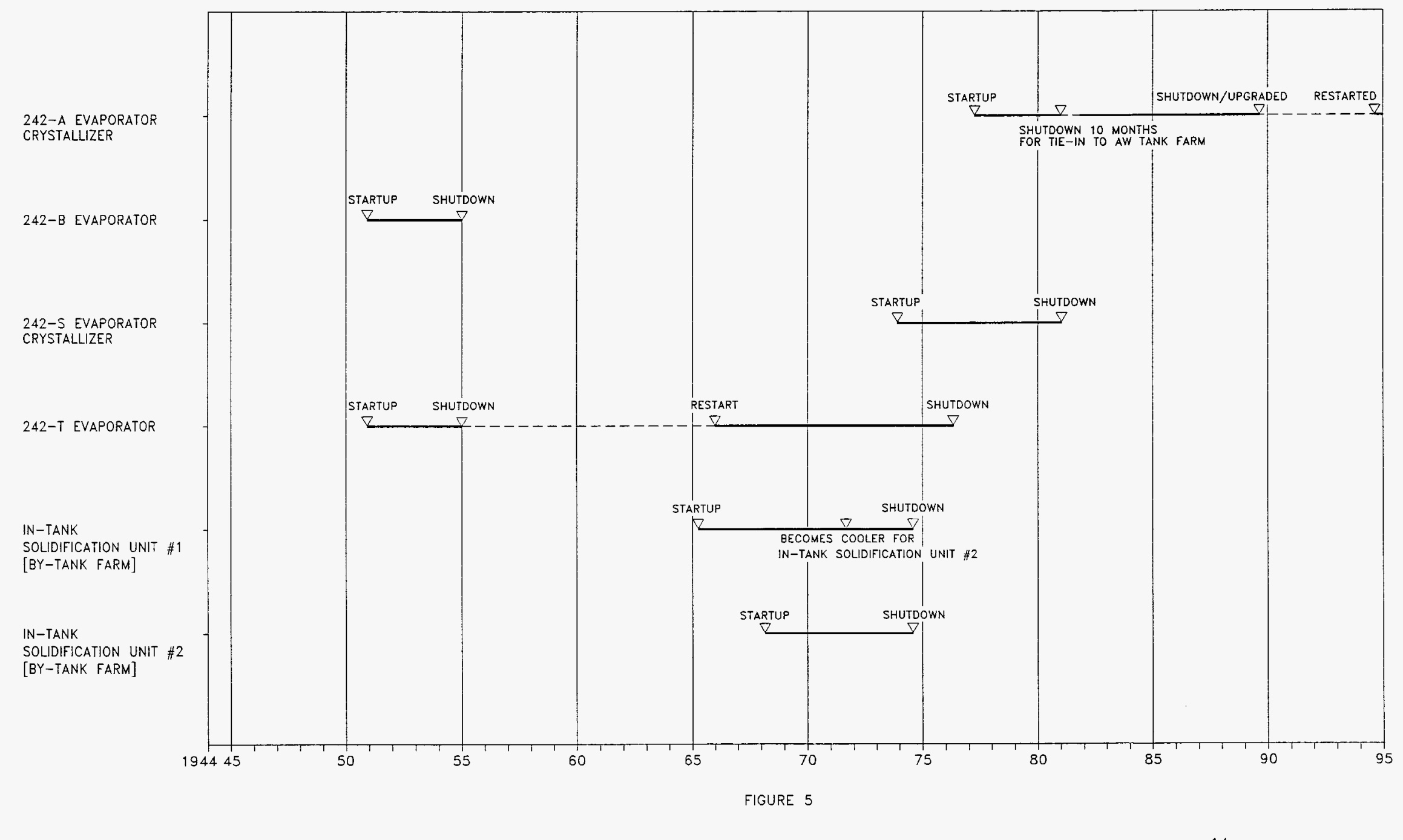




\section{THIS PAGE INTENTIONALLY}

LEFT BLANK 


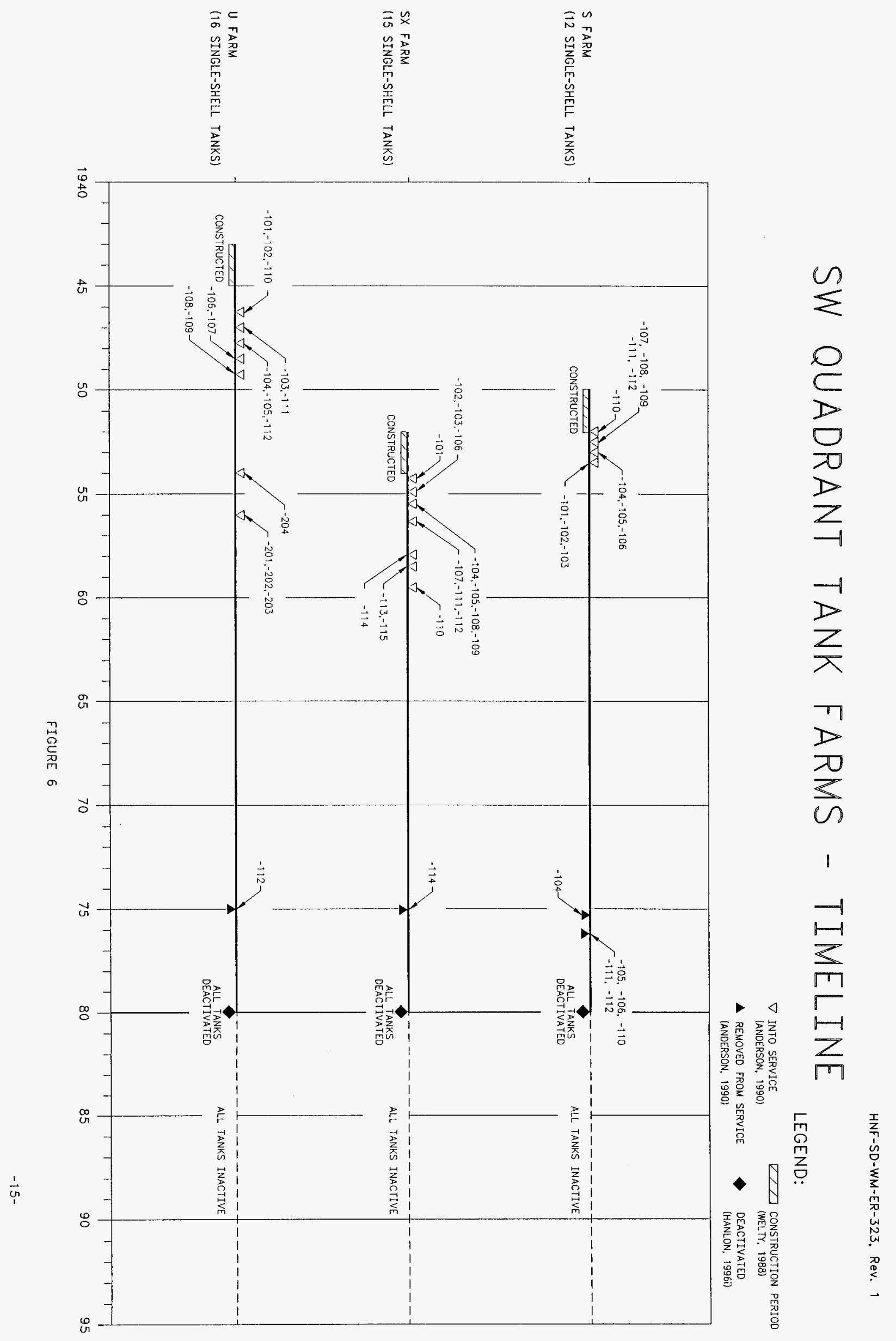


THIS PAGE INTENTIONALLY LEFT BLANK 


\subsection{Waste and Level History}

The Waste and Level History section is presented by a combination of two methods and is represented by sketches shown in Appendix C. The first method presents a graph of waste levels versus time for each tank. The waste levels graphed include the total waste level, solid waste level and, when available, the interstitial liquid level (ILL). The waste level graphs also include information on transfers, stabilization, intrusion prevention (isolation), salt-well and jet-pumping activities, level adjustments, $\mathrm{pH}$, photographs, and a few other miscellaneous items. The second method presents a time line that is made up of two parts. One part of the time line shows how the classification of the waste has changed for each tank and the other part of the time line shows the different waste types that were added to each tank. The time line and the waste level graphs for a given tank have been arranged so that the time axis for each method correlates with one another.

\subsubsection{Source of Data}

The references used to create the total waste level graph and the solid waste level graph for each level history graph are listed below in chronological order beginning with the oldest documents. Anderson (1990) was the source used for level information from when the tanks entered service until the end of 1980 . Level information from 1981 to the present was taken from a series of documents that basically contain the same type of information. These documents have been given various titles over the years but they all reflect the monthly waste status (i.e., waste volumes) for all the tanks. Beginning in 1981, these "monthly waste status reports" have been authored by the following people: O. C. Mudd; O. C. Mudd and D. C. McCann; D. C. McCann; D. C. McCann and T. S. Vail; T. S. Vail; T. S. Vail and G. D. Murry; T. S. Vail and G. J. Carter; G. J. Carter; G. A. Escobar; J. M. Thurman; and B. M. Hanlon. The last "monthly waste status report" reviewed was for June 30, 1996 (Hanlon, 1996i). See Appendix B for more complete reference information. All monthly waste status reports after January 1981 are included in the references.

The reference for the interstitial liquid level (ILL) graph for the applicable level history graphs is from Lockheed Martin Hanford Corporation's Surveillance Analysis Computer System (SACS), formerly maintained by Westinghouse Hanford Company. SACS is a database that stores ILL data along with other types of surveillance data. PCSACS software on a personal computer is the user interface to the SACS database via the Hanford Local Area Network (HLAN). The SACS database was queried back to 1950 for ILL data.

The only reference for the transfer information is Anderson (1990). Anderson contains information for all the tanks through 1980. Transfers that may have occurred after 1980 have not been identified on the Waste and Level History sketches. For more transfer information not included in this document, see Waste Status and Transaction Record Summary for the Southwest Quadrant (Agnew et al., 1995).

The reference for stabilization information is Hanlon (1996i).

For tanks that were intrusion prevented before June 1988, the reference is Welty (1988). For tanks that were intrusion prevented after June 1988, the references are various monthly waste status reports. For more complete reference information on intrusion prevention after June 1988, refer to 
the Waste and Level History sketches in Appendix C, where the references for intrusion prevention have been identified.

The reference for the salt-well pumping completion dates and jet-pumping completion dates is Welty (1988). Salt-well and jet-pumping activities that may have occurred after the release of Welty's document have not been identified on the Waste and Level History sketches.

Level adjustment dates were taken from various monthly waste status reports beginning in 1981 and from Anderson (1990) prior to 1981. Anderson's document does not contain a complete listing of the level adjustments prior to 1981 . For more complete information on level adjustments, refer to the individual "monthly waste status reports." For more complete reference information on level adjustments after 1980, refer to the Waste and Level History sketches in Appendix C where the references for these level adjustments have been identified.

The reference source for the $\mathrm{pH}$ information is Borsheim and Kirch (1991). The $\mathrm{pH}$ information after the release of Borsheim and Kirch's document has not been identified on the Waste and Level History sketches.

The photographic information was taken from Appendix $\mathrm{G}$ of this document.

The information on the time lines came from two sources. The reference for the Waste Types Time Line was Anderson (1990). The reference for the Primary Additions Time Line was Agnew et al. (1995).

\subsubsection{Development of Data}

The total waste level graphs and the solids waste level graphs were developed from waste volume information from Anderson (1990) and the "monthly waste status reports." Anderson compiled a listing of total waste volumes and solids waste volumes for all the tanks on a quarteriy basis prior to January 1981. Since Anderson's document is a compilation of the monthly waste status reports prior to January 1981, specific monthly waste status reports were reviewed when typographical errors were found. In order to continue the compilation of data on a quarterly basis after January 1981, the total waste volumes and the solids waste volumes were taken from the March, June, September, and December editions of the reports. The waste volumes were converted into equivalent waste levels based upon the Hanford Site accepted formula for S Farm. The following Hanford Site accepted formula has been applied for all volume to surface level conversions:

$$
\frac{\text { Total Gallons }-12,500 \text { Gallons }}{2750 \frac{\text { Gallons }}{\text { Inch }}}+12 \text { Inches }=\text { Total Inches }
$$

The "0" reference point for the total waste levels and the solids waste levels are at the bottom of the dish, inside of the tank. The waste levels have been rounded to the nearest thousand gallons (Kgal). In the event that the total waste level and the solids waste level were the same, the reported volumes were reviewed to determine whether the reported volumes were the same. If the volumes were 
the same, only the solids level was graphed. If the volumes were not the same, then both the total waste level and the solids waste level were graphed. The quarterly waste volumes and associated waste levels have been arranged in tables and are titled the "Level History" tables. These tables were developed within Microsoft Excel ${ }^{\oplus}$ and are presented in Appendix C.

The interstitial liquid level (ILL) graphs were developed from ILL data retrieved from SACS, that were imported into Microsoft Excel ${ }^{\circledast}$ spreadsheets. The SACS database custodians labeled the IIL data either as good or suspect. Only the data labeled as good were used for the ILL graphs. The SACS data that were labeled as good were reviewed on a quarterly basis. The quarters were set to match the quarterly system set up for the monthly waste status reports (i.e., the last month for each quarter was March, June, September, and December). The last data reading available from each quarter was the data reading used for the $\mathbb{L} L$ graphs. The data from SACS have been rounded off to the nearest whole number. The "0" reference point for the SACS ILL data is the bottom inside of the tank. The reviewed quarterly ILL data were added to the appropriate quarter in the Level History tables presented in Appendix $\mathbf{C}$.

The total waste level graphs, the solids waste level graphs, and the interstitial liquid level graphs were all created within AutoCAD ${ }^{\infty}$. In order to expedite the creation of these graphs, script files were generated from the information contained within the Level History tables. The script files were generated by arranging the waste level information and the corresponding dates from the Level History tables into a Cartesian coordinate system (i.e., $\mathrm{x}, \mathrm{y}$ coordinates). The script files allowed AutoCAD to automatically generate the graphs on the Waste and Level History sketches.

Transfer information was taken from the "Remarks" column of the Waste Status Summary tables in Anderson (1990). Transfer information was available on a quarterly basis. However, due to the scale of the time axis on the Waste and Level History sketch, the transfer information was placed near the total waste level graph corresponding to the appropriate year. Because of space limitations on the sketches, not all the transfer information available within Anderson's document could be included. For more details about the transfer information, see Anderson (1990).

Intrusion prevention (isolation) dates were taken from Welty (1988) and various monthly waste status reports. However, Welty and the various monthly waste status reports issued before 1993 use the old terminology of interim isolated. In 1993, the term "interim isolated" was replaced with "intrusion prevention." In order to remain consistent with current terms, the Waste and Level History sketches have used the interim isolation dates given by Welty and changed the terminology to intrusion prevention.

The Waste Types Time Line information was taken from the "Type Waste" column of the Waste Status Summary tables from Anderson (1990). The information taken from Anderson (1990) represents the waste type classification for the waste in the tanks. Since Anderson's document is a compilation of the monthly waste status reports prior to January 1981 , specific monthly waste status reports were reviewed when typographical errors were found. The vertical lines on the time line are boundaries between which the waste in the tank was classified as a certain type. It does not necessarily indicate that the waste in the tank was classified as a certain type over the entire period of time. For more details on the waste type classifications, see Anderson (1990). The vertical lines are spaced a minimum of three years apart. 
The Primary Additions Time Line information was taken from the spreadsheets located in Appendix D of the Waste Status and Transaction Record Summary for the Southwest Quadrant (Agnew et al., 1995). Two columns in the spreadsheet were reviewed to determine the information that would appear on the time line. The first column reviewed was the "Type" column. The Type column describes the type of transaction that occurred in a tank. The type of transactions that were reviewed were the transactions that Agnew et al. labeled as "XIN" or "xin." Agnew et al. used these two labels to indicate an addition of primary waste to a tank. According to Agnew et al., XIN is an addition of primary waste from a plant and xin is a transaction that was derived. If the Type column indicated either an XIN or xin, then the "DWXT" column was reviewed for the type of waste added to the tank. The waste types defined in the DWXT column that corresponded to an XIN or xin from the Type column were the waste types added to the time line. The vertical lines on the Primary Additions Time Line are boundaries between which the types of wastes identified have been added to the tanks at least once. It does not necessarily indicate that the types of wastes identified were added to the tank over the entire period of time. For more details on the waste types added, see Agnew et al.(1995). The vertical lines are spaced a minimum of three years apart.

\subsubsection{Assumptions}

An assumption was required in order to begin developing the total waste level graphs and the solid waste level graphs. The assumption was that the tanks did not contain waste prior to the time that Anderson (1990) started recording information.

The waste volume information taken from Anderson (1990) and the various monthly waste status reports required an assumption in order to apply the waste volume information to waste level formulas. The actual total waste surface and the actual solid waste surface were assumed to be flat and level.

The total waste level graphs and the solid waste level graphs required an assumption in order to make complete graphs. There were many cases within the Waste Status Summary tables (Anderson, 1990) where the tables lacked waste volume information for one or more consecutive quarters. When these omissions occurred, it was assumed that the waste volume followed an increasing, decreasing, or horizontal linear trend across these quarters for which Anderson lacked the volume information. Because of the linear nature of the waste-volume-to-waste-level formulas used to convert waste volumes into waste levels, a linear trend in the volumes results in a linear trend in waste levels on the Waste and Level History sketches.

The interstitial liquid in the tanks is the liquid that fills the interstitial spaces of the solid waste. The supernatant liquid in the tanks is located above the solid waste. Once the supernatant liquid has been removed from the tanks, the liquid that remains is the interstitial liquid. The interstitial liquid level (ILL) in most tanks is unknown unless a liquid observation well (LOW) has been installed. The LOW allows access for instrumentation to record ILL. Unfortunately, in many tanks, the LOW's were not installed and the ILL's were not recorded until some time after the supernatant liquid was removed. Therefore, the ILL graphs required some assumptions in order to define the ILL graph from the point where the supernatant liquid was removed from the tank to the point where ILL data were started. One assumption was that the ILL followed a decreasing linear trend from the point where the supernatant liquid was removed from the tank to the point where salt-well pumping was completed. Another 
assumption was that the $\mathbb{L L}$ followed a horizontal linear trend from the point where salt-well pumping was completed to the point where the first ILL data were recorded on SACS.

\subsubsection{Quality of Data}

The total waste level graphs and the solids waste level graphs on the Waste and Level History sketches were developed by using the Hanford Site accepted waste-volume-to-waste-level formulas. However, there are some limitations with the formulas that affect the waste level results. The formulas do not yield realistic results when the waste volume is less than the volume that can be held below the top of the tank knuckle. The formulas do not account for construction tolerances on the tanks, the knuckle geometry on the tanks, the true geometric configuration of the tanks, and the irregularities in the surface of the solid wastes.

The total waste level graphs were developed from the volume data from Anderson (1990) and from the "monthly waste status reports." The frequency with which these references have their volume information updated is not consistent with the frequency with which the waste surface level readings of the SACS database are updated. Therefore, a discrepancy may be noticed between the total waste level graphs of the Waste and Level History sketches in Appendix C and the waste surface level graphs in Appendix E.

The interstitial liquid level (ILL) graphs were developed from data contained within the SACS database. The SACS custodians have qualified the data as either good or suspect. Only the data labeled as good have been plotted. However, for some tanks, the ILL data plotted showed that the ILL exceeded the total amount of waste as plotted from the total waste level data. This situation is a contradiction to the definition of interstitial liquid. Even though the SACS custodians have labeled the data as good, the data quality seems questionable. The questionable data were graphed anyway. It was not the function of this document to judge or qualify the $\Pi L L$ data nor to discuss in detail this situation.

\section{$1.4 \quad$ Temperatures}

\subsubsection{Surveillance Techniques}

Interior tank temperatures of the single-shell tanks in S Tank Farm are monitored with thermocouples. Thermocouples are simple devices that develop a millivoltage when parts of the thermocouple are exposed to temperature differentials. The millivoltage can be converted to a temperature reading based upon a specific voltage-versus-temperature curve inherent to the type of thermocouple being used. Thermocouples are attached to a fabricated assembly called a thermocouple tree. The number of thermocouples attached to the tree varies as a function of the depth of the tank as well as the thermocouple tree design. For thermocouple trees with multiple thermocouples, the thermocouples are spaced at intervals along the tree so that a vertical temperature profile of the tank contents can be developed. The thermocouple tree is installed in a riser and left in place inside the tank. If necessary, the thermocouple tree can be removed from the tank.

\subsubsection{Source of Data}

The source of the interior tank temperature data is from the Lockheed Martin Hanford Corporation's Surveillance Analysis Computer System (SACS) formerly maintained by Westinghouse 
Hanford Company. SACS is a database that stores temperature data along with other types of surveillance data. PCSACS software on a personal computer is the user interface to the SACS database via the Hanford Local Area Network (HLAN). The SACS database was queried back to 1950 for temperature data.

\subsubsection{Development of Data}

Interior tank temperature data imported from SACS into spreadsheets (Microsoft Excel ${ }^{\Phi}$ ) were rearranged onto separate spreadsheets depending on the data qualifier assigned by SACS custodians. The SACS database custodians labeled the interior tank temperature data using three data qualifiers or categories. The categories are good (G), transcribed (T), and suspect (S). Only the $G$ and the $T$ data were imported from SACS. The imported $\mathrm{G}$ and $\mathrm{T}$ data were used to develop graphs of individual thermocouple data. The graphs were developed within Microsoft Excel ${ }^{\infty}$ spreadsheets. There were two conditions about the temperature data that were evaluated before the graphs of individual thermocouple data were developed. The first condition evaluated was the number of data points from a particular thermocouple. If a thermocouple had five or fewer data points, then a graph was not developed for that particular thermocouple. The second condition evaluated was the time span between consecutive data points. If the time span between consecutive data points was greater than 36 months, then the graph was shown as discontinuous across the span (see Appendix D).

The thermocouple elevations identified on the individual thermocouple graphs were determined from design drawings listed in the narratives and from the Thermocouple Status Single Shell and Double Shell Tanks (Tran, 1993). Tran's document contains design drawing references along with thermocouple elevations. If the design drawings listed in Tran's document could be verified for the individual tanks, then the thermocouple elevations listed by Tran were used. If the design drawings listed in Tran's document could not be verified for an individual tank or if design drawings could not be located, then the thermocouple elevations were labeled as unknown. If Tran's document lacked information about thermocouple elevations for a particular tank and design drawings were located, then the thermocouple elevations were labeled as approximate.

Undocumented, as well as some documented changes, and/or modifications to the thermocouple tree designs may have occurred at some tanks. Consideration of these changes and/or modifications, however, depended on whether proper documentation on the change and/or modification was located. Proper documentation of a change and/or modification is documentation that has been recorded and filed with the appropriate Hanford Site document control stations. If proper documentation of a change and/or modification could not be located, then the changes and/or modifications were not considered.

\subsubsection{Assumptions}

The transcribed data points are data points that have not been verified or validated by Lockheed Martin Hanford Corporation (or by Westinghouse Hanford Company). Transcribed data were assumed to be good data and were included on the graphs of individual thermocouple data and in the statistics. Individual judgements were not made on particular transcribed data points even though they had a high probability of being suspect. Verification and/or validation of data is not the function of this document.

The design drawings listed in each of the tank temperature narratives are assumed to be the design drawings that reflect the thermocouple tree design considered in this document. 


\subsubsection{Quality of Data}

The quality of the interior tank temperature data is noted by the three category labels assigned by the custodians of the SACS database. The good and suspect data points have been verified and/or validated by Lockheed Martin Hanford Corporation (or by Westinghouse Hanford Company, the previous contractor). The transcribed data points have not been reviewed by Lockheed Martin Hanford Corporation (or by Westinghouse Hanford Company). The transcribed data could be classified as either good or suspect at a later date.

This document has treated the transcribed data as good data. However, an area where the transcribed data points have a high probability of being suspect is when the temperature data values are below 45 to $50^{\circ} \mathrm{F}$. The approximate temperature of the soil surrounding the tanks is 45 to $50^{\circ} \mathrm{F}$ and the soil will prevent the temperature of the tank from dropping below this point. Some of the tanks have many data points below the 45 to $50^{\circ} \mathrm{F}$ range, and these data points should be evaluated carefully as to whether or not they should be considered as good data points.

\subsection{Waste Surface Level}

\subsubsection{Surveillance Techniques}

One of four types of waste surface level devices is used to monitor waste levels in the single-shell tanks in S Tank Farm: a level indicating transmitter or Food Instrument Corporation (FIC) gauge; a level indicator assembly or manual tape; a high-level detector (an FIC gauge in intrusion mode); and the ENRAF 854 ATG Liquid Level Indicator/Transmitter.

The Food Instrument Corporation gauge is based on conductivity. A plummet is lowered into the tank. When the plummet contacts an electrically conducting surface that is in contact with the edge of the tank, a circuit is completed between the probe and the tank which is grounded to the instrument. This triggers the drive motor to stop and the motor brake to engage. The brake is held for 60 seconds, before the motor raises the plummet. The plummet is raised until the circuit is broken. This instrument is used both as a high-level detector and as an actual level detector. The high-level mode is used to detect intrusions (i.e., any unexplained addition to the tank like rainwater) into the tank. The actual level mode can be read automatically, manually, or both. The automatic FIC reading is automatically read in the field and loaded on to the Surveillance Analysis Computer System (SACS). FIC readings are also read manually in the field and entered into the SACS.

The manual tape flake boxes are used for measuring waste levels manually if other devices fail or do not exist. A hand crank on the flake box is used to lower the tape probe until a electrical conducting surface is contacted and a circuit is completed between the tank and the instruments (similar to the FIC gauge). If the circuit is not completed, the probe is lowered until the tape is slack; then, a measurement is recorded.

The ENRAF 854 ATG Liquid Level Indicator/Transmitter has been installed on several tanks and will eventually replace the old level measurement devices. The ENRAF 854 ATG is a microprocessor controlled surface level gauge. Level detection is based on the principle of buoyancy of a non-floating polyethylene displacer. The displacer is attached to a stainless steel measuring wire. The measuring wire is attached to a measuring drum which is fixed to a riser of known elevation. The 
weight of the displacer is entered into the memory of a force transducer. A second weight of about 0.35 to 0.53 ounces less than the actual weight of the displacer is entered into the transducer as the control point. An electronic servomechanism turns the measuring drum causing the displacer to move. As the displacer comes in contact with the surface in question, the displacer will exert a smaller force on the transducer due to buoyancy. The displacer is lowered until the force exerted on the transducer is equal to the control point. By knowing the elevations of the riser and tank bottom, and the distance from the riser to the surface of the waste, the surface level of the waste can be determined. If the surface level changes, the displacer will be raised or lowered by the measuring drum depending on the force exerted on the transducer relative to the control point. The ENRAF ${ }^{\circledR}$ can be read automatically, manually or both. The automatic ENRAF ${ }^{\star}$ reading is loaded on the Lockheed Martin Hanford Corporation Surveillance Analysis Computer System (SACS) formerly maintained by Westinghouse Hanford Company. Manual ENRAF ${ }^{\star}$ readings maybe taken at any time of day and are manually entered into the SACS.

\subsubsection{Source of Data}

The data recorded from January 1, 1991, to October 3, 1996, for the waste surface levels were obtained from the SACS database. PCSACS software on a personal computer is the user interface to the SACS database. The information was parsed in a spreadsheet in Microsoft Excel ${ }^{\otimes}$ software and displayed on graphs. Since the intrusion FIC is fixed at a certain elevation, it only records that elevation unless there is an unexplained addition. The device does not truly measure the waste surface level so the data were not displayed.

\subsubsection{Development of Data}

Waste surface level data imported from the SACS database into spreadsheets (Microsoft Excel ${ }^{\text {* }}$ ) were rearranged onto separate spreadsheets depending on the data qualifier assigned by SACS custodians. The SACS database custodians label the waste surface level data using three data qualifiers or categories. The categories are good $(\mathrm{G})$, transcribed $(\mathrm{T})$, and suspect $(\mathrm{S})$. The waste surface level data were then filtered to remove all the $S$ data, leaving only the $G$ and $T$ data. All devices with good or transcribed data, were graphed on one graph to show the possible measurement differences between devices. If the tank has more than one device to measure the waste surface level, an individual graph was made to display the data from each device. The graphs show waste level versus time. The data are displayed using the best representative scale on the y axis. The safety limit maximum waste level is placed in the title of each graph (Dougherty, 1995). The current information on the waste surface levels is in Appendix E. The maximum and minimum waste surface levels, along with the respective dates, are summarized in each tanks Current Status Section.

\subsubsection{Assumptions}

The data obtained from PCSACS database are the best available data. The data quality designation, instrument type, and level measurement are accurate. The devices are in good condition and give accurate readings if the following assumptions are made: internal tank temperature changes do not cause the tape, wire, or probe to change length; the tape, wire, and probe are straight; the surface profile of the waste is flat; and changes in atmospheric temperature do not affect the portions of the measuring device exposed to the atmosphere. 


\subsubsection{Quality of Data}

Waste surface level readings may be affected by plummet (i.e., manual tape) error, flushing water accumulation, waste surface irregularities, or gas generation. Crystalline wastes (i.e., salt cake) can build up gradually on the end of the plummet and contact the waste which indicates a false surface level increase. Significant level discrepancies occur when the encrusting waste breaks off or when the measuring instrument plummet is flushed to remove the encrusting salt cake. Flushing the FIC gauge, manual tapes, or any other equipment may cause accumulated wash water to collect under the plummet which can also indicate a false increase in the overall volume of waste within the tank. Waste surface level readings are often difficult to obtain from tanks with a relatively dry waste surface of salt cake. Some tanks have crystalline waste built up on internal tank equipment (e.g., pumps, thermocouples, and other protruding equipment). As the supernatant liquid is pumped from the tanks, the crystalline structure may remain attached to the equipment and be suspended above the liquid. Therefore, an accurate waste surface level measurement would be difficult if the breakup of the crystalline structure were inconsistent and a nonuniform waste surface were created. Steel tapes or wires that are bent or warped from operation or those discarded on the waste surface are other sources of altered waste surface level readings.

Data from the SACS database were obtained electronically from the Lockheed Martin Hanford Corporation surveillance group (formerly of Westinghouse Hanford Company) and were plotted. The data are actual waste surface levels recorded from the surveillance equipment and may not match the data used in the Waste and Level History sketches of Appendix C. If the surveillance equipment in a particular tank riser has been removed from service, the readings may show a level change when a new instrument and/or riser is used, especially if the waste surface shows severe heterogeneity.

The data used to produce the plots and the data obtained from the surveillance group have been verified as identical. However, errors in the data prior to the exchange of information could still exist. Employees of Lockheed Martin Hanford Corporation (formerly of Westinghouse Hanford Company) qualified the data with G, S, and T for good, suspect, and transcribed, respectively. Data that is labeled transcribed has not been validated or verified by Lockheed Martin Hanford Corporation. The criteria for determining data labeled good or suspect are unknown.

\subsection{Riser Configuration}

\subsubsection{Source of Data}

The riser configuration sketches and tables in this document were compiled from design and/or as-built drawings including engineering change notices dated before September 30, 1996, the Riser Configuration Document for Single-Shell Waste Tanks (Alstad, 1993), and Waste Tank Risers Available for Sampling (Lipnicki, 1996).

\subsubsection{Development of Data}

There are two sketches and a table for each tank in Appendix $\mathrm{F}$ that show the approximate riser locations, construction materials, dimensions, and riser function. The sketches were developed from the design and/or as-built drawings. Alstad (1993) was used as a guide; however, the design and/or as-built drawings and engineering change notices take precedence. An engineering change notice 
(ECN, $\mathrm{CEO}, \mathrm{FCN}$ ) is a tool used to change a drawing without creating a new drawing revision. If an above ground survey of the tank has been done, Soil Load above Hanford Waste Storage Tanks (Pianka, 1995) was used as the grade elevation; otherwise, the design and/or as-built drawings grade elevation was used. The tables in Appendix F contain the riser number, diameter; sampling, and description of each riser and nozzle. The riser number and diameter on the design and/or as-built drawings' figure is compared to the design and/or as-built drawings' table and Alstad (1993). If there were any discrepancies between the design and/or as-built drawings and Alstad (1993), the design and/or as-built drawings and engineering change notices take precedence; however, a note was made below the table briefly describing the discrepancy. If there was a discrepancy between the design and/or as-built drawings' figure and the design and/or as-built drawings' table, a note was made below the table briefly describing the discrepancy. The sampling column lists risers that are tentatively available for sampling (Lipnicki, 1996). The description and comment column describes the riser's intended use and, if applicable, gives in parentheses the date, number and a brief explanation of the pertaining engineering change notices. The intent of the description and comment column was to provide a historical use of the riser along with providing the current use of the riser.

\subsubsection{Assumptions}

The design and/or as-built drawings are the best available data. All the engineering change notices written against the referenced drawings are released and accurate. Since figures or sources were not listed in Lipnicki (1996), the riser numbers labeled tentatively available for sampling are assumed to be the same as the riser numbers listed in Alstad (1993).

\subsubsection{Quality of Data}

The riser configuration section of this document is a mixture of data from three main sources: design and/or as-built drawings, Riser Configuration Document for Single-Shell Waste Tanks (Alstad, 1993), and Waste Tank Risers Available for Sampling (Lipnicki, 1996). The three sources do not always agree. Alstad (1993) and Lipnicki (1996) reference the design and/or as-built drawings in their respective documents. The design and/or as-built drawings contain a plan view of the tank dome and a table explaining the function of each riser. Sometimes the plan view and the table do not match. If there was a discrepancy, a comment was made below the table for that tank. Other design and/or as-built drawings show a cross-sectional view of the tank. Changes made to the structure of a tank may not have been documented by an engineering change notice. The sketches and tables are intended to give the reader information as to: approximate location of risers and equipment; approximate dimensions; construction material; diameter of risers; number of risers; and what equipment each riser might contain. The sketches are not to scale.

\subsection{Photographs and Montages}

\subsubsection{Source of Data}

The photographs include an aerial photograph of the tank farm and a photographic montage of each tank interior, if available (see Appendix G). All of the photographs were obtained from Boeing Computer Services Richland-Photography (now Lockheed Martin Services, Inc.-Photography). The aerial photographs were reviewed in January 1996 to determine the clearest and most recent representation of the $S$ Tank Farm to be used in this document. The montages were created from sets 
of interior tank photographs. These sets were also reviewed in January 1996 to determine which ones were the clearest and most recent photographs available. Only interior tank photographs representing the waste surface were used. In some cases, existing montages were the clearest and most recent; therefore, a new montage was not created.

\subsubsection{Development of Data}

The tank farm aerial photograph was labeled to show tank orientation, identifiable equipment, and structures. Arrows were placed on the tank farm aerial photograph to indicate the cascade overflows. The clearest and most recent montage of the tank interior was labeled to show identifiable monitoring equipment, piping, and risers. A table was also developed listing aerial photograph numbers, interior tank montage numbers, photographic set numbers, and the date (see Appendix G).

\subsubsection{Quality of Data}

The interior tank photographs may not represent the actual colors of the waste surface due to possible alteration of colors during copying of the original photographs. To see the colors of the original montage, an original may be ordered through Lockheed Martin Services, Inc.-Photography. Radiation can also affect the film distorting the apparent colors of the waste. Some tanks had fogging problems in the vapor space which prevented use of the latest photographs. The montage may not reflect the current waste level and waste type due to pumping, additions, mixing, and/or settling of the waste after the photographs were taken. Also, the equipment configuration may not reflect tank upgrades and/or maintenance.

\subsection{Inventory Estimates}

The Inventory Estimates developed by Los Alamos National Laboratory (LANL) are presented in Appendix $\mathrm{H}$ along with LANL's approach and development sections. The Inventory Estimates presented in this document represent an estimated waste content of the tanks in S Tank Farm based on an inventory estimate date of December 31, 1993.

\subsubsection{Source of Data}

The Inventory Estimates, and their approach and development sections presented in this document are from the Hanford Tank Chemical and Radionuclide Inventories: HDW Model Rev. 4 (Agnew et al., 1997). For further information about the Inventory Estimates refer to Agnew's document.

\subsubsection{Development of Data}

The data presented in Appendix $\mathrm{H}$ were selectively taken from the Hanford Tank Chemical and Radionuclide Inventories: HDWModel Rev. 4 (Agnew et al., 1997) document to include the Inventory Estimates for S Tank Farm. The introduction to Agnew's document is included in its entirety. The Inventory Estimates (Agnew et al., 1997, Appendix E) were included for only S Tank Farm. 
THIS PAGE INTENTIONALLY LEFT BLANK 


\section{FARM INFORMATION}


THIS PAGE INTENTIONALLY LEFT BLANK 


\section{$2.0 \quad$ S Tank Farm}

\subsubsection{S Tank Farm Information}

The S Tank Farm is located west of Beliot Avenue and north of 13th Street in the 200-West Area. The farm contains twelve 100 series, dish bottom design, single-shell tanks built in 1950 and 1951 (Welty, 1988). The 100 series tanks are 75 feet in diameter with an operating capacity of 758,000 gallons (Hanlon, 1996i) (see sketches in Appendix F). The tanks are constructed of a reinforced concrete shell with a steel liner on the interior bottom and sides. The tanks were designed to hold nonboiling waste at a fluid temperature of $220^{\circ} \mathrm{F}$ (Leach and Stahl, 1993). The dome of each tank is penetrated by risers varying in diameter from 4 to 42 inches. All of the tanks have at least six feet of earth cover.

The twelve tanks were constructed with connecting overflow lines that allowed waste to cascade from tank to tank. There are four cascade sequences in the S Tank Farm. One cascade sequence is from Tank 241-S-101 to -102 to -103 . The second is from Tank 241-S-104 to -105 to -106 . The third is from Tank $241-\mathrm{S}-107$ to -108 to -109 , and the fourth is from Tank $241-\mathrm{S}-110$ to -111 to -112 .

\subsubsection{S Tank Farm Waste and Level History}

The Waste and Level History sketches in Appendix C present the waste history and level history of S Tank Farm.

\subsubsection{S Tank Farm Temperature History}

Interior tank temperature data for S Tank Farm are quite limited compared to the span of time in which the tanks have been operating. Information about the various temperature monitoring devices and their locations throughout history is also quite limited. The information that was available came from the Surveillance Analysis Computer System (SACS) database. Data from the files were available as far back as early 1988 .

\subsubsection{S Tank Farm Occurrences}

Only the occurrences determined as significant are included. The reports presented are incomplete because not all of the documentation on occurrences for $S$ Tank Farm could be located.

\subsubsection{S Tank Farm Current Status}

The tanks in S Tank Farm entered service from 1952 to 1953 (Anderson, 1990). The dates the tanks entered service are based on when the tank first received waste or test water. This date may vary in other documents. The total waste volume for all of the tanks is approximately 5,510,000 gallons as of June 30, 1996. All tanks are out of service. Tanks 241-S-101, -102, -103, -105, -106, -107, -108, $-109,-110,-111$ and -112 are categorized as sound, and Tank 241-S-104 is an assumed leaker. Tanks 241-S-102, -111, and -112 are on watch lists (Hanlon, 1996i). See Appendix E for waste surface level graphs. The risers tentatively available for sampling are listed in Appendix F. 


\subsubsection{S Tank Farm Photograph and Montages}

The photographs for S Tank Farm include an aerial photograph of the farm and a montage of interior tank photographs for each tank. The aerial photograph shows the tank orientation, equipment, and structures. Arrows were placed between the tanks to represent the cascade overflow lines and the flow directions. The interior tank photographs were arranged into montages to show the waste surface, monitoring equipment, piping, and risers in each tank. The photographs and a table listing the photographs, montage numbers, photograph set numbers, and dates of the photographs in this document are in Appendix G.

\subsubsection{S Tank Farm Inventory Estimates}

The Inventory Estimates from the Hanford Tank Chemical and Radionuclide Inventories: HDW Model Rev. 4 (Agnew et al., 1997) for S Tank Farm are presented in Appendix H. 


\section{$2.1 \quad$ Tank $241-\mathrm{S}-101$}

\subsubsection{Waste and Level History of Tank 241-S-101}

The Waste and Level History sketch in Appendix C presents the waste history and level history of Tank 241-S-101.

\subsubsection{Temperature History of Tank 241-S-101}

Interior tank temperature data for Tank 241-S-101 were recorded by 6 thermocouples attached to one thermocouple tree. Drawing H-2-90344, Rev. 3, indicates that the thermocouple tree is located in riser 14 and is designed as shown on Drawing H-2-90342, Sht. 2, Rev. 2 with 6 thermocouples. The Surveillance Analysis Computer System (SACS) indicates that the thermocouple tree is also located in riser 14. In the past, other risers and equipment may have been used to monitor the temperature in the tank. However, the thermocouple tree located at riser 14 was the only source of temperature data for this document. The temperature data were obtained from the SACS database on October 1, 1996. The earliest data retrieved from the SACS were from early January 1988

- Graphs of individual thermocouple data are presented in Appendix D. A graph was created for each thermocouple. The following statistical information was taken from all 6 thermocouples. The maximum temperature was $136.8^{\circ} \mathrm{F}$ taken by thermocouple 1 on February 6,1989 . The minimum temperature was $82.8^{\circ} \mathrm{F}$ taken by thermocouple 6 on July 25,1994 . The maximum and minimum temperatures are labeled as good data points within the SACS. The average temperature for all the thermocouples is $110^{\circ} \mathrm{F}$.

\subsubsection{Occurrences for Tank 241-S-101}

No significant occurrences are associated with Tank 241-S-101.

\subsubsection{Current Status of Tank 241-S-101}

Tank 241-S-101 entered service during the third quarter of 1953 (Anderson, 1990) and as of June 30, 1996, stores approximately 427,000 gallons of waste (Hanlon, 1996i). The minimum waste surface level was 160.82 inches on March 27 and 28, 1995. The maximum waste surface level was 162.4 inches on numerous dates from August 31, 1992 through September 23, 1992. See Appendix E for details on waste surface level. The tank is out of service with partial interim isolation completed. The tank is listed as sound and is passively ventilated (Hanlon, 1996i). A plan view in Appendix F depicts the approximate riser locations as of September 30, 1996. Tank 241-S-101 has 12 risers with four tentatively available for sampling: three 4-inch risers (Nos. 4, 11 and 16) and one 12-inch riser (No. 6) (Lipnicki, 1996).

\subsubsection{Interior Montage of Tank 241-S-101}

The clearest and most recent set of interior tank photographs was taken on March 18, 1988. Other interior tank photographs are available, but only the photographs showing the waste surface were used to create a montage. The montage has labels identifying some of the monitoring equipment, piping, and risers in the tank. The montage and photographic information are shown in Appendix G. 
HNF-SD-WM-ER-323, Rev. 1

\subsubsection{Inventory Estimate of Tank 241-S-101}

The Inventory Estimate from the Hanford Tank Chemical and Radionuclide Inventories: HDW Model Rev. 4 (Agnew et al., 1997) for Tank 241-S-101 is presented in Appendix H. 


\subsection{Tank $241-\mathrm{S}-102$}

\subsubsection{Waste and Level History of Tank 241-S-102}

The Waste and Level History sketch in Appendix C presents the waste history and level history of Tank 241-S-102.

\subsubsection{Temperature History of Tank 241-S-102}

Interior tank temperature data for Tank 241-S-102 were recorded by 6 thermocouples attached to one thermocouple tree. Drawing H-2-90344, Rev. 3, indicates that the thermocouple tree is located in riser 3 and is designed as shown on Drawing H-2-90342, Sht. 2, Rev. 2 with 6 thermocouples. The Surveillance Analysis Computer System (SACS) indicates that the thermocouple tree is also located in riser 3. In the past, other risers and equipment may have been used to monitor the temperature in the tank. However, the thermocouple tree located at riser 3 was the only source of temperature data for this document. The temperature data were obtained from the SACS database on October 1, 1996. The earliest data retrieved from the SACS were from early January 1988.

Graphs of individual thermocouple data are presented in Appendix D. A graph was created for each thermocouple. The following statistical information was taken from all 6 thermocouples. The maximum temperature was $122^{\circ} \mathrm{F}$ taken by thermocouple 2 on May 2, 1988 and June 6, 1988, thermocouple 3 on May 2, 1988, and by thermocouple 4 on May 2, 1988. The minimum temperature was $71.2^{\circ} \mathrm{F}$ taken by thermocouple 6 on April 18, 1992. The maximum and minimum temperatures are labeled as good data points within the SACS. The average temperature for all the thermocouples is $102^{\circ} \mathrm{F}$.

\subsubsection{Occurrences for Tank 241-S-102}

No significant occurrences are associated with Tank 241-S-102.

\subsubsection{Current Status of Tank 241-S-102}

Tank 241-S-102 entered service during the third quarter of 1953 (Anderson, 1990) and as of June 30, 1996, stores approximately 549,000 gallons of waste (Hanlon, 1996i). The minimum waste surface level was 202 inches on January 21, 1991. The maximum waste surface level was 206.1 inches on June 13, 1994. See Appendix E for details on waste surface level. Tank 241-S-102 was added to the Organics Watch List and the Hydrogen Watch List in January 1991. The tank is out of service with partial interim isolation completed. The tank is listed as sound and is passively ventilated (Hanlon, 1996i). A plan view in Appendix F depicts the approximate riser locations as of September 30, 1996. Tank $241-\mathrm{S}-102$ has 12 risers with five tentatively available for sampling: four 4-inch risers (Nos. 1, 11, 14, and 16) and one 12-inch riser (No. 7) (Lipnicki, 1996).

\subsubsection{Interior Montage of Tank 241-S-102}

The clearest and most recent set of interior tank photographs was taken on March 18, 1988. Other interior tank photographs are available, but only the photographs showing the waste surface were 
used to create a montage. The montage has labels identifying some of the monitoring equipment, piping, and risers in the tank. The montage and photographic information are shown in Appendix $G$.

2.2.6 Inventory Estimate of Tank 241-S-102

The Inventory Estimate from the Hanford Tank Chemical and Radionuclide Inventories: HDW Model Rev. 4 (Agnew et al., 1997) for Tank 241-S-102 is presented in Appendix H. 


\subsection{Tank 241-S-103}

\subsubsection{Waste and Level History of Tank 241-S-103}

The Waste and Level History sketch in Appendix C presents the waste history and level history of Tank 241-S-103. The interstitial liquid level data are questionable since they exceed the total waste level in the tank.

\subsubsection{Temperature History of Tank 241-S-103}

Interior tank temperature data for Tank 241-S-103 were recorded by 6 thermocouples attached to one thermocouple tree. Drawing H-2-90344, Rev. 3, indicates that the thermocouple tree is located in riser 4 and is designed as shown on Drawing H-2-90342, Sht. 2, Rev. 2 with 6 thermocouples. The Surveillance Analysis Computer System (SACS) indicates that the thermocouple tree is also located in riser 4 . In the past, other risers and equipment may have been used to monitor the temperature in the tank. However, the thermocouple tree located at riser 4 was the only source of temperature data for this document. The temperature data were obtained from the SACS database on October 1, 1996. The earliest data retrieved from the SACS were from early January 1988.

Graphs of individual thermocouple data are presented in Appendix D. A graph was created for each thermocouple. The following statistical information was taken from all 6 thermocouples. The maximum temperature was $98^{\circ} \mathrm{F}$ taken by thermocouple 1 on November 7,1988 . The minimum temperature was $63.7^{\circ} \mathrm{F}$ taken by thermocouple 5 on July 2,1994 . The maximum and minimum temperatures are labeled as good data points within the SACS. The average temperature for all the thermocouples is $82^{\circ} \mathrm{F}$.

\subsubsection{Occurrences for Tank 241-S-103}

No significant occurrences are associated with Tank 241-S-103.

\subsubsection{Current Status of Tank 241-S-103}

Tank 241-S-103 entered service during the fourth quarter of 1953 (Anderson, 1990) and as of June 30, 1996, stores approximately 248,000 gallons of waste (Hanlon, 1996i). The minimum waste surface level was 102.5 inches on March 7 and 8, 1991. The maximum waste surface level was 106 inches on May 13, 1996. See Appendix E for details on waste surface level. The tank is out of service with partial interim isolation completed. The tank is listed as sound and is passively ventilated (Hanlon, 1996i). A plan view in Appendix F depicts the approximate riser locations as of September 30,1996 . Tank $241-\mathrm{S}-103$ has 12 risers with five tentatively available for sampling: three 4-inch risers (Nos. 11, 14 and 16) and two 12-inch risers (Nos. 6, and 8) (Lipnicki, 1996).

\subsubsection{Interior Montage of Tank 241-S-103}

The clearest and most recent set of interior tank photographs was taken on June 1, 1989. Other interior tank photographs are available, but only the photographs showing the waste surface were used to create a montage. The montage has labels identifying some of the monitoring equipment, piping, and risers in the tank. The montage and photographic information are shown in Appendix $\mathrm{G}$. 
2.3.6 Inventory Estimate of Tank 241-S-103

The Inventory Estimate from the Hanford Tank Chemical and Radionuclide Inventories: HDW Model Rev. 4 (Agnew et al., 1997) for Tank 241-S-103 is presented in Appendix H. 


\subsection{Tank 241-S-104}

\subsubsection{Waste and Level History of Tank 241-S-104}

The Waste and Level History sketch in Appendix C presents the waste history and level history of Tank 241-S-104.

\subsubsection{Temperature History of Tank 241-S-104}

Interior tank temperature data for Tank 241-S-104 were recorded by 14 thermocouples attached to one thermocouple tree. Documentation of the design configuration of the thermocouple tree was not located. The design of the thermocouple tree is unclear and the elevations of the individual thermocouples are unknown. The Surveillance Analysis Computer System (SACS) indicates that the thermocouple tree is located in riser 4 . In the past, other risers and equipment may have been used to monitor the temperature in the tank. However, the thermocouple tree located at riser 4 is the only source of temperature data for this document. The temperature data were obtained from the SACS database on October 1, 1996. The earliest data retrieved from the SACS were from mid November 1991.

Graphs of individual thermocouple data are presented in Appendix D. A graph was created for each thermocouple. The following statistical information was taken from all 14 thermocouples. The maximum temperature was $114^{\circ} \mathrm{F}$ taken by thermocouple 3 on January 4,1995 . The minimum temperature was $83.1^{\circ} \mathrm{F}$ taken by thermocouples 6 and 7 on July 2,1993 , and thermocouple 14 on February 13, 1993. The maximum and minimum temperatures are labeled as good data points within the SACS. The average temperature for all the thermocouples is $98^{\circ} \mathrm{F}$.

\subsubsection{Occurrences for Tank 241-S-104}

No significant occurrences are associated with Tank 241-S-104.

\subsubsection{Current Status of Tank 241-S-104}

Tank 241-S-104 entered service during the first quarter of 1953 (Anderson, 1990) and as of June 30,1996 , stores approximately 294,000 gallons of waste (Hanlon, 1996i). The minimum waste surface level was 111.25 inches on numerous dates from April 3, 1995 through January 2, 1996. The maximum waste surface level was 114 inches on numerous dates from January 3, 1991 through February 11, 1991. See Appendix E for details on waste surface level. The tank is out of service with interim stabilization and intrusion prevention completed. The tank is listed as a assumed leaker and is passively ventilated (Hanlon, 1996i). A plan view in Appendix F depicts the approximate riser locations as of September 30, 1996. Tank 241-S-104 has 9 risers with four tentatively available for sampling: two 4-inch risers (Nos. 1 and 3) and two 12-inch risers (Nos. 7 and 8) (Lipnicki, 1996).

\subsubsection{Interior Montage of Tank 241-S-104}

The clearest and most recent set of interior tank photographs was taken on December 12, 1984. Other interior tank photographs are available, but only the photographs showing the waste surface were 
used to create a montage. The montage has labels identifying some of the monitoring equipment, piping, and risers in the tank. The montage and photographic information are shown in Appendix G.

2.4.6 Inventory Estimate of Tank 241-S-104

The Inventory Estimate from the Hanford Tank Chemical and Radionuclide Inventories: HDW Model Rev. 4 (Agnew et al., 1997) for Tank 241-S-104 is presented in Appendix H. 


\subsection{Tank 241-S-105}

\subsubsection{Waste and Level History of Tank 241-S-105}

The Waste and Level History sketch in Appendix C presents the waste history and level history of Tank 241-S-105.

\subsubsection{Temperature History of Tank 241-S-105}

Interior tank temperature data for Tank 241-S-105 were recorded by 14 thermocouples attached to one thermocouple tree. Drawing H-2-46175, Rev. 3, indicates that the thermocouple tree is designed as shown on Drawing H-2-34304, Sht. 1, Rev. 6 with 14 thermocouples. The Surveillance Analysis Computer System (SACS) indicates that the thermocouple tree is located in riser 4. In the past, other risers and equipment may have been used to monitor the temperature in the tank. However, the thermocouple tree located at riser 4 is the only source of temperature data for this document. The temperature data were obtained from the SACS database on October 1, 1996. The earliest data retrieved from the SACS were from early January 1991.

Graphs of individual thermocouple data are presented in Appendix D. A graph was created for each thermocouple. The following statistical information was taken from all six thermocouples. The maximum temperature was $82.58^{\circ} \mathrm{F}$ taken by thermocouple 1 on January 30,1996 . The minimum temperature was $58.64^{\circ} \mathrm{F}$ taken by thermocouple 6 on May 20,21 , and 22, 1996. The maximum and minimum temperatures are labeled as good data points within the SACS. The average temperature for all the thermocouples is $73^{\circ} \mathrm{F}$.

\subsubsection{Occurrences for Tank 241-S-105}

No significant occurrences are associated with Tank 241-S-105.

\subsubsection{Current Status of Tank 241-S-105}

Tank 241-S-105 entered service during the second quarter of 1953 (Anderson, 1990) and as of June 30, 1996, stores approximately 456,000 gallons of waste (Hanlon, 1996i). The minimum waste surface level was 155.1 inches on July 8 and 29, 1991. The maximum waste surface level was 158 inches on April 11, 1995. See Appendix E for details on waste surface level. The tank is out of service with interim stabilization and intrusion prevention completed. The tank is listed as sound and is passively ventilated (Hanlon, 1996i). A plan view in Appendix F depicts the approximate riser locations as of September 30, 1996. Tank 241-S-105 has 12 risers with five tentatively available for sampling: three 4-inch risers (Nos. 2, 11 and 16) and two 12-inch risers (Nos. 6 and 8) (Lipnicki, 1996).

\subsubsection{Interior Montage of Tank 241-S-105}

The clearest and most recent set of interior tank photographs was taken on April 12, 1989. Other interior tank photographs are available, but only the photographs showing the waste surface were used to create a montage. The montage has labels identifying some of the monitoring equipment, piping, and risers in the tank. The montage and photographic information are shown in Appendix $G$. 
HNF-SD-WM-ER-323, Rev. 1

2.5.6 Inventory Estimate of Tank 24I-S-105

The Inventory Estimate from the Hanford Tank Chemical and Radiomuclide Inventories: HDW Model Rev. 4 (Agnew et al., 1997) for Tank 241-S-105 is presented in Appendix H. 


\subsection{Tank 241-S-106}

2.6.1 Waste and Level History of Tank 241-S-106

The Waste and Level History sketch in Appendix C presents the waste history and level history of Tank 241-S-106.

\subsubsection{Temperature History of Tank 241-S-106}

Interior tank temperature data for Tank 241 -S-106 were recorded by 14 thermocouples located on one thermocouple tree. Drawing H-2-46175, Rev. 3, indicates that the thermocouple tree is designed as shown on Drawing H-2-34304, Sht. 1, Rev. 6 with 14 thermocouples. The Surveillance Analysis Computer System (SACS) indicates that the thermocouple tree is located in riser 2. In the past, other risers and equipment may have been used to monitor the temperature in the tank. However, the thermocouple tree located at riser 2 is the only source of temperature data for this document. The temperature data were obtained from the SACS database on October 1, 1996. The earliest data retrieved from the SACS were from early January 1991.

Graphs of individual thermocouple data are presented in Appendix D. A graph was created for each thermocouple. The following statistical information was taken from all 14 thermocouples. The maximum temperature was $87^{\circ} \mathrm{F}$ taken by thermocouple 3 on January 1,1992 . The minimum temperature was $62.06^{\circ} \mathrm{F}$ taken by thermocouple 10 on March 19, 1996, March 21, 1996, March 24, 1996, and thermocouple 9 on March 24, 1996. The maximum and minimum temperatures are labeled as good data points within SACS. The average temperature for all the thermocouples is $76^{\circ} \mathrm{F}$.

\subsubsection{Occurrences for Tank 241-S-106}

No significant occurrences are associated with Tank 241-S-106.

\subsubsection{Current Status of Tank 241-S-106}

Tank 241-S-106 entered service during the second quarter of 1953 (Anderson, 1990) and as of June 30, 1996, stores approximately 479,000 gallons of waste (Hanlon, 1996i). The minimum waste surface level was 174.5 inches on April 8 and 22,1991. The maximum waste surface level was 181.06 inches on December 13, 1995. See Appendix E for details on waste surface level. The tank is out of service with partial interim isolation completed. The tank is listed as sound and is passively ventilated (Hanlon, 1996i). A plan view in Appendix F depicts the approximate riser locations as of September 30, 1996. Tank $241-\mathrm{S}-106$ has 12 risers with five tentatively available for sampling: three 4-inch risers (Nos. 11, 14 and 16) and twol2-inch risers (Nos. 6 and 8) (Lipnicki, 1996).

\subsubsection{Interior Montage of Tank 241-S-106}

The clearest and most recent set of interior tank photographs was taken on April 5, 1979. Other interior tank photographs are available, but only the photographs showing the waste surface were used to create a montage. The montage has labels identifying some of the monitoring equipment, piping, and risers in the tank. The montage and photographic information are shown in Appendix $G$. 
HNF-SD-WM-ER-323, Rev. 1

2.6.6 Inventory Estimate of Tank 24l-S-106

The Inventory Estimate from the Hanford Tank Chemical and Radionuclide Inventories: HDW Model Rev. 4 (Agnew et al., 1997) for Tank 241-S-106 is presented in Appendix H. 


\subsection{Tank 241-S-107}

\subsubsection{Waste and Level History of Tank 241-S-107}

The Waste and Level History sketch in Appendix C presents the waste history and level history of Tank 241-S-107

\subsubsection{Temperature History of Tank 241-S-107}

Interior tank temperature data for Tank 241-S-107 were recorded by 6 thermocouples attached to one thermocouple tree. Drawing H-2-90344, Rev. 3, indicates that the thermocouple tree is located in riser 4 and is designed as shown on Drawing H-2-90342, Sht. 2, Rev. 2 with 6 thermocouples. The Surveillance Analysis Computer System (SACS) indicates that the thermocouple tree is also located in riser 4 . In the past, other risers and equipment may have been used to monitor the temperature in the tank. However, the thermocouple tree located at riser 4 was the only source of temperature data for this document. The temperature data were obtained from the SACS database on October 1, 1996. The earliest data retrieved from SACS were from early January 1988.

Graphs of individual thermocouple data are presented in Appendix D. A graph was created for each thermocouple. The following statistical information was taken from all 6 thermocouples. The maximum temperature was $123^{\circ} \mathrm{F}$ taken by thermocouple 1 on May 2, 1988 . The minimum temperature was $78.3^{\circ} \mathrm{F}$ taken by thermocouple 6 on February 13, 1993. The maximum temperature is labeled as a good data point and the minimum temperature is labeled as a good data point within the SACS. The average temperature for all the thermocouples is $101^{\circ} \mathrm{F}$.

\subsubsection{Occurrences for Tank 241-S-107}

An occurrence report was issued in July 1976 because of a liquid level decrease. The decrease was attributed to temperature and evaporative losses.

\subsubsection{Current Status of Tank 241-S-107}

Tank 241-S-107 entered service during the fourth quarter of 1952 (Anderson, 1990) and as of June 30, 1996, stores approximately 376,000 gallons of waste (Hanlon, 1996i). The minimum waste surface level was 142.1 inches on numerous dates from January 1, 1991 through May 16, 1991. The maximum waste surface level was 146.49 inches on September 15, 1996. See Appendix E for details on waste surface level. The tank is out of service with partial interim isolation completed. The tank is listed as sound and is passively ventilated (Hanlon, 1996i). A plan view in Appendix F depicts the approximate riser locations as of September 30, 1996. Tank 241-S-107 has 12 risers with five tentatively available for sampling: four 4 -inch risers (Nos. 2, 11, 14 and 16) and one 12-inch riser (No. 6) (Lipnicki, 1996).

\subsubsection{Interior Montage of Tank 241-S-107}

The clearest and most recent set of interior tank photographs was taken on March 12, 1987 . Other interior tank photographs are available, but only the photographs showing the waste surface were 
used to create a montage. The montage has labels identifying some of the monitoring equipment, piping, and risers in the tank. The montage and photographic information are shown in Appendix $G$.

2.7.6 Inventory Estimate of Tank 241-S-107

The Inventory Estimate from the Hanford Tank Chemical and Radionuclide Inventories: HDW Model Rev. 4 (Agnew et al, 1997) for Tank 241-S-107 is presented in Appendix H. 


\subsection{Tank 241-S-108}

\subsubsection{Waste and Level History of Tank 241-S-108}

The Waste and Level History sketch in Appendix C presents the waste history and level history of Tank 241-S-108.

\subsubsection{Temperature History of Tank 241-S-108}

Interior tank temperature data for Tank $241-S-108$ were recorded by 14 thermocouples located on one thermocouple tree. Drawing H-2-46175, Rev. 3, indicates that the thermocouple tree is designed as shown on Drawing H-2-34304, Sht. 1, Rev. 6 with 14 thermocouples. The Surveillance Analysis Computer System (SACS) indicates that the thermocouple tree is located in riser 4 . In the past, other risers and equipment may have been used to monitor the temperature in the tank. However, the thermocouple tree located at riser 4 is the only source of temperature data for this document. The temperature data were obtained from the SACS database on October 1, 1996. The earliest data retrieved from the SACS were from early January 1991

Graphs of individual thermocouple data are presented in Appendix D. A graph was created for each thermocouple. The following statistical information was taken from all 14 thermocouples. The maximum temperature was $94.64^{\circ} \mathrm{F}$ taken by thermocouple 6 on December 15, 1995. The minimum temperature was $57^{\circ} \mathrm{F}$ taken by thermocouple 14 on July 2,1994 . The maximum and minimum temperatures are labeled as good data points within the SACS. The average temperature for all the thermocouples is $81^{\circ} \mathrm{F}$

\subsubsection{Occurrences for Tank 241-S-108}

No significant occurrences are associated with Tank 241-S-108.

\subsubsection{Current Status of Tank 241-S-108}

Tank 241-S-108 entered service during the fourth quarter of 1952 (Anderson, 1990) and as of June 30, 1996, stores approximately 604,000 gallons of waste (Hanlon, 1996i). The minimum waste surface level was 158.8 inches on April 3, 1995. The maximum waste surface level was 191 inches on February 4, 1991. See Appendix E for details on waste surface level. The tank is out of service with partial interim isolation completed. The tank is listed as sound and is passively ventilated (Hanlon, 1996i). A plan view in Appendix F depicts the approximate riser locations as of September 30, 1996. Tank 241-S-108 has 12 risers with four tentatively available for sampling: two 4-inch risers (Nos. 11 and 14) and two 12-inch risers (Nos. 7, and 8) (Lipnicki, 1996).

\subsubsection{Interior Montage of Tank 241-S-108}

The clearest and most recent set of interior tank photographs was taken on March 12, 1987. Other interior tank photographs are available, but only the photographs showing the waste surface were used to create a montage. The montage has labels identifying some of the monitoring equipment, piping, and risers in the tank. The montage and photographic information are shown in Appendix $G$. 
HNF-SD-WM-ER-323, Rev. 1

2.8.6 Inventory Estimate of Tank 241-S-108

The Inventory Estimate from the Hanford Tank Chemical and Radionuclide Inventories: HDW Model Rev. 4 (Agnew et al., 1997) for Tank 24I-S-108 is presented in Appendix H. 


\subsubsection{Waste and Level History of Tank 241-S-109}

The Waste and Level History sketch in Appendix C presents the waste history and level history of Tank 241-S-109.

\subsubsection{Temperature History of Tank 241-S-109}

Interior tank temperature data for Tank 241-S-109 were recorded by 14 thermocouples located on one thermocouple tree. Drawing H-2-46175, Rev. 3, indicates that the thermocouple tree is designed as shown on Drawing H-2-34304, Sht. 1, Rev. 6 with 14 thermocouples. The Surveillance Analysis Computer System (SACS) indicates that the thermocouple tree is located in riser 4 . In the past, other risers and equipment may have been used to monitor the temperature in the tank. However, the thermocouple tree located at riser 4 is the only source of temperature data for this document. The temperature data were obtained from the SACS database on October 1, 1996. The earliest data retrieved from the SACS were from early January 1991.

Graphs of individual thermocouple data are presented in Appendix D. A graph was created for each thermocouple except for thermocouples 11,13 and 14. Thermocouples Nos. 11, 13 and 14 were not graphed due to a lack of data. The following statistical information was taken from all 14 thermocouples. The maximum temperature was $89.42^{\circ} \mathrm{F}$ taken by thermocouple 3 on January 30, 1996, February 1, 1996, and February 2, 1996. The minimum temperature was $55.3^{\circ} \mathrm{F}$ taken by thermocouple 11 on February 13, 1993. The maximum and minimum temperatures are labeled as good data points within the SACS. The average temperature for all the thermocouples is $79^{\circ} \mathrm{F}$.

\subsubsection{Occurrences for Tank 241-S-109}

No significant occurrences are associated with Tank 241-S-109.

\subsubsection{Current Status of Tank 241-S-109}

Tank 241-S-109 entered service during the fourth quarter of 1952 (Anderson, 1990) and as of June 30,1996 , stores approximately 568,000 gallons of waste (Hanlon, 1996i). The minimum waste surface level was 171.1 inches on July 1, 1995. The maximum waste surface level was 172.8 inches on September 6, 1993. See Appendix E for details on waste surface level. The tank is out of service with partial interim isolation completed. The tank is listed as sound and is passively ventilated (Hanlon, 1996i). A plan view in Appendix F depicts the approximate riser locations as of September 30, 1996. Tank $241-\mathrm{S}-109$ has 12 risers with five tentatively available for sampling: four 4-inch risers (Nos. 2, 11, 14 and 16) and one 12-inch riser (No. 6) (Lipnicki, 1996).

\subsubsection{Interior Montage of Tank 241-S-109}

The clearest and most recent set of interior tank photographs was taken on August 24, 1984. Other interior tank photographs are available, but only the photographs showing the waste surface were used to create a montage. The montage has labels identifying some of the monitoring equipment, piping, and risers in the tank. The montage and photographic information are shown in Appendix $G$. 
HNF-SD-WM-ER-323, Rev. 1

2.9.6 Inventory Estimate of Tank 241-S-109

The Inventory Estimate from the Hanford Tank Chemical and Radionuclide Inventories: HDW Model Rev. 4 (Agnew et al., 1997) for Tank 241-S-109 is presented in Appendix H. 


\subsection{Tank 241-S-110}

\subsubsection{Waste and Level History of Tank 241-S-110}

The Waste and Level History sketch in Appendix C presents the waste history and level history of Tank 241-S-110.

\subsubsection{Temperature History of Tank 241-S-110}

Interior tank temperature data for Tank 241-S-110 were recorded by 6 thermocouples attached to one thermocouple tree. Drawing H-2-90344, Rev. 3, indicates that the thermocouple tree is located in riser 4 and is designed as shown on Drawing H-2-90342, Sht. 2, Rev. 2 with 6 thermocouples. The Surveillance Analysis Computer System (SACS) indicates that the thermocouple tree is also located in riser 4. In the past, other risers and equipment may have been used to monitor the temperature in the tank. However, the thermocouple tree located at riser 4 was the only source of temperature data for this document. The temperature data were obtained from the SACS database on October 1, 1996. The earliest data retrieved from the SACS were from early January 1988.

Graphs of individual thermocouple data are presented in Appendix D. A graph was created for each thermocouple. The following statistical information was taken from all 6 thermocouples. The maximum temperature was $136^{\circ} \mathrm{F}$ taken by thermocouple 1 on May 2, 1988 . The minimum temperature was $72.6^{\circ} \mathrm{F}$ taken by thermocouple 6 on February 13, 1993. The maximum and minimum temperatures are labeled as good data points within the SACS. The average temperature for all the thermocouples is $106^{\circ} \mathrm{F}$.

\subsubsection{Occurrences for Tank 241-S-110}

No significant occurrences are associated with Tank 241-S-110.

\subsubsection{Current Status of Tank 241-S-110}

Tank 241-S-1 10 entered service during the second quarter of 1952 (Anderson, 1990) and as of June 30, 1996, stores approximately 390,000 gallons of waste (Hanlon, 1996i). The minimum waste surface level was 148.2 inches on October 19, 1992. The maximum waste surface level was 149.52 inches on numerous dates from August 26, 1995, through August 30, 1995. See Appendix E for details on waste surface level. The tank is out of service with partial interim isolation completed. The tank is listed as sound and is passively ventilated (Hanlon, 1996i). A plan view in Appendix $F$ depicts the approximate riser locations as of September 30, 1996. Tank 241-S-110 has 12 risers with five tentatively available for sampling: three 4-inch risers (Nos. 11, 14 and 16) and two 12-inch risers (Nos. 7 and 8) (Lipnicki, 1996)

\subsubsection{Interior Montage of Tank 241-S-110}

The clearest and most recent set of interior tank photographs was taken on March 12, 1987. Other interior tank photographs are available, but only the photographs showing the waste surface were used to create a montage. The montage has labels identifying some of the monitoring equipment, piping, and risers in the tank. The montage and photographic information are shown in Appendix $\mathrm{G}$. 
HNF-SD-WM-ER-323, Rev. 1

2.10.6 Inventory Estimate of Tank 241-S-110

The Inventory Estimate from the Hanford Tank Chemical and Radionuclide Inventories: HDW Model Rev. 4 (Agnew et al., 1997) for Tank 241-S-110 is presented in Appendix H. 


\subsection{Tank 241-S-111}

\subsubsection{Waste and Level History of Tank 241-S-111}

The Waste and Level History sketch in Appendix C presents the waste history and level history of Tank 241-S-111.

\subsubsection{Temperature History of Tank 241-S-111}

Interior tank temperature data for Tank 241-S-111 were recorded by 14 thermocouples attached to one thermocouple tree. Drawing H-2-46175, Rev. 3, indicates that the thermocouple tree is designed as shown on Drawing H-2-34304, Sht. 1, Rev. 6 with 14 thermocouples. The Surveillance Analysis Computer System (SACS) indicates that the thermocouple tree is located in riser 4 . In the past, other risers and equipment may have been used to monitor the temperature in the tank. However, the thermocouple tree located at riser 4 is the only source of temperature data for this document. The temperature data were obtained from the SACS database on October 1, 1996. The earliest data retrieved from the SACS were from early January 1991.

Graphs of individual thermocouple data are presented in Appendix D. A graph was created for each thermocouple. The following statistical information was taken from all 14 thermocouples. The maximum temperature was $97^{\circ} \mathrm{F}$ taken by thermocouple 1 on March 9, 1991, March 24, 1991, March 31, 1991, April 6, 1991, April 14, 1991, December 14, 1991, December 22, 1991, January 5, 1992, March 1, 1992; thermocouple 2 on March 9, 1991, March 24, 1991, December 14, 1991, December 22, 1991, January 5, 1992, March 1, 1992; thermocouple 3 on March 9, 1991, December 14, 1991, December 22, 1991, January 5, 1992, March 1, 1992. The minimum temperature was $65.2^{\circ} \mathrm{F}$ taken by thermocouple 14 on April 18, 1992. The maximum and minimum temperatures are labeled as good data points within the SACS. The average temperature for all the thermocouples is $83^{\circ} \mathrm{F}$.

\subsubsection{Occurrences for Tank 241-S-111}

No significant occurrences are associated with Tank 241-S-111.

\subsubsection{Current Status of Tank 241-S-111}

Tank 241-S-111 entered service during the third quarter of 1952 (Anderson, 1990) and as of June 30, 1996, stores approximately 596,000 gallons of waste (Hanlon, 1996i). The minimum waste surface level was 202.5 inches on numerous dates from February 11, 1991 through March 13, 1994. The maximum waste surface level was 204.255 inches on December 12, 1995. See Appendix E for details on waste surface level. Tank 241-S-111 was added to the Hydrogen Watch List in January 1991 and Organics Watch List in May 1994. The tank is out of service with partial interim isolation completed. The tank is listed as sound and is passively ventilated (Hanlon, 1996i). A plan view in Appendix F depicts the approximate riser locations as of September 30, 1996. Tank 241-S-111 has 12 risers with five tentatively available for sampling: two 4-inch risers (Nos. 11 and 14) and three 12-inch risers (Nos. 6, 7 and 8) (Lipnicki, 1996). 
2.11.5 Interior Montage of Tank 241-S-111

The clearest and most recent set of interior tank photographs was taken on August 10, 1989 . Other interior tank photographs are available, but only the photographs showing the waste surface were used to create a montage. The montage has labels identifying some of the monitoring equipment, piping, and risers in the tank. The montage and photographic information are shown in Appendix $G$.

2.11.6 Inventory Estimate of Tank 241-S-111

The Inventory Estimate from the Hanford Tank Chemical and Radionuclide Inventories: HDW Model Rev. 4 (Agnew et al., 1997) for Tank 241-S-111 is presented in Appendix H. 


\subsection{Tank 241-S-112}

2.12.1 Waste and Level History of Tank 241-S-112

The Waste and Level History sketch in Appendix C presents the waste history and level history of Tank 241-S-112.

\subsubsection{Temperature History of Tank 241-S-112}

Interior tank temperature data for Tank 241-S-112 were recorded by 14 thermocouples attached to one thermocouple tree. Drawing H-2-46175, Rev. 3, indicates that the thermocouple tree is designed as shown on Drawing H-2-34304, Sht. 1, Rev. 6 with 14 thermocouples. The Surveillance Analysis Computer System (SACS) indicates that the thermocouple tree is located in riser 4. In the past, other risers and equipment may have been used to monitor the temperature in the tank. However, the thermocouple tree located at riser 4 is the only source of temperature data for this document. The temperature data were obtained from the SACS database on October 1, 1996. The earliest data retrieved from SACS were from late March 1991.

Graphs of individual thermocouple data are presented in Appendix D. A graph was created for each thermocouple. The following statistical information was taken from the 14 thermocouples that had temperature data. The maximum temperature was $92^{\circ} \mathrm{F}$ taken by thermocouple 4 on April 18, 1991, and thermocouple 6 on March 26, 1992. The minimum temperature was $50^{\circ} \mathrm{F}$ taken by thermocouple 14 on March 19,1992. The maximum and minimum temperatures are labeled as good data points within the SACS. The average temperature for all the thermocouples is $78^{\circ} \mathrm{F}$.

\subsubsection{Occurrences for Tank 241-S-112}

No significant occurrences are associated with Tank 241-S-112.

\subsubsection{Current Status of Tank 241-S-112}

Tank 241-S-112 entered service during the third quarter of 1952 (Anderson, 1990) and as of June 30, 1996, stores approximately 523,000 gallons of waste (Hanlon, 1996i). The minimum waste surface level was 194.9 inches on July 22,1991 . The maximum waste surface level was 197.8 inches on April 11, 1995. See Appendix E for details on waste surface level. Tank 241-S-112 was added to the Hydrogen Watch List in January 1991. The tank is out of service with partial interim isolation completed. The tank is listed as sound and is passively ventilated (Hanlon, 1996i). A plan view in Appendix F depicts the approximate riser locations as of September 30, 1996. Tank 241-S-112 has 12 risers with five tentatively available for sampling: four 4-inch risers (Nos. 2, 11, 14 and 16) and one 12-inch riser (No. 6) (Lipnicki, 1996).

\subsubsection{Interior Montage of Tank 241-S-112}

The clearest and most recent set of interior tank photographs was taken on March 24, 1987. Other interior tank photographs are available, but only the photographs showing the waste surface were used to create a montage. The montage has labels identifying some of the monitoring equipment, piping, and risers in the tank. The montage and photographic information are shown in Appendix G. 
2.12.6 Inventory Estimate of Tank 241-S-112

The Inventory Estimate from the Hanford Tank Chemical and Radionuclide Inventories: $H D W$ Model Rev. 4 (Agnew et al., 1997) for Tank 241-S-112 is presented in Appendix H. 


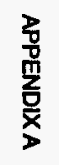


THIS FAGE INTENTIONALLY LEFT BLANK 


\section{GLOSSARY}

This glossary of Hanford terminology has been compiled from numerous sources. A lot of the terms have come from Anderson (1990), Jungfleisch (1984) and Agnew et al. (1997). These definitions may conflict with other sources.

$1 \mathrm{C}$

$1 \mathrm{Cl}$

$1 \mathrm{C} 2$

$1 \mathrm{CFeCN}$

224

$2 \mathrm{C}$

$2 \mathrm{C} 1$

$2 \mathrm{C} 2$

AlSltCk

A2SItSlr

Active Drywell
First-cycle decontamination waste from the bismuth phosphate $\left(\mathrm{BiPO}_{4}\right)$ process at $\mathrm{B}$ and $\mathrm{T}$ Plants consisting of by-products co-precipitated from a solution containing plutonium (contains $10 \%$ of the original fission product activity and $2 \%$ of the products). By-product cake solution was mixed with product waste and neutralized with $50 \%$ caustic. Coating waste from removing aluminum fuel element cladding was added and comprised about $24 \%$ of the waste.

First-cycle decontamination waste from the bismuth phosphate $\left(\mathrm{BiPO}_{4}\right.$ ) process, 1944-49 (LANL defined waste \#3)

First-cycle decontamination waste from the bismuth phosphate $\left(\mathrm{BiPO}_{4}\right.$ ) process, 1950-56 (LANL defined waste \#4)

Ferrocyanide sludge produced by in-plant scavenging of $1 \mathrm{C}$ supernatant wastes (LANL defined waste \#12)

224-U Waste. $\mathrm{LaF}_{3}$ finishing waste from $\mathrm{BiPO}_{4}$ process and uranium recovery in the 224 buildings by T Plant and B Plant and the Plutonium Finishing Plant (LANL defined waste \#7)

Second-cycle decontamination waste from the bismuth phosphate $\left(\mathrm{BiPO}_{4}\right)$ process at $\mathrm{B}$ and T Plants (see second-cycle decontamination waste)

Second-cycle decontamination waste from the bismuth phosphate $\left(\mathrm{BiPO}_{4}\right)$ process, 1944-49 (LANL defined waste \#5)

Second-cycle decontamination waste from the bismuth phosphate $\left(\mathrm{BiPO}_{4}\right)$ process, $1950-56$ (LANL defined waste \#6)

Salt cake waste generated from the 242-A EvaporatorCrystallizer from 1977 until 1980.

Salt slurry waste generated from the 242-A EvaporatorCrystallizer from 1981 until 1994.

Drywell in which radiation readings of greater than 50 counts per second are detected. The readings must be consistent as to 
depth and radiation level for repeated readings to be considered active.

Airlift Circulator

AR

Assumed Leaker

B

BG

BL

BM

BNW

BSLTCK

BYSLTCK

Cascade

Catch Tanks
A device installed in aging waste tanks to promote mixing of the supernate. By maintaining motion within the body of the liquid, the circulators minimize superheat buildup and, consequently, minimize burping

Washed PUREX sludge from the 244-AR Vault (LANL defined waste \#31)

A waste storage tank for which past surveillance data has indicated a loss of liquid attributed to a breach of integrity. In 1984, the designations of "suspect leaker," "questionable integrity," "confirmed leaker," "declared leaker," "dormant", and "borderline" were merged into one category called "assumed leaker."

High-level waste from PUREX acidified waste processed through B Plant to extract strontium (LANL defined waste \#32)

Below grade

B Plant low-level waste beginning 1968 (LANL defined waste \#33)

Bench mark

Battelle Northwest Laboratory waste

Salt cake waste generated from the 242-B Evaporator, 1951-53 (LANL defined waste \#41)

Salt cake waste generated from in-tank solidification units 1 and 2 in BY Tank Farm, 1965-74 (LANL defined waste \#44)

Eleven of the single-shell tank farms (all except the AX Tank Farm) were equipped with overflow lines between tanks. The tanks were connected in series and were placed at different elevations creating a downhill gradient for liquids to flow (cascade) from one tank to another. Thus, multiple tanks could be filled with one pump.

Small capacity single-shell tanks associated with diversion boxes and diverter stations. The tanks are designed to receive 
$\mathrm{CC}$

CEM

Crib

CSR

$\mathrm{CW}$

CWP1

CWP2

CWP/ZR

CWR1

CWR2

CWZRI

CWZR2

$\mathrm{DE}$ any transfer line clean out, spills or leakage from the boxes, or leakage from the adjacent pipe encasement.

Complexant concentrate waste or concentrated complexant; concentrated product from evaporating dilute complexed waste which contained high concentrations of organic complexants, such as HEDTA, EDTA, and citric acid.

Cement (LANL defined waste \#37)

An underground structure filled with aggregate designed to receive liquid waste, usually through a perforated pipe. The filtration and ion exchange properties of the soil in and around the crib were used to contain the radionuclides.

Waste (supernate) from cesium recovery of tank supernate at B Plant (LANL defined waste \#35)

Coating (cladding) waste produced at PUREX from dissolution of Zircaloy or aluminum fuel cladding.

Coating (cladding) waste (PUREX); (LANL defined waste \#21, CWP/Al, 1956-60)

Cladding (coating) waste (PUREX), (LANL defined waste \#22, CWP/Al, 1961-72)

Now called PD or NCRW

REDOX cladding (coating) waste, (LANL defined waste \#15, CWR/Al, 1952-60)

Coating (cladding) waste (REDOX), (LANL defined waste \#16, CWL/Al with some Zr, 1961-72)

Coating (cladding) waste (PUREX), Zircaloy cladding; 1968-72 (LANL defined waste \#23)

Coating (cladding) waste (PUREX), Zircaloy cladding; 1983-88 (LANL defined waste \#47); see NCRW and PD; also known as CWP/ZR2

Diatomaceous Earth; Diatomite $\left(\mathrm{SiO}_{2}\right)$; a light friable siliceous material derived from diatom (algal) remains; added to some underground waste storage tanks to absorb residual liquids. (LANL defined waste \#36) 
Ditch

Diversion Box

DSSF

Drywell

DW

EB

EF

EVAP

Evaporator Feed

Ferrocyanide

FIC

HDRL

HS

ILL
A linear excavation often used for the temporary diversion or disposal of process waste streams.

A below grade, concrete enclosure containing the remotely maintained jumpers and spare nozzles for routing waste solution to storage tank farms.

Double-shell slurry feed; Waste concentrated in evaporators until the solution is nearly saturated with sodium aluminate without exceeding receiver tank composition limits. This form is not as concentrated as double-shell slurry.

A steel casing, generally 6-inch internal diameter, drilled into the ground to various depths (but do not reach the water table) and used to insert monitoring instruments for measuring the presence of radioactivity or moisture content.

Decontamination waste; a wash solution from equipment decontamination at T Plant (LANL defined waste \#39)

Evaporator bottoms; a slurry from the evaporators

Evaporator feed; various supernatant liquids whose composition depends on the source

Evaporator feed (post 1976 designation)

Any waste liquid that can be concentrated to form salt cake; e.g., aged waste, low heat waste, dilute interstitial liquor, and other radioactive waste solutions.

An ion composed of iron and cyanide with the chemical formula of $\mathrm{Fe}(\mathrm{CN})_{6}^{-4}$.

Food Instrument Corporation, waste surface level device

Hanford defense residual liquor; late 1970s designation for terminal liquors remaining after evaporation; includes complexed and noncomplexed waste, partially neutralized waste, and DSSF (see RESID).

Hot Semiworks (C Plant); a pilot facility with a variety of operations. Also, Hot or Strontium Semiworks waste (LANL defined waste \#28); see SSW.

Interstitial liquid level 
Inactive Tank

Interim Isolation

Interim Stabilization

Interstitial Liquid

Intrusion Prevention

IX

Knuckle

Level Adjustment

Liquid Observation Well (LOW)

LW

MH
A tank that has been removed from liquid-processing service, has been pumped to less than 33,000 gallons of waste, and will be, or is in the process of being, stabilized followed by intrusion prevention. This includes all tanks not in active or active-restricted categories. Also included are inactive spare tanks that would be used if an active tank failed.

An administrative designation reflecting the completion of the physical effort required to minimize the addition of liquids into an inactive storage tank, process vault, sump, catch tank, or diversion box. (In June 1993, "interim isolation" was replaced by "intrusion prevention".)

A tank which contains less than 50,000 gallons of drainable interstitial liquid and less than 5,000 gallons of supernate. If a jet pump was used to achieve interim stabilization, then the jet pump flowrate must have been at or below 0.05 gallons per minute before interim stabilization was completed

The interstitial liquid within the tanks is the liquid that fills the interstitial (voids) spaces of the solid waste.

An administrative designation reflecting the completion of the physical effort required to minimize the addition of liquids into an inactive storage tank, process vault, catch tank, sump, or diversion box.

Ion exchange waste from the cesium recovery process at B Plant

Point where the side wall and the bottom curved surface of a tank meet.

Any update in the waste inventory (or tank level) in a tank. The adjustments usually result from surveillance observations or historical investigations.

A liquid observation well is a fiber glass or tefzel-reinforced epoxy-polyester resin, $89 \mathrm{~mm}$ ( 3.5 inches) diameter pipe that is capped on the bottom end. This end is placed within $25 \mathrm{~mm}$ ( 1 inch) above the bottom portion of the steel tank liner. Three types of probes are used in the LOW to monitor changes in the interstitial liquid level: acoustic, gamma, and neutron.

Laboratory waste from the 222-S Building

Manhole 
Mixed Waste

MW

MW1

MW2

$\mathrm{N}$

NCAW

NCPLX

NCRW

NIT

Non-Complexed

OBSV Port

Out-of-Service-Tank
Waste containing both radioactive and hazardous (dangerous as defined in WAC 173-303) waste.

Waste from the bismuth phosphate process (which extracted plutonium) containing all the uranium, approximately $90 \%$ of the original fission product activity, and approximately $1 \%$ of the product. This waste was brought to the neutral point with $50 \%$ caustic and then treated with an excess of sodium carbonate. This procedure yielded almost completely soluble waste at a minimum total volume. The exact composition of the carbonate compounds was not known, but was assumed to be a uranium phosphate carbonate mixture. The term "metal" was the code word for plutonium.

Metal waste from $\mathrm{BiPO}_{4}, 1944-49$ (LANL defined waste \#1, same as MW)

Metal waste from $\mathrm{BiPO}_{4}, 1950-56$ (LANL defined waste $\# 2$, same as MW)

Phosphate decontamination waste from N Reactor (LANL defined waste \#40)

Neutralized current acid waste, primary high-level waste stream from PUREX process (LANL defined waste $\# 45$, formerly P3, 1983-88)

Non-complexed waste; general term for supernates and salt well liquors that did not contain organic complexants.

Neutralized cladding removal waste, same as CWP/Zr (LANL defined waste \# 47, formerly CWZr2).

$\mathrm{HNO}_{3} / \mathrm{KMNO}_{4}$ solution added during evaporator operation (LANL defined waste \#38)

General waste term applied to all Hanford Site liquors not identified as complexed (containing organics).

Observation Port

A tank that does not meet the definition of an in-service tank Before September 1988, these tanks were defined as inactive. (Note: All single-shell tanks are out of service.) 
OWW

OWW1

OWW2

OWW3

P

P1

P2

P2'

P3

Partial Interim Isolation

PASF

PFeCN1

PFeCN2

$\mathrm{pH}$

PL

PL1

PL2
Organic Wash Waste; The solvent used in PUREX was treated before reuse by washing with potassium permanganate and sodium carbonate, followed by dilute nitric acid.

Organic wash waste, 1956-62, also known as CARB (LANL defined waste \#24)

Organic wash waste, 1963-67 (LANL defined waste \#25)

Organic wash waste, 1968-72 (LANL defined waste \#26)

High-level neutralized acid waste from PUREX

PUREX high-level waste, 1956-62 (LANL defined waste \#17)

PUREX high-level waste, 1963-67 (LANL defined waste \#18)

1968-1972, assigned to P2. (LANL defined waste \#19)

1983-1988, now called PXNAW or NCAW. (LANL defined waste \#45)

The administrative designation for completing the physical effort required for interim isolation, except for isolating the risers and piping that will be required for jet pumping or for other methods of stabilization.

PUREX ammonia scrubber feed (LANL defined waste \#48)

Ferrocyanide sludge produced by in-plant scavenging (using $0.005 \mathrm{M}$ ferrocyanide) of waste from uranium recovery (LANL defined waste \#9)

Same as PFeCN1 except $0.0025 \mathrm{M}$ ferrocyanide used (LANL defined waste \#10)

A measure of the hydrogen ion concentration in solution.

Low-level waste from PUREX

PUREX low-level waste (LANL defined waste \#20)

1983-88, now called PXMSC, among other things. (LANL defined waste \#46) 
PNF

Primary Addition

PXMSC

PXNAW

R

R1

R2

RESID

Riser

RIX

RSLTCK

S1SltCk

S2SltSlr

SACS

Salt Cake

Salt Well

Scavenged Waste
Partial Neutralization Feed. Indicates addition of nitric acid at an evaporator in an attempt to produce more salt cake during volume reduction.

An addition of waste from a specific plant or process vault

Dilute, non-complexed waste from PUREX misc. streams (LANL defined waste \#46, formerly PL2)

Aging waste from PUREX high level waste; see NCAW (LANL defined waste \#45, formerly P3, 1983-88)

High-level waste from REDOX

REDOX waste, 1952-57 (LANL defined waste \#13)

REDOX waste, 1958-66 (LANL defined waste \#14)

Hanford defense residual liquor (see HDRL)

A vertical pipe through a tank dome (access to the tank interior).

REDOX ion exchange waste produced at B Plant by extracting cesium from REDOX supernate

Salt-cake waste from the REDOX concentrator (LANL defined waste \#43)

Salt cake waste generated from the 242-S Evaporator/Crystallizer from 1973 until 1976.

Salt cake waste generated from the 242-S Evaporator/Crystallizer from 1977 until 1980.

Surveillance analysis computer system

Crystallized nitrate and other salts deposited in waste tanks, usually after the waste is concentrated by evaporation.

A hole drilled or sluiced into salt cake and lined with a cylindrical screen to permit drainage and jet pumping of interstitial liquids.

Waste which has been treated with ferrocyanide to remove cesium from the supernate by precipitating it into a sludge. 
Self-Concentrating Waste

Sluicing, or sluiced

Slurry

SMM

SMP

SMMSI

SMMS2

SMMT2

Sound

SRR

SST

Stabilization

Supernatant or Supernate

T1SLTCK

T2SLTCK

Tank Farm
Liquid, high-level radioactive waste whose decaying radionuclides heat the solution sufficiently to boil off (i.e., evaporate) the water, thus concentrating the waste

To wash with water. At Hanford, this has meant to dissolve or suspend waste in solution using a high pressure water stream.

Insoluble material suspended in water or aqueous solution.

Supernatant Mixing Model (created at LANL) that calculates the composition of tank liquids and concentrates as linear combinations of supernates

Sludge measuring port

Solids from concentrate caiculated by SMM.

Solids from concentrate calculated by SMM.

Solids from concentrate calculated by SMM.

The integrity classification of a waste storage tank for which surveillance data indicate no loss of liquid attributed to a breach of integrity.

Sluiced PUREX sludge from A and AX Tank Farms sent to B Plant to recover strontium from 1967-76 (LANL defined waste \#34). The sludge returned from $B$ Plant was sent to the AR Vault and the supernate was sent to 241-C-105.

Single-shell tank

The removal or immobilization, as completely as possible, of the liquid contained in a radioactive waste storage tank by pumping via a salt well, adding diatomaceous earth, etc.

Liquid floating above the solids in the waste storage tanks. Supernate is usually derived by subtracting the solids level measurement from the liquid level measurement.

Salt-cake waste generated from the 242-T Evaporator, 1951-56 (LANL defined waste \#42)

Salt-cake waste generated from the 242-T Evaporator, 1965-76

An area containing underground storage tanks for storing waste. 
Terminal Liquor

$\mathrm{TFeCN}$

TH1

TH2

Thermocouple

Thermocouple Tree

Thorium

TL

TLM

Trench

TRUEX
The concentrated supernatant liquid decanted from the evaporator bottoms (produced by the evaporators), which may not be concentrated further without forming solids that was unacceptable for storage in single-shell tanks (see HDRL). Terminal liquor is characterized by a caustic concentration of approximately $5.5 \mathrm{M}$ (the caustic molarity was lower if the aluminum salt saturation was reached first).

Ferrocyanide sludge produced by in-tank or in-farm scavenging (LANL defined waste \#11)

Thoria high-level or cladding waste, 1966 (LANL defined waste \#29, formerly TH66)

Thoria high-level or cladding waste, 1970 (LANL defined waste $\# 30$, formerly $\mathrm{TH} 70$ )

Thermocouples are simple devices that develop a millivoltage when parts of the thermocouple are exposed to temperature differentials. The millivoltage can be converted to a temperature reading based upon a specific voltage versus temperature curve inherent to the type of thermocouple being used. Thermocouples are attached to a fabricated assembly called a thermocouple tree

Thermocouples are attached to a fabricated assembly called a thermocouple tree. The number of thermocouples attached to the tree varies as a function of the depth of the tank as well as the thermocouple tree design. The thermocouples are spaced at intervals, along trees that have many thermocouples, so that a vertical temperature profile of the tank contents can be developed. The thermocouple tree is installed in a riser and left in place inside the tank.

A chemical element which is fertile material. Fertile means that when it is subjected to radiation in a nuclear reactor, it will be converted, in this case, to ${ }^{233} \mathrm{U}$, a potential fuel.

Terminal Liquor

Tank Layer Model (created at LANL and derived from Waste Status and Transaction Record Summary (Agnew et al., 1995) database) models the volumes of wastes in the tanks.

A linear excavation used for the disposal of solid waste.

Transuranic extraction process 
UNK

UR

Watch List Tank

WC

WESF

WTR

Wyden Amendment

Z
Unknown waste type (LANL defined waste)

Uranium recovery operation in U Plant, 1952-57. Created uranium recovery waste (UR) (LANL defined waste \#8), also known as tributyl phosphate (TBP) waste, and FeCN (scavenging wastes). See TFeCN and PFeCN.

An underground storage tank requiring special safety precautions because the tank potentially could release highlevel radioactive waste if uncontrolled increases in pressure or temperature occur. Special restrictions have been placed on the tanks by "Safety Measures for Waste Tanks at Hanford Nuclear Reservation," Section 3137, National Defense Authorization Act for Fiscal Year 1991, November 5, 1990, Public Law 101-501 (also called the Wyden Amendment).

Weather cover

Waste Encapsulation and Storage Facility

Water; flush water from miscellaneous sources.

See watch list tank.

Waste discharged from Z Plant (PFP) (LANL defined waste \#27) 



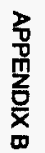


THIS PAGE INTENTIONALLY LEFT BLANK 


\section{REFERENCES}

Agnew, S.F., R.A. Corbin, T.B. Duran, K.A. Jurgensen, T.P. Ortiz, and B.L. Young; September 1995; Waste Status and Transaction Record Summary for the Southwest Quadrant; WHC-SD-WM-TI-614, Rev. 1; Los Alamos National Laboratory, Los Alamos, New Mexico.

Agnew, S.F., J. Boyer, R.A. Corbin, T.B. Duran, J. FitzPatrick, K.A. Jurgensen, T.P. Ortiz, and B.L. Young; 1997; Hanford Tank Chemical and Radionuclide Inventories: HDW Model Rev. 4; LA-UR-96-3860; Los Alamos National Laboratory, Los Alamos, New Mexico.

Alstad, A.T.; December 1993; Riser Configuration Document for Single-Shell Waste Tanks; WHC-SD-RE-TI-053, Rev. 9; Westinghouse Hanford Company, Richland, Washington.

Anderson, J.D.; June 1990; A History of the 200 Areas Tank Farms; WHC-MR-0132; Westinghouse Hanford Company, Richland, Washington.

Ballinger, M.Y., and R.B. Hall; March 1991; A History of Major Hanford Facilities and Processes Involving Radioactive Material; PNL-6964 HEDR; Pacific Northwest Laboratory, Richland, Washington.

Borsheim, G.L., and N.W. Kirch; March 1991; Summary of Single-Shell Tank Waste Stability; WHC-EP-0347; Westinghouse Hanford Company, Richland, Washington.

Brevick, C.H., and J. L. Stroup; January 1997a; Supporting Document for the Historical Tank Content Estimate for SX Tank Farm; HNF-SD-WM-ER-324, Rev. 1; Fluor Daniel Northwest, Inc., Richland, Washington.

Brevick, C.H., and J. L. Stroup; January 1997b; Supporting Dacument for the Historical Tank Content Estimate for U Tank Farm; HNF-SD-WM-ER-325, Rev. 1; Fluor Daniel Northwest, Inc., Richland, Washington.

Brevick, C.H., and J. L. Stroup; January 1997c; Historical Tank Content Estimate for the Southwest Quadrant of the Hanford 200 West Area; HNF-SD-WM-ER-352, Rev. 1; Fluor Daniel Northwest, Inc., Richland, Washington.

Brevick, C.H., L. A. Gaddis, and W. W. Pickett; June 1994; Historical Tank Content Estimate for the Southwest Quadrant of the Hanford 200 West Area; WHC-SD-WM-ER-352, Rev. Ob; ICF Kaiser Hanford, Richland, Washington.

Brevick, C.H., L.A. Gaddis, and A. C. Walsh; June 1994; Supporting Document for the Historical Tank Content Estimate for S Tank Farm; WHC-SD-WM-ER-323, Rev. 0; ICF Kaiser Hanford, Richland, Washington.

Carter, G.J.; May 1986a; Waste Status Summary, April 1986; RHO-RE-SR-14; Rockwell Hanford Operations, Richland, Washington.

Carter, G.J.; June 1986b; Waste Status Summary, May 1986; RHO-RE-SR-14; Rockwell Hanford Operations, Richland, Washington. 
Carter, G.J.; July 1986c; Waste Status Summary, June 1986; RHO-RE-SR-14; Rockwell Hanford Operations, Richland, Washington.

Carter, G.J ; August 1986d; Waste Status Summary, July 1986; RHO-RE-SR-14; Rockwell Hanford Operations, Richland, Washington.

Carter, G.J.; September 1986e; Waste Status Summary, August 1986; RHO-RE-SR-14; Rockwell Hanford Operations, Richland, Washington.

Carter, G.J.; October 1986f; Waste Status Summary, September 1986; RHO-RE-SR-14; Rockwell Hanford Operations, Richland, Washington.

Caudill H.L.; June 1965; Design Criteria for a Second In-Tank Waste Solidification System; RL-SEP-499; General Electric Company, Richland, Washington.

Caudill H.L.; March 1967; Design Criteria Modifications for Increased Capacity in the First In-Tank Solidification System; ISO-869; Isochem, Inc., Richland, Washington.

Dougherty, L.F.; March 23, 1995; Single-Shell Tank Interim Operational Safety Requirements; WHC-SD-WM-OSR-005, Rev. OB; Westinghouse Hanford Company, Richland, Washington.

Duncan, D.R., B.A. Mayancsik, J.A. Pottmeyer, E.J. Vejvoda, J.A. Reddick, K.M. Sheldon, and M.I. Weyns; February 1993; Characterization of Past and Present Solid Waste Streams from the Plutonium Finishing Plant; WHC-EP-0621; Westinghouse Hanford Company, Richland, Washington.

Eberlein, S. J., J. E. Meacham, H. Babad, R. J. Cash, G. T. Dukelow, D. W. Hamilton, G. D. Johnson, J. W. Osborne, M. A. Payne, and D. A. Sherwood; March 1995; Approach for Tank Safety Characterization of Hanford Site Waste; WHC-EP-0843; Westinghouse Hanford Company, Richland, Washingtion.

Escobar, G.A.; December 1986a; Waste Status Summary, October 1986; RHO-RE-SR-14; Rockwell Hanford Operations, Richland, Washington.

Escobar, G.A; December 1986b; Waste Status Summary, November 1986; RHO-RE-SR-14; Rockwell Hanford Operations, Richland, Washington.

Escobar, G.A.; January 1987a; Waste Status Summary, December 1986; RHO-RE-SR-14; Rockwell Hanford Operations, Richland, Washington.

Escobar, G.A.; February 1987b; Waste Status Summary, January 1987; RHO-RE-SR-14; Rockwell Hanford Operations, Richland, Washington.

Gerber, M.S.; September 1992; Legend and Legacy: Fifty Years of Defense Production at the Hanford Site; WHC-MR-0293, Rev. 2; Westinghouse Hanford Company, Richland, Washington.

Gerber, M.S ; October 1993a; The Hanford Site: An Anthology of Early Histories; WHC-MR-0435; Westinghouse Hanford Company, Richland, Washington. 
Gerber, M.S.; November 1993b; A Brief History of the PUREX and UO Facilities; WHC-MR-0437; Westinghouse Hanford Company, Richland, Washington.

Gerber, M.S.; April 1994; Dramatic Change at T Plant; WHC-MR-0452; Westinghouse Hanford Company, Richland, Washington.

Gerber, M.S.; 1996; Correspondence to C.V. Salois of ICF Kaiser Hanford; Westinghouse Hanford Company, Richland, Washington.

Godfrey, W.L.; June 1965; 242-T Evaporator Facility, Information Manual; RL-SEP-396; Hanford Atomic Products Operation, General Electric Company, Richland, Washington.

Hanlon, B.M.; March 1990a; Tank Farm Surveillance and Waste Status Summary Report for November 1989; WHC-EP-0182-20; Westinghouse Hanford Company, Richland, Washington.

Hanlon, B.M.; March 1990b; Tank Farm Surveillance and Waste Status Summary Report for December 1989; WHC-EP-0182-21; Westinghouse Hanford Company, Richland, Washington.

Hanlon, B.M.; March 1990c; Tank Farm Surveillance and Waste Status Summary Report for Jamuary 1990; WHC-EP-0182-22; Westinghouse Hanford Company, Richland, Washington.

Hanlon, B.M; April 1990d; Tank Farm Surveillance and Waste Status Summary Report for February 1990; WHC-EP-0182-23; Westinghouse Hanford Company, Richland, Washington.

Hanlon, B.M.; June 1990e; Tank Farm Surveillance and Waste Status Summary Report for March 1990; WHC-EP-0182-24; Westinghouse Hanford Company, Richland, Washington.

Hanlon, B.M.; June 1990f, Tank Farm Surveillance and Waste Status Summary Report for April 1990; WHC-EP-0182-25; Westinghouse Hanford Company, Richland, Washington.

Hanlon, B.M; August 1990g; Tank Farm Surveillance and Waste Status Summary Report for May 1990; WHC-EP-0182-26; Westinghouse Hanford Company, Richland, Washington

Hanlon, B.M.; September 1990h; Tank Farm Surveillance and Waste Status Summary Report for June 1990; WHC-EP-0182-27; Westinghouse Hanford Company, Richland, Washington.

Hanlon, B.M.; November 1990i; Tank Farm Surveillance and Waste Status Summary Report for July 1990; WHC-EP-0182-28; Westinghouse Hanford Company, Richland, Washington.

Hanlon, B.M; January 1991a; Tank Farm Surveillance and Waste Status Summary Report for August 1990; WHC-EP-0182-29; Westinghouse Hanford Company, Richland, Washington.

Hanlon, B.M.; January 1991b; Tank Farm Surveillance and Waste Status Summary Report for November 1990; WHC-EP-0182-32; Westinghouse Hanford Company, Richland, Washington.

Hanlon, B.M.; February 1991c; Tank Farm Surveillance and Waste Status Summary Report for September 1990; WHC-EP-0182-30; Westinghouse Hanford Company, Richland, Washington. 
Hanlon, B.M.; February 1991d; Tank Farm Surveillance and Waste Status Summary Report for October 1990; WHC-EP-0182-31; Westinghouse Hanford Company, Richland, Washington.

Hanlon, B.M; February 1991e; Tank Farm Surveillance and Waste Status Summary Report for Jamuary 1991; WHC-EP-0182-34; Westinghouse Hanford Company, Richland, Washington.

Hanlon, B.M.; April 1991f, Tank Farm Surveillance and Waste Status Summary Report for December 1990; WHC-EP-0182-33; Westinghouse Hanford Company, Richland, Washington.

Hanlon, B.M; May 1991g; Tank Farm Surveillance and Waste Status Summary Report for February 1991; WHC-EP-0182-35; Westinghouse Hanford Company, Richland, Washington.

Hanlon, B.M; June 1991h; Tank Farm Surveillance and Waste Status Summary Report for March 1991; WHC-EP-0182-36; Westinghouse Hanford Company, Richland, Washington.

Hanlon, B.M.; July 1991i; Tank Farm Surveillance and Waste Status Summary Report for April 1991; WHC-EP-0182-37; Westinghouse Hanford Company, Richland, Washington.

Hanlon, B.M.; August 1991j; Tank Farm Surveillance and Waste Status Summary Report for May 1991; WHC-EP-0182-38; Westinghouse Hanford Company, Richland, Washington.

Hanlon, B.M.; September 1991k; Tank Farm Surveillance and Waste Status Summary Report for June 1991; WHC-EP-0182-39; Westinghouse Hanford Company, Richland, Washington.

Hanlon, B.M:; September 19911; Tank Farm Surveillance and Waste Status Summary Report for July 1991; WHC-EP-0182-40; Westinghouse Hanford Company, Richland, Washington.

Hanlon, B.M.; October 1991m; Tank Farm Surveillance and Waste Status Summary Report for August 1991; WHC-EP-0182-41; Westinghouse Hanford Company, Richland, Washington.

Hanlon, B.M.; November 1991n; Tank Farm Surveillance and Waste Status Summary Report for September 1991; WHC-EP-0182-42; Westinghouse Hanford Company, Richland, Washington.

Hanlon, B.M; January 1992a; Tank Farm Surveillance and Waste Status Summary Report for October 1991; WHC-EP-0182-43; Westinghouse Hanford Company, Richland, Washington.

Hanlon, B.M; February 1992b; Tank Farm Surveillance and Waste Status Summary Report for November 1991; WHC-EP-0182-44; Westinghouse Hanford Company, Richland, Washington.

Hanlon, B.M.; March 1992c; Tank Farm Surveillance and Waste Status Summary Report for December 1991; WHC-EP-0182-45; Westinghouse Hanford Company, Richland, Washington.

Hanlon, B.M.; April 1992d; Tank Farm Surveillance and Waste Status Summary Report for January 1992; WHC-EP-0182-46; Westinghouse Hanford Company, Richland, Washington.

Hanlon, B.M.; May 1992e; Tank Farm Surveillance and Waste Status Summary Report for February 1992; WHC-EP-0182-47; Westinghouse Hanford Company, Richland, Washington. 
Hanlon, B.M.; June 1992f; Tank Farm Surveillance and Waste Status Summary Report for March 1992; WHC-EP-0182-48; Westinghouse Hanford Company, Richland, Washington.

Hanlon, B.M.; July 1992g; Tank Farm Surveillance and Waste Status Summary Report for April 1992; WHC-EP-0182-49; Westinghouse Hanford Company, Richland, Washington.

Hanlon, B.M.; August 1992h; Tank Farm Surveillance and Waste Status Summary Report for May 1992; WHC-EP-0182-50; Westinghouse Hanford Company, Richland, Washington.

Hanlon, B.M.; September 1992i; Tank Farm Surveillance and Waste Status Summary Report for June 1992; WHC-EP-0 182-51; Westinghouse Hanford Company, Richland, Washington.

Hanlon, B.M.; October 1992j; Tank Farm Surveillance and Waste Status Summary Report for July 1992; WHC-EP-0182-52; Westinghouse Hanford Company, Richland, Washington.

Hanlon, B.M.; November 1992k; Tank Farm Surveillance and Waste Status Summary Report for August 1992; WHC-EP-0182-53; Westinghouse Hanford Company, Richland, Washington.

Hanlon, B.M.; December 19921; Tank Farm Surveillance and Waste Status Summary Report for September 1992; WHC-EP-0182-54; Westinghouse Hanford Company, Richland, Washington.

Hanlon, B.M.; January 1993a; Tank Farm Surveillance and Waste Status Summary Report for October 1992; WHC-EP-0182-55; Westinghouse Hanford Company, Richland, Washington.

Hanlon, B.M.; February 1993b; Tank Farm Surveillance and Waste Status Summary Report for November 1992; WHC-EP-0182-56; Westinghouse Hanford Company, Richland, Washington.

Hanlon, B.M.; February 1993c; Tank Farm Surveillance and Waste Status Summary Report for December 1992; WHC-EP-0182-57; Westinghouse Hanford Company, Richland, Washington.

Hanlon, B.M.; March 1993d; Tank Farm Surveillance and Waste Status Summary Report for January 1993; WHC-EP-0182-58; Westinghouse Hanford Company, Richland, Washington.

Hanlon, B.M.; April 1993e; Tank Farm Surveillance and Waste Status Summary Report for February 1993; WHC-EP-0182-59; Westinghouse Hanford Company, Richland, Washington.

Hanlon, B.M.; May 1993f; Tank Farm Surveillance and Waste Status Summary Report for March 1993; WHC-EP-0182-60; Westinghouse Hanford Company, Richland, Washington.

Hanlon, B.M.; July 1993g; Tank Farm Surveillance and Waste Status Summary Report for April 1993, WHC-EP-0182-61; Westinghouse Hanford Company, Richland, Washington.

Hanlon, B.M.; August 1993h; Tank Farm Surveillance and Waste Status Summary Report for May 1993; WHC-EP-0182-62; Westinghouse Hanford Company, Richland, Washington.

Hanlon, B.M; October 1993i; Tank Farm Surveillance and Waste Status Summary Report for June 1993; WHC-EP-0182-63; Westinghouse Hanford Company, Richland, Washington. 
Hanlon, B.M.; November 1993j; Tank Farm Surveillance and Waste Status Summary Report for July 1993; WHC-EP-0182-64; Westinghouse Hanford Company, Richland, Washington.

Hanlon, B.M; December 1993k; Tank Farm Surveillance and Waste Status Summary Report for August 1993; WHC-EP-0182-65; Westinghouse Hanford Company, Richland, Washington.

Hanlon, B.M.; January 1994a; Tank Farm Surveillance and Waste Status Summary Report for September 1993; WHC-EP-0182-66; Westinghouse Hanford Company, Richland, Washington.

Hanlon, B.M.; January 1994b; Tank Farm Surveillance and Waste Status Summary Report for October 1993; WHC-EP-0182-67; Westinghouse Hanford Company, Richland, Washington.

Hanlon, B.M.; February 1994c; Tank Farm Surveillance and Waste Status Summary Report for November 1993; WHC-EP-0182-68; Westinghouse Hanford Company, Richland, Washington.

Hanlon, B.M; April 1994d; Tank Farm Surveillance and Waste Status Summary Report for December 1993; WHC-EP-0182-69; Westinghouse Hanford Company, Richland, Washington.

Hanlon, B.M.; May 1994e; Tank Farm Surveillance and Waste Status Summary Report for January 1994; WHC-EP-0182-70; Westinghouse Hanford Company, Richland, Washington.

Hanlon, B.M; May 1994f; Tank Farm Surveillance and Waste Status Summary Report for February 1994; WHC-EP-0182-71; Westinghouse Hanford Company, Richland, Washington.

Hanlon, B.M; June 1994g; Tank Farm Surveillance and Waste Status Summary Report for March 1994; WHC-EP-0182-72; Westinghouse Hanford Company, Richland, Washington.

Hanlon, B.M; July 1994h; Tank Farm Surveillance and Waste Status Summary Report for April 1994; WHC-EP-0182-73; Westinghouse Hanford Company, Richland, Washington.

Hanlon, B.M; August 1994i; Tank Farm Surveillance and Waste Status Summary Report for May 1994; WHC-EP-0182-74; Westinghouse Hanford Company, Richland, Washington.

Hanlon, B.M.; September 1994j; Tank Farm Surveillance and Waste Status Summary Report for June 1994; WHC-EP-0182-75; Westinghouse Hanford Company, Richland, Washington.

Hanlon, B.M; November 1994k; Waste Tank Summary for Month Ending July 31, 1994; WHC-EP-0182-76; Westinghouse Hanford Company, Richland, Washington.

Hanlon, B.M; November 19941; Waste Tank Summary for Month Ending August 31, 1994; WHC-EP-0182-77; Westinghouse Hanford Company, Richland, Washington.

Hanlon, B.M.; November 1994m; Waste Tank Summary for Month Ending September 30, 1994; WHC-EP-0182-78; Westinghouse Hanford Company, Richland, Washington

Hanlon, B.M.; December 1994n; Waste Tank Summary for Month Ending October 31, 1994; WHC-EP-0182-79; Westinghouse Hanford Company, Richland, Washington. 
Hanlon, B.M; January 1995a; Waste Tank Summary for Month Ending November 30, 1994; WHC-EP-0182-80; Westinghouse Hanford Company, Richland, Washington.

Hanlon, B.M; February 1995b; Waste Tank Summary for Month Ending December 31, 1994; WHC-EP-0182-81; Westinghouse Hanford Company, Richland, Washington.

Hanlon, B.M:; March 1995c; Waste Tank Summary for Month Ending January 31, 1995; WHC-EP-0182-82; Westinghouse Hanford Company, Richland, Washington.

Hanlon, B.M; April 1995d; Waste Tank Summary for Month Ending February 28, 1995; WHC-EP-0182-83; Westinghouse Hanford Company, Richland, Washington.

Hanlon, B.M; May 1995e; Waste Tank Summary for Month Ending March 31, 1995; WHC-EP-0182-84; Westinghouse Hanford Company, Richland, Washington.

Hanlon, B.M; June 1995f; Waste Tank Summary for Month Ending April 30, 1995; WHC-EP-0182-85; Westinghouse Hanford Company, Richland, Washington.

Hanlon, B.M; July 1995g; Waste Tank Summary for Month Ending May 31, 1995; WHC-EP-0182-86; Westinghouse Hanford Company, Richland, Washington.

Hanlon, B.M; August 1995h; Waste Tank Summary for Month Ending June 30, 1995; WHC-EP-0182-87; Westinghouse Hanford Company, Richland, Washington.

Hanlon, B.M.; September 1995i; Waste Tank Summary for Month Ending July 31, 1995; WHC-EP-0182-88; Westinghouse Hanford Company, Richland, Washington.

Hanlon, B.M; October 1995j; Waste Tank Summary for Month Ending August 31, 1995; WHC-EP-0182-89; Westinghouse Hanford Company, Richland, Washington

Hanlon, B.M.; December 1995k; Waste Tank Summary for Month Ending September 30, 1995; WHC-EP-0182-90; Westinghouse Hanford Company, Richland, Washington.

Hanlon, B.M; January 1996a; Waste Tank Summary for Month Ending October 31, 1995; WHC-EP-0182-91; Westinghouse Hanford Company, Richland, Washington.

Hanlon, B.M; February 1996b; Waste Tank Summary for Month Ending November 30, 1995; WHC-EP-0182-92; Westinghouse Hanford Company, Richland, Washington.

Hanlon, B.M.; February 1996c; Waste Tank Summary for Month Ending December 31, 1995; WHC-EP-0182-93; Westinghouse Hanford Company, Richland, Washington.

Hanlon, B.M; April 1996d; Waste Tank Summary for Month Ending January 31, 1996; WHC-EP-0182-94; Westinghouse Hanford Company, Richland, Washington.

Hanlon, B.M; April 1996e; Waste Tank Summary for Month Ending February 29, 1996; WHC-EP-0182-95; Westinghouse Hanford Company, Richland, Washington. 
Hanlon, B.M.; May 1996f; Waste Tank Summary for Month Ending March 31, 1996; WHC-EP-0182-96; Westinghouse Hanford Company, Richland, Washington.

Hanlon, B.M.; June 1996g; Waste Tank Summary for Month Ending April 30, 1996, WHC-EP-0182-97; Westinghouse Hanford Company, Richland, Washington.

Hanlon, B.M.; August 1996h; Waste Tank Summary for Month Ending May 31, 1996; WHC-EP-0182-98; Westinghouse Hanford Company, Richland, Washington.

Hanlon, B.M.; September 1996i; Waste Tank Summary for Month Ending June 30, 1996; WHC-EP-0182-99; Westinghouse Hanford Company, Richland, Washington.

HEW; January 11, 1944; CVI, Specifications for Construction of Composite Storage Tanks Bldg. No. 241, Project 9536; CVI 73550; Hanford Engineer Works, Richland, Washington.

Jungfleisch, F.M.; March 1984; Preliminary Estimation of the Waste Inventories in Hanford Tanks Through 1980; SD-WM-TI-057; Rockwell Hanford Operations, Richland, Washington.

Leach, C.E., and S.M. Stahl; August 1993; Hanford Site Tank Farm Facilities Interim Safety Basis, Vols. 1 \& 2; WHC-SD-WM-ISB-001; Westinghouse Hanford Company, Richland, Washington.

Lipnicki, J.; June 1996; Waste Tank Risers Available for Sampling; WHC-SD-WM-TI-710, Rev. 3; Westinghouse Hanford Company, Richland, Washington.

McCann, D.C.; August 1981a; Waste Status Summary, July 1981; RHO-CD-14; Rockwell Hanford Operations, Richland, Washington.

McCann, D.C.; September 1981b; Waste Status Summary, August 1981; RHO-CD-14; Rockwell Hanford Operations, Richland, Washington.

McCann, D.C.; October 1981c; Waste Status Summary, September 1981; RHO-CD-14; Rockwell Hanford Operations, Richland, Washington.

McCann, D.C.; November 1981d; Waste Status Summary, October 1981; RHO-CD-14; Rockwell Hanford Operations, Richland, Washington.

McCann, D.C.; December 1981e; Waste Status Summary November 1981; RHO-RE-SR-14; Rockwell Hanford Operations, Richland, Washington.

McCann, D.C.; April 1982a; Waste Status Summary, March 1982; RHO-RE-SR-14; Rockwell Hanford Operations, Richland, Washington.

McCann, D.C.; May 1982b; Waste Status Summary, April 1982; RHO-RE-SR-14; Rockwell Hanford Operations, Richland, Washington.

McCann, D.C.; June 1982c; Waste Status Summary, May 1982; RHO-RE-SR-14; Rockwell Hanford Operations, Richland, Washington. 
McCann, D.C.; July 1982d; Waste Status Summary, June 1982; RHO-RE-SR-14; Rockwell Hanford Operations, Richland, Washington.

McCann, D.C.; August 1982e; Waste Status Summary, July 1982; RHO-RE-SR-14; Rockwell Hanford Operations, Richland, Washington.

McCann, D.C.; September 1982f; Waste Status Summary, August 1982; RHO-RE-SR-14; Rockwell Hanford Operations, Richland, Washington.

McCann, D.C.; October 1982g; Waste Status Summary, September 1982; RHO-RE-SR-14; Rockwell Hanford Operations, Richland, Washington.

McCann, D.C.; November 1982h; Waste Status Summary, October 1982; RHO-RE-SR-14; Rockwell Hanford Operations, Richland, Washington.

McCann, D.C; December 1982i; Waste Status Summary, November 1982; RHO-RE-SR-14; Rockwell Hanford Operations, Richland, Washington.

McCann, D.C.; January 1983a; Waste Status Summary, December 1982; RHO-RE-SR-14; Rockwell Hanford Operations, Richland, Washington.

McCann, D.C.; February 1983b; Waste Status Summary, January 1983; RHO-RE-SR-14; Rockwell Hanford Operations, Richland, Washington.

McCann, D.C.; March 1983c; Waste Status Summary, February 1983; RHO-RE-SR-14; Rockwell Hanford Operations, Richland, Washington.

McCann, D.C.; April 1983d; Waste Status Summary, March 1983; RHO-RE-SR-14; Rockwell Hanford Operations, Richland, Washington.

McCann, D.C.; May 1983e; Waste Status Summary, April 1983; RHO-RE-SR-14; Rockwell Hanford Operations, Richland, Washington.

McCann, D.C.; June 1983f; Waste Status Summary, May 1983; RHO-RE-SR-14; Rockwell Hanford Operations, Richland, Washington.

McCann, D.C.; July 1983g; Waste Status Summary, June 1983; RHO-RE-SR-14; Rockwell Hanford Operations, Richland, Washington.

McCann, D.C.; August 1983h; Waste Status Summary, July 1983; RHO-RE-SR-14; Rockwell Hanford Operations, Richland, Washington.

McCann, D.C.; September 1983i; Waste Status Summary, August 1983; RHO-RE-SR-14; Rockwell Hanford Operations, Richland, Washington.

McCann, D.C.; October 1983j; Waste Status Summary, September 1983; RHO-RE-SR-14; Rockwell Hanford Operations, Richland, Washington. 
McCann, D.C.; November 1983k; Waste Status Summary, October 1983; RHO-RE-SR-14; Rockwell Hanford Operations, Richland, Washington.

McCann, D.C.; December 19831; Waste Status Summary, November 1983; RHO-RE-SR-14; Rockwell Hanford Operations, Richland, Washington.

McCann, D.C.; January 1984a; Waste Status Summary, December 1983; RHO-RE-SR-14; Rockwell Hanford Operations, Richland, Washington.

McCann, D.C.; February 1984b; Waste Status Summary, January 1984; RHO-RE-SR-14; Rockwell Hanford Operations, Richland, Washington.

McCann, D.C.; March 1984c; Waste Status Summary, February 1984; RHO-RE-SR-14; Rockwell Hanford Operations, Richland, Washington.

McCann, D.C.; April 1984d; Waste Status Summary, March 1984; RHO-RE-SR-14; Rockwell Hanford Operations, Richland, Washington.

McCann, D.C.; May 1984e; Waste Status Summary, April 1984; RHO-RE-SR-14; Rockwell Hanford Operations, Richland, Washington.

McCann, D.C.; June 1984f; Waste Status Summary, May 1984; RHO-RE-SR-14; Rockwell Hanford Operations, Richland, Washington.

McCann, D.C.; July 1984g; Waste Status Summary, June 1984; RHO-RE-SR-14; Rockwell Hanford Operations, Richland, Washington.

McCann, D.C.; August 1984h; Waste Status Summary, July 1984; RHO-RE-SR-14; Rockwell Hanford Operations, Richland, Washington.

McCann, D.C., and T.S. Vail; September 1984i; Waste Status Summary, August 1984; RHO-RE-SR-14; Rockwell Hanford Operations, Richland, Washington.

McCann, D.C., and T.S. Vail; October 1984j; Waste Status Summary, September 1984; RHO-RE-SR-14; Rockwell Hanford Operations, Richland, Washington.

McCann, D.C., and T.S. Vail; November 1984k; Waste Status Summary, October 1984; RHO-RE-SR-14; Rockwell Hanford Operations, Richland, Washington.

McCann, D.C., and T.S. Vail; December 19841; Waste Status Summary, November 1984; RHO-RE-SR-14; Rockwell Hanford Operations, Richland, Washington.

McCann, D.C., and T.S. Vail; January 1985a; Waste Status Summary, December 1984; RHO-RE-SR-14; Rockwell Hanford Operations, Richland, Washington.

McCann, D.C., and T.S. Vail; February 1985b; Waste Status Summary, January 1985; RHO-RE-SR-14; Rockwell Hanford Operations, Richland, Washington. 
McCann, D.C., and T.S. Vail; March 1985c; Waste Status Summary, February 1985; RHO-RE-SR-14; Rockwell Hanford Operations, Richland, Washington.

Mudd, O.C.; February 1981a; Waste Status Summary, January 1981; RHO-CD-14; Rockwell Hanford Operations, Richland, Washington.

Mudd, O.C.; March 1981b; Waste Status Summary, February 1981; RHO-CD-14; Rockwell Hanford Operations, Richland, Washington.

Mudd, O.C.; April 1981c; Waste Status Summary, March 1981; RHO-CD-14; Rockwell Hanford Operations, Richland, Washington.

Mudd, O.C.; May 1981d; Waste Status Summary, April 1981; RHO-CD-14; Rockwell Hanford Operations, Richland, Washington.

Mudd, O.C.; June 1981e; Waste Status Summary, May 1981; RHO-CD-14; Rockwell Hanford Operations, Richland, Washington.

Mudd, O.C., and D.C. McCann; July 1981f; Waste Status Summary, June 1981; RHO-CD-14; Rockwell Hanford Operations, Richland, Washington.

Pianka, E.W; January 24, 1995; Soil Load above Hanford Waste Storage Tanks; WHC-SD-WM-TI-665, Rev. 0A; Westinghouse Hanford Company, Richland, Washington.

PNL; 1991; Resource Book - Decommissioning of Contaminated Facilities at Hanford; PNL-7008 (originally BNWL-MA-88 in 1975, then PNL-MA-588); Battelle Pacific Northwest Laboratories, Richland, Washington.

Rockwell; August 1985; 200 Areas Fact Book, Rockwell Hanford Company, Richland, Washington.

Rodenhizer, D.G.; 1987; Hanford Waste Tank Sluicing History; SD-WM-TI-302; Westinghouse Hanford Company, Richland, Washington.

Shefcik, J.J.; December 1964; Process Specifications for In-Tank Solidification of Radiochemical Wastes; RL-SEP-115; General Electric Company, Richland, Washington.

Thurman, J.M.; March 1987a; Waste Status Summary, February 1987; RHO-RE-SR-14; Rockwell Hanford Operations, Richland, Washington.

Thurman, J.M.; April 1987b; Waste Status Summary, March 1987; RHO-RE-SR-14; Rockwell Hanford Operations, Richland, Washington.

Thurman, J.M.; May 1987c; Waste Status Summary, April 1987; RHO-RE-SR-14; Rockwell Hanford Operations, Richland, Washington.

Thurman, J.M.; June 1987d; Waste Status Summary, May 1987; RHO-RE-SR-14; Rockwell Hanford Operations, Richland, Washington. 
Thurman, J.M.; July 1987e; Waste Status Summary, June 1987; RHO-RE-SR-14; Rockwell Hanford Operations, Richland, Washington.

Thurman, J.M.; August 1987f, Waste Status Summary for July 1987; WHC-SP-0038-1; Westinghouse Hanford Company, Richland, Washington.

Thurman, J.M.; September 1987g; Waste Status Summary for August 1987; WHC-SP-0038-2; Westinghouse Hanford Company, Richland, Washington.

Thurman, J.M.; October 1987h; Waste Status Summary for September 1987; WHC-SP-0038-3; Westinghouse Hanford Company, Richland, Washington.

Thurman, J.M.; November 1987i; Waste Status Summary for October 1987; WHC-SP-0038-4; Westinghouse Hanford Company, Richland, Washington.

Thurman, J.M.; December 1987j; Waste Status Summary for November 1987; WHC-SP-0038-5; Westinghouse Hanford Company, Richland, Washington.

Thurman, J.M.; January 1988a; Waste Status Summary for December 1987; WHC-SP-0038-6; Westinghouse Hanford Company, Richland, Washington.

Thurman, J.M.; February 1988b; Waste Status Summary for January 1988; WHC-SP-0038-7; Westinghouse Hanford Company, Richland, Washington.

Thurman, J.M.; March 1988c; Waste Status Summary for February 1988; WHC-SP-0038-8; Westinghouse Hanford Company, Richland, Washington.

Thurman, J.M.; April 1988d; Waste Status Summary for March 1988; WHC-SP-0038-9; Westinghouse Hanford Company, Richland, Washington.

Thurman, J.M.; June 1988e; Tank Farm Surveillance and Waste Status Summary Report for April 1988; WHC-EP-0182-1; Westinghouse Hanford Company, Richland, Washington.

Thurman, J.M.; August 1988f; Tank Farm Surveillance and Waste Status Summary Report for May 1988; WHC-EP-0182-2; Westinghouse Hanford Company, Richland, Washington.

Thurman, J.M.; August 1988g; Tank Farm Surveillance and Waste Status Summary Report for June 1988; WHC-EP-0182-3; Westinghouse Hanford Company, Richland, Washington.

Thurman, J.M.; September 1988h; Tank Farm Surveillance and Waste Status Summary Report for July 1988; WHC-EP-0182-4; Westinghouse Hanford Company, Richland, Washington.

Thurman, J.M.; December 1988i; Tank Farm Surveillance and Waste Status Summary Report for August 1988; WHC-EP-0182-5; Westinghouse Hanford Company, Richland, Washington.

Thurman, J.M.; December 1988j; Tank Farm Surveillance and Waste Status Summary Report for September 1988; WHC-EP-0182-6; Westinghouse Hanford Company, Richland, Washington. 
Thurman, J.M.; December 1988k; Tank Farm Surveillance and Waste Status Summary Report for October 1988; WHC-EP-0182-7; Westinghouse Hanford Company, Richland, Washington.

Thurman, J.M; January 1989a; Tank Farm Surveillance and Waste Status Summary Report for November 1988; WHC-EP-0182-8; Westinghouse Hanford Company, Richland, Washington.

Thurman, J.M; January 1989b; Tank Farm Surveillance and Waste Status Summary Report for December 1988, WHC-EP-0182-9; Westinghouse Hanford Company, Richland, Washington.

Thurman, J.M.; March 1989c; Tank Farm Surveillance and Waste Status Summary Report for Jaruary 1989; WHC-EP-0182-10; Westinghouse Hanford Company, Richland, Washington.

Thurman, J.M.; April 1989d; Tank Farm Surveillance and Waste Status Summary Report for February 1989; WHC-EP-0182-11; Westinghouse Hanford Company, Richland, Washington.

Thurman, J.M; May 1989e; Tank Farm Surveillance and Waste Status Summary Report for March 1989; WHC-EP-0182-12; Westinghouse Hanford Company, Richland, Washington

Thurman, J.M.; June 1989f; Tank Farm Surveillance and Waste Status Summary Report for April 1989; WHC-EP-0182-13; Westinghouse Hanford Company, Richland, Washington.

Thurman, J.M; July 1989g; Tank Farm Surveillance and Waste Status Summary Report for May 1989; WHC-EP-0182-14; Westinghouse Hanford Company, Richland, Washington.

Thurman, J.M; August 1989h; Tank Farm Surveillance and Waste Status Summary Report for June 1989; WHC-EP-0182-15; Westinghouse Hanford Company, Richland, Washington.

Thurman, J.M.; September 1989i; Tank Farm Surveillance and Waste Status Summary Report for July 1989; WHC-EP-0182-16; Westinghouse Hanford Company, Richland, Washington.

Thurman, J.M.; October 1989j; Tank Farm Surveillance and Waste Status Summary Report for August 1989; WHC-EP-0182-17; Westinghouse Hanford Company, Richland, Washington.

Thurman, J.M; November 1989k; Tank Farm Surveillance and Waste Status Summary Report for September 1989; WHC-EP-0182-18; Westinghouse Hanford Company, Richland, Washington.

Thurman, J.M.; February 1990; Tank Farm Surveillance and Waste Status Summary Report for October 1989; WHC-EP-0182-19; Westinghouse Hanford Company, Richland, Washington.

Tran, T.T.; April 27, 1993; Thermocouple Status Single-Shell and Double-Shell Waste Tanks; WHC-SD-WM-TI-553; Westinghouse Hanford Company, Richland, Washington.

U.S. DOE; December 1987; EIS, Disposal of Hanford Defense High Level, Transuranic, and Tank Wastes, Vols. 1-5; DOE/EIS-0113; Department of Energy, Richland, Washington.

Vail, T.S.; April 1985a; Waste Status Summary, March 1985; RHO-RE-SR-14; Rockwell Hanford Operations, Richland, Washington. 
Vail, T.S.; May 1985b; Waste Status Summary, April 1985; RHO-RE-SR-14; Rockwell Hanford Operations, Richland, Washington.

Vail, T.S.; June 1985c; Waste Status Summary, May 1985; RHO-RE-SR-14; Rockwell Hanford Operations, Richland, Washington.

Vail, T.S.; July 1985d; Waste Status Summary, June 1985; RHO-RE-SR-14; Rockwell Hanford Operations, Richland, Washington.

Vail, T.S.; August 1985e; Waste Status Summary, July 1985; RHO-RE-SR-14; Rockwell Hanford Operations, Richland, Washington.

Vail, T.S.; September 1985f; Waste Status Summary, August 1985; RHO-RE-SR-14; Rockwell Hanford Operations, Richland, Washington.

Vail, T.S; October 1985g; Waste Status Summary, September 1985; RHO-RE-SR-14; Rockwell Hanford Operations, Richland, Washington.

Vail, T.S.; November 1985h; Waste Status Summary, October 1985; RHO-RE-SR-14; Rockwell Hanford Operations, Richland, Washington.

Vail, T.S., and G.D. Murry; December 1985i; Waste Status Summary, November 1985; RHO-RE-SR-14; Rockwell Hanford Operations, Richland, Washington.

Vail, T.S., and G.D. Murry; January 1986a; Waste Status Summary, December 1985; RHO-RE-SR-14; Rockwell Hanford Operations, Richland, Washington.

Vail, T.S., and G.D. Murry; February 1986b; Waste Status Summary, Jamuary 1986; RHO-RE-SR-14; Rockwell Hanford Operations, Richland, Washington.

Vail, T.S., and G.J. Carter; March 1986c; Waste Status Summary, February 1986; RHO-RE-SR-14; Rockwell Hanford Operations, Richland, Washington.

Vail, T.S., and G.J. Carter; April 1986d; Waste Status Summary, March 1986; RHO-RE-SR-14; Rockwell Hanford Operations, Richland, Washington.

Welty, R.K; September 1988; Waste Storage Tank Status and Leak Detection Criteria; WHC-SD-WM-TI-356, Vols. 1 and 2; Westinghouse Hanford Company, Richland, Washington.

Wilson, G.R., and I.E. Reep; December 1991; A Plan to Implement Remediation of Waste Tank Safety Issues at the Hanford Site; WHC-EP-0422, Rev. 1; Westinghouse Hanford Company, Richland, Washington. 
1. :

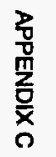


THISI.

LEI GLAirk 


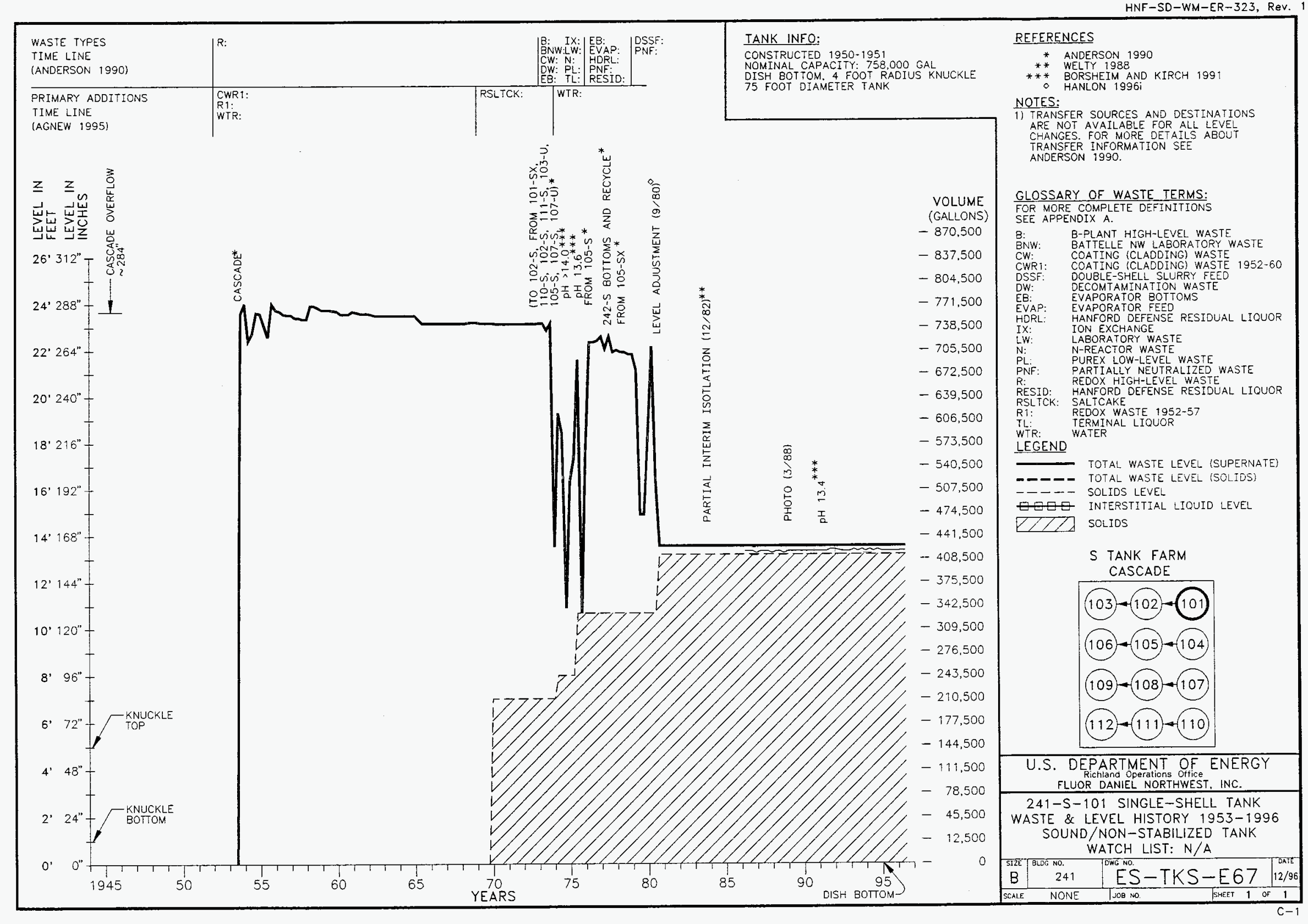




\section{THIS PAGE INTENTIONALLY}

LEFT BLANK 


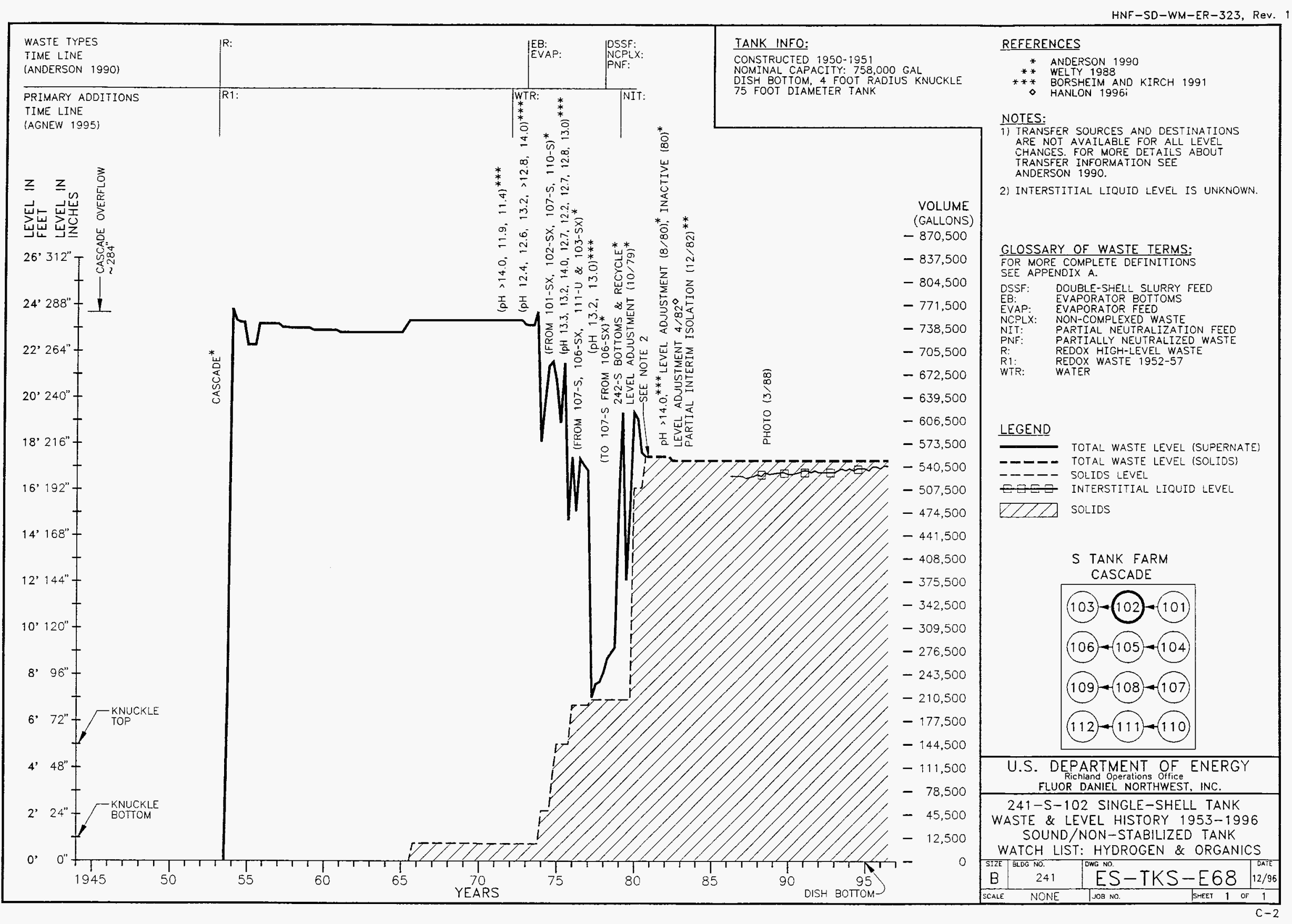


THIS PAGE INTENTIONALLY LEFT BLANK 


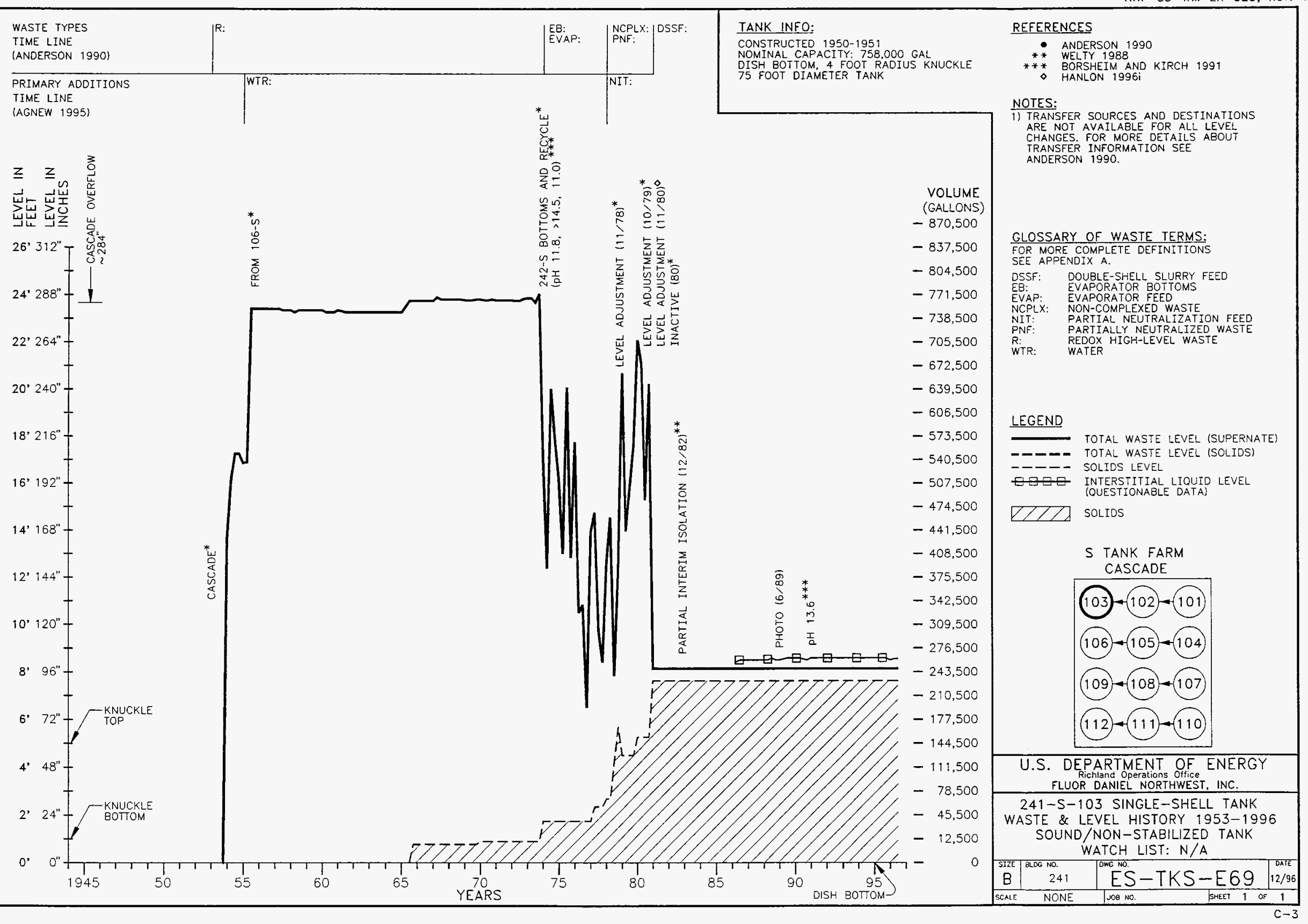


THIS PAGE INTENTIONALLY LEFT BLANK 


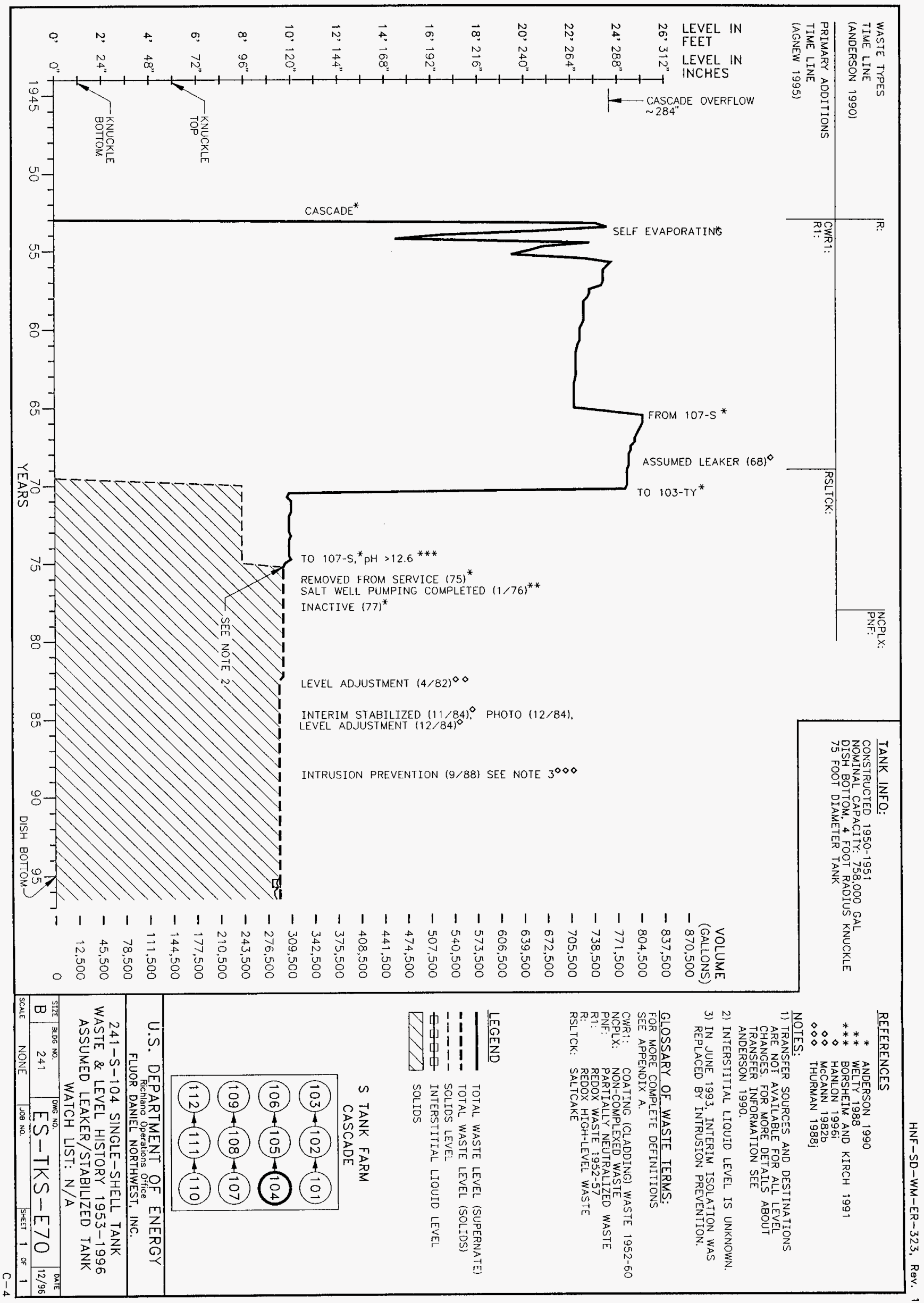


THIS PAGE INTENTIONALLY LEFT BLANK 


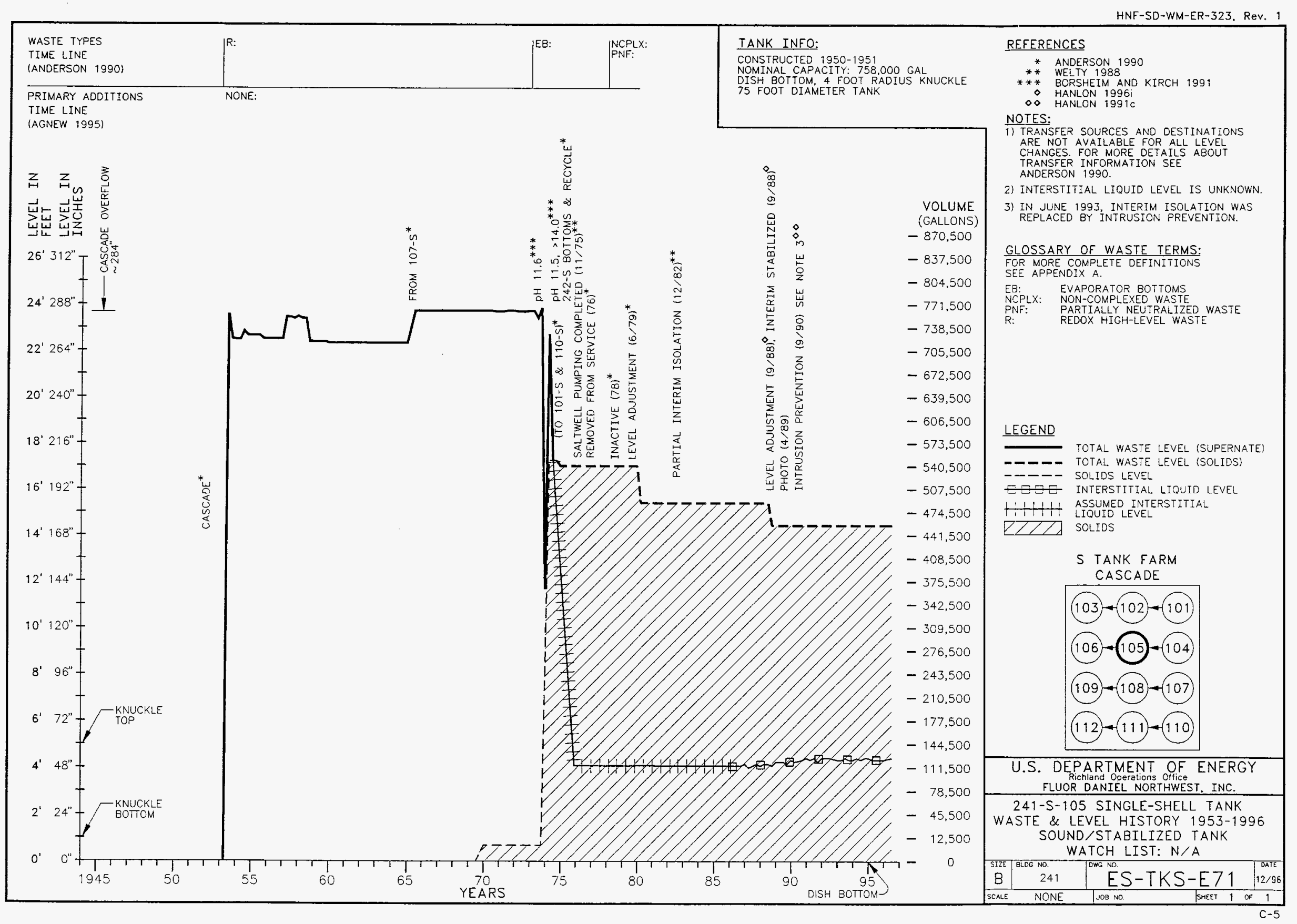


THIS PAGE INTENTIONALLY LEFT BLANK 


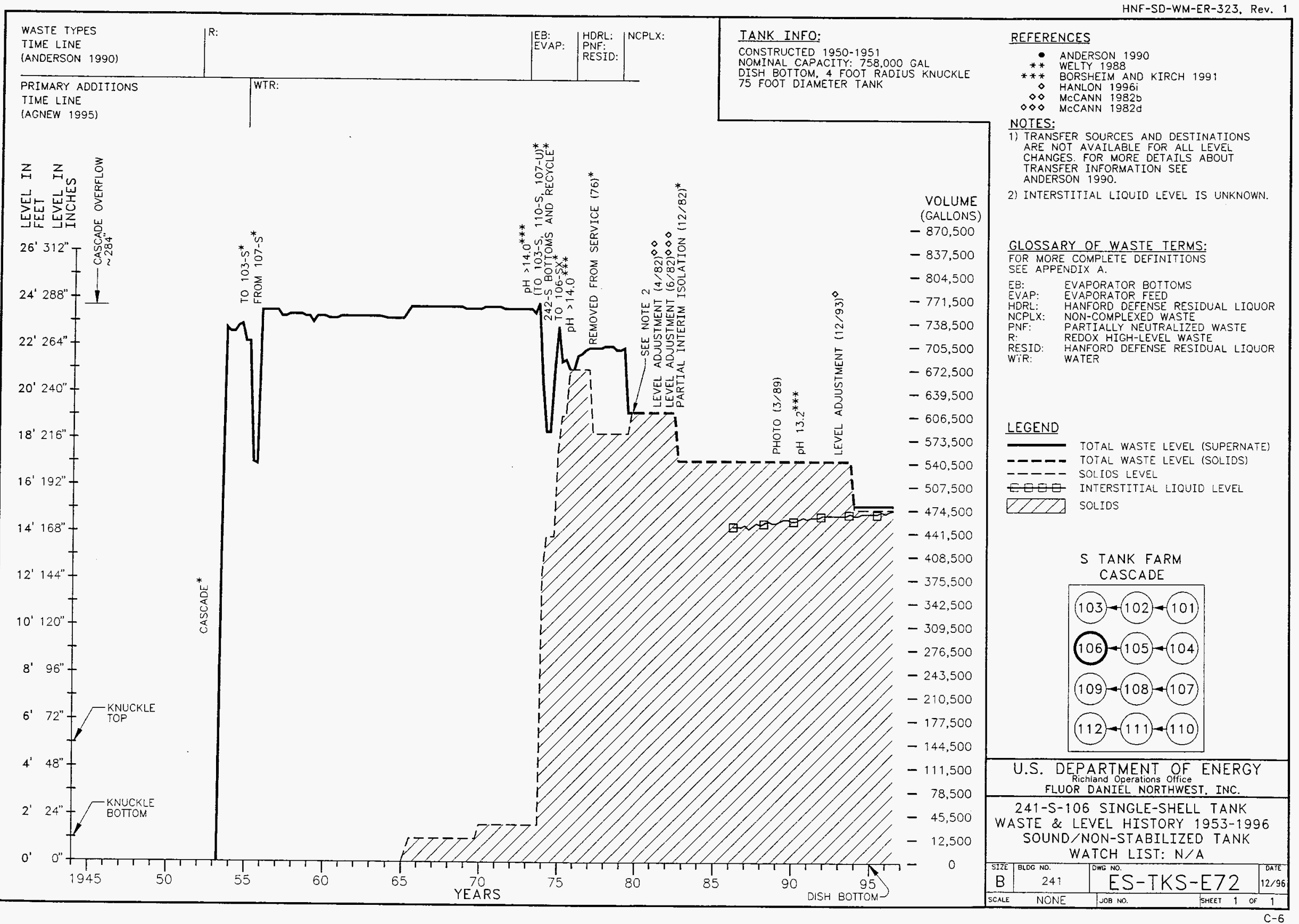


THIS PAGE INTENTIONALLY LEFT BLANK 


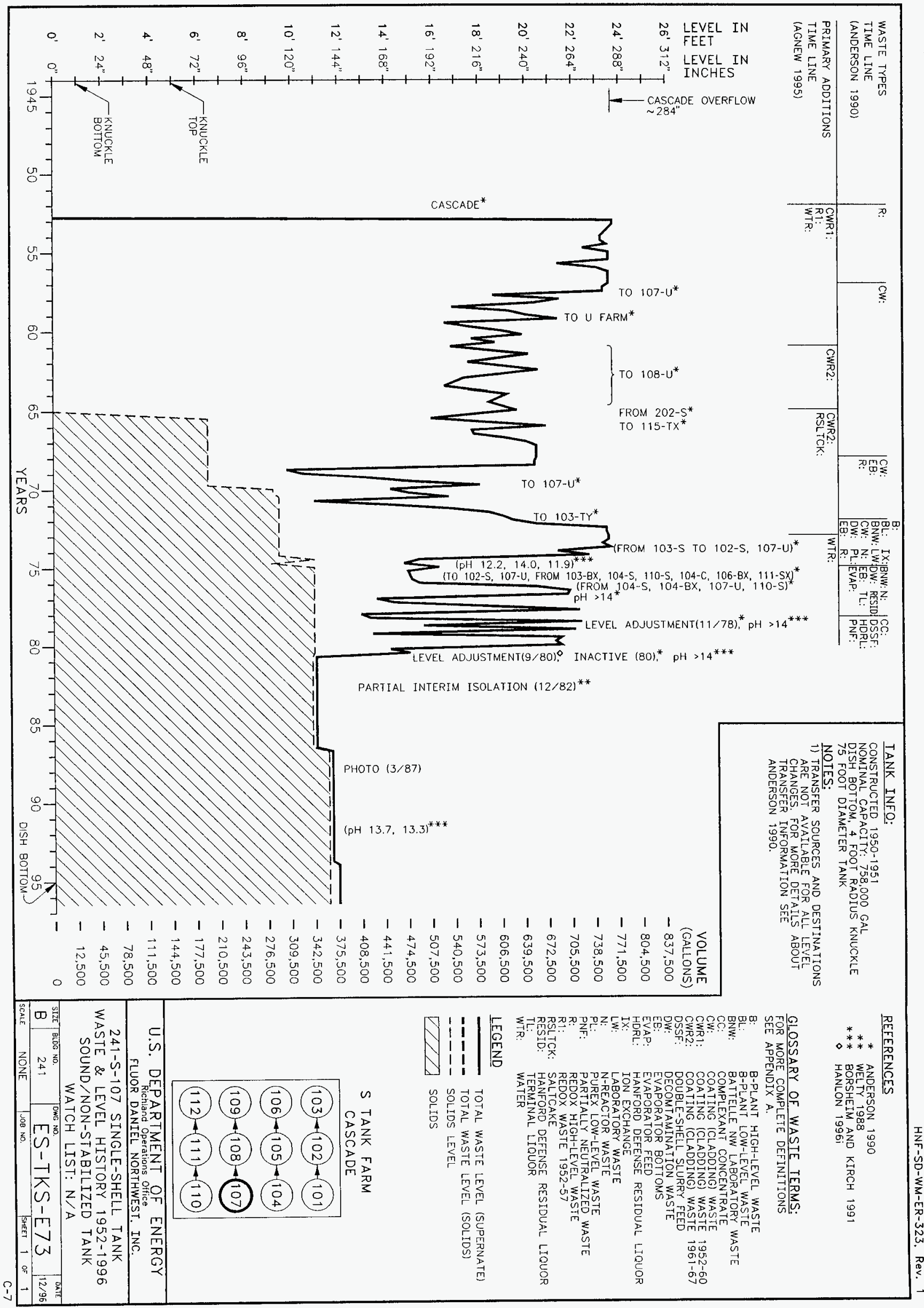


THIS PAGE INTENTIONALLY LEFT BLANK 


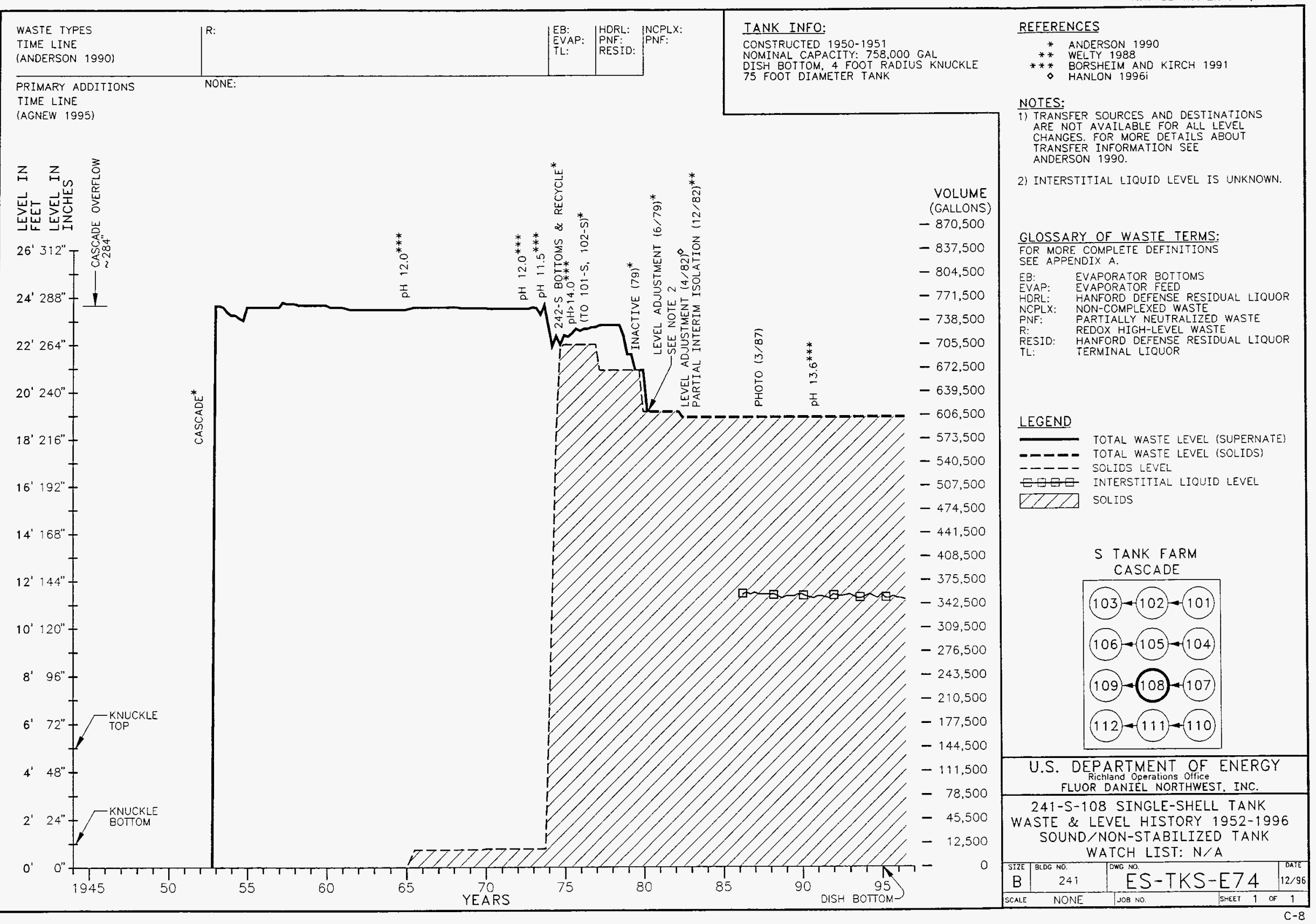


THIS PAGE INTENTIONALLY LEFT BLANK 
HNF-SD-WM-ER-323, Rev.

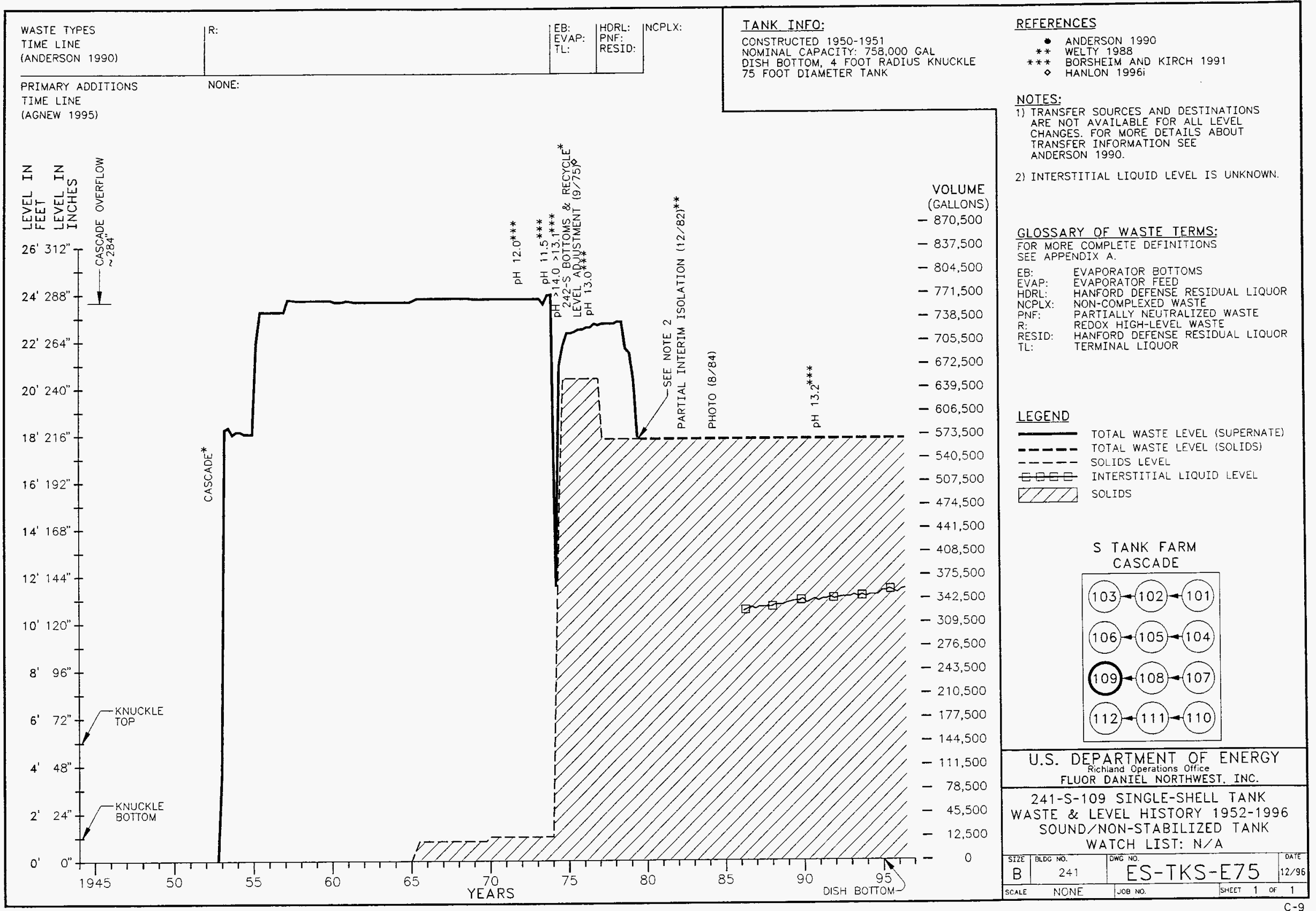


THIS PAGE INTENTIONALLY

LEFT BLANK 


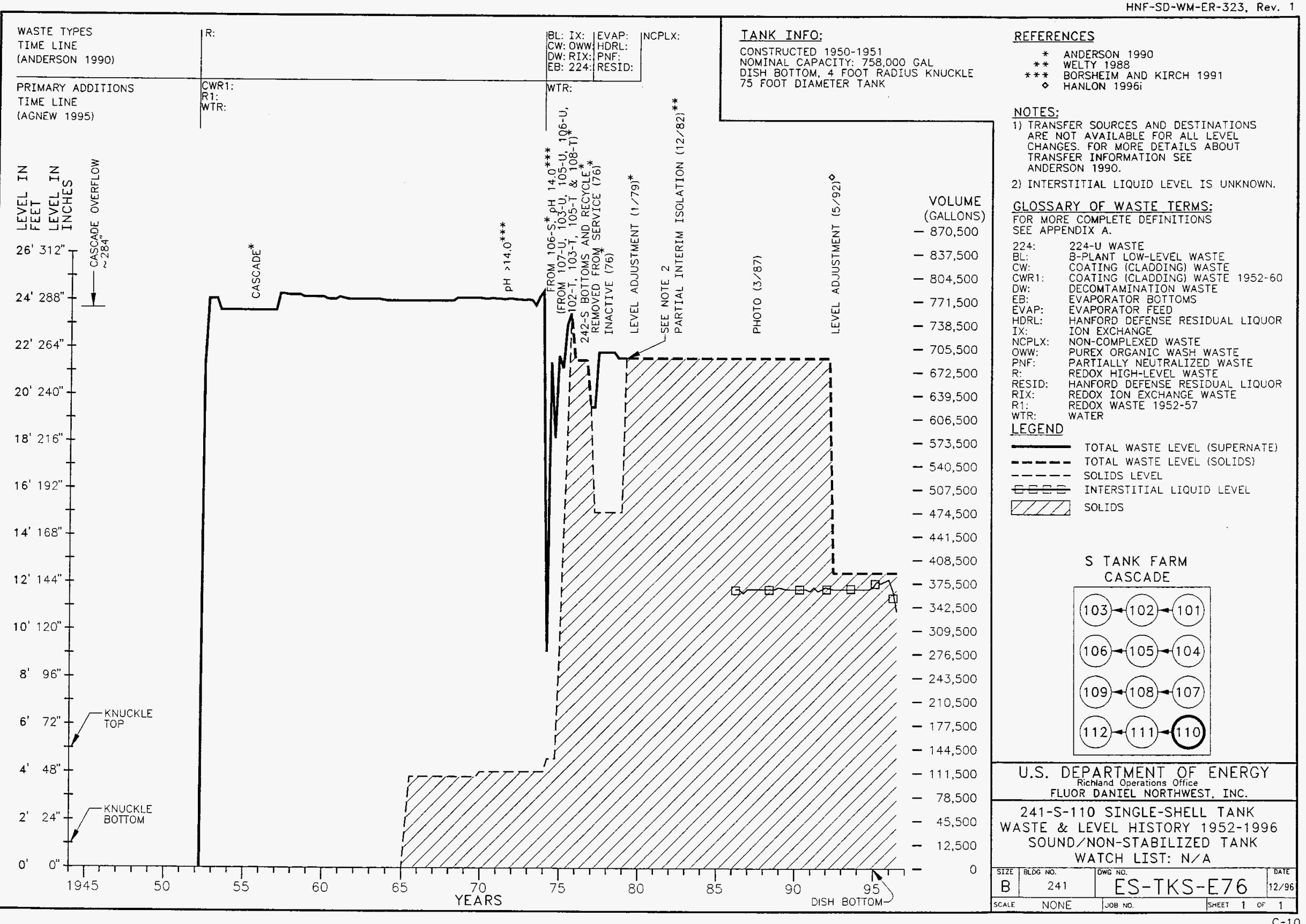


THIS PAGE INTENTIONALLY

LEFT BLANK 
HNF-SD-WM-ER-323. Rev. 1

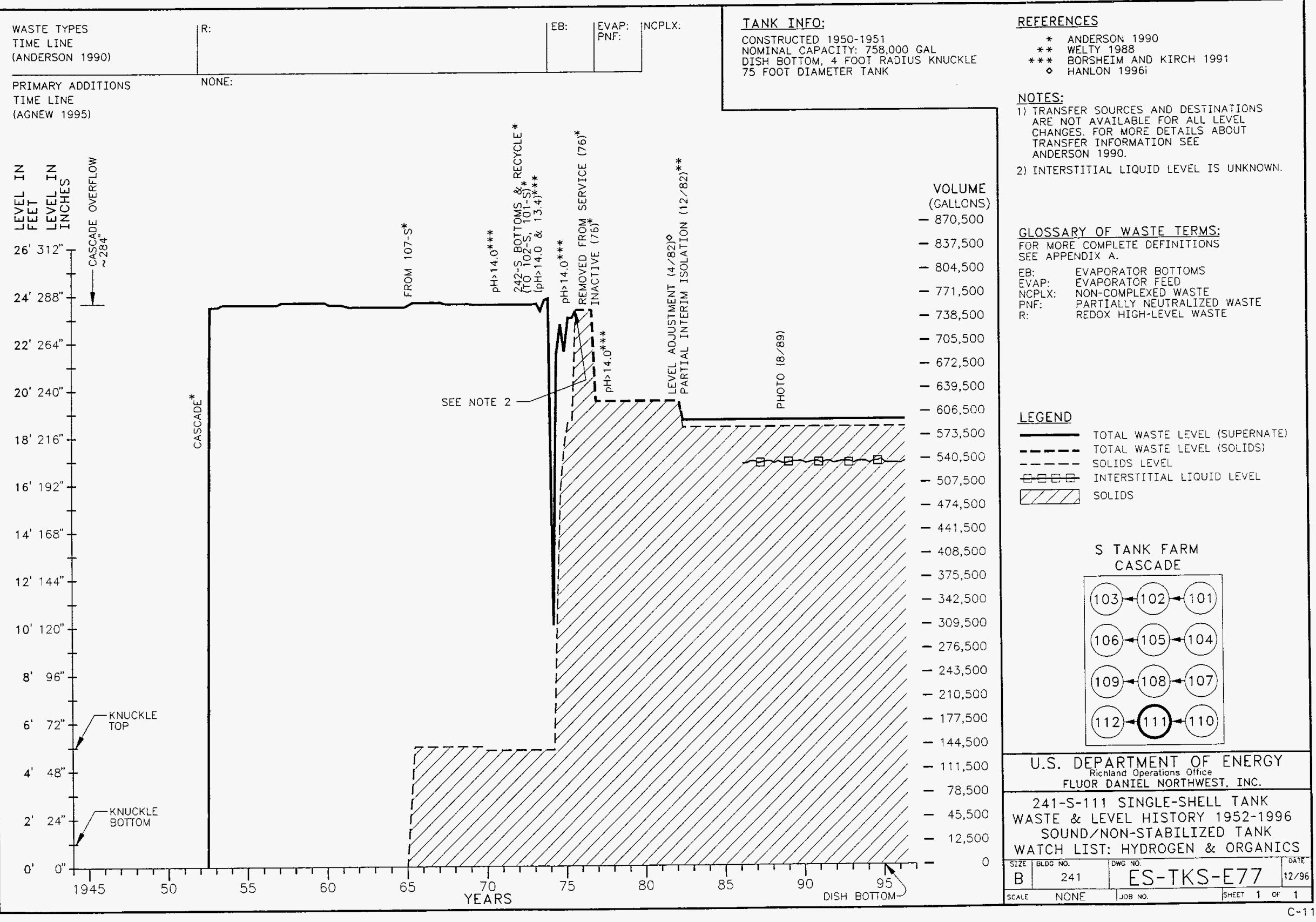


THIS PAGE INTENTIONALLY

LEFT BLANK 


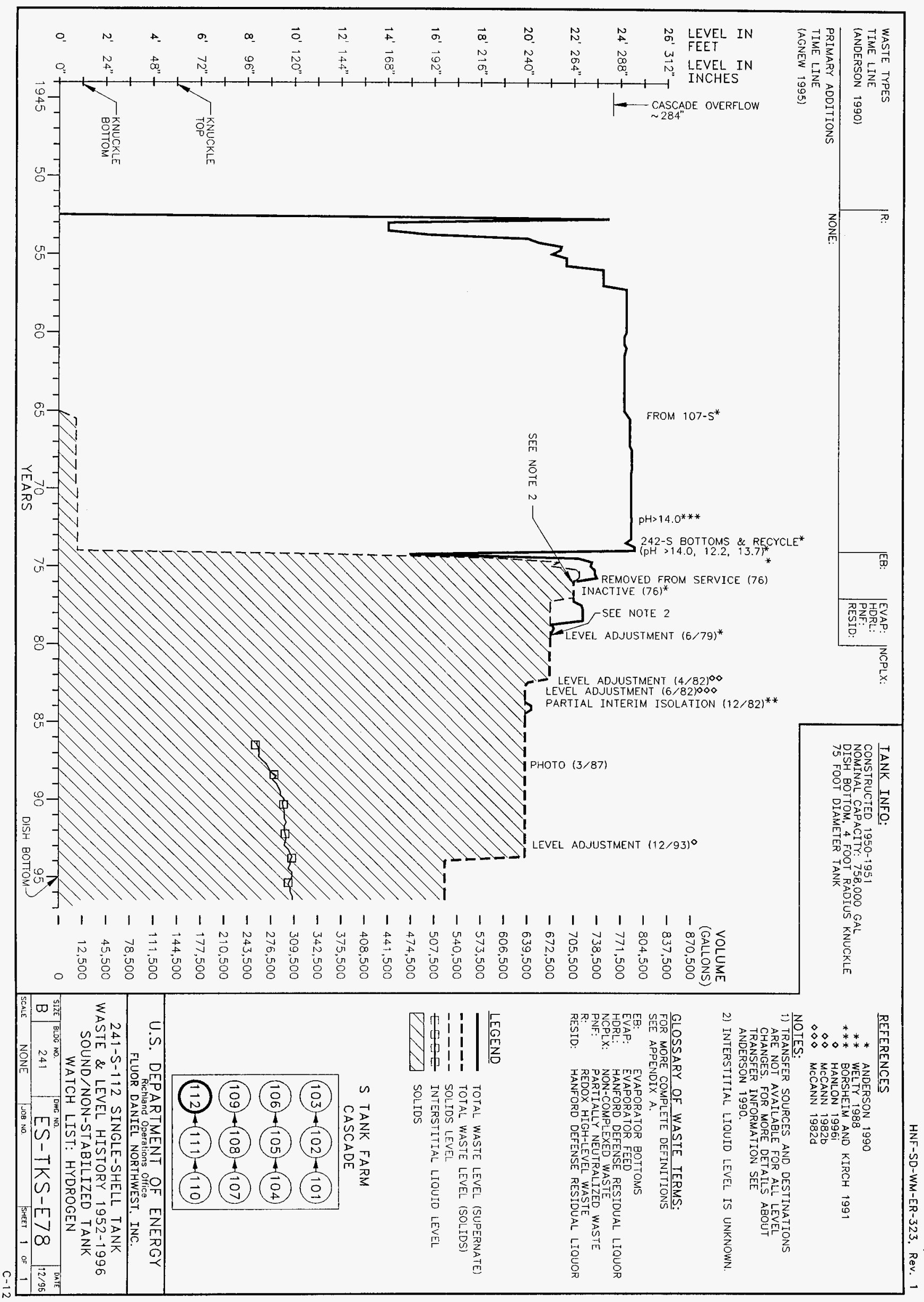



241-S-101 LEVEL HISTORY

\begin{tabular}{|c|c|c|c|c|c|}
\hline Year & Total & Total & Solids & Solids & ILL \\
\hline & (K gal) & (in) & (K gal) & (in) & (in) \\
\hline \multirow{2}{*}{\multicolumn{6}{|c|}{1.1952}} \\
\hline & & & & & \\
\hline \multicolumn{6}{|l|}{2} \\
\hline \multicolumn{6}{|l|}{3} \\
\hline \multicolumn{6}{|l|}{4} \\
\hline \multicolumn{6}{|l|}{$1-1953$} \\
\hline \multicolumn{6}{|l|}{2} \\
\hline 3 & 758 & 283 & 0 & 0 & \\
\hline 4 & 772 & 288 & 0 & 0 & \\
\hline 1.1954 & 719 & 269 & 0 & 0 & \\
\hline 2 & 730 & 273 & 0 & 0 & \\
\hline 3 & 759 & 283 & 0 & 0 & \\
\hline 4 & 758 & 283 & 0 & 0 & \\
\hline $1-1955$ & 741 & 277 & 0 & 0 & \\
\hline 2 & $7 \overline{25}$ & 271 & 0 & 0 & \\
\hline 3 & 772 & 288 & 0 & 0 & \\
\hline 4 & 763 & 285 & 0 & 0 & \\
\hline $1-1956$ & 761 & 284 & 0 & 0 & \\
\hline 2 & 757 & 283 & 0 & 0 & \\
\hline 3 & 756 & 282 & 0 & 0 & \\
\hline 4 & 756 & 282 & 0 & 0 & \\
\hline $1-1957$ & 752 & 281 & 0 & 0 & \\
\hline 2 & 752 & 281 & 0 & 0 & \\
\hline 3 & 750 & 280 & 0 & 0 & \\
\hline 4 & 750 & 280 & 0 & 0 & \\
\hline 1-1958 & 769 & 287 & 0 & 0 & \\
\hline 2 & 769 & 287 & 0 & 0 & \\
\hline 3 & 766 & 286 & 0 & 0 & \\
\hline 4 & 763 & 285 & 0 & 0 & \\
\hline 1-1959 & 763 & 285 & 0 & 0 & \\
\hline 2 & 763 & 285 & 0 & 0 & \\
\hline 3 & 762 & 285 & 0 & 0 & \\
\hline 4 & 761 & 284 & 0 & 0 & \\
\hline $1-1960$ & 757 & 283 & 0 & 0 & \\
\hline 2 & 757 & 283 & 0 & 0 & \\
\hline 3 & 757 & 283 & 0 & 0 & \\
\hline 4 & 761 & 284 & 0 & 0 & \\
\hline \multicolumn{6}{|l|}{$1-1961$} \\
\hline 2 & 758 & 283 & 0 & 0 & \\
\hline \multicolumn{6}{|l|}{3} \\
\hline 4 & 758 & 283 & 0 & 0 & \\
\hline \multicolumn{6}{|l|}{$1-1962$} \\
\hline 2 & 755 & 282 & 0 & 0 & \\
\hline \multicolumn{6}{|l|}{3} \\
\hline 4 & 755 & 282 & 0 & 0 & \\
\hline \multicolumn{6}{|l|}{$1-1963$} \\
\hline 2 & 755 & 282 & 0 & 0 & \\
\hline
\end{tabular}


241-S-101 LEVEL HISTORY

HNF-SD-WM-ER-323, Rev . I

\begin{tabular}{|c|c|c|c|c|c|}
\hline Year & Total & Total & Solids & Solids & ILL \\
\hline & (K gal) & (in) & (K gal) & (in) & (in) \\
\hline \multirow{2}{*}{\multicolumn{6}{|c|}{3}} \\
\hline 4 & & & & & \\
\hline \multirow{2}{*}{\multicolumn{6}{|c|}{$1-1964$}} \\
\hline & & & & & \\
\hline 2 & 755 & 282 & 0 & 0 & \\
\hline \multicolumn{6}{|l|}{3} \\
\hline 4 & 755 & 282 & 0 & 0 & \\
\hline \multicolumn{6}{|l|}{$1-1965$} \\
\hline 2 & 744 & 278 & 0 & 0 & \\
\hline 3 & 744 & 278 & 0 & 0 & \\
\hline 4 & 744 & 278 & 0 & 0 & \\
\hline 1-1966 & 744 & 278 & 0 & 0 & \\
\hline 2 & 744 & 278 & 0 & 0 & \\
\hline 3 & 744 & 278 & 0 & 0 & \\
\hline 4 & 744 & 278 & 0 & 0 & \\
\hline 1-1967 & 744 & 278 & 0 & 0 & \\
\hline 2 & 744 & 278 & 0 & 0 & \\
\hline 3 & 744 & 278 & 0 & 0 & \\
\hline 4 & 744 & 278 & 0 & 0 & \\
\hline $1-1968$ & 744 & 278 & 0 & 0 & \\
\hline 2 & 745 & 278 & 0 & 0 & \\
\hline 3 & 745 & 278 & 0 & 0 & \\
\hline 4 & 745 & 278 & 0 & 0 & \\
\hline 1-1969 & 744 & 278 & 0 & 0 & \\
\hline 2 & 744 & 278 & 0 & 0 & \\
\hline 3 & 744 & 278 & 0 & 0 & \\
\hline 4 & 744 & 278 & 211 & 84 & \\
\hline $1-1970$ & 743 & 278 & 211 & 84 & \\
\hline 2 & 743 & 278 & 211 & 84 & \\
\hline 3 & 743 & 278 & 211 & 84 & \\
\hline 4 & 743 & 278 & 211 & 84 & \\
\hline 1-1971 & 743 & 278 & 211 & 84 & \\
\hline 2 & 743 & 278 & 211 & 84 & \\
\hline 3 & 743 & 278 & 211 & 84 & \\
\hline 4 & 743 & 278 & 211 & 84 & \\
\hline 1-1972 & 743 & 278 & 211 & 84 & \\
\hline 2 & 743 & 278 & 211 & 84 & \\
\hline 3 & 743 & 278 & 211 & 84 & \\
\hline 4 & 744 & 278 & 211 & 84 & \\
\hline $1-1973$ & 744 & 278 & 211 & 84 & \\
\hline 2 & 734 & 274 & 211 & 84 & \\
\hline 3 & 744 & 278 & 211 & 84 & \\
\hline 4 & 426 & 162 & 211 & 84 & \\
\hline $1-1974$ & 616 & 231 & 244 & 96 & \\
\hline 2 & 588 & 221 & 244 & 96 & \\
\hline 3 & 339 & 131 & 244 & 96 & \\
\hline 4 & 519 & 196 & 244 & 96 & \\
\hline
\end{tabular}


241-S-101 LEVEL. HISTORY

HNF-SD-WM-ER-323, Rev . 1

\begin{tabular}{|c|c|c|c|c|c|}
\hline Year & Total & Total & Solids & Solids & ILL \\
\hline & (K gal! & (in) & [K gal) & (in) & (in) \\
\hline $1-1975$ & 552 & 208 & 244 & $9 \overline{6}$ & \\
\hline 2 & $\widehat{692}$ & 259 & 332 & 128 & \\
\hline 3 & 332 & 128 & 332 & 128 & \\
\hline 4 & 560 & 211 & 332 & 128 & \\
\hline $1-1976$ & 717 & 268 & 332 & 128 & \\
\hline 2 & 717 & 268 & 332 & 128 & \\
\hline 3 & $7 \longdiv { 7 1 9 }$ & 269 & 332 & 128 & \\
\hline 4 & 725 & 271 & 332 & 128 & \\
\hline 1.1977 & 708 & $2 \overline{65}$ & 332 & 128 & \\
\hline 2 & 725 & 271 & 332 & 128 & \\
\hline 3 & 703 & 263 & 332 & 128 & \\
\hline 4 & 706 & 264 & 332 & 128 & \\
\hline $1-1978$ & 703 & 263 & 332 & 128 & \\
\hline 2 & 703 & $2 \overline{63}$ & 332 & $12 \overline{8}$ & \\
\hline 3 & 700 & 262 & 332 & 128 & \\
\hline 4 & 700 & 262 & 332 & 128 & \\
\hline $1-1979$ & 678 & 254 & 332 & 128 & \\
\hline 2 & 472 & 179 & 332 & 128 & \\
\hline 3 & 472 & 179 & 332 & 128 & \\
\hline 4 & 585 & 220 & 332 & 128 & \\
\hline $1-1980$ & 711 & 266 & 332 & 128 & \\
\hline 2 & 520 & 197 & 332 & 128 & \\
\hline 3 & 427 & 163 & 415 & 158 & \\
\hline 4 & 427 & 163 & 415 & 158 & \\
\hline 1-1981 & 427 & 163 & 415 & 158 & \\
\hline 2 & 427 & 163 & 415 & 158 & \\
\hline 3 & 427 & 163 & 415 & 158 & \\
\hline 4 & 427 & 163 & 415 & 158 & \\
\hline $1-1982$ & 427 & 163 & 415 & 158 & \\
\hline 2 & 427 & 163 & 415 & 158 & \\
\hline 3 & 427 & 163 & 415 & 158 & \\
\hline 4 & 427 & 163 & 415 & 158 & \\
\hline 1-1983 & 427 & 163 & $\overline{415}$ & 158 & \\
\hline 2 & 427 & 163 & 415 & 158 & \\
\hline 3 & 427 & 163 & 415 & 158 & \\
\hline 4 & 427 & 163 & 415 & 158 & \\
\hline $1-1984$ & 427 & 163 & 415 & 158 & \\
\hline 2 & 427 & 163 & 415 & 158 & \\
\hline 3 & 427 & 163 & 415 & 158 & \\
\hline 4 & 427 & 163 & 415 & 158 & \\
\hline $1-1985$ & 427 & $\overline{163}$ & 415 & 158 & \\
\hline 2 & 427 & 163 & 415 & 158 & \\
\hline 3 & 427 & 163 & 415 & 158 & \\
\hline 4 & 427 & $16 \overline{3}$ & 415 & 158 & \\
\hline $1-1986$ & 427 & $1 \overline{63}$ & 415 & 158 & 160 \\
\hline 2 & 427 & 163 & 415 & 158 & 160 \\
\hline
\end{tabular}


241-S-101 LEVEL. HISTORY

HNF-SD-WM-ER-323, Rev . 1

\begin{tabular}{|c|c|c|c|c|c|}
\hline Year & Total & Total & Solids & Solids & ILL \\
\hline & (K gal) & (in) & (K gal) & (in) & (in) \\
\hline 3 & 427 & 163 & 415 & 158 & 160 \\
\hline 4 & 427 & 163 & 415 & 158 & 159 \\
\hline 1-1987 & 427 & 163 & 415 & 158 & 160 \\
\hline 2 & 427 & 163 & 415 & 158 & \\
\hline 3 & 427 & 163 & 415 & 158 & 160 \\
\hline 4 & 427 & 163 & 415 & 158 & 159 \\
\hline 1-1988 & 427 & 163 & 415 & 158 & 160 \\
\hline 2 & 427 & 163 & 415 & 158 & 160 \\
\hline 3 & 427 & 163 & 415 & 158 & 159 \\
\hline 4 & 427 & 163 & 415 & 158 & 160 \\
\hline 1-1989 & 427 & 163 & 415 & 158 & \\
\hline 2 & 427 & 163 & 415 & 158 & 160 \\
\hline 3 & 427 & 163 & 415 & 158 & 160 \\
\hline 4 & 427 & 163 & 415 & 158 & 160 \\
\hline $1-1990$ & 427 & 163 & 415 & 158 & 160 \\
\hline 2 & 427 & 163 & 415 & 158 & \\
\hline 3 & 427 & 163 & 415 & 158 & 160 \\
\hline 4 & 427 & 163 & 415 & 158 & 160 \\
\hline 1-1991 & 427 & 163 & 415 & 158 & 160 \\
\hline 2 & 427 & 163 & 415 & 158 & 160 \\
\hline 3 & 427 & 163 & 415 & 158 & 161 \\
\hline 4 & 427 & 163 & 415 & 158 & 161 \\
\hline $1-1992$ & 427 & 163 & 415 & 158 & \\
\hline 2 & 427 & 163 & 415 & 758 & 160 \\
\hline 3 & 427 & 163 & 415 & 158 & \\
\hline 4 & 427 & 163 & 415 & 158 & 160 \\
\hline $1-1993$ & 427 & 163 & 415 & 158 & 161 \\
\hline 2 & 427 & 163 & 415 & 158 & 160 \\
\hline 3 & 427 & 163 & 415 & 158 & 161 \\
\hline 4 & 427 & 163 & 415 & 158 & 160 \\
\hline 1-1994 & 427 & 163 & 415 & 158 & 161 \\
\hline 2 & 427 & 163 & 415 & 158 & 160 \\
\hline 3 & 427 & 163 & 415 & 158 & 161 \\
\hline 4 & 427 & 163 & 415 & 158 & 160 \\
\hline $1-1995$ & 427 & 163 & 415 & 158 & 160 \\
\hline 2 & 427 & 163 & 415 & 158 & 160 \\
\hline 3 & 427 & 163 & 415 & 158 & 160 \\
\hline 4 & 427 & 163 & 415 & 158 & 160 \\
\hline $1-1996$ & 427 & 163 & 415 & 158 & 160 \\
\hline 2 & 427 & 163 & 415 & 158 & 160 \\
\hline
\end{tabular}


241-S-102 LEVEL. HISTORY

\begin{tabular}{|c|c|c|c|c|c|}
\hline Year & Total & Total & Solids & Solids & ILL \\
\hline & (K gal) & (in) & (K gal) & (in) & (in) \\
\hline & & & & & \\
\hline \multicolumn{6}{|l|}{$1-1952$} \\
\hline \multicolumn{6}{|l|}{2} \\
\hline \multicolumn{6}{|l|}{3} \\
\hline \multicolumn{6}{|l|}{4} \\
\hline \multicolumn{6}{|l|}{$1-1953$} \\
\hline \multicolumn{6}{|l|}{2} \\
\hline 3 & 239 & 94 & 0 & 0 & \\
\hline 4 & 766 & 286 & 0 & 0 & \\
\hline 1-1954 & 750 & 280 & 0 & 0 & \\
\hline 2 & 747 & 279 & 0 & 0 & \\
\hline 3 & 747 & 279 & 0 & 0 & \\
\hline 4 & 715 & 267 & 0 & 0 & \\
\hline $1-1955$ & 715 & 267 & 0 & 0 & \\
\hline 2 & 715 & 267 & 0 & 0 & \\
\hline 3 & 745 & 278 & 0 & 0 & \\
\hline 4 & 745 & 278 & 0 & 0 & \\
\hline 1-1956 & 745 & 278 & 0 & 0 & \\
\hline 2 & 745 & 278 & 0 & 0 & \\
\hline 3 & 745 & 278 & 0 & 0 & \\
\hline 4 & 745 & 278 & 0 & 0 & \\
\hline 1.1957 & 740 & 277 & 0 & 0 & \\
\hline 2 & 740 & 277 & 0 & 0 & \\
\hline 3 & 739 & 276 & 0 & 0 & \\
\hline 4 & 739 & 276 & 0 & 0 & \\
\hline $1-1958$ & 739 & 276 & 0 & 0 & \\
\hline 2 & 739 & 276 & 0 & 0 & \\
\hline 3 & 739 & 276 & 0 & 0 & \\
\hline 4 & 739 & 276 & 0 & 0 & \\
\hline $1-1959$ & 736 & 275 & 0 & 0 & \\
\hline 2 & 736 & 275 & 0 & 0 & \\
\hline 3 & 736 & 275 & 0 & 0 & \\
\hline 4 & 736 & 275 & 0 & 0 & \\
\hline $1-1960$ & 736 & 275 & 0 & 0 & \\
\hline 2 & 736 & 275 & 0 & 0 & \\
\hline 3 & 736 & 275 & 0 & 0 & \\
\hline 4 & 733 & 274 & 0 & 0 & \\
\hline \multicolumn{6}{|l|}{ 1-1961 } \\
\hline 2 & 733 & 274 & 0 & 0 & \\
\hline \multicolumn{6}{|l|}{3} \\
\hline 4 & 733 & 274 & 0 & 0 & \\
\hline \multicolumn{6}{|l|}{$1-1962$} \\
\hline 2 & 733 & 274 & 0 & 0 & \\
\hline \multicolumn{6}{|l|}{3} \\
\hline 4 & 733 & 274 & 0 & 0 & \\
\hline \multicolumn{6}{|l|}{$1-1963$} \\
\hline 2 & 733 & 274 & 0 & 0 & \\
\hline
\end{tabular}


241-S-102 LEVEL HISTORY

\begin{tabular}{|c|c|c|c|c|c|}
\hline Year & Total & Total & Solids & Solids & ILL \\
\hline & (K gal) & (in) & (K gal) & (in) & (in) \\
\hline \multirow{2}{*}{\multicolumn{6}{|c|}{3}} \\
\hline & & & & & \\
\hline 4 & 733 & 274 & 0 & 0 & \\
\hline \multicolumn{6}{|l|}{ 1-1964 } \\
\hline 2 & 733 & 274 & 0 & 0 & \\
\hline \multicolumn{6}{|l|}{3} \\
\hline 4 & 733 & 274 & 0 & 0 & \\
\hline \multicolumn{6}{|l|}{$1-1965$} \\
\hline 2 & 750 & 280 & 0 & 0 & \\
\hline 3 & 750 & 280 & 5 & 9 & \\
\hline 4 & 750 & 280 & 5 & 9 & \\
\hline $1-1966$ & 750 & 280 & 5 & 9 & \\
\hline 2 & 750 & 280 & 5 & 9 & \\
\hline 3 & 750 & 280 & 5 & 9 & \\
\hline 4 & 750 & 280 & 5 & 9 & \\
\hline $1-1967$ & 750 & 280 & 5 & 9 & \\
\hline 2 & 750 & 280 & 5 & 9 & \\
\hline 3 & 750 & 280 & 5 & 9 & \\
\hline 4 & 750 & 280 & 5 & 9 & \\
\hline $1-1968$ & 750 & 280 & 5 & 9 & \\
\hline 2 & 750 & 280 & 5 & 9 & \\
\hline 3 & 750 & 280 & 5 & 9 & \\
\hline 4 & 750 & 280 & 5 & 9 & \\
\hline $7-1969$ & 750 & 280 & 5 & 9 & \\
\hline 2 & 750 & 280 & 5 & 9 & \\
\hline 3 & 750 & 280 & 5 & 9 & \\
\hline 4 & 750 & 280 & 4 & 9 & \\
\hline $1-1970$ & 750 & 280 & 4 & 9 & \\
\hline 2 & 750 & 280 & 4 & 9 & \\
\hline 3 & 750 & 280 & 4 & 9 & \\
\hline 4 & 750 & 280 & 4 & 9 & \\
\hline 1-1971 & 750 & 280 & 4 & 9 & \\
\hline 2 & 750 & 280 & 4 & 9 & \\
\hline 3 & 750 & 280 & 4 & 9 & \\
\hline 4 & 750 & 280 & 4 & 9 & \\
\hline $1-1972$ & 750 & 280 & 4 & 9 & \\
\hline 2 & 750 & 280 & 4 & 9 & \\
\hline 3 & 750 & 280 & 4 & 9 & \\
\hline 4 & 744 & 278 & 4 & 9 & \\
\hline $1-1973$ & 743 & 278 & 4 & 9 & \\
\hline 2 & 743 & 278 & 4 & 9 & \\
\hline 3 & 762 & 285 & 4 & 9 & \\
\hline 4 & 577 & 217 & 51 & 26 & \\
\hline $1-1974$ & 634 & 238 & 51 & 26 & \\
\hline 2 & 685 & 257 & 51 & $\overline{26}$ & \\
\hline 3 & 691 & 259 & 103 & 45 & \\
\hline 4 & 662 & 248 & 145 & 60 & \\
\hline
\end{tabular}


241-S-102 LEVEL HISTORY

\begin{tabular}{|c|c|c|c|c|c|}
\hline Year & Total & Total & Solids & Solids & ILL \\
\hline & (K gal) & (in) & (K gal) & (in) & (in) \\
\hline $1-1975$ & 604 & 227 & 145 & 60 & \\
\hline 2 & 689 & 258 & 145 & 60 & \\
\hline 3 & 464 & 176 & 145 & 60 & \\
\hline 4 & 554 & 209 & 200 & 80 & \\
\hline $1-1976$ & 477 & 181 & 200 & 80 & \\
\hline 2 & 552 & 208 & 200 & 80 & \\
\hline 3 & 543 & 205 & 200 & 80 & \\
\hline 4 & 535 & 202 & 200 & 80 & \\
\hline $1-1977$ & 211 & 84 & 208 & 83 & \\
\hline 2 & 230 & 91 & 208 & 83 & \\
\hline 3 & 233 & 92 & 208 & 83 & \\
\hline 4 & 246 & 97 & 208 & 83 & \\
\hline $1-1978$ & 266 & 104 & 208 & 83 & \\
\hline 2 & 274 & 107 & 208 & 83 & \\
\hline 3 & 282 & 110 & 208 & 83 & \\
\hline 4 & 483 & 183 & 208 & 83 & \\
\hline 1-1979 & 618 & $2 \overline{3} \overline{2}$ & 208 & 83 & \\
\hline 2 & 378 & 145 & 208 & 83 & \\
\hline 3 & 480 & 182 & 208 & 83 & \\
\hline 4 & 618 & 232 & 510 & 193 & \\
\hline 1.1980 & 609 & 229 & 510 & 193 & \\
\hline 2 & 561 & 211 & 510 & 193 & \\
\hline 3 & 555 & 209 & 555 & 209 & \\
\hline 4 & 555 & 209 & 555 & 209 & \\
\hline $1-1981$ & 555 & 209 & 555 & 209 & \\
\hline 2 & 555 & 209 & 555 & 209 & \\
\hline 3 & 555 & 209 & 555 & 209 & \\
\hline 4 & 555 & 209 & 555 & 209 & \\
\hline $1-1982$ & 555 & 209 & 555 & 209 & \\
\hline 2 & 549 & 207 & 549 & 207 & \\
\hline 3 & 549 & 207 & 549 & 207 & \\
\hline 4 & 549 & 207 & 549 & 207 & \\
\hline $1-1983$ & 549 & 207 & 549 & 207 & \\
\hline 2 & 549 & 207 & 549 & 207 & \\
\hline 3 & 549 & 207 & 549 & 207 & \\
\hline 4 & 549 & 207 & 549 & 207 & \\
\hline 1-1984 & 549 & 207 & 549 & 207 & \\
\hline 2 & 549 & 207 & 549 & 207 & \\
\hline 3 & 549 & 207 & 549 & 207 & \\
\hline 4 & 549 & 207 & 549 & 207 & \\
\hline $1-1985$ & 549 & 207 & 549 & 207 & \\
\hline 2 & 549 & 207 & 549 & 207 & \\
\hline 3 & 549 & 207 & 549 & 207 & \\
\hline 4 & 549 & 207 & 549 & 207 & \\
\hline $1-1986$ & 549 & 207 & 549 & 207 & 199 \\
\hline 2 & 549 & 207 & 549 & 207 & 199 \\
\hline
\end{tabular}


241-S-102 LEVEL. HISTORY

HNF-SD-WM-ER-323, Rev. 1

\begin{tabular}{|c|c|c|c|c|c|}
\hline Year & Total & Total & Solids & Solids & ILL \\
\hline & (K gal) & (in) & (K gal) & (in) & (in) \\
\hline 3 & 549 & 207 & 549 & 207 & 199 \\
\hline 4 & 549 & 207 & 549 & 207 & $19 \overline{9}$ \\
\hline 1-1987 & 549 & 207 & 549 & 207 & 198 \\
\hline 2 & 549 & 207 & 549 & 207 & \\
\hline 3 & 549 & 207 & 549 & 207 & 199 \\
\hline 4 & 549 & 207 & 549 & 207 & 200 \\
\hline 1-1988 & 549 & 207 & 549 & 207 & 199 \\
\hline 2 & 549 & 207 & 549 & 207 & 201 \\
\hline 3 & 549 & 207 & 549 & 207 & 200 \\
\hline 4 & 549 & 207 & 549 & 207 & \\
\hline $1-1989$ & 549 & 207 & 549 & 207 & \\
\hline 2 & 549 & 207 & 549 & 207 & 201 \\
\hline 3 & 549 & 207 & 549 & 207 & 201 \\
\hline 4 & 549 & 207 & 549 & 207 & 200 \\
\hline $1-1990$ & 549 & 207 & 549 & 207 & 200 \\
\hline 2 & 549 & 207 & 549 & 207 & \\
\hline 3 & 549 & 207 & 549 & 207 & 201 \\
\hline 4 & 549 & 207 & 549 & 207 & 202 \\
\hline 1-1991 & 549 & 207 & 549 & 207 & 202 \\
\hline 2 & 549 & 207 & 549 & 207 & 201 \\
\hline 3 & 549 & 207 & 549 & 207 & 202 \\
\hline 4 & 549 & 207 & 549 & 207 & 201 \\
\hline $1-1992$ & 549 & 207 & 549 & 207 & \\
\hline 2 & 549 & 207 & 549 & 207 & 201 \\
\hline 3 & 549 & 207 & 549 & 207 & \\
\hline 4 & 549 & 207 & 549 & 207 & 201 \\
\hline 1-1993 & 549 & 207 & 549 & 207 & 202 \\
\hline 2 & 549 & 207 & 549 & 207 & 203 \\
\hline 3 & 549 & 207 & 549 & 207 & 202 \\
\hline 4 & 549 & 207 & 549 & 207 & 203 \\
\hline 1-1994 & 549 & 207 & 549 & 207 & 202 \\
\hline 2 & 549 & 207 & 549 & 207 & 203 \\
\hline 3 & 549 & 207 & 549 & 207 & 203 \\
\hline 4 & 549 & 207 & 549 & 207 & 203 \\
\hline 1-1995 & 549 & 207 & 549 & 207 & 202 \\
\hline 2 & 549 & 207 & 549 & 207 & 204 \\
\hline 3 & 549 & 207 & 549 & 207 & 204 \\
\hline 4 & 549 & 207 & 549 & 207 & 203 \\
\hline 1-1996 & 549 & 207 & 549 & 207 & 205 \\
\hline 2 & 549 & 207 & 549 & 207 & 204 \\
\hline
\end{tabular}


241-S-103 LEVEL HISTORY

HNF-SD-WM-ER-323, Rev, 1

\begin{tabular}{|c|c|c|c|c|c|}
\hline Year & Total & Total & Solids & Solids & ILL \\
\hline & (K gal) & (in) & (K gal) & (in) & (in) \\
\hline \multicolumn{6}{|l|}{$1-1952$} \\
\hline \multicolumn{6}{|l|}{2} \\
\hline \multicolumn{6}{|l|}{3} \\
\hline \multicolumn{6}{|l|}{4} \\
\hline \multicolumn{6}{|l|}{$1-1953$} \\
\hline \multicolumn{6}{|l|}{2} \\
\hline 3 & 0 & 0 & 0 & 0 & \\
\hline 4 & 430 & 164 & 0 & 0 & \\
\hline 1-1954 & 514 & 194 & 0 & 0 & \\
\hline 2 & 549 & 207 & 0 & 0 & \\
\hline 3 & 549 & 207 & 0 & 0 & \\
\hline 4 & 536 & 202 & 0 & 0 & \\
\hline $1-1955$ & 537 & 203 & 0 & 0 & \\
\hline 2 & 752 & 281 & 0 & 0 & \\
\hline 3 & 752 & 281 & 0 & 0 & \\
\hline 4 & 752 & 281 & 0 & 0 & \\
\hline 1-1956 & 752 & 281 & 0 & 0 & \\
\hline 2 & 752 & 281 & 0 & 0 & \\
\hline 3 & 752 & 281 & 0 & 0 & \\
\hline 4 & 752 & 281 & 0 & 0 & \\
\hline 1-1957 & 752 & 281 & 0 & 0 & \\
\hline 2 & 750 & 280 & 0 & 0 & \\
\hline 3 & 750 & 280 & 0 & 0 & \\
\hline 4 & 750 & 280 & 0 & 0 & \\
\hline $1-1958$ & 747 & 279 & 0 & 0 & \\
\hline 2 & 750 & 280 & 0 & 0 & \\
\hline 3 & 750 & 280 & 0 & 0 & \\
\hline 4 & 750 & 280 & 0 & 0 & \\
\hline $1-1959$ & 750 & 280 & 0 & 0 & \\
\hline 2 & 750 & 280 & 0 & 0 & \\
\hline 3 & 750 & 280 & 0 & 0 & \\
\hline 4. & 750 & 280 & 0 & 0 & \\
\hline $1-1960$ & 747 & 279 & 0 & 0 & \\
\hline 2 & 747 & 279 & 0 & 0 & \\
\hline 3 & 747 & 279 & 0 & 0 & \\
\hline 4 & 750 & 280 & 0 & 0 & \\
\hline \multicolumn{6}{|l|}{$1-1961$} \\
\hline 2 & 747 & 279 & 0 & 0 & \\
\hline \multicolumn{6}{|l|}{3} \\
\hline 4 & $7 \overline{47}$ & 279 & 0 & 0 & \\
\hline \multicolumn{6}{|l|}{ 1-1962 } \\
\hline 2 & 747 & 279 & 0 & 0 & \\
\hline \multicolumn{6}{|l|}{3} \\
\hline 4 & 747 & 279 & 0 & 0 & \\
\hline \multicolumn{6}{|l|}{$1-1963$} \\
\hline 2 & 747 & 279 & 0 & 0 & \\
\hline
\end{tabular}


241-S-103 LEVEL HISTORY

HNF-SD-WM-ER-323, Rev. 1

\begin{tabular}{|c|c|c|c|c|c|}
\hline Year & Total & Total & Solids & Solids & ILL \\
\hline & (K gal) & (in) & (K gal) & (in) & (in) \\
\hline & & & & & \\
\hline \multicolumn{6}{|l|}{3} \\
\hline 4 & 747 & 279 & 0 & 0 & \\
\hline \multicolumn{6}{|l|}{$1-1964$} \\
\hline 2 & 747 & 279 & 0 & 0 & \\
\hline \multicolumn{6}{|l|}{3} \\
\hline 4 & 747 & 279 & 0 & 0 & \\
\hline \multicolumn{6}{|l|}{$1-1965$} \\
\hline 2 & 763 & 285 & 0 & 0 & \\
\hline 3 & 763 & 285 & 5 & 9 & \\
\hline 4 & 763 & 285 & 5 & 9 & \\
\hline $1-1966$ & 763 & 285 & 5 & 9 & \\
\hline 2 & $7 \overline{763}$ & 285 & 5 & 9 & \\
\hline 3 & 763 & 285 & 5 & 9 & \\
\hline 4 & 763 & 285 & 5 & 9 & \\
\hline $1-1967$ & 768 & 287 & 5 & 9 & \\
\hline 2 & 765 & 286 & 5 & 9 & \\
\hline 3 & 765 & 286 & 5 & 9 & \\
\hline 4 & 765 & 286 & 5 & 9 & \\
\hline $1-1968$ & 765 & 286 & 5 & 9 & \\
\hline 2 & 765 & 286 & 5 & 9 & \\
\hline 3 & 765 & 286 & 5 & 9 & \\
\hline 4 & 764 & 285 & 5 & 9 & \\
\hline $1-1969$ & 764 & 285 & 5 & 9 & \\
\hline 2 & 764 & 285 & 5 & 9 & \\
\hline 3 & 765 & 286 & 5 & 9 & \\
\hline 4 & 765 & 286 & 9 & 11 & \\
\hline $1-1970$ & 764 & 285 & 9 & 11 & \\
\hline 2 & 764 & 285 & 9 & 11 & \\
\hline 3 & 765 & 286 & 9 & 11 & \\
\hline 4 & 764 & 285 & 9 & 11 & \\
\hline 1-1971 & 764 & 285 & 9 & 11 & \\
\hline 2 & 764 & 285 & 9 & 11 & \\
\hline 3 & 764 & 285 & 9 & 11 & \\
\hline 4 & 764 & 285 & 9 & 11 & \\
\hline 1-1972 & 763 & 285 & 9 & 11 & \\
\hline 2 & 763 & 285 & 9 & 11 & \\
\hline 3 & 766 & 286 & 9 & 11 & \\
\hline 4 & 767 & 286 & 9 & 11 & \\
\hline $1-1973$ & 767 & 286 & 9 & 11 & \\
\hline 2 & 761 & 284 & 9 & 11 & \\
\hline 3 & 773 & 289 & 9 & 11 & \\
\hline 4 & 549 & 207 & 37 & 21 & \\
\hline $1-1974$ & 388 & 149 & 37 & 21 & \\
\hline 2 & 640 & 240 & 37 & 21 & \\
\hline 3 & 570 & 215 & 37 & 21 & \\
\hline 4 & 513 & 194 & 37 & 21 & \\
\hline
\end{tabular}


241-S-103 LEVEL HISTORY

HNF-SD-WM-ER-323, Rev. 1

\begin{tabular}{|c|c|c|c|c|c|}
\hline Year & Total & Total & Solids & Solids & ILL \\
\hline & (K gal) & (in) & ( $\mathrm{K}$ gal) & (in) & (in) \\
\hline 1-1975 & 409 & 156 & 37 & 21 & \\
\hline 2 & 642 & 241 & 37 & 21 & \\
\hline 3 & 403 & 154 & 37 & 21 & \\
\hline 4 & 565 & 213 & 37 & 21 & \\
\hline $1-1976$ & 326 & 126 & 37 & 21 & \\
\hline 2 & 337 & 130 & 37 & 21 & \\
\hline 3 & 194 & 78 & 37 & 21 & \\
\hline 4 & 439 & 167 & 37 & 21 & \\
\hline $1-1977$ & 466 & 177 & 57 & 28 & \\
\hline 2 & 301 & 117 & 57 & 28 & \\
\hline 3 & 257 & 101 & 57 & 28 & \\
\hline 4 & 395 & 151 & 68 & 32 & \\
\hline $1-1978$ & 459 & 174 & 68 & 32 & \\
\hline 2 & 238 & 94 & 120 & 51 & \\
\hline 3 & 387 & 148 & 167 & 68 & \\
\hline 4 & 662 & 248 & 128 & 54 & \\
\hline $1-1979$ & 440 & 167 & 128 & 54 & \\
\hline 2 & 499 & 189 & 128 & 54 & \\
\hline 3 & 560 & 211 & 128 & 54 & \\
\hline 4 & 708 & 265 & 153 & 63 & \\
\hline $1-1980$ & 675 & 253 & 153 & 63 & \\
\hline 2 & 484 & 183 & 153 & 63 & \\
\hline 3 & 647 & 243 & 153 & 63 & \\
\hline 4 & 248 & 98 & 231 & 91 & \\
\hline $1-1981$ & 248 & 98 & 231 & 91 & \\
\hline 2 & 248 & 98 & 231 & 91 & \\
\hline 3 & 248 & 98 & 231 & 91 & \\
\hline 4 & 248 & 98 & 231 & 91 & \\
\hline 1-1982 & 248 & 98 & 231 & 91 & \\
\hline 2 & 248 & 98 & 231 & 91 & \\
\hline 3 & 248 & 98 & 231 & 91 & \\
\hline 4 & 248 & 98 & 231 & 91 & \\
\hline $1-1983$ & 248 & 98 & 231 & 91 & \\
\hline 2 & 248 & 98 & 231 & 91 & \\
\hline 3 & 248 & 98 & 231 & 91 & \\
\hline 4 & 248 & 98 & 231 & 91 & \\
\hline $1-1984$ & 248 & 98 & 231 & 91 & \\
\hline 2 & 248 & 98 & 231 & 91 & \\
\hline 3 & 248 & 98 & 231 & 91 & \\
\hline 4 & 248 & 98 & 231 & 91. & \\
\hline $1-1985$ & 248 & 98 & 231 & 91 & \\
\hline 2 & 248 & 98 & 231 & 91 & \\
\hline 3 & 248 & 98 & 231 & 91 & \\
\hline 4 & 248 & 98 & 231 & 91 & \\
\hline 1-1986 & 248 & 98 & 231 & 91 & 101 \\
\hline 2 & 248 & 98 & 231 & 91 & 102 \\
\hline
\end{tabular}


241-S-103 LEVEL HISTORY

HNF-SD-WM-ER-323, Rev. 1

\begin{tabular}{|c|c|c|c|c|c|}
\hline Year & Total & Total & Solids & Solids & ILL \\
\hline & (K gal) & (in) & (K gal) & (in) & (in) \\
\hline 3 & 248 & 98 & 231 & 91 & 102 \\
\hline 4 & 248 & 98 & 231 & 91 & 102 \\
\hline 1-1987 & 248 & 98 & 231 & 91 & 102 \\
\hline 2 & 248 & 98 & 231 & 91 & \\
\hline 3 & 248 & 98 & 231 & 91 & 102 \\
\hline 4 & 248 & 98 & 231 & 91 & 102 \\
\hline $1-1988$ & 248 & 98 & 231 & 91 & 102 \\
\hline 2 & 248 & 98 & 231 & 91 & 103 \\
\hline 3 & 248 & 98 & 231 & 91 & 102 \\
\hline 4 & 248 & 98 & 231 & 91 & 102 \\
\hline 1-1989 & 248 & 98 & 231 & 91 & \\
\hline 2 & 248 & 98 & 231 & 91 & 103 \\
\hline 3 & 248 & 98 & 231 & 91 & 103 \\
\hline 4 & 248 & 98 & 231 & 91 & 103 \\
\hline 1-1990 & 248 & 98 & 231 & 91 & 103 \\
\hline 2 & 248 & 98 & 231 & 91 & \\
\hline 3 & 248 & 98 & 231 & 91 & 102 \\
\hline 4 & 248 & 98 & 231 & 91 & 103 \\
\hline \begin{tabular}{|l|}
$1-1991$ \\
\end{tabular} & 248 & 98 & 231 & 91 & 103 \\
\hline 2 & 248 & 98 & 231 & 91 & 103 \\
\hline 3 & 248 & 98 & 231 & 91 & 103 \\
\hline 4 & 248 & 98 & 231 & 91 & 103 \\
\hline $1-1992$ & 248 & 98 & 231 & 91 & \\
\hline 2 & 248 & 98 & 231 & 91 & \\
\hline 3 & 248 & 98 & 231 & 91 & \\
\hline 4 & 248 & 98 & 231 & 91 & 103 \\
\hline 1.1993 & 248 & 98 & 231 & 91 & 103 \\
\hline 2 & 248 & 98 & 231 & 91 & 103 \\
\hline 3 & 248 & 98 & 231 & 91 & 103 \\
\hline 4 & 248 & 98 & 231 & 91 & 103 \\
\hline $1-1994$ & 248 & 98 & 231 & 91 & 103 \\
\hline 2 & 248 & 98 & 231 & 91 & 103 \\
\hline 3 & 248 & 98 & 231 & 91 & 103 \\
\hline 4 & 248 & 98 & 231 & 91 & 103 \\
\hline 1-1995 & 248 & 98 & 231 & 91 & 103 \\
\hline 2 & 248 & 98 & 231 & 91 & 103 \\
\hline 3 & 248 & 98 & 231 & 91 & 103 \\
\hline 4 & 248 & 98 & 231 & 91 & 102 \\
\hline $1-1996$ & 248 & 98 & 231 & 91 & 103 \\
\hline 2 & 248 & 98 & 231 & 91 & 103 \\
\hline
\end{tabular}


241-S-104 LEVEL HISTORY

HNF-SD-WM-ER-323, Rev. 1

\begin{tabular}{|c|c|c|c|c|c|}
\hline Year & Total & Total & Solids & Solids & ILL \\
\hline & (K gal) & (in) & (K gal) & (in) & (in) \\
\hline \multicolumn{6}{|l|}{ 1-1952 } \\
\hline \multirow{2}{*}{\multicolumn{6}{|c|}{2}} \\
\hline \multirow{2}{*}{\multicolumn{6}{|c|}{3}} \\
\hline \multirow{2}{*}{\multicolumn{6}{|c|}{4}} \\
\hline & & & & & \\
\hline $1-1953$ & 741 & 277 & 0 & 0 & \\
\hline 2 & 758 & 283 & 0 & 0 & \\
\hline 3 & $6 \overline{63}$ & 249 & 0 & 0 & \\
\hline 4 & 524 & 198 & 0 & 0 & \\
\hline $1-1954$ & 458 & 174 & 0 & 0 & \\
\hline 2 & 734 & 274 & 0 & 0 & \\
\hline 3 & 667 & 250 & 0 & 0 & \\
\hline 4 & 648 & 243 & 0 & 0 & \\
\hline 1-1955 & 623 & 234 & 0 & 0 & \\
\hline 2 & 725 & 271 & 0 & 0 & \\
\hline 3 & 763 & 285 & 0 & 0 & \\
\hline 4 & $7 \overline{59}$ & 283 & 0 & 0 & \\
\hline $1-1956$ & 752 & 281 & 0 & 0 & \\
\hline 2 & 753 & 281 & 0 & 0 & \\
\hline 3 & 753 & 281 & 0 & 0 & \\
\hline 4 & 753 & 281 & 0 & 0 & \\
\hline $1-1957$ & 750 & 280 & 0 & 0 & \\
\hline 2 & 732 & 274 & 0 & 0 & \\
\hline 3 & 732 & 274 & 0 & 0 & \\
\hline 4 & 730 & 273 & 0 & 0 & \\
\hline $1-1958$ & 725 & 271 & 0 & 0 & \\
\hline 2 & 725 & 271 & 0 & 0 & \\
\hline 3 & 725 & 271 & 0 & 0 & \\
\hline 4 & 725 & 271 & 0 & 0 & \\
\hline $1-1959$ & 725 & 271 & 0 & 0 & \\
\hline 2 & 725 & 271 & 0 & 0 & \\
\hline 3 & 721 & 270 & 0 & 0 & \\
\hline 4 & 719 & 269 & 0 & 0 & \\
\hline $1-1960$ & 719 & 269 & 0 & 0 & \\
\hline 2 & 719 & 269 & 0 & 0 & \\
\hline 3 & 719 & 269 & 0 & 0 & \\
\hline 4 & 716 & 268 & 0 & 0 & \\
\hline \multicolumn{6}{|l|}{$1-1961$} \\
\hline 2 & 714 & 267 & 0 & 0 & \\
\hline \multicolumn{6}{|l|}{3} \\
\hline 4 & 714 & 267 & 0 & 0 & \\
\hline \multicolumn{6}{|l|}{$1-1962$} \\
\hline 2 & 714 & 267 & 0 & 0 & \\
\hline \multicolumn{6}{|l|}{3} \\
\hline 4 & 714 & 267 & 0 & 0 & \\
\hline \multicolumn{6}{|l|}{$1-1963$} \\
\hline 2 & 711 & 266 & 0 & 0 & \\
\hline
\end{tabular}


241-S-104 LEVEL HISTORY

HNF-SD-WM-ER-323, Rev. 1

\begin{tabular}{|c|c|c|c|c|c|}
\hline Year & Total & Total & Solids & Solids & ILL \\
\hline & (K gal) & (in) & (K gal) & (in) & (in) \\
\hline \multirow{2}{*}{\multicolumn{6}{|c|}{3}} \\
\hline & & & & & \\
\hline 4 & 711 & 266 & 0 & 0 & \\
\hline \multicolumn{6}{|l|}{1.1964} \\
\hline 2 & 711 & 266 & 0 & 0 & \\
\hline \multicolumn{6}{|l|}{3} \\
\hline 4 & 711 & 266 & 0 & 0 & \\
\hline \multicolumn{6}{|l|}{$1-1965$} \\
\hline 2 & 807 & 301 & 0 & 0 & \\
\hline 3 & 807 & 301 & 0 & 0 & \\
\hline 4 & 807 & 301 & 0 & 0 & \\
\hline $1-1966$ & 805 & 300 & 0 & 0 & \\
\hline 2 & 802 & 299 & 0 & 0 & \\
\hline 3 & 799 & 298 & 0 & 0 & \\
\hline 4 & 796 & 297 & 0 & 0 & \\
\hline 1-1967 & 796 & 297 & 0 & 0 & \\
\hline 2 & 791 & 295 & 0 & 0 & \\
\hline 3 & 790 & 295 & 0 & 0 & \\
\hline 4 & 789 & 294 & 0 & 0 & \\
\hline $1-1968$ & 787 & 294 & 0 & 0 & \\
\hline 2 & 787 & 294 & 0 & 0 & \\
\hline 3 & 787 & 294 & 0 & 0 & \\
\hline 4 & 785 & 293 & 0 & 0 & \\
\hline $1-1969$ & 785 & 293 & 0 & 0 & \\
\hline 2 & 784 & 293 & 0 & 0 & \\
\hline 3 & 784 & 293 & & & \\
\hline 4 & 784 & 293 & 241 & 95 & \\
\hline $1-1970$ & 783 & 292 & 241 & 95 & \\
\hline 2 & 307 & 119 & 241 & 95 & \\
\hline 3 & 304 & 118 & 241 & 95 & \\
\hline 4 & 310 & 120 & 241 & 95 & \\
\hline $1-1971$ & 310 & 120 & 241 & 95 & \\
\hline 2 & 309 & 120 & 241 & 95 & \\
\hline 3 & 309 & 120 & 241 & 95 & \\
\hline 4 & 308 & 119 & 241 & 95 & \\
\hline 1-1972 & 308 & 119 & 241 & 95 & \\
\hline 2 & 308 & 119 & 241 & 95 & \\
\hline 3 & 308 & 119 & 241 & 95 & \\
\hline 4 & 308 & 119 & 241 & 95 & \\
\hline $1-1973$ & 309 & 120 & 241 & 95 & \\
\hline 2 & 307 & 119 & 241 & 95 & \\
\hline 3 & 308 & 119 & 241 & 95 & \\
\hline 4 & 307 & 119 & 241 & 95 & \\
\hline $1-1974$ & 307 & 119 & 241 & 95 & \\
\hline 2 & 307 & 119 & 241 & 95 & \\
\hline 3 & 309 & 120 & 241 & 95 & \\
\hline 4 & 301 & 117 & 241 & 95 & \\
\hline
\end{tabular}


241-S-104 LEVEL HISTORY

\begin{tabular}{|c|c|c|c|c|c|}
\hline Year & Total & Total & Solids & Solids & ILL \\
\hline & (K gal) & (in) & (K gal) & (in) & (in) \\
\hline $1-1975$ & 299 & 116 & 299 & 116 & \\
\hline 2 & 299 & 116 & 299 & 116 & \\
\hline 3 & 299 & 116 & 299 & 116 & \\
\hline 4 & 299 & 116 & 299 & 116 & \\
\hline 1-1976 & 299 & 116 & 299 & 116 & \\
\hline 2 & 299 & 116 & 299 & 116 & \\
\hline 3 & 299 & 116 & 299 & 116 & \\
\hline 4 & 299 & 116 & 299 & 116 & \\
\hline 1-1977 & 299 & 116 & 299 & 116 & \\
\hline 2 & 299 & 116 & 299 & 116 & \\
\hline 3 & 299 & 116 & 299 & 116 & \\
\hline 4 & 299 & 116 & 299 & 116 & \\
\hline $1-1978$ & 299 & 116 & 299 & 116 & \\
\hline 2 & 299 & $11 \overline{6}$ & 299 & 116 & \\
\hline 3 & 299 & 116 & 299 & 116 & \\
\hline 4 & 299 & 116 & 299 & 116 & \\
\hline 1.1979 & 299 & 116 & 299 & 116 & \\
\hline 2 & 299 & 116 & 299 & 116 & \\
\hline 3 & 299 & 116 & 299 & 116 & \\
\hline 4 & 299 & 116 & 299 & 116 & \\
\hline $1-1980$ & 299 & 116 & 299 & 116 & \\
\hline 2 & 299 & 116 & 299 & 116 & \\
\hline 3 & 299 & 116 & 299 & 116 & \\
\hline 4 & 299 & 116 & 299 & 116 & \\
\hline 1-1981 & 299 & 116 & 299 & 116 & \\
\hline 2 & 299 & 116 & 299 & 116 & \\
\hline 3 & 299 & 116 & 299 & 116 & \\
\hline 4 & 299 & 116 & 299 & 116 & \\
\hline $1-1982$ & 299 & 116 & 299 & 116 & \\
\hline 2 & 294 & 114 & 293 & 114 & \\
\hline 3 & 294 & 114 & 293 & 114 & \\
\hline 4 & 294 & 114 & 293 & 114 & \\
\hline 1-1983 & 294 & 114 & 293 & 114 & \\
\hline 2 & 294 & 114 & 293 & 114 & \\
\hline 3 & 294 & 114 & 293 & 114 & \\
\hline 4 & 294 & 114 & 293 & 114 & \\
\hline 1-1984 & 294 & 114 & 293 & 114 & \\
\hline 2 & 294 & 114 & 293 & 114 & \\
\hline 3 & 294 & 114 & 293 & 114 & \\
\hline 4 & 294 & 114 & 293 & 114 & \\
\hline $1-1985$ & 294 & 114 & 293 & 114 & \\
\hline 2 & 294 & 114 & 293 & 114 & \\
\hline 3 & 294 & 114 & 293 & 114 & \\
\hline 4 & 294 & 114 & 293 & 114 & \\
\hline $1-1986$ & 294 & 114 & 293 & 114 & \\
\hline 2 & 294 & 114 & 293 & 114 & \\
\hline
\end{tabular}


241-S-104 LEVEL. HISTORY

\begin{tabular}{|c|c|c|c|c|c|}
\hline Year & Total & Total & Solids & Solids & ILL \\
\hline & (K gal) & (in) & (K gal) & (in) & (in) \\
\hline 3 & 294 & 114 & 293 & 114 & \\
\hline 4 & 294 & 114 & 293 & 114 & \\
\hline 1-1987 & 294 & 114 & 293 & 114 & \\
\hline 2 & 294 & 114 & 293 & 114 & \\
\hline 3 & 294 & 114 & 293 & 114 & \\
\hline 4 & 294 & 114 & 293 & 114 & \\
\hline $1-1988$ & 294 & 114 & 293 & 114 & \\
\hline 2 & 294 & 114 & 293 & 114 & \\
\hline 3 & 294 & 114 & 293 & 114 & \\
\hline 4 & 294 & 114 & 293 & 114 & \\
\hline 1-1989 & 294 & 114 & 293 & 114 & \\
\hline 2 & 294 & 114 & 293 & 114 & \\
\hline 3 & 294 & 114 & 293 & 114 & \\
\hline 4 & 294 & 114 & 293 & 114 & \\
\hline $1-1990$ & 294 & 114 & 293 & 114 & \\
\hline 2 & 294 & 114 & 293 & 114 & \\
\hline 3 & 294 & 114 & 293 & 114 & \\
\hline 4 & 294 & 114 & 293 & 114 & \\
\hline $1-1991$ & 294 & 114 & 293 & 114 & \\
\hline 2 & 294 & 114 & 293 & 114 & \\
\hline 3 & 294 & 114 & 293 & 114 & \\
\hline 4 & 294 & 114 & 293 & 114 & \\
\hline $1-1992$ & 294 & 114 & 293 & 114 & \\
\hline 2 & 294 & 114 & 293 & 114 & \\
\hline 3 & 294 & 114 & 293 & 114 & \\
\hline 4 & 294 & 114 & 293 & 114 & \\
\hline $1-1993$ & 294 & 114 & 293 & 114 & \\
\hline 2 & 294 & 114 & 293 & 114 & \\
\hline 3 & 294 & 114 & 293 & 114 & \\
\hline 4 & 294 & 114 & 293 & 114 & \\
\hline $1-1994$ & 294 & 114 & 293 & 114 & \\
\hline 2 & 294 & 114 & 293 & 114 & \\
\hline 3 & 294 & 114 & 293 & 114 & \\
\hline 4 & 294 & 114 & 293 & 114 & 114 \\
\hline 1-1995 & 294 & 114 & 293 & 114 & 113 \\
\hline 2 & 294 & 114 & 293 & 114 & 112 \\
\hline 3 & 294 & 114 & 293 & 114 & 113 \\
\hline 4 & 294 & 114 & 293 & 114 & 111 \\
\hline $1-1996$ & 294 & 114 & 293 & 114 & 111 \\
\hline 2 & 294 & 114 & 293 & 114 & 112 \\
\hline
\end{tabular}


241-S-105 LEVEL HISTORY

HNF-SD-WM-ER-323, Rev. 1

\begin{tabular}{|c|c|c|c|c|c|}
\hline Year & Total & Total & Solids & Solids & ILL \\
\hline & (K gal) & (in) & (K gal) & (in) & (in) \\
\hline \multirow{2}{*}{\multicolumn{6}{|c|}{ 1-1952 }} \\
\hline \multirow{2}{*}{\multicolumn{6}{|c|}{2}} \\
\hline \multirow{2}{*}{\multicolumn{6}{|c|}{3}} \\
\hline \multirow{2}{*}{\multicolumn{6}{|c|}{4}} \\
\hline \multirow{2}{*}{\multicolumn{6}{|c|}{ 1-1953 }} \\
\hline 2 & & & & & \\
\hline 2 & 758 & 283 & 0 & 0 & \\
\hline 3 & 723 & 270 & 0 & 0 & \\
\hline 4 & 722 & 270 & 0 & 0 & \\
\hline 1-1954 & 722 & 270 & 0 & 0 & \\
\hline 2 & 734 & 274 & 0 & 0 & \\
\hline 3 & 728 & 272 & 0 & 0 & \\
\hline 4 & 728 & 272 & 0 & 0 & \\
\hline 1-1955 & 728 & 272 & 0 & 0 & \\
\hline 2 & 728 & 272 & 0 & 0 & \\
\hline 3 & 723 & 270 & 0 & 0 & \\
\hline 4 & 723 & 270 & 0 & 0 & \\
\hline 1-1956 & 723 & 270 & 0 & 0 & \\
\hline 2 & 723 & 270 & 0 & 0 & \\
\hline 3 & 723 & 270 & 0 & 0 & \\
\hline 4 & 723 & 270 & 0 & 0 & \\
\hline $1-1957$ & 754 & 282 & 0 & 0 & \\
\hline 2 & 754 & 282 & 0 & 0 & \\
\hline 3 & 752 & 281 & 0 & 0 & \\
\hline 4 & 755 & 282 & 0 & 0 & \\
\hline 1-1958 & 752 & 281 & 0 & 0 & \\
\hline 2 & 752 & 281 & 0 & 0 & \\
\hline 3 & 719 & 269 & 0 & 0 & \\
\hline 4 & 719 & 269 & 0 & 0 & \\
\hline $1-1959$ & 719 & 269 & 0 & 0 & \\
\hline 2 & 719 & 269 & 0 & 0 & \\
\hline 3 & 719 & 269 & 0 & 0 & \\
\hline 4 & 717 & 268 & 0 & 0 & \\
\hline $1-1960$ & 717 & 268 & 0 & 0 & \\
\hline 2 & 717 & 268 & 0 & 0 & \\
\hline 3 & 717 & 268 & 0 & 0 & \\
\hline 4 & 717 & 268 & 0 & 0 & \\
\hline \multicolumn{6}{|l|}{1.1961} \\
\hline 2 & 717 & 268 & 0 & 0 & \\
\hline \multicolumn{6}{|l|}{3} \\
\hline 4 & 717 & 268 & 0 & 0 & \\
\hline \multicolumn{6}{|l|}{$1-1962$} \\
\hline 2 & 717 & 268 & 0 & 0 & \\
\hline \multicolumn{6}{|l|}{3} \\
\hline 4 & 717 & 268 & 0 & 0 & \\
\hline \multicolumn{6}{|l|}{$1-1963$} \\
\hline 2 & 717 & 268 & 0 & 0 & \\
\hline
\end{tabular}


241-S-105 LEVEL HISTORY

HNF-SD-WM-ER-323, Rev. 1

\begin{tabular}{|c|c|c|c|c|c|}
\hline Year & Total & Total & Solids & Solids & ILL \\
\hline & (K gal) & (in) & (K gal) & (in) & (in) \\
\hline \multicolumn{6}{|l|}{3} \\
\hline 4 & 717 & 268 & 0 & 0 & \\
\hline \multicolumn{6}{|l|}{$1-1964$} \\
\hline 2 & 717 & 268 & 0 & 0 & \\
\hline \multicolumn{6}{|l|}{3} \\
\hline 4 & 717 & 268 & 0 & 0 & \\
\hline \multicolumn{6}{|l|}{$1-1965$} \\
\hline 2 & 763 & 285 & 0 & 0 & \\
\hline 3 & 763 & 285 & 0 & 0 & \\
\hline 4 & 763 & 285 & 0 & 0 & \\
\hline $1-1966$ & 763 & 285 & 0 & 0 & \\
\hline 2 & 763 & 285 & 0 & 0 & \\
\hline 3 & 763 & 285 & 0 & 0 & \\
\hline 4 & 763 & 285 & 0 & 0 & \\
\hline $1-1967$ & 763 & 285 & 0 & 0 & \\
\hline 2 & 763 & 285 & 0 & 0 & \\
\hline 3 & 763 & 285 & 0 & 0 & \\
\hline 4 & 763 & 285 & 0 & 0 & \\
\hline $1-1968$ & 763 & 285 & 0 & 0 & \\
\hline 2 & 763 & 285 & 0 & 0 & \\
\hline 3 & 763 & 285 & 0 & 0 & \\
\hline 4 & 763 & 285 & 0 & 0 & \\
\hline $1-1969$ & 763 & 285 & 0 & 0 & \\
\hline 2 & 763 & 285 & 0 & 0 & \\
\hline 3 & 763 & 285 & & & \\
\hline 4 & 763 & 285 & 2 & 8 & \\
\hline $1-1970$ & 763 & 285 & 2 & 8 & \\
\hline 2 & 763 & 285 & 2 & 8 & \\
\hline 3 & 762 & 285 & 2 & 8 & \\
\hline 4 & 762 & 285 & 2 & 8 & \\
\hline $1-1971$ & 762 & 285 & 2 & 8 & \\
\hline 2 & 763 & 285 & 2 & 8 & \\
\hline 3 & 763 & 285 & 2 & 8 & \\
\hline 4 & 763 & 285 & 2 & 8 & \\
\hline $1-1972$ & 762 & 285 & 2 & 8 & \\
\hline 2 & 762 & 285 & 2 & 8 & \\
\hline 3 & 762 & 285 & 2 & 8 & \\
\hline 4 & 763 & 285 & 2 & 8 & \\
\hline $1-1973$ & 763 & 285 & 2 & 8 & \\
\hline 2 & 753 & 281 & 2 & 8 & \\
\hline 3 & 767 & 286 & 2 & 8 & \\
\hline 4 & 365 & 140 & 249 & 98 & \\
\hline 1-1974 & 730 & 273 & 549 & 207 & \\
\hline 2 & 549 & 207 & 549 & 207 & \\
\hline 3 & 549 & 207 & 549 & 207 & \\
\hline 4 & 541 & 204 & 541 & 204 & \\
\hline
\end{tabular}


241-S-105 LEVEL HISTORY

HNF-SD-WM-ER-323, Rev. 1

\begin{tabular}{|c|c|c|c|c|c|}
\hline Year & Total & Total & Solids & Solids & ILL \\
\hline & (K gal) & (in) & (K gal) & (in) & (in) \\
\hline $1-1975$ & 541 & 204 & 541 & 204 & \\
\hline 2 & 541 & 204 & 541 & 204 & \\
\hline 3 & 541 & 204 & 541 & 204 & \\
\hline 4 & 541 & 204 & 541 & 204 & \\
\hline $1-1976$ & 541 & 204 & 541 & 204 & \\
\hline 2 & 541 & 204 & 541 & 204 & \\
\hline 3 & 541 & 204 & 541 & 204 & \\
\hline 4 & 541 & 204 & 541 & 204 & \\
\hline $1-1977$ & 541 & 204 & 541 & 204 & \\
\hline 2 & 541 & 204 & 541 & 204 & \\
\hline 3 & 541 & 204 & 541 & 204 & \\
\hline 4 & 541 & 204 & 541 & 204 & \\
\hline \begin{tabular}{|l|l|}
1978 \\
\end{tabular} & 541 & 204 & 541 & 204 & \\
\hline 2 & 541 & 204 & 541 & 204 & \\
\hline 3 & 541 & 204 & 541 & 204 & \\
\hline 4 & 541 & 204 & 541 & 204 & \\
\hline $1-1979$ & 541 & 204 & 541 & 204 & \\
\hline 2 & 541 & 204 & 541 & 204 & \\
\hline 3 & 541 & 204 & 541 & 204 & \\
\hline 4 & 541 & 204 & 541 & 204 & \\
\hline $1-1980$ & 488 & 185 & 488 & 185 & \\
\hline 2 & 488 & 185 & 488 & 185 & \\
\hline 3 & 488 & 185 & 488 & 185 & \\
\hline 4 & 488 & 185 & 488 & 185 & \\
\hline $1-1981$ & 488 & 185 & 488 & 185 & \\
\hline 2 & 488 & 185 & 488 & 185 & \\
\hline 3 & 488 & 185 & 488 & 185 & \\
\hline 4 & 488 & 185 & 488 & 185 & \\
\hline $1-1982$ & 488 & 185 & 488 & 185 & \\
\hline 2 & 488 & 185 & 488 & 185 & \\
\hline 3 & 488 & 185 & 488 & 185 & \\
\hline 4 & 488 & 185 & 488 & 185 & \\
\hline $1-1983$ & 488 & 185 & 488 & 185 & \\
\hline 2 & 488 & 185 & 488 & 185 & \\
\hline 3 & 488 & 185 & 488 & 185 & \\
\hline 4 & 488 & 185 & 488 & 185 & \\
\hline $1-1984$ & 488 & 185 & 488 & 185 & \\
\hline 2 & 488 & 185 & 488 & 185 & \\
\hline 3 & 488 & 185 & 488 & 185 & \\
\hline 4 & 488 & 185 & 488 & 185 & \\
\hline 1-1985 & 488 & 185 & 488 & 185 & \\
\hline 2 & 488 & 185 & 488 & 185 & \\
\hline 3 & 488 & 185 & 488 & 185 & \\
\hline 4 & 488 & 185 & 488 & 185 & \\
\hline 1-1986 & 488 & 185 & 488 & 185 & 49 \\
\hline 2 & 488 & 185 & 488 & 185 & 49 \\
\hline
\end{tabular}


241-S-105 LEVEL HISTORY

HNF-SD-WM-ER-323, Rev. 1

\begin{tabular}{|c|c|c|c|c|c|}
\hline Year & Total & Total & Solids & Solids & ILL \\
\hline & (K gal) & (in) & (K gal) & (in) & (in) \\
\hline 3 & 488 & 185 & 488 & 185 & 49 \\
\hline 4 & 488 & 185 & 488 & 185 & 50 \\
\hline 1-1987 & 488 & 185 & 488 & 185 & 48 \\
\hline 2 & 488 & 185 & 488 & 185 & \\
\hline 3 & 488 & 185 & 488 & 185 & 50 \\
\hline 4 & 488 & 185 & 488 & 185 & 49 \\
\hline $1-1988$ & 488 & 185 & 488 & 185 & 50 \\
\hline 2 & 488 & 185 & 488 & 185 & 50 \\
\hline 3 & 456 & 173 & 456 & 173 & 49 \\
\hline 4 & 456 & 173 & 456 & 173 & $\overline{51}$ \\
\hline 1-1989 & 456 & 173 & 456 & 173 & \\
\hline 2 & 456 & 173 & 456 & 173 & 51 \\
\hline 3 & 456 & 173 & 456 & 173 & 50 \\
\hline 4 & 456 & 173 & 456 & 173 & 52 \\
\hline $1-1990$ & 456 & 173 & 456 & 173 & 51 \\
\hline 2 & 456 & 173 & 456 & 173 & \\
\hline 3 & 456 & 173 & 456 & 173 & 52 \\
\hline 4 & 456 & 173 & 456 & 173 & 53 \\
\hline 1-1991 & 456 & 173 & 456 & 173 & 52 \\
\hline 2 & 456 & 173 & 456 & 173 & 53 \\
\hline 3 & 456 & 173 & 456 & 173 & 51 \\
\hline 4 & 456 & 173 & 456 & 173 & 53 \\
\hline 1-1992 & 456 & 173 & 456 & 173 & \\
\hline 2 & 456 & 173 & 456 & 173 & 53 \\
\hline 3 & 456 & 173 & 456 & 173 & \\
\hline 4 & 456 & 173 & 456 & 173 & 52 \\
\hline $1-1993$ & 456 & 173 & 456 & 173 & 52 \\
\hline 2 & 456 & 173 & 456 & 173 & 53 \\
\hline 3 & 456 & 173 & 456 & 173 & 52 \\
\hline 4 & 456 & $17 \overline{3}$ & 456 & 173 & 52 \\
\hline $1-1994$ & 456 & 173 & 456 & 173 & 53 \\
\hline 2 & 456 & 173 & 456 & 173 & 52 \\
\hline 3 & 456 & 173 & 456 & 173 & 53 \\
\hline 4 & 456 & 173 & 456 & 173 & 53 \\
\hline $1-1995$ & 456 & 173 & 456 & 173 & 52 \\
\hline 2 & 456 & 173 & 456 & 173 & 52 \\
\hline 3 & 456 & 173 & 456 & 173 & 52 \\
\hline 4 & 456 & 173 & 456 & 173 & 52 \\
\hline $1-1996$ & 456 & 173 & 456 & 173 & 53 \\
\hline 2 & 456 & 173 & 456 & 173 & 53 \\
\hline
\end{tabular}


241-S-106 LEVEL HISTORY

HNF-SD-WM-ER-323, Rev. 1

\begin{tabular}{|c|c|c|c|c|c|}
\hline Year & Total & Total & Solids & Solids & ILL \\
\hline & (K gal) & (in) & (K gal) & (in) & (in) \\
\hline \multicolumn{6}{|l|}{1.1952} \\
\hline \multicolumn{6}{|l|}{2} \\
\hline \multicolumn{6}{|l|}{3} \\
\hline \multicolumn{6}{|l|}{4} \\
\hline \multicolumn{6}{|l|}{ 1-1953 } \\
\hline 2 & 426 & 162 & 0 & 0 & \\
\hline 3 & 729 & 273 & 0 & 0 & \\
\hline 4 & 725 & 271 & 0 & 0 & \\
\hline $1-1954$ & 725 & 271 & 0 & 0 & \\
\hline 2 & 733 & 274 & 0 & 0 & \\
\hline 3 & 737 & 275 & 0 & 0 & \\
\hline 4 & 712 & 266 & 0 & 0 & \\
\hline $1-1955$ & 711 & 266 & 0 & 0 & \\
\hline 2 & 541 & 204 & 0 & 0 & \\
\hline 3 & 539 & 203 & 0 & 0 & \\
\hline 4 & 754 & 282 & 0 & 0 & \\
\hline 1-1956 & 754 & 282 & 0 & 0 & \\
\hline 2 & 754 & 282 & 0 & 0 & \\
\hline 3 & 754 & 282 & 0 & 0 & \\
\hline 4 & 754 & 282 & 0 & 0 & \\
\hline $1-1957$ & 748 & 279 & 0 & 0 & \\
\hline 2 & 748 & 279 & 0 & 0 & \\
\hline 3 & 750 & 280 & 0 & 0 & \\
\hline 4 & 750 & 280 & 0 & 0 & \\
\hline 1-1958 & 750 & 280 & 0 & 0 & \\
\hline 2 & 750 & 280 & 0 & 0 & \\
\hline 3 & 747 & 279 & 0 & 0 & \\
\hline 4 & 747 & 279 & 0 & 0 & \\
\hline 1-1959 & 739 & 276 & 0 & 0 & \\
\hline 2 & 747 & 279 & 0 & 0 & \\
\hline 3 & 747 & 279 & 0 & 0 & \\
\hline 4 & 747 & 279 & 0 & 0 & \\
\hline $1-1960$ & 744 & 278 & 0 & 0 & \\
\hline 2 & 744 & 278 & 0 & 0 & \\
\hline 3 & 744 & 278 & 0 & 0 & \\
\hline 4 & 747 & 279 & 0 & 0 & \\
\hline \multicolumn{6}{|l|}{ 1-1961 } \\
\hline 2 & 747 & 279 & 0 & 0 & \\
\hline \multicolumn{6}{|l|}{3} \\
\hline 4 & 747 & 279 & 0 & 0 & \\
\hline \multicolumn{6}{|l|}{ 1-1962 } \\
\hline 2 & 747 & 279 & 0 & 0 & \\
\hline \multicolumn{6}{|l|}{3} \\
\hline 4 & 747 & 279 & 0 & 0 & \\
\hline \multicolumn{6}{|l|}{\begin{tabular}{|l|}
$1-1963$ \\
\end{tabular}} \\
\hline 2 & 747 & 279 & 0 & 0 & \\
\hline
\end{tabular}




\section{1-S-106 LEVEL HISTORY}

\begin{tabular}{|c|c|c|c|c|c|}
\hline Year & Total & Total & Solids & Solids & $\mathbf{I L L}$ \\
\hline & (K gal) & (in) & (K gal) & (in! & (in) \\
\hline & & & & & \\
\hline \multicolumn{6}{|l|}{3} \\
\hline 4 & 744 & 278 & 0 & 0 & \\
\hline \multicolumn{6}{|l|}{1.1964} \\
\hline 2 & 744 & 278 & 0 & 0 & \\
\hline \multicolumn{6}{|l|}{3} \\
\hline 4 & 744 & 278 & 0 & 0 & \\
\hline \multicolumn{6}{|l|}{$1-1965$} \\
\hline 2 & 761 & 284 & 13 & 12 & \\
\hline 3 & 761 & 284 & 13 & 12 & \\
\hline 4 & 761 & 284 & 13 & 12 & \\
\hline $1-1966$ & $\overline{761}$ & 284 & 13 & 12 & \\
\hline 2 & 761 & 284 & 13 & 12 & \\
\hline 3 & 761 & 284 & 13 & 12 & \\
\hline 4 & 761 & 284 & 13 & 72 & \\
\hline $1-1967$ & 761 & 284 & 13 & 12 & \\
\hline 2 & 761 & 284 & 13 & 12 & \\
\hline 3 & 761 & 284 & 13 & 12 & \\
\hline 4 & 761 & 284 & 73 & 12 & \\
\hline $1-1968$ & 761 & 284 & 13 & 12 & \\
\hline 2 & $7 \overline{761}$ & 284 & 13 & 12 & \\
\hline 3 & 761 & 284 & 13 & 12 & \\
\hline 4 & 758 & 283 & 13 & 12 & \\
\hline $1-1969$ & 759 & 283 & 13 & 12 & \\
\hline 2 & 759 & 283 & 13 & 12 & \\
\hline 3 & 759 & 283 & 13 & 12 & \\
\hline 4 & 759 & 283 & 32 & 19 & \\
\hline 1.1970 & 760 & 284 & 32 & 19 & \\
\hline 2 & 760 & 284 & 32 & 19 & \\
\hline 3 & 760 & 284 & 32 & 19 & \\
\hline 4 & 760 & 284 & 32 & 19 & \\
\hline 1.1971 & 760 & 284 & 32 & 19 & \\
\hline 2 & 759 & 283 & 32 & 19 & \\
\hline 3 & 759 & 283 & 32 & 19 & \\
\hline 4 & 759 & 283 & 32 & 19 & \\
\hline $1-1972$ & 759 & 283 & 32 & 19 & \\
\hline 2 & 759 & 283 & 32 & 19 & \\
\hline 3 & 759 & 283 & 32 & 19 & \\
\hline 4 & 759 & 283 & 32 & 19 & \\
\hline $1-1973$ & 759 & 283 & 32 & 19 & \\
\hline 2 & 753 & 281 & 32 & 19 & \\
\hline 3 & 766 & 286 & 32 & 19 & \\
\hline 4 & 657 & 246 & 384 & 147 & \\
\hline $1-1974$ & 585 & 220 & 436 & 166 & \\
\hline 2 & 584 & 220 & 436 & 166 & \\
\hline 3 & 669 & 251 & 436 & 166 & \\
\hline 4 & 733 & 274 & 557 & 210 & \\
\hline
\end{tabular}


241-S-106 LEVEL HISTORY

\begin{tabular}{|c|c|c|c|c|c|}
\hline Year & Total & Total & Solids & Solids & ILL \\
\hline & (K gal) & (in) & (K gal) & (in) & (in) \\
\hline $1-1975$ & 684 & 256 & 607 & 228 & \\
\hline 2 & 686 & 257 & 607 & 228 & \\
\hline 3 & 673 & 252 & 673 & 252 & \\
\hline 4 & 673 & 252 & 673 & 252 & \\
\hline $1-1976$ & 692 & 259 & 673 & 252 & \\
\hline 2 & 695 & 260 & 673 & 252 & \\
\hline 3 & 700 & 262 & 673 & 252 & \\
\hline 4 & 703 & 263 & 673 & 252 & \\
\hline $1-1977$ & 703 & 263 & 582 & 219 & \\
\hline 2 & 703 & 263 & 582 & 219 & \\
\hline 3 & 703 & 263 & 582 & 219 & \\
\hline 4 & 706 & 264 & 582 & 219 & \\
\hline $1-1978$ & 706 & 264 & 582 & 219 & \\
\hline 2 & 706 & 264 & 582 & 219 & \\
\hline 3 & 700 & 262 & 582 & 219 & \\
\hline 4 & 700 & 262 & 582 & 219 & \\
\hline $1-1979$ & 703 & 263 & 582 & 219 & \\
\hline 2 & 612 & 230 & 582 & 219 & \\
\hline 3 & 612 & 230 & 612 & 230 & \\
\hline 4 & 612 & 230 & 612 & 230 & \\
\hline $1-1980$ & 612 & 230 & 612 & 230 & \\
\hline 2 & 612 & 230 & 612 & 230 & \\
\hline 3 & 612 & 230 & 612 & 230 & \\
\hline 4 & 612 & 230 & 612 & 230 & \\
\hline $1-1981$ & 612 & 230 & 612 & 230 & \\
\hline 2 & 612 & 230 & 612 & 230 & \\
\hline 3 & 612 & 230 & 612 & 230 & \\
\hline 4 & 612 & 230 & 612 & 230 & \\
\hline $1-1982$ & 612 & 230 & 612 & 230 & \\
\hline 2 & 612 & 230 & 611 & 230 & \\
\hline 3 & 543 & 205 & 543 & 205 & \\
\hline 4 & 543 & 205 & 543 & 205 & \\
\hline $1-1983$ & 543 & 205 & 543 & 205 & \\
\hline 2 & 543 & 205 & 543 & 205 & \\
\hline 3 & 543 & 205 & 543 & 205 & \\
\hline 4 & 543 & 205 & 543 & 205 & \\
\hline $1-1984$ & 543 & 205 & 543 & 205 & \\
\hline 2 & 543 & 205 & 543 & 205 & \\
\hline 3 & 543 & 205 & 543 & 205 & \\
\hline 4 & 543 & 205 & 543 & 205 & \\
\hline $1-1985$ & 543 & 205 & 543 & 205 & \\
\hline 2 & 543 & 205 & 543 & 205 & \\
\hline 3 & 543 & 205 & 543 & 205 & \\
\hline 4 & 543 & 205 & 543 & 205 & \\
\hline $1-1986$ & 543 & 205 & 543 & 205 & 171 \\
\hline 2 & 543 & 205 & 543 & 205 & 171 \\
\hline
\end{tabular}


241-S-106 LEVEL HISTORY

HNF-SD-WM-ER-323, Rev. 1

\begin{tabular}{|c|c|c|c|c|c|}
\hline Year & Total & Total & Solids & Solids & ILL \\
\hline & (K gal) & (in) & (K gal) & (in) & (in) \\
\hline & & & & & \\
\hline 3 & 543 & 205 & 543 & 205 & 171 \\
\hline 4 & 543 & 205 & 543 & 205 & 172 \\
\hline $1-1987$ & 543 & 205 & 543 & 205 & 170 \\
\hline 2 & 543 & 205 & 543 & 205 & \\
\hline 3 & 543 & 205 & 543 & 205 & 173 \\
\hline 4 & 543 & 205 & 543 & 205 & 172 \\
\hline $1-1988$ & 543 & 205 & 543 & 205 & 173 \\
\hline 2 & 543 & 205 & 543 & 205 & 174 \\
\hline 3 & 543 & 205 & 543 & 205 & 173 \\
\hline 4 & 543 & 205 & 543 & 205 & 173 \\
\hline 1-1989 & 543 & 205 & 543 & 205 & \\
\hline 2 & 543 & 205 & 543 & 205 & 175 \\
\hline 3 & 543 & 205 & 543 & 205 & 175 \\
\hline 4 & 543 & 205 & 543 & 205 & 174 \\
\hline $1-1990$ & 543 & 205 & 543 & 205 & 174 \\
\hline 2 & 543 & 205 & 543 & 205 & \\
\hline 3 & 543 & 205 & 543 & 205 & 176 \\
\hline 4 & 543 & 205 & 543 & 205 & 175 \\
\hline 1-1991 & 543 & 205 & 543 & 205 & 176 \\
\hline 2 & 543 & 205 & 543 & 205 & 176 \\
\hline 3 & 543 & 205 & 543 & 205 & 176 \\
\hline 4 & 543 & 205 & 543 & 205 & 177 \\
\hline 1-1992 & 543 & 205 & $54 \overline{3}$ & 205 & \\
\hline 2 & 543 & 205 & 543 & 205 & 177 \\
\hline 3 & 543 & 205 & 543 & 205 & \\
\hline 4 & 543 & 205 & 543 & 205 & 177 \\
\hline $1-1993$ & 543 & 205 & 543 & 205 & 177 \\
\hline 2 & 543 & 205 & 543 & 205 & 177 \\
\hline 3 & 543 & 205 & 543 & 205 & 178 \\
\hline 4 & 479 & 182 & 475 & 180 & 177 \\
\hline 1-1994 & 479 & 182 & 475 & 180 & 177 \\
\hline 2 & 479 & 182 & 475 & 180 & 178 \\
\hline 3 & 479 & 182 & 475 & 180 & 178 \\
\hline 4 & 479 & 182 & 475 & 180 & 178 \\
\hline 1-1995 & 479 & 182 & 475 & 180 & 178 \\
\hline 2 & 479 & 182 & 475 & 180 & 178 \\
\hline 3 & 479 & 182 & 475 & 180 & 179 \\
\hline 4 & 479 & 182 & 475 & 180 & 178 \\
\hline 1-1996 & 479 & 182 & 475 & 180 & 179 \\
\hline 2 & 479 & 182 & 475 & 180 & 180 \\
\hline
\end{tabular}


241-S-107 LEVEL HISTORY

HNF-SD-WM-ER-323, Rev. I

\begin{tabular}{|c|c|c|c|c|c|}
\hline Year & Total & Total & Solids & Solids & ILL \\
\hline & (K gal) & (in) & (K gal) & (in) & (in) \\
\hline \multirow{2}{*}{\multicolumn{6}{|c|}{$1-1952$}} \\
\hline & & & & & \\
\hline \multicolumn{6}{|l|}{2} \\
\hline \multicolumn{6}{|l|}{3} \\
\hline 4 & 763 & 285 & 0 & 0 & \\
\hline $1-1953$ & 763 & 285 & 0 & 0 & \\
\hline 2 & 758 & 283 & 0 & 0 & \\
\hline 3 & 751 & $\overline{281}$ & 0 & 0 & \\
\hline 4 & 748 & 279 & 0 & 0 & \\
\hline 1-1954 & 748 & 279 & 0 & 0 & \\
\hline 2 & 754 & 282 & 0 & 0 & \\
\hline 3 & 722 & 270 & 0 & 0 & \\
\hline 4 & 758 & 283 & 0 & 0 & \\
\hline 1-1955 & 758 & 283 & 0 & 0 & \\
\hline 2 & 758 & 283 & 0 & 0 & \\
\hline 3 & 685 & 257 & 0 & 0 & \\
\hline 4 & 741 & 277 & 0 & 0 & \\
\hline $1-1956$ & 757 & 283 & 0 & 0 & \\
\hline 2 & 757 & 283 & 0 & 0 & \\
\hline 3 & 757 & 283 & 0 & 0 & \\
\hline 4 & 757 & 283 & 0 & 0 & \\
\hline $1-1957$ & 750 & 280 & 0 & 0 & \\
\hline 2 & 750 & 280 & 0 & 0 & \\
\hline 3 & 596 & 224 & 0 & 0 & \\
\hline 4 & 689 & 258 & 0 & 0 & \\
\hline $1-1958$ & 653 & 245 & 0 & 0 & \\
\hline 2 & 538 & 203 & 0 & 0 & \\
\hline 3 & 618 & 232 & 0 & 0 & \\
\hline 4 & 642 & 241 & 0 & 0 & \\
\hline 1.1959 & 686 & 257 & 0 & 0 & \\
\hline 2 & 528 & 199 & 0 & 0 & \\
\hline 3 & 562 & 212 & 0 & 0 & \\
\hline 4 & 607 & 228 & 0 & 0 & \\
\hline 1.1960 & 637 & 239 & 0 & 0 & \\
\hline 2 & 564 & 213 & 0 & 0 & \\
\hline 3 & 598 & 225 & 0 & 0 & \\
\hline 4 & 536 & 202 & 0 & 0 & \\
\hline \multicolumn{6}{|l|}{ 1-1961 } \\
\hline 2 & 645 & 242 & 0 & 0 & \\
\hline \multicolumn{6}{|l|}{3} \\
\hline 4 & 560 & 211 & 0 & 0 & \\
\hline \multicolumn{6}{|l|}{$1-1962$} \\
\hline 2 & 659 & 247 & 0 & 0 & \\
\hline \multicolumn{6}{|l|}{3} \\
\hline 4 & 555 & 209 & 0 & 0 & \\
\hline \multicolumn{6}{|l|}{$1-1963$} \\
\hline 2 & 527 & 199 & 0 & 0 & \\
\hline
\end{tabular}


241-S-107 LEVEL HISTORY

\begin{tabular}{|c|c|c|c|c|c|}
\hline Year & Total & Total & Solids & Solids & ILL \\
\hline & (K gal) & (in) & (K gal) & (in) & (in) \\
\hline & & & & & \\
\hline \multicolumn{5}{|l|}{3} & \\
\hline 4 & 615 & 231 & 0 & 0 & \\
\hline \multicolumn{6}{|l|}{$1-1964$} \\
\hline 2 & 590 & 222 & 0 & 0 & \\
\hline \multicolumn{6}{|l|}{3} \\
\hline 4 & 628 & 236 & 0 & 0 & \\
\hline \multicolumn{6}{|l|}{$1-1965$} \\
\hline 2 & 508 & 192 & 194 & 78 & \\
\hline 3 & 587 & 221 & 194 & 78 & \\
\hline 4 & 669 & 251 & 194 & 78 & \\
\hline $1-1966$ & 565 & 213 & 194 & 78 & \\
\hline 2 & 568 & 214 & 194 & 78 & \\
\hline 3 & 613 & 230 & 194 & 78 & \\
\hline 4 & 642 & 241 & 194 & 78 & \\
\hline $1-1967$ & 656 & 246 & 194 & 78 & \\
\hline 2 & 656 & 246 & 194 & 78 & \\
\hline 3 & 656 & 246 & 194 & 78 & \\
\hline 4 & 656 & 246 & 194 & 78 & \\
\hline $1-1968$ & 654 & 245 & 194 & 78 & \\
\hline 2 & 654 & 245 & 194 & 78 & \\
\hline 3 & 305 & 118 & 194 & 78 & \\
\hline 4 & 325 & 126 & 194 & 78 & \\
\hline $1-1969$ & 415 & 158 & 194 & 78 & \\
\hline 2 & 476 & 181 & 194 & 78 & \\
\hline 3 & 577 & 217 & 194 & 78 & \\
\hline 4 & 451 & 171 & 285 & 111 & \\
\hline $1-1970$ & 477 & 181 & 285 & 111 & \\
\hline 2 & 532 & 201 & 293 & 114 & \\
\hline 3 & 343 & 132 & 293 & 114 & \\
\hline 4 & 449 & 171 & 293 & 114 & \\
\hline 1-1971 & 536 & 202 & 293 & 114 & \\
\hline 2 & 589 & 222 & 293 & 114 & \\
\hline 3 & 609 & 229 & 293 & 114 & \\
\hline 4 & 622 & 234 & 293 & 114 & \\
\hline $1-1972$ & 655 & 246 & 293 & 114 & \\
\hline 2 & 756 & 282 & 293 & 114 & \\
\hline 3 & 756 & 282 & 293 & 114 & \\
\hline 4 & 757 & 283 & 293 & 114 & \\
\hline $1-1973$ & 757 & 283 & 293 & 114 & \\
\hline 2 & 749 & 280 & 293 & 114 & \\
\hline 3 & 761 & 284 & 293 & 114 & \\
\hline 4 & 686 & 257 & 293 & 114 & \\
\hline $1-1974$ & 731 & 273 & 293 & 114 & \\
\hline 2 & 492 & 186 & 343 & 132 & \\
\hline 3 & 468 & 178 & 282 & 110 & \\
\hline 4 & 519 & 196 & 343 & 132 & \\
\hline
\end{tabular}


241-S-107 LEVEL HISTORY

HNF-SD-WM-ER-323, Rev. 1

\begin{tabular}{|c|c|c|c|c|c|}
\hline Year & Total & Total & Solids & Solids & ILL \\
\hline & (K gal) & (in) & (K gal) & (in) & (in) \\
\hline $1-1975$ & 475 & 180 & 343 & 132 & \\
\hline 2 & 477 & 181 & 343 & 132 & \\
\hline 3 & 480 & 182 & 343 & 132 & \\
\hline 4 & 491 & 186 & 343 & 132 & \\
\hline $1-1976$ & 656 & 246 & 343 & 132 & \\
\hline 2 & 703 & $26 \overline{3}$ & 343 & 132 & \\
\hline 3 & 700 & 262 & 343 & 132 & \\
\hline 4 & 431 & 164 & 343 & 132 & \\
\hline $1-1977$ & 455 & 173 & 343 & 132 & \\
\hline 2 & 585 & 220 & 343 & 132 & \\
\hline 3 & 717 & 268 & 343 & 132 & \\
\hline 4 & 409 & 156 & 343 & 132 & \\
\hline $1-1978$ & 422 & 161 & 343 & 132 & \\
\hline 2 & 719 & 269 & 343 & 132 & \\
\hline 3 & 497 & 188 & 343 & 132 & \\
\hline 4 & 711 & 266 & 343 & 132 & \\
\hline $1-1979$ & 425 & 162 & 343 & 132 & \\
\hline 2 & 692 & 259 & 343 & 132 & \\
\hline 3 & 684 & 256 & 343 & 132 & \\
\hline 4 & 692 & 259 & 343 & 132 & \\
\hline $1-1980$ & 450 & 171 & 343 & 132 & \\
\hline 2 & 476 & 181 & 343 & 132 & \\
\hline 3 & 346 & 133 & 340 & 131 & \\
\hline 4 & 346 & 133 & 340 & 131 & \\
\hline 1-1981 & 346 & 133 & 340 & 131 & \\
\hline 2 & 346 & 133 & 340 & 131 & \\
\hline 3 & 346 & 133 & 340 & 131 & \\
\hline 4 & 346 & 133 & 340 & 131 & \\
\hline $1-1982$ & 346 & 133 & 340 & 131 & \\
\hline 2 & 346 & 133 & 340 & 131 & \\
\hline 3 & 346 & 133 & 340 & 131 & \\
\hline 4 & 346 & 133 & 340 & 131 & \\
\hline $1-1983$ & 346 & 133 & 340 & 131 & \\
\hline 2 & 346 & 133 & 340 & 131 & \\
\hline 3 & 346 & 133 & 340 & 131 & \\
\hline 4 & 346 & 133 & 340 & 131 & \\
\hline $1-1984$ & 346 & 133 & 340 & 131 & \\
\hline 2 & 346 & 133 & 340 & 131 & \\
\hline 3 & 346 & 133 & 340 & 131 & \\
\hline 4 & 346 & 133 & 340 & 131 & \\
\hline $1-1985$ & 346 & 133 & 340 & 131 & \\
\hline 2 & 346 & 133 & 340 & 131 & \\
\hline 3 & 346 & 133 & 340 & 131 & \\
\hline 4 & 346 & 133 & 340 & 131 & \\
\hline $1-1986$ & 346 & 133 & 340 & 131 & \\
\hline 2 & 346 & 133 & 340 & 131 & \\
\hline
\end{tabular}


241-S-107 LEVEL HISTORY

HNF-SD-WM-ER-323, Rev. 1

\begin{tabular}{|c|c|c|c|c|c|}
\hline Year & Total & Total & Solids & Solids & $\overline{\mathrm{ILL}}$ \\
\hline & (K gal) & (in) & (K gai) & (in) & (in) \\
\hline$\overline{3}$ & 368 & 141 & 362 & 139 & \\
\hline 4 & 368 & 141 & 362 & 139 & \\
\hline 1-1987 & 368 & 141 & 362 & 139 & \\
\hline 2 & 368 & 141 & 362 & 139 & \\
\hline 3 & 368 & 141 & 362 & 139 & \\
\hline 4 & 368 & 141 & 362 & 139 & \\
\hline $1-1988$ & 368 & 141 & 362 & 139 & \\
\hline 2 & 368 & $\overline{141}$ & 362 & 139 & \\
\hline 3 & 368 & 141 & 362 & 139 & \\
\hline 4 & 368 & 141 & 362 & 139 & \\
\hline 1-1989 & 368 & 141 & 362 & 139 & \\
\hline 2 & 368 & 141 & 362 & 139 & \\
\hline 3 & 368 & 141 & 362 & 139 & \\
\hline 4 & 368 & 141 & 362 & 139 & \\
\hline $1-1990$ & 368 & 141 & 362 & 139 & \\
\hline 2 & 368 & 141 & 362 & 139 & \\
\hline 3 & 368 & 141 & 362 & 139 & \\
\hline 4 & 368 & 141 & 362 & 139 & \\
\hline 1-1991 & 368 & 141 & 362 & 139 & \\
\hline 2 & 368 & 141 & 362 & 139 & \\
\hline 3 & 368 & 141 & 362 & 139 & \\
\hline 4 & 368 & 141 & 362 & 139 & \\
\hline 1-1992 & 368 & 141 & 362 & 139 & \\
\hline 2 & 368 & 141 & 362 & 139 & \\
\hline 3 & 368 & 141 & 362 & 139 & \\
\hline 4 & 368 & 141 & 362 & 139 & \\
\hline 1-1993 & 368 & 141 & 362 & 139 & \\
\hline 2 & 368 & 141 & 362 & 139 & \\
\hline 3 & 368 & 141 & 362 & 139 & \\
\hline 4 & 376 & 144 & 362 & 139 & \\
\hline \begin{tabular}{|l|}
$1-1994$ \\
\end{tabular} & 376 & 144 & 362 & 139 & \\
\hline 2 & 376 & 144 & 362 & 139 & \\
\hline 3 & 376 & 144 & 362 & 139 & \\
\hline 4 & 376 & 144 & 362 & 139 & \\
\hline 1.1995 & 376 & 144 & 362 & 139 & \\
\hline 2 & 376 & 144 & 362 & 139 & \\
\hline 3 & 376 & 144 & 362 & 139 & \\
\hline 4 & 376 & 144 & 362 & 139 & \\
\hline 1-1996 & 376 & 144 & 362 & 139 & \\
\hline 2 & 376 & 144 & 362 & 139 & \\
\hline
\end{tabular}


241-S-108 LEVEL HISTORY

HNF-SD-WM-ER-323, Rev. 1

\begin{tabular}{|c|c|c|c|c|c|}
\hline Year & Total & Total & Solids & Solids & ILL \\
\hline & (K gal) & (in) & (K gal) & (in) & (in) \\
\hline & & & & & \\
\hline \multicolumn{6}{|l|}{$1-1952$} \\
\hline \multicolumn{6}{|l|}{2} \\
\hline \multicolumn{6}{|l|}{3} \\
\hline 4 & 760 & 284 & 0 & 0 & \\
\hline 1-1953 & 760 & 284 & 0 & 0 & \\
\hline 2 & 758 & 283 & 0 & 0 & \\
\hline 3 & 751 & 281 & 0 & 0 & \\
\hline 4 & 747 & 279 & 0 & 0 & \\
\hline 1-1954 & 747 & 279 & 0 & 0 & \\
\hline 2 & 743 & 278 & 0 & 0 & \\
\hline 3 & 740 & 277 & 0 & 0 & \\
\hline 4 & 758 & 283 & 0 & 0 & \\
\hline $1-1955$ & 758 & 283 & 0 & 0 & \\
\hline 2 & 758 & 283 & 0 & 0 & \\
\hline 3 & 758 & 283 & 0 & 0 & \\
\hline 4 & 758 & 283 & 0 & 0 & \\
\hline $1-1956$ & 758 & 283 & 0 & 0 & \\
\hline 2 & 758 & 283 & 0 & 0 & \\
\hline 3 & 758 & 283 & 0 & 0 & \\
\hline 4 & 758 & 283 & 0 & 0 & \\
\hline $1-1957$ & 765 & 286 & 0 & 0 & \\
\hline 2 & 763 & 285 & 0 & 0 & \\
\hline 3 & 763 & 285 & 0 & 0 & \\
\hline 4 & 763 & 285 & 0 & 0 & \\
\hline $1-1958$ & 761 & 284 & 0 & 0 & \\
\hline 2 & 761 & 284 & 0 & 0 & \\
\hline 3 & 761 & 284 & 0 & 0 & \\
\hline 4 & 761 & 284 & 0 & 0 & \\
\hline 1-1959 & 761 & 284 & 0 & 0 & \\
\hline 2 & 761 & 284 & 0 & 0 & \\
\hline 3 & 761 & 284 & 0 & 0 & \\
\hline 4 & 761 & 284 & 0 & 0 & \\
\hline $1-1960$ & 758 & 283 & 0 & 0 & \\
\hline 2 & 758 & 283 & 0 & 0 & \\
\hline 3 & 758 & 283 & 0 & 0 & \\
\hline 4 & 758 & 283 & 0 & 0 & \\
\hline \multicolumn{6}{|l|}{$1-1961$} \\
\hline 2 & 755 & 282 & 0 & 0 & \\
\hline \multicolumn{6}{|l|}{3} \\
\hline 4 & 755 & 282 & 0 & 0 & \\
\hline \multicolumn{6}{|l|}{ 1-1962 } \\
\hline 2 & 755 & 282 & 0 & 0 & \\
\hline \multicolumn{6}{|l|}{3} \\
\hline 4 & 755 & 282 & 0 & 0 & \\
\hline \multicolumn{6}{|l|}{$1-1963$} \\
\hline 2 & 755 & 282 & 0 & 0 & \\
\hline
\end{tabular}


241-S-108 LEVEL HISTORY

\begin{tabular}{|c|c|c|c|c|c|}
\hline Year & Total & Total & Solids & Solids & ILL \\
\hline & (K gal) & (in) & (K gal) & (in) & (in) \\
\hline & & & & & \\
\hline \multicolumn{6}{|l|}{3} \\
\hline 4 & 755 & 282 & 0 & 0 & \\
\hline \multicolumn{6}{|l|}{1.1964} \\
\hline 2 & 755 & 282 & 0 & 0 & \\
\hline \multicolumn{6}{|l|}{3} \\
\hline 4 & 755 & 282 & 0 & 0 & \\
\hline \multicolumn{6}{|l|}{ 1-1965 } \\
\hline 2 & 758 & 283 & 4 & 9 & \\
\hline 3 & 758 & 283 & 4 & 9 & \\
\hline 4 & 758 & 283 & 4 & 9 & \\
\hline $1-1966$ & 758 & 283 & 4 & 9 & \\
\hline 2 & 758 & 283 & 4 & 9 & \\
\hline 3 & 758 & 283 & 4 & 9 & \\
\hline 4 & 758 & 283 & 4 & 9 & \\
\hline $1-1967$ & 758 & 283 & 4 & 9 & \\
\hline 2 & 758 & 283 & 4 & 9 & \\
\hline 3 & 758 & 283 & 4 & 9 & \\
\hline 4 & 758 & 283 & 4 & 9 & \\
\hline 1.1968 & 758 & 283 & 4 & 9 & \\
\hline 2 & 757 & 283 & 4 & 9 & \\
\hline 3 & 757 & 283 & 4 & 9 & \\
\hline 4 & 757 & 283 & 4 & 9 & \\
\hline 1-1969 & 757 & 283 & 4 & 9 & \\
\hline 2 & 757 & 283 & 4 & 9 & \\
\hline 3 & 757 & 283 & 4 & 9 & \\
\hline 4 & 757 & 283 & 5 & 9 & \\
\hline $1-1970$ & 756 & 282 & 5 & 9 & \\
\hline 2 & 756 & 282 & 5 & 9 & \\
\hline 3 & 756 & 282 & 5 & 9 & \\
\hline 4 & 756 & 282 & 5 & 9 & \\
\hline $1-1971$ & 756 & 282 & 5 & 9 & \\
\hline 2 & 756 & 282 & 5 & 9 & \\
\hline 3 & 756 & 282 & 5 & 9 & \\
\hline 4 & 756 & 282 & 5 & 9 & \\
\hline $1-1972$ & 756 & 282 & 5 & 9 & \\
\hline 2 & 756 & 282 & 5 & 9 & \\
\hline 3 & 756 & 282 & 5 & 9 & \\
\hline 4 & 758 & 283 & 5 & 9 & \\
\hline $1-1973$ & 757 & 283 & 5 & 9 & \\
\hline 2 & 748 & 279 & 5 & 9 & \\
\hline 3 & 761 & 284 & 5 & 9 & \\
\hline \multicolumn{6}{|l|}{4} \\
\hline $1-1974$ & 704 & 263 & 373 & 143 & \\
\hline 2 & 718 & 269 & 585 & 220 & \\
\hline 3 & 706 & 264 & 702 & 263 & \\
\hline 4 & 719 & 269 & 706 & 264 & \\
\hline
\end{tabular}


241-S-108 LEVEL HISTORY

HNF-SD-WM-ER-323, Rev. 1

\begin{tabular}{|c|c|c|c|c|c|}
\hline Year & Total & Total & Solids & Solids & ILL \\
\hline & (K gal) & (in) & (K gal) & (in) & (in) \\
\hline 1-1975 & 717 & 268 & 706 & 264 & \\
\hline 2 & 722 & 270 & 706 & 264 & \\
\hline 3 & 728 & 272 & 706 & 264 & \\
\hline 4 & 725 & 271 & 706 & 264 & \\
\hline $1-1976$ & 728 & 272 & 706 & 264 & \\
\hline 2 & 728 & 272 & 706 & 264 & \\
\hline 3 & 730 & 273 & 706 & 264 & \\
\hline 4 & 730 & 273 & 706 & 264 & \\
\hline $1-1977$ & 733 & 274 & 670 & 251 & \\
\hline 2 & 733 & 274 & 670 & 251 & \\
\hline 3 & 733 & 274 & 670 & 251 & \\
\hline 4 & 733 & 274 & 670 & 251 & \\
\hline $1-1978$ & 733 & 274 & 670 & 251. & \\
\hline 2 & 733 & 274 & 670 & 251 & \\
\hline 3 & 719 & 269 & 670 & 251 & \\
\hline 4 & 692 & 259 & 670 & 251 & \\
\hline $1-1979$ & 692 & 259 & 670 & 251 & \\
\hline 2 & 670 & 251 & 670 & 251 & \\
\hline 3 & 670 & 251 & 670 & 251 & \\
\hline 4 & 670 & 251 & 612 & 230 & \\
\hline $1-1980$ & 612 & 230 & 612 & 230 & \\
\hline 2 & 612 & 230 & 612 & 230 & \\
\hline 3 & 612 & 230 & 612 & 230 & \\
\hline 4 & 612 & 230 & 612 & 230 & \\
\hline 1-1981 & 612 & 230 & 612 & 230 & \\
\hline 2 & 612 & 230 & 612 & 230 & \\
\hline 3 & 612 & 230 & 612 & 230 & \\
\hline 4 & 612 & 230 & 612 & 230 & \\
\hline 1-1982 & 612 & 230 & 612 & 230 & \\
\hline 2 & 604 & 227 & 604 & 227 & \\
\hline 3 & 604 & 227 & 604 & 227 & \\
\hline 4 & 604 & 227 & 604 & 227 & \\
\hline 1-1983 & 604 & 227 & 604 & 227 & \\
\hline 2 & 604 & 227 & 604 & 227 & \\
\hline 3 & 604 & 227 & 604 & 227 & \\
\hline 4 & 604 & 227 & 604 & 227 & \\
\hline 1-1984 & 604 & 227 & 604 & 227 & \\
\hline 2 & 604 & 227 & 604 & 227 & \\
\hline 3 & 604 & 227 & 604 & 227 & \\
\hline 4 & 604 & 227 & 604 & 227 & \\
\hline 1-1985 & 604 & 227 & 604 & 227 & \\
\hline 2 & 604 & 227 & 604 & 227 & \\
\hline 3 & 604 & 227 & 604 & 227 & \\
\hline 4 & 604 & 227 & 604 & 227 & \\
\hline $1-1986$ & 604 & 227 & 604 & 227 & 137 \\
\hline 2 & 604 & 227 & 604 & 227 & 138 \\
\hline
\end{tabular}


241-S-108 LEVEL, HISTORY

HNF-SD-WM-ER-323, Rev. 1

\begin{tabular}{|c|c|c|c|c|c|}
\hline Year & Total & Total & Solids & Solids & $\mathrm{ILL}$ \\
\hline & (K gal) & (in) & (K gal) & (in) & (in) \\
\hline 3 & 604 & 227 & 604 & 227 & 137 \\
\hline 4 & 604 & 227 & 604 & 227 & 138 \\
\hline $1-1987$ & 604 & 227 & 604 & 227 & 137 \\
\hline 2 & 604 & 227 & 604 & 227 & \\
\hline 3 & 604 & 227 & 604 & 227 & 137 \\
\hline 4 & 604 & 227 & 604 & 227 & 137 \\
\hline $1-1988$ & 604 & 227 & 604 & 227 & 137 \\
\hline 2 & 604 & 227 & 604 & 227 & 136 \\
\hline 3 & 604 & 227 & 604 & 227 & 135 \\
\hline 4 & 604 & 227 & 604 & 227 & 136 \\
\hline $1-1989$ & 604 & 227 & 604 & 227 & \\
\hline 2 & 604 & 227 & 604 & 227 & 136 \\
\hline 3 & 604 & 227 & 604 & 227 & 137 \\
\hline 4 & 604 & 227 & 604 & 227 & 137 \\
\hline $1-1990$ & 604 & 227 & 604 & 227 & 136 \\
\hline 2 & 604 & 227 & 604 & 227 & \\
\hline 3 & 604 & 227 & 604 & 227 & 135 \\
\hline 4 & 604 & 227 & 604 & 227 & 136 \\
\hline 1-1991 & 604 & 227 & 604 & 227 & 136 \\
\hline 2 & 604 & 227 & 604 & 227 & \\
\hline 3 & 604 & 227 & 604 & 227 & 135 \\
\hline 4 & 604 & 227 & 604 & 227 & 137 \\
\hline $1-1992$ & 604 & 227 & 604 & 227 & \\
\hline 2 & 604 & 227 & 604 & 227 & 136 \\
\hline 3 & 604 & 227 & 604 & 227 & \\
\hline 4 & 604 & 227 & 604 & 227 & 137 \\
\hline $1-1993$ & 604 & 227 & 604 & 227 & 136 \\
\hline 2 & 604 & 227 & 604 & 227 & 135 \\
\hline 3 & 604 & 227 & 604 & 227 & 135 \\
\hline 4 & 604 & 227 & 604 & 227 & 136 \\
\hline 1-1994 & 604 & 227 & 604 & 227 & 137 \\
\hline 2 & 604 & 227 & 604 & 227 & 136 \\
\hline 3 & 604 & 227 & 604 & 227 & 135 \\
\hline 4 & 604 & 227 & 604 & 227 & 137 \\
\hline 1-1995 & 604 & 227 & 604 & 227 & 135 \\
\hline 2 & 604 & 227 & 604 & 227 & 135 \\
\hline 3 & 604 & 227 & 604 & 227 & 136 \\
\hline 4 & 604 & 227 & 604 & 227 & 135 \\
\hline 1-1996 & 604 & 227 & 604 & 227 & 135 \\
\hline 2 & 604 & 227 & 604 & 227 & 134 \\
\hline
\end{tabular}


241-S-109 LEVEL HISTORY

\begin{tabular}{|c|c|c|c|c|c|}
\hline Year & Total & Total & Solids & Solids & ILL \\
\hline & (K gal) & (in) & (K gal) & (in) & (in) \\
\hline \multicolumn{6}{|l|}{ 1-1952 } \\
\hline \multicolumn{6}{|l|}{2} \\
\hline \multicolumn{6}{|l|}{3} \\
\hline 4 & 103 & 45 & & & \\
\hline $1-1953$ & 582 & 219 & & & \\
\hline 2 & 585 & 220 & 0 & 0 & \\
\hline 3 & 575 & 217 & 0 & 0 & \\
\hline 4 & 579 & 218 & 0 & 0 & \\
\hline $1-1954$ & 579 & 218 & 0 & 0 & \\
\hline 2 & 576 & 217 & 0 & 0 & \\
\hline 3 & 576 & 217 & 0 & 0 & \\
\hline 4 & 576 & 217 & 0 & 0 & \\
\hline 1-1955 & 704 & 263 & 0 & 0 & \\
\hline 2 & 747 & 279 & 0 & 0 & \\
\hline 3 & 747 & 279 & 0 & 0 & \\
\hline 4 & 747 & 279 & 0 & 0 & \\
\hline $1-1956$ & 747 & 279 & 0 & 0 & \\
\hline 2 & 747 & 279 & 0 & 0 & \\
\hline 3 & 747 & 279 & 0 & 0 & \\
\hline 4 & 747 & 279 & 0 & 0 & \\
\hline $1-1957$ & 764 & 285 & 0 & 0 & \\
\hline 2 & 764 & 285 & 0 & 0 & \\
\hline 3 & 763 & 285 & 0 & 0 & \\
\hline 4 & 763 & 285 & 0 & 0 & \\
\hline $1-1958$ & 763 & 285 & 0 & 0 & \\
\hline 2 & 763 & 285 & 0 & 0 & \\
\hline 3 & 763 & 285 & 0 & 0 & \\
\hline 4 & 763 & 285 & 0 & 0 & \\
\hline $1-1959$ & 763 & 285 & 0 & 0 & \\
\hline 2 & 763 & 285 & 0 & 0 & \\
\hline 3 & 763 & 285 & 0 & 0 & \\
\hline 4 & 763 & 285 & 0 & 0 & \\
\hline $1-1960$ & 761 & 284 & 0 & 0 & \\
\hline 2 & 761 & 284 & 0 & 0 & \\
\hline 3 & 761 & 284 & 0 & 0 & \\
\hline 4 & 763 & 285 & 0 & 0 & \\
\hline \multicolumn{6}{|l|}{$1-1961$} \\
\hline 2 & 761 & 284 & 0 & 0 & \\
\hline \multicolumn{6}{|l|}{3} \\
\hline 4 & 761 & 284 & 0 & 0 & \\
\hline \multicolumn{6}{|l|}{$1-1962$} \\
\hline 2 & 761 & 284 & 0 & 0 & \\
\hline \multicolumn{6}{|l|}{3} \\
\hline 4 & 761 & 284 & 0 & 0 & \\
\hline \multicolumn{6}{|l|}{$1-1963$} \\
\hline 2 & 761 & 284 & 0 & 0 & \\
\hline
\end{tabular}


241-S-109 LEVEL HISTORY

\begin{tabular}{|c|c|c|c|c|c|}
\hline Year & Total & Total & Solids & Solids & $\mathrm{ILL}$ \\
\hline & (K gal) & (in) & (K gal) & (in) & (in) \\
\hline \multicolumn{6}{|l|}{3} \\
\hline 4 & 761 & 284 & 0 & 0 & \\
\hline \multicolumn{6}{|l|}{$1-1964$} \\
\hline 2 & 761 & 284 & 0 & 0 & \\
\hline \multicolumn{6}{|l|}{3} \\
\hline 4 & 761 & 284 & 0 & 0 & \\
\hline \multicolumn{6}{|l|}{$1-1965$} \\
\hline 2 & 766 & 286 & 7 & 10 & \\
\hline 3 & 766 & 286 & 7 & 10 & \\
\hline 4 & 766 & 286 & 7 & 10 & \\
\hline 1-1966 & 766 & 286 & 7 & 10 & \\
\hline 2 & 766 & 286 & 7 & 10 & \\
\hline 3 & 766 & 286 & 7 & 10 & \\
\hline 4 & 766 & 286 & 7 & 10 & \\
\hline $1-1967$ & 766 & 286 & 7 & 10 & \\
\hline 2 & 766 & 286 & 7 & 10 & \\
\hline 3 & 766 & 286 & 7 & 10 & \\
\hline 4 & 766 & 286 & 7 & 10 & \\
\hline $1-1968$ & 766 & 286 & 7 & 10 & \\
\hline 2 & 766 & 286 & 7 & 10 & \\
\hline 3 & 766 & 286 & 7 & 10 & \\
\hline 4 & 765 & 286 & 7 & 10 & \\
\hline $1-1969$ & 765 & 286 & 7 & 10 & \\
\hline 2 & 765 & 286 & 7 & 10 & \\
\hline 3 & 765 & 286 & 7 & 10 & \\
\hline 4 & 765 & 286 & 13 & 12 & \\
\hline $1-1970$ & 765 & 286 & 13 & 12 & \\
\hline 2 & 765 & 286 & 13 & 12 & \\
\hline 3 & 765 & 286 & 13 & 12 & \\
\hline 4 & 765 & 286 & 13 & 12 & \\
\hline $1-1971$ & 765 & 286 & 13 & 12 & \\
\hline 2 & 765 & 286 & 13 & 12 & \\
\hline 3 & 765 & 286 & 13 & 12 & \\
\hline 4 & 765 & 286 & 13 & 12 & \\
\hline $1-1972$ & 765 & 286 & 13 & 12 & \\
\hline 2 & 765 & 286 & 13 & 12 & \\
\hline 3 & 765 & 286 & 13 & 12 & \\
\hline 4 & 765 & 286 & 13 & 12 & \\
\hline $1-1973$ & 765 & 286 & 13 & 12 & \\
\hline 2 & 758 & 283 & 13 & 12 & \\
\hline 3 & 770 & 287 & 13 & 12 & \\
\hline 4 & 771 & 288 & 13 & 12 & \\
\hline 1-1974 & 361 & 139 & 263 & 103 & \\
\hline 2 & 672 & 252 & 488 & 185 & \\
\hline 3 & 700 & 262 & 653 & 245 & \\
\hline 4 & 717 & 268 & 653 & 245 & \\
\hline
\end{tabular}


241-S-109 LEVEL HISTORY

HNF-SD-WM-ER-323, Rev. 1

\begin{tabular}{|c|c|c|c|c|c|}
\hline Year & Total & Total & Solids & Solids & ILL \\
\hline & (K gal) & (in) & (K gal) & (in) & (in) \\
\hline & & & & & \\
\hline $1-1975$ & 717 & $2 \overline{68}$ & 653 & 245 & \\
\hline 2 & 719 & $2 \overline{69}$ & 653 & 245 & \\
\hline 3 & 722 & 270 & 653 & 245 & \\
\hline 4 & 722 & 270 & 653 & $\overline{245}$ & \\
\hline 1-1976 & 725 & 271 & 653 & 245 & \\
\hline 2 & 725 & 271 & 653 & 245 & \\
\hline 3 & 730 & 273 & 653 & 245 & \\
\hline 4 & 728 & 272 & 653 & 245 & \\
\hline $1-1977$ & 730 & 273 & 568 & 214 & \\
\hline 2 & 730 & 273 & 568 & 214 & \\
\hline 3 & 730 & 273 & 568 & 214 & \\
\hline 4 & 730 & 273 & 568 & 214 & \\
\hline 1-1978 & 733 & 274 & 568 & 214 & \\
\hline 2 & 733 & 274 & 568 & 214 & \\
\hline 3 & 695 & 260 & 568 & 214 & \\
\hline 4 & 689 & 258 & 568 & 214 & \\
\hline 1-1979 & 651 & 244 & 568 & 214 & \\
\hline 2 & 568 & 214 & 568 & 214 & \\
\hline 3 & 568 & 214 & 568 & 214 & \\
\hline 4 & 568 & 214 & 568 & 214 & \\
\hline $1-1980$ & 568 & 214 & 568 & 214 & \\
\hline 2 & 568 & 214 & 568 & 214 & \\
\hline 3 & 568 & 214 & 568 & 214 & \\
\hline 4 & 568 & 214 & 568 & 214 & \\
\hline $1-1981$ & 568 & 214 & 568 & 214 & \\
\hline 2 & 568 & 214 & 568 & 214 & \\
\hline 3 & 568 & 214 & 568 & 214 & \\
\hline 4 & 568 & 214 & 568 & $21 \overline{4}$ & \\
\hline $1-1982$ & 568 & 214 & 568 & 214 & \\
\hline 2 & 568 & 214 & 568 & 214 & \\
\hline 3 & 568 & 214 & 568 & 214 & \\
\hline 4 & 568 & 214 & 568 & 214 & \\
\hline $1-1983$ & 568 & 214 & 568 & 214 & \\
\hline 2 & 568 & 214 & 568 & 214 & \\
\hline 3 & 568 & 214 & 568 & 214 & \\
\hline 4 & 568 & 214 & 568 & 214 & \\
\hline $1-1984$ & 568 & 214 & 568 & 214 & \\
\hline 2 & 568 & 214 & 568 & 214 & \\
\hline 3 & 568 & 214 & 568 & 274 & \\
\hline 4 & 568 & 214 & 568 & 214 & \\
\hline $1-1985$ & 568 & 214 & 568 & 214 & \\
\hline 2 & 568 & 214 & 568 & 214 & \\
\hline 3 & 568 & 214 & 568 & 214 & \\
\hline 4 & 568 & 214 & 568 & 214 & \\
\hline $1-1986$ & 568 & 214 & 568 & 214 & 126 \\
\hline 2 & 568 & 214 & 568 & 214 & 127 \\
\hline
\end{tabular}


241-S-109 LEVEL HISTORY

\begin{tabular}{|c|c|c|c|c|c|}
\hline Year & Total & Total & Solids & Solids & ILL \\
\hline & (K gal) & (in) & ( $\mathrm{K}$ gal) & (in) & (in) \\
\hline 3 & 568 & 214 & 568 & 214 & 128 \\
\hline 4 & 568 & 214 & 568 & 214 & 127 \\
\hline $1-1987$ & 568 & 214 & 568 & 214 & 128 \\
\hline 2 & 568 & 214 & 568 & 214 & \\
\hline 3 & 568 & 214 & 568 & 214 & 128 \\
\hline 4 & 568 & 214 & 568 & 214 & 128 \\
\hline 1-1988 & 568 & 214 & $5 \overline{68}$ & 214 & 129 \\
\hline 2 & 568 & 214 & 568 & 214 & 129 \\
\hline 3 & 568 & 214 & 568 & 214 & 129 \\
\hline 4 & 568 & 214 & 568 & 214 & 130 \\
\hline $1-1989$ & 568 & 214 & 568 & 214 & \\
\hline 2 & 568 & 214 & 568 & 214 & 131 \\
\hline 3 & 568 & 214 & 568 & 214 & 131 \\
\hline 4 & 568 & 214 & 568 & 214 & 132 \\
\hline $1-1990$ & 568 & 214 & 568 & 214 & 130 \\
\hline 2 & 568 & 214 & 568 & 214 & \\
\hline 3 & 568 & 214 & 568 & 214 & 132 \\
\hline 4 & 568 & 214 & 568 & 214 & 131 \\
\hline $1-1991$ & 568 & 214 & 568 & 214 & 132 \\
\hline 2 & 568 & 214 & 568 & 214 & 132 \\
\hline 3 & 568 & 214 & 568 & 214 & 133 \\
\hline 4 & 568 & 214 & 568 & 214 & 132 \\
\hline 1-1992 & 568 & 214 & 568 & 214 & \\
\hline 2 & 568 & 214 & 568 & 214 & 133 \\
\hline 3 & 568 & 214 & 568 & 214 & \\
\hline 4 & 568 & 214 & 568 & 214 & 133 \\
\hline 1-1993 & 568 & 214 & 568 & 214 & 134 \\
\hline 2 & 568 & 214 & 568 & 214 & 133 \\
\hline 3 & 568 & 214 & 568 & 274 & 134 \\
\hline 4 & 568 & 214 & 568 & 214 & 133 \\
\hline $1-1994$ & 568 & 214 & 568 & 214 & 134 \\
\hline 2 & 568 & 214 & 568 & 214 & 134 \\
\hline 3 & 568 & 214 & 568 & 214 & 134 \\
\hline 4 & 568 & 214 & 568 & 214 & 136 \\
\hline $1-1995$ & 568 & 214 & 568 & 214 & 136 \\
\hline 2 & 568 & 214 & 568 & 214 & 137 \\
\hline 3 & 568 & 214 & 568 & 214 & 136 \\
\hline 4 & 568 & 214 & 568 & 214 & 135 \\
\hline $1-1996$ & 568 & 214 & 568 & 214 & 137 \\
\hline 2 & 568 & 214 & 568 & 214 & 137 \\
\hline
\end{tabular}


241-S-110 LEVEL HISTORY

\begin{tabular}{|c|c|c|c|c|c|}
\hline Year & Total & Total & Solids & Solids & ILL \\
\hline & (K gal) & (in) & (K gal) & (in) & (in) \\
\hline \multirow{2}{*}{\multicolumn{6}{|c|}{ 1-1952 }} \\
\hline & & & & & \\
\hline 2 & 681 & 255 & 0 & 0 & \\
\hline 3 & 774 & 289 & 0 & 0 & \\
\hline 4 & 774 & 289 & 0 & 0 & \\
\hline 1-1953 & 774 & 289 & 0 & 0 & \\
\hline 2 & 758 & 283 & 0 & 0 & \\
\hline 3 & 758 & 283 & 0 & 0 & \\
\hline 4 & 758 & 283 & 0 & 0 & \\
\hline $1-1954$ & 758 & 283 & 0 & 0 & \\
\hline 2 & 758 & 283 & 0 & 0 & \\
\hline 3 & 758 & 283 & 0 & 0 & \\
\hline 4 & 758 & 283 & 0 & 0 & \\
\hline 1-1955 & 758 & 283 & 0 & 0 & \\
\hline 2 & 758 & 283 & 0 & 0 & \\
\hline 3 & 758 & 283 & 0 & 0 & \\
\hline 4 & 758 & 283 & 0 & 0 & \\
\hline $1-1956$ & 758 & 283 & 0 & 0 & \\
\hline 2 & 758 & 283 & 0 & 0 & \\
\hline 3 & 758 & 283 & 0 & 0 & \\
\hline 4 & 758 & 283 & 0 & 0 & \\
\hline $1-1957$ & 781 & 291 & 0 & 0 & \\
\hline 2 & 781 & 291 & 0 & 0 & \\
\hline 3 & 780 & 291 & 0 & 0 & \\
\hline 4 & 780 & 291 & 0 & 0 & \\
\hline 1-1958 & 780 & 291 & 0 & 0 & \\
\hline 2 & 780 & 291 & 0 & 0 & \\
\hline 3 & 777 & 290 & 0 & 0 & \\
\hline 4 & 777 & 290 & 0 & 0 & \\
\hline $1-1959$ & 777 & 290 & 0 & 0 & \\
\hline 2 & 777 & 290 & 0 & 0 & \\
\hline 3 & 777 & 290 & 0 & 0 & \\
\hline 4 & 777 & 290 & 0 & 0 & \\
\hline $1-1960$ & 774 & 289 & 0 & 0 & \\
\hline 2 & 774 & 289 & 0 & 0 & \\
\hline 3 & 774 & 289 & 0 & 0 & \\
\hline 4 & 777 & 290 & 0 & 0 & \\
\hline \multicolumn{6}{|l|}{$1-1961$} \\
\hline 2 & 774 & 289 & 0 & 0 & \\
\hline \multicolumn{6}{|l|}{3} \\
\hline 4 & 774 & 289 & 0 & 0 & \\
\hline \multicolumn{6}{|l|}{$1-1962$} \\
\hline 2 & 774 & 289 & 0 & 0 & \\
\hline \multicolumn{6}{|l|}{3} \\
\hline 4 & 774 & 289 & 0 & 0 & \\
\hline \multicolumn{6}{|l|}{$1-1963$} \\
\hline & 774 & 289 & 0 & 0 & \\
\hline
\end{tabular}


241-S-110 LEVEL HISTORY

HNF-SD-WM-ER-323, Rev. 1

\begin{tabular}{|c|c|c|c|c|c|}
\hline Year & Total & Total & Solids & Solids & ILL \\
\hline & (K gal) & (in) & (K gal) & (in) & (in) \\
\hline \multicolumn{6}{|l|}{3} \\
\hline 4 & 772 & 288 & 0 & 0 & \\
\hline \multicolumn{5}{|l|}{$1-1964$} & \\
\hline 2 & 772 & 288 & 0 & 0 & \\
\hline \multicolumn{6}{|c|}{3} \\
\hline 4 & 772 & 288 & 0 & 0 & \\
\hline \multicolumn{6}{|l|}{ 1-1965 } \\
\hline 2 & 772 & 288 & 106 & 46 & \\
\hline 3 & 772 & 288 & 106 & 46 & \\
\hline 4 & 772 & 288 & 106 & 46 & \\
\hline 1.1966 & 772 & 288 & 106 & 46 & \\
\hline 2 & 772 & 288 & 106 & 46 & \\
\hline 3 & 772 & 288 & 106 & 46 & \\
\hline 4 & 772 & 288 & 106 & 46 & \\
\hline $1-1967$ & 772 & 288 & 106 & 46 & \\
\hline 2 & 772 & 288 & 106 & 46 & \\
\hline 3 & 772 & 288 & 106 & 46 & \\
\hline 4 & 772 & 288 & 106 & 46 & \\
\hline $1-1968$ & 772 & 288 & 106 & 46 & \\
\hline 2 & 776 & 290 & 106 & 46 & \\
\hline 3 & 776 & 290 & 106 & 46 & \\
\hline 4 & 776 & 290 & 106 & 46 & \\
\hline $1-1969$ & 776 & 290 & 106 & 46 & \\
\hline 2 & 776 & 290 & 106 & 46 & \\
\hline 3 & 776 & 290 & 106 & 46 & \\
\hline 4 & 776 & 290 & 113 & 49 & \\
\hline $1-1970$ & 775 & 289 & 113 & 49 & \\
\hline 2 & 775 & 289 & 113 & 49 & \\
\hline 3 & 776 & 290 & 113 & 49 & \\
\hline 4 & 775 & 289 & 113 & 49 & \\
\hline 1.1971 & 775 & 289 & 113 & 49 & \\
\hline 2 & 774 & 289 & 113 & 49 & \\
\hline 3 & 775 & 289 & 113 & 49 & \\
\hline 4 & 775 & 289 & 113 & 49 & \\
\hline $1-1972$ & 774 & 289 & 113 & 49 & \\
\hline 2 & 774 & 289 & 113 & 49 & \\
\hline 3 & 773 & 289 & 113 & 49 & \\
\hline 4 & 774 & 289 & 113 & 49 & \\
\hline 1.1973 & 773 & 289 & 113 & 49 & \\
\hline 2 & 766 & 286 & 113 & 49 & \\
\hline 3 & 778 & 290 & 113 & 49 & \\
\hline 4 & 786 & 293 & 113 & 49 & \\
\hline $1-1974$ & 280 & 109 & 131 & 55 & \\
\hline 2 & 686 & 257 & 131 & 55 & \\
\hline 3 & 580 & 218 & 131 & 55 & \\
\hline 4 & 695 & 260 & 255 & 100 & \\
\hline
\end{tabular}


241-S-110 LEVEL HISTORY

HNF-SD-WM-ER-323, Rev. 1

\begin{tabular}{|c|c|c|c|c|c|}
\hline Year & Total & Total & Solids & Solids & ILL \\
\hline & (K gal) & (in) & (K gal) & (in) & (in) \\
\hline $1-1975$ & 678 & 254 & 414 & 158 & \\
\hline 2 & 739 & 276 & 560 & 211 & \\
\hline 3 & 755 & 282 & 755 & 282 & \\
\hline 4 & 689 & 258 & 689 & 258 & \\
\hline $1-1976$ & 689 & 258 & 689 & 258 & \\
\hline 2 & 689 & 258 & 689 & 258 & \\
\hline 3 & 689 & 258 & 689 & 258 & \\
\hline 4 & 623 & 234 & 623 & 234 & \\
\hline $1-1977$ & 623 & 234 & 475 & 180 & \\
\hline 2 & 700 & 262 & 475 & 180 & \\
\hline 3 & 700 & 262 & 475 & 180 & \\
\hline 4 & 700 & 262 & 475 & 180 & \\
\hline $1-1978$ & 700 & 262 & 475 & 180 & \\
\hline 2 & 700 & 262 & 475 & 180 & \\
\hline 3 & 692 & 259 & 475 & 180 & \\
\hline 4 & 692 & 259 & 475 & 180 & \\
\hline 1-1979 & 692 & 259 & 692 & 259 & \\
\hline 2 & 692 & 259 & 692 & 259 & \\
\hline 3 & 692 & 259 & 692 & 259 & \\
\hline 4 & 692 & 259 & 692 & 259 & \\
\hline $1-1980$ & 692 & 259 & 692 & 259 & \\
\hline 2 & 692 & 259 & 692 & 259 & \\
\hline 3 & 692 & 259 & 692 & 259 & \\
\hline 4 & 692 & 259 & 692 & 259 & \\
\hline $1-1981$ & 692 & 259 & 692 & 259 & \\
\hline 2 & 692 & 259 & 692 & 259 & \\
\hline 3 & 692 & 259 & 692 & 259 & \\
\hline 4 & 692 & 259 & 692 & 259 & \\
\hline 1-1982 & 692 & 259 & 692 & 259 & \\
\hline 2 & 692 & 259 & 692 & 259 & \\
\hline 3 & 692 & 259 & 692 & 259 & \\
\hline 4 & 692 & 259 & 692 & 259 & \\
\hline 1-1983 & 692 & 259 & 692 & 259 & \\
\hline 2 & 692 & 259 & 692 & 259 & \\
\hline 3 & 692 & 259 & 692 & 259 & \\
\hline 4 & 692 & 259 & 692 & 259 & \\
\hline 1-1984 & 692 & 259 & 692 & 259 & \\
\hline 2. & 692 & 259 & 692 & 259 & \\
\hline 3 & 692 & 259 & 692 & 259 & \\
\hline 4 & 692 & 259 & 692 & 259 & \\
\hline 1-1985 & 692 & 259 & 692 & 259 & \\
\hline 2 & 692 & 259 & 692 & 259 & \\
\hline 3 & 692 & 259 & 692 & 259 & \\
\hline 4 & 692 & 259 & 692 & 259 & \\
\hline $1-1986$ & 692 & 259 & 692 & 259 & 141 \\
\hline 2 & 692 & 259 & 692 & 259 & 141 \\
\hline
\end{tabular}


241-S-110 LEVEL HISTORY

HNF-SD-WM-ER-323, Rev. 1

\begin{tabular}{|c|c|c|c|c|c|}
\hline Year & Total & Total & Solids & Solids & ILL \\
\hline & (K gal) & (in) & (K gal) & (in) & (in) \\
\hline 3 & 692 & 259 & 692 & 259 & 139 \\
\hline 4 & 692 & 259 & 692 & 259 & 141 \\
\hline $1-1987$ & 692 & 259 & 692 & 259 & 141 \\
\hline 2 & 692 & 259 & 692 & 259 & \\
\hline 3 & 692 & 259 & 692 & 259 & 141 \\
\hline 4 & 692 & 259 & 692 & 259 & 141 \\
\hline $1-1988$ & 692 & 259 & 692 & 259 & 141 \\
\hline 2 & 692 & 259 & 692 & 259 & 141 \\
\hline 3 & 692 & 259 & 692 & 259 & 141 \\
\hline 4 & 692 & 259 & 692 & 259 & 142 \\
\hline $1-1989$ & 692 & 259 & 692 & 259 & \\
\hline 2 & 692 & 259 & 692 & -259 & 141 \\
\hline 3 & 692 & 259 & 692 & 259 & 141 \\
\hline 4 & 692 & 259 & 692 & 259 & 141 \\
\hline $1-1990$ & 692 & 259 & 692 & 259 & 141 \\
\hline 2 & 692 & 259 & 692 & 259 & \\
\hline 3 & 692 & 259 & 692 & 259 & 141 \\
\hline 4 & 692 & 259 & 692 & 259 & 140 \\
\hline $1-1991$ & 692 & 259 & 692 & 259 & 142 \\
\hline 2 & 692 & 259 & 692 & 259 & 140 \\
\hline 3 & 692 & 259 & 692 & 259 & 141 \\
\hline 4 & 692 & 259 & 692 & 259 & 142 \\
\hline $1-1992$ & 692 & 259 & 692 & 259 & \\
\hline 2 & 390 & 149 & 390 & 149 & 141 \\
\hline 3 & 390 & 149 & 390 & 149 & \\
\hline 4 & 390 & 149 & 390 & 149 & 141 \\
\hline $1-1993$ & 390 & 149 & 390 & 149 & 141 \\
\hline 2 & 390 & 149 & 390 & 149 & \\
\hline 3 & 390 & 149 & 390 & 149 & 141 \\
\hline 4 & 390 & 149 & 390 & 149 & 141 \\
\hline 1-1994 & 390 & 149 & 390 & 149 & 141 \\
\hline 2 & 390 & 149 & 390 & 149 & 141 \\
\hline 3 & 390 & 149 & 390 & 149 & 141 \\
\hline 4 & 390 & 149 & 390 & 149 & 142 \\
\hline 1-1995 & 390 & 149 & 390 & 149 & 145 \\
\hline 2 & 390 & 149 & 390 & 149 & 144 \\
\hline 3 & 390 & 149 & 390 & 149 & 145 \\
\hline 4 & 390 & 149 & 390 & 149 & 146 \\
\hline $1-1996$ & 390 & 149 & 390 & 149 & 140 \\
\hline 2 & 390 & 149 & 390 & 149 & 130 \\
\hline
\end{tabular}


241-S-111 LEVEL HISTORY

\begin{tabular}{|c|c|c|c|c|c|}
\hline Year & Total & Total & Solids & Solids & ILL \\
\hline & (K gal) & (in) & (K gal) & (in) & (in) \\
\hline \multirow{2}{*}{\multicolumn{6}{|c|}{$1-1952$}} \\
\hline & & & & & \\
\hline \multicolumn{6}{|l|}{2} \\
\hline 3 & 755 & 282 & 0 & 0 & \\
\hline 4 & 755 & 282 & 0 & 0 & \\
\hline $1-1953$ & 755 & 282 & 0 & 0 & \\
\hline 2 & 758 & 283 & 0 & 0 & \\
\hline 3 & 758 & 283 & 0 & 0 & \\
\hline 4 & 758 & 283 & 0 & 0 & \\
\hline 1-1954 & 758 & 283 & 0 & 0 & \\
\hline 2 & 758 & 283 & 0 & 0 & \\
\hline 3 & 758 & 283 & 0 & 0 & \\
\hline 4 & 758 & 283 & 0 & 0 & \\
\hline $1-1955$ & 758 & 283 & 0 & 0 & \\
\hline 2 & 758 & 283 & 0 & 0 & \\
\hline 3 & 758 & 283 & 0 & 0 & \\
\hline 4 & 758 & 283 & 0 & 0 & \\
\hline 1.1956 & 758 & 283 & 0 & 0 & \\
\hline 2 & 758 & 283 & 0 & 0 & \\
\hline 3 & 758 & 283 & 0 & 0 & \\
\hline 4 & 758 & 283 & 0 & 0 & \\
\hline 1-1957 & 761 & 284 & 0 & 0 & \\
\hline 2 & 761 & 284 & 0 & 0 & \\
\hline 3 & 761 & 284 & 0 & 0 & \\
\hline 4 & 761 & 284 & 0 & 0 & \\
\hline $1-1958$ & 761 & 284 & 0 & 0 & \\
\hline 2 & 761 & 284 & 0 & 0 & \\
\hline 3 & 761 & 284 & 0 & 0 & \\
\hline 4 & 761 & 284 & 0 & 0 & \\
\hline $1-1959$ & 761 & 284 & 0 & 0 & \\
\hline 2 & 761 & 284 & 0 & 0 & \\
\hline 3 & 761 & 284 & 0 & 0 & \\
\hline 4 & 761 & 284 & 0 & 0 & \\
\hline $1-1960$ & 758 & 283 & 0 & 0 & \\
\hline 2 & 758 & 283 & 0 & 0 & \\
\hline 3 & 758 & 283 & 0 & 0 & \\
\hline 4 & 758 & 283 & 0 & 0 & \\
\hline \multicolumn{6}{|l|}{$1-1961$} \\
\hline 2 & 755 & 282 & 0 & 0 & \\
\hline \multicolumn{6}{|l|}{3} \\
\hline 4 & 755 & 282 & 0 & 0 & \\
\hline \multicolumn{6}{|l|}{ 1-1962 } \\
\hline 2 & 755 & 282 & 0 & 0 & \\
\hline \multicolumn{6}{|l|}{3} \\
\hline 4 & 755 & 282 & 0 & 0 & \\
\hline \multicolumn{6}{|l|}{$1-1963$} \\
\hline 2 & 755 & 282 & 0 & 0 & \\
\hline
\end{tabular}


HNF-SD-WM-ER-323, Rev. I

241-S-111 LEVEL HISTORY

\begin{tabular}{|c|c|c|c|c|c|}
\hline Year & Total & Total & Solids & Solids & ILL. \\
\hline & (K gal) & (in) & (K gal) & (in) & (in) \\
\hline \multirow{2}{*}{\multicolumn{6}{|c|}{3}} \\
\hline & & & & & \\
\hline 4 & 755 & 282 & 0 & 0 & \\
\hline \multicolumn{6}{|l|}{$1-1964$} \\
\hline 2 & 755 & 282 & 0 & 0 & \\
\hline \multicolumn{6}{|l|}{3} \\
\hline 4 & 755 & 282 & 0 & 0 & \\
\hline \multicolumn{6}{|l|}{$1-1965$} \\
\hline 2 & 761 & 284 & 144 & 60 & \\
\hline 3 & 761 & 284 & 144 & 60 & \\
\hline 4 & 761 & 284 & 144 & 60 & \\
\hline $1-1966$ & 761 & 284 & 144 & 60 & \\
\hline 2 & 761 & 284 & 144 & 60 & \\
\hline 3 & 761 & 284 & 144 & 60 & \\
\hline 4 & 761 & 284 & 144 & 60 & \\
\hline 1-1967 & 761 & 284 & 144 & 60 & \\
\hline 2 & 759 & 283 & 144 & 60 & \\
\hline 3 & 759 & 283 & 144 & 60 & \\
\hline 4 & 759 & 283 & 144 & 60 & \\
\hline $1-1968$ & 759 & 283 & 144 & 60 & \\
\hline 2 & 759 & 283 & 144 & 60 & \\
\hline 3 & 759 & 283 & 144 & 60 & \\
\hline 4 & 759 & 283 & 144 & 60 & \\
\hline $1-1969$ & 758 & 283 & 144 & 60 & \\
\hline 2 & 758 & 283 & 144 & 60 & \\
\hline 3 & 758 & 283 & 144 & 60 & \\
\hline 4 & 758 & 283 & 139 & 58 & \\
\hline $1-1970$ & 758 & 283 & 139 & 58 & \\
\hline 2 & 758 & 283 & 139 & 58 & \\
\hline 3 & 758 & 283 & 139 & 58 & \\
\hline 4 & 758 & 283 & 139 & 58 & \\
\hline $1-1971$ & 758 & 283 & 139 & 58 & \\
\hline 2 & 758 & 283 & 139 & 58 & \\
\hline 3 & 758 & 283 & 139 & 58 & \\
\hline 4 & 758 & 283 & 139 & 58 & \\
\hline 1.1972 & 758 & 283 & 139 & 58 & \\
\hline 2 & 758 & 283 & 139 & 58 & \\
\hline 3 & 758 & 283 & 139 & 58 & \\
\hline 4 & 758 & 283 & 139 & 58 & \\
\hline 1.1973 & 759 & 283 & 139 & 58 & \\
\hline 2 & 749 & 280 & 139 & 58 & \\
\hline 3 & 764 & 285 & 139 & 58 & \\
\hline 4 & 766 & 286 & 139 & 58 & \\
\hline $1-1974$ & 311 & 121 & 139 & 58 & \\
\hline 2 & 689 & 258 & 334 & 129 & \\
\hline 3 & 730 & 273 & 502 & 190 & \\
\hline 4 & 692 & 259 & 563 & 212 & \\
\hline
\end{tabular}


241-S-111 LEVEL HISTORY

HNF-SD-WM-ER-323, Rev. 1

\begin{tabular}{|c|c|c|c|c|c|}
\hline Year & Total & Total & Solids & Solids & ILL \\
\hline & (K gal) & (in) & (K gal) & (in) & (in) \\
\hline $1-1975$ & 739 & 276 & 596 & 224 & \\
\hline 2 & 739 & 276 & 596 & 224 & \\
\hline 3 & 750 & 280 & 750 & 280 & \\
\hline 4 & 750 & 280 & 750 & 280 & \\
\hline 1-1976 & 750 & 280 & 750 & 280 & \\
\hline 2 & 750 & 280 & 750 & 280 & \\
\hline 3 & 750 & 280 & 750 & 280 & \\
\hline 4 & 623 & 234 & 623 & 234 & \\
\hline 1-1977 & 623 & 234 & 623 & 234 & \\
\hline 2 & 623 & 234 & 623 & 234 & \\
\hline 3 & 623 & 234 & 623 & 234 & \\
\hline 4 & 623 & 234 & 623 & 234 & \\
\hline $1-1978$ & 623 & 234 & 623 & 234 & \\
\hline 2 & 623 & 234 & 623 & 234 & \\
\hline 3 & 623 & 234 & 623 & 234 & \\
\hline 4 & 623 & 234 & 623 & 234 & \\
\hline $1-1979$ & 623 & 234 & 623 & 234 & \\
\hline 2 & 623 & 234 & 623 & 234 & \\
\hline 3 & 623 & 234 & 623 & 234 & \\
\hline 4 & 623 & 234 & 623 & 234 & \\
\hline $1-1980$ & 623 & 234 & 623 & 234 & \\
\hline 2 & 623 & 234 & 623 & 234 & \\
\hline 3 & 623 & 234 & 623 & 234 & \\
\hline 4 & 623 & 234 & 623 & 234 & \\
\hline 1-1981 & 623 & 234 & 623 & 234 & \\
\hline 2 & 623 & 234 & 623 & 234 & \\
\hline 3 & 623 & 234 & 623 & 234 & \\
\hline 4 & 623 & 234 & 623 & 234 & \\
\hline 1-1982 & 623 & 234 & 623 & 234 & \\
\hline 2 & 596 & 224 & 586 & 221 & \\
\hline 3 & 596 & 224 & 586 & 221 & \\
\hline 4 & 596 & 224 & 586 & 221 & \\
\hline 1-1983 & 596 & 224 & 586 & 221 & \\
\hline 2 & 596 & 224 & 586 & 221 & \\
\hline 3 & 596 & 224 & 586 & 221 & \\
\hline 4 & 596 & 224 & 586 & 221 & \\
\hline 1-1984 & 596 & 224 & 586 & 221 & \\
\hline 2 & 596 & 224 & 586 & 221 & \\
\hline 3 & 596 & 224 & 586 & 221 & \\
\hline 4 & 596 & 224 & 586 & 221 & \\
\hline $1-1985$ & 596 & 224 & 586 & 221 & \\
\hline 2 & 596 & 224 & 586 & 221 & \\
\hline 3 & 596 & 224 & 586 & 221 & \\
\hline 4 & 596 & 224 & 586 & 221 & \\
\hline $1-1986$ & 596 & 224 & 586 & 221 & 202 \\
\hline 2 & 596 & 224 & 586 & 221 & 202 \\
\hline
\end{tabular}


241-S-111 LEVEL HISTORY

\begin{tabular}{|c|c|c|c|c|c|}
\hline Year & Total & Total & Solids & Solids & ILL \\
\hline & (K gal) & (in) & ( $K$ gal) & (in) & (in) \\
\hline 3 & 596 & 224 & 586 & 221 & 203 \\
\hline 4 & 596 & 224 & 586 & 221 & 203 \\
\hline $1-1987$ & 596 & 224 & 586 & 221 & 202 \\
\hline 2 & 596 & 224 & 586 & 221 & \\
\hline 3 & 596 & 224 & 586 & 221 & 203 \\
\hline 4 & 596 & 224 & 586 & 221 & 203 \\
\hline $1-1988$ & 596 & 224 & 586 & 221 & 202 \\
\hline 2 & 596 & 224 & 586 & 221 & 203 \\
\hline 3 & 596 & 224 & 586 & 221 & 202 \\
\hline 4 & 596 & 224 & 586 & 221 & 202 \\
\hline 1-1989 & 596 & 224 & 586 & 221 & \\
\hline 2 & 596 & 224 & 586 & 221 & 203 \\
\hline 3 & 596 & 224 & 586 & 221 & 203 \\
\hline 4 & 596 & 224 & 586 & 221 & 203 \\
\hline $1-1990$ & 596 & 224 & 586 & 221 & 202 \\
\hline 2 & 596 & 224 & 586 & 221 & \\
\hline 3 & 596 & 224 & 586 & 221 & 203 \\
\hline 4 & 596 & 224 & 586 & 221 & 203 \\
\hline 1-1991 & 596 & 224 & 586 & 221 & 202 \\
\hline 2 & 596 & 224 & 586 & 221 & 203 \\
\hline 3 & 596 & 224 & 586 & 221 & 203 \\
\hline 4 & 596 & 224 & 586 & 221 & 202 \\
\hline \multicolumn{6}{|l|}{ 1-1992 } \\
\hline 2 & 596 & 224 & 586 & 221 & 203 \\
\hline 3 & 596 & 224 & 586 & 221 & \\
\hline 4 & 596 & 224 & 586 & 221 & 203 \\
\hline $1-1993$ & 596 & 224 & 586 & 221 & 202 \\
\hline 2 & 596 & 224 & 586 & 221 & 203 \\
\hline 3 & 596 & 224 & 586 & 221 & 203 \\
\hline 4 & 596 & 224 & 586 & 221 & 203 \\
\hline $1-1994$ & 596 & 224 & 586 & 221 & 202 \\
\hline 2 & 596 & 224 & 586 & 221 & 203 \\
\hline 3 & 596 & 224 & 586 & 221 & 203 \\
\hline 4 & 596 & 224 & 586 & 221 & 204 \\
\hline 1-1995 & 596 & 224 & 586 & 221 & 202 \\
\hline 2 & 596 & 224 & 586 & 221 & 202 \\
\hline 3 & 596 & 224 & 586 & 221 & 202 \\
\hline 4 & 596 & 224 & 586 & 221 & 202 \\
\hline 1-1996 & 596 & 224 & 586 & 221 & 202 \\
\hline 2 & 596 & 224 & 586 & 221 & 203 \\
\hline
\end{tabular}


241-S-112 LEVEL HISTORY

HNF-SD-WM-ER-323, Rev. 1

\begin{tabular}{|c|c|c|c|c|c|}
\hline Year & Total & Total & Solids & Solids & ILL \\
\hline & (K gal) & (in) & (K gal) & (in) & (in) \\
\hline \multirow{2}{*}{\multicolumn{6}{|c|}{ 1-1952 }} \\
\hline & & & & & \\
\hline \multicolumn{6}{|l|}{2} \\
\hline 3 & 755 & 282 & 0 & 0 & \\
\hline 4 & 442 & 168 & 0 & 0 & \\
\hline $1-1953$ & 442 & 168 & 0 & 0 & \\
\hline 2 & 442 & 168 & $\overline{0}$ & 0 & \\
\hline 3 & 498 & 189 & 0 & 0 & \\
\hline 4 & 640 & 240 & $\overline{0}$ & 0 & \\
\hline $1-1954$ & 656 & 246 & 0 & 0 & \\
\hline 2 & 688 & 258 & 0 & 0 & \\
\hline 3 & 686 & 257 & 0 & 0 & \\
\hline 4 & 675 & 253 & 0 & 0 & \\
\hline $1-1955$ & 695 & 260 & 0 & 0 & \\
\hline 2 & 695 & 260 & 0 & 0 & \\
\hline 3 & 695 & 260 & 0 & 0 & \\
\hline 4 & 747 & 279 & 0 & 0 & \\
\hline $1-1956$ & 747 & 279 & 0 & 0 & \\
\hline 2 & 747 & 279 & 0 & 0 & \\
\hline 3 & 747 & 279 & 0 & 0 & \\
\hline 4 & 747 & 279 & 0 & 0 & \\
\hline 1-1957 & 780 & 291 & 0 & 0 & \\
\hline 2 & 780 & 291 & 0 & 0 & \\
\hline 3 & 780 & 291 & 0 & 0 & \\
\hline 4 & 780 & 291 & 0 & 0 & \\
\hline $1-1958$ & 780 & 291 & 0 & 0 & \\
\hline 2 & 780 & 291 & 0 & 0 & \\
\hline 3 & 780 & 291 & 0 & 0 & \\
\hline 4 & 780 & 291 & 0 & 0 & \\
\hline 1-1959 & 780 & 291 & 0 & 0 & \\
\hline 2 & 780 & 291 & 0 & 0 & \\
\hline 3 & 780 & 291 & 0 & 0 & \\
\hline 4 & 780 & 291 & 0 & 0 & \\
\hline $1-1960$ & 777 & 290 & 0 & 0 & \\
\hline 2 & 777 & 290 & 0 & 0 & \\
\hline 3 & 777 & 290 & 0 & 0 & \\
\hline 4 & 780 & 291 & 0 & 0 & \\
\hline \multicolumn{6}{|l|}{ 1-1961 } \\
\hline 2 & 777 & 290 & 0 & 0 & \\
\hline \multicolumn{6}{|l|}{3} \\
\hline 4 & 777 & 290 & 0 & 0 & \\
\hline \multicolumn{6}{|l|}{$1-1962$} \\
\hline 2 & 777 & 290 & 0 & 0 & \\
\hline \multicolumn{6}{|l|}{3} \\
\hline 4 & 777 & 290 & 0 & 0 & \\
\hline \multicolumn{6}{|l|}{$1-1963$} \\
\hline 2 & 777 & 290 & 0 & 0 & \\
\hline
\end{tabular}


241-S-112 LEVEL HISTORY

HNF-SD-WM-ER-323, Rev. 1

\begin{tabular}{|c|c|c|c|c|c|}
\hline Year & Total & Total & Solids & Solids & ILL \\
\hline & (K gal) & (in) & (K gal) & (in) & (in) \\
\hline \multicolumn{6}{|l|}{3} \\
\hline 4 & 777 & 290 & 0 & 0 & \\
\hline \multicolumn{6}{|l|}{$1-1964$} \\
\hline 2 & 777 & 290 & 0 & 0 & \\
\hline \multicolumn{6}{|l|}{3} \\
\hline 4 & 777 & 290 & 0 & 0 & \\
\hline \multicolumn{6}{|l|}{$1-1965$} \\
\hline 2 & 785 & 293 & 4 & 9 & \\
\hline 3 & 785 & 293 & 4 & 9 & \\
\hline 4 & 785 & 293 & 4 & 9 & \\
\hline 1-1966 & 785 & 293 & 4 & 9 & \\
\hline 2 & 785 & 293 & 4 & 9 & \\
\hline 3 & 785 & 293 & 4 & 9 & \\
\hline 4 & 785 & 293 & 4 & 9 & \\
\hline $1-1967$ & 785 & 293 & 4 & 9 & \\
\hline 2 & 788 & 294 & 4 & 9 & \\
\hline 3 & 788 & 294 & 4 & 9 & \\
\hline 4 & 788 & 294 & 4 & 9 & \\
\hline $1-1968$ & 788 & 294 & 4 & 9 & \\
\hline 2 & 788 & 294 & 4 & 9 & \\
\hline 3 & 788 & 294 & 4 & 9 & \\
\hline 4 & 787 & 294 & 4 & 9 & \\
\hline $1-1969$ & 787 & 294 & 4 & 9 & \\
\hline 2 & 787 & 294 & 4 & 9 & \\
\hline 3 & 787 & 294 & 4 & 9 & \\
\hline 4 & 787 & 294 & 6 & 10 & \\
\hline $1-1970$ & 787 & 294 & 6 & 10 & \\
\hline 2 & 787 & 294 & 6 & 10 & \\
\hline 3 & 787 & 294 & 6 & 10 & \\
\hline 4 & 787 & 294 & 6 & 10 & \\
\hline 1-1971 & 787 & 294 & 6 & 10 & \\
\hline 2 & 787 & 294 & 6 & 10 & \\
\hline 3 & 787 & 294 & 6 & 10 & \\
\hline 4 & 788 & 294 & 6 & 10 & \\
\hline 1-1972 & 787 & 294 & 6 & 10 & \\
\hline 2 & 787 & 294 & 6 & 10 & \\
\hline 3 & 787 & 294 & 6 & 10 & \\
\hline 4 & 787 & 294 & 6 & 10 & \\
\hline $1-1973$ & 787 & 294 & 6 & 10 & \\
\hline 2 & 780 & 291 & 6 & 10 & \\
\hline 3 & 792 & 295 & 6 & 10 & \\
\hline 4 & 792 & 295 & 6 & 10 & \\
\hline $1-1974$ & 473 & 179 & 367 & 141 & \\
\hline 2 & 713 & 267 & 598 & 225 & \\
\hline 3 & 732 & 274 & 686 & 257 & \\
\hline 4 & 730 & 273 & 673 & 252 & \\
\hline
\end{tabular}


241-S-112 LEVEL HISTORY

\begin{tabular}{|c|c|c|c|c|c|}
\hline Year & Total & Total & Solids & Solids & ILL \\
\hline & (K gal) & (in) & (K gal) & (in) & (in) \\
\hline $1-1975$ & 736 & 275 & 714 & 267 & \\
\hline 2 & 736 & 275 & 714 & 267 & \\
\hline 3 & 739 & 276 & 714 & 267 & \\
\hline 4 & 706 & 264 & 706 & 264 & \\
\hline $1-1976$ & 706 & 264 & 706 & 264 & \\
\hline 2 & 706 & 264 & 706 & 264 & \\
\hline 3 & 706 & 264 & 706 & 264 & \\
\hline 4 & 706 & 264 & 706 & 264 & \\
\hline $1-1977$ & 706 & 264 & 673 & 252 & \\
\hline 2 & 717 & 268 & 673 & 252 & \\
\hline 3 & 719 & 269 & 673 & 252 & \\
\hline 4 & 719 & 269 & 673 & 252 & \\
\hline $1-1978$ & 719 & 269 & 673 & 252 & \\
\hline 2 & 719 & 269 & 673 & 252 & \\
\hline 3 & 675 & 253 & 673 & 252 & \\
\hline 4 & 678 & 254 & 673 & 252 & \\
\hline $1-1979$ & 675 & 253 & 673 & 252 & \\
\hline 2 & 673 & 252 & 673 & 252 & \\
\hline 3 & 673 & 252 & 673 & 252 & \\
\hline 4 & 673 & 252 & 673 & 252 & \\
\hline $1-1980$ & 673 & 252 & 673 & 252 & \\
\hline 2 & 672 & 252 & 672 & 252 & \\
\hline 3 & 672 & 252 & 672 & 252 & \\
\hline 4 & 672 & 252 & 672 & 252 & \\
\hline $1-1981$ & 672 & 252. & 672 & 252 & \\
\hline 2 & 672 & 252 & 672 & 252 & \\
\hline 3 & 672 & 252 & 672 & 252 & \\
\hline 4 & 672 & 252 & 672 & 252 & \\
\hline $1-1982$ & 672 & 252 & 672 & 252 & \\
\hline 2 & 641 & 241 & 641 & 241 & \\
\hline 3 & 637 & 239 & 637 & 239 & \\
\hline 4 & 637 & 239 & 637 & 239 & \\
\hline $1-1983$ & 637 & 239 & 637 & 239 & \\
\hline 2 & 637 & 239 & 637 & 239 & \\
\hline 3 & 637 & 239 & 637 & 239 & \\
\hline 4 & 646 & 242 & 637 & 239 & \\
\hline $1-1984$ & 646 & 242 & 637 & 239 & \\
\hline 2 & 637 & 239 & 637 & 239 & \\
\hline 3 & 637 & 239 & 637 & 239 & \\
\hline 4 & 637 & 239 & 637 & 239 & \\
\hline $1-1985$ & 637 & 239 & 637 & 239 & \\
\hline 2 & 637 & 239 & 637 & 239 & \\
\hline 3 & 637 & 239 & 637 & 239 & \\
\hline 4 & 637 & 239 & 637 & 239 & \\
\hline 1-1986 & 637 & 239 & 637 & 239 & 101 \\
\hline 2 & 637 & 239 & 637 & 239 & 101 \\
\hline
\end{tabular}


HNF-SD-WM-ER-323, Rev. 1

241-S-112 LEVEL HISTORY

\begin{tabular}{|c|c|c|c|c|c|}
\hline Year & Total & Total & Solids & Solids & ILL \\
\hline & (K gal) & (in) & (K gal) & (in) & (in) \\
\hline 3 & 637 & 239 & 637 & 239 & 102 \\
\hline 4 & 637 & 239 & 637 & 239 & 102 \\
\hline 1-1987 & 637 & 239 & 637 & 239 & 102 \\
\hline 2 & 637 & 239 & 637 & 239 & \\
\hline 3 & 637 & 239 & 637 & 239 & 106 \\
\hline 4 & 637 & 239 & 637 & 239 & 107 \\
\hline 1-1988 & 637 & 239 & 637 & 239 & 108 \\
\hline 2 & 637 & 239 & 637 & 239 & 111 \\
\hline 3 & 637 & 239 & 637 & 239 & 109 \\
\hline 4 & 637 & 239 & 637 & 239 & 111 \\
\hline $1-1989$ & 637 & 239 & 637 & 239 & \\
\hline 2 & 637 & 239 & 637 & 239 & 113 \\
\hline 3 & 637 & 239 & 637 & 239 & 113 \\
\hline 4 & 637 & 239 & 637 & 239 & 115 \\
\hline $1-1990$ & 637 & 239 & 637 & 239 & 114 \\
\hline 2 & 637 & 239 & 637 & 239 & \\
\hline 3 & 637 & 239 & 637 & 239 & 115 \\
\hline 4 & 637 & 239 & 637 & 239 & 115 \\
\hline 1-1991 & 637 & 239 & 637 & 239 & 115 \\
\hline 2 & 637 & 239 & 637 & 239 & 116 \\
\hline 3 & 637 & 239 & 637 & 239 & 115 \\
\hline 4 & 637 & 239 & 637 & 239 & 115 \\
\hline 1-1992 & 637 & 239 & 637 & 239 & \\
\hline 2 & 637 & 239 & 637 & 239 & 116 \\
\hline 3 & 637 & 239 & 637 & 239 & \\
\hline 4 & 637 & 239 & 637 & 239 & 115 \\
\hline $1-1993$ & 637 & 239 & 637 & 239 & 117 \\
\hline 2 & 637 & 239 & 637 & 239 & 118 \\
\hline 3 & 637 & 239 & 637 & 239 & 119 \\
\hline 4 & 523 & 198 & 523 & 198 & 118 \\
\hline $1-1994$ & 523 & 198 & 523 & 198 & 118 \\
\hline 2 & 523 & 198 & 523 & 198 & 117 \\
\hline 3 & 523 & 198 & 523 & 198 & 119 \\
\hline 4 & 523 & 198 & 523 & 198 & 119 \\
\hline 1-1995 & 523 & 198 & 523 & 198 & 118 \\
\hline 2 & 523 & 198 & 523 & 198 & 117 \\
\hline 3 & 523 & 198 & 523 & 198 & 118 \\
\hline 4 & 523 & 198 & 523 & 198 & 118 \\
\hline $1-1996$ & 523 & 198 & 523 & 198 & 119 \\
\hline 2 & 523 & 198 & 523 & 198 & 119 \\
\hline
\end{tabular}


THIS PAGE INTENTIONALLY LEFT BLANK 


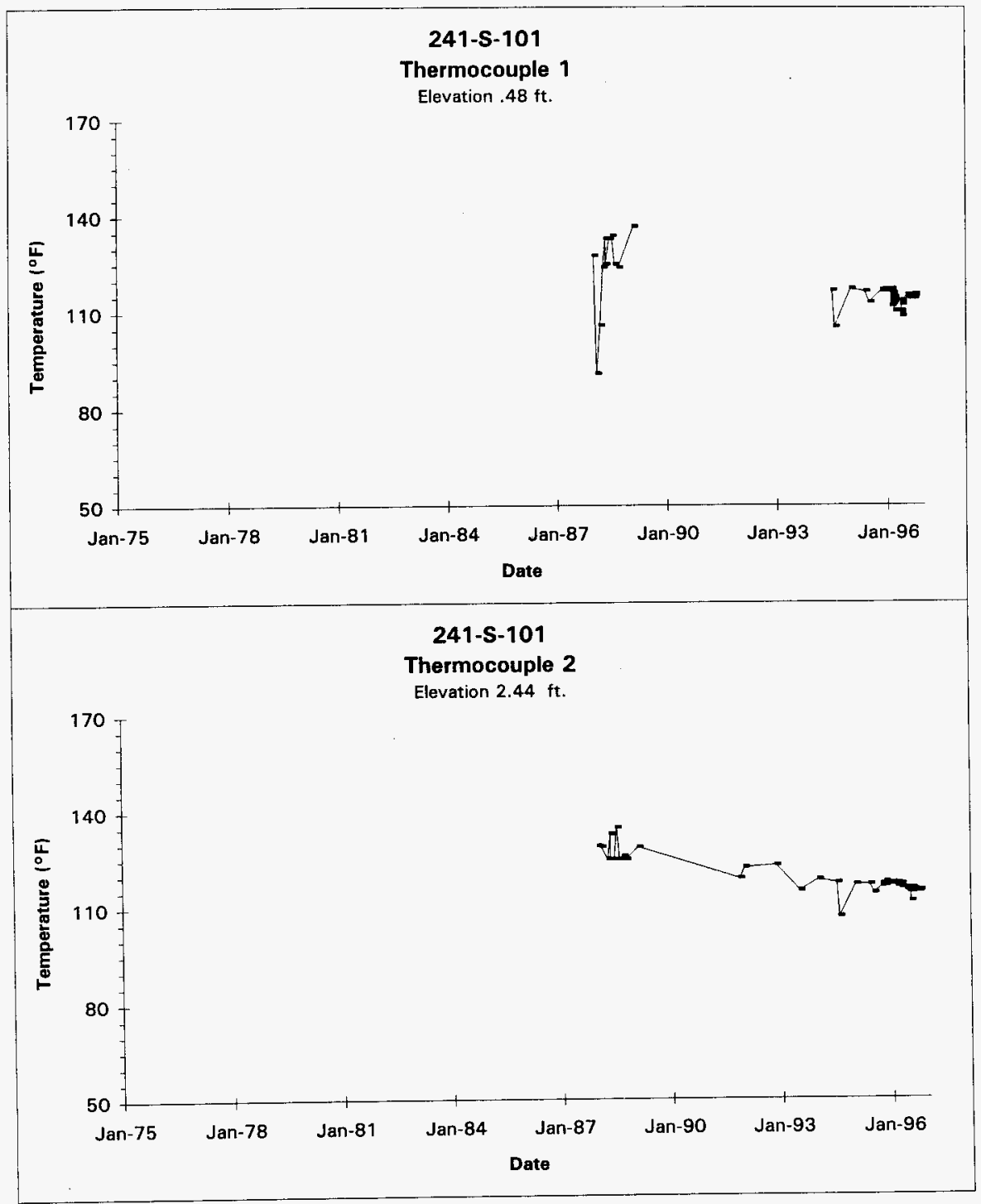

Data obtained from LMHC Surveillance Analysis Computer System (SACS), Oct 1, 1996.

D-1 


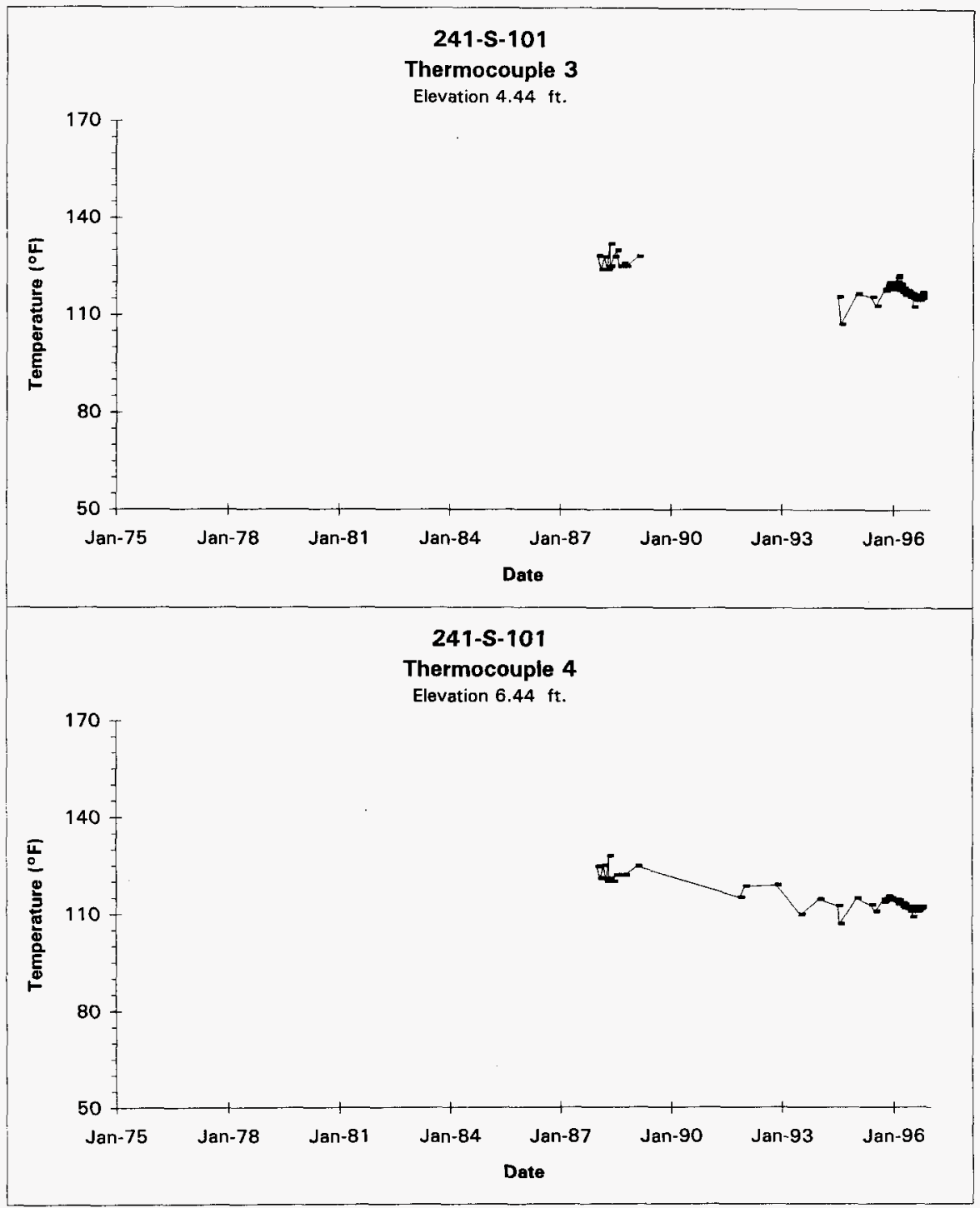

Data obtained from LMHC Surveillance Analysis Computer System (SACS), Oct 1, 1996.

D-2 


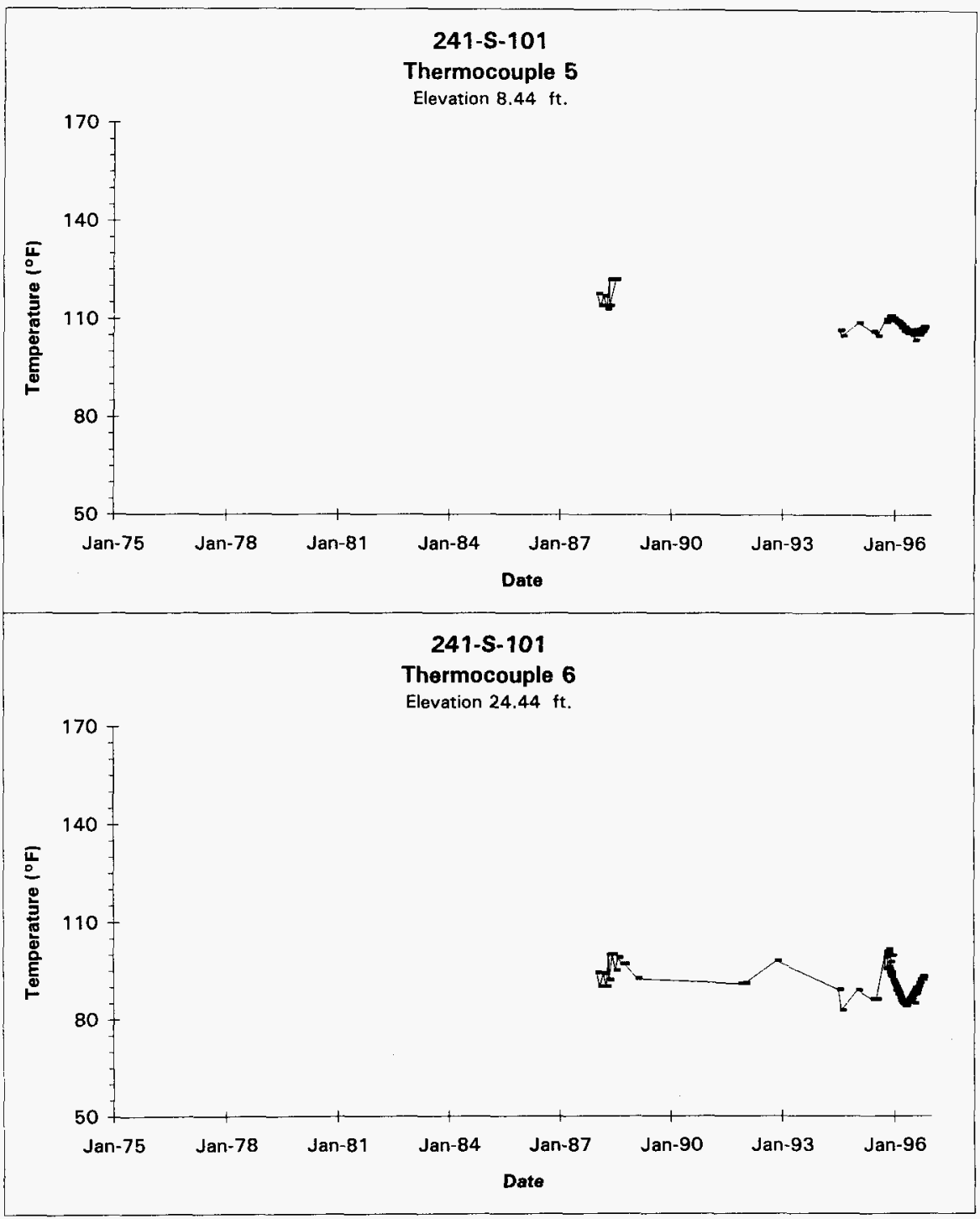

Data obtained from LMHC Surveillance Analysis Computer System (SACS), Oct 1, 1996.

D-3 


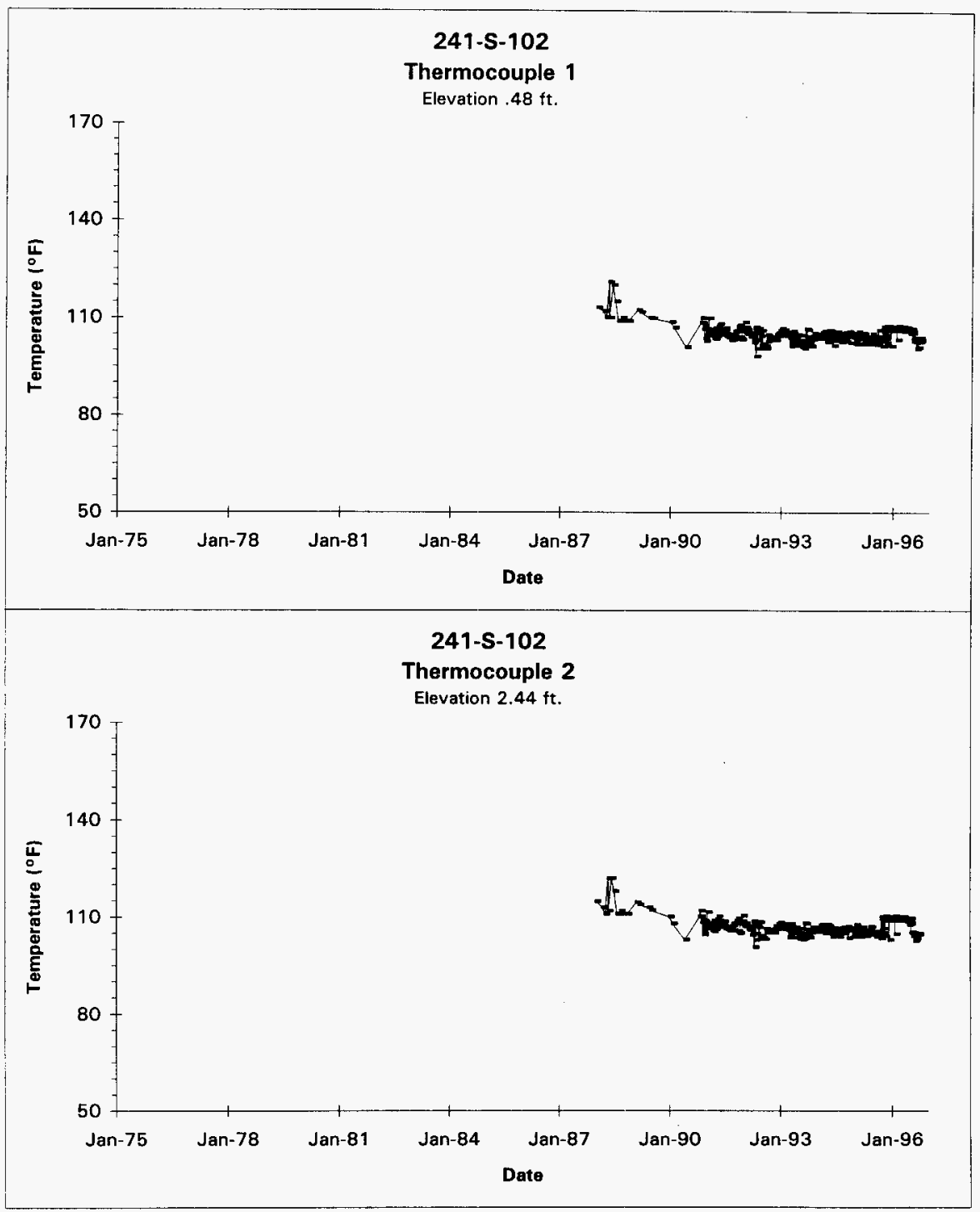

Data obtained from LMHC Surveillance Analysis Computer System (SACS), Oct 1, 1996.

D-4 


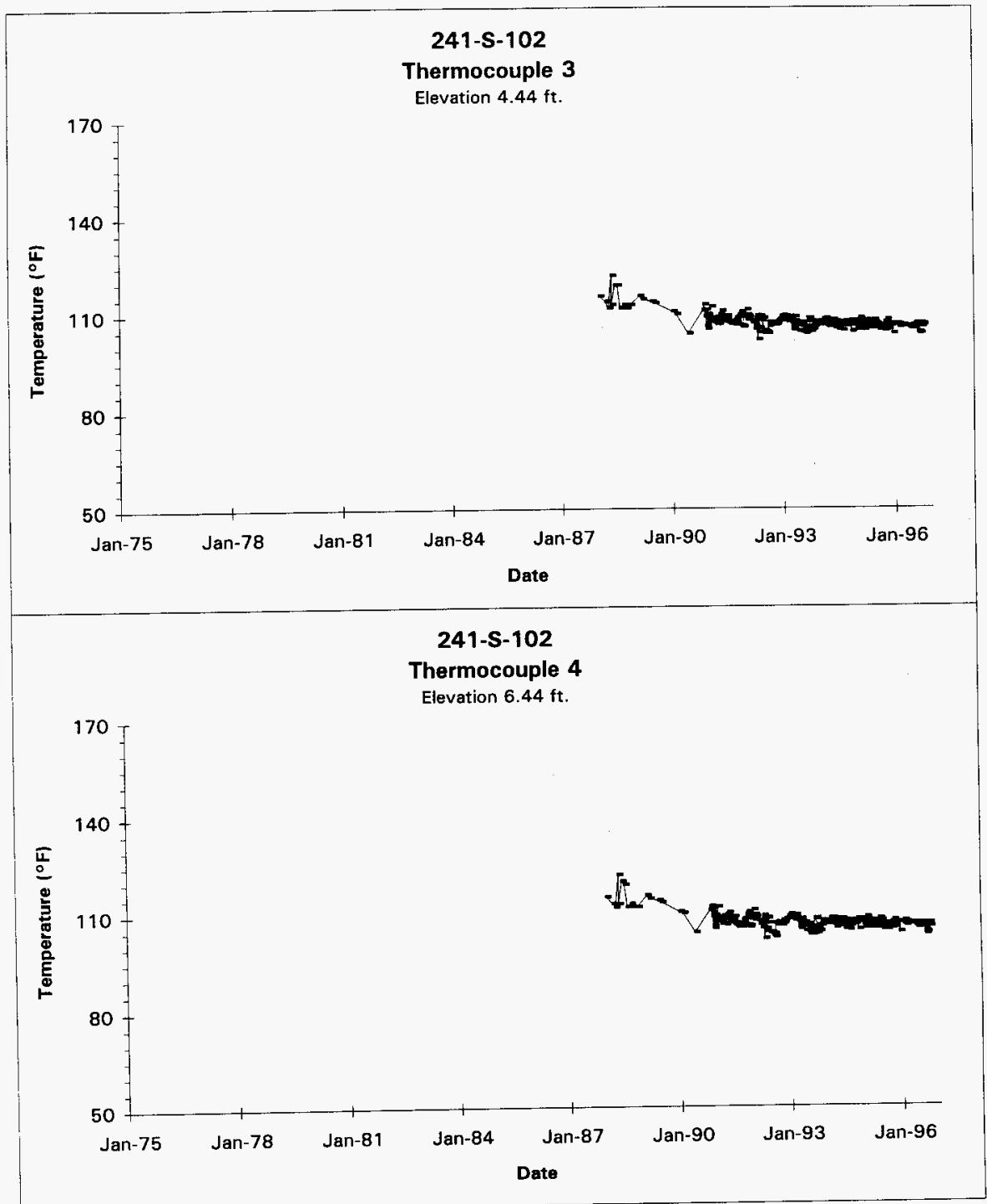

Data obtained from LMHC Surveillance Analysis Computer System (SACS), Oct 1, 1996.

D-5 


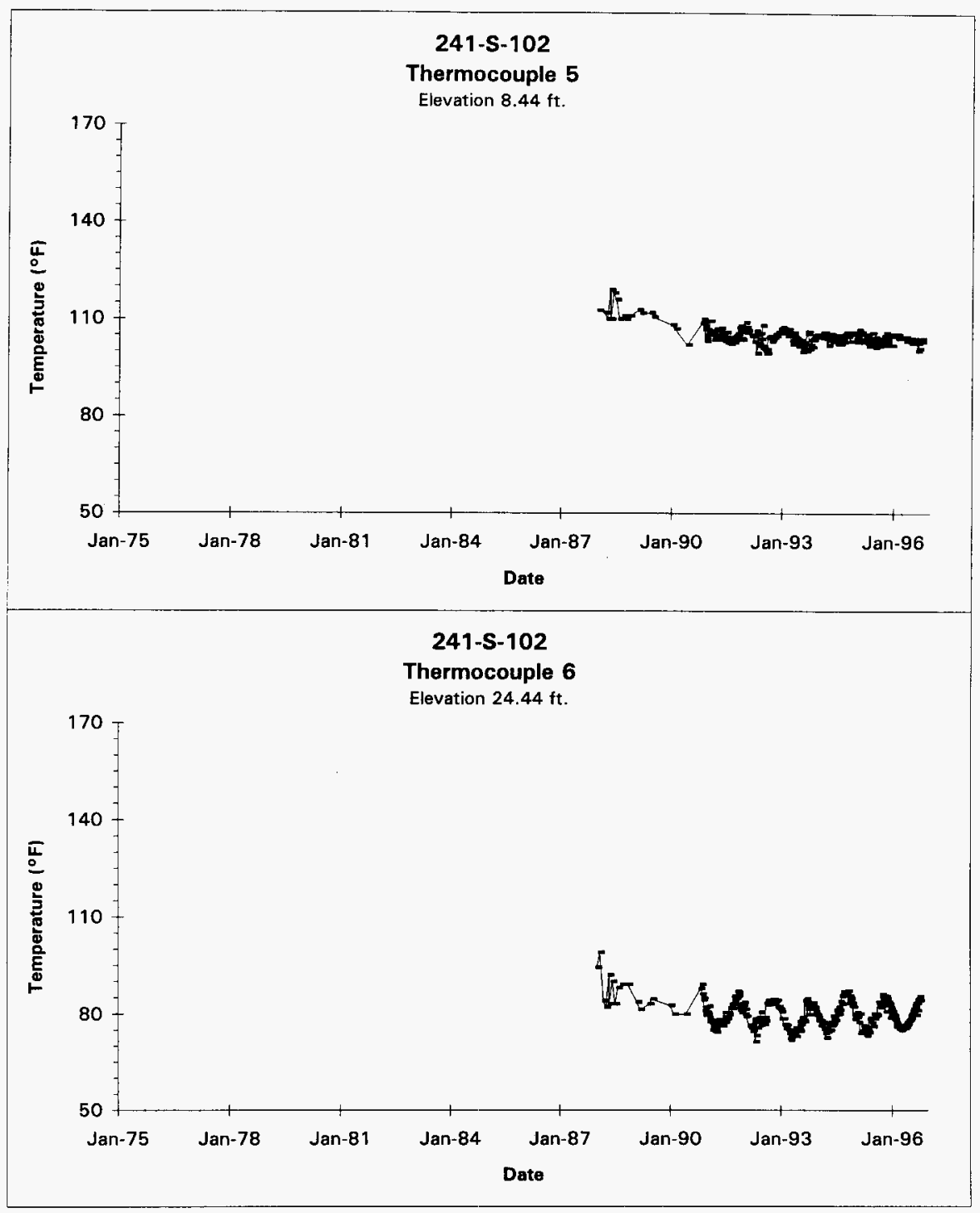

Data obtained from LMHC Surveillance Analysis Computer System (SACS), Oct 1, 1996.

D-6 


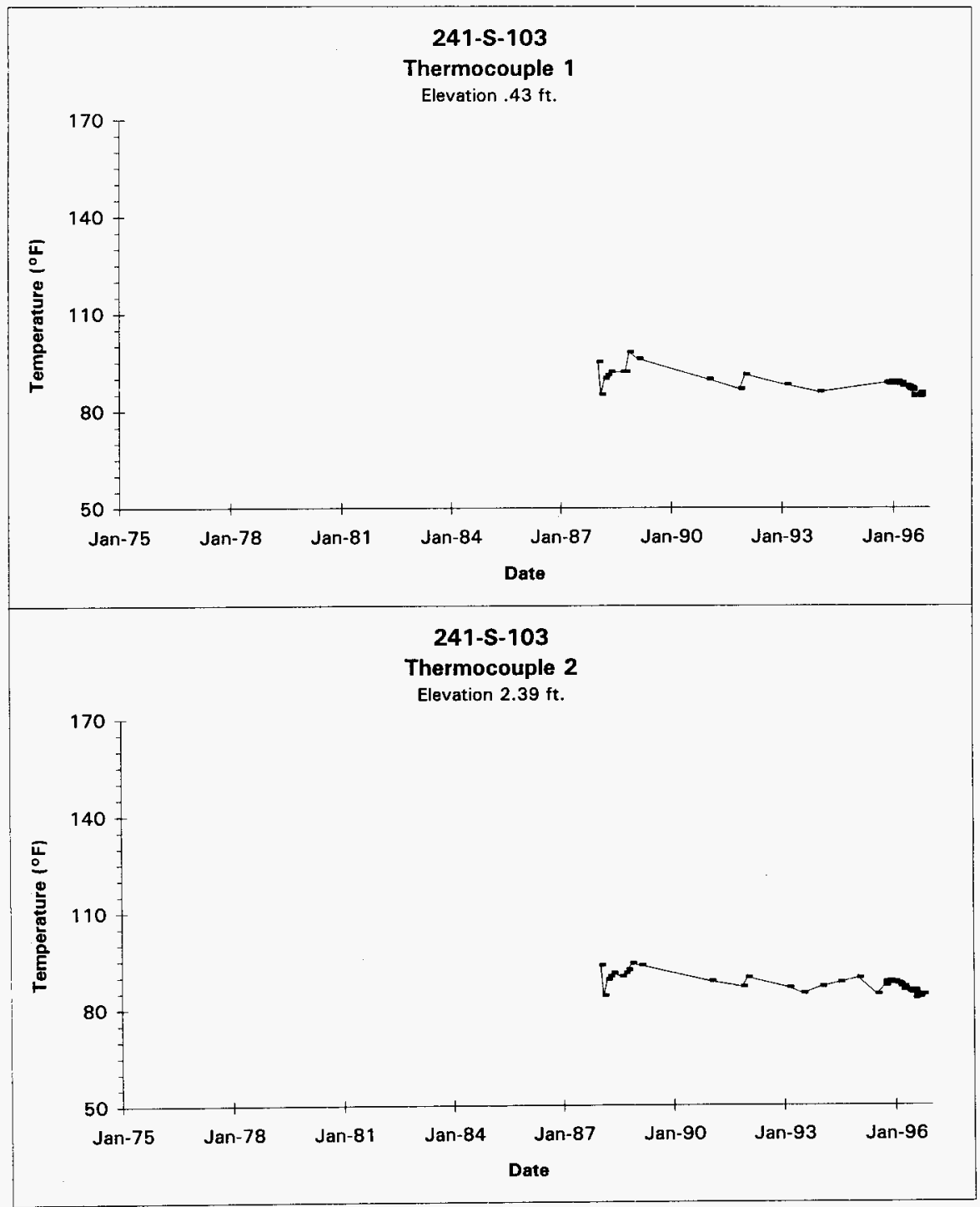

Data obtained from LMHC Surveillance Analysis Computer System (SACS), Oct 1, 1996.

D-7 


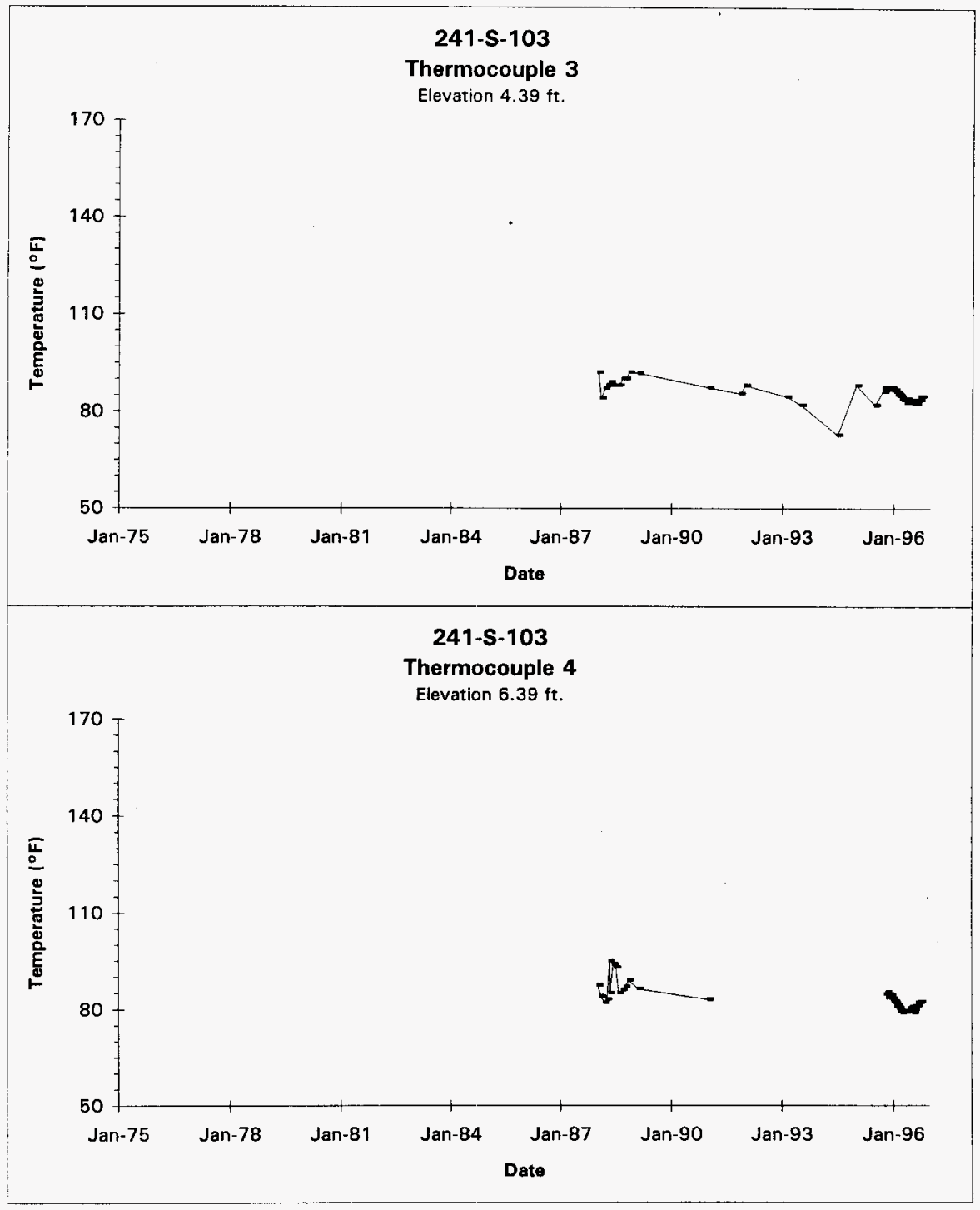

Data obtained from LMHC Surveillance Analysis Computer System (SACS), Oct 1, 1996. 


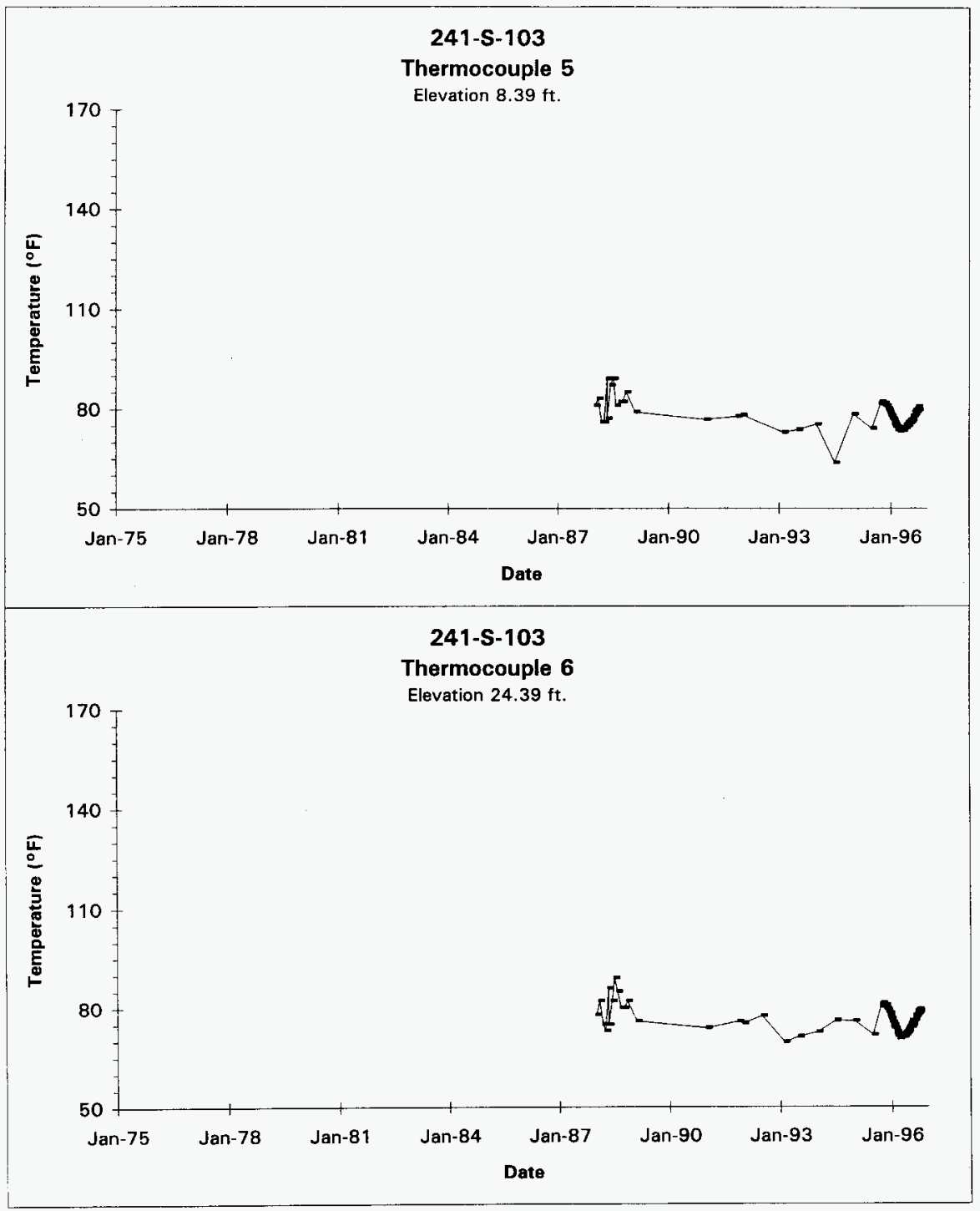

Data obtained from LMHC Surveillance Analysis Computer System (SACS), Oct 1, 1996.

$$
\text { D-9 }
$$




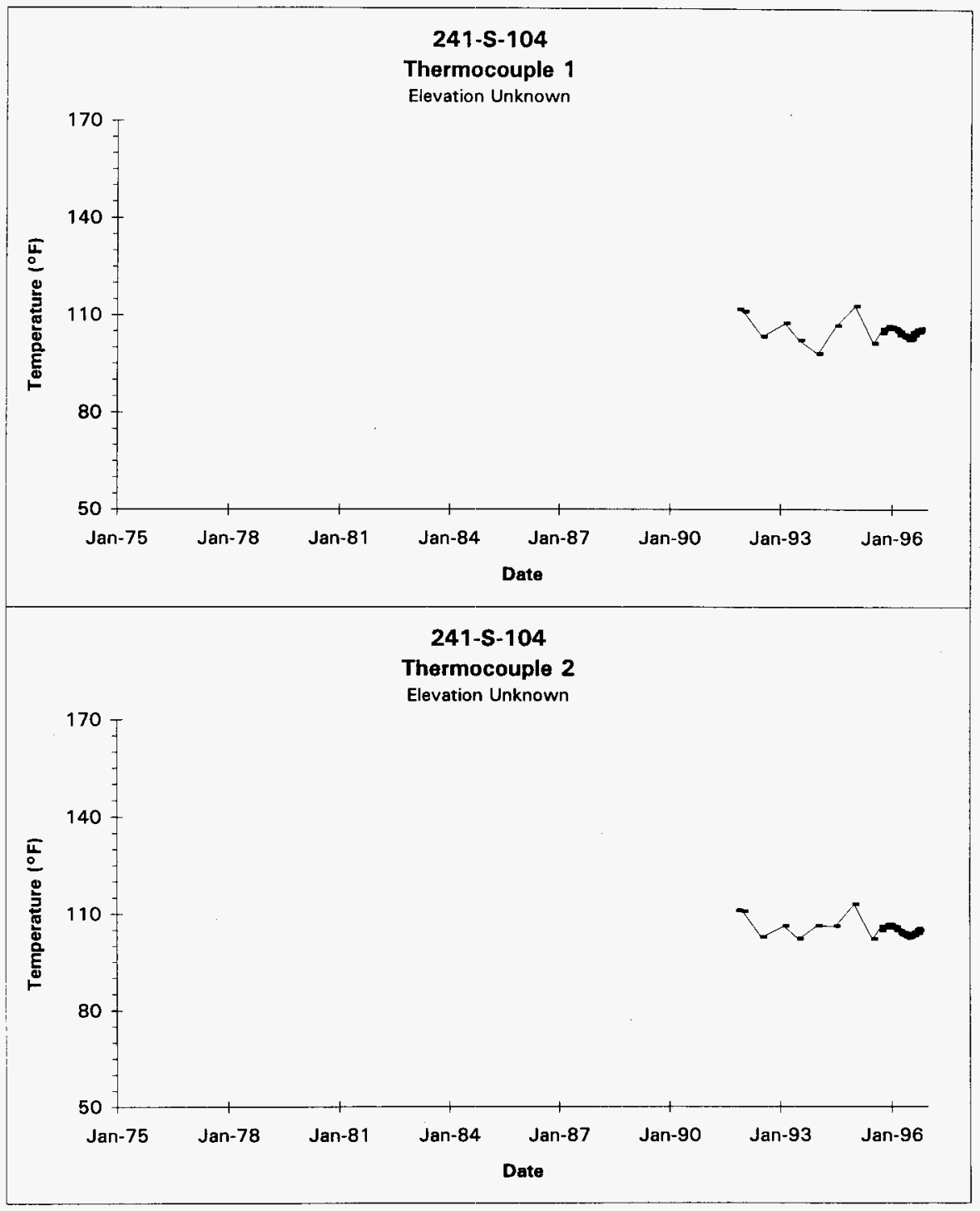

Data obtained from LMHC Surveillance Analysis Computer System (SACS), Oct 1, 1996.

D-10 


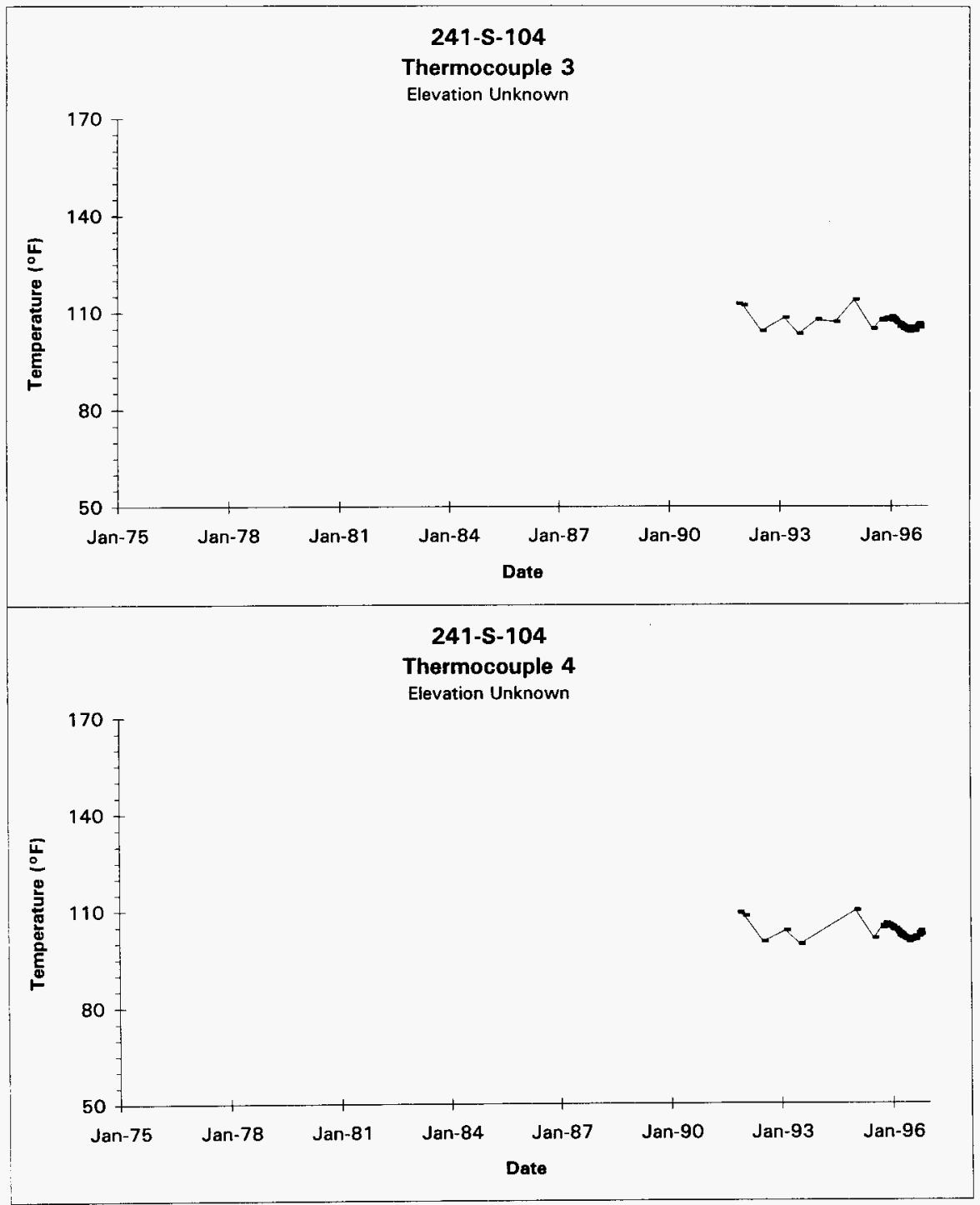

Data obtained from LMHC Surveillance Analysis Computer System (SACS), Oct 1, 1996.

D-11 


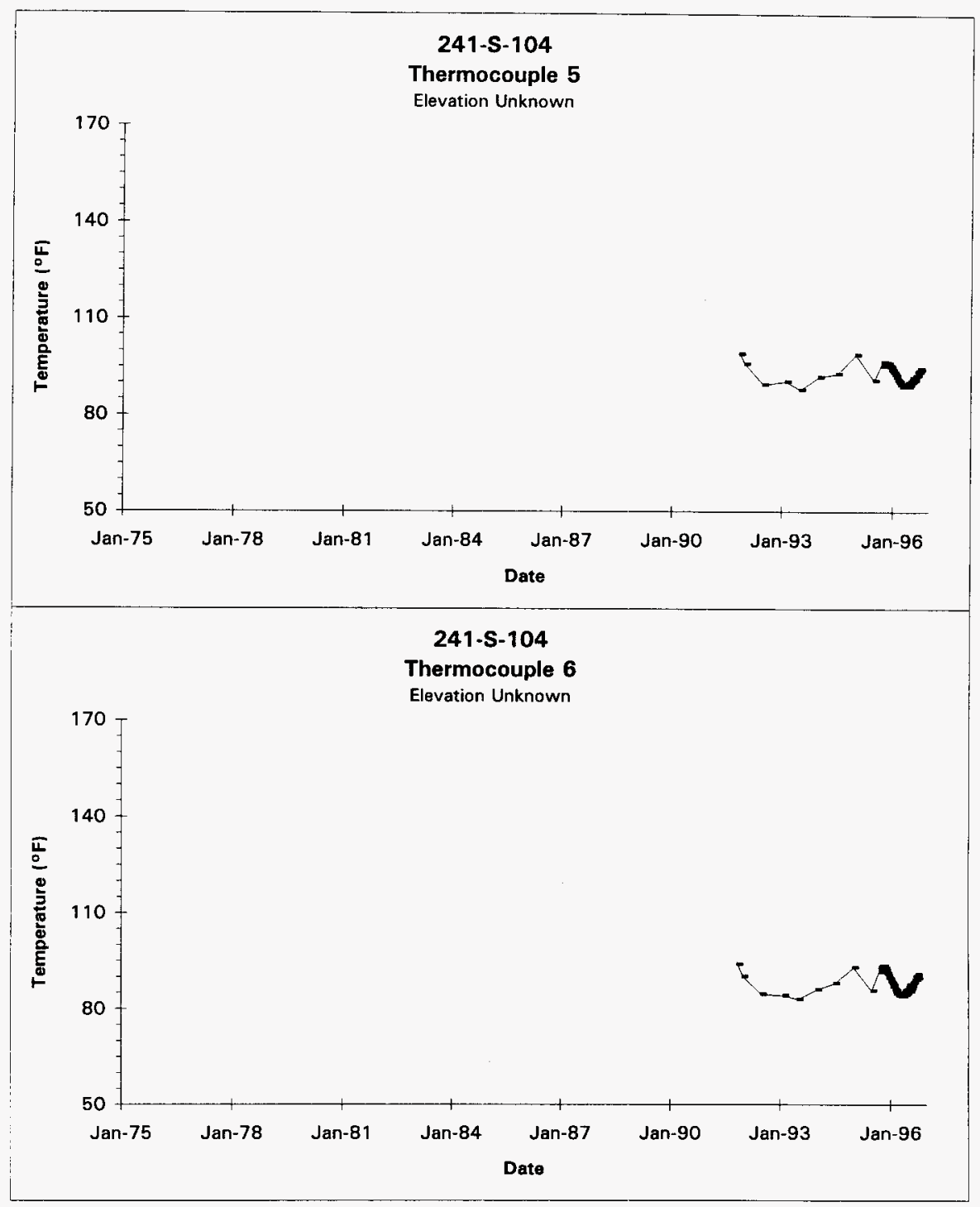

Data obtained from LMHC Surveillance Analysis Computer System (SACS), Oct 1, 1996.

D-12 
HNF-SD-WM-ER-323, Rev. 1

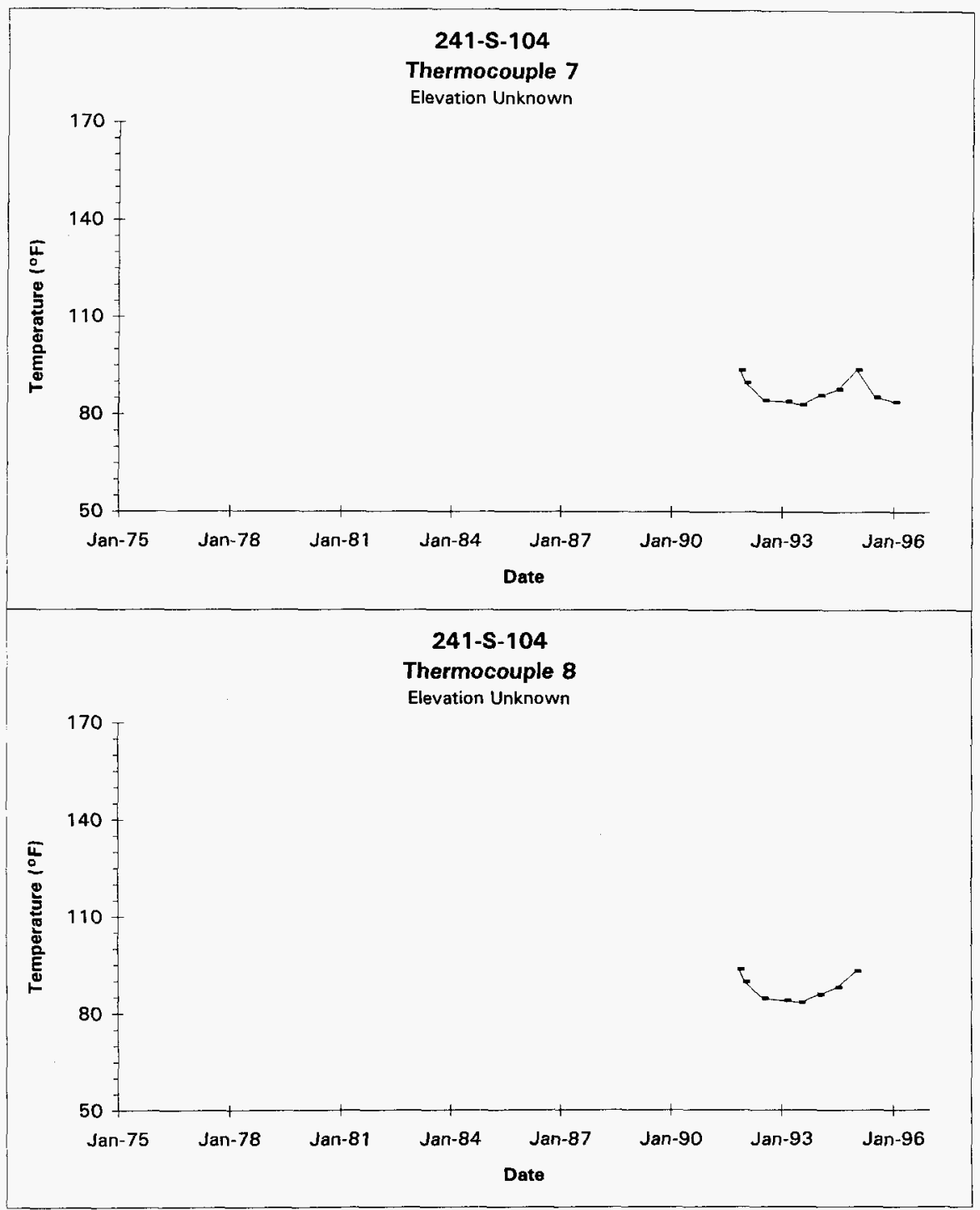

Data obtained from LMHC Surveillance Analysis Computer System (SACS), Oct 1, 1996.

D-13 


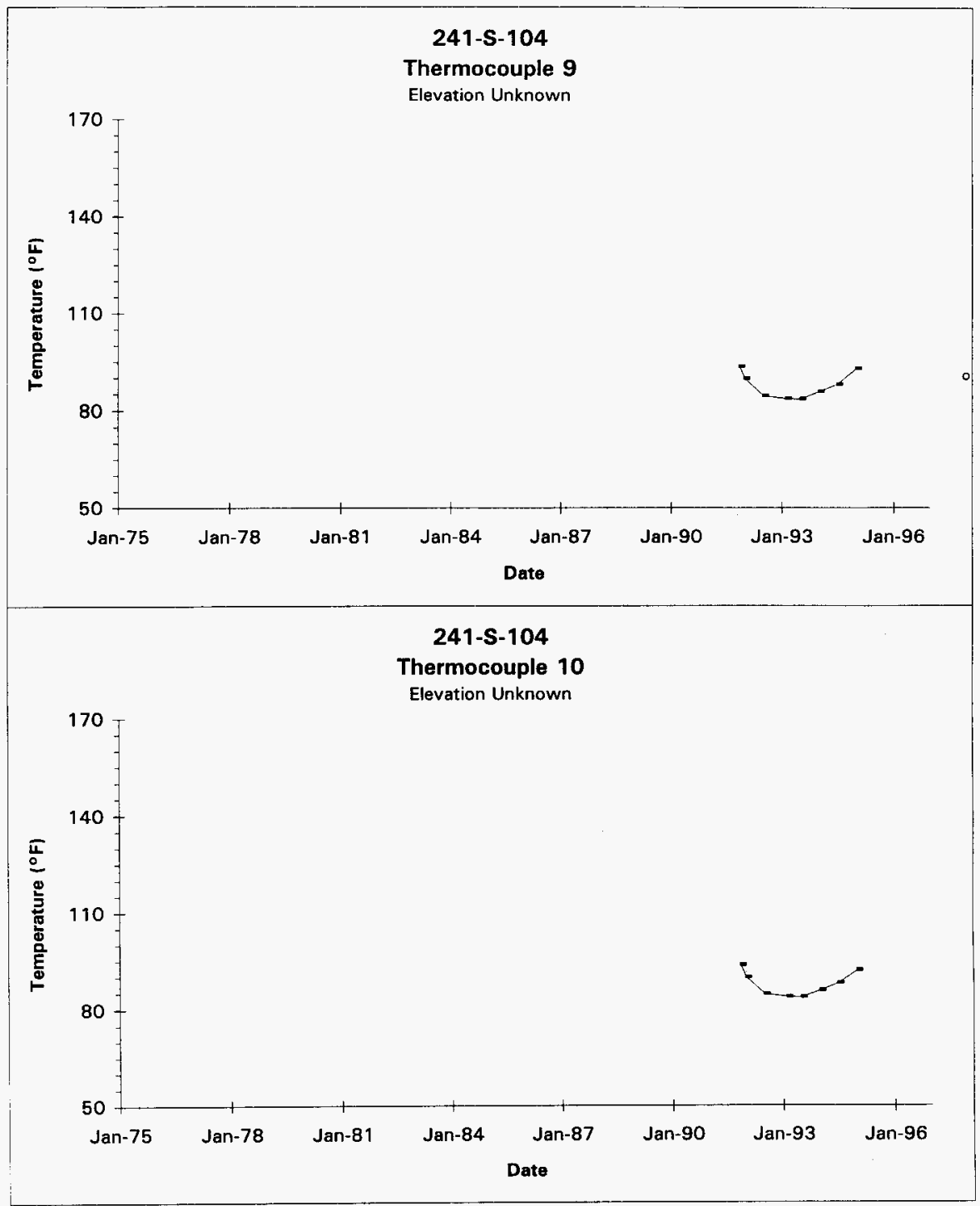

Data obtained from LMHC Surveillance Analysis Computer System (SACS), Oct 1, 1996.

$$
\text { D-14 }
$$




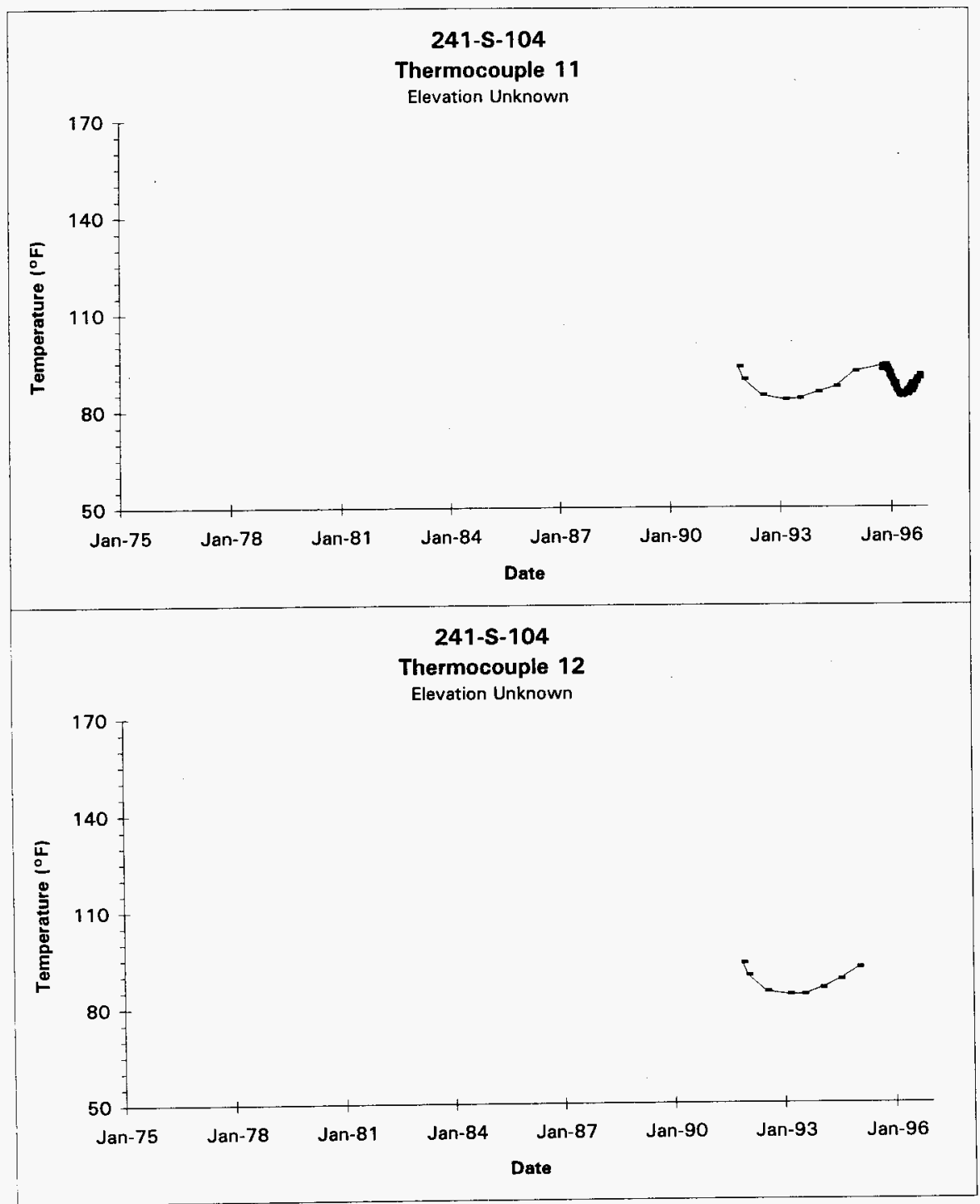

Data obtained from LMHC Surveillance Analysis Computer System (SACS), Oct 1, 1996.

D-15 


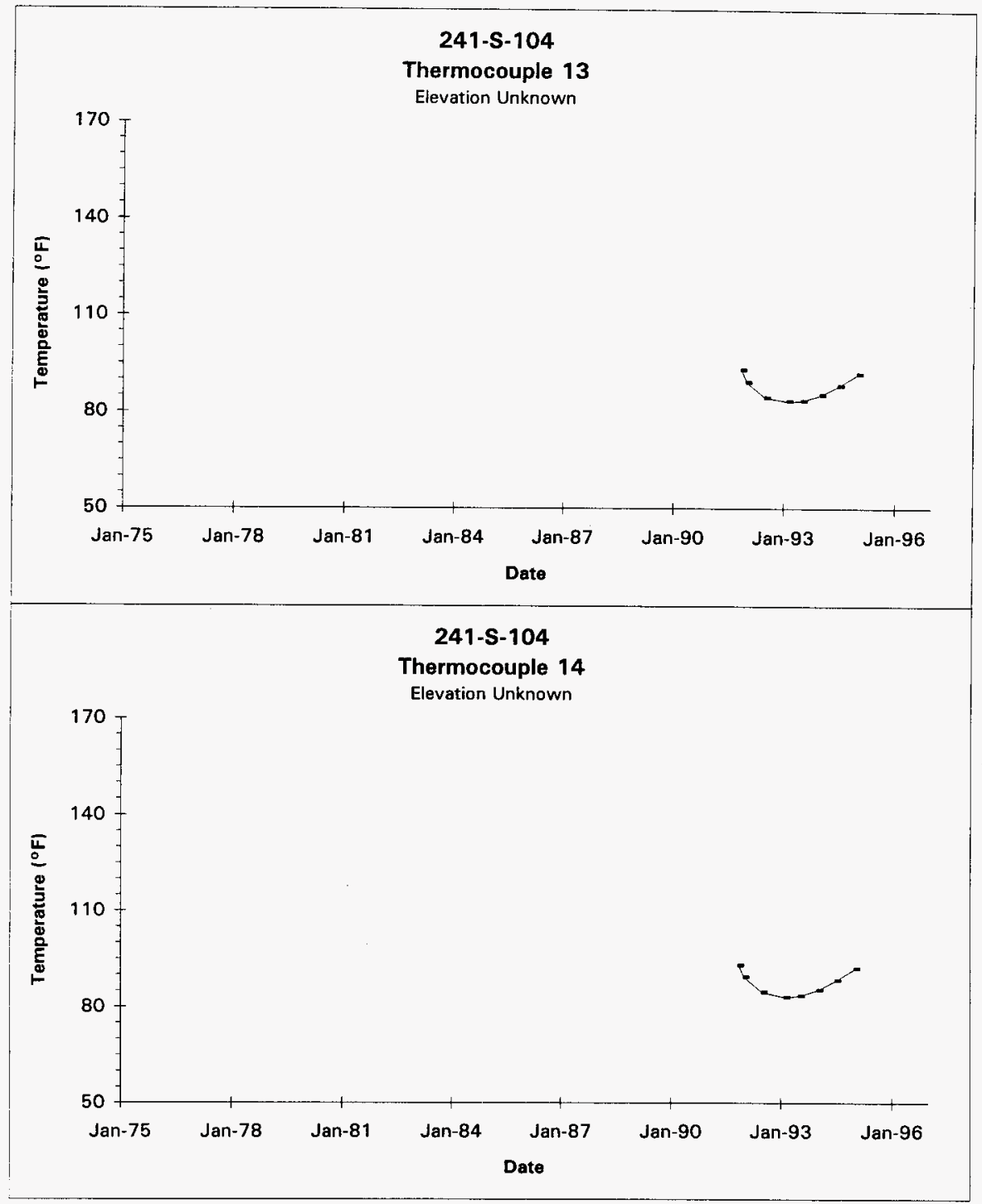

Data obtained from LMHC Surveillance Analysis Computer System (SACS), Oct 1, 1996.

$$
\text { D-16 }
$$




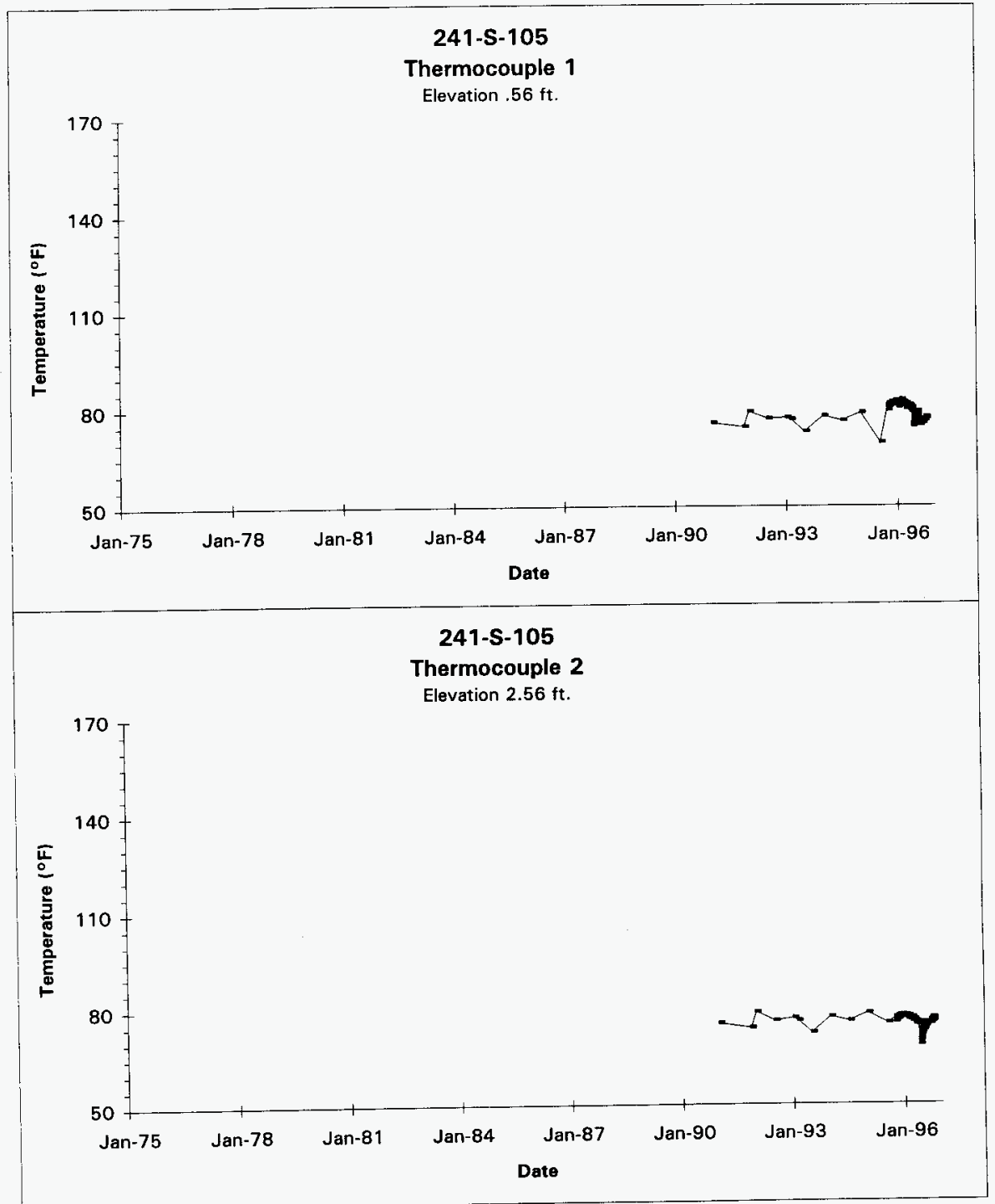

Data obtained from LMHC Surveillance Analysis Computer System (SACS), Oct 1, 1996.

D-17 


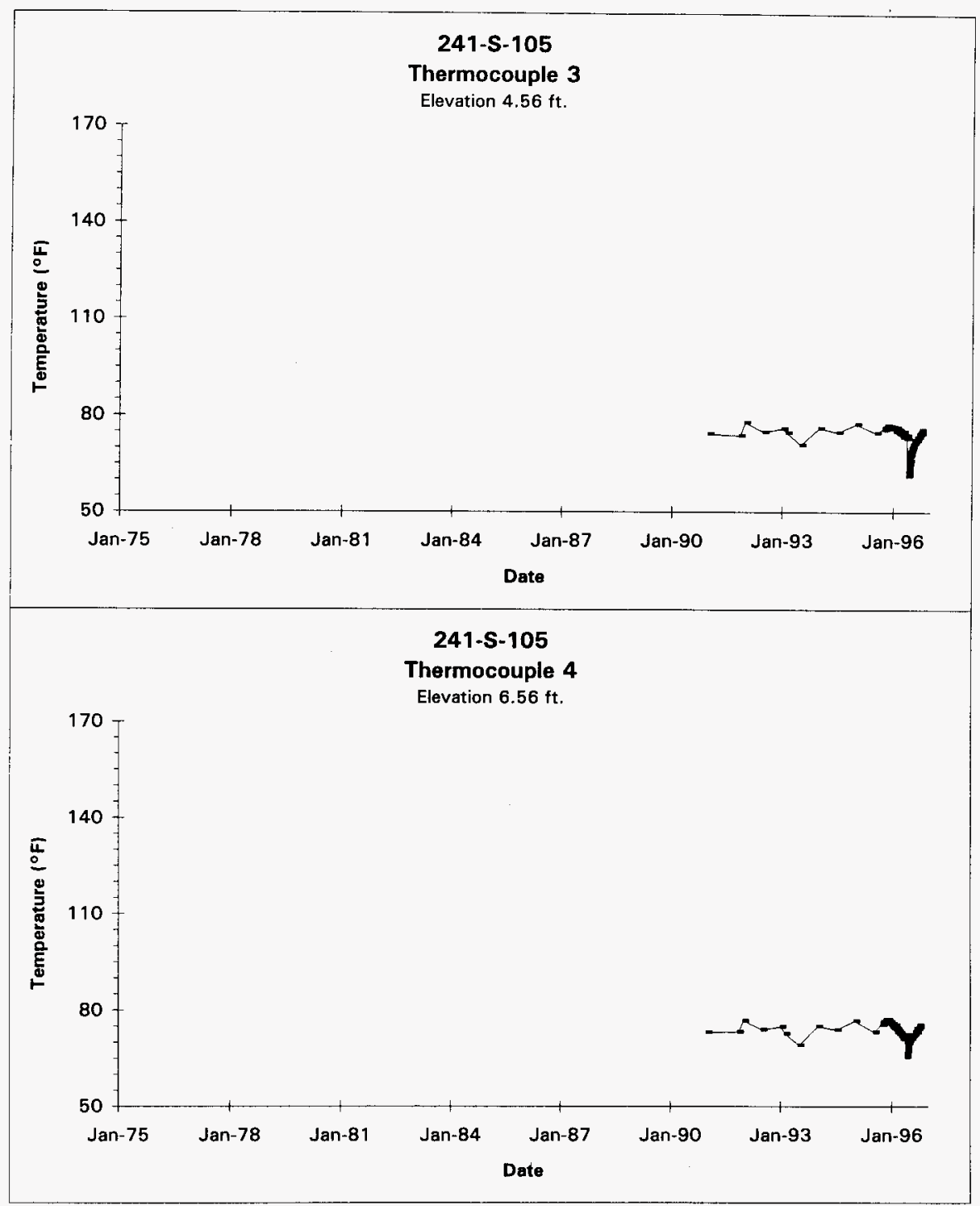

Data obtained from LMHC Surveillance Analysis Computer System (SACS), Oct 1, 1996.

D-18 


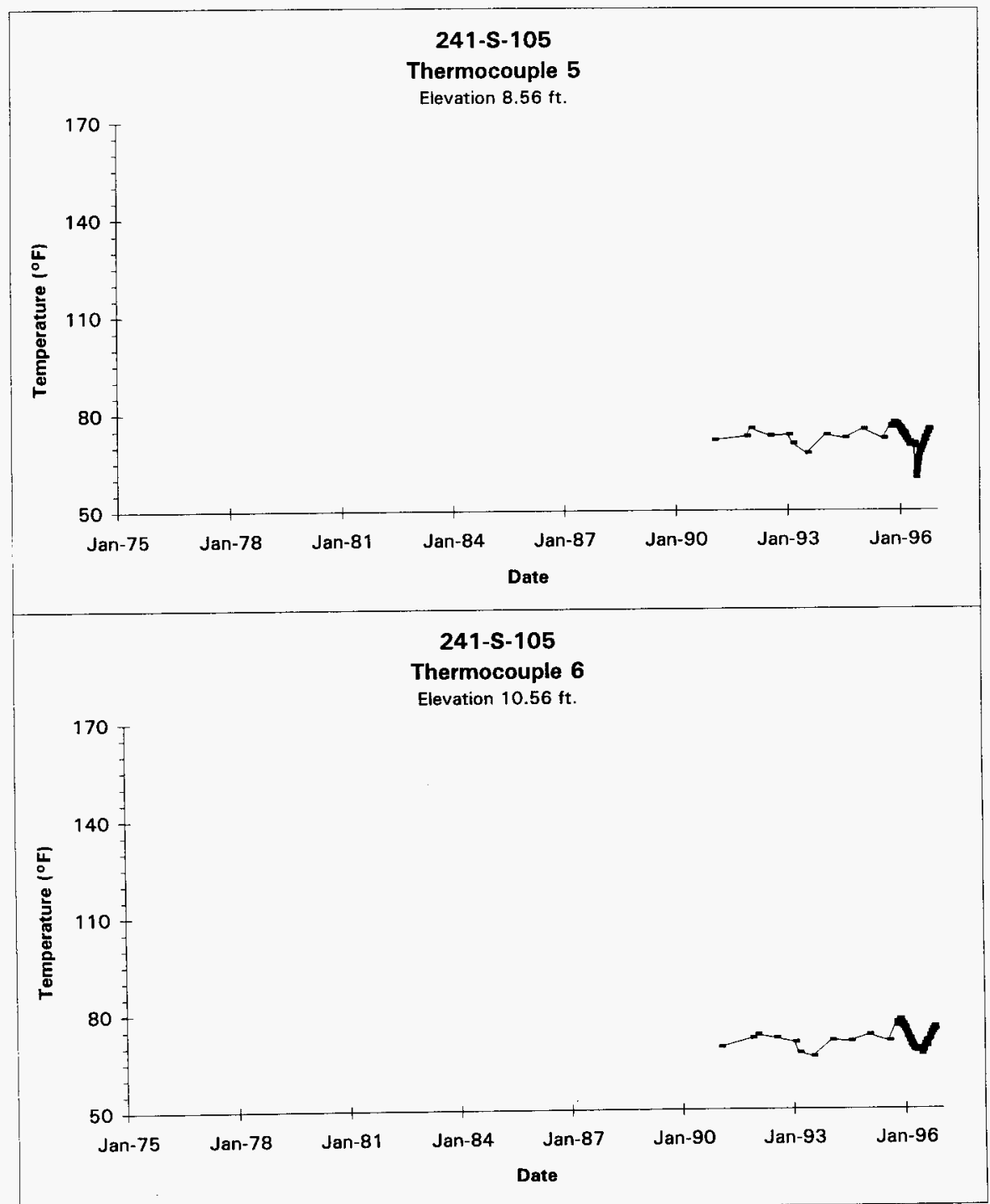

Data obtained from LMHC Surveillance Analysis Computer System (SACS), Oct 1, 1996. D-19 


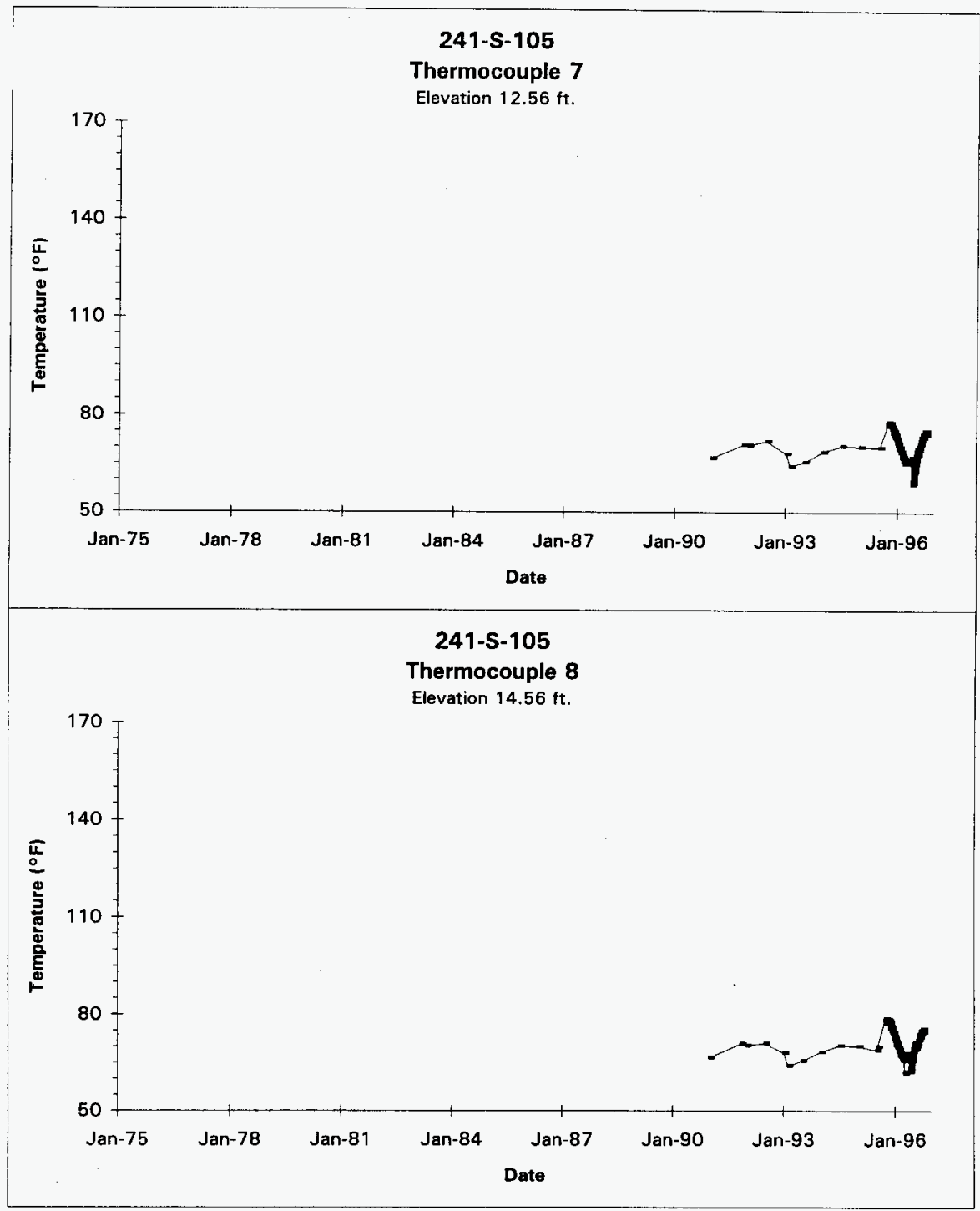

Data obtained from LMHC Surveillance Analysis Computer System (SACS), Oct 1, 1996. 
HNF-SD-WM-ER-323, Rev. 1

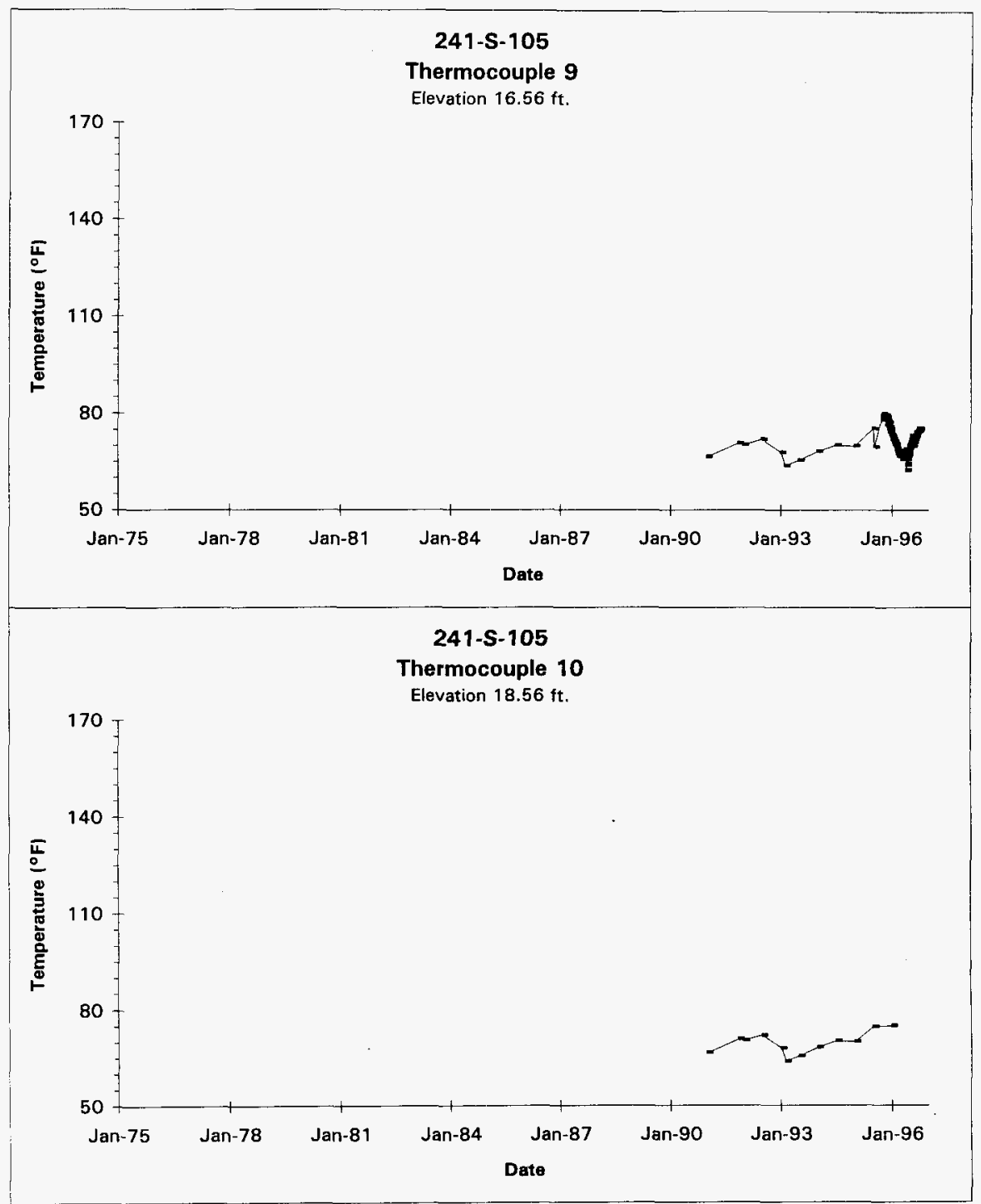

Data obtained from LMHC Surveillance Analysis Computer System (SACS), Oct 1, 1996.

D-21 


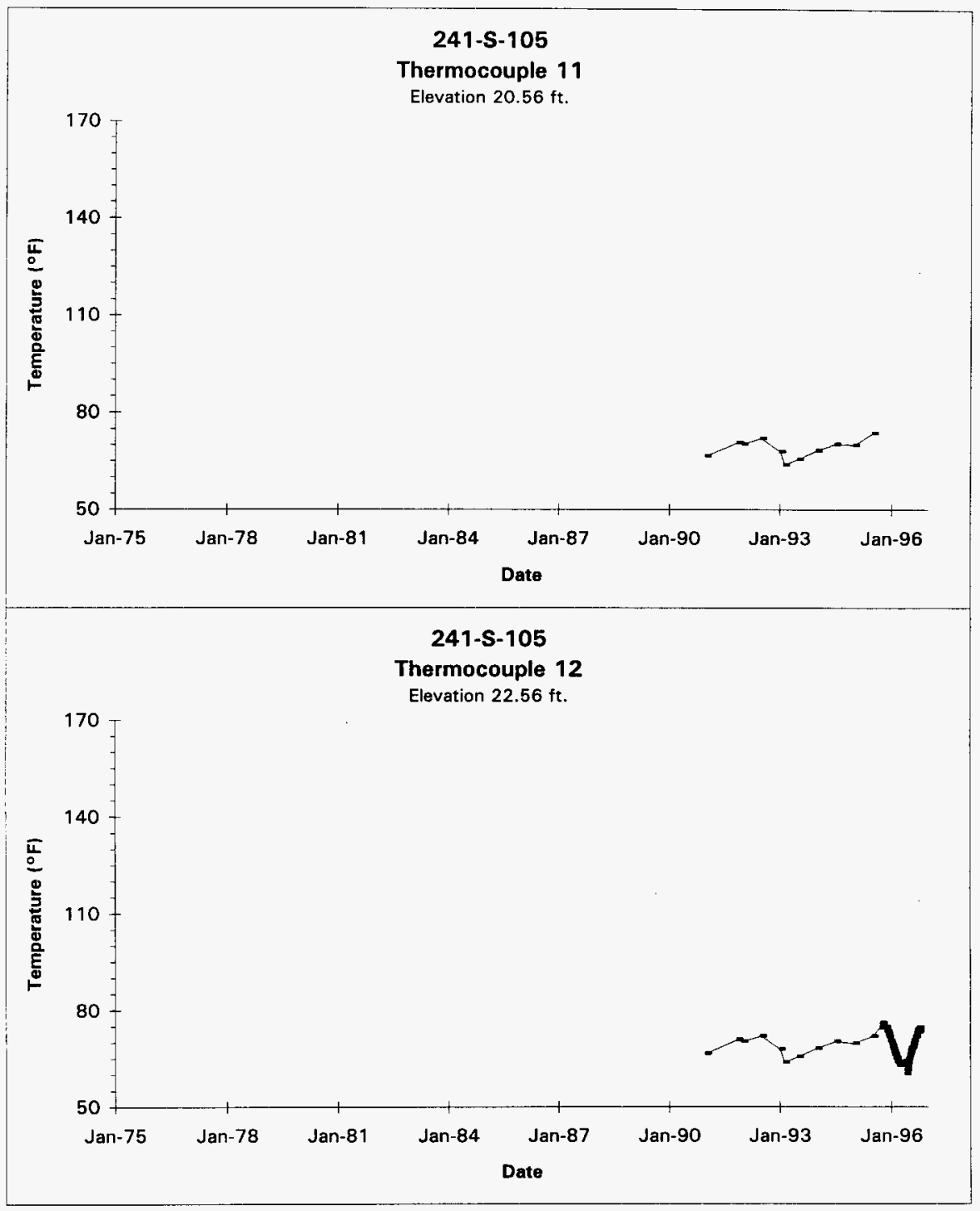

Data obtained from LMHC Surveillance Analysis Computer System (SACS), Oct 1, 1996.

D-22 


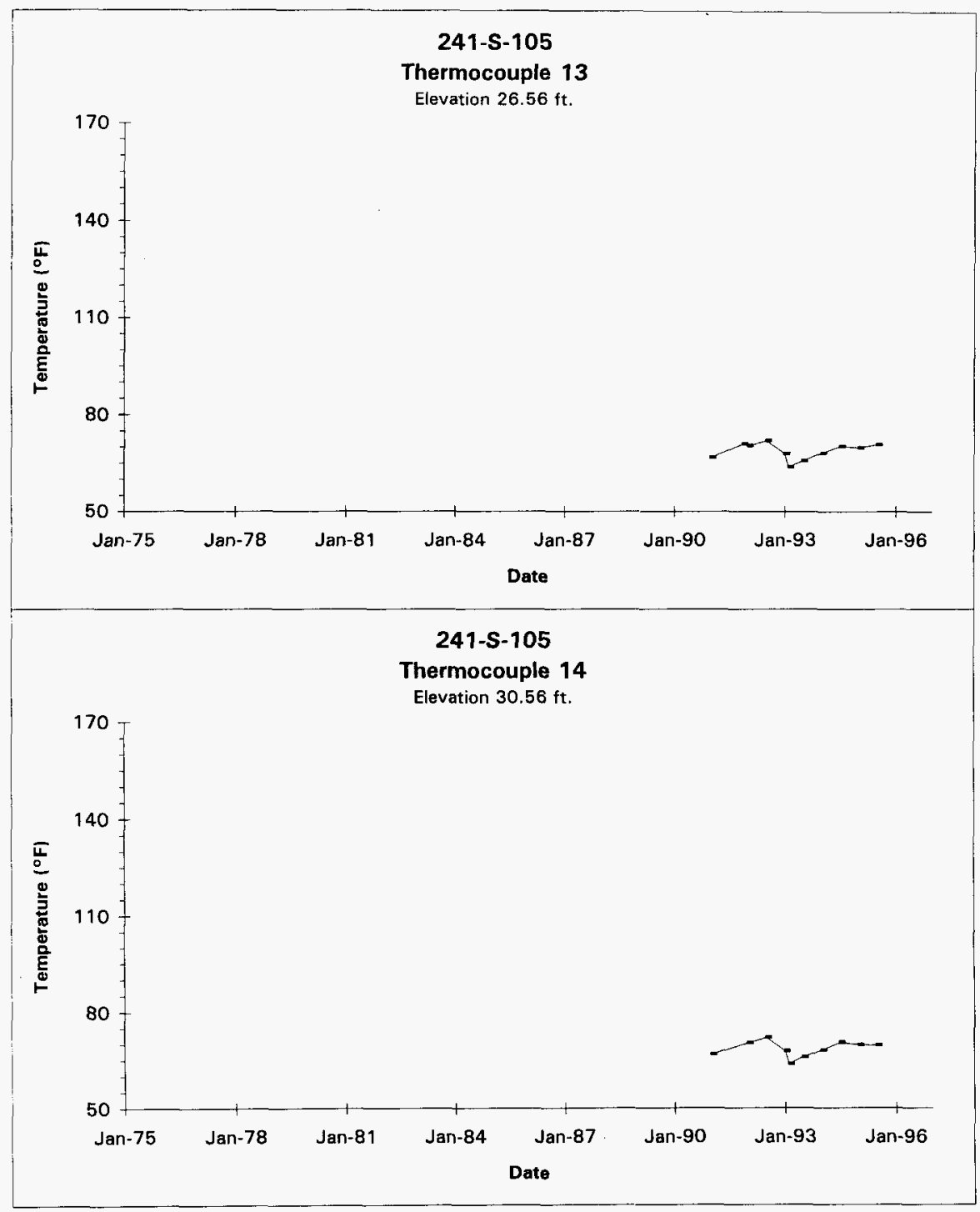

Data obtained from LMHC Surveillance Analysis Computer System (SACS), Oct 1, 1996. 


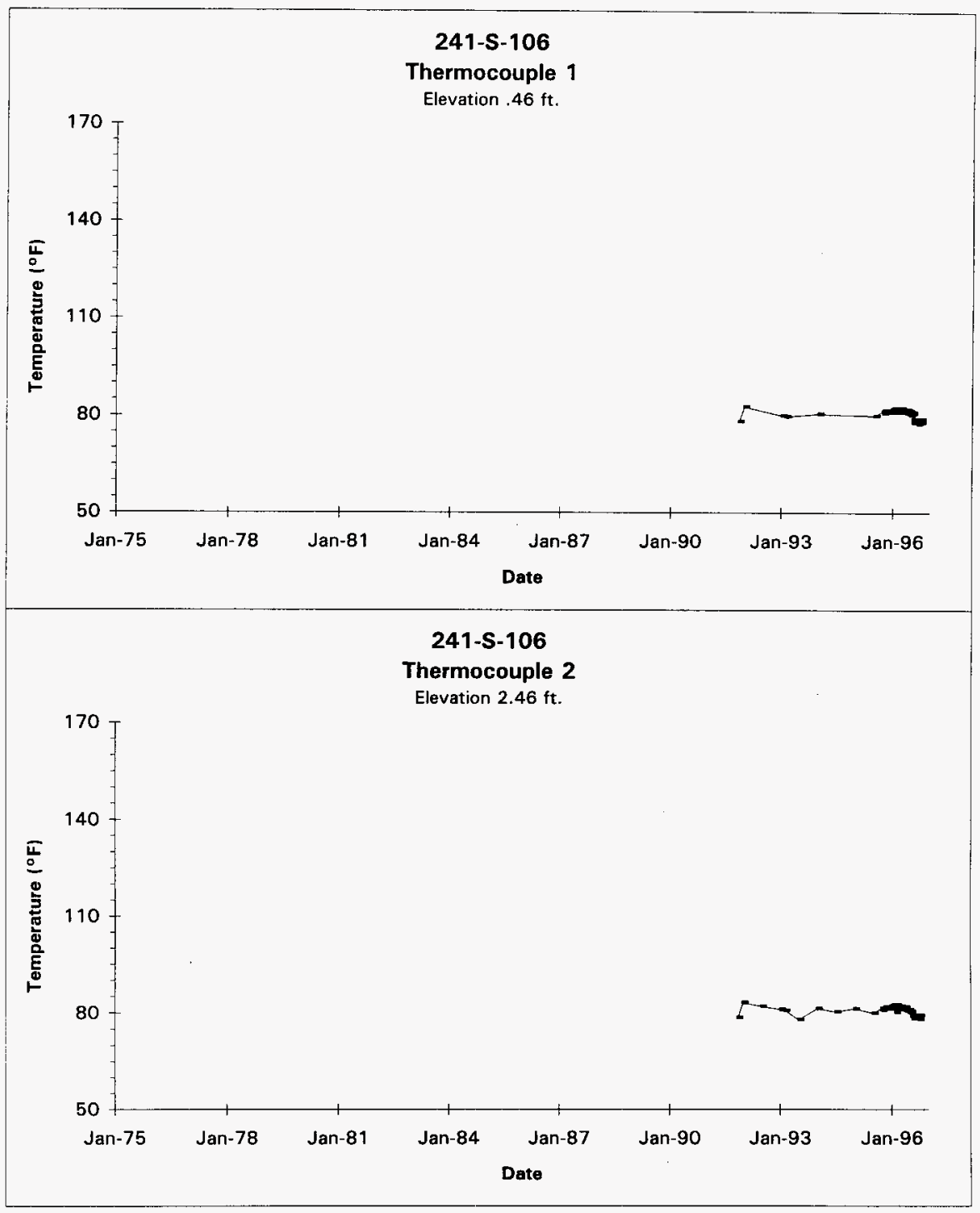

Data obtained from LMHC Surveillance Analysis Computer System (SACS), Oct 1, 1996.

$$
\text { D-24 }
$$




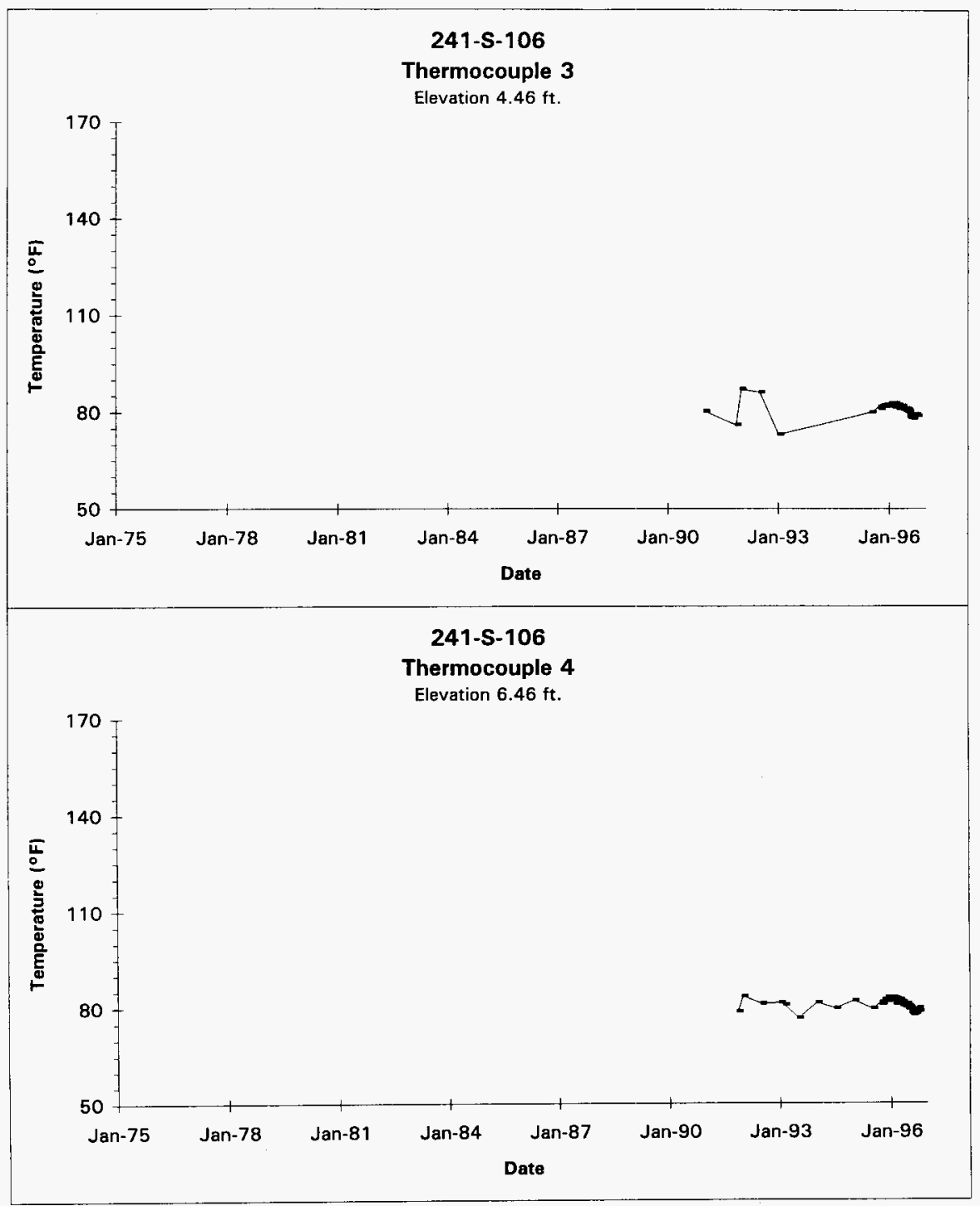

Data obtained from LMHC Surveillance Analysis Computer System (SACS), Oct 1, 1996.

D-25 


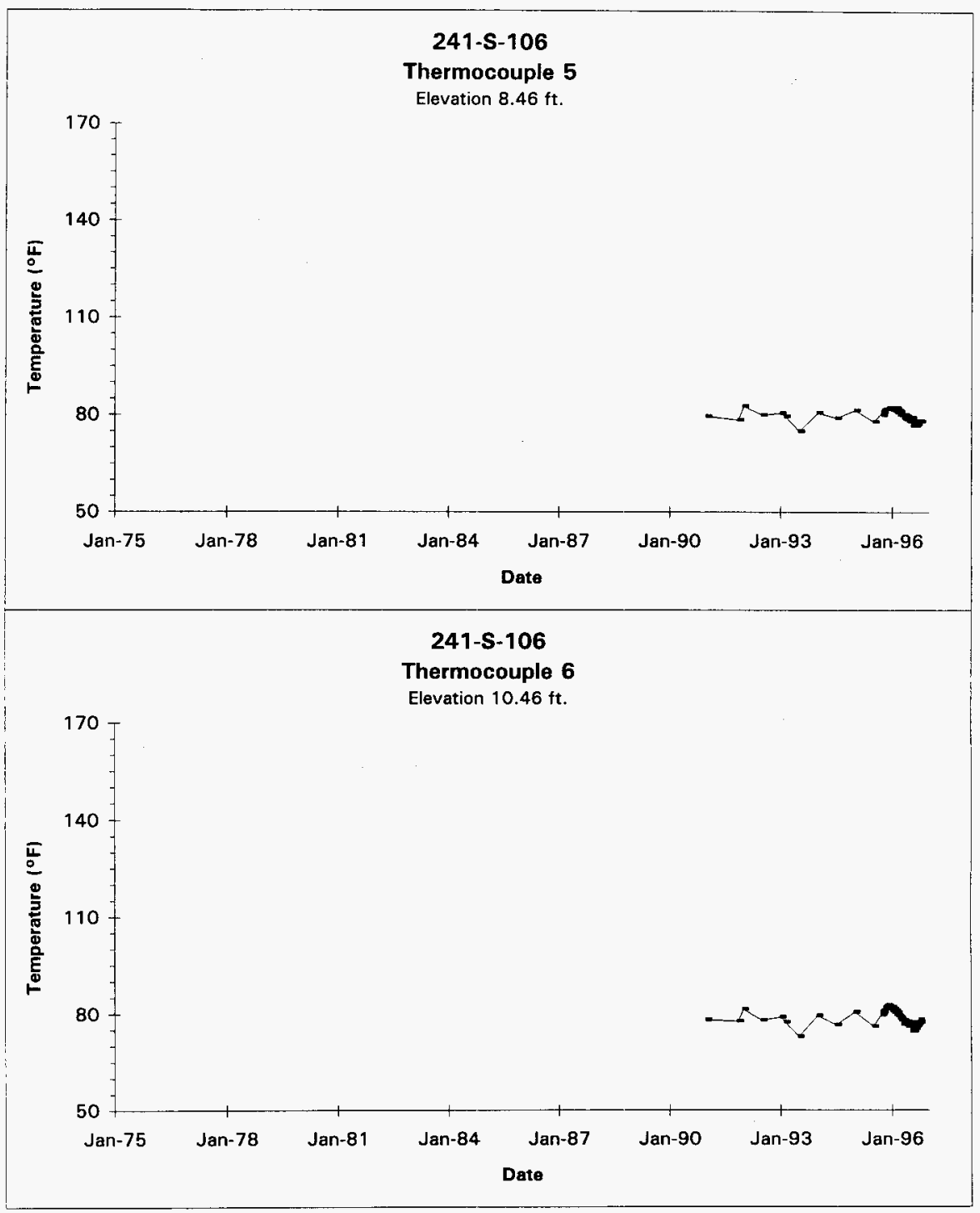

Data obtained from LMHC Surveillance Analysis Computer System (SACS), Oct 1, 1996.

D-26 


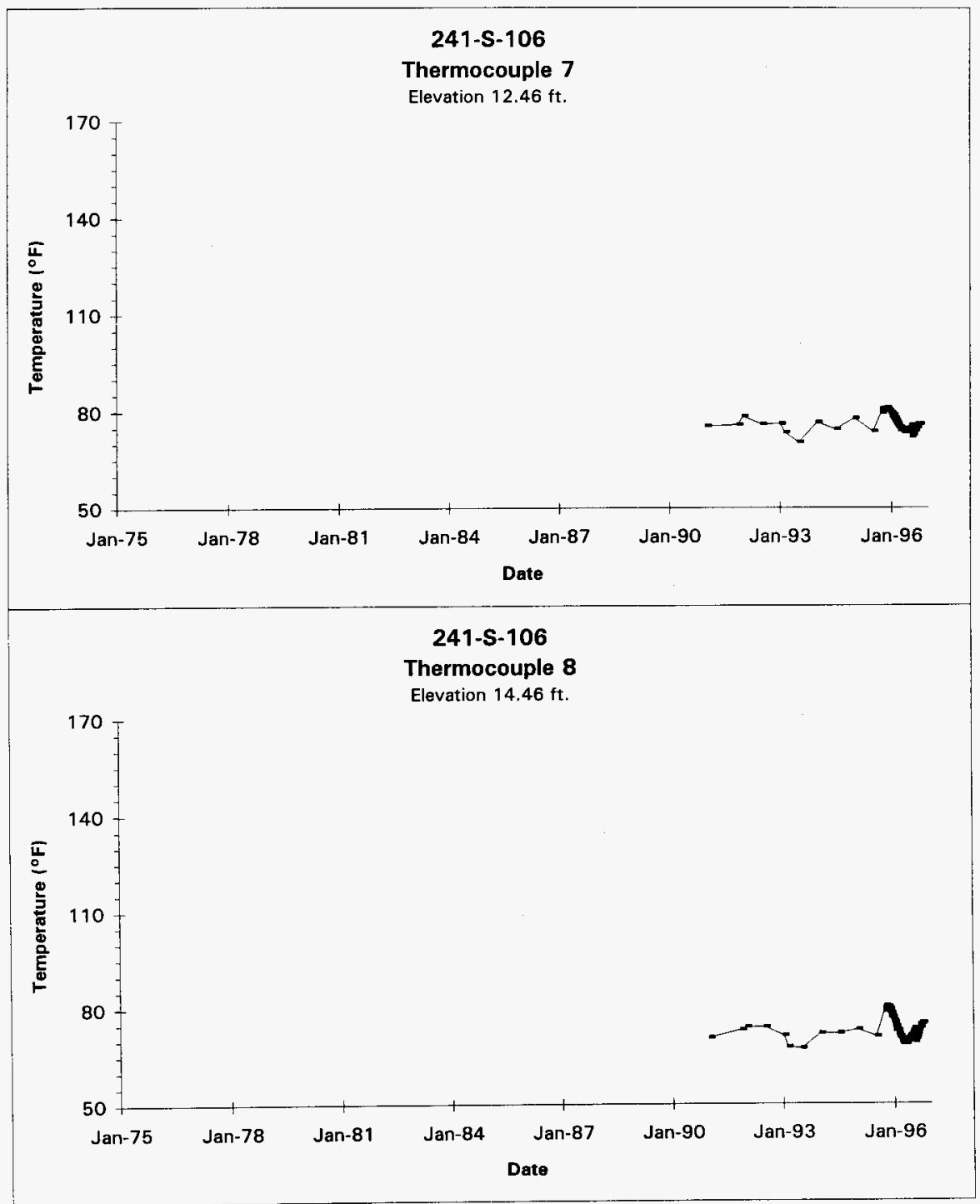

Data obtained from LMHC Surveillance Analysis Computer System (SACS), Oct 1, 1996. 


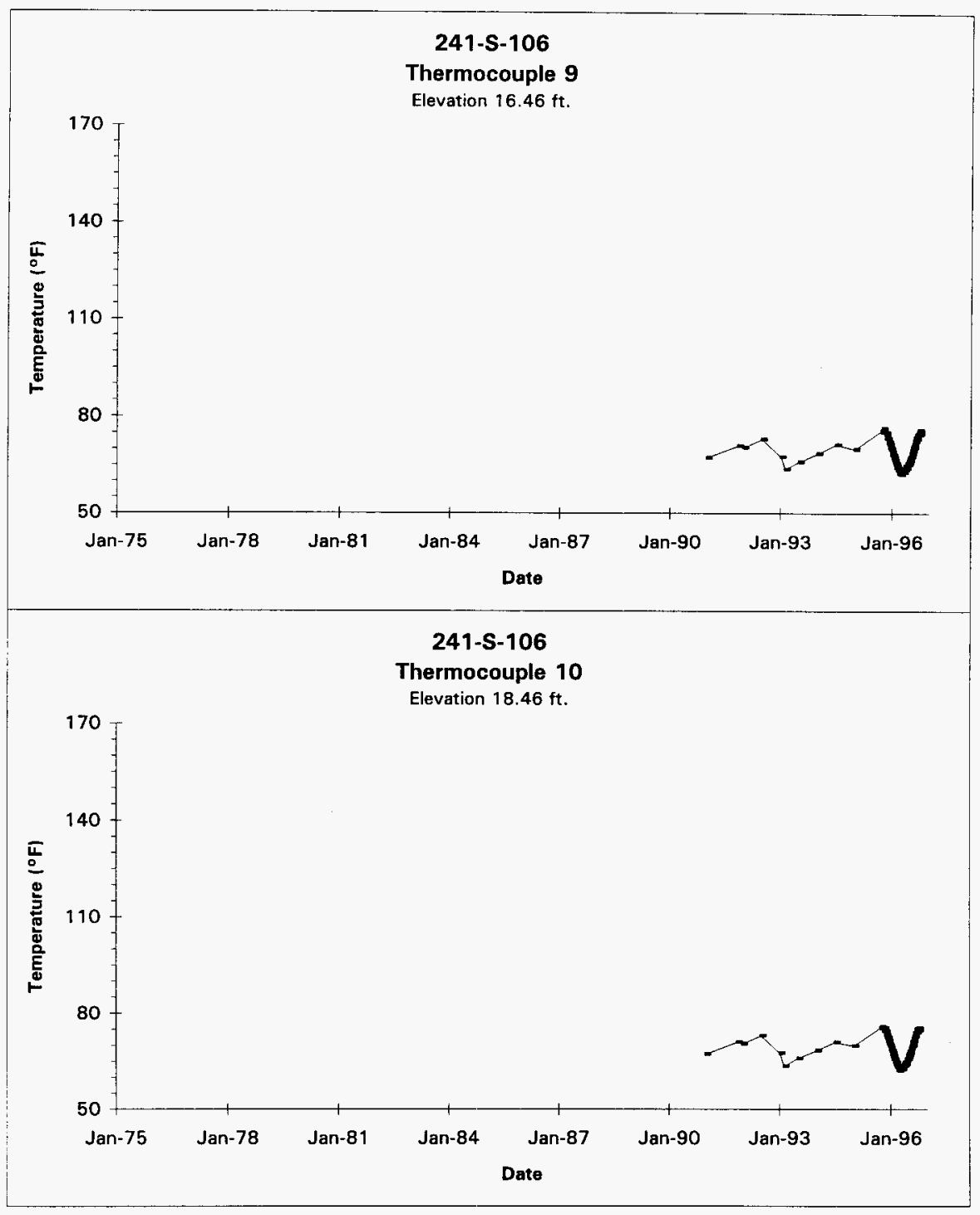

Data obtained from LMHC Surveillance Analysis Computer System (SACS), Oct 1, 1996.

D-28 


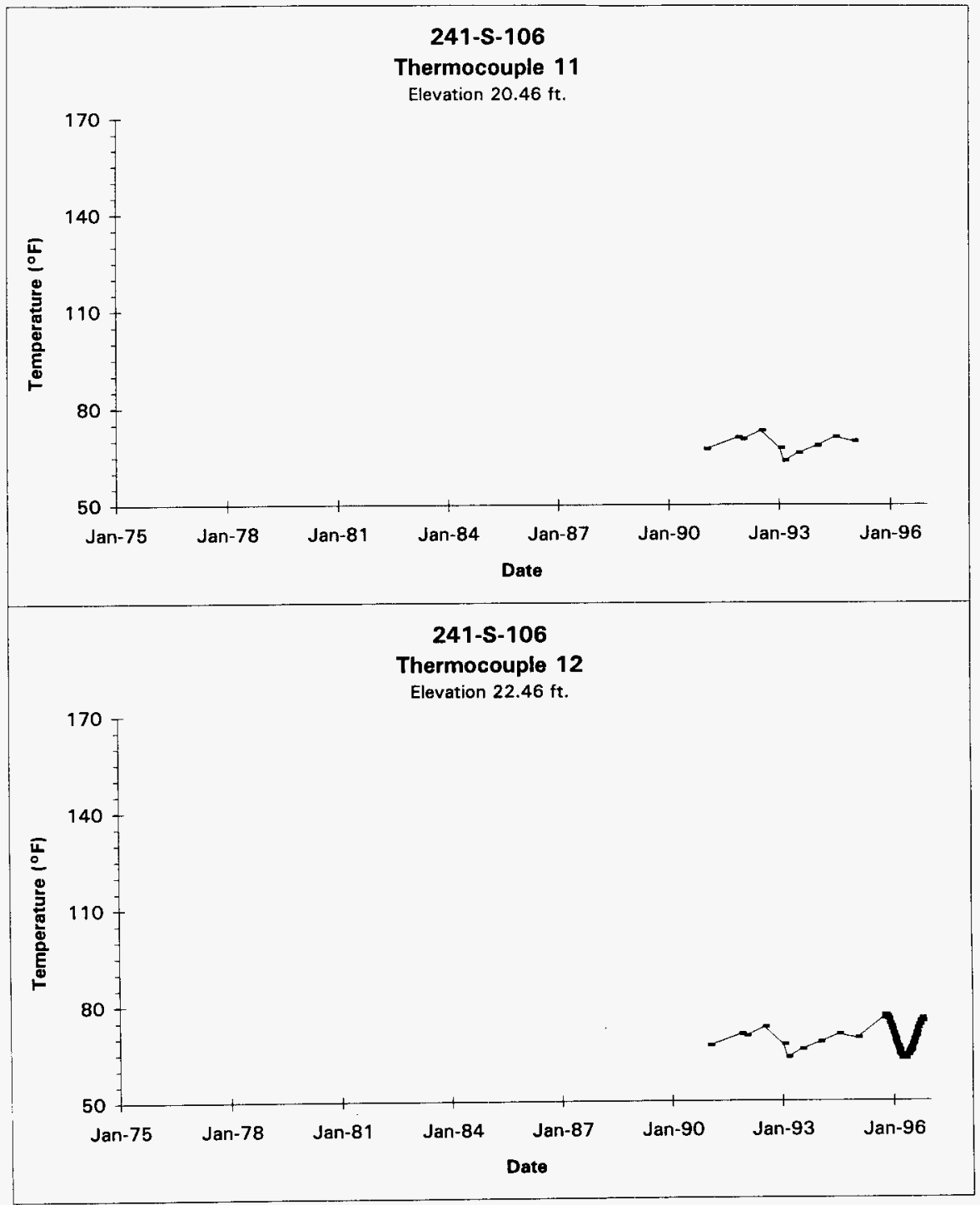

Data obtained from LMHC Surveillance Analysis Computer System (SACS), Oct 1, 1996.

$$
\text { D-29 }
$$




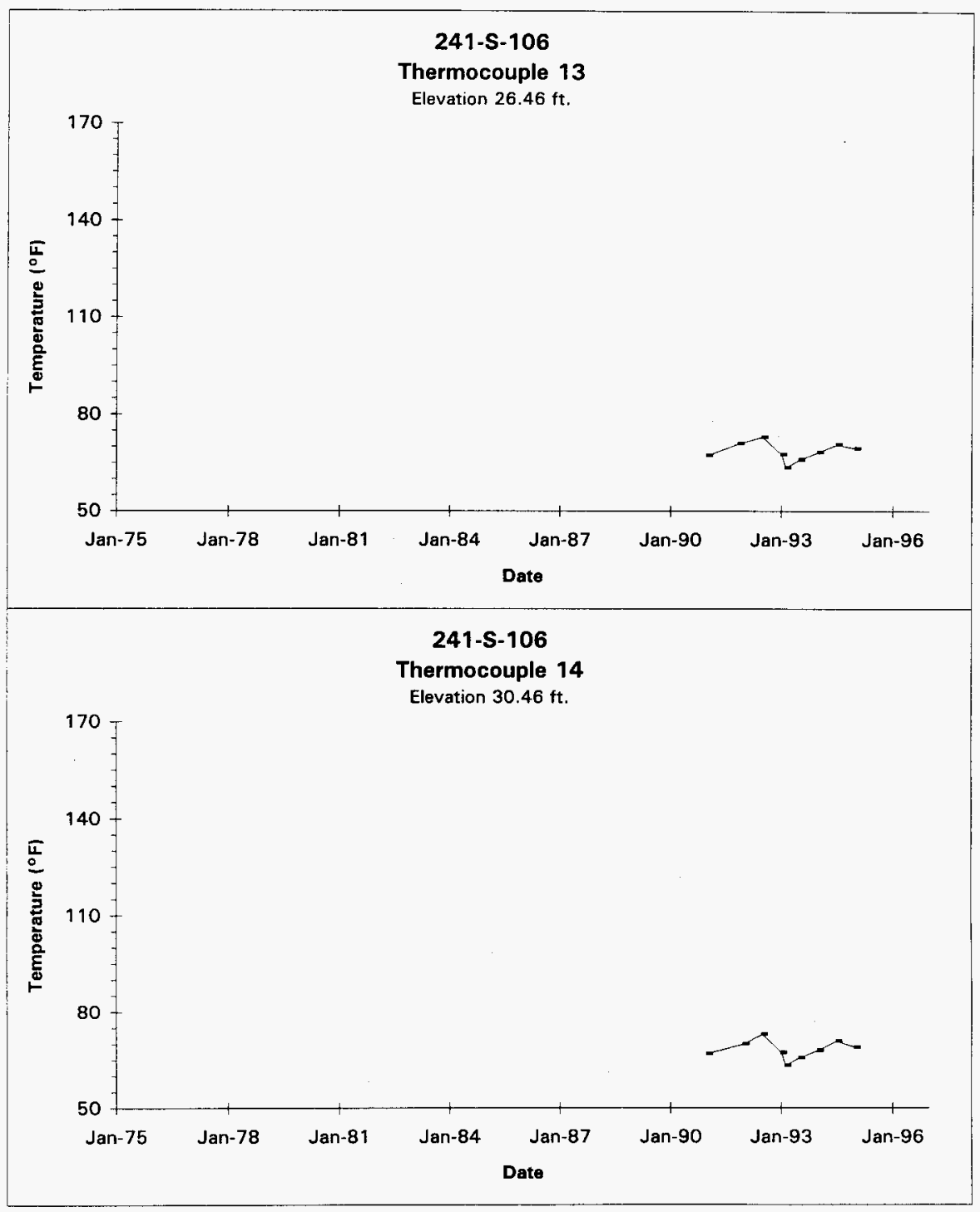

Data obtained from LMHC Surveillance Analysis Computer System (SACS), Oct 1, 1996.

D-30 
HNF-SD-WM-ER-323, Rev. 1

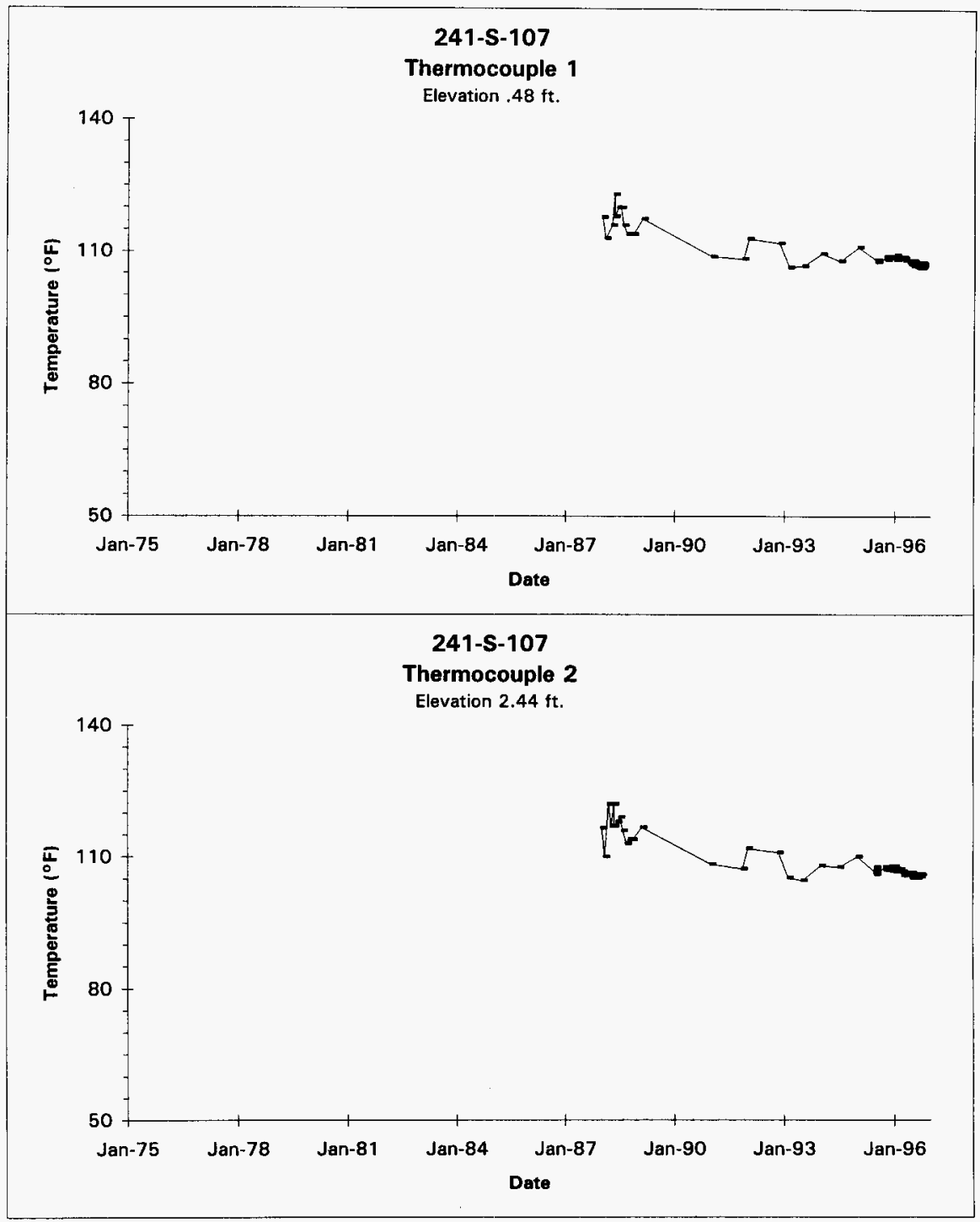

Data obtained from LMHC Surveillance Analysis Computer System (SACS), Oct 1, 1996.

\section{D-31}




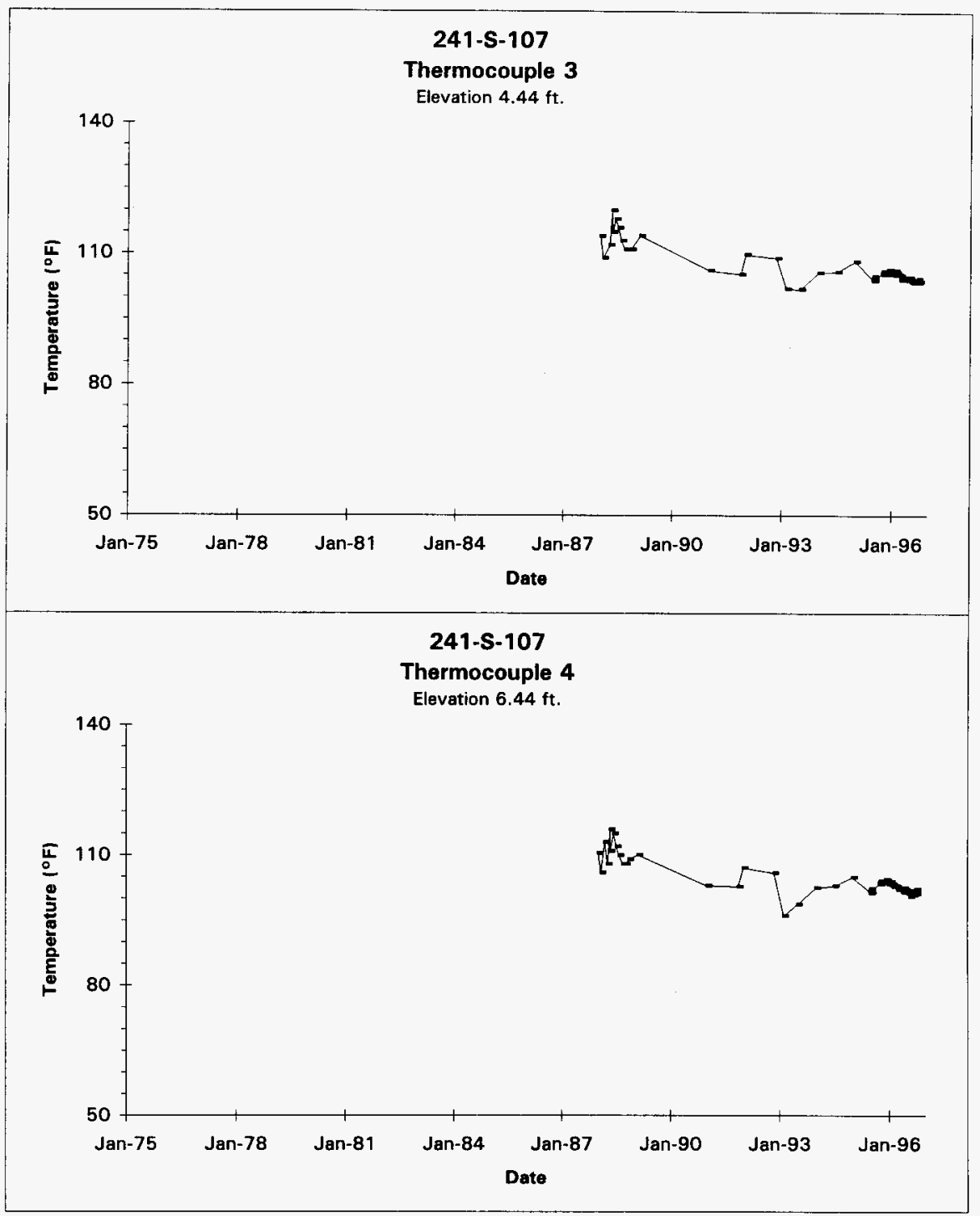

Data obtained from LMHC Surveillance Analysis Computer System (SACS), Oct 1, 1996.

$$
\text { D-32 }
$$




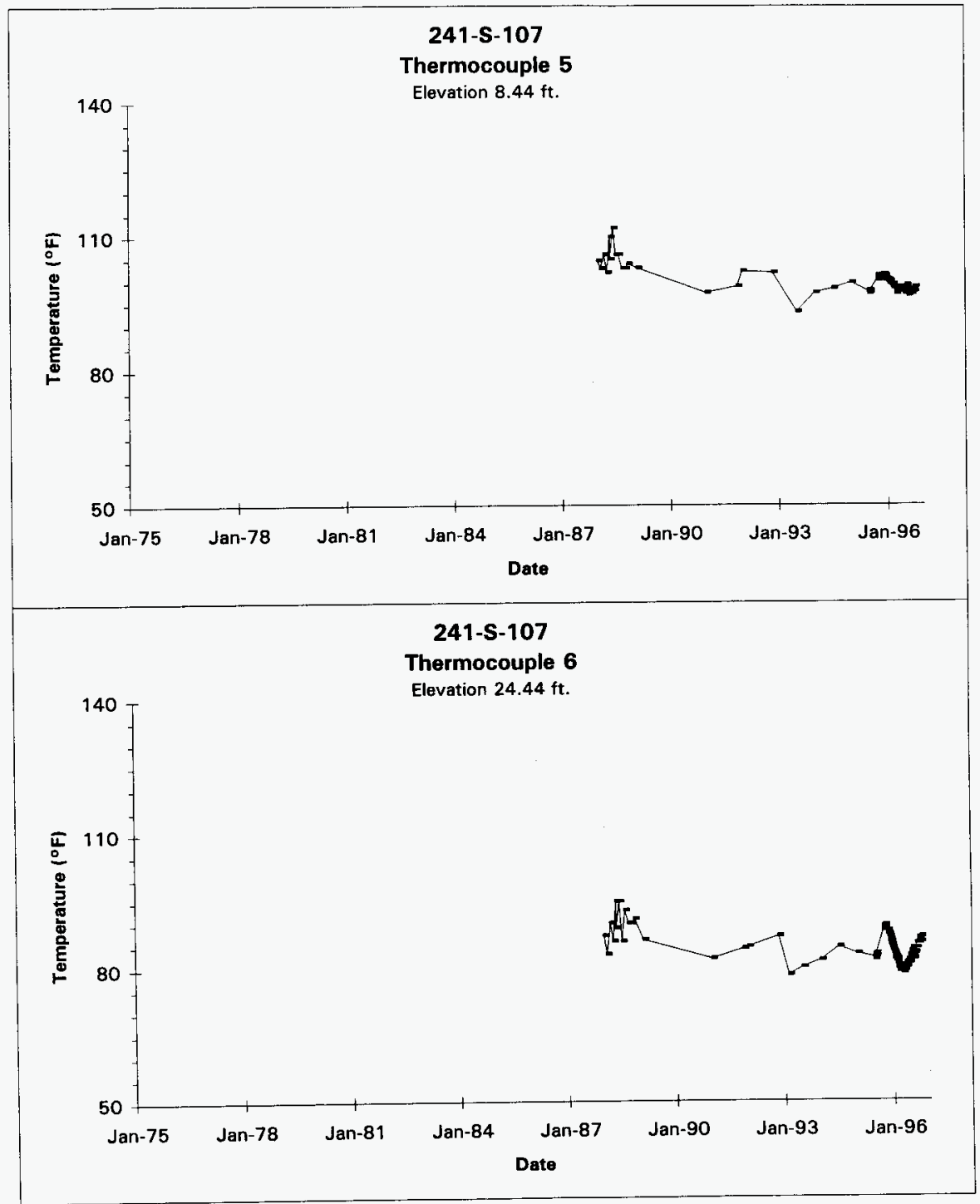

Data obtained from LMHC Surveillance Analysis Computer System (SACS), Oct 1, 1996.

D-33 


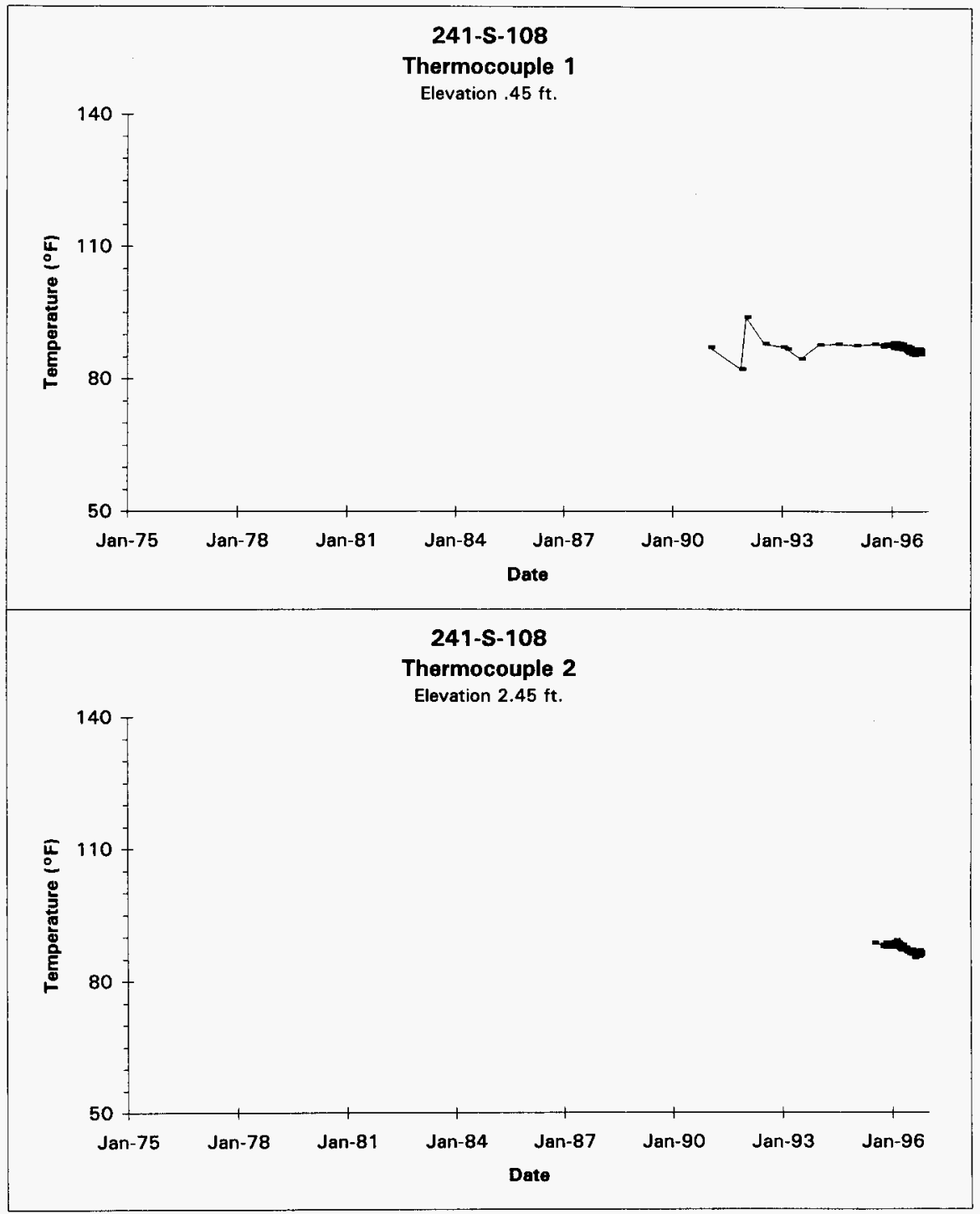

Data obtained from LMHC Surveillance Analysis Computer System (SACS), Oct 1, 1996.

D-34 


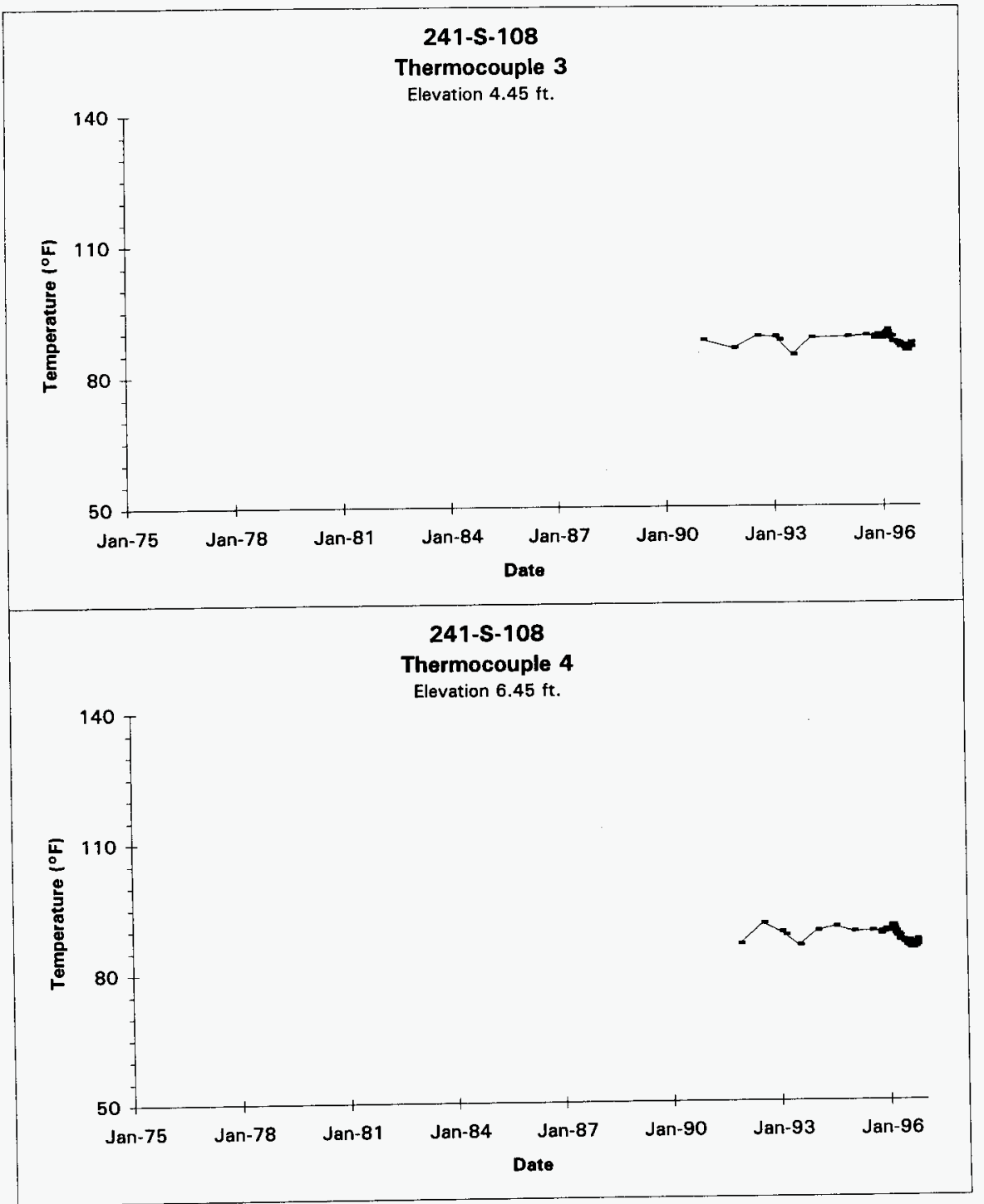

Data obtained from LMHC Surveillance Analysis Computer System (SACS), Oct 1, 1996.

D-35 


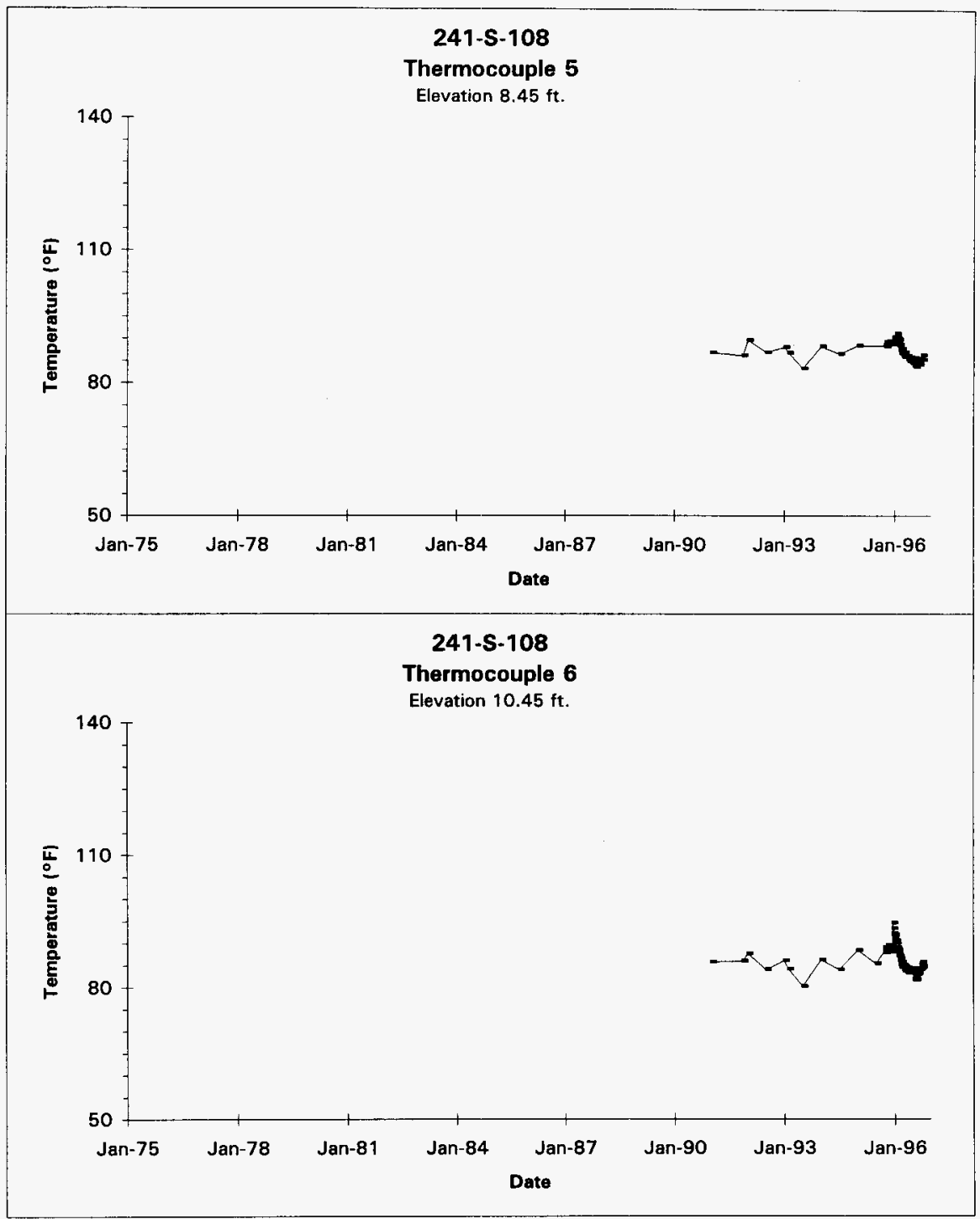

Data obtained from LMHC Surveillance Analysis Computer System (SACS), Oct 1, 1996.

D-36 


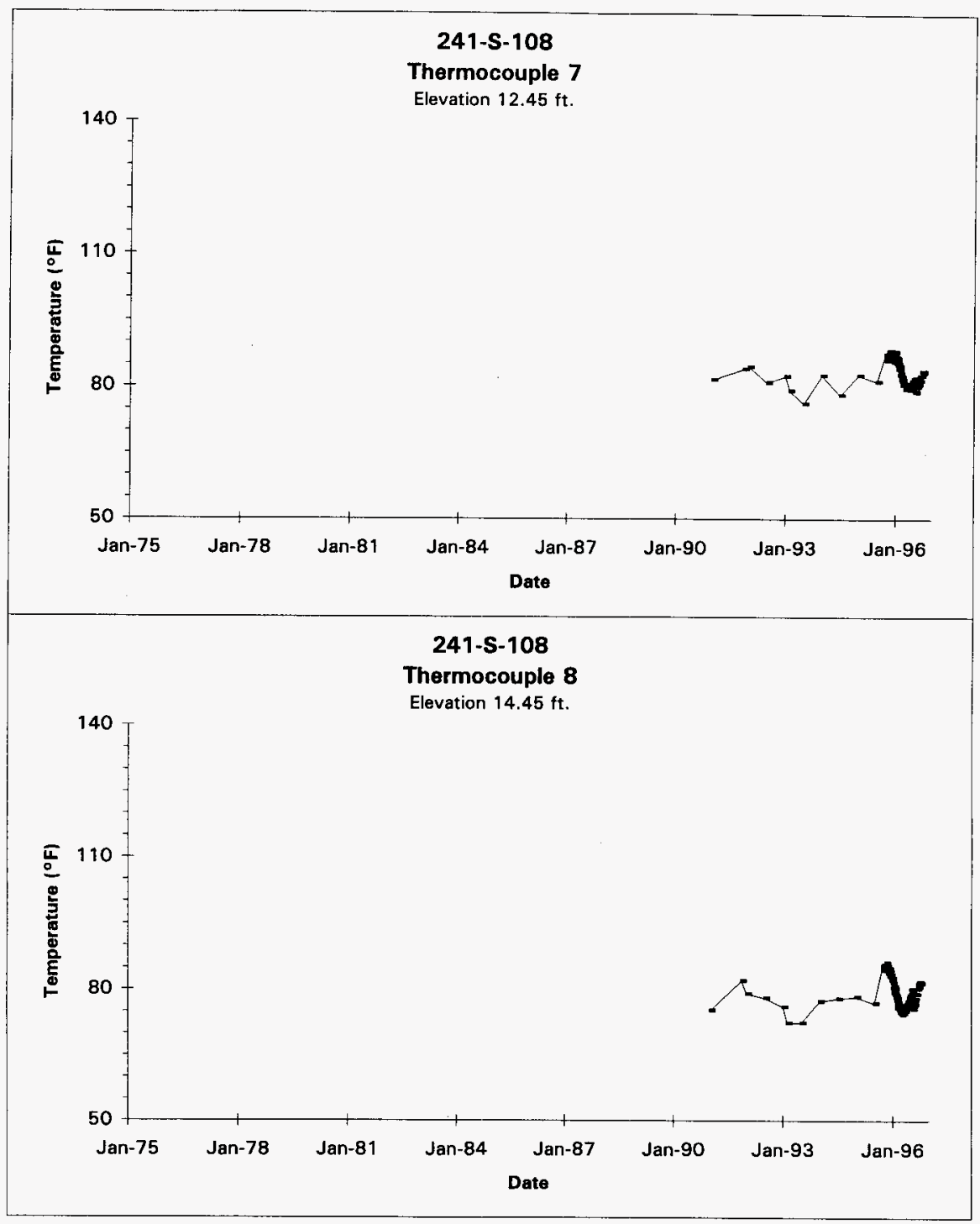

Data obtained from LMHC Surveillance Analysis Computer System (SACS), Oct 1, 1996. 


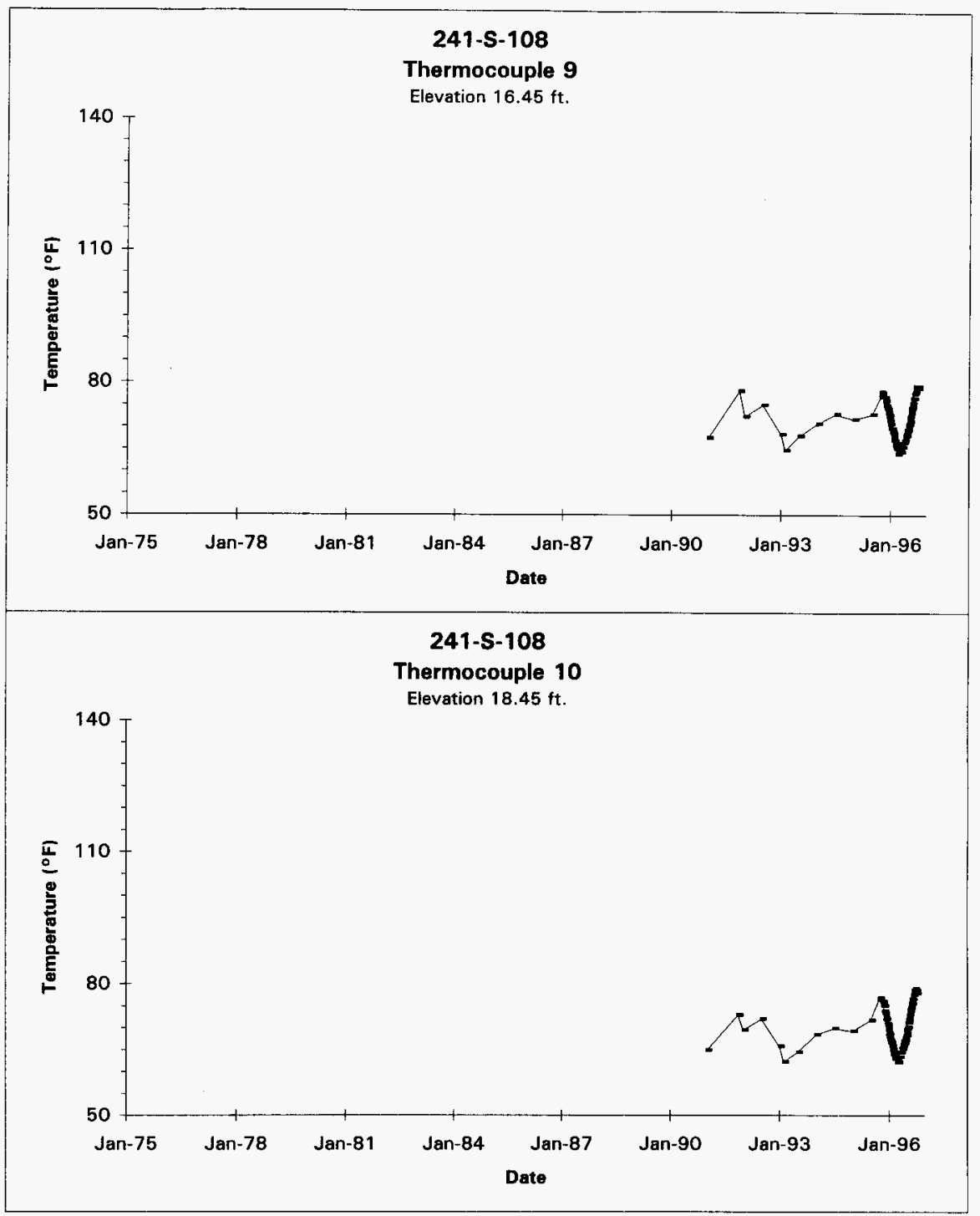

Data obtained from LMHC Surveillance Analysis Computer System (SACS), Oct 1, 1996. 


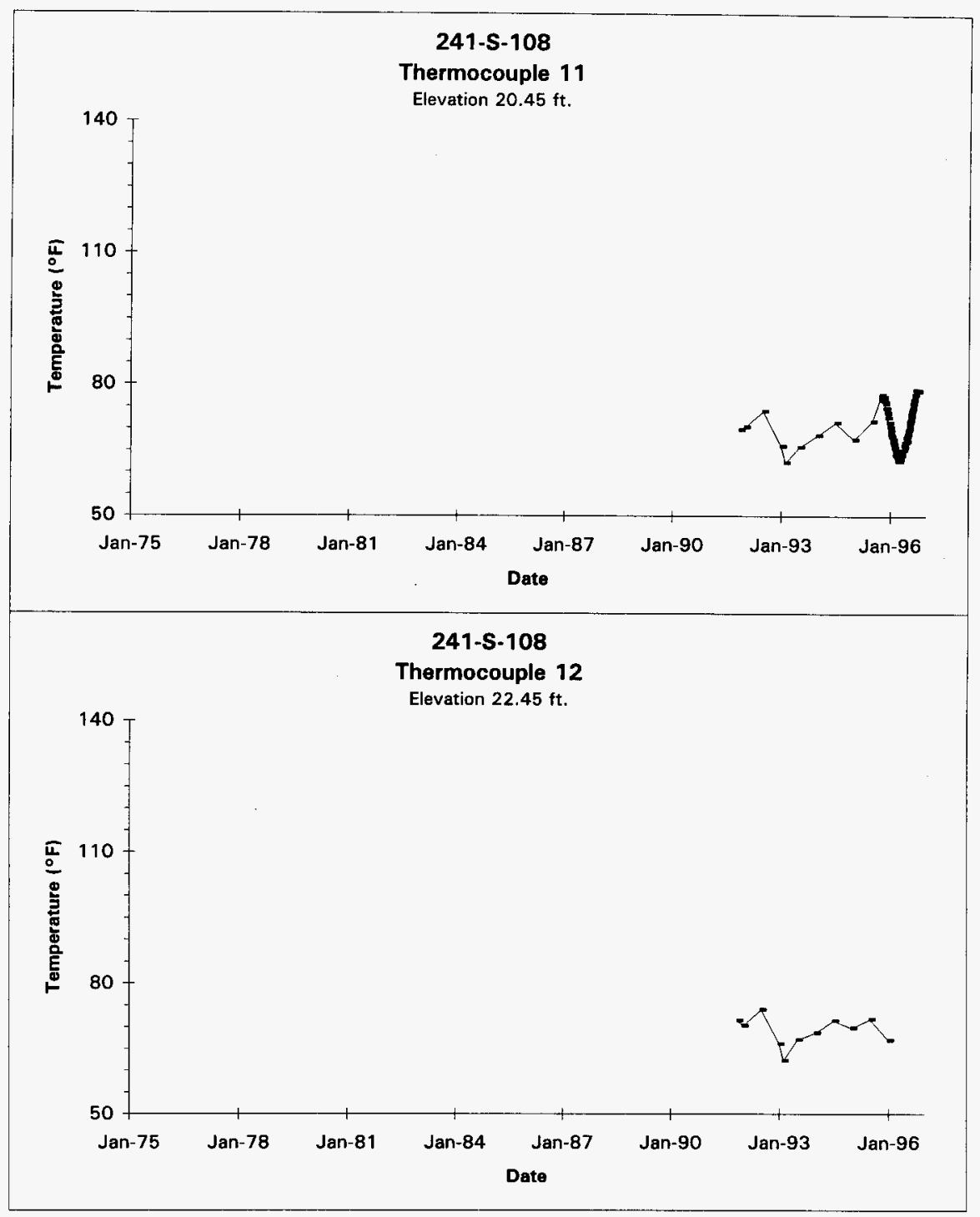

Data obtained from LMHC Surveillance Analysis Computer System (SACS), Oct 1, 1996.

$$
\text { D-39 }
$$


HNF-SD-WM-ER-323, Rev. 1

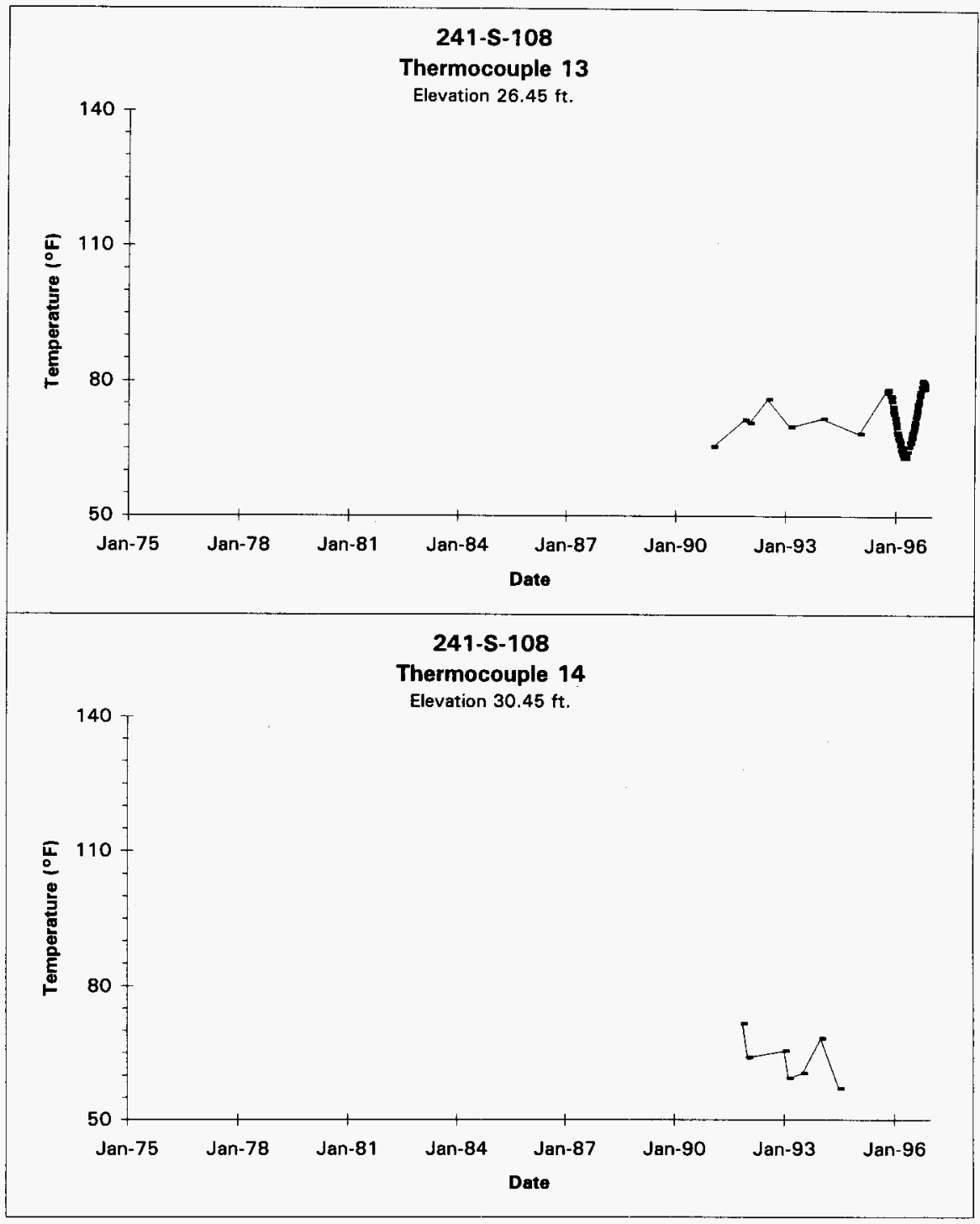

Data obtained from LMHC Surveillance Analysis Computer System (SACS), Oct 1, 1996.

$$
\text { D-40 }
$$




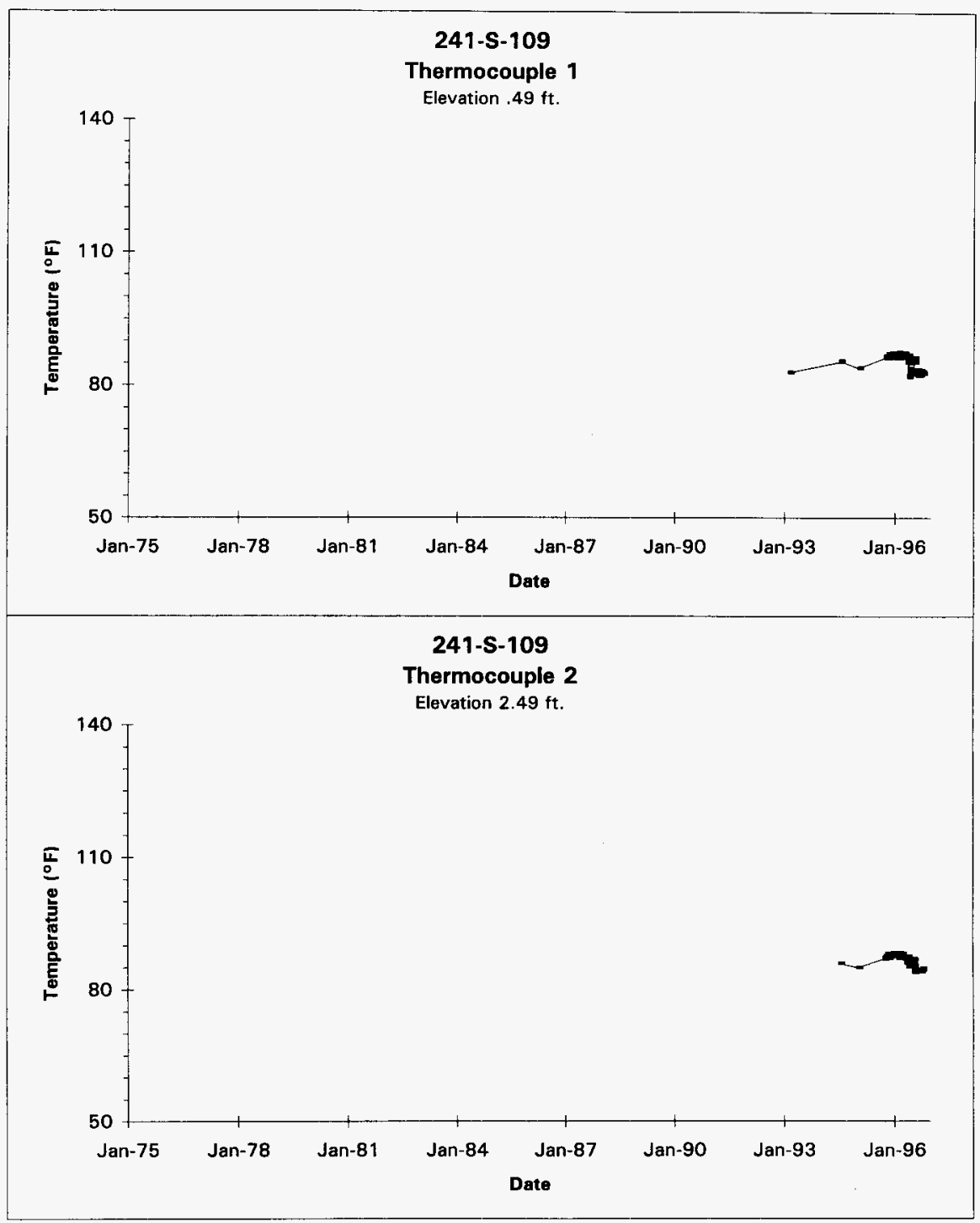

Data obtained from LMHC Surveillance Analysis Computer System (SACS), Oct 1, 1996.

D-41 


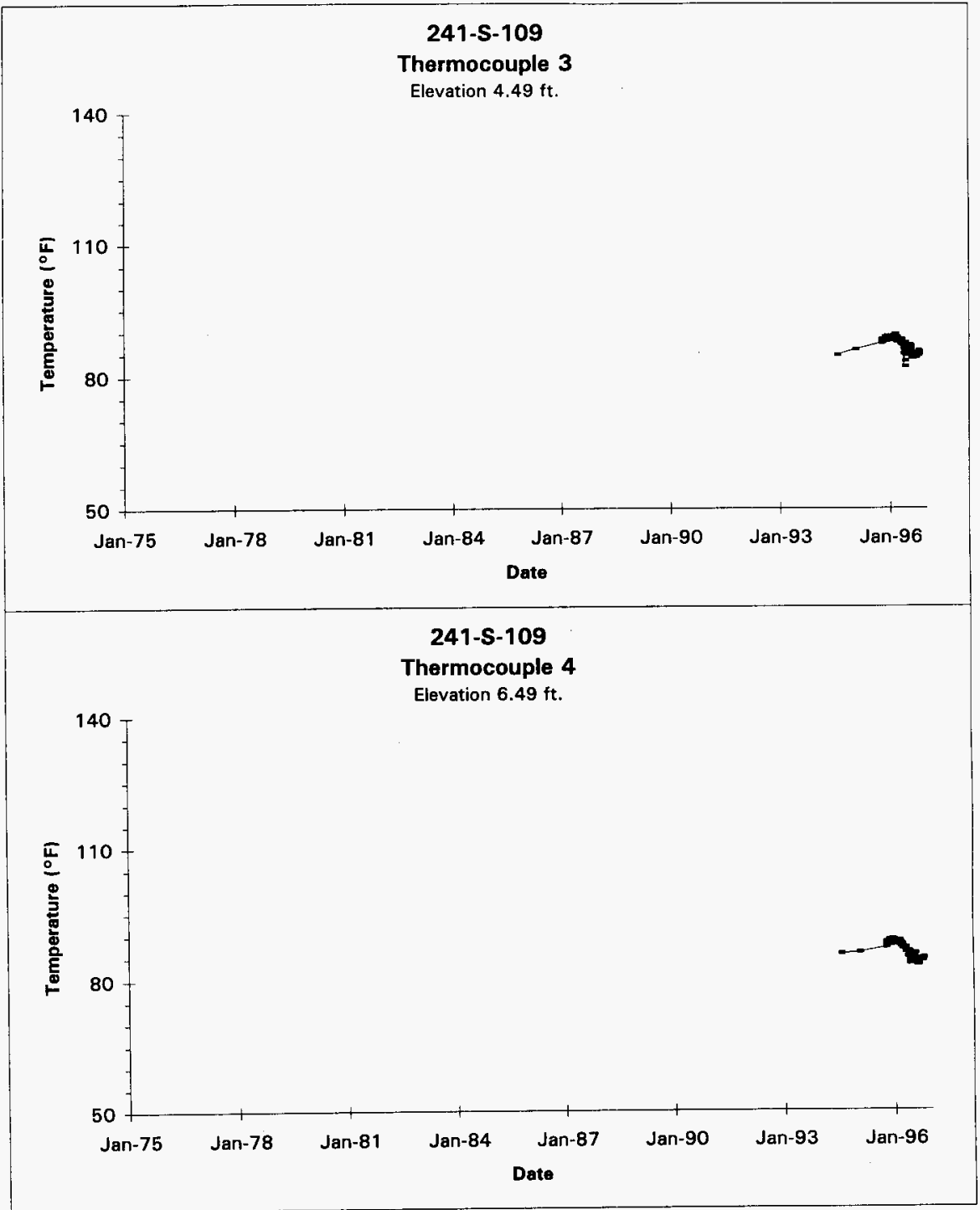

Data obtained from LMHC Surveillance Analysis Computer System (SACS), Oct 1, 1996.

$$
\text { D-42 }
$$




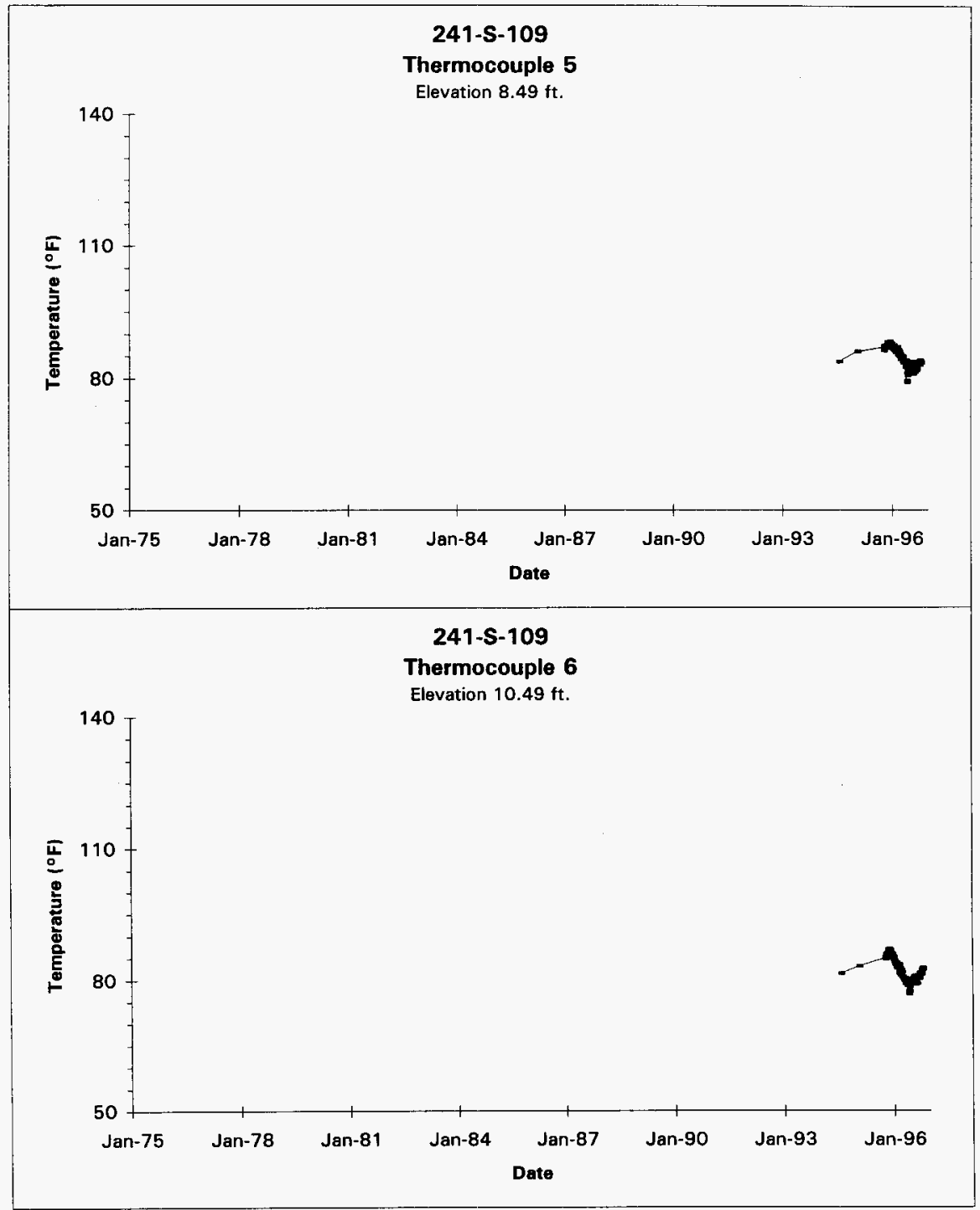

Data obtained from LMHC Surveillance Analysis Computer System (SACS), Oct 1, 1996. 


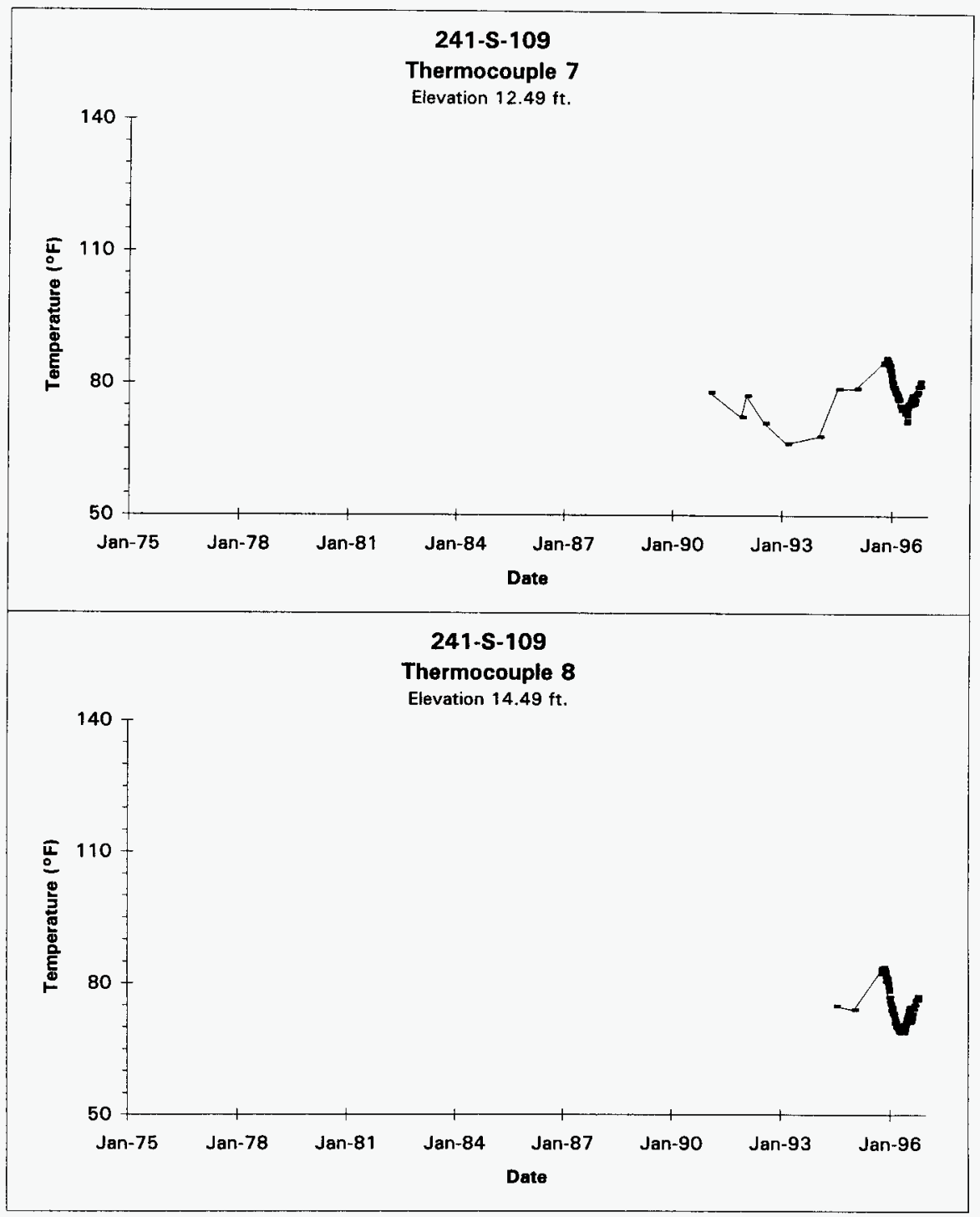

Data obtained from LMHC Surveillance Analysis Computer System (SACS), Oct 1, 1996.

$$
\text { D-44 }
$$




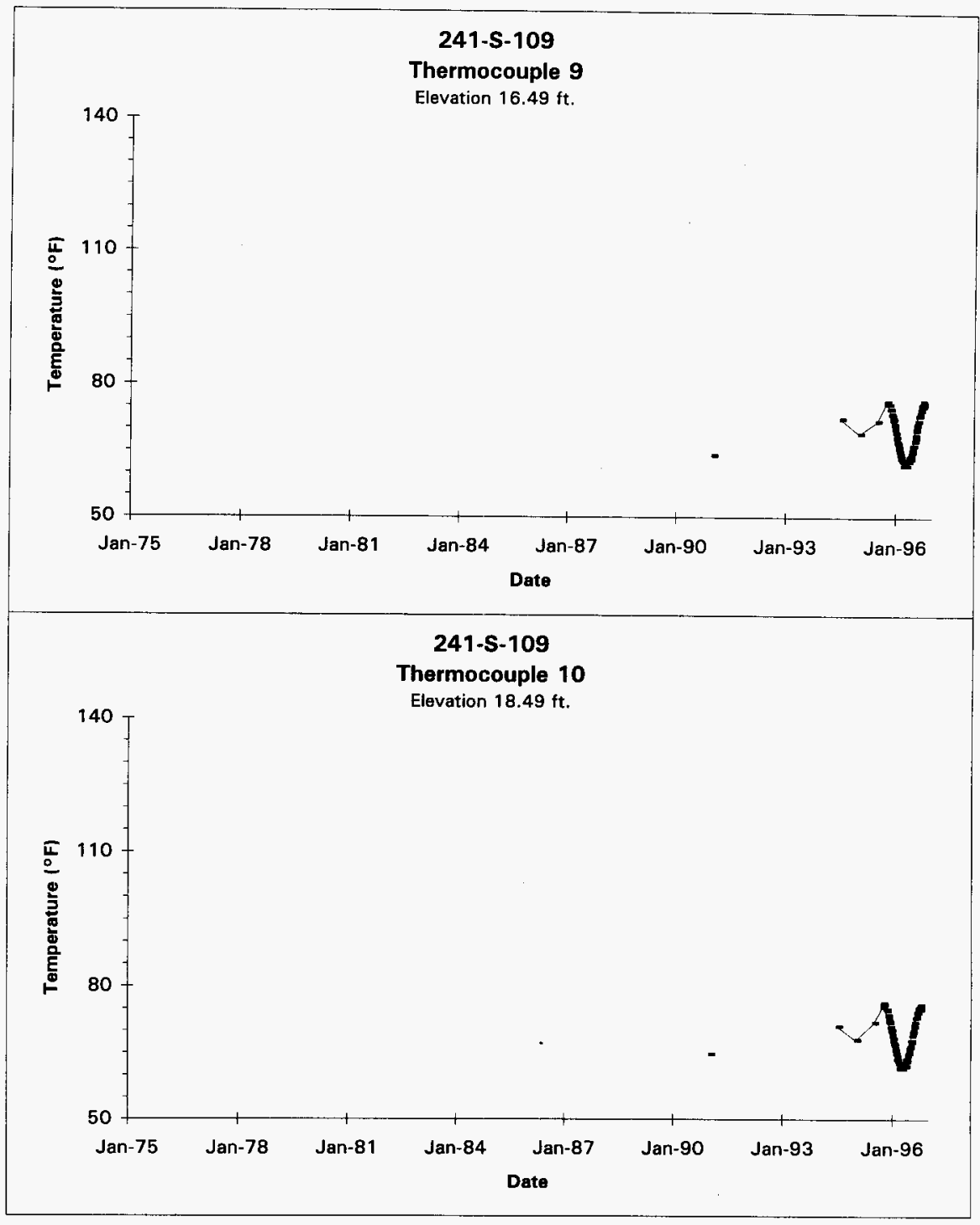

Data obtained from LMHC Surveillance Analysis Computer System (SACS), Oct 1, 1996 


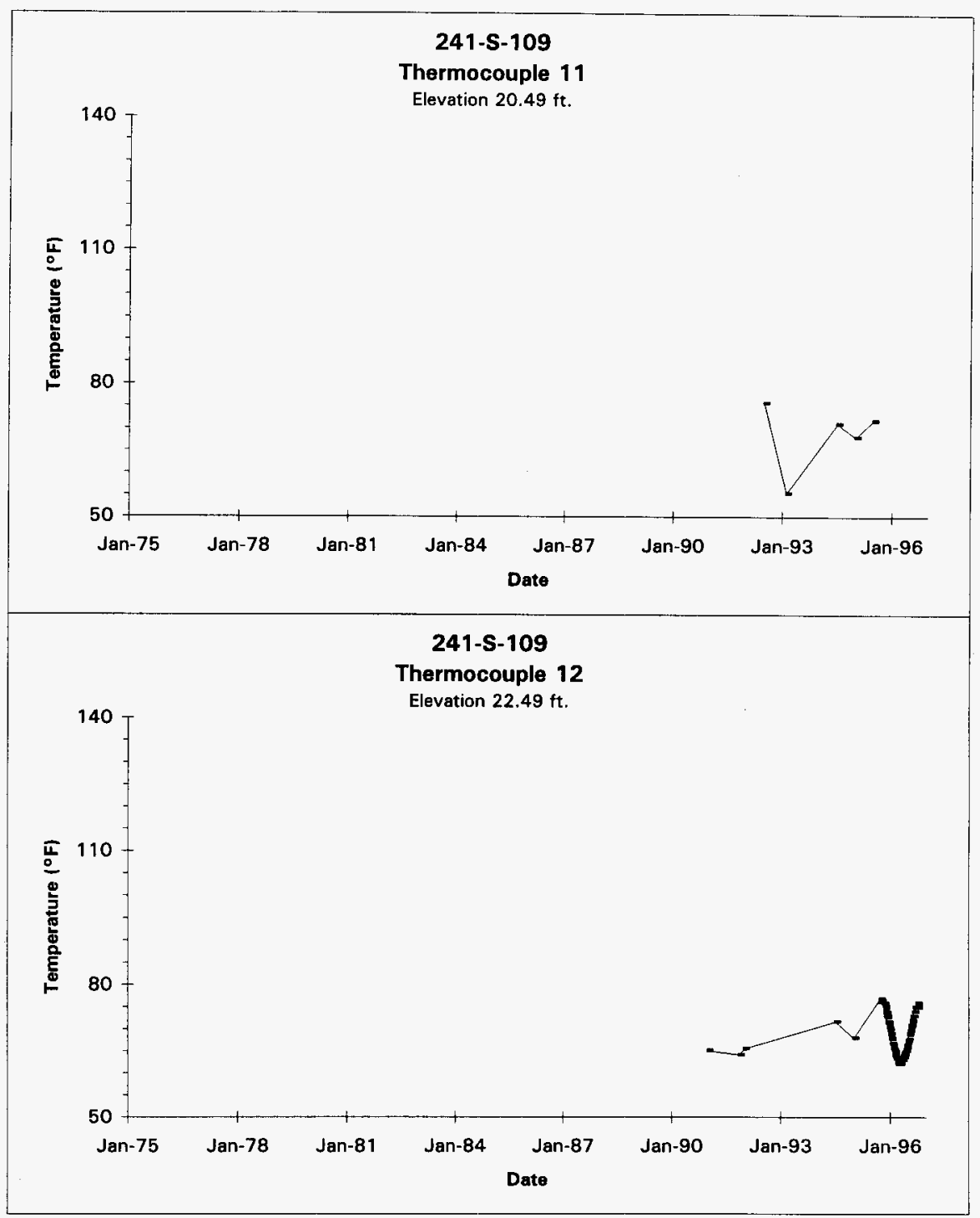

Data obtained from LMHC Surveillance Analysis Computer System (SACS), Oct 1, 1996.

$$
\text { D-46 }
$$




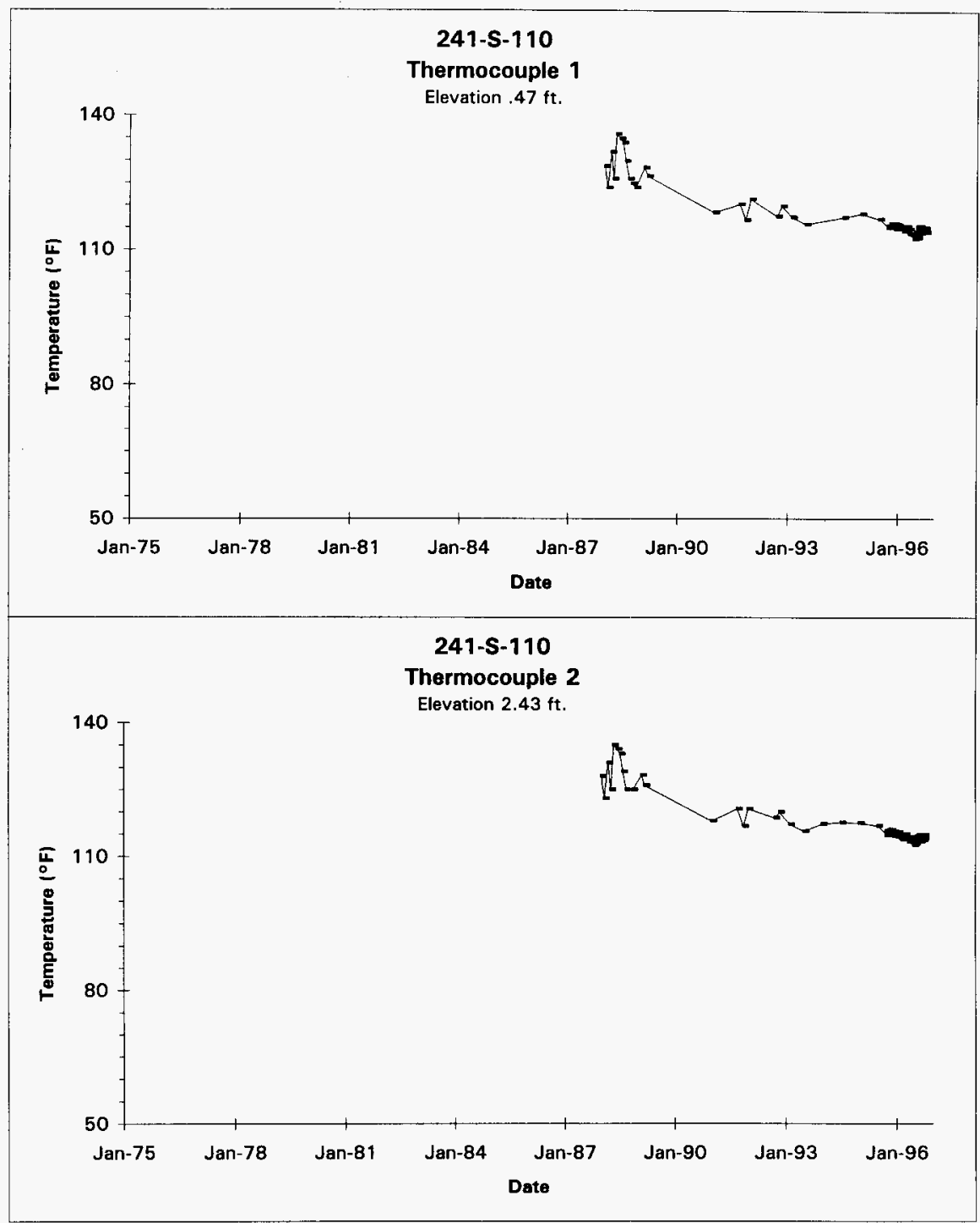

Data obtained from LMHC Surveillance Analysis Computer System (SACS), Oct 1, 1996. 


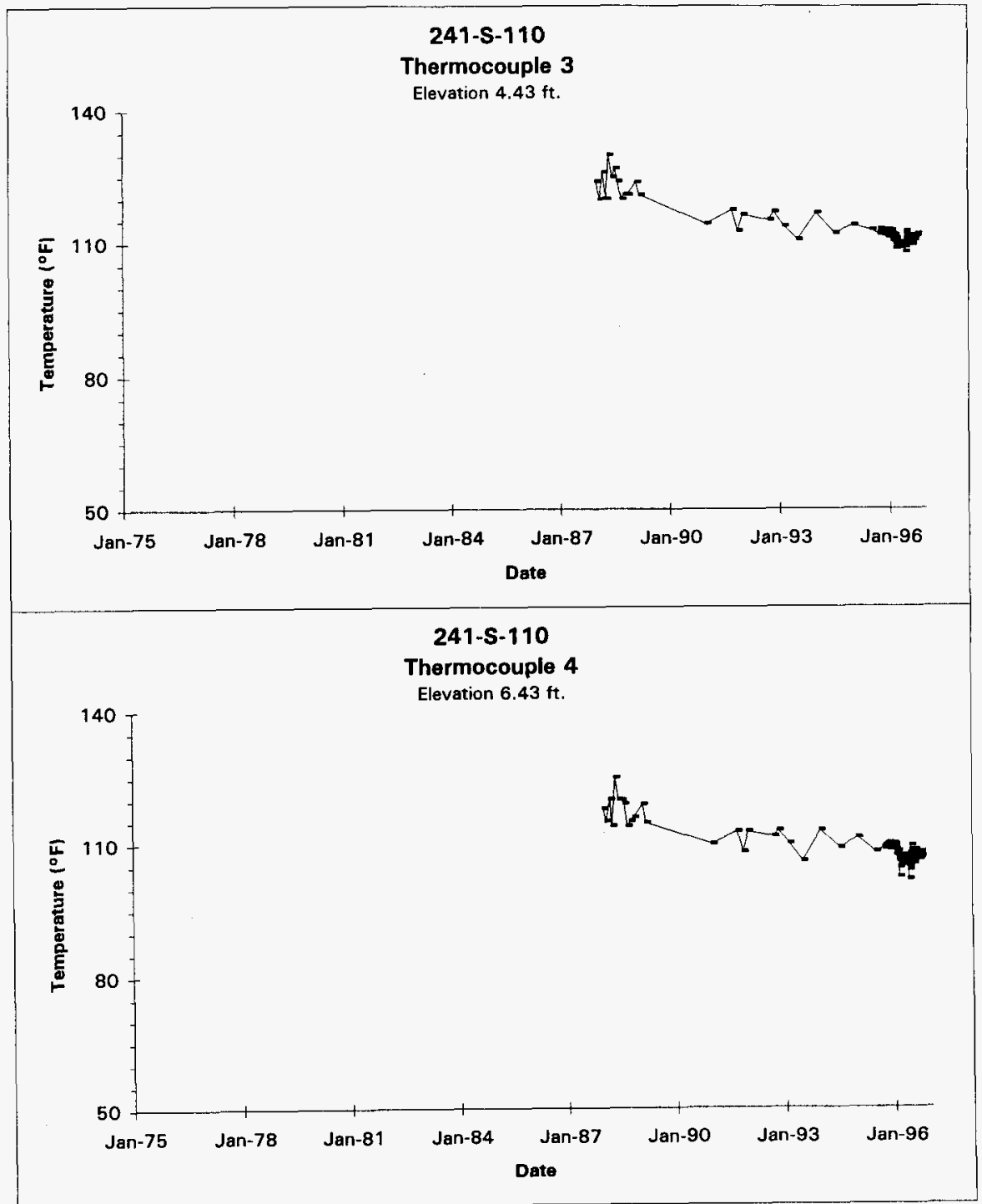

Data obtained from LMHC Surveillance Analysis Computer System (SACS), Oct 1, 1996. 


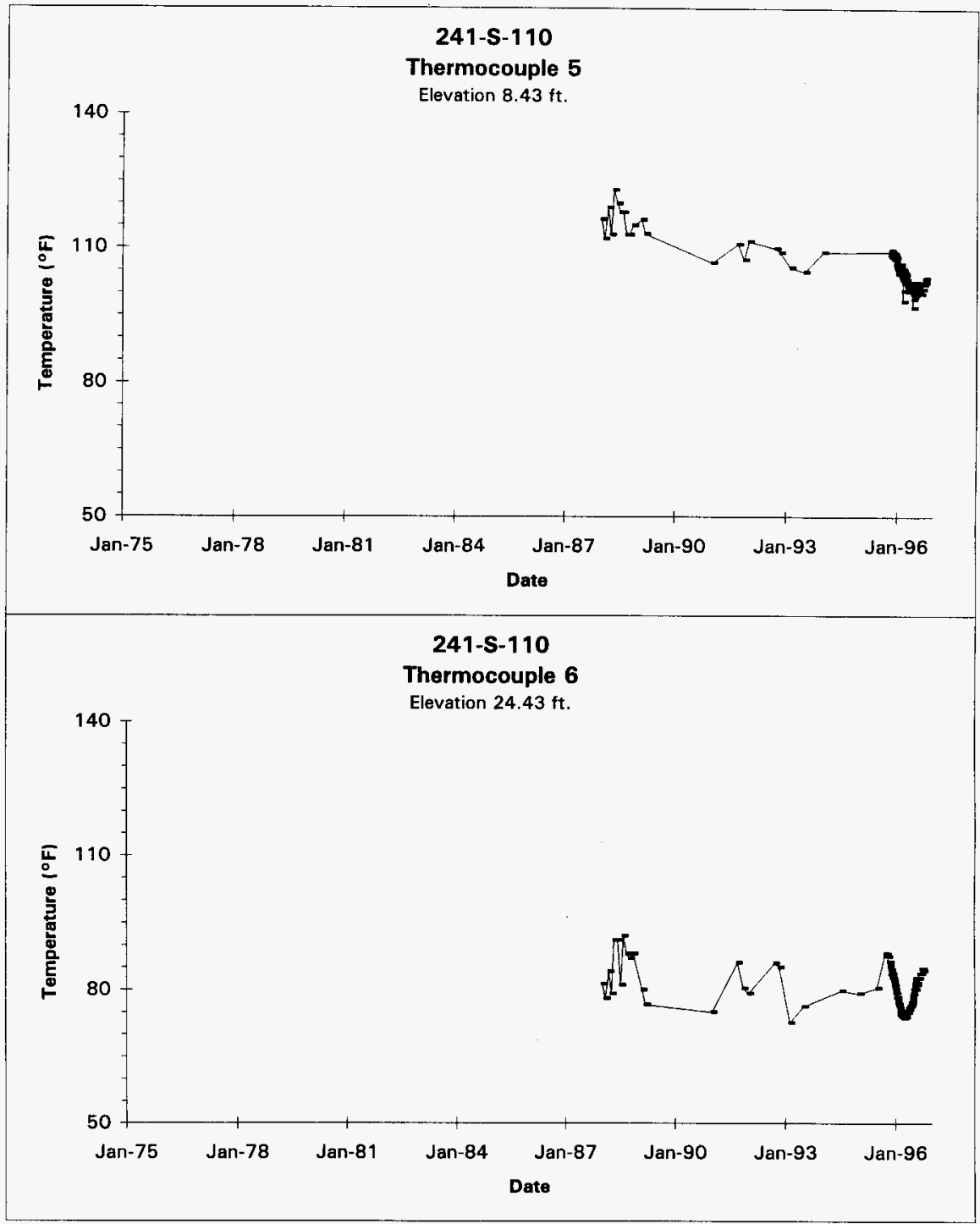

Data obtained from LMHC Surveillance Analysis Computer System (SACS), Oct 1, 1996. D-49 


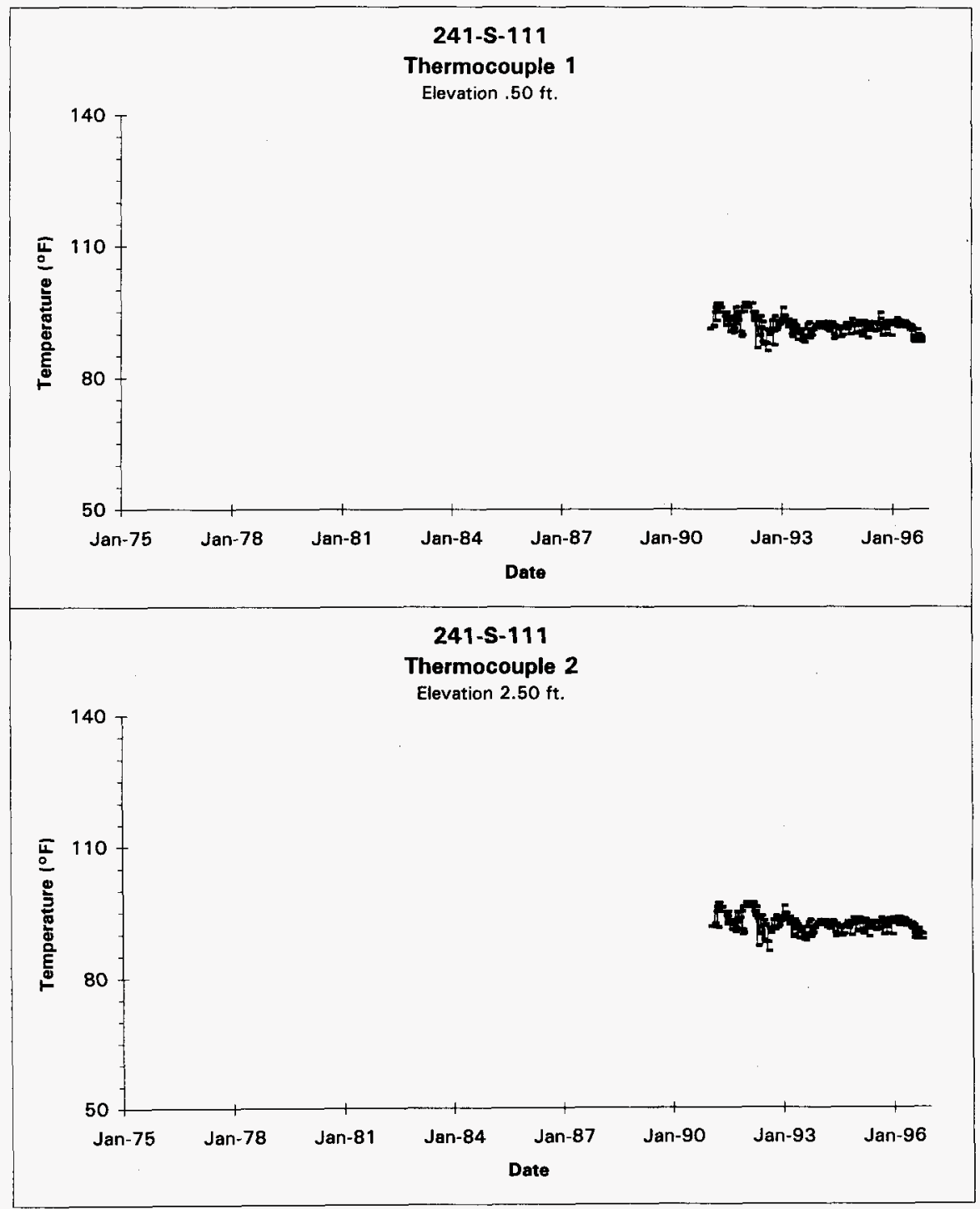

Data obtained from LMHC Surveillance Analysis Computer System (SACS), Oct 1, 1996. 


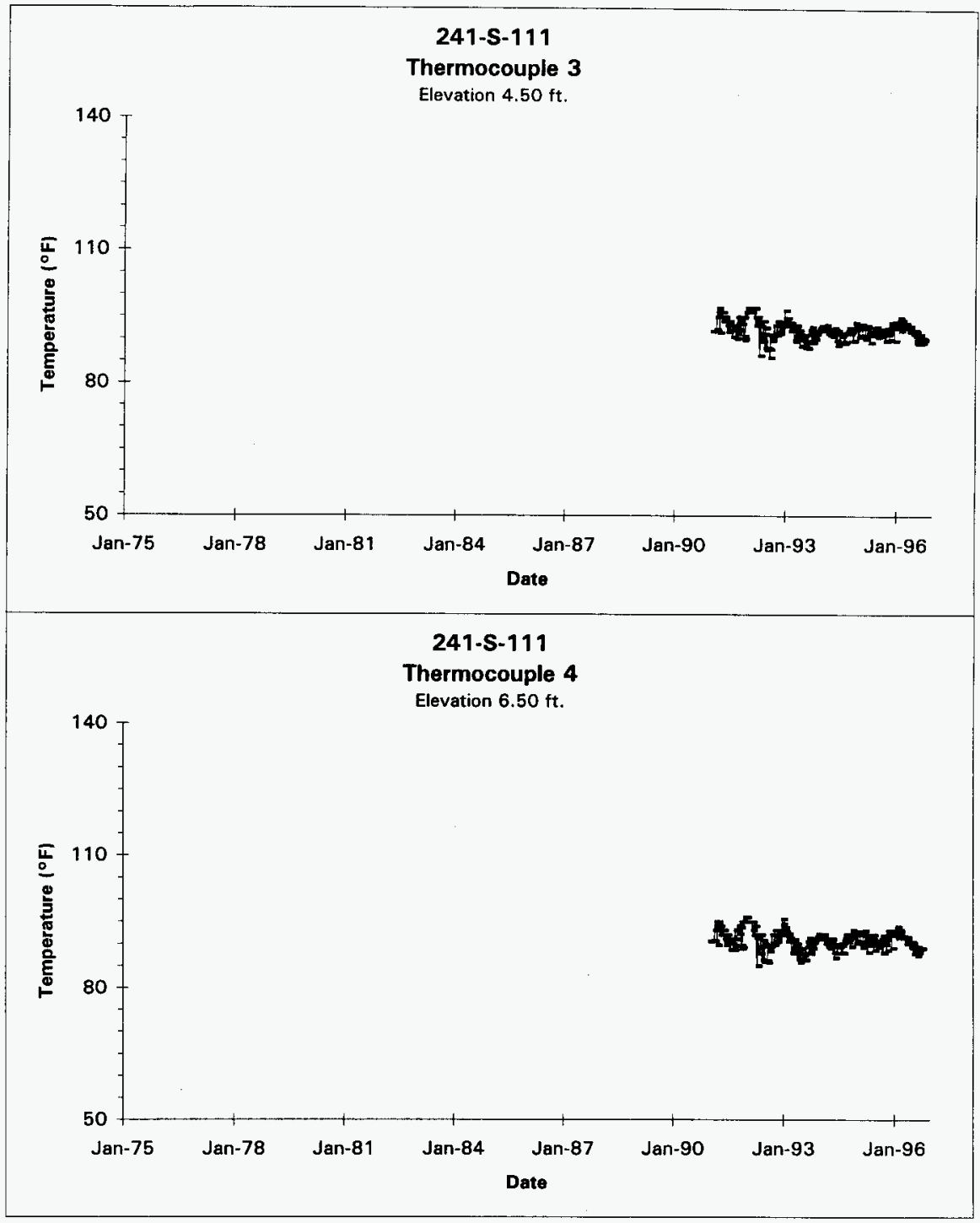

Data obtained from LMHC Surveillance Analysis Computer System (SACS), Oct 1, 1996. 


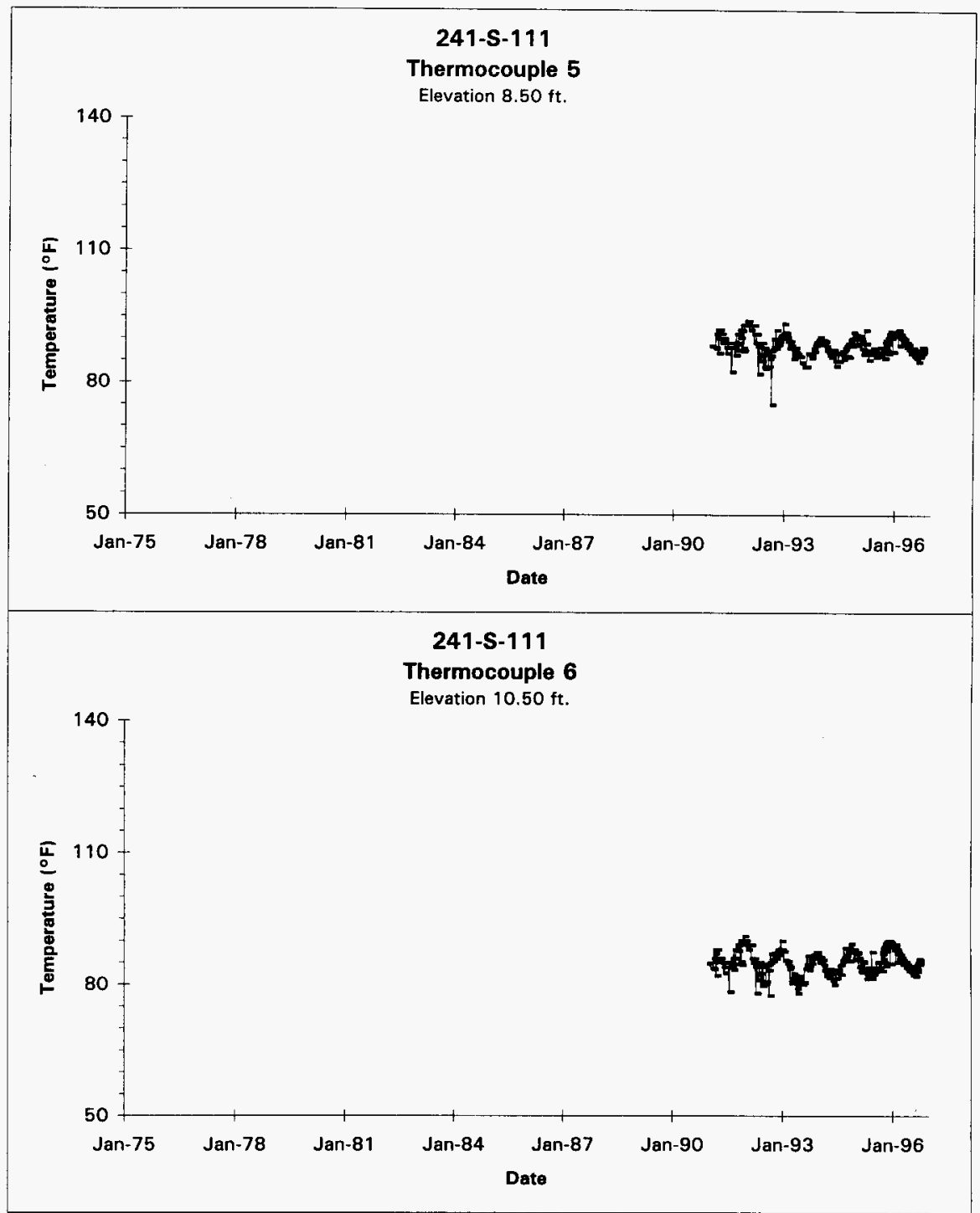

Data obtained from LMHC Surveillance Analysis Computer System (SACS), Oct 1, 1996.

D-52 


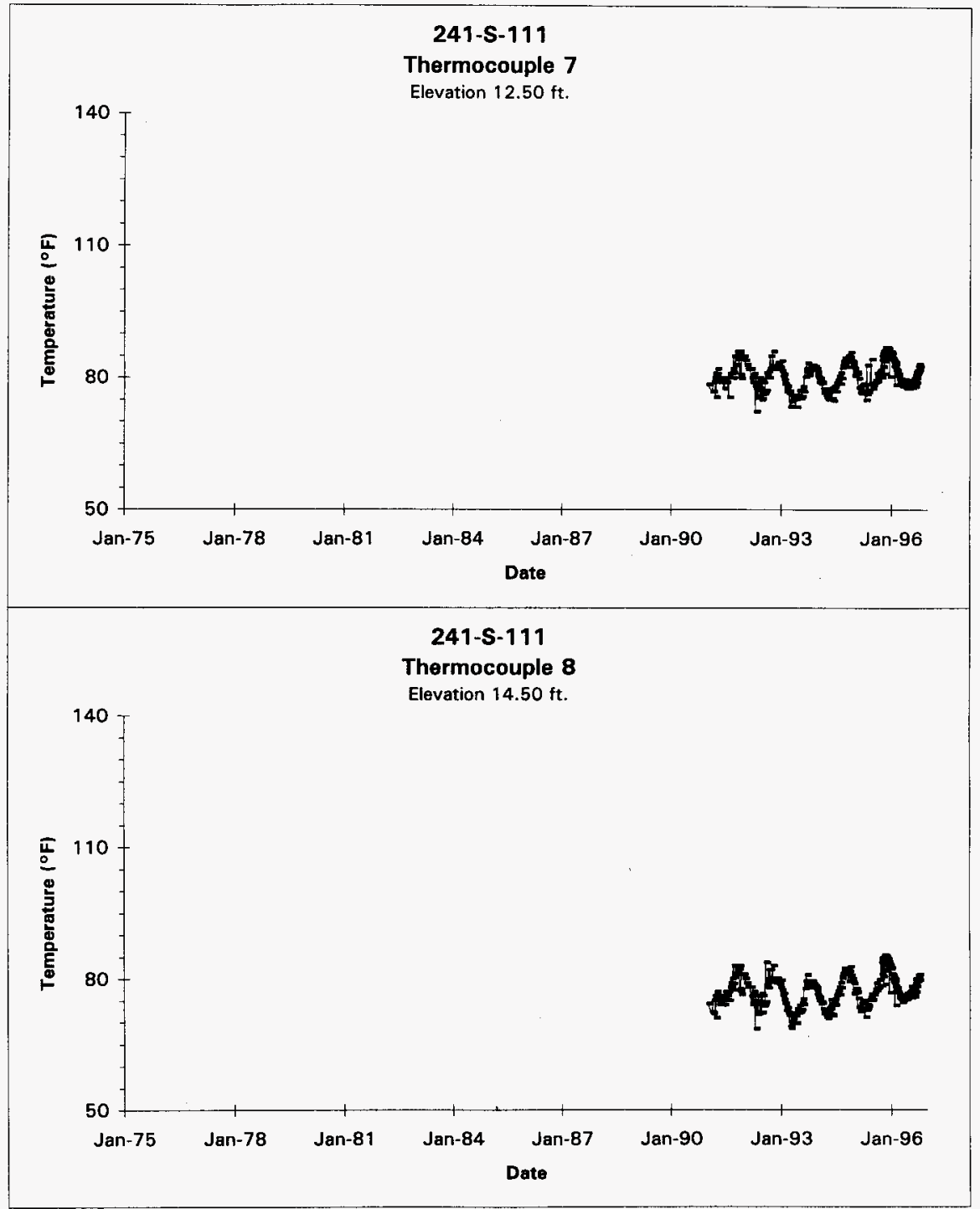

Data obtained from LMHC Surveillance Analysis Computer System (SACS), Oct 1, 1996.

D-53 


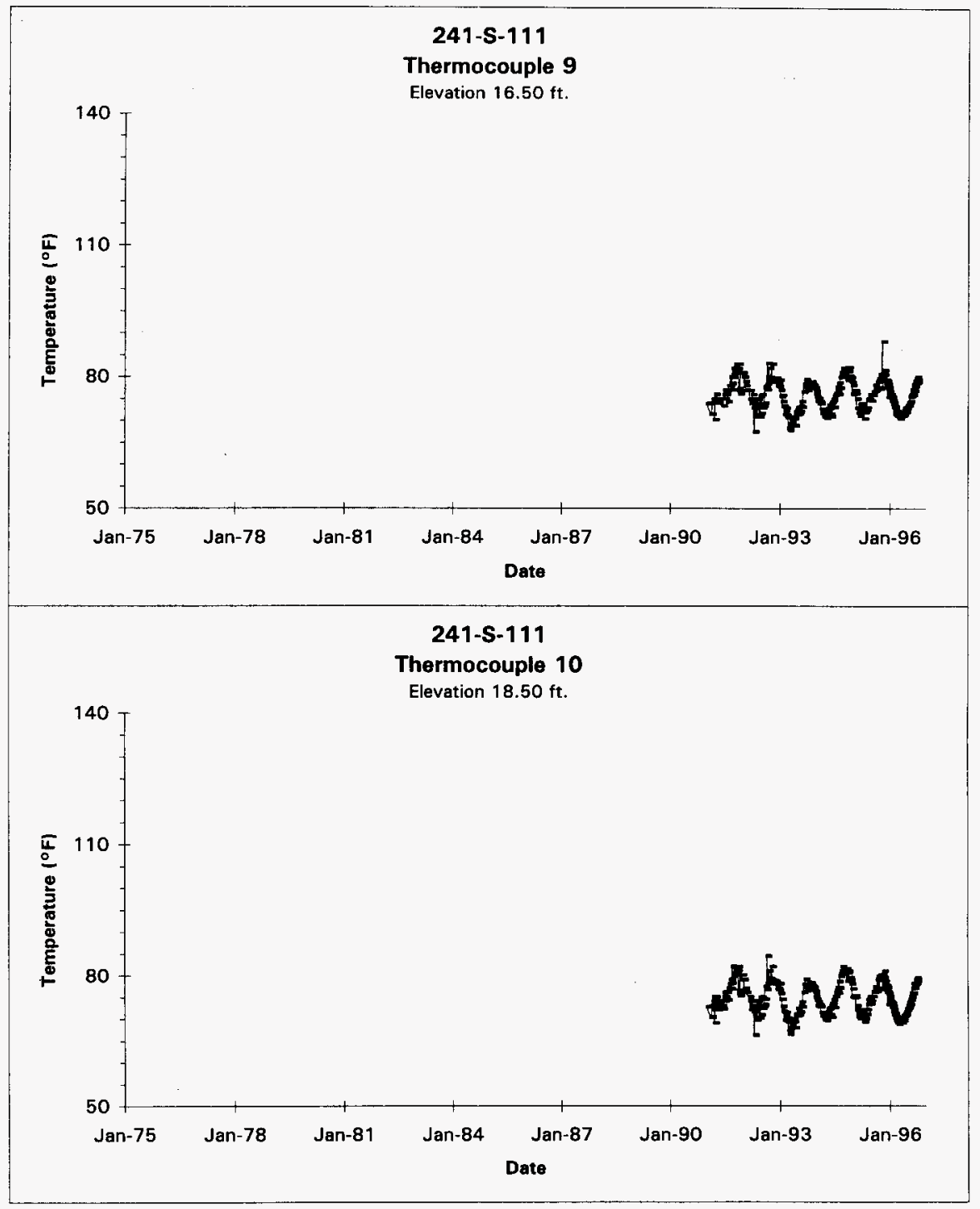

Data obtained from LMHC Surveillance Analysis Computer System (SACS), Oct 1, 1996.

D-54 


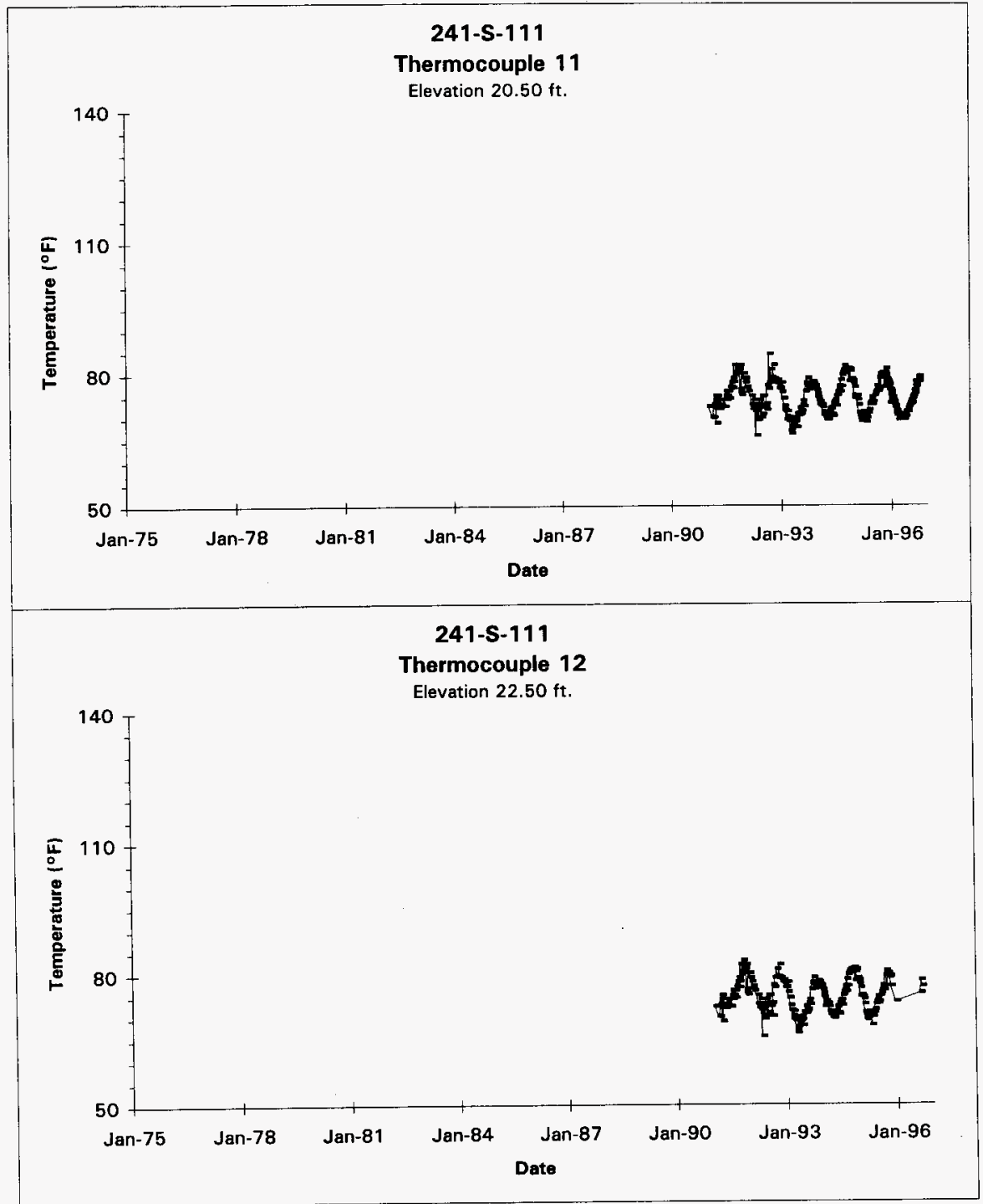

Data obtained from LMHC Surveillance Analysis Computer System (SACS), Oct 1, 1996.

D-55 


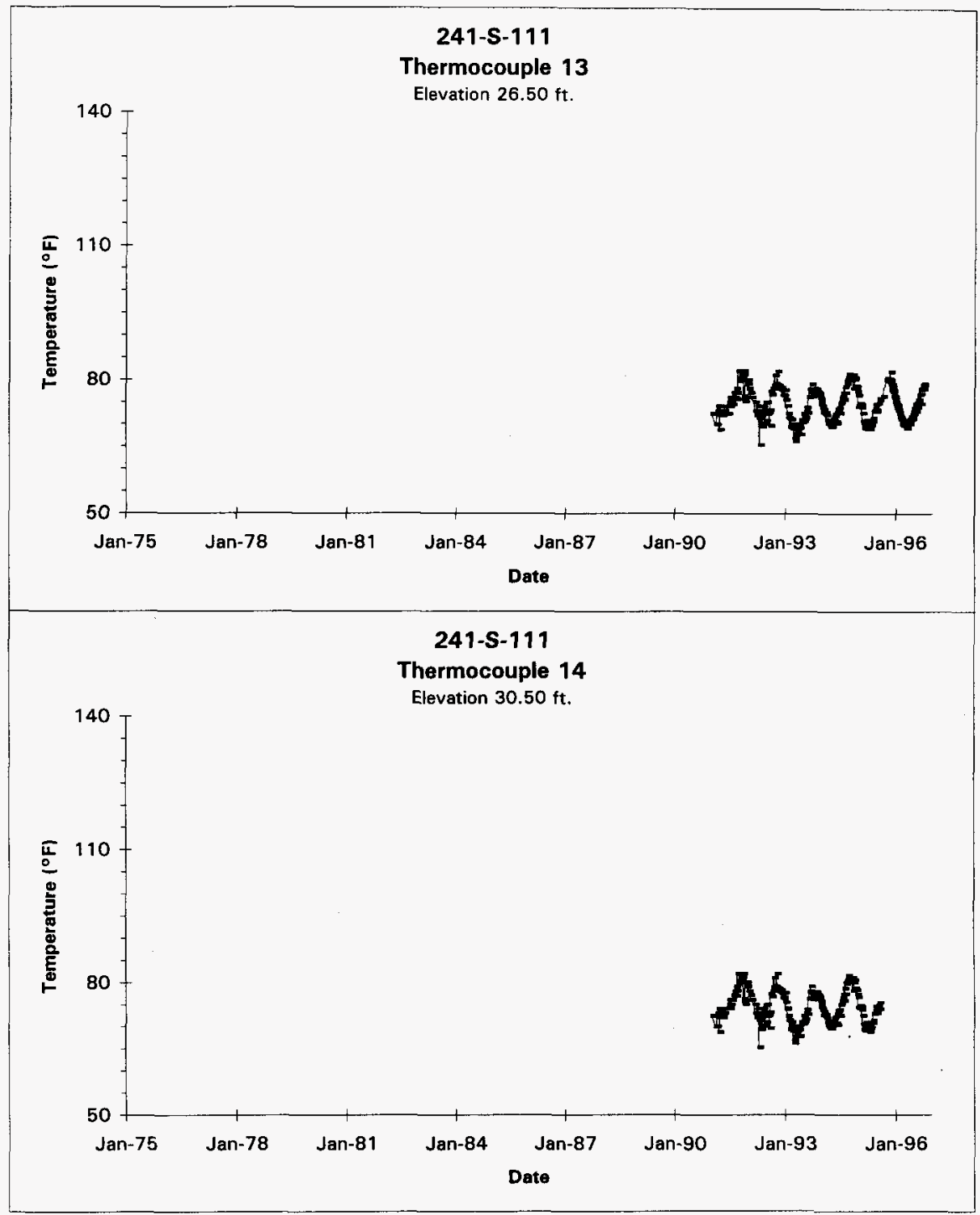

Data obtained from LMHC Surveillance Analysis Computer System (SACS), Oct 1, 1996.

$$
\text { D-56 }
$$




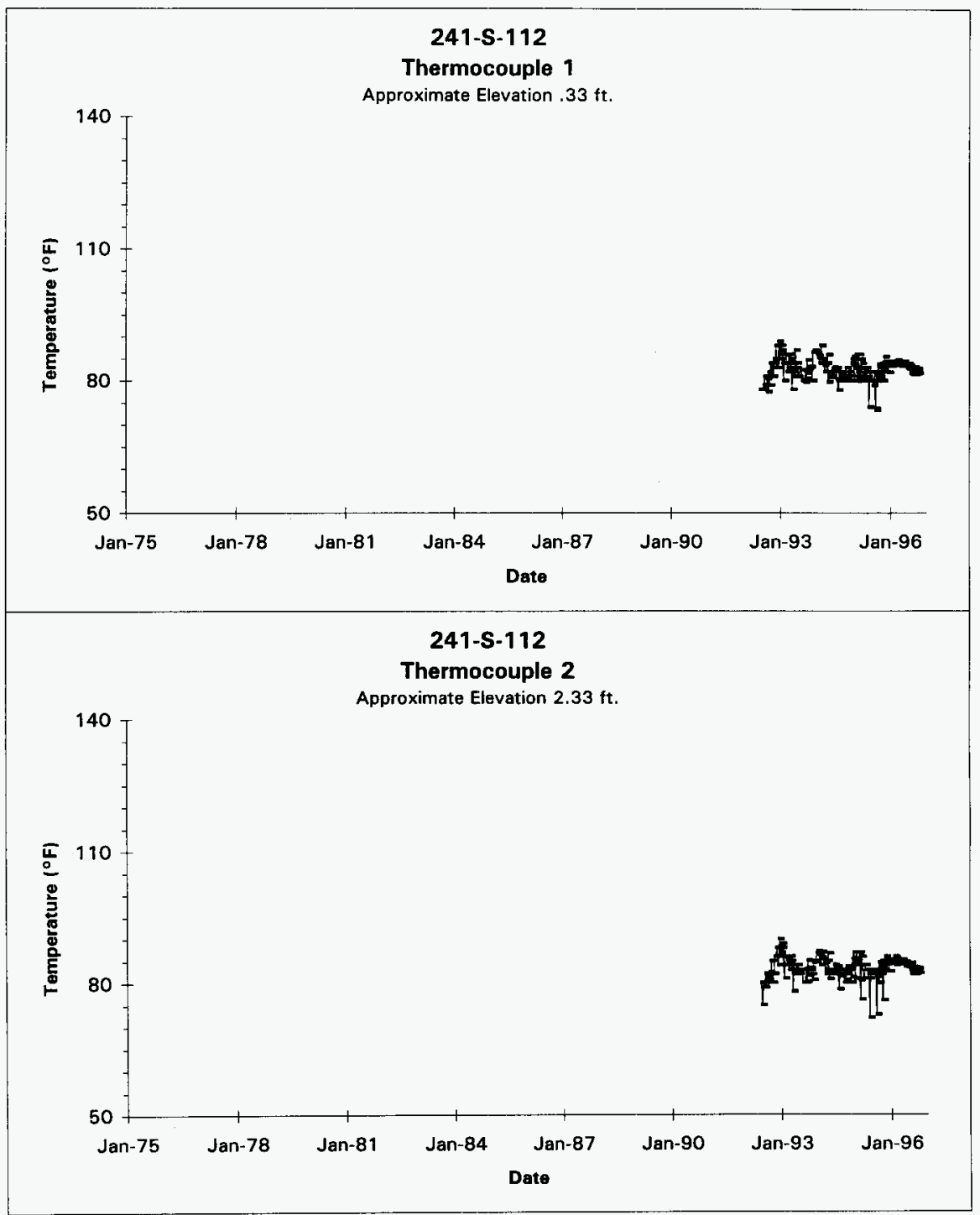

Data obtained from LMHC Surveillance Analysis Computer System (SACS), Oct 1, 1996.

$$
\text { D-57 }
$$




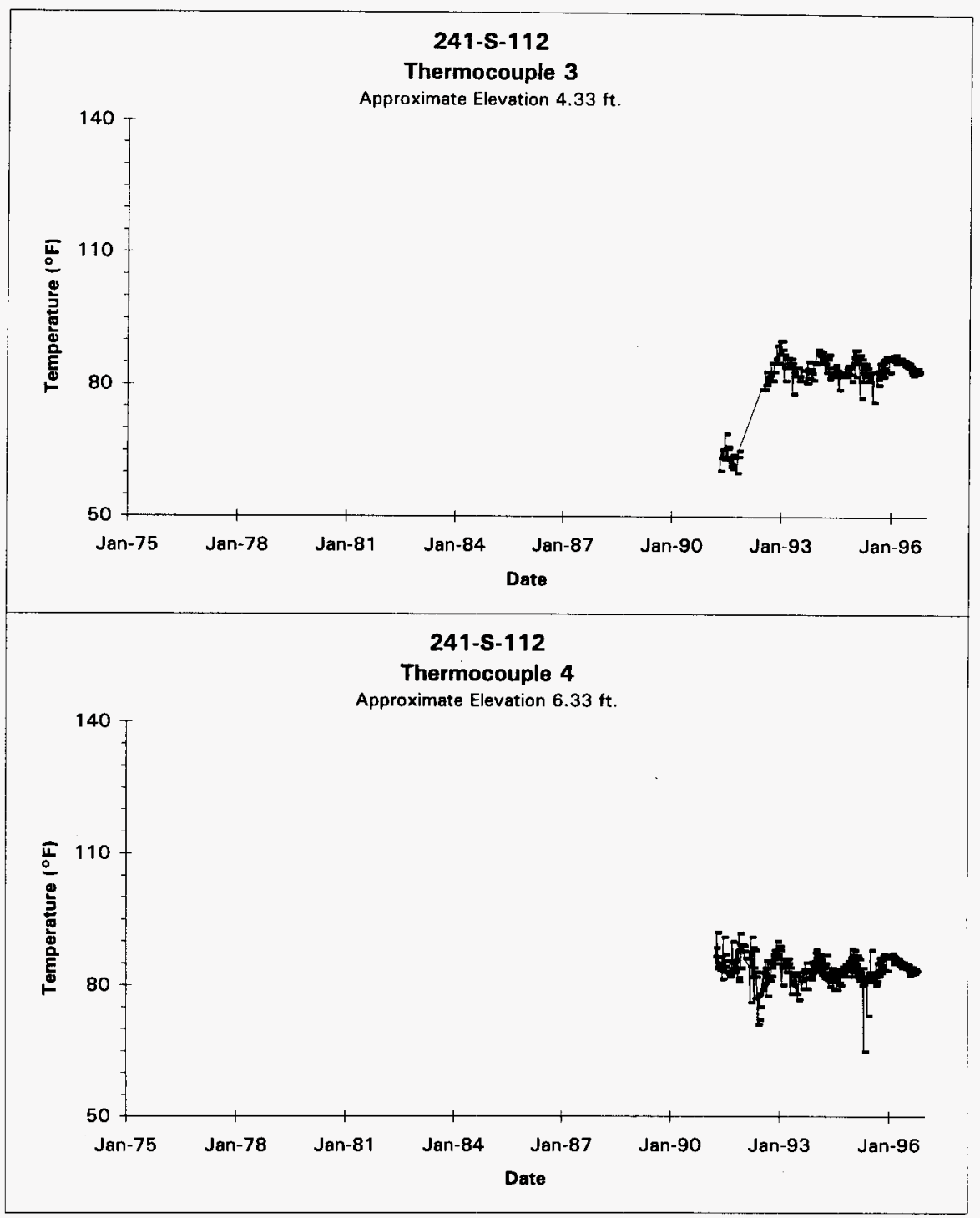

Data obtained from LMHC Surveillance Analysis Computer System (SACS), Oct 1, 1996.

D-58 


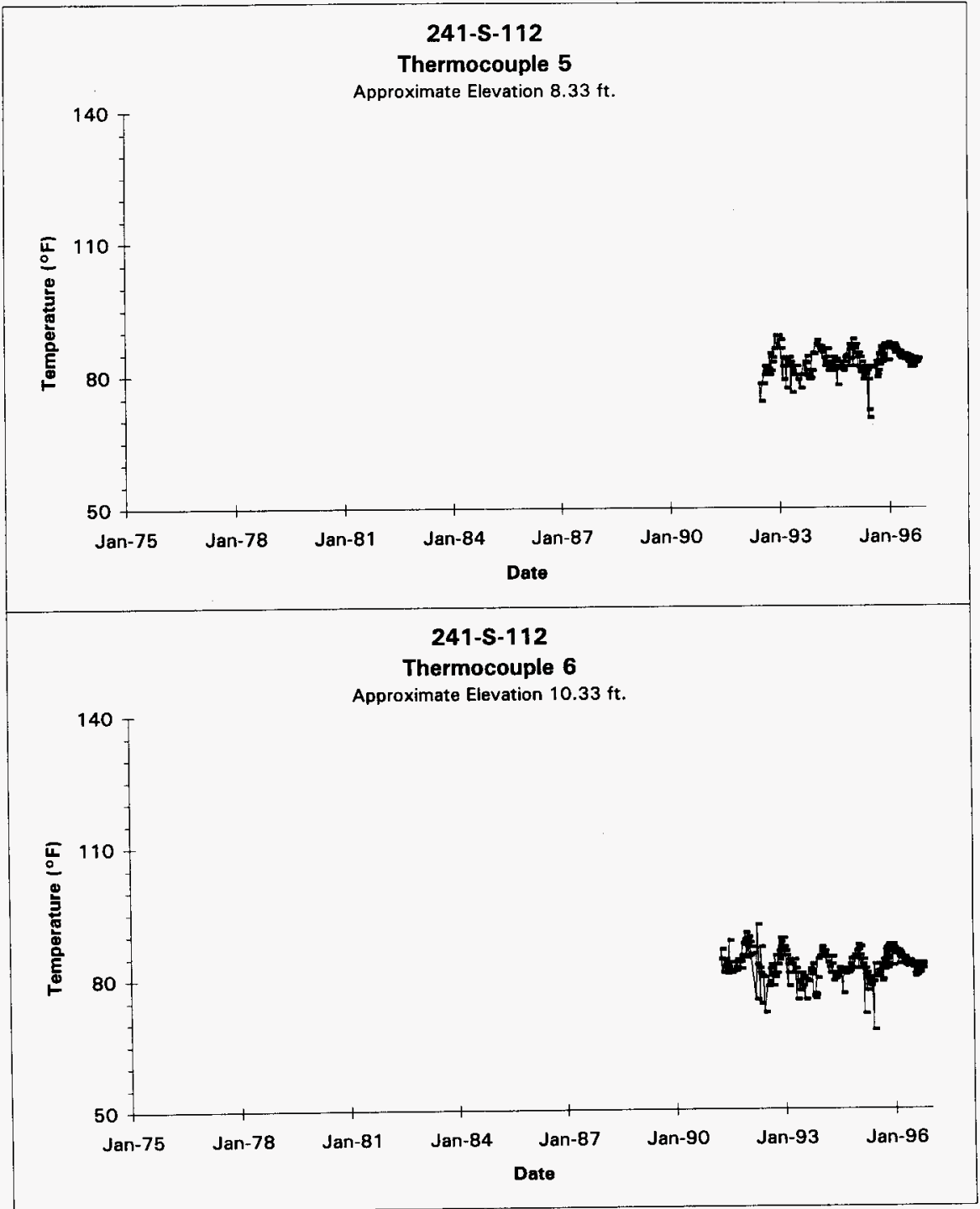

Data obtained from LMHC Surveillance Analysis Computer System (SACS), Oct 1, 1996.

D-59 


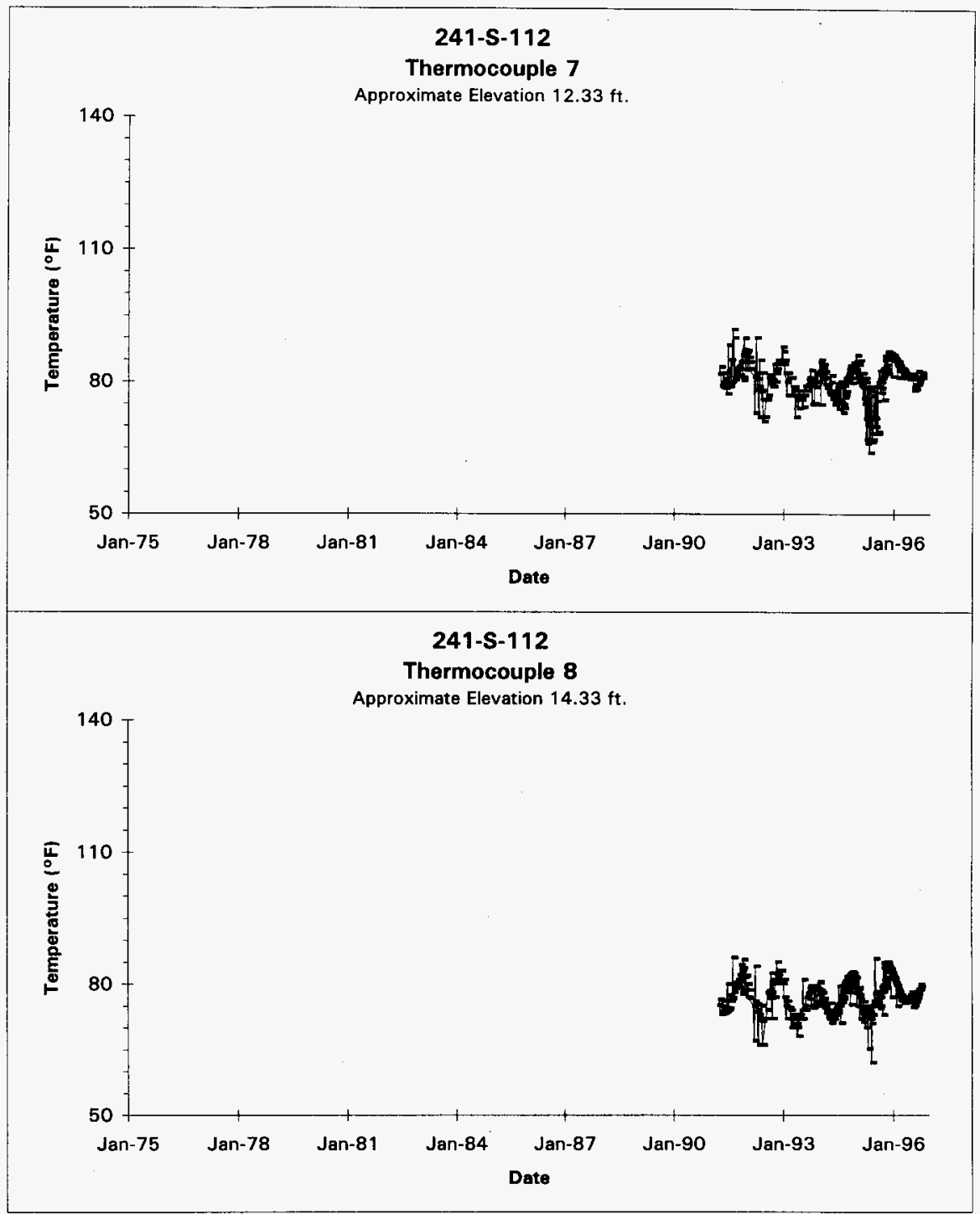

Data obtained from LMHC Surveillance Analysis Computer System (SACS), Oct 1, 1996.

D-60 


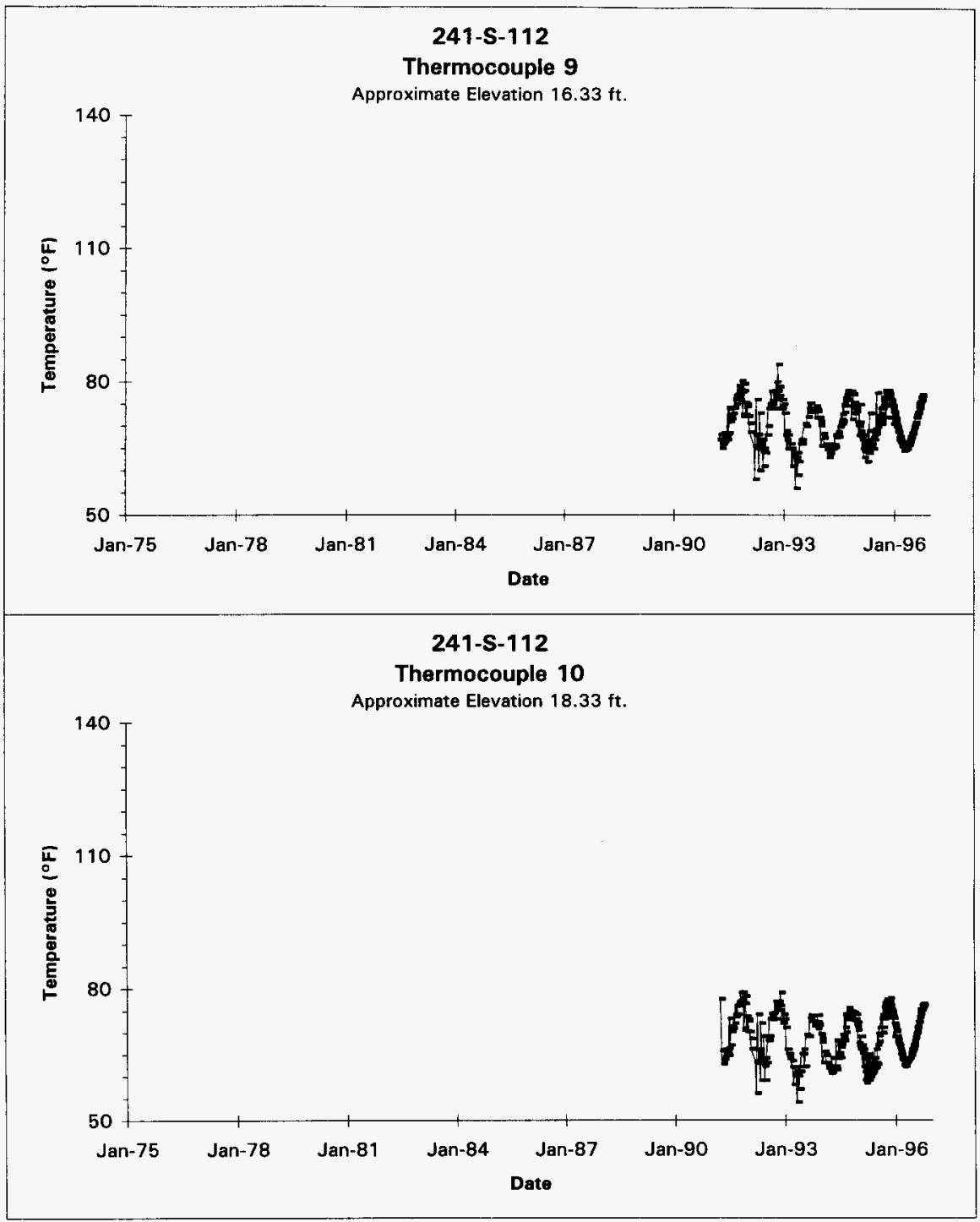

Data obtained from LMHC Surveillance Analysis Computer System (SACS), Oct 1, 1996.

D-61 


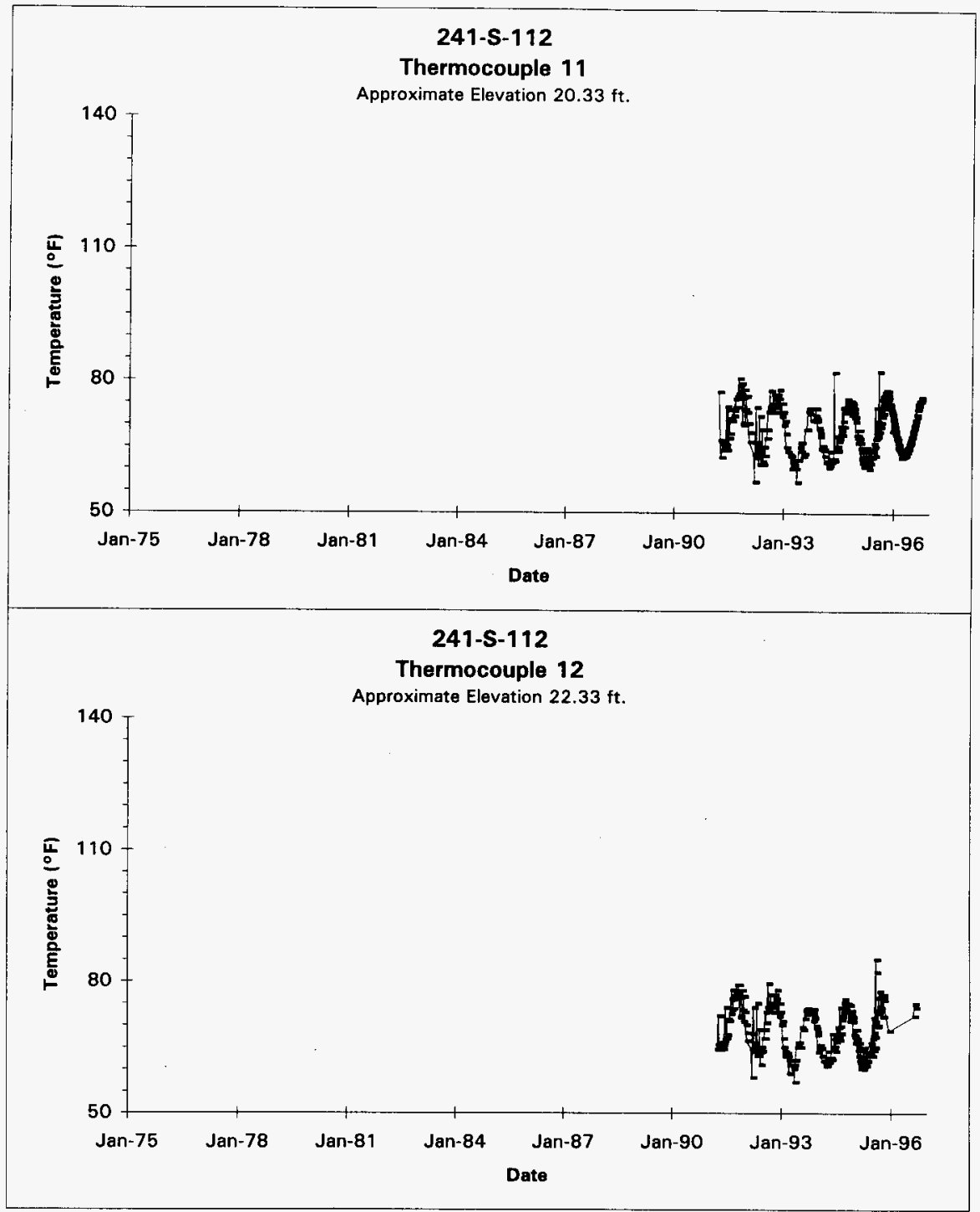

Data obtained from LMHC Surveillance Analysis Computer System (SACS), Oct 1, 1996. 
HNF-SD-WM-ER-323, Rev. I

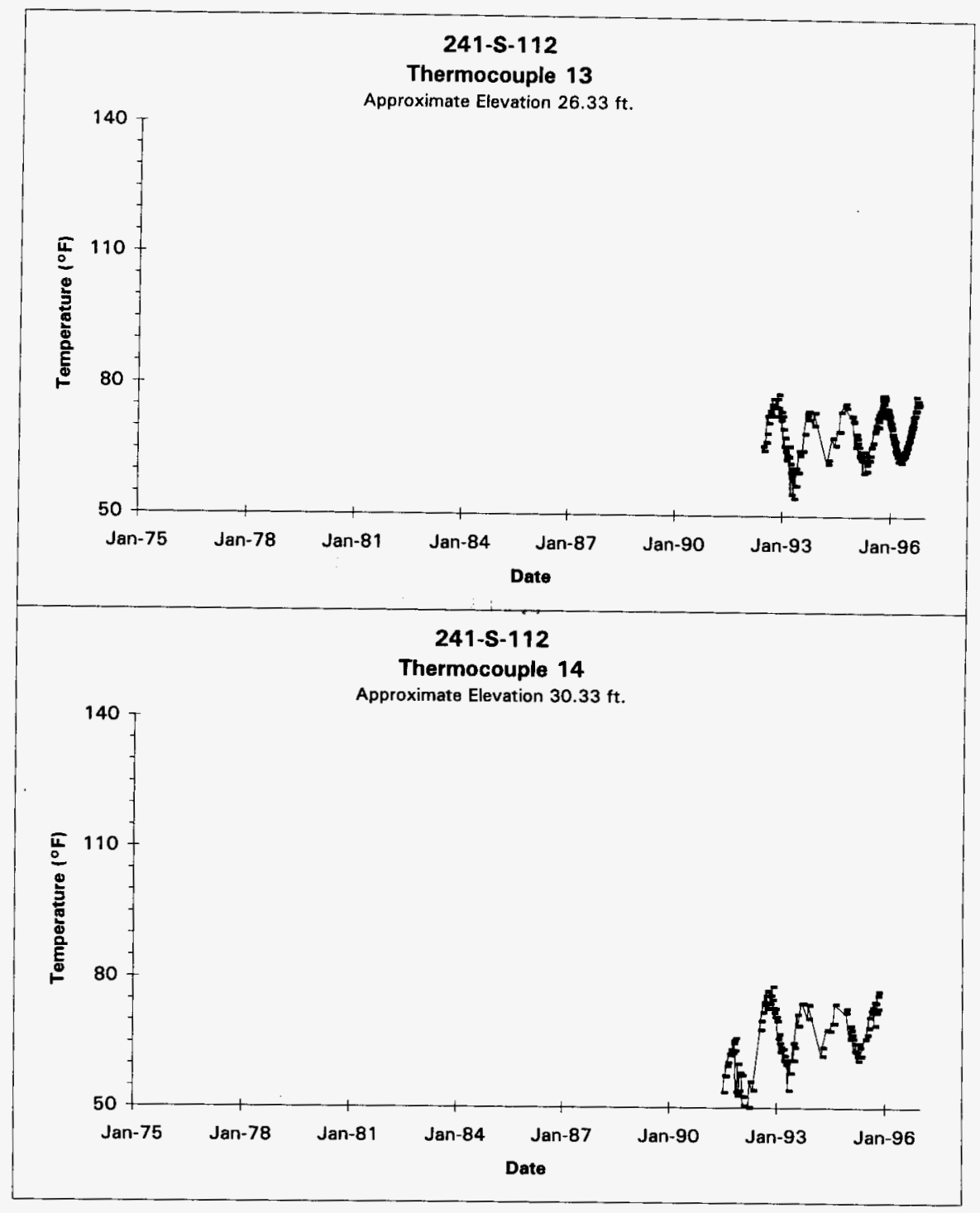

Data obtained from LMHC Surveillance Analysis Computer Systern (SACS), Oct 1, 1996 D-63 
THIS PAGE INTENTIONALLY LEFT BLANK 
另
离
$\frac{\mathbf{z}}{\mathbf{x}}$
m 
THIS P, GE INTENTIONALLY LEFT BLANK 
HNF-SD-WM-ER-323, Rev. 1

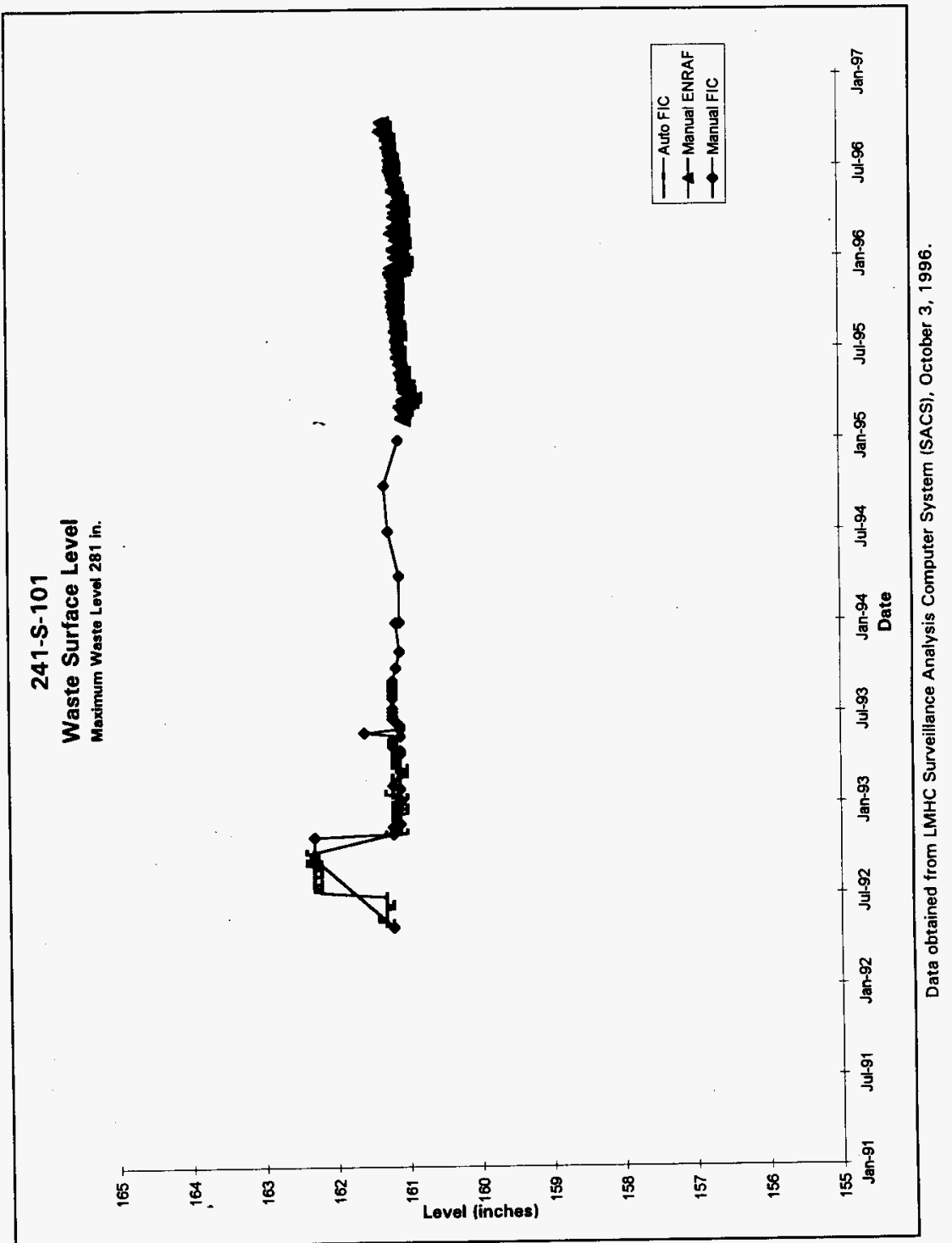

E-1 


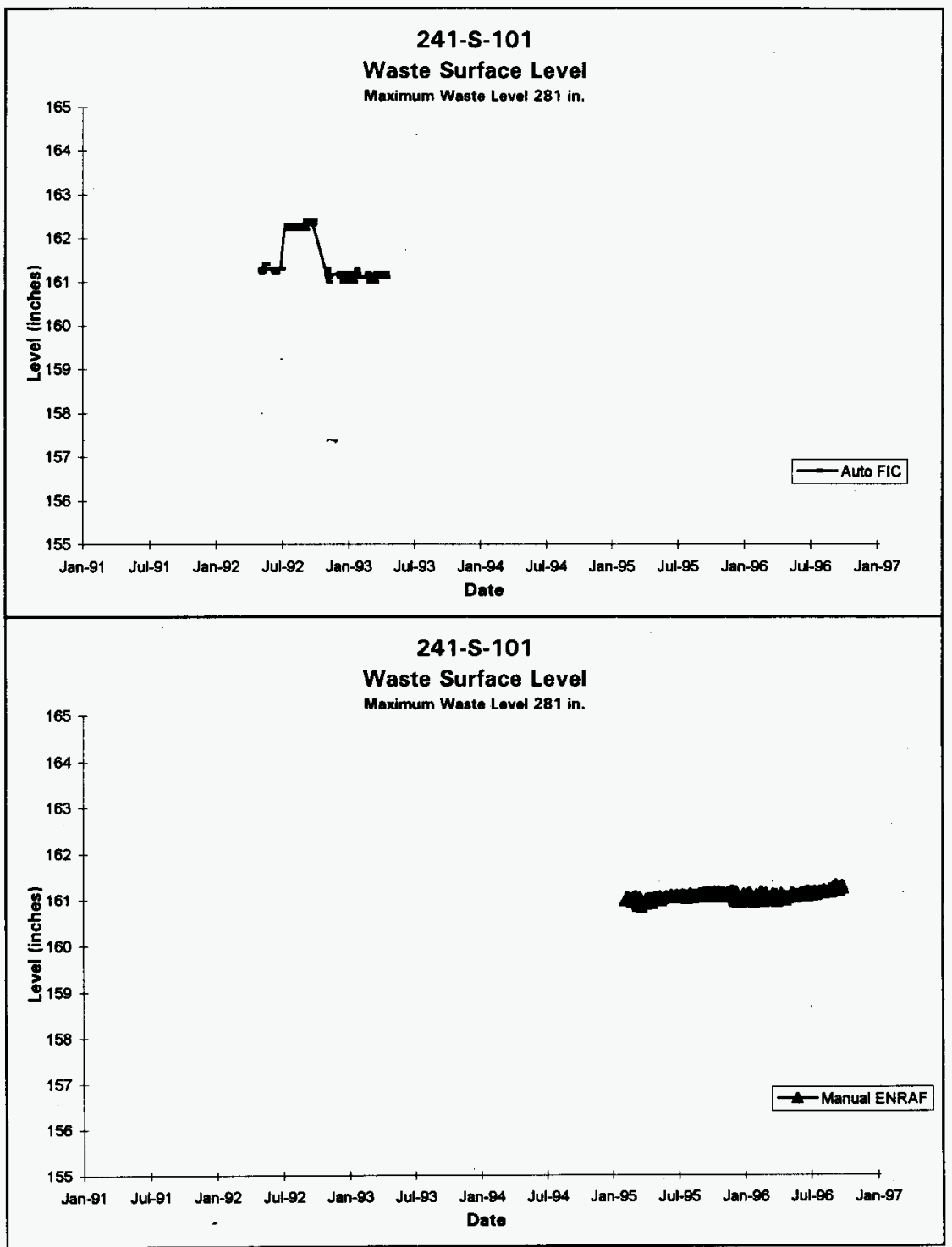

Data obtained from LMHC Surveillance Analysis Computer System (SACS), October 3, 1996.

E-2 


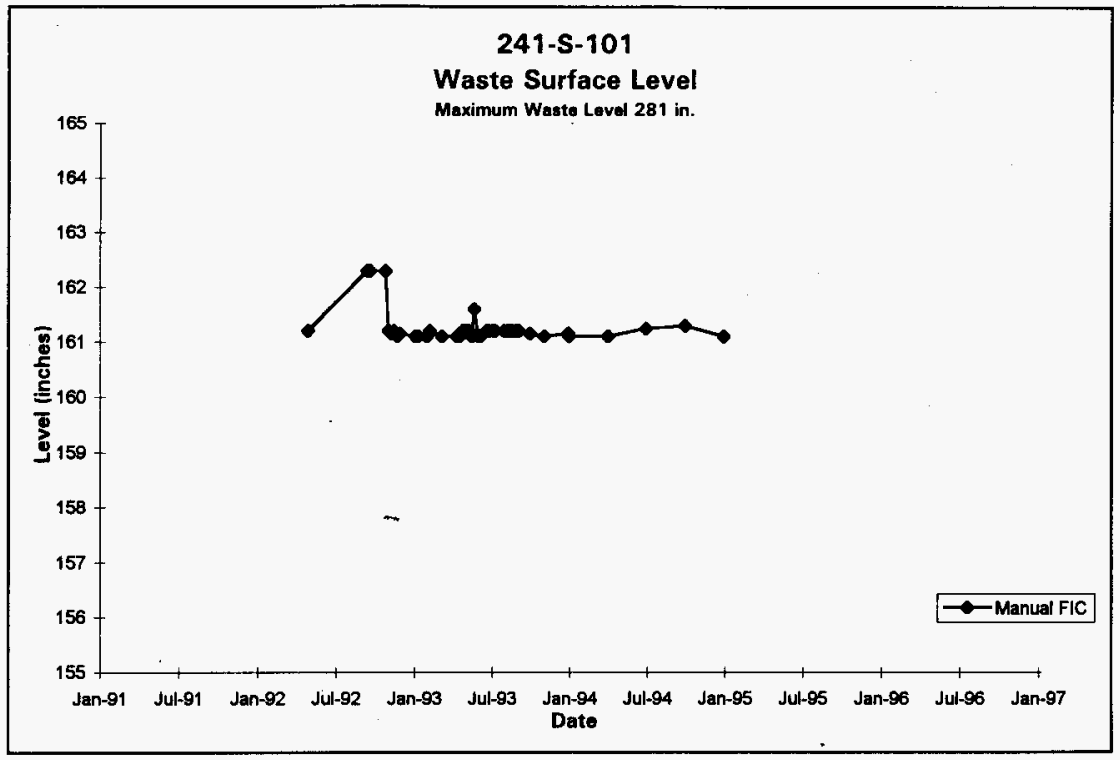

Data obtained from LMHC Surveillance Analysis Computer System (SACS), October 3, 1996. 


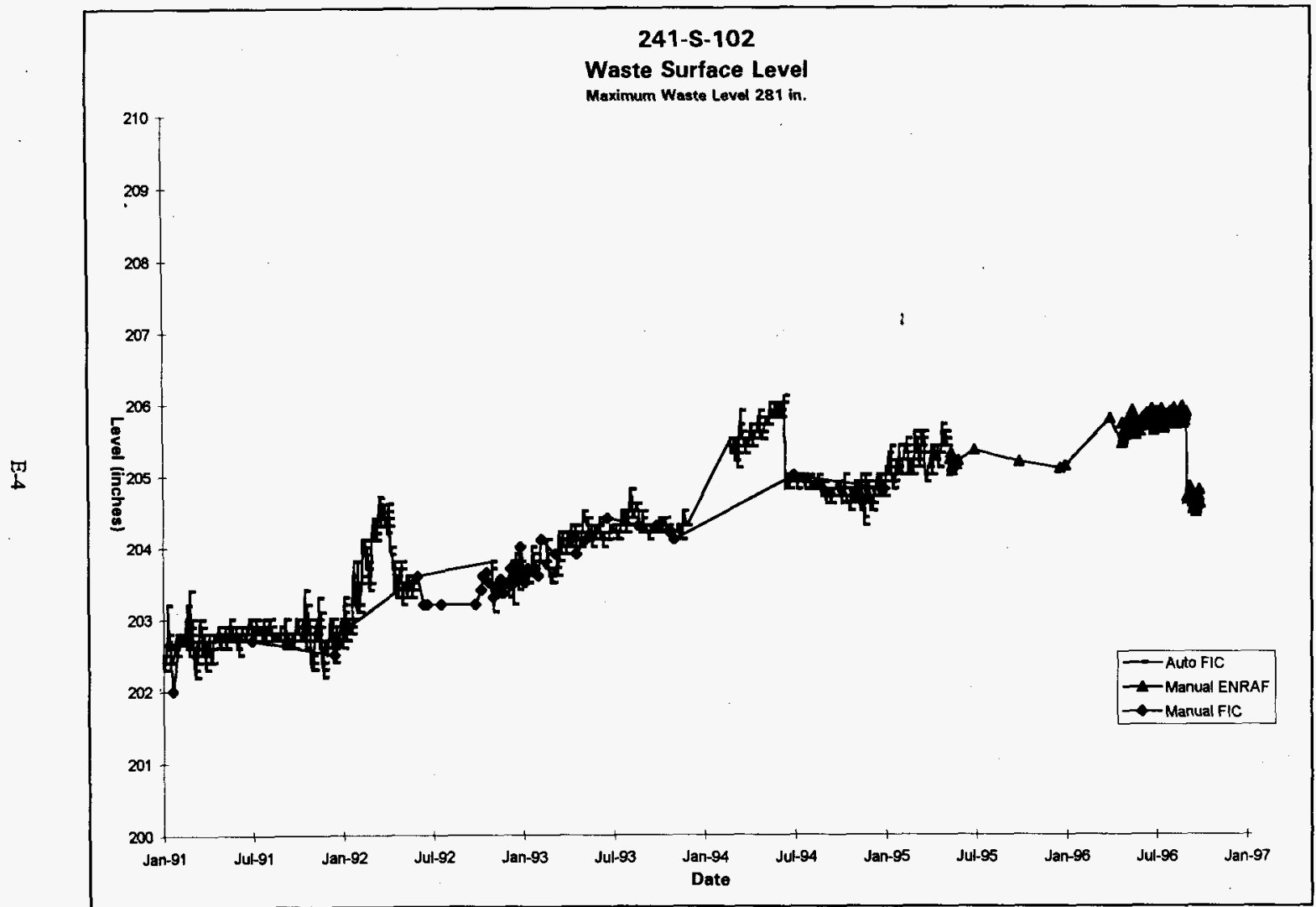

Data obtained from LMHC Surveillance Analysis Computer System (SACS), October 3, 1996. 


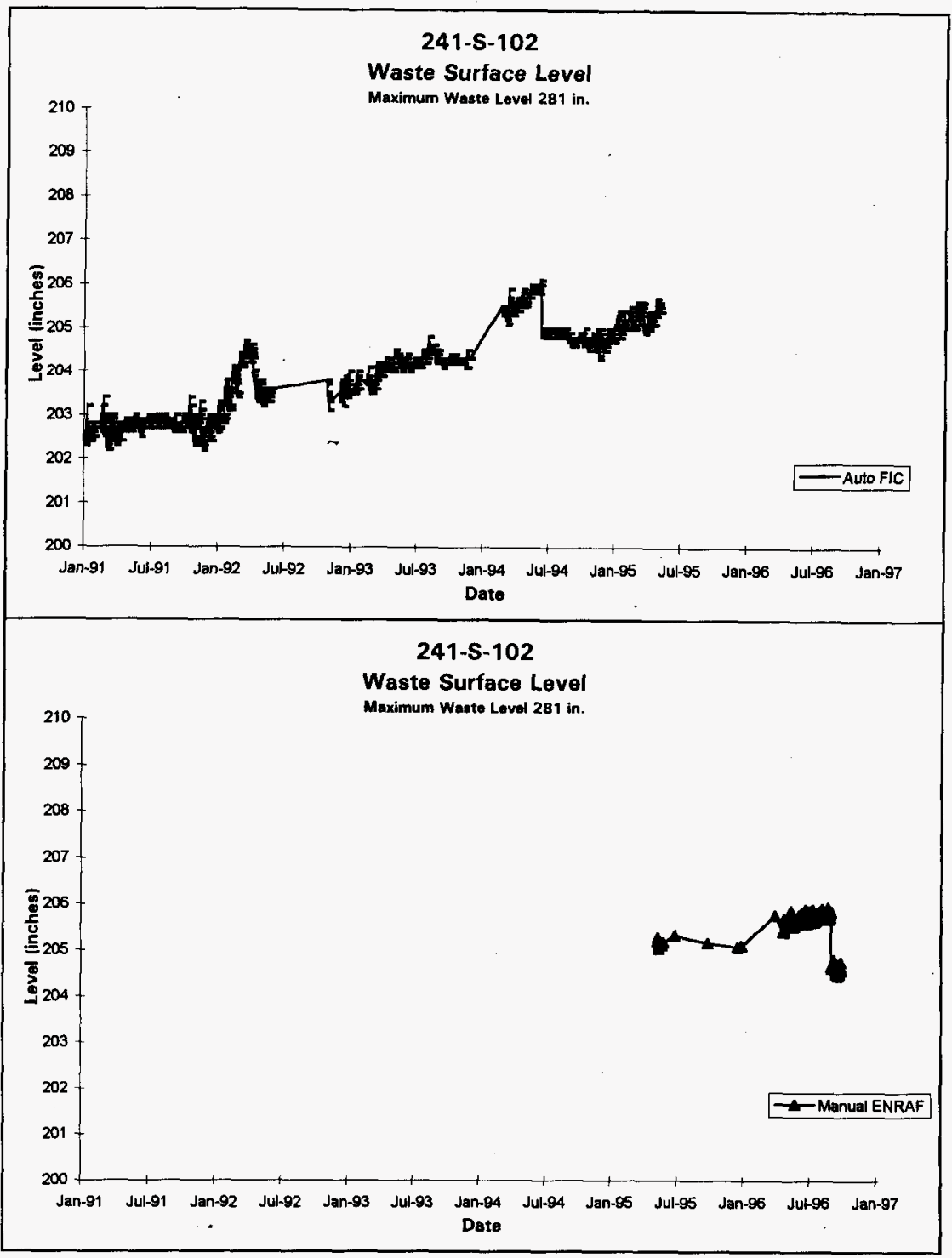

Data obtained from LMHC Surveillance Analysis Computer System (SACS), October 3, 1996. 


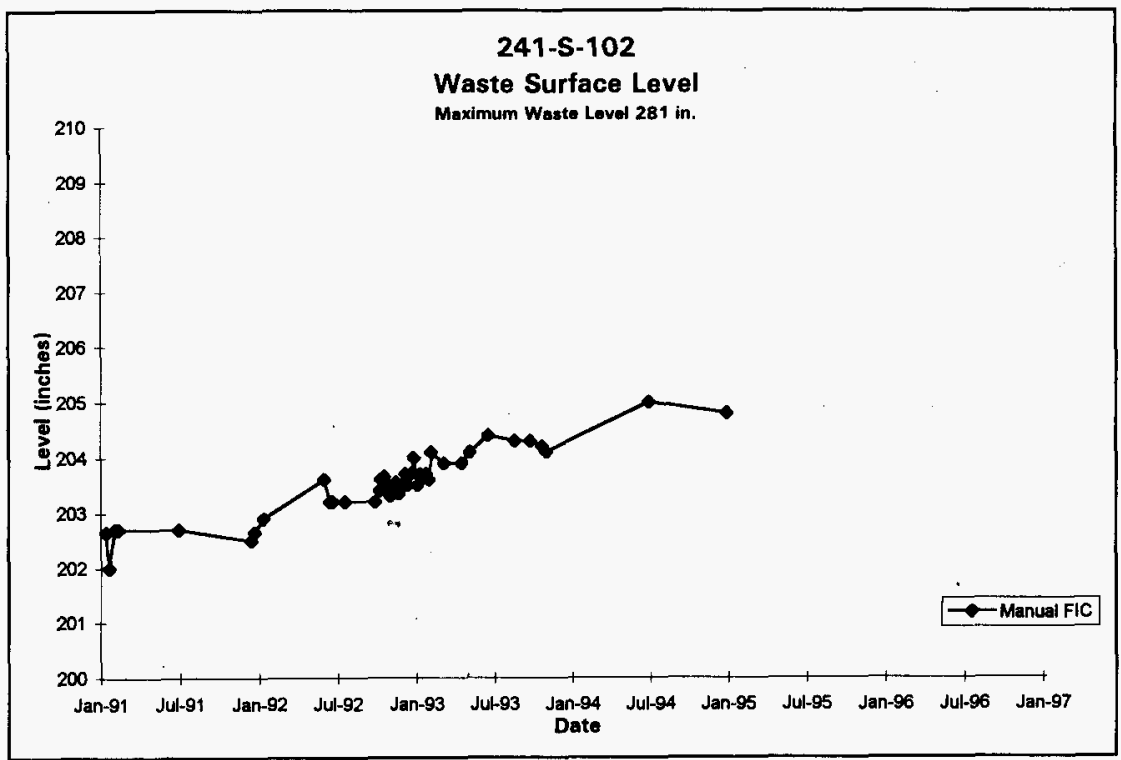

Data obtained from LMHC Surveillance Analysis Computer System (SACS), October 3, 1996. 


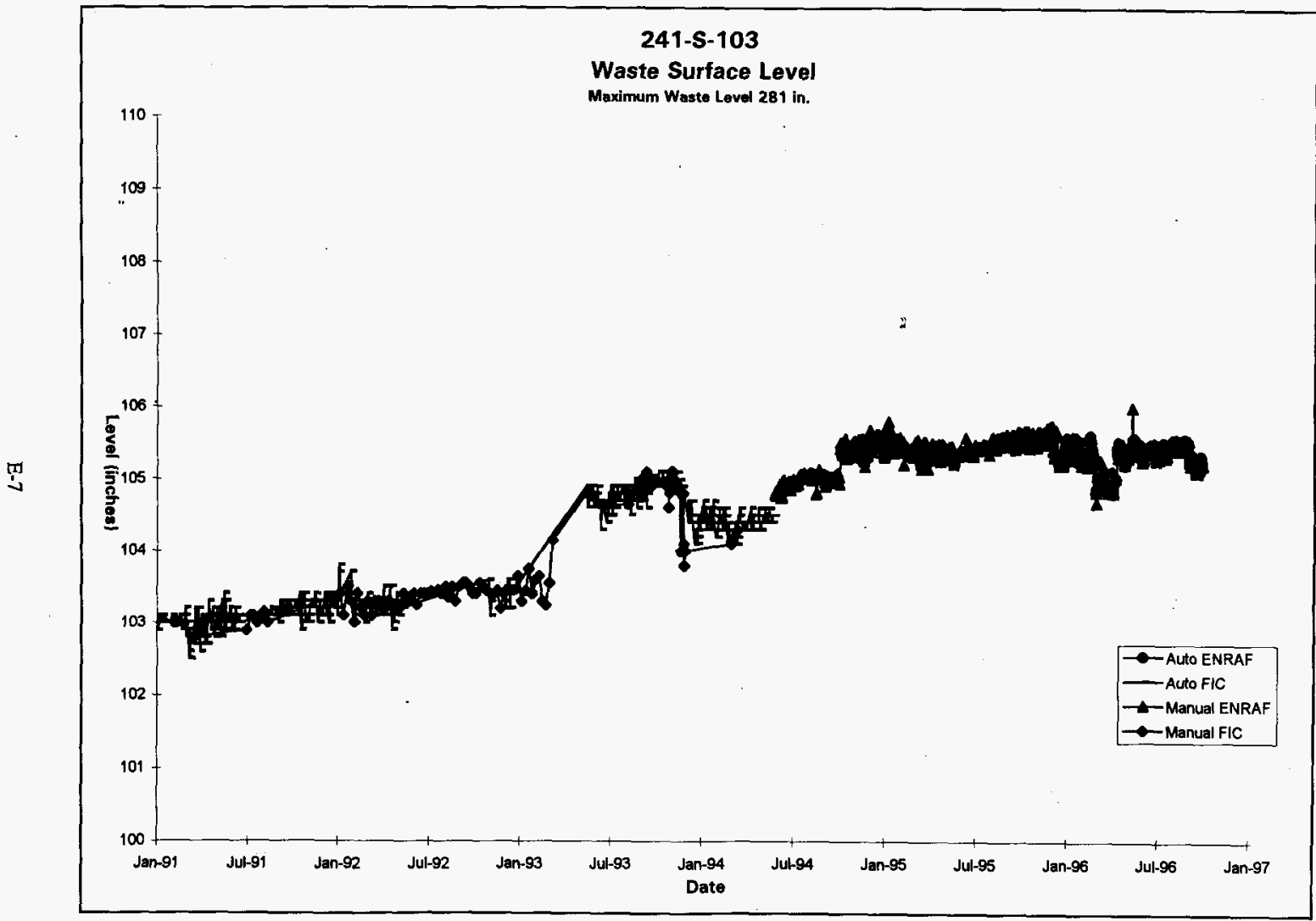

Data obtained from LMHC Surveillance Analysis Computer Systern (SACS), October 3, 1996. 


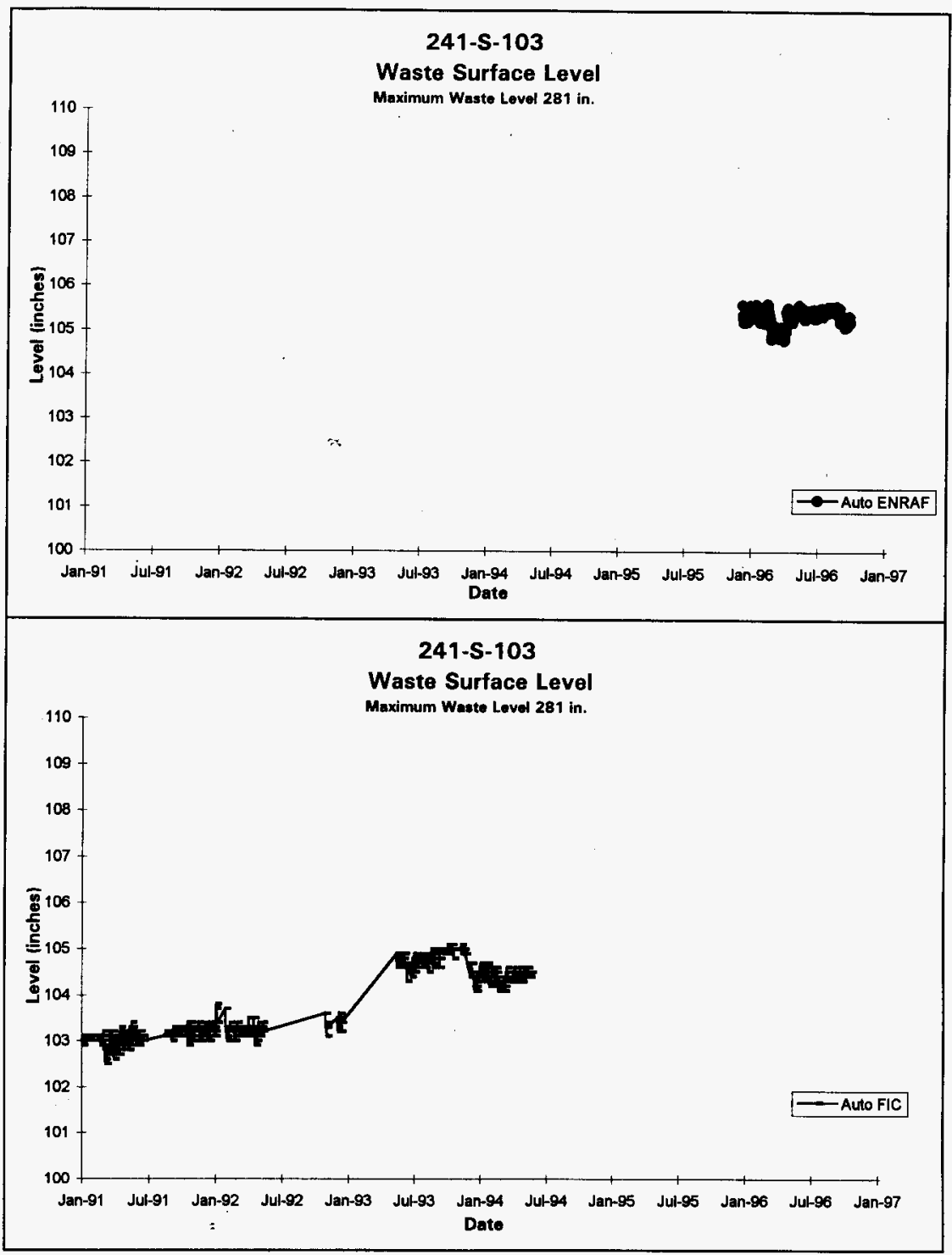

Data obtained from LMHC Surveillance Analysis Computer System (SACS), October 3, 1996. 


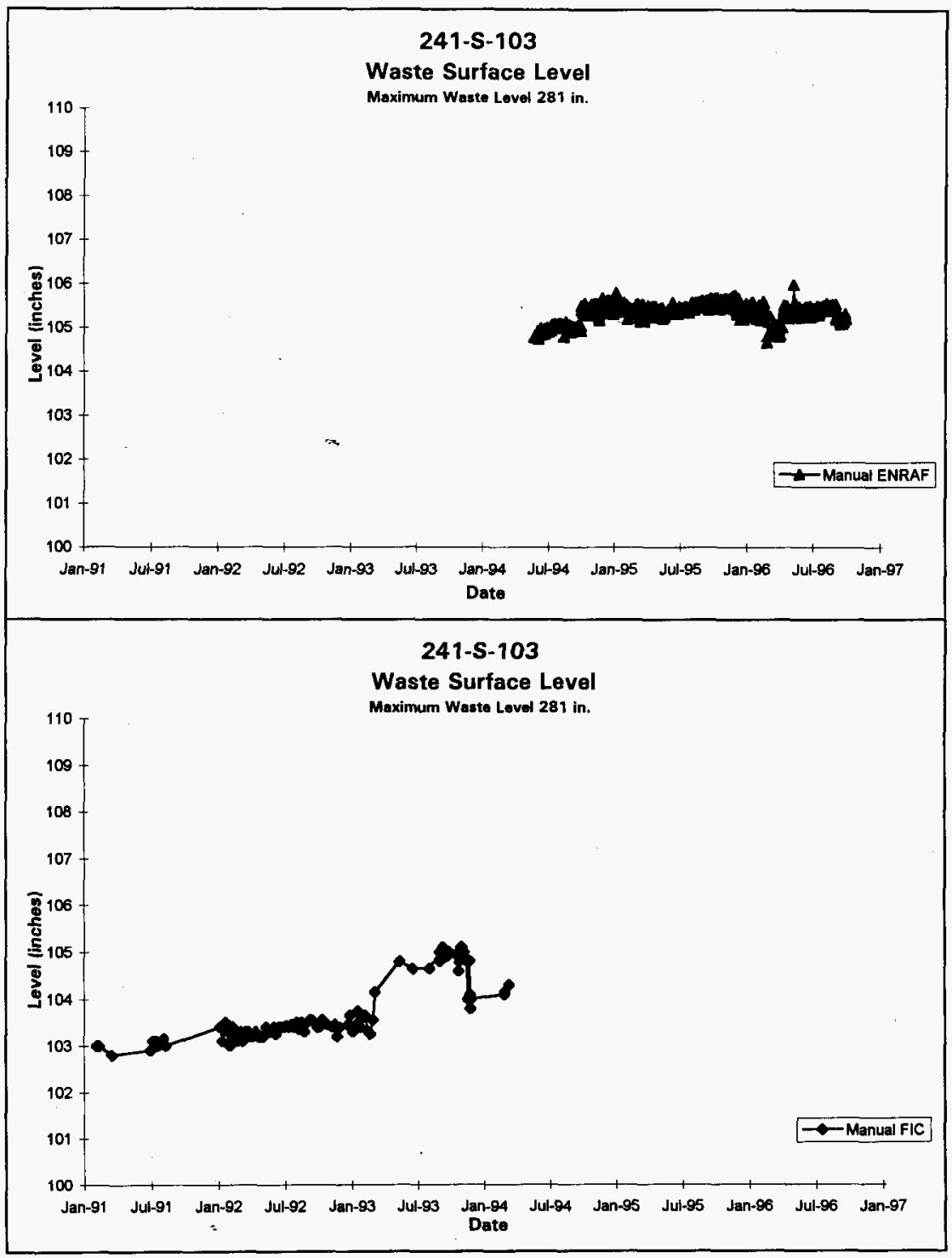

Data obtained from LMHC Surveillance Analysis Computer System (SACS), October 3, 1996. 


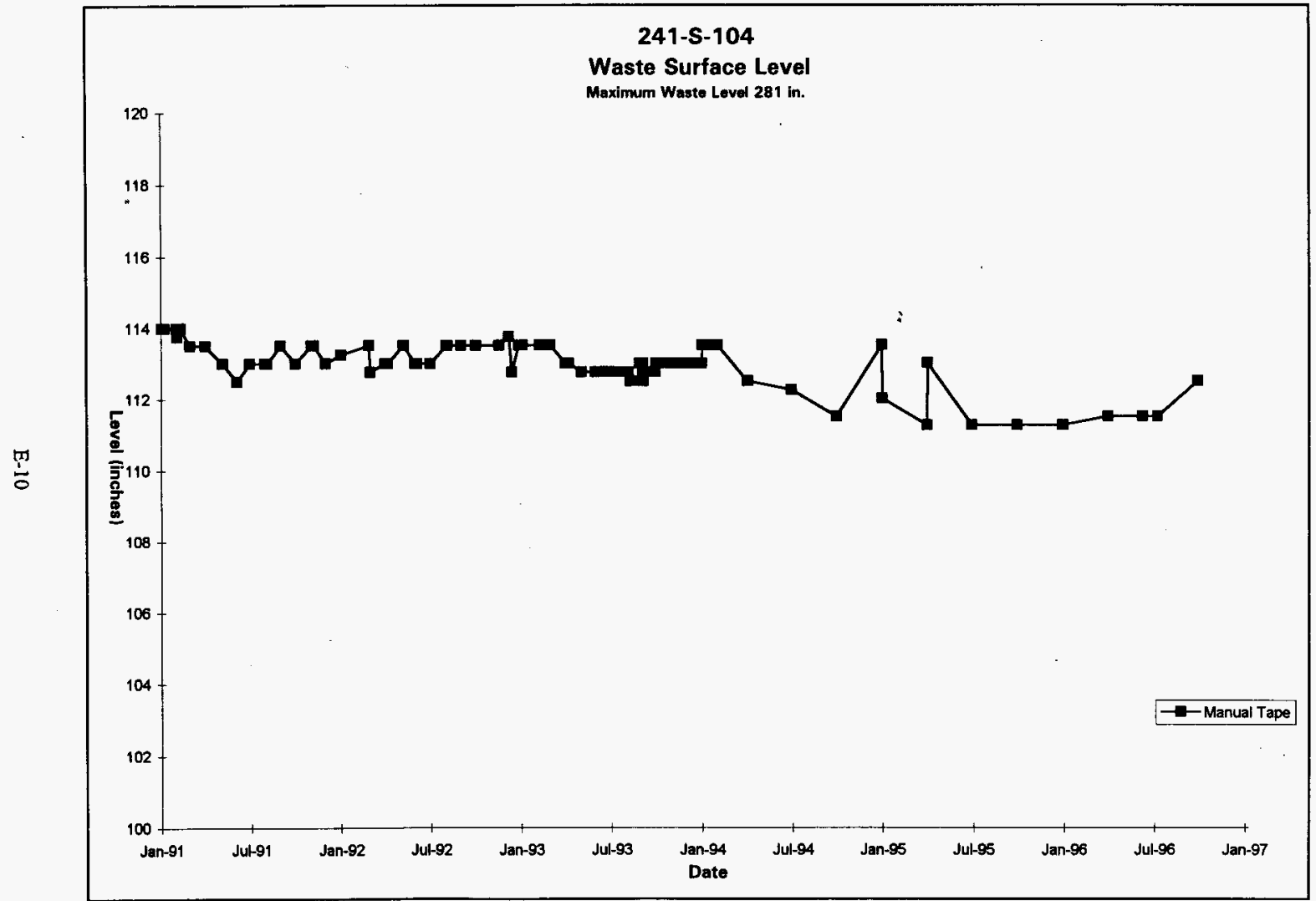




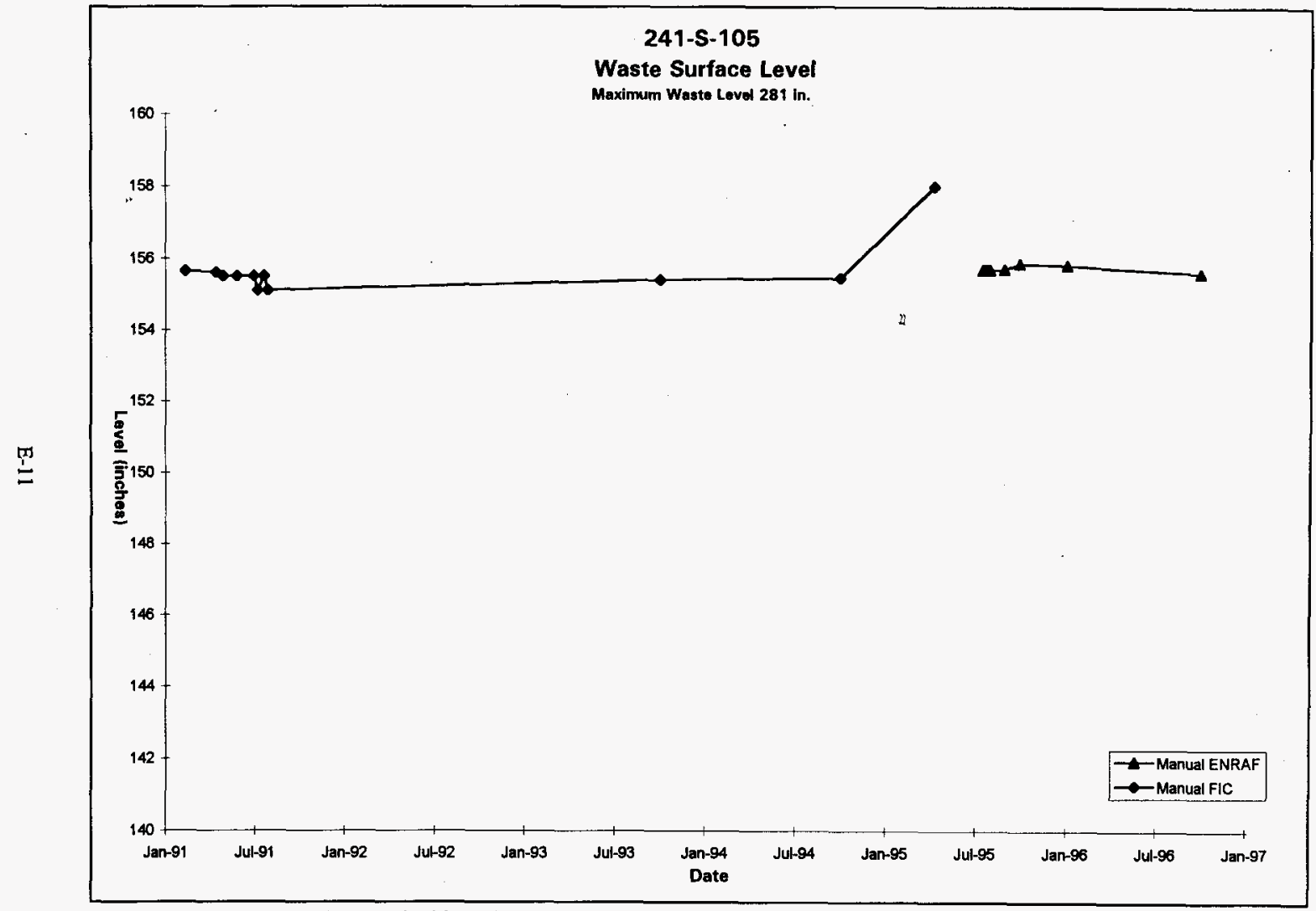




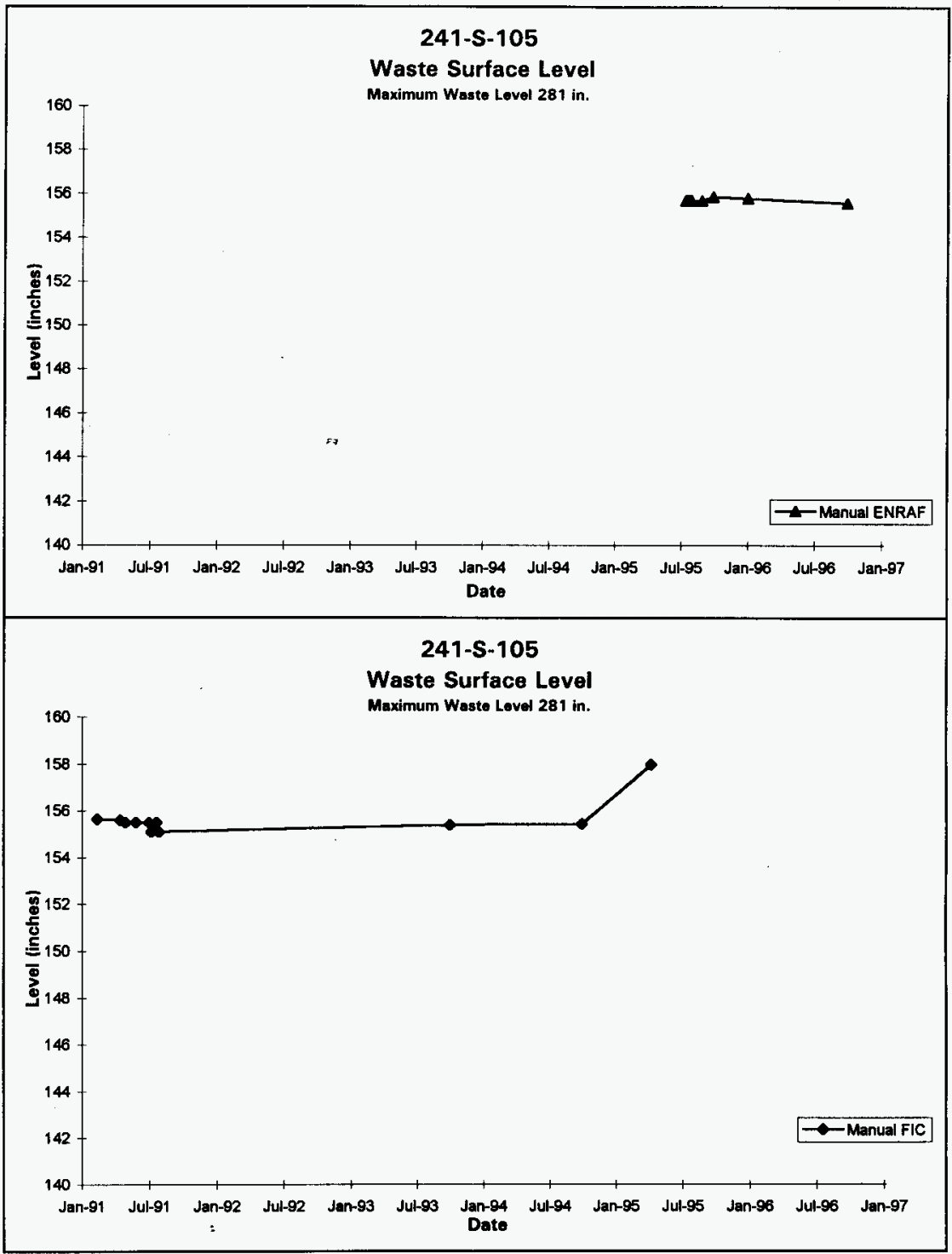

Data obtained from LMHC Surveillance Analysis Computer System (SACS), October 3, 1996.

E-12 


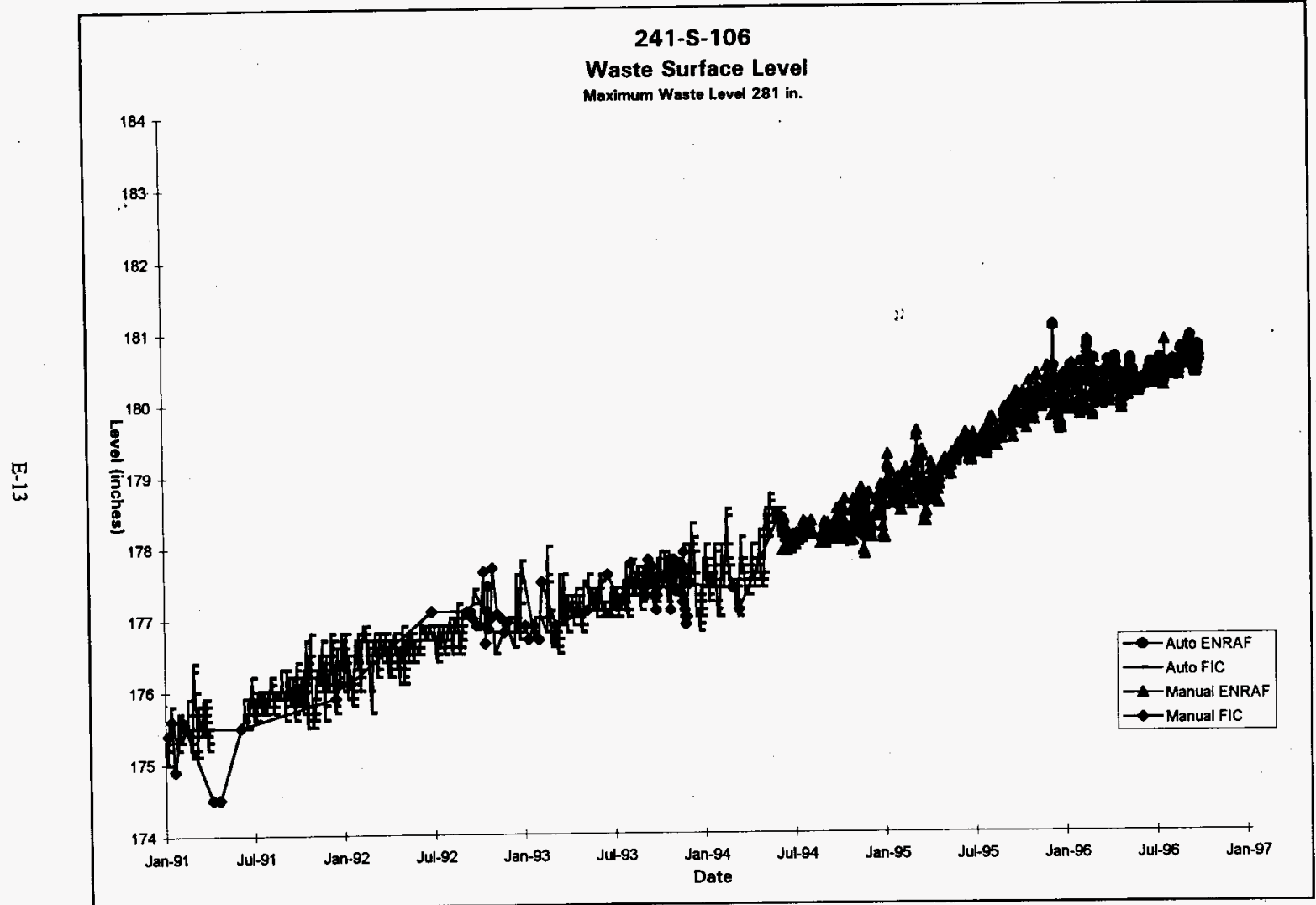

Data obtained from LMHC Surveillance Analysis Computer System (SACS), October 3, 1996. 


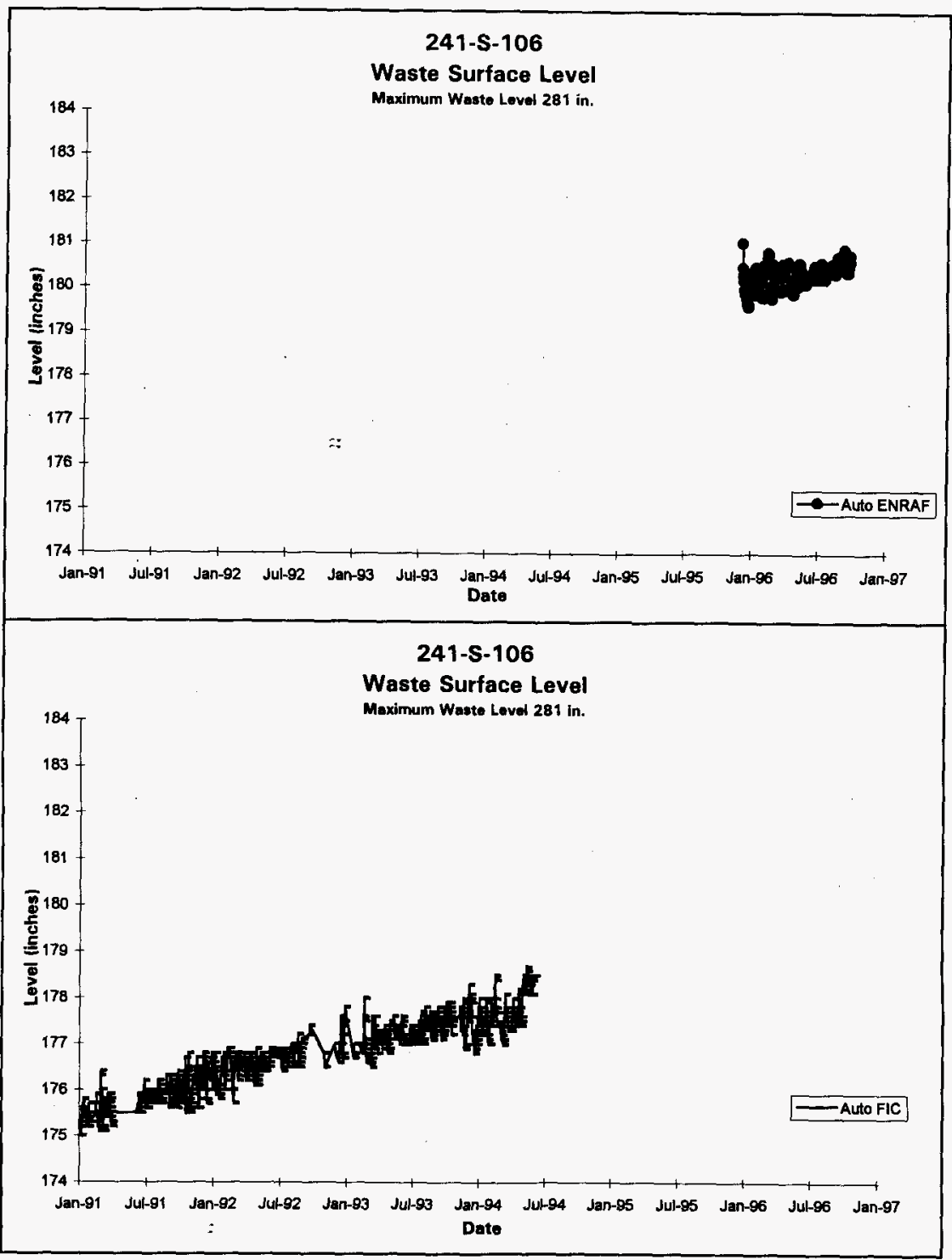

Data obtained from LMHC Surveillance Analysis Computer System (SACS), October 3, 1996. 


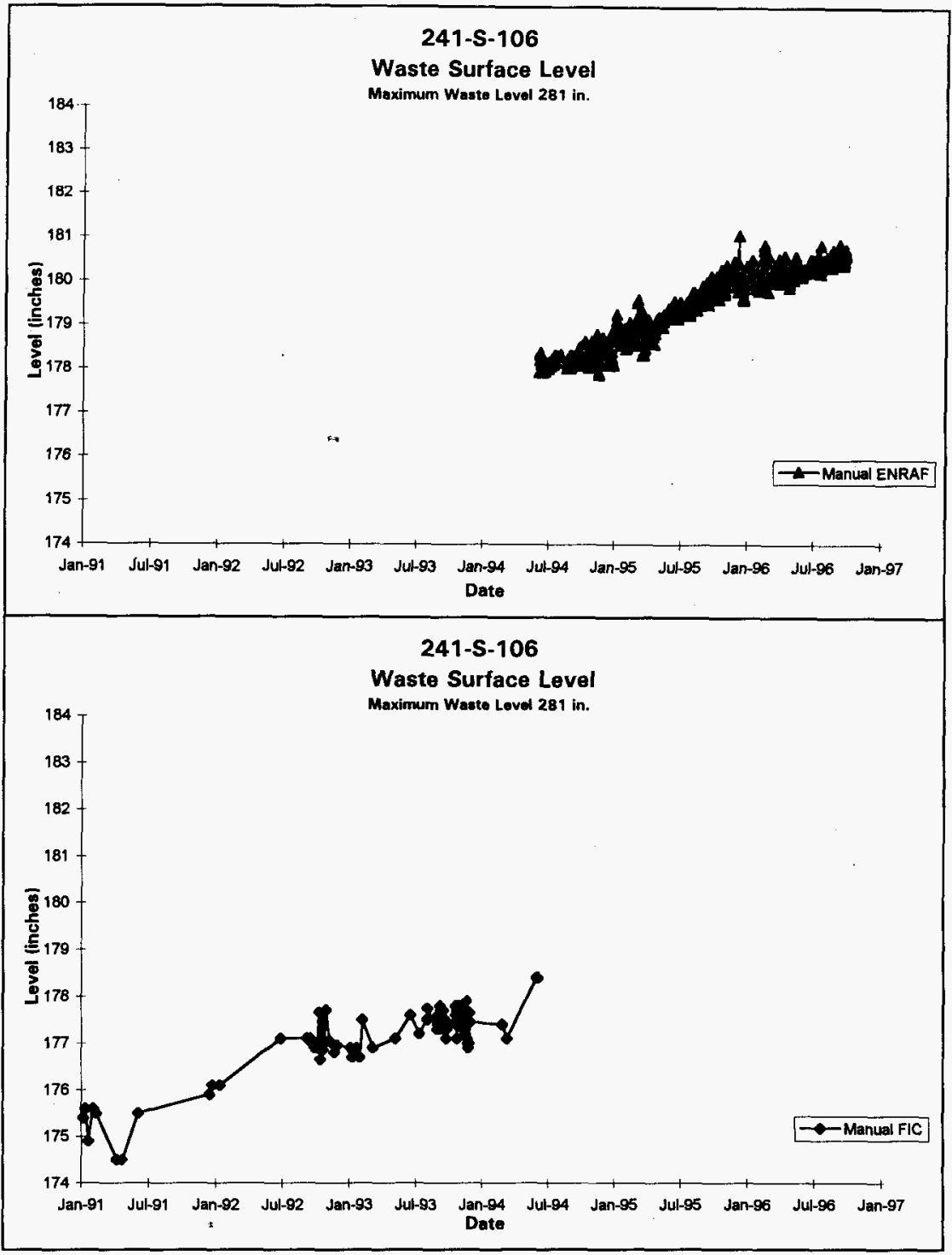

Data obtained from LMHC Surveillance Analysis Computer System (SACS), October 3, 1996.

E-15 
HNF-SD-WM-ER-323, Rev. 1

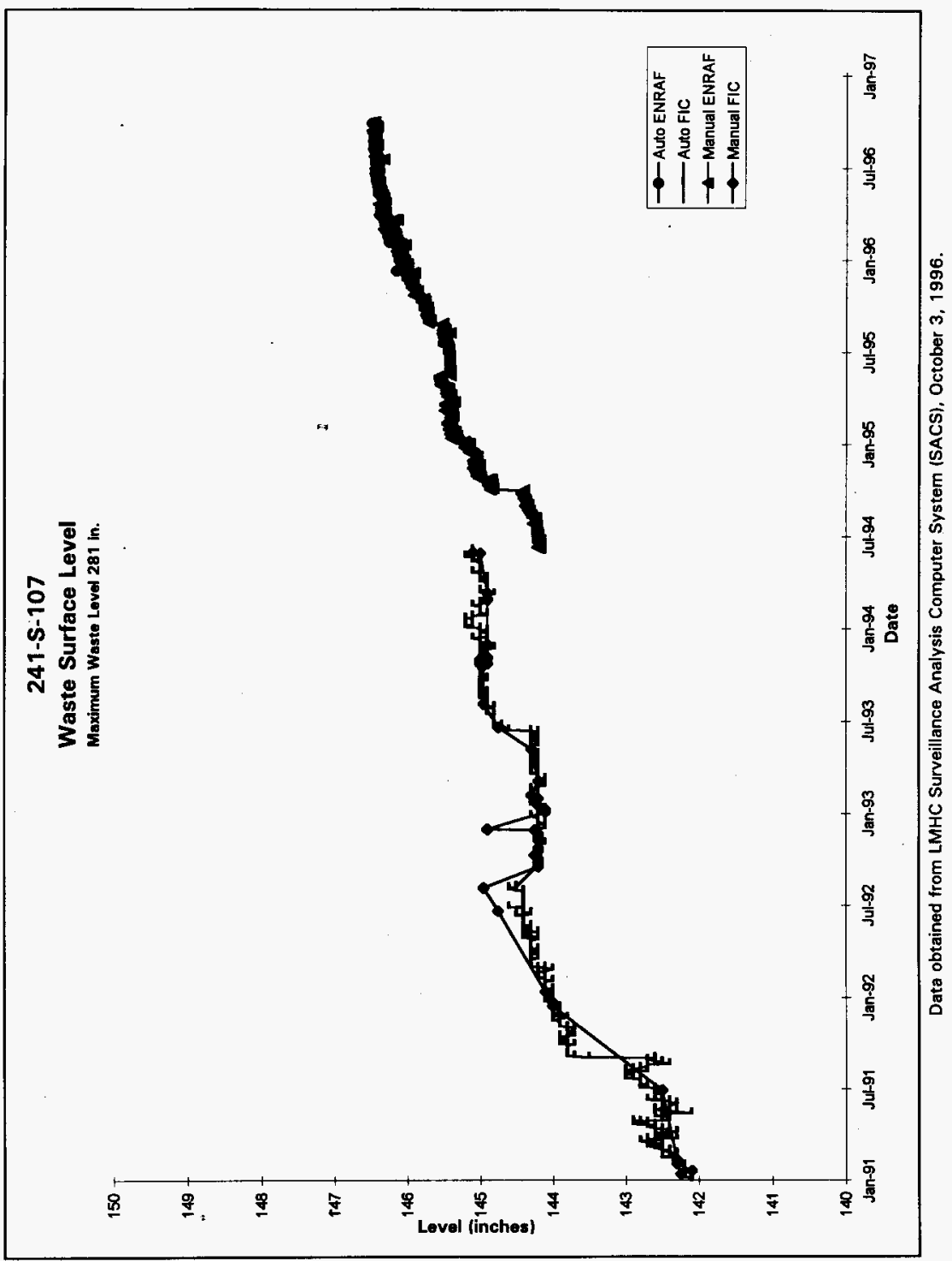




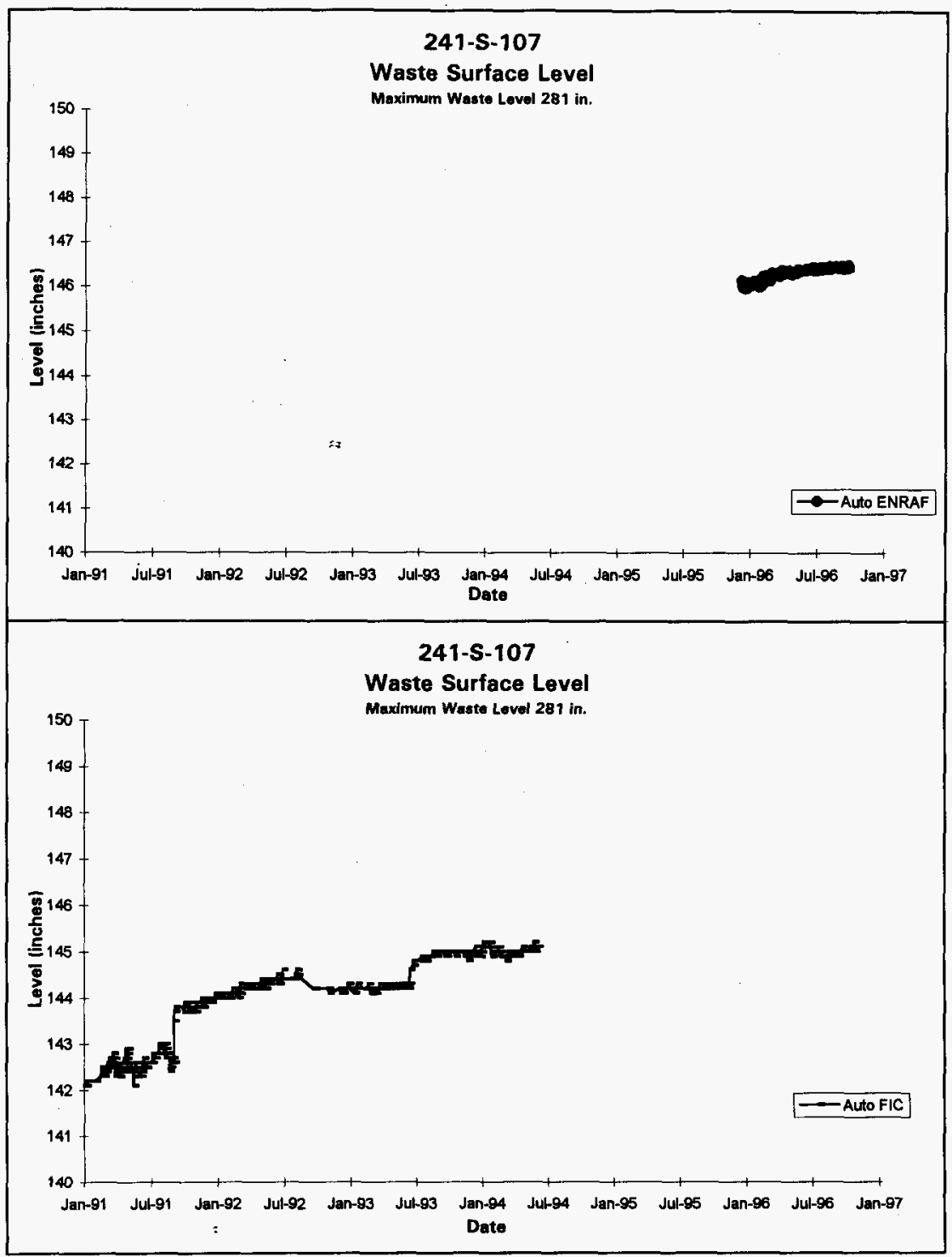

Data obtained from LMHC Surveillance Analysis Computer System (SACS), October 3, 1996. 


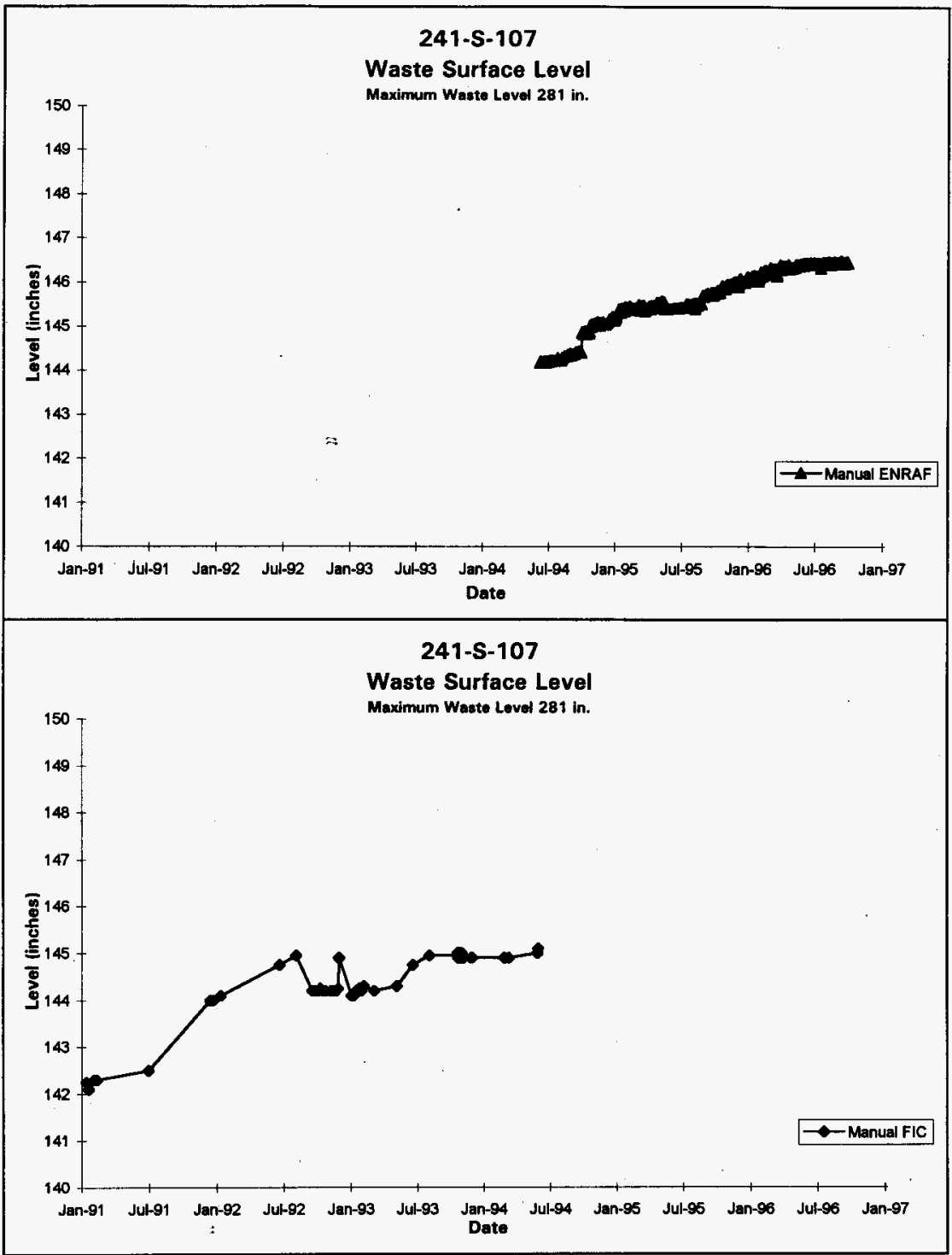

Data obtained from LMHC Surveillance Analysis Computer System (SACS), October 3, 1996. 


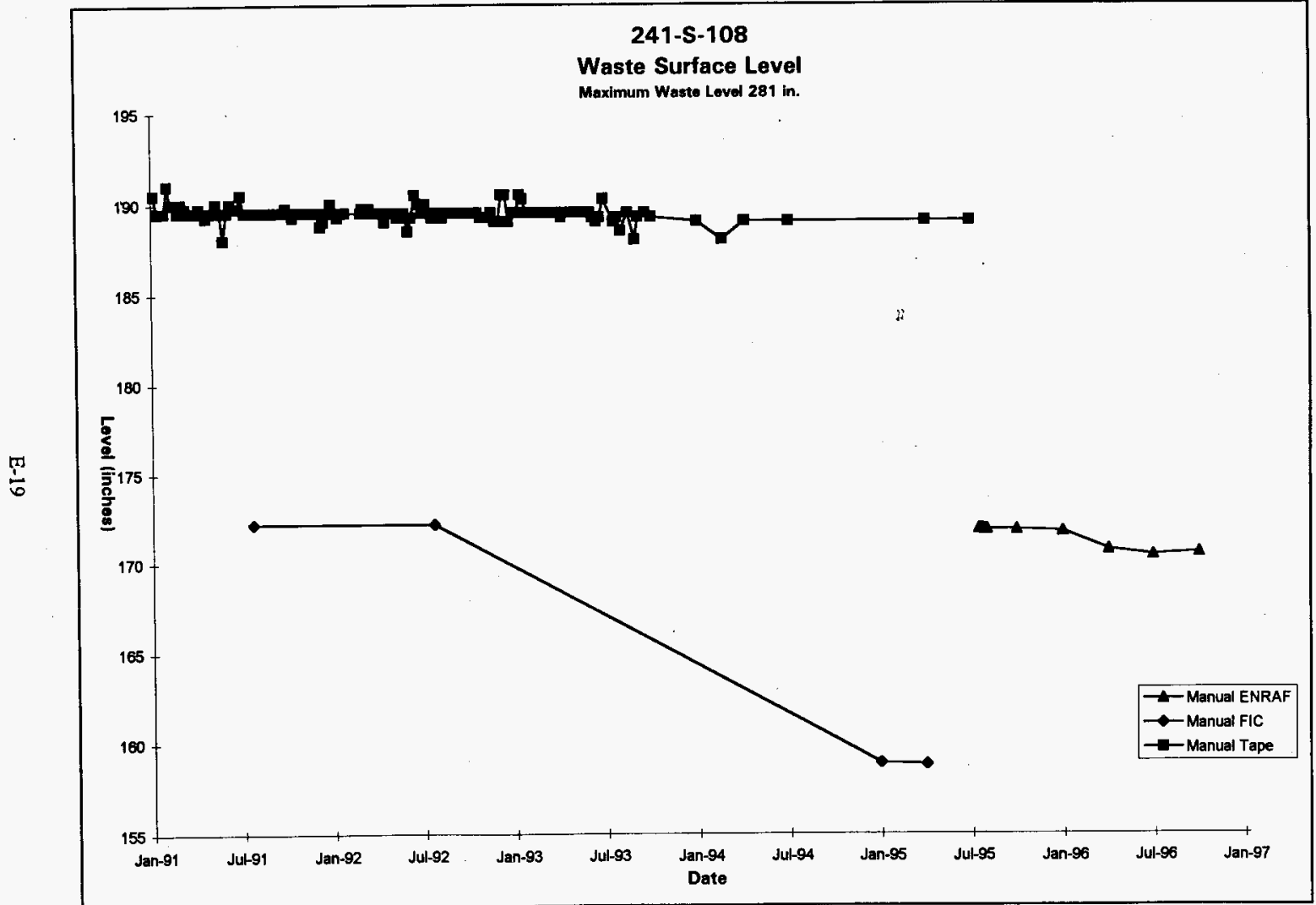

Data obtained from LMHC Surveillance Analysis Computer System (SACS), October 3, 1996. 


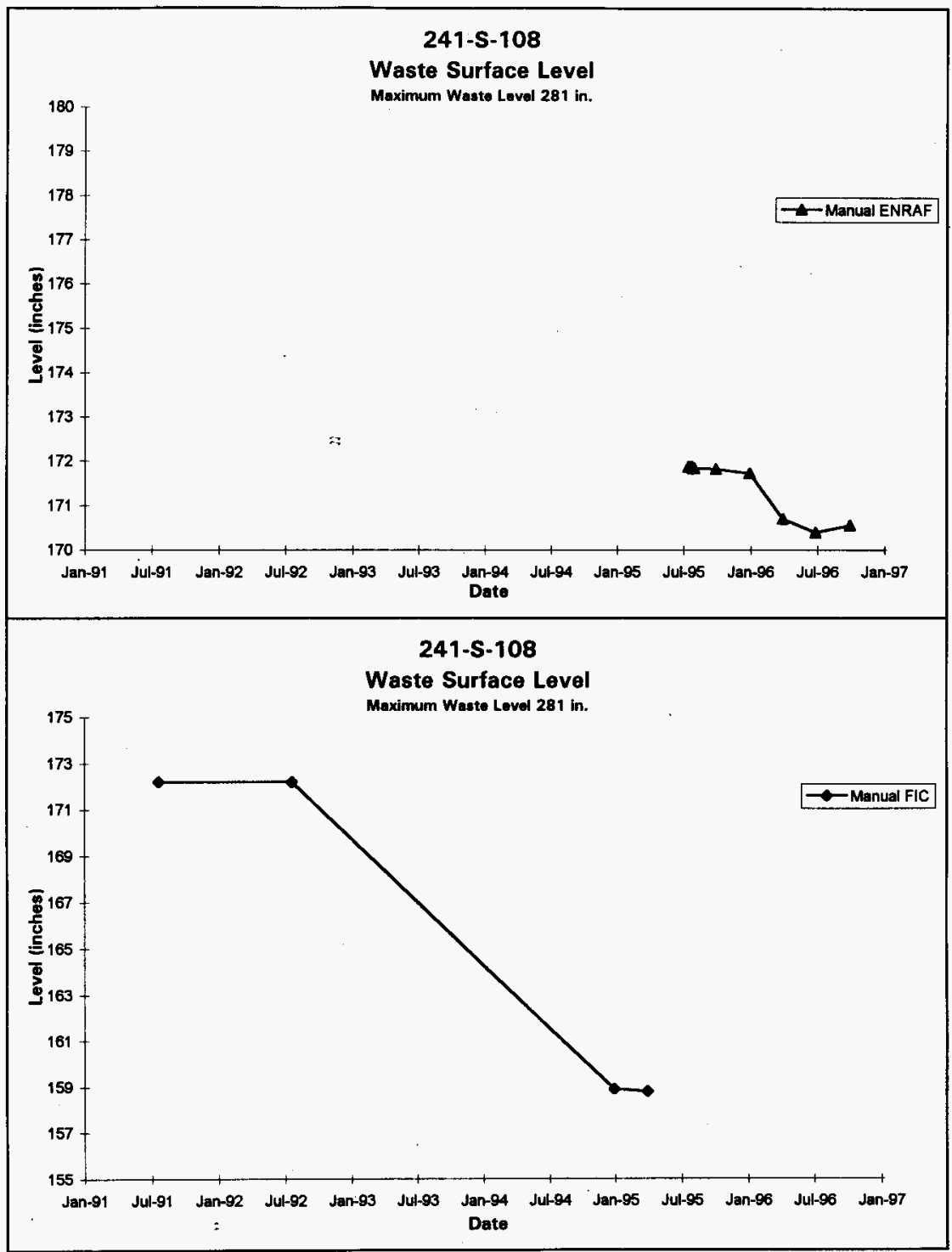

Data obtained from LMHC Surveillance Analysis Computer System (SACS), October 3, 1996. 


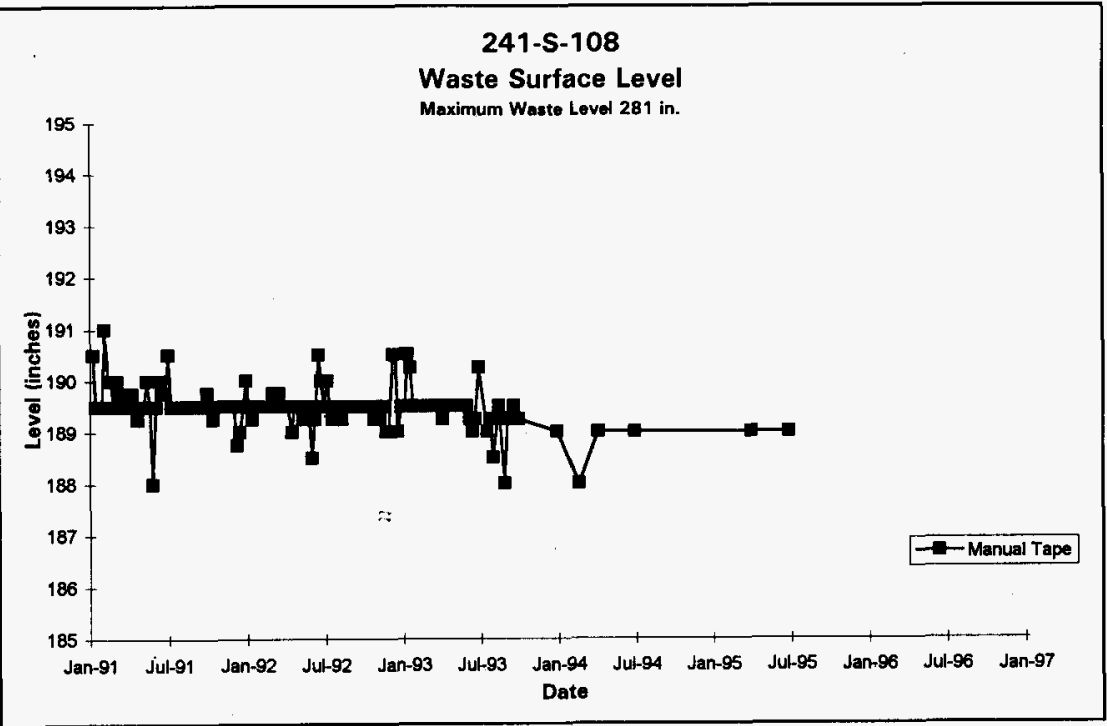

Data obtained from LMHC Surveillance Analysis Computer System (SACS), October 3, 1996. 
HNF-SD-WM-ER-323, Rev. 1

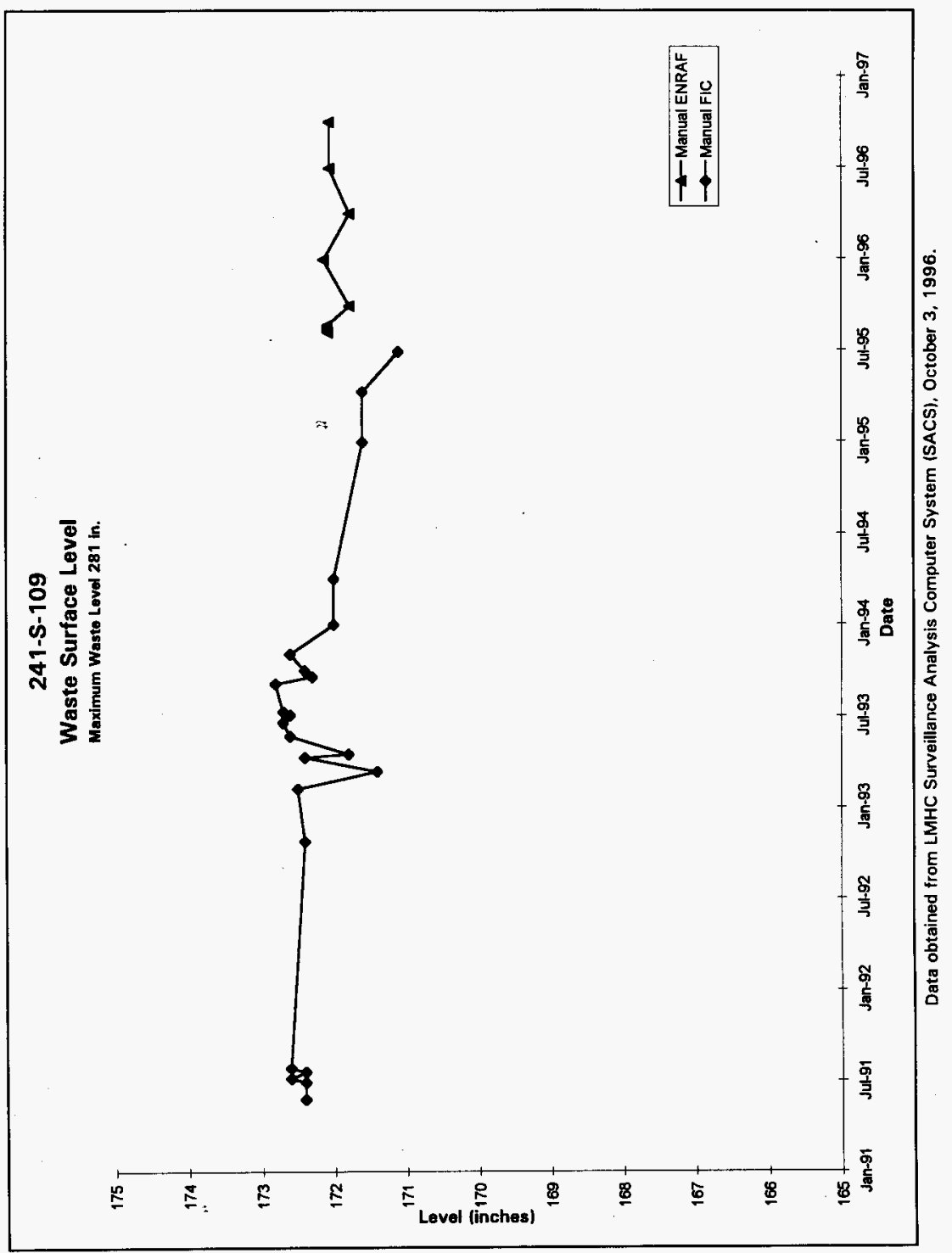




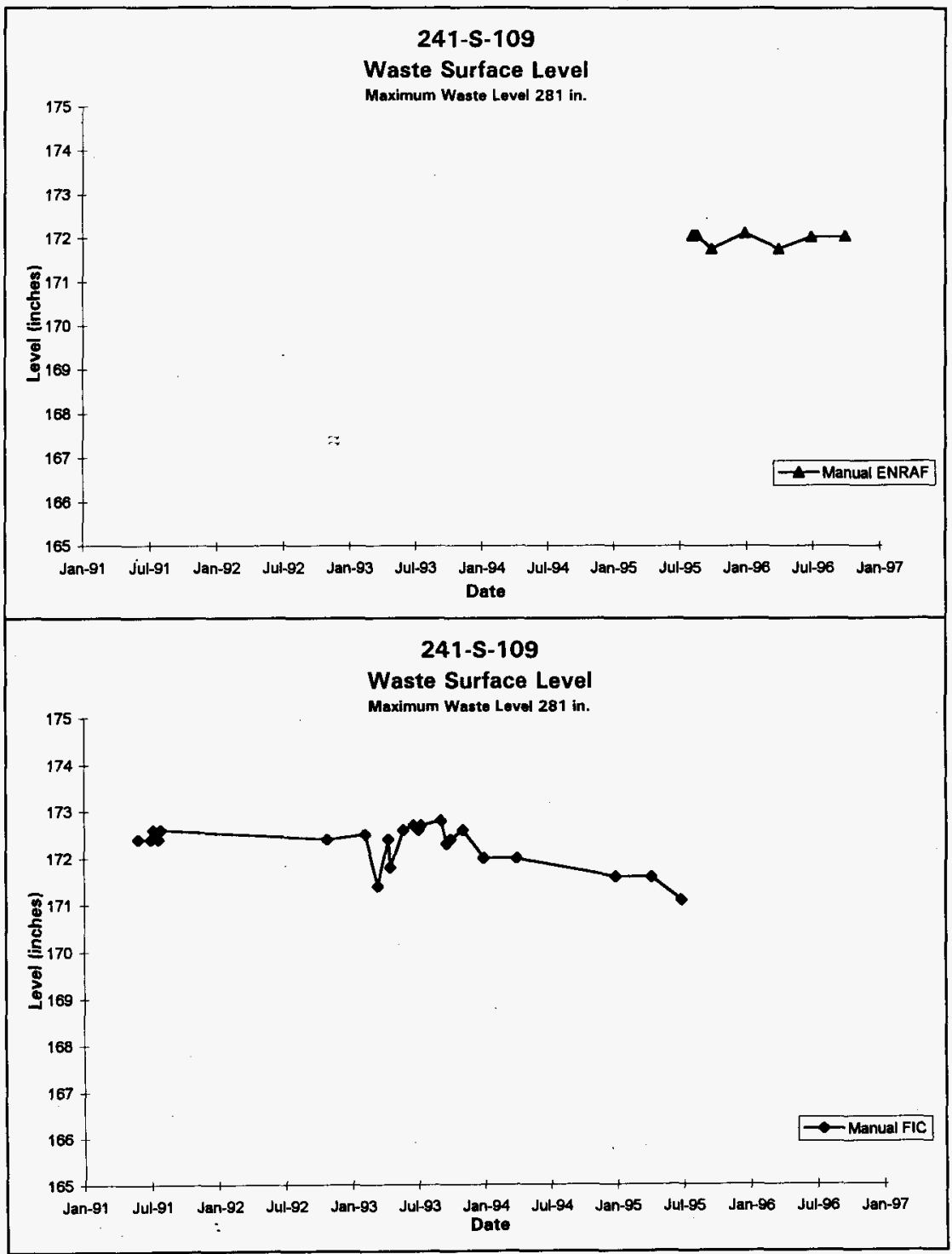

Data obtained from LMHC Surveillance Analysis Computer System (SACS), October 3, 1996. 


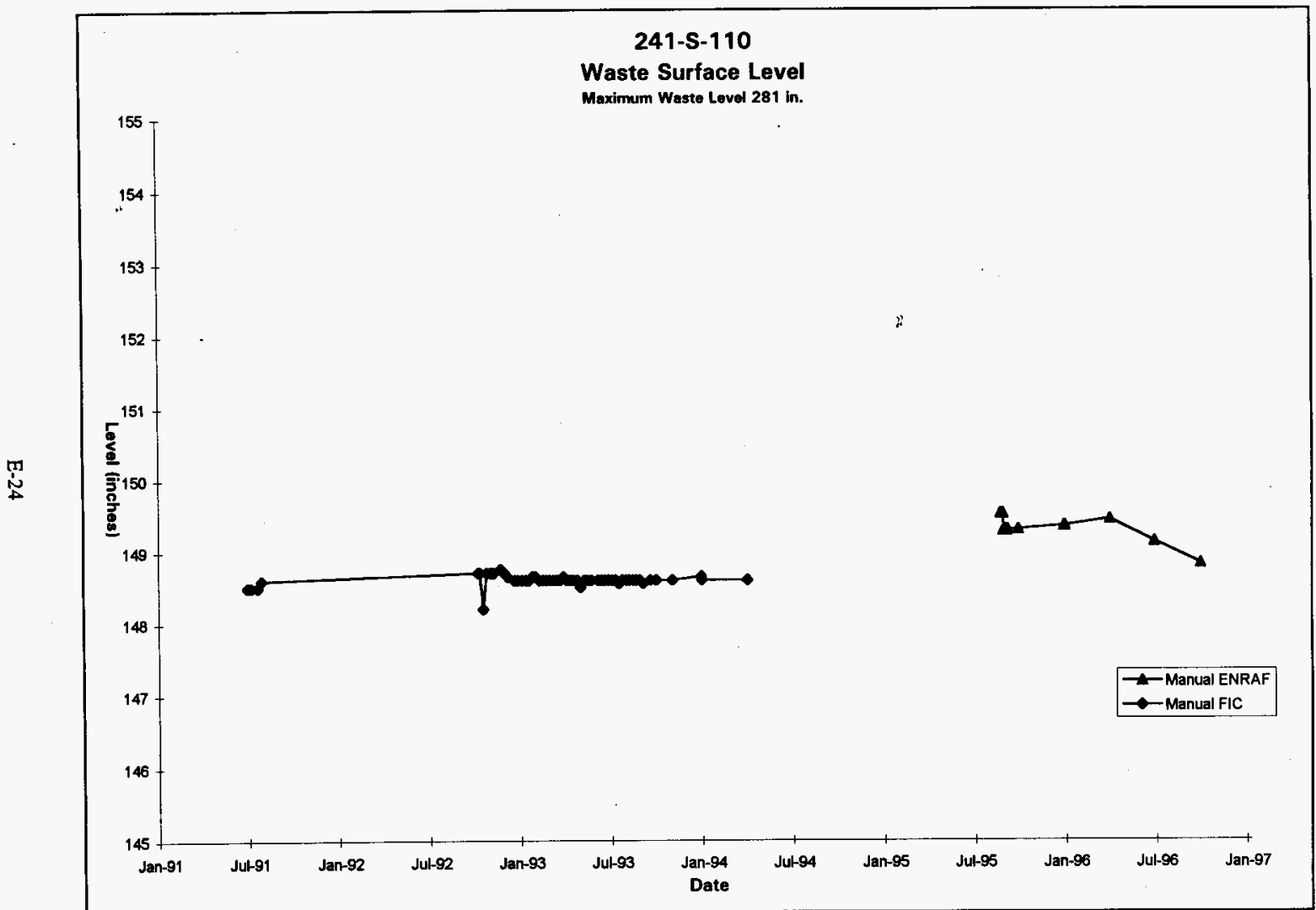

Data obtained from LMHC Surveillance Analysis Computer System (SACS), October 3, 1996 


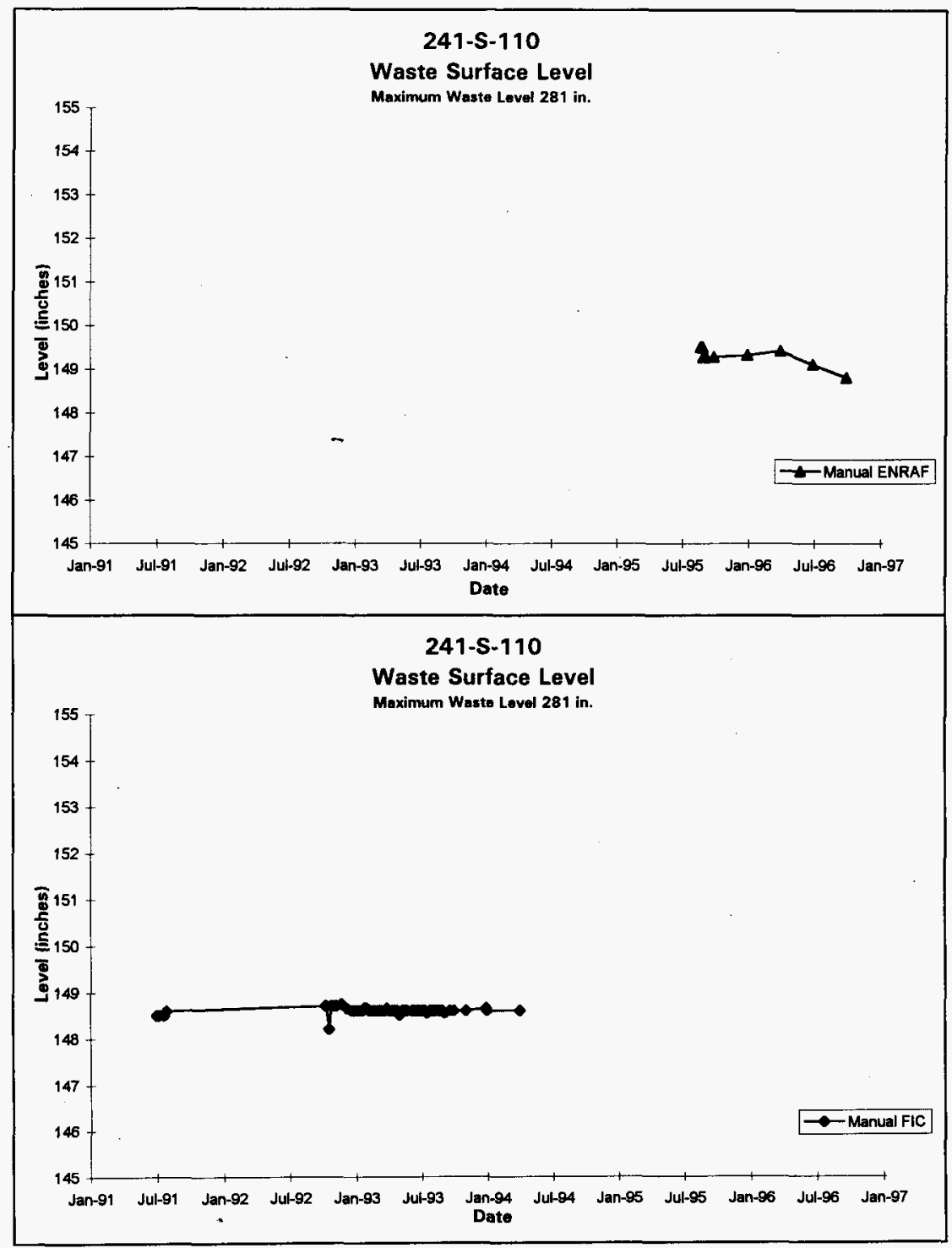

Data obtained from LMHC Surveillance Analysis Computer System (SACS), October 3, 1996. 


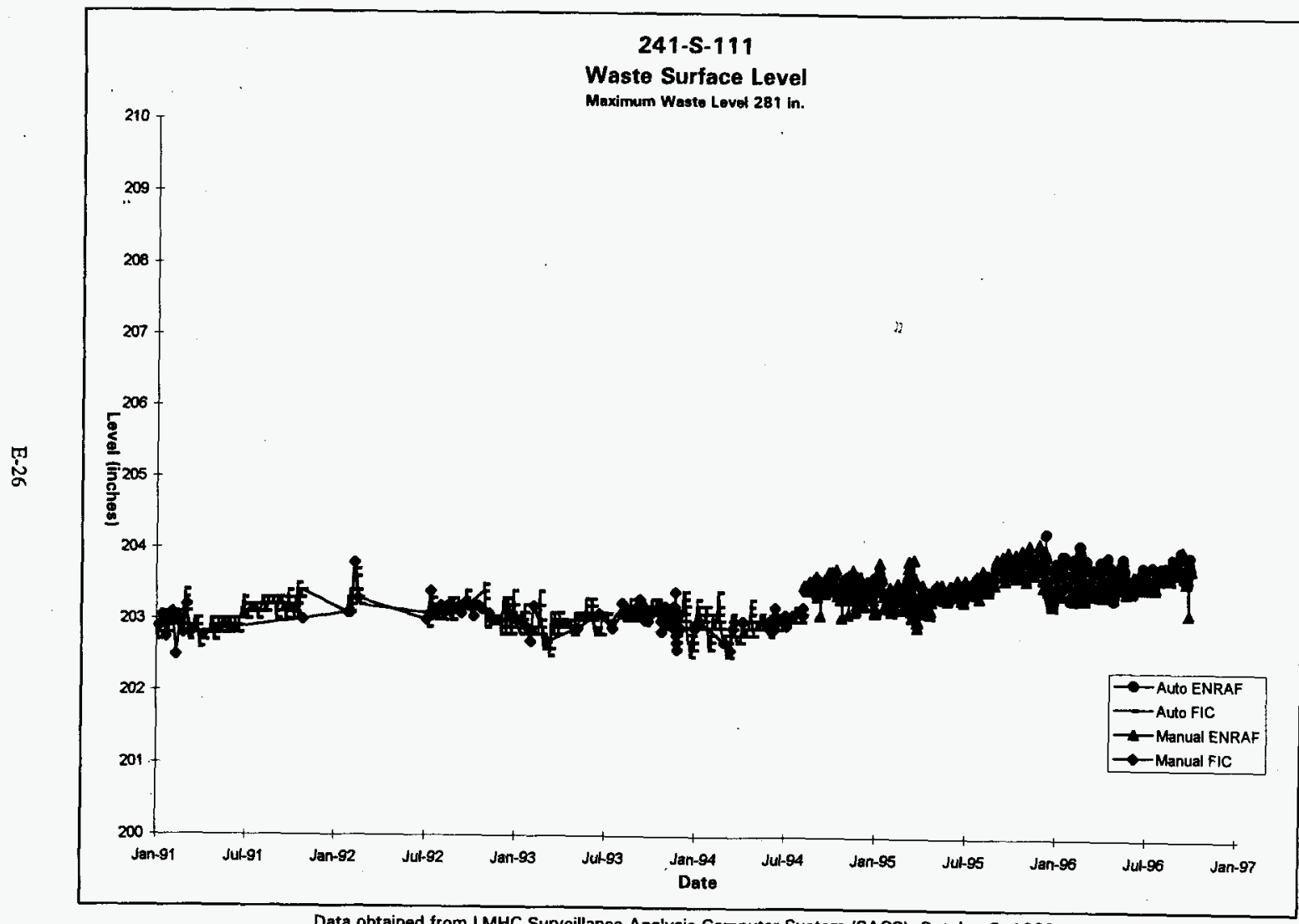

Data obtained from LMHC Surveillance Analysis Computer System (SACS), October 3, 1996.

究 


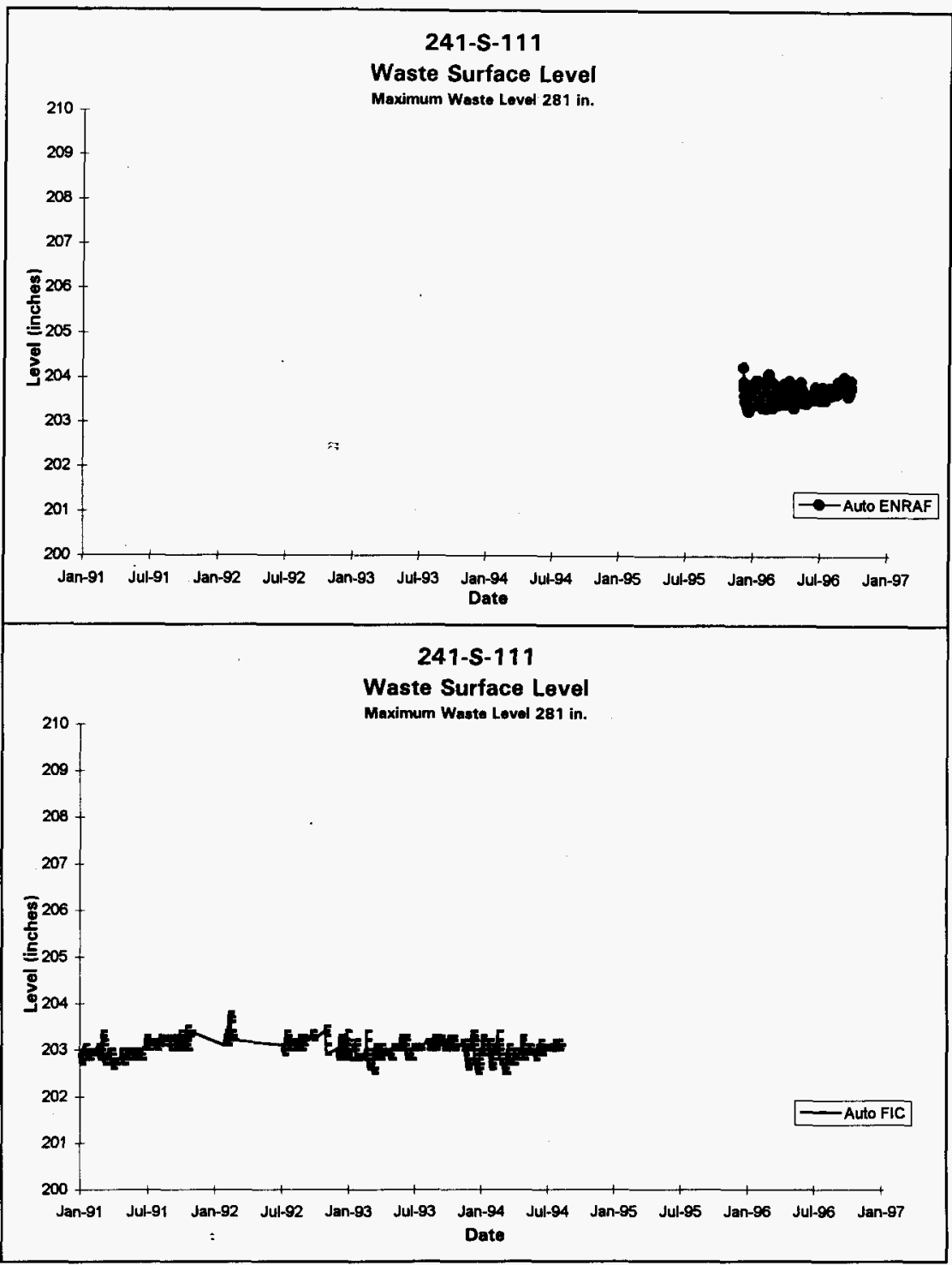

Data obtained from LMHC Surveillance Analysis Computer System (SACS), October 3, 1996. 
HNF-SD-WM-ER-323, Rev. 1

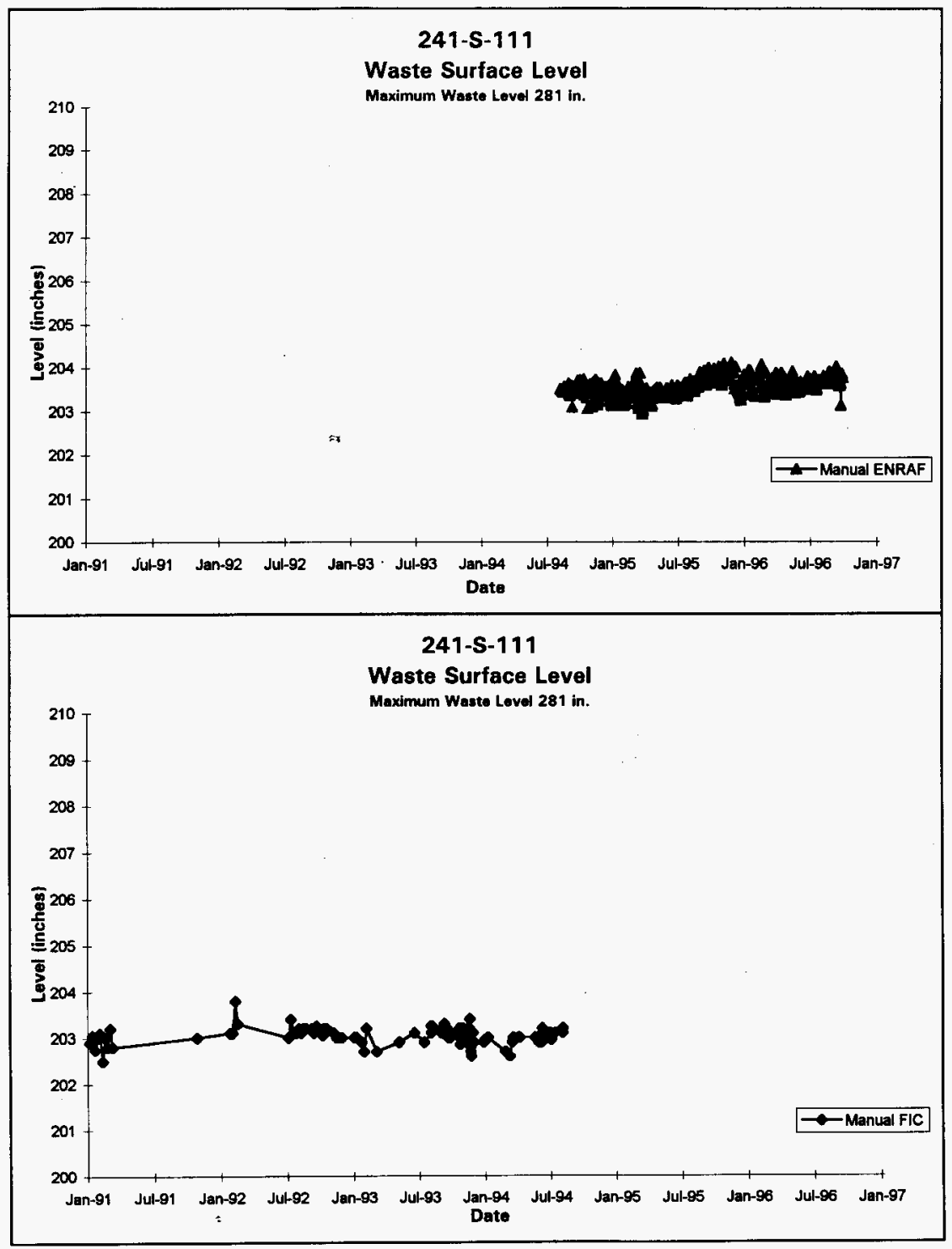

Data obtained from LMHC Surveillance Analysis Computer System (SACS), October 3, 1996.

E-28 


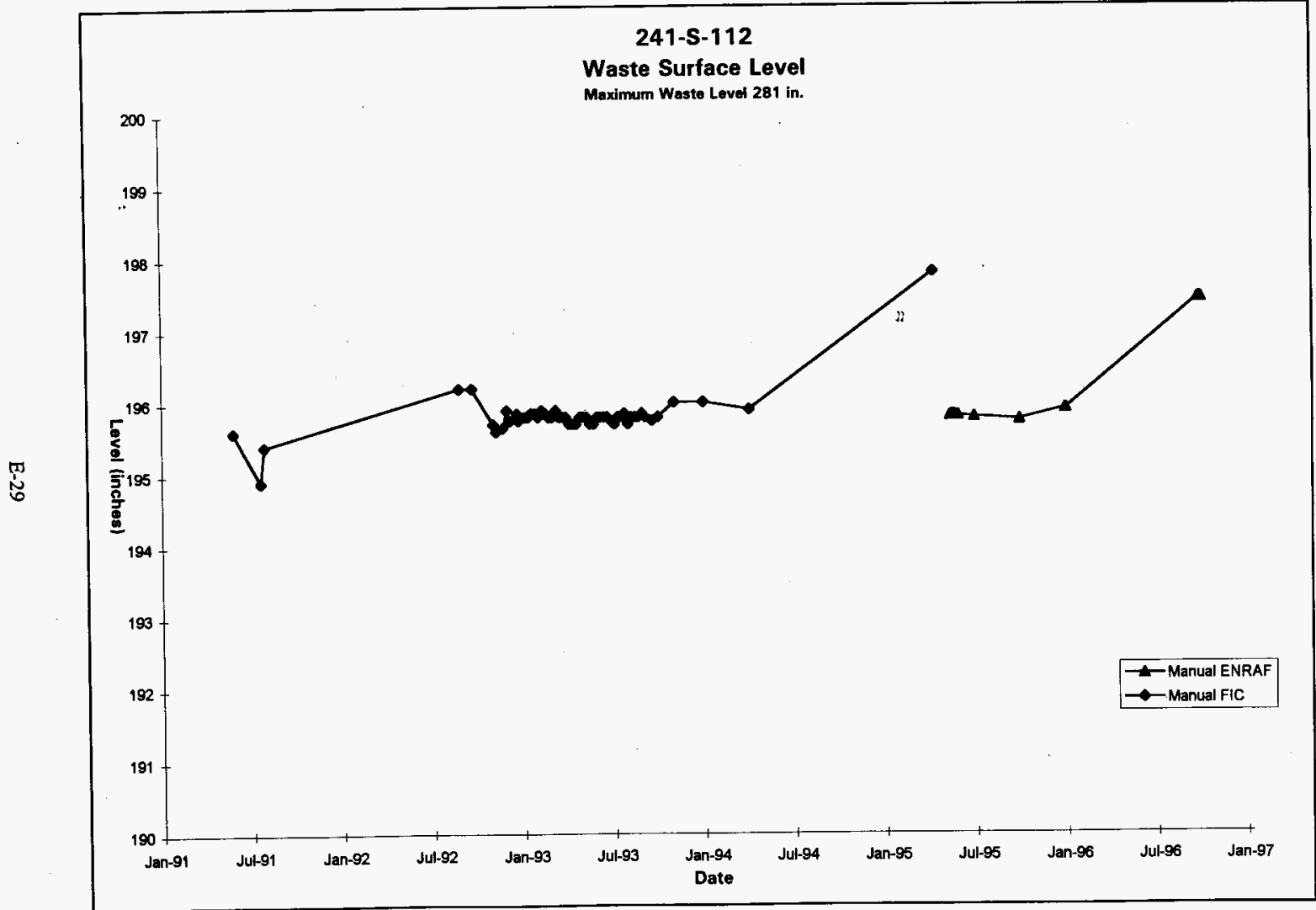

Data obtained from LMHC Surveillance Analysis Computer System (SACS), October 3, 1996. 


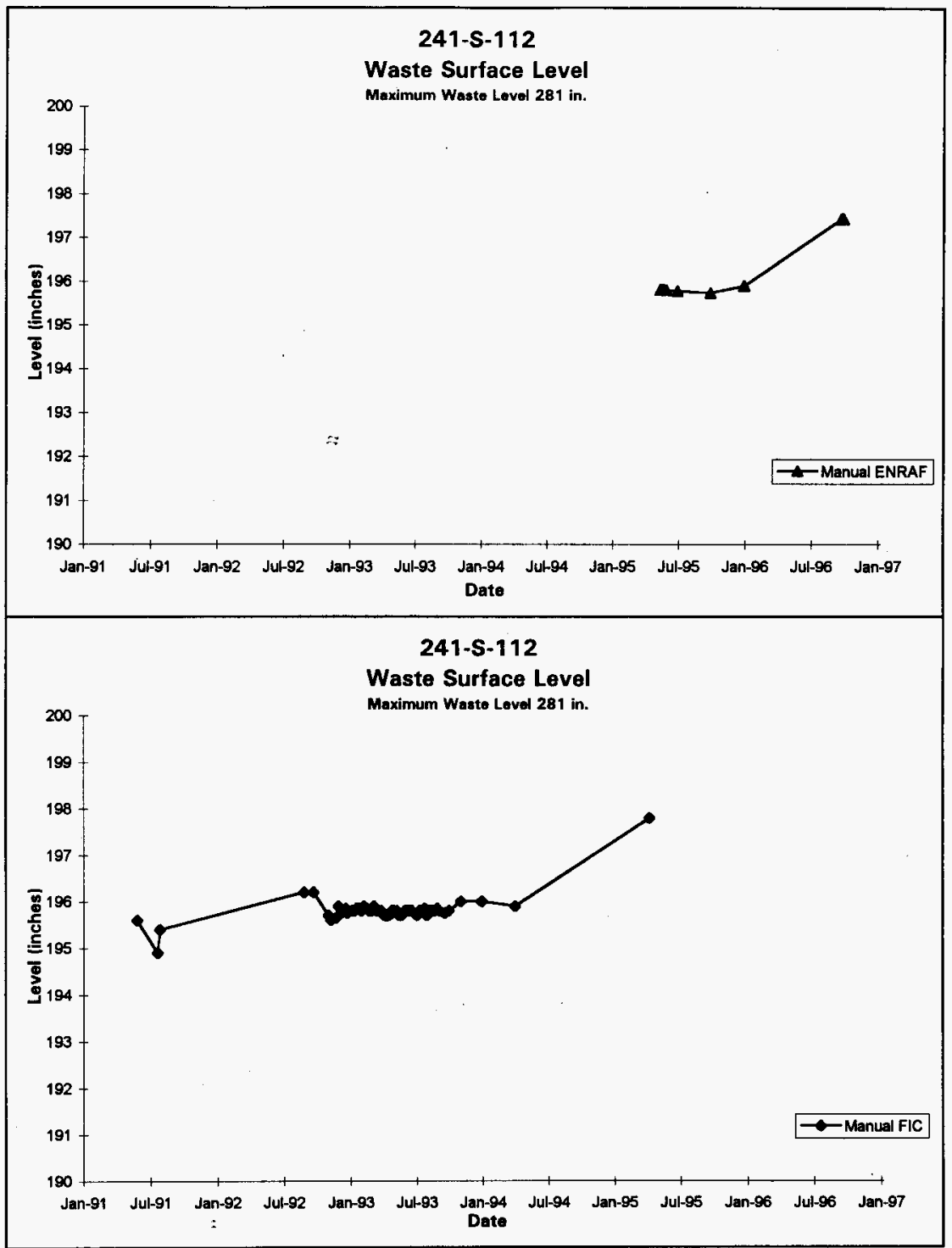

Data obtained from LMHC Surveillance Analysis Computer System (SACS), October 3, 1996. 
咅 
THIS PAGE INTENTIONALLY LEFT BLANK 
HNF-SD-WM-ER-323, Rev. I

Intentionally Jeft blank. 


\section{$241-S-101$}

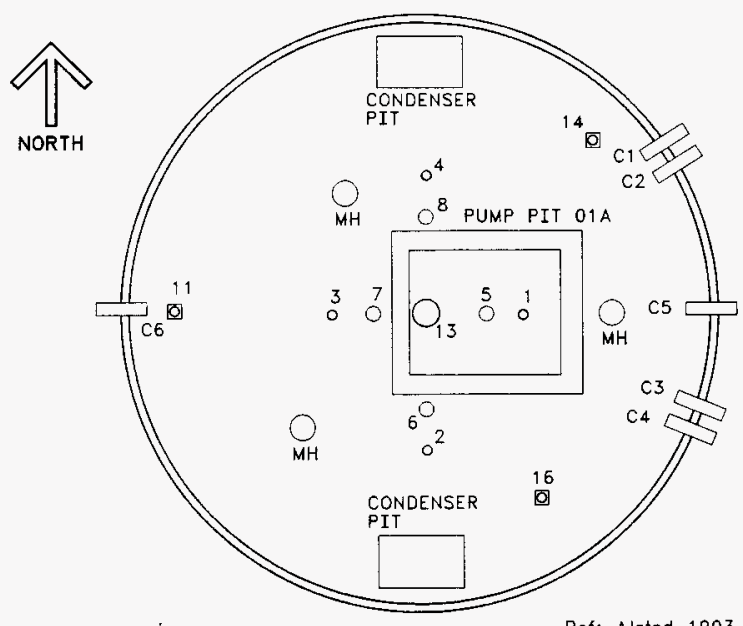

Ref: Alstad 1993

H-2-73181. Rev. 4

H-2-37524, Rev. 1

\section{TANK RISER LOCATION}

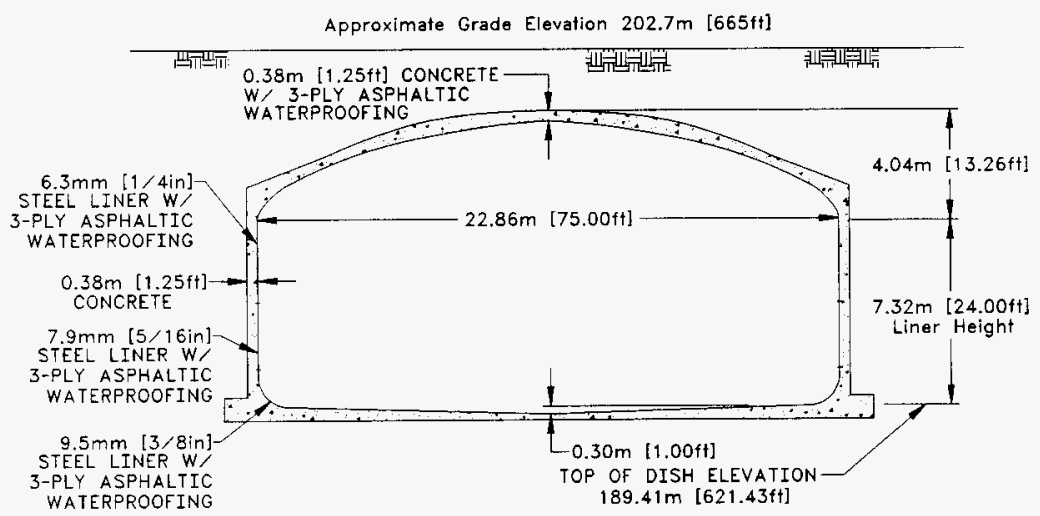

NOT TO SCALE

$\mathrm{F}-\mathrm{2}$
Ref: H-2-1783. Rev. 3 H-2-46293, Rev. 3 H-2-1784, Rev. 2 


\begin{tabular}{|c|c|c|c|}
\hline NO. & DIA. & SAMPLING* & DESCRIPTION AND COMMENTS \\
\hline 1 & $4^{\prime \prime}$ & & CONN NOZZLE \\
\hline 2 & $4^{\prime \prime}$ & & B-436 LOW [BM CEO-36911 12/11/86] \\
\hline 3 & $4 "$ & & FIC [ENRAF ECN-617149 12/21/94] \\
\hline 4 & $4^{\prime \prime}$ & $\bar{x}$ & TEMPERATURE PROBE [SPARE CE0-41054 2/27/87] \\
\hline 5 & $12^{\prime \prime}$ & & SALTWELL SCREEN AND PUMP \\
\hline 6 & $12^{\prime \prime}$ & $\bar{x}$ & B-222 OBSV PORT \\
\hline 7 & $12 "$ & & $\begin{array}{l}\text { VENTILATION [BLANK CE0-41054 2/27/87] [DUCT } \\
\text { REMOVED \& RISER CAPPED ECN-706501 } \\
\end{array}$ \\
\hline 8 & $12^{\prime \prime}$ & & B-222 OBSRV PORT \\
\hline 11 & $4^{\prime \prime}$ & $\bar{x}$ & EXHAUSTER \\
\hline 13 & $42^{\prime \prime}$ & & SLURRY DISTRIBUTOR \\
\hline 14 & 4" & & $\begin{array}{l}\text { SMP [B-221 TEMPERATURE PROBE CE0-41054 } \\
2 / 27 / 87]\end{array}$ \\
\hline 16 & $4^{\prime \prime}$ & $x$ & $\begin{array}{l}\text { SMP [BM CEO-36911 } 12 / 11 / 86] \text { [BREATHER FILTER } \\
\text { CE0-41054 } 2 / 27 / 87] \text { [MODIFY HEATED VAPOR PROBE } \\
\text { TO STANDARD HYDROGEN MONITORING SYSTEM } \\
\text { ECN-624411 10/23/95] [INSTALL BREATHER FILTER } \\
\text { AND SPOOL PIECE ONTO OFFSET ADAPTER ECN- } \\
626617 \quad 11 / 10 / 95]\end{array}$ \\
\hline C1 & 3" & & SPARE NOZZLE, CAPPED \\
\hline $\mathrm{C} 2$ & 3" & & SPARE NOZZLE, CAPPED \\
\hline C3 & $3^{\prime \prime}$ & & SPARE NOZZLE, CAPPED \\
\hline C4 & 3" & & SPARE NOZZLE, CAPPED \\
\hline C5 & 3" & & $\begin{array}{l}\text { INLET LINE V541, SEALED IN DIVERSION BOX 241-S- } \\
151\end{array}$ \\
\hline $\mathrm{C} 6$ & $3^{\prime \prime}$ & & OUTLET \\
\hline
\end{tabular}

Ref: Alstad 1993, Lipnicki 1996, \& H-2-73181, Rev. 4.

If there was a discrepancy between the documents and the drawings, the drawings shall take precedence.

Comments placed in [] denote Engineering Change Notices (ECN) made against the reference drawings.

* Denotes risers tentatively available for sampling (Lipnicki 1996) 


\section{$241-5-102$}

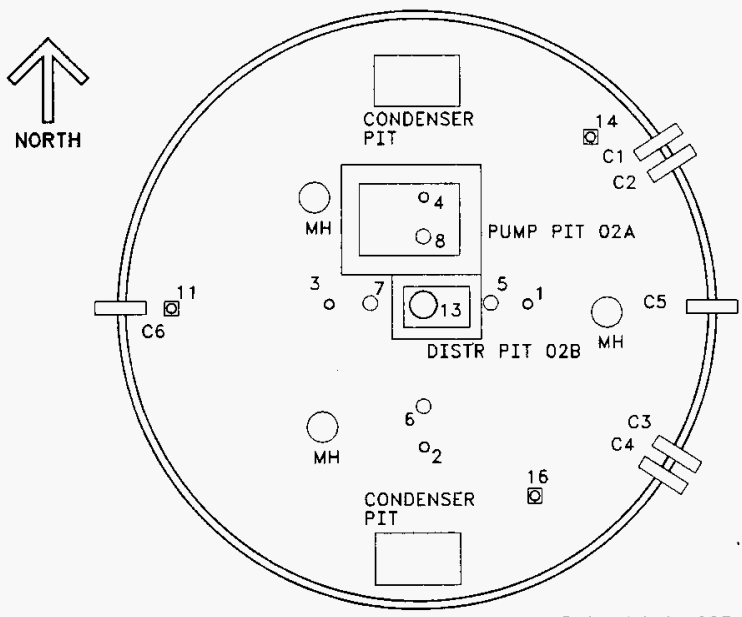

Ref: Alstad 1993

$\mathrm{H}-2-73182$. Rev, 4

$\mathrm{H}-2-37525$, Rev, 1

\section{TANK RISER LOCATION}

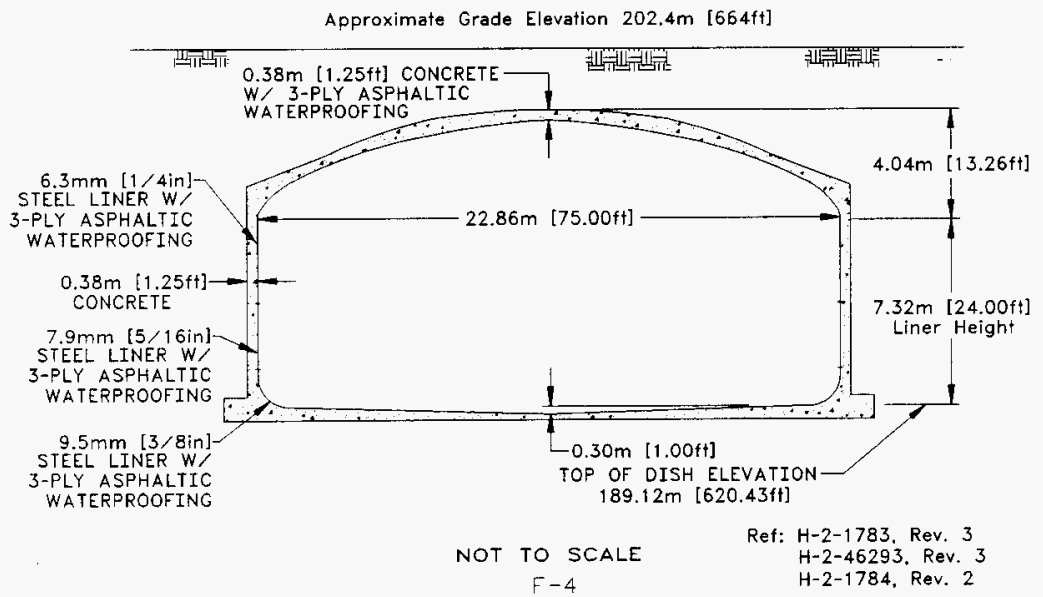




\begin{tabular}{|c|c|c|c|}
\hline NO. & DIA. & SAMPLING* & DESCRIPTION AND COMMENTS \\
\hline 1 & 4" & $x$ & 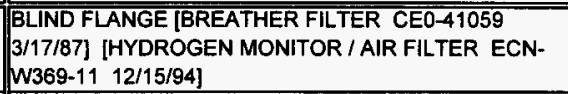 \\
\hline 2 & 4" & & $\begin{array}{l}\text { FIC [BM CEO-36910 12/11/86] [ENRAF ECN-620751 } \\
2 / 27 / 95]\end{array}$ \\
\hline 3 & 4" & & B-221 TEMPERATURE PROBE \\
\hline 4 & 4" & & SPARE [UNUSABLE CE0-41059 $3 / 17 / 87$ ] \\
\hline 5 & $12^{\prime \prime}$ & & B-346 LOW [BM CEO-36910 12/11/86] \\
\hline 6 & $12^{\prime \prime}$ & & $\begin{array}{l}\text { VENTILATION [BLANK CEO-41059 } 3 / 17 / 87] \text { [DUCT } \\
\text { REMOVED \& RISER CAPPED ECN-706501 8/29/95] }\end{array}$ \\
\hline 7 & $12^{\prime \prime}$ & $x$ & $\begin{array}{l}\text { B-222 OBSRV PORT [INSTALL MULTIPORT ADAPTER } \\
\text { W/ HEATED VAPOR PROBE IN HIGHEST PORT ECN- } \\
62873512 / 19 / 95]\end{array}$ \\
\hline 8 & $12^{\prime \prime}$ & & PUMP MOUNT \\
\hline 11 & 4" & $x$ & SMP \\
\hline 13 & $42^{\prime \prime}$ & & SALTWELL SCREEN AND PUMP \\
\hline 14 & $4 "$ & $\bar{x}$ & SMP \\
\hline 16 & 4" & $x$ & SMP [BM CEO-36910 12/11/86] \\
\hline $\mathrm{C1}$ & 3" & & SPARE NOZZLE, CAPPED \\
\hline $\mathrm{C} 2$ & 3" & & SPARE NOZZLE, CAPPED \\
\hline $\mathrm{C3}$ & 3" & & SPARE NOZZLE, CAPPED \\
\hline $\mathrm{C} 4$ & $3 "$ & & SPARE NOZZLE, CAPPED \\
\hline C5 & 3" & & INLET \\
\hline $\mathrm{C6}$ & $3 "$ & & OUTLET \\
\hline
\end{tabular}

Ref: $\quad$ Alstad 1993, Lipnicki 1996, \& H-2-73182, Rev. 4.

If there was a discrepancy between the documents and the drawings, the drawings shall take precedence.

Comments placed in $\square$ denote Engineering Change Notices (ECN) made against the reference drawings.

- Denotes risers tentatively available for sampling (Lipnicki 1996) 


\section{$241-5-103$}

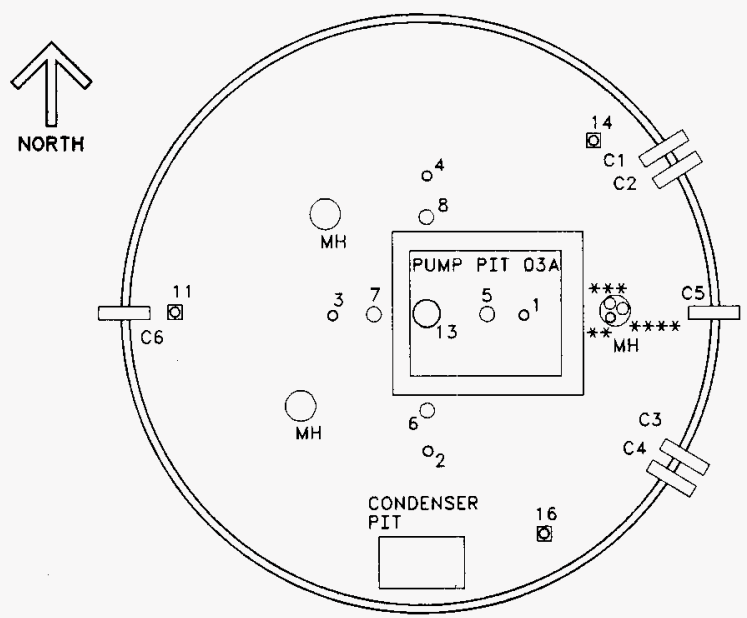

Ref: Alstad 1993

H-2-73183, Rev, 4

H-2-37526, Rev. 1

\section{TANK RISER LOCATION}

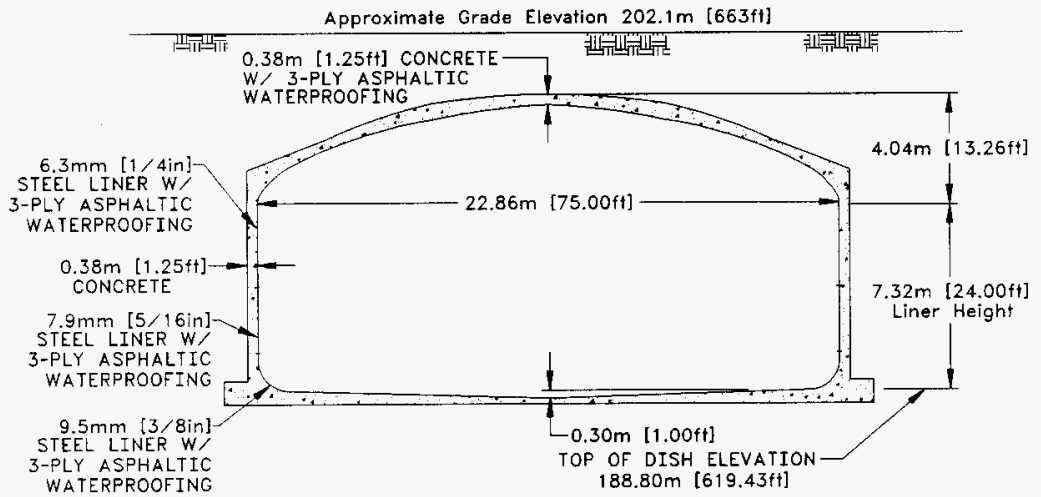

$$
\begin{gathered}
\text { NOT TO SCALE } \\
F-6
\end{gathered}
$$

Ref: $\mathrm{H}-2-1783$. Rev. 3 H-2-46293. Rev. 3 $\mathrm{H}-2-1784$, Rev. 2 


\begin{tabular}{|c|c|c|c|}
\hline NO. & DIA. & SAMPLING* & DESCRIPTION AND COMMENTS \\
\hline 1 & 4" & & CONN NOZZLE \\
\hline 2 & $4 "$ & & B-436 LOW [BM CEO-36909 12/11/86] \\
\hline 3 & $4 "$ & & FIC [ENRAF ECN-607985 4/8/94] \\
\hline 4 & 4" & & B-221 TEMPERATURE PROBE \\
\hline 5 & $12^{\prime \prime}$ & & SALTWEUL SCREEN AND PUMP \\
\hline 6 & $12 "$ & $x$ & $\begin{array}{l}\text { SPARE [UNUSABLE, CONCRETE PLUG WI LIFTING } \\
\text { BALE CE0-41060 } 3 / 17 / 87 \text { ] }\end{array}$ \\
\hline 7 & $12^{\prime \prime}$ & & $\begin{array}{l}\text { VENTILATION [BLANK CE0-41060 3/17/87] [DUCT } \\
\text { REMOVED \& RISER CAPPED ECN-706501 8/29/95] }\end{array}$ \\
\hline 8 & $12^{\prime \prime}$ & $\bar{x}$ & B-222 OBSRV PORT \\
\hline 11 & 4" & $\bar{x}$ & SMP \\
\hline 13 & $42^{\prime \prime}$ & & SLURRY DISTRIBUTOR \\
\hline 14 & 4" & $\mathbf{x}$ & $\begin{array}{l}\text { SMP [BREATHER FILTER CE0-41060 3/17/87] } \\
\text { [INSTALL BREATHER FILTER ON 4" X 4" X 4" OFFSET } \\
\text { ADAPTER ECN-628728 12/20/95] }\end{array}$ \\
\hline 16 & $4 "$ & $\mathrm{x}$ & SMP [BM CEO-36909 12/11/86] \\
\hline ** & $6^{\prime \prime}$ & & DRAIN LINE 343 FROM 242-S \\
\hline *n* & $10^{\prime \prime}$ & & DRAIN LINE 334 FROM 242-S \\
\hline ***** & $10^{\prime \prime}$ & & DRAIN LINE 335 FROM 242-S \\
\hline $\mathrm{C} 1$ & 3" & & SPARE NOZZLE, CAPPED \\
\hline $\mathrm{C} 2$ & $3^{\prime \prime}$ & & SPARE NOZZLE, CAPPED \\
\hline $\mathrm{C3}$ & $3^{\prime \prime}$ & & SPARE NOZZLE, CAPPED \\
\hline $\mathrm{C4}$ & 3" & & SPARE NOZZLE, CAPPED \\
\hline $\mathrm{C} 5$ & 3" & & NLET \\
\hline $\mathrm{C} 6$ & 3" & & OUTLET, CAPPED \\
\hline
\end{tabular}

Ref: Alstad 1993, Lipnicki 1996, \& H-2-73183, Rev. 4.

If there was a discrepancy between the documents and the drawings, the drawings shall take precedence.

Comments placed in $\square$ denote Engineering Change Notices (ECN) made against the reference drawings.

* Denotes risers tentatively available for sampling (Lipnicki 1995)

** Not in Lipnicki 1996, Alstad 1993, or the table in H-2-73183 Rev. 4.

*** Not in Lipnicki 1996, Alstad 1993, or the table in H-2-73183 Rev. 4.

**** Not in Lipnicki 1996, Alstad 1993, or the table in H-2-73183 Rev. 4. 


$$
241-5-104
$$

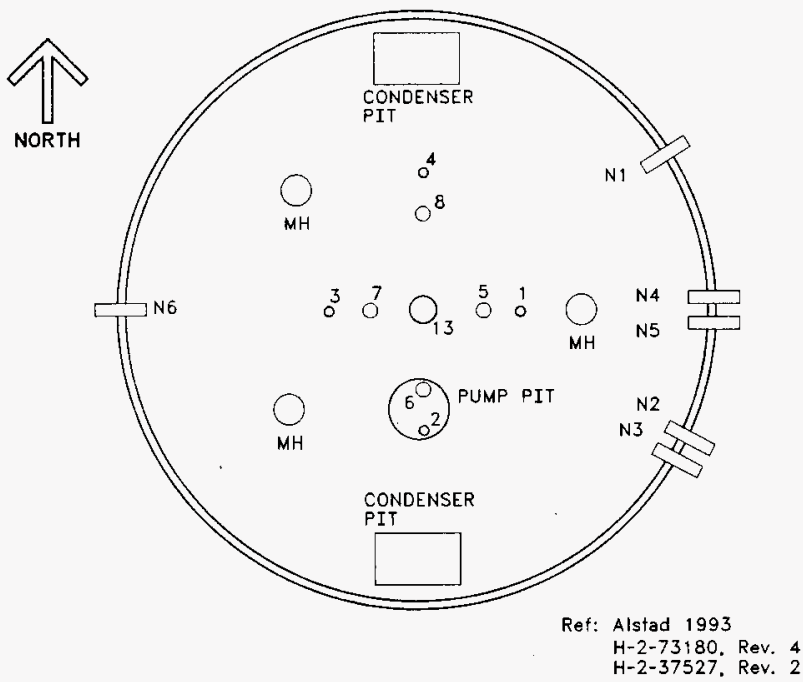

\section{TANK RISER LOCATION}

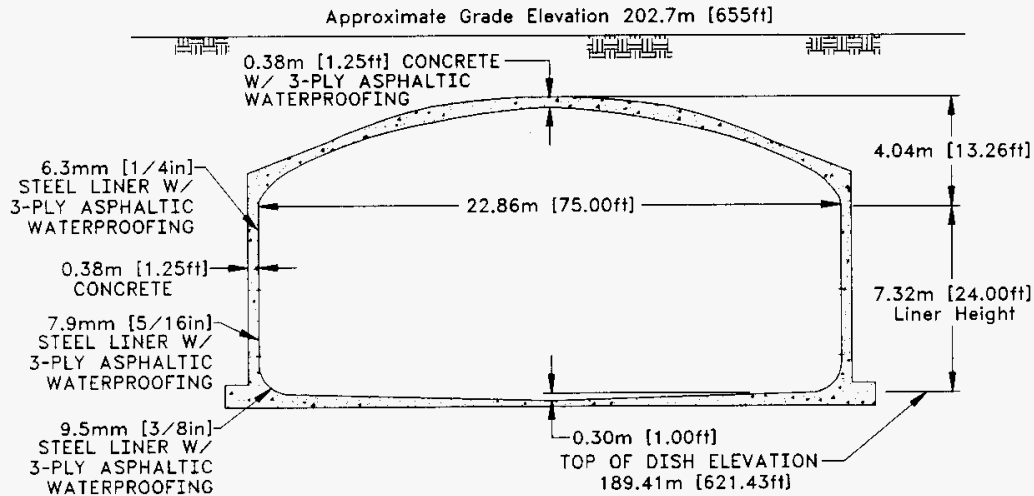

NOT TO SCALE
F-8

Ref: H-2-1783, Rev, 3

$\mathrm{H}-2-46293$, Rev. 3

H-2-1784, Rev. 2 
HNF-SD-WM-ER-323, Rev. 1

\begin{tabular}{|c|c|c|c|}
\hline NO. & DIA. & SAMPLING* & DESCRIPTION AND COMMENTS \\
\hline 1 & 4" & $\bar{x}$ & TEMPERATURE PROBE [BLANK CE0-41057 2/27/87] \\
\hline 2 & 4" & & $\begin{array}{l}\text { CAPPED [BM CEO-36908 12/11/86] [STEEL LOW ECN } \\
6141809 / 27 / 94]\end{array}$ \\
\hline 3 & $4^{\prime \prime}$ & $\mathbf{x}$ & $\begin{array}{l}\text { BREATHER FILTER [BREATHER FILTER CE0-41057 } \\
\text { 2/27/87] [BREATHER FILTER / VAPOR SAMPLING ASSY } \\
\text { ECN-601024 8/10/93] }\end{array}$ \\
\hline 4 & $4 "$ & & TEMPERATURE PROBE \\
\hline 5 & $12^{\prime \prime}$ & & $\begin{array}{l}\text { LIQUID LEVEL REEL [BM CEO-36908 12/11/86 \& ECN- } \\
61418410 / 31 / 94]\end{array}$ \\
\hline 6 & $12 "$ & & SALTWELL SCREEN AND PUMP \\
\hline 7 & $12^{\prime \prime}$ & $\mathrm{x}$ & $\begin{array}{l}\text { B-222 OBSRV PORT [BM CEO-36908 12/11/86] [BM } \\
\text { ECN-614184 10/31/94] }\end{array}$ \\
\hline 8 & $12^{\prime \prime}$ & $\mathbf{x}$ & BLIND FLANGE \\
\hline 13 & $42^{\prime \prime}$ & & PUMP, WC IN PIT \\
\hline N1 & $3^{\prime \prime}$ & & SPARE NOZZLE, CAPPED \\
\hline N2 & $3^{\prime \prime}$ & & SPARE NOZZLE, CAPPED \\
\hline N3 & 3" & & SPARE NOZZLE, CAPPED \\
\hline N4 & 3" & & $\begin{array}{l}\text { INLET LINE V539, SEALED IN DIVERSION BOX 241-S- } \\
151\end{array}$ \\
\hline N5 & **2" & & $\begin{array}{l}\text { INLET LINE V538, SEALED IN DIVERSION BOX 241-S- } \\
151\end{array}$ \\
\hline N6 & $3^{\prime \prime}$ & & OVERFLOW \\
\hline
\end{tabular}

Ref: $\quad$ Alstad 1993, Lipnicki 1996, \& H-2-73180, Rev. 4.

If there was a discrepancy between the documents and the drawings, the drawings shall take precedence.

Comments placed in $[$ denote Engineering Change Notices (ECN) made against the reference drawings.

- Denotes risers tentatively available for sampling (Lipnicki 1996)

** H-2-73180 Rev. 4 table shows a 2 inch nozzle and the figure shows a 3 inch nozzl 


\section{$241-S-105$}

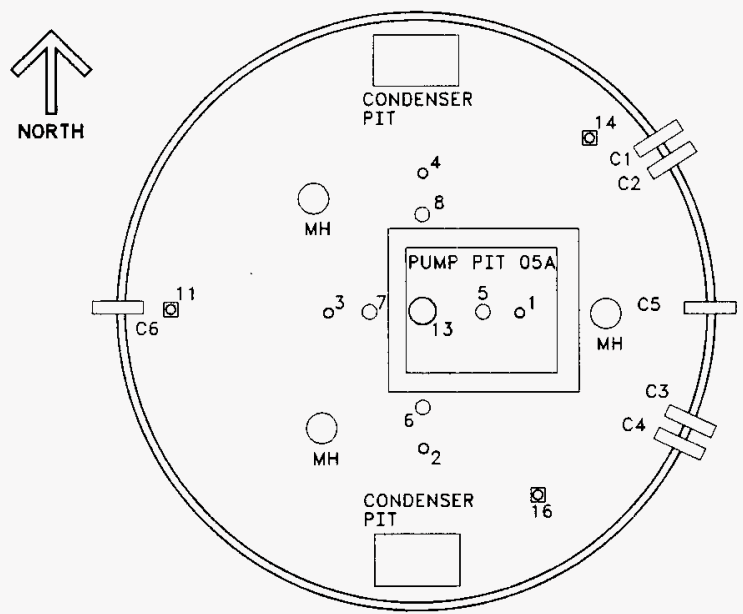

Ref: Alstad 1993

H-2-73184, Rev. 4

$\mathrm{H}-2-37528$, Rev. 2

\section{TANK RISER LOCATION}

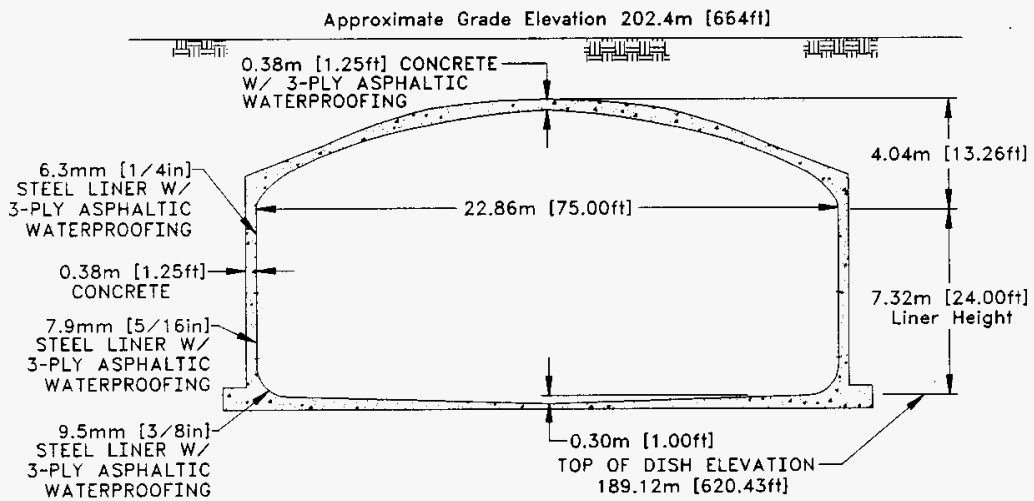

NOT TO SCALE

$$
F-10
$$

Ref: H-2-1783, Rev. 3 $\mathrm{H}-2-46293$. Rev. 3 $\mathrm{H}-2-1784$, Rev. 2 


\begin{tabular}{|c|c|c|c|}
\hline NO. & DIA. & SAMPLING* & DESCRIPTION AND COMMENTS \\
\hline$\overline{\overline{1}}$ & $4 "$ & & CONN NOZZLE \\
\hline 2 & $4^{\prime \prime}$ & $x$ & $\begin{array}{l}\text { BLIND FLANGE [BM CEO-36907 12/11/86] [BLANK } \\
\text { CEO-41061 3/17/87] [INSTALL HEATED VAPOR PROBE } \\
\text { \& REMOVE WITHIN } 90 \text { DAYS (NOT ON RISER 16) ECN- } \\
626621 \text { L 12/1/95] }\end{array}$ \\
\hline 3 & 4" & & FIC [ENRAF ECN-622487 4/21/95] \\
\hline$\overline{4}$ & 4" & & TEMPERATURE PROBE, WC \\
\hline 5 & $12 "$ & & SALTWELL SCREEN AND PUMP \\
\hline 6 & $12^{\prime \prime}$ & $x$ & $\begin{array}{l}\text { OBSRV PORT [FLANGE WI LIFTING BALE CE0-41061 } \\
3 / 17 / 87 \text { ] }\end{array}$ \\
\hline 7 & $12 "$ & & $\begin{array}{l}\text { VENTILATION [BLANK CE0-41061 3/17/87] [DUCT } \\
\text { REMOVED \& RISER CAPPED ECN-706501 8/29/95] }\end{array}$ \\
\hline 8 & $12^{\prime \prime}$ & $\bar{x}$ & SPARE [B-222 OBSRV PORT CE0-41061 3/17/87] \\
\hline 11 & 4" & $\mathrm{x}$ & SMP \\
\hline 13 & 42" & & SLURRY DISTRIBUTOR \\
\hline 14 & $4^{\prime \prime}$ & & B-436 LOW \\
\hline 16 & 4" & $\mathrm{x}$ & $\begin{array}{l}\text { SMP [BM CEO-36907 12/11/86] [BREATHER FILTER } \\
\text { CE0-41061 3/17/87] [INSTALL HEATED VAPOR PROBE } \\
\text { \& REMOVE WITHIN 90 DAYS ECN-626612L 11/20/95] }\end{array}$ \\
\hline C1 & $3^{\prime \prime}$ & & SPARE NOZZLE, CAPPED \\
\hline C2 & $3 "$ & & SPARE NOZZLE, CAPPED \\
\hline $\mathrm{C3}$ & 3" & & SPARE NOZZLE, CAPPED \\
\hline $\mathrm{C4}$ & $3 "$ & & SPARE NOZZLE, CAPPED \\
\hline $\mathrm{C5}$ & $3 "$ & & INLET \\
\hline C6 & 3" & & OUTLET \\
\hline
\end{tabular}

Ref: $\quad$ Alstad 1993, Lipnicki 1996, \& H-2-73184, Rev. 4.

If there was a discrepancy between the documents and the drawings, the drawings shall take precedence.

Comments placed in [] denote Engineering Change Notices (ECN) made against the reference drawings.

* Denotes risers tentatively available for sampling (Lipnicki 1996) 


\section{$241-S-106$}

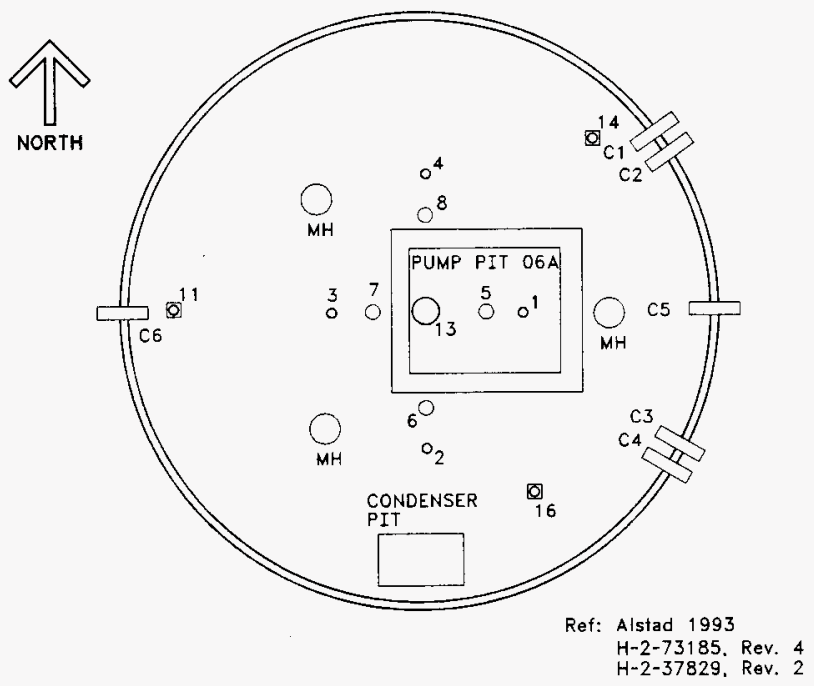

TANK RISER LOCATION

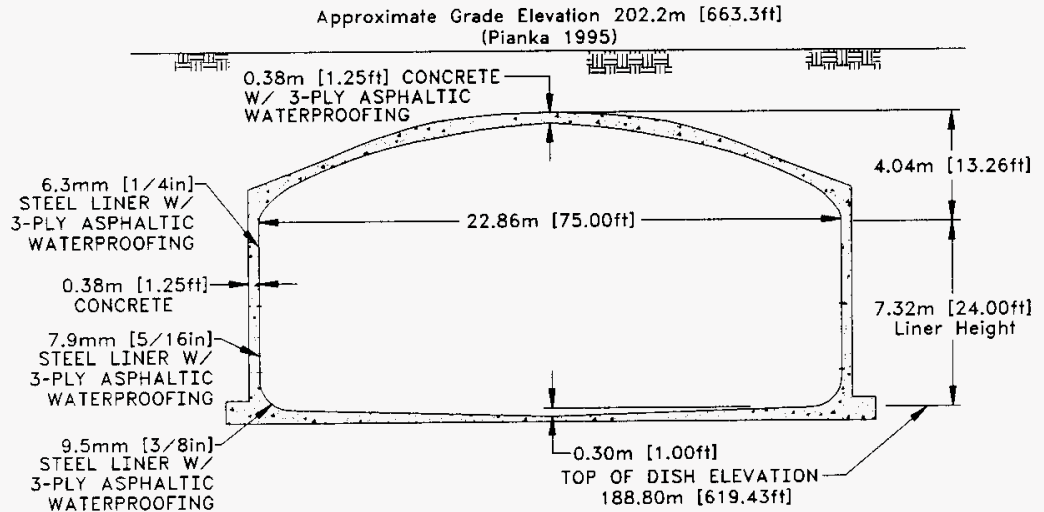

NOT TO SCALE

$F-1 e$
Ref: H-2-1783, Rev. 3 H-2-46293. Rev. 3 $\mathrm{H}-2-1784$, Rev. 2 


\begin{tabular}{|c|c|c|c|}
\hline NO. & DIA. & SAMPLING* & DESCRIPTION AND COMMENTS \\
\hline$\overline{11}$ & 4" & & CONN NOZZLE \\
\hline 2 & $4 "$ & & TEMPERATURE PROBE [BM CEO-36907 12/11/86] \\
\hline 3 & 4" & & FIC [ENRAF ECN-608115 5/12/94] \\
\hline 4 & $4 "$ & & $B-436$ LOW \\
\hline 5 & $12^{\prime \prime}$ & & SALTWELL SCREEN AND PUMP \\
\hline 6 & $12^{n}$ & $\bar{x}$ & SPARE [UNUSABLE CE0-41062 $3 / 17 / 87$ ] \\
\hline 7 & $12^{n}$ & & $\begin{array}{l}\text { VENTILATION [BLANK CE0-41062 } 3 / 17 / 87] \text { [DUCT } \\
\text { REMOVED \& RISER CAPPED ECN-706501 8/29/95] }\end{array}$ \\
\hline 8 & $12^{\prime \prime}$ & $\bar{x}$ & B-222 OBSRV PORT \\
\hline 11 & 4" & $\bar{x}$ & SMP \\
\hline 13 & $42^{\prime \prime}$ & & SLURRY DISTRIBUTOR \\
\hline 14 & $4 "$ & $\bar{x}$ & SMP \\
\hline 16 & 4" & $x$ & $\begin{array}{l}\text { SMP [BM CEO-36907 12/11/86] [BREATHER FILTER } \\
\text { CE0-41062 } 3 / 17 / 87]\end{array}$ \\
\hline C1 & $3 "$ & & SPARE NOZZLE, CAPPED \\
\hline $\mathrm{C2}$ & 3 & & SPARE NOZZLE, CAPPED \\
\hline $\mathrm{C3}$ & $3 "$ & & SPARE NOZZLE, CAPPED \\
\hline $\mathrm{C4}$ & 3" & & SPARE NOZZLE, CAPPED \\
\hline $\mathrm{C5}$ & 3" & & INLET \\
\hline C6 & $3^{\prime \prime}$ & & OUTLET, CAPPED \\
\hline
\end{tabular}

Ref: $\quad$ Alstad 1993, Lipnicki 1996, \& H-2-73185, Rev. 4.

If there was a discrepancy between the documents and the drawings, the drawings shall take precedence.

Comments placed in $[$ denote Engineering Change Notices (ECN) made against the reference drawings.

- Denotes risers tentatively available for sampling (Lipnicki 1996) 


\section{$241-5-107$}

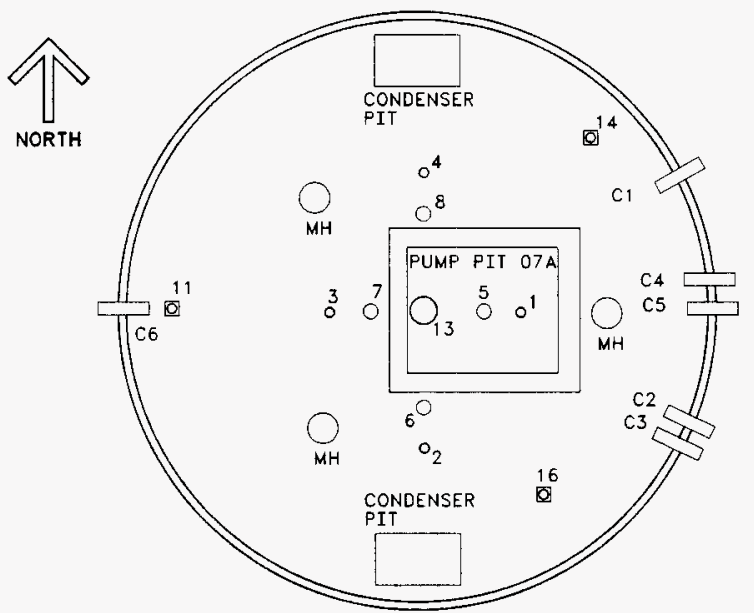

Ref: Alstad 1993

$\mathrm{H}-2-73: 86$, Rev. 4

$\mathrm{H}-2-37530$, Rev. 2

\section{TANK RISER LOCATION}

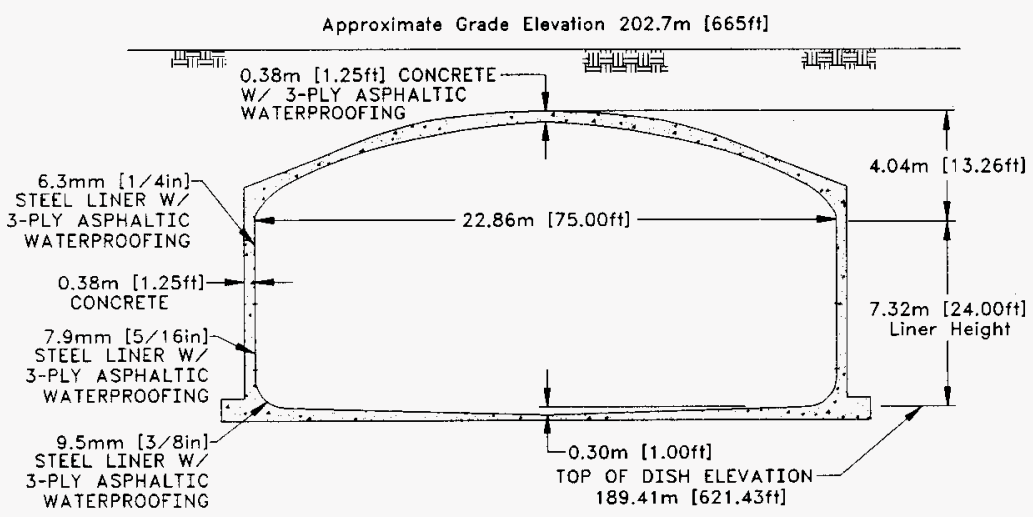

NOT TO SCALE
$F-14$

Ref: H-2-1783, Rev. 3 $\mathrm{H}-2-46293$. Rev. 3 $\mathrm{H}-2-1784$, Rer. 2 


\begin{tabular}{|c|c|c|c|}
\hline NO. & DIA. & SAMPLING* & DESCRIPTION AND COMMENTS \\
\hline 1 & 4" & & CONN NOZZLE \\
\hline 2 & $4^{\prime \prime}$ & $x$ & $\begin{array}{l}\text { BLIND FLANGE [BM CEO-36907 } 12 / 11 / 86] \\
\text { [BREATHER FILTER ECN-706164 } \\
\text { S/9/95] }\end{array}$ \\
\hline 3 & 4" & & FIC [ENRAF ECN-608115 5/12/94] \\
\hline 4 & 4" & & B-221 TEMPERATURE PROBE \\
\hline 5 & $12^{\prime \prime}$ & & SALTWELL SCREEN AND PUMP \\
\hline 6 & $12 "$ & $\mathbf{x}$ & $\begin{array}{l}\text { B-222 OBSRV PORT [4" BALL VALVE \& 4" BLIND } \\
\text { FLANGE ECN-6081155/12/94] }\end{array}$ \\
\hline 7 & $12 "$ & & $\begin{array}{l}\text { VENTILATION [BLANK CEO-41055 2/27/87] [DUCT } \\
\text { REMOVED \& RISER CAPPED ECN-706501 8/29/95] }\end{array}$ \\
\hline 8 & $12^{\prime \prime}$ & & OBSRV PORT [UNUSABLE CE0-41055 2/27/87] \\
\hline 11 & $4 "$ & $\mathrm{x}$ & SMP \\
\hline 13 & $42^{\prime \prime}$ & & SLURRY DISTRIBUTOR \\
\hline 14 & $4^{\prime \prime}$ & $\bar{x}$ & SMP \\
\hline 16 & $4^{\prime \prime}$ & $\mathbf{x}$ & $\begin{array}{l}\text { SMP [BM CEO-36907 12/11/86] [BREATHER FILTER } \\
\text { CE0-41055 2/27/87] [SPARE ECN-706164 5/9/95] }\end{array}$ \\
\hline C1 & $3^{\prime \prime}$ & & SPARE NOZZLE, CAPPED \\
\hline $\mathrm{C2}$ & 3" & & SPARE NOZZLE, CAPPED \\
\hline $\mathrm{C3}$ & 3" & & SPARE NOZZLE, CAPPED \\
\hline $\mathrm{C} 4$ & $3 "$ & & $\begin{array}{l}\text { INLET LINE V537, SEALED IN DIVERSION BOX 241-S- } \\
151\end{array}$ \\
\hline C5 & $3^{\prime \prime}$ & & $\begin{array}{l}\text { INLET LINE V536, SEALED IN DIVERSION BOX 241-S- } \\
151\end{array}$ \\
\hline $\mathrm{C6}$ & 3" & & OUTLET \\
\hline
\end{tabular}

Ref: $\quad$ Alstad 1993, Lipnicki 1996, \& H-2-73186, Rev. 4.

If there was a discrepancy between the documents and the drawings, the drawings shall take precedence.

Comments placed in [] denote Engineering Change Notices (ECN) made against the reference drawings.

- Denotes risers tentatively available for sampling (Lipnicki 1996) 


\section{$241-5-108$}

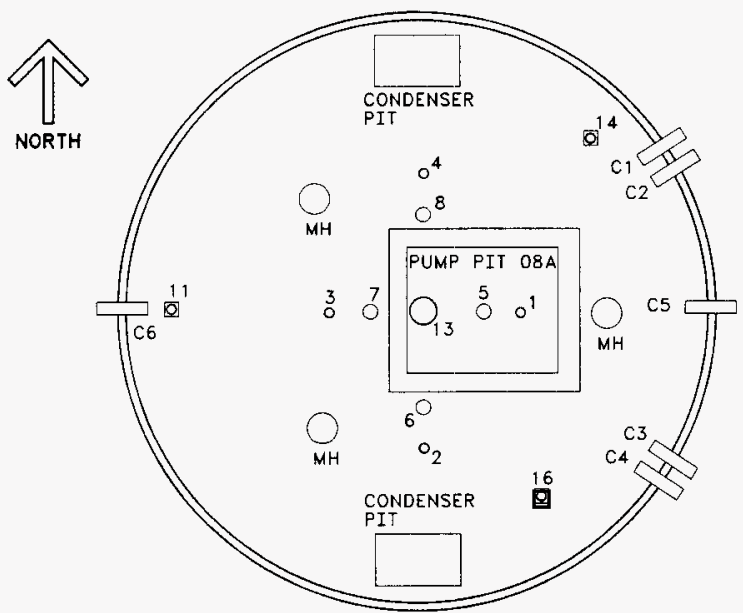

Ref: Alstad 1993

$\mathrm{H}-2-73187$, Rev. 4

H-2-37531, Rev. 2

\section{TANK RISER LOCATION}

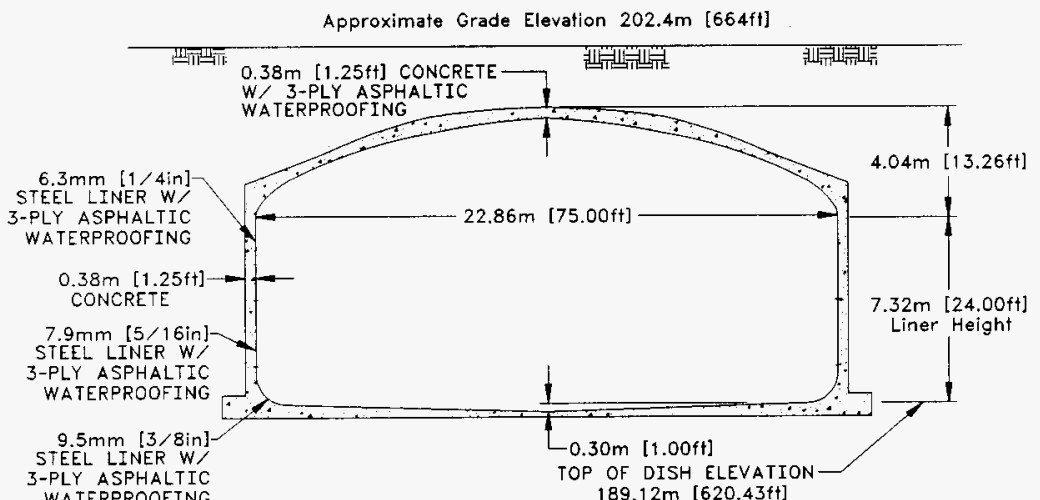

WATERPROOFING

$189.12 \mathrm{~m} \mathrm{[620.43 \textrm {t } t ]}$

$$
\begin{gathered}
\text { NOT TO SCALE } \\
F-16
\end{gathered}
$$

Ref: H-2-1783, Rev. 3 $\mathrm{H}-2-46293$. Rev. 3 $\mathrm{H}-2-1784$, Rev. 2 


\begin{tabular}{|c|c|c|c|}
\hline NO. & DIA. & SAMPLING* & DESCRIPTION AND COMMENTS \\
\hline 1 & 4" $^{\prime \prime}$ & & CONN NOZZLE \\
\hline 2 & $4^{\prime \prime}$ & & 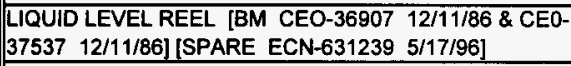 \\
\hline 3 & $4^{\prime \prime}$ & & FIC [ENRAF ECN-622487 $4 / 21 / 95]$ \\
\hline 4 & $4 "$ & & TEMPERATURE PROBE \\
\hline 5 & $12^{\prime \prime}$ & & SALTWELL SCREEN AND PUMP \\
\hline 6 & $12^{\prime \prime}$ & & $\begin{array}{l}\text { VENTILATION [BLANK CE0-41063 3/17/87] [DUCT } \\
\text { REMOVED \& RISER CAPPED ECN-706501 8/29/95] }\end{array}$ \\
\hline 7 & $12^{\prime \prime}$ & $x$ & B-222 OBSRV PORT \\
\hline 8 & $12^{\prime \prime}$ & $\bar{x}$ & CORROSION PROBE \\
\hline 11 & 4" & $\bar{x}$ & SMP \\
\hline 13 & 42" & & SLURRY DISTRIBUTOR \\
\hline 14 & $4^{\prime \prime}$ & $x$ & $\begin{array}{l}\text { SMP [BREATHER FILTER CE0-41063 } 3 / 17 / 87] \text { [4" X 4" } \\
\times 4 " \text { OFFSET ADAPTER W/ BREATHER FILTER ECN- } \\
62661311 / 16 / 95]\end{array}$ \\
\hline 16 & $4^{\prime \prime}$ & & $\begin{array}{l}\text { B-436 LOW [BM CEO-36907 12/11/86 \& CE0-37537 } \\
12 / 11 / 86]\end{array}$ \\
\hline $\mathrm{C} 1$ & 3" & & SPARE NOZZLE, CAPPED \\
\hline $\mathrm{C2}$ & $3^{\prime \prime}$ & & SPARE NOZZLE, CAPPED \\
\hline C3 & $3^{\prime \prime}$ & & SPARE NOZZLE, CAPPED \\
\hline $\mathrm{C} 4$ & $3^{\prime \prime}$ & & SPARE NOZZLE, CAPPED \\
\hline $\mathrm{C5}$ & $3^{\prime \prime}$ & & INLET \\
\hline C6 & $3^{\prime \prime}$ & & OUTLET \\
\hline
\end{tabular}

Ref: Alstad 1993, Lipnicki 1996, \& H-2-73187, Rev. 4.

If there was a discrepancy between the documents and the drawings, the drawings shall take precedence.

Comments placed in [] denote Engineering Change Notices (ECN) made against the reference drawings.

- Denotes risers tentatively available for sampling (Lipnicki 1996) 


\section{1-S-109}

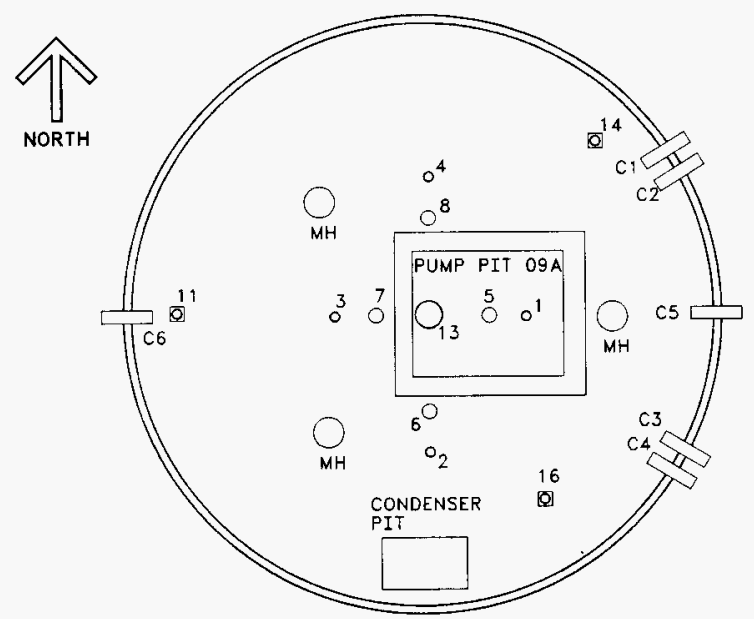

Ref: Alstad 1993

$\mathrm{H}-2-73188$. Rev. 4

$\mathrm{H}-2-37532$, Rev. 2

\section{TANK RISER LOCATION}

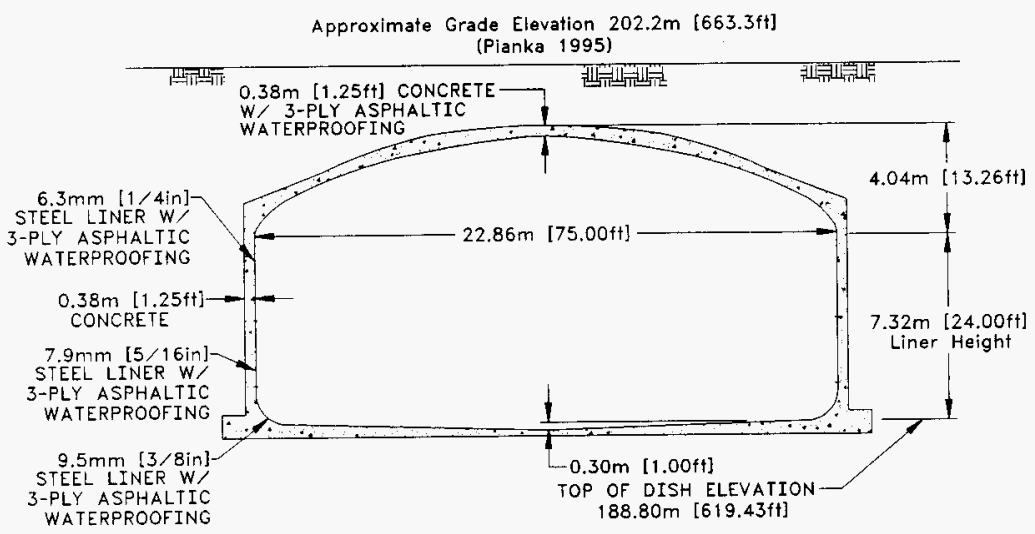

$$
\begin{gathered}
\text { NOT TO SCALE } \\
F-18
\end{gathered}
$$

Ref: $\mathrm{H}-2-1783$. Rev. 3 H-2-46293. Rev. 3 $\mathrm{H}-2-1784$. Rev. 2 


\begin{tabular}{|c|c|c|c|}
\hline NO. & DIA. & SAMPLING* & DESCRIPTION AND COMMENTS \\
\hline 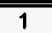 & $4 "$ & & CONN NOZZLE \\
\hline 2 & 4" & $x$ & $\begin{array}{l}\text { BLIND FLANGE [BM CEO-36907 12/11/86] [BREATHER } \\
\text { FILTER CEO-41064 } 3 / 17 / 87 \text { ] }\end{array}$ \\
\hline 3 & $4 "$ & & FIC [ENRAF ECN-622487 4/21/95] \\
\hline 4 & $4 "$ & & TEMPERATURE PROBE \\
\hline 5 & $12^{\prime \prime}$ & & SALTWELL SCREEN AND PUMP \\
\hline 6 & $12^{\prime \prime}$ & $x$ & SPARE [B-222 OBSRV PORT CE0-41064 3/17/87] \\
\hline 7 & $12^{\prime \prime}$ & & $\begin{array}{l}\text { VENTILATION [BLANK CEO-41064 3/17/87] [DUCT } \\
\text { REMOVED \& RISER CAPPED ECN-706501 8/29/95] }\end{array}$ \\
\hline 8 & $12^{\prime \prime}$ & & B-436 LOW \\
\hline 11 & $4 "$ & $\bar{x}$ & SMP \\
\hline 13 & $42^{\prime \prime}$ & & SLURRY DISTRIBUTOR \\
\hline 14 & 4" & $\underline{x}$ & SMP \\
\hline 16 & 4" & $\bar{x}$ & SMP [BM CEO-36907 12/11/86] \\
\hline C1 & $3 "$ & & SPARE NOZZLE, CAPPED \\
\hline $\mathrm{C} 2$ & 3" & & SPARE NOZZLE, CAPPED \\
\hline $\mathrm{C3}$ & 3 & & SPARE NOZZLE, CAPPED \\
\hline $\mathrm{C4}$ & $3^{\prime \prime}$ & & SPARE NOZZLE, CAPPED \\
\hline $\mathrm{C5}$ & $3^{\prime \prime}$ & & INLET \\
\hline C6 & $3 "$ & & OUTLET, CAPPED \\
\hline
\end{tabular}

Ref: Alstad 1993, Lipnicki 1996, \& H-2-73188, Rev. 4.

If there was a discrepancy between the documents and the drawings, the drawings shall take precedence.

Comments placed in $\square$ denote Engineering Change Notices (ECN) made against the reference drawings.

- Denotes risers tentatively available for sampling (Lipnicki 1996) 


\section{$241-S-110$}

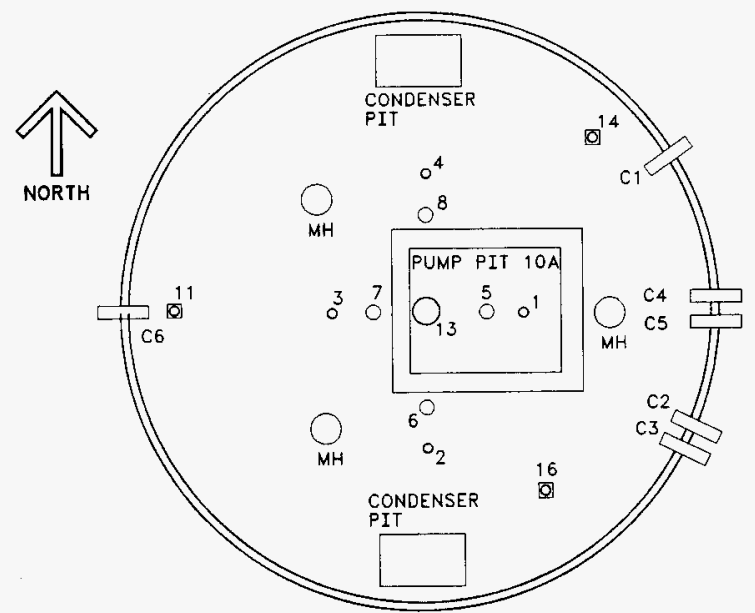

Ref: Alstad 1993

$\mathrm{H}-2-73189$, Rev. 4

$\mathrm{H}-2-37533$. Rev. 2

\section{TANK RISER LOCATION}

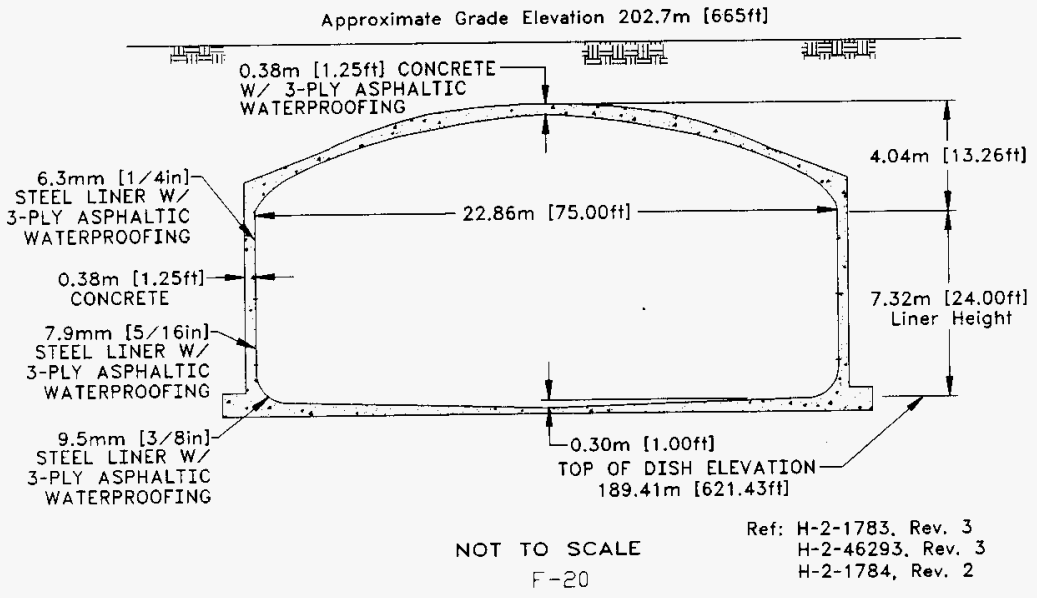




\begin{tabular}{|c|c|c|c|}
\hline NO. & $\overline{\mathrm{DIA}}$. & SAMPLING* & DESCRIPTION AND COMMENTS \\
\hline 1 & $4 "$ & & CONN NOZZLE \\
\hline 2 & $4^{\prime \prime}$ & & B-436 LOW [BM CEO-36907 12/11/86] \\
\hline 3 & $4^{\prime \prime}$ & & FIC [ENRAF ECN-623969 7/18/95] \\
\hline 4 & $4 "$ & & B-221 TEMPERATURE PROBE \\
\hline 5 & 12" & & SALTWELL SCREEN AND PUMP \\
\hline 6 & $12^{\prime \prime}$ & & $\begin{array}{l}\text { VENTILATION [BLANK CE0-41056 2/27/87] [DUCT } \\
\text { REMOVED \& RISER CAPPED ECN-706501 8/29/95] } \\
\end{array}$ \\
\hline 7 & $12^{\prime \prime}$ & $\bar{x}$ & B-222 OBSRV PORT \\
\hline 8 & $12^{\prime \prime}$ & $x$ & B-221 OBSRV PORT [SPARE CE0-41056 2/27/87] \\
\hline 11 & $4 "$ & $\bar{x}$ & SMP [BLANK ECN-195060 2/4/93] \\
\hline 13 & $42^{\prime \prime}$ & & SLURRY DISTRIBUTOR \\
\hline 14 & 4" & $\bar{x}$ & SMP [BLANK ECN-195060 2/4/93] \\
\hline 16 & 4" & $x$ & $\begin{array}{l}\text { SMP [BM CEO-36907 } 12 / 11 / 86] \text { [BREATHER FILTER } \\
\text { CEO-41056 2/27/87] [INSTALL HEAT VAPOR PROBE \& } \\
\text { REMOVE WITHIN } 90 \text { DAYS ECN-624409L 9/26/95] } \\
\text { [OFFSET ADAPTER W/ BREATHER FILTER ECN- } \\
624410 \text { 9/26/95 \& ECN-626610 } 11 / 10 / 95 \text { ] }\end{array}$ \\
\hline $\mathrm{C} 1$ & $3^{\prime \prime}$ & & SPARE NOZZLE, CAPPED \\
\hline $\mathrm{C2}$ & $3^{\prime \prime}$ & & SPARE NOZZLE, CAPPED \\
\hline $\mathrm{C3}$ & $3^{\prime \prime}$ & & SPARE NOZZLE, CAPPED \\
\hline C4 & 3" & & $\begin{array}{l}\text { INLET LINE V535, SEALED IN DIVERSION BOX 241-S- } \\
151\end{array}$ \\
\hline C5 & 3" & & $\begin{array}{l}\text { INLET LINE V534, SEALED IN DIVERSION BOX 241-S- } \\
151\end{array}$ \\
\hline $\mathrm{C6}$ & 3" & & OUTLET \\
\hline
\end{tabular}

Ref: $\quad$ Alstad 1993, Lipnicki 1996, \& H-2-73189, Rev. 4.

If there was a discrepancy between the documents and the drawings, the drawings shall take precedence.

Comments placed in 0 denote Engineering Change Notices (ECN) made against the reference drawings.

- Denotes risers tentatively available for sampling (Lipnicki 1996) 


\section{$241-5-111$}
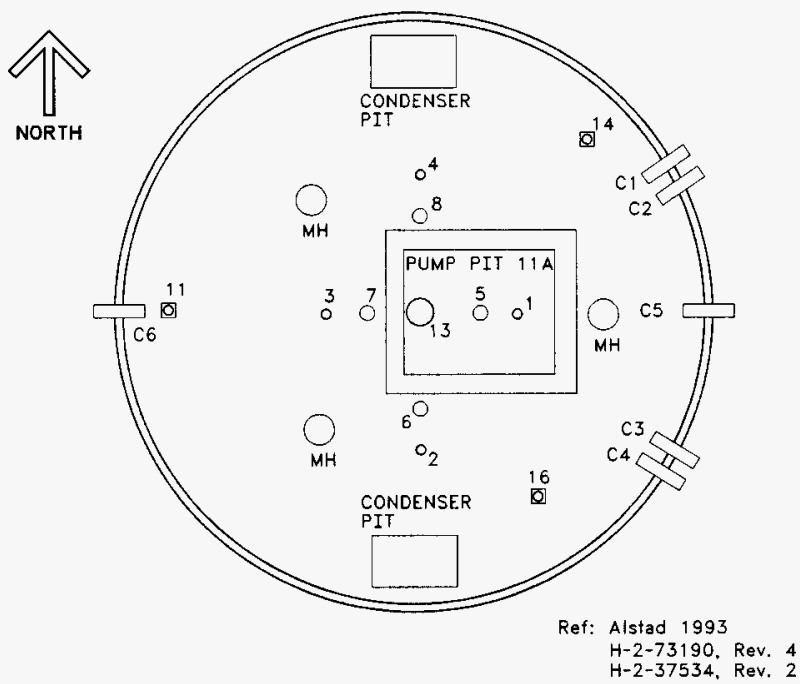

\section{TANK RISER LOCATION}

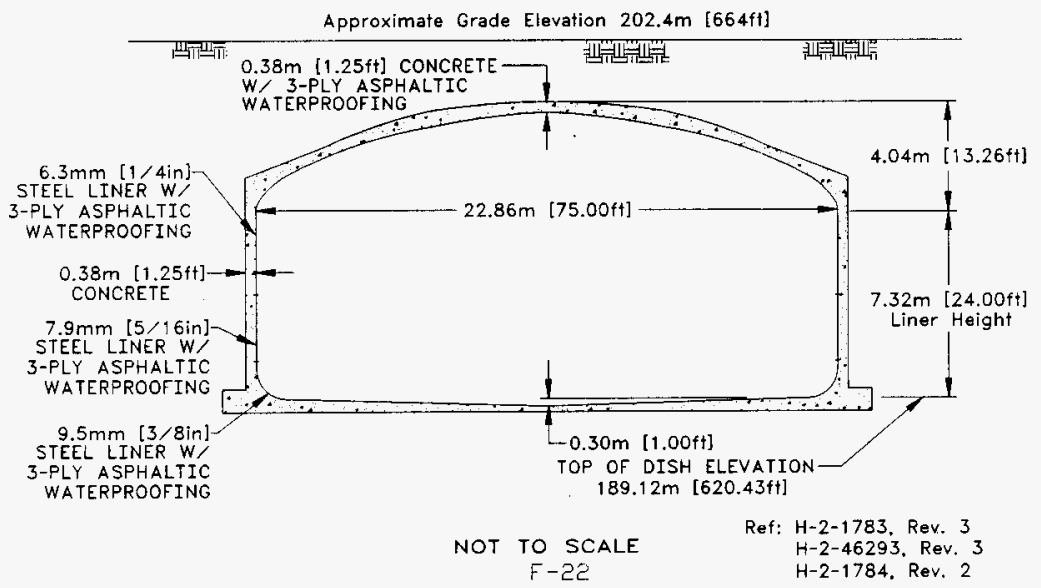




\begin{tabular}{|c|c|c|c|}
\hline NO. & DIA. & SAMPLING* & DESCRIPTION AND COMMENTS \\
\hline 1 & $4^{\prime \prime}$ & & CONN NOZZLE \\
\hline 2 & 4" & & $\begin{array}{l}\text { FIC [BM CEO-36907 12/11/86] [SPARE ECN-631237 } \\
5 / 13 / 96]\end{array}$ \\
\hline 3 & $4 "$ & & FIC [ENRAF ECN-613546 $8 / 3 / 94$ ] \\
\hline 4 & 4" & & TEMPERATURE PROBE \\
\hline 5 & $12^{\prime \prime}$ & & SALTWELL SCREEN AND PUMP \\
\hline 6 & $12^{\prime \prime}$ & $x$ & \begin{tabular}{|lll} 
VENTILATION [BLANK CE0-41065 & $3 / 17 / 87]$ \\
REMOVED \& RISER CAPPED ECN-706501 & $8 / 29 / 95]$
\end{tabular} \\
\hline 7 & $12^{\prime \prime}$ & $\bar{x}$ & PROTOTYPE SALTWELL \\
\hline 8 & $12^{\prime \prime}$ & $x$ & $\begin{array}{l}\text { B-222 OBSRV PORT [TEMPORARY AIR FILTER } \\
\text { INSTALLED TO ALLOW FOR CORE SAMPLING IN } \\
\text { RISER } 14 \text { ECN-632402L 5/10/96] }\end{array}$ \\
\hline 11 & 4" & $x$ & PROTOTYPE SALTWELL \\
\hline 13 & 42" & & SLURRY DISTRIBUTOR \\
\hline 14 & 4" & $x$ & $\begin{array}{l}\text { BLIND FLANGE [BREATHER FILTER CE0-41065 } \\
3 / 17 / 87] \text { [HYDROGEN MONITOR I AIR FILTER ECN- } \\
\text { W369-11 12/15/94] [TEMPORARILY REMOVE } \\
\text { HYDROGEN MONITOR I AIR FILTER TO ALLOW FOR } \\
\text { CORE SAMPLING ECN-632402L 5/10/96] } \\
\end{array}$ \\
\hline 16 & 4" & & B-436 LOW [BM CEO-36907 12/11/86] \\
\hline C1 & $3^{\prime \prime}$ & & SPARE NOZZLE, CAPPED \\
\hline $\mathrm{C2}$ & $3 "$ & & SPARE NOZZLE, CAPPED \\
\hline C3 & 3" & & SPARE NOZZLE, CAPPED \\
\hline $\mathrm{C4}$ & $3 "$ & & SPARE NOZZLE, CAPPED \\
\hline $\mathrm{C5}$ & $3 "$ & & INLET \\
\hline C6 & $3^{\prime \prime}$ & & OUTLET \\
\hline
\end{tabular}

Ref: $\quad$ Alstad 1993, Lipnicki 1996, \& H-2-73190, Rev. 4.

If there was a discrepancy between the documents and the drawings, the drawings shall take precedence.

Comments placed in $\square$ denote Engineering Change Notices (ECN) made against the reference drawings.

* Denotes risers tentatively available for sampling (Lipnicki 1996) 


\section{$241-S-112$}

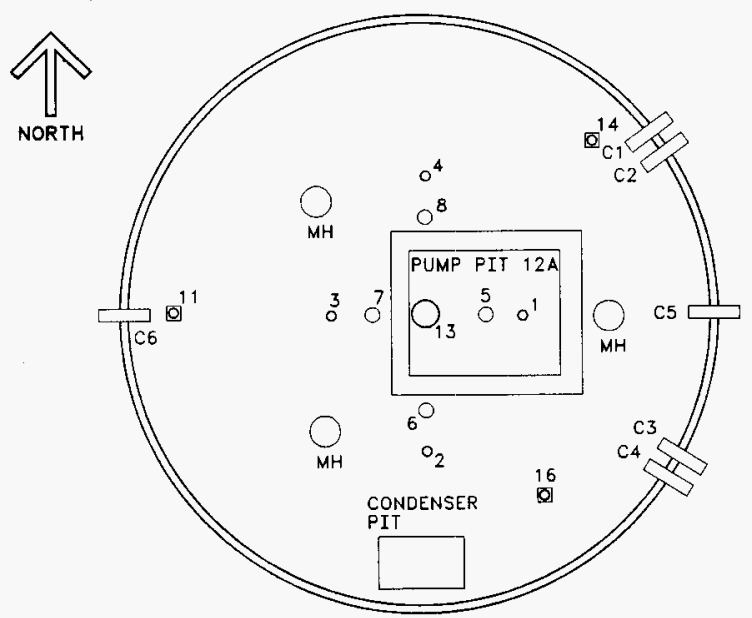

Ref: Alstad 1993

$\mathrm{H}-2-73191$, Rev. 4

$\mathrm{H}-2-37535$, Rev. 2

\section{TANK RISER LOCATION}

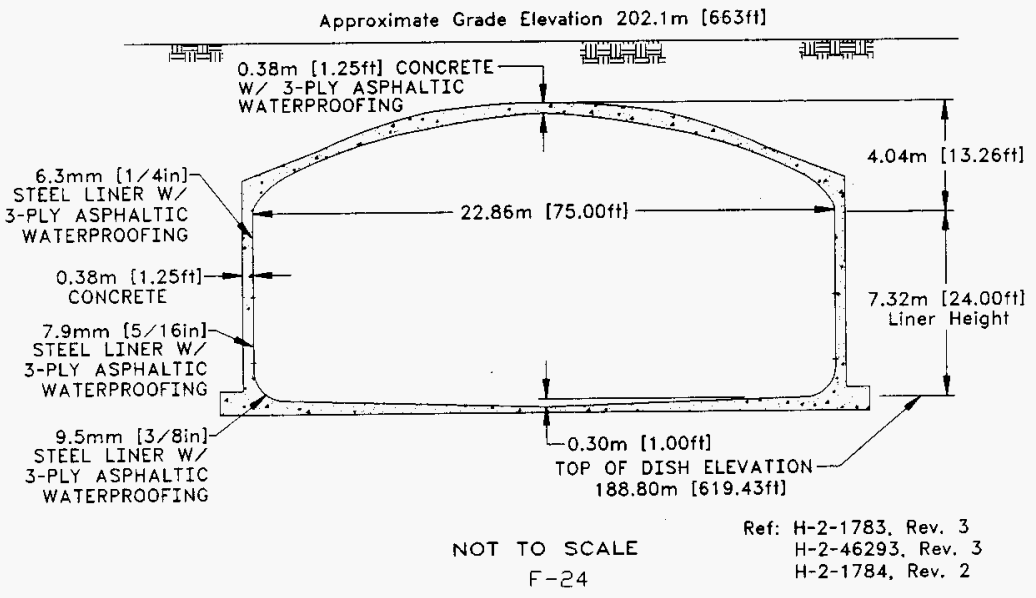




\begin{tabular}{|c|c|c|c|}
\hline NO. & DIA. & SAMPLING* & DESCRIPTION AND COMMENTS \\
\hline 1 & 4" & & CONN NOZZLE \\
\hline 2 & 4" & $x$ & $\begin{array}{l}\text { BLIND FLANGE [BM CEO-36907 12/11/86] [BLANK } \\
\text { CE0-41066 } 3 / 17 / 87]\end{array}$ \\
\hline 3 & 4" & & FIC [ENRAF ECN-620751 2/27/95] \\
\hline 4 & 4" & & TEMPERATURE PROBE \\
\hline 5 & $12^{\prime \prime}$ & & SALTWELL SCREEN AND PUMP \\
\hline 6 & $12^{\prime \prime}$ & $\bar{x}$ & B-222 OBSRV PORT \\
\hline 7 & $12^{\prime \prime}$ & & \begin{tabular}{|l|l} 
VENTILATION [BLANK CE0-41066 3/17/87] [DUCT \\
REMOVED \& RISER CAPPED ECN-706501 & $8 / 29 / 95]$ \\
\end{tabular} \\
\hline 8 & $12 "$ & & B-436 LOW \\
\hline 11 & 4" & $\bar{x}$ & SMP \\
\hline 13 & $42^{\prime \prime}$ & & SLURRY DISTRIBUTOR \\
\hline 14 & 4" & $x$ & $\begin{array}{l}\text { SMP [BREATHER FILTER CE0-41066 3/17/87] } \\
\text { [HYDROGEN MONITOR / AIR FILTER ECN-W369-11 } \\
\text { 12/15/94] }\end{array}$ \\
\hline 16 & 4" & $\bar{x}$ & SMP [BM CEO-36907 12/11/86] \\
\hline $\mathrm{C} 1$ & $3 "$ & & SPARE NOZZLE, CAPPED \\
\hline $\mathrm{C2}$ & $3^{\prime \prime}$ & & SPARE NOZZLE, CAPPED \\
\hline $\mathrm{C3}$ & $3^{\prime \prime}$ & & SPARE NOZZLE, CAPPED \\
\hline $\mathrm{C4}$ & 3" & & SPARE NOZZLE, CAPPED \\
\hline C5 & 3" & & INLET \\
\hline $\mathrm{C6}$ & 3" & & OUTLET, CAPPED \\
\hline
\end{tabular}

Ref: $\quad$ Alstad 1993, Lipnicki 1996, \& H-2-73191, Rev. 4.

If there was a discrepancy between the documents and the drawings, the drawings shall take precedence.

Comments placed in $[$ denote Engineering Change Notices (ECN) made against the reference drawings.

- Denotes risers tentatively available for sampling (Lipnicki 1996) 
THIS PAGE INTENTIONALLY LEFT BLANK 


$$
\begin{aligned}
& \text { Z } \\
& \mathbf{0} \\
& \mathbf{m} \\
& \frac{\mathbf{n}}{\mathbf{x}} \\
& \mathbf{\Omega}
\end{aligned}
$$


THIS PAGE INTENTIONALLY LEFT BLANK 


\section{S TANK FARM PHOTOS}

\begin{tabular}{|c|c|c|c|}
\hline 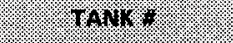 & MOMHAEE & 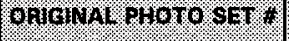 & $\operatorname{mat}$ \\
\hline S Farm Aerial Photo & N/A & $93030994-235 \mathrm{CN}$ & N/A \\
\hline 241-S-101 & $94041033-40 \mathrm{CN}$ & 8801412 & $3 / 18 / 88$ \\
\hline 241-S-102 & $94041033-39 \mathrm{CN}$ & 8801411 & $3 / 18 / 88$ \\
\hline 241-S-103 & $94041033-26 \mathrm{CN}$ & 89060148 & $6 / 1 / 89$ \\
\hline 241-S-104 & $94041033-25 \mathrm{CN}$ & 8408365 & $12 / 12 / 84$ \\
\hline 241-S-105 & $94041033-22 \mathrm{CN}$ & 89041307 & $4 / 12 / 89$ \\
\hline 241-S-106 & $94041033-23 \mathrm{CN}$ & 89032049 & $3 / 17 / 89$ \\
\hline 241-S-107 & $94041033-24 \mathrm{CN}$ & 8701464 & $3 / 12 / 87$ \\
\hline 241-S-108 & $94041033-32 \mathrm{CN}$ & 8701462 & $3 / 12 / 87$ \\
\hline 241-S-109 & $94041033-18 \mathrm{CN}$ & 8405333 & $8 / 24 / 84$ \\
\hline 241-S-110 & $94041033-19 \mathrm{CN}$ & 8701463 & $3 / 12 / 87$ \\
\hline 241-S-111 & $94041033-17 \mathrm{CN}$ & 89081043 & $8 / 10 / 89$ \\
\hline $241-S-112$ & $94041033-30 \mathrm{CN}$ & 8701812 & $3 / 24 / 87$ \\
\hline
\end{tabular}


THIS PAGE INTENTIONALLY LEFT BLANK 


\section{STANK FARM}

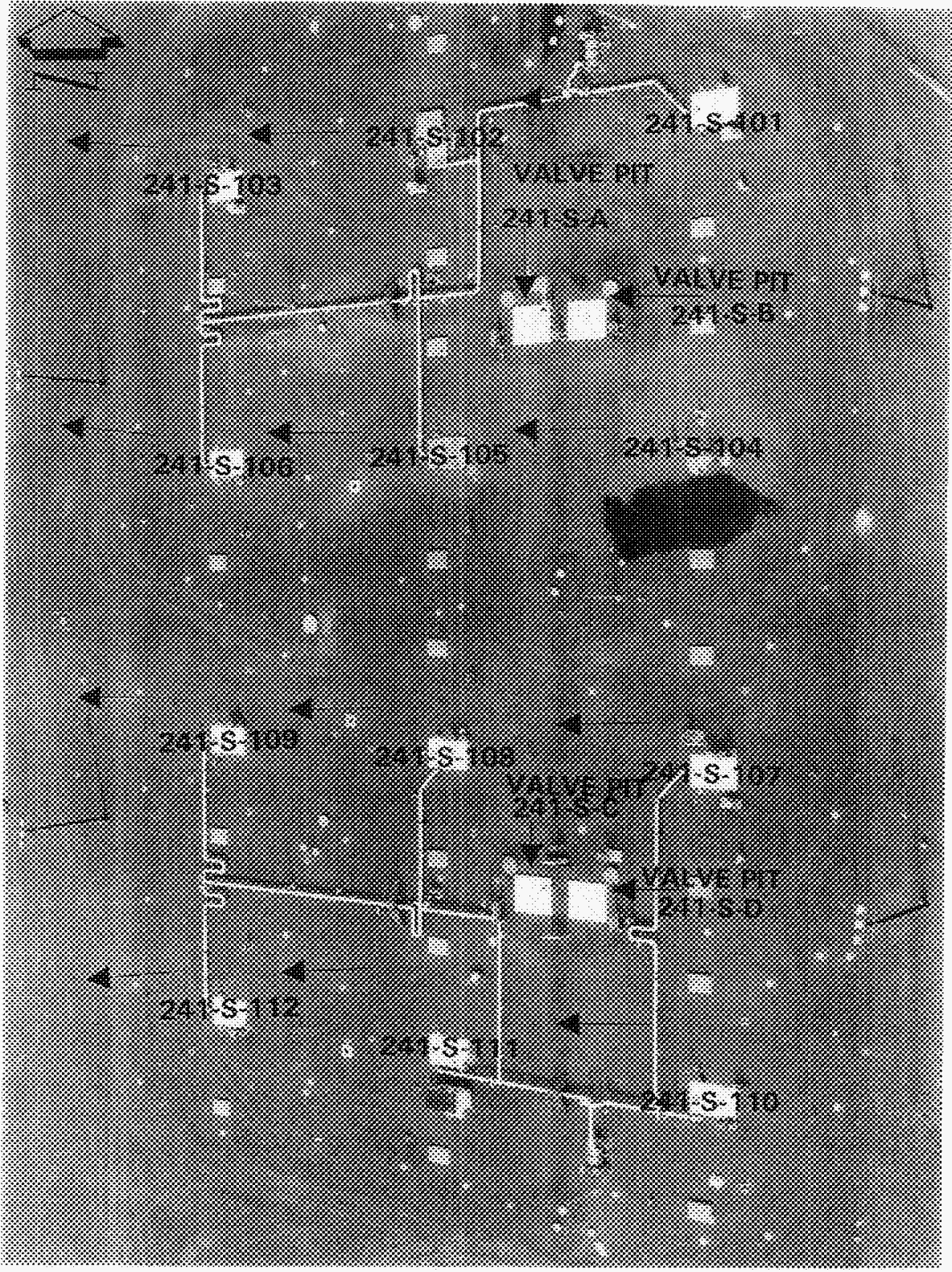


THIS PAGE INTENTIONALLY LEFT BLANK 


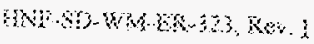

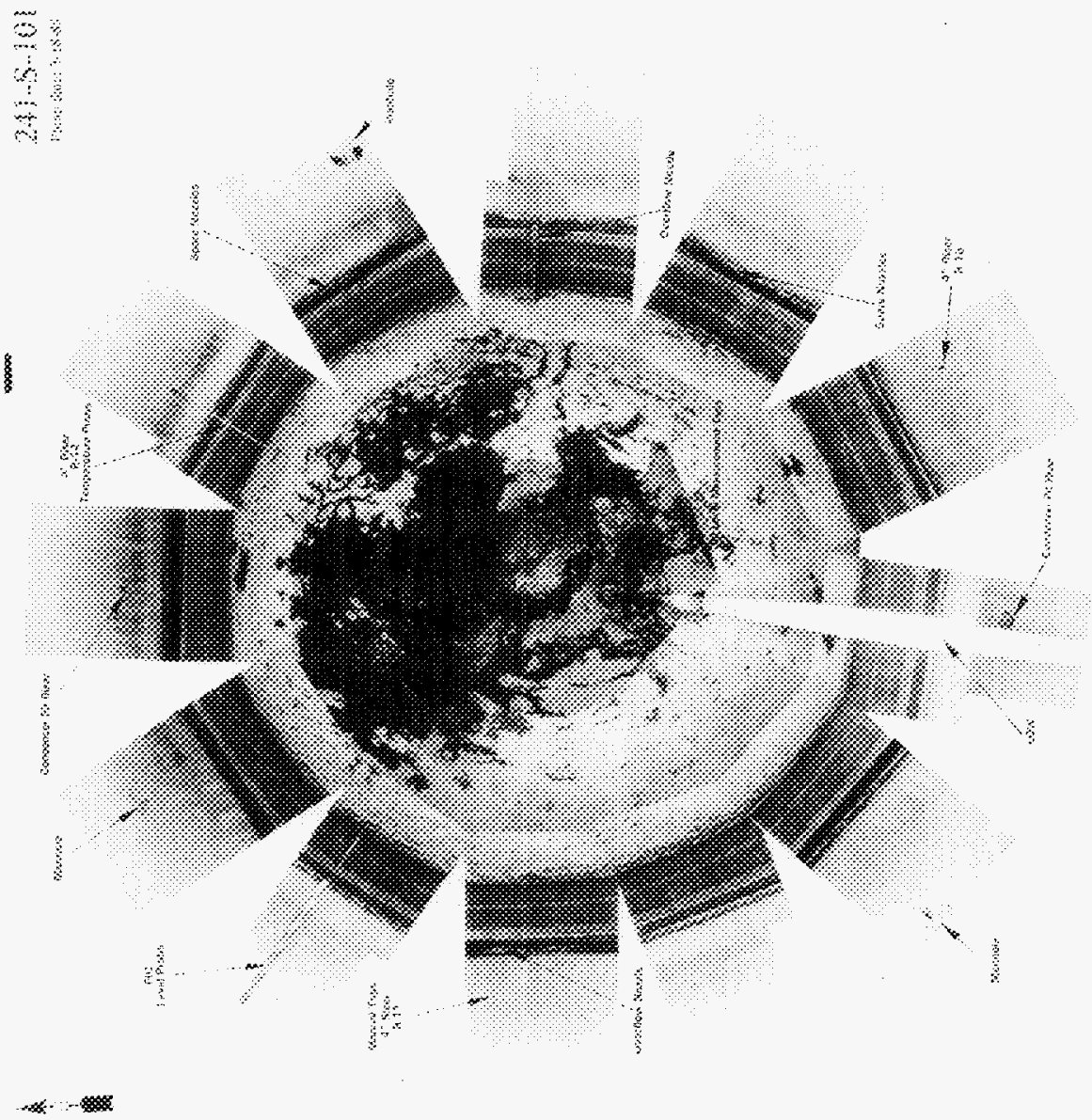


THIS PAGE INTENTIONALLY

LEFT BLANK 


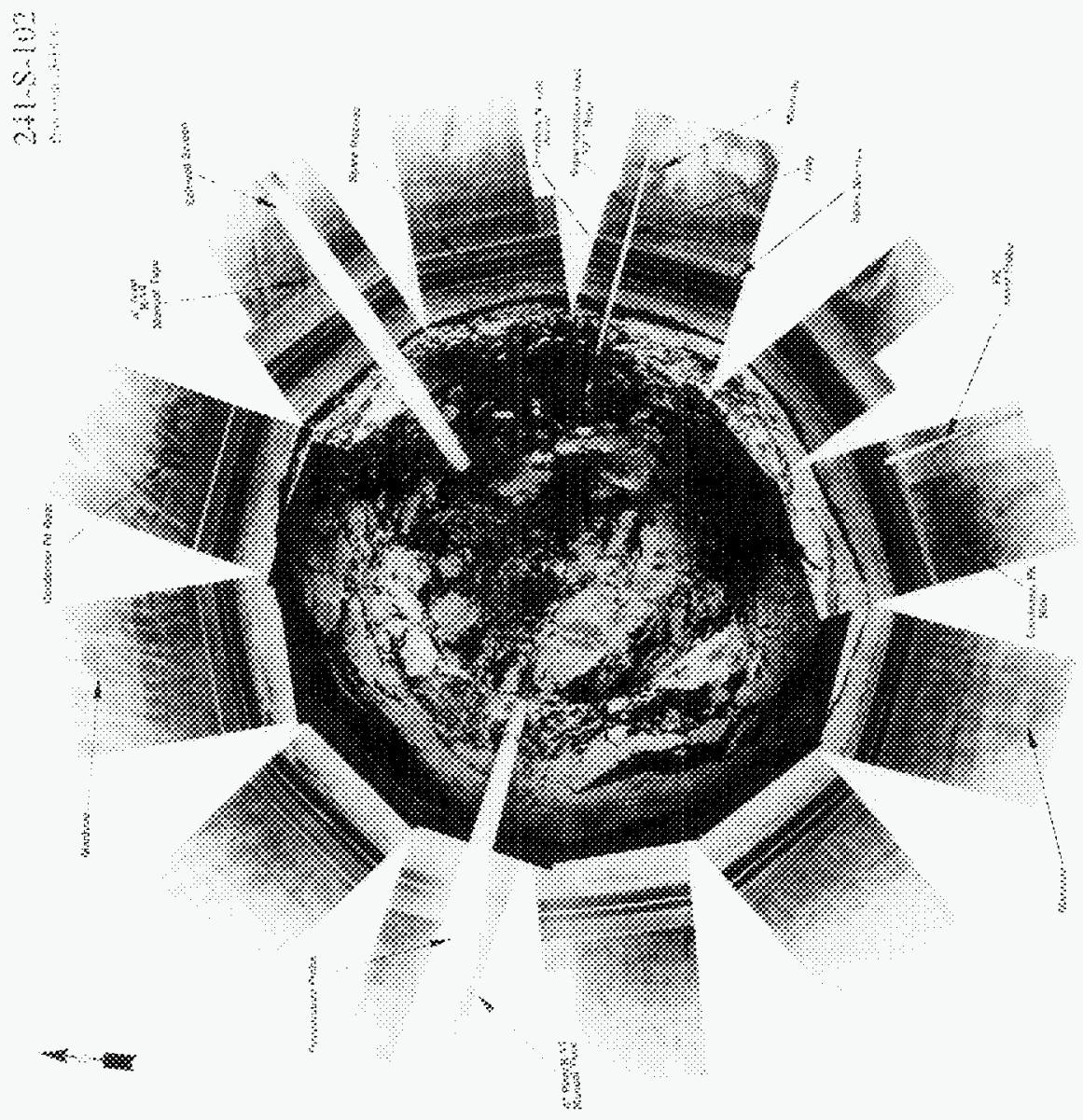


THIS PAGE INTENTIONALLY LEFT BLANK 
$\frac{3}{3}$

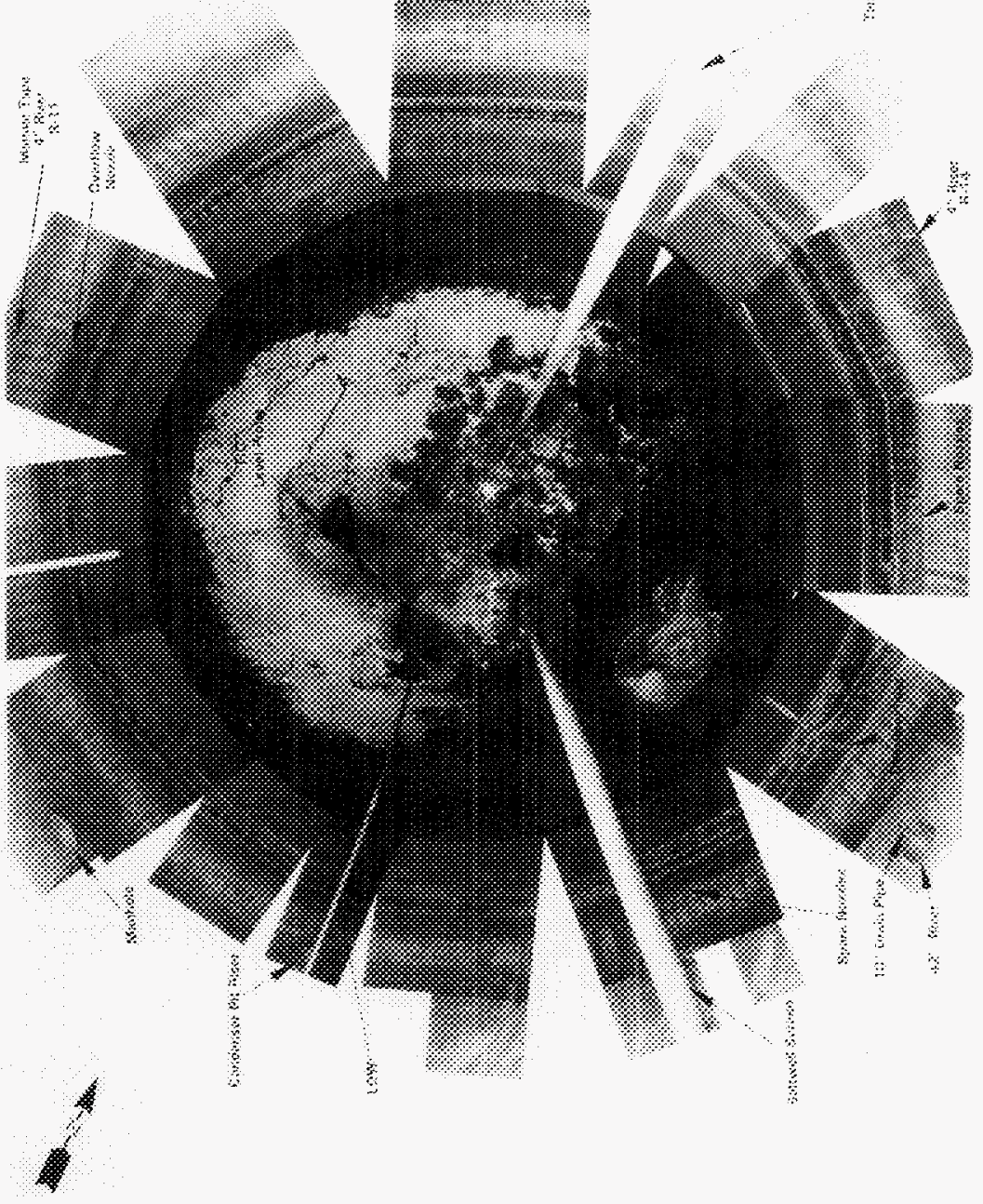


THIS PAGE INTENTIONALLY LEFT BLANK 
要

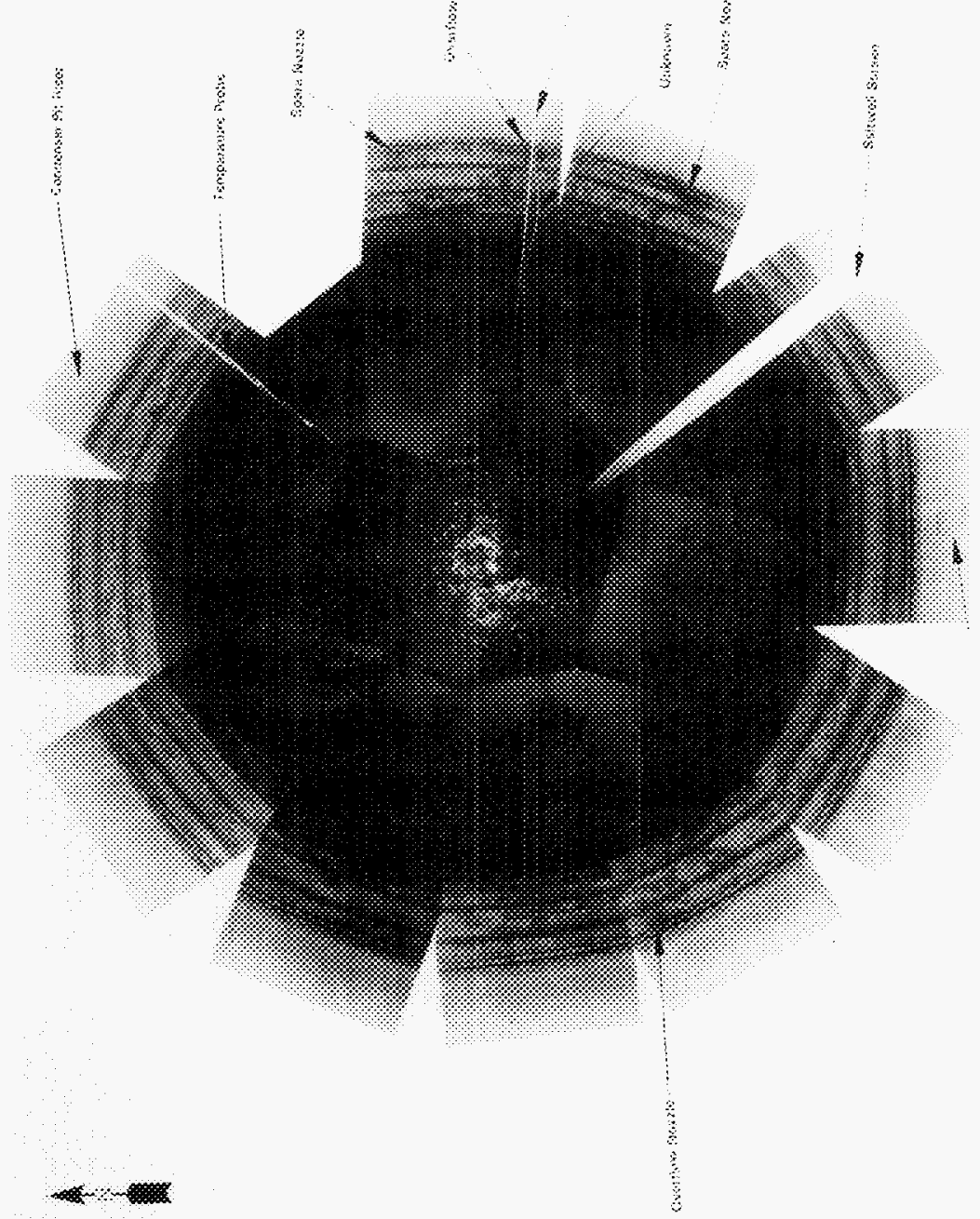


THIS PAGE INTENTIONALLY

LEFT BLANK 


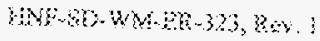

$\begin{array}{ll}3 & \vdots \\ m & \vdots \\ \vdots & \vdots \\ \vdots & \vdots \\ \cdots & \vdots \\ \cdots & \vdots\end{array}$

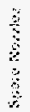

$\vdots$
$\vdots$
$\vdots$
$\vdots$
$\vdots$

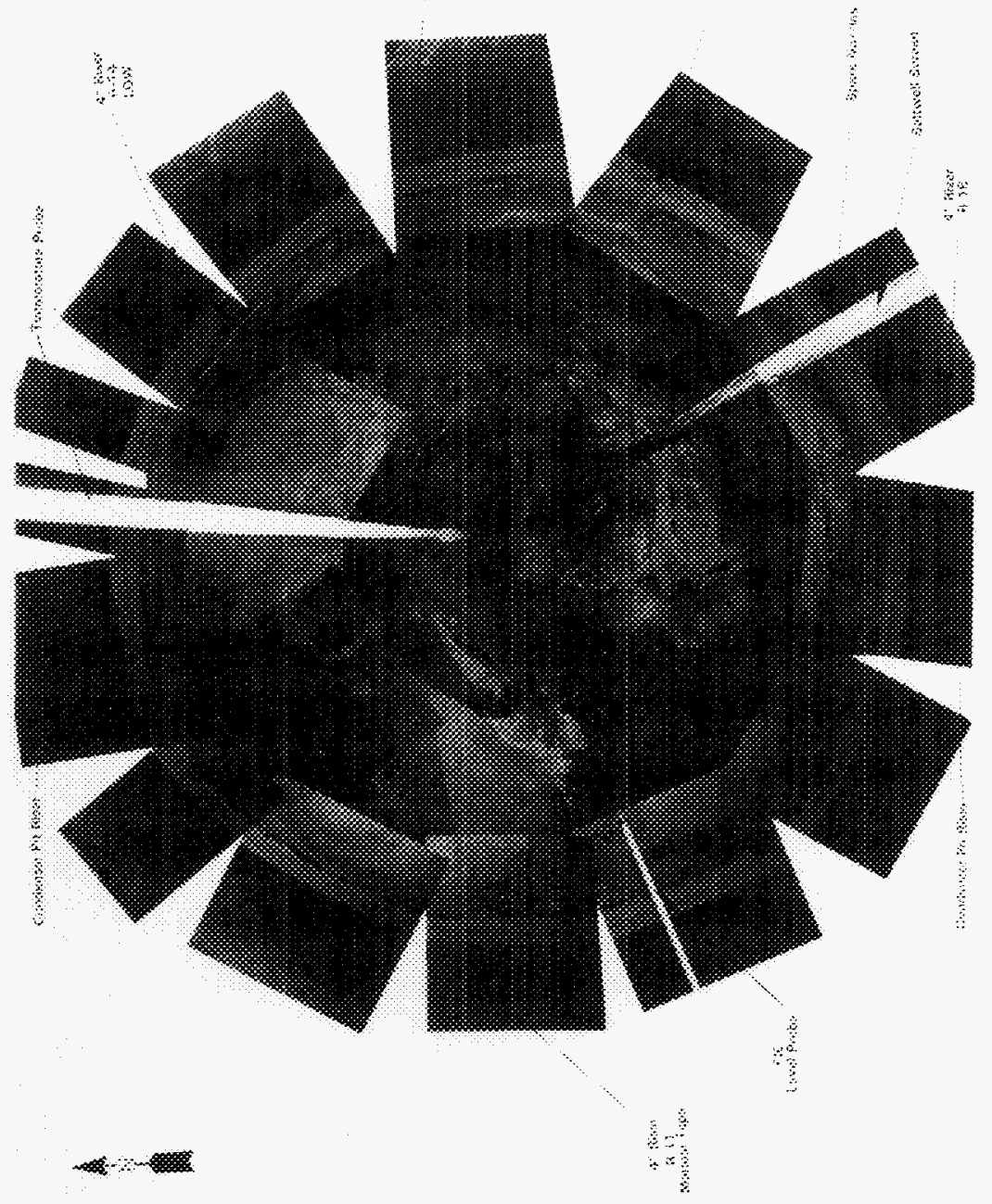


THIS PAGE INTENTIONALLY LEFT BLANK 
$\frac{8}{3}$

0
3
3
3
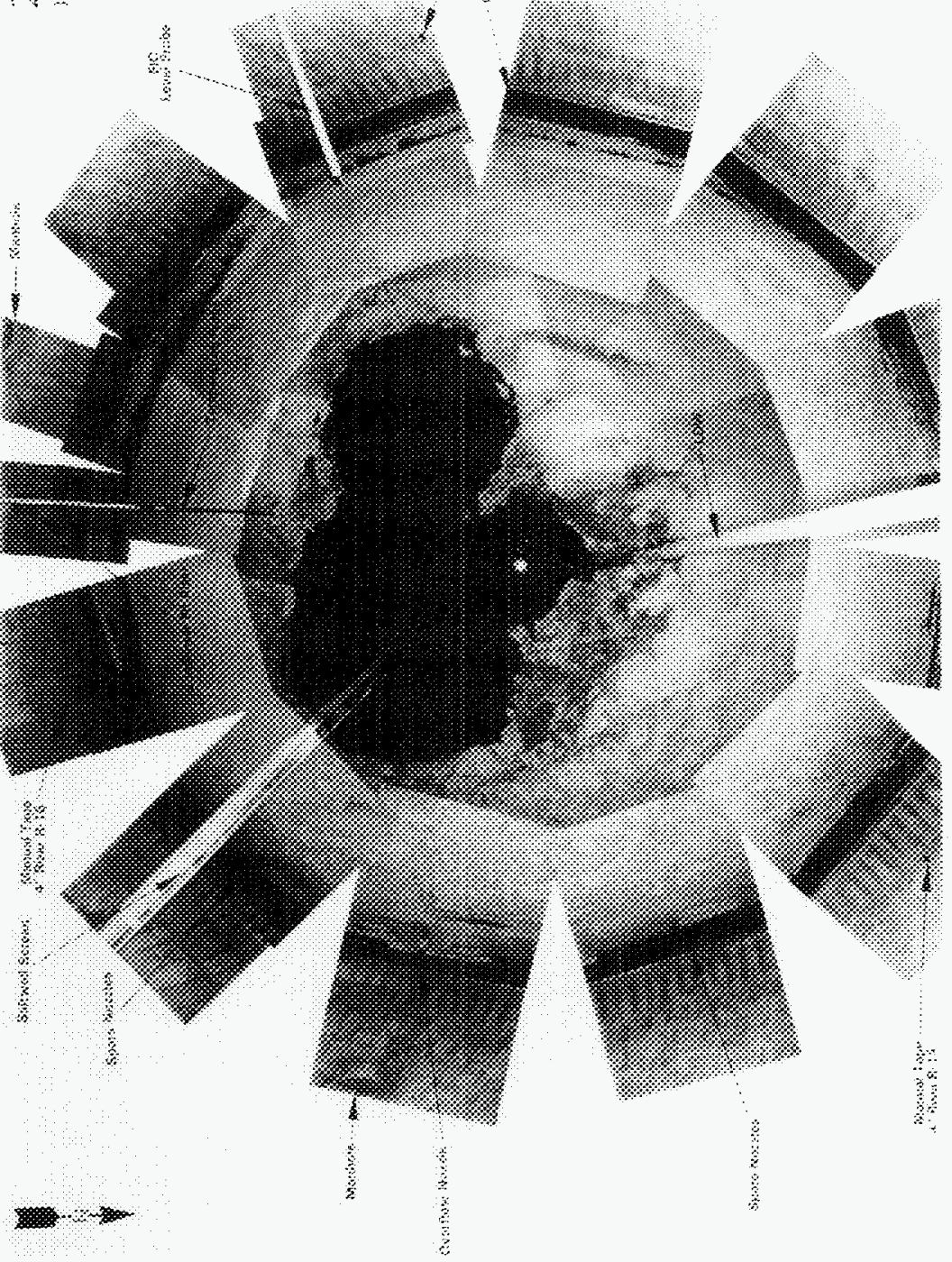

\%

- . 3 \% $\%$

\%

\% $8 \% 8$

$8 \%$

\%
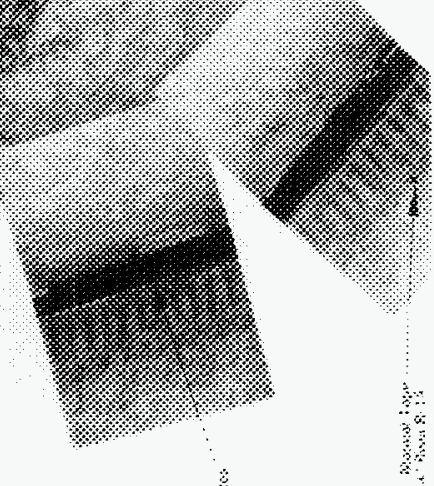


\section{THIS PAGE INTENTIONALLY}

LEFT BLANK 


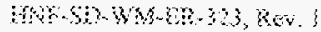

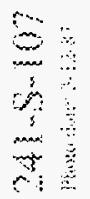

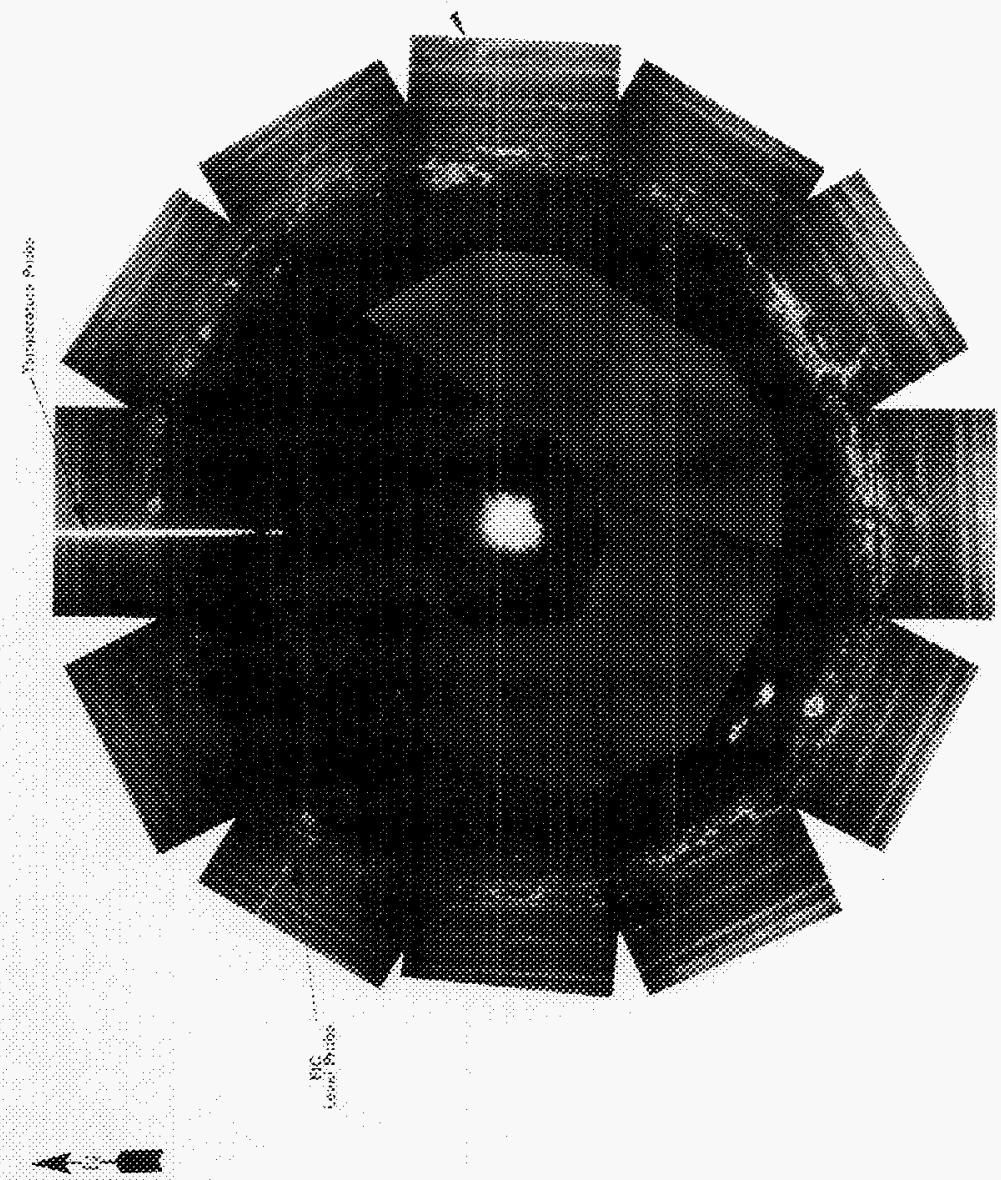


THIS PAGE INTENTIONALLY LEFT BLANK 


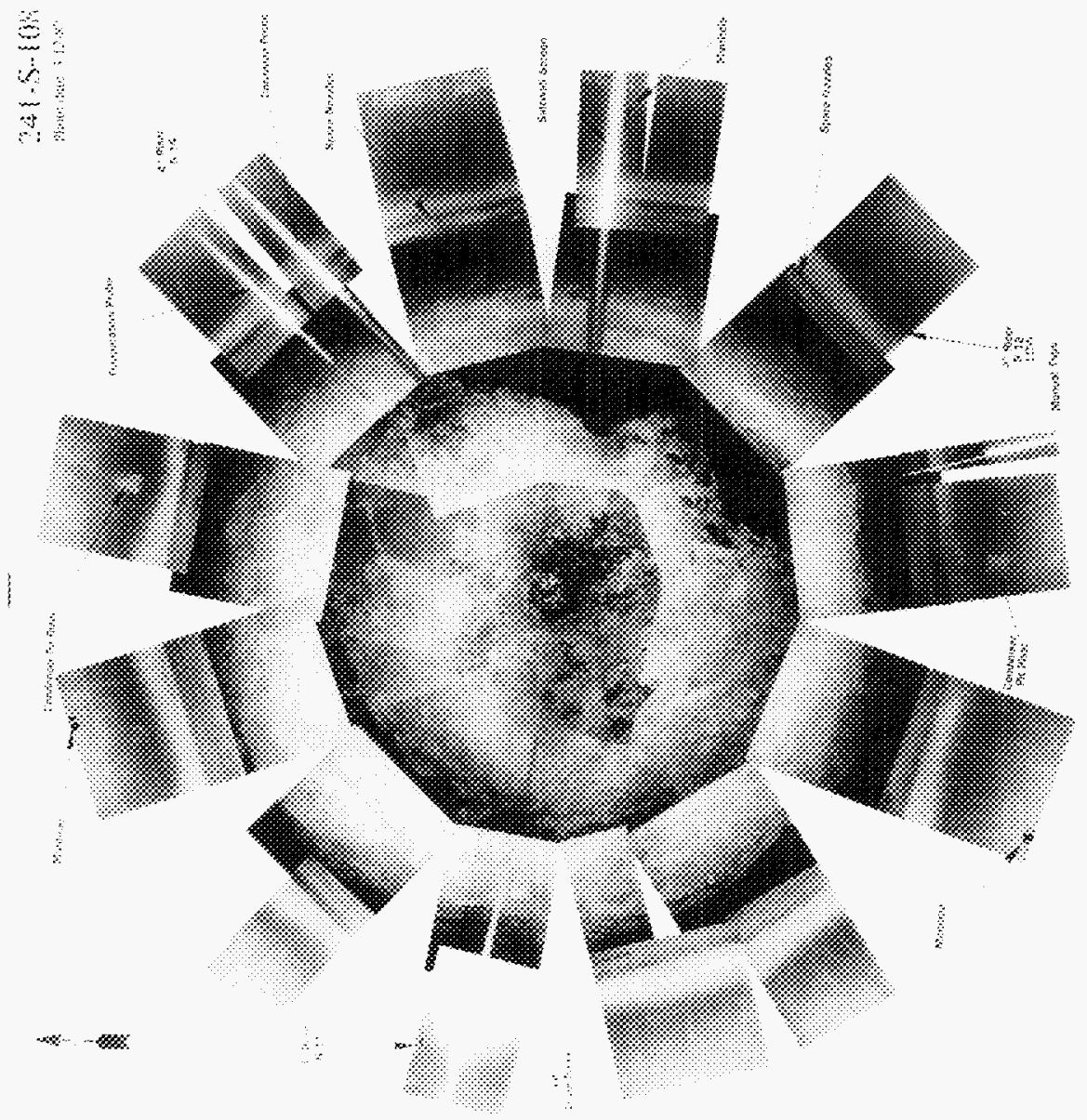


THIS PAGE INTENTIONALLY LEFT BLANK 
के
के

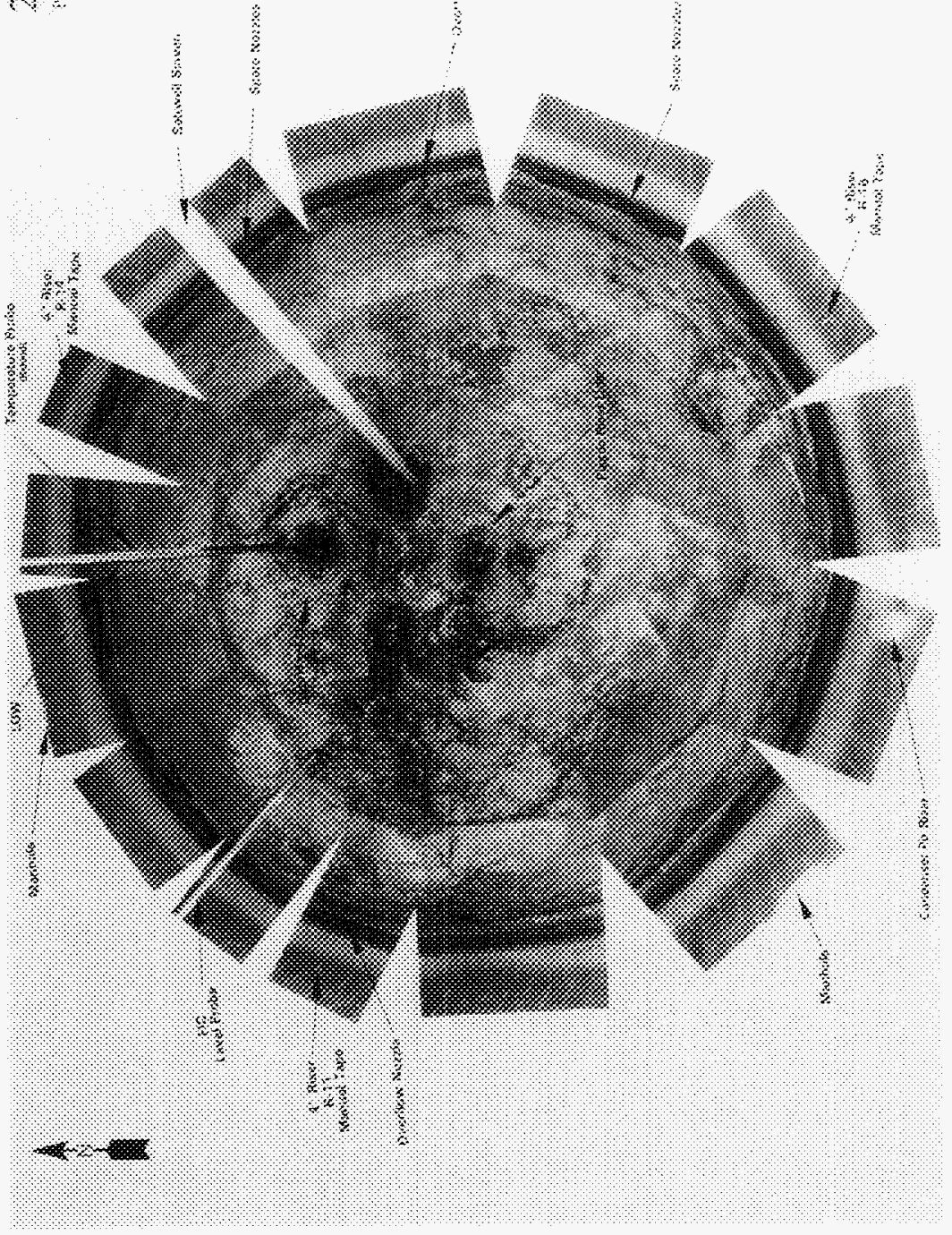


THIS PAGE INTENTIONALLY LEFT BLANK 


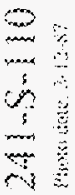

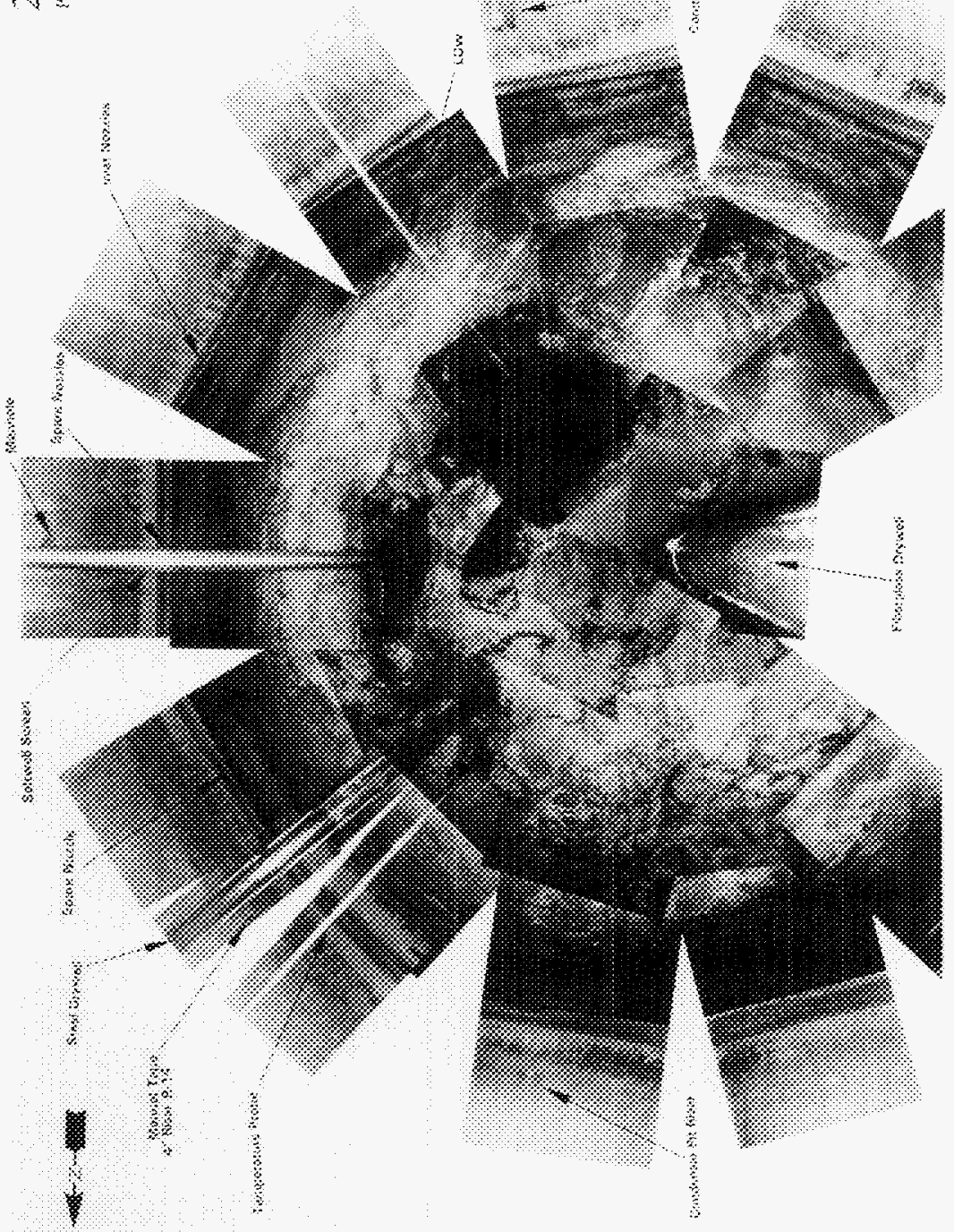


THIS PAGE INTENTIONALLY LEFT BLANK 


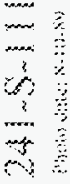
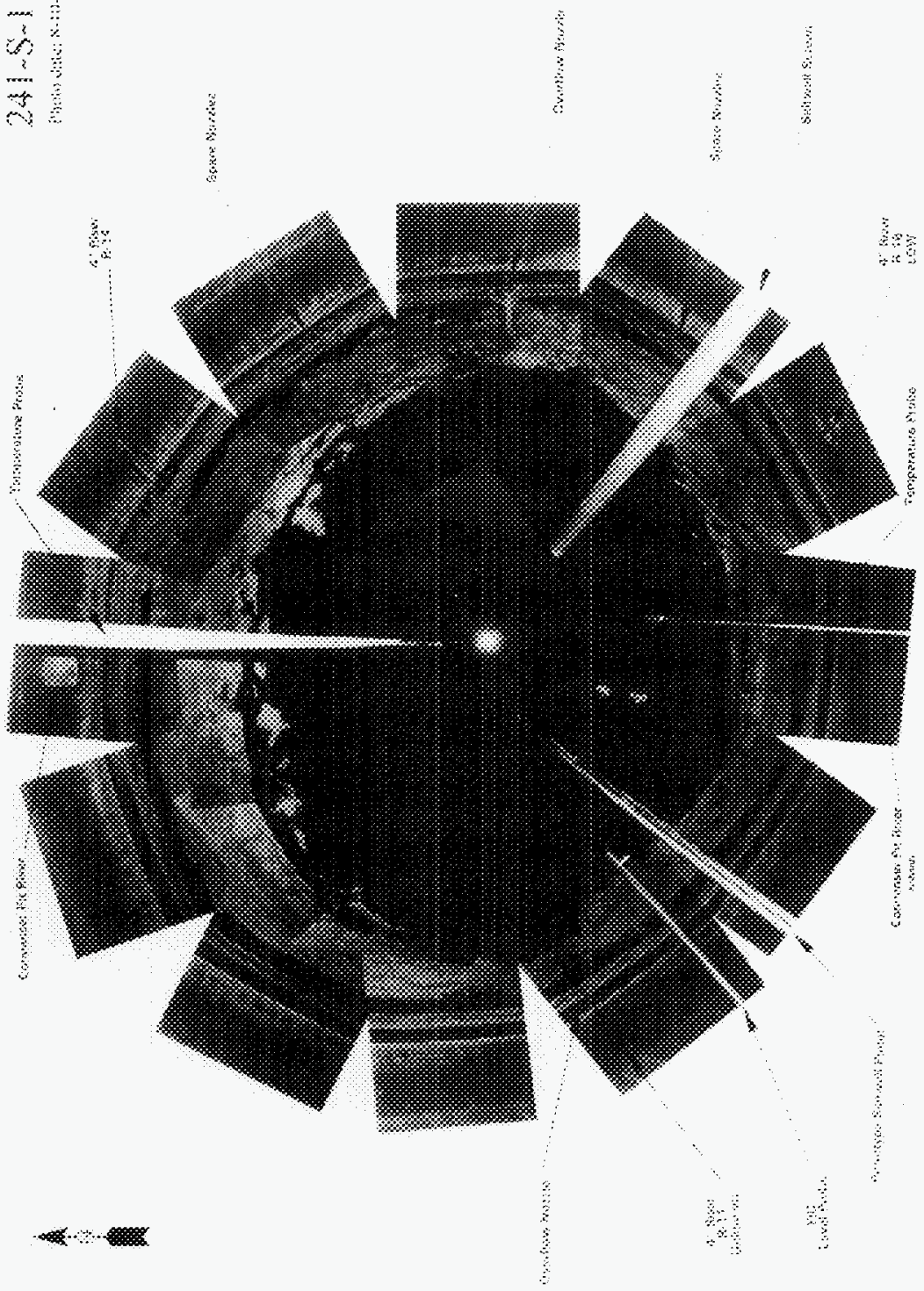
THIS PAGE INTENTIONALLY LEFT BLANK 


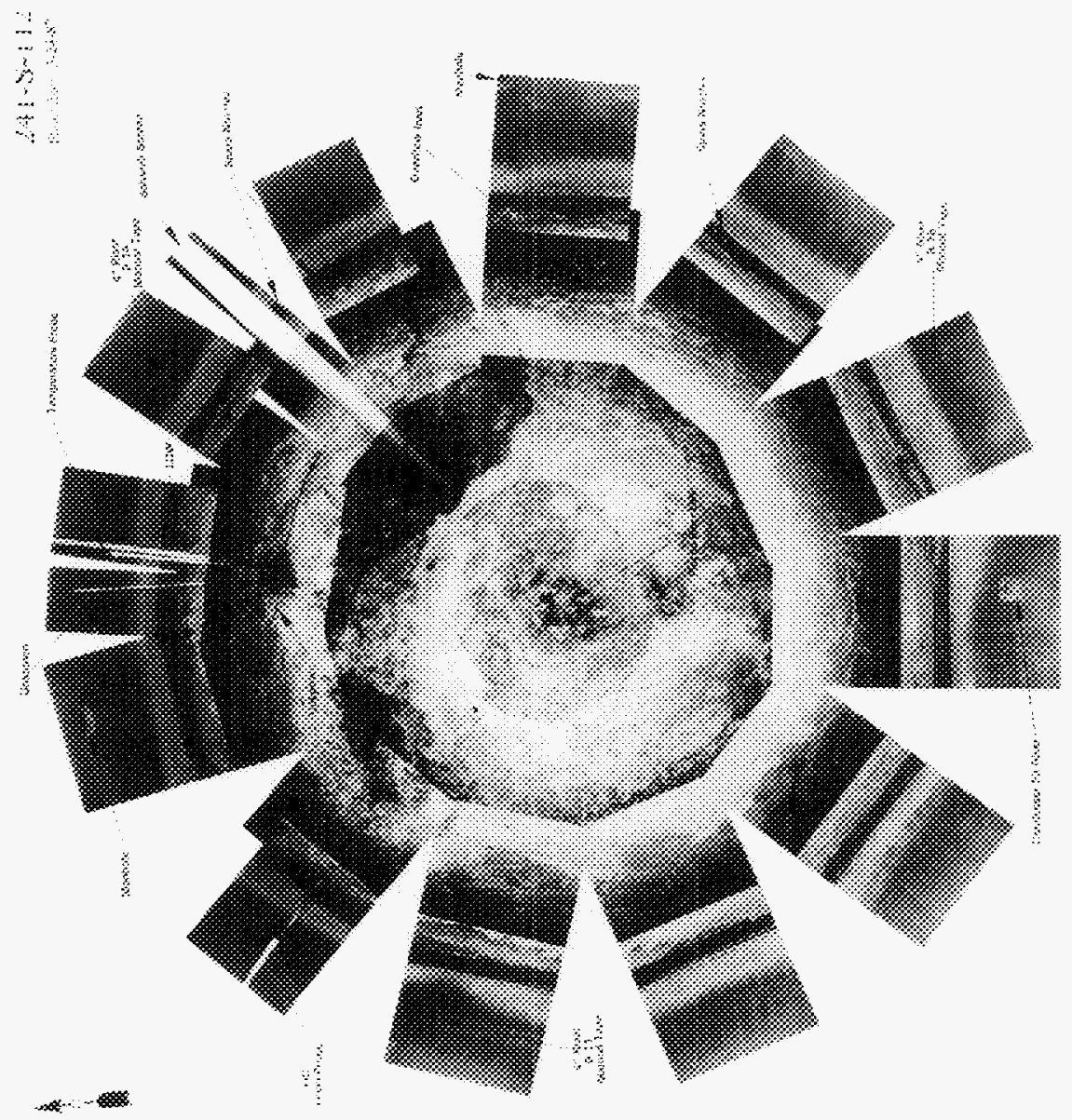


THIS PAGE INTENTIONALLY LEFT BLANK 


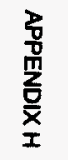


THIS PAGE INTENTIONALLY

LEFT BLANK 


\title{
Hanford Tank Chemical and Radionuclide Inventories: HDW Model Rev. 4
}

\author{
Stephen F. Agnew \\ James Boyer \\ Robert A. Corbin \\ Tomasita B. Duran \\ John R. FitzPatrick \\ Kenneth A. Jurgensen \\ Theodore P. Ortiz \\ Bonnie L. Young \\ Chemical Science and Technology Division \\ Los Alamos National Laboratory \\ Los Alamos, NM 87545 \\ Jamuary 1997

\section{Executive Summary}

This estimate for the chemical and radionuclide compositions of the 177 Hanford High Level Waste storage tanks is the fourth major revision in a developing model called the Hanford Defined Waste (HDW) model. The particular aspects of Rev. 4 are an expansion of the radionuclides considered from four to forty-six and the inclusion of variability estimates for each analyte within the predictions. All estimates are valid up to $1-1-94$ for transactions and radionuclide decay. The HDW model is composed of four parts:

1) compilation of transaction records for all the tanks called the Waste Status and Transaction Record Summary (WSTRS);

2) derivation called the Tank Layer Model (TLM) of solids histories for each tank based an primary additions of waste;

3) calculation of supernatant blending and concentration with the Supernatant Mixing Model (SMM); and

4) combination of process information along with some transaction information to derive compositions for about fifty Hanford Defined Wastes (HDW's), each of which has both sludge and supernatant layers.

All of this information is combined together in a spreadsheet format to produce total chemical and radionuclide compositions for each tank's waste as well as a composition for its TLM and SMM blends. Furthermore, each tank's inventory is also represented by a linear combination of TLM sludges and SMM supernatants, each expressed in kgal of original waste. Thus, the genealogy of each tank's waste can be traced back to the plant and process from which it derived. These estimates comprise some 33 nonradioactive species, 4 properties (density, water $w t \%$, TOC wt\%, sludge void fraction), and 46 radionuclides. The 33 non-radioactive species in the model are $\mathrm{Na}, \mathrm{Al}, \mathrm{Fe}, \mathrm{Cr}, \mathrm{Bi}, \mathrm{La}, \mathrm{Hg}, \mathrm{Zr}, \mathrm{Pb}, \mathrm{Ni}$, $\mathrm{Sr}(\mathrm{stable}), \mathrm{Mn}, \mathrm{Ca}, \mathrm{K}, \mathrm{OH}$, nitrate, nitrite, carbonate, phosphate, sulfate, silicate, $\mathrm{F}, \mathrm{Cl}$, citrate, EDTA, HEDTA, glycolate, acetate, oxalate, DBP, butanol, ammonia, and ferrocyanide. The forty-six radionuclides are: $\mathrm{H}-3, \mathrm{C}-14$, Ni-59, Ni-63, Co-60, Se-79, Sr-90, Y-90, Zr-93, Nb-93m, Tc-99, Ru106, Cd-113m, Sb-125, Sn-126, l-129, Cs-134, Cs-137, Ba-137m, Sm-151, Eu-152, Eu-154, Eu155, Ra-226, Ra-228, Ac-227, Pa-231, Th-229, Th-232, U-232, U-233, U-234, U-235, U-236, U238, U-Total (M), Np-237, Pu-238, Pu-239, Pu-240, Pu-241, Pu-242, Pu-Total (g/L), Am-241, Am243. Cm-242, Cm-243, Cm-244. Note that $\mathrm{Y}-90$ and $\mathrm{Ba}-137 \mathrm{~m}$ are both short-lived daughters of $\mathrm{Sr}-90$ and $\mathrm{Cs}-137$, respectively, and that total $\mathrm{Pu}$ is calculated as $\mathrm{g} / \mathrm{L}$ and total $\mathrm{U}$ as $\mathrm{mol} / \mathrm{L}$. The five radionuclides $\mathrm{H}-3, \mathrm{C}-14, \mathrm{Ni}-59, \mathrm{Ni}-63$, and $\mathrm{Co}-60$ are impurities activated by neutron capture in the reactor fuel or fuel cladding.

Also reported are total site inventories for Double Shell (DST's), Single Shell (SST's), as well as the total inventory of waste placed into cribs and trenches from the waste tanks during the history of Hanford. These estimates do not cover all waste additions to cribs since many streams went into the cribs directly from the plants. Such streams as stack scrubbing and process condensates were often sent directly to cribs from the plants. 


\section{Acknowledgments}

A project of this nature would not be possible were it not for the help of a great number of people. They are Todd Brown, Brett Simpson, and Bob Watrous (LMHC) for the help with data gathering, Stacey Hartley (PNNL) for comments on model results, Ray Daubert and Richard Anema (Ogden Envir.) for data validation, and Jerry Koreski and Jim Strode (LMHC) for the Operational Waste Volume Projection document, George Borsheim (WHC-retired) for many useful suggestions, as well as a great number of other people at PMHC and PNNL for their generous help.

This work was performed under the auspices of the Department of Energy and was funded by DOE-RL under the Tank Waste Remediation Program's Characterization Project at Project Management Hanford Company.

Microsoft, Excel, and Visual C++ are all registered trademarks of Microsoft, Inc. 


\section{Table of Contents}

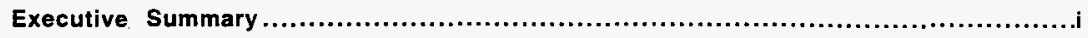

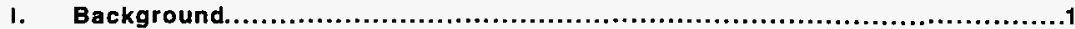

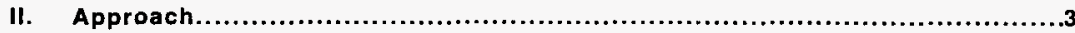

Ila. Approach-Waste Status and Transaction Record Summary...................5 Cascade transfers Transaction ordering HDW evaporator model

Resolution of unknown transactions Level discrepancies Verification of WSTRS

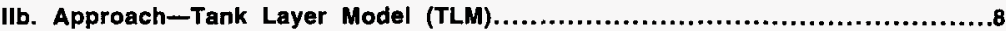
Sludge accumulation from primary waste

Saltcake accumulation Diatomaceous earth/cement Solids not included in TLM

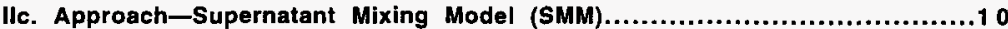
SMM and TLM output tables Implementation of SMM Transaction processing Ancillary information

IId. Approach-Hanford Defined Wastes (HDW) Compositions....................13 Three methods for establishing defined wastes Knowledge Of Process Chemicais Used / Waste Volume Produced Analysis of Characteristic Waste Method used for HDW model

Review of Campaigns.........................................................16

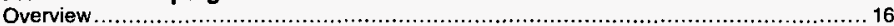

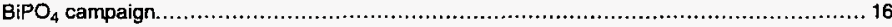

Uranium recovery............................................................................ 20

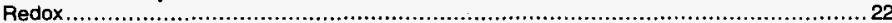

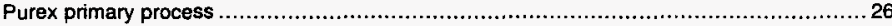

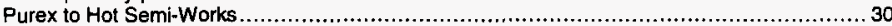

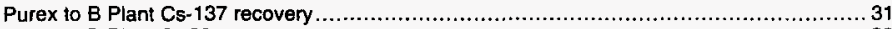

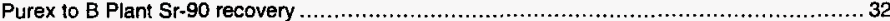

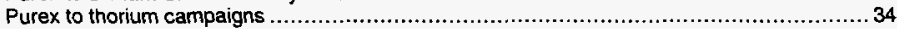

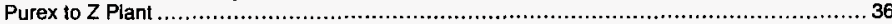

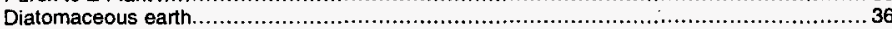

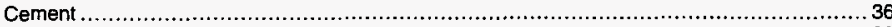

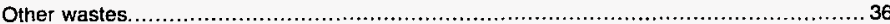

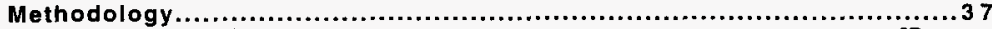

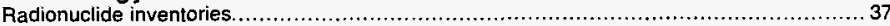

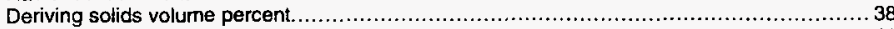

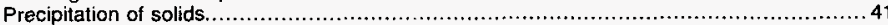

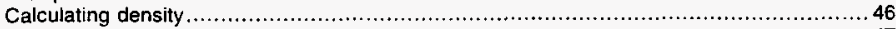

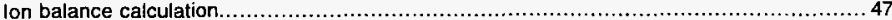

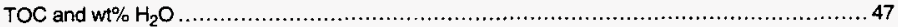

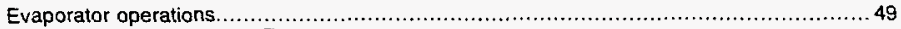
242-B and First 242-T

$S$ and $S X$ Farms self-concentration $A$ and $A X$ Fams self-concentration 
In Tank Solidification (ITS) campaign in BY Farm

Acid additions during evaporator runs

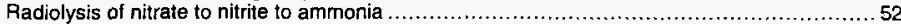

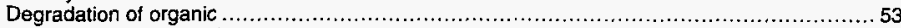

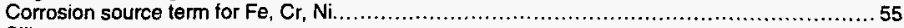

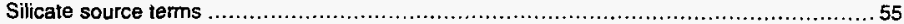

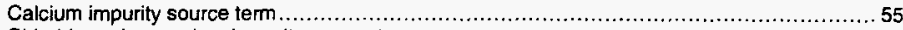

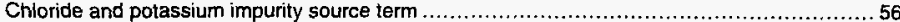

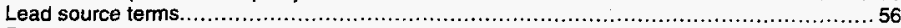

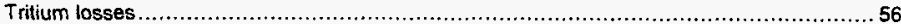

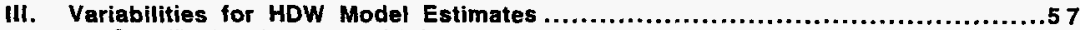
Quantification of process variability

HDW variability due to variations in analyte solubilities Transaction variability Variability due to waste heterogeneity

IV. Calculating Tank Inventories from HDW Compositions .........................6 6

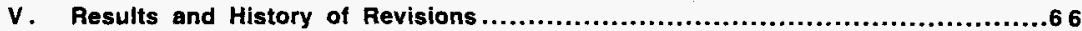

VI. Uses and Limitations of HDW Model Estimates ...............................68

Appendices
A. HDW List
B. HDW Compositions Spreadsheet
C. TLM Working Spreadsheet
D. SMM and TLM Composition Tables for all Tanks
E. HDW Model Inventories for 177 Tanks
F. Glossary of Hanford Terminology
G. HDW Compositions Spreadsheet Equations
H. Tank Waste Transaction Statistics 


\section{Background}

One of the most important tasks associated with the Hanford waste tanks is an adequate estimation of those tank's contents. Such estimates are very important for four reasons: 1) To establish safety limits during intrusive activities; 2) To establish a planning basis for future disposal; 3) To allow assays from one tank's waste to be used to validate, compare, and assess hazards among other tank's with similar waste inventories; and 4) To make sense out of the highly variable results that often come from a tank's waste assays, it is necessary to couch assay results in terms of the particular process and storage history of each tank.

It is clear that direct assays of tank wastes will always be an important and ongoing need for the Hanford tanks. However, it is equally clear that it will not be possible to adequately address all waste issues by sampling and assay alone. Obtaining a set of samples that is representative of the waste heterogeneity within a tank is undoubtedly the most difficult aspect of deriving tank inventories from assays alone. Both the extremely heterogeneous nature of tank waste and the limited access provided by riser pathways to waste in these seventy-five foot diameter underground tanks contribute to difficulties in using assays alone to derive tank inventories. There are safety issues, such as elevated amounts of soluble organic in dry nitrate waste in inaccessible regions of a tank that are difficult to address by sampling alone.

The High Level radioactive Waste (HLW) generated at Hanford from 1945 until 1989 all derived from the chemical dissolution and extraction of plutonium and uranium (and some thorium and neptunium) from reactor fuel elements (see Hanford Timeline Fig. 1). Over these years, the extractions evolved through three different processes. The first process was a bismuth phosphate $\left(\mathrm{BiPO}_{4}\right)$ precipitation, which operated from 1945 until 1956 in B and T Plants. This method was eventually supplanted by a more efficient method that involved contacting a methyl isobutyl ketone (hexone) organic phase with an aqueous aluminum nitrate solution of plutonium and uranium from dissolved fuel slugs. This process was known as Redox and operated from 1952 to 1966 in the Redox or S Plant. The Redox process was later replaced by Purex, a much-improved solvent extraction based on an organic phase that was a mixture of NPH (normal-paraffinic hydrocarbon or kerosene) and TBP (tributyl phosphate) contacting an aqueous nitric acid solution of plutonium and uranium. Purex began in 1956 at Hanford, and ran until 1972, then restarted in 1983 and ran until 1988. All Purex operations were performed in the Purex Plant, or A Plant.

The wastes from each of these three processes were neutralized and placed into $75^{\circ}$ and $25^{\prime}$ diameter storage tanks, but a variety of further processing occurred with the wastes after this initial disposal. This further processing, which was usually concentration of waste by evaporation of water, resulted in new wastes that were then returned to the tanks. The difficulty of using only process plant knowledge and tank transaction records to estimate the contents of each of the waste tanks is obvious, and is compounded by the often inadequate and conflicting records that have been kept for Hanford tanks.

There are over a thousand analytical assays of existing tank waste with the assays for supernatants being most numerous. Analytical assays of solids layers within a given tank have proven to be quite variable as solid wastes within the tanks are often highly stratified, with both vertical and horizontal heterogeneities. Unless a tank's waste is homogeneous, one needs to determine the vertical and lateral distributions of those waste layers within the tank-otherwise it is not possible to derive a tank inventory from assays. The distribution of waste layers derives from the fact that the tank "remembers" when and how waste solids were added and removed as well as how subsequent operations affected the waste layers that were already within the tank.

Thus, two tanks could very well have identical inventories but different waste distributions, which could tead to a misinterpretation of sample results. Without independent knowledge of those waste distributions, it would be impossible to know that the two tanks actually had identical inventories. The difficulties in deriving tank inventories from limited sample information are compounded by other factors, such as less than $100 \%$ recovery for cores, the limited number of sampling points for each tank, and the fact that the same risers that are available for sampling are the fill and removal points of the tank. The fill and removal points are exactly where the greatest waste heterogeneities often lie. 


\section{Hanford Timeline}

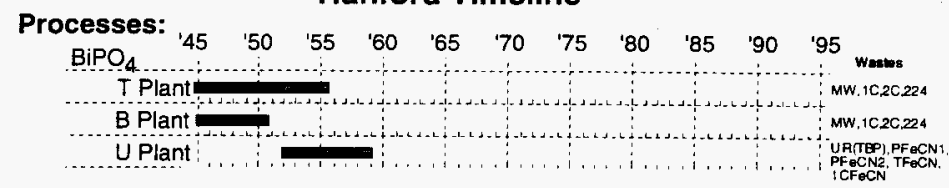

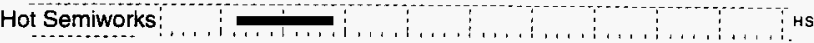

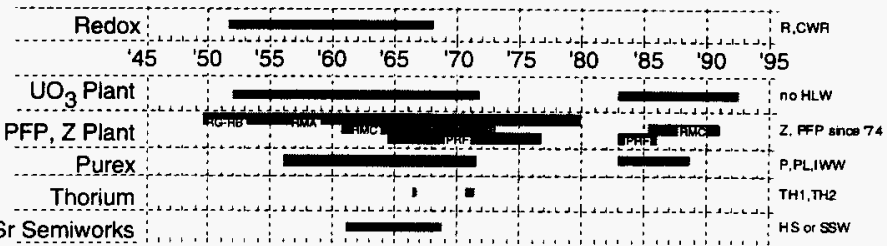

Sr Semiworks

-B Plant-

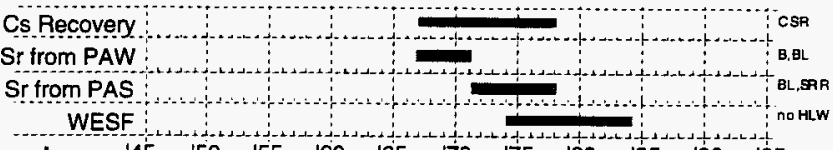

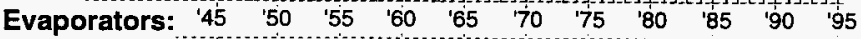
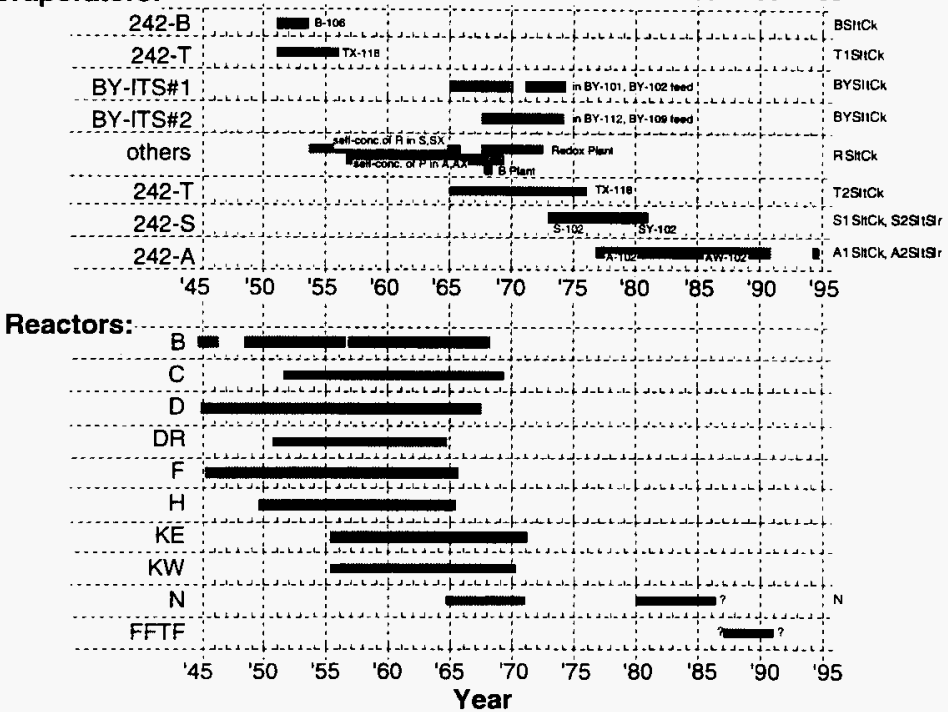

Fig. 1. Hanford Timeline. 


\section{Approach}

The Hanford Defined Waste (HDW) model is described in Fig. 2 (Schematic of Overall Strategy). The model begins with a process and transaction dataset that derives from a variety of sources. From this dataset, a balanced tank-by-tank quarterly summary transaction spreadsheet is derived called the Waste Status and Transaction Record Summary (WSTRS). At the end of each quarter, all tanks' volumes are reconciled with their reported status at that time and in the process, unknown transactions are recorded to accommodate otherwise unexplained gains or losses at the end of each quarter.

Using these fill records, the Tank Layer Model (TLM) provides a definition of the sludge and satt cake layers within each tank. The TLM is a volumetric and chronological description of tank inventory based on a defined set of waste solids layers. Each solids layer is attributed to a particular waste addition or process, and any solids layers that have unknown origin are assigned as such and contribute to the uncertainty of that tank's inventory. The TLM simply associates each layer of sludge within a tank with a process waste addition. As indicated in (Fig. 2), the TLM analysis depends only on information from WSTRS.

The Supernatant Mixing Model (SMM) is an algorithm written in $\mathrm{C}++$ and installed as a spreadsheet macro under Excel (a Dynamic Linked Library or dll module) that calculates the supematant concentrates within each of the tanks. The SMM uses information from both WSTRS and the TLM and describes supematant concentrates in terms of original waste volume in $\mathrm{kgal}$ ( $1 \mathrm{kgal}=1,000 \mathrm{gal}$ ) for each of the process waste additions. The original waste volume in addition to the present tank volume give the amount of concentration (water removal) or dilution that a tank has seen in its history.

The WSTRS, TLM, and SMM altogether determine each tank's waste inventory as a linear combination of HDW sludge and supernatant volumes (TLM and SMM volumes). In order to provide information on the elemental composition of each tank, the HDW compositions describe each waste stream based on process historical information. Each HDW has both supernatant and sludge layers and derives its total waste volume from WSTRS and its sludge volume from the TLM. Thus, the HDW compositions depend on all prior model components-process/transaction dataset, WSTRS, TLM, and SMM.

Each tank's total inventory is then calculated as superposition of waste components as

$$
\operatorname{tank}_{i}=\frac{\sum_{j} \operatorname{tlm}_{i j} h d w_{j}^{s l}}{s l V o l_{i}}+\frac{\sum_{j} s m m_{i j} h d w_{j}^{s u}}{\operatorname{suVol}_{i}}
$$

where

$$
\begin{aligned}
& \text { tank } \mathbf{i}=\text { composition vector for tank } \mathbf{i} \\
& h \mathbf{h w}_{\mathbf{j}} \mathbf{s l}^{\mathrm{I}} \quad=\text { composition vector for HDW sludge } \mathrm{j} \\
& h \mathbf{d w}_{\mathrm{j}}^{\mathrm{su}} \quad=\text { composition vector for HDW supernatant } \mathrm{j} \\
& \mathrm{tlm}_{\mathrm{ij}} \quad=\mathrm{kgal} \text { of hdw sludge } \mathrm{j} \text { for tank } \mathrm{i} \\
& \mathrm{smm}_{\mathrm{ij}} \quad=\mathrm{kgal} \text { of hdw supernatant } \mathrm{j} \text { for tank } \mathrm{i} \\
& \text { slVoli } \quad=\text { sludge } \mathrm{kgal} \text { for tank } \mathrm{i} \\
& \text { suVoli }=\text { supernatant concentrate kgal for } \tan \mathrm{k} \mathrm{i} \text {. }
\end{aligned}
$$




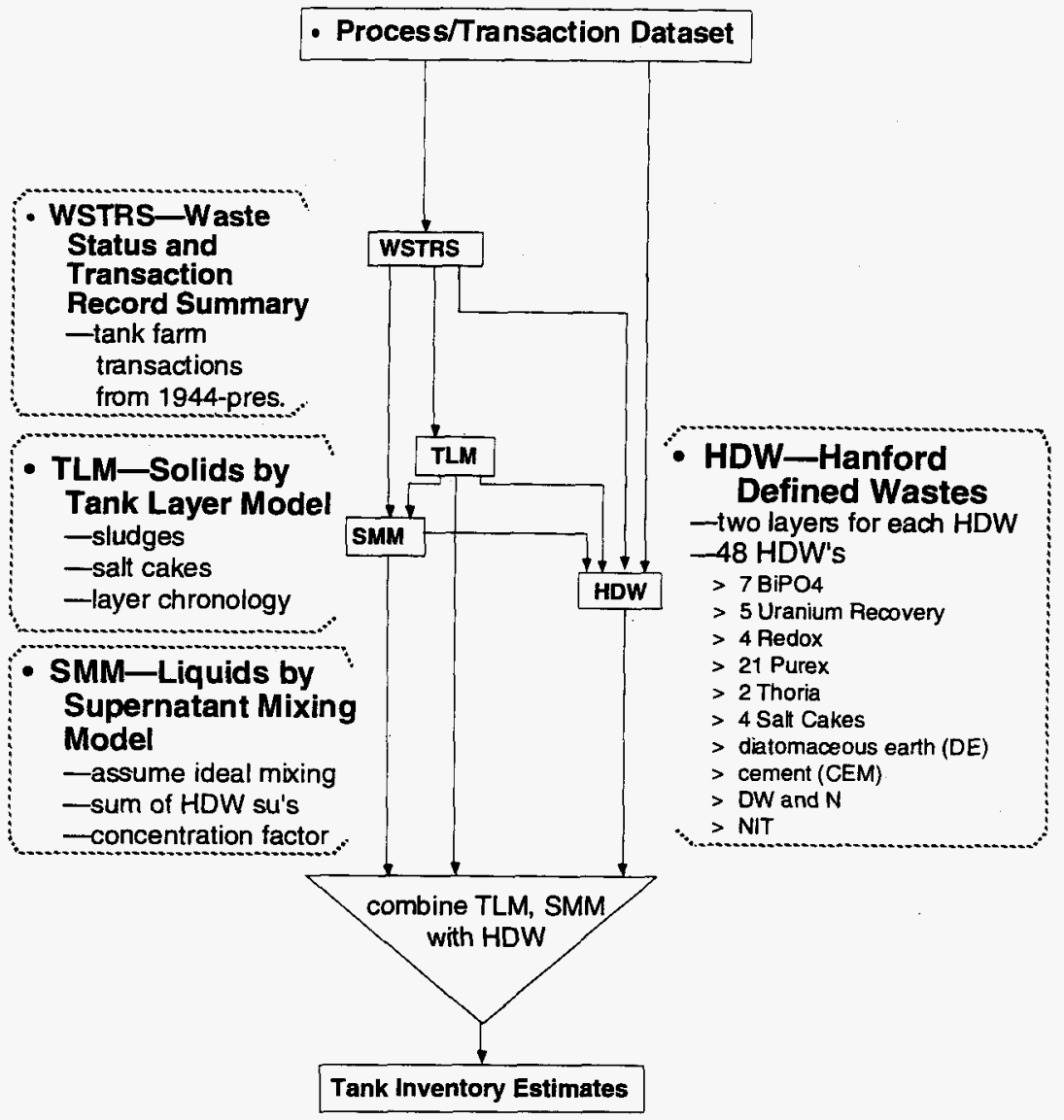

Fig. 2. Schematic of overall strategy.

The first term is the TLM solids inventory and is reported as

$$
\operatorname{tank}_{\mathrm{j}}^{s \mathrm{I}}=\frac{\sum_{\mathrm{j}} \operatorname{tlm}_{\mathrm{ij}} \mathrm{hd} \mathbf{w}_{\mathrm{j}}^{\mathrm{s} I}}{\mathrm{slVol}_{\mathrm{i}}}
$$

while the second term is the SMM inventory reported as 
HNF-SD-WM-ER-323, Rev. 1

$$
\operatorname{tank}_{\mathrm{i}}^{s u}=\frac{\sum_{j} \operatorname{smm}_{\mathrm{ij}} \mathbf{h d w _ { j } \mathbf { s }}}{\text { suVol}_{\mathrm{i}}} .
$$

These inventory estimates for each tank also appear in the Historical Tank Content Estimate (HTCE) reports for each of four quadrants. ${ }^{1}$

\section{lla. Approach-Waste Status and Transaction Record Summary}

The WSTRS is an Excel spreadsheet of qualified fill records ${ }^{2}$ with information extracted from Jungfleisch $-83^{3}$ and Anderson-914, and checked by Ogden Environmental and LANL against quarterly summary reports and the Logbook Dataset. ${ }^{5}$ The WSTRS reports, although largely representative of the waste histories of the tanks, are nevertheless incomplete in that there are a number of unrecorded transactions that have occurred for many tanks. Included within the WSTRS report, then, is a comparison of the tank volume that is calculated based on the fill records that are present in WSTRS with the measured volume of each tank. This comparison is made for each quarter to record any unknown waste additions or removals that may have occurred during that quarter. transactions.

After 1980, the Waste Volume Projection (WVP) project has provided an excellent set of ordered

The Rev. 3 and 4 estimates include new information from the Logbook Dataset that was not included with previous versions and have extensive changes in the latter four evaporator campaigns: 242-S (S1 and S2) and 242-A (A1 and A2). The Logbook Dataset contains extremely detailed tank level information from about 1975 to 1992 and has allowed Rev. 3/4 to accommodate the blending that occurred during these campaigns. In Rev. 1, each campaign's waste was blended over many years of operation, then concentrated in one single step and distributed over all the bottoms receivers. In contrast, Rev. $3 / 4$ blends the evaporator concentrates on about a quarterly basis thereby providing much better representation of these evaporator campaigns.

\section{- Cascade transfers}

Cascade lines were underground 3" pipes between tanks that were generally offset one foot of elevation between tanks. These lines allowed a tank to overfiow into the next tank in the cascade series, and then from that tank to the next, and so on, from two to six tanks total in a given cascade series. WSTRS includes explicit transactions for each cascade transfer based on the following rules. If a tank's Total_vol exceeds its rated capacity, then check to see if a CSEND SET and CREC SET pair are present in the records of Tank_ $n$ and Trans_tank, respectively. If a pair is present, insert a "send" and "rec" pair of transactions of the appropriate volume. When cascading out to a crib "send" and "outx" pair are inserted. In the SE Quadrant (i.e. for DST's) there is no cascading.

\section{-Transaction ordering}

The chronological ordering of the transactions in WSTRS prior to 1981 is not clearly defined. Hanford's quarteriy summarization of transactions tended to truncate much of the individual transaction ordering information. From 1976 to 1981 , the Logbook Dataset was used to help re-establish transaction

\footnotetext{
${ }^{1}$ Brevick, C. H., et al., "Historical Tank Content Estimate of the Northeast (Southwest, Northwest, Southeast) Quadrant of the Hanford 200 East Area," WHC-SD-WM-ER-349 thru 352, Rev. 0, June 1994.

2 (a) Agnew, S. F., et al., "Waste Status and Transaction Record Summary for the NE Quadrant" WHC-SD-WM-TI615, Rev. 1, October 1994. (b) Agnew, S. F., et al. "Waste Status and Transaction Record Summary for the SW Quadrant, "WHC-SD-WM-TI-614, Rev. 1, October 1994. (c) Agnew, S. F., et al. "Waste Status and Transaction Record Summary for the NW Quadrant, "WHC-SD-WM-TI-669, Rev. 1, October 1994.

${ }^{3}$ (a) Jungfleisch, F. M. "Hantord High-Level Defense Waste Characterization-A Status Report," RH-CD-1019, July 1980. (b) Jungfleisch, F. M. "Supplementary Information for the Preliminary Estimation of Waste Tank Inventories in Hanford Tanks through 1980," SD-WM-TI-058, June 1983. (c) Jungfleisch, F. M. "Preliminary Estimation of Waste Tank Inventories in Hanford Tanks through 1980," SD-WM-TI-057, March 1984.

${ }^{4}$ Anderson, J. D. "A History of the 200 Area Tank Farms," WHC-MR-0132, June 1990.

${ }^{5}$ Agnew, S.F.; Corbin, R.A.; Duran, T.B.; Jurgensen, K.A.; Ortiz, T.P. "Hanford Tank Logbook Dataset," LA-UR-96. 3387, September 1996.
} 
order and to resolve many other transaction questions. Transactions with no additional information (i.e. pre-1976) were arranged in the following order for each quarter:

1) Xin's from primary sources

2) Tank to tank transfers not involved in evaporator operations

3) Tank to tank transfers involved in evaporator feeds

4) Concentration of wastes involved in evaporators

5) Tank to tank transfers for the bottoms receivers

6) Outx's to processes and cribs (no condensates)

Some corrections to this initial order were required to prevent the total volume of the taniks from going negative and to minimize tank overfills. Further corrections will be necessary as more information as to the segregation of the organic wastes is compiled.

The transaction order for post-1980 transactions were left as represented by the WVP report. Many of these dates are summaries of transactions and some are nominal, so there exists the possibility that some reordering may be necessary as more information on these transactions surface.

-HDW evaporator model

An essential part of defining the waste history of Hanford wastes is understanding the operation of the many evaporator campaigns at Hanford, since the greatest uncertainties within WSTRS are associated with evaporator campaigns. In other words, the volume reductions and continuous transfers of concentrates and condensates that occurred during these campaigns are not very well represented in WSTRS.

Much of the transaction information associated with evaporator operations was derived by Jungfieisch-83 with several models for various evaporator campaigns that were embedded within the WSTRS Rev. 1 data set.

In the WVP data set, the evaporation model transferred a volume from the feed tank to a bottoms receiver tank. The volume received by the bottoms receiver tank, however, would be less than the volume sent from the feed tank. This difference was the condensate that was evaporated, which was not specifically included.

In WSTRS Rev. 2, 3 and 4, all evaporator transactions are assumed to take place from the evaporator feed tank. Therefore, all implicit condensate that is evaporated from the feed tank is explicitly included as transactions from the feed tank to a crib. We have inserted these condensate transactions for the feed tank and have changed the transaction volume (when necessary) to be equal to the volume received in the bottoms tank. This same model has been imposed on all evaporator operations at Hanford within WSTRS. The Logbook Dataset has given much more detailed insight into the transactions in the later evaporator campaigns.

This evaporator model reduces significantly the unknown transactions for the history of Hanford operations. One must bear in mind, though, that the assumptions that have been made are meant to be approximations that allow the bounding of waste compositions for all site operations. We have found, for example, that the transaction order within each quarter is not well defined and our assumptions about that order are very approximate.

\section{-Resolution of unknown transactions}

Transactions from the Logbook Dataset were added to WSTRS to resolve the many unexplained level changes for each quarter according to a set of rules resulting in an updated WSTRS that is known as Rev. 4. This unknown transaction resolution was only completed for all unknowns larger than $50 \mathrm{kgal}$, although many smaller transaction unknowns were accommodated as well. The following rules were used for unknown transaction resolution for the various transaction types: 


\section{Evaporator feed and bottoms receivers:}

During an evaporator campaign, unknown waste transfers at the end of each quarter are resolved by sending wastes to or receiving wastes from an evaporator feed tank for tanks identified as either bottoms receivers or feed tanks for those campaigns. ${ }^{6}$

\section{Self-concentrating tanks:}

Certain tanks in S, SX, A, and AX farms were allowed to self-concentrate. Any losses or additions to these tanks are assigned to condensate or water, respectively.

\section{Sluicing receivers:}

For tanks associated with a sluicing campaign (either UR or SRR), unknown transactions are resolved by either sending or receiving from the sluicing receiver tank for that campaign. Unassigned losses from the sluicing receivers, then, are sent directly to the process.

\section{Salt-well pumping and stabilization:}

If an unknown loss occurs during salt well pumping stabilization of a tank, then the unknown is resolved by sending waste to the active salt well receiver at that time.

\section{Historical use of tank:}

If none of the above rules apply, then the historical use of the tank is used to assign the transaction. For example, C-105 was used as a supernatant feed tank for the CSR campaign and supplied $-1,500 \mathrm{kgal}$ per quarter for several years. However, there is one quarter (1971q2) where $\mathrm{C}-105$ loses $1,748 \mathrm{kgal}$ without an assigned transaction. Because of $\mathrm{C}-105$ 's process history, this transaction is assigned to CSR feed. Likewise, there are a number of large supernatant losses in $A$ and $A X$ Farms during sluicing for sludge recovery. These supernatant losses are assigned as feed to AR, which are the slurries transferred to AR Vault for solids separation, washing, dissolution, and feed to SRR.

There are volume reductions among the $S$ and $S X$ Farm tanks in the fifties and early sixties. We attribute these losses to the Redox waste self-concentrating tanks and these tanks also accumulated solids as they concentrated, which we assign as Redox salt cake. Likewise, the Purex waste tanks in $A$ and AX Farms were self-concentrating, but no salt cake formed from this self-concentration. Volume losses for such tanks are assigned as condensate transactions out of the tank and additions are assigned as water in.

The two Purex cladding waste (CWP) cascades, B-103 and B-109 had large unknown transfers in 1963q4. These were resolved by transferring supernatants to $A-102$, which was the staging tank for $A$ Farm sluicing as well as a feed for the self-boiling tanks in $A$ and $A X$ Farms. Excess volume added to $A$ 102 amounts to over $2,000 \mathrm{kgal}$ in ' $63 \mathrm{q} 4$, which is assumed to have been associated with the sluicing required for initial feed to HS or SSW (Hot or Strontium Semiworks), and eventually ended up blended into the A/AX self-boiling tanks. Likewise, other later excess losses that accumulated in A-102 are assumed to have been used as feed for the self-boiling tanks in AVAX Farms.

Sluicing of $A$ and $A X$ Farms resulted in many unrecorded transactions for these tanks during that period. As shown in Evaporation and Cs/Sr Extraction Campaigns (Table 9), we have resolved all large transactions by creating transactions between tanks and the sluicing receiver in $A$ and $A X$ Farms as indicated in (Table 9). The sluicing receivers evolved from A-102, A-106, to AX-103 over the course of the campaign.

For each of the evaporator campaigns, we have identified the feed and bottoms receivers. All unknown transactions for the feed and bottoms receivers are sent to or received from the evaporator feed tank. This allows us to resolve all evaporator campaign transactions and to therefore track the waste compositions as a function of time.

${ }^{6}$ Agnew, S.F.; Corbin, R. A.; Boyer, J.; Duran, T.; Jurgensen, K.; Ortiz, T.; Young, B. "History of Organic Carbon in Hanford HLW Tanks: HDW Model Rev. 3," LA-UR-96-989, March 1996. 
Cross-site transfer tanks S-107 to/from BX-104 and BX-105 were used to transfer wastes back and forth to and from west and east areas prior to 1981. We assume this linkage was used for most cross site transactions between East and West areas.

- Level discrepancies C-96.

There are a certain number of level discrepancies that have occurred and are noted in App. C, p.

\section{-Verification of WSTRS}

Validation for the WSTRS and WVP datasets was performed by Ogden Environmental of Richland, WA. Reference documentation was provided for each transaction that Ogden verified. Validation information for all tanks is shown in (Table 1) with the numbers and percents validated for transactions and transaction voiumes in all quadrants prior to Jan. 1981. Validation for DST's in after Jan. 1981 is provided by the Waste Volume Projection data source.

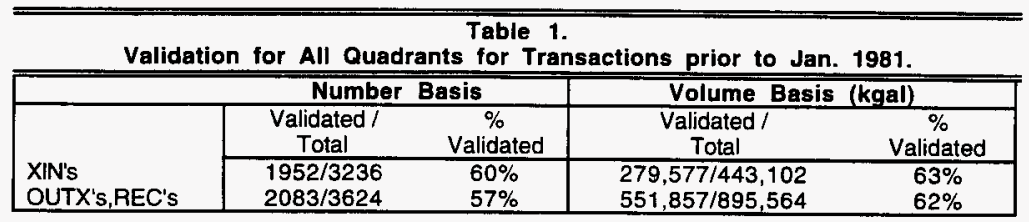

- Other statistics for WSTRS transactions are shown in App. $\mathrm{H}$, Table $\mathrm{H}-1$.

\section{Ilb. Approach-Tank Layer Model (TLM)}

The TLM uses the past fill history of each tank to derive an estimate of the types of solids that reside within those tanks. The $T L M^{7,8}$ is generated by reconciling the reported solids levels from WSTRS for each tank as shown in (App. C) with the solids volume per cent expected for each primary waste addition (App. A). Note that a solid's model has already been extensively used at Hanford to estimate sludge and salt cake accumulation, the results of which are reported monthly in the Tank Farm Surveillance and Waste Status Summary Report for November $1993^{\circ}$.

There are some tanks that the HDW model assumes a different waste inventory than that reported in Hanlon. These differences come about because of the difficulties that are often encountered in determining the remaining inventory in tanks with large surface heterogeneities. Also shown in (App. C), then, are a list of tanks for with their Hanlon volumes and their adjusted volumes used for the HDW estimates. The sources of these discrepancies are a series of reports about stabilized tanks. 10

The TLM is a volumetric and chronological description of tank inventory based on the HDW sludges and salt cakes. Each solids layer is attributed to a particular waste addition or process, and any solids layers that have unknown origin are assigned as such and contribute to the uncertainty of that tank's inventory. The TLM simply associates layers of solids within each tank with a waste addition or a process campaign.

${ }^{7}$ (a) Brevick, C. H., et al., "Supporting Document for the Historical Tank Content Estimate for A Tank Farm," WHCSD-WM-ER-308, Rev. O, June 1994. Likewise, reports and numbers for each farm are as follows: $A X$ is $309, B$ is $310, B X$ is $311, B Y$ is $312, C$ is $313, S$ is $323, S X$ is 324 , and $U$ is 325 . These supporting documents contain much of the detailed information for each tank farm in a concise format, all released as Rev. 0 in June 1994.

${ }^{8}$ Agnew, S. F., et al. "Tank Layer Model (TLM) for Northeast, Southwest, and Northwest Quadrants," LA-UR-944269, February 1995.

${ }^{9}$ Hanton, B. M. "Tank Farm Surveillance and Waste Status and Summary Report for November 1993, "WHC-EP. 0182-68, February 1994, published monthly.

${ }^{10}$ (a) Swaney, S. L. "Waste Level Discrepancies between Manual Level Readings and Current Waste inventory for Single-Shell Tanks," Internal Memo 7C242-93-038, Dec. 10, 1993. (b) Boyles, V. C. Boyles "Single Shell Tank Stabilization Record," SD-RE-TI-178 Rev. 3, July 1992. (c) Welty, R. K. "Waste Storage Tank Status and Leak Detection Criteria," SD-WM-TI-356, September 1988. 
The TLM uses the information obtained from the transaction history for each tank to predict solids accumulations. These predictions are made for three categories of waste tanks.

The first category involves primary waste additions, which are the waste additions from process plants directly into a waste tank. The primary waste transactions are used along with solids volume reports for each tank to derive an average volume per cent solids for each HDW type. The solids accumulations are then assigned to a particular HDW for the tanks where the solids information is missing or inconsistent.

A second category of waste is that where solids accumulate as a result of evaporative concentration of supernatants. All solids that accumulate in such tanks occur after they have been designated as "bottoms" receivers. These solids are assigned to one of four salt cakes, which are defined as blends over entire evaporator campaigns. The four salt cakes are BSItCk, T1SitCk, BYSItCk, and RSItCk, and are all defined as HDW's. The latter five evaporator campaigns $T 2, S 1, S 2, A 1$, and $A 2$ all result in waste concentrates that are defined differently for each tank within the SMM. These solids are explained in the section "Solids not included in TLM."

The third category of waste is where solids accumulate due to tank to tank transfers of solids. This category allows solids to cascade from tank to tank, or accounts for solids lost during routine transfers as was common with decladding wastes CWR and CWP as well as $1 \mathrm{C}$ and FeCN sludges.

The results of the TLM analysis are a description of each tank's solids in terms of sludge and salt cake layers. Although interstitial liquid is incorporated within the composition for sludges and salt cakes, any residual supernatants that reside in these tanks above the solids are described by the SMM. The output of the TLM, then, can only be used to predict the inventory of the sludges and each of four salt cakes that reside within waste tanks. These TLM results are inserted into the WSTRS record and are used by the SMM in considering excluded volumes for mixing of waste supernatants.

Not all of the transactions that have occurred in the past are faithfully recorded by the WSTRS data set. Therefore, WSTRS is an incomplete document with many missing transactions. However, the two critical pieces of information that are used in the TLM analysis are the primary waste additions and the solids level measurements, both of which are well represented in WSTRS.

The missing transactions largely involve tank-to-tank transfers within WSTRS. These missing transactions, which are salt cake, salt slurry, and supernatant, do lead to a larger uncertainty for the compositions of the concentrated products from evaporator operations. As many as $25 \%$ of all transactions may be missing from this data set, perhaps as many as $60-80 \%$ of these missing transactions are associated with the evaporator operations. Although this information might be recovered in the future, the HDW model strategy at this time resolves as many of these unknown transactions as possible with the rules stated above.

\section{-Sludge accumulation from primary waste}

The TLM analysis associates a solids volume percent (vol\%) with each primary waste stream. These solids vol\% are those that are consistent with the solids volumes reported in Anderson-91 by comparing those solids accumulations with the primary waste additions that are recorded in WSTRS. The result of this analysis is a solids volume percent for each waste type with a range of uncertainty associated with the inherent variability of the process.

Not all of the waste types have adequate solids reports associated with them. For these waste types, a nominal value is assigned based on similarity to other waste types where there exists a solids vol\%. For example, a total of $810 \mathrm{kgal}$ of Hot Semi-Works waste (HS) was added to several tanks in C Farm, but these additions only constituted a small fraction of the total solids present in any of these tanks. Therefore, a nominal 5 vol\% solids is assigned for that waste type.

Each TLM spreadsheet table shows the primary waste additions and the solids from those additions based on the characteristic vol\% for that waste type. The TLM compares this prediction with the solids level reported for the tank and indicates either an unknown gain or loss for this tank. Once a layer is "set" in the tank, its volume appears in the "Pred. layer" column and the type in "Layer type" column, thus 
comprising a chronological layer order from the bottom of a tank to the top, where each layer is described in terms of a volume and a type. Note that lateral variations are not accounted for in this model, and only derive an average layer thickness. The lateral distribution, in some cases, can be quite extreme.

There are two main sources for variations in the solids vol\% for each waste type. First, there is an inherent variability in each process stream, which is largely attributable to process variations. Second, solids can be added to or removed from tanks by inadvertent (or purposeful) entrainment during other supernatant transfers. In addition to these sources of variation, there are a number of other minor sources of solids changes such as compaction, subsidence following removal of salt well liquid, and dissolution of soluble salts by later dilute waste additions. Other solids variations may be due to metathesis and other chemical reactions within the tanks, such as degradation of organic complexants over time.

The TLM assigns solids changes to variability when they fall within the range established. If a change in solids falls outside of this range, the TLM associates the gain or loss of solids with a waste transfer to or from another tank or to dissolution of soluble salts in the upper existing solids layers.

\section{-Sattcake accumulation}

Once a tank becomes a "bottoms" receiver, the TLM assumes from that point on that any solids that accumulate are salt cake or salt slurry. Salt cake can be any one of four different types, depending on which evaporator campaign created it. These are: 1) B for $242-\mathrm{B}, 2$ ) $\mathrm{T} 1$ for early $242-\mathrm{T}, 3$ ) BY for ITS \#1 and \#2 in BY Farm, and 4) $R$ for Redox self-concentrating tanks. Overview of Hanford Waste Volume (Table 3 ) describes the various evaporator campaigns that resulted in concentration of waste and precipitation of solids at Hanford. For salt cake accumulation, the TLM assumes that all of the solids reported are salt cake. Two other minor evaporation campaigns involved use of Redox and B Plant evaporators for tank wastes. These minor campaigns have been associated with T2 or S1 campaigns, respectively.

The HDW model assigns waste of the five later campaigns for 242-T, 242-S, and 242-A evaporators as concentrates within the SMM. These later concentrates correspond roughly to what is known as double-shell slurry (DSS) or double-shell slurry feed (DSSF), although their early concentrates are often referred to as salt cake as well.

\section{-Diatomaceous earth/cement}

Diatomaceous Earth, an effective and efficient waste sorbent material, was added to the following waste storage tanks BX-102 (1971), SX-113 (1972), TX-116 (1970), TX-117 (1970), TY-106 (1972), and U-104 (1972). The additions of diatomaceous earth were used to immobilize residual supernatant liquid in tanks where the liquid removal by pumping was not feasible. The conversion factor in the TLM for Diatomaceous Earth (DE) is $0.16 \mathrm{kgal} /$ ton and Cement (CEM) or (CON) is $0.12 \mathrm{kgal} / \mathrm{ton}$. The CEM waste was only added to one tank, BY-105 (1977).

\section{-Solids not included in TLM}

The TLM predicts the salt cake layers for the B, early $T$, BY, and $R$ evaporator campaigns. These are HDW wastes and are defined on a campaign basis. The concentrated supernatants associated with the $A, S$, and latter $T$ evaporator campaigns are treated as free supernatants and thus defined by the SMM. The SMM tracks the concentration and mixing of these supernatants on a tank-by-tank quarterly basis. This is necessary due to the complexity of the latter evaporator scenarios and waste types.

\section{IIc. Approach-Supernatant Mixing Model (SMM)}

The SMM calculates the composition of supernatant concentrates within each tank. The result is a table of waste in $\mathrm{kgal}$ of original HDW supernatants for each of the tanks (see App. D). The SMM is a very critical part of the definition of waste in double-shell tanks (DST's) where a large fraction of supernatants now reside. The Supernatant Mixing Model Block Diagram (Fig.3) illustrates the SMM approach. A fundamental assumption within the SMM is the ideal and complete mixing of each tank's supernatant following each transaction. In this approach, the volume of TLM solids layers within each tank are excluded from mixing with any supernatant additions. However, any solids that form as a result of waste concentration within the SMM are treated as if they were liquid. 


\section{Supernatant Mixing Model Block Diagram}

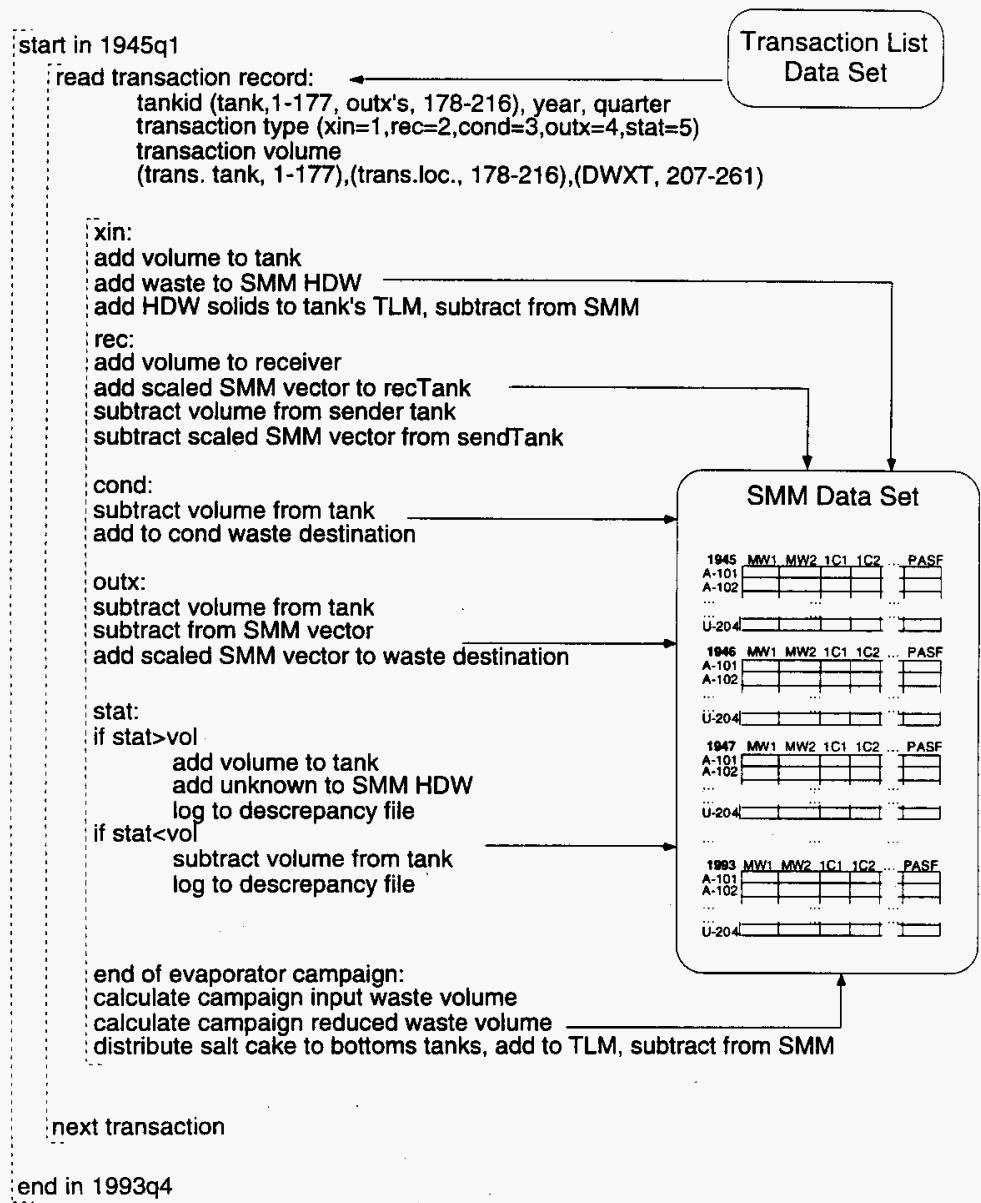

Fig. 3. Block Diagram of SMM algorithm.

The SMM module reads transaction information from WSTRS, sorts it to an absolute chronological order, and performs a transaction by transaction accounting of all of the tank waste supernatant for the history of Hanford. The SMM provides a description of each tank's supernatant concentrate as a linear combination of Hanford Defined Waste (HDW) supernatants. The HDW type is specified within WSTRS for each transaction from plant to tank. This HDW supernatants within a tank 
represent a total volume that is usually larger (and sometimes smaller) than the actual volume of supernatant concentrate within that tank. This is due to active evaporation (or dilution) of the waste during its history. Each tank's SMM waste is expressed in terms of a linear combination of HDW supernatants (a waste vector), which in turn are used to calculate a chemical and radionuclide inventory with compositions for each tank.

\section{- SMM and TLM output tables}

The output of the SMM is a table or matrix whose column headings are the HDW's and auxiliary wastes and whose rows are the waste tanks and processes. The auxiliary wastes are water, unk, swliq, and gas and do not appear as an HDW. These auxiliary wastes are used for tracking of unknowns, evaporator runs, and gas retention in waste concentrates. The SMM columns (App. D) show the HDW distribution among the tanks and processes and are given in kgal of original HDW supernatant.

The TLM tables also appear in App. D and follow roughly the same format as the SMM tables. There are no concentration effects with TLM solids and so the row sum for each tank within the TLM is equal to the total TLM volume for that tank.

The Tank Composition tables in App. D are a percentage representation of each HDW's contribution to a tank's supernatant composition. This gives a quick quantitative reference in terms of HDW's of a tanks' particular makeup. The HDW Distribution tables are a percentage representation of the entire location of a particular HDW. This gives a quick quantitative reference of the final history of a waste stream. This not only gives information on the quantity in the 177 waste tanks, but also the amounts that were cribbed, reprocessed, or concentrated into saltcakes.

\section{-Implementation of SMM}

The SMM is implemented as two Microsoft Excel workbooks and an extension to Excel (called a Dynamic Linked Library). Figure 4 shows the relationship of the SMM components to each other as well as to the overall tank inventory prediction strategy. The transaction data source for the SMM program is the WSTRS workbook, which is not modified during operation. A second workbook (SMM-TLM.xIs) provides additional input, program control, and storage for formatted SMM output. The smm.dll is written in the 'C++' programming language.

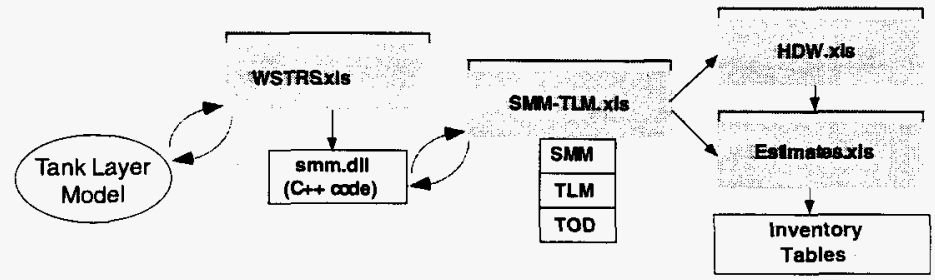

Fig. 4 Diagram of the HDW model components.

\section{-Transaction processing}

The smm.dll tracks the tank waste contents (in HDW component basis) based on transaction bookkeeping. The model achieves ideal mixing by assuring that a withdrawal from a tank is an HDW vector proportional to the HDW vector of the tank supernatant contents. The general algorithm is to process the information on waste transfers and level measurements in transaction order while keeping track of the HDW components in each tank. The processing details are determined by the transaction type. Since transactions are summarized rather than detailed, there can be apparent overfills.

If there is insufficient supernatant in a source tank, the shortage is accounted for by addition of an unknown waste type. The additions of unknowns are treated as if they were HDW waste additions. These unknowns measure one uncertainty in the tank HDW vector. Apparent overfills are less important as they generally result from miss-phasing due to the granularity of the dataset and tend to correct themselves. Transaction processing is relatively straight forward. There are five basic transaction types. 
1) The XIN and xin transactions represent the transfer of waste from process to storage and simply record the transier of HDW waste to the storage tanks. The xin designation identifies an assumed rather than a documented transaction. 2)The REC and rec transactions are tank to tank or tank to process transactions with the rec designating an assumed transaction. 3)The outx is a transfer to process or CRIB and is essentially another variation of the REC transaction. The last three transactions move HDW waste vectors between tanks and processes. 4)The cond transaction removes water from the tank without changing the HDW waste vector. 5)The stat transaction serves to realign the tank volume with physical measurements.

\section{-Ancillary information}

In addition to the tank compositions, the SMM also generates a variety of other important information. These include volumes of waste from assumed transactions, total traftic in a tank, traffic from assumed transactions, and various other information.

\section{Ild. Approach-Hanford Defined Wastes (HDW) Compositions}

The determination of chemical and radionuclide concentrations for each of the HDW's begin with inputs of radionuclides and stable chemicals, both of which are used to define the total species in each waste stream as shown by the Block Diagram of HDW Spreadsheet (Fig. 5). These total species are then separated into two layers, a sludge and a supernatant, that result in different concentrations of species for the two layers.

Each species is precipitated according to a single point solubility. lons precipitated in more than one salt are simply successively precipitated. Thus, the solids that precipitate are merely representative and are not meant to reflect the actual solids distribution. Because the supernatant is also present in the interstices of the sludge layer, this "supernatant" is included as part of the sludge composition. The solubility of each species is set by a macro that when run on the HDW spreadsheet, adjusts the fraction precipitated parameter so that the supernatant concentration is equal to or less than the target solubility.

The sludge and supernatant compositions are each expressed in mol/L for the stable chemicals, with water and $T O C$ as $w \%$ and radionuclides in $\mu \mathrm{Ci} / g$ and $\mathrm{Ci} / \mathrm{L}$, respectively. Each waste is kept in ion and mass balance according to the oxidation states assumed for that species. The sludge and supernatant layers are also expressed in terms of ppm composition. However, the mass balances are limited by differences among water, oxide, and hydroxide with the various solids to only within $\pm 2 \%$.

\section{-Three methods for establishing defined wastes}

There are three distinct methods that have been used by previous workers to set the concentrations of each component in the various waste streams at Hanford. These three methods are based on either one or a combination of (1)knowledge of process, (2)chemical used and waste volume produced, and (3)analysis of characteristic waste. However, while these methods provide necessary information for waste stream definition, these methods do not provide sufficient information to define the waste streams.

That is, once the component concentrations (i.e. source terms) are determined for each of the HDW's, one still needs to determine two critical pieces of information to calculate the actual compositions of solids and supernatant components within each tank. That is, one needs to know the solubility of each component in the supernatant as well as the total solids volume percent for that waste stream. With these two additional parameters the waste stream source terms can be related to what is in each tank that received those wastes.

Furthermore, the model that we have developed for the HDW compositions uses representative values for the speciation of the components in the solids phases. In other words, the solids that precipitate within the HDW model are those listed in Methodology (see below). For example, phosphate solubility is determined by the ranges observed in tank supernatants, but the solid phases that precipitate are limited to a combination of $\mathrm{BiPO}_{4}, \mathrm{Na}_{3} \mathrm{PO}_{4} \cdot 10 \mathrm{H}_{2} \mathrm{O}$, and $\mathrm{Na}_{3} \mathrm{PO}_{4} \cdot 12 \mathrm{H}_{2} \mathrm{O}$, depending on the circumstances of the waste stream. We argue that this approach adequately approximates the major features of each solids layer without the complexity that undoubtedly exists in the actual waste sludge. 


\section{Block Diagram of HDW Spreadsheet}

Campaign Information, pp. $1^{*}-3$
- fuel processed
-exposure
- total waste
-average waste rate
- Pu extraction efficiency
-nuclide source terms
- solids vol\%
-CSR input

Chemicals Added, pp. 4-6
- mostly based on tons fuel
-others concentration
- NaOH added to neutralize

"p. 1 refers to Appendix pages with HDW spreadsheets.

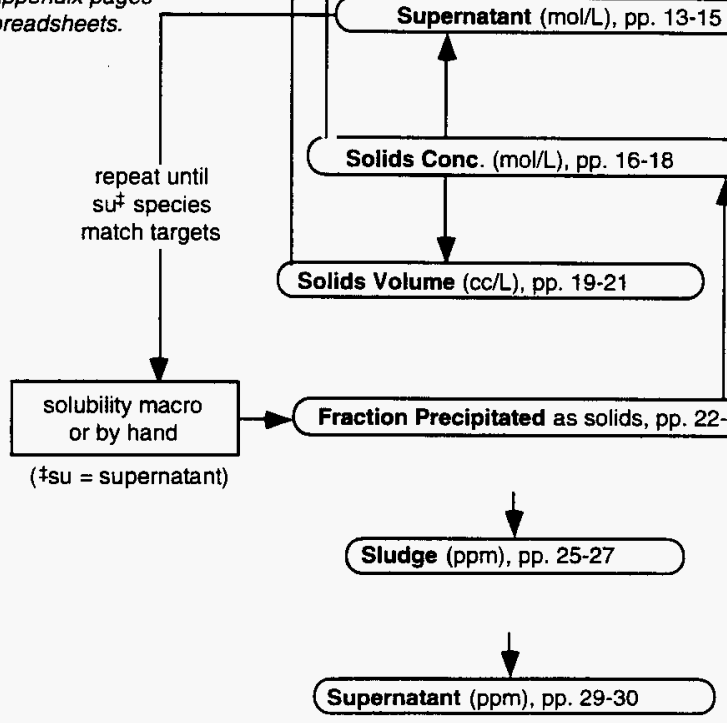

Supernatant to Evaporator Campaigns, Nitrate to Nitrite to Ammonia Radiolysis, AR and CSR Waste Blends, pp. 31-33

Fig. 5. Block Diagram of HDW spreadsheet . 
Another example is Cs-137, which is precipitated within our model formally as the zero valent metal, when in fact we fully realize that it is actually precipitated as a monopositive cation as some kind of salt.

\section{-Knowledge of Process}

Basically, this strategy uses process information, such as flowsheets, to derive waste compositions based on a process driver. At Hanford, the driver for HLW was tons of uranium fuel processed per quarter. Therefore, based on this feed, a certain amount of waste was generated for a given Hanford process, such as Purex, and expressed as gallons of waste per ton fuel.

The advantages of this strategy are its simplicity and straightforward application to waste streams. The disadvantages are that often the flowsheet information is incorrect or incomplete. In fact, ancillary or cleanup operations not described explicitly within the flowsheet can end up creating larger waste volumes, and add different constituents to the waste as well.

Specifically, actual waste volumes usually differ from predicted waste volumes based on flowsheet information, and it is not clear how to scale the composition of the primary stream. For example, if a Purex flowsheet indicates that $51 \mathrm{gal}$. of $P$ waste is generated per ton of uranium, but actually 275 gal/ton occurred, how do the constituents within the stream scale? is the additional volume just water?

\section{-Chemicals Used/Waste Volume Produced}

This approach uses observed waste stream volumes, both liquid and settled solids, to establish an actual waste generation rate in gallons of waste per ton of uranium processed for each waste stream. Thus, volume conservation is enforced from the beginning. Then, total chemicals added during processing allows one to unambiguously derive average waste compositions. Thus, total mass is always conserved with this approach. (Note that this is the approach that Allen used ${ }^{11}$ in his report in 1976.)

The disadvantage of this approach is that it concentrates on only the total amount of chemical used during a campaign. There is often limited information about how the chemicals used changed during a campaign. As a result, while this Chemicals Used/Waste Produced approach provides an accurate average waste composition, variations in the compositions of the waste streams are not represented unless one has independent information about the amounts of chemicals used during each of those process variations within the campaigns.

\section{-Analysis of Characteristic Waste}

Deriving waste inventories from analytical information of course begs the whole issue of determining a waste type composition. If a tank's waste is fairly homogeneous, straightforward sampling and analysis will provide an inventory for that tank. The real issue, then, is how can the information from an analysis of tank A's waste be used to predict the contents of tank B. On the one hand, direct analytical information constitutes the bottom line for any tank inventory. On the other hand, there are mitigating factors that show that this approach also has severe limitations.

Sampling has been and will continue to be a very uncertain source of waste composition information for many different reasons, not the least of which is that it is a very difficult and expensive thing to achieve for 177 tanks. For example, pulling a sample from a waste tank is in and of itself a very difficult task, but even when that is done, the question of whether that sample is representative of the tank waste is sometimes impossible to answer-even with additional sampling.

Then, there are the inevitable analytical errors that derive from the procedures used to homogenize, dissolve, and finally analyze each sample. And there is the over arching uncertainty, once these other issues are resolved, of exactly which waste type a tank's waste is representative. In other words, given that SY-101 is a mixture of DSS and CC wastes, what does the analysis of that tank's waste tell us about either of these two waste types?

- Method used for HDW model

We believe that the Chemicals Used/Waste Produced approach is the best for defining waste compositions, therefore, will favor that method. However, much use will be made of Knowledge of

${ }_{11}^{11}$ Allen, G. K. "Estimated Inventories of Chemicats Added to Underground Waste Tanks, 1944 through 1977," ARHCD-6108, March 1976. 
Process, since it is necessary to change the manner in which the chemicals are partitioned in the waste stream over the years in order to account for changes in the process. Untortunately, these two sources of information are often in severe disagreement. For example, there was $3.3 \mathrm{Mmol}(1 \mathrm{Mmol}=$ one million moles) of citrate reportedly used in B Plant, but the flowsheets suggest that 32 Mmol would have been used, based on the flowsheet concentration and volumes of waste produced. We do not feel that it will ever be possible to resolve all of these discrepancies between these two sources of information. Therefore, we will only use Knowledge of Process to account for notable changes in the waste processes. For example, in 1962, the Purex waste (termed P) decreased from 844 gal/ton to 288 gal/ton-a factor of 2.9 reduction. Obviously there was a change in the process in 1962 that had a dramatic affect on the volume of $P$ waste that was generated. At that same time, CWP volume stayed at $291 \mathrm{gal} / \mathrm{ton}$, but OWW volume went from $108 \mathrm{gal} / \mathrm{ton}$ to $336 \mathrm{gal} / \mathrm{ton}$, an increase of a factor of 3.1 .

\section{Review of Campaigns \\ -Overview}

Some 496 million gallons of waste was placed into single-shell and double-shell tanks at Hanford from 1944 to 1988 , shown in an overview of Hanford waste volumes Table 2. This amount includes Metal Waste (MW) that was reprocessed in U Plant from 1952-56 and Purex sludge and supernatants that were reprocessed in B Plant 1967-76. After reprocessing and water additions, there was a net of 347 million gallons of waste placed into various tanks. Then, 301 million gallons were removed by evaporation. The condensate was placed in cribs while 86 million gallons of waste was placed into cribs either directly or following scavenging operations, leaving 46 million gallons of waste in the tanks from the 1944-80 era.

Since that time, another 30 million gallons of waste has been generated and concentrated to 15 million gallons of waste as of January 1994, leaving 61 million gallons of waste in Hantord waste tanks. Of this amount, 36 million gallons of waste are now in single-shell tanks (all derive from the early era), while the 25 million gallons of waste reside in the double-shell tanks (deriving from both eras), as shown in an Overview of Hanford Waste Volumes (Table 2).

\section{- $\mathrm{BiPO}_{4}$ campaign}

The bismuth phosphate process began in T Plant in December 1944, and in B Plant in April 1945. This process ran until 1952 in B Plant and until $1956 \mathrm{in} T$ Plant and generated some $98,000 \mathrm{kgal}$ of MW, 1C, 2C, and 224 wastes. The Farms T, TX, and TY were used for wastes from $T$ Plant, while B, BX, and BY were used for wastes from B Plant. The term Metal Waste (MW) derived from the code word for plutonium during the war years, "metal." The other terms $1 C, 2 C$, and 224 represent the wastes from first cycie, second cycle, and the plutonium finishing operation in building "224."

These wastes were generated from 1944 to 1956 and the HDW compositions reflect those of Anderson, Jungfleisch, Schneider, and Place. A summary of the wastes generated during this campaign are shown in App. B and a synopsis for the BiPO, process is illustrated in Bismuth Phosphate Process Synopsis (Fig. 6).

Anderson-91 reported that, starting in September 1947, second cycle decontamination waste

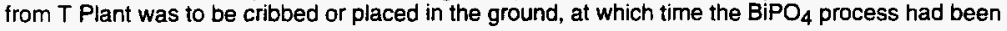
running some two-and-a-half years. Then in February 1948, second cycle wastes from $B$ Plant were directly cribbed as well. Thus, the $-2,400$ gal/ton waste rate shown in (App. B) for $2 C$ waste probably reflects the fact that a large portion of the $2 \mathrm{C}$ waste had already gone into the ground at $\mathrm{T}$ Plant.

Around 1950 , there was an abrupt change in both the $1 \mathrm{C}$ and $2 \mathrm{C}$ waste rates. The $2 \mathrm{C}$ rate increased from $-2,400$ to an average of $\sim 4,600 \mathrm{gal} / \mathrm{ton}$, nearly twice the volume rate that it had been. Moreover, in 1954 the rate actually peaked at 25;000 gal/ton, as shown in Waste rates for $\mathrm{BiPO}_{4}(\mathrm{Fig}$. 7). During this same period, the actual amount of fuel that was processed was quite low, as shown in the $\mathrm{BiPO}_{4}$ Waste History (Fig. 8)., therefore, there is not a large effect on the average rate over the period 1950-56. Anderson-91 noted that canyon cell drainage waste, previously disposed of to dry well via 361 Settling Tank, was added to $2 \mathrm{C}$ after 1951 , and that stack drainage (from decladding off gas?) was combined with $2 \mathrm{C}$ up until 1951. If this change in volume were due to this drainage waste, it suggests that some $12,043 \mathrm{kgal}$ of drainage waste was cribbed at B and T Plants from 1945-51. Likewise, there was a change in the solids volume per cent for $2 \mathrm{C}$ waste, which we estimate to have been 6.8 vol\% from $1945-49$ and 3.4 vol\% from $1950-56$. 


\begin{tabular}{|c|c|c|c|}
\hline \multirow{2}{*}{\multicolumn{2}{|c|}{$\begin{array}{c}\text { Table } 2 . \\
\text { Overview of Hanford Waste }\end{array}$}} & \multicolumn{2}{|l|}{ Volumes* } \\
\hline & & kgal & kgal \\
\hline \multirow{2}{*}{\multicolumn{2}{|c|}{\begin{tabular}{l|l} 
total waste generated $\mathbf{1 9 4 4 - 8 0}$ \\
less MW sluiced
\end{tabular}}} & & 496,200 \\
\hline & less MW sluiced & 35,800 & \\
\hline \multicolumn{2}{|r|}{ less $P$ sludge sluiced } & 900 & \\
\hline \multicolumn{2}{|r|}{ less $\mathrm{P} / \mathrm{R} / \mathrm{B}$ supernatants to $\mathrm{B}$ Plant } & 26,500 & \\
\hline \multirow[t]{4}{*}{ net } & waste after reprocessing & & 433,000 \\
\hline & less $2 \mathrm{C}$ and 224 to crib & 30,000 & \\
\hline & less $1 C$ to crib & 12,400 & \\
\hline & less 1C/UR/FeCN scavenged to crib & 43,300 & \\
\hline \multirow[t]{4}{*}{ net } & waste after cribbing & & 347,300 \\
\hline & less water added and evaporated & 74,500 & \\
\hline & less condensate (recorded) water to crib & 43,900 & \\
\hline & less further evaporator reduction & 182,900 & \\
\hline \multirow[t]{4}{*}{ net } & waste remaining from $1944-80$ & & 46,000 \\
\hline & sludge & 14,000 & \\
\hline & salt cake & 19,000 & \\
\hline & other liquids & 13,000 & \\
\hline \multirow{2}{*}{$\frac{10 t a l}{11}$} & waste generated 1981-88 & & 30,000 \\
\hline & less volume evaporated & 15,000 & \\
\hline \multirow[t]{4}{*}{ net } & waste remaining & & 15,000 \\
\hline & sludge from ' $83-88$ & 1,100 & \\
\hline & other liquids from ' $83-88$ & 13,900 & \\
\hline & waste from $1944-80$ & 46,000 & \\
\hline \multicolumn{2}{|c|}{ total waste $1-1-94$} & & 61,000 \\
\hline & SST & 36,000 & \\
\hline & DST & 25,000 & \\
\hline
\end{tabular}

*As of 1-1-1994.

The main receiver of $2 C$ waste from $T$ Plant was $T-110$, which cascaded to $T-111$ and $T-112$, and then to various cribs. From $B$ Plant, $2 C$ waste was placed into $B-110$, which cascaded to $B-111$ and $B-$ 112. All told, $21,000 \mathrm{kgal}$ of $2 \mathrm{C}$ was cribbed from $\mathrm{T}-112$, and 4,700 cribbed from $B-112$.

The $1 \mathrm{C}$ vol\% solids increased rather dramatically from 14 vol\% trom $1945-47$ to 25 vol\% from 1947-51 as shown in the section Methodology (Table 5), while the waste rate only increased from $\sim 3,700 \mathrm{gal} / \mathrm{ton}$ to $\sim 4,900 \mathrm{gal} / \mathrm{ton}$ from pre 1950 to post 1950 (App. B) and Waste Rates for BiPO (Fig. 7). We do not completely understand this change in $1 \mathrm{C}$ solids per cent, but it is important in the solids layering predictions. We assume in our model that the change in solids volume per cent was due to a reduction in sodium hydroxide added to neutralize the waste stream. With less sodium hydroxide, more aluminum precipitates as the oxide and less remains in solution as aluminate. Accordingly, we adjust the caustic downward for $1 \mathrm{C} 2$ versus $1 \mathrm{C} 1$, and adjust the traction aluminum precipitated as oxide accordingly. Note that the $1 \mathrm{C} 2$ waste rate was quite variable during its period, ranging from 1,800 to $6,800 \mathrm{gal} . /$ ton fuel, while the $1 \mathrm{C} 1$ waste rate ranged from 2,000 to $4,000 \mathrm{gal} . /$ ton during its period.

Anderson-91 reported that alkaline coating removal waste $(\mathrm{CW})$ was combined with $1 \mathrm{C}$ for storage, and that stack drainage was combined with $1 \mathrm{C}$ after May 1951. Whether either of these wastes had anything to do with the waste rate changes is uncertain at this time. In any event, $1 \mathrm{C}$ wastes were largely added to $\mathrm{T}-107, \mathrm{U}-107, \mathrm{~B}-107$, and $\mathrm{C}-107$ overfilied to the corresponding tanks in a three tank cascade. 
Bismuth Phosphate Process Synopsis

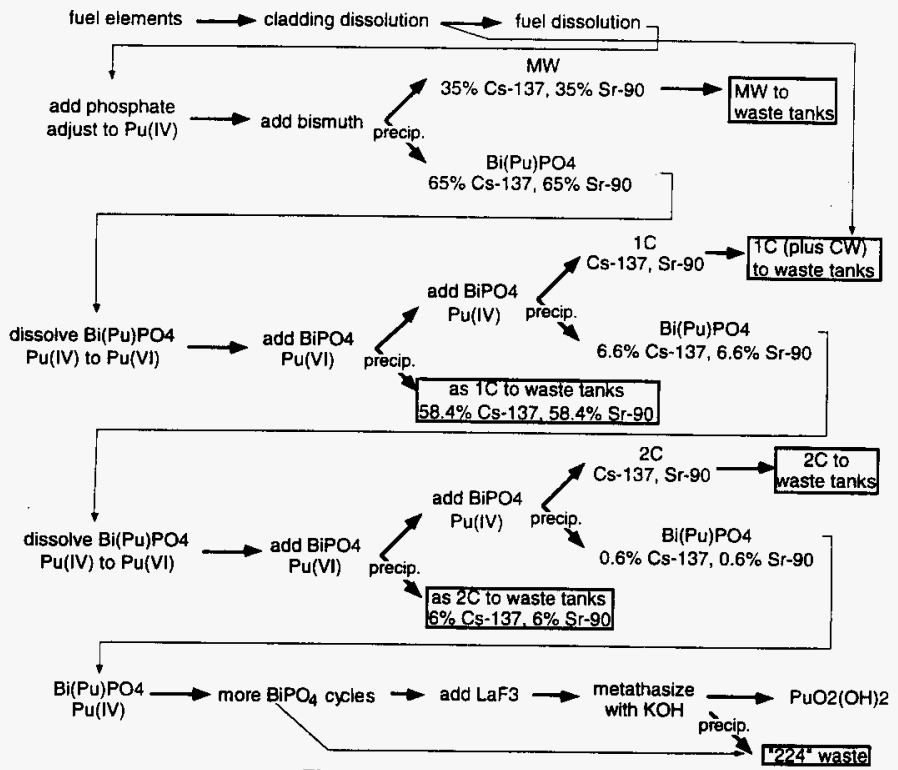

Fig. 6. Diagram of $\mathrm{BiPO}_{4}$ process.

Prior to being welded in their jackets, the fuel slugs were coated with a bronze layer ( $\mathrm{Cu}$ and $\mathrm{Sn}$ ) up until about 1952 or so and neither of these elements are currently within the HDW chemicals added. During most of the aluminum cladding operation, though, lead dips instead of bronze were used for fuel slugs which is included with the cladding waste definition.

The $1 \mathrm{C}$ supernatant was not generally cribbed, although Anderson-91 reported that 4,807 kgal of $1 \mathrm{C}$ supematant and $1,938 \mathrm{kgal}$ of $1 \mathrm{C}$ evaporator bottoms were cribbed. The SMM and TLM composition tables (App. D) show some 12,439 kgal of original 1C HDW supernatants were placed into cribs during this time. This increased volume of original supernatants over and above the actual volume sent to the crib is because the cribbing of concentrated $1 \mathrm{C}$ supernatants. Other $1 \mathrm{C}$ supernatants were "scavenged" in TY Farm during the ferrocyanide campaign. These scavenging operations resulted in production of ferrocyanide sludges that are termed $1 \mathrm{CFeCN}$ in the defined waste list HDW Composition Spreadsheets (App. B) in waste type \#12 and now reside in TY-101 and TY-103.

Waste from the plutonium concentration facility, so-called "224" waste, was generated by a plutonium finishing process known as the Lanthanum Fluoride process. According to Anderson-91, all the 224 wastes were cribbed at the plant, and therefore never entered the waste farm. However, WSTRS (and even Anderson-91 waste summaries) show that some "224" waste was placed into the farms. In particular, WSTRS reported $1,220 \mathrm{kgal}$ of "224" waste was added to T-110, $-111,-112$ from $1951-53,173 \mathrm{kgal}$ was added to T-201, $-202,-203$, and -204 , and another $372 \mathrm{kgal}$ was added to B-204, $-203,-202$ from 1952-56. It is not clear why only this amount of "224" ended up in the farms. It is possible, for example, that only accumulated studges and not the all of the waste was placed into waste tanks, or perhaps there was some process upset that occurred. 


\section{Waste Rates for BiPO4}

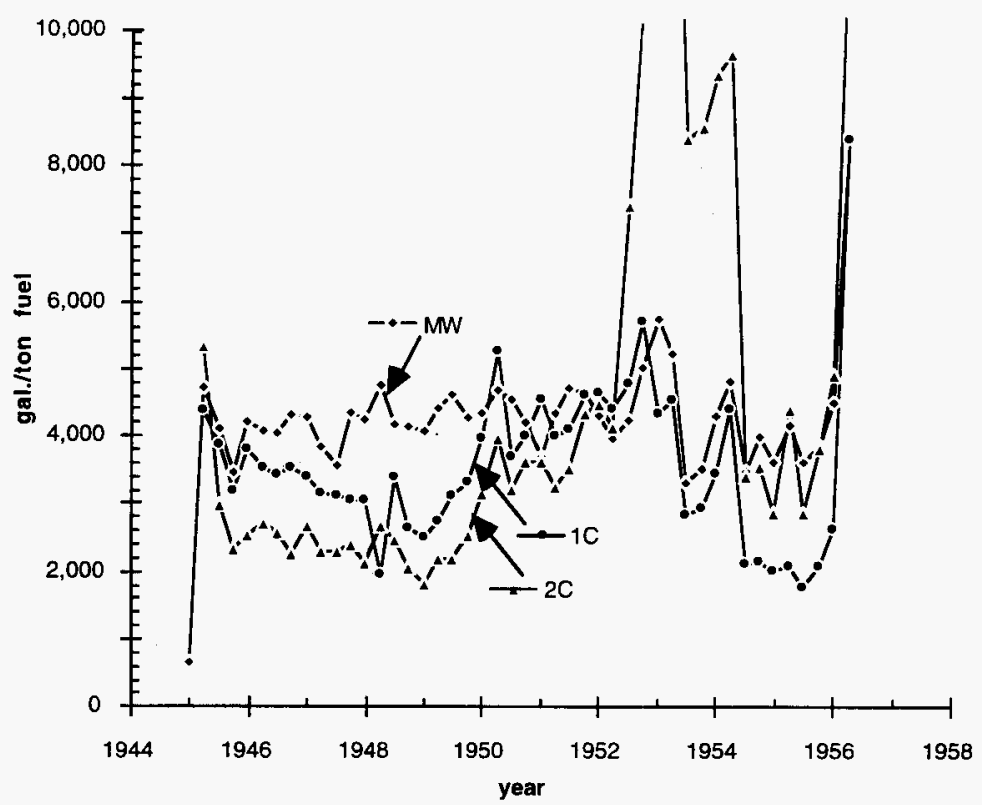

Fig. 7. Waste volume rates for $\mathrm{BiPO}_{4}$ campaign.

The solids amount for "224" waste is very uncertain at this time, but an estimate for the solids fraction comes from the percent water reported 12 from an analysis of T-111 waste. Note that some 154 $\mathrm{kgal}$ of solids accumulated in B-204, $-203,-202$, and -201 , while only $372 \mathrm{kgal}$ was recorded as having been added to this cascade by WSTRS, for a 41 vol\% solids. This solids content is very high and it is probable that much more than $372 \mathrm{kgal}$ was actually added to this cascade. In particular, estimates of per cent solids obtained by using an 80 wt\% water would be 4 vol\%, which would suggest that more like $3,900 \mathrm{kgal}$ was added to this cascade. Likewise, some $124 \mathrm{kgal}$ solids accumulated in T-203, $-202,-201$, -204 , while only $173 \mathrm{kgal}$ "224" is recorded as having been added to this cascade. Using a nominal 4 vol\% solids, this actuat volume added to the T-200 cascade would have been more like $4,300 \mathrm{kgal}$. Thus, we have assumed that a total of $8,300 \mathrm{kgal}$ of "224" waste was placed into these various cascades over the history of this process.

The compositions of the $\mathrm{BiPO}_{4}$ wastes came from Anderson-91, Jungfleisch-84, Allen-76, and other information. ${ }^{13^{\prime} 14}$ Anderson states that $1 \mathrm{C}$ waste contained $10 \%$ of fission products and $2 \mathrm{C}$ had

\footnotetext{
12Preliminary analysis of T-111 courtesy of Roger Bean, PNL

13 no author "Hanford Technical Manual," HW-10475, May 1944.
} 
$1 \%$, but this description does not further define the partitioning of fission products, such as Sr-90 versus Cs-137. Therefore, we have partitioned both $\mathrm{C}-137$ and $\mathrm{Sr}-90$ as $35 \%$ into $\mathrm{MW}, 58.6 \%$ into $1 \mathrm{C}$ waste, $6 \%$ into $2 \mathrm{C}$, and $0.6 \%$ into 224 . The remaining radionuclides are partitioned as $88.9,10,1.0$, and $0.1 \%$, respectively.

\section{BiPO4 Waste History}

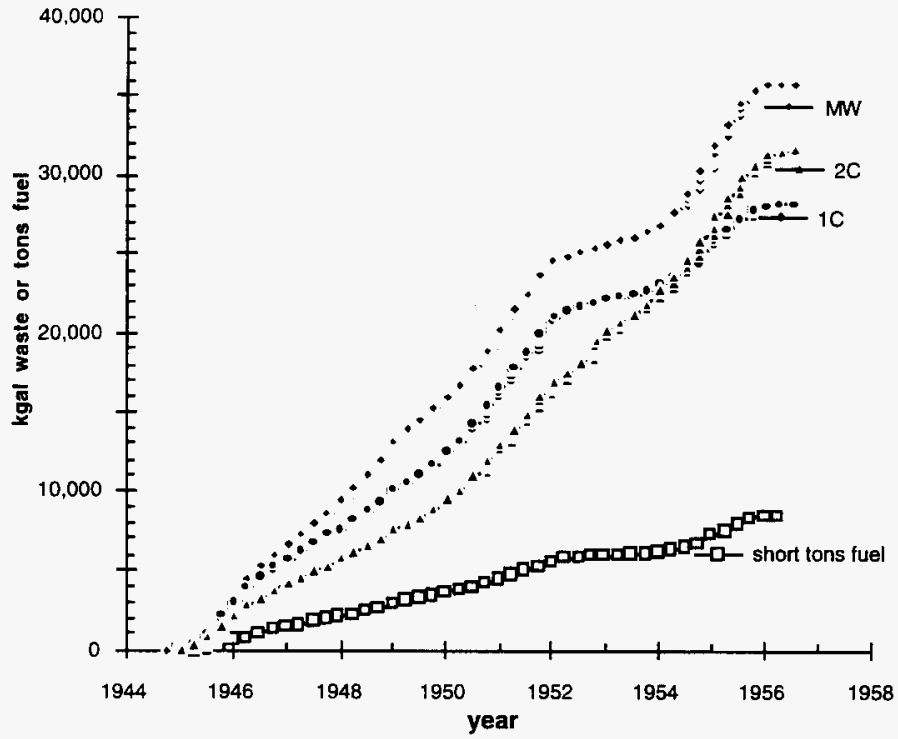

Fig. 8. Total waste volumes from $\mathrm{BiPO}_{4}$ campaign

\section{-Uranium recovery}

The $\mathrm{BiPO}_{4}$ process did not remove uranium from the process stream. A second campaign, the uranium recovery campaign, began that involved sluicing the MW tanks and extracting the uranium from those wastes. This uranium campaign involved $U$ Plant and a process that was based on a TBPNPH solvent extraction, and produced a waste that has been referred to as TBP. This report, however, it is termed UR waste to make clear the distinction among the process wastes that involved the chemical, TBP. A description of the overall uranium recovery process is shown in (Fig. 9).

The uranium recovery campaign began in 1954 and recovered the $M W$ that was stored in $B, C$, $B X, B Y, T, T X$, and $U$ Farms. This campaign reportedly produced about two gallons of waste for each gallon of MW that was processed (actually -2.5 by WSTRS, adding UR, PFeCN1, PFeCN2). Therefore, more waste was created than could be accommodated by the tank farms and a scavenging program based on the precipitation of $\mathrm{Na}_{2} \mathrm{NiFe}(\mathrm{CN})_{6}$ within the waste stream to scavenge or entrap the $\mathrm{Cs}-137$ began. With the Cs-137 precipitated in the sludge, the supernatant was then placed into the ground in cribs or trenches. As a result, about $30,000 \mathrm{kgal}$ of scavenged waste was sent to the cribs following an in-

\footnotetext{
14 Kupter, M.J.; Boldt, A.L; Higley, B.A.; Lambert, S.L.; Orme, R.M.; Place, D.E.; Shelton, L.W.; Watrous, R.A.; Borsheim, G.L.; Colton, N.G.; LeClair, M.D.; Schulz, W.W.; Hedengren, D.C.; Winward, R.T. "Standard Inventories of Chemicals and Radionuclides in Hanford Site Tank Wastes," WHC-SD-WM-TI-7.40 Draft, September 1996.
} 
plant addition of ferrocyanide and $12,000 \mathrm{kgal}$ was sent to the crib with in-farm or CR-Vault addition of ferrocyanide see Overview of Hantord Waste Volumes, Table 2.

\section{Uranium Recovery Process Synopsis}

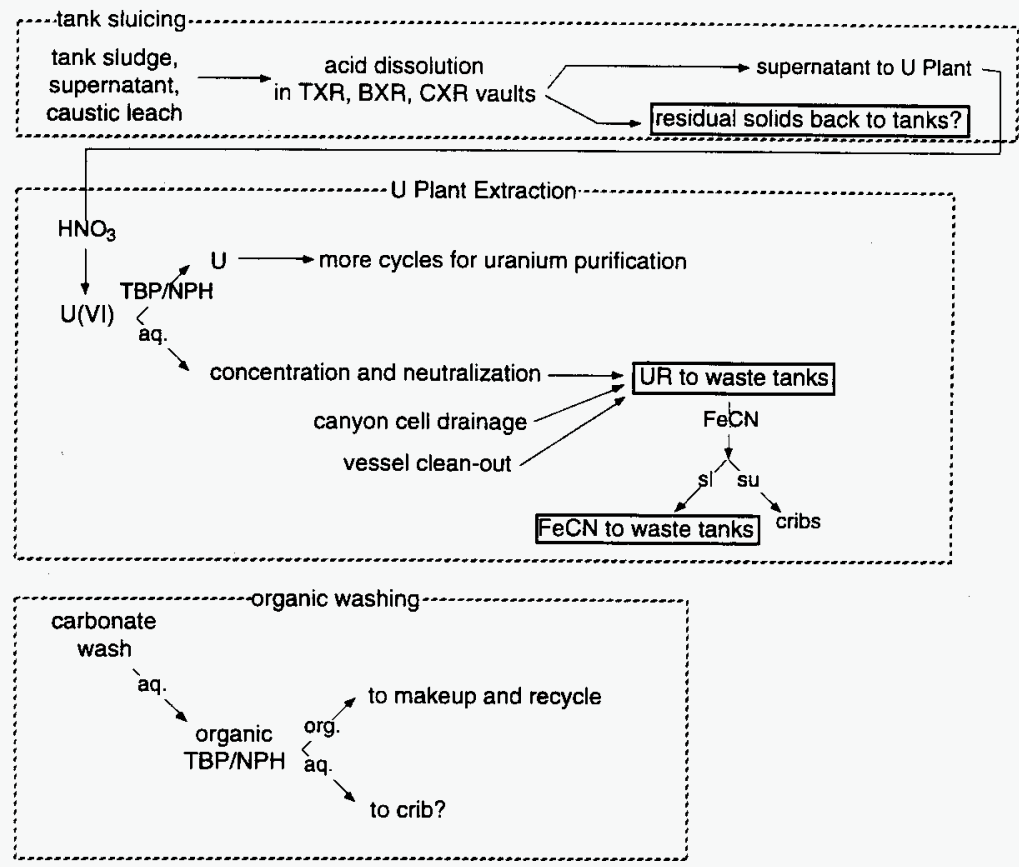

Fig. 9. Uranium Recovery process synopsis.

Evidently, there were heel remnants of MW solids in many of the tanks following sluicing and as much as $40 \mathrm{kgal}$ of solids remained in some tanks following sluicing operations. We assume that some of these heels were left behind because of expediency and not because the solids weren't sluicable. However, there were reports of hard pan forming in well-aged MW sludges and the suggestion was that a very hard uranium carbonate phase was formed in aged MW sludges. The Uranium Recovery manual states ${ }^{15}$ that although sluicing and then acid digesting MW sludge in tank farm vaults was the baseline process, dissolution of uranium in caustic water and/or sodium carbonate solutions would also be attempted within the waste tanks. It is not clear, though, what happened to the sludge remnants that would have resulted following acid digestion in TXR, BXR, and CR tank farm vaults during feed preparation of recovered MW sludge. We have found that more than $15-20 \%$ of the expected $4,309 \mathrm{kgal}$ of MW sludge still remain as heels within various tanks. We have used the reported sludge levels for MW as $12 \mathrm{vol} \%$, although values as high as 25 vol\% were mentioned in the Uranium Recovery manual.

\footnotetext{
${ }^{15}$ no author "Uranium Recovery Technical Manual," HW-19140, November 1951, section 1.1, p. 109.
} 
Although the solubility of the uranium/carbonate complex in MW supernatants was reported in the UR manual to be $0.11 \mathrm{M}$, that same source also claimed that $75 \%$ of the uranium was present as a solid in the sludge. Our calculations show that if $75 \%$ of the uranium were in the sludge, a supernatant of only $0.04 \mathrm{M} \mathrm{U}$ would result. If we assume that the $0.11 \mathrm{M}$ solubility point is correct (uranium solubility is $-0.004 \mathrm{M}$ for typical tank waste supernatants), this would have meant that only $35 \%$ of the uranium was in MW sludge. Thus, we suggest that caustic sludge leaching may have been performed in the later stages of the Uranium Recovery Campaign in lieu of actual sludge removal and acid digestion in the vaults.

For these MW heels, we have assumed that $80 \%$ of the plutonium and strontium and $95 \%$ of the cesium associated with MW sludge were removed and ended up in the uranium recovery wastes as either UR, PFeCN1, and PFeCN2 sludges, while the remainder of the plutonium and strontium remained with the MW heels. There were frequent problems with pump failures and other difficulties sluicing the socalled "hard-pan" out of tanks with well-aged MW, as previously detailed. ${ }^{16}$ It is possible that these difficulties resulted in some expediency with regards to the $\mathrm{MW}$ heel remnants that were left within the tanks.

For UR waste, there is a problem with the composition-it is not consistent with expectations based on the flowsheet. This is shown by the phosphate levels, for example, which lead to a solids volume per cent that is much larger than that observed for this waste. We assume that the decision to leave many MW solids in the waste tanks was based on the leachability of uranium from the solids. That is, agitation of the solids with a basic carbonate solution should have been sufficient to leach the uranium out of the solids and into the supernatant. The supernatant could then be passed on to Uranium Recovery for processing, and therefore preventing the unnecessary transfer of large amounts of solids.

Since only supernatants were scavenged for TFeCN and $1 \mathrm{CFeCN}$ wastes, there were very small amounts of plutonium and $\mathrm{Sr}-90$ in these wastes and we have neglected them. We have used a Cs-137 concentration identical to that of the supernatant of UR and $1 \mathrm{C}$ wastes, respectively.

The compositions of the UR wastes were taken from Anderson-91 and Jungfleisch-84, but the ferrocyanide sludges were defined ${ }^{17}$ from Borscheim and Simpson. Also used to some extent was the Uranium Recovery Manual. ${ }^{18}$ However, we have found that there is evidently some double counting of species within the MW and UR waste streams. That is, the 810 Mmois of $\mathrm{NaNO}_{3}$ mentioned by Allen evidently included the $\mathrm{NaNO}_{3}$ added during MW. Therefore, many estimates of the total sodium used for these two processes are in error since they add these two source terms together. Likewise, Allen shows $50 \mathrm{Mmols}$ of $\mathrm{Na}_{3} \mathrm{PO}_{4}$ added during UR, when no phosphate at all was actually added during the uranium recovery campaign. All of the phosphate was actually carried over from the $\mathrm{BiPO}_{4}$ operation. Therefore, there has been some double counting of added chemicals in past estimates of site inventories.

\section{-Redox}

The Redox process was based on the extraction or salting out of plutonium and uranium from an aqueous aluminum nitrate solution into an organic phase, methyl isobutyl ketone also known as hexone in the Redox Process Synopsis (Fig. 10). Anderson-91 describes the various stages in the development of the Redox process, which began in January 1952 at $S$ or Redox Plant.

According to Anderson, waste was originally generated at 4,378 gal/ton in 1952 , and that rate was reduced to 594 gal/ton in 1966 . We have found by analyzing the fill records, on the other hand, that the waste rate peaked at around $4,600 \mathrm{gal} /$ ton in 1952 and after around 1958 , leveled off to around $1,100 \mathrm{gal} / \mathrm{ton}$.

\footnotetext{
16Podenhizer, D. G. "Hanford Waste Tank Sluicing History," WM-T1-302, September 1987.

17 Borsheim, G. L. and Simpson, R. C. "An Assessment of the Inventories of the Ferrocyanide Watchlist Tanks," WHC-SD-WM-ER-133, October 1991.

18 no author "Uranium Recovery Technical Manual," HW-19140, November 1951.
} 


\section{Redox Process Synopsis}

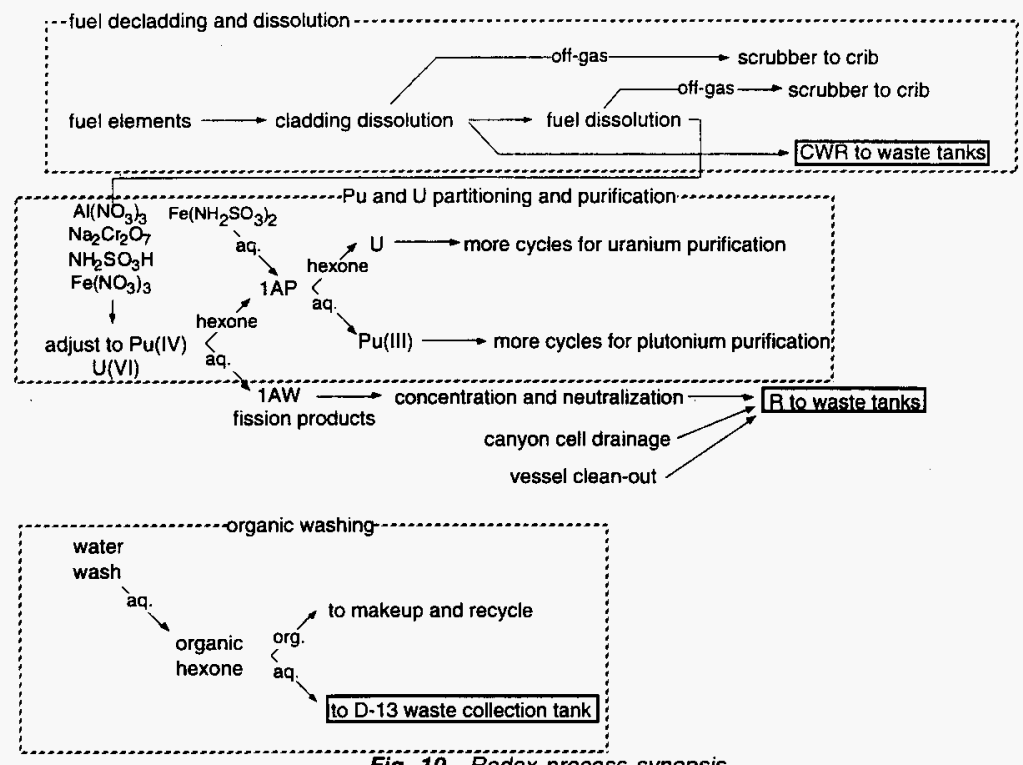

Fig. 10. Redox process synopsis.

Thus, there were essentially two eras for the Redox waste, the first era from 1952.58 averaged $2,106 \mathrm{gal} / \mathrm{ton}$, followed by a reduction to $1,119 \mathrm{gal} / \mathrm{ton}$ from 1959-66 as shown in the Waste Volume Rates for Redox Campaign (Fig. 11) and the HDW Model Inventories for 177 Tanks (App. E). We do find a waste rate as low as 500 gal/ton in the last quarter of 1966, but averaged for all 1966, the last full year of operation, Redox generated waste at the rate of $1,085 \mathrm{gal} / \mathrm{ton}$.

We have also found that the cladding waste generation rate CWR was fairly constant at $266 \pm 30$ gal/ton over the entire history of Redox, as opposed to a remark by Anderson-91, that cladding waste volumes were cut in half in 1956-57. We have found no such decrease in CWR waste rates averaged for any year of operation over the entire Redox campaign. There are some $980 \mathrm{kgal}$ of CWR that is reported by WSTRS after all fuel was no longer processed in Redox in mid 1966 see Total Waste Volumes for Redox Campaign (Fig. 12). We assume that the fuel slugs from this decladding operation were actually processed in Purex Plant.

Prior to being welded in their jackets, the fuel slugs were coated with a bronze layer ( $\mathrm{Cu}$ and $\mathrm{Sn}$ ) up until 1952. Neither of these elements are currently within the HDW chemicals added and so are not included in the cladding waste estimates. Subsequently, lead dips were used instead of bronze for fuel slugs and lead is in the cladding waste chemicals added.

Anderson-91 also mentions that Redox processed some Zircaloy cladded fuel, which came from $\mathrm{N}$-Reactor. However, Jungileisch indicated that the first Zircaloy cladding waste was created in Sept. 1967, and Redox plant shut down in 1966. Other sources (HWN-1991, p. 130) have indicated, on the other hand, that some 269 tons of Zircaloy clad fuel was indeed processed in Redox in 1966 . Since the last cladding waste from Redox (CWR) was place in S-107 in 1967qt, and we expect that some $18 \mathrm{kgal}$ of CWZ 1 sludge would be in the layers of this tank. 
The early solids accumulation in Redox waste tanks during 1952-8 is associated with the era where the Redox waste rate underwent substantial change, as noted before. These tanks were also selfconcentrating the waste, which increases the tanks'solids load even further. We have used a value of solids volume per cent of 4.4 vol\%, which is based on accumulations in SX-105 and SX-111, neither of which tanks were reported to have undergone significant seff-concentration over the period in question. These solids per cents are derived based on consistency with the 2.3 vol\% that we have found for the second Redox period, R2.

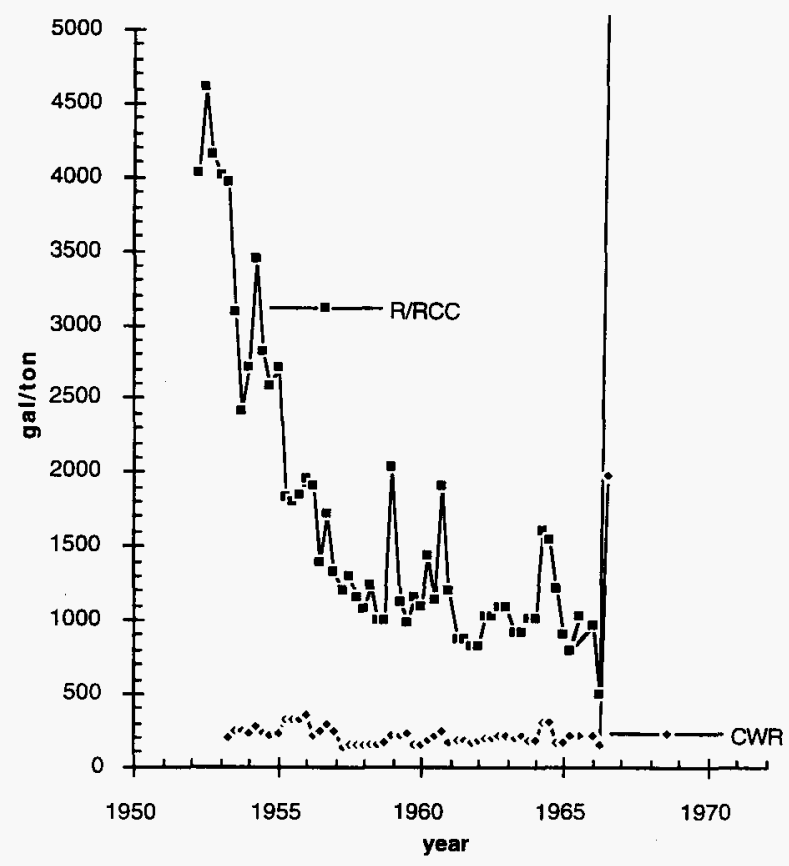

Fig. 11. Waste volume rates for Redox campaign.

For the second Redox period, solids accumulation in Redox waste dropped to $2.3 \pm 1.3$ vol\%, even as the waste rate dropped from 2,106 to 1,119 gal/ton from R1 to R2 (App. B).

Many tanks in S and SX Farms were altowed to self-concentrate therefore accumulated solids in excess of those from the primary additions. In particular, S-101, S-104, and S-107 were all primary receivers of R1 waste and were reported as self-concentrating waste tanks. Unfortunately, we do not have enough information to always differentiate between the two types of solids accumulation within the waste tanks. However S-110 was also a primary receiver of R1, but never was reported to have reached boiling. If we assume that the solids for R1 were actually $4.5 \mathrm{vol} \%$, that would provide an estimate for the 
concentrated solids, RSItCk, in S-101, S-104, and S-107. Thus, we assume that R waste has an implicit component within it that we attribute to the concentrate.

Tank SX-109 accumulated 14 vol\% solids from its $1,756 \mathrm{kgal}$ Redox waste. An analysis ${ }^{20}$ of the fill history of this tank reveals that it self-concentrated the Redox supernatants, and therefore deposited salt cake. Consequently, we attribute much of the solids accumulation in SX-109 to this salt accumulation and not to Redox sludge. We find that a series of tanks accumulated this Redox salt cake, which amounted to $1,065 \mathrm{kgal}$ in a number of tanks in $S$ and $S X$ Farms. This resulted in a particular waste type, RSItCk, which is \#43 in (App. B).

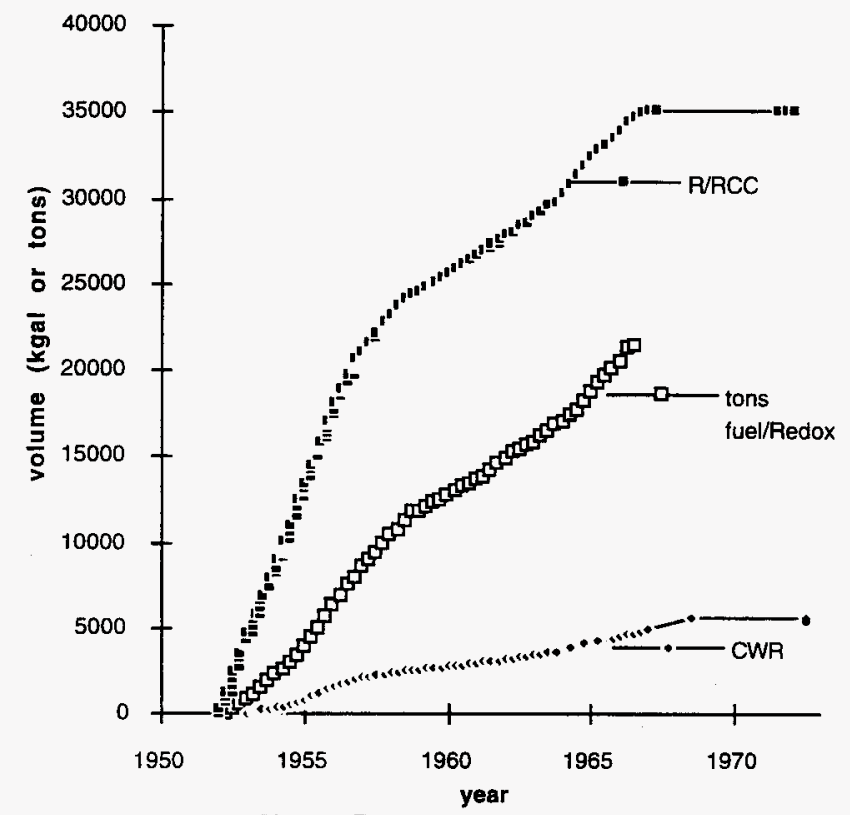

Fig. 12. Total waste volumes for Redox campaign.

Compositions of Redox wastes were taken from Anderson-91, Jungfleisch-84, Allen-76, as well as published fiowsheets. ${ }^{19}$ However, there is a difficulty in the amount of silica that is present in the Redox waste tanks is far greater than the amount that is listed as being present in the flowsheet. We have found a similar excessive silica source for Purex and other processes. Thus, we have added an amount of silica to the Redox waste that amounts to $50 \mathrm{~mol}$ Si per ton of fuel processed. The fuel that

19 (a) no author "Redox Technical Manual," HW-18700, July 1951. (b) Crawley, D. T.; Harmon, M. K. "Redox Chemical Flowsheet HW-No.6," October 1960, HW-66203. (c) Isaacson, R. E. "Redox Chemical Flowsheets HW No.7 and HW No.8." RL-SEP-243, January 1965. (d) Jenkins, C. E.; Foster, C. B. "Synopsis of Redox Plant Operations," RHO-CD-505-RD-DEL, July 1978, declassified with deletions. 
was processed did actually have a silica component, which is listed in the published flowsheets as 21 mol Si/ton. At the present time, we cannot explain why the silica is actually much larger.

The amount of iron present in Redox sludge reflects the process vessel corrosion source term that we have found is a significant contribution to the Purex sludges. We have not found any information about the process vessel corrosion rates during the Redox campaign and have therefore assumed that the rates are identical with Purex.

\section{-Purex primary process}

The Purex process was based on the extraction or salting out of an aqueous plutonium and uranium nitric acid solution into a tributyl phosphate/normal paraffinic hydrocaroon (TBP/NPH) organic phase Purex Process Synopsis (Fig. 13). Purex came on line in January 1956 in A Plant or Purex Plant after having run as a pilot in the Hot Semi-Works (C Plant). The Purex campaigns and the subsequent processing that occurred in B Plant produced the most complicated combination of wastes at Hanford. We have found twenty-one distinct waste types that have derived from the Purex campaign from 195688.

\section{Purex Process Synopsis}

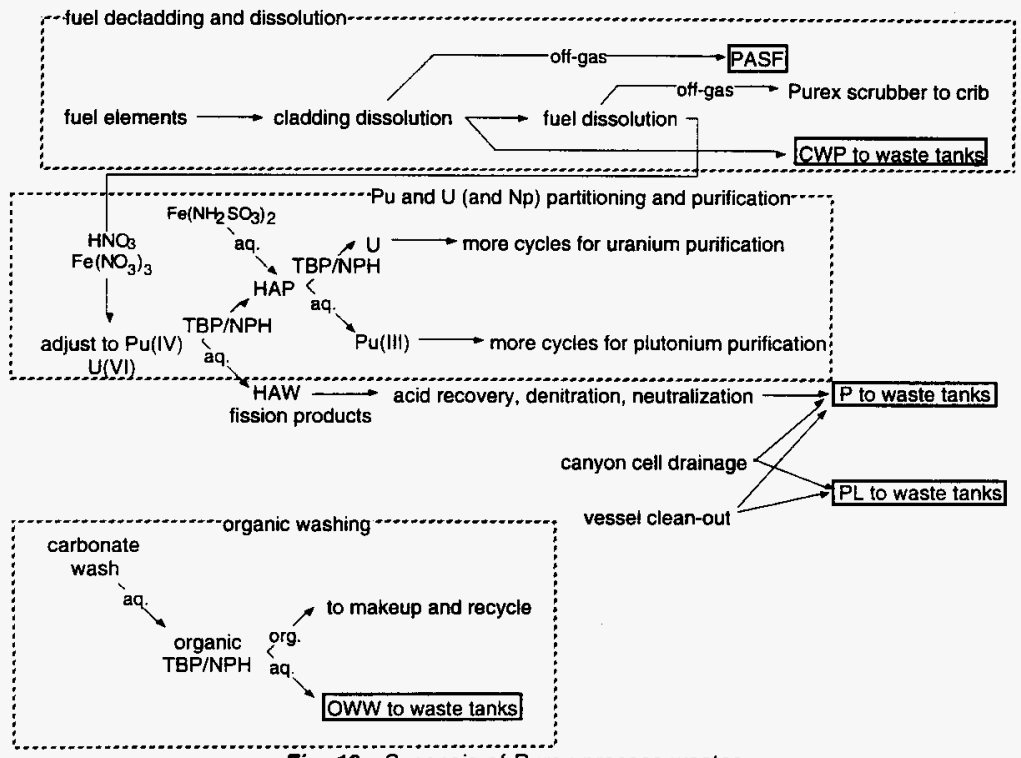

Fig. 13. Synopsis of Purex process wastes.

An analysis of the waste history shows that there were three eras for the Purex process. From 1956 until 1962, the Purex high level waste rate averaged 877 gal/ton, OWW 150 gal/ton and CWP 346 gal/ton, although there is a large variability associated with the $P$ waste rate. Also in this period, we are missing OWW (actually called CARB) for the years 1959 and 1961. During this period, there was a large variability in the waste rate of Purex as compared with other operations as well as other periods of Purex. The waste rate for $P$ waste peaked at $1,400 \mathrm{gal} / \mathrm{ton}$ in 1960 , and again at $1,000 \mathrm{gal} / \mathrm{ton}$. These waste rates suggest that the OWW wastes were actually routed to $P$ waste receivers in 1959 and 1961 . In fact, 
Anderson-91 mentions that OWW were added to boiling waste tanks until 1969, and Jungfleisch-84 states that for some unspecified period, $\mathrm{P}$ and $\mathrm{OWW}$ were mixed together.

In 1960-61, it was reported 20 that a sulfate strike (i.e. precipitation) was used in Purex Plant to precipitate $\mathrm{Sr}-90$ and separate it from the other fission products in HAW. The $P$ waste rate reportedly went from $53 \mathrm{gal} /$ ton to $193 \mathrm{gal} /$ ton during this time. Thus, we suggest that the increase in waste rates in 1960-61 may have been due to a combination of this sulfate strike and redirection of the OWW streams. We have not, however, included any strontium depletion for this P waste. Evidently, this impure product was worked up later in either HS or B Plant operations.

The Purex waste receivers in 1959-61 were A and AX Farms. We suggest that the redirection of the OWW to these farms was due to the desire to concentrate these wastes by self-boiling. However, successive failures of two tanks in A Farm due to overheating evidently modified that strategy, and from then on, OWW was placed into other receivers and reduced in separate operations. There was a sizable self-concentration that occurred in A and AX Farms as a result of this self-boiling. However, unlike the Redox self-concentration campaign, no salt cake accumutated as a result of Purex waste selfconcentration. Therefore, the volume reduction that occurred is accounted in other evaporator campaigns.

From 1963-67, the average waste rate for Purex decreased by a factor of two from 877 to 378 gal/ton while that for OWW rose by a factor of three from 150 to 391 gal/ton as compared to the period 1956-62. These changes in waste rates coincided with two fundamental changes for the Purex process. In Sept. 1962, the solutions from the second cycle uranium extraction were recycled instead of sent to the waste tanks and in Sept. 1963, sugar denitration was introduced, which reduced the nitric acid in HAW and therefore the amount of caustic that was needed to neutralize the waste. During 1962-67, the Strontium Semi-Works facility was directly processing PAW as well. The variability of the Purex waste rate remained fairly high during this period of operation. The inherent amount of high level waste generated by the Purex operation (i.e. HAW) was quite small, on the order of $50 \mathrm{gal} / \mathrm{ton}$, and we suggest that other more a periodic sources, such as vessel cleanout and canyon drainage wastes, now began to dominate over the primary process waste.

Another spike in the $P$ waste rate occurred in 1966, where the rate climbed to $900 \mathrm{gal} / \mathrm{ton}$. We do not yet know why. See Waste Volume Rates for Purex Campaign (Fig. 14).

Finally, from 1968-72 the Purex HAW stream was sent directly to B Plant for strontium removal. and so very little $P$ waste was added to the tank farms after 1967. Eventually, this waste appears as B Plant high and low level wastes (B and $B L)$. On the other hand, OWW increased once again during this latter era to $575 \mathrm{gal} / \mathrm{ton}$.

We assume that the CWP/Al waste rate for the period $1961-72$ remained at $346 \mathrm{gal} / \mathrm{ton}$, which is the rate that occurred for 1956-60. During the period 1968-72, some 708 tons of Zircaloy N-Reactor fuel was processed in Purex (and 269 tons went to Redox, as noted above), and the waste rate for this Zirflex process (termed CW/Zr) was much higher than CWP/AI. In fact, a later flowsheet projected $21 \mathrm{~g}^{27} \mathrm{gal} / \mathrm{ton}^{2}$ for Zirflex waste, although the rate we calculate from WSTRS would have been 1,650 gal/ton, provided that the CWP/Al rate was $346 \mathrm{gal} /$ ton. Since the two types of cladding wastes, CWP/AI and CWP/Zr were not segregated, i.e. both were added to C-104 during 1968-72, we have simply proportioned the $\mathrm{CWP} / \mathrm{Al}$ and CWPRr waste solids accordingly over this period.

\footnotetext{
20 Monthly summaries, 1959-60.

21 Allen, G. K.; Jacobs, L. L.; Reberger, D.W. "Purex Flowsheel-Reprocessing N Reactor Fueis," PFD-P-02000001, Sept. 1982.
} 


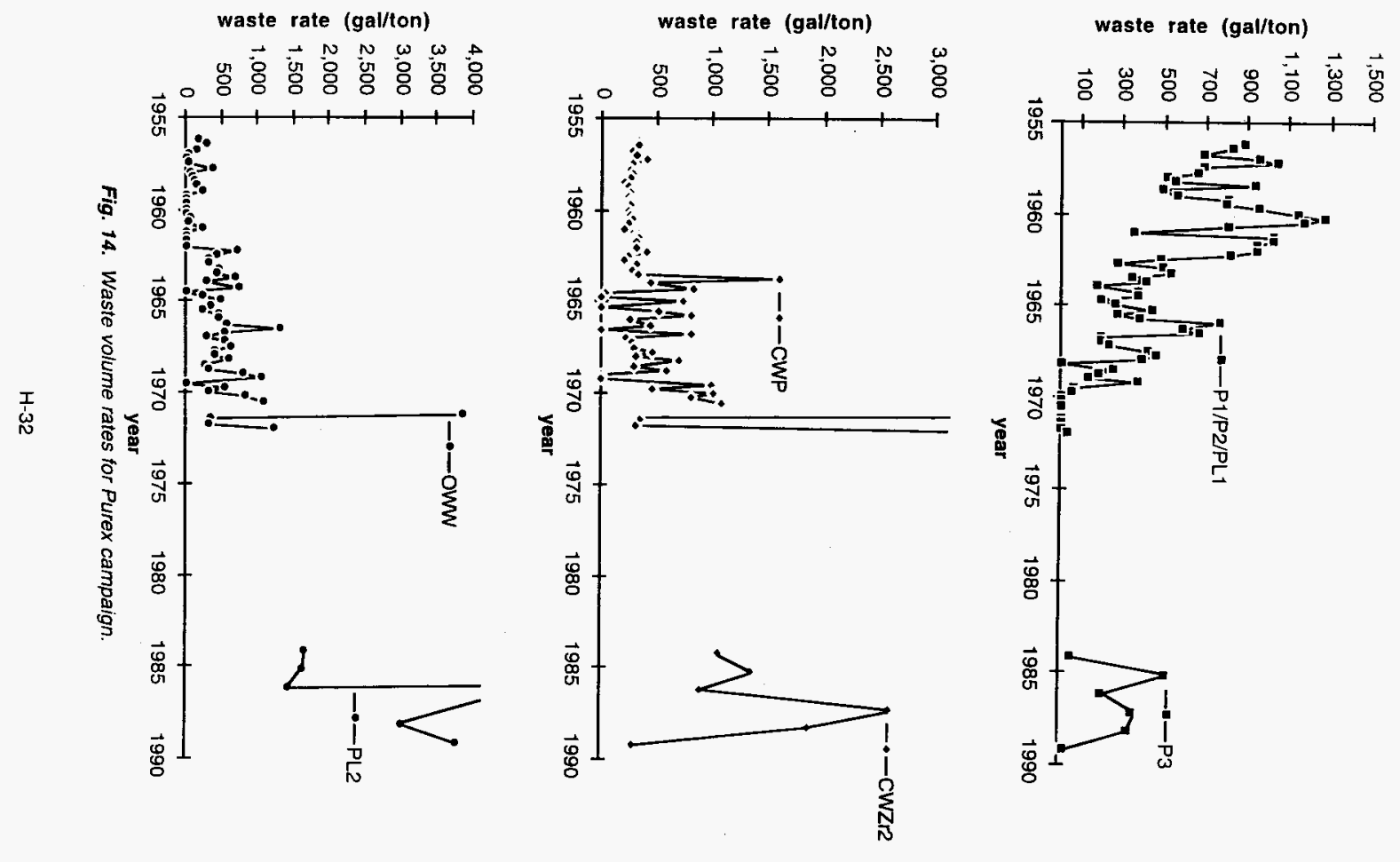


Lead dips were used for coating fuel slugs before placing them into aluminum jackets. As a result, lead is a part of the cladding waste chemicals added.

The solids volume per cent for the CWZR1 waste of this period is assumed to be the same as CWZR2, which was 10.5 vol\%, for the ' $83-' 88$ campaign. Moreover, there are indications that significant amounts of mercury were added to the dissolver solution to limit the emission of $1-131$ during dissolution of the fuel cladding. We have included mercury additions in our definition of cladding wastes. For total waste volumes for Purex campaign see (Fig.15).

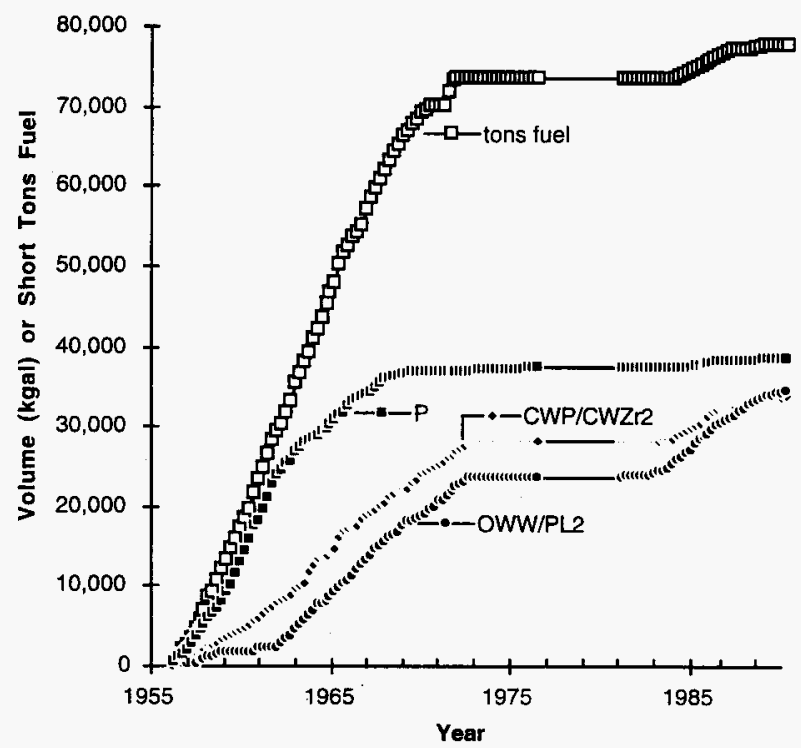

Fig. 15. Total waste volumes for Purex campaign.

The composition of Purex waste was taken trom several sources. An early flowsheet 22 was used for the first era, a second flowsheet ${ }^{23}$ was used for $\mathrm{N}$ Reactor fuels, and a later flowsheet ${ }^{24}$ was used that updated the Purex process for N Reactor fuets. These compositions were adjusted to account for the changes in waste volume that are recorded in WSTRS combined with the total chemicals used reported by Allen-76. Basically, we have taken the waste rate of $50 \mathrm{gal} /$ ton for all of the process chemicals except iron and silica, and diluted those chemicals to the observed waste rates of either 877 or $378 \mathrm{gal} / \mathrm{ton}$.

\footnotetext{
${ }^{22}$ Switt, W. H.; Irish, E. R. "Purex Two Cycle Flowsheet, Revision No.1," October 1957, HW-52389-DEL, declassified with deletions.

23Jeppson, D. W. "Purex Flowsheet Reprocessing N Reactor Fuels," November 1976, ARH-F-103.

${ }^{24}$ Allen, G. K.; Jacobs, L. L. "Purex Flowsheet Reprocessing N Reactor Fuels," September 1982, PFD-P-0200001.
} 
For both iron and silica, we have used Allen-76 to set the total chemicals used and the Chemicals Used/Waste Produced approach to define the waste compositions for these two species. This $\mathrm{Si}$ amount is on the order of $130-160 \mathrm{~mol} \mathrm{Si} /$ ton fuel which is much larger than the $21 \mathrm{~mol}$ Si/ton fuel reported in various flow sheets. The concentration of $\mathrm{SiO}_{2}$ in the waste stream from the plants amounts to 3,000 to $6,000 \mathrm{ppm}$, which is very large compared to a normal impurity level that is expected from hard water, which is on the order of $20-30 \mathrm{ppm}$.

Estimates of organic loss during solvent processing 25 have shown 8.4 gal organic per ton of fuel processed during Purex. For a $70 / 30$ mixture of NPH/TBP, this suggests a loss of $2.5 \mathrm{gal}$ TBP/ton fuel processed. We then assume that all of this TBP is hydrolyzed to DBP and butanol and place those in the corresponding waste streams.

The waste rate for PL2 (PXMISC in WVP notation) amounts to nearly 3,000 gal/ton of fuel processed for the ' $83-' 88$ campaign. Since no OWW was reported at all during this period (flowsheet values suggest -400 gal/ton fuel), we assume that PL2 is actually a combination of both PL1 and OWW, despite the fact that the $3,000 \mathrm{gal} /$ ton value is substantially larger than the $400 \mathrm{gal} / \mathrm{ton}$ expected from the flowsheet. The weighting that we have used is as follows:

$$
\text { PL2 chemicals added }=(2100 * P L 1+800 * \text { OWW } 3) / 2900
$$

The P3 waste (Neutralized Current Acid Waste, NCAW) is derived from flowsheet values that are scaled to the actual waste volume. For example, neutralized ZAW flowsheets predict 160 gal/ton, while the actual volume sent to the tanks averaged $288 \mathrm{gal} / \mathrm{ton}$. We therefore scale all neutralized ZAW flowsheet chemicals by $160 / 288=0.556$ (i.e. assumed dilution).

The sugar denitration of acid ZAW used a sugar solution that would have resulted in about $25 \mathrm{~g}$ TOC/L of waste had it not reacted with the nitric acid. Complete consumption of the sugar was assumed, which reduced the $\mathrm{HNO}_{3}$ from 2.8 to $0.95 \mathrm{M}$ in the waste, with the carbon lost as $\mathrm{CO}_{2}$. An estimate of the amount of sugar used in this process is that $160 \mathrm{gal} / \mathrm{ton}$ of fuel processed at $0.18 \mathrm{~mol} / \mathrm{L}$ and $47,767 \mathrm{tons}$ fuel processed is $5.2 e 6$ mols sucrose, or 1,800 MT. During this denitration, an anti-foam agent was used to keep the solution from foaming. The amount added was $2.2 \mathrm{fl} .0 \mathrm{z} / \mathrm{ton}$ fuel, which amounts to about $6 \mathrm{e}-$ $5 \mathrm{M} \mathrm{Si}$ in the waste stream, assuming the anti-foam agent is $3 \mathrm{w} \% \mathrm{Si}$. However, we have previously found that the Si amounts in Purex waste are significantly greater than this. Accordingly, we use a value of $0.092 \mathrm{M} \mathrm{Si}$ in the P3 waste stream.

\section{-Purex to Hot Semi-Works}

The Hot Semi-Works or C Plant was used as a pilot for both Redox and Purex in the 1950's and was then used as a pilot for strontium extraction in the 1960's prior to B Plant as shown in the Hanford Timeline (Fig. 1). Purex HAW was processed ${ }^{26}$ with this pilot and only a fairly small amount of waste was generated, amounting to $1,003 \mathrm{kgal}$ over the years 1962-67. We do not know exactly how much HAW was processed for the whole campaign, but for the first hot run in 1962, $16 \mathrm{kgal}$ of HAW was processed resuiting in $50 \mathrm{kgal}$ of waste. If we assume that the rest of the campaign progressed with the same waste rates, then $1,003 / 50 \times 16=321 \mathrm{kgal}$ of PAW were processed in the Hot Semi-Works Plant. corresponding to $321 \mathrm{kgal} / 0.3 \mathrm{kgal} / \mathrm{ton}=1,070$ tons of fuel. During the period $1962-67$, on the other hand, about 25,000 tons of fuel were processed, so the amount of PAW processed in the Semi-Works amounted to a relatively small fraction of the total PAW waste.

According to the report for this first hot run, about $1.04 \mathrm{MCi}$ Sr-90 was extracted from $1.2 \mathrm{MCi} \mathrm{Sr}-$ 90 total within the crude PAW (it not clear if this includes the $\gamma-90$ daughter) leaving $0.14 \mathrm{MC}$ i Sr-90 in the waste. Scaling this by the ratio of overall waste with just this run $(1,003 / 50)$, suggests that $2.8 \mathrm{MCi} \mathrm{Sr}-90$ were left in the waste over the entire campaign, or $1.4 \mathrm{MCi}$, decayed to 1994 .

\footnotetext{
${ }^{25}$ Camaioni, D. M.; Samuels, W. D.; Lenihan, B. D.; Clauss, S. A.; Wahl, K.L.; Campbell, J. A. "Organic Tanks Safety Program Waste Aging Studies," PNL-10161, Nov. 1994.

${ }^{26}$ Richardson, G. L.; Schultz, W. W.; Mendel, J. E.; Burns, P. E.; Rushbrook, P. R.; Alford, M. D.; Cooley, C. R. "Hot Semiworks Strontium-90 Recovery Program, Part1: Program Synopsis, Pant 2: Technical Basis, Part 3: Laboratory Studies, Part 4: Cold Semiworks, Part 5: Hot Semiworks Runs" HW-72666-RD, February-November 1962.
} 
Another indication of the Sr-90 concentration in HS waste comes from a report of C-112 sampling in 1993, the very top layer of which was SSW sludge and was $\sim 6 \mathrm{Cl} / \mathrm{L}$. If we assume that all HS sludge was nominally $6 \mathrm{Ci} / \mathrm{L} \mathrm{Sr}-90$, this results in an HS waste inventory of $0.45 \mathrm{MCi} \mathrm{Sr}-90$ (decayed to 1994) in HS sludge, which is a factor of three less than the amount estimated by the hot run report.

The chemical composition of HS from the Lucas ${ }^{27}$ draft report, disagrees with the information from the first hot run report, and we have used this hot run report to define our HS waste. However, there is no Pu or Cs-137 in this waste, and the Pb concentration of $0.034 \mathrm{M}$ results in a very high $\mathrm{Pb}$ value in the sludge. Therefore, we arbitrarily choose to adjust the $\mathrm{Pb}$ down by a factor of ten to $0.0034 \mathrm{M}$. We have also added an amount of Pu to the waste that is volume-scaled to the P2 waste stream.

Although there are records of HS and SSW wastes being added to C-107,-108,-109,-111,-112, there was another addition of $200 \mathrm{kgal}$ of these semi-works wastes to the C-200 series tanks as well. Anderson-91 assigns the waste in tanks C-201, -202, -203, and -204 to SSW and HS and notes starting dates for HS additions in 1955 and 1956, even though we have otherwise no information about the fill history of the C Farm 200-series tanks following the MW sluicing. Therefore, there were undoubtedly unrecorded additions of HS as early as May 1955, which would have been during the Purex pilot operation in the C Plant. Further additions occurred in 1966 trom SSW to C-204, which were presurnably decontamination operations for C Plant, and then all of the C-200 tanks were pumped to their sludge heels in 1970 and 1977.

\section{-Purex to B Plant Cs-137 recovery}

Although B Plant was used from 1945-1952 for the $\mathrm{BiPO}_{4}$ process, it was later reconfigured for the cesium and strontium extraction campaign. From 1967 to 1976, B Plant extracted strontium from both Purex acid waste (PAW) (from Purex Plant) and Purex sludges (sluiced from A and AX Farms), and extracted cesium from a variety of neutralized supernatants taken from the tank farms.

Our model for the cesium recovery approximates the feed for this campaign by deriving volumes of waste supernatant that was processed from the tank farms. In (App. B) we show the nominal composition of the feed for CSR aiong with the sum of supematants from which it was derived. Note that many concentrated wastes were processed during this campaign and we find that the average concentration factor was $\sim 2.7$ for the CSR feed. The following equation represents the majority of the contributions to the CSR waste in terms of other HDW supernatants:

$$
\begin{gathered}
\text { CSR in }= \\
0.34 \mathrm{P} 1+0.11 \mathrm{P} 2+0.11 \mathrm{~B}+0.05 \mathrm{AR}+0.07 \mathrm{R} 1+0.03 \mathrm{R} 2+0.21 \mathrm{OWW}
\end{gathered}
$$

as well as 0.08 as other wastes (see App. D). This equation represents a deconvolution of wastes that are actually partof concentrated waste blends such as RSItCK and BYSItCK.

Supernatants were fed to the cesium recovery process through C-105, which was the staging tank for the caustic sludge washing in AR vault. All total, $45 \%$ of the CSR feed was Purex supernatants, $21 \%$ organic wash waste, and $10 \%$ Redox supernatants, with the balance being other wastes. The CSR waste blend was concentrated on average by a factor of 2.7 prior to CSR processing but the HDW model does not account for any solids in the CSR feed. As a result, the CSR blend is only an approximation of the true feed for CSR.

There were two different processes for extracting Cs-137. The first was a chromatographic extraction of Cs-137 onto zeolites from the caustic supernatants that were drawn from various tank wastes. We find that $92 \%$ of the Cs-137 was extracted from these supernatants with this process. The second process by which Cs-137 was extracted was by precipitation of Cs-137 with phosphotungstic acid added to the HAW acid feed that was obtained directly from Purex plant. This process evidently produced the $B$ and $B L$ wastes. Finally, the crude cesium product purification produced additional $B$ and $\mathrm{BL}$ wastes as well.

27Lucas, G. E. "Waste Types in Hanford Single-Shell Tanks," WHC-SD-ER-TI-001, draft report, 1989. 
HNF-SD-WM-ER-323, Rev. 1

\section{-Purex to B Plant Sr-90 recovery}

The strontium recovery operation was much more complex than the cesium recovery as shown in (Fig. 16). The origin of the complexant wastes at Hanford are attributed to this process. The waste volumes from the various processes are shown in (Fig. 17). Note that the production of $B$ and BL wastes evidently derived from PAW (or HAW) processing, although we do not know the volume processed. We estimate that about 4,000 kgal of PAW would have been processed in B Plant from 1968-72, which resulted in the production of $11,763 \mathrm{kgal}$ of $B$ and $4,000 \mathrm{kgal}$ of $B L$ wastes (App. E). There was about $397 \mathrm{kgal}$ of $P$ and $1,233 \mathrm{kgal}$ of $\mathrm{PL}$ placed in waste tanks during this time, some of which was then sluiced for the later Sr-90 extraction from PAS (Purex Acidified Sludge).

Although sluicing operations began to send sludge to AR Vault in 1967, the production of SPR waste did not begin until 1973. We cannot explain this time difference, but note the SRR waste came primarily from the purification of the Sr product. The Cs-137 recovery operation, on the other hand, ran fairly consistently from 1967 to 1973, and then slowed down substantially after that time. There were two different processes for separating cesium from waste, depending on whether the waste was alkaline or acidic. For neutralized or alkaline supernatants Purex Sludge Supernatant (PSS), resins were used to extract cesium. For acidic supernatants, phosphotungstic acid was used to precipitate the cesium before neutralization.
B waste from PAW
BL low level waste from all operations
AR solids "washed" P sludge. Also used to derive SRR.
SRR Strontium recovery waste from sluiced $P$ sludge - based on washed Purex sludge plus added EDTA, HEDTA, and glycolate.
CSR waste from cesium recovery from supernatants-not a characteristic waste type, but rather a supernatant from which the ${ }^{137} \mathrm{Cs}$ has been removed. Need only to add citrate to supernatants to track this component.

The amount of Cs-137 and $\mathrm{Sr}-90$ extracted during this campaign has been reported 28 and is shown in Table 3 The Partitioning Following CSR and SRR. The difference is very important, since that is the residual Cs-137 and Sr-90 that is present in the waste tanks. We have assumed that the residual Sr90 is distributed among $P, A R, S R R, B, B L$, and HS wastes, while the residual Cs-137 is distributed among CSR and $B$ wastes.

Overall, the campaign succeeded in extracting $42 \%$ of the $5 r-90$ from various wastes. Since B waste derived from the extraction of $\mathrm{Sr}-90$ from PAW, we assume that $80 \%$ of that $\mathrm{Sr}-90$ was extracted and $20 \%$ ended up in B waste. With this assumption in hand, we find that $27 \%$ of the $\mathrm{Sr}-90$ in neutralized Purex sludge ended up in the WESF capsules, while the remaining was distributed as shown in Table 3.

There are some 4.3 MCi Sr-90 (decayed to 1993q4) for which we can not yet account. It is not clear at this time if this "missing" Sr-90 was actually extracted from the sludge and shipped oft site or is otherwise present on the site and its apparent absence indicates an inaccuracy in our model. For example, increasing the solubility of the $\mathrm{Sr}-90$ in the wastes would naturally accommodate more $\mathrm{Sr}-90$ in concentrates of waste supernatants. Another possibility is that there is unaccounted Sr-90 within and underneath damaged tank A-105. That there is a significant heat source underneath this tank is indicated by the very high temperatures within a lateral well underneath the tank as well as the high dome space temperature for the tank itself. The "missing" $4.3 \mathrm{MCi}$ would amount to a $-100,000 \mathrm{Btu} / \mathrm{hr}$ heat source, which would presumably be more than enough to raise the lateral-well temperature to the $-212^{\circ} \mathrm{F}$ reported today. Therefore, some or all of the missing Sr-90 may be in or under A-105.

\footnotetext{
${ }^{28}$ ORNL document (no author), "Integrated Data Base for 1991: U. S. Spent Fuel and Radioactive Waste inventories. Projections, and Characteristics." DOE/RW-0006, Rev. 7, 1991, Mike Cooney is Hanford contact.
} 
Two tanks that are key in containing the amount of Sr-90 in BL waste are C-106 and B-101. Independent estimates of tank waste heat loads from tank temperatures suggest that $C-106$ is $100,000 \pm 20,000 \mathrm{Btu} / \mathrm{hr}$, while B-101 is around $14,000 \pm 6,000 \mathrm{Btu} / \mathrm{hr}$. Strontium-90 levels in BL also impact the heat load in AY-102, since this tank was a primary receiver for B Plant non-complexed waste in the years $1981-8$. We have assigned this B Plant waste as BL. The solvent washing waste from B Plant operations evidently ended up as BL waste. Therefore, the solvent residues from this process ended up in these tanks.

In particular, recent assays of sludge grab samples from C-106 show residual di-2-ethyl hexylphosphate in $\mathrm{BL}$ sludge. Evidently, this extractant preferentially remained with the sludge particles upon neutralization of waste from B Plant, since the amounts of TBP and NPH residues in the sludge are much less than the proportions used in the process.

\section{Strontium Recovery Process Synopsis}
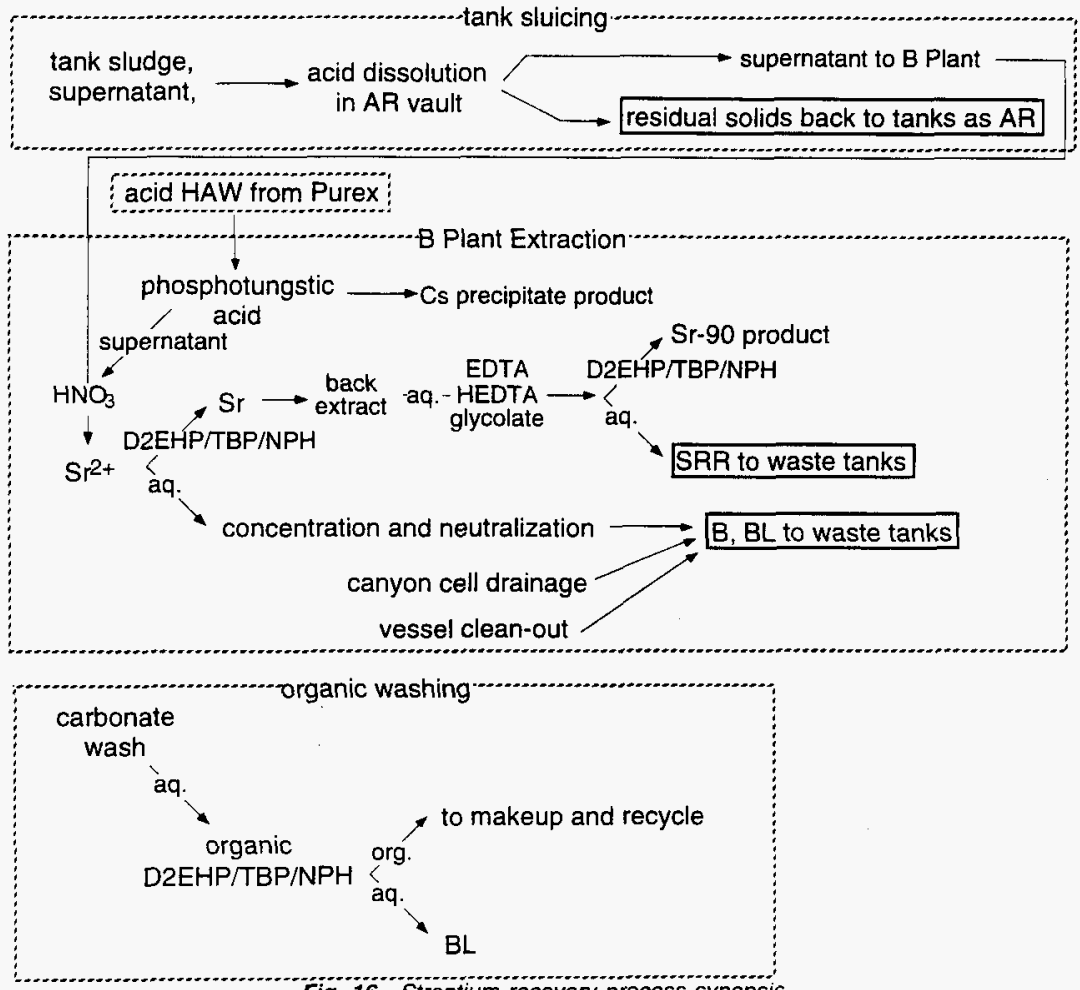

Fig. 16. Strontium recovery process synopsis.

The strontium extraction process as applied to tank sludges required the sluicing and acid dissolution of Purex sludges in AR vault. The dissolution of these sludges did not proceed exactly as 
planned, in that the sedimentation rates of the washed sludges in tank AR-002 evidently varied widely, which resulted in the inadvertent transfer of AR solids from AR-002 10 C-106. Furthermore, there were some acid insoluble solids that resulted which had to be neutralized and returned to the tank farms as well. These AR solids had very high levels of strontium, which resulted in high heat loads being placed into C-106, C-103, and A-106. We have defined AR waste Defined Waste \#31, (App. A) as essentially a Purex sludge with all of the soluble components removed. We have estimated that about $166 \mathrm{kgal}$ of AR sludge still remains distributed around $A, A X$, and $C$ Farms.

Of the $981 \mathrm{kgal}$ of P/PL sludge that was placed into the tanks, $99 \mathrm{kgal}$ remains as P/PL sludge and $166 \mathrm{kgal}$ remains as AR sludge, leaving $716 \mathrm{kgal}$ of sludge processed as PAS in B Plant. However, we estimate that only $201 \mathrm{kgal}$ of solids accumulated from BL and SRR waste additions in the tank farm and therefore can only account for roughly $28 \%$ of the solids that were processed in B Plant. At this time it is not clear whether the complexants present in SSR waste mitigated sludge formation for this waste or if we simply have an incomplete record of the transfers for this type of waste.

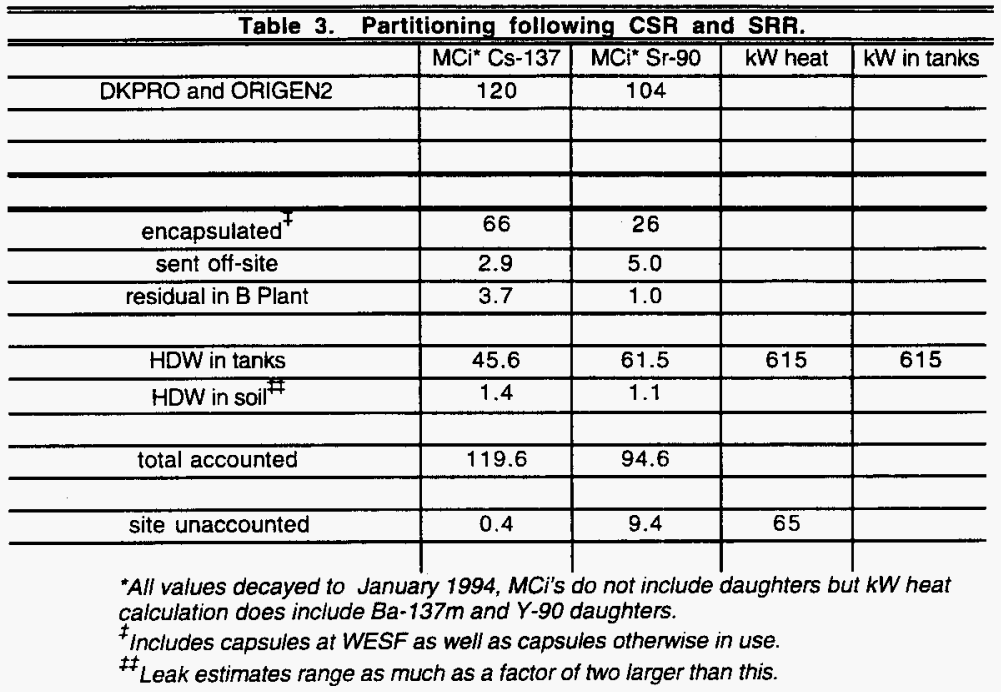

Compositions of B Plant waste streams are derived from Jungfleisch-84, as well as published flowsheet information and total chemicals used reported in Allen-76. The solubility of $\mathrm{Sr}-90, \mathrm{Pu}$, and $\mathrm{Fe}$ have been increased in SRR and CSR wastes by a factor of four (see Partitioning following CSR and SRR, Table 3), which is consistent with the presence of complexants in these two waste streams. We have not increased the solubility of any other components in these waste streams although we expect that many other metal ions will also have enhanced solubility in these wastes.

\section{-Purex to thorium campaigns}

There were two thorium campaigns that ran in the Purex facility. The first ran in 1966 and involved a very low burnup (1.7 MWD/ton) of only 191 tons of fuel and generated $443 \mathrm{kgal}$ of waste, all of which was placed into $\mathrm{C}-102$. The second campaign ran in 1970 and involved 390 tons of fuel at a very high burnup of 1,606 MWD/ton, generated $912 \mathrm{kgal}$ of waste, and was all placed into C-104. All of the Pu values in (App. B) for $\mathrm{TH} 1$ and $\mathrm{TH} 2$ wastes are actually for $\mathrm{U}-233$, not $\mathrm{Pu}$, while the $\mathrm{U}$ concentrations refer to Th-232, not $U-238$, the normal isotope of uranium. The details of these thorium campaigns are not clear enough to discern the amount of fission products present within each fuel. Therefore, the fission products are added in the same manner as with other HLW. 

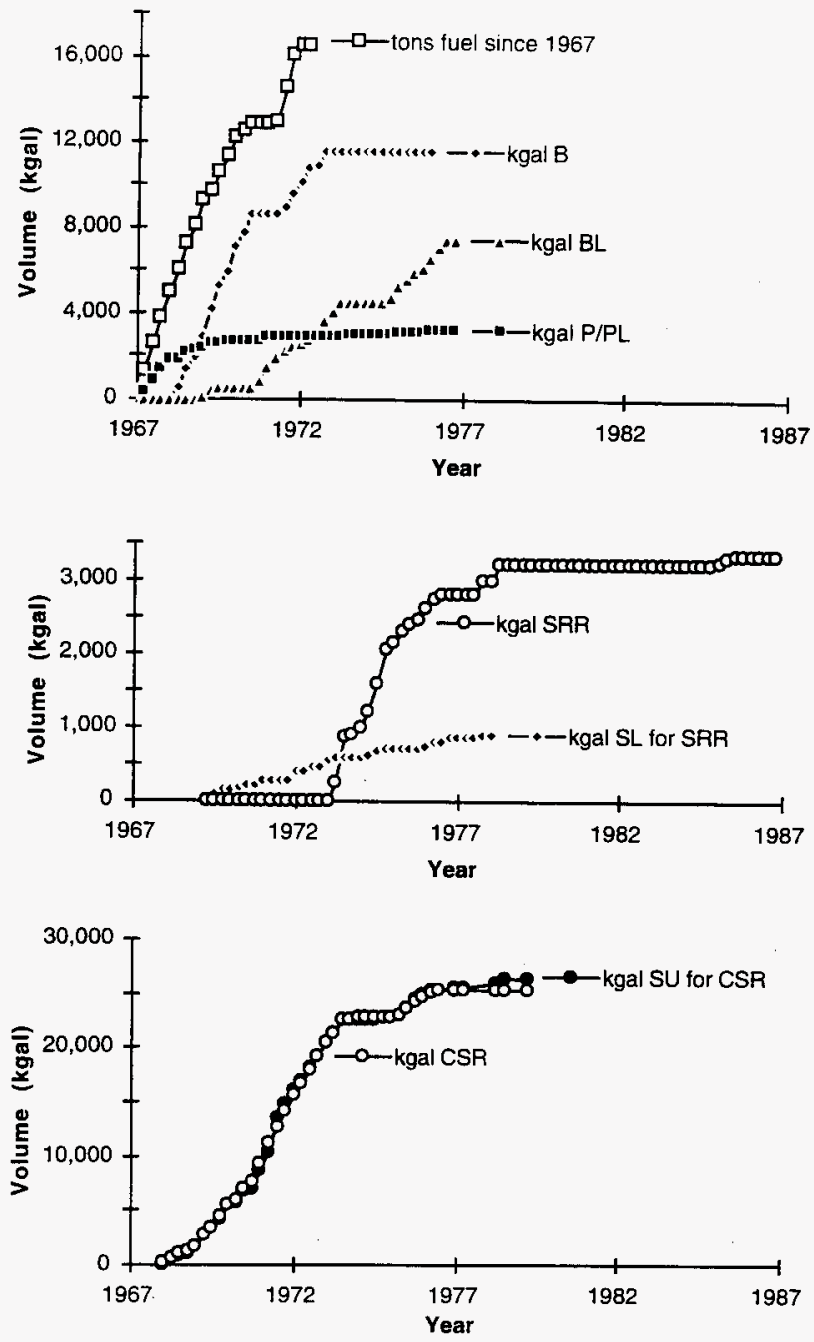

Fig. 17. Total waste volumes for B Plant campaign. 
This leaves $0.9 \mathrm{MCi}$ of $\mathrm{Sr}-90$ into $\mathrm{C}-104$, which increases this tanks heating by a factor of three or four. The predicted heat load based on this amount of $\mathrm{Sr}-90$ is $31,000 \mathrm{Btu} / \mathrm{hr}$ as opposed to the predicted $8,000 \mathrm{Btu} / \mathrm{hr}$ based on tank temperatures. At this time, we do not understand this discrepancy.

The volume per cent solids for these thoria wastes is uncertain, since such small amounts were generated. Therefore, a nominal value of 5.8 vol\% is assumed, which corresponds to a void fraction of 0.63 in the precipitated sludge layer.

\title{
-Purex to Z Plant
}

Estimates for $Z$ Plant wastes are complicated by the fact that the two receiver tanks, SY-102 and $T X-118$, were both evaporator feed tanks and therefore the $Z$ waste was co-mingled with precipitated salt accumulations from recycle additions to these feed tanks. Some $1,910 \mathrm{kgal}$ of $Z$ waste was added to TX118 from $1973-76$ and during this time, TX-118 was an active feed tank for 242-T evaporator. Therefore, it is likely that any sludge from the $Z$ waste would have been distributed around the TX Farm bottoms receivers.

From $1981-88,1,656 \mathrm{kgal} Z$ waste was added to SY-102. During that time, $S Y-102$ was no longer an active feed tank for $242-S$, but only a cross site supernatant transfer tank for to the $242-A$ evaporator feed tank, AW-102. Thus, the sludge that accumulated in SY-102 from Z waste largely still remains within this tank. However, a bottom remnant of accumulated salts still remains in $S Y-102$ from the recycle additions during the 1976-80 242-S operation. The addition of this dilute $Z$ waste evidently eroded or redissolved a substantial layer of this precipitated salt as well as mingling the $Z$ sludge with that salt layer.

\section{-Diatomaceous earth}

Diatomaceous earth is a highly effective and inexpensive absorbent and was used in six tanks at Hanford in an attempt to sequester residual liquids within those tanks. These tanks are BX-102, SX-113, U-104, TX-116, TX-117, and TY-106. We have used a reported ${ }^{29}$ composition and density to establish the composition of DE layer, which is included within the TLM. This reference suggests that the DE is $0.651 \mathrm{kgal} /$ ton (packed) and composed aimost entirely of silica, $\left(\mathrm{SiO}_{2}\right)$, with some minor amounts of $\mathrm{Al}$, $\mathrm{Fe}$, and $\mathrm{Ca}$. However, we have included two equivalents of $\mathrm{NaOH}$ in the composition, since our model assumes the form of silica in all tanks is waterglass, $\mathrm{Na}_{2} \mathrm{SiO}_{3}$. Note that the authors of the diatomaceous earth report also found that after two years, the form of silica within each of this tanks converted from $\mathrm{SiO}_{2}$ to waterglass and some small amount of cancrinite, $\mathrm{NaAlSiO}_{4}$.

\section{- Cement}

We have used a composition for Type 1 Portland cement ${ }^{30}$ to represent the 63 tons of cement that was added to BY-105 in 1972 . This cement was added in an attempt to sequester the residual fluids within BY-105 tank, which was a suspected leaker. Evidently, the cement did not set in the high-caustic, high-salt liquid and no further additions of cement were made to this or any other tank. Type 1 Portland cement is $46 \mathrm{wt} \% \mathrm{Ca}, 10 \mathrm{wt} \% \mathrm{Si}$, with the balance being oxygen, $\mathrm{Al}, \mathrm{Fe}, \mathrm{Ca}, \mathrm{Mg}$, sulfate, and water. Since the basic constituent of cement is calcium silicate, $\left(\mathrm{CaSiO}_{3}\right)$, we are able to adapt it to our composition vectors. We assume that the cement was added with a specific volume of $0.13 \mathrm{kgal} / \mathrm{ton}$, for a total amount of $8 \mathrm{kgal}$ added to BY-105. As far as we know, this is the only addition of cement to any tank at Hanford.

\author{
-Other wastes: \\ $\mathrm{N} \quad$ phosphate waste from N-Reactor decontamination \\ DW various decontamination wastes, mainly from T Plant.
}

\footnotetext{
${ }^{29}$ Buckingham, J. S.; Metz, W. P. "Characterization of the Effects of Diatomaceous Earth Additions to Hanford Tanks," WHC-MR-0302 (ARH-CD-222), Dec. 1974.

30 Heimuth, R. A.; Milier, F. M.; O'Conner, T. R.; Greening, N.R. "Cement," Encyclopedia of Composite Materials and Components, M. Grayson, Ed., 1983, p. 273.
} 
Salt Siury estimated from a chemical model by precipitation of soluble salts following concentration via evaporator. DSS derives from the supernatants of a variety of wastes following evaporation of water.

For certain evaporator campaigns, an average blend is derived, concentrated, and redistributed among the bottoms receivers as salt cake and supernatant. These wastes are:

$$
\begin{aligned}
& \text { BSItCk } \\
& \text { T1SItCk } \\
& \text { RSItCk } \\
& \text { BYSitCk }
\end{aligned}
$$

Other blended concentrates are listed in the HDW rev. 2, but not used as an HDW in the inventory estimates. The SMM keeps track of all of later concentrates on a tank by tank basis. (These were used in HDW Rev. 1.)

$$
\begin{aligned}
& \text { T2SitCk } \\
& \text { S1SltCk } \\
& \text { S2SItslr } \\
& \text { A1SitCk } \\
& \text { A2Sitslr } \\
& \text { BP/Cplx Complexant waste from B Plant 1981-8, assigned SRR. }
\end{aligned}
$$$$
\text { BP/NCplx non-complexant waste from B Plant 1981-8, assigned BL. }
$$

There are various other waste designations that appear in Hanford documentation. Here is a list and to what they have been assigned.

$\begin{array}{ll}\text { LW, HLO, BNW various lab wastes, assigned to water } \\ \text { CSKW } & \text { don't know what this is yet, assigned to water } \\ \text { CARB } & \text { same as OWW55-62 } \\ \text { EB } & \text { same as salt cakes or SMM concentrates } \\ \text { folded into salt cakes, same as CSR } \\ \text { NCPL } & \text { not actually a waste type } \\ \text { TL } & \text { Terminal Liquor, same as DSS } \\ \text { HDRL } & \text { Hanford Defense Residual Liquor, same as DSS } \\ \text { RESD } & \text { residual liquor, can be same as DSS } \\ \text { RIX } & \text { Redox ion exchange, same as CSR } \\ \text { RSN } & \text { same as supernatant from R, sent to CSR } \\ \text { SIX } & \text { S Plant ion exchange, same as RIX, assigned to CSR } \\ \text { SRS } & \text { Sr Solids, same as AR solids }\end{array}$

DSS

DSSF

CPLX

$\mathrm{CC}$

\section{Methodology}

\section{- Radionuclide inventories}

The radionuclide sources terms are those published ${ }^{31}$ as a result of an ORIGEN2 calculation for each of $\sim 1,400$ fuel batches that have been processed at Hanford in the past. This calculation included some extraction and other processing as a secondary model termed DKPRO. In particular, the partitioning of Am-241 is somewhat complicated by the fact that its primary parent is Pu-241. Therefore, Am-241 in waste derives from both Am-241 present in the fuel being processed as well as the Pu-241 residue in the waste at the time of processing. Most of the Pu-241 (T1/2 =14 year) isotope, which is the primary parent of Am-241, was extracted by the process and passed on to other facilities within the Weapons Complex. Therefore, only the residual Pu-241 within the waste that has subsequently

\footnotetext{
31 Watrous, R.A.; Schmitroth, F. "Radionuclide Sources Term for Hanford Site," in preparation.
} 
decayed to Am-241 (along with Am-241 originally present in the waste) now constitute the Am-241 total in waste tanks.

The starting point in the ORIGEN2 calculation are the tons of fuel processed in each fuel batch and the MWD's exposure for that fuel. Radionuclides associated with certain campaign periods are then summed as per the HDW campaigns shown in App. B with various reprocessing factors included in App. $B$, which show how various radionuclides partitioned within each of the processes.

One difficulty in assigning radionuclides to various HDW's is the fact that two large waste reprocessing operations occurred at Hanford. The radionuclides in $\mathrm{BiPO} 4$ waste were partitioned among MW1, MW2, 1C1, 1C2, 2C1, 2C2, and 224 HDW's, where $1 \mathrm{C}$ waste by and large also contained the cladding waste radionuclides. The Uranium Recovery campaign, the first reprocessing, then retrieved MW sludge and supernatant from the BiPO4 process and produced a new set of wastes as a result.

The radionuclides in MW were then distributed among the MW remnants as well as the Uranium Recovery campaign HDW's: UR, PFeCN1, PFeCN2, TFeCN, and $1 \mathrm{CFeCN}$. Further complicating this partitioning is the fact that TFeCN was actually derived from UA waste supernatants, i.e. it represents yet another reprocessing step.

The second major reprocessing was the sluicing of Purex sludges in A and AX Farms, their dissolution in AR Vault and the further extraction of Sr-90 and Cs-137 in B Plant. Thus, the radionuclides from Purex operation were passed on to these reprocessing streams and were partitioned into CSR, $B$, $\mathrm{BL}, \mathrm{AR}$, and SRR wastes as shown in the partitioning table. The original Purex process also produced PL, PL2, OWW1, OWW2, OWW3, CWR1, CWR2, CWZr1, and CWZr2. These wastes also received some share of the original waste radionuclides.

The HDW model does not partition any radionuclides to OWW1, OWW2, and OWW3 (organic wash wastes). Since these wastes were nearly always combined with $\mathrm{P1}$ and $\mathrm{P2}$ wastes in the tanks, this does not result in a very large error. In contrast, during the '83-'88 Purex campaign, the organic wash waste termed PL2, was kept segregated from P3. The HDW does therefore partition $\mathrm{Pu}$ and $\mathrm{U}$ to this waste as shown in the radionuclide partition table in App. B..

Plutonium losses to waste increased dramatically for the Zirflex process. While the Pu losses to cladding and HAW were each about $0.4 \%$, totaling roughly $1.0 \%$ for aluminum clad fuel, the total losses for the Zirlex process were around $3.0 \%$. The HDW model assumes a $1 \%$ loss to each of $\mathrm{P3}, \mathrm{CWZ} \mathrm{r} 1$, CWZr2, and PL2 for the Pu processed with Zirflex. This loss suggests that the sludge in AZ-101 should be on the order of $12 \mu \mathrm{Ci} / \mathrm{g}$ Pu-239/240, whereas limited sample results suggest only 2-4 $\mu \mathrm{Ci} / \mathrm{g}$ (Rapko, Lametta and Wagner, Sept. 1996, show $6.1 \mu \mathrm{Ci} / \mathrm{g}$ dry, $\mathrm{HDW}$ is $55 \mathrm{wt} \%$ water yielding $2.7 \mu \mathrm{Ci} / \mathrm{g}$ wet). If these sample results are representative of the mean sludge value, it would suggest much lower $\mathrm{Pu}$ tosses to waste than $1 \%$.

\begin{tabular}{c|c|c}
\hline \multicolumn{2}{c}{ Table 4. Plutonium (fuel slug) Loss During Decladding. } \\
\hline Waste & Pu \% loss & source \\
\hline BiPO4-CW & 0.4 & assumed \\
\hline & & 1965 flowsheet \\
\hline CWR & 0.4 & \\
\hline CWP & 0.4 & assumed \\
\hline CWZr1 and CWZr2 & 1.0 & assumed
\end{tabular}

\section{-Deriving solids volume percent}

It is necessary to derive or assign a characteristic solids or sludge volume per cent for each waste type. This is done by one of two means. First, we attempt to use the fill and solids volume histories of various tanks to derive a characteristic solids vol\% for a given waste type. Following the reported solids volumes for those tanks as a function of total primary waste added, then, gives a vol\% solids in a straightforward manner as shown in (Tables 5a-f). 
For waste types with insufficient solids information, we begin the TLM analysis with a nominal vol\% solids for those waste types and at the end of the analysis, produce a total solids for those waste types which is usually distributed among several tanks. This total solids value then forms the basis of an adjusted solids vol\%, and we repeat the analysis until the values converge producing our best estimate for the solids vol\%. The wastes AR, HS, B, BL, and SRR all have very small solids remnants distributed among a handful of tanks. The resultant solids vol\% has a greater uncertainty for these waste types.

The solids vol\% is a very important parameter, since it determines the sludge void fraction and therefore the amount of interstitial liquid within each waste sludge. It also bounds the amount of precipitated solids, since it is very unlikely that a sludge will have a void traction any less than about 0.30 0.40 .

Certain wastes, DW, N, OWW1, OWW2, OWW3, and CSR have no solids by definition. No solids are precipitated in these waste streams and therefore all their material is carried by their supernatants into concentrate receivers as determined by the SMM.

\begin{tabular}{|c|c|c|c|c|c|c|c|c|}
\hline $\tan k$ & start & $q t r$ & end & qtr. & waste type & pri.vol. & acc.sol. & vol\% \\
\hline B-107 & 1945 & 2 & 1946 & 2 & $1 \mathrm{C}$ & 1590 & 220 & 13.8 \\
\hline C-110 & 1946 & 2 & 1947 & 4 & $1 \mathrm{C}$ & 1589 & 231 & 14.5 \\
\hline $\bar{T}-107$ & 1945 & 1 & 1947 & 4 & 10 & 1590 & 201 & 12.6 \\
\hline avg. & 1945 & 1 & 1947 & 4 & $1 \mathrm{C}$ & 4769 & 652 & 13.7 \\
\hline & & & & & & & & \\
\hline$\overline{B X-107}$ & 1948 & 3 & 1951 & 2 & $1 \mathrm{C}$ & 1590 & 437 & 27.5 \\
\hline $\mathrm{C}-107$ & 1947 & 1 & 1947 & 4 & $1 \mathrm{C}$ & 1588 & 399 & 25.1 \\
\hline$T X-109$ & 1949 & 1 & 1950 & 2 & $1 \mathrm{C}$ & 3032 & 722 & 23.8 \\
\hline $\mathrm{U}-110$ & 1946 & 3 & 1951 & 1 & $1 \mathrm{C}$ & 1394 & 336 & 24.1 \\
\hline avg. & 1947 & 1 & 1951 & 2 & $1 \mathrm{C}$ & 7604 & 1894 & 24.9 \\
\hline
\end{tabular}

\section{Table 5b. Redox Solids.}

\begin{tabular}{|c|c|c|c|c|c|c|c|c|}
\hline $\operatorname{tank} n$ & year & qtr & $\begin{array}{c}\text { lineal } \\
\text { date }\end{array}$ & $\begin{array}{l}\text { kgal } \\
\text { CWR }\end{array}$ & $\begin{array}{c}\text { kgal } \\
\text { Redox }\end{array}$ & $\begin{array}{l}\text { Acc. } \\
\text { solids }\end{array}$ & vol\% & comments \\
\hline SX-105 & 1955 & 2 & & & 961 & 43 & 4.5 & \\
\hline \multirow[t]{3}{*}{ SX-111 } & 1956 & 2 & & & 963 & 41 & 4.3 & \\
\hline & & & & & & & & \\
\hline & & & & & & & 4.4 & $4.5 \mathrm{R} 1$ \\
\hline SX-110 & 1966 & 2 & 1966.3 & & 1621 & 62 & 3.8 & \\
\hline $\mathrm{SX}-113$ & 1958 & 2 & 1958.3 & & 487 & 10 & 2.1 & \\
\hline$S x-115$ & 1960 & 3 & 1960.5 & & 967 & 10 & 1.0 & \\
\hline & & & & & 3075 & 82 & 2.7 & $2.3 R 2$ \\
\hline
\end{tabular}


Table 5c. In Plant PFeCN/1 and PFeCN/2 Ferrocyanide Sludges.

\begin{tabular}{c|c|c|c|c|c|c}
\hline \hline & PFeCN/1 & PFeCN/2 & units & HDW totals & B\&S totals & units \\
\hline FeCN M & 0.005 & 0.0025 & $\mathrm{M}$ & & & \\
\hline pri. vol. & 10901 & 22460 & $\mathrm{kgal}$ & 33361 & 33861 & $\mathrm{kgal}$ \\
\hline acc.sed. & 403 & 718 & $\mathrm{kgal}$ & 1115 & 1393 & $\mathrm{kgal}$ \\
\hline vol\% sed. & 3.7 & 3.2 & $\mathrm{vol} \%$ & 3.4 & 4.1 & $\mathrm{vol} \%$ \\
\hline FeCN sed. & 0.14 & 0.078 & $\mathrm{M}$ & & & \\
\hline density & 1.45 & 1.45 & $\mathrm{~g} / \mathrm{cm} 3$ & & & \\
\hline $\begin{array}{c}\text { pred. wet } \\
\text { exotherm }\end{array}$ & 42 & 24 & $\mathrm{cal} / \mathrm{g}$ & & & \\
\hline $\begin{array}{c}\text { pred. dry } \\
\text { exotherm }\end{array}$ & 106 & 61 & $\mathrm{cal} / \mathrm{g}$ & & &
\end{tabular}

\begin{tabular}{c|c|c|c|c}
\hline \multicolumn{4}{|c}{ Table 5d. In-Tank (or in-farm) TFeCN Waste vol\% Solids. } \\
\hline \hline waste type & tank & $\begin{array}{c}\text { primary } \\
\text { volume }\end{array}$ & $\begin{array}{c}\text { accumul. } \\
\text { solids }\end{array}$ & vol\% solids \\
\hline TFeCN & $\mathrm{C}-108$ & 1034 & 15 & 1.5 \\
\hline & $\mathrm{C}-109$ & 2954 & 44 & 1.5 \\
\hline & $\mathrm{C}-111$ & 2732 & 35 & 1.3 \\
\hline & $\mathrm{C}-112$ & 4442 & 67 & 1.5 \\
\hline TFeCN & & & & 1.4 \\
\hline
\end{tabular}

\begin{tabular}{c|c|c|c|c|c|c|c|c}
\hline \multicolumn{10}{c}{ Table 5e. } & \multicolumn{1}{c}{ Purex P Waste vol\% Solids. } \\
\hline \hline tank & start & qtr. & end & qtr. & waste type & pri.vol. & acc.sol. & vol\% \\
\hline A-101 & 1956 & 1 & 1973 & 4 & P & 4545 & 83 & 1.8 \\
\hline A-102 & 1956 & 1 & 1961 & 3 & P & 7138 & 102 & 1.4 \\
\hline A-103 & 1956 & 2 & 1960 & 3 & P & 3813 & 102 & 2.7 \\
\hline A-104 & 1959 & 3 & 1961 & 4 & P & 6765 & 171 & 2.5 \\
\hline AX-104 & 1966 & 3 & 1969 & 2 & P & 1202 & 47 & 3.9 \\
\hline & & & & & & & & \\
\hline avg. & 1956 & 1 & 1973 & 4 & P & $\mathbf{2 3 4 6 3}$ & $\mathbf{5 0 5}$ & $\mathbf{2 . 2}$ \\
\hline & & & & & & & & \\
\hline A-106 & 1960 & 4 & 1962 & 2 & P & 1460 & 118 & 8.1 \\
\hline AX-101 & 1968 & 2 & 1969 & 2 & P & 40 & $? ?$ & $? ?$ \\
\hline AY-101 & 1971 & 2 & 1971 & 4 & P & 14 & $? ?$ & $? ?$ \\
\hline C-104 & 1970 & 4 & 1976 & 2 & P & 91 & $? ?$ & $? ?$
\end{tabular}




\begin{tabular}{|c|c|c|c|c|c|c|c|c|}
\hline \multicolumn{2}{|c|}{ Table $5 f}$. & \multicolumn{4}{|c|}{ Purex Cladding Waste (CWP) } & \multicolumn{2}{|c|}{ Waste vol\% Solids. } & \multirow[b]{2}{*}{ vol\% } \\
\hline $\operatorname{tank}$ & start & $q$ tr & end & qtr & waste type & pri.vol. & acc.sol. & \\
\hline C-101 & 1960 & 4 & 1962 & 2 & CWP/AI & 660 & 56 & 8.5 \\
\hline$C-103$ & 1960 & 2 & 1960 & 4 & CWP/Al & 479 & 35 & 7.3 \\
\hline C-104 & 1956 & 1 & 1957 & 2 & CWP/Al & 1118 & 90 & 8.1 \\
\hline C.105 & 1957 & 3 & 1960 & 2 & CWP/AI & 3130 & 262 & 8.4 \\
\hline C-106 & 1958 & 2 & 1960 & 2 & CWP/AI & 420 & 28 & 6.7 \\
\hline avg. & 1956 & 1 & 1965 & 2 & CWP/AI & 5807 & 471 & 8.1 \\
\hline C-102 & 1960 & 3 & 1965 & 2 & CWP/Al & 5355 & 184 & 3.4 \\
\hline C.104 & 1969 & $\overline{4}$ & 1970 & 1 & $\mathrm{CWP} Z \mathrm{Zr}$ & 535 & & \\
\hline C-104 & 1970 & 2 & 1972 & 3 & CWP/AI & 3816 & 108 & 2.5 \\
\hline C-102 & 1965 & 3 & 1969 & 4 & CWP/AI\&Zr & 6448 & $?$ & ?? \\
\hline C.107 & 1961 & 3 & 1962 & 2 & CWP/Al & 1364 & $? ?$ & ? \\
\hline C.108 & 1961 & 2 & 1961 & 2 & CWPIAl & 502 & $?$ & ?? \\
\hline C-111 & 1957 & 1 & 1960 & 4 & CWP/AI & 347 & $?$ & $? ?$ \\
\hline C-112 & 1960 & 3 & 1961 & 2 & CWP/AI & 254 & $?$ & $? ?$ \\
\hline
\end{tabular}

-Precipitation of solids

The solids that precipitate in each waste is set by adjusting the fraction precipitated parameter so that the solubility of that component falls within the correct range. That range is set for each component by an analysis of data from supernatant samples from the tank farm and evaporator operations. By plotting the concentrations of species that have been measured for tank supernatants, we obtain a limiting solubility ${ }^{32}$ of a species as well as its range of solubility. These values provide the method by which we partition the solids in the waste into supernatant and sludge fractions. However, the concentration of those solids in the sludge layer is dependent on the solids volume per cent for that waste as well. The concentration of each component in the sludge depends on a combination of three factors - concentration of precipitated solids, concentration of supernatant, and volume per cent solids.

Aluminum is a special case and is precipitated in two stages. We assume that during neutralization, a set fraction of the aluminum precipitates as aluminum oxy/hydroxide before the soluble aluminum ends up as $\mathrm{Al}(\mathrm{OH})_{4}-$ in solution. Therefore, the fraction of aluminum precipitated as oxide is adjusted in our model, both to produce reasonable void fractions $(0.6$ to 0.7$)$ in the precipitate and to correspond to sludge analyses for those waste types. The aluminum remains in solution as aluminate and only precipitates when the aluminate solubility limit is reached see Solubility Limits (Table 7). This occurs following concentration of waste as a result of evaporator operation.

Jungfleisch-84 referenced a report by Barney ${ }^{33}$ that said below $1.6 \mathrm{M}$ hydroxide, $\mathrm{Al}(\mathrm{OH})_{3}$ precipitates, while above $1.6 \mathrm{M}$ hydroxide, sodium aluminate precipitates. Later work reported ${ }^{34}$ that, for the range 2.0-6.5 M hydroxide, the aluminate solubility decreased as the square of the hydroxide molarity (with no correction for activity). Aluminate solubility in this report ranged from a high of $2.3 \mathrm{M}$ with $2 \mathrm{M}$ hydroxide to a low of $-0.9 \mathrm{M}$ with $6 \mathrm{M}$ hydroxide. This suggests that while $\mathrm{Al}(\mathrm{OH})_{3}$ precipitates at neutral $\mathrm{pH}$, as hydroxide increases, aluminate solubility peaks at hydroxide concentrations between 1.6 and 2.0

${ }^{32}$ Agnew, S. F. and Watkin, J. G. "Estimation of Limiting Solubilities for lonic Species in Hanford Waste Tank Supernates," LA-UR-94-3590, October 1994.

${ }^{33}$ Barney. G. S. "Vapor-Liquid Solids Phase Equilibrium of Radioactive Sodium Salt Waste at Hanford," ARH-ST133, January 1976.

${ }^{34}$ Reynolds, D. A.; Herting, D. L. "Solubilities of Sodium Nitrate, Sodium Nitrite, and Sodium Aluminate in Simulated Nuclear Waste," RHO-RE-ST-14P, May 1984. 
M. At its maximum, then, aluminate concentration lies between 2.0 and $2.5 \mathrm{M}$. But note that as aluminate precipitates, one equivalent of sodium hydroxide also precipitates. Therefore, as the solution is concentrated and the aluminate solubility limit is reached, the hydroxide and sodium concentrations are butfered at that concentration by the aluminate.

These rules for aluminum are necessary since we are often lacking the exact details associated with the waste neutralization process for each of the waste types. Depending on the rate of addition, the stirring time, the excess hydroxide, and so on, very different fractions of the aluminum will precipitate as oxyhydroxides. The wastes that are most affected by this rule are $1 \mathrm{C}, \mathrm{R}, \mathrm{CWR}$, and CWP.

Once the solid volume per cent is derived, we use it along with the precipitated solids to calculate the void fraction for a given sludge. The composition of each sludge, then, is a combination of precipitated solids and interstitial liquid, while the composition of the supernatant is simply what remains in solution. The supernatant and the interstitial liquid are one and the same at the time of precipitation. After the sludge is placed into a tank, we assume that the interstitial liquid remembers what it was, even if the supernatant layer has been altered by later waste additions or removals.

The solubility limit for Sr-90 in SRR and CSR was increased from 0.034 to $0.091 \mathrm{Ci} / \mathrm{L}$. This was done because analyses of complexant waste tanks clearly show greater solubility for a number of cations, including $\mathrm{Sr}-90$. Such an increase in $\mathrm{Sr}-90$ solubility is also consistent with the heat distribution in tanks with SRR sludge. 


\begin{tabular}{|c|c|c|c|c|}
\hline \multirow{3}{*}{$\begin{array}{l}\text { chemicals } \\
\text { added } \\
\text { HNO3 }\end{array}$} & \multirow{3}{*}{\begin{tabular}{|l|}
$\begin{array}{l}\text { defined } \\
\text { precipitates }\end{array}$ \\
NaNO3
\end{tabular}} & \multirow[t]{3}{*}{ OH'S } & \multirow[t]{2}{*}{ H2O's } & \multirow{2}{*}{ comments } \\
\hline & & & & \\
\hline & & & 0 & $\begin{array}{l}\text { Sodium nitrate precipitates as a result of evaporator } \\
\text { concentration of neutralized wastes. }\end{array}$ \\
\hline \multirow[t]{2}{*}{$\begin{array}{l}\mathrm{Al}(\mathrm{NO} 3)_{3} \\
\mathrm{NaAlO2}\end{array}$} & $(\mathrm{Al} 2 \mathrm{O} 3.3 \mathrm{H} 2 \mathrm{O}) / 2$ & 1.5 & $\overline{3}$ & $\begin{array}{l}\text { A set fraction of aluminum always precipitates as } \\
\text { oxide upon neutralization, generally about } 7 \% \text {, but } \\
\text { as high as } 60 \% \text { for ciadding waste. }\end{array}$ \\
\hline & $\mathrm{NaAlO} 2$ & 4 & 0 & $\begin{array}{l}\text { Only after concentration does aluminate } \\
\text { precipitate. }\end{array}$ \\
\hline $\begin{array}{l}\mathrm{Fe}\left(\mathrm{HSO}_{4}\right)^{2} \\
\mathrm{Fe}(\mathrm{NO} 3)_{3}\end{array}$ & $\begin{array}{l}\mathrm{FeO}(\mathrm{OH}) \\
(\mathrm{Na} 2 \mathrm{SO} 4)\end{array}$ & 3 & 0.5 & $\begin{array}{l}\text { Iron is added as } \mathrm{Fe}(\mathrm{II}) \text { and } \mathrm{Fe}(\mathrm{III}) \text { but is precipitated } \\
\text { only as } \mathrm{Fe}(\mathrm{III}) \text {, producing hydrogen and hydroxide. } \\
\text { Note iron also in } \mathrm{FeCN} \text {. }\end{array}$ \\
\hline $\mathrm{Na2CrO4}$ & $\mathrm{Cr}(\mathrm{OH}) 3$ & 3 & 1.5 & $\begin{array}{l}\text { Chromium is added as VI, but precipitated as III, } \\
\text { consuming water. }\end{array}$ \\
\hline \multirow[t]{3}{*}{ BiPO4 } & $\mathrm{BiPO4}$ & & $\overline{0}$ & $\begin{array}{l}\text { Phosphate precipitates first as BiPO4, then as the } \\
\text { sodium } 12 \text { hydrate. After evaporator } \\
\text { concentration, the sodium } 10 \text { hydrate precipitates. }\end{array}$ \\
\hline & $\mathrm{Na3PO} 4.10 \mathrm{H} 2 \mathrm{O}$ & & 10 & \\
\hline & $\mathrm{Na} 3 \mathrm{PO} 4.12 \mathrm{H} 2 \mathrm{O}$ & & 12 & \\
\hline ZrO(OH)2 & $\mathrm{ZrO}(\mathrm{OH}) 2$ & & $\overline{2}$ & $\begin{array}{l}\text { Zirconium actually derives from Zirconium alloy } \\
\text { cladding. }\end{array}$ \\
\hline \multirow[t]{2}{*}{ NISO4 } & $\mathrm{Ni}(\mathrm{OH}) 2$ & 2 & 1 & $\begin{array}{l}\text { Nickel first precipitated as ferrocyanide, then as } \\
\text { hydroxide. }\end{array}$ \\
\hline & $\begin{array}{l}\mathrm{Na} 2 \mathrm{NiFe}(\mathrm{CN}) \cdot 6.6 \mathrm{H} \\
2 \mathrm{O}\end{array}$ & & 6.6 & \\
\hline $\mathrm{NaOH}$ & & & 0.5 & Not precipitated. \\
\hline NaNO2 & NaNO2 & & 0 & $\begin{array}{l}\text { Precipitates as a result of evaporator } \\
\text { concentration. }\end{array}$ \\
\hline \multirow[t]{2}{*}{$\mathrm{Na2CO3}$} & $\mathrm{Na2CO} 3.7 \mathrm{H} 2 \mathrm{O}$ & & 7 & $\begin{array}{l}\text { Only the sodium seven and calcium six hydrates } \\
\text { precipitate in this model. }\end{array}$ \\
\hline & $\mathrm{CaCO} 3.6 \mathrm{H} 2 \mathrm{O}$ & & 6 & \\
\hline \multirow[t]{3}{*}{$\mathrm{Na3PO4}$} & $\mathrm{BiPO4}$ & & 0 & See BiPO4 for phosphate details. \\
\hline & $\mathrm{Na3PO} 4.10 \mathrm{H} 2 \mathrm{O}$ & & 10 & $\begin{array}{l}\mathrm{Na} \text { is used as surrogate for whatever cation actually } \\
\text { precipitates. }\end{array}$ \\
\hline & $\mathrm{Na3PO} 4.12 \mathrm{H} 2 \mathrm{O}$ & & 12 & $\begin{array}{l}\mathrm{Na} \text { is used as surrogate for whatever cation actually } \\
\text { precipitates. }\end{array}$ \\
\hline $\mathrm{Na2SO}_{4}$ & $\mathrm{Na} 2 \mathrm{SO} 4$ & & 10 & $\begin{array}{l}\text { Even though metathesis is likely, } \mathrm{Na} \text { is used as } \\
\text { cation surrogate. }\end{array}$ \\
\hline $\mathrm{Na2SiO3}$ & $\mathrm{Na2SiO3}$ & 6 & 0 & Once again, $\mathrm{Na}$ is used as surrogate for cation. \\
\hline Na2SiF6 & $\begin{array}{l}\mathrm{Na2SiO3} \\
\mathrm{NaF}\end{array}$ & & 0 & Assume all SiF hydrolyzes to silicate. \\
\hline NaF & $\mathrm{NaF}$ & & 0 & Use Na as cation surrogate. \\
\hline $\mathrm{NaCl}$ & $\mathrm{NaCl}$ & & 0 & Not precipitated. \\
\hline La(NO3)3 & LaF3 & & 0 & Precipitated as La2O3 "224" waste. \\
\hline NH3 & & & 0 & Not precipitated. \\
\hline
\end{tabular}




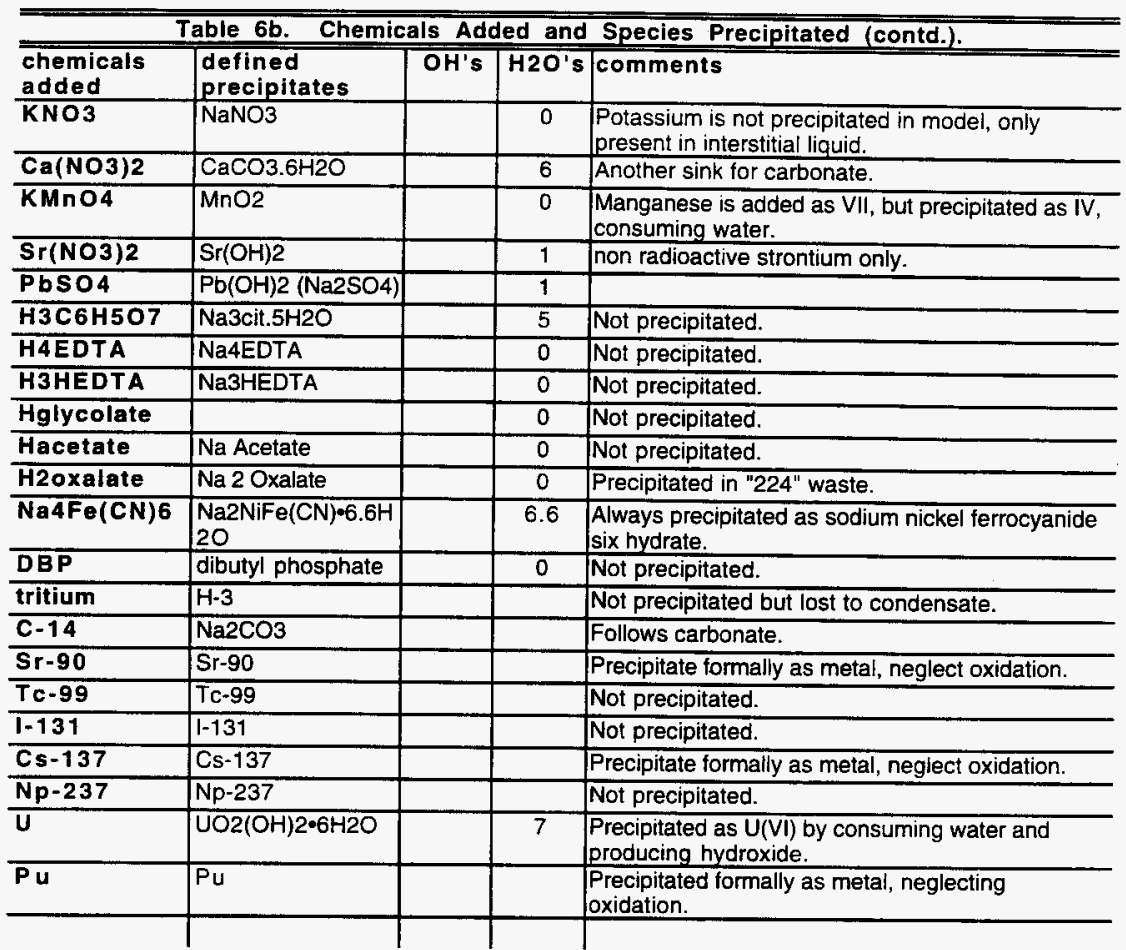




\begin{tabular}{|c|c|c|c|c|c|c|c|c|}
\hline solids & nota & sol. limit & & rsd & $-1 \mathrm{rsd}$ & $1 \mathrm{rsd}$ & $-1.96 \mathrm{rsd}$ & 1.96 \\
\hline NaNO3 & 1 & 2.8 & $\bar{M}$ & $9.69 \%$ & $2.53 \mathrm{E}+00$ & $3.07 \mathrm{E}+00$ & $2.27 \mathrm{E}+00$ & $3.33 \bar{E}+00$ \\
\hline $\mathrm{NaNO} 2$ & 1 & 3.1 & M & $8.75 \%$ & $2.83 E+00$ & $3.37 E+00$ & $2.57 E+00$ & $3.63 E+00$ \\
\hline $\mathrm{NaCl}$ & & & & & & & & \\
\hline $\mathrm{NaF}$ & 2 & 0.24 & $M$ & $16.13 \%$ & 2.01E-01 & 2.79E-01 & $1.64 \mathrm{E}-01$ & $3.16 \mathrm{E}-01$ \\
\hline $\mathrm{Sr}(\mathrm{OH}) 2$ & 2,7 & 0.002 & $\bar{M}$ & $5.11 \%$ & $1.90 \mathrm{E}-03$ & $2.10 \mathrm{E}-03$ & $1.80 \mathrm{E}-03$ & $2.20 \mathrm{E}-03$ \\
\hline $\mathrm{Na} 2 \mathrm{CO} 3$ & 2 & 0.4 & M & $6.07 \%$ & $3.76 \mathrm{E}-01$ & 4.24E-01 & $3.52 \mathrm{E}-01$ & $4.48 \mathrm{E}-01$ \\
\hline $\mathrm{Na3PO} 4$ & 2 & 0.15 & M & $7.75 \%$ & $1.38 \mathrm{E}-01$ & $1.62 E-01$ & 1.27E-01 & $1.73 E-01$ \\
\hline $\mathrm{Na} 2 \mathrm{SO}_{4}$ & 2 & 0.35 & $M$ & $10.87 \%$ & 3.12E-01 & $3.88 E-01$ & 2.75E-01 & $4.25 \mathrm{E}-01$ \\
\hline $\mathrm{Na2SiO3}$ & 2 & 0.034 & $\bar{M}$ & $25.22 \%$ & $2.54 \mathrm{E}-02$ & 4.26E-02 & $1.72 \mathrm{E}-02$ & $5.08 \mathrm{E}-02$ \\
\hline $\mathrm{NaAlO} 2$ & 1 & 1.6 & M & $3.77 \%$ & $1.54 E+00$ & $1.66 \mathrm{E}+00$ & $1.48 \mathrm{E}+00$ & $1.72 E+00$ \\
\hline $\mathrm{FeO}(\mathrm{OH})$ & 2,6 & 0.002 & M & $22.83 \%$ & $1.54 \mathrm{E}-03$ & $2.46 \mathrm{E}-03$ & $1.11 \mathrm{E}-03$ & $2.89 E-03$ \\
\hline $\mathrm{Cr}(\mathrm{OH}) 3$ & 3 & 0.12 & M & $21.93 \%$ & $9.37 \mathrm{E}-02$ & $1.46 \mathrm{E}-01$ & 6.84E-02 & 1.72E-01 \\
\hline $\mathrm{MnO} 2$ & 2 & 0.009 & $\bar{M}$ & $15.71 \%$ & $7.59 \mathrm{E}-03$ & 1.04E-02 & $6.23 E-03$ & $1.18 \mathrm{E}-02$ \\
\hline BiPO4 & 2 & 0.004 & M & $7.93 \%$ & $3.68 \mathrm{E}-03$ & $4.32 E-03$ & $3.38 \mathrm{E}-03$ & 4.62E-03 \\
\hline $\begin{array}{l}\mathrm{Pb}(\mathrm{OH}) 2 \\
\mathrm{La2O} 3\end{array}$ & 2 & $\begin{array}{r}0.0016 \\
0\end{array}$ & $\begin{array}{l}M \\
M\end{array}$ & $\%$ & $=-03$ & E-03 & E-04 & -03 \\
\hline $\mathrm{HgO}$ & 2,7 & 0.00001 & $\bar{M}$ & $5.11 \%$ & $9.49 E-06$ & $1.05 \mathrm{E}-05$ & $9.00 \mathrm{E}-06$ & $1.10 \mathrm{E}-05$ \\
\hline $\mathrm{Na} 2$ Oxalate & 4,7 & 0.005 & $M$ & $5.83 \%$ & $4.71 E-03$ & $5.29 \mathrm{E}-03$ & $4.43 \mathrm{E}-03$ & 5.57E-03 \\
\hline $\mathrm{CaCO} 3.6 \mathrm{H} 2 \mathrm{O}$ & 2 & 0.009 & M & $19.90 \%$ & $7.21 E-03$ & $1.08 E-02$ & $5.49 E-03$ & $1.25 \mathrm{E}-02$ \\
\hline $\mathrm{Ni}(\mathrm{OH}) 2$ & 2,7 & 0.0018 & M & $5.11 \%$ & $1.71 \mathrm{E}-03$ & $1.89 \mathrm{E}-03$ & 1.62E-03 & $1.98 \mathrm{E}-03$ \\
\hline $\mathrm{ZrO}(\mathrm{OH}) 2$ & 2 & 0.003 & $\bar{M}$ & $6.68 \%$ & $2.80 \mathrm{E}-03$ & $3.20 \mathrm{E}-03$ & $2.61 \mathrm{E}-03$ & $3.39 \mathrm{E}-03$ \\
\hline Co-60 & 5 & $1.00 \mathrm{E}-04$ & $\mathrm{Ci} / \mathrm{L}$ & $36.18 \%$ & $6.38 \mathrm{E}-05$ & 1.36E-04 & 2.91E-05 & 1.71E-04 \\
\hline $\mathrm{Se}-79$ & 5 & $2.00 E-06$ & $\mathrm{Ci} / \mathrm{L}$ & $36.18 \%$ & $1.28 \mathrm{E}-06$ & $2.72 \mathrm{E}-06$ & 5.82E-07 & 3.42E-06 \\
\hline Sr-90 & 2,6 & 0.034 & $\mathrm{Ci} / \mathrm{L}$ & $7.36 \%$ & $3.15 \mathrm{E}-02$ & $3.65 \mathrm{E}-02$ & 2.91E-02 & 3.89E-02 \\
\hline $\mathrm{Zr}-93$ & 5 & $1.00 \mathrm{E}-05$ & $\mathrm{Ci} / \mathrm{L}$ & $8 \%$ & $6.38 \mathrm{E}-06$ & 1.36E-05 & $2.91 \mathrm{E}-06$ & $1.71 \mathrm{E}-05$ \\
\hline $\mathrm{Nb} .93 \mathrm{~m}$ & 5 & $7.00 \mathrm{E}-06$ & $\mathrm{Ci} / \mathrm{L}$ & $18 \%$ & 4.47E-06 & 9.53E-06 & $2.04 E-06$ & 1.20E-05 \\
\hline$R u-106$ & 5 & $5.00 \mathrm{E}-09$ & $\mathrm{Ci} / \mathrm{L}$ & $8 \%$ & $3.19 \mathrm{E}-09$ & $6.81 \mathrm{E}-09$ & $1.45 \mathrm{E}-09$ & 8.55E-09 \\
\hline $\mathrm{Cd}-113 \mathrm{~m}$ & 5 & $6.00 \mathrm{E}-05$ & $\mathrm{Ci} / \mathrm{L}$ & $18 \%$ & 3.83E-05 & 8.17E-05 & $1.75 \mathrm{E}-05$ & 1.03E-04 \\
\hline $\mathrm{Sb}-125$ & 5 & $1.90 \mathrm{E}-02$ & $\mathrm{Ci} / \mathrm{L}$ & $36.18 \%$ & $1.21 \mathrm{E}-02$ & $2.59 E-02$ & 5.53E-03 & $3.25 \mathrm{E}-02$ \\
\hline Sn-126 & 5 & $3.00 \mathrm{E}-06$ & $\mathrm{ci} / \mathrm{L}$ & $18 \%$ & $1.91 E-06$ & 4.09E-06 & 8.73E-07 & $5.13 E-06$ \\
\hline Sm-151 & 5 & 0.007 & $\mathrm{Ci} / \mathrm{L}$ & $18 \%$ & $4.47 \mathrm{E}-03$ & EE-03 & $2.04 \mathrm{E}-03$ & $1.20 \mathrm{E}-02$ \\
\hline Eu- 154 & 5 & $5.00 \mathrm{E}-04$ & $\mathrm{Ci} / \mathrm{L}$ & $36.18 \%$ & $3.19 \mathrm{E}-04$ & $6.81 \mathrm{E}-04$ & 1.45E-04 & 8.55E-04 \\
\hline Ra-226 & 5 & $6.20 \mathrm{E}-11$ & $\mathrm{Ci} / \mathrm{L}$ & $36.18 \%$ & $3.96 \mathrm{E}-11$ & $8.44 \mathrm{E}-11$ & $1.80 \mathrm{E}-11$ & $1.06 \mathrm{E}-10$ \\
\hline AC- 227 & 5 & $3.60 \mathrm{E}-10$ & $\mathrm{Ci} / \mathrm{L}$ & $36.18 \%$ & $2.30 \mathrm{E}-10$ & $4.90 \mathrm{E}-10$ & 1.05E-10 & $6.15 \mathrm{E}-10$ \\
\hline $\mathrm{Pa}-231$ & 5 & $2.00 \mathrm{E}-09$ & $\mathrm{Ci} / \mathrm{L}$ & $8 \%$ & $1.28 \mathrm{E}-09$ & 2.72E-09 & $5.82 \mathrm{E}-10$ & $3.42 \mathrm{E}-09$ \\
\hline Th-232 & 5 & 7.00E-07 & $\mathrm{Ci} / \mathrm{L}$ & $36.18 \%$ & 4.47E-07 & 9.53E-07 & 2.04E-07 & 1.20E-06 \\
\hline U-Total & 2,7 & 0.004 & $M$ & $5.11 \%$ & $3.80 \mathrm{E}-03$ & $4.20 \mathrm{E}-03$ & $3.60 \mathrm{E}-03$ & $4.40 \mathrm{E}-03$ \\
\hline Pu-Total & 2,6 & $1.48 \mathrm{E}-04$ & $g / L$ & $36.18 \%$ & $9.45 E-05$ & $2.02 E-04$ & $4.31 \mathrm{E}-05$ & $2.53 E-04$ \\
\hline Am-241 & 2,6 & $3.00 \mathrm{E}-05$ & $\mathrm{Ci} / \mathrm{L}$ & $36.18 \%$ & $1.91 E-05$ & 4.09E-05 & $8.73 E-06$ & 5.13E-05 \\
\hline $\mathrm{Cm}-244$ & 5 & $1.00 \mathrm{E}-07$ & $\mathrm{Ci} / \mathrm{L}$ & $36.18 \%$ & $6.38 \mathrm{E}-08$ & 1.36E-07 & $2.91 \mathrm{E}-08$ & $1.71 E-07$ \\
\hline \multicolumn{9}{|c|}{$\begin{array}{l}\text { 1Solubility limit set by assays of concentrates (Ret. 32). Variabilities of nitrate and nitrite determined by variation of } \\
\text { upper quartile while variation of aluminate set by variation of Na. } \\
2 \text { Solubility limits set by solubility report (Ref. } 32 \text { ) and variabilities determined by upper quartile statistics. } \\
3 \text { Chromium solubitity set at four times value in Rev. } 3 \text {. This is consistent with chromium as chomate. } \\
4 \text { Oxalate set by S. Barney, "Solubilities of Organic Carbon" report, } 1996 \text {. } \\
5 \text { Solubility limit set by arbitrarily distributing radionuclide approximately } 50 \% \text { in sludges and } 50 \% \text { in concentrates, } \\
\text { while variabilities set by Pu. } \\
\text { 6) Solubilities increased by factor of four for SRR and CSR wastes to account for presence of complexants. } \\
\text { 7) Variabilites for cations based on hydroxide and anions on sodium, scaled as a solubility product. }\end{array}$} \\
\hline
\end{tabular}




\section{- Calculating density}

An equation derived from previous work to calculate the density of the supernatant is based on fitting the densities of a series of solutions reported by Herting and Reynolds ${ }^{35}$ with a minimal parameter set. This equation is:

$$
\text { density }\left(\mathrm{g} / \mathrm{cm}^{3}\right)=1+0.038 *\left[\mathrm{Na}^{+}\right]+0.07 *\left[\mathrm{Al}(\mathrm{OH})_{4}\right]-0.015 *\left[\text { free } \mathrm{OH}^{-}\right] \text {. }
$$

All concentrations are in mol $/ \mathrm{L}$ and are uncorrected for activity. This expression calculates density within $\pm 0.2 \mathrm{~g} / \mathrm{cm}^{3}$ for 400 of 400 analytical results.

An alternative caiculation for density that is much more accurate $\left( \pm 0.01 \mathrm{~g} / \mathrm{cm}^{3}\right)$ is as follows (all concentrations in $\mathrm{mol} / \mathrm{L}$ ):

$$
\begin{aligned}
\text { density }\left(\mathrm{g} / \mathrm{cm}^{3}\right) & =1+0.0206\left[\mathrm{Na}^{+}\right]-9.96 \mathrm{e}-4\left[\mathrm{Na}^{+}\right]^{2} \\
& +0.0794[\mathrm{Al}]-0.0200[\mathrm{Al}]^{2} \\
& +8.52 \mathrm{e}-4\left[\mathrm{OH}^{-}\right]+0.00404\left[\mathrm{OH}^{-}\right]^{2} \\
& +0.0394\left[\mathrm{NO}_{3}^{-}\right]+2 \mathrm{e}-4\left[\mathrm{NO}_{3}^{-}\right]^{2} \\
& +0.074\left[\mathrm{NO}_{2}^{-}\right]-0.0146\left[\mathrm{NO}_{2}^{-}\right]^{2}
\end{aligned}
$$

However, this expression comes with ten parameters as opposed to just three for the first expression. We will use the simpler expression for the HDW.

The density of the sludge phase is calculated using that of the interstitial liquid, its fraction, and each of the solid phases with their corresponding densities. The void fraction is calculated by summing the volumes of all of the solids that have precipitated and subtracting that from the solids volume per cent parameter for that waste type. Generally, void fractions in the range 0.2 to 0.8 are possible for sludges, with the range 0.4-0.7 most likely. Void fractions below this range are highly suspect, while those above the range imply an increasingly flocculant precipitate.

Examples of the spreadsheet calculation for aluminum in supernatant and sludge are shown as

$$
\begin{aligned}
& \text { SUAI }=\text { inAl * }\left(1-\text { frAIO2 +frAl2O3) } /\left(1-\left(1-\text { voidFr) }{ }^{*} \text { vol\%Solids } / 100\right)\right.\right. \\
& \text { sIAI }=\text { sIAIO2 + sIAI2O3 + suAI * voidFr }
\end{aligned}
$$

where
suAl
s|A|
frAl2O3
mol/L of $A l$ in supernatant
fr $\mathrm{AlO} 2$
$\mathrm{mol} / \mathrm{h}$ of $\mathrm{Al}$ in sludge
inAl
voidFr
vol\%Solids
SIAIO2
fraction of aluminum precipitated as $\mathrm{Al}_{2} \mathrm{O}_{3}$
SIAI2O3 fraction of aluminum precipitated as $\mathrm{NaAlO}_{2}$ $\mathrm{mol} / \mathrm{L}$ of total aluminum in waste stream liquid void fraction of sludge volume percent solids in waste stream $\mathrm{mol} / \mathrm{L}$ of $\mathrm{NaAlO}_{2}$ solids precipitated in sludge mol/L of aluminum in precipitated alumina solids, $\mathrm{Al}_{2} \mathrm{O}_{3}$.

In cases where there is tittle or no aluminum precipitate, the aluminum concentration in the supernatant is actually greater than that in the total waste stream. This occurs because of the excluded volume of the solids within the sludge layer reduces the total volume of supernatant in the waste, thereby increasing the concentration of all soluble species over that in the total waste stream. This volume exclusion induced increase in concentration is a part of the HDW model.

\footnotetext{
${ }^{35}$ Reynolds, D. A.; Herting, D. L. "Solubilities of Sodium Nitrate, Sodium Nitrite, and Sodium Aluminate in Simulated Nuclear Waste," RHO-RE-ST-14, May 1984.
} 


\section{-Ion balance calculation}

Ion balance is calculated for each HDW sludge and supernatant. The result is shown in the row labeled "balance." The ion balance for the sludge layers is calculated as:

$$
\begin{aligned}
& \text { sludge ion balance }=\mathrm{Na}+\mathrm{Al}^{*} 3+\mathrm{Fe}^{*} 3+\mathrm{Cr}^{*} 3+\mathrm{Bi}^{*} 3+\mathrm{La}^{*} 3+\mathrm{Hg}^{*} 2+\mathrm{Zr}^{*} 4 \\
&+\mathrm{Pb}^{*} 2+\mathrm{Ni}^{*} 2+\mathrm{Sr}^{*} 2+\mathrm{Mn}^{*} 4+\mathrm{Ca}^{*} 2+\mathrm{K}+\mathrm{U}^{*} 6 \\
&-\left[\mathrm{OH}(\text { total })+\mathrm{NO}_{3}+\mathrm{NO}_{2}+\mathrm{CO}_{3}^{* 2}+\mathrm{PO}_{4}^{*} 3+\mathrm{SO}_{4}^{* 2}+\mathrm{SiO}_{3}^{* 2}+\mathrm{F}+\mathrm{Cl}+\mathrm{C}_{6} \mathrm{H}_{5} \mathrm{O}_{7} * 3\right. \\
&\left.+\mathrm{EDTA}^{*} 4+\mathrm{HEDTA}^{*} 3+\text { glycolate+acetate+oxalate } 2+\mathrm{Fe}(\mathrm{CN})_{6}{ }^{*} 4\right]
\end{aligned}
$$

Note in particular that silicon is always counted as silicate, $\mathrm{SiO}_{3}{ }^{2-}$, zirconium is counted at the free $4+i o n$, not zirconyl, $\mathrm{ZrO}^{2-}$, aluminum is counted as the $3+$ cation, not as aluminate, and uranium is counted as $6+$, not uranyl, $\mathrm{UO}_{2}{ }^{2+}$. Therefore, the total hydroxide reported within the siudge includes hydroxides bound to all species except $\mathrm{Si}$. The free hydroxide value reponted for sludges is the hydroxide concentration associated with only the interstitial liquid.

The ion balance for the supernatant is calculated slightly differently since the hydroxide value in the supernatant does not include the hydroxide ion complexed to the aluminum. The supernatant ion balance is calculated as:

$$
\begin{aligned}
& \text { supernatant ion balance }=\mathrm{Na}-\mathrm{Al}+\mathrm{Fe}^{*} 3+\mathrm{Cr}^{*} 3+\mathrm{Bi}^{*} 3+\mathrm{La}^{*} 3+\mathrm{Hg}^{*} 2+\mathrm{Zr}^{*} 4 \\
& +\mathrm{Pb}^{*} 2+\mathrm{Ni}^{*} 2+\mathrm{Sr}^{*} 2+\mathrm{Mn}^{*} 4+\mathrm{Ca}^{*} 2+\mathrm{K}+\mathrm{U}^{*} 6 \\
& -\left[\mathrm{OH}+\mathrm{NO} 3+\mathrm{NO}_{2}+\mathrm{CO}^{*} 2+\mathrm{PO}_{4}^{*} 3+\mathrm{SO}_{4}^{*} 2+\mathrm{SiO}^{*} 2+\mathrm{F}+\mathrm{Cl}+\mathrm{C}_{6} \mathrm{H}_{5} \mathrm{O}_{7}^{*} 3\right. \\
& \left.+{\text { EDTA* } 4+\text { HEDTA* }^{*}+\text { glycolate + acetate + oxalate }}^{*} 2\right]
\end{aligned}
$$

Therefore, the hydroxide reported for the supernatants does not include either that bound to the aluminate nor that bound to silicate. All other ligated hydroxides are included within the supematant hydroxide value. The supernatant hydroxide within the interstices of the sludge appears as free hydroxide.

\section{- TOC and $\mathrm{wt}_{\mathrm{H}} \mathrm{H}_{2} \mathrm{O}$}

These values are calculated in a straightforward manner. Note that the wt\% water for a solution can be derived from its density and the total grams of dissolved species. The wt\% of the sludge that of the solution as well as the various solid phases have specific states of hydration for the various solid phases that are defined within the speciation. The equation for the solution is:

$$
w t \% \mathrm{H}_{2} \mathrm{O}=(1-\text { grams dissolved species / grams per } L \text { solution }) * 100
$$

while that of the solids is that of the interstitial liquid plus the waters of hydration of the solids. Note that silicate and aluminate will consume or produce water depending on their speciation. For example,

$$
\begin{gathered}
\mathrm{Al}(\mathrm{OH})_{4}^{*} \longrightarrow \mathrm{AlO}_{2}^{*}+2 \mathrm{H}_{2} \mathrm{O} \\
\mathrm{SiO}_{3}^{2-}+\mathrm{H}_{2} \mathrm{O} \longrightarrow \mathrm{SiO}_{2}(\mathrm{OH})_{2}^{2-} \\
\mathrm{SiF}_{6}^{2-}+6 \mathrm{OH}^{-} \longrightarrow \mathrm{SiO}_{2}(\mathrm{OH})_{2}^{2-}+2 \mathrm{H}_{2} \mathrm{O}+6 \mathrm{~F}^{-}
\end{gathered}
$$

The two equivalents of water for $\mathrm{Al}(\mathrm{OH}) 4$ are counted in the supernatant water wt\%, since the molecular weight used is for $\mathrm{AlO}_{2}{ }^{-}$. Likewise, one half equivalent of water from dissolved hydroxide is included, since

$$
\mathrm{NaOH} \longrightarrow 1 / 2 \mathrm{Na}_{2} \mathrm{O}+1 / 2 \mathrm{H}_{2} \mathrm{O} .
$$

For the precipitated solids, the waters of hydration that are included for the sludge wt\% water are those shown in Table 5e. The sludge wt\% water comprises both the waters of hydration of precipitated solids and the water from the interstitial liquid, which is that calculated for the supernatant. 
state. These are

Other species either consume or produce water or hydroxide because of changes in oxidation

$$
\begin{gathered}
\mathrm{CrO}_{4}^{2-}+5 / 2 \mathrm{H}_{2} \mathrm{O} \rightarrow \mathrm{Cr}(\mathrm{OH})_{3}+2 \mathrm{OH}^{-}+3 / 4 \mathrm{O}_{2} \\
\mathrm{Fe}(\mathrm{HSO} 4)_{2}+4 \mathrm{OH}^{-} \rightarrow \mathrm{Fe}(\mathrm{OH})_{3}+2 \mathrm{SO}_{4}^{2-}+\mathrm{H}_{2} \mathrm{O}+\mathrm{H}_{2} \\
\mathrm{MnO}_{4}^{-}+1 / 2 \mathrm{H}_{2} \mathrm{O} \rightarrow \mathrm{MnO}_{2}+\mathrm{OH}^{-}+3 / 4 \mathrm{O}_{2} .
\end{gathered}
$$

Note that the stable form for $\mathrm{Cr}$ in caustic nitrate is in fact the chromate, $\mathrm{Cr}(\mathrm{VI}) \mathrm{O}_{4}{ }^{2}$, not $\mathrm{Cr}(\mathrm{III})(\mathrm{OH})_{4}$ (see reaction A). In the HDW model, however, all chromium is assumed to be present as $\mathrm{Cr}$ (III). Also, in high hydroxide and nitrate simulants, there are reports ${ }^{36}$ of $\mathrm{NO}_{2}$ production upon addition of $\mathrm{Fe} 3+$. One explanation for this observation is that these waste concentrates oxidize $\mathrm{Fe} 3+$ to Fe6+ resulting in the ferrate ion $\mathrm{Fe}(\mathrm{VI}) \mathrm{O}_{4}{ }^{2-}$, not ferric $\mathrm{Fe}(\mathrm{III}) \mathrm{O}_{2}{ }^{-}$as shown in reaction $\mathrm{B}$ since this results in production of $\mathrm{NO}_{x}$ (i.e. $\mathrm{N}_{2} \mathrm{O}_{4}$ ).

$$
\begin{array}{cl}
\mathrm{Cr}(\mathrm{III})(\mathrm{OH})_{4}^{+}+4 \mathrm{OH}^{-} \rightarrow \mathrm{Cr}(\mathrm{VI}) \mathrm{O}_{4}{ }^{2-}+4 \mathrm{H}_{2} \mathrm{O}+3 \mathrm{e}^{-} & 0.13 \mathrm{~V}(1 \\
3 / 2\left(\mathrm{NO}_{3}+\mathrm{H}_{2} \mathrm{O}+2 \mathrm{e}^{-} \rightarrow \mathrm{NO}_{2}^{-}+2 \mathrm{OH}^{-}\right. & 0.01 \mathrm{~V})
\end{array}
$$

A.

$$
\begin{array}{cl}
\hline \mathrm{Cr}(\mathrm{III})(\mathrm{OH})_{4}^{-}+3 / 2 \mathrm{NO}_{3}^{-}+\mathrm{OH}^{-} \rightarrow \mathrm{Cr}(\mathrm{VI}) \mathrm{O}_{4}{ }^{2-}+3 / 2 \mathrm{NO}_{2}^{-}+5 / 2 \mathrm{H}_{2} \mathrm{O} & 0.145 \mathrm{~V}(1 \mathrm{~N} \mathrm{NaOH}) \\
\mathrm{Fe}(\mathrm{III}) \mathrm{O}_{2}^{-}+4 \mathrm{OH}^{-} \rightarrow \mathrm{Fe}(\mathrm{VI}) \mathrm{O}_{4}{ }^{2-}+2 \mathrm{H}_{2} \mathrm{O}+3 \mathrm{e}^{-} & -0.55 \mathrm{~V}(10 \mathrm{~N} \mathrm{NaOH}) \\
1 / 2\left(\mathrm{NO}_{3}^{-}+\mathrm{H}_{2} \mathrm{O}+2 \mathrm{e}^{-} \rightarrow \mathrm{NO}_{2}^{-}+2 \mathrm{OH}^{-}\right. & 0.01 \mathrm{~V}) \\
2\left(\mathrm{NO}_{3}^{-}+2 \mathrm{H}^{+}+\mathrm{e}^{-} \rightarrow 1 / 2 \mathrm{~N}_{2} \mathrm{O}_{4}+\mathrm{H}_{2} \mathrm{O}\right. & 0.405 \mathrm{~V})
\end{array}
$$

B. $\mathrm{Fe}(\mathrm{III}) \mathrm{O}_{2}^{-}+5 / 2 \mathrm{NO}_{3}^{-}+1 / 2 \mathrm{H}_{2} \mathrm{O} \rightarrow \mathrm{Fe}\left(\mathrm{VI}^{-} \mathrm{O}_{4}{ }^{2-}+\mathrm{N}_{2} \mathrm{O}_{4}+1 / 2 \mathrm{NO}_{2}^{-}+\mathrm{OH}^{-} 0.26 \mathrm{~V}(10 \mathrm{~N} \mathrm{NaOH})\right.$

where relevant and related standard reduction potentials are (by CRC)

$$
\begin{array}{cl}
\mathrm{NO}_{3}^{-}+\mathrm{H}_{2} \mathrm{O}+2 \mathrm{e}^{-} \rightarrow \mathrm{NO}_{2}^{-}+2 \mathrm{OH}^{-} & 0.01 \mathrm{~V} \\
\mathrm{NO}_{2}^{-}+\mathrm{H}_{2} \mathrm{O}+2 \mathrm{e}^{-} \rightarrow 1 / 2 \mathrm{~N}_{2} \mathrm{O}_{2}^{-}+2 \mathrm{OH}^{-} & -0.09 \mathrm{~V} \\
1 / 2 \mathrm{H}_{2} \mathrm{~N}_{2} \mathrm{O}_{2}+3 \mathrm{H}^{+}+2 \mathrm{e}^{-} \rightarrow \mathrm{N}(\mathrm{OH}) \mathrm{H}_{3}^{+} & 0.25 \mathrm{~V} \\
\mathrm{~N}\left(\mathrm{OH}^{+} \mathrm{H}_{3}^{+}+2 \mathrm{H}^{+}+2 \mathrm{e}^{-} \rightarrow \mathrm{NH}_{4}^{+}+\mathrm{H}_{2} \mathrm{O}\right. & 1.35 \mathrm{~V} \\
\mathrm{NO}_{3}^{-}+2 \mathrm{H}^{+}+\mathrm{e}^{-} \rightarrow 1 / 2 \mathrm{~N}_{2} \mathrm{O}_{4}+\mathrm{H}_{2} \mathrm{O} & 0.405 \mathrm{~V} \\
\mathrm{NO}_{3}^{-}+\mathrm{H}_{2} \mathrm{O}+\mathrm{e}^{-} \rightarrow 1 / 2 \mathrm{~N}_{2} \mathrm{O}_{4}+2 \mathrm{OH}^{-} & -0.425 \mathrm{~V} \\
\mathrm{H}_{2} \mathrm{O}+\mathrm{e}^{-} \rightarrow \mathrm{H}_{2}+\mathrm{OH}^{-} & -0.414 \mathrm{~V} \\
\mathrm{H}^{+}+\mathrm{e}^{-} \rightarrow \mathrm{H}_{2} & 0.00 \mathrm{~V} \\
\mathrm{Fe}(\text { III }) \mathrm{O}_{2}^{-}+4 \mathrm{OH}^{-} \rightarrow \mathrm{Fe}(\mathrm{Vl}) \mathrm{O}_{4}^{2-}+2 \mathrm{H}_{2} \mathrm{O}+3 \mathrm{e}^{-} & -0.55 \mathrm{~V}(10 \mathrm{~N} \mathrm{NaOH})
\end{array}
$$

36 Scheele, R.A., personal communication. 


$$
\mathrm{Cr}(\mathrm{III})(\mathrm{OH})_{4}^{-}+4 \mathrm{OH}^{-} \rightarrow \mathrm{Cr}(\mathrm{Vl}) \mathrm{O}_{4}^{2-}+4 \mathrm{H}_{2} \mathrm{O}+3 \mathrm{e}^{-} \quad 0.13 \mathrm{~V}(1 \mathrm{~N} \mathrm{NaOH})
$$

The implication with these observations is that chromate and ferrate are the thermodynamicaily more stable forms of these ions in the concentrated caustic/nitrate wastes typical of Hanford. There is a competition between the precipitation of iron(III) oxyhydroxide and its oxidation to ferrate during neutralization of acid waste prior to placing into waste tanks. If the iron(III) oxyhydroxide is trapped in a sludge, later reaction with concentrated caustic/nitrate solutions will produce NOx. Therefore, future sludge washing under caustic conditions should be considering the possibility of such an occurrence.

The TOC is calculated using the equivalent of organic carbon present in each molecule as shown in Table 8. Also shown is a list of tentative ratios of measured to predicted TOC's to facilitate comparison of HDW calculated TOC's with measured values. For example, only roughly one half of the organic carbon that is predicted in ferrocyanide actually shows up in a measured TOC.

\begin{tabular}{c|c|c|c}
\hline \hline & Table 8. Grams Organic Carbon per mol Species. \\
\hline species & $\begin{array}{c}\text { g organic carbon } \\
\text { per mol }\end{array}$ & meas./pred. & $\begin{array}{c}\text { measured g carbon } \\
\text { per mol }\end{array}$ \\
\hline EDTA & 120 & 0.8 & 96 \\
\hline HEDTA & 120 & 0.8 & 96 \\
\hline glycolate & 24 & 1.0 & 24 \\
\hline citrate & 72 & 1.0 & 72 \\
\hline acetate & 24 & 1.0 & 24 \\
\hline Oxalate & 24 & 1.0 & 12 \\
\hline TBP & 144 & 1.0 & 144 \\
\hline NPH & 144 & 1.0 & 144 \\
\hline hexone & 60 & 1.0 & 60 \\
\hline FeCN & 72 & 0.5 & 72
\end{tabular}

\section{-Evaporator operations}

There have been a variety of evaporator operations at Hanford, as shown in Table 9. These campaigns involved various facilities and tanks as follows:

- separate in-farm evaporators (242-B, 242-T, 242-S, or 242-A);

- use of either $B$ or Redox Plant evaporators for tank supernatants;

- in-tank heaters as in BY Farm; and

- boiling waste self-concentration in S, SX, A, and AX Farms.

Each of these operations involved heating the waste and accumulating and separately disposing the condensate. The concentrate (or bottoms) are then transferred to various waste tanks (bottoms tanks) and the salts within the concentrate are allowed to accumulate in those tanks (as salt cake).

We have adopted a strategy with the 242-T (1950's), 242-B, Redox self-concentrates, and BY Farm ITS campaigns wherein all waste input to each of these campaigns is blended to produce one salt cake and one supernatant for each campaign. The HDW blends for each of these campaigns is shown in (App. B). These definitions allow for later reconcentration of previously concentrated supernatants from each campaign.

For all of the other evaporator campaigns, we have used the SMM to allow the prediction of a concentrated blend of HDW supernatants for each tank. However, we have nevertheless calculated the blended feeds for other evaporator campaigns as shown in App. B.

In principle, there are two states of hydration for each of the salt cakes-wet (i.e. as created) and in various stages of hydration. In fact, Allen reported two sets of values for salt cakes from 242-S (S1SitCk) bottoms receivers-one set for low water contents (3-5 wt\%) and a second set for larger water contents $(-15-30 w t \%)$. However, Allen did not report density measurements for any of his samples, and 
therefore we do not know the solid fraction of any of those samples. All salt cakes reported here are kept with the water contents that resulted when they were formed. I.e., the HDW model does not include any drying of the waste in the waste tanks other than that associated with one of the evaporator campaigns.

\begin{tabular}{|c|c|c|c|c|c|c|c|c|}
\hline \multicolumn{9}{|c|}{$\begin{array}{c}\text { Table } 9 . \\
\text { Evaporation and } \mathrm{Cs} / \mathrm{Sr} \text { Extraction Campaigns }\end{array}$} \\
\hline Evaporator & st.date & & en.date & & $\operatorname{Tank}(\mathrm{s})$ & kgal out & $\begin{array}{c}\text { kgal } \\
\text { reduction }\end{array}$ & $\begin{array}{c}\text { kgal salt } \\
\text { cake }\end{array}$ \\
\hline 242-T & 1951 & 2 & 1955 & 3 & TX-118 & 8,060 & 7.849 & 764 \\
\hline $242-B$ & 1951 & 4 & 1954 & 4 & B-106 & 8,048 & 7,861 & 786 \\
\hline $\begin{array}{l}\text { Redox self- } \\
\text { conc. }\end{array}$ & 1952 & & 1965 & & S/SX Farms & 8,240 & 8,400 & 514 \\
\hline $\begin{array}{l}\text { A/AX self- } \\
\text { conc. }\end{array}$ & 1960 & & 1965 & & $\bar{A}$ AX Farms & & & \\
\hline HS or SSW & 1961 & 4 & 1965 & 2 & $\begin{array}{c}\mathrm{C}-109 \\
\mathrm{C}-111, \mathrm{C}-112\end{array}$ & & & \\
\hline Redox Plant & 1967 & & 1972 & & S-107 feed & & & \\
\hline ITS proto. & 1965 & 1 & 1966 & 3 & BY-101 & & & \\
\hline ITS\#1 & 1966 & 4 & 1971 & 2 & $\begin{array}{c}\text { BY-102, } \\
\text { BY-103 feed }\end{array}$ & & & \\
\hline ITS\#2 & 1967 & 4 & 1976 & 1 & $\begin{array}{c}\text { BY-112, } \\
\text { BY-109 feed }\end{array}$ & 9,585 & 38,111 & 3887 \\
\hline $\begin{array}{l}\text { B Plant Cell } \\
23\end{array}$ & 1967 & 4 & 1967 & 4 & $\begin{array}{l}\text { B-112 feed, } \\
\text { B-111 bottoms }\end{array}$ & & & \\
\hline 242-T & 1965 & \begin{tabular}{|l|}
4 \\
\end{tabular} & 1976 & 1 & TX-118 feed & 20,014 & 42,242 & 5874 \\
\hline $242-5$ & 1972 & 4 & 1977 & 1 & S-102 feed & 21,126 & 34,642 & 5123 \\
\hline 242-A & 1976 & 4 & 1980 & 4 & A-102 feed & 20.465 & 7,405 & 1073 \\
\hline $242-S$ & 1977 & 2 & 1980 & 4 & SY-102 feed & 7,793 & 7,000 & \\
\hline 242-A & 1981 & 1 & 1991 & & AW-102 feed & 10,794 & 8,053 & \\
\hline $\begin{array}{l}\text { A-102 } \\
\text { sluicing }\end{array}$ & 1963 & 4 & 1969 & 1 & $\overline{A-102}$ & & & \\
\hline $\begin{array}{l}\text { A-106 } \\
\text { sluicing }\end{array}$ & 1969 & 2 & 1973 & 4 & $\begin{array}{l}\text { A-106 sl to AR, su } \\
\text { feeds C-105 }\end{array}$ & & & \\
\hline $\begin{array}{l}\text { AX-103 } \\
\text { sluicing } \\
\end{array}$ & 1974 & 1 & 1977 & 3 & $\begin{array}{c}\mathrm{AX}-103 \mathrm{sl} \text { to } \mathrm{AR}, \mathrm{su} \\
\text { feeds C-105 }\end{array}$ & & & \\
\hline CSR & 1967 & 4 & 1979 & 1 & C-105 su feed & & & \\
\hline SRA & 1969 & 1 & 1977 & 2 & A-106 sl feed & & & \\
\hline totals & & & & & & & 161,563 & 18,937 \\
\hline
\end{tabular}

$-242-B$ and First 242-T

These evaporators both began in 1951 with $242-\mathrm{B}$ running through 1953, and 242-T running through 1956. They were primarily used to concentrate $1 \mathrm{C}$ and UR waste supernatants. Anderson- 91 reported $242-\mathrm{B}$ reduced 6,027 to $1,151 \mathrm{kgal}(80.9$ vol\% reduction), while $242-\mathrm{T}$, in two passes, reduced 8,638 to $1,546 \mathrm{kgal}(82.1$ vol\% reduction).

The WSTRS transaction records, on the other hand, show different total numbers. WSTRS shows that $242-\mathrm{B}$ reduced 15,089 to $7,240 \mathrm{kgal}(52.0$ vol\% reduction) and that $242-\mathrm{T}$ reduced 18,191 to $8,330 \mathrm{kgal}$ ( $54.2 \mathrm{vol} \%$ reduction). Anderson-91 actually reports the total waste volume reduction for 242$\mathrm{B}$ as $7,172 \mathrm{kgal}$, and that for $242-\mathrm{T}$ as $9,181 \mathrm{kgal}$, or a total volume reduction of $16,353 \mathrm{kgal}$, while our volume reduction is $17,710 \mathrm{kgal}$ for this same period. Our main difference with Anderson-91 is with the total volume processed. Note that our estimates are not broken down by $1 \mathrm{C}, 2 \mathrm{C}$, or UR supernatants. 
Our estimate in fact comprise all three. We have found that some $786 \mathrm{kgal}$ salt cake was formed in B Farm as a result of $242-\mathrm{B}$ operation, or $6.1 \mathrm{vol} \%$ of the original volume, and $764 \mathrm{kgal}$ was formed for $242-\mathrm{T}$.

\section{- S and SX Farms self-concentration}

As shown in Table $5 b$, tanks in $S$ and SX Farms that had been filled with waste from the Redox plant were allowed to boil and self-concentrate. We have found that there are a number of tanks in which the salts from this concentration accumulated and we have termed that waste RSItCk, Redox Salt Cake. These sixteen tanks are: S-101, -104, -107, -110, and SX-101, -102, -103, -104, -107, -108, -109, -110, $-111,-112,-114,-115$. The composition of RSItCk is a blend of all of the supernatants that were fed into any of these tanks during the years noted in Table 5b. Thus, this blend of the concentrate will actually be distributed among all of these tanks.

\section{- $A$ and $A X$ Farms self-concentration}

Corresponding to the self-boiling tanks in S and SX Farms for Redox wastes, there were a series of selt-boiling tanks in A and AX Farms as well. However, no salt precipitates formed as a result of their concentration and so there are no salt cake remnants formed. However, the supernatants did concentrate appreciably. Then, the supernatants were recovered; their Cs-137 extracted; and the supernatants returned to the waste tanks, where they were then concentrated. The self-concentrating tanks are: $A-101,-102,-103,-104,-105$, while the remaining tanks in $A$ and $A X$, although equipped for boiling waste, never actually boiled.

\section{- In Tank Solidification (ITS) campaign in BY Farm}

In 1965, a prototype heater was placed into BY-101 and the demonstration of evaporation driven by in tank heaters was performed. In 1966, a second heater was placed into BY-102 with tank BY-103 acting as a primary feed tank. The strategy of this campaign was to circulate the feed to the heated tank and then from there transferred to other tanks in BY Farm. As the concentrate cooled, the idea was to solidify an entire series of waste tanks by continuously recycling the concentrate around this loop.

Finally, in 1966 a third heater was placed into BY-112 with BY-109 acting as primary feed. During this third heater's operation, the heater in BY-102 was used as a cooler instead and hot concentrate from BY-112 was routed then to BY-102. The ITS campaign ended in 1976 and resulted in about 38 million gallons of volume reduction and the formation of $3,887 \mathrm{kgal}$ of salt cake, BYSItCK.

\section{- Acid additions during evaporator runs}

From 1977 through 1980 , a series of acid/permanganate additions were performed during 242-S evaporator runs which were designated NIT (neutralization in transfer) by Jungfleisch or PNF (partial neutralization of feed) by Anderson. Evidently, this campaign was an attempt to precipitate more sodium as the nitrate salt and thereby enhance the solidification of the waste within the bottoms receivers. For each NIT transaction, Jungfleisch started with that volume of the receiving tank, adjusted the hydroxide of that volume to a maximum of $0.9 \mathrm{M}$ by adding nitric acid, and finally increased the concentration of $\mathrm{NaNO}_{3}$ by $0.3 \mathrm{M}$ and $\mathrm{KMnO}_{4}$ by $1.3 \mathrm{e}-3 \mathrm{M}$. This volume was then added back to the tank and mixed with the waste already in the tank. This model was meant to simulate the actual NIT additions that occurred continuously during an evaporator run. For example, WSTRS reports that $52 \mathrm{kgal}$ of NIT was added to SY-102 during this campaign see (Tabie 10) and NIT was also added to S-102, S-103, SX-106, U-102, U103, U-107, U-111.

These transactions also added to each waste stream a variable amount of nitric acid that depended on the tank waste composition at the time of the addition. In the HDW, we have assumed that the composition of the NIT was on average $0.5 \mathrm{M} \mathrm{HNO}_{3}$, as well as the other two components, $0.3 \mathrm{M}$ $\mathrm{NaNO}_{3}$ and $0.0013 \mathrm{M} \mathrm{KMnO}_{4}$. It is not clear how much nitric acid was actually added during each of these runs nor is it clear that the hydroxide that is bound to the aluminate ion is included in this neutralization scheme. 


\begin{tabular}{c|c|c|c}
\hline \multicolumn{4}{c}{ Table 10. } \\
Evaporator Partial & Neutralization (PNF) Campaign \\
\hline \hline tank & kgal NIT & from & to \\
\hline$S Y-102$ & 52 & 1977.75 & 1977.75 \\
\hline$U-102$ & 29 & 1977.75 & 1978 \\
\hline$U-103$ & 26 & 1977.75 & 1977.75 \\
\hline$S-103$ & 220 & 1978 & 1980.75 \\
\hline$U-107$ & 109 & 1978 & 1980.75 \\
\hline S-102 & 63 & 1979.5 & 1979.75 \\
\hline$U-111$ & 17 & 1979.5 & 1979.5 \\
\hline SX-106 & 138 & 1980.5 & 1980.75 \\
\hline kgal Total & $\mathbf{6 5 4}$ & $\mathbf{1 9 7 7 . 7 5}$ & $\mathbf{1 9 8 0 . 7 5}$
\end{tabular}

-Radiolysis of nitrate to nitrite to ammonia

A previous repor ${ }^{37}$ has shown a yield of 4.5 molecules of nitrite in $2 \mathrm{M}$ nitrate solutions per 100 $\mathrm{eV}$ of absorbed dose $(\mathrm{G}=4.5)$. If we express this in terms of cesium and/or strontium radiolysis, a $1 \mathrm{Ci} /$ (or $3.7 \mathrm{e} 10 \mathrm{~Bq} / \mathrm{L}$ ) solution of these species woutd correspond to 4.5 molecules nitrite / $100 \mathrm{eV} /(2 \mathrm{M}$ nitrate) $\times 1.1 \mathrm{e} 6 \mathrm{eV} /$ decay $\times 1 \mathrm{Ci} / \mathrm{L} \times 3.7 \mathrm{e} 10$ decays / sec / Ci $\times 3.16 \mathrm{e} 7 \mathrm{secs} /$ year / 6.023e23 molecules / mol which is

$$
=0.048 \mathrm{~mol} \mathrm{NO}_{2}^{-} / \mathrm{mol} \mathrm{NO}_{3}-\mathrm{Ci} / \mathrm{yr} \text {. }
$$

For a waste tank at $0.5 \mathrm{Ci} / \mathrm{L}$ and $2.5 \mathrm{M}$ nitrate, this would amount to $0.06 \mathrm{M}$ nitrite produced per year. For SY-101, this suggests that from 1981 to present, there has been $\sim 0.8 \mathrm{M}$ nitrite created by radiolysis. Of course, the decay of the radionuclides must also be taken into account for any long term radiolysis.

We have not been able to locate a $G$ value for ammonia production from nitrite. We assume therefore that there is some channel for which nitrite undergoes further radiolysis to hyponitrite, hydroxlammine, and finally ammonia as

$$
\mathrm{NO}_{3}{ }^{-} \rightarrow \mathrm{NO}_{2}{ }^{-} \rightarrow \mathrm{NOd} \stackrel{\text { rad. }}{\rightarrow} \mathrm{H}_{2} \mathrm{NOH} \rightarrow \mathrm{NH}_{3} \text {. }
$$

Ammonia production for SY-101 has been reported 38 to be 2.4 mol NH$_{3} /$ year, which suggests that the actual ammonia production from radiolysis of nitrite is only $7 \%$ that of the radiolysis of nitrate to nitrite. We further assume that ammonia production for SY-101 exceeds that of radiolysis alone by factor of three, which is the amount of hydrogen gas production over that of radiolysis alone (because of the presence of complexant). Therefore, we derive a value of

$$
1.2 \mathrm{e}-3 \mathrm{~mol} \mathrm{NH}_{3} / \mathrm{mol} \mathrm{NO}_{2}-/ \mathrm{Ci} / \mathrm{yr} \text {. }
$$

Production of ammonia from nitrite is assumed to proceed as

$$
\mathrm{NO}_{2}^{-}+2 \mathrm{H}_{2} \mathrm{O} \rightarrow \mathrm{NH}_{3}+\mathrm{OH}^{-}+3 / 2 \mathrm{O}_{2}
$$

where the hyponitrite and hydroxylamine intermediates are presumed to be short lived. Therefore, for each mol ammonia produced, two mols water are consumed and one mol of hydroxide and three halves mols oxygen are produced. Ammonia production is accumulated in each waste as $\mathrm{NH}_{3}$ and no solubility limit is imposed. Therefore, all of the ammonia that has been produced for the entire history of a tank's waste remains within that waste in the HDW model.

${ }^{37}$ Hyder, M. L. "The Radiolysis of Aqueous Nitrate Solutions," J. Phys. Chem.. 69, 1858-65, 1965.

${ }^{38}$ Norton, J. D. and Pederson, L.R. "Ammonia in Simulated Hanford Double-Shell Tank Wastes: Solubility and Effects on Surface Tension," PNL-10173, Sept. 1994 
HNF-SD-WM-ER-323, Rev. 1

To account for decay of the Cs-137 and Sr-90, we have used the expression

$$
\left.=(C s-137+S r-90)^{*} t_{1 / 2} / L N(2)^{*}\left(\operatorname{EXP}(1994-c a m p . y r .){ }^{*} \operatorname{LN}(2) / t_{1 / 2}\right)-1\right)
$$

and have used an average halt life of 29.15 years. This expression provides the total dose of a waste in Ci.-yr.'s when the Cs-137 and Sr-90 are expressed in 1994 curies. This does not account for any other radionuclide source terms besides $\mathrm{Cs}-137, \mathrm{Sr}-90$, and their daughters. For example, we have not accourted for any of the short-lived fission products in green waste.

\section{-Degradation of organic}

The HDW model does not include any degradation of organic residues. However, there is ample indication that the organic residues have degraded in tanks over time. Presumably, the degradation of organic complexants proceeds at different rates for the different organics and their decomposition products and many of these rates are uncertain see Degradation of Organics in Waste Tanks (Table 11). There has been much suggestion that the organics that were passed through the 242-T evaporator were substantially degraded. This evaporator operated at $100^{\circ} \mathrm{C}$ and the residence times were on the order of 13 hours.

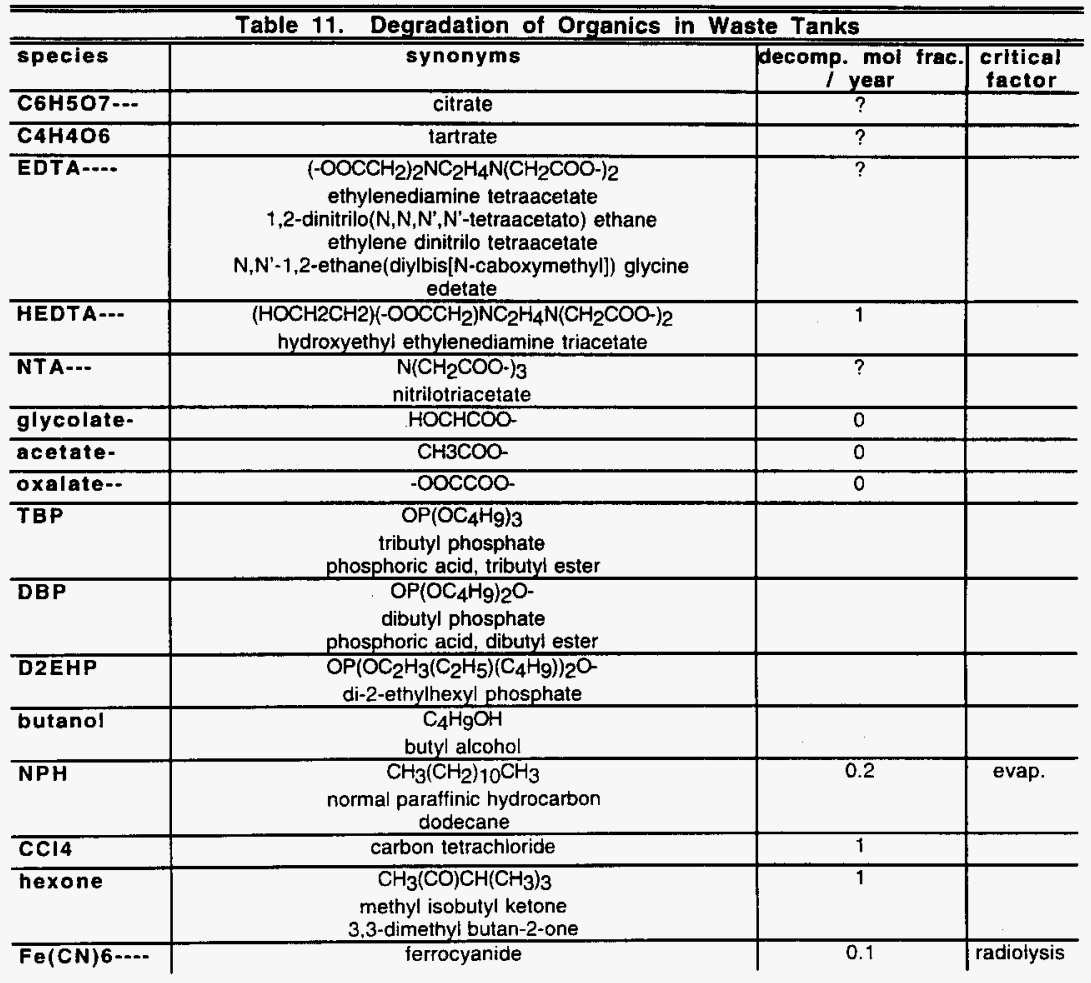


Based on three measurements for tank AN-107 over a 10 year period, we can estimate an organic decomposition as mol fraction TOC per $\mathrm{Ci} / \mathrm{L}-\mathrm{yr}$ of dose. This number is $0.06 \mathrm{~mol}$ fraction per $\mathrm{Ci} / \mathrm{L}$ yr of dose.

A scheme that uses a minimum set of "representative reactions" to represent the degradation of organics is shown below. Note that the reactions of this scheme do not need to be "real," but only representative of the overall system. Such a scheme is more amenable to using partial information and naturally allows the conservation of mass, once the reactions are balanced. Such a minimum set might be, for example, the following (which are not balanced):

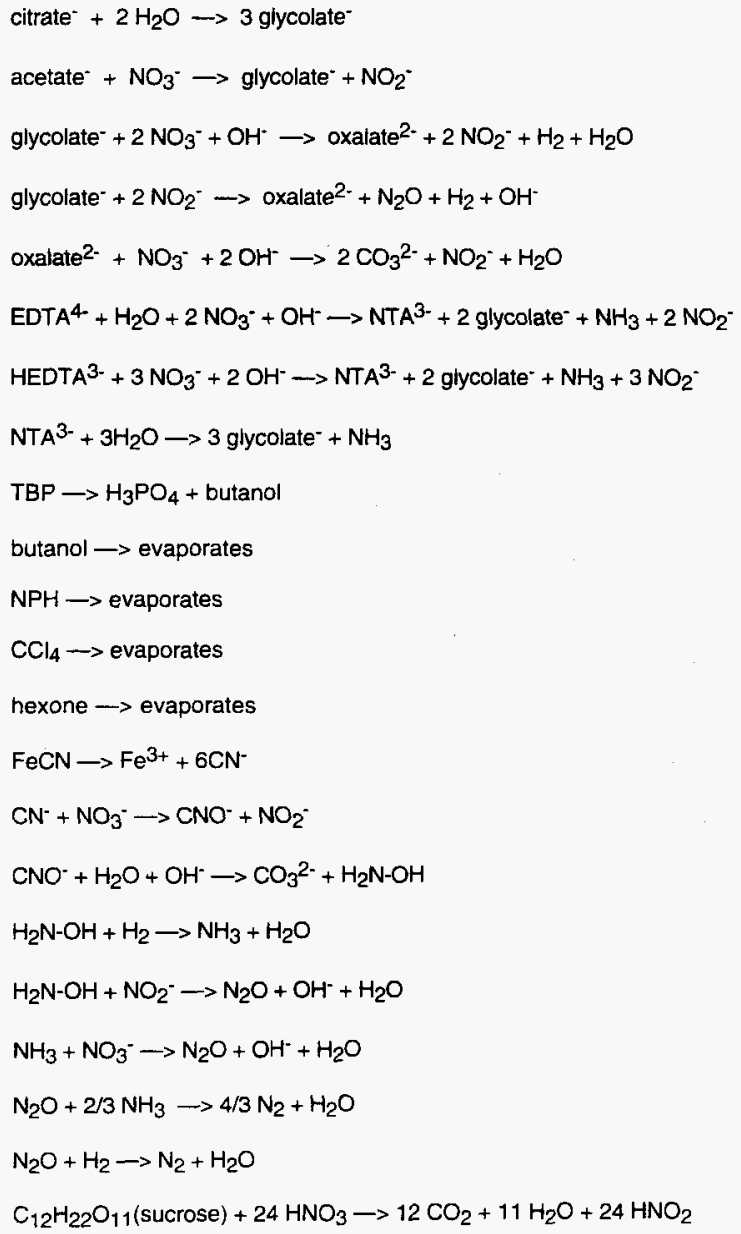




$$
\begin{aligned}
& \mathrm{C}_{12} \mathrm{H}_{22} \mathrm{O}_{11} \text { (sucrose) }+24 \mathrm{HNO}_{2} \rightarrow 12 \mathrm{CO}_{2}+23 \mathrm{H}_{2} \mathrm{O}+12 \mathrm{~N}_{2} \mathrm{O} \\
& \mathrm{C}_{12} \mathrm{H}_{22} \mathrm{O}_{11} \text { (sucrose) }+24 \mathrm{~N}_{2} \mathrm{O} \rightarrow 12 \mathrm{CO}_{2}+11 \mathrm{H}_{2} \mathrm{O}+24 \mathrm{~N}_{2}
\end{aligned}
$$

- Corrosion source term for $\mathrm{Fe}, \mathrm{Cr}$, $\mathrm{Ni}$

We have found that the iron concentrations in Hanford sludges are much higher than can be accounted for with the iron that was added during processing. Therefore, we have added an additional iron source that we attribute to corrosion of process vessels and lines primarily within a plant. In order to add this source term, we needed to derive a corrosion source term.

A previous report ${ }^{39}$ has suggested that about $1,200 \mathrm{~g}$ of iron are produce per ton of fuel processed, with about one half of this coming from iron in the fuel element itself while the other half derives largely from corrosion of various process equipment that has been in contact with the solution. Over the history of Hanford, approximately 108,000 tons of fuel have been processed. This would suggest that about $2.3 \mathrm{Mmol}$ of Fe would be due to this source.

We have found, on the other hand, that the "extra" source of iron is much larger than this. In fact, we estimate that $10-15 \mathrm{Mmol}$ of iron, which is about as much as was added during processing, must have come from various pieces of equipment that were used in processing and transporting waste. This is equivalent to $0.04 \mathrm{M}$ iron in the $390,000 \mathrm{kgal}$ of waste that has been generated at Hanford totaling 341 metric tons of iron.

With the iron fixed at $0.04 \mathrm{M}$, we can estimate both chromium and nickel assuming that the source term is stainless steel 304 . We have used $\mathrm{Cr}=0.2 \times \mathrm{Fe}$ while $\mathrm{Ni}=0.1 \times \mathrm{Fe}$, which is close to reported ranges for this alloy. 40

We have reduced the corrosion source term for the $\mathrm{BiPO}_{4}$ and all decladding processes to $40 \%$ of this value. This value was chosen such that analytical results for these classes of wastes agreed with predictions generated by the model and also because we expect that these processes would have produced much less corrosion because of the involved lower acid or caustic processing.

\section{-Silicate source terms}

There were many different sources of silicate in waste streams. We have found, though, that a particular source that has been often overlooked is the silicate that derived from silicone anti-foaming agents used in the sugar denitration of the Purex acid waste prior to its neutralization and disposal to tanks.

\section{- Calcium impurity source term}

The primary source of calcium in the waste is another mystery at this time. We estimate that there is some $28 \mathrm{Mmol}$ of calcium in Hanford wastes (based on extrapolation of analytical data on sludges to all tanks), but have only accounted for $4 \mathrm{Mmol}$ by flowsheet additions. Thus, we do not have a source term for $24 \mathrm{Mmol}$ of calcium. In order to provide an estimate for calcium, we have considered three additional sources.

First, the calcium may have derived from the fuel elements themselves. This amount of calcium in 108,000 tons of fuel would amount to $1 \mathrm{wt} \% \mathrm{Ca}$, which is not listed at all as a component of $\mathrm{N}$-Reactor fuel. ${ }^{41}$ Calcium would be a potential component of the silica binder that is used in the fuel elements, but we have not found any reference to calcium in the fuel.

A second source of calcium would be that added as needed during operation to control the $\mathrm{Sr}$ 90 in solution. Although such additions are not documented in any of the flowsheets that we have examined, there are anecdotal references to calcium additions to precipitate as a phosphate or carbonate, enhancing $\mathrm{Sr}-90$ decontamination of solutions.

\footnotetext{
${ }^{39}$ Van der Cook, R. E. and Walser, R. L. "Purex Alternate Reductant Study," ARH-1649, June 1970.

${ }^{40} \mathrm{CRC}$ Handbook of Chemistry and Physics, 58th ed., 1977-78.

${ }^{41}$ Chapter five "Fuel Element Dissolution and Waste Treatment Technology," WHC-SP-0479.
} 
Another possible source for the calcium is a larger than expected amount of $\mathrm{Ca}$ in the sodium hydroxide that was used to neutralize the waste stream. There were approximately $1,927 \mathrm{Mmol} \mathrm{NaOH}$ added to the various waste streams. We have assumed that it was added as a 50 wt\% caustic solution at $30 \mathrm{ppm} \mathrm{Ca}(0.0012 \mathrm{M})$, which only amounts to a fraction of a Mmol Ca. If, on the other hand, we assumed that all of the $\mathrm{Ca}$ came from $\mathrm{NaOH}$ solution, the solution would have been $5 \mathrm{w} \% \mathrm{Ca}$. This seems like an unreasonably large amount of $\mathrm{Ca}$ in the neutralization stream.

Crushed limestone $\left(\mathrm{CaCO}_{3}\right)$ rock was often used in holding tanks prior to release of condensate and other water sources to cribs and trenches. However, we have no indication that any of this limestone ended up in waste tanks. Twenty $\mathrm{Mmol}_{\text {of }} \mathrm{CaCO}_{3}$ would amount to 2,000 metric tons of limestone.

A final calcium source would be that in normal water. The level of calcium in ground water at Hanford is $20-40 \mathrm{ppm}$ (that of the Columbia River is $-30 \mathrm{ppm}$ ) which is equivalent to $7.5 \mathrm{e}-4 \mathrm{M} \mathrm{Ca}$. The process solutions used in plant operation were normally deionized, but there were undoubtedly many flush and cleaning water additions that used simple tap water. The volume of waste at Hanford excluding reprocessing is $433,000 \mathrm{kgal}$ (Table 2). We estimate that there is on the order of $24 \mathrm{Mmol} \mathrm{Ca}$ in the Hanford waste tanks, which amounts to $0.015 \mathrm{M} \mathrm{Ca}(-600 \mathrm{ppm})$. Therefore, the amount of calcium is greater than a factor of twenty greater than we can explain based on caicium in hard water. Combined with the fact that the process solutions, which constitute a large fraction of the total waste produced, were deionized is inconsistent with hard water as being the calcium source.

We will nevertheless assume for the purposes of our estimates that the calcium source was distributed across most all process solutions and that it amounted to $0.015 \mathrm{M} \mathrm{Ca}$ in these original wastes. We suggest that the most probable source for this calcium was rinse and flush water, with added calcium for Sr-90 decontamination being a second factor.

\section{-Chloride and potassium impurity source terms}

The primary source of chloride and potassium in the waste is from the added sodium hydroxide. The reported chloride amounts are added in each waste stream according to the reported ppm of $\mathrm{Cl}^{-}$in the $\mathrm{NaOH}$ feed. Chloride impurities in $\mathrm{NaOH}$ are reported to be $1 \mathrm{wt} \%$. This amounts to a chloride inventory of $28 \mathrm{Mmol}$, as opposed to the chloride in process additions-around $1 \mathrm{Mmol} \mathrm{Cl}^{-}$added during the Uranium Recovery campaign.

We use a value of potassium in $\mathrm{NaOH}$ of around 0.5 wt\%, which results in a potassium inventory of 15 Mmois for all tanks.

\section{- Lead source terms (and neglect of copper and zinc)}

The only recorded use of lead in process was with the Hot Semi-Works or C Plant. Thus, there is lead in HS waste. We have also determined, though, that after 1954 the fuel slugs were dipped into a lead bath prior to their being dipped into the aluminum silicide bonding agent and then welded into the cladding. This bonding agent, then, contained some amount of lead, which we have estimated based on descriptions of this process and assuming a one micron layer of residuai $\mathrm{Pb}$ on the fuel siugs. This added an additional source term for lead in CWR1, CWR2, CWP1, and CWP2. Since prior to 1954 a bronze dip ( $\mathrm{Cu}$ and $\mathrm{Zn}$ ) was used in place of the lead dip, no adjustments were made to the $1 \mathrm{C} 1 \mathrm{or} 1 \mathrm{C2}$, which include the cladding waste from those periods. Note that the HDW model does not include either copper or zinc and so this source term is completely neglected for $1 \mathrm{C} 1$ and $1 \mathrm{C} 2$.

\section{-Tritium losses}

There are a variety of sources for tritium in the fuel that was processed at Hanford. The HDW model assumes that all tritium is present as tritiated water, HTO, and therefore is lost as condensate whenever waste is concentrated by active evaporation. The tritium in each HDW supernatant is therefore reduced by ratioing the sodium concentration of the supernatant to $12 \mathrm{M}$. This method then assumes a water loss that is in direct proportion to a final waste that is $12 \mathrm{M} \mathrm{Na}$. Note that all HDW sludges retain the original amount of tritium (decayed to 1-1-94) without any other losses. 
HNF-SD-WM-ER-323, Rev. 1

\section{Variabilities for HDW Model Estimates}

There are four different potential sources for disagreement between HDW model and assay estimates for tank waste: model, parameter, transaction, and sampling:

- Model: Approximations and assumptions used within the HDW model result in model dependent variability, termed model errors. This variability is the only irreducible source of variability within the HDW model. In other words, if there are incorrect data or parameters within the HDW model, its estimates will be "wrong" but the model itself may very well still be "valid." Specifically, a discrepancy between HDW model and assay results may be due to the assumption of single-point solubility for an analyte, which would be a model error. As long as the solubility parameter represented the mean solubility point for all HDW wastes, the parameter itself would be correct.

- Parameter: A parameter within the HDW model source term may be incorrect, such as an incorrect analyte solubility limit. Each solubility limit represents the mean value for all HDW wastes. For example, the Pu solubility is assumed to be $30 \mu \mathrm{Ci} / \mathrm{L}$ for all HDW Rev. 3 , but new information suggests that $9 \mu \mathrm{Ci} / \mathrm{L}$ (the value used in Rev. 4) is a more representative single point solubility for $\mathrm{Pu}$ in the absence of complexants. This error, then, would be a solubility point parameter error. Another kind of parameter error is associated with HDW model source terms. For example, the HDW Rev. 3 used a value of $0.22 \mathrm{M}$ for $\mathrm{Al}$ in $1 \mathrm{C}$ waste, which suggests an aluminum amount of 2,500 mols Al/short ton fuel processed. However, other process knowledge suggests that the actual amount of aluminum present in the cladding waste was 1,100 mols Al/short ton fuel (adjusted value for Rev. 4). This difference amounts to a source term parameter error.

- Transaction: This is a symmetric volume error that involves at least a pair of tanks. If waste from a tank or process did not go into the designated tank, then it has to go into some other tank. Therefore, two or more tanks are affected by such a waste transaction error. This type of error tends to average out for supernatants in the limit of large numbers of transaction (shown below). On the other hand, it can have very dramatic consequences on tank sludge inventory.

- Sampling: A particularly vexing source of discrepancy in comparisons of HDW model estimates with assays is caused by waste heterogeneity, termed a sampling error. Waste heterogeneity can make it difficult if not impossible to obtain a set of waste samples from a tank that adequately represent that tank's heterogeneity. Not only are there vertical layers of waste within each tank, but the distribution of those waste layers about the tank can be extremely irregular as well. Waste heterogeneity not only makes comparisons with HDW model results difficult, it also bedevils attempts to use waste assays to establish tank inventories. In addition, limited access by risers and incomplete recovery during core sampling further complicate the interpretation of sample assays.

The HDW model Rev. 4 only includes two sources of variability, process and solubility. These two sources of variability shouid account for the vast majority of variation outside of heterogeneity. The HDW model calculates the variation of each of 33 processes (see Table 12) at \pm 1.00 RSD to provide 2 scenarios. Then, it calculates another 2 scenarios for 24 analyte solubilites varied as a group by $\pm t$ RSD along with 16 analyte solubilities varied independently (see Table 7), for another 32 scenarios. In all, 36 scenarios of $48 \mathrm{HDW}$ 's provides 1,728 variations for each analyte for each waste tank at $\pm 1 \mathrm{RSD}$. The maximum and minimum variations are selected from this set to represent the \pm 1 RSD variation and then another 1,728 variations are calculated for $\pm 1.96 \mathrm{RSD}(95 \% \mathrm{Cl})$ and their maximum and minimum then determine the \pm 1.96 RSD.

Among these trials, -12 of the 1,728 variations for \pm 1.96 resulted in unphysical waste sludges and were therefore rejected. That is, the amount of material in the waste stream for certain trials exceeded the solids volume for those wastes given the solubility of its constituents. Within the HDW model, a negative void fraction is an indication of too many solids for the specified solids volume percent of the waste.

-Quantification of process variability

Each of the forty-eight HDW compositions varies with time. This variation in composition has a variety of causes but can be represented as a linear trend and a variation about that trend. The variation 
of each process is assumed to be caused by process waste dilution although there is undoubtedly a component of rework processing, for the purposes of the HDW model, rework variability is ignored.

This variation is particular for each waste and is volume dependent. That is, the larger the volume of an HDW that exists within a given tank, the smaller will be the variability of that estimate and the more representative will that tanks volume be of that waste type. As an example, see Fig. 18, which is a plot of waste rates for the Redox campaign. There are fourteen tanks in S and SX Farms that hold nearly all of the A1 sludge, averaging $75 \mathrm{kgal}$ each. Thus, each tank's sludge represents about two quarters worth of accumulation and the variability is $12 \%$ at one RSD, ranging from $10-16 \%$ per tank depending on exactly how much sludge is in a given tank.

Most of the R2 waste sludge is on average distributed $30 \mathrm{kgal}$ each among 7 tanks. At $30 \mathrm{kgal}$, the variability will be $13 \%$, and will range from $10-16 \%$ for that set of tanks as well. It is interesting to note that despite the very different Redox campaigns, the waste rate variabilities are very similar between $\mathrm{R} 1$ and R2.

The waste rate variability represents a dilution of all species while the waste rate trend does not change the chemical composition at all, since chemicals added remain proportional to waste volume. On the other hand, there will be a bias in the radionuclide concentration through a campaign as a result of the waste rate trend. Radionuclides will be more dilute early in the campaign and more concentrated late in the campaign. Thus, there is an extra source of variability for radionuclides within each campaign that is tied to the waste rate trend parameter.

For example, the waste rate trend for $\mathbf{R} 1$ is $\pm 73 \%$ of the mean over the campaign, which places an effective RSD for the radionuclides at $\pm 50 \%$. Thus, while the chemical composition variability for these tanks is within an RSD of $\pm 12 \%$, the radionuclides vary with an RSD of $\pm 50 \%$. The HDW model does not account for this extra variation in radionuclides. Since only R1 waste shows this kind of extreme waste rate trend, the neglect of this effect should not be a bounding source of variability for any waste except R1.

Starting with the hypothesis that the waste rate variability is the most direct measure of process variability and therefore of HDW compositional variability, the two sources of waste rate variability are:

1) Rework processing. For a given amount of fuel processed during a campaign, early batches needed to be reworked more often than later batches because the separations failed to achieve the necessary decontamination or separation factors. Note that for rework, the chemicals in the waste scale linearly with the waste volume but the radionuclides will be diluted by increasing rework;

2) Ancillary processing resulting in primary waste dilution. There are many ancillary waste streams that derive from various cell cleanup and vessel cleanout activities. These activities by and large add very little or no chemicals or radionuclides to the waste stream. Therefore, to a first approximation, this variability simply dilutes or concentrates the waste stream. This dilution or concentration simply changes the relative supernatant and sludge inventories of each component.

The HDW approach completely neglects chemical source term variability, which derives from measurement errors during processing. This variability is in the range $3-5 \%$ and should therefore be bounded by the two variability sources noted above.

The variability of every process waste rate will actually be a combination of rework and ancillary processing and there is little information about the precise nature of this combination. Assuming that the amount of chemicals used scales linearly with the volume of the waste produced for rework processing, the waste compositions within each tank will actually be independent of the amount of process rework (radionuclides, as noted above, will be reduced in concentration by the increase in rework.) 


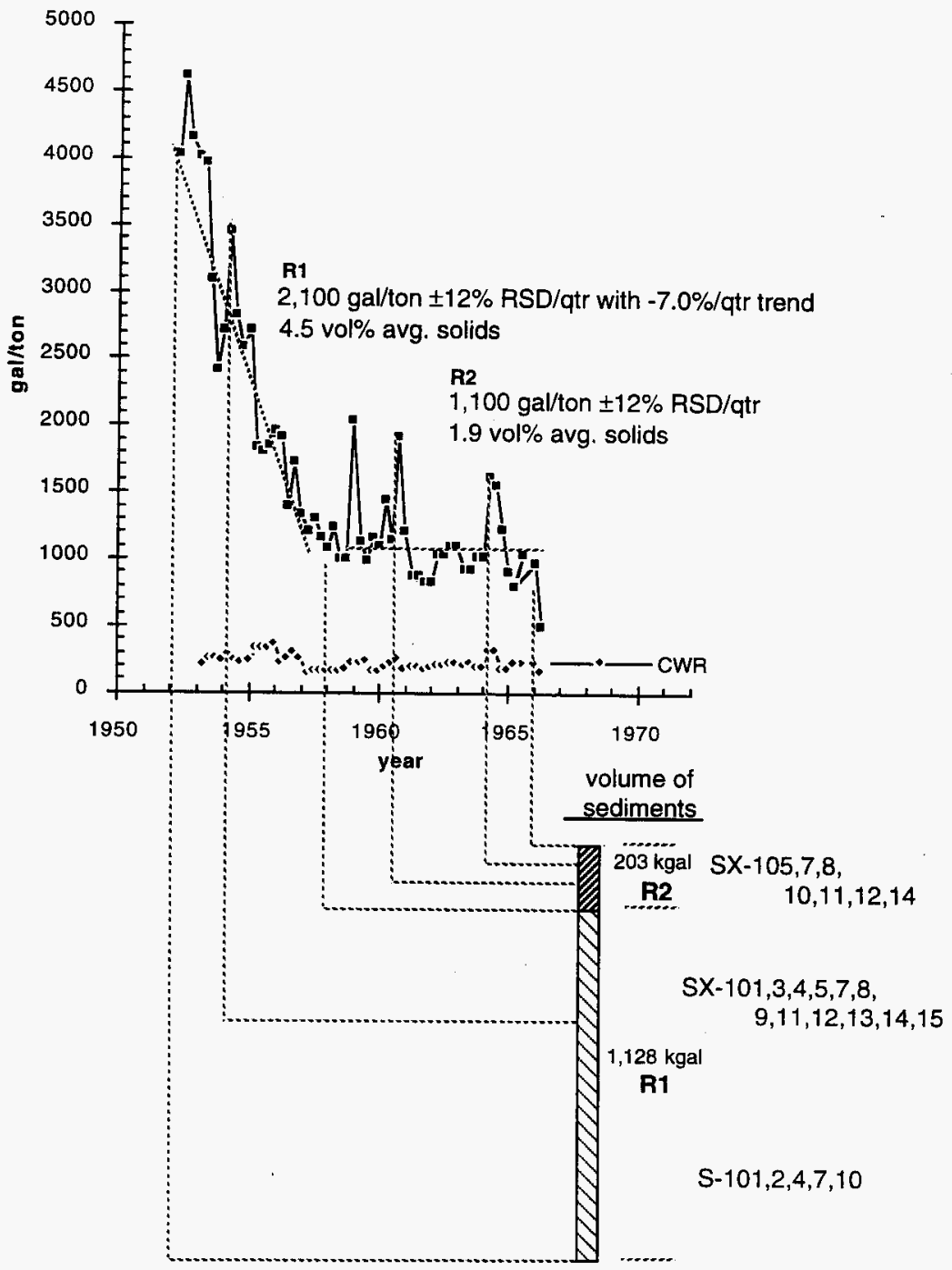

Fig. 18. Derivation of process variability and trend and relation to sludge layers. 
This approach subtracts a linear trend from each waste rate due to rework over the period of a campaign and makes the assumption that the resultant variability of the waste composition is wholly attributable to ancillary processing. This results in waste composition variabilities that should be equal to or greater than the true waste composition variabilities. In principle, the HDW model would need more information to assign the correct fraction of waste rate variability to process rework.

With these assumptions in hand, an uncertainty for each HDW can be derived by resolving each analyte for its upper and lower limits. An RSD (Relative Standard Deviation) for each HDW results in a set of upper and lower compositions for each component of each HDW. Note that these relative variabilities will be different in general from the overall RSD for each HDW. This is because of the fact that the solution concentrations of semi-soluble species are directly linked to their sludge inventories. The set of process variabilities is shown in Table 12.

\begin{tabular}{|c|c|c|c|c|c|c|}
\hline HDW type & $\begin{array}{c}1 \text { RSD } \\
\text { per } 52 \text { kgal* }\end{array}$ & $\begin{array}{c}1.96 \text { RSD } \\
\text { per } 52 \text { kgal* }\end{array}$ & $\begin{array}{l}1 \text { RSD } \\
\text { per qtr }\end{array}$ & $\begin{array}{l}\text { trend } \\
\text { per atr }\end{array}$ & $\begin{array}{c}\text { waste rate } \\
\text { kgal/gtr }\end{array}$ & $\begin{array}{c}\text { solids rate } \\
\text { kgal/gtr }\end{array}$ \\
\hline MW1 & $7.9 \%$ & $15.5 \%$ & $7.0 \%$ & $0.4 \%$ & 766 & 92.0 \\
\hline MW2 & $12.6 \%$ & $24.7 \%$ & $11.3 \%$ & $-0.6 \%$ & 817 & 98.0 \\
\hline $1 \mathrm{C1}$ & $15.6 \%$ & $30.6 \%$ & $14.4 \%$ & $-1.3 \%$ & 588 & 80.6 \\
\hline $1 \mathrm{C} 2$ & $26.0 \%$ & $51.0 \%$ & $22.8 \%$ & $-3.6 \%$ & 632 & 157 \\
\hline $2 \mathrm{Cl}$ & $15.4 \%$ & $30.2 \%$ & $23.6 \%$ & $-1.9 \%$ & 448 & 30.5 \\
\hline $2 \mathrm{C} 2$ & $12.6 \%$ & $24.7 \%$ & $15.5 \%$ & $0.2 \%$ & 1000 & 34.0 \\
\hline 224 & $14.0 \%$ & $27.4 \%$ & \pm & & & \\
\hline UR/TBP & $63.9 \%$ & $99.0 \%$ & $61.5 \%$ & $7.4 \%$ & 2090 & 58.6 \\
\hline $\mathbf{R} 1$ & $13.3 \%$ & $26.1 \%$ & $14.2 \%$ & $-5.7 \%$ & 929 & 41.8 \\
\hline $\mathbf{R} 2$ & $7.9 \%$ & $15.5 \%$ & $25.1 \%$ & $-1.1 \%$ & 325 & 6.2 \\
\hline CWA1 & $16.6 \%$ & $32.5 \%$ & $30.4 \%$ & $-6.0 \%$ & 100 & 8.1 \\
\hline CWR2 & $0.5 \%$ & $1.0 \%$ & $6.7 \%$ & $3.8 \%$ & 67.9 & 2.0 \\
\hline P1 & $26.8 \%$ & $52.5 \%$ & $32.6 \%$ & $-0.4 \%$ & 956 & 21.0 \\
\hline $\mathrm{P2}$ & $33.9 \%$ & $66.4 \%$ & $43.4 \%$ & $0.6 \%$ & 463 & 18.0 \\
\hline PL1 & $11.1 \%$ & $21.8 \%$ & $65.0 \%$ & $-16.2 \%$ & 132 & 2.9 \\
\hline CWP1 & $9.6 \%$ & $18.8 \%$ & $13.3 \%$ & $-1.9 \%$ & 305 & 24.7 \\
\hline CWP2 & $36.5 \%$ & $71.5 \%$ & $96.7 \%$ & $3.7 \%$ & 556 & 16.1 \\
\hline $\mathrm{CWZr1}$ & $36.5 \%$ & $71.5 \%$ & $\neq$ & & & \\
\hline OWW1 & $16.8 \%$ & $32.9 \%$ & $76.5 \%$ & $7.3 \%$ & 255 & 0.3 \\
\hline OWW2 & $6.7 \%$ & $13.1 \%$ & $48.9 \%$ & $1.0 \%$ & 582 & 0.6 \\
\hline OWW3 & $10.8 \%$ & $21.2 \%$ & $102.6 \%$ & $8.7 \%$ & 433 & 0.4 \\
\hline $\bar{Z}$ & $29.9 \%$ & $58.6 \%$ & $107.5 \%$ & $-5.7 \%$ & 80.5 & 1.9 \\
\hline $\mathrm{HS}$ & $9.7 \%$ & $19.0 \%$ & $\neq$ & & & \\
\hline TH1 & $33.9 \%$ & $66.4 \%$ & $\ddagger$ & & & \\
\hline $\mathrm{TH} 2$ & $33.9 \%$ & $66.4 \%$ & \pm & & & \\
\hline$B$ & $43.7 \%$ & $85.7 \%$ & $163.6 \%$ & $14.0 \%$ & 128 & 0.6 \\
\hline $\mathrm{BL}$ & $27.9 \%$ & $54.7 \%$ & $65.1 \%$ & $-2.0 \%$ & 183 & 1.2 \\
\hline SRA & $39.0 \%$ & $76.4 \%$ & $93.8 \%$ & $-7.2 \%$ & 153 & 4.0 \\
\hline $\mathrm{P3}$ & $15.3 \%$ & $30.0 \%$ & $72.2 \%$ & $10.5 \%$ & 63.1 & 2.5 \\
\hline PL2 & $29.5 \%$ & $57.8 \%$ & $91.5 \%$ & $4.3 \%$ & 377 & 7.5 \\
\hline CWZr2 & $50.3 \%$ & $98.6 \%$ & $77.8 \%$ & $2.6 \%$ & 262 & 27.5 \\
\hline PASF & $7.0 \%$ & $13.7 \%$ & $96.0 \%$ & $-3.5 \%$ & 528 & 3.2 \\
\hline
\end{tabular}

"Given a $19^{\prime \prime}$ core segment, equivalent to $52 \mathrm{kgal}$ average volume in 75 ' dia. tank.

¥Variabilities for 224, CWZr1, HS, TH1, and TH2 assigned to $2 \mathrm{C}, \mathrm{CWP} 2, \mathrm{SRR}, \mathrm{P2}$, and P2, respectively.

Finally, there is a fundamental correspondence that relates a tank's waste volume to a corresponding waste stream variability. That is, if a tank contains $75 \mathrm{kgal}$ of a waste sludge, then the waste rate variability must be calculated for the time that it took to deposit that $75 \mathrm{kgal}$ of sludge.

This is a very important point. A manifestation of waste heterogeneity within a tank is that the larger the waste sample taken from a tank, the more representative that sample will be to the mean value for that waste type. The waste rate variability quantifies that relationship. It means that the smaller the sample of 
waste in an assay, the larger the margin will be for comparison of that assay to the HDW estimates. Conversely, the larger the amount of waste sampled, the better it will represent the tank's waste and the smaller will be the margin for HDW estimate comparisons.

Because the variations of some materials result in changes in precipitated fractions, there is the possibility that an analyte distribution will "fold" and that the 1.96 RSD variation results in less analyte variation in a waste sludge than does a 1.0 RSD variation. This can occur for soluble analytes in sludges when a waste stream process variation results in a decrease in void fraction. Such a reduction in void fraction then works against the increase in analyte concentration, reducing whatever increase occurred.

The expression for a sludge analyte is

$$
h d w_{j}^{\text {slAnal }}=\frac{h d w_{j}^{\text {totAnal }} f_{j}^{\text {Anal }}}{1000 \rho_{j}^{\text {sl }} f_{j}^{\text {sl }}}+\frac{h d w_{j}^{\text {suAnal }} f_{j}^{\text {void }}}{1000 \rho_{j}^{\text {su }}}
$$

and for soluble analytes, $\mathrm{f}_{\mathrm{j}}^{\mathrm{Anal}}=0$ and so the first term is zero. In the second term, as long as the siudge liquid void traction does not change when the waste stream analyte concentration is increased, the soluble analyte will experience the same variation as all the other species within the waste. However, if the void fraction decreases as a result of a change in the fraction precipitated of semi-soluble species, then an increase in the analyte concentration for that waste will be partially mitigated by a corresponding decrease in void fraction. For extremely severe decreases in void fraction, void fraction reduction can actually dominate and decrease the soluble analyte concentration in the sludge layer.

\section{-HDW variability due to variations in analyte solubilities}

The HDW model assumes a single point solubility for each analyte regardless of what other species are present in solution. In reality a given analyte can potentially have quite different solubilities for different waste streams and as a result, the assumption of single point solubilities produces a source of variability within the HDW model.

For a given waste stream, the solubility variability of each component within that stream is not significant compared to the variation of analyte solubilities among HDW's. The variability for each analyte's solubility, then, represents the potential ranges of that analyte's solubility among HDW's. The HDW model assumption of a single solubility for each analyte among all HDW's dominates the variability of the HDW estimates for those analytes. Those variabilities are shown in Table 7.

The solubility variation is assumed to affect wastes volumetrically and randomly. As long as the particular HDW supematant is adequately represented in the solubility dataset, its true solubility should fall within the uncertainty calculation for all tanks. A solubility variation of one RSD represents the uncertainty of predicting an anaiyte among the set of HDW's and therefore among the set of waste tanks.

Each of the analytes and radionuclides has a variability that can be calculated based on either one of two parameters: one RSD (relative standard deviation) of process variability or one RSD of analyte solubility. Depending on the particular details of each waste stream, either one of these sources of variability can dominate the variability of any of the HDW's species. If the concentration basis is inventory limited (i.e. no analyte is precipitated) then the process variability dominates that species' variability.

Therefore, it is necessary to partition all analytes according to their upper and lower limits at one RSD of either solubility or process dilution variabilities. In addition, we need to derive a 1.95 SD or $95 \%$ confidence limit for each of these variabilities. This is because of the sometimes extremely non-normal distributions that result for particular cases. For example, lowering Pu solubility from 30 to $6 \mu \mathrm{Ci} / \mathrm{L}$ for $2 \mathrm{C} 1$, a factor of six, raises the sludge Pu concentration by a factor of twelve, from 0.011 to $0.13 \mu \mathrm{Ci} / \mathrm{g}$ in $2 \mathrm{C} 1$ sludge. This is caused by the fact that no Pu precipitates in the sludge at all at $30 \mu \mathrm{Ci} / \mathrm{L}$ while $60 \%$ is precipitated at $6 \mu \mathrm{C} / \mathrm{L}$ solubility. As an analyte solubility decreases to the point where precipitation occurs for a given HDW, the analyte sludge concentration increases roughly in proportion to the ratio of total waste to sludge volumes for that HDW. This means that the concentration of a sludge analyte can vary quite dramatically provided the solubility limit is exceeded and the sludge volume fraction is small. 


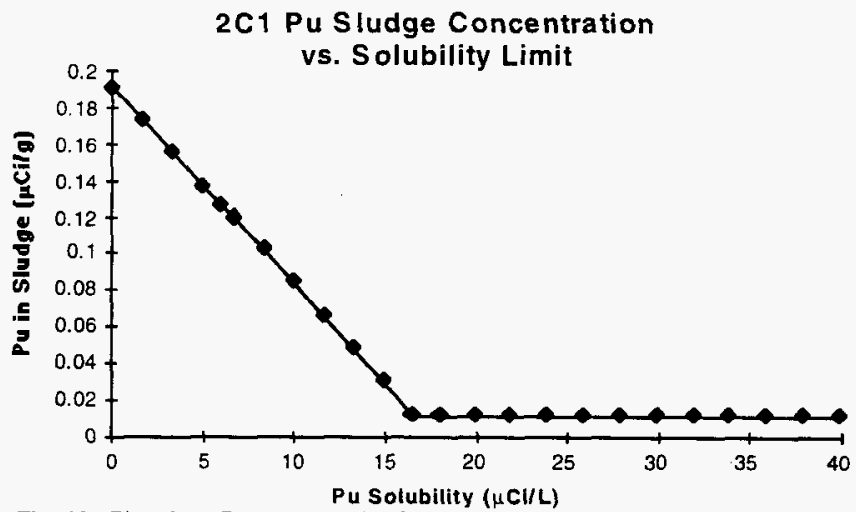

Fig. 19. Plot of the Pu concentration for $2 C 1$ sludge versus the Pu solubility limit.

This is illustrated in (Fig. 19), where a plot of the Pu concentration for $2 \mathrm{C} 1$ sludge versus the $\mathrm{Pu}$ solubility limit shows that the sludge concentration remains constant until Pu begins to precipitate at 16.3 $\mu \mathrm{Ci} / \mathrm{L}$. In the solubility regime above $16.3 \mu \mathrm{Ci} / \mathrm{L}, \mathrm{Pu}$ is present only in the interstitial liquid of the sludge. In the Pu solubility regime below $16.3 \mu \mathrm{Ci} / \mathrm{L}$, Pu partitions increasingly to the sludge and the sludge $\mathrm{Pu}$ concentration is directly proportional to the solubility limit with a slope proportional to the inverse of the solids fraction. Thus, changes in the Pu solubility for a waste once it precipitates result in changes in the Pu sludge concentration that are proportional to the inverse of the solids fraction for that waste. The calculation for the HDW supernatant Pu concentrations is

$$
h d w_{j}^{\text {suPu }}=\frac{h d w_{j}^{\text {lotPu }}\left(1-f_{j}^{P u}\right)}{1-\left(1-f_{j}^{\text {void }}\right) f_{j}^{\text {sl }}} .
$$

This expression defines the supernatant concentration following precipitation of $\mathrm{f}^{\mathrm{Pu}} \mathrm{j}_{\mathrm{j}}$ fraction of total Pu for the jth HDW. The denominator accounts for the excluded volume of the solids that precipitate from the waste stream and therefore are no longer part of the solution. The sludge concentration is calculated as

$$
h d w_{j}^{s l P u}=\frac{h d w_{j}^{\text {totPu }} f_{j}^{\text {Pu }}}{1000 \rho_{j}^{\text {sl }} f_{j}^{s l}}+\frac{h d w_{j}^{\text {suPu }} f_{j}^{\text {void }}}{1000 \rho_{j}^{\text {su }}}
$$

where the first term is the contribution to the sludge Pu due to Pu solids and the second term is the contribution to the sludge $P$ u due to interstitial hiquid. The sludge concentration is expressed on a mass basis, $\mu \mathrm{Ci} / \mathrm{g}$, and the inverse dependence on the sludge volume fraction, $\mathrm{fs}^{\mathrm{s}}$, , occurs in the first term.

For Pu concentrations that fall below its solubility limit, the first term is zero since the fraction precipitated is zero as

$$
\mathrm{f}_{\mathrm{j}}^{\mathrm{Pu}}=0, \text { hdw }_{\mathrm{j}}^{\text {totPu }}<\mathrm{Pu} \text { solubility } .
$$

If, on the other hand, the Pu concentration exceeds its solubility limit, the first term is non zero since the fraction precipitated is greater than zero as 
where

$$
f_{j}^{P l}=1-\frac{h d w_{j}^{s u P u}}{h d w_{j}^{\text {lotPu }}}\left[1-\left(1-f_{j}^{\text {void }}\right) f_{j}^{s l}\right],
$$

and

$$
h_{\mathrm{d}} \mathrm{w}_{\mathrm{j}}^{\mathrm{totPu}} \geq \mathrm{Pu} \text { solubility }
$$

$$
\begin{aligned}
& h d w_{j} \mathrm{slPu}=\text { Pu concentration for HDW sludge } \mathrm{j} \text { in } \mu \mathrm{Ci} / \mathrm{g} \text {. } \\
& n d w_{j} \text { suPu }=\text { Pu concentration for } \mathrm{HDW} \text { supematant } \mathrm{j} \text { in } \mu \mathrm{Ci} / \mathrm{L} \text {, } \\
& \text { hdw } w_{j}^{\text {totPu }}=\text { total Pu concentration for } H D W \mathrm{j} \text { in } \mu \mathrm{Ci} / \text {, } \\
& \mathrm{Pu}_{\mathrm{j}}=\text { fraction of total Pu precipitated for HDW j. } \\
& \text { froid }=\text { liquid void fraction for jth HDW sludge, } \\
& \mathrm{f}^{5 \mathrm{j}}=\text { solids volume traction for HDW } \mathrm{j} \text {, } \\
& \rho^{\text {s.j }}=\text { sludge density for HDW } \mathrm{j} \text {, } \\
& \rho^{s u j}=\text { supernatant density for HDW } \mathrm{j} \text {. }
\end{aligned}
$$

The above expressions all neglect the volume and mass of the Pu solids within the sludge.

Reported Pu concentrations for B-110 sludge, which contains $2 \mathrm{C} 1$ and $2 \mathrm{C} 2$ sludges are 0.13 $\mu \mathrm{Ci} / g$. Therefore, this particular tank's sludge is consistent with a Pu solubility of $-6 \mu \mathrm{Ci} / L$.

The upper quartile of the supematant solubility data set is assumed to represent saturation conditions for the entire population of HDW wastes. By further assuming that this upper quartile represents a normal distribution with a corresponding mean and standard deviation, these solubility statistics can be applied to each HDW in order to propagate a variability due to this solubility.

This distribution is assumed to be valid for HDW's that correspond to the mean total waste volume per waste, $9,028 \mathrm{kgal}$ or $2.2 \%$. The variation in solubility is for the population of HDW's and if any HDW supernatant is not well represented, then its distribution may exceed the nominal values used here. If the sampling rate of each HDW supernatant is proportional to its volume fraction of site wastes, there will be an increase in uncertainty for wastes with small volume fractions and a decrease in uncertainty for wastes with larger volume fractions. For example, since $2 \mathrm{C} 1$ waste represents $2.2 \%$ of the total HDW volume, the solubility data variability should represent this waste without adjustment.

Any comparison of HDW estimates with assay data must compare data across more than one tank in order to effectively sample the same population of wastes for which the HDW model solubility variabilities are derived. As a result, quantitative comparisons of HDW model variability must be made across more than one tank. In addition, comparisons must be performed on both concentration and inventory bases. This is because the population upon which solubility variability is based represents all tanks, those with large inventories as well as those with small inventories. Only comparisons among all tanks, then, will test this source of variability.

\section{-Transaction variability}

There are three contributions to transaction variability; evaporator blending, concentrate carryover, and of course, inaccurate transaction information. As regards to inaccurate information, it is not possible to derive meaningful uncertainty estimates about what is not known. Therefore, variability estimates are only possible for the first two contributions.

Evaporator blending and concentrate carryover are now both approximations used within the HDW model. Evaporator blending assumes that all of the waste feed for a given time can be blended together and reduced in volume as a blend and then transferred to a bottoms receiver. In reality, this process was continuous volume reduction with continuous feed.

Concentrate carryover is the result of an approximation within the HDW model whereby a liquid that is removed and recycled to an evaporator from a bottoms receiver is assumed to be homogeneous 
mixture of the entire concentrate inventory of that tank. This approximation is very good for dilute wastes but increasingly invalid as wastes are reconcentrated in subsequent campaigns. In reality, waste concentrates are returned to bottoms receivers from an evaporator and allowed to cool, sediment, and gel. As a result, the residual liquid that is removed from these tanks, reblended, and further concentrated is not identical with a homogeneous blend. The HDW model then allows concentrated waste to be "carried over" into later receivers because of its blending assumptions. This represents a second major source of variability within the model, but it only affects concentrated wastes.

In fact, the SMM model variability is strongly associated with concentrate carry over. Sending more concentrated waste from slurry receivers into the next generation of evaporator receivers increases sodium and nitrate concentrations in later waste receivers over that that actually occurs. For example, suppose concentration of a waste blend results in a product with an average sodium concentration of 12 $M$. Following addition of this mixture to a slurry receiver, precipitation and sedimentation would produce two layers of waste: perhaps two-thirds of the volume as a settled solids layer at $13 \mathrm{M} \mathrm{Na}$ and one-third of the volume as a supernatant layer at $10 \mathrm{M} \mathrm{Na}$.

The supernatant layer is then removed, reconcentrated, and placed into another tank. The result is a $20 \%$ over estimation by the HDW model of $\mathrm{Na}(12 \mathrm{M}$ instead of $10 \mathrm{M}$ ) in this second generation of concentrates and an $8 \%$ underestimation in the slurry remnant (12 versus $13 \mathrm{M})$. If the overconcentration bias were propagated again, this third generational error would be $44 \%\left(1.2^{2}\right)$ even though the tank wastes of previous generations would still each only be subject to an $8 \%$ underestimate (12 $\mathrm{M}$ instead of $13 \mathrm{M}$ ). The concentrate carry over error manifests itself primarily in overconcentration in second and third generation slurry receivers that are removed and distinct from the original slurry receivers. For example, tank $\mathrm{AN}-105$ has an HDW estimated $\mathrm{Na}$ of $18.1 \mathrm{M}$ and was involved in blending and concentrating second and third generation concentrates. Therefore, the HDW model estimate may be greater than is now actually in the tank by $20-44 \%$.

The effect of random transaction errors is largely damped as the number of transactions increases as shown below. HDW model estimate is based upon the sum products of the SMM and TLM matrices within the corresponding HDW composition vectors as

$$
\operatorname{tank}_{\mathrm{i}}=\frac{\sum_{\mathrm{j}} \operatorname{tlm}_{\mathrm{ij}} \mathbf{h d w _ { j }}{ }_{\mathrm{j}}^{\mathrm{sl}}}{\text { slVol }_{\mathrm{i}}}+\frac{\sum_{\mathbf{j}} \mathrm{smm}_{\mathrm{ij}} \mathbf{h d w}_{\mathrm{j}}^{\text {su }}}{\text { suVol }_{\mathrm{i}}}
$$

where

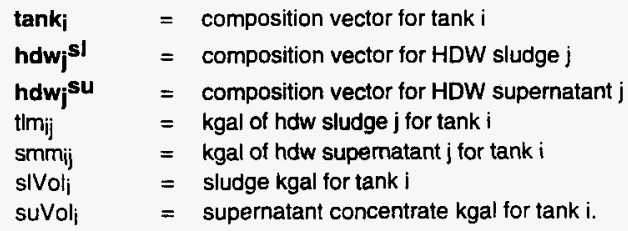

Introducing variabilities into the calculation for each analyte results in

$$
\operatorname{tank}_{i}^{s u N a}+\Delta_{i}^{s u N a}=\frac{\sum_{j}\left(s m m_{i j}+\delta_{i j}^{s m m}\right)\left(h_{d w} w_{j}^{s u N a}+\delta_{j}^{s u N a}\right)}{\text { suVol }_{i}}
$$

where

$$
\begin{array}{lll}
i & = & \text { th tank, } \\
\mathrm{i} & = & \mathrm{jth} \mathrm{HDW}, \\
\tan \mathrm{k}_{\mathrm{i}} \text { suNa } & =\mathrm{Na} \text { concentration in supernatant concentrate for ith tank, }
\end{array}
$$




\begin{tabular}{|c|c|c|}
\hline$\Delta j^{\text {SuNa }}$ & $=$ & variation in SMM Na for ith tank, \\
\hline$s m m_{i j}$ & $=$ & kgal of jth hdw supernatant for ith tank, \\
\hline $\mathrm{d}_{\mathrm{j} j}^{\mathrm{smm}}$ & $=$ & Im volume for ith tank and jth $\mathrm{HDW}$, \\
\hline $\begin{array}{l}\text { hdwj suNa } \\
\mathrm{dj}_{\text {suNa }}^{\text {suNa }} \\
\text { suvol }\end{array}$ & $\begin{array}{l}= \\
= \\
=\end{array}$ & $\begin{array}{l}\text { Na concentration for jth HDW supernatant, } \\
\text { variation in supernatant } \mathrm{Na} \text { for jth } \mathrm{HDW} \text {, } \\
\text { supernatant concentrate kgal for ith tank. }\end{array}$ \\
\hline
\end{tabular}

Insofar as incomplete blending and transaction misdirection act as random variations among the components of an SMM matrix column vector, the sum of those variations is zero,

$$
\sum_{i} \delta_{i j}^{s m m}=0
$$

This is simple a statement that if waste volume doesn't go to one tank, it must go to another. With further transactions, these random variations average to zero for each tank's SMM components. If we suppose multiple transactions result in an accumulation of these random blending errors over $k$ transactions, the result is

$$
\operatorname{tank}_{i}^{s u N a}+\Delta_{i}^{s u N a}=\frac{\sum_{j}\left(s m m_{i j}+\sum_{k} \delta_{i j}^{s m m_{k}}\right)\left(h d w_{j}^{s u N a}+\delta_{j}^{s u N a}\right)}{s u V o l_{i}}
$$

\begin{tabular}{|c|c|c|}
\hline & $\begin{array}{l}= \\
=\end{array}$ & $\begin{array}{l}\text { ith tank, } \\
\text { jth HDW, } \\
\text { kth transaction, }\end{array}$ \\
\hline tanki suNa & $=$ & Na concentration in supernatant concentrate for ith tank, \\
\hline$\Delta_{i}^{\text {suNa }}$ & $=$ & variation in SMM Na for ith tank, \\
\hline & $=$ & $\begin{array}{l}\text { kgal of jth hdw supernatant for ith tank, } \\
\text { variation in smm volume for ith tank and jth HDW, }\end{array}$ \\
\hline hdwj suNa & $=$ & Na concentration for jth HDW supernatant, \\
\hline$d_{i j}$ suNa & $=$ & ant $\mathrm{Na}$ for jth $\mathrm{HC}$ \\
\hline
\end{tabular}

where

For $k$ sufficiently large, we expect that

$$
\sum_{\mathrm{k}} \delta_{\mathrm{ij}}^{\mathrm{smm}}=0
$$

or that the sum of random blending and misdirection errors for a sufficiently large number of transactions will be zero. incomplete waste blending, then, leads to a source of variability that is actually minimized as the number of transactions associated with those waste elements increases.

An increase in the reported liquid level of a tank that is not associated with a waste transaction is assigned to an unknown waste addition, which results in an accumulation of an UNK waste type within the SMM. Such unknowns can be due to a number of factors including undocumented waste or water additions, thermal expansion, sturry growth due to gas entrapment, apparent liquid increases due to solids slumping from higher levels, rainwater intrusions, or simply due to errors in measurement of waste volume. Within the HDW model estimates, these unknown assignments are simply attributed to water.

Unexplained decreases in tank inventory are always assumed to be due to loss of water (i.e. concentration of the waste) or gas. Therefore, no inventory except water is ever lost because of 
unassigned inventory losses. Unassigned losses from waste tanks are due to undocumented waste removal, water evaporation, thermal contraction due to cooling, collapse following entrapped gas venting from slurries, subsidence of waste following salt well pumping, undocumented leaks, and of course, measurement errors. Note that leaks that have been assigned to inventory losses are tracked within the HDW model.

\section{-Variability due to waste heterogeneity}

Two important geometric demarcations of waste heterogeneity are vertical and lateral within each tank. Although vertical and lateral heterogeneities are manifestations of the same waste fragments, it is nevertheless useful to discuss them separately.

Vertical heterogeneity: The primary causes of vertical heterogeneity are changes in kinds of waste that are added to each tank. A secondary source of vertical heterogeneity is due to process variations within each HDW layer. However, much of this source of variability is already accounted by inclusion of process variability in the HDW model.

Lateral heterogeneity: There are many causes for lateral heterogeneity within each waste tank but it is important to recognize that lateral heterogeneity is simply another manifestation of vertical heterogeneity. That is, as the process history changed and evolved for each tank, the lateral distribution of waste fragments around a tank also changed and evolved. Several specific origins of lateral waste heterogeneity are:

- salt-well pumping and subsequent solids slumping,

- localized sedimentation and slumping.

- redissolution of precipitated salts,

- salt domes, holes, and floating crusts,

- liquid pools under risers, and

- wall scale and ledges.

It is important to recognize the factthat the HDW model does not account for much of this source of waste variation. Some of the components of vertical variation are implicit in the TLM and SMM layer descriptions, but there is no allowance for lateral heterogeneity within the HDW model at all.

\section{Calculating Tank Inventories from HDW Compositions}

Final tank inventory estimates are derived by using amounts for each of the HDW sludge and supernatants present in each tank. These amounts are derived separately for the tank sludges and supernatants. The sludge layers are assigned by the Tank Layer Model (TLM), where the total volumes of waste types and corresponding solids volume per cent for each of those wastes are used. This results in layers of sludges that are expressed in $\mathrm{kgal}$ and have a chronology or order within the tank. However, the lateral heterogeneity present in many tanks precludes interpreting these layers as necessarily flat and level. For the supernatants and their concentrates, the Supernatant Mixing Model (SMM) provides a composition in terms of a combination of HDW supernatants. Unlike the TLM, though, there is no chronology to the HDW componentswithin the SMM since the SMM assumes ideal and complete mixing within each tank following a transaction.

These derived compositions can then be compared to analytical results from sampling events, taking into account the unsampled dish volume, as well as any segment recoveries less then $100 \%$-merely weight that particular layer with a lower factor. Lateral inhomogeneities, however, are still a big problem when a comparison between the historical fill data and measured data is performed. Once again, the HDW model does not account for lateral heterogeneities.

\section{Results and History of Revisions}

Appendix E shows the composition and inventory for each of the 177 Hanford Waste tanks. Each tank is described by three tables and each table comprises three columns of information. Two columns describe the analyte concentrations as $\mathrm{mol} / \mathrm{L}$ and $\mathrm{ppm}$ and the third column expresses the tank inventory in $\mathrm{kg}$ or $\mathrm{MCi}(1 \mathrm{MCi}=1 \mathrm{e} 6 \mathrm{Ci}$ ). The three tables represent TLM solids blend, SMM liquids blend, and total composite tank concentrations and inventories. The TLM solids composition and inventory represents the volume average blend of all of the TLM solids layers. Note that among the TLM solids 
HNF-SD-WM-ER-323, Rev. 1

definitions are four salt cake concentrates: $\mathrm{B}, \mathrm{T1}, \mathrm{R}$, and BY. Therefore, the TLM solids inventory definition includes sludges and some salt cake.

The second table for each tank represents the SMM composite inventory for liquids and concentrates. This table represents inventories from evaporator concentrates termed T2, S1, S2, Ai, and A2. Note that these concentrates actually include a lot of solids but are treated nevertheless as homogeneous mixtures that can be pumped, blended, and moved to other tanks as though they were liquid.

The HDW model provided its first estimates in June of 1994 as Rev, 0 for the NE and SW quadrants. This early revision was based on single waste types for salt cake and salt slurry for the entire site. Revision 1 was actually the first complete site inventory and was completed in Fall of 1994 for the three SST quadrants, NE, SW, and NW, while Rev. 1 for the DST SE quadrant was completed in March 1995. These estimates included many bug fixes and other corrections and also included additions for process vessel corrosion source terms (adds $\mathrm{Fe}, \mathrm{Cr}$, and $\mathrm{Ni}$ ) as well as a hard water $\mathrm{Ca}$ source term. However, the Cs-137 and Sr-90 inventories were calculated too high by about $20 \%$ and all evaporator campaigns were blended into multi-year composites. These evaporator blends were an improvement over the single waste types for salt cake and salt slurry in Rev. 0 , but still represented an approximation for individual tanks. Essentially, these evaporator blends were excellent representations of the total waste into a campaign and its total volume reduction, but were distributed across perhaps ten or twenty different slurry receivers that were involved in each campaign.

The next step with the HDW Estimates, Rev. 2, was an attempt to express the five later evaporator campaigns on a tank by tank basis. The SMM provided the waste concentrate history step by step throughout each of the evaporator campaigns. Revision 2.1 represents a bug fix in the spreadsheet that incorrectly calculated water and TOC and another problem with miscalculation in SX Farm. This revision was based on the HDW Rev. 2 compositions, which had improved the Cs-137 and Sr-90 inventory calculation and had included chloride and potassium source terms that piggyback on the $\mathrm{NaOH}$ additions. Various other bug fixes and changes and additions were a mercury source term used in the decladding process, adjustments on the wastes from UR (Uranium Recovery), slight realignments of $1 \mathrm{C}$ and $2 \mathrm{C}$ waste campaigns, and other minor changes. Revision 2 also reduced the process vessel corrosion source term ( $\mathrm{Fe}, \mathrm{Ni}, \mathrm{Cr}$ ) for early BiPO4 wastes and decladding wastes consistent with the fact that these processes were much less corrosive than either Purex or Redox.

The Rev. 2.1 estimates nevertheless had some problems. The most significant problem was the incomplete transaction records for the later evaporator campaigns caused incorrect distribution of waste concentrates. In particular, some tanks were impossibly over concentrated ( $\mathrm{Na}$ in excess of 16-17 mol/L), while other slurry receivers were more dilute than they should have been. It was clear that there were severe problems in waste misdirection with Rev. 2.

To correct these problems, the Rev. 3 extensively modified WSTRS by adjusting the evaporator transactions to blend on a per quarter basis and for some quarters, wastes have been blended on an even finer time scale. This improvement in the transaction record was largely accomplished by use of the Logbook Dataset ${ }^{5}$, derived from date provided by ICF Kaiser for WHC. Aiso used is an extensive set of reports from evaporator operations for 242-S and 242-T. Unfortunately, there was a lack of detailed information about the 242-T evaporator operation.

The overall inventories for the analytes have not changed significantly except for lead, manganese, and oxalate. Lead site inventories increased dramatically in Rev. 3 since these estimates included the lead coating that covered each fuel slug. This turns out to be a major source of lead in the waste tanks and the total lead inventory increased from 3 to $280 \mathrm{MT}$. There was also an error in the concentration of manganese in OWW2, which upon correction lowered the manganese site inventory from 219 to $39 \mathrm{MT}$. The oxalate inventory increased from 23 to $69 \mathrm{MT}$ because of a decrease in its solubility limit. Since 224 waste supernatant was all cribbed, decreasing oxalate solubility retains more in the waste tanks and this waste was the only process oxalate source term. 


\section{Uses and Limitations of HDW Model Estimates}

The HDW Model Rev. 4 estimates represent a Hanford site inventory based on process history that is compatible with the waste types, compositions, and processing history of the site. These total site estimates will not change appreciably in the future unless the waste source terms for the various waste streams change, but it is possible that corrections in the transaction record will alter the inventory estimates of individual tanks. All estimates are valid as of 1-1-94 and $\mathrm{Sr}-90$ and $\mathrm{Cs}-137$ are both decayed to the same date. Therefore, these estimates do not account for the latest evaporator campaign in '95'96, which moved and blended large amounts of waste supernatants in the DST's.

The HDW estimates are the first complete, total, ion, and mass balanced inventory estimates yet provided on a per tank basis. As such, they have immediately shown that site sodium inventory has been traditionally overestimated by about one third. Whereas previous site estimates for sodium were around $71,000 \mathrm{MT}$ (MT = metric tonnes), the HDW estimate show only $48,000 \mathrm{MT}$ are actually now in either the DST's or the SST's. This difference is largely due to the large amount of waste supernatant that was sent to crib, which contained some $20,000 \mathrm{MT}$ of $\mathrm{Na}$, but is also due to more subtle double counting of waste stream chemicals that has occurred in past site estimates.

The HDW model estimates have also shown an increase in the iron and chromium inventories, which the HDW model now estimates at 1,830 MT (1,610 in the SST's and 220 in the DST's) as compared to previous site estimates of 710-730 MT. These total site estimates are shown in (App. E) along with estimates for individual tanks.

The site inventory estimates include totals for waste sent to the cribs as well as totals for leaks with measurable volume losses. Note that the leaks from waste tanks are a small fraction of the total inventory sent to the ground, constituting only $10 \%$ of the $2.2 \mathrm{MCi}$ of $\mathrm{Cs} / \mathrm{Sr}$ activity and only $2 \%$ of the 48 $\mathrm{kg}$ of $\mathrm{Pu}$ that was sent to the soil column. Thus, the amount of activity intentionally sent to the soil column is much greater than the activity inadvertently placed into the ground by leaks and spills. However, recent work has suggested that some leak inventories have been severely underestimated. 42

There are still problems with these estimates. The evaporator blending and SMM approaches naturally produce blended averages for waste supernatants that were processed during each quarter. The actual blending that occurred during these quarters may not be represented very well in this approximation. This biending error then contributes to the overall variability in the waste predictions.

Another problem with the HDW model is that precipitated solids from waste concentration do not remain in the slurry receiver during evaporator runs. That is, liquid that is drawn from each bottoms tank following cooling is always removed as a blend of the total concentrate. This leads to an under concentration of the bottoms receiver and correspondingly an over concentration of tanks that receive and further blend and concentrate the recycled liquors. This effect systematically shifts concentrate from early receivers to later receivers and therefore increases the variability of the estimates by introducing a systematic bias in early versus late concentrates.

There are a number of chemical and physical constraints within the HDW model. For example, ion and mass balance are imposed, waste neutralization is assumed for all wastes (except NIT), void fraction must never fall below some reasonable minimum value $(-0.20)$, and the water wt\% must also be some reasonable amount ( $>-20 \mathrm{wt} \%)$. It is useful to also realize that both nitrate and nitrite are precipitated as per their original concentrations. Subsequently, nitrate and nitrite both undergo radiolysis at a rate that depends on the activity of the waste. Therefore, the nitrate and nitrite concentrations will both be lower than that for when they first precipitated. In general, then, nitrate will be increasingly undersaturated and nitrite will be increasingly over its saturation point.

As noted above (see water wt\%), the HDW estimates do not include any drying of the waste that may have occurred within a tank but not part of an evaporator campaign. Notably, tanks in SX Farm have been on active ventilation to keep the sludges cool for a number of years and therefore have dried out considerably. The combination of active ventilation and high heat load combine with these tanks to

\footnotetext{
42 Agnew, S.F. and Corbin, R.A. "Analysis of SX Farm Leak Histories-Historical Leak Model (HLM)," LA-UR-963537, October 1996.
} 
produce some of the driest waste inventories at Hanford. This phenomenon is not represented at all within the HDW model.

The derivation of tank inventories from tank assay information is no less a challenge than that undertaken here with the HDW model. The extremely heterogeneous wastes within each tank complicate the collection of a representative set of samples, compounded by limited access to the tank waste. Theretore, comparisons between inventory estimates based on waste assays with those of the HDW model actually involve comparing one model with another model and both models have significant variabilities. Whereas assay variabilities are derived for one tank's waste, HDW estimate variabilities actually cover groups of tanks. Therefore, comparisons between the HDW model and assays are more effective if they are made among groups of tanks with similar process histories. Such tank grouping strategies can be very important in comparisons between assay data and HDW predictions.

Determinations of variability for waste assays involve the variation of analyte concentration as a function of position within each waste tank. Normally, this variation is assumed to represent a random population and is reported on a log-normal scale. This distribution of analyte concentrations is only valid in principle for that tank's waste. The HDW analyte variation on the other hand really pertains to a group of tanks with similar process histories. As a result, HDW variations do not have quite the same meaning as do variations derived from tank waste assays. 
HDW Model Rev. 4

\begin{tabular}{|c|c|c|c|c|c|c|c|}
\hline \multicolumn{8}{|c|}{ Single-Shell Tank 241-S-101 } \\
\hline & \multicolumn{7}{|c|}{ TLM Solids Composite Inventory Estimate* } \\
\hline $\begin{array}{l}\text { Physical } \\
\text { Properties }\end{array}$ & & & & $-9 \mathrm{~S} \mathrm{CI}$ & $-67 \mathrm{CI}$ & $+67 \mathrm{CI}$ & 495 CI \\
\hline Total TLM Wast & $1.39 \mathrm{E}+06(\mathrm{~kg})$ & $(211 \mathrm{kgs})$ & 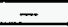 & $\cdots$ & $\ldots$ & $+\cdots$ & - \\
\hline Heat Land & $3.39(\mathrm{~kW})$ & $(1.16 \mathrm{E}+04 \mathrm{BTU} / \mathrm{hc})$ & $=$ & 2.77 & 3.13 & 3.56 & 3.64 \\
\hline Buik Density & $1.74(g / c)$ & $\cdots$ & - & 1.02 & 1.64 & 1.89 & 1.95 \\
\hline Void Fraction & 0.658 & $=$ & - & 0.450 & 0.519 & 0.746 & 0.748 \\
\hline Water $w t \%$ & 29.9 & $\cdots$ & - & 17.4 & 21.4 & 36.4 & 38.2 \\
\hline TOC wt\% C (wet & $2.21 \mathrm{E}-03$ & $\ldots$ & - & 1.97E-03 & $2.03 \mathrm{E}-03$ & $2.38 \mathrm{E}-03$ & 2.57E-03 \\
\hline $\begin{array}{l}\text { Chemical } \\
\text { Constituents }\end{array}$ & moleh & ppm & $\mathbf{k g}$ & $\begin{array}{l}-95 \mathrm{Cl} \\
\text { (motili) }\end{array}$ & $\begin{array}{r}67 \mathrm{CI} \\
\text { (mole/L) }\end{array}$ & $\begin{array}{l}+67 \mathrm{Cl} \\
\text { (tilolen })\end{array}$ & $\begin{array}{c}+95 \mathrm{CI} \\
\text { (mole/t) }\end{array}$ \\
\hline $\mathrm{Nat}$ & 10.4 & $1.37 \mathrm{E}+05$ & $1,90 \mathrm{E}+05$ & 8.15 & 8.37 & 13.2 & 148 \\
\hline$A 13+$ & 6.03 & $936 \mathrm{E}+04$ & $1.30 \mathrm{E}+05$ & 5.20 & 5.44 & 6.67 & 7.32 \\
\hline $\mathrm{Fec}^{3+(\operatorname{tata} \mathrm{Fe})}$ & 0,472 & $1.51 E+04$ & $2.10 E+04$ & 0.464 & 0.468 & 0.476 & 0.480 \\
\hline $\mathrm{Cr} 3+$ & 0.378 & 1.13E+04 & $1.57 \mathrm{E}+04$ & 0.232 & 0.302 & 0.578 & 0.813 \\
\hline $\mathrm{Bi3+}$ & 2.93E-06 & 0.352 & 0489 & $2.48 E-06$ & $2.70 \mathrm{E}-06$ & 3.23E-06 & $3.55 \mathrm{E}-06$ \\
\hline Lot+ & $7.22 \mathrm{E}-12$ & $5.76 \mathrm{E}-07$ & $8.010-07$ & $6.33 \mathrm{E}-12$ & $6.63 \mathrm{E}-12$ & $7.98 \mathrm{E}-12$ & \$.7TE-12 \\
\hline $\mathrm{Hg} 2+$ & $5.61 E-04$ & 64.7 & 89.9 & $5.53 \mathrm{E}-04$ & $5.58 \mathrm{E}-04$ & $5.64 \mathrm{E}-04$ & 5.65E-04 \\
\hline $\mathrm{Zr}(\operatorname{as~} \mathrm{ZrO}(\mathrm{OH}) 2)$ & $2.93 E-07$ & $1.53 \mathrm{E}-02$ & $2.13 \mathrm{E}-02$ & $2.69 \mathrm{E}-07$ & $2806-07$ & $3.07 \mathrm{E}-07$ & $3.31 \mathrm{E}-07$ \\
\hline $\mathrm{Pb2+}$ & $1.62 E-02$ & $1.93 \mathrm{E}+03$ & $2.69 \mathrm{E}+03$ & 1.49E-02 & $1,57 \mathrm{E}-02$ & $1.68 \mathrm{E}-02$ & $1.74 E-02$ \\
\hline $\mathrm{Ni2t}$ & $2.87 \mathrm{E}-02$ & 968 & $1.34 \mathrm{E}+03$ & $2.25 \mathrm{E}-02$ & $2,60 \mathrm{E}-02$ & $3.07 \mathrm{E}-02$ & $3.03 \mathrm{E}-02$ \\
\hline $\mathrm{Sr} 2+$ & 0 & 0 & 0 & 0 & 0 & 0 & o \\
\hline $\operatorname{Mn} 4+$ & $2.13 \mathrm{E}-05$ & 0.674 & 0.936 & 1.52E-05 & $1.82 \mathrm{E}-03$ & 2.45E-05 & $2.76 \mathrm{E}-05$ \\
\hline $\mathrm{Ca} 2+$ & 0.145 & $3.35 E+03$ & $4.65 \mathrm{E}+03$ & 0.113 & 0.129 & 0.162 & 0.178 \\
\hline $\mathrm{K+}$ & $1.88 \mathrm{E}-02$ & 422 & 586 & $1.44 \mathrm{E}-02$ & $1.62 \mathrm{E}-02$ & $2.05 \mathrm{E}-02$ & $2.13 \mathrm{E}-02$ \\
\hline OH. & 24.9 & $2,43 \mathrm{E} * 05$ & $3,38 E+05$ & 21.0 & 22.3 & 27.6 & 30.3 \\
\hline NO3- & 4.62 & $1.65 E+05$ & $2.29 \mathrm{E}+05$ & 2.35 & 2.35 & 8.19 & 10.1 \\
\hline NO2. & 1.57 & $4.14 E+04$ & $5.75 \mathrm{E}+04$ & 0.987 & 1.19 & 187 & 1.87 \\
\hline CO32- & 0.147 & $5.08 E+03$ & $7.06 \mathrm{E}+03$ & 0.115 & 0.131 & 0.164 & 0.180 \\
\hline PO43. & $1.90 \mathrm{E}-04$ & 10.4 & 14.4 & $1.76 \mathrm{E}-04$ & $.1 .84 \mathrm{E}-04$ & $1.97 \mathrm{E}-04$ & 2.15E- 04 \\
\hline SOA2:- & $2.29 \mathrm{E}-02$ & $1.27+03$ & $1.76 E+03$ & $1.89 \mathrm{E}-02$ & $2.05 E-02$ & 2.45E-02 & $2.6 \mathrm{E}-02$ \\
\hline $\mathrm{Si}$ (as $\mathrm{SiO}_{2}-$ ) & 6.17E-02 & 997 & $1.38 E+03$ & 4.14E-02 & $5.40 \mathrm{E}-02$ & $7,02 \mathrm{E}-02$ & $831 \mathrm{E}-02$ \\
\hline F* & I.SIE-04 & 1.65 & 2.29 & $1.29 \mathrm{E}-04$ & $1.42 \mathrm{E}-04$ & 1.66E-04 & 1. T9E-04 \\
\hline $\mathrm{Cl}-$ & $8.04 \vec{E}-02$ & $1.64 \mathrm{E}+03$ & $2.21 \mathrm{E}+0.3$ & $5.41 \mathrm{E}-02$ & $6.70 \mathrm{E}-02$ & 8.84E-02 & 0.126 \\
\hline C6H5O71- & $1.56 \mathrm{E}-04$ & 17.0 & 23.6 & $1.51 \mathrm{E}-04$ & $1.54 \mathrm{E}-04$ & $1.63 \mathrm{E}-04$ & $1.77 \mathrm{E}-04$ \\
\hline EDTA4- & 6.08E-06 & 1.01 & 1.40 & $4.28 E-06$ & $5.26 \mathrm{E}-06$ & $7.16 \mathrm{E}-06$ & 8.24E-06 \\
\hline HEDTA3. & 5.05E-06 & $0.7 \%$ & T.11 & $1.62 \mathrm{E}-06$ & $3.47 \mathrm{E}-06$ & 6.74E-06 & $8.39 \mathrm{E}-06$ \\
\hline glycolale- & $220 \mathrm{E}-04$ & 9.51 & 13.2 & $1.20 \mathrm{E}-04$ & $1.74 \mathrm{E}-04$ & 2.74E-04 & $3.26 \mathrm{E}-04$ \\
\hline Letate- & $2.29 \mathrm{E}-05$ & 0.776 & 1.08 & $2.22 \mathrm{E}-05$ & $2.25 \mathrm{E}-05$ & 2.43E-05 & $2.60 \mathrm{E}-05$ \\
\hline oxalate?- & $946 \mathrm{E}-12$ & T.78E-07 & 6.6SE-07 & 8.38E-12 & $8.90 \mathrm{E}-12$ & 1,04E-11 & $1.14 \mathrm{E}-11$ \\
\hline DBP & $1.39 \mathrm{E}-04$ & 16.7 & 23.3 & $1.29 \mathrm{E}-04$ & $1,35 \mathrm{E}-04$ & $1,50 \mathrm{E}-04$ & $1.62 \mathrm{E}-04$ \\
\hline butanol & 1.39E-04 & 590 & 8.20 & 1.29E-04 & 1.35E-04 & 1.50E-04 & 1.62E-04 \\
\hline $\mathrm{NH3}$ & 0.127 & $1.24 \mathrm{E}+03$ & $1.72 \mathrm{E}+03$ & $4.40 \mathrm{E}-02$ & $5.57 \mathrm{E}-02$ & 0.133 & 0.139 \\
\hline Fo(CN)64- & $\overline{0}$ & 0 & 0 & 0 & 0 & 0 & 0 \\
\hline
\end{tabular}

"Unknowns in tank solids inventory are assigned by Tunk Layering Model (TLM) 
HDW Model Rov, 4

\begin{tabular}{|c|c|c|c|c|c|c|c|}
\hline & \multicolumn{7}{|c|}{ Single-Shell Tank 241-S-101 } \\
\hline & \multicolumn{7}{|c|}{ SMM Cornposite Inventory Estimate } \\
\hline Physical & & & & & & & \\
\hline Properties & & & $\because \because$ & $.95 \mathrm{CI}$ & $-67 \mathrm{CI}$ & $+67 \mathrm{CI}$ & $+95 \mathrm{CI}$ \\
\hline Total SMM Wast & $1.26 E+06(\mathrm{~kg})$ & $(216 \mathrm{kgal})$ & - & - & - & $=$ & - \\
\hline Heat Lood & $1.51(\mathrm{~kW})$ & (5.17E+03 BTU/hr) & $=$ & 1.42 & 1.47 & 1.56 & 1.60 \\
\hline Bulk Density* & $1.54(\mathrm{~g} / \mathrm{cc})$ & $=$ & - & 1.49 & 151 & 1.55 & 1.57 \\
\hline & & & & & & & \\
\hline Water wt\% & 38.2 & - & --- & 35.5 & 36.7 & 39.7 & 416 \\
\hline TOC wt\% C (wel & 0.692 & $m$ & - & 0.466 & 0.577 & 0.808 & 0.921 \\
\hline $\begin{array}{l}\text { Chemical : } \\
\text { Constituents }\end{array}$ & molel. & pmin & $\mathbf{k o}$ & $\begin{array}{r}95 \mathrm{CI} \\
\text { (molef })\end{array}$ & $\begin{array}{r}67 \mathrm{CI} \\
\text { (mole/L) }\end{array}$ & $\begin{array}{l}+67 \mathrm{Cl} \\
(\text { mold } / \mathrm{L})\end{array}$ & $\begin{array}{l}+95 \mathrm{Cl} \\
\text { (mole/L) }\end{array}$ \\
\hline $\mathrm{Nat}$ & 12.5 & $188 \mathrm{E}+05$ & $2.36 \mathrm{E}+0 \mathrm{~S}$ & 11.4 & 120 & 13,0 & 13.5 \\
\hline $\mathrm{Al} 3+$ & 1.49 & $2.62 \mathrm{E}+04$ & $3.29 \mathrm{E}+04$ & 1.38 & 1.44 & 1.56 & 1.60 \\
\hline $\mathrm{Fe}^{\mathrm{j}}+($ total Fe $)$ & $9.64 \mathrm{E}-03$ & 351 & 440 & 7.79E-03 & 8.70E-03 & $106 \mathrm{E}-02$ & 1.15E-02 \\
\hline $\mathrm{Cr} 3+$ & 0.133 & $4.52 \mathrm{E}+03$ & $5.67 \mathrm{E}+03$ & 0.111 & 0.123 & 0.137 & 0.141 \\
\hline $\mathrm{Bi}+$ & T.09E-03 & 148 & 186 & $9.94 \mathrm{E}-04$ & $1.04 \mathrm{E}-0 \mathrm{3}$ & $1.14 \mathrm{E}-03$ & $1.18 \mathrm{E}-03$ \\
\hline Lajt & $3.30 \mathrm{E}-0 \mathrm{~S}$ & 2.99 & 3.75 & 2.39 E-05 & $283 \mathrm{E}-05$ & $3.77 \mathrm{E}-05$ & $4.22 \mathrm{E}-0.5$ \\
\hline $\mathrm{H}_{32+}$ & $7.80 \mathrm{E}-06$ & 1.02 & 1.28 & $7.30 \mathrm{E}-06$ & $7.58 \mathrm{E}-06$ & $7.92 \mathrm{E}-06$ & $8.03 \mathrm{E}-06$ \\
\hline Zr (as Zro(OH)2] & $200 E-04$ & 11.9 & 14.9 & $1.83 \mathrm{E}-04$ & $1.89 \mathrm{E}-04$ & 2.07E-04 & $2.17 \mathrm{E}-04$ \\
\hline Pb2+ & $8.81 E-04$ & 19 & 149 & $7.15 \mathrm{E}-04$ & $7.97 \mathrm{E}-04$ & 9.665004 & $1.05 \mathrm{E}-03$ \\
\hline $\mathrm{Ni2}+$ & $5.65 \mathrm{E}-03$ & 216 & 271 & $5.40 \mathrm{E}-03$ & $5.52 \mathrm{E}-03$ & $5.72 \mathrm{E}-0.03$ & $5.79 \mathrm{E}-03$ \\
\hline Sr2t & 0 & 0 & 0 & 0 & 0 & 0 & 0 \\
\hline Mndt & $3,4 \overline{4 E}-03$ & 123 & 154 & 2.55E-03 & $2.98 \mathrm{E}-03$ & $3,89 E-03$ & 4.32E-03 \\
\hline C.24 & $2.96 \mathrm{E}-02$ & 772 & 970 & 2.69E -02 & $2.82 \mathrm{E}-02$ & 3.09E-02 & 3.22E-02 \\
\hline$\overline{K+}$ & $5.73 F-02$ & $1.4 \mathrm{E}+03$ & $1.84 E+03$ & $5.21 \mathrm{E}-02$ & $5.47 \mathrm{E}-02$ & $605 \mathrm{E}-02$ & $6.33 \mathrm{E}-02$ \\
\hline क्म- & 9,00 & $997 \mathrm{E}+04$ & $1.25 \mathrm{E}+05$ & 8.32 & 8.64 & 9.37 & 9.57 \\
\hline $\mathrm{NO3-}$ & 4.33 & $175 E+05$ & $2.20 \mathrm{E}+05$ & 4.03 & 4.21 & 4.40 & 4.45 \\
\hline NO2- & 2.20 & $6.59 \mathrm{E}+04$ & $8.27 \mathrm{E}+04$ & 1.82 & 1.99 & 2.40 & 2.58 \\
\hline CO32- & 0.379 & $1.48 \mathrm{E}+04$ & $1.86 \mathrm{E}+04$ & 0.343 & 0.361 & 0.403 & 0.410 \\
\hline PO43. & $7.74 \mathrm{E}-02$ & $47 \mathrm{SE}+03$ & $6.01 E+03$ & $6.73 \mathrm{E}-02$ & $7.08 \mathrm{E}-02$ & $7,92 \mathrm{E}-02$ & $8.09 E-02$ \\
\hline $5042-$ & 0.213 & $1.34 \mathrm{E}+04$ & $1,68 \mathrm{E}+04$ & 0.164 & 0.188 & 0.240 & 0.263 \\
\hline $\mathrm{Si}$ (As SiO32-) & $6.88 \mathrm{E}-02$ & $1.26 \mathrm{E}+03$ & $1.58 E+63$ & 5.61E-02 & $6.23 \mathrm{E}-02$ & $7.33 \mathrm{E}-02$ & 15E-02 \\
\hline $\mathrm{F}-$ & $5.34 \mathrm{E}-02$ & 661 & 830 & 4.52E-0? & $4.86 \mathrm{E}-02$ & $5.75 \mathrm{E}-02$ & 6.07E-02 \\
\hline $\mathrm{Cl}-$ & 0.215 & $4.97 \mathrm{E}+03$ & $6.24 E+03$ & 0.194 & 0.204 & 0.225 & 0.234 \\
\hline C6HSO73- & $2.57 \mathrm{E}-02$ & $3.17 \mathrm{E}+0 \mathrm{03}$ & 3.98E+03 & $2.37 \mathrm{E}-02$ & $2.47 \mathrm{~F}, 02$ & $2.68 E-02$ & $2.78 \mathrm{E}-02$ \\
\hline EDTA4- & $1.26 E-02$ & $2,37 \mathrm{E}+03$ & $2.97 \mathrm{E}+03$ & $401 E-03$ & $8.22 \mathrm{E}-03$ & 1.71E-02 & $2.14 \mathrm{E}-02$ \\
\hline HEDTA3. & $2.33 \mathrm{E}-02$ & $4.15 E+03$ & $5,22 E+03$ & $6.06 \mathrm{E}-03$ & $1.45 \mathrm{E}-02$ & $3.21 \mathrm{E}-02$ & $408 \mathrm{E}-02$ \\
\hline glycolate- & $7,68 \mathrm{E}-02$ & $3.75 \mathrm{E}+03$ & $4.71 E+03$ & $499 \mathrm{E}-02$ & $6,30 \mathrm{E}-02$ & $9.06 \mathrm{E}-02$ & 0.104 \\
\hline acetate- & $6.32 \mathrm{E}-03$ & 243 & 305 & $5.16 \mathrm{E}-03$ & $5.73 \mathrm{E} \cdot 03$ & $6.91 \mathrm{E}-03$ & $7.47 \mathrm{E}-03$ \\
\hline oxalate2. & 4.32E-05 & 2.48 & 3.11 & $3.83 \mathrm{E}-05$ & 4.07E-05 & 4.59E-05 & $4.82 E-05$ \\
\hline$\overline{\mathrm{DBP}}$ & $1.72 \mathrm{E}-02$ & $235 E+03$ & $2.95 E+03$ & $1.40 \mathrm{E}-02$ & $1.56 \mathrm{E}-02$ & $1.67 \mathrm{E}-02$ & $2.02 E-02$ \\
\hline butanol & $1.72 \mathrm{E}-02$ & 829 & $1.04 \mathrm{E}+03$ & $1.40 \mathrm{E}-02$ & $156 \mathrm{E}-02$ & $1.87 \mathrm{E}-02$ & $2.02 \mathrm{E}-02$ \\
\hline $\mathrm{NH}$ & $7.38 \mathrm{~B}=02$ & 817 & $1.03 \mathrm{E}+03$ & $5.94 \mathrm{E}-02$ & $6.51 E-02$ & 8.48E-02 & $9.77 \mathrm{E}-02$ \\
\hline Fe(CN)64- & 0 & 0 & 0 & 0 & 0 & 0 & 0 \\
\hline
\end{tabular}

"Density is calculated based on $\mathrm{Na}, \mathrm{OH}-$, and $\mathrm{AlO} 2$.

+Water wt\% derived from the difference of density and total dissolved species. 


\begin{tabular}{|c|c|c|c|c|c|c|c|}
\hline \multirow[b]{3}{*}{$\begin{array}{l}\text { Physical } \\
\text { Propertics }\end{array}$} & \multicolumn{7}{|c|}{ Single-Shell Tank 241-S-101 } \\
\hline & \multicolumn{7}{|c|}{ Total Inventory Estimate* } \\
\hline & & & سم & $-95 \mathrm{CI}$ & $-67 \mathrm{CI}$ & $+67 \mathrm{CI}$ & $+95 \mathrm{CI}$ \\
\hline Total Waste & $2.64 E+06(\mathrm{~kg})$ & $(427 \mathrm{kgal})$ & $\cdots$ & - & - & - & --- \\
\hline Hear Load & $4.90(\mathrm{~kW})$ & (1.67E+04 BTU/hs) & - & 4.24 & 4.62 & 5.10 & 5.14 \\
\hline Bulk Densityt & $1.64(\mathrm{~g} / \mathrm{cc})$ & - & $=$ & 1.57 & 1,58 & 1.72 & 1.74 \\
\hline & & & & & & & \\
\hline Water wt\%t & 33.8 & $m$ & - & 27.0 & 28.8 & 37.6 & 38.4 \\
\hline TOC wt\% C (wet) & 0.330 & $\cdots$ & $\ldots$ & 0.222 & 0.275 & 0.385 & 0.439 \\
\hline $\begin{array}{c}\text { Chemical } \\
\text { Constituents }\end{array}$ & moled & ppin & kg: & $\begin{array}{c}95 \mathrm{CI} \\
\text { (molesiL) }\end{array}$ & $\begin{array}{l}-67 \mathrm{CI} \\
(\mathrm{mole} / \mathrm{L})\end{array}$ & $\begin{array}{l}+67 \mathrm{CI} \\
\text { (mole/A) }\end{array}$ & $\begin{array}{l}+95 \mathrm{CI} \\
\text { (mole/L) }\end{array}$ \\
\hline $\mathrm{Nat}$ & 115 & $1.61 E+05$ & $4.26 \mathrm{E}+05$ & 10.1 & 10.3 & 13.0 & 13.5 \\
\hline $\mathrm{A} / 3+$ & 3.74 & 6. $16 \mathrm{E}+04$ & $1.63 \mathrm{E}+05$ & 3.31 & 3.45 & 4.05 & 4.37 \\
\hline $\mathrm{Fe} 3+(\operatorname{total} \mathrm{Fe})$ & 0.238 & $8.12 E+03$ & $2.15 \mathrm{E}+04$ & 0.234 & 0.236 & 0.240 & 0.242 \\
\hline $\mathrm{Cr} 3+$ & 0.254 & $8.08 \mathrm{E}+03$ & $2.14 \mathrm{E}+04$ & 0.182 & 0.217 & 0.348 & 0.458 \\
\hline Bi3+ & $553 E-04$ & 70.6 & 187 & $5.05 \mathrm{E}-04$ & 328E-04 & $5.7 \% \mathrm{E}-04$ & $6.01 \mathrm{E}-04$ \\
\hline $\mathrm{Laj+}$ & $1.67 \mathrm{E}-05$ & 1.42 & 3.73 & $1.21 \mathrm{E}-05$ & $1.43 \mathrm{E}-0 \mathrm{~S}$ & $1.91 \mathrm{E}-05$ & 2. $13 \mathrm{E}-05$ \\
\hline $\mathrm{Hg} 2+$ & 2.8IE-04 & 34.5 & 91.2 & $277 \mathrm{E}-04$ & 2.80E-04 & $2.82 \mathrm{E}-04$ & $2.83 E-04$ \\
\hline $\mathrm{Zr}(\mathrm{As} \mathrm{ZrO}(\mathrm{OH}) 2)$ & 1.01E-04 & 5.65 & 14.9 & $9.26 \mathrm{E}-05$ & 9.57E-05 & $1.05 E-04$ & 1. $10 \mathrm{E}-04$ \\
\hline Pb2t & $8.47 \mathrm{E}-03$ & $107 E+03$ & $2.84 E+03$ & 7.82E-03 & $8.20 \mathrm{E}-03$ & $8.74 E-03$ & $9.00 \mathrm{E}-03$ \\
\hline $\mathrm{Ni2+}$ & $1.70 \mathrm{E}-02$ & 611 & $1.62 \mathrm{E}+03$ & 1.40E-02 & $1.57 \mathrm{E}-02$ & $1.80 \mathrm{E}-02$ & $1.78 \mathrm{E}-02$ \\
\hline Sr2+ & 0 & 0 & 0 & 0 & 0 & 0 & 0 \\
\hline Min4t & $1.75 \mathrm{E}-03$ & 58.7 & 155 & $1.30 \mathrm{E}-03$ & $1,52 \mathrm{E}-03$ & $1.98 \mathrm{E}-03$ & $2.20 \mathrm{E}-03$ \\
\hline $\mathrm{Ca}$ ?t+ & $8.67 \mathrm{E}-02$ & $2.12 \mathrm{E}+03$ & $5.62 \mathrm{E}+03$ & $7.13 \mathrm{E}-02$ & $7,90 \mathrm{E}-02$ & $9.45 \mathrm{E}-02$ & 0.102 \\
\hline $\mathrm{K}+$ & $3.84 \mathrm{E}-02$ & 917 & $2.43 \mathrm{E}+03$ & $3.56 \mathrm{E}-02$ & $3.69 \mathrm{E}-02$ & $3.99 \mathrm{E}-02$ & 4. $13 E+02$ \\
\hline OH- & 16.8 & $1.75 E+05$ & $4.63 \mathrm{E}+05$ & 14.8 & 15.5 & 18.2 & 19.6 \\
\hline NO3- & 4.48 & $7.70 E+05$ & 4.49E+0S & 3.30 & 3.34 & 6.22 & 7.11 \\
\hline $\mathrm{NO}$ - & 1.89 & $3,30 \mathrm{E}+04$ & $1.40 \mathrm{E}+05$ & 156 & $1.7 !$ & 2.05 & 2.08 \\
\hline Cos2- & 0.265 & $9.71 E+03$ & $2.57 \mathrm{E}+04$ & 0.246 & 0.255 & 0.277 & 0.280 \\
\hline PO43. & $3.92 \mathrm{E}-02$ & $2.28 \mathrm{E}+03$ & $6.02 E+03$ & $3.41 \mathrm{E}-02$ & $3.59 \mathrm{E}-02$ & 4.01E-02 & 4. $10 E-02$ \\
\hline SO42- & 0.119 & $7.00 \mathrm{E}+03$ & 1.85E+04 & $9.45 \mathrm{E}-02$ & 0.106 & 0.133 & 0.144 \\
\hline $\mathrm{Si}\left(4 \mathrm{SiO}_{2}-\right)$ & $6.53 \mathrm{E}-02$ & $1.12 \mathrm{E}+03$ & $2.97 \mathrm{E}+03$ & $5.66 \mathrm{E}-02$ & $6.15 \mathrm{E}-02$ & $6.93 \mathrm{E}-02$ & $7.52 \mathrm{E}-02$ \\
\hline F. & 2.7TE-02 & 315 & 832 & $2.29 \mathrm{E}-02$ & $2.47 \mathrm{E}-02$ & $2.92 \mathrm{E}-02$ & $3.08 \mathrm{E}-02$ \\
\hline Ci. & 0.149 & $3.22 \mathrm{E}+03$ & $8.51 \mathrm{E}+03$ & 0.129 & 0.139 & 0.154 & 0.170 \\
\hline C6HSO73- & 1.31E-02 & $1.31 \mathrm{E}+03$ & $4,00 \mathrm{E}+03$ & $1.21 \mathrm{E}-02$ & $1.26 \mathrm{E}-02$ & $1.36 \mathrm{E}-02$ & $1.41 \mathrm{E}-02$ \\
\hline EDTA4 & 6.39E-03 & $1.12 \mathrm{E}+03$ & $29 \sqrt{E}+03$ & 2.03E-03 & $4,16 \mathrm{E}-03$ & $8.63 \mathrm{E}-03$ & $1.08 \mathrm{E}-02$ \\
\hline HEDTA3:- & $1.18 E-02$ & $1.97 \mathrm{E}+03$ & $5.22 \mathrm{E}+03$ & $3.07 \mathrm{E}-03$ & $7.32 \mathrm{E}-03$ & $1.63 \mathrm{E}-02$ & $2.06 \mathrm{E}-02$ \\
\hline glycolate: & $3.90 \mathrm{E}-02$ & $1.79 \mathrm{E}+03$ & $4.72 \mathrm{E}+03$ & $2.53 \mathrm{E}-02$ & $3.20 \mathrm{E}-02$ & $4.60 \mathrm{E}-02$ & $5.26 \mathrm{E}-02$ \\
\hline acetate- & $3.21 \mathrm{E}-03$ & 116 & 306 & 2.62E- 03 & $2.91 \mathrm{E}-03$ & 3.SIE-03 & $3.79 \mathrm{E}-03$ \\
\hline oxalate2- & 2.19E-0S & 1.18 & 3.11 & $1.94 \mathrm{E}-05$ & $2.06 \mathrm{E}-05$ & 2.31E-05 & $2.44 \mathrm{E}-05$ \\
\hline DBP & $8.75 \mathrm{E}-03$ & $1.12 \mathrm{E}+03$ & $2.97 E+03$ & 7.1 E-03 & 7.95E-03 & 9.54E-0.7 & $1.03 \mathrm{E}-02$ \\
\hline butanol & 8.75E-03 & 397 & $1.05 \mathrm{E}+03$ & $7.17 \mathrm{E}-03$ & $7.95 \mathrm{E}-03$ & $9.54 \mathrm{E}-03$ & $1.03 \mathrm{E} \cdot 02$ \\
\hline $\mathrm{NH3}$ & $1.00 \mathrm{E}-0 \mathrm{I}$ & $1.04 E+03$ & $2.75 E+03$ & $5.75 \mathrm{E}-02$ & $6.41 \mathrm{E}-02$ & 0.106 & 0.112 \\
\hline Fo(CN) 64 . & 0 & 0 & 0 & 0 & 0 & 01 & 0 \\
\hline
\end{tabular}

*Unknowns in tank solids inventory are assigned by Tank Layering Model (TLM).

† Water we\% derived from the difference of density and total dissolved apecies. 
HNF-SD-WM-ER-323, Rev. 1

HDW Madel Rov, 4

\begin{tabular}{|c|c|c|c|c|c|c|c|}
\hline & & Sing & e-Shell Tar & $241-5-101$ & & & \\
\hline & & TLM Solids & Composite & nventory Es & timate ${ }^{*}$ & & \\
\hline & & & & $95 \mathrm{Cl}$ & & & \\
\hline \begin{tabular}{|l|} 
Properties \\
Total TLM Wast
\end{tabular} & $1.39 \mathrm{E}+06(\mathrm{~kg})$ & (211 kgal) & $\ldots$ & $\frac{95 . \mathrm{Cl}}{-}$ & $-67 \mathrm{CI}$ & $+67 \mathrm{Cl}$ & $495 \mathrm{CI}$. \\
\hline Heat Load & $3.39(\mathrm{~kW})$ & $(1.16 \mathrm{E}+04 \mathrm{BTU} / \mathrm{hr})$ & $\ldots$ & 2.77 & 3.13 & 3.56 & 3.64 \\
\hline Bulk Density & $1.74(g / c)$ & $\ldots$ & - & 1.62 & 1.64 & 1.89 & 1.95 \\
\hline Void Fraction & 0.658 & - & - & 0.450 & 0.519 & 0746 & 0.748 \\
\hline Water wt\% & 29.9 & - & - & 17.4 & 21.4 & 36.4 & 38.2 \\
\hline ToC wt \% C (wet & $2.21 \mathrm{E}-03$ & $\ldots$ & - & $197 \mathrm{E}-0.3$ & 2.03E-03 & $2.38 E-03$ & $2.57 \mathrm{E}-03$ \\
\hline $\begin{array}{l}\text { Radiological } \\
\text { Constituents }\end{array}$ & $\mathrm{Ci} / \mathrm{L}$ & $\mathrm{HC} / \mathrm{s}$ & a & $\begin{array}{l}-9 \mathrm{CCI} \\
\text { (CVL) }\end{array}$ & $\begin{array}{l}67 \mathrm{Cl} \\
\text { (C)L) }\end{array}$ & $\begin{array}{l}+67 \mathrm{CI} \\
(\mathrm{Cin})\end{array}$ & $\begin{array}{r}+95 \mathrm{CI} \\
\text { (CUL) }\end{array}$ \\
\hline $\mathrm{H}-3$ & $1.08 \mathrm{E}-04$ & $6.21 E-02$ & 86.2 & 1.29E-05 & $1.29 \mathrm{E}-05$ & $1.13 \mathrm{E}-04$ & I.21E-04 \\
\hline$C-14$ & 5.88E-06 & $3.38 \mathrm{E}-03$ & 4.70 & $1,00 E-106$ & $100 \mathrm{E}-06$ & $6.23 \mathrm{E}-06$ & $6.53 \mathrm{E}-06$ \\
\hline Ni-59 & $9.39 \mathrm{E}-06$ & $5.40 \mathrm{E}-03$ & 7.50 & $6.48 \mathrm{E}-06$ & $648 E-06$ & $9.99 \mathrm{E}-06$ & 9.88E-06 \\
\hline $\mathrm{Ni} \cdot 63$ & $8.88 E-04$ & 0.510 & 709 & $6.05 \mathrm{E}-04$ & $605 \mathrm{E}-04$ & 9.44E-04 & $9,33 \mathrm{E}-04$ \\
\hline $\mathrm{Co}-60$ & 4.79E-06 & $2.75 \mathrm{E}-03$ & 3.82 & $3.96 \mathrm{E}-07$ & $3.96 \mathrm{E}-07$ & $4.98 \mathrm{E}-06$ & $5.38 E-06$ \\
\hline Se-79 & $3.18 \mathrm{E}-06$ & $1.83 \mathrm{E}-03$ & 2.54 & 2.13E-07 & 2.13E-07 & $3.25 \mathrm{E}-06$ & $6.09 \mathrm{E}-06$ \\
\hline Sr-90 & 0.517 & 297 & $4.13 \mathrm{E}+05$ & 0.401 & 0.467 & 0.556 & 0.563 \\
\hline Y-90 & 0.517 & 297 & $4.13 \mathrm{E}+05$ & 0.401 & 0405 & 0.556 & 0.363 \\
\hline $2 s-93$ & $1.50 \mathrm{E}-05$ & $8.64 \mathrm{E}-03$ & 12.0 & $1.00 E-06$ & $1.00 \mathrm{E}-06$ & $1.54 \mathrm{E}-05$ & $273 \mathrm{E}-05$ \\
\hline Nb-93m & 1.22E-05 & $7.02 \mathrm{E}-03$ & 9.75 & $8.21 \mathrm{E}-07$ & 8.21E-07 & $1.25 \mathrm{E} .05$ & $2.54 \mathrm{E}-05$ \\
\hline Tc-99 & 4.49E-05 & $2.58 \mathrm{E}-02$ & 35.9 & 3.88E-05 & $4.13 E-05$ & $4.78 \mathrm{E}-05$ & $5.11 E-05$ \\
\hline Ru-106 & 1.02E-09 & 5.84E-07 & $8.11 E-04$ & $1.13 E-12$ & $1.13 E-12$ & $1.06 \mathrm{E}-09$ & $1.15 \mathrm{E}-09$ \\
\hline $\mathrm{Cd}-113 \mathrm{~m}$ & $2.29 \mathrm{E}-05$ & $1.31 \mathrm{E}-02$ & 18.3 & $3.10 \mathrm{E}-06$ & $3.10 E-06$ & 4.70E-05 & 4.55E-0.5 \\
\hline Sb-125 & 1.63E-05 & $9.35 \mathrm{E}-03$ & 13.0 & $5.83 \mathrm{E}-07$ & $5.83 E-07$ & $1.70 \mathrm{E}-0 \mathrm{~S}$ & $1.84 \mathrm{E} .05$ \\
\hline $\mathrm{Sn}_{\mathrm{n}-126}$ & $4.88 \mathrm{E}-06$ & $2.80 E-03$ & 3,89 & $3.27 E-07$ & $3.27 \mathrm{E}-07$ & $4.99 \mathrm{E}-06$ & $9.55 \mathrm{E}-06$ \\
\hline $1-129$ & 8.54E-08 & 4.91E-05 & $6.82 E-02$ & $7.37 \mathrm{E}-08$ & $7.84 \mathrm{E}-08$ & $9.09 \mathrm{E}-08$ & $9.71 E-08$ \\
\hline Cs-134 & $9.93 \mathrm{E}-07$ & $5.71 E-04$ & 0.793 & $1.21 \mathrm{E}-08$ & $1.21 \mathrm{E}-08$ & $1.03 \mathrm{E}-06$ & $1.11 \mathrm{E}-06$ \\
\hline $\mathrm{Cs}-137$ & 0.163 & \begin{tabular}{l|l|}
93.6 & \\
\end{tabular} & $1.30 \mathrm{E}+05$ & 0.145 & 0.152 & 0.173 & 0.184 \\
\hline$B a-137 m$ & 0.154 & 88.6 & $1.23 \mathrm{E}+05$ & $2.34 E-02$ & $2.34 E-02$ & 0.161 & 0.169 \\
\hline $\mathrm{Sm}-151$ & $1.13 \mathrm{E}-02$ & 6.51 & $9.05 E+03$ & $7.59 \mathrm{E}-04$ & $7.59 \mathrm{E}-04$ & $1.16 \mathrm{E}-02$ & $2.21 \mathrm{E}-02$ \\
\hline Eu-152 & $6.86 \mathrm{E}-06$ & $3.94 E-03$ & 5.48 & $3.08 E-06$ & $3.08 \mathrm{E}-06$ & 6.89E- -66 & $6.93 \mathrm{E}-06$ \\
\hline$E_{u-154}$ & $1.15 \mathrm{E}-04$ & $6.58 \mathrm{E}-02$ & 91.5 & 9.57E-06 & $9.57 \mathrm{E}-06$ & 1. $19 \mathrm{E}-04$ & $1.48 \mathrm{E}-04$ \\
\hline Eu-155 & $3.37 \mathrm{E}-04$ & 0.194 & 269 & $1.45 \mathrm{E}-04$ & $1.45 \mathrm{E}-04$ & $3.38 \mathrm{E}-04$ & $3.41 \mathrm{E}-04$ \\
\hline Ra.226 & $6.72 \mathrm{E}=10$ & $3.86 \mathrm{E}-07$ & $5.36 \mathrm{E}-04$ & 2.65E-10 & $4.64 E-10$ & $8.79 \mathrm{E}-10$ & $1.08 \mathrm{E}-09$ \\
\hline Ra-228 & $6.72 \mathrm{E}-09$ & $3.86 \mathrm{E}-06$ & $5,37 \mathrm{E}-03$ & 4.08E-15 & $4.08 E_{1} 15$ & $6.78 \mathrm{E}-09$ & $6.86 \mathrm{E}-09$ \\
\hline Ac-227 & $3.23 \mathrm{E}-09$ & $1.87 \mathrm{E}-06$ & $2.60 \mathrm{E}-03$ & 1.31E-09 & 2.04E-09 & 4.46E-09 & $5.61 E-09$ \\
\hline Pa-231 & 4.73E-09 & 2.72E-06 & $378 \mathrm{E}-03$ & $3.18 \mathrm{E}-10$ & $3.18 \mathrm{E}-10$ & $5,31 E-09$ & $1.17 \mathrm{E}-08$ \\
\hline$T \mathrm{~h}-229$ & $1.62 \mathrm{E}-10$ & $9,30 E-08$ & 1.29E-04 & $7.76 E-13$ & $7.76 \mathrm{E}-13$ & $1.036-10$ & $1.65 \mathrm{E}-10$ \\
\hline $\mathrm{Th}-232$ & 9.01E-1! & $5.18 E-08$ & $7.19 \mathrm{E}-03$ & $2.63 \mathrm{E}-16$ & $2.63 \mathrm{E}-16$ & $1.14 \mathrm{E}-10$ & $1.37 \mathrm{E}-10$ \\
\hline $\mathrm{U}-232$ & 3.01E-08 & $1.73 \mathrm{E}-05$ & $2.40 \mathrm{E}-02$ & $1.07 \mathrm{E}-08$ & $1.97 \mathrm{E}-08$ & $4.29 \mathrm{E}-08$ & $5.71 E-08$ \\
\hline $\mathrm{U}-233$ & 1.15E-07 & $6.60 \mathrm{E}-05$ & $9.18 \mathrm{E}-02$ & $4.06 \mathrm{E}-08$ & $7.50 \mathrm{E}-08$ & 1.64E- -97 & $2.18 \mathrm{E}-07$ \\
\hline $\mathrm{U}-234$ & $2.63 \mathrm{E}-06$ & 1.51E-03 & 2.10 & $2.32 \mathrm{E}-06$ & $2.47 \mathrm{E}-06$ & $2.80 \mathrm{E}-106$ & $2.97 \mathrm{E}-06$ \\
\hline $\mathrm{U}-235$ & 1. IIE-07| & $6.36 \mathrm{E}-05$ & $8.83 \mathrm{E}-02$ & $9.82 \mathrm{E}-08$ & $104 \mathrm{E}-07$ & $1.17 \mathrm{E}-07$ & $1.24 E-07$ \\
\hline U-236 & 6.81E-08 & 3.91E-05 & $5.44 \mathrm{E}-02$ & $5.59 \mathrm{E}-08$ & $6.19 \mathrm{E}-08$ & $7.45 \mathrm{E}-08$ & 8.09E-08 \\
\hline $\mathrm{U}-238$ & $2.51 \mathrm{E}-06$ & $1.44 \mathrm{E}-03$ & 2.00 & 2.24E- 06 & $2.37 \mathrm{E}-06$ & $2.66 \mathrm{E}-106$ & $2.81 \mathrm{E}-06$ \\
\hline Np-237 & \begin{tabular}{l|l|}
$2.11 \mathrm{E}-07$ & \\
\end{tabular} & $1.21 E-04$ & 0.169 & $1.71 \mathrm{E}-07$ & $1.87 \mathrm{E}-07$ & $2.2 \mathrm{E}-07$ & $2.40 \mathrm{E}-07$ \\
\hline $\mathrm{Pu}-238$ & $7.34 \mathrm{E}-06$ & 4.22E-03 & 5.86 & 6.53E-06 & $6.92 \mathrm{E}-06$ & $7.75 \mathrm{E}-106$ & 8. $15 E-06$ \\
\hline Pu-239 & $4.596-04$ & 0.264 & 367 & 4.01E-04 & $4.30 \mathrm{E}-04$ & $4.89 \mathrm{E}-04$ & $5.17 E-04$ \\
\hline Pu-240 & $6.60 \mathrm{E}-05$ & $379 \mathrm{E}-02$ & 52.7 & 5.77E-05 & 6.18E-05 & $7.03 E-05$ & 7.43E-05 \\
\hline Pu-24! & 4.15E-04 & 0.239 & 332 & $3.66 \mathrm{E}-04$ & $3.90 \mathrm{E}-04$ & 4.40E-04 & $4.64 \mathrm{E}-04$ \\
\hline Pu-242 & $1.88 \mathrm{E}-09$ & $1.08 \mathrm{E}-06$ & $1.50 \mathrm{E}-03$ & $1.65 \mathrm{E}-09$ & $1.76 \mathrm{E}-09$ & $1.99 \mathrm{E}-09$ & $2.10 E .09$ \\
\hline Am-241 & 5.34E-0S & $3.07 E-02$ & 42.6 & $3.18 \mathrm{E}-05$ & $4.66 \mathrm{E}-05$ & $6.06 \mathrm{E}-05$ & $1.12 \mathrm{E}-04$ \\
\hline Am-243 & $1.62 \mathrm{E}-09$ & $\begin{array}{l}9.33 \mathrm{E}-07 \\
\end{array}$ & 1,30E-03 & $7.64 \mathrm{E}-10$ & $1.41 \mathrm{E}-09$ & $1.78 \mathrm{E}-09$ & $2,17 E-09$ \\
\hline $\mathrm{Cm}-242$ & $7.14 \mathrm{E}-08$ & 4. $10 \mathrm{E}-05$ & $5.70 \mathrm{E}-02$ & $6.99 \mathrm{E}-08$ & 7.05E-08 & $7.20 \mathrm{E}-08$ & $7.20 \mathrm{E}-08$ \\
\hline $\mathrm{Cm}-243$ & 1.63E-09 & 9.39E-07 & $1.30 \mathrm{E}-03$ & $1.60 \mathrm{E}-09$ & $1.61 \mathrm{E}-09$ & $1.65 \mathrm{E}-09$ & $1.65 \mathrm{E}-09$ \\
\hline $\mathrm{Cm}-244$ & $1.28 \mathrm{E}-09$ & 7.34E-07 & 1.02E-03 & $2.33 \mathrm{E}-10$ & $6.51 \mathrm{E}-10$ & $1.70 \mathrm{E}-09$ & 1.70E-09 \\
\hline Totals: & $\mathbf{M}$ & wor & kg. & $\begin{array}{l}9 \mathrm{Cl} \\
\text { (Mor } \\
\text { /C) }\end{array}$ & $\begin{array}{c}67 \mathrm{Cl}(\mathrm{M} \\
\text { or g/L) }\end{array}$ & $\begin{array}{c}+67 \mathrm{Cl}(\mathrm{M} \\
\text { or } \mathrm{g} / \mathrm{l})\end{array}$ & $\begin{array}{c}+95 \mathrm{CI} \\
(\mathrm{M} \mathrm{or} \\
g / L)\end{array}$ \\
\hline $\mathbf{P u}$ & $7.68 \mathrm{E}-03(\mathrm{~g} \mathrm{~L})$ & - & 6.13 & $6.71 \mathrm{E}-03$ & $7.18 \mathrm{E}-03$ & $8.17 \mathrm{E}-03$ & $8.65 \mathrm{E}-03$ \\
\hline $\bar{U}$ & $3.15 \mathrm{E}-02$ & $4.31 \mathrm{E}+03$ & $5.98 \mathrm{E}+03$ & $2.81 \mathrm{E}-02$ & $2.97 \mathrm{E}-02$ & $3.33 \mathrm{E}-02$ & $3.52 \mathrm{E}-02$ \\
\hline
\end{tabular}


HNF-SD-WM-ER-323, Rev. 1

HDW Model Rev. 4

\begin{tabular}{|c|c|c|c|c|c|c|c|}
\hline \multirow{4}{*}{$\begin{array}{l}\text { Physical } \\
\text { Properties }\end{array}$} & \multicolumn{7}{|c|}{ Single-Shell Tank $241-5-101$} \\
\hline & \multicolumn{7}{|c|}{ SMM Composite lnventory Estimate } \\
\hline & & & & & & & \\
\hline & & & & $-95 \mathrm{Cl}$ & $-67 \mathrm{CI}$ & $+67 \mathrm{CI}$ & $+95 \mathrm{CI}$ \\
\hline Total SMM Wast & $1.26 \mathrm{E}+06(\mathrm{~kg})$ & $(216 \mathrm{kgal})$ & - & $\cdots$ & - & $\cdots$ & - \\
\hline Heat Load & $1.51(\mathrm{~kW})$ & (5.17E+03 BTU/hr) & - & 1.42 & 1.47 & 1.56 & 1.60 \\
\hline Bulk Density* & $1.54\left(g^{\prime} / c\right)$ & - & - & 1.49 & 1.51 & 1.55 & 1.57 \\
\hline Water $w t^{0} \dagger$ & 38.2 & $\ldots$ & - & 35.5 & 36.7 & 39.7 & 41.6 \\
\hline TOC wi\% C (wet & 0.692 & $m$ & - & 0.466 & 0.577 & 0.808 & 0.921 \\
\hline $\begin{array}{l}\text { Radiologicil } \\
\text { Constituents }\end{array}$ & CUL & $\mu \mathrm{C} / \mathrm{s}$ & Ci & $\begin{array}{l}-95 \mathrm{CI} \\
\text { (CLL) }\end{array}$ & $\begin{array}{l}-67 \mathrm{CI} \\
\text { (CVL) }\end{array}$ & $\begin{array}{l}+67 \mathrm{Cl} \\
(\mathrm{CV} / \mathrm{L}) \\
\end{array}$ & $\begin{array}{r}+95 \mathrm{CI} \\
(\mathrm{CU} / \mathrm{L})\end{array}$ \\
\hline $\mathrm{H}+3$ & $2.29 \mathrm{E}-04$ & 0.149 & 187 & 1.15E-04 & $1.15 E-04$ & $2.35 \mathrm{E}-04$ & $2.44 \mathrm{E}-04$ \\
\hline $\mathrm{C}-14$ & $3.31 \mathrm{E}-05$ & $2.16 \mathrm{E}-02$ & 27.1 & 9.45E-96 & $9.45 \mathrm{E}-06$ & 3.36E-05 & 3.40E-05 \\
\hline $\mathrm{Ni} .59$ & $2.10 \mathrm{E}-06$ & $1.37 \mathrm{E}-03$ & 1.72 & 9.85E-07 & $9.85 E-07$ & $2.16 \mathrm{E}-06$ & $2.21 E-06$ \\
\hline $\mathrm{Ni}-63$ & $2.06 \mathrm{E}-04$ & 0.134 & 168 & $9.55 \mathrm{E}-05$ & 9.55E-05 & $2.11 E-04$ & 2.17E-04 \\
\hline $\mathrm{Co-60}$ & 3.67E-05 & $2.39 \mathrm{E}-02$ & 30.0 & $9.56 \mathrm{E}-06$ & 9.56E-06 & $3.73 \mathrm{E}-05$ & 3.79E-05 \\
\hline Se-79 & $3.23 \mathrm{E}-06$ & $2.11 \mathrm{E}-03$ & 2.64 & $1.72 E-06$ & $1.72 \mathrm{E}-06$ & $3.78 \mathrm{E}-06$ & $431 \mathrm{E}-06$ \\
\hline Sr-90 & 0.107 & 70,0 & $8.79 \mathrm{E}+04$ & 0.100 & 0.104 & 0.111 & 0.115 \\
\hline$Y-90$ & 0.108 & 70.0 & $8.79 \mathrm{E}+04$ & $5.52 \mathrm{E}-02$ & $5.52 \mathrm{E}-02$ & 0.111 & 0.115 \\
\hline $2 r-93$ & $1.59 \mathrm{E}-05$ & $1.03 \mathrm{E}-02$ & 13.0 & D.31E-06 & 8.31E-06 & $1.86 \mathrm{E}-05$ & $2.12 E-05$ \\
\hline $\mathrm{Nb}-93 \mathrm{~m}$ & 1.15E-05 & $7.50 \mathrm{E}-03$ & 9.42 & $6.22 \mathrm{E}-06$ & $6.22 \mathrm{E}-06$ & 1.34E-05 & $1.53 \mathrm{E}-05$ \\
\hline Tc- 99 & $2.36 \mathrm{E}-04$ & 0.154 & 193 & L.48E-04 & 1.91E-04 & $2.81 E-04$ & 3.25E-04 \\
\hline Ru-106 & $645 \mathrm{E}-09$ & $4.20 \mathrm{E}-106$ & $5.27 \mathrm{E}-03$ & 2.94E-09 & $2.94 \mathrm{E}-09$ & $7.23 \mathrm{E}-09$ & $7.95 \mathrm{E}-09$ \\
\hline $\mathrm{Cd}-113 \mathrm{~m}$ & $8.36 \mathrm{E}-0 \mathrm{~S}$ & $5,44 \mathrm{E}-02$ & 68.3 & 3.82E-05 & 3.82E-05 & 1.00E-04 & $1.16 \mathrm{E}-04$ \\
\hline$S b-125$ & $1.58 \mathrm{~B}-04$ & 0.103 & 129 & $4.02 \mathrm{E}-05$ & $4.02 \mathrm{E}-05$ & $1.60 \mathrm{E}-04$ & $1.63 \mathrm{E}-04$ \\
\hline $5 n-126$ & $4.89 \mathrm{E}-06$ & $3.18 \mathrm{E}-03$ & 4.00 & $2.62 \mathrm{E}-06$ & $2.62 \mathrm{E}-06$ & $5.71 \mathrm{E}-06$ & 6.49E-06 \\
\hline $1-129$ & 4.55E-07 & $2.96 \mathrm{E}-04$ & 0372 & 2.84E-07 & $3.68 \mathrm{E}-07$ & 5.43E-07 & $6.2 \mathrm{RE}-07$ \\
\hline Cs-134 & $2.31 \mathrm{E}-06$ & $1.51 \mathrm{E}-03$ & 1.89 & $1.66 \mathrm{E}-06$ & $1,91 \mathrm{E}-06$ & $2.65 \mathrm{E}-06$ & $2.98 \mathrm{E}-06$ \\
\hline $\mathrm{C} 8.137$ & 0.240 & 157 & $1.97 \mathrm{E}+05$ & 0.218 & 0.229 & 0.252 & 0.263 \\
\hline$B a-137 m$ & 0.227 & 149 & $1.86 \mathrm{E}+05$ & 0.174 & 0.174 & 0.238 & 0.249 \\
\hline$S_{m 1}=151$ & $1.14 \mathrm{E}-02$ & 741 & $9.31 \mathrm{E}+03$ & $6.09 \mathrm{E}-03$ & $6.09 E-03$ & $1.33 \mathrm{E}-02$ & $1,51 \mathrm{E}-02$ \\
\hline Eu-152 & $3.70 \mathrm{E}-06$ & $2.41 \mathrm{E}-03$ & 3.03 & 1.83E-06 & 1.83E-06 & $3.94 \mathrm{E}-06$ & $4.17 \mathrm{E}-06$ \\
\hline Eu=154 & 5.91E-04 & 0.385 & 483 & 2.13E-04 & $2.13 \mathrm{E}-04$ & $7.28 \mathrm{E}-04$ & $782 E-04$ \\
\hline Eu-155 & 2.18E-04 & 0.142 & 179 & 1.07E-04 & $1,07 \mathrm{E}-04$ & 2.33E-04 & $247 E-04$ \\
\hline $\mathrm{Ra}-226$ & $1.36 \mathrm{E}-10$ & B.8BE-08 & $1.12 \mathrm{E}-04$ & 8.95E-11 & $8.95 \mathrm{E}-11$ & $1.53 \mathrm{E}-10$ & 1.70E-10 \\
\hline $\operatorname{Ra}-228$ & $1.26 \mathrm{E}-07$ & $8.20 \mathrm{E}-05$ & 0.103 & $5.28 \mathrm{E}-08$ & $8.85 \mathrm{E}-08$ & 1.69E-07 & $2.15 E-07$ \\
\hline Ac-227 & $8.62 \mathrm{E}-10$ & $3.62 \mathrm{E}-07$ & $7.05 \mathrm{E}-04$ & $5.90 \mathrm{E}-10$ & $3.90 \mathrm{E}-10$ & $9.61 \mathrm{E}-10$ & $106 \mathrm{E}-09$ \\
\hline $\mathrm{Pa}-231$ & $3.98 \mathrm{E}-09$ & 2.59E-0.6 & $3.26 \mathrm{E}-03$ & 2.47E-09 & $2.47 \mathrm{E}-09$ & $4.53 \mathrm{E}-09$ & 5.06E-09 \\
\hline Th-229 & $296 \mathrm{E}-09$ & $1.93 \mathrm{E}-06$ & 2.42E-03 & $1,36 \mathrm{E}-09$ & $2.14 E-09$ & $3.91 \mathrm{E}-09$ & $4.93 \mathrm{E}-09$ \\
\hline Th-232 & 8.44E-09 & $5.50 \mathrm{E}-06$ & $6.90 \mathrm{E}-03$ & 4.40E-09 & $6.3 \mathrm{EE}-09$ & $1.05 \mathrm{E}-08$ & $1.25 \mathrm{E}-08$ \\
\hline U-232 & 6.55E-07 & $4.26 \mathrm{E}-\mathrm{-04}$ & 0.535 & $3,62 E-07$ & $5.05 E-07$ & 8.28E-07 & 1.01E-00 \\
\hline U-233 & 2.51E-06 & $1.63 \mathrm{E}-03$ & 2.05 & $1.39 \mathrm{E}-06$ & $1.94 \mathrm{E}-06$ & $3.18 \mathrm{E}-06$ & $388 E-06$ \\
\hline U-234 & $7.62 \mathrm{E}-07$ & $4.96 \mathrm{E}-04$ & 0.623 & 7,34E-07 & $7.47 E-07$ & $7.78 \mathrm{E}-07$ & $7.88 \mathrm{E}-07$ \\
\hline U-235 & 3.09E- 08 & 2.02E-05 & $2.53 \mathrm{E}-02$ & 2.97E-08 & $3.03 \mathrm{E}-08$ & 3.16E-08 & $3.20 \mathrm{E}-08$ \\
\hline$U=236$ & $2.38 \mathrm{E}-08$ & 1.55E-05 & $1.94 \mathrm{E}-02$ & $2.29 \mathrm{E}-08$ & $2.34 \mathrm{E}-08$ & $2.42 E-08$ & $2.46 \mathrm{E}-08$ \\
\hline $\mathrm{U}-238$ & 8.82E-07 & 5.75E-04 & 0.721 & 8.53E-07 & 8.67E-07 & 8.99E-07 & $9.06 \mathrm{E}-07$ \\
\hline $\mathrm{Np}-237$ & 8.61E-07 & $5,61 \mathrm{E}-04$ & 0.704 & 5.73E-07 & $7.13 \mathrm{E}-07$ & $1.01 \mathrm{E}-06$ & $1.15 \mathrm{E}-06$ \\
\hline $\mathrm{P} u=238$ & $1.32 \mathrm{E}-166$ & $8.59 \mathrm{E}-04$ & 1.08 & $9.80 E-07$ & $1.15 \mathrm{E}-06$ & $1.49 \mathrm{E}-06$ & $1.66 \mathrm{E}-06$ \\
\hline Pu-239 & $4.49 \mathrm{E}-05$ & 2.93E-02 & 36.7 & $3.62 \mathrm{E}-05$ & $4.05 \mathrm{E}-05$ & 4.94E-05 & $5.36 \mathrm{E}-05$ \\
\hline Pu-240 & 7.64E-06 & $4.97 \mathrm{E}-03$ & 6.24 & $6.02 \mathrm{E}-06$ & $6.81 \mathrm{E}-06$ & $8.46 \mathrm{E}-06$ & $925 \mathrm{E}-06$ \\
\hline Pu-24! & 3.93E-05 & $5.82 \mathrm{E}-02$ & 73.0 & $6.62 \mathrm{E}-05$ & $7.75 \mathrm{E}-05$ & 1.01E-04 & $1.12 \mathrm{E}-04$ \\
\hline Pu-242 & $\$ 91 \mathrm{E}-10$ & $3.20 \mathrm{E}-07$ & 401E-04 & $3.53 \mathrm{E}-10$ & $4.20 \mathrm{E}-10$ & $5.61 \mathrm{E}-10$ & $6.29 \mathrm{E}-10$ \\
\hline Am-241 & 5.44E-05 & $3.54 \mathrm{E}-02$ & 44.5 & 3.83E-05 & $4.62 \mathrm{E}-05$ & $6.26 \mathrm{E}-05$ & $7.05 \mathrm{E}-05$ \\
\hline Am-243 & $1.90 \mathrm{E}-09$ & $1.23 \mathrm{E}-06$ & $1.55 E-03$ & $1.39 \mathrm{E}-09$ & $1.63 \mathrm{E}-09$ & 2.16E-09 & $2.42 \mathrm{E}-09$ \\
\hline $\mathrm{Cm}-242$ & $1.40 E-07$ & $9.14 E-05$ & 0.115 & $6.34 \mathrm{E}-08$ & 6.34E- 08 & $1.49 \mathrm{E}-07$ & $1.58 \mathrm{E}-07$ \\
\hline $\mathrm{Cm}-243$ & 1.30E-08 & $8.48 \mathrm{E}-06$ & 106E-02 & $5.69 \mathrm{E}-09$ & $5.69 \mathrm{E}-\mathrm{DP}$ & 1.38E-08 & $1.46 \mathrm{E}-08$ \\
\hline $\mathrm{Cm}=244$ & 1.30E-07 & 8.45E-05 & 0.106 & S.41E-08 & $541 \mathrm{E}-08$ & 1.55E-07 & $1.72 \mathrm{E}-07$ \\
\hline Totals. & $\mathbf{M}$ & $+/ s$ & les: & $\begin{array}{r}95 \text { CI } \\
\text { (Mor } \\
\text { /L) }\end{array}$ & $\begin{array}{r}67 \mathrm{CT}(\mathrm{M} \\
\operatorname{org} \mathrm{L})\end{array}$ & $\begin{array}{l}+67 \mathrm{CI}(\mathrm{M} \\
\text { or } / \mathrm{L})\end{array}$ & $\begin{array}{r}+95 \mathrm{CI} \\
(\mathrm{M} \text { or } \\
\mathrm{g} / \mathrm{L})\end{array}$ \\
\hline $\mathrm{Pu}$ & $5.60 \mathrm{E}-04(\mathrm{~g} / \mathrm{L})$ & - & 0.458 & 3.95E-04 & $4.76 E-04$ & $6,43 \mathrm{E}-04$ & $7.23 \mathrm{E}-04$ \\
\hline $\mathrm{U}$ & $8.75 \mathrm{E}-03$ & $1.36 \mathrm{E}+03$ & 1. $70 \mathrm{E}+03$ & 8.38E-03 & 8.56k-03 & 8.95E-03 & 9.05E-03 \\
\hline
\end{tabular}

- Density is calculated based on $\mathrm{Na}, \mathrm{OH}$ - and $\mathrm{AJO}$ -

tWater wt\% derived from the difference of density and total dissolved species. 


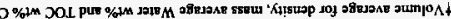

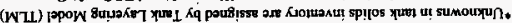

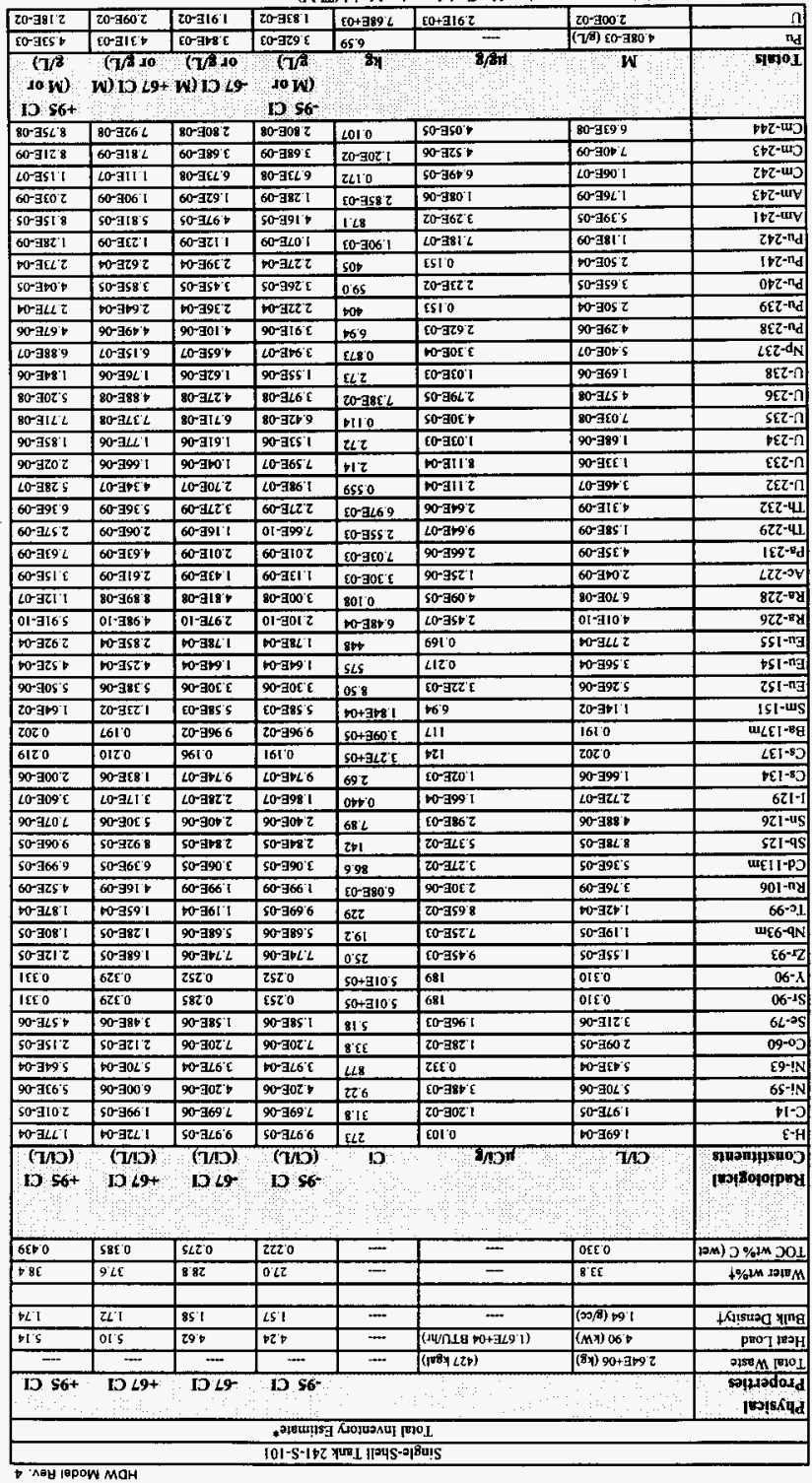


HNF-SD-WM-ER-323, Rev. 1

HDW Model Rav, 4

\begin{tabular}{|c|c|c|c|c|c|c|c|}
\hline \multirow{4}{*}{$\begin{array}{l}\text { Physical } \\
\text { Properties: }\end{array}$} & \multicolumn{7}{|c|}{ Single-Shell Tank 241-S-102 } \\
\hline & \multicolumn{7}{|c|}{ TLM Solids Composite Inventory Estimate* } \\
\hline & & & & & & & \\
\hline & & & & $-95 \mathrm{Cl}$ & $-67 \mathrm{CI}$ & $+67 \mathrm{CI}$ & $+95 \mathrm{CI}$ \\
\hline Total TLM Wast & $2.66 \mathrm{E}+04(\mathrm{~kg})$ & $(4.00 \mathrm{kgal})$ & - & - & $m$ & $\ldots$ & $\ldots$ \\
\hline Heat Lond & $9.86 \mathrm{E}-02(\mathrm{~kW})$ & (j37 BTU/br) & - & $7.15 \mathrm{E}-02$ & 8.74E-02 & 0.106 & 0.109 \\
\hline Bulk Density & $1.76(\mathrm{~g} / \mathrm{cc})$ & - & $\cdots$ & 1.51 & 1.53 & 2.11 & 2.24 \\
\hline Void Fraction & 0.536 & - & $\ldots$ & $6.00 \mathrm{E}-02$ & 0.217 & 0.737 & 0.743 \\
\hline Water wt $\%$ & 27.0 & - & $\because$ & 2.64 & 10.0 & 42.6 & 44.2 \\
\hline TOC wt\% C (wet & 0 & -- & $=$ & 0 & 0 & 0 & 0 \\
\hline $\begin{array}{l}\text { Chemicel } \\
\text { Constituents }\end{array}$ & mole/L & Ppm & ki & $\begin{array}{l}-95 \mathrm{CI} \\
\text { (mole/L) }\end{array}$ & $\begin{array}{c}67 \mathrm{CI} \\
\text { (mole/L) }\end{array}$ & $\begin{array}{l}+67 \mathrm{CI} \\
\text { (mole/L) }\end{array}$ & $\begin{array}{c}+95 \mathrm{CI} \\
(\mathrm{mole} / \mathrm{L})\end{array}$ \\
\hline Nat & 8.13 & $1.06 E+05$ & $283 \mathrm{E}+03$ & 3.05 & 3.56 & 14.7 & 18.2 \\
\hline $\mathrm{A} \mid 3+$ & 6.99 & $1.07 \mathrm{E}+05$ & $2.85 E+03$ & 6.58 & 6.74 & 7.15 & 7.15 \\
\hline Fe3+ (total Fe) & 1.01 & $3.22 E+04$ & 856 & 0.994 & 100 & 1.02 & 1.03 \\
\hline $\mathrm{Cr} 3+$ & 6.19E- 02 & $1.83 \mathrm{E}+03$ & 48.7 & 7.0BE-03 & 3.39E-02 & 0.522 & 1.06 \\
\hline$\overline{\mathrm{B} 3 \mathrm{3}+}$ & 0 & 0 & 0 & 0 & 0 & 0 & 0 \\
\hline Lo3+ & 0 & 0 & 0 & 0 & 0 & 0 & 0 \\
\hline $\mathrm{Hg}_{2+}$ & 0 & 0 & 0 & 0 & 0 & 0 & 0 \\
\hline $\operatorname{Zr}(\mathrm{as} \mathrm{ZrO}(\mathrm{OH}) 2)$ & 0 & 0 & 0 & 0 & 0 & 0 & 0 \\
\hline $\mathrm{Pb2t}$ & 0 & 0 & 0 & 0 & 0 & 0 & 0 \\
\hline $\mathrm{Ni} 2+$ & 5,07E-02 & $1.69 E+03$ & 45.0 & $3.66 \mathrm{E}-02$ & 4.45E-02 & $5.54 \mathrm{E}-02$ & $5.45 \mathrm{E}-02$ \\
\hline Sr2+ & 0 & 0 & 0 & 0 & 0 & 0 & 0 \\
\hline Mndt & 0 & 0 & 0 & 0 & 0 & 0 & 0 \\
\hline $\mathrm{Ca} 2+$ & 0.220 & $5.02 \mathrm{E}+03$ & 133 & 0.146 & 0.182 & 0.258 & 0.294 \\
\hline $\mathbf{K}+$ & $1.12 \mathrm{E}-02$ & 250 & 6.64 & 1.2BE-03 & $5.25 \mathrm{E}-03$ & $1.52 \mathrm{E}-02$ & $1.52 \mathrm{E}-02$ \\
\hline OH- & 25.6 & $2.48 \mathrm{E}+05$ & $6.59 \mathrm{E}+0 \mathrm{3}$ & 23.3 & 24.3 & 26.9 & 28.4 \\
\hline NO3. & 5.29 & $1.87 E+05$ & $4.96 \mathrm{E}+03$ & $6.03 \mathrm{E}-02$ & $6.03 \mathrm{E}-02$ & 13.5 & 17.9 \\
\hline NO2. & 1.46 & $3.82 \mathrm{E}+04$ & T.OIE+03 & 0.132 & 0.595 & 2.15 & 2.15 \\
\hline CO32- & 0.220 & $7.51 \mathrm{E}+03$ & 200 & 0.146 & 0.182 & 0.258 & 0.294 \\
\hline PO43- & 0 & 0 & 0 & 0 & 0 & 0 & 0 \\
\hline SO42- & $1.04 \mathrm{E}-02$ & 569 & 15.1 & 1.19E-03 & 4.8TE- 03 & $141 \mathrm{E}-02$ & 1.41E-02 \\
\hline Si ( $(\mathrm{sz}$ SiO32-) & $8.06 \mathrm{E}-03$ & 129 & 3.42 & 9.21E-04 & $3.77 \mathrm{E}-03$ & $1.09 \mathrm{E}-02$ & $1.09 \mathrm{E}-02$ \\
\hline F- & 0 & 0 & 0 & 0 & 0 & 0 & 0 \\
\hline Cl- & $5.16 \mathrm{E}-02$ & $1.04 \mathrm{E}+03$ & 27.7 & $5.91 \mathrm{E}-03$ & 2.42E-02 & $7,00 \mathrm{E}-02$ & 0.157 \\
\hline C6HSO73. & 0 & 0 & 0 & 0 & 0 & 0 & 0 \\
\hline EDTA4- & 0 & 0 & 0 & 0 & 0 & 0 & 0 \\
\hline HEDTA3- & 0 & 0 & 0 & 0 & 0 & 0 & 0 \\
\hline glyoolnte- & 0 & 0 & 0 & 0 & 0 & 0 & 0 \\
\hline acetate- & 0 & 0 & 0 & 0 & 0 & 0 & 0 \\
\hline oxalate2- & 0 & 0 & 0 & 0 & 0 & 0 & 0 \\
\hline $\mathrm{DBP}$ & 0 & 0 & 0 & 0 & 0 & 0 & 0 \\
\hline butanol & 0 & 0 & 0 & 0 & 0 & 0 & 0 \\
\hline $\mathrm{NH3}$ & 0.256 & $2.47 \mathrm{E}+03$ & 65.7 & 6.5SE- 02 & $9.25 \mathrm{E}-02$ & 0.270 & 0.284 \\
\hline Fe(CN)64- & 0 & 0 & 0 & 0 & 0 & 0 & 0 \\
\hline
\end{tabular}

"Unknowns in tank eolids inventoty are sesigned by Tunk Layering Model (TLM) 
HDW Model Rev. 4

\begin{tabular}{|c|c|c|c|c|c|c|c|}
\hline \multicolumn{8}{|c|}{ Single-Shell Tank 241-S-102 } \\
\hline \multicolumn{8}{|c|}{ SMM Composite Inventory Estimate } \\
\hline $\begin{array}{l}\text { Physical } \\
\text { Properties }\end{array}$ & & & & $-95 \mathrm{CI}$ & $-67 \mathrm{CI}$ & $+67 \mathrm{CI}$ & $+95 \mathrm{CI}$ \\
\hline Total SMM Wast & $2.64 \mathrm{E}+06(\mathrm{~kg})$ & $(545 \mathrm{kgal})$ & - & $-\cdots$ & $\ldots$ & $\rightarrow$ & $\cdots$ \\
\hline Heat Load & $1.99(\mathrm{kw})$ & (6.80E +03 BTU/hs) & - & 1.88 & 1.93 & 2.05 & 2.11 \\
\hline Bulk Density* & $1.28(g / c)$ & - & - & 1.26 & 1.27 & 1.29 & 1.30 \\
\hline Water wt\% & 61.4 & -- & $\ldots$ & $\$ 9.5$ & 60.4 & 62.5 & 63.7 \\
\hline TOC wi\% C (wet & 0.413 & $\cdots$ & יביר & 0.274 & 0.342 & 0.484 & 0.553 \\
\hline $\begin{array}{l}\text { Chemical } \\
\text { Constituents }\end{array}$ & molel: & ppin & kg & $\begin{array}{l}\text {-95 CI } \\
\text { (molen) }\end{array}$ & $\begin{array}{l}-67 \mathrm{CI} \\
\text { (mole/L) }\end{array}$ & $\begin{array}{l}+67 \mathrm{CI} \\
\text { (mole/L) }\end{array}$ & $\begin{array}{l}+95 \mathrm{CI} \\
(\mathrm{mole} / \mathrm{L}) \\
\end{array}$ \\
\hline $\mathrm{Nat}$ & 6.50 & $1.17 \mathrm{E}+05$ & $3,08 \mathrm{E}+05$ & 5.95 & 6.22 & 6.73 & 6.95 \\
\hline $\mathrm{Al3+}$ & 0.779 & $1.64 \mathrm{E}+04$ & $4.33 E+04$ & 0.719 & 0747 & 0.813 & 0.830 \\
\hline $\mathrm{Fet}^{3+}+(\mathrm{tot} \mathrm{s} \mathrm{Fe})$ & $4.88 \mathrm{E}-03$ & 213 & 562 & $3,98 \mathrm{E}-03$ & 4.42E-03 & $5.34 \mathrm{E}-03$ & $5.78 \mathrm{E}-03$ \\
\hline $\mathrm{Cr} 3+$ & $7.01 \mathrm{E}-02$ & $2.85 E+03$ & $7.52 \mathrm{E}+03$ & $5.79 \mathrm{E}-02$ & $6.45 \mathrm{E}-02$ & $7.23 \mathrm{E}-02$ & $7,44 \mathrm{E}-02$ \\
\hline $\mathrm{Bi} 3+$ & $5.71 \mathrm{E}-04$ & 93.3 & 246 & 5.19E-04 & $5,44 \mathrm{E}-04$ & $5.97 \mathrm{E}-04$ & $6.23 \mathrm{E}-04$ \\
\hline L-23+ & $1.54 \mathrm{E}-05$ & 1.67 & 4.41 & 1.11E-05 & $1.32 \mathrm{E}-05$ & $1.76 \mathrm{E}-05$ & $1.96 \mathrm{E}-05$ \\
\hline $\mathrm{Hg} 2+$ & $3.99 \mathrm{E}-46$ & 0.626 & 1.65 & 3.72E-06 & $387 \mathrm{E}_{-06}$ & $4.05 \mathrm{E}-06$ & 4. $10 E-06$ \\
\hline $\mathrm{Zr}(\mathrm{as} \mathrm{ZrO}(\mathrm{OH}) 2)$ & $1.07 \mathrm{E}-04$ & 7.66 & 20.2 & $9.79 \mathrm{E}-05$ & $101 E-04$ & 1.11E-04 & $1.1 \mathrm{TE}-04$ \\
\hline $\mathrm{Pb} 2+^{2}$ & $4.43 \mathrm{E}-64$ & 71.8 & 189 & $3.61 \mathrm{E}-04$ & 401E-04 & $4.84 \mathrm{E}-04$ & $5.24 \mathrm{E}-04$ \\
\hline $\mathrm{Ni2+}$ & $2.91 \mathrm{E}-03$ & 133 & 352 & $2.78 E-03$ & 2.84E- 03 & $2.94 \mathrm{E}-03$ & $2.9 \pi \mathrm{E}-03$ \\
\hline Sr2+ & 0 & 0 & 0 & 0 & 0 & 0 & 0 \\
\hline Mn4+ & $1.75 \mathrm{E}-03$ & 75.2 & 198 & I.32E-03 & $1.53 \mathrm{E}-03$ & $1.97 \mathrm{E}-03$ & $2.18 \mathrm{E}-03$ \\
\hline $\mathrm{Ca}+$ & $1.52 \mathrm{E}-02$ & 477 & $1.26 \mathrm{E}+03$ & 1.39E-02 & $1.45 \mathrm{E}-02$ & 1.59E-02 & 1.65E-02 \\
\hline $\mathrm{K+}$ & $2.97 E-02$ & 908 & $2.39 \mathrm{E}+03$ & $2.70 \mathrm{E}-02$ & $2.83 \mathrm{E}-02$ & 3.11E-02 & $3.25 \mathrm{E}-02$ \\
\hline $\mathrm{OH}$ - & 4.67 & $6.21 E+04$ & $1,64 \mathrm{E}+05$ & 4.30 & 447 & 4.88 & 4.95 \\
\hline NO3- & 2.29 & $1.11 \mathrm{E}+05$ & $2.93 \mathrm{E}+05$ & 2.12 & 2.22 & 2.33 & 2.34 \\
\hline NO2- & 1.14 & $409 E+04$ & $1.08 \mathrm{E}+05$ & 0.952 & 1.04 & 1.23 & 1.32 \\
\hline $\cos 2$. & 0.191 & $895 E+03$ & $2.36 \mathrm{E}+04$ & 0.173 & 0.180 & 0.204 & 0.206 \\
\hline PO43- & 3.91E-02 & $2.91 E+03$ & $7.66 \mathrm{E}+03$ & $337 \mathrm{E}-02$ & $3.56 \mathrm{E}-02$ & $4.01 \mathrm{E}-02$ & 4. $10 \mathrm{E}-02$ \\
\hline SO42- & 0.108 & $8[3 E+0\}$ & $2.14 E+04$ & $8.44 E-02$ & $9.57 \mathrm{E}-02$ & $0.12 \mathrm{t}$ & 0.132 \\
\hline $\mathrm{Si}(\mathrm{ar} \mathrm{SiO} 32-)$ & $3.52 \mathrm{E}-02$ & 774 & $2.04 \mathrm{E}+03$ & $2.90 \mathrm{E}-02$ & $3.20 \mathrm{E}-02$ & 3.84E-02 & 4. $14 \mathrm{E}-02$ \\
\hline F. & $2.80 \mathrm{E}-02$ & 416 & $1.10 \mathrm{E}+03$ & 2.34E-02 & $2.53 \mathrm{E}-0.2$ & $3.02 \mathrm{E}-02$ & $3.19 \mathrm{E}-02$ \\
\hline $\mathrm{Cl}-$ & 0.112 & $3.10 \mathrm{E}+03$ & $8.16 \mathrm{E}+03$ & 0.101 & 0.106 & 0.117 & 0.121 \\
\hline C6HSO73 & $1.25 \mathrm{E}-02$ & 1. BSE+03 & $4.89 E+03$ & L.15E-02 & $1.20 \mathrm{E}-02$ & 1.30E-02 & $1.3 \mathrm{SE}-02$ \\
\hline EDTA4- & $6.31 E-03$ & $1.42 \mathrm{E}+03$ & $3.75 \mathrm{E}+03$ & 1.95E-03 & 4.08E-03 & $8.56 \mathrm{E}-03$ & $1.03 \mathrm{E}-02$ \\
\hline HEDTA3- & $1.18 E-02$ & $2.53 \mathrm{E}+03$ & $6.67 \mathrm{E}+03$ & $3,00 \mathrm{E}-03$ & $7.32 \mathrm{E}-03$ & $1.63 \mathrm{E}-02$ & $2.07 \mathrm{E}-02$ \\
\hline glycolate- & 3.83E-02 & $2.25 E+03$ & $5.93 \mathrm{E}+03$ & $2.49 \mathrm{E}-02$ & $3.15 \mathrm{E}-02$ & $4.51 \mathrm{E}-02$ & $5.17 \mathrm{E}-02$ \\
\hline acetate- & $267 \mathrm{E}-03$ & 123 & 325 & 2 19E-03 & $2.43 \mathrm{E}-0.3$ & $2.92 \mathrm{E}-03$ & 3.16E-03 \\
\hline oxalate2:- & 2.01E-0S & 1.39 & 3.66 & 1.78E-05 & $1,90 \mathrm{E}-05$ & 2.13E-05 & 2.24E-05 \\
\hline DBP & 8.44E-03 & $1.39 \mathrm{E}+03$ & $3.60 \mathrm{E}+03$ & $6.92 \mathrm{E}-03$ & $7.67 \mathrm{E}-03$ & $9.20 \mathrm{E}-03$ & $9.93 \mathrm{E}-03$ \\
\hline butanol & 8.44E-03 & 490 & $1.29 \mathrm{E}+03$ & $6.92 \mathrm{E}-03$ & $7.67 \mathrm{E}-03$ & $9.20 \mathrm{E}-03$ & $9.93 \mathrm{E}-03$ \\
\hline NH3 & 3.84E-02 & 511 & $1.35 \mathrm{E}+03$ & $3.14 \mathrm{E}-02$ & $3.42 \mathrm{E}-02$ & $4.38 \mathrm{E}-02$ & $5.01 \mathrm{E}-02$ \\
\hline Fe(CN)64 & 0 & 0 & 0 & 0 & 0 & 0 & 0 \\
\hline
\end{tabular}

*Density is calculated based on $\mathrm{Na}, \mathrm{OH}-$, and $\mathrm{AlO} 2$ -

tWater wt $\%$ derived from the difference of density and total dissolved species. 
HNF-SD-WM-ER-323, Rev. I

HDW Model Rev. 4

\begin{tabular}{|c|c|c|c|c|c|c|c|}
\hline \multirow[b]{3}{*}{$\begin{array}{l}\text { Physical : } \\
\text { Properties }\end{array}$} & \multicolumn{7}{|c|}{ Single-Shell Tank 24I-S-102 } \\
\hline & \multicolumn{7}{|c|}{ Iotal laventory Estimate" } \\
\hline & & & & $.95 \mathrm{CI}$ & $-67 \mathrm{CI}$ & $+67 \mathrm{CI}$ & $+95 \mathrm{CI}$ \\
\hline Total Waste & $2.66 \mathrm{E}+06(\mathrm{~kg})$ & (549 kgal) & - & $\ldots$ & $\ldots$ & $\cdots$ & $\cdots$ \\
\hline Heat Load & $2.09(\mathrm{kw})$ & (7.13E+03 8TU/hr) & $\ldots$ & 1.97 & 2.03 & 2.15 & 220 \\
\hline Builk Density' & $1.28(g / c)$ & $\cdots$ & $\cdots$ & 1.26 & 1.27 & 129 & 1.30 \\
\hline Water wt\%t & 61.1 & - & - & 59.2 & 600 & 62.1 & 634 \\
\hline TOC wi\% C (wet & 0.409 & - & - & 0.271 & 0.338 & 0.479 & 0.547 \\
\hline $\begin{array}{l}\text { Chemical } \\
\text { Constituents }\end{array}$ & mole/L :...: & Ppm & $\mathrm{kg}$ & $\begin{array}{l}-95 \mathrm{Cl} \\
\text { (mole } \mathrm{L})\end{array}$ & $\begin{array}{l}67 \mathrm{CI} \\
\text { (mole/L) }\end{array}$ & $\begin{array}{l}+67 \mathrm{Cl} \\
\text { (maler ) }\end{array}$ & $\begin{array}{l}+95 \mathrm{CI} \\
\text { (mole/L) }\end{array}$ \\
\hline $\mathrm{Na}+$ & 6.51 & $1,17 \mathrm{E}+05$ & $3.11 \mathrm{E}+05$ & 5.9 & 6.24 & 6.74 & 6.95 \\
\hline Al3t & 0.824 & $1.73 \mathrm{E}+04$ & $4.62 \mathrm{E}+04$ & 0.764 & 0.793 & 0.856 & 0.874 \\
\hline $\mathrm{Fe}^{3+}($ total $\mathrm{Fe})$ & $1.22 \mathrm{E}-02$ & 533 & 1.42E+03 & $1.13 \mathrm{E}-02$ & $1.18 \mathrm{E}-02$ & $1.27 \mathrm{E}-02$ & 1.31E-02 \\
\hline $\bar{C} 3+$ & $7.01 \mathrm{E}-02$ & $2.84 E+03$ & $7.57 \mathrm{E}+03$ & $6.21 \mathrm{E}-02$ & 6.59E- 02 & $7.28 \mathrm{E}-02$ & 7.43E- 02 \\
\hline $\mathrm{Bi3+}$ & $5.67 \mathrm{E}-04$ & 92.4 & 246 & $5.15 \mathrm{E}-04$ & $5,40 \mathrm{E}-04$ & $3.93 \mathrm{E}-04$ & $6.18 \mathrm{E}-04$ \\
\hline $2.3+$ & $1.53 \mathrm{E}-0 \mathrm{~S}$ & 1.65 & 4.41 & $1.11 \mathrm{E}-05$ & $1.31 \mathrm{E}-05$ & $1,74 E-0 S$ & 1.95E-05 \\
\hline $\mathrm{Hg}+$ & $396 \mathrm{E}-06$ & 0.620 & 1.65 & $3.69 E-06$ & 3. $84 E-06$ & $4.02 \mathrm{E}-06$ & $407 \mathrm{E}-06$ \\
\hline $\mathrm{Zr}(\triangle \mathrm{ZrO}(\mathrm{OH}) 2)$ & 1.07E-04 & 7.59 & 20.2 & 9.71E-05 & $1.01 \mathrm{E} .04$ & $1.10 \mathrm{E}-04$ & $16 \mathrm{E}-04$ \\
\hline $\mathrm{Pb} 2+$ & $4.39 \mathrm{E}-04$ & 71.0 & 189 & 3.58E-04 & $3.98 \mathrm{E}-04$ & $4.81 \mathrm{E}-04$ & $5.21 E-04$ \\
\hline $\mathrm{Ni2+}$ & $3.25 \mathrm{E}-03$ & 149 & 397 & $3.13 \mathrm{E}-03$ & 3.19E-03 & $3.29 \mathrm{E}-03$ & $3.32 \mathrm{E}-03$ \\
\hline Sriz+ & 0 & 0 & $\overline{0}$ & 0 & 0 & 0 & 0 \\
\hline Mn4t & $1.74 \mathrm{E}-03$ & 74.5 & T98 & $1.31 \mathrm{E}-03$ & $1.52 E-03$ & $1.96 \mathrm{E}-03$ & $2.17 \mathrm{E}-03$ \\
\hline $\mathrm{Cos}^{2}$ & $1.67 \mathrm{E}-02$ & 522 & $1.39 \mathrm{E}+03$ & 1.54E-02 & $1.60 \mathrm{E}-02$ & $1.74 \mathrm{E}-02$ & $1.80 \mathrm{E}-02$ \\
\hline $\mathrm{K}+$ & $2.95 \mathrm{E}-02$ & 901 & $2.40 \mathrm{E}+03$ & $2.69 \mathrm{E}-02$ & $282 E-02$ & $3.10 \mathrm{E}-02$ & $3.23 \mathrm{E}-02$ \\
\hline OH- & 4.82 & $6.40 \mathrm{E}+04$ & $1.70 \mathrm{E}+05$ & 4.45 & 4.63 & 5.02 & 5.10 \\
\hline NO3- & 2.31 & $1.12 \mathrm{E}^{105}$ & $2.97 \mathrm{E}+05$ & 2.14 & 2.24 & 2.35 & 2.36 \\
\hline NO2- & 1.14 & $4.09 \mathrm{E}+04$ & $1,09 \mathrm{E}+05$ & 0.956 & 1.04 & 1.23 & 1.33 \\
\hline $\cos 2$. & 0.191 & $8.94 \mathrm{E}+03$ & $2,38 E+04$ & 0.173 & 0.181 & 0.204 & 0.206 \\
\hline P043. & $3.88 \mathrm{E} 02$ & $2.88 \mathrm{E}+03$ & $7.66 \mathrm{E}+03$ & $3.35 \mathrm{E}-02$ & $3.53 \mathrm{E}-02$ & $3.98 \mathrm{E}-02$ & $4.07 \mathrm{E}-02$ \\
\hline SO42- & 0.107 & $8.06 E+03$ & $2.15 E+04$ & 8.38E-02 & $9.51 E-02$ & 0.120 & 0.131 \\
\hline Si (as $\mathrm{SiO}_{2}-$ ) & $3.50 \mathrm{E}-02$ & 767 & $2.04 \mathrm{E}+03$ & $2.89 \mathrm{E}-02$ & 3.19E-02 & $3.81 \mathrm{E}-02$ & $4.11 E-02$ \\
\hline F. & $2.78 E-02$ & 412 & $1.10 \mathrm{E}+03$ & $2.33 \mathrm{E}-02$ & $2.52 \mathrm{E}-02$ & $3.00 \mathrm{E}-02$ & $3.17 E-02$ \\
\hline CI. & 0.111 & $3.0 \mathrm{EE}+03$ & $8.19 \mathrm{E}+03$ & 0.101 & 0.105 & 0.116 & 0.120 \\
\hline C6H5073- & $1,24 E-02$ & $1.84 \mathrm{E}+03$ & $4.89 t+03$ & $1.14 \mathrm{E}-02$ & $1.19 \mathrm{E}-02$ & $1.30 \mathrm{E}-02$ & $1.34 \mathrm{E}-62$ \\
\hline EDTA4- & $6.27 \mathrm{E}-03$ & $1.41 E+03$ & $3.75 E+03$ & $1.93 \mathrm{E}-03$ & $405 \mathrm{E}-03$ & $8.50 \mathrm{E}-03$ & $1.07 \mathrm{E}-02$ \\
\hline HEDTA3- & 1.17E-02 & $2.50 \mathrm{E}+03$ & $6.67 \mathrm{E}+03$ & $3.03 \mathrm{E}-0.03$ & $7.27 \mathrm{E}-03$ & $1.62 \mathrm{E}-02$ & $2.05 \mathrm{E}-02$ \\
\hline Glycolste- & $3.80 \mathrm{E}-02$ & $2.23 \mathrm{E}+03$ & $5.93 \mathrm{E}+03$ & 2.48E-02 & 3. $12 \mathrm{E}-02$ & $4.48 \mathrm{E}-02$ & $5.13 E-02$ \\
\hline ncente- & $2.65 \mathrm{E}-03$ & 122 & 325 & $2.17 \mathrm{E}-03$ & $2.41 E-03$ & $290 \mathrm{E}-03$ & $3.14 E-03$ \\
\hline oxalate2. & $2.00 \mathrm{E}-05$ & 1.37 & 3.66 & $1.77 \mathrm{E}-05$ & $1.98 \mathrm{E}-05$ & $2.12 \mathrm{E}-05$ & $2.23 \mathrm{E}-0 \mathrm{~s}$ \\
\hline DBP & 8. $35 \mathrm{E}-03$ & $1.37 \mathrm{E}+03$ & $3.66 \mathrm{E}+03$ & 6.87E-03 & $7.61 \mathrm{E}-03$ & $9.14 \mathrm{E}-03$ & 9.86E-03 \\
\hline butand & 8.38E-03 & 485 & $1.29 \mathrm{E}+03$ & $6.87 \mathrm{E}-03$ & $7.61 \mathrm{E}-03$ & 9 $14 E-03$ & $9.86 \mathrm{E}-\mathrm{C} 3$ \\
\hline $\mathrm{NH}$ & $4.00 \mathrm{E}-02$ & 530 & $1.41 E+03$ & $3.31 \mathrm{E}-02$ & $3.58 \mathrm{E}-02$ & $4.53 \mathrm{E}-02$ & 5. $13 \mathrm{E}-02$ \\
\hline $\mathrm{Fe}(\mathrm{CN}) 64-$ & 0 & 0 & 0 & 0 & 0 & 0 & 0 \\
\hline
\end{tabular}

- Unknowns in tank solids inventory are assigned by Tank Layering Model (TLM).

tWater wi\% derived from the diffetence of density and total dissolved species. 
HDW Model Rev. 4

\begin{tabular}{|c|c|c|c|c|c|c|c|}
\hline \multirow{4}{*}{$\begin{array}{l}\text { Physical } \\
\text { Properties }\end{array}$} & \multicolumn{7}{|c|}{ Single-Shell Tank 241-S-102 } \\
\hline & \multicolumn{7}{|c|}{ TLM Solids Composite Inventory Estimate* } \\
\hline & & & & & & & \\
\hline & & & & $95 \mathrm{Cl}$ & $-67 \mathrm{CI}$ & $+67 \mathrm{CI}$ & $+95 \mathrm{CI}$ \\
\hline Total TLM Wast & $2.66 \mathrm{E}+04(\mathrm{~kg})$ & $(4.00 \mathrm{kgal})$ & -- & $\cdots$ & --. & $\rightarrow$ & $\cdots$ \\
\hline Heat Load & $9.86 \mathrm{E}-02(\mathrm{~kW})$ & (337 BTU/hr) & $=$ & $7.15 \mathrm{E}-02$ & $8.74 \mathrm{E}-02$ & 0.106 & 0.109 \\
\hline Bulk Density & $1.76(\mathrm{~g} / \mathrm{cc})$ & $\cdots$ & --- & 1.51 & 1.53 & 2.11 & 2.24 \\
\hline Void Fraction & 0.536 & $\ldots$ & $\rightarrow$ & $6.00 \mathrm{E}-02$ & 0.217 & 0.737 & 0.743 \\
\hline Water wt\% & 27.0 & - & $\cdots$ & 2.64 & 10.0 & 42.6 & 44.2 \\
\hline ToC wt \% C (wet & 0 & - & - & 0 & 0 & 0 & 0 \\
\hline $\begin{array}{l}\text { Radiologlcal } \\
\text { Constituents }\end{array}$ & $\mathrm{Cu} / \mathrm{L}$ & $\mu \mathrm{Co} g$ & $\mathbf{C}$ & $\begin{array}{r}-95 \mathrm{CI} \\
\text { (CL/L) }\end{array}$ & $\begin{array}{l}67 \mathrm{CI} \\
(\mathrm{Ci} / \mathrm{L})\end{array}$ & $\begin{array}{l}+67 \mathrm{CI} \\
(\mathrm{CV} / \mathrm{L}) \\
\end{array}$ & $\begin{array}{c}+95 \mathrm{CI} \\
(\mathrm{Cl} / \mathrm{L})\end{array}$ \\
\hline $\mathrm{H}-3$ & $2.92 \mathrm{E}-05$ & $1.67 \mathrm{E}-02$ & 0.443 & $3.05 \mathrm{E}-06$ & 1.47E-05 & $4.10 \mathrm{E}-05$ & $4.10 \mathrm{E}-05$ \\
\hline C-14 & $2.26 \mathrm{E}-06$ & $1.29 \mathrm{E}-0.3$ & $3.43 \mathrm{E}-02$ & $2.59 \mathrm{E}-07$ & $1.06 \mathrm{E}-06$ & $3.07 \mathrm{E}-06$ & $3.07 \mathrm{E}-06$ \\
\hline Ni-59 & $1.49 \mathrm{E}-05$ & $8.46 \mathrm{E}-03$ & 0.225 & $1.07 \mathrm{E}-05$ & $1.31 \mathrm{E}-05$ & $162 \mathrm{E}-05$ & $1.60 \mathrm{E}-05$ \\
\hline Ni-63 & $139 \mathrm{E}-03$ & 0.790 & 21.0 & $1.00 \mathrm{E}-03$ & $1.22 \mathrm{E}-03$ & $1.52 \mathrm{E}-03$ & 1.49E-03 \\
\hline $\mathrm{Co}-60$ & 8.94E-07) & 5.0.5E-04 & t.35E-02 & $1.02 \mathrm{E}-07$ & 4.18E-07 & $1.21 \mathrm{E}-06$ & $1.21 \mathrm{E}_{-06}$ \\
\hline Se-79 & $4.81 E \cdot 07$ & $2.74 \mathrm{E}-04$ & $7.27 \mathrm{E}-03$ & $5.50 \mathrm{E}-0 \mathrm{~B}$ & 2.25E-07 & $6.51 E-07$ & 7. $15 \mathrm{E}-06$ \\
\hline Sr-90 & 0.928 & $\$ 29$ & $140 \mathrm{E}+04$ & 0.662 & 0.812 & 1.02 & 1.03 \\
\hline Y-90 & 0.929 & 529 & $1.41 \mathrm{E}+04$ & 0.662 & 0.812 & 1.02 & 1.03 \\
\hline $2 x-93$ & 2.27E- -66 & $1.29 \mathrm{E}-03$ & $3.4 \mathrm{E}-02$ & $2.59 \mathrm{E}-07$ & $106 E-06$ & $3.08 \mathrm{E}-06$ & 3.03E-05 \\
\hline $\mathrm{Nb} .93 \mathrm{~m}$ & $1.85 \mathrm{E}-06$ & $1.06 \mathrm{E}-03$ & $2.81 E-02$ & $2.12 \mathrm{E}-07$ & $868 \mathrm{E} \cdot 07$ & $2.51 \mathrm{E}-06$ & $3.21 \mathrm{E}-05$ \\
\hline Tc-99 & $1.58 E-0.5$ & 9.02E-03 & 0.240 & 1.81E-06 & $7.42 \mathrm{E}-06$ & $2.15 \mathrm{E}-05$ & 2. $15 \mathrm{E}-05$ \\
\hline Ru=106 & $2.50 \mathrm{E}+12$ & $1.42 \mathrm{E}-09$ & 3.78E-08 & $2.86 \mathrm{E}-13$ & 1.17E-12 & $3.38 \mathrm{E}-12$ & $3.38 \mathrm{E}-12$ \\
\hline Cd-113m & $6.99 \mathrm{E}-06$ & $3.98 \mathrm{E}-03$ & 0.106 & $8.00 \mathrm{E}-07$ & 3 27E-06 & 9.48E-06 & $9.48 \mathrm{E}-06$ \\
\hline$S b-125$ & $1.31 E-06$ & $7.47-0.4$ & $1.99 \mathrm{E}-02$ & 1.50E-07 & 6. 14E-07 & $1.78 \mathrm{E}-06$ & $1.78 \mathrm{E}-06$ \\
\hline Sn-126 & $7.39 \mathrm{E}-07$ & $4.21 \mathrm{E}-04$ & T.12E- 02 & 8.45E-08 & $346 \mathrm{E}-07$ & 1.00E-06 & $1.14 \mathrm{E}-05$ \\
\hline 1.129 & $3,04 E-08$ & $1.73 \mathrm{E}-0 \mathrm{~s}$ & 4.61E-04 & $3,48 \mathrm{E}-09$ & $1.42 E-08$ & 4.13E-08 & 4.13E-08 \\
\hline Cs-134 & $2.72 E-08$ & $1.55 \mathrm{E}-0 \mathrm{~s}$ & 4.11E-04 & 7.19E-09 & $1.52 \mathrm{E}-08$ & $3.52 E-08$ & $3.52 \mathrm{E}-0.8$ \\
\hline Cs-137 & $5.60 \mathrm{E}-02$ & 31.9 & 848 & $1.48 \mathrm{E}-02$ & $3.13 \mathrm{E}-02$ & $7.26 \mathrm{E}-02$ & $7.26 \mathrm{E}-02$ \\
\hline Ba-137m & $5.30 \mathrm{E}-02$ & 30.2 & 802 & $1.40 \mathrm{E}-02$ & $296 \mathrm{E}-02$ & $6.87 \mathrm{E}-02$ & $6.87 \mathrm{E}-02$ \\
\hline $5 m-151$ & $1.71 \mathrm{E}-03$ & 0.976 & 260 & $1.96 \mathrm{E}-04$ & 8.03E-04 & 2.33E-03 & 2.64E-02 \\
\hline Eu-152 & $7.00 \mathrm{E}-06$ & $3.98 \mathrm{E}-03$ & 0.106 & 6.85E-06 & $6.91 \mathrm{E}-06$ & 7.06E-06 & $7.06 \mathrm{E}-\infty 6$ \\
\hline Eu-154 & $2.16 \mathrm{E}-05$ & $1.23 \mathrm{E}-02$ & 0.327 & 2.47E-06 & 1.01E-05 & 2.93E-105 & 2.93E-05 \\
\hline Eu-155 & $3.30 \mathrm{E}_{-24}$ & 0.188 & 5.00 & $3.23 \mathrm{E}-04$ & 3.26E-04 & $3.33 \mathrm{E}-04$ & $3.33 \mathrm{E}-04$ \\
\hline $\mathrm{Ra}-226$ & $1.08 \mathrm{E}-\infty 9$ & $6.15 \mathrm{E}-07$ & $1.63 \mathrm{E}-05$ & 1.48E-10 & $6.04 \mathrm{E}-10$ & $1.56 \mathrm{E}-09$ & $201 E-09$ \\
\hline $\mathrm{Ra}-228$ & $9.26 \mathrm{E}-15$ & $5.28 \mathrm{E}-12$ & $1.40 \mathrm{E}-10$ & $9.07 \mathrm{E}-15$ & $9.15 \mathrm{E}-15$ & $9.34 \mathrm{E} \cdot 15$ & 9.34E-15 \\
\hline Ac- 227 & $4.76 \mathrm{E}-09$ & $2.71 \mathrm{E}-06$ & $7.20 \mathrm{E}-03$ & 3.06E-10 & $2,00 \mathrm{E}-09$ & $7.52 \mathrm{E}-09$ & $102 \mathrm{E}-0 \mathrm{~B}$ \\
\hline $\mathrm{Pa}-231$ & $7.18 \mathrm{E}-10$ & $4.09 \mathrm{E}-07$ & $1,09 \mathrm{E}-05$ & $8.21 \mathrm{E}-11$ & $3.36 \mathrm{E}-10$ & $2.04 \mathrm{E}-09$ & $1.68 \mathrm{E}-0 \mathrm{~B}$ \\
\hline Th-229 & $1.77 \mathrm{E}-12$ & $1.01 \mathrm{E}-09$ & $2.67 \mathrm{E}-08$ & $1.73 \mathrm{E}-12$ & $1.75 \mathrm{E}-12$ & $1.78 \mathrm{E} \cdot 12$ & $178 \mathrm{E}-12$ \\
\hline Th-232 & $5.91 \mathrm{E}-16$ & $3.36 \mathrm{E}-13$ & 8.94E-12 & $6.76 \mathrm{E}-17$ & $2.77 \mathrm{E}-16$ & $8.01 \mathrm{E}-16$ & $801 E-16$ \\
\hline $\mathrm{U}-232$ & $5.24 \mathrm{E}-12$ & $2.98 \mathrm{E}-09$ & $7.92 \mathrm{E}-08$ & $5.99 \mathrm{E}-13$ & 2.45E- 12 & $7.10 \mathrm{E} \cdot 12$ & 7.10E-12 \\
\hline $\mathrm{U}-233$ & $2.00 \mathrm{E}-13$ & $1.14 \mathrm{E}-10$ & $3.02 E-09$ & $2.28 \mathrm{E}-14$ & $9,34 \mathrm{E}-14$ & $2.71 \mathrm{E}-13$ & 27 IE-13 \\
\hline $\mathrm{U}-234$ & $1.19 \mathrm{E}-07$ & $6.78 \mathrm{E}-05$ & $1.80 \mathrm{E}-03$ & $1.36 \mathrm{E}-08$ & $5.58 \mathrm{E}-08$ & $1.62 \mathrm{E}-07$ & $162 \mathrm{E}-107$ \\
\hline $\mathrm{U}-235$ & $5.13 \mathrm{E}-\infty$ & $2.92 \mathrm{E}-06$ & $7.76 \mathrm{E}-05$ & $5.86 \mathrm{E}-10$ & $2.40 \mathrm{E}-09$ & $6,95 \mathrm{E}-09$ & 6.95E-09 \\
\hline $\mathrm{U} \cdot 236$ & $1.88 \mathrm{E}-69$ & $1.07 \mathrm{E}-06$ & 2.85E-05 & 2.16E-10 & $8.82 \mathrm{E}-10$ & $2.56 \mathrm{E} .69$ & $2.56 \mathrm{E}-109$ \\
\hline U-238 & $1.22 \mathrm{E}-07$ & $6.92 \mathrm{E}-05$ & 1.84E-03 & $1.39 \mathrm{E}-0 \mathrm{~g}$ & $5.69 \mathrm{E}-08$ & $1.65 \mathrm{E}-07$ & $1.65 \mathrm{E}-07$ \\
\hline$N p-237$ & $1.03 \mathrm{E}-07$ & $5.86 \mathrm{E}-05$ & $1.56 \mathrm{E}-03$ & 1.18E-0.8 & $4.81 \mathrm{E}-08$ & 1.39E-07 & 139E-07 \\
\hline Pu-238 & $0.61 \mathrm{E}-00$ & $3.77 \mathrm{E}-03$ & 0.100 & 4.75E-06 & $5.60 \mathrm{E}-06$ & $7.57 \mathrm{~T}-06$ & $8.48 E-06$ \\
\hline Pu-239 & 4. $72 \mathrm{E}-04$ & 0.269 & 7.14 & $3.39 \mathrm{E}-04$ & $404 \mathrm{E}-04$ & $5.40 \mathrm{E}-04$ & $605 \mathrm{E}-04$ \\
\hline Pu-240 & $6.76 \mathrm{E}-05$ & 3.85E-02 & 1.02 & 4.85E-05 & $5.78 \mathrm{E}-05$ & 7.73E-05 & 8. 66E-05 \\
\hline Pu-241 & $3.97 \mathrm{E}-94$ & 0.226 & 6.01 & $2.85 \mathrm{E}-04$ & $340 \mathrm{E}-04$ & 4.55E-04 & $509 \mathrm{E}-04$ \\
\hline \begin{tabular}{|l|}
$\mathrm{Pu} \cdot 242$ \\
\end{tabular} & $1.82 \mathrm{E}-09$ & $1,04 E-06$ & 2.76E-05 & $1,31 \mathrm{E}-09$ & $1.56 \mathrm{E}-09$ & $2.08 \mathrm{E}-09$ & $2.34 \mathrm{E}-09$ \\
\hline Am-241 & 8.09E-06 & $461 \mathrm{E}-03$ & 0.122 & $9.26 \mathrm{E}-07$ & $3.79 \mathrm{E}-06$ & $1.10 \mathrm{E}-05$ & 1.43E-04 \\
\hline Am-243 & $7,57 \mathrm{E}-11$ & 4.31E-08 & 1.15E-06 & $8.66 \mathrm{E}-12$ & 3.54E-11 & $1.03 \mathrm{E}-10$ & 1.34E- 09 \\
\hline $\mathrm{Cm}-242$ & 1.6tE-0? & $9.16 \mathrm{E}-05$ & 2.44E-03 & $1.58 \mathrm{E}-67$ & $1.59 \mathrm{E}-07$ & $1.62 \mathrm{E}-07$ & 162E-07 \\
\hline$C m-243$ & $3.69 \mathrm{E}-09$ & $2.10 \mathrm{E}-06$ & 5.58E-05 & $3.61 \mathrm{E}-09$ & $3.64 \mathrm{E}-09$ & 3.72E-09 & 3. $72 \mathrm{E}-09$ \\
\hline Cm-244 & $2.70 \mathrm{E}-09$ & 1.54E-06 & 4.09E-05 & $3.09 \mathrm{E}-10$ & $1.27 \mathrm{E}-09$ & 3.67E-09 & $3.67 \mathrm{E}=09$ \\
\hline Totals: & $\mathbf{M}$ & 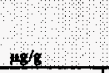 & Le & $\begin{array}{l}+95 \mathrm{Cl} \\
(\mathrm{M} \mathrm{er} \\
\mathrm{dL}\end{array}$ & $\begin{array}{l}67 \mathrm{Cl}(\mathrm{M} \\
\text { or } \mathrm{g} / \mathrm{L})\end{array}$ & $\begin{array}{c}+67 C \mathrm{Cl}(\mathrm{M} \\
\text { or } \mathrm{g} / \mathrm{L}) \\
\end{array}$ & $\begin{array}{c}+95 \mathrm{CI} \\
(\mathrm{M} \mathrm{or} \\
\left.\mathrm{g}^{\prime} \mathrm{L}\right)\end{array}$ \\
\hline Pu & 7.89E-03 (gL) & $\ldots$ & 0.119 & $5.67 \mathrm{E}-03$ & $6.76 \mathrm{E}-03$ & $9.03 \mathrm{E}-03$ & $1.01 \mathrm{E}-02$ \\
\hline $\mathrm{U}$ & $1.53 \mathrm{E}-03$ & 207 & 5.51 & 1.75E-04 & 7.16E- 04 & $2.07 \mathrm{E}-03$ & $2.07 \mathrm{E}-03$ \\
\hline
\end{tabular}

"Unknowns in tank solids inventory are assigned by Tark Layering Model (TLM). 
HNF-SD-WM-ER-323, Rev. 1

HDW Modal Rav.

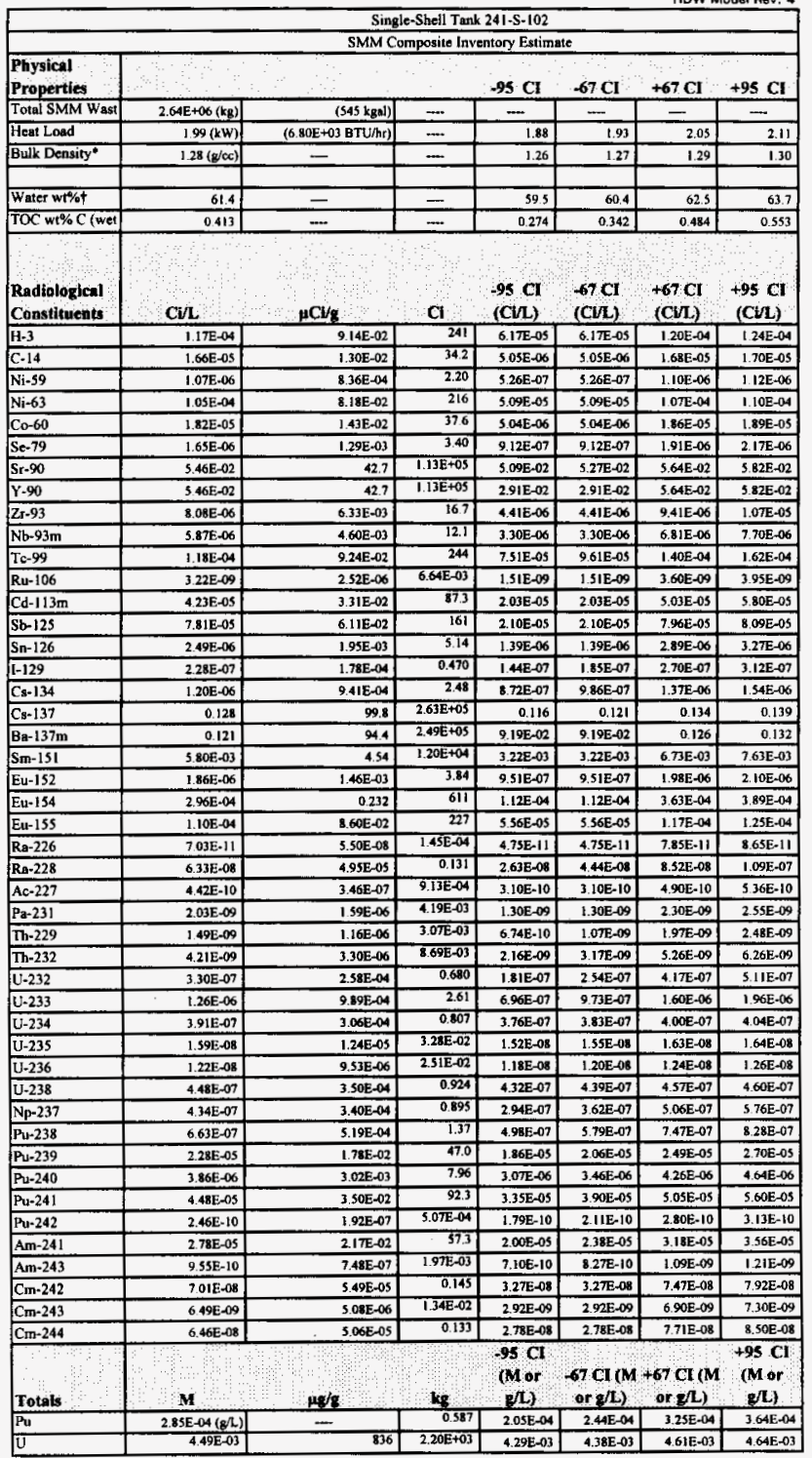

"Density is calculated based on $\mathrm{Na}, \mathrm{OH}$-, and AlO2.

tWater wt\% derived from the difference of density and total dissolved species. 
HDW Model Rov. 4

\begin{tabular}{|c|c|c|c|c|c|c|c|}
\hline \multirow[b]{3}{*}{$\begin{array}{l}\text { Physical } \\
\text { Propertles }\end{array}$} & \multicolumn{7}{|c|}{ Single-Shell Tank 241-S.102 } \\
\hline & \multicolumn{7}{|c|}{ Total Inventory Estimsic* } \\
\hline & & & & $-95 \mathrm{CI}$ & $-67 \mathrm{CI}$ & $+67 \mathrm{CI}$ & $+95 \mathrm{CI}$ \\
\hline Total Wastc & $2.66 \mathrm{E}+06(\mathrm{~kg})$ & (549 kgal) & $m$ & - & $\ldots$ & $\cdots$ & $\cdots$ \\
\hline Heat Load & $2.09(\mathrm{~kW})$ & $(7.13 \mathrm{E}+03 \mathrm{BTU} / \mathrm{hr})$ & $\ldots$ & 1.97 & 2.03 & 2.15 & 2.20 \\
\hline Bulk Density & $1.28(\mathrm{~g} / \mathrm{cc})$ & $\ldots$ & $=$ & 1.26 & 1.27 & 1.29 & 1.30 \\
\hline & & & & & & & \\
\hline Water wt\% $\%$ & 61.1 & 一 & - & 59.2 & 60.0 & 62.1 & 63,4 \\
\hline TOC wt $\%$ C (wet & 0.409 & $=$ & - & 0.271 & 0.338 & 0.479 & 0.547 \\
\hline $\begin{array}{l}\text { Radiological } \\
\text { Consttuents }\end{array}$ & $\mathrm{Cu}$ & $\mu C y$ & $\mathrm{Ci}$ & $\begin{array}{l}-95 \mathrm{CI} \\
(\mathrm{CVL})\end{array}$ & $\begin{array}{l}-67 \mathrm{CI} \\
(\mathrm{CUL}) \\
\end{array}$ & $\begin{array}{l}+67 \mathrm{CI} \\
(\mathrm{Ci} / \mathrm{L}) \\
\end{array}$ & $\begin{array}{r}+95 \mathrm{Ct} \\
(\mathrm{CU} / \mathrm{L})\end{array}$ \\
\hline $\mathrm{H}-3$ & $1.16 \mathrm{E}-04$ & $9.07 E-02$ & 241 & $6.15 E-05$ & $6.15 E-05$ & $1.20 \mathrm{E}-04$ & $1.24 \mathrm{E}+04$ \\
\hline $\mathrm{C}-14$ & $1.65 \mathrm{E}-05$ & $129 \mathrm{E}-02$ & 34.2 & $5,03 E-06$ & $5.03 \mathrm{E}-06$ & $1.67 \mathrm{E}-05$ & $1.69 \mathrm{E}-05$ \\
\hline Ni-59 & $1.17 \mathrm{E}-06$ & $9,12 E-04$ & 2.43 & $6.30 \mathrm{E}-07$ & $6.30 \mathrm{E}-07$ & $1.20 E-06$ & $1.22 \mathrm{E}-06$ \\
\hline $\mathrm{Ni}-63$ & $1.14 \mathrm{E}-04$ & $8.89 \mathrm{E}-02$ & 237 & $6.07 \mathrm{E}-05$ & $6.07 \mathrm{E}-0 \mathrm{~S}$ & $1.17 \mathrm{E}-04$ & 1. 19E-04 \\
\hline $\mathrm{Co}-60$ & $1.81 E-05$ & $1.41 \mathrm{E}-02$ & 37.6 & $5.01 \mathrm{E}-06$ & $5.01 \mathrm{E}-06$ & 1. 8dE-0S & $1.87 E-05$ \\
\hline Se-79 & 1.64E-A6 & 1.28E-03 & 3.41 & $9.08 \mathrm{E}-07$ & $9.08 \mathrm{E}-07$ & $1.90 \mathrm{E}-06$ & $2.16 E-06$ \\
\hline Sr-90 & $6.09 \mathrm{E}-02$ & 47.5 & $12 \pi+05$ & $5.73 \mathrm{E} \cdot 02$ & $5.91 E-02$ & $6.2 T E-02$ & 6.45E.02 \\
\hline$Y-90$ & $6.09 \mathrm{E}-02$ & 47.5 & $1.27 \mathrm{E}+05$ & $3.57 \mathrm{E}-02$ & $3.576-02$ & $6.28 \mathrm{E}-02$ & 6.45E-02 \\
\hline $2 r-93$ & $8,04 \mathrm{E}-06$ & $6.28 \mathrm{E}-03$ & 16.7 & $439 \mathrm{E}-06$ & $4.39 \mathrm{E}-106$ & 9.36E- 06 & $1.06 \mathrm{E}-0 \mathrm{~S}$ \\
\hline $\mathrm{Nb}-93 \mathrm{~m}$ & SBSE-06 & 4.56E-03 & 12.1 & $3.29 \mathrm{E}-06$ & $3.29 \mathrm{E}-06$ & 6.77E-06 & $7.66 \mathrm{E}-06$ \\
\hline Tc-99 & $1.17 \mathrm{E}-04$ & $9.16 \mathrm{E}-02$ & 244 & $7.47 \mathrm{E}-05$ & $9.55 \mathrm{E}-05$ & $139 \mathrm{E}-04$ & $1.61 \mathrm{E}-04$ \\
\hline$R_{\mathrm{H}-106}$ & $3.20 \mathrm{E}-09$ & $2.49 \mathrm{E}-06$ & $6.64 \mathrm{E}-03$ & $1.50 \mathrm{E}-09$ & $1.50 \mathrm{E}-09$ & $3.57 \mathrm{E}-09$ & $3.92 \mathrm{E}-09$ \\
\hline $\mathrm{Cd}-113 \mathrm{~m}$ & 4.21E-05 & 3.28E-02 & 87.4 & $2.02 \mathrm{E}-0 \mathrm{~S}$ & $2.02 \mathrm{E}-05$ & $5,00 E-05$ & $5.76 \mathrm{E}-05$ \\
\hline$S b-125$ & $7.76 \mathrm{E}-05$ & $6.05 \mathrm{E}-02$ & 161 & $2.09 \mathrm{E}-05$ & 2.09E-0S & $7.90 \mathrm{E}-05$ & 8.04E-05 \\
\hline $5 n-126$ & $2.48 \mathrm{E}-06$ & $1.93 \mathrm{E}-03$ & 5.15 & $1,39 \mathrm{E}-06$ & $1.38 \mathrm{E}-06$ & $2.87 \mathrm{E}-06$ & $3.25 \mathrm{E}-06$ \\
\hline 1.129 & $2.26 \mathrm{E}-07$ & $1.77 \mathrm{E}-04$ & $\begin{array}{l}0.470 \\
\end{array}$ & $1,44 \mathrm{E}-07$ & 1.84E-07 & $2.69 \mathrm{E}-07$ & 3.10E-07 \\
\hline $\mathrm{C}_{\mathrm{B}-134}$ & $1.19 \mathrm{E}-06$ & $932 \mathrm{E}-04$ & 2.48 & 8.66E-07 & $9.79 \mathrm{E}-\mathrm{Al}$ & $1.36 \mathrm{E}-06$ & $1.53 \mathrm{E}-06$ \\
\hline Cs-137 & 0.120 & 99.1 & $2.64 \mathrm{E}+05$ & 0.115 & 0.121 & 0.133 & 0.139 \\
\hline $\mathrm{Ba}-137 \mathrm{~m}$ & 0.120 & 93.8 & $2.50 \mathrm{E}+05$ & $9.16 \mathrm{E}-02$ & $9.16 \mathrm{E}-02$ & 0.126 & 0.131 \\
\hline Sm-151 & 5.77E-03 & 4.50 & $1.20 \mathrm{E}+04$ & $3.21 \mathrm{E}-03$ & $3.21 \mathrm{E}, 03$ & $6.70 \mathrm{E}-03$ & $7.58 \mathrm{E}-03$ \\
\hline Eu-152 & 1.90E-106 & $1.48 \mathrm{E}-03$ & 3.95 & $9.95 \mathrm{E}-07$ & 9.95E-0? & $2.02 \mathrm{E}-06$ & $2.14 \mathrm{E}-06$ \\
\hline Eu-154 & $2.94 \mathrm{E}-04$ & \begin{tabular}{l|l|}
0.229 \\
\end{tabular} & 611 & 1.11E-04 & 1.11E-04 & $3.60 \mathrm{E}-04$ & $3.86 \mathrm{E}-04$ \\
\hline Eu-155 & $1.11 \mathrm{E}-04$ & 8. $70 \mathrm{E}-02$ & 232 & $5.76 \mathrm{E}-05$ & $5.76 \mathrm{E}-05$ & $1.19 E-04$ & $1.26 \mathrm{E}-04$ \\
\hline $\mathrm{Ra}-226$ & $7.76 \mathrm{E}-11$ & $0.06 \mathrm{E}-08$ & 1.6JE-04 & $5.50 \mathrm{E}-11$ & $5.50 \mathrm{E}-11$ & 8.58E-11 & $937 \mathrm{E}-11$ \\
\hline $\mathrm{Ra}-228$ & $6.28 \mathrm{E}-08$ & $490 \mathrm{E}-0 \mathrm{~S}$ & 0.131 & 2.61E-08 & 441E-08 & $8.46 \mathrm{E}-08$ & $1.08 \mathrm{E}-07$ \\
\hline$A c-227$ & $4.74 \mathrm{E}-10$ & $3.70 \mathrm{E}-07$ & 9.85E-04 & $3.42 \mathrm{E}-10$ & 3.42E-10 & $5.21 \mathrm{E}-10$ & $5.67 \mathrm{E}-10$ \\
\hline $\mathrm{Pa}-231$ & $2.02 \mathrm{E}-09$ & $1.58 E-06$ & $4.205-03$ & 1.29E-49 & $1.29 \mathrm{E}-09$ & $2.29 \mathrm{E}-09$ & $2.54 \mathrm{E}-09$ \\
\hline Th-229 & $1.48 E-09$ & $1.15 \mathrm{E}-06$ & $3.07 \mathrm{E}-03$ & $6.69 \mathrm{E}-10$ & $1.06 \mathrm{E}-09$ & $1.95 \mathrm{E}-09$ & $2.46 \mathrm{E}-09$ \\
\hline Th-232 & $4.18 \mathrm{E}-09$ & $3.26 \mathrm{E}-06$ & 8.69E-03 & $2.15 \mathrm{E}-09$ & 3.14E-09 & $5.22 \mathrm{E}-09$ & $6.21 \mathrm{E}-09$ \\
\hline $\mathrm{U}-232$ & $327 \mathrm{E}-07$ & 2.55E-04 & $\begin{array}{l}0.650 \\
\end{array}$ & $1.80 \mathrm{E}-07$ & $2.52 \mathrm{E}-07$ & $4.14 \mathrm{E}-07$ & 5.07E-07 \\
\hline $\mathrm{U}-233$ & $1.25 \mathrm{E}-06$ & 9.79E-04 & 2.61 & 6.91E-07 & $966 \mathrm{E}-07$ & 1.59E-06 & $1,94 \mathrm{E}-0.6$ \\
\hline U-234 & $3.89 \mathrm{E}-07$ & $3.04 E_{-04}$ & 0.809 & $3.74 \mathrm{E}-07$ & 3.81E-07 & 3.98E-07 & $4.01 E-07$ \\
\hline U-235 & t.58E-08 & $1.23 \mathrm{E}-05$ & $3.29 \mathrm{E}-02$ & 1.52E-08 & $155 \mathrm{E}-08$ & $1.62 E-08$ & $1.63 \mathrm{E}-08$ \\
\hline$U-236$ & $1.2 \mathrm{JE}-0 \mathrm{~g}$ & 9,45E-06 & $252 \mathrm{E}-02$ & 1.17E-08 & $1.19 \mathrm{E}-08$ & $1.23 E-08$ & $1.25 \mathrm{E}-08$ \\
\hline $\mathrm{U}-238$ & 4.45E-07 & $3.48 \mathrm{E}-04$ & 0.925 & 4.30E-07 & 437E- -97 & 4.54E-07 & $457 \mathrm{E}-07$ \\
\hline $\mathrm{Np}-237$ & $4.31 \mathrm{E}-07$ & $3.3 \pi \mathrm{E}-04$ & $\begin{array}{l}0.897 \\
\end{array}$ & $2.92 \mathrm{E}-07$ & $3.60 \mathrm{E}-07$ & 5.03E-07 & $5.73 \mathrm{E}-07$ \\
\hline $\mathrm{Pu}-238$ & $7,06 \mathrm{E}-07$ & $5.51 \mathrm{E}-04$ & 1.47 & $5.42 \mathrm{E}-07$ & $623 \mathrm{E}-07$ & 7.90E-07 & $8.70 \mathrm{E}-07$ \\
\hline Pu-239 & $2.61 \mathrm{E}-05$ & $2,03 \mathrm{E}-02$ & 54.2 & $2.19 \mathrm{E}-05$ & $239 \mathrm{E}-0 \mathrm{~s}$ & $2.82 \mathrm{E}-0 \mathrm{5}$ & 3.02E-05 \\
\hline $\mathrm{Pu}-240$ & 4.32E- 06 & $3.37 \mathrm{E}-03$ & 8.98 & $3.54 E-06$ & $393 \mathrm{E}-06$ & $4.72 \mathrm{E}-06$ & $5.10 \mathrm{E}-06$ \\
\hline Pu-241 & 4.73E-05 & $3.69 \mathrm{E}-02$ & 98.4 & $3.62 \mathrm{E}-05$ & $4.16 \mathrm{E}-05$ & $\begin{array}{l}5.30 \mathrm{E}-05 \\
\end{array}$ & $5.85 \mathrm{E}-0 \mathrm{~s}$ \\
\hline Pu-242 & $2.57 \mathrm{E} \cdot 10$ & 2.01E-07 & 5.34E-04 & $1.91 \mathrm{E}-10$ & $2.23 \mathrm{E}-10$ & $2.91 \mathrm{E} \cdot 10$ & $3.24 \mathrm{E}-10$ \\
\hline Ann-241 & $2.76 \mathrm{E}-05$ & $2.16 \mathrm{E}-02$ & \begin{tabular}{l|l}
57.4 \\
\end{tabular} & $1.99 \mathrm{E}-05$ & $237 \mathrm{E}-05$ & $3.16 \mathrm{E}-05$ & 3.54E-05 \\
\hline Am-243 & $9.49 \mathrm{E}-10$ & $7.41 \mathrm{E}-07$ & $1.97 \mathrm{E}-03$ & $7.05 \mathrm{E}-10$ & $8.22 \mathrm{E}-10$ & $1.08 \mathrm{E}-09$ & $1.20 \mathrm{E}-109$ \\
\hline $\mathrm{Cm}-242$ & $7.0 \mathrm{BE}-08$ & $5,52 \mathrm{E}-05$ & 0.147 & 3.37E-08 & 3.37E-08 & $7.54 \mathrm{E}-0 \mathrm{~s}$ & $7.98 \mathrm{E}-08$ \\
\hline Cm-243 & $6.47 \mathrm{E}-09$ & $5.05 \mathrm{E}-06$ & $1.34 \mathrm{E}-02$ & 2.92E-09 & $2,92 E-09$ & $6.88 E-09$ & $7.28 \mathrm{E} .99$ \\
\hline $\mathrm{Cm}-244$ & $6.42 \mathrm{E}-08$ & 5.01E-05 & 0.133 & $2.76 \mathrm{E}-0 \mathrm{~s}$ & $2.76 \mathrm{E}-08$ & $7.65 \mathrm{E}-0 \mathrm{~B}$ & $8.44 \mathrm{E}-0 \mathrm{~S}$ \\
\hline Totats & $\mathbf{M}$ & Hers & kg & $\begin{array}{c}-95 \mathrm{CI} \\
(\mathrm{M} \mathrm{or} \\
\text { /L) }\end{array}$ & $\begin{array}{l}67 \mathrm{CI}(\mathrm{M} \\
\text { or } \mathrm{el})\end{array}$ & $\begin{array}{r}+67 \mathrm{CI}(\mathrm{M} \\
\operatorname{er} \mathrm{g} / \mathrm{L})\end{array}$ & $\begin{array}{r}+95 \mathrm{CI} \\
\text { (M or } \\
\mathrm{uLl}\end{array}$ \\
\hline $\mathrm{Pu}$ & 3,40E-04 (g) & $\ldots$ & 0.707 & $2.6 ! \mathrm{E}-04$ & $3.00 \mathrm{E}-04$ & $3.80 \mathrm{E}-04$ & $4.19 \mathrm{E}-04$ \\
\hline $\mathrm{U}$ & $4.47 \mathrm{E}-03$ & 830 & $2.21 \mathrm{E}+03$ & $4.27 \mathrm{~T}-03$ & $4.37 \mathrm{E}-03$ & 4.58E-03 & 461E-03 \\
\hline
\end{tabular}

Unknowns in tank solids inventory are assigned by Tank Layering Model (TLM)

†Volume average for density, mass average Water wr\% and TOC wt\% C 
HDW Model Rev. 4

\begin{tabular}{|c|c|c|c|c|c|c|c|}
\hline & \multicolumn{7}{|c|}{ Single-Shell Tank 241-S-103 } \\
\hline & \multicolumn{7}{|c|}{ TLM Solids Composite Inventory Estimate* } \\
\hline $\begin{array}{l}\text { Physical } \\
\text { Properties }\end{array}$ & & & & -9S CI & $-67 \mathrm{CI}$ & $+67 \mathrm{CI}$ & $+95 \mathrm{CI}$ \\
\hline Total TLM Wast & $5.98 \mathrm{E}+04(\mathrm{~kg})$ & $(900 \mathrm{kgal})$ & 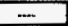 & $\ldots$ & $\ldots$ & $\ldots$ & - \\
\hline Heat Lond & $0.222(\mathrm{kw})$ & (758 BTU/ar) & - & 0.161 & 0.197 & 0.238 & 0.246 \\
\hline Bulk Density & $1.76(\mathrm{~g} / \mathrm{cc})$ & - & - & 1.51 & 1.53 & 2.11 & 224 \\
\hline Void Fraction & 0.536 & - & - & $6.00 E-02$ & 0.217 & 0.737 & 0.743 \\
\hline Water w $\%$ & 27.0 & $\ldots$ & - & 2.64 & 100 & 42.6 & 44.2 \\
\hline TOC wt\% C (wet & 0 & - & $\overline{-}$ & 0 & 0 & 0 & 0 \\
\hline $\begin{array}{c}\text { Chentical } \\
\text { Constituents }\end{array}$ & molert & ppin & $\mathrm{kg}$ & $\begin{array}{l}\text {-9s Cl } \\
\text { (mole/L) }\end{array}$ & $\begin{array}{r}-67 \mathrm{CI} \\
\text { (mole/L) }\end{array}$ & $\begin{array}{l}+67 \text { CI } \\
\text { (molerL) }\end{array}$ & $\begin{array}{l}+95 \mathrm{Cl} \\
(\mathrm{mole} / \mathrm{L})\end{array}$ \\
\hline $\mathrm{Nat}$ & 8.13 & $1.06 \mathrm{E}+05$ & $6.36 \mathrm{E}+03$ & 3.05 & 3.56 & 14.7 & 18.2 \\
\hline$\hat{A l 3+}$ & 6.99 & $1.07 \mathrm{E}+05$ & $6.42 E+03$ & 6.58 & 6.74 & 7.15 & 7.15 \\
\hline Fe3+(total Fe) & 1.01 & $3.22 \mathrm{E}+0 \mathrm{~K}$ & $1.93 \mathrm{E}+03$ & 0.994 & 1.00 & 1.02 & 1.03 \\
\hline $\mathrm{Cr} 3+$ & $019 \mathrm{E}-02$ & $1.83 \mathrm{E}+03$ & 110 & $7.08 \mathrm{E}-0 \mathrm{~s}$ & 3.39E-02 & 0522 & 1.06 \\
\hline $\mathrm{B}$ i3t & 0 & 0 & $\bar{D}$ & 0 & 0 & 0 & 0 \\
\hline Lejt & 0 & 0 & 0 & 0 & 0 & 0 & 0 \\
\hline $\mathrm{Hg}+$ & 0 & 0 & 0 & 0 & 0 & 0 & 0 \\
\hline $\mathrm{Zr}(\mathrm{m} \mathrm{ZrO}(\mathrm{OH}) 2)$ & 0 & 0 & 0 & 0 & 0 & 0 & 0 \\
\hline $\mathrm{Pb} 2+$ & 0 & 0 & 0 & 0 & 0 & 0 & 0 \\
\hline $\mathrm{Ni2+}$ & $5.07 \mathrm{E}-02$ & $1.69 \mathrm{E}+03$ & 101 & $3,66 \mathrm{E}-02$ & 4.45E- 02 & $3.54 \mathrm{E}-02$ & $5.45 \mathrm{E}-02$ \\
\hline $5.2+$ & 마 & 0 & 0 & 0 & 0 & 0 & 0 \\
\hline Mnst+ & 0 & 0 & 0 & 0 & 0 & 0 & 0 \\
\hline $\mathrm{CA}^{2+}$ & 0.220 & $5.02 E+03$ & 300 & 0.146 & 0.182 & 0.258 & 0.294 \\
\hline$\overline{\mathrm{K}+}$ & $112 \mathrm{E}-02$ & 250 & 14.9 & $1.28 \mathrm{E}-03$ & $5.25 \mathrm{E}-03$ & $1.52 \mathrm{E}-02$ & $1.52 \mathrm{E}-02$ \\
\hline $\mathrm{OH}-$ & 25.6 & $2.48 \mathrm{E}+05$ & $1.48 \mathrm{E}+04$ & 23.1 & 24.3 & 26.9 & 28.4 \\
\hline NO3- & 5.29 & $1.87 \mathrm{~F}+05$ & $1.12 \mathrm{E}+04$ & $6.03 \mathrm{E}-02$ & $6.03 \mathrm{E}-02$ & 13.5 & 17.9 \\
\hline NO2- & 1.46 & $3.82 \mathrm{E}+04$ & $2.202+03$ & 0.132 & 0.595 & 2.15 & 2.15 \\
\hline $\cos 2$. & 0.220 & $7.51 E+03$ & 449 & 0.146 & 0.182 & 0.258 & 0.294 \\
\hline PO43- & 0 & 0 & 0 & 0 & 0 & 0 & a \\
\hline 5042- & 1.04E-02 & 569 & 34,0 & 1.19E-03 & $487 \mathrm{E}-03$ & $1.41 \mathrm{E}-02$ & 14 !E-02 \\
\hline Si (as SiO32-) & $8.06 E-03$ & 129 & 7.71 & $9.21 \mathrm{E}-04$ & $3.77 \mathrm{E}-03$ & 1.09E-02 & 1.09E-02 \\
\hline$\vec{F}$ : & 0 & 0 & 0 & 0 & 0 & 0 & 0 \\
\hline CI- & $3.161,02$ & $1.04 \mathrm{E}+03$ & 52.3 & $5.91 E-03$ & $2.42 \mathrm{E}-02$ & $7,00 \mathrm{E}-02$ & 0.157 \\
\hline C6H5073. & 0 & 0 & 0 & 0 & 0 & 0 & 0 \\
\hline EDTA4- & 0 & 6 & 0 & 0 & 0 & 0 & 0 \\
\hline HEDT A 3. & 0 & 0 & 0 & c & 0 & 0 & 0 \\
\hline glycolate- & 0 & 0 & 0 & 0 & 0 & 0 & 0 \\
\hline scente- & 0 & 0 & 0 & 0 & 0 & 0 & 0 \\
\hline Oxalate2- & 0 & 0 & 0 & 0 & 0 & 0 & 0 \\
\hline$\overline{\mathrm{DBP}}$ & 0 & 0 & 0 & 0 & 0 & 0 & 0 \\
\hline butanol & 0 & 0 & 0 & 0 & 0 & 0 & 0 \\
\hline$\overline{\mathrm{NH} 3}$ & 0.256 & $247 \mathrm{E}+03$ & 148 & 6.55E- -92 & $9.25 \mathrm{E}-02$ & 0.270 & 0.284 \\
\hline $\mathrm{Fe}(\mathrm{CN}) 64$ & 0 & 0 & $\overline{0}$ & 0 & $\overline{0}$ & 0 & 0 \\
\hline
\end{tabular}

"Lnknownz in tank solids inventory are assigned by Tank Leyering Model (TLM). 
HNF-SD-WM-ER-323, Rev. 1

HDW Model Rev. 4

\begin{tabular}{|c|c|c|c|c|c|c|c|}
\hline \multirow[b]{3}{*}{$\begin{array}{l}\text { Physical } \\
\text { Propertites }\end{array}$} & \multicolumn{7}{|c|}{ Single-Shell Tank 241-S-103 } \\
\hline & \multicolumn{7}{|c|}{ SMM Composite Inventory Estimate } \\
\hline & & & & $-95 \mathrm{CI}$ & $-67 \mathrm{CI}$ & $+67 \mathrm{CI}$ & $+95 \mathrm{CI}$ \\
\hline Total SMM Wast & $1.52 \mathrm{E}+06(\mathrm{~kg})$ & (239 kgnl) & $\cdots$ & - & $=$ & $\ldots$ & $\ldots$ \\
\hline Heat Load & $2.12(\mathrm{~kW})$ & $(7.25 \mathrm{E}+03 \mathrm{BTU} / \mathrm{hr})$ & $\cdots$ & 1.99 & 2.06 & 2.19 & 2.26 \\
\hline Bulk Density* & $1.67(g / c c)$ & -- & $\ldots$ & 1.62 & 1.65 & 1.70 & 1.72 \\
\hline Water wt $\%$ & 28.3 & - & $\ldots$ & 25.7 & 26.8 & 29.8 & 31.7 \\
\hline TOC wt\% C (wet & 0.798 & $\ldots$ & - & 0.523 & 0.658 & 0.938 & 1.07 \\
\hline $\begin{array}{l}\text { Chemilcat } \\
\text { Constituents }\end{array}$ & mole/L & ppin & $\mathrm{kg}$ & $\begin{array}{l}-95 \mathrm{CI} \\
\text { (molol } \mathrm{L})\end{array}$ & $\begin{array}{r}-67 \mathrm{CI} \\
(\mathrm{mole} / \mathrm{L})\end{array}$ & $\begin{array}{l}+67 \mathrm{Ct} \\
\text { (mole/ })\end{array}$ & $\begin{array}{l}+95 \mathrm{CI} \\
(\mathrm{mole} / \mathrm{L})\end{array}$ \\
\hline $\mathrm{Na}+$ & 15.8 & $2.17 \mathrm{E}+0 \mathrm{~S}$ & $3.28 E+05$ & 14.5 & 15.1 & 16.4 & 16.9 \\
\hline $\mathrm{Al}+\mathbf{+}$ & 1.86 & $2.99 \mathrm{E}+04$ & $453 \mathrm{E}+04$ & 1.72 & 1.79 & 1.93 & 1.99 \\
\hline $\mathrm{Fe}_{\mathrm{e}}+$ + (10ta) Fe) & $1.21 \mathrm{E}-02$ & 403 & 611 & $9.90 E-03$ & $1.10 \mathrm{E}-02$ & $1.32 E-02$ & $1.43 \mathrm{E}-02$ \\
\hline $\mathrm{Cr} 3+$ & 0.165 & $5.12 E+03$ & $7.75 E+03$ & 0.137 & 0.152 & 0.170 & 0.174 \\
\hline $\mathrm{Bi3+}$ & $1.52 \mathrm{E}-03$ & 190 & 288 & 1.38E-03 & 1 45E-03 & $1.59 \mathrm{E}-03$ & $1.66 \mathrm{E}-03$ \\
\hline $\mathrm{Ln} 3+$ & $4.56 \mathrm{E}-05$ & 3.78 & 5.72 & $3.30 \mathrm{E}-05$ & $3.91 \mathrm{E}-05$ & $520 \mathrm{E}-05$ & $5.82 \mathrm{E}-05$ \\
\hline $\mathrm{Hg} 2+$ & $1.03 \mathrm{E}-05$ & \begin{tabular}{l|l|}
1.23 \\
\end{tabular} & 1.86 & $9.52 \mathrm{E}-06$ & $994 E-06$ & $1.04 \mathrm{E} \cdot 05$ & $1.05 \mathrm{E}-05$ \\
\hline$\overline{\mathrm{ZT}(\mathrm{AS} \mathrm{ZrO}(\mathrm{OH}) 2)}$ & $2.87 \mathrm{E}-04$ & 15.6 & 23.7 & 2.6 IE-04 & $270 \mathrm{E}-04$ & 2.97E-04 & 3.12E-04 \\
\hline Pb2+ & $1.12 \mathrm{E}-03$ & 138 & 209 & $9.06 \mathrm{E}-04$ & $101 \mathrm{E}-03$ & $1.22 \mathrm{E}-03$ & $1.33 \mathrm{E}-03$ \\
\hline Ni2+ & $7.21 \mathrm{E}-03$ & \begin{tabular}{|l|l|}
253 \\
\end{tabular} & 383 & $6.89 \mathrm{E}-03$ & 7.05E- 03 & $7.29 \mathrm{E}-03$ & $7.37 \mathrm{E}-03$ \\
\hline $5 r_{2}+$ & 0 & 0 & 0 & 0 & 0 & 0 & 0 \\
\hline Mndt & $4.31 \mathrm{E}-03$ & 141 & 214 & $3.26 \mathrm{E}-03$ & $378 \mathrm{E}-03$ & $4.85 \mathrm{E}-03$ & 5.36E-03 \\
\hline $\mathrm{Ca2}+$ & $3.78 \mathrm{E}-02$ & 905 & $1,37 \mathrm{E}+03$ & $3.47 \mathrm{E}-02$ & $362 \mathrm{E}-02$ & $3.94 \mathrm{E}-02$ & $4.09 \mathrm{E}-02$ \\
\hline K+ & $7.32 \mathrm{E}-02$ & $1.7 \mathrm{E}+03$ & $2.59 E+03$ & $6.68 \mathrm{E}-02$ & $698 \mathrm{E}-02$ & 7.67E-02 & $8.00 \mathrm{E}-02$ \\
\hline OH- & 15.1 & $1.13 \mathrm{E}+05$ & $1.71 \mathrm{E}+05$ & 10.2 & 10.6 & 11.6 & 11.8 \\
\hline No3. & 5.64 & $2.09 \mathrm{E}+0 \mathrm{~s}$ & $3,16 \mathrm{E}+05$ & 5.19 & 5.45 & 5.73 & 5.78 \\
\hline NO2- & 2.72 & $7.47 E+04$ & $1.13 \mathrm{E}+05$ & 2.27 & 2.48 & 2.95 & 3.17 \\
\hline $\cos 2$ & 0.482 & \begin{tabular}{l|}
$.73 E+04$ \\
\end{tabular} & $2.62 E+04$ & 0.437 & 0454 & 0.516 & 0.522 \\
\hline $\mathrm{PO43-}$ & 0.101 & $5,74 \mathrm{E}+03$ & $870 \mathrm{E}+03$ & $8.67 \mathrm{E}-02$ & $9.18 \mathrm{E}-02$ & 0.104 & 0.106 \\
\hline SO42- & 0.272 & $1.56 \mathrm{E}+04$ & $2.36 \mathrm{E}+04$ & 0.214 & 0.241 & 0.303 & 0.330 \\
\hline Si (as SiO32-) & $8.66 \mathrm{E}-02$ & $1.45 E+03$ & $2.20 \mathrm{E}+03$ & 7.15E-02 & $7.89 \mathrm{E}-02$ & $9.42 \mathrm{E}-02$ & 0.102 \\
\hline F- & \begin{tabular}{l|l}
$7.47 \mathrm{E}-02$ \\
\end{tabular} & 847 & $1.28 \mathrm{E}+03$ & $6,26 \mathrm{E}-02$ & $6.7 \mathrm{E}-02$ & $8.07 \mathrm{E}-02$ & 8.54E-02 \\
\hline $\mathrm{Cl}$ - & 0.273 & $5.77 \mathrm{E}+03$ & B.75E+03 & 0.247 & 0.257 & 0.285 & 0.295 \\
\hline C6H5O73- & $3.07 \mathrm{E}-02$ & $3,46 \mathrm{E}+03$ & $5 . \overline{24 \mathrm{E}+03}$ & $2.81 \mathrm{E}-02$ & $2.94 \mathrm{E}-02$ & $3.19 \mathrm{E}-02$ & $3.32 \mathrm{E}-02$ \\
\hline EDTA4: & $1.64 \mathrm{E}-02$ & $2.83 \mathrm{E}+03$ & 4.28E+03 & $5.03 \mathrm{E}-03$ & $1.06 \mathrm{E}-02$ & $2.23 \mathrm{E}-02$ & $2.80 \mathrm{E}-02$ \\
\hline HEDTA3- & $3.07 \mathrm{E}-02$ & $5.03 \mathrm{E}+03$ & $7.62 E+03$ & $7.92 \mathrm{E}-03$ & $1.91 \mathrm{E}-02$ & 4.25E-02 & $5.39 \mathrm{E}-02$ \\
\hline glycolate- & $9.70 \mathrm{E}-02$ & $4.34 \mathrm{E}+03$ & $6.58 E+03$ & 6.35E-02 & $7.99 \mathrm{E}-02$ & 0.114 & 0.131 \\
\hline acetale- & $6.90 \mathrm{E}-03$ & 243 & 368 & $5.66 \mathrm{E}-03$ & 6.2JE-03 & $7.53 \mathrm{E}-03$ & 8. 14E-03 \\
\hline oxalate 2- & $5.97 \mathrm{E}-05$ & 3.14 & 4.75 & 5.29E- 05 & $5.62 \mathrm{~F}-05$ & $6.31 \mathrm{E}-05$ & $6.65 \mathrm{E}-05$ \\
\hline DBP & $2.09 \mathrm{E}-02$ & $2.62 \mathrm{E}+03$ & $3.97 \mathrm{E}+03$ & $1.72 \mathrm{E}-02$ & $1.90 \mathrm{E}-02$ & $2.27 \mathrm{E}-02$ & $245 \mathrm{E}-02$ \\
\hline butenol & $2.09 \mathrm{E}-02$ & 923 & $1.40 E+03$ & $1.72 \mathrm{E}-02$ & $1.90 \mathrm{E}-02$ & $2.27 \mathrm{E}-02$ & $2.45 \mathrm{E}-02$ \\
\hline $\mathrm{NH3}$ & $9.63 \mathrm{E}-02$ & 97 & $1.48 \mathrm{E}+03$ & 7.94E-02 & 8.60E-02 & 0.109 & 0.125 \\
\hline Fe(CN)64- & 0 & 0 & 0 & 0 & 0 & 0 & 0 \\
\hline
\end{tabular}

- Density is calculated based on $\mathrm{Na}, \mathrm{OH}$-, and AlO2-.

tWater wt\% derived from the difference of density and total dissolved species. 


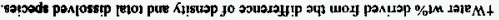

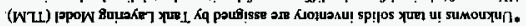

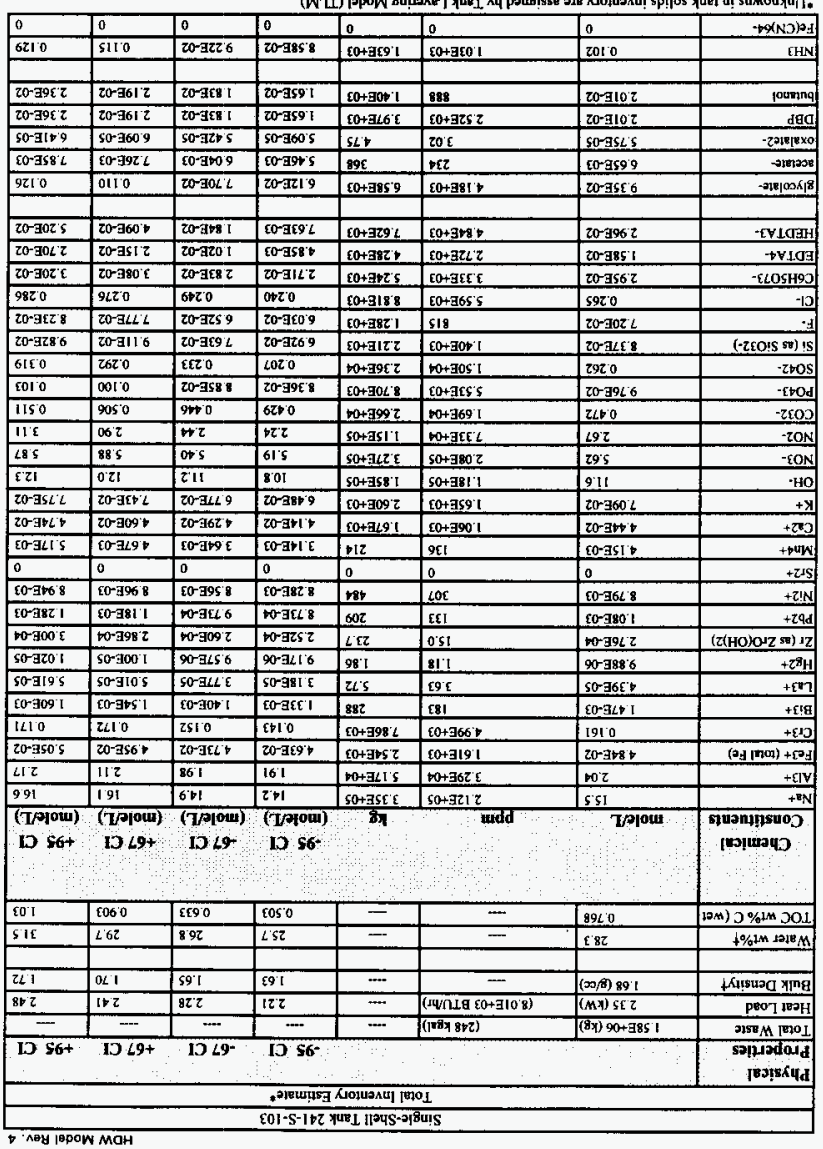


HDW Model Rav. 4

\begin{tabular}{|c|c|c|c|c|c|c|c|}
\hline \multirow[b]{3}{*}{$\begin{array}{l}\text { Physical } \\
\text { Praperties }\end{array}$} & \multicolumn{7}{|c|}{ Single-Shell Tank 241-S-103 } \\
\hline & \multicolumn{7}{|c|}{ TLM Solids Composite Inventory Estimate" } \\
\hline & & & & $-95 \mathrm{CI}$ & $-67 \mathrm{CI}$ & $+67 \mathrm{CI}$ & $+95 \mathrm{CI}$ \\
\hline Total TLM Wast & $5.98 \mathrm{E}+04(\mathrm{~kg})$ & $(9, \infty$ kgal $)$ & $--\cdot$ & $m$ & $\cdots$ & $\ldots$ & -- \\
\hline Heat Lasd & $0.222(\mathrm{~kW})$ & (758 8TU/hr) & $\rightarrow$ & 0.161 & 0.197 & 0.238 & 0.246 \\
\hline Bulk Density & $1.76(\mathrm{~g} / \mathrm{cc})$ & - & $\ldots$ & 1.51 & 1.53 & 2.11 & 2.24 \\
\hline Void Fraction & 0.536 & $\cdots$ & - & $6.00 \mathrm{E}-02$ & 0.217 & 0.737 & 0.743 \\
\hline Water wt\% & 27.0 & $\cdots$ & + & 2.64 & 10.0 & 42.6 & 44.2 \\
\hline TOC wt\% C (wet & 0 & - & -- & 0 & 0 & 0 & 0 \\
\hline $\begin{array}{l}\text { Radiological } \\
\text { Constitulientis: }\end{array}$ & CiA & $\mu \mathrm{Cu} / \mathrm{g}$ & $\mathbf{C i}$ & $\begin{array}{r}-95 \mathrm{CI} \\
(\mathrm{Ci} / \mathrm{L})\end{array}$ & $\begin{array}{l}67 \mathrm{CI} \\
(\mathrm{Ci} / \mathrm{L})\end{array}$ & $\begin{array}{l}+67 \mathrm{CI} \\
\text { (CVL) }\end{array}$ & $\begin{array}{c}+95 \mathrm{CI} \\
(\mathrm{CV} / \mathrm{L})\end{array}$ \\
\hline $\mathrm{H}-3$ & $2.92 \mathrm{E}-05$ & $1.67 \mathrm{E}-02$ & 0.996 & $3.05 \mathrm{E}-06$ & $1.4 \pi \mathrm{E}-05$ & 4.10E-05 & 4.10E-05 \\
\hline $\mathrm{C}-14$ & $2.26 E .06$ & $129 \mathrm{E}-03$ & $7.71 \mathrm{E}-02$ & 2.59E-07 & $1.06 \mathrm{E}-06$ & 3.07E- 06 & $3.07 E+06$ \\
\hline Ni-59 & $1.49 \mathrm{E}-05$ & $8.46 \mathrm{E}-03$ & 0.506 & 1.07E-05 & $1.31 \mathrm{E}-05$ & 1.62E-05 & $1.60 \mathrm{E}-05$ \\
\hline $\mathrm{Ni}-63$ & $1,39 \mathrm{~F}-03$ & 0.790 & 47.2 & $1.00 \mathrm{E}-03$ & $1.22 \mathrm{E}-03$ & $1.52 \mathrm{E}=03$ & $1.49 \mathrm{E}-03$ \\
\hline $\mathrm{Co}-60$ & $8.94 \mathrm{E}-07$ & 5.09E-04 & $304 E-02$ & $1.02 \mathrm{E}-07$ & $4.18 \mathrm{E}-07$ & $1.21 \mathrm{E}-06$ & $1.21 \mathrm{E}-06$ \\
\hline Se-79 & $4.81 \mathrm{E}-07$ & $2.74 \mathrm{E}+04$ & $1.64 \mathrm{E}-02$ & $5.50 \mathrm{E}-08$ & $7.25 \mathrm{E}-07$ & 6.51E-07 & $7.15 \mathrm{E}-06$ \\
\hline Sr-90 & 0.928 & 529 & $3.16 \mathrm{E}+04$ & 0.662 & 0.812 & 1.02 & 1.03 \\
\hline$Y-90$ & 0.929 & 529 & $3.16 \mathrm{E}+04$ & 0.662 & 0.812 & 1.02 & 1.03 \\
\hline $2 \mathrm{~T}-93$ & 2.27E- 06 & $1.29 \mathrm{E}-03$ & $7.73 \mathrm{E}-02$ & 2.59E-07 & $1.06 \mathrm{E}-06$ & $3.08 \mathrm{E}-06$ & $3.03 \mathrm{E}-05$ \\
\hline $\mathrm{Nb}-93 \mathrm{~m}$ & 1.85E-06 & $1.06 \mathrm{E}-03$ & $6.32 \mathrm{E}-02$ & 2.12E-07 & $8.68 \mathrm{E}-07$ & $251 E-06$ & 3.21E-05 \\
\hline Tc-99 & $1.58 \mathrm{E}-05$ & 9.02E-03 & 0.540 & $1.81 \mathrm{E}-06$ & $7.42 \mathrm{E}-06$ & 2.15E-05 & $2.15 \mathrm{E}-05$ \\
\hline Ru-106 & $2.50 \mathrm{E}-12$ & $1.42 E-09$ & $8,50 \mathrm{E}-0 \mathrm{8}$ & $2.86 \mathrm{E}-13$ & $1.17 \mathrm{E} .12$ & $3.38 E-12$ & $3.38 \mathrm{E} \cdot 12$ \\
\hline $\mathrm{Cd}-113 \mathrm{~m}$ & $6.99 \mathrm{E}-06$ & $3.98 \mathrm{E}-03$ & 0.238 & $8,00 \mathrm{E}-07$ & $3.27 \mathrm{E}-06$ & 9.4BE-06 & $9.48 \mathrm{E}-06$ \\
\hline $\mathrm{Sb}-125$ & $1,31 \mathrm{E}-06$ & $7.47 \mathrm{E}-04$ & $4.47 \mathrm{E}-02$ & $1.50 \mathrm{E}-07$ & $6.14 \mathrm{E}_{-}-07$ & 1.7BE- 06 & 1.78E-06 \\
\hline$S n-126$ & $7.39 \mathrm{E}-07$ & A.21E-04 & $252 \mathrm{E}-02$ & 8.45E-08 & $7.46 \mathrm{E}-07$ & $1.00 \mathrm{E}-06$ & 1. $14 \mathrm{E}-05$ \\
\hline $1-129$ & $3.04 \mathrm{E}-08$ & $1.73 \mathrm{E}-05$ & $104 E-03$ & 3.48E-09 & $1.42 \mathrm{E}-0 \mathrm{~B}$ & 4.13E-08 & $4.13 \mathrm{E}-08$ \\
\hline Cs=134 & $2.72 \mathrm{E}-08$ & 1.55E-05 & $926 \mathrm{E}-14$ & 7.19E-09 & $1.52 \mathrm{E}-08$ & 3.52E-08 & $3.52 \mathrm{E}-08$ \\
\hline Cs-137 & $5,60 \mathrm{E}-02$ & 31.9 & $0.91 \mathrm{E}+03$ & $1,48 E-02$ & $3.13 \mathrm{E}-02$ & $7.26 \mathrm{E}-02$ & $7.26 \mathrm{E}-02$ \\
\hline$B a-137 m$ & $5.30 \mathrm{E}-02$ & 30.2 & $1.80 \mathrm{E}+03$ & $1.40 \mathrm{E}-02$ & $2.96 \mathrm{E}-02$ & $6.87 \mathrm{E}-02$ & 6.8TE-02 \\
\hline Sm-151 & $1.71 \mathrm{E}-03$ & 0.976 & 58.4 & $1.96 \mathrm{E}-04$ & 1.03E-04 & 2.33E-03 & $2.64 \mathrm{E}-02$ \\
\hline Eu-152 & $100 \mathrm{E}-06$ & $3.98 \mathrm{E}-03$ & 0.238 & $6.85 \mathrm{E}-06$ & 6.91E-06 & $7.06 \mathrm{E}-06$ & $706 \mathrm{E}-06$ \\
\hline Eu-154 & 2.16E-05 & $1.23 \mathrm{E}-02$ & 0.735 & $2.47 \mathrm{E}-06$ & $1.01 \mathrm{E}-05$ & $2.93 \mathrm{E}-\mathrm{OS}$ & $2.93 \mathrm{E}-05$ \\
\hline Eu-155 & $3,30 \mathrm{E}-04$ & 0.188 & 11.3 & 3.23E-04 & $3.26 \mathrm{E}-04$ & $3.33 \mathrm{E}-04$ & $3.33 \mathrm{E}-04$ \\
\hline $\operatorname{Ra}-226$ & $1.08 \mathrm{E}-09$ & $6.15 \mathrm{E}-07$ & $3.68 \mathrm{E}-05$ & $1.48 \mathrm{E}-10$ & $6.04 \mathrm{E}-10$ & 1.56E-09 & $2.01 \mathrm{E}-09$ \\
\hline $\mathrm{Ra}-228$ & $9.26 \mathrm{E}-15$ & $5.28 \mathrm{E}-12$ & 3.16E-10 & $9.07 \mathrm{E}-15$ & $9.15 \mathrm{E}-15$ & $9.34 \mathrm{E}-15$ & $9.34 \mathrm{E}-15$ \\
\hline$A c-227$ & $4.76 \mathrm{E}-09$ & $2.71 \mathrm{E}-06$ & $1.62 \mathrm{E}-04$ & $3.06 \mathrm{E}-10$ & $200 \mathrm{E}-09$ & $7.52 \mathrm{E}-09$ & $1.02 E-68$ \\
\hline Pa-231 & $7.18 \mathrm{E}-10$ & 4.09E-07 & $2.44 E-05$ & $8.21 \mathrm{E}-11$ & $3.36 \mathrm{E}-10$ & $204 \mathrm{E}-09$ & $1.68 \mathrm{E}-08$ \\
\hline Th-229 & $1.77 \mathrm{E}-12$ & $1.01 \mathrm{E}-09$ & $6.02 E-08$ & $1.73 \mathrm{E}-12$ & $1.75 \mathrm{E}-12$ & $1.78 \mathrm{E}-12$ & $1.78 \mathrm{E}-12$ \\
\hline Th-232 & $5,91 \mathrm{E}-16$ & $3.36 \mathrm{E}-13$ & $2.01 \mathrm{E}-11$ & $6.76 \mathrm{E}-17$ & $2.77 \mathrm{E}-16$ & $8.01 \mathrm{E}-16$ & $8.01 \mathrm{E}-16$ \\
\hline U.232 & $5.24 \mathrm{E}-12$ & 2.98E-09 & $1.7 \mathrm{BE}-07$ & $5.99 \mathrm{E}-13$ & 2.45E-12 & $7.10 \mathrm{E}-12$ & $7.10 \mathrm{E}-12$ \\
\hline U-233 & $2.00 \mathrm{E}-13$ & 1.14E-10 & 6.80E-09 & $2.2 \mathrm{BE}-14$ & 9.34E-14 & $2.71 \mathrm{E}-13$ & $2.71 \mathrm{E}-13$ \\
\hline $\mathrm{U}-234$ & 1.19E-07 & $6.73 E-05$ & 406E-03 & $1.36 \mathrm{E}-08$ & 5.58E-08 & 1.62E-07 & $1.62 \mathrm{E}-07$ \\
\hline $\mathrm{U}-235$ & $5.13 \mathrm{E}-09$ & $2.92 \mathrm{E}-06$ & $1.75 \mathrm{E}-04$ & $5.86 \mathrm{E}-10$ & $2.40 \mathrm{E}-09$ & $6.95 \mathrm{E}-09$ & $6.95 \mathrm{E}-09$ \\
\hline U-236 & 1.88E- -09 & $1.0 \pi \mathrm{E}-06$ & $6.42 \mathrm{E}-05$ & 2.16E-10 & $8.82 E-10$ & $2.56 \mathrm{E}-09$ & 2.56E- 09 \\
\hline \begin{tabular}{|l|} 
U-238 \\
\end{tabular} & $1.22 \mathrm{E}-07$ & $6.92 \mathrm{E}-05$ & $4.14 \mathrm{E}-03$ & $1.39 \mathrm{E}-08$ & $569 \mathrm{E}-\mathrm{OP}$ & $1.65 \mathrm{E}-07$ & $1.65 \mathrm{E}-07$ \\
\hline$N p=237$ & $1.03 \mathrm{E}-07$ & $5.86 \mathrm{E}-05$ & 3.50E-03 & $1.18 \mathrm{E}-08$ & $4.81 \mathrm{E}-08$ & $1.39 \mathrm{E}-07$ & $1.39 \mathbf{E}-07$ \\
\hline \begin{tabular}{|l|} 
Pu-238 \\
\end{tabular} & $6.61 \mathrm{E}-06$ & $3.77 \mathrm{E}-03$ & 0.225 & 4.75E-06 & $5.66 \mathrm{E}-06$ & $7.57 \mathrm{E}-06$ & $8.48 \mathrm{E}-06$ \\
\hline $\mathrm{Pu}-239$ & $4.72 \mathrm{E}-04$ & 0.269 & 161 & $3.39 \mathrm{E}-04$ & 4,04E-04 & $5.40 \mathrm{E}-04$ & $6.05 \mathrm{E}-04$ \\
\hline$P u-240$ & 6.76E-05 & 3. $85 E-02$ & 2.30 & 4.85E-05 & 5.78E-0S & $7.73 \mathrm{E}-05$ & $866 \mathrm{E}-05$ \\
\hline $\mathrm{Pu}-24$ & $3.97 \mathrm{E}-04$ & 0.226 & 135 & 2.85E-04 & 3. 40E-04 & 4.55E-04 & $5.09 \mathrm{E}-04$ \\
\hline $\mathrm{Pu}-242$ & $1.82 \mathrm{E}-09$ & 1.04E-06 & $6.21 \mathrm{E}-05$ & $1.31 \mathrm{E}-09$ & $1.56 \mathrm{E}-09$ & $2.08 \mathrm{E}-09$ & $2.34 \mathrm{E}-09$ \\
\hline Am-241 & 8.09E-06 & 4.61E-03 & 0.276 & $9.26 \mathrm{E}-07$ & $3.79 \mathrm{E}-06$ & $1.10 \mathrm{E}-0 \mathrm{~S}$ & $1.43 \mathrm{E}-04$ \\
\hline Am-243 & $7.57 \mathrm{E}-11$ & $4.31 \mathrm{E}-08$ & $2.58 E-06$ & B.66E-12 & 3.54E-11 & 1.03E-10 & 1.34E-09 \\
\hline $\mathrm{Cm}-242$ & $1.61 \mathrm{E}-07$ & $9.16 \mathrm{E}-05$ & $3.48 \mathrm{E}-03$ & 1.58E-07 & $1.59 \mathrm{E}-07$ & $1.62 \mathrm{E}-02$ & $1.62 \mathrm{E}-07$ \\
\hline $\mathrm{Cm}-243$ & $3.69 \mathrm{E}-09$ & $2.10 \mathrm{E}-06$ & $1.26 \mathrm{E}-04$ & $3.61 \mathrm{E}-09$ & 3. $64 \mathrm{E}-09$ & $3.72 \mathrm{E}-109$ & 3.72E-09 \\
\hline $\mathrm{Cm} \cdot 244$ & $2.70 \mathrm{E}-09$ & $1.54 \mathrm{E}-06$ & $9.21 \mathrm{E}-05$ & $3.096-10$ & 1.27E-09 & $3.67 \mathrm{E} \cdot 09$ & $3.6 \mathrm{~J}-09$ \\
\hline Totals & $\mathbf{M}$ & $\mu / d$ & ks & $\begin{array}{l}95 \mathrm{CL} \\
(\mathrm{Mor} \\
\mathrm{B} / \mathrm{L})\end{array}$ & $\begin{array}{l}6 \mathrm{CT}(\mathrm{M} \\
\text { or } \mathrm{g} / \mathrm{A})\end{array}$ & $\begin{array}{l}+67 \mathrm{CT}(\mathrm{M} \\
\quad \text { or } \mathrm{g} / \mathrm{L})\end{array}$ & $\begin{array}{l}+95 \mathrm{Cl} \\
(\mathrm{M} \text { or } \\
\mathrm{g} / \mathrm{L})\end{array}$ \\
\hline $\mathrm{Pu}$ & $7.89 \mathrm{E}-03(g h)$ & $=$ & 0.269 & $567 \mathrm{E}-03$ & $6.76 \mathrm{E}-03$ & $903 E-03$ & $1.01 \mathrm{E}-02$ \\
\hline $\mathrm{U}$ & $1.53 \mathrm{E}-03$ & 207 & 12.4 & $1.75 \mathrm{E}-04$ & $7.16 \mathrm{E}-04$ & $2.07 \mathrm{E}-03$ & $2.07 \mathrm{E}-03$ \\
\hline
\end{tabular}

\footnotetext{
- Unknowns ir tank solids inventory are assigned by Tank Layering Model (TLM)
} 
HDW Madel Rev. 4

\begin{tabular}{|c|c|c|c|c|c|c|c|}
\hline \multirow[b]{3}{*}{$\begin{array}{l}\text { Physical } \\
\text { Properties }\end{array}$} & \multicolumn{7}{|c|}{ Single-Shell Tank 241-S-103 } \\
\hline & \multicolumn{7}{|c|}{ SMM Composite lnventory Estimate } \\
\hline & & & & $-95 \mathrm{Cl}$ & $-67 \mathrm{CI}$ & $+67 \mathrm{CI}$ & $+95 \mathrm{CI}$ \\
\hline Total SMM Wast & $1.52 \mathrm{E}+06(\mathrm{~kg})$ & $(239 \mathrm{kgal})$ & $=$ & $\rightarrow$ & $=$ & $=$ & $\ldots$ \\
\hline Heat Load & $2.12(\mathrm{~kW})$ & (7.25E+03 gTU/Ar) & - & 1.99 & 2.00 & 2.19 & 2.26 \\
\hline Bulk Density" & $1.67(g / c)$ & $\cdots$ & 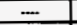 & 1.62 & 1.65 & 1.70 & 1.72 \\
\hline Water wi \%" & 28.3 & $\ldots$ & $\cdots$ & 25.7 & 26.8 & 29.8 & 31.7 \\
\hline TOC wro C (wet & 0.798 & $\ldots$ & 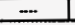 & 0.523 & 0.658 & 0.938 & 1.07 \\
\hline $\begin{array}{l}\text { Radiological } \\
\text { Constituients }\end{array}$ & $\mathrm{CM}$ & $\mathrm{nCl} / \mathrm{s}$ & CI & $\begin{array}{l}-95 \mathrm{CI} \\
\text { (CiL) }\end{array}$ & $\begin{array}{l}-67 \mathrm{Cl} \\
(\mathrm{CV} / \mathrm{L})\end{array}$ & $\begin{array}{l}+67 \mathrm{CI} \\
(\mathrm{CUL} / \mathrm{)} \\
\end{array}$ & $\begin{array}{r}+95 \mathrm{C} \\
\text { (CVL) }\end{array}$ \\
\hline $\mathrm{H}-3$ & $2.80 E-04$ & 0.167 & 253 & $146 E-04$ & $1.46 \mathrm{E}-04$ & 2.89E-C4 & $3.00 \mathrm{E}-04$ \\
\hline $\mathrm{C}=14$ & $4.03 \mathrm{E}-0 \mathrm{~S}$ & $241 \mathrm{E}-02$ & 36.4 & $1.23 \mathrm{E}-05$ & $1.23 E-05$ & $4.09 \mathrm{E}-05$ & $4.15 \mathrm{E}-05$ \\
\hline $\mathrm{Ni}-59$ & $2.61 \mathrm{E}-06$ & $1.56 \mathrm{E}-03$ & 236 & $1.29 \mathrm{E}-06$ & 1. 29E.06 & $267 \mathrm{E}-06$ & $2.74 \mathrm{E}-06$ \\
\hline $\mathrm{Ni}-63$ & $255 \mathrm{E}-04$ & 0.153 & 231 & $1.25 \mathrm{E}-04$ & $1,25 \mathrm{E}-04$ & $2.62 E-04$ & $2.68 \mathrm{E}-04$ \\
\hline $\mathrm{Co}-60$ & $4.46 \mathrm{E}-05$ & $2.66 \mathrm{E} \cdot 02$ & 40.3 & $1.26 \mathrm{E}-05$ & $1.26 \mathrm{E}-05$ & 4.54E-05 & $4,625-05$ \\
\hline Se-79 & $4,00 \mathrm{E}-06$ & $239 \mathrm{E}-03$ & 3.62 & $2.21 E-06$ & $2.21 \mathrm{E}-06$ & 4.65E-06 & $5.27 \mathrm{E}-06$ \\
\hline $5 r \cdot 90$ & 0.133 & 79.6 & $1.21 \mathrm{E}+05$ & 0.124 & 0.129 & 0.138 & 0.142 \\
\hline$Y=90$ & 0.133 & 79.6 & $1.21 \mathrm{E}+05$ & $7.16 E-02$ & $7.16 \mathrm{E}-02$ & 0.138 & 0.142 \\
\hline Zr-93 & $1.96 \mathrm{E}-05$ & $1.17 \mathrm{E}-02$ & 17.8 & $1.07 \mathrm{E}-0 \mathrm{~S}$ & 1.07EAS & 2.29F-05 & $2.60 \mathrm{E}-05$ \\
\hline $\mathrm{Nb}-93 \mathrm{~m}$ & 1.43E-05 & $8.51 \mathrm{E} .03$ & 12.9 & 8.01E-06 & $8.01 E-06$ & $1.65 \mathrm{E}-05$ & $1.87 \mathrm{E}-0 \mathrm{~S}$ \\
\hline Te-99 & $2.87 \mathrm{E}-04$ & 0.171 & 260 & $1.83 \mathrm{E}-04$ & $2.34 \mathrm{E}-04$ & 3.41E-04 & $3.92 \mathrm{E}-04$ \\
\hline Ru-106 & 7.93E-09 & $4.73 \mathrm{E}-06$ & 7.17E-03 & 3.79E-09 & $379 \mathrm{E}-19$ & B.85E-09 & $9.69 \mathrm{E}-09$ \\
\hline $\mathrm{Cd} .113 \mathrm{~m}$ & $1.03 \mathrm{E}-04$ & $6.15 E-02$ & 93.2 & 4.94E-0S & $4,94 \mathrm{E}-05$ & $1.22 \mathrm{E}-04$ & $1.41 \mathrm{E}-04$ \\
\hline $\mathrm{sb}-125$ & $1.925-04$ & 0.114 & 173 & $5.30 \mathrm{E}-0 \mathrm{~S}$ & $5.30 \mathrm{E}-0 \mathrm{~s}$ & $1.95 \mathrm{E}-\mathrm{CA}$ & $1.99 \mathrm{E}-04$ \\
\hline $5 n-126$ & $6.05 \mathrm{E}-86$ & $3.61 \mathrm{E}-03$ & 5,47 & $3.37 \mathrm{E}-06$ & $3.37 \mathrm{E}-06$ & $7,02 \mathrm{E}-06$ & $7.94 \varepsilon-06$ \\
\hline 1.129 & $5.53 \mathrm{E}-07$ & $3.30 \mathrm{e}-04$ & $0 \$ 50$ & $3.51 \mathrm{E}-07$ & $4.50 \mathrm{E}-07$ & $6.57 \mathrm{E}-07$ & $7.57 \mathrm{~T}-07$ \\
\hline $\mathrm{Cs}+134$ & $3.03 \mathrm{E}-06$ & $1.81 \mathrm{E}-03$ & 2.74 & $2.16 \mathrm{E}-16$ & $2.55 \mathrm{E}-06$ & $3.47 \mathrm{E}-06$ & $390 \mathrm{E}-06$ \\
\hline Cs-137 & 0.310 & 185 & $2.80 E+05$ & 0.279 & 0.294 & 0.325 & 0.341 \\
\hline Ba-137m & 0.293 & 175 & $2.65 \mathrm{E}+05$ & 0.230 & 0.230 & 0.308 & 0.322 \\
\hline $5 m-151$ & $1.41 \mathrm{E}-02$ & 8.41 & $1.27 \mathrm{E}+04$ & $7.84 \mathrm{E}-03$ & $784 \mathrm{E}-03$ & $1.63 \mathrm{E}-02$ & 1.85E-02 \\
\hline Eu-152 & $4.61 \mathrm{E}-06$ & $2.75 \mathrm{E}-03$ & 4.17 & $2.40 \mathrm{E}-06$ & $2,40 E-06$ & $4.93 \mathrm{E}-06$ & $5.24 \mathrm{E}-06$ \\
\hline Eu-154 & $2.23 \mathrm{E}-04$ & 0.432 & 654 & 2.77E-OA & $2.77 \mathrm{E}-04$ & 8.85E-04 & $9.49 \mathrm{E}-04$ \\
\hline Et-155 & $2.73 \mathrm{E}-04$ & 0.163 & 247 & $1.41 E-04$ & $1.41 \mathrm{E}-04$ & $2.92 \mathrm{E}-04$ & $3.11 E-04$ \\
\hline $\mathrm{ka} a-226$ & $1.71 E=10$ & $1.02 \mathrm{E}-07$ & $1.55 \mathrm{E}-04$ & $1.16 \mathrm{E}-10$ & $1.16 \mathrm{E}-10$ & $1.91 \mathrm{E}-10$ & $2.10 \mathrm{E}-10$ \\
\hline Ra-228 & $1.07 \mathrm{E}-07$ & 9.95E-05 & 0,151 & $6.98 \mathrm{E}-08$ & 1.1TE-07 & $2.24 t-07$ & $2.85 \mathrm{E}-07$ \\
\hline Ac-22? & $1.08 \mathrm{E}-09$ & $6.46 \mathrm{E}-07$ & 9.79E-04 & $7.61 \mathrm{E}-10$ & $7.61 \mathrm{E}-10$ & $1.20 \mathrm{E}-09$ & 1.31E-09 \\
\hline $\mathrm{Pa}-231$ & $4.96 \mathrm{E}-09$ & $2.96 \mathrm{E}-06$ & 4.49E-03 & $3.18 \mathrm{E}-09$ & $3.18 \mathrm{E}-0 \mathrm{9}$ & $5.61 \mathrm{E}-09$ & $6.23 \mathrm{E}-09$ \\
\hline Th-229 & $3.91 \mathrm{E}-09$ & $2.33 \mathrm{E}-06$ & 3.54E-03 & 1.78E-09 & $2.82 \mathrm{E}-09$ & 5.17E-09 & $6.52 \mathrm{E}-09$ \\
\hline Th-232 & $1.11 \mathrm{E}-08$ & $6.63 \mathrm{E}-06$ & $1.00 \mathrm{E}-02$ & $5,74 E-09$ & $8.37 \mathrm{E}-09$ & $1.38 \mathrm{E}-0 \mathrm{~s}$ & $1.65 \mathrm{E}-08$ \\
\hline $\mathrm{U}-232$ & $8.58 E-07$ & $5.12 \mathrm{E}-04$ & 0.776 & 4.69E-A7 & $6.59 \mathrm{E}-07$ & $1.09 E-06$ & $1.33 \mathrm{E}-0.6$ \\
\hline U-233 & $3.29 \mathrm{E}-06$ & $1.96 \mathrm{E}-03$ & 2.97 & I.MOE-06 & $2.53 \mathrm{E}-06$ & 4.17E-06 & $5.11 \mathrm{E}-06$ \\
\hline $\mathrm{U}-234$ & 9.64E-07 & 5.76E-04 & 0.872 & 9.28E-07 & $9.46 \mathrm{E}-07$ & 9.84E-07 & $9.94 \mathrm{E}-07$ \\
\hline U.235 & $3.91 \mathrm{E}-08$ & 2.34E-05 & $3.54 \mathrm{E}-02$ & $3.76 \mathrm{E}-0 \mathrm{~B}$ & $3.83 \mathrm{E}-0 \mathrm{~s}$ & $4.00 \mathrm{e}-0 \mathrm{~s}$ & 4.04E-08 \\
\hline$U-236$ & $3.02 \mathrm{E}-0 \mathrm{8}$ & $1.80 \mathrm{E}-0 \mathrm{~s}$ & $2.73 E-02$ & 2.91E-08 & $2.97 \mathrm{E}-08$ & $306 \mathrm{E}-08$ & 3.11E-08 \\
\hline $\mathrm{U}-238$ & $1.12 \mathrm{E}-06$ & $6.67 \mathrm{E}-04$ & 101 & 1.08E- 06 & $1.10 \mathrm{E}-06$ & 1. $14 \mathrm{E}-06$ & $1.15 \mathrm{E}-06$ \\
\hline $\mathrm{Np}-237$ & 1.05E-06 & $6.26 \mathrm{E}-04$ & 0.948 & $7,07 \mathrm{E}-07$ & $8.74 \mathrm{E}-07$ & $1.22 E-16$ & $1.39 \mathrm{E}-06$ \\
\hline$P u-2,38$ & $1.63 \mathrm{E}-06$ & $9.75 \mathrm{E}-04$ & 1.48 & $1.23 \mathrm{E}-06$ & $1.43 \mathrm{E}-06$ & 1.84E-06 & $2.03 \mathrm{E}-06$ \\
\hline Pu-239 & $5.61 \mathrm{E}-05$ & $3.35 \mathrm{E}-02$ & 30.8 & 4.58E-05 & $5.09 \mathrm{E}-05$ & 6.13E-05 & $6.63 \mathrm{E}-05$ \\
\hline $\mathrm{Pu}-240$ & 9.51E.06 & $\$ .68 \mathrm{E}-03$ & 860 & 7,60E- -16 & $8.54 \mathrm{E}-06$ & 1.05E-05 & $1.14 \mathrm{E}-05$ \\
\hline Pu-241 & $1.10 \mathrm{E}-04$ & $6.59 \mathrm{E}-02$ & 99.8 & $8.31 E-05$ & $9.64 \mathrm{E}-0 \mathrm{~S}$ & $1.24 \mathrm{E} \cdot 09$ & $1.38 E-04$ \\
\hline $\mathrm{Pu}-242$ & $6.06 \mathrm{E}-10$ & 3.62E-07 & 5.48E-04 & $4,43 \mathrm{E}-10$ & $5.23 \mathrm{E}-10$ & $6.89 \mathrm{E}-10$ & $7.69 \mathrm{E}-10$ \\
\hline Ann-241 & $6.81 \mathrm{E}-0 \mathrm{~S}$ & $4.06 \mathrm{E}-02$ & 61,6 & 4.9IE-0S & $5.84 \mathrm{E}-05$ & $7.78 \mathrm{E}-05$ & 8.71E-05 \\
\hline $\mathrm{Am}-243$ & $2.36 \mathrm{E}-09$ & $1.41 \mathrm{E}-06$ & $2.13 \mathrm{E}-03$ & $1.76 E .09$ & $2.05 \mathrm{E}-09$ & $2.68 \mathrm{E}-09$ & $2.98 \mathrm{E}-09$ \\
\hline $\mathrm{Cm}-242$ & 1.75E-07 & $1.04 \mathrm{E}-04$ & 0.158 & 8.40E-08 & $8.40 \mathrm{E}-0 \mathrm{~B}$ & $1.87 \mathrm{E}-07$ & $1.99 \mathrm{E}-07$ \\
\hline $\mathrm{Cm}-243$ & $1.62 \mathrm{E}-08$ & 9.66E-06 & $1.46 \mathrm{E}-02$ & 7.52E-09 & $7.52 \mathrm{E}-09$ & 1.73E- 68 & $1.83 \mathrm{E}-08$ \\
\hline $\mathrm{Cm}-244$ & $1.60 \mathrm{E}-07$ & $9.55 E-05$ & 0.145 & $2.07 \mathrm{E}-0 \mathrm{~B}$ & $7.07 \mathrm{E}-0 \mathrm{~B}$ & $1.90 \mathrm{E}-07$ & 2. $10 \mathrm{E}+07$ \\
\hline Totals & $\mathbf{M}$ & $\mu q / \mathrm{s}$ & tos: & $\begin{array}{l}-95 \mathrm{CI} \\
\mathrm{M} \text { or } \\
\\
\mathrm{C} / \mathrm{C}\end{array}$ & $\begin{array}{l}-69 \mathrm{Cl}(\mathrm{M} \\
\text { or } / \mathrm{L})\end{array}$ & $\begin{array}{c}+67 C I(M \\
\text { or } N / L)\end{array}$ & $\begin{array}{r}-95 \mathrm{Cl} \\
\text { (M or } \\
\mathrm{g} / \mathrm{L})\end{array}$ \\
\hline $\mathrm{Pu}$ & $7.03 \mathrm{E}+04(\mathrm{~g} / \mathrm{L})$ & $=$ & 0.636 & $5.09 \mathrm{E}-04$ & $6.04 E-04$ & $8.02 \mathrm{E}-04$ & 8.96E-114 \\
\hline U & $110 \mathrm{E}-02$ & $1.57 \mathrm{E}+03$ & $2.38 \mathrm{E}+03$ & $1.06 \mathrm{E}-02$ & $1.05 \mathrm{E}-02$ & 1. $13 E-02$ & $1.14 \mathrm{E}-02$ \\
\hline
\end{tabular}

-Density is calculated based on $\mathrm{Na}, \mathrm{OH}$, and $\mathrm{AlO} 2$ -

+ Water wt $\%$ derived from the difference of density and total dissolved species. 


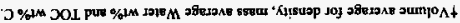

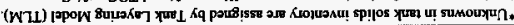

\begin{tabular}{|c|c|c|c|c|c|c|c|}
\hline $20-\Xi 011$ & $70-\mathbf{3 6 0} I$ & $20-150 \mathrm{I}$ & $20 . \mathrm{AE0} t$ & $\varepsilon 0+36 \varepsilon z$ & t0+7zS I & $20-3 \angle 01$ & n \\
\hline to-GSI I & c0-490 I & $00-3698$ & $50-3 L L L$ & 5060 & - & $(7 / 8)+0-71096$ & $\mathrm{rnd}$ \\
\hline $\begin{array}{r}(T / \mathrm{h} \\
\mathrm{10} \mathrm{W}) \\
1096+\end{array}$ & $\begin{array}{l}\text { (T/2 50 } \\
\text { w) } 10 \text { is }\end{array}$ & $\begin{array}{l}\text { (T/4) } \\
\text { Wh } 150\end{array}$ & $\begin{array}{l}(1 / 2 \\
\text { sow) } \\
\text { iD } 96\end{array}$ & का & $870+1$ & W: & 무인 \\
\hline$\angle 0-a z O^{\prime} Z$ & (0) & $80-9589$ & $80-7589$ & $s+10$ & $50-70 z^{\prime} 6$ & Lo-ats I & $\nabla \nabla z$-45 \\
\hline $80-3861$ & $80-389 \mathrm{I}$ & 60-385 $L$ & $60^{\circ} \mathrm{A8C} L$ & $20-9 p_{1} 1$ & 90 alc 6 & $80-3151$ & cbz-uว \\
\hline$\angle 0-7 \angle 61$ & $40-7981$ & $80-9898$ & $90-3898$ & Fol 0 & $10-7601$ & $\angle 0$ - $B \geqslant L$ I & $z+z-40$ \\
\hline $60-3882$ & $60-9852$ & $60-3861$ & \$0-70L I & fo: 1012 & $90 \mathrm{G9C1}$ & $60-78 z z$ & $\mathrm{Ebz}$-urv \\
\hline $50-32+8$ & $50-325 ' L$ & S0- 7996 & 50-39L'b & 819 & $z 0-3 E 6 \mathrm{C}$ & $50-3659$ & 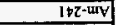 \\
\hline $01-3808$ & $01-5052$ & $0 \mathrm{t}-906 \mathrm{~s}$ & $01-3466^{\prime}$ & 100-9019 & 20-988 $\mathrm{c}$ & $01-3059$ & $\left.z+2-n_{d}\right]$ \\
\hline to- $3<t-1$ & botate 1 & to- 1201 & $50-3566$ & EII & 20-302 L & 100-3IZ: & {$\left[+2=-n_{d}\right.$} \\
\hline şo-ESC I & $50-3921$ & $50-\mathrm{a} \angle 0^{\circ} 1$ & $90.78 \mathrm{C}^{\prime} 6$ & 601 & $80-7269$ & $50-39 I^{\prime} 1$ & $0+2-n_{d}$ \\
\hline 50-701'8 & $50-3292$ & $50-1299$ & 50-3EI'9 & 899 & $z 0-36 z+$ & so-JZIL $L$ & $62^{-6 n} \mathrm{~d}$ \\
\hline $90-302 z$ & $90-3102$ & $90-3291$ & 90-aEt I & $0 L I$ & $50-780^{\prime} 1$ & $90-318 \mathrm{I}$ & $8 \varepsilon t-n_{\mathrm{d}}$ \\
\hline 90-3551 & 90-381 t & $20-39+8$ & $20-7989$ & 2550 & $+0-3109$ & 90-310 I & $\angle E Z^{-d} \mathrm{~N}$ \\
\hline $90-3117$ & 903011 & $90-590^{\prime} 1$ & $90-3501$ & 101 & po-3its & $90-980 \mathrm{t}$ & $8 \varepsilon 2-n$ \\
\hline $80-300 ' t$ & $80-3962$ & $80-3182 z$ & $80-3182$ & $20-3 b c t$ & So-anL I & $80-3162$ & $9 \varepsilon 2 * 1\}$ \\
\hline $80-916 \mathrm{C}$ & 80.398' ⿷ & so-glL' & Bo-3to' & 20-G95: & 50-392? & $80-36 L E$ & sร2-n \\
\hline 40.9296 & co-315 6 & L0-991 6 & $\angle 0-9668$ & $9 \angle 80$ & torgas's & Lo-3rE 6 & $\mathrm{DEZ}-\Omega$ \\
\hline $900126 \%$ & 90-320' & $90-a b+\tau$ & $90-37 L ' \mathrm{I}$ & $\angle 62$ & $50-368 \mathrm{I}$ & $90-\mathrm{ALI} E$ & 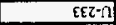 \\
\hline 90-7621 & $90-3501$ & $\angle 0$-g5e 9 & Lo-Gzs b & 9LO & to-386t & $\angle 0^{-} 3 \angle 28$ & $z \varepsilon 2-\Omega$ \\
\hline 803651 & $80-3 \varepsilon \varepsilon 1$ & 6007008 & 60-J5s's & $20-9001$ & 90-A8E 9 & $80-3 \angle 0^{\prime} 1$ & $2 \varepsilon z-41$. \\
\hline $60-3829$ & $60-986$ & 60-72L'2 & 60- $92 L 1$ & co-ghst & 90-3szZ & $60-3 \angle L \mathrm{E}$ & 6ZZ-4I \\
\hline $60-3509$ & $60-350$ '5 & $60-760 \mathrm{C}$ & $60-360 \%$ & co-gest & $90-1<8 t$ & $00-9180$ & $1 E t^{-8} d$ \\
\hline $60-3 b t 1$ & 60-3โC I & 01-790'6 & $01-390^{\prime} 6$ & $60-4 \times 11$ & $\angle 0-9 S Z L$ & $60-322 \mathrm{I}$ & $\angle z Z \cdot V V$ \\
\hline$\angle 0^{\circ} \mathrm{BS} \angle \tau$ & $\angle 0^{\circ} 3912$ & Lo-gEI't & sorazc 9 & ISIO & $50-3856$ & $\angle 0^{-3} 19 \mathrm{t}$ & $8 z \tau-$ - \\
\hline $0 t-\pi z+z$ & 01.3522 & 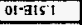 & $01-315 \mathrm{I}$ & $00-9261$ & L0-3zz! & 01-3noz & $9 z^{-x} y$ \\
\hline porazle & Do- 5862 & to-G8t I & bo- & $85 t$ & $\$ 910$ & $00-\exists S L^{\prime} z$ & $\mathrm{sst} \cdot \mathrm{ng}$ \\
\hline 50.7916 & nor $35 s 8$ & $50-789 z$ & $60-7892$ & 559 & 9160 & t01-3869 & $\left.75 I^{-110}\right]$ \\
\hline 90-70rs & $90-300 ' 5$ & 90-3LS' & 90-3LS'2 & 160 & $80-908 \tau$ & $90-30 \mathrm{C}^{\prime} \mathrm{b}$ & $2 s t^{-n} \mathrm{~g}$ \\
\hline $20-1627$ & $20-3851$ & EO-GI9 L & co-3igi & $60+38 Z^{\prime} I$ & $E 18$ & 20-99E: 1 & ISI-US \\
\hline Etco & 6620 & $\operatorname{cec} 0$ & EโZO & $50+7 \angle 9=2$ & 691 & 5820 & $\mathrm{u}_{L E I-\varepsilon g}$ \\
\hline 1650 & 9150 & $\operatorname{sen} 0$ & $I L Z^{\prime} 0$ & so+gzgz & $6<1$ & $00 E 0$ & $\langle[1-s]$ \\
\hline $96-396 \mathrm{C}$ & 90 -JSE' ह & $90-39+z$ & $90-360 \%$ & 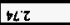 & co-anL I & $90-9262$ & $\left.\nabla \varepsilon 1^{-5}\right]$ \\
\hline$\angle O A I C L$ & $20-3+69$ & LOTSE' & $\angle 0-30 b^{\prime} \mathrm{E}$ & 1050 & $60-381^{\prime} \mathrm{C}$ & $\angle 0-\exists \supset E ' S$ & $621-1$ \\
\hline $90-189 \mathrm{~L}$ & $90-3649$ & $90-\mathrm{I} \angle \tau \mathrm{L}$ & $90-\exists \perp Z 6$ & $6 t 5$ & $50-36+\varepsilon$ & 90-7ss's & $9 z 1^{-u S}$ \\
\hline 50.9261 & 603881 & So-AII's & So-zitl's & $\varepsilon 41$ & 0110 & torass I & $521-95$ \\
\hline to-39:1 & Do-38t't & 50-364t & 50-366 $C^{\circ}$ & $+\varepsilon 6$ & $20-356 \mathrm{~s}$ & $50-3566$ & Ш气I I-PO \\
\hline $60-76[6$ & $60-3558$ & $60-759 \mathrm{\varepsilon}$ & $60-359 \mathrm{c}$ & co- $\$ 41 L$ & 90-asst & $60-3+92$ & $90 t^{-n y}$ \\
\hline $50.362 \mathrm{C}$ & por-36z $\varepsilon$ & $\hbar 0-192 \tau$ & w. 3141 & $09 \pi$ & 5910 & $60 \cdot \exists \angle C Z$ & $66^{-2} \mathrm{I}$ \\
\hline S0-3I8 I & $50-3091$ & $90-78 L L$ & $90-98 L L$ & 0 EI & $60-3 z_{8} 8$ & 50-98EI & 표 6 -9N \\
\hline so-3isz & $50 \cdot 312 Z 2$ & 50091001 & Sorjpo' I & $8 \angle 1$ & $20-3511$ & S0-306 I & E6-'ZZ \\
\hline 1610 & 9910 & 5010 & EOFO & sotzzs: & 996 & $29 \mathrm{I}^{\circ} 0$ & $06-\lambda$ \\
\hline 1610 & $99[0$ & 8510 & 2510 & sorgzs! & 996 & 2910 & $06-5 \mathrm{~S}$ \\
\hline $90-7605$ & $90-305$ & $90-$ asiz & $90-35 I^{\prime 2}$ & Hec & Eo-giez & $90-9 \angle 8 \mathrm{E}$ & $6 L-9$ \\
\hline SO- 990 & $50-3856$ & soralz I & S0-3IZI & pot & toots 6 & So-50ED & $09-00$ \\
\hline $10-360 \varepsilon$ & $60-350 \mathrm{E}$ & bo-alli & DOE-GILI & $8 L Z$ & $\angle L I 0$ & $60-3 \angle 62$ & Eg-!N \\
\hline $90-781 \mathrm{C}$ & 90-3ZIE & $90-3821$ & $90-38 \div 1$ & $9 \mathrm{~g}^{\prime} z$ & 60-3281 & $90750 \varepsilon$ & $6 S=! N$ \\
\hline so- $110 t$ & $50-356 \varepsilon$ & $50-361 \%$ & $50-361$ t & $59 \varepsilon$ & zo-aze $\tau$ & Sor-368' 5 & $\mathrm{DI}-\mathrm{O}$ \\
\hline 503062 & $p 0-36 L Z$ & ro-Azt I & $10-3 z+1$ & $\operatorname{csz}$ & 1910 & $60-31 L z$ & $\varepsilon=\mathrm{H}$ \\
\hline $\begin{array}{l}(7 / / 0) \\
19567\end{array}$ & $\begin{array}{l}(\mathrm{T} / \mathrm{l}) \\
1 \mathrm{D} / 9+\end{array}$ & $\begin{array}{l}(\mathrm{T} / \mathrm{S}) \\
13<9\end{array}$ & $\begin{array}{l}(7 / 5) \\
1096\end{array}$ & D & $4 \mathrm{O}^{\mathrm{f}}$ & $7 / 3$ & $\begin{array}{l}\text { spumpinao } \\
\text { [propolpwad }\end{array}$ \\
\hline tot & 5060 & Eह9'0 & fos'o & $\cdots$ & - & $89 L^{\prime} 0$ & $19 M) J \%^{1 M} \supset O L$ \\
\hline $51 E$ & 462 & 892 & $\operatorname{cst}$ & $=$ & - & $\varepsilon 8 z$ & $4 \% 1 \mathrm{M}$ xoIs $\mathrm{M}$ \\
\hline$z L^{\prime} I$ & 041 & $\$ 9^{\circ} 1$ & EqI & - & - & $(0 / A) 89 \mathrm{I}$ & Kolsusa *ing \\
\hline $8+2$ & 162 & $82 z$ & $i z z$ & 二- & $(14 / 218$ fo+GIo \&) & $(M 1)$ sद $\tau$ & peo' IRTH \\
\hline- & - & - & $\cdots$ & $=$ & $(189918+z)$ & $(8 y) 90+3855$ & OLST $M$ [E10I \\
\hline \multicolumn{7}{|c|}{$1056+\quad$ ID $\angle 9+$ ID $\angle 9 \quad$ IP $\$ 6^{\circ}$} & 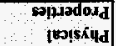 \\
\hline \multicolumn{8}{|c|}{ 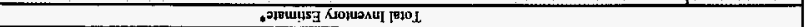 } \\
\hline \multicolumn{8}{|c|}{ 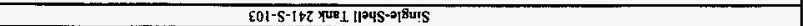 } \\
\hline
\end{tabular}


HNF-SD-WM-ER-323, Rev. 1

HOW Model Rev. 4

\begin{tabular}{|c|c|c|c|c|c|c|c|}
\hline \multirow[b]{3}{*}{$\begin{array}{l}\text { Physical } \\
\text { Properties }\end{array}$} & \multicolumn{7}{|c|}{ Singie-Shell Tank 241-S-104 } \\
\hline & \multicolumn{7}{|c|}{ TLM Solids Composite Inventory Estimate" } \\
\hline & & $\therefore$ & & $-95 \mathrm{Cl}$ & $-67 \mathrm{CI}$ & $\begin{array}{r}+57 \mathrm{CI} \\
\end{array}$ & $+95 \mathrm{CI}$ \\
\hline Total TLM Wast & $1.92 \mathrm{E}+0.06(\mathrm{~kg})$ & $(293 \mathrm{kgal})$ & 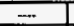 & - & - & 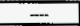 & - \\
\hline Heat Laad & $4.67(\mathrm{kw})$ & $(1.59 \mathrm{E}+04 \mathrm{BTU} / \mathrm{hr})$ & $\ldots$ & 3.94 & 4.37 & 4.87 & 4.96 \\
\hline Bulk Density & $1.73(g / c)$ & - & $\cdots$ & 1.58 & 1.65 & 1.86 & 1.91 \\
\hline Void Fraction & 0.682 & $\cdots$ & - & 0.507 & 0.565 & 0.750 & 0.788 \\
\hline Water wt\% & 31.0 & - & - & 20.1 & 23.6 & 36.5 & 42.0 \\
\hline TOC wt\% C (wet) & $2.85 \mathrm{E}-03$ & $\ldots$ & $\overline{-}$ & $2.58 E-03$ & $2.65 \mathrm{E}-0.03$ & $3.06 E-03$ & $3.33 \mathrm{E}-03$ \\
\hline $\begin{array}{c}\text { Chemical } \\
\text { Consthtiuents }\end{array}$ & malefL & ppip & kg : & $\begin{array}{c}-95 \mathrm{CI} \\
\text { (mole/l) }\end{array}$ & $\begin{array}{l}67 \mathrm{CI} \\
(\text { mole } / \mathrm{L})\end{array}$ & $\begin{array}{r}+67 \mathrm{CI} \\
\text { (molel })\end{array}$ & $\begin{array}{r}+95 \mathrm{CI} \\
(\mathrm{mole} / \mathrm{L})\end{array}$ \\
\hline $\mathrm{Na}+$ & 11.0 & $1.46 \mathrm{E}+05$ & $2.81 \mathrm{E}+05$ & 8.34 & 935 & 13.5 & 14.7 \\
\hline Al3+ & 5.36 & $8.34 E+04$ & $1.60 \mathrm{E}+05$ & 4.28 & 4.60 & 6.18 & 7.01 \\
\hline Fej+(tats $F e)$ & 0.397 & $1.28 \mathrm{E}+04$ & $2.46 \mathrm{E}+04$ & 0.390 & 0.393 & 0.400 & 0.404 \\
\hline $\mathrm{Cr} 3+$ & 0475 & $142 \mathrm{E}+04$ & $2.74 \mathrm{E}+04$ & 0.287 & 0.376 & 0.644 & 0.842 \\
\hline Bijt & $3.58 \mathrm{E}-06$ & 0.455 & 0.875 & 3.19E-06 & $3.48 \mathrm{E}-06$ & 4. $16 \mathrm{E}-06$ & $457 \mathrm{E}-06$ \\
\hline Last & $9.30 \mathrm{E}-12$ & 7.45E-07 & T.43E.06 & 8.15E-12 & $8.80 \mathrm{E}-12$ & 1.03E-11 & 1.13E-1! \\
\hline Hg2+ & $3.35 \mathrm{E}-04$ & 36.7 & 74.5 & $3.30 \mathrm{E}-04$ & $3.33 \mathrm{E}-04$ & $3.36 \mathrm{E}-04$ & 3.37E-04 \\
\hline $\mathrm{Zr}(\mathrm{AB} \mathrm{ZrO}(\mathrm{OH}) 2)$ & $3.77 \mathrm{E}-07$ & $1.98 \mathrm{E}-02$ & $3.81 \mathrm{E}-02$ & 3.46E-97 & $3.6 \mathrm{JE}-07$ & $3.95 \mathrm{E}-07$ & $4.26 \mathrm{E}-07$ \\
\hline $\mathrm{Pb} 2+$ & $9.75 \mathrm{E}-03$ & $116 E+03$ & $2.24 E+03$ & $8.95 \mathrm{E}-03$ & $9.38 \mathrm{E}-63$ & $1.01 E-02$ & $1.04 \mathrm{E}-02$ \\
\hline $\mathrm{Ni2+}$ & $2.71 \mathrm{E}-02$ & 917 & $1.76 \mathrm{E}+03$ & 2. 19E-02 & $2.48 \mathrm{E}-02$ & 2.88E-02 & $2.83 \mathrm{E}-02$ \\
\hline $5 r^{2+}$ & 0 & 0 & 0 & 0 & 0 & 0 & 0 \\
\hline Mn4t & $2.75 \mathrm{E}-05$ & 0.871 & 1.67 & 1.96E-05 & $2.33 \mathrm{E}-05$ & 3.15E-05 & 3.55E-05 \\
\hline $\mathrm{Cos}_{2+}$ & 0.133 & $3.08 \mathrm{E}+03$ & $3,92 E+03$ & 0.106 & 0.119 & 0.147 & 0.161 \\
\hline $\mathrm{K}+$ & $218 \mathrm{E}-02$ & 493 & 947 & $1.82 \mathrm{E}-02$ & $1.96 \mathrm{E}-02$ & $2.34 E-02$ & $2.51 \mathrm{E}-02$ \\
\hline $\mathrm{OH}$ & 23.1 & $2.27 \bar{E}+05$ & $436 E+05$ & 18.2 & 19.8 & 26.7 & 30.2 \\
\hline NC3- & 4.88 & $1.75 \mathrm{E}+0 \mathrm{~S}$ & $3.36 \mathrm{E}+05$ & 2.96 & 2.96 & 7.89 & 9.53 \\
\hline NO2- & 164 & $4.36 \mathrm{E}+04$ & $8.39 E+04$ & 1.15 & 1.33 & 1.90 & 1.90 \\
\hline CO32- & 0.136 & $4.70 E+03$ & $904 E+03$ & 0.108 & 0.122 & 0.130 & 0.163 \\
\hline PO43- & 2.44E-04 & 13.4 & 25.7 & $2.27 \varepsilon-04$ & $2.37 \mathrm{E}-04$ & $2.54 \mathrm{E}-04$ & $2.77 \mathrm{E}-04$ \\
\hline SO42- & $2.67 \mathrm{E}-02$ & $1.48 \mathrm{E}+03$ & $2.85 \mathrm{E}+03$ & $2.33 \mathrm{E}-02$ & $2.47 E-02$ & $2.87 \mathrm{E}-02$ & $3.08 \mathrm{E}-02$ \\
\hline Si (as Sio32-) & $7.61 \mathrm{E}-02$ & $1.23 \mathrm{E}+03$ & $2.37 \mathrm{E}+03$ & $4.98 \mathrm{E}-02$ & $6.6 \mathrm{tE}-02$ & $8.60 \mathrm{E}-02$ & 957E-02 \\
\hline F- & $1.95 \mathrm{E}-04$ & 2.13 & 4.10 & $1.66 \mathrm{E}-04$ & $1.83 \mathrm{E}-04$ & $2.14 \mathrm{E}-04$ & 2.J1E-04 \\
\hline $\mathrm{Cl}-$ & $9.29 \mathrm{E}-02$ & $1.90 \mathrm{E}+03$ & $365 E+03$ & $5.90 \mathrm{E}-02$ & $7.56 \mathrm{E}-02$ & 0.100 & 0.132 \\
\hline C6 65073. & 2.01E-04 & 21.9 & 42.2 & 1.95E-04 & $1.98 \mathrm{E}-04$ & $2.10 \mathrm{E}-04$ & $2.28 \mathrm{E}-04$ \\
\hline EDTA4- & $7.84 E-06$ & 1.30 & 2.50 & $5.51 E-06$ & 6. $72 \mathrm{E}-0 \mathrm{~s}$ & $9.23 \mathrm{E}-06$ & $1.06 \mathrm{E}-05$ \\
\hline ḦEDTA3- & $6.51 \mathrm{E}-06$ & 1.03 & 1.98 & $2.09 \mathrm{E}-06$ & 447E-06 & 8.69E- 06 & $108 \mathrm{E}-05$ \\
\hline glycolate- & $2.84 \mathrm{E}-04$ & 12.3 & 23.6 & 1.54E-04 & 2.25E-04 & $3,5,3 \mathrm{E}=04$ & 4.19E-04 \\
\hline acetate- & $2.95 \mathrm{E}-05$ & 100 & 1.93 & $2.85 \mathrm{E}-0.5$ & $2.90 \mathrm{E}-05$ & 3.13E-05 & $3.35 \mathrm{E} \cdot 05$ \\
\hline oxalate2- & $1.22 \mathrm{E}-11$ & 6.19E-07 & $1.19 \mathrm{E}-0 \mathrm{C}$ & $1.08 \mathrm{E}-11$ & $1.15 \mathrm{E}-11$ & $1.34 \mathrm{E}-11$ & 1.47E-11 \\
\hline$\overline{\mathrm{DBP}}$ & $1.78 E-04$ & 21.6 & 41.6 & $1.67 \mathrm{E}-04$ & $1.74 \mathrm{E}-04$ & 1.93E-04 & $2.09 \mathrm{E}-04$ \\
\hline butanol & $1.78 E-04$ & 7.63 & 14.7 & $1.67 E-04$ & $1.74 E-04$ & $1.93 \mathrm{E}-04$ & 2.08E-04 \\
\hline NIS & 0.114 & $1.12 \mathrm{E}+03$ & 2.15E+03 & $4.406-02$ & $5.39 \mathrm{E}-02$ & 0.119 & 0.125 \\
\hline $\mathrm{Fe}[\mathrm{CN} ; 64-$ & 0 & 0 & 0 & 0 & 0 & 0 & 0 \\
\hline
\end{tabular}

"Unknowns in tank solids inventory are aurigned by Tank Layering Model (TLM). 
HNF-SD-WM-ER-323, Rev. 1

HDW Model Rev. 4

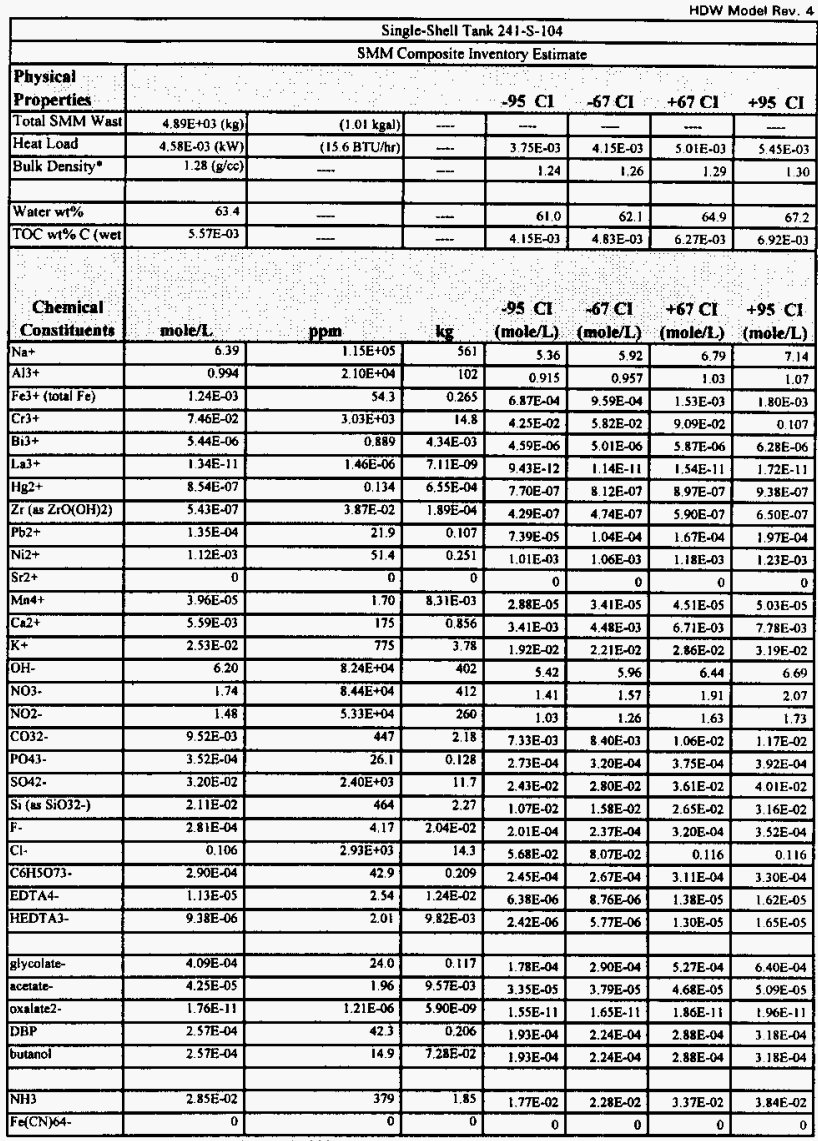

*Density is calculated based on $\mathrm{Na}$, $\mathrm{OH}-$, and $\mathrm{AlO} 2-$.

+Water wt\% derived from the difference of density and total dissolved species. 


\begin{tabular}{|c|c|c|c|c|c|c|c|}
\hline \multirow{3}{*}{\begin{tabular}{|l|} 
\\
Physical \\
Properties \\
\end{tabular}} & \multicolumn{7}{|c|}{ Single-Shell Tank 24I-S-104 } \\
\hline & \multicolumn{7}{|c|}{ Total Inventory Estimate* } \\
\hline & & & & $.95 \mathrm{Cl}$ & $67 \mathrm{CI}$ & & \\
\hline Total Waste & $1.93 \mathrm{E}+06(\mathrm{~kg})$ & $(294 \mathrm{kgal})$ & - & - & - & - & -2 \\
\hline Heat Load & $4.67(\mathrm{~kW})$ & (1.60E+0. BTU/hr) & - & 3.94 & 4.37 & 4.87 & 4.96 \\
\hline Bulk Densityt & $173(\mathrm{~g} / \mathrm{ccc})$ & - & $-\ldots$ & 1.58 & 1.65 & 1.86 & 1.91 \\
\hline & & & & & & & \\
\hline Water wt $\% t$ & 31.1 & - & - & 20.2 & 23.7 & 36.6 & 42.1 \\
\hline TOC wi\% C (wet & $2.86 \mathrm{E}-03$ & - & - & $2.59 \mathrm{E}-03$ & $2.66 \mathrm{E}-03$ & $3.07 \mathrm{E}-03$ & $3.33 \mathrm{E}-03$ \\
\hline $\begin{array}{l}\text { Chemical } \\
\text { Constituents }\end{array}$ & mole/ & ppm & kg & $\begin{array}{l}-95 \mathrm{CI} \\
\text { (motell) }\end{array}$ & $\begin{array}{l}67 \mathrm{Cl} \\
\text { (mole/L) }\end{array}$ & $\begin{array}{c}+67 \mathrm{CI} \\
\text { (mole/ } / \mathrm{L})\end{array}$ & $\begin{array}{l}+95 \mathrm{Cl} \\
\text { (mole/L) }\end{array}$ \\
\hline $\mathrm{Na}+$ & 110 & $1.46 \mathrm{E}+05$ & $282 \mathrm{E}+05$ & 8.33 & 9.34 & 134 & 14.7 \\
\hline $\mathrm{A} \sqrt{3}+$ & 5.34 & B.32E+04 & $1.60 E+05$ & 4.27 & 4.59 & 6.16 & 6.99 \\
\hline Fe3+(10tal Fe) & 0.395 & $1.27 \mathrm{E}+04$ & $2.46 E+04$ & 0.388 & 0.392 & 0.399 & 0.402 \\
\hline $\mathrm{Cr}+3+^{+}$ & 0.473 & $1.42 \mathrm{E}+04$ & $2.74 E+04$ & 0.286 & 0.375 & 0842 & 0.840 \\
\hline$\overline{B i 3+}$ & $3.78 \mathrm{E}=06$ & 0.456 & 0.879 & $3.195-06$ & $3.48 \mathrm{E}-66$ & $416 \mathrm{E}-06$ & $4.58 \mathrm{E}-06$ \\
\hline L.3+ & $9.31 \mathrm{E}-12$ & $7.47 \mathrm{E}-07$ & $1.44 \mathrm{E}-06$ & 8. $16 E-12$ & $8,81 E-12$ & $1.03 \mathrm{E}-11$ & $1.13 \mathrm{E}-11$ \\
\hline $\mathrm{H}_{\mathrm{g} 2}{ }^{2+}$ & $3.34 \mathrm{E}-04$ & 38.6 & 74.5 & $329 \mathrm{E}-24$ & $3.32 \mathrm{E}-04$ & $3.35 \mathrm{E} .04$ & $3.36 \mathrm{E}-04$ \\
\hline $\mathrm{Zr}(\mathrm{AS} \mathrm{ZrO}(\mathrm{OH}) 2)$ & $3.77 \mathrm{E}-07$ & $1.99 \mathrm{E}-02$ & $3.83 E-02$ & $3.47 \mathrm{E}-07$ & $3.62 \mathrm{E}-07$ & $3.96 \mathrm{E}-07$ & $4.27 \mathrm{E}-07$ \\
\hline $\mathrm{Pb2+}$ & $9.70 \mathrm{E}-03$ & $1.16 \mathrm{E}+03$ & $2.24 E+03$ & $8.92 E-03$ & $9.35 \mathrm{E}-03$ & $1,00 \mathrm{E}-02$ & $1.04 E-02$ \\
\hline Ni2+ & $2.70 \mathrm{E}-02$ & 914 & $1.76 \mathrm{E}+03$ & $2.18 E-02$ & $2.47 E-02$ & $2.87 \mathrm{E}-02$ & $2.84 \mathrm{E}-02$ \\
\hline Sr2t & 0 & 0 & 0 & 0 & 0 & 0 & 0 \\
\hline Mn4t+ & $2.75 \mathrm{SE}-05$ & 0.873 & 1.68 & $1.97 \mathrm{E}-05$ & $2.35 \mathrm{E}-0 \mathrm{~S}$ & $3.16 \mathrm{E}-05$ & $3.56 \mathrm{E}-05$ \\
\hline $\mathrm{Ca} 2+$ & 0.133 & $3.07 \mathrm{E}+03$ & $3.92 \mathrm{E}+03$ & 0.105 & 0.119 & 0.147 & 0.160 \\
\hline $\mathrm{K}+$ & $2.19 \mathrm{E}-02$ & 494 & S51 & $1.82 E-02$ & 1.97E-02 & $2.34 \mathrm{E}-02$ & $2.52 \mathrm{E}-02$ \\
\hline DH- & 23.1 & $2.26 \mathrm{E}+05$ & $4.36 \mathrm{E}+05$ & 18.1 & 197 & 26.6 & 30.1 \\
\hline NO3- & 4.87 & $1.74 E+05$ & $3.36 \mathrm{E}+05$ & 2.95 & 2.95 & 7.87 & 9.50 \\
\hline NO2- & 1.64 & $4.36 \mathrm{E}+04$ & $8.41 E+04$ & 1.16 & 1.33 & 1.90 & 1.90 \\
\hline $\cos 2-$ & 0.135 & $4.69 \mathrm{E}+03$ & $9.04 \mathrm{E}+03$ & 0.108 & 0.121 & 0.149 & 0.163 \\
\hline PO43- & 2.4SE-04 & 13.4 & 25.9 & $2.27 \mathrm{E}-04$ & $2.37 \mathrm{E}-04$ & $2.55 E_{-}-4$ & $2.77 E-04$ \\
\hline SO42- & $2.68 \mathrm{E}-02$ & $1.48 \mathrm{E}+03$ & $2.86 \mathrm{E}+03$ & $2.34 E-02$ & $247 \mathrm{E}-02$ & $2.87 \mathrm{E}-02$ & 3.08E-02 \\
\hline $5 \mathrm{si}(28 \mathrm{SiO} 2-)$ & $7,59 \mathrm{E}-02$ & $1.23 \mathrm{E}+03$ & $2.37 \mathrm{E}+0 \mathrm{3}$ & $4.97 \mathrm{E}-02$ & $6.59 \mathrm{E}-02$ & $8.58 \mathrm{E} .02$ & $9.54 \mathrm{E}-02$ \\
\hline F. & 1.95E-04 & 2.14 & 4.12 & $1.66 \mathrm{E}-04$ & $1.84 \mathrm{E}-04$ & $2.14 E-04$ & $2.31 \mathrm{E}-04$ \\
\hline Cl. & $9.30 \mathrm{E}-02$ & $1.90 \mathrm{E}+03$ & $3.67 \mathrm{E}+03$ & $5.90 \mathrm{E}-02$ & $7.56 \mathrm{E}-02$ & 0.100 & 0.132 \\
\hline C6H5O73- & $2.02 E-04$ & 22.0 & 42.4 & $1.95 \mathrm{E}-04$ & $1.98 \mathrm{E}-04$ & $2.10 \mathrm{E}-04$ & $2.29 \mathrm{E}-04$ \\
\hline EDTA4- & $7.85 E-06$ & 1.31 & 2.52 & $5.52 E-06$ & $6.79 \mathrm{E}-06$ & $9.24 \mathrm{E} \cdot 06$ & $1.06 \mathrm{E}-05$ \\
\hline HEDTA3- & $6.52 \mathrm{E}-06$ & 1.03 & 1.99 & $2.09 \mathrm{E}-06$ & $4.48 E-06$ & 8.70E- 06 & $1.08 \mathrm{E}-05$ \\
\hline glycolste- & $2.84 \mathrm{E}-04$ & 12.3 & 23.7 & $144 E_{-04}$ & $255 \mathrm{E}-0$ & TEF & $400=0$ \\
\hline scetulte- & 2.95E-05 & 1.01 & 1.94 & $2.86 \mathrm{E}-05$ & $290 \mathrm{E}-05$ & $3.14 \mathrm{E}-05$ & 3.35E-05 \\
\hline oxalate2- & $1.22 \mathrm{E}=1 \mathrm{II}$ & $6.20 \mathrm{E}-07$ & 1.19E-06 & $1.08 E-11$ & $1.15 \mathrm{E}-11$ & $1.34 \mathrm{E}-11$ & $1.47 \mathrm{E}-11$ \\
\hline$\overline{\mathrm{DBP}}$ & $1.79 \mathrm{E}-94$ & 21.7 & 41.8 & $1.67 \mathrm{E}-04$ & $1.74 \mathrm{E}-04$ & $1.93 \mathrm{E}-04$ & $2.09 \mathrm{E}-04$ \\
\hline butanol & $1.79 \mathrm{E}-04$ & 7.65 & 147 & $1.67 \mathrm{~T}-04$ & $1.74 \mathrm{E}-04$ & $1.93 \mathrm{E}-04$ & $2.09 \mathrm{E}-04$ \\
\hline & & & & & & & \\
\hline $\mathrm{NH3}$ & 0.114 & T.12E+03 & $2.15 \mathrm{E}+03$ & $4.39 \mathrm{E}-02$ & $5.38 E-02$ & 0.119 & 0.124 \\
\hline $\mathrm{Fe}(\mathrm{CN}) 64$ & 0 & 0 & 0 & 0 & 0 & 0 & 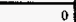 \\
\hline
\end{tabular}

"Unknowns in tank solids inventory are assigned by Tank Layering Model (TLM).

† Water wt\% derived from the difference of density and total dissolved species. 
HNF-SD-WM-ER-323, Rev. 1

HDW Model Rev. 4

\begin{tabular}{|c|c|c|c|c|c|c|c|}
\hline \multirow[b]{3}{*}{$\begin{array}{l}\text { Physical : } \\
\text { Properties }\end{array}$} & \multicolumn{7}{|c|}{ Single-Shell Tank 24 J-S-104 } \\
\hline & \multicolumn{7}{|c|}{ TLM Solids Composite Inventory Estimate* } \\
\hline & & . & & $-95 \mathrm{CI}$ & $\begin{array}{l}-67 \mathrm{Cl} \\
\end{array}$ & $+67 \mathrm{CI}$ & $+95 \mathrm{CI}$ \\
\hline Total TLM Wast & $1.92 \mathrm{E}+06(\mathrm{~kg})$ & (293 kgal) & $\ldots$ & $\ldots$ & r. & - & - \\
\hline Heat Lond & $4,67(\mathrm{~kW})$ & (1.59E+04 BTU/hr) & $\ldots$ & 3.94 & 4.37 & 4.87 & 4.96 \\
\hline Bulk Densiry & $1.73(\mathrm{~g} / \mathrm{cc})$ & $\cdots$ & $\ldots$ & 1.58 & 1.65 & 1.86 & 1.91 \\
\hline Void Fraction & $\begin{array}{ll}0.682 \\
\end{array}$ & $\cdots$ & - & 0.507 & 0.565 & 0.756 & 0.788 \\
\hline Water wt\% & 31.0 & - & - & 20.1 & 23.6 & \begin{tabular}{l|l}
36.5 \\
\end{tabular} & 42.0 \\
\hline TOC wt $\%$ C (wet & $2.85 \mathrm{E}-03$ & - & $=$ & 2.58E-03 & $2,65 \mathrm{E}-03$ & $3.06 \mathrm{E}-03$ & $3.33 \mathrm{E}-03$ \\
\hline $\begin{array}{l}\text { Radiologienil } \\
\text { Constifuents }\end{array}$ & CWL & $\mathrm{HCV} / \mathrm{s}$ & CI & $\begin{array}{l}95 \mathrm{Cl} \\
(\mathrm{CUL})\end{array}$ & $\begin{array}{l}467 \mathrm{Cl} \\
\text { (Ci/L) }\end{array}$ & $\begin{array}{l}+67 \mathrm{CI} \\
(\mathrm{CVI})\end{array}$ & $\begin{array}{l}+95 \mathrm{CI} \\
(\mathrm{Cu} / \mathrm{L})\end{array}$ \\
\hline $\mathrm{H}-3$ & $1.33 \mathrm{E}-04$ & $7.69 \mathrm{E}-02$ & 148 & $1.09 \mathrm{E}-05$ & $1.09 \mathrm{E}-05$ & $139 E-04$ & $1.51 \mathrm{E}-04$ \\
\hline C-14 & $7.13 \mathrm{E}-06$ & 4.11E-03 & 7.90 & $\$, 43 E-07$ & $8.43 \mathrm{E}-07$ & $7,42 \mathrm{E}-06$ & $7.96 \mathrm{E}-06$ \\
\hline Ni-59 & $9.22 \mathrm{E}-96$ & $5.32 \mathrm{E}-0.3$ & 10.2 & $5.48 \mathrm{E}-106$ & $5.48 \mathrm{E}-46$ & $973 \mathrm{E}-06$ & $9.64 \mathrm{E}-06$ \\
\hline $\mathrm{Ni}-63$ & 8.75E-04 & 0.505 & 971 & $5.12 E-04$ & $5.12 \mathrm{E}-04$ & $9.23 \mathrm{E}-04$ & $9.14 \mathrm{E}-04$ \\
\hline Co-60 & $5.99 \mathrm{E}-06$ & $3.46 \mathrm{E}-03$ & 6.64 & 3.33E-07 & $3.33 \mathrm{E}-47$ & $6.23 \mathrm{E}-06$ & 6.7SE.06 \\
\hline Se-79 & $4.00 \mathrm{E}-06$ & $2.31 \mathrm{E}-03$ & 4.43 & $1.79 \mathrm{E}-07$ & $1.79 \mathrm{E}-07$ & $4.06 \mathrm{E}-06$ & $6.46 \mathrm{E}-06$ \\
\hline Sr-90 & 0487 & 281 & $5.40 \mathrm{E}+05$ & 0.389 & 0.444 & 0.520 & 0.525 \\
\hline$Y-90$ & 0.487 & 281 & $5.40 E+05$ & 0.342 & \begin{tabular}{l|l}
0.342 \\
\end{tabular} & 0.520 & 0.525 \\
\hline$Z r-93$ & $1.89 \mathrm{E}-05$ & 1.09E-02 & 21.0 & 8.45E-07 & 8.45E-07 & $1.92 \mathrm{E}-05$ & $2.93 \mathrm{E}-05$ \\
\hline $\mathrm{Nb}-93 \mathrm{~m}$ & $1.54 \mathrm{E}-05$ & 8.96E-03 & 17.0 & $6.91 E-07$ & $6.91 \mathrm{E}-07$ & $1.56 \mathrm{E}-05$ & $2.65 \mathrm{E}-05$ \\
\hline Tc-99 & $5.48 \mathrm{E}-05$ & 3.16E-02 & 60.7 & $496 \mathrm{E}-05$ & $5.16 \mathrm{E}-05$ & 5.84E-05 & $6.26 \mathrm{E}-05$ \\
\hline$R u-106$ & $1,31 \mathrm{E}-09$ & $7.55 \mathrm{E}-07$ & 1.45E-03 & $9,43 \mathrm{E}-13$ & $9.43 \mathrm{E}-13$ & $1.37 \mathrm{E}-09$ & 1.49E-09 \\
\hline $\mathrm{Cd} .113 \mathrm{~m}$ & $2.81 \mathrm{E}-05$ & $1.62 \mathrm{E}-02$ & 31.1 & 2.61E-06 & $2.61 \mathrm{E}-06$ & $5.91 \mathrm{E}-05$ & $5.72 \mathrm{E}-05$ \\
\hline Sb-125 & $2.07 \mathrm{E}-05$ & $1.19 \mathrm{E}-02$ & 23.0 & $4.90 \mathrm{E}-07$ & $4.90 \mathrm{E}-07$ & $2.16 \mathrm{E}-05$ & $2.34 \mathrm{E}-05$ \\
\hline$S n-126$ & $6.14 E-06$ & $3.54 E-03$ & 6.80 & $2.75 \mathrm{E}-07$ & 2.75E-07 & $6.23 \mathrm{E}-06$ & $101 \mathrm{E}-05$ \\
\hline I-129 & $1,04 \mathrm{E}-07$ & $6.00 \mathrm{E}-05$ & 0.115 & $9.41 \mathrm{E}-08$ & $9.81 \mathrm{E}-08$ & $1.11 \mathrm{E}-07$ & I. 19E-07 \\
\hline Cs=134 & $1.27 \mathrm{E}-06$ & $7.35 \mathrm{E}-04$ & 141 & $102 \mathrm{E}-08$ & $1.02 \mathrm{E}-0 \mathrm{~g}$ & $1.32 \mathrm{E}-06$ & $1.42 \mathrm{E}-06$ \\
\hline Cs-137 & 0.199 & 115 & $2.20 \bar{E}+05$ & 0.184 & 0.190 & 0.212 & 0.226 \\
\hline $\mathrm{Ba}=137 \mathrm{~m}$ & 0.188 & 108 & $2.09 \mathrm{E}+05$ & $1.97 \mathrm{E}-02$ & $1.97 \mathrm{E}-02$ & 0194 & 0.208 \\
\hline $5 m-151$ & $1.43 \mathrm{E}-02$ & 8.22 & $1.58 E+04$ & $6.39 \mathrm{E}-04$ & $6,39 \mathrm{E}-04$ & $1.45 E-02$ & $2.33 \mathrm{E}-02$ \\
\hline Eu-152 & $7.47 \mathrm{E}-06$ & $4.31 \mathrm{E}-03$ & 8.28 & $2.59 \mathrm{E}-06$ & $2.59 \mathrm{E}-06$ & 7 50E-06 & $7.56 \mathrm{E}-06$ \\
\hline Eu-154 & $1.43 \mathrm{E}-04$ & 8.27E-02 & 159 & $8.05 \mathrm{E}-06$ & 8.05E-05 & $1.49 \mathrm{E}-04$ & $1.86 \mathrm{E}-04$ \\
\hline Eu-155 & $3.70 \mathrm{E}-04$ & 0.213 & 410 & $1.22 \mathrm{E}-04$ & $1.22 E-04$ & $3.71 \mathrm{E}-04$ & $3.74 \mathrm{E}-04$ \\
\hline $\mathrm{R} 8-226$ & $6.56 \mathrm{E}-10$ & 3.79E-07 & $7.28 \mathrm{E}-104$ & 3.13E-10 & $3.98 \mathrm{E}-10$ & $8.32 \mathrm{E}-10$ & $1.00 \mathrm{E}-09$ \\
\hline Ra-228 & $8.66 \mathrm{E}-09$ & $4,99 \mathrm{E}-06$ & $9.60 \mathrm{E}-03$ & $344 \mathrm{E}-15$ & $3.44 \mathrm{E}-15$ & $8.74 \mathrm{E}-09$ & $8.84 \mathrm{E}-109$ \\
\hline Ac-227 & 3.27E- 09 & $1.88 \mathrm{E}-06$ & 3.62E-03 & $1,62 \mathrm{E}-09$ & $1.76 \mathrm{E}-09$ & 4.28E-09 & $5.26 \mathrm{E}-09$ \\
\hline $\mathrm{Pa}-231$ & 5.95E-09 & $3.43 \mathrm{E}-06$ & $6.60 \mathrm{E}-03$ & $2.67 \mathrm{E}-10$ & $2.67 \mathrm{E} \cdot 10$ & $6.44 \mathrm{E}_{\mathrm{m}} 09$ & $1.19 \mathrm{E}-08$ \\
\hline Th-229 & $2.08 \mathrm{E}-10$ & $1.20 \mathrm{E}-07$ & $2.31 \mathrm{E}-0 \mathrm{~A}$ & 6.55E-13 & $0.55 \mathrm{E}-13$ & $2.10 \mathrm{E}-10$ & $2.12 \mathrm{E}-10$ \\
\hline Th-232 & 1.16E-10 & $6.69 \mathrm{E}-08$ & $1.296-04$ & $2.21 \mathrm{E}-16$ & $2.21 \mathrm{E} \cdot 16$ & 1.46E-to & $176 \mathrm{E}-10$ \\
\hline U-232 & $3.87 E-08$ & 2.23E-05 & $4.29 \mathrm{E}-02$ & 1.37E-08 & $2.53 \mathrm{E}-0 \mathrm{~s}$ & $5.52 \mathrm{E}-08$ & $7,34 \mathrm{E}-0 \mathrm{~s}$ \\
\hline U-233 & $1.48 E-07$ & 1.54E-05 & 0.164 & $5.23 \mathrm{E}-08$ & $9.66 \mathrm{E}-08$ & $2.11 \mathrm{E}=07$ & $2.81 \mathrm{E}-07$ \\
\hline U-234 & $1.96 \mathrm{E}-06$ & 1.13E-03 & 2.17 & 1.56E-06 & $1.75 \mathrm{E}-06$ & 2. $17 E-06$ & 2.39E-06 \\
\hline $\mathrm{U}-235$ & $8.16 \mathrm{E}-08$ & $4.71 \mathrm{E}-05$ & 9.0SE-02 & $6.57 \mathrm{E}-0 \mathrm{~s}$ & $7.34 \mathrm{E}-08$ & $9.05 \mathrm{E}-08$ & $9.94 \mathrm{E}-08$ \\
\hline U-236 & $5.63 \mathrm{E}-08$ & $3.25 \mathrm{E}-05$ & $6.25 \mathrm{SE}-02$ & 4,06E-08 & 4.84E-08 & 6.46E-08 & $7.28 \mathrm{E}-08$ \\
\hline $\mathrm{U}-238$ & $1.84 \mathrm{E}-06$ & $1.06 \mathrm{E}-03$ & 2.04 & $1.49 \mathrm{E}-06$ & 1.66E-06 & $2.03 \mathrm{E}-06$ & $2.23 \mathrm{E}-06$ \\
\hline $\mathrm{Np}-237$ & $2.51 \mathrm{E}-07$ & $1.45 \mathrm{E}-04$ & \begin{tabular}{ll|}
0.279 \\
\end{tabular} & 2.18E-07 & $2.31 \mathrm{E}-07$ & $2695-07$ & $2.89 \mathrm{E}-07$ \\
\hline Pu-238 & $5.79 \mathrm{E}-06$ & $3.34 E-03$ & 6.42 & $4.88 \mathrm{E}-06$ & $5.32 E-06$ & $625 \mathrm{E}-06$ & $6.70 \mathrm{E}-06$ \\
\hline$P u=239$ & $3.48 E-04$ & 0.200 & 385 & $2.98 \mathrm{E}-04$ & $3.23 \mathrm{E}-04$ & $3.73 \mathrm{E}-04$ & $3.97 \mathrm{E}-04$ \\
\hline Pu-240 & $5.04 \mathrm{E}-05$ & $2.91 \mathrm{E}-02$ & 55.9 & 4.34E-05 & $4.68 E-05$ & $5.40 \mathrm{E}-05$ & $5.74 \mathrm{E}-05$ \\
\hline Pu-241 & $3.26 \mathrm{E}-04$ & 0.183 & 362 & $2.80 \mathrm{E}-04$ & $3,03 \mathrm{E}-04$ & 3.50E-04 & $3.73 \mathrm{E}-04$ \\
\hline$P u-242$ & L.51E-09 & $8.69 \mathrm{E}-07$ & $1.67 \mathrm{E}-03$ & $1.26 \mathrm{E}-09$ & $138 \mathrm{E}-09$ & $163 \mathrm{E}-09$ & $1.75 \mathrm{E}-(99$ \\
\hline Am-241 & $6.71 \mathrm{E}-05$ & $3.87 \mathrm{E}-02$ & 74.5 & $3.94 \mathrm{E}-05$ & $5.85 \mathrm{E}-05$ & $7.65 \mathrm{E}-0 \mathrm{~S}$ & $1.17 \mathrm{E}-04$ \\
\hline $\mathrm{Am}-243$ & $2.07 \mathrm{E}-09$ & $1.20 \mathrm{E}-06$ & $230 \mathrm{E}-03$ & $9.69 \mathrm{E}-10$ & $1,80 \mathrm{E}-09$ & $2.28 \mathrm{E}-09$ & $2.54 \mathrm{E}-09$ \\
\hline $\mathrm{Cm}-242$ & $6.05 \mathrm{E}-08$ & $3.49 \mathrm{E}-05$ & $6.71 \mathrm{E}-02$ & $5.92 \mathrm{E}-0 \mathrm{~B}$ & $5.96 \mathrm{E}-0 \mathrm{~B}$ & 6. $10 \mathrm{E}-0 \mathrm{~s}$ & $6.10 \mathrm{E}-0 \mathrm{~s}$ \\
\hline $\mathrm{Cm}-243$ & $1.38 \mathrm{E}-09$ & $7.99 \mathrm{E}-07$ & 1.54E-03 & $1.36 \mathrm{E}-09$ & $1.37 \mathrm{E}-09$ & 1.40E-09 & $140 \mathrm{E}-09$ \\
\hline $\mathrm{Cm}-244$ & $1.11 \mathrm{E}-09$ & $6,41 \mathrm{E}-07$ & $1.23 \mathrm{E}-03$ & $2.29 \mathrm{E}-10$ & $5.81 \mathrm{E}-10$ & $1.47 \mathrm{E}-09$ & $147 \mathrm{E}-09$ \\
\hline Totals: & $\mathbf{M}$ & $\mathrm{Mg} / \mathrm{g}$ & ky & $\begin{array}{l}\text { CS CI } \\
\mathrm{M} / \mathrm{r} \\
\mathrm{I} / \mathrm{L})\end{array}$ & $\begin{array}{l}-67 \mathrm{CI}(\mathrm{M} \\
\text { or } \mathrm{M})\end{array}$ & $\begin{array}{l}467 \mathrm{CI}(\mathrm{M} \\
\mathrm{or} / \mathrm{L})\end{array}$ & $\begin{array}{r}+95 \mathrm{Cl} \\
\text { (Mor } \\
\mathrm{gL} / \mathrm{L})\end{array}$ \\
\hline $\mathrm{Pu}$ & $5.81 \mathrm{E}-03(\mathrm{~g} / \mathrm{L})$ & - & 6.44 & $4.99 \mathrm{E}-03$ & $539 E-03$ & $6.23 E-03$ & 6.63E-03 \\
\hline U & $2.30 \mathrm{E}-02$ & $3.16 \mathrm{E}+03$ & $6.07 E+03$ & $1.87 \mathrm{E}-02$ & $2.08 \mathrm{E}-02$ & $2.54 \mathrm{E}-02$ & $2.78 \mathrm{E}-02$ \\
\hline
\end{tabular}

"Unknowns in tank solids inventory are assigned by Tank Layering Model (TLM). 
HNF-SD-WM-ER-323, Rev. 1

\begin{tabular}{|c|c|c|c|c|c|c|c|}
\hline \multirow[b]{3}{*}{$\begin{array}{l}\text { Physical } \\
\text { Properties: }\end{array}$} & \multicolumn{7}{|c|}{ Single-Shell Tank 241-S-104 } \\
\hline & \multicolumn{7}{|c|}{ SMM Composite Inventory Estimale } \\
\hline & & & & $-95 \mathrm{Cl}$ & $-67 \mathrm{CI}:$ & $+67 \mathrm{CI}$ & $+95 \mathrm{Cl}$ \\
\hline Total SMM Wast & $489 \mathrm{E}+03(\mathrm{~kg})$ & $(1.01 \mathrm{kgat})$ & - & $\ldots$ & - & - & 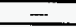 \\
\hline Heat Load & $4.58 \mathrm{E}-03(\mathrm{~kW})$ & $(15.6 \mathrm{BTU} / \mathrm{hr})$ & - & $3.73 \mathrm{E}-03$ & $4.15 E-03$ & $5.01 \mathrm{E}-03$ & $5.45 \mathrm{E}-03$ \\
\hline Bulk Density* & $1.28(\mathrm{~g} / \mathrm{cc})$ & - & - & 1.24 & 1.26 & 1.29 & 1.30 \\
\hline & & & & & & & \\
\hline Water wt\%† & 63.4 & - & - & 61.0 & 62.1 & 64.9 & 67.2 \\
\hline TOC wt \% C (wet & 5.57E-03 & - & - & $4.15 E-03$ & $4.83 E-03$ & $6.27 \mathrm{E}-03$ & $6.92 \mathrm{E}-03$ \\
\hline $\begin{array}{l}\text { Radiological } \\
\text { Constituents }\end{array}$ & $\mathrm{Cl} / \mathrm{L}$ & $\mu \mathrm{CV}_{\mathrm{g}}$ & ci & $\begin{array}{l}-95 \mathrm{CI} \\
\text { (CVL) }\end{array}$ & $\begin{array}{l}-67 \mathrm{CI} \\
(\mathrm{CVL})\end{array}$ & $\begin{array}{l}+67 \mathrm{CI} \\
\text { (CiL) }\end{array}$ & $\begin{array}{l}+95 \mathrm{Cl} \\
(\mathrm{CI} / \mathrm{L})\end{array}$ \\
\hline $\mathrm{H}-3$ & $1.76 \mathrm{E}-04$ & 0.138 & 0.674 & $9.18 \mathrm{E}-10$ & 9.18E-10 & $1.77 \mathrm{E}-04$ & $1.77 \mathrm{E}-04$ \\
\hline$C-14$ & $9.06 \mathrm{E}-06$ & $7,08 \mathrm{E}-03$ & $3.46 \mathrm{E}-02$ & $7.13 \mathrm{E}-11$ & $7.13 E \cdot 11$ & $9.14 \mathrm{E}-06$ & $9.22 \mathrm{E}-06$ \\
\hline $\mathrm{Ni}-59$ & 3.05E-07 & $3.95 \mathrm{E}-04$ & $1.93 \mathrm{E}-03$ & 9.02E-12 & $9.02 \mathrm{E}-12$ & 5.32E-07 & $5.58 \mathrm{E}-07$ \\
\hline $\mathrm{Ni}-63$ & $490 \mathrm{E}-05$ & 3.83E-02 & 0.187 & \begin{tabular}{|c|}
$8.43 E-10$ \\
\end{tabular} & $8.43 \mathrm{E}-10$ & $5.17 \mathrm{E}-0 \mathrm{~S}$ & $5.42 E-05$ \\
\hline $\mathrm{Co}-60$ & $815 E-06$ & 6.37E-03 & 3.1.1E-02 & $2.84 \mathrm{E}-11$ & $2.84 \mathrm{E}-11$ & $8.22 E-06$ & 8.29E-06 \\
\hline Se-79 & $1.24 E-06$ & $9.72 \mathrm{E}-04$ & $4.73 \mathrm{E}-03$ & $1.51 \mathrm{E}-11$ & $1.51 \mathrm{E}-11$ & $1.69 \mathrm{E}-06$ & $2.09 \mathrm{E}-06$ \\
\hline Sr.90 & $2.11 \mathrm{E}-02$ & 16.5 & 80.7 & I.8IE-02 & $1.96 \mathrm{E}-02$ & $2.27 \mathrm{E}-02$ & $2.42 E-02$ \\
\hline Y-90 & $2.11 \mathrm{E}-02$ & 16.5 & 80.7 & \begin{tabular}{|c|}
$5.85 E-07$ \\
\end{tabular} & 5.85E-07 & $2.27 \mathrm{E}-02$ & $2.42 \mathrm{E}-02$ \\
\hline $2 r-93$ & $6.22 \mathrm{E}-06$ & $4.866 \mathrm{E}-03$ & $2.37 \mathrm{E}-02$ & $7.15 \mathrm{SE}-11$ & $7.15 \mathrm{E}-11$ & $8.46 \mathrm{E}-46$ & $9.97 \mathrm{E}-06$ \\
\hline $\mathrm{Nb}-93 \mathrm{~m}$ & $4.35 \mathrm{E}-06$ & $3.40 \mathrm{E}-03$ & $1.66 \mathrm{E}-02$ & $584 \mathrm{E}-11$ & S.84E-11 & $5.92 \mathrm{E}-06$ & $7,43 \mathrm{E}-06$ \\
\hline$T c-99$ & $7.04 \mathrm{E}-05$ & $5.50 \mathrm{E}-02$ & 0.269 & 5.49E-09 & $6.24 \mathrm{E}-05$ & $7.84 \mathrm{E}-05$ & 8. $65 E-05$ \\
\hline $\mathrm{Ru}-106$ & $1.88 \mathrm{E}-09$ & $1.47 \mathrm{E}-06$ & 7.19E-06 & $1.78 \mathrm{E}-16$ & $1.78 E-16$ & $1.90 \mathrm{E}-09$ & $1.92 \mathrm{E}-09$ \\
\hline$C d-113 m$ & $3.6 \pi-05$ & $2.8 \pi \mathrm{E}-02$ & 0.140 & $2.21 \mathrm{E}-10$ & $2.21 \mathrm{E}-10$ & 3.70E-05 & $3.73 \mathrm{E}-05$ \\
\hline Sb-125 & 2.91E-05 & $2.28 \mathrm{EE}-02$ & 0.111 & $4.27 \mathrm{E}-11$ & $4.27 \mathrm{E}-11$ & $2.93 \mathrm{E}-05$ & $2.96 \mathrm{E}-0 \mathrm{~s}$ \\
\hline$S_{n-126}$ & $1.86 \mathrm{E}-06$ & $1.46 \mathrm{E}-03$ & $7.12 \mathrm{E}-03$ & $2.33 \mathrm{E}-11$ & $2.33 \mathrm{E}-11$ & $2.34 \mathrm{E}-06$ & $3.06 \mathrm{E}-\mathrm{A} 6$ \\
\hline $1-129$ & $1.34 \mathrm{E}-07$ & $1.04 \mathrm{E}-04$ & $5.10 \mathrm{E}-04$ & \begin{tabular}{|l|}
$1.04 E-07$ \\
\end{tabular} & 1.18E-07 & $1.49 E-07$ & 1.64E-07 \\
\hline C\&-134 & $1.60 \mathrm{E}-06$ & $1.25 \mathrm{E}-03$ & 6. I2E- 03 & $7.87 \mathrm{E}-13$ & $7.87 \mathrm{E}-13$ & $1.62 \mathrm{E}-96$ & $163 \mathrm{E}-16$ \\
\hline Cs.137 & 0.226 & 176 & 862 & 0.179 & 0.201 & 0.250 & 0.274 \\
\hline $\mathrm{Ba}-137 \mathrm{~m}$ & 0.213 & 167 & 813 & $1.39 \mathrm{E}-06$ & $1.39 \mathrm{E}-06$ & 0.215 & 0.217 \\
\hline Sm-151 & $4.35 \mathrm{E}-03$ & 3.40 & 16.6 & $5.41 \mathrm{E}-08$ & $5.41 \mathrm{E}-08$ & $5.92 \mathrm{E}-03$ & $7,38 \mathrm{E}-03$ \\
\hline Eu-152 & $7.06 \mathrm{E}-07$ & $5.52 \mathrm{E}-04$ & $2.70 \mathrm{E}-03$ & 5.34E-12 & 5.30E-12 & $7.12 \mathrm{E}-07$ & $7.19 E-07$ \\
\hline Eu-154 & $1.95 \mathrm{SE}-04$ & \begin{tabular}{l|l|}
0.152 \\
\end{tabular} & 0.744 & 6.85E-10 & $6.85 \mathrm{E}-10$ & $1.96 \mathrm{E}-04$ & $1.98 \mathrm{E}-04$ \\
\hline Eu-155 & $3.58 \mathrm{E}-0.5$ & $2.80 \mathrm{E}-02$ & 0.137 & $2.53 E-10$ & $2.53 \mathrm{E}-10$ & $3.61 \mathrm{E}-05$ & $3.65 \mathrm{E}-05$ \\
\hline Ra-226 & $3.85 \mathrm{E}-11$ & 3.01E-08 & 1.47E-07 & 1.05E-15 & 1.05E-15 & $5.25 \mathrm{E}-11$ & $658 \mathrm{E}-11$ \\
\hline Ra-228 & $1.25 E-09$ & 9.80E-07 & 4.79E-06 & $7.12 E-21$ & $7.12 \mathrm{E}-21$ & $1.27 \mathrm{E}-09$ & $1.28 \mathrm{E}-09$ \\
\hline Ac-227 & $2.24 \mathrm{E}-10$ & $1.75 \mathrm{E}-07$ & $8.53 E-07$ & $6.10 \mathrm{E}-15$ & 6.10E-15 & $3.05 E_{10} 10$ & 3 B2E-10 \\
\hline $\mathrm{Pa}-231$ & $1.24 \mathrm{E}-09$ & $9.72 E-07$ & 4.75E-06 & $2.26 \mathrm{E}-14$ & $2.26 \mathrm{E}-14$ & $1.69 \mathrm{E}-09$ & $2.12 \mathrm{E}-09$ \\
\hline Th-229 & 3.01E-11 & $2.35 \mathrm{E}-08$ & T.1SE-07 & $1.34 \mathrm{E}-18$ & $134 \mathrm{E}-18$ & $3.04 \mathrm{E}-11$ & $306 \mathrm{E}-11$ \\
\hline Th-232 & $1.67 \mathrm{E}-10$ & $1.31 \mathrm{E}-07$ & $6.35 \mathrm{E}-07$ & $1.89 \mathrm{E}-20$ & $1.89 \mathrm{E}-20$ & $2.09 \mathrm{E}-10$ & $2.49 \mathrm{E}-10$ \\
\hline $\mathrm{v}-232$ & $1.27 \mathrm{E}-08$ & $9.94 \mathrm{E}-06$ & 483E-05 & $1.04 E-08$ & $1.17 \mathrm{E}-08$ & $1.37 \mathrm{FE}-08$ & $147 \mathrm{E}-0 \mathrm{~B}$ \\
\hline $\mathrm{U}-233$ & $4.86 \mathrm{E}-08$ & $3.80 E-05$ & 1.86E- 04 & $3.97 \mathrm{E}-08$ & $4.49 \mathrm{E}-08$ & 5.25E-08 & 5.61E-08 \\
\hline $\mathrm{U}-234$ & $2.30 \mathrm{E}-07$ & $1.80 \mathrm{E}-04$ & $877 E_{-14}$ & \begin{tabular}{|c|}
$2.07 \mathrm{E}-07$ \\
\end{tabular} & $2.18 \mathrm{E}-07$ & $2.41 E .07$ & $2.52 E-07$ \\
\hline U.235 & 9.2BE-09 & $7.25 \mathrm{E}-06$ & 3.54E-05 & $8.35 \mathrm{E}-09$ & 8. $80 \mathrm{E}-09$ & $9,74 E-09$ & $1.02 \mathrm{E}-08$ \\
\hline U-236 & $9.51 \mathrm{E}-09$ & $7.44 E-06$ & $3.63 \mathrm{E}-03$ & $8.54 \mathrm{E}-09$ & 9.01E-09 & $1,00 E-08$ & $1.05 E-08$ \\
\hline $\mathrm{U}-2.38$ & $2.01 \mathrm{E}-07$ & $1.57 \mathrm{E}-\mathrm{OA}$ & $7.69 \mathrm{E}-\mathrm{OA}$ & $1.81 \mathrm{E}-07$ & $1,91 \mathrm{E}-07$ & $2.11 \mathrm{E}-07$ & $2.21 \mathrm{E}-07$ \\
\hline $\mathrm{Np}-237$ & $3.07 \mathrm{E}-07$ & $2.40 \mathrm{E}-04$ & $1.17 \mathrm{E}-03$ & $2.36 \mathrm{E}-07$ & $2.70 \mathrm{E}-07$ & \begin{tabular}{|c|}
$3.45 E-07$ \\
\end{tabular} & $382 E-07$ \\
\hline $\begin{array}{l}\text { Pu-238 } \\
\end{array}$ & $1.71 \mathrm{E}-07$ & $1,34 E-04$ & $6.53 \mathrm{E}-04$ & $5.09 \mathrm{E}-08$ & $1.10 \mathrm{E}-07$ & $2.32 E-01$ & 2.91E-07 \\
\hline Pu-239 & $5.522-06$ & $4.32 \mathrm{E}-03$ & $2.11 \mathrm{E}-02$ & $1.66 \mathrm{E}-06$ & $3.55 E-06$ & $7.50 \mathrm{E}-06$ & $9.39 E-06$ \\
\hline Pu-240 & $9.16 \mathrm{E}-07$ & $7,16 \mathrm{E}-04$ & $3,50 \mathrm{E}-03$ & \begin{tabular}{|l|}
$2.74 E-07$ \\
\end{tabular} & $5.89 \mathrm{E}-07$ & $1.24 E-06$ & 1.56E-06 \\
\hline Pu-241 & 8.77E-06 & $6.86 \mathrm{E}-03$ & 3.35E-02 & $2.63 \mathrm{E}-96$ & $5.64 \mathrm{E}-16$ & $1.19 \mathrm{E}-05$ & $1.49 \mathrm{E}-05$ \\
\hline Pu-242 & $4.65 \mathrm{E}-11$ & $3.64 E-08$ & 1.78E-07 & $1.39 \mathrm{E}-11$ & $2.99 \mathrm{E}-11$ & $6.32 E-11$ & $7.91 \mathrm{E}-11$ \\
\hline $\mathrm{Am}-241$ & $1.86 \mathrm{E}-05$ & $1.46 \mathrm{E}-02$ & 7.12E-02 & 5.42E-96 & $1.19 \mathrm{E}-0 \mathrm{~s}$ & $254 \mathrm{E}-05$ & $3.19 \mathrm{E}-05$ \\
\hline$A m-243$ & $5.95 \mathrm{E}-10$ & $4.65 \mathrm{E}-07$ & $2.27 \mathrm{E}-06$ & $1.40 \mathrm{E}-10$ & $3.33 \mathrm{E}-10$ & \begin{tabular}{ll|}
$3.72 \mathrm{E}-10$ \\
\end{tabular} & 1.15E-09 \\
\hline $\mathrm{Cm}-242$ & $1.26 \mathrm{E}-10$ & $9,86 \mathrm{E}-08$ & 481E-07 & $1.22 \mathrm{E}-13$ & $1.22 \mathrm{E}-13$ & $\begin{array}{l}.28 \mathrm{E}-10 \\
\end{array}$ & $129 \mathrm{E}-10$ \\
\hline Cm-243 & $2.58 \mathrm{E}-12$ & $2.01 E-0 s$ & $9.84 E-09$ & $2.80 \mathrm{E}-15$ & $2.80 \mathrm{E}-15$ & $2.61 \mathrm{E}-12$ & $2.63 \mathrm{E}-12$ \\
\hline Cm-244 & $1.50 \mathrm{E}-10$ & $1.17 \mathrm{E}-07$ & 5.73E-67] & $8.52 \mathrm{E}-14$ & 8.52E-14 & $1.52 \mathrm{E}-10$ & $1.53 \mathrm{E}-10$ \\
\hline Totals & $\mathbf{M}$ & $\mu / / \beta$ & 28 & $\begin{array}{l}95 \mathrm{Cl} \\
(\mathrm{M} \text { or } \\
\mathrm{gL})\end{array}$ & $\begin{array}{r}67 \mathrm{Cl}(\mathrm{M} \\
\text { or } g / \mathrm{L})\end{array}$ & $\begin{array}{c}+67 \mathrm{CI}(\mathrm{M} \\
\text { orph) }\end{array}$ & $\begin{array}{c}+95 \mathrm{Cl} \\
\mathrm{M} \mathrm{or} \\
\mathrm{g} / \mathrm{L})\end{array}$ \\
\hline $\mathbf{P u}$ & $9.20 \mathrm{E}-05(\mathrm{~g} / \mathrm{L})$ & - & $3.51 \mathrm{E}-04$ & $2.68 E-05$ & $5.87 E-05$ & $1.25 \mathrm{EE}-04$ & $1.57 \mathrm{E}-04$ \\
\hline U & 2.49E-03 & 463 & 2.26 & \begin{tabular}{|c|}
$2.24 \mathrm{E}-03$ \\
\end{tabular} & $2.36 \mathrm{E}-03$ & $2.61 \mathrm{E}-03$ & $273 \mathrm{E}-03$ \\
\hline
\end{tabular}

"Density is calculated based on $\mathrm{Na}, \mathrm{OH}-$, and $\mathrm{AlO}_{2}$.

+Water wo \% derived from the difference of density and total dissolved species. 
HNF-SD-WM-ER-323, Rev. 1

HDW Model Rev. 4

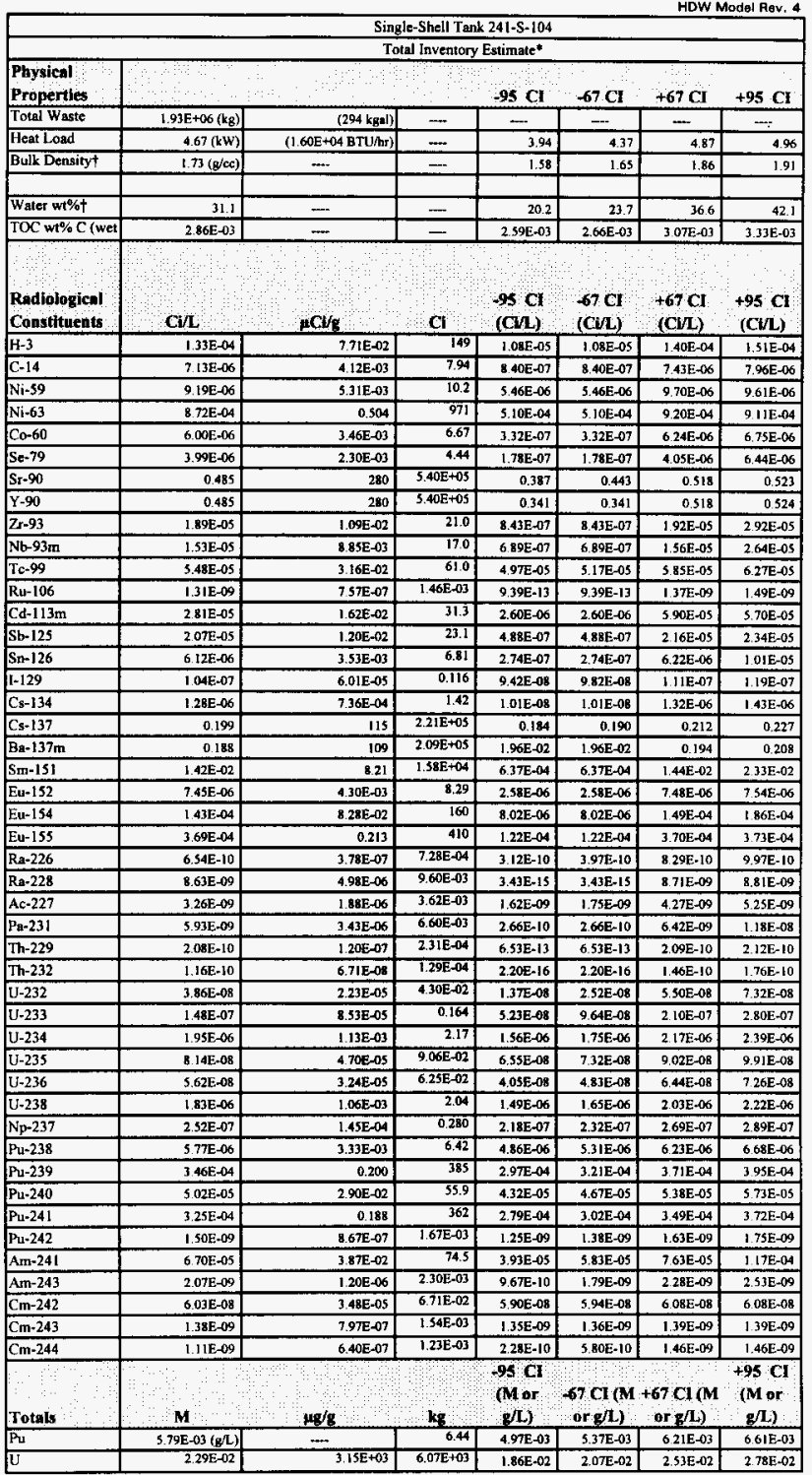

"Unknowns in tank solids inventory are assigned by Tank Layering Model (TLM).

+Volume average for density, mass average Water wt\% and TOC wi\% C. 
HNF-SD-WM-ER-323, Rev. 1

HoW Model Rev. 4

\begin{tabular}{|c|c|c|c|c|c|c|c|}
\hline \multirow[b]{3}{*}{$\begin{array}{l}\text { Physical } \\
\text { Properties }\end{array}$} & \multicolumn{7}{|c|}{ Single-Shell Tank 241-5-105 } \\
\hline & \multicolumn{7}{|c|}{ TI.M Solids Composite Inventory Estimate* } \\
\hline & . & & & $-95 \mathrm{Cl}$ & $-67 \mathrm{Cl}$ & $+67 \mathrm{Cl}$ & $+95 \mathrm{Cl}$ \\
\hline Total TLM Wast & $1.33 \mathrm{E}+04(\mathrm{~kg})$ & $(2.00 \mathrm{kgal})$ & -- & -- & $\ldots$ & - & - \\
\hline Heat Losd & $4.93 \mathrm{E}-02(\mathrm{~kW})$ & (168 BTU/hr) & $\rightarrow$ & $3.57 \mathrm{E}-02$ & $4.37 \mathrm{E}-02$ & $529 \mathrm{E}-02$ & $5.46 E-02$ \\
\hline Bulk Density & $1.76(\mathrm{gcc})$ & $m$ & $m$ & 151 & 1.53 & 2.11 & 2.24 \\
\hline Void Fraction & 0.536 & $\cdots$ & - & $600 \mathrm{E}-02$ & 0.217 & 0.737 & 0.743 \\
\hline Water wt\% & 27.0 & - & - & 2.64 & 10.0 & 42.6 & 44.2 \\
\hline TOC wt $\% \mathrm{C}$ (wet) & 3 & - & - & 0 & 0 & 0 & 0 \\
\hline $\begin{array}{c}\text { Chemical } \\
\text { Constituents }\end{array}$ & mole/L & ppmi & $\mathrm{kg}$ & $\begin{array}{c}95 \text { CI } \\
\text { (mole/L) }\end{array}$ & $\begin{array}{l}67 \mathrm{CI} \\
\text { (molel) }\end{array}$ & $\begin{array}{l}+67 \mathrm{CI} \\
\text { (mole/L) }\end{array}$ & $\begin{array}{l}+95 \mathrm{CI} \\
\text { (mole/L) }\end{array}$ \\
\hline Nat & 8.13 & $1.06 E+05$ & $1.41 \mathrm{E}+03$ & 3.05 & 3.56 & 14.7 & 18.2 \\
\hline $\mathrm{Al3+}$ & 699 & $1.07 \mathrm{E}+05$ & $1.43 E+03$ & 6.58 & 6.74 & 7.15 & 7.15 \\
\hline Fej+(totsl Fe) & 1.01 & $3.22 \mathrm{E}+04$ & 428 & 0.994 & 1.00 & 102 & 1.03 \\
\hline $\mathrm{Cr} 3+$ & $6.19 \mathrm{E}-02$ & $1.83 \mathrm{E}+03$ & 243 & $7.08 \mathrm{E}-03$ & $3.39 \mathrm{E}-\mathrm{n} 2$ & 0.522 & 1.06 \\
\hline $\mathrm{Bi} 3 \div$ & 0 & 可 & 0 & 0 & 0 & 0 & 0 \\
\hline $\mathrm{Laj}^{3+}$ & 0 & 0 & 0 & 0 & 0 & 0 & 0 \\
\hline $\mathrm{Hg} 2+$ & 0 & 0 & 0 & 0 & 0 & 0 & i \\
\hline $\mathrm{Zr}(\mathrm{as} \mathrm{ZrO}(\mathrm{OH}) 2)$ & 0 & 0 & 0 & 0 & 0 & 0 & 0 \\
\hline $\mathrm{Pb2+}$ & 0 & 0 & 0 & 0 & 0 & 0 & 0 \\
\hline $\mathrm{Ni2+}$ & 5.07E-02 & $1.69 \mathrm{E}+03$ & 22.5 & $360 \mathrm{E}-02$ & 4.45E-02 & $554 \mathrm{E}-02$ & $5.45 E-02$ \\
\hline $\mathrm{Si2+}$ & 0 & 0 & 0 & 0 & 0 & 0 & 0 \\
\hline Mr4t & 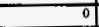 & 0 & 8 & 0 & 0 & 0 & 0 \\
\hline $\operatorname{Cn} 2 t$ & 0.220 & $5.02 \mathrm{E}+03$ & 66.6 & 0.146 & 0.182 & 0.258 & 0.294 \\
\hline $\mathrm{K}+$ & $1.12 \mathrm{E}-02$ & 250 & 3.32 & $2.28 \mathrm{E}-03$ & $5.25 \mathrm{E}-03$ & $1.52 \mathrm{E}-12$ & 1.52E-02 \\
\hline Ö- & 25.6 & $2.48 \mathrm{E}+05$ & $3.296+03$ & 23.1 & 24.3 & 26.9 & 28.4 \\
\hline No3- & 5.29 & $1.87 \mathrm{~F}+\mathrm{CS}$ & $2.48 \mathrm{E}+03$ & $6.03 \mathrm{E}-02$ & $6.03 \mathrm{E}-02$ & 13.5 & 17.9 \\
\hline NO2- & 1.46 & $3.52 \mathrm{E}+04$ & 507 & 0.132 & 0.595 & 2.15 & 2.15 \\
\hline Cos3: & 0.220 & $7.51 E+0 \overline{3}$ & 99.8 & 0.146 & 0.182 & 0.258 & C.294 \\
\hline PO43: & 0 & 0 & 0 & 0 & 3 & 0 & 0 \\
\hline SOA2- & $1.04 \mathrm{E}-02$ & 569 & 7.56 & $1.19 \mathrm{E}-03$ & $4.87 \mathrm{E}-03$ & $1.41 \mathrm{E}-02$ & $141 \mathrm{E}-02$ \\
\hline Si (4 SiO32-) & $8.06 \mathrm{E}-03$ & 129 & 1.71 & \$21E-04 & $3.77 E-03$ & $1,09 \mathrm{E}-42$ & $1.09 \mathrm{E}-02$ \\
\hline F. & 0 & 0 & 0 & 0 & 0 & 0 & 0 \\
\hline Cl- & 5. $16 \mathrm{E}-02$ & $1,04 E+03$ & 13.8 & $5.91 \mathrm{E}-07$ & $2.42 \mathrm{E}-02$ & $7.00 \mathrm{E}-02$ & 0.157 \\
\hline $\mathrm{C} 6 \mathrm{H} 5073$. & 0 & 0 & 0 & 0 & 0 & 0 & 0 \\
\hline EDTA4- & 0 & 0 & 0 & 0 & 0 & 0 & 0 \\
\hline HEDTA3- & 0 & 0 & $\ddot{0}$ & 0 & $\mathrm{c}$ & 0 & 0 \\
\hline glycolate: & 0 & 0 & 2 & a & 0 & 0 & 0 \\
\hline scetate- & 0 & 0 & 0 & 0 & 0 & 0 & 0 \\
\hline oxalate2. & 0 & 0 & 0 & 0 & 0 & 0 & $\overrightarrow{0}$ \\
\hline DBP & 0 & 0 & 0 & 0 & 0 & 0 & 0 \\
\hline butanol & 0 & 0 & 0 & 0 & 0 & 0 & 0 \\
\hline NH3 & 0.250 & $2.47 \mathrm{E}+03$ & 32.9 & 6.5SE-02 & $9.25 \mathrm{E}-02$ & 0.270 & 0.284 \\
\hline Fe(CN)64- & 0 & 0 & 0 & 0 & 0 & 0 & 0 \\
\hline
\end{tabular}

tinknowns in tank solids inventory are ausigned by Tank Layering Model (TLM). 
HNF-SD-WM-ER-323, Rev. 1

HDW Mode! Rev. 4

\begin{tabular}{|c|c|c|c|c|c|c|c|}
\hline \multirow[b]{3}{*}{$\begin{array}{l}\text { Plyysical } \\
\text { Properties }\end{array}$} & \multicolumn{7}{|c|}{ Single-Shell Tank 241-S-105 } \\
\hline & \multicolumn{7}{|c|}{ SMM Composite Inventory Estimate } \\
\hline & & & & $-95 \mathrm{CI}$ & $-67 \mathrm{CI}$ & $\begin{array}{l}+67 \mathrm{Cl} \\
+\end{array}$ & $+95 \mathrm{CI}$ \\
\hline Total SMM Wast & $2.46 \mathrm{E}+06(\mathrm{~kg})$ & (405 kgal) & - & - & $\rightarrow$ & $\ldots$ & - \\
\hline Heat Load & $2.89(\mathrm{~kW})$ & $(9.88 \mathrm{E}+03 \mathrm{BTU} / \mathrm{hr})$ & - & 2.69 & 2.79 & 3.01 & 3.01 \\
\hline Bulk Density & $1.61\left(\mathrm{~g}^{\prime} \mathrm{cc}\right)$ & -- & - & 1.54 & 1.58 & 163 & 1.63 \\
\hline & & & & & & & \\
\hline Water wt\% & 34.2 & - & $\ldots$ & 32.4 & 33.1 & 35.9 & 385 \\
\hline TOC wt\% C (wet & 0.264 & + & -- & 0.235 & 0.249 & 0.278 & 0.292 \\
\hline $\begin{array}{l}\text { Chernical } \\
\text { Constitients }\end{array}$ & mole/t. & ppin & kg & $\begin{array}{l}95 \text { CI } \\
\text { (moles) }\end{array}$ & $\begin{array}{l}-67 \mathrm{CI} \\
\text { (moleh })\end{array}$ & $\begin{array}{l}+67 \mathrm{CI} \\
\text { (mole/L) }\end{array}$ & $\begin{array}{r}+95 \mathrm{Cl} \\
\text { (mole/L) }\end{array}$ \\
\hline $\mathrm{Nat}$ & 13.9 & $1.99 \mathrm{E}+05$ & $4.90 \mathrm{E}+05$ & 12.4 & 13.2 & 14.4 & 14.6 \\
\hline$\overline{\mathrm{Al} 3+}$ & 197 & $3.32 \mathrm{E}+04$ & $8.17 \mathrm{E}+04$ & 1.70 & 1.83 & 2.13 & 2.03 \\
\hline Fet $3+$ (total $F e)$ & $8.26 \mathrm{E}-03$ & 287 & 707 & $6.92 \mathrm{E}-03$ & 7. 58E-03 & $8.93 \mathrm{E}-03$ & $9.59 \mathrm{E}-03$ \\
\hline$\overline{\mathrm{Cr}}+$ & 0.204 & $6.59 \mathrm{E}+03$ & $1.62 \mathrm{E}+04$ & 0.147 & 0.178 & 0.209 & 0.213 \\
\hline $\mathrm{Bi3+}$ & $7.36 \mathrm{E}-04$ & 95.7 & 236 & $6.61 \mathrm{E}-04$ & $6.97 \mathrm{E}-04$ & $7.74 \mathrm{E}-04$ & 8.11E-04 \\
\hline LaJ+ & $9.36 \mathrm{E}-10$ & $8.09 \mathrm{E}-05$ & $1.99 \mathrm{E}-04$ & $7.17 \mathrm{E}-10$ & 8.25E-10 & 1.05E- 09 & $1.16 \mathrm{E}-09$ \\
\hline Hg2t & $6.14 \mathrm{E}-96$ & 0.767 & 1.89 & $5.75 \mathrm{E}-06$ & $5.97 \mathrm{E}-06$ & $6.23 \mathrm{E}-106$ & $6.31 \mathrm{E}-06$ \\
\hline $\mathrm{Zr}(\mathrm{as} \mathrm{ZrOHOH}) 2)$ & $1.54 \mathrm{E}-04$ & 8.72 & 21.5 & $1.40 \mathrm{E}-\mathrm{04}$ & 1.45E-04 & $1.59 \mathrm{E}-04$ & $1.67 \mathrm{E}_{0}-04$ \\
\hline $\mathrm{Pb} 2+$ & $7.23 \mathrm{E}-04$ & 93.2 & 230 & 5.99E-04 & $6.60 \mathrm{E}-04$ & $7.86 \mathrm{E}-04$ & $8.47 \mathrm{E}-04$ \\
\hline $\mathrm{Ni24}$ & $5.5 \mathrm{~T}-03$ & 203 & 501 & $5.35 E-03$ & $5.45 E_{-03}$ & $5.68 \mathrm{E}-03$ & $5.78 E-03$ \\
\hline$\overline{512+}$ & 0 & 0 & 0 & 0 & 0 & 0 & 0 \\
\hline Mn4t & $2.39 \mathrm{E}-03$ & 81.6 & 201 & $1.75 \mathrm{E}-03$ & $2.06 \mathrm{E}-03$ & $2.71 \mathrm{E}-03$ & $303 \mathrm{E}-03$ \\
\hline $\mathrm{Ca}^{2+}$ & $2,84 \mathrm{E}-02$ & 709 & $1.75 \mathrm{E}+03$ & $2.42 \mathrm{E}-02$ & $2.63 \mathrm{E}-02$ & $3.06 \mathrm{E}-02$ & $3.26 \mathrm{E}-02$ \\
\hline $\mathrm{K}+$ & $5.80 \mathrm{E}-02$ & $1.41 \mathrm{E}+03$ & $3.48 E+03$ & $5.13 \mathrm{E}-02$ & $5.45 \mathrm{E}-02$ & $6.20 \mathrm{E}-02$ & $6.22 \mathrm{E}-02$ \\
\hline $\mathrm{OH}$. & 11.9 & $1.26 \mathrm{E}+05$ & $3.10 \mathrm{E}+05$ & 10.2 & 11.0 & 12.9 & 12.3 \\
\hline NO3- & 4.70 & $1.81 \mathrm{E}+05$ & $4.47 \mathrm{E}+05$ & 4.26 & 4.48 & 4.88 & 4.88 \\
\hline NO2- & 262 & $7.50 \mathrm{E}+04$ & $1.85 \mathrm{E}+05$ & 2.25 & 2.46 & 2.76 & 2.90 \\
\hline CO32- & 0.234 & $8.74 \mathrm{E}+03$ & $2.15 \mathrm{E}+04$ & 0.212 & 0.219 & 0.253 & 0.255 \\
\hline $\mathrm{PO4} 3$. & $5.27 E-02$ & $3.11 \mathrm{E}+03$ & $7.67 \mathrm{E}+03$ & $4.62 \mathrm{E}-02$ & $4.85 \mathrm{E}-02$ & $5.41 \mathrm{E}-02$ & 5.54E-02 \\
\hline SO42- & 0.161 & $9.60 \mathrm{E}+03$ & $2.36 \mathrm{E}+04$ & 0.125 & 0.142 & 0.180 & 0.196 \\
\hline $\mathrm{Si}($ as SiO32-) & $6.2 \mathrm{TE}-62$ & $1.10 \mathrm{E}+03$ & $2.70 \mathrm{E}+03$ & $5.36 \mathrm{E}-02$ & $5.80 \mathrm{E}-02$ & $6.74 \mathrm{E}-92$ & $7.19 \mathrm{E}-02$ \\
\hline F. & $3.52 \mathrm{E}-62$ & 417 & $1.03 \mathrm{E}+03$ & 2.87E-02 & $3.14 E-02$ & 3.85E-02 & 4. $106-02$ \\
\hline C. & 0.236 & $5.19 \mathrm{E}+03$ & $1.28 \mathrm{E}+04$ & 0.204 & 0.219 & 0.253 & 0249 \\
\hline C6H3073- & $171 \mathrm{E}-42$ & $2.01 E+03$ & $4.96 \mathrm{E}+03$ & $1.58 \mathrm{E}-02$ & $1.64 \mathrm{E}-02$ & $1.78 \mathrm{E}-02$ & 1.84E-02 \\
\hline EDTA4- & $1.92 \mathrm{E}-03$ & 344 & 846 & $9.55 \mathrm{E}-04$ & $1.42 \mathrm{E}-03$ & $2.4 ! \mathrm{E}-03$ & $289 E-03$ \\
\hline HEDTA3- & $3.07 \mathrm{E}-63$ & 523 & $1.29 \mathrm{E}+03$ & $1.15 \mathrm{E}-03$ & $2.08 \mathrm{E}-03$ & $4.06 \mathrm{E}-03$ & 5.02E-03 \\
\hline glycolate- & 2.65E-02 & $1.24 E+03$ & $3.04 \mathrm{E}+03$ & $1.53 \mathrm{E}-02$ & $2.08 \mathrm{E}-02$ & $3.22 \mathrm{E}-02$ & $3.76 \mathrm{E}-02$ \\
\hline acetate- & $2.46 \mathrm{E}-03$ & 90.4 & 223 & $1.99 \mathrm{E}-\mathrm{B3}$ & $2.22 \mathrm{E}-03$ & $2.70 \mathrm{E}-03$ & $293 \mathrm{E}-03$ \\
\hline oxalate2- & $123 \mathrm{E}-09$ & 6.72E-05 & $1.65 \mathrm{E}-04$ & $1.11 \mathrm{E}-09$ & $1.17 E-09$ & 1.29E-09 & 1.34E-09 \\
\hline DBP & $1.19 \mathrm{E}-02$ & $1.55 \mathrm{E}+03$ & $3.83 \mathrm{E}+03$ & $9.63 \mathrm{E}-03$ & $1.07 \mathrm{E}-02$ & $130 \mathrm{E}-02$ & $143 \mathrm{E}-02$ \\
\hline butanol & I.19E-02 & 548 & $1.35 \mathrm{E}+03$ & $9.63 \mathrm{E}-03$ & 1.076-02 & 1.30E-02 & $1.41 \mathrm{E}-02$ \\
\hline NH3 & $6.88 \mathrm{E}-02$ & 728 & $1.79 \mathrm{E}+03$ & 5.8SE-02 & $6.26 \mathrm{E}-02$ & $7.68 \mathrm{E}-02$ & 3.61E-02 \\
\hline Fe(CN)64- & 0 & 0 & 0 & 0 & 0 & 0 & 0 \\
\hline
\end{tabular}

- Density is calculated based on $\mathrm{Na}, \mathrm{OH}_{-}$, and $\mathrm{AlO2}$ -

tWater wt\% derived from the difference of density and total dissolved species. 
HOW Model Rov. 4

\begin{tabular}{|c|c|c|c|c|c|c|c|}
\hline \multirow{3}{*}{\begin{tabular}{|l|} 
\\
Physical \\
Properties \\
\end{tabular}} & \multicolumn{7}{|c|}{ Single-Shell Tank 241-S-105 } \\
\hline & \multicolumn{7}{|c|}{ Total Inventory Estimate* } \\
\hline & $\begin{array}{l}\therefore \\
\therefore\end{array}$ & & & $.95 \mathrm{CI}$ & $-67 \mathrm{CI}$ & $+67 \mathrm{CI}$ & $+95 \mathrm{Cl}$ \\
\hline Total Waste & $2.48 \mathrm{E}+06(\mathrm{~kg})$ & $(407 \mathrm{kgal})$ & - & - & $=$ & $\cdots$ & -- \\
\hline Heat Load & $2.94(\mathrm{~kW})$ & $(1,00 E+04$ BTU/hr $)$ & $\ldots$ & 2.73 & 2.83 & $3 . \infty 6$ & 306 \\
\hline Bulk Density ${ }^{\dagger}$ & $1.61(\mathrm{~g} / \mathrm{cec})$ & - & - & 1.54 & 1.58 & 1.63 & 1.63 \\
\hline & & & & & & & \\
\hline Water $w t \% \dagger$ & 34.2 & $\rightarrow$ & - & 32.3 & 33.0 & 35.9 & 38.6 \\
\hline TOC wt\% C (wet & 0.262 & $\cdots$ & - & 0.233 & 0.247 & 0.277 & 0.290 \\
\hline $\begin{array}{c}\text { Chemical } \\
\text { Constituents }\end{array}$ & mole $/ \mathrm{L}$ : & Ppm & Le & $\begin{array}{c}-95 \mathrm{Cl} \\
\text { (mole/L) }\end{array}$ & $\begin{array}{l}-67 \mathrm{CI} \\
\text { (molel) }\end{array}$ & $\begin{array}{r}+67 \mathrm{CI} \\
\text { (mole } / \mathrm{L} \text { ) }\end{array}$ & $\begin{array}{l}+93 \mathrm{Cl} \\
(\text { mole/L) }\end{array}$ \\
\hline $\mathrm{Na}+$ & 13.9 & $1.99 \mathrm{E}+05$ & $4.92 E+05$ & 12.3 & 13.2 & 14.4 & 14.5 \\
\hline $\bar{A} \mid 3^{+}$ & 200 & $3.36 \mathrm{E}+04$ & $8.31 E+04$ & 1.72 & 1.85 & 2.16 & 2.05 \\
\hline$F_{4} 3+$ (LOLA Fe) & $1.32 \mathrm{E}-02$ & 458 & $1.13 \mathrm{E}+03$ & 1.19E-02 & $1.25 \mathrm{E}-02$ & 1.39E-02 & $1.45 \mathrm{E}-02$ \\
\hline $\mathrm{Cr} 3+$ & 0.203 & $6.56 E+03$ & $1.63 \mathrm{E}+04$ & 0.152 & 0.179 & 0.209 & 0.212 \\
\hline $\mathrm{Bi3+}$ & $7.32 E-04$ & 95.2 & 236 & $6.57 \mathrm{E}-04$ & $6.94 \mathrm{E}-04$ & $7.70 \mathrm{E}-04$ & 8.07E-04 \\
\hline L.34 & $9.32 \mathrm{E} \cdot 10$ & 8.05E-05 & $1.99 \mathrm{E}-04$ & $7.14 E-10$ & 8.20E-10 & $1.04 \mathrm{E}-09$ & $1.15 E-09$ \\
\hline $\mathrm{Hg2+}$ & $6.11 E-06$ & 0.763 & 1.89 & $5.72 E-06$ & $5.95 \mathrm{E}-06$ & $6.20 \mathrm{E}-06$ & $6.28 E-06$ \\
\hline $\mathrm{Zr}(\mathrm{as} \mathrm{ZO}(\mathrm{OH}) 2)$ & 1.53E-04 & 867 & 21.5 & $1.39 \mathrm{E}-04$ & $144 \mathrm{E}-04$ & $1.58 \mathrm{E}-04$ & $1.66 \mathrm{E}-04$ \\
\hline $\mathrm{Pb} 2+$ & $7.19 \mathrm{E}-04$ & 92.7 & 230 & $5.96 \mathrm{E}-04$ & $6.56 \mathrm{E}-04$ & $7.82 \mathrm{E}-04$ & $8.43 \mathrm{E}-04$ \\
\hline$\sqrt{12+}$ & $5.79 \mathrm{E}=03$ & 211 & 523 & $5.59 \mathrm{E}-03$ & $5.69 \mathrm{E}-03$ & $5.89 \mathrm{E}_{0} 03$ & $5.98 \mathrm{E}-03$ \\
\hline $\mathrm{Sr2t}$ & 0 & 0 & 0 & 0 & 0 & 0 & 0 \\
\hline Mn4+ & $2.37 \mathrm{E}-03$ & 81.2 & 201 & $1.74 \mathrm{E}-03$ & $2.05 \mathrm{E}-03$ & $2.70 E-03$ & 3.01E-03 \\
\hline Ca2t & $2.94 \mathrm{E}-02$ & 733 & $1.81 E+03$ & 2.55E-02 & $2.74 \mathrm{E}-02$ & $3.13 \mathrm{E}-02$ & $3.32 \mathrm{E}-02$ \\
\hline $\mathrm{K}+$ & $5.78 \mathrm{E}-02$ & $1.41 E+03$ & $3.48 E+03$ & $5.11 \mathrm{E}-02$ & $5.43 \mathrm{E}-02$ & $6,17 \mathrm{E}-02$ & $6.19 \mathrm{E}-02$ \\
\hline $\mathrm{OH}-$ & 12.0 & $1.27 E+05$ & $3.13 E+05$ & 10.2 & 11.1 & 12.9 & 124 \\
\hline No3. & 4.71 & $1.82 \mathrm{E}+05$ & $4.49 E+05$ & 4.33 & 4,51 & 4.86 & 4.86 \\
\hline NO2. & 2.61 & $748 \mathrm{E}+04$ & $1.85 \mathrm{E}+05$ & 2.25 & 2.46 & 2.75 & 2.89 \\
\hline Co32- & \begin{tabular}{l|l|}
0.234 \\
\end{tabular} & $73 \mathrm{E}+03$ & $2.16 \mathrm{E}+04$ & 0.212 & 0.219 & 0.252 & 0255 \\
\hline PO43- & 5.24E-02 & $3.10 E+03$ & $7.67 \mathrm{E}+03$ & $4.60 \mathrm{E}-02$ & $4.82 \mathrm{E}-02$ & $5.3 \mathrm{BE}-02$ & $5.52 \mathrm{E}-02$ \\
\hline SO42- & 0.160 & 9.55E+03 & $2.36 \mathrm{E}+04$ & 0.125 & 0.141 & 0.179 & 0.195 \\
\hline Si (as Sio32-) & $6.24 E-02$ & $1.09 \mathrm{E}+03$ & $2.70 \mathrm{E}+03$ & $5.33 \mathrm{E}-02$ & $578 \mathrm{E}-02$ & $6.31 \mathrm{E}-02$ & $7.16 \mathrm{E}-02$ \\
\hline F- & 3.51E-02 & 415 & $1.03 \mathrm{E}+03$ & $2,86 \mathrm{E}-02$ & $3.13 \mathrm{E}-02$ & $3.83 \mathrm{E}-02$ & 4.08E-02 \\
\hline Cl- & 0.235 & $5.17 E+03$ & $1.28 \mathrm{E}+04$ & 0.204 & 0.218 & 0.252 & 0.248 \\
\hline C6H5073- & $1.70 \mathrm{E}-02$ & $2.00 \mathrm{E}+03$ & $4.96 \mathrm{E}+03$ & $1.57 \mathrm{E}-02$ & $1.64 \mathrm{E}-02$ & $1.77 \mathrm{E}-02$ & 1.83E-02 \\
\hline EDTA4. & 1.91E-03 & 342 & 246 & $9.51 \mathrm{E}-04$ & $142 \mathrm{E}-03$ & 2.40E- 03 & $288 \mathrm{E}-03$ \\
\hline HEDTA3: & 3.05E-03 & 520 & $1.29 \mathrm{E}+03$ & $1.14 \mathrm{E}-03$ & $207 \mathrm{E}-03$ & $4.04 \mathrm{E}-03$ & $500 \mathrm{E}-03$ \\
\hline glycolate- & $2.63 E-02$ & $1.23 \mathrm{E}+03$ & $3.04 \mathrm{E}+03$ & $1,53 \mathrm{E}-02$ & $207 \mathrm{E}-02$ & $3.20 \mathrm{E}-02$ & $3.74 \mathrm{E}-02$ \\
\hline acetalt- & 2.45E-03 & 89,9 & 223 & 1.98E-03 & $2.21 \mathrm{E}-03$ & $2.69 \mathrm{E}-03$ & $291 \mathrm{E}-03$ \\
\hline oxalate2- & 1.22E-09 & $6.68 \mathrm{E}-05$ & $165 \mathrm{E}-04$ & 1. $10 \mathrm{E}-09$ & 1. $16 \mathrm{E}-09$ & $1.28 \mathrm{E}-09$ & $134 \mathrm{E}-09$ \\
\hline DBP & 1.18E-02 & $1.5,5 \mathrm{E}+03$ & $3.83 \mathrm{E}+0.3$ & $9.58 \mathrm{E}-03$ & $107 E-02$ & $1.29 \mathrm{E}-02$ & $140 \mathrm{E}-02$ \\
\hline butanol & I.18E-02 & 345 & 1.35E+03 & $9.58 \mathrm{E}-03$ & 1.07E-02 & $1.29 \mathrm{E}-02$ & $140 \mathrm{E}-02$ \\
\hline & & & & & & & \\
\hline NH3 & $6.97 \mathrm{E}-02$ & 737 & $1.83 E+03$ & $5.95 \mathrm{E}-02$ & $6.35 \mathrm{E}-02$ & $7.76 \mathrm{E}-02$ & $8.69 \mathrm{E}-02$ \\
\hline Fe(CN)64- & 0 & 0 & 0 & 0 & 0 & 0 & 0 \\
\hline
\end{tabular}

"Unknowns in tank solids inventory are assigned by Tank Lnyering Model (TTM)

tWater wt\% derived from the difference of density and tokal dissolved species. 
HNF-SD-WM-ER-323, Rev. 1

HDW Model Rev. 4

\begin{tabular}{|c|c|c|c|c|c|c|c|}
\hline \multirow[b]{3}{*}{$\begin{array}{l}\text { Physical } \\
\text { Properties }\end{array}$} & \multicolumn{7}{|c|}{ Single-Shell Tarik 241-S-105 } \\
\hline & \multicolumn{7}{|c|}{ TLM Solids Composite Inventory Estimate" } \\
\hline & & & & $-95 \mathrm{Cl}$ & $-67 \mathrm{Cl}$ & $+67 \mathrm{CI}$ & $+95 \mathrm{CI}$ \\
\hline Total TLM Wast & $133 \mathrm{E}+04(\mathrm{~kg})$ & $(2.00 \mathrm{kgal})$ & $=$ & $\cdots$ & - & - & $\rightarrow$ \\
\hline Heat Loud & $4.93 \mathrm{E}-02(\mathrm{~kW})$ & (168 BTU/hr) & $=$ & $3.57 \mathrm{E}-02$ & $4.37 \mathrm{E}-02$ & $5.29 \mathrm{E}-02$ & $5.46 \mathrm{E}-02$ \\
\hline Bulk Density & $1,76(g / c c)$ & $一$ & - & 1.51 & 1.53 & 2.11 & 2.24 \\
\hline Void Fraction & 0.536 & - & 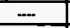 & $600 \mathrm{E}-02$ & 0.217 & 0.737 & 0.743 \\
\hline Water wt\% & 27.0 & - & 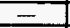 & 2.64 & 100 & 42.6 & 44.2 \\
\hline TOC wt\% C (wet & 0 & $\ldots$ & $\cdots$ & 0 & 0 & 0 & 0 \\
\hline $\begin{array}{l}\text { Radiologicil } \\
\text { Constifinents }\end{array}$ & $\mathrm{C} n \mathrm{n}$ & $\mathrm{HCW}$ & Ci. & $\begin{array}{l}-95 \mathrm{CI} \\
\text { (CV) }\end{array}$ & $\begin{array}{l}-67 \mathrm{CI} \\
\text { (CUL) }\end{array}$ & $\begin{array}{l}+67 \mathrm{CI} \\
\text { (CVI) }\end{array}$ & $\begin{array}{l}+95 \mathrm{CI} \\
(\mathrm{ClL})\end{array}$ \\
\hline $\mathrm{H}-3$ & 2.92E-05 & $1.67 \mathrm{E}-02$ & 0.221 & $305 E-06$ & $1.47 \mathrm{E}-0 \mathrm{~S}$ & $4.10 \mathrm{E}-05$ & 4.10E-05 \\
\hline C. 14 & $2.26 \mathrm{E}-06$ & $1.29 \mathrm{E}-03$ & $1.71 \mathrm{E}-02$ & $2.596-07$ & $1.06 \mathrm{E}-06$ & $3.07 E-06$ & 3.07E-06 \\
\hline $\mathrm{Ni}-59$ & $1.49 \mathrm{E}-05$ & B.46E-03 & 0.112 & $107 \mathrm{E}-05$ & $1.31 \mathrm{E}-05$ & $1.62 \mathrm{E}-05$ & $1.60 \mathrm{E}-05$ \\
\hline $\mathrm{Ni}-63$ & $1.39 \mathrm{E}-03$ & 0.790 & 10.5 & $1.00 \mathrm{E}-03$ & $1.22 \mathrm{E}-03$ & $1.52 \mathrm{E}-03$ & 1.49E-03 \\
\hline $\mathrm{Co}-60$ & $8.94 \mathrm{E}-07$ & $5.09 \mathrm{E}-04$ & $6.76 \mathrm{E}-03$ & $1.02 \mathrm{E}-07$ & 4. 18E-07 & $1.21 \mathrm{E}-06$ & $1.21 \mathrm{E}-06$ \\
\hline Se-79 & $4.81 \mathrm{E}-07$ & $2.74 \mathrm{E}-04$ & $3.63 \mathrm{E}-03$ & $5.50 \mathrm{E}-0 \mathrm{~B}$ & $2.25 \mathrm{E}-07$ & $6.51 \mathrm{E}-07$ & $7.15 \mathrm{E}-06$ \\
\hline$S r-90$ & 0.928 & 529 & $7,02 \mathrm{E}+03$ & 0.662 & 0.812 & 1.02 & 1.03 \\
\hline Y 90 & 0.929 & 529 & $7.02 E+03$ & 0,662 & 0.812 & 1.02 & 1.03 \\
\hline Zr-93 & $2.27 \mathrm{E}-06$ & $1.29 E-03$ & $1.72 \mathrm{E}-02$ & 2.59E-07 & $1.06 \mathrm{E}-06$ & $3.08 \mathrm{E}-06$ & $3.03 \mathrm{E}-05$ \\
\hline $\mathrm{Nb}-93 \mathrm{~m}$ & $1.85 \mathrm{E}-06$ & $1.06 \mathrm{E}-03$ & $1.40 \mathrm{E}-02$ & $2.12 \mathrm{E}-07$ & 8.68E-07 & $2.51 \mathrm{E}-06$ & $3.21 \mathrm{E}-05$ \\
\hline Tc-99 & $1.58 \mathrm{E}-05$ & $9.02 \mathrm{E}-03$ & 0.120 & $1.81 \mathrm{E}-06$ & $7.42 \mathrm{E}-06$ & $2.15 E_{-} 05$ & $2.15 \mathrm{E}-05$ \\
\hline$R u=106$ & $2.50 \mathrm{E}-12$ & $1.42 \mathrm{E}-09$ & $1.89 \mathrm{E}-08$ & $2.86 \mathrm{E}-13$ & $1.17 \mathrm{E}-12$ & $3.38 \mathrm{E}-12$ & $3.39 \mathrm{E}-12$ \\
\hline $\mathrm{Cd}-113 \mathrm{~m}$ & $6.99 E-06$ & $3.98 \mathrm{E}-03$ & $5.29 \mathrm{E}-02$ & $8,00 \mathrm{E}-07$ & $3.27 \mathrm{E}-06$ & $9.48 E-06$ & $9.48 E-06$ \\
\hline $5 b-125$ & 1.31E-06 & $7.47 \mathrm{E}-04$ & 9.92E-03 & J.50E-07 & 6.14E-07 & $1.78 \mathrm{E}-06$ & 1.78E-06 \\
\hline $5 n-126$ & 7.39E-07 & $4.21 \mathrm{E}-04$ & $5.59 \mathrm{E}-03$ & B. $45 \mathrm{E}-08$ & $3.46 \mathrm{E}-07$ & $1.00 \mathrm{E}-06$ & 1.14E-05 \\
\hline I-129 & $3.04 \mathrm{E}-08$ & $1.73 \mathrm{E}-05$ & $2.30 \mathrm{E}-04$ & $3.48 \mathrm{E}-09$ & $1.42 \mathrm{E}-08$ & 4.13E-08 & $4.13 E-08$ \\
\hline Cs-134 & $2.72 \mathrm{E}-08$ & $1.55 \mathrm{E}-05$ & 2.06E-04 & 7.19E-09 & 1.52E-08 & $3.52 \mathrm{E}-0 \mathrm{~B}$ & $3.52 \mathrm{E}-0.08$ \\
\hline Cs-137 & $5.60 \mathrm{E}-02$ & 31.9 & 424 & $1,48 \mathrm{E}-02$ & 3.13E-02 & $7.26 \mathrm{E}-02$ & $7.26 \mathrm{E}-02$ \\
\hline Ba-137m & $5.30 \mathrm{E}-02$ & 30.2 & 401 & $1.40 \mathrm{E}-02$ & $296 \mathrm{E}-02$ & $6.87 \mathrm{E}-02$ & $6.87 \mathrm{E}-02$ \\
\hline $5 \mathrm{~m}-151$ & $1.71 \mathrm{E}-03$ & 0.976 & 13.0 & $1.96 \mathrm{E}-04$ & $8.03 \mathrm{E}-04$ & $2.33 E-03$ & 2.64E-02 \\
\hline Eu=152 & $7.00 \mathrm{E}-06$ & $3.98 E-03$ & $5.29 \mathrm{E}-02$ & 6.85E-06 & $6.91 \mathrm{E}-06$ & $7,06 \mathrm{E}-06$ & $7.06 \mathrm{E}-06$ \\
\hline Eu-154 & $2.16 \mathrm{E}-05$ & $1.23 \mathrm{E}-02$ & 0.163 & $2.47 \mathrm{E}-06$ & 1.01E-05 & 2.93E-05 & $2.93 \mathrm{E}-05$ \\
\hline Eu-155 & $3.30 \mathrm{E}-04$ & 0.188 & 2.50 & $3.23 \mathrm{E}-04$ & $3.26 \mathrm{E}-04$ & $3.33 \mathrm{E}-04$ & $3.33 \mathrm{E}-04$ \\
\hline $\mathrm{Ra}-226$ & $1.08 \mathrm{E}-09$ & 6. $15 \mathrm{E}-07$ & $8.17 \mathrm{E}-06$ & 1.48E- 10 & $604 \mathrm{E}-10$ & $1.56 \mathrm{E}-09$ & $2.01 \mathrm{E}-09$ \\
\hline Ra-228 & $9.26 \mathrm{E}-15$ & $5.28 \mathrm{E}-12$ & 7.01E-11 & $9,07 \mathrm{E}-15$ & $9.15 \mathrm{E}-15$ & 9.34E-15 & $9.34 \mathrm{E}-15$ \\
\hline$A c-227$ & $4.76 E-09$ & $271 \mathrm{E}-06$ & $3.60 \mathrm{E}-05$ & $3.06 \mathrm{E}=10$ & $200 \mathrm{E}-09$ & $7.52 \mathrm{E}-09$ & $102 \mathrm{E}-08$ \\
\hline $\mathrm{Pa}-231$ & $7.18 \mathrm{E}-10$ & 4.09E-07 & $5.43 \mathrm{E}-06$ & $8.21 \mathrm{E}-11$ & $3.36 \mathrm{E}-10$ & $2.04 \mathrm{E}-09$ & $1.68 \mathrm{E}-0 \mathrm{~B}$ \\
\hline Th-229 & $177 \mathrm{E}-12$ & $1.01 \mathrm{E}-09$ & $1,34 \mathrm{E}-08$ & $1.73 \mathrm{E}-12$ & $1.75 \mathrm{E}-12$ & 1.78E-12 & $1.78 \mathrm{E}-12$ \\
\hline Th-232 & $5.91 \mathrm{E}-16$ & $3.36 \mathrm{E}-13$ & $4.47 \mathrm{E}-12$ & $6.76 \mathrm{E}-17$ & $2.77 \mathrm{E}-16$ & $8.01 \mathrm{E}-16$ & 8.01E-16 \\
\hline$U-232$ & $5.24 \mathrm{E} \cdot 12$ & $2.98 \mathrm{E}-19$ & $3.96 \mathrm{E}-0 \mathrm{~B}$ & $5.99 \mathrm{E}-13$ & $245 \mathrm{E}-12$ & $7.10 \mathrm{E}-12$ & $7.10 \mathrm{E}-12$ \\
\hline U-233 & $2.00 \mathrm{E}-13$ & $1.14 \mathrm{E}-10$ & 1.51E-09 & $2.28 \mathrm{E}-14$ & $9.34 \mathrm{E}-14$ & 2.71E-13 & $2.71 \mathrm{E}-13$ \\
\hline U-234 & I.19E-07 & 6.78E- 05 & $9.01 \mathrm{E}-0 \mathrm{~A}$ & 1.36E-As & $5.58 \mathrm{E}-08$ & $1.62 \mathrm{E}-07$ & 1.62E-07) \\
\hline $\mathrm{U}-235$ & $5.13 \mathrm{E}-09$ & $2.92 \mathrm{E}-06$ & 3. $38 E-05$ & $5.86 \mathrm{E}-10$ & $2.40 \mathrm{E}-09$ & $6.95 \mathrm{E}-09$ & 6.95E-09 \\
\hline U. 236 & $1.88 \mathrm{E}-09$ & $1.07 \mathrm{E}-06$ & 1.43E-0s & $2.16 \mathrm{E}-10$ & $8.82 \mathrm{E}-10$ & $2.56 \mathrm{E}-09$ & $2.36 \mathrm{E}-09$ \\
\hline U-238 & 1.22E-07 & $6.92 \mathrm{E}-05$ & $9.20 \mathrm{E}-04$ & 1.39E-09 & $5.69 \mathrm{E}-0 \mathrm{~B}$ & $1.65 \mathrm{E}-07$ & $1.65 \mathrm{E}-07$ \\
\hline $\mathrm{Np}-237$ & $1.03 \mathrm{E}-07$ & $5.86 \mathrm{E}-05$ & $7.78 \mathrm{E}-04$ & $1.18 \mathrm{E}-\mathrm{AB}$ & $4.81 E-0 B$ & 1.39E-07 & 1.39E-07 \\
\hline Pu-238 & $6.61 \mathrm{E}-06$ & $3.77 \mathrm{E}-03$ & $5.00 \mathrm{E}-02$ & 4.75E-05 & $5.66 \mathrm{E}-06$ & $7.57 \mathrm{E}-06$ & $8.48 \mathrm{E}-06$ \\
\hline$P_{11-239}$ & $4.72 \mathrm{E}-0 \mathrm{~d}$ & 0.269 & 3,57 & $3.39 \mathrm{E}-04$ & $4.04 E-04$ & $5.40 \mathrm{E}-04$ & 6.05E-04 \\
\hline$P u-240$ & $6.76 \mathrm{E}-05$ & $3.85 E-02$ & 0.511 & $4.85 \mathrm{E}-05$ & $5.78 \mathrm{E}-0 \mathrm{~S}$ & $7.73 \mathrm{E}-05$ & $8.66 \mathrm{E}-05$ \\
\hline Pu-241 & $3.97 \mathrm{E}-04$ & 0.226 & 3.01 & $2.85 \mathrm{E}-04$ & $3.40 E-04$ & $4.55 \mathrm{E}-04$ & 5.09E-04 \\
\hline Pu-242 & $1.82 \mathrm{E}-09$ & $104 \mathrm{E}-06$ & $1.38 \mathrm{E}-05$ & $1.31 \mathrm{E}-09$ & $1,56 \mathrm{E}-09$ & 2.08E-09 & $2.34 \mathrm{E}-09$ \\
\hline Ams 241 & $8.09 \mathrm{E}-06$ & 461E-03 & $6.12 \mathrm{E}-02$ & $9.26 \mathrm{E}-07$ & 3.79E-06 & $1.10 \mathrm{E}-05$ & 1.43E-04 \\
\hline Ann-243 & $7.57 \mathrm{E}-11$ & $431 E-08$ & $5.72 \mathrm{E}-07$ & $8.66 \mathrm{E}-12$ & 3.54E-11 & $1.03 \mathrm{E}-10$ & $1.34 \mathrm{E}-09$ \\
\hline $\mathrm{Cm}=242$ & $1.61 \mathrm{E}-07$ & 9.16E-05 & $1.22 \mathrm{E}-03$ & $1.58 \mathrm{E}-07$ & 1.59E-07 & 1.62E- 07 & $1.62 \mathrm{E} \cdot 07$ \\
\hline $\mathrm{Cm}-243$ & 3.69E-09 & $2.10 \mathrm{E}-06$ & $2.79 \mathrm{E}-05$ & $3.61 E-09$ & $3.64 \mathrm{E}-09$ & $3.72 \mathrm{E}-09$ & 3.72E-09 \\
\hline $\mathrm{Cm}-244$ & $2.70 \mathrm{E}-09$ & $1.54 \mathrm{E}-06$ & 2.05E-05 & $3.09 \mathrm{E}-10$ & $1.27 \mathrm{E}-09$ & $3.67 E-09$ & $367 \mathrm{E}-09$ \\
\hline Totals & $\mathbf{M}$ & $\mu / \mathrm{g}$ & ks & $\begin{array}{l}95 \mathrm{Ct} \\
(\mathrm{M} \mathrm{dr} \\
\text { (ft) }\end{array}$ & $\begin{array}{r}67 \mathrm{Cl}(\mathrm{M} \\
0 \mathrm{or} / \mathrm{L})\end{array}$ & $\begin{array}{c}+67 \mathrm{Cl}(\mathrm{M} \\
0 \mathrm{r} / \mathrm{L})\end{array}$ & $\begin{array}{l}+95 \mathrm{CI} \\
(\mathrm{M} \mathrm{or} \\
\mathrm{g} / \mathrm{L})\end{array}$ \\
\hline $\mathrm{Pu}$ & $7.89 \mathrm{E}-03(\mathrm{~g} / \mathrm{L})$ & - & $5.97 E-02$ & $5.6 \mathrm{TE}-03$ & $6.76 \mathrm{E}-03$ & $9.03 \mathrm{E}-03$ & $1.01 \mathrm{E}-02$ \\
\hline $\mathrm{U}$ & 153E-13 & 207 & 2.75 & 1.75E-04 & $7.16 \mathrm{E}-04$ & 2.07E-03 & 207E-03 \\
\hline
\end{tabular}

tUnknowns in tank solids inventory are assigned by Tank Layering Model (TL.M) 
HOW Model Rev. 4

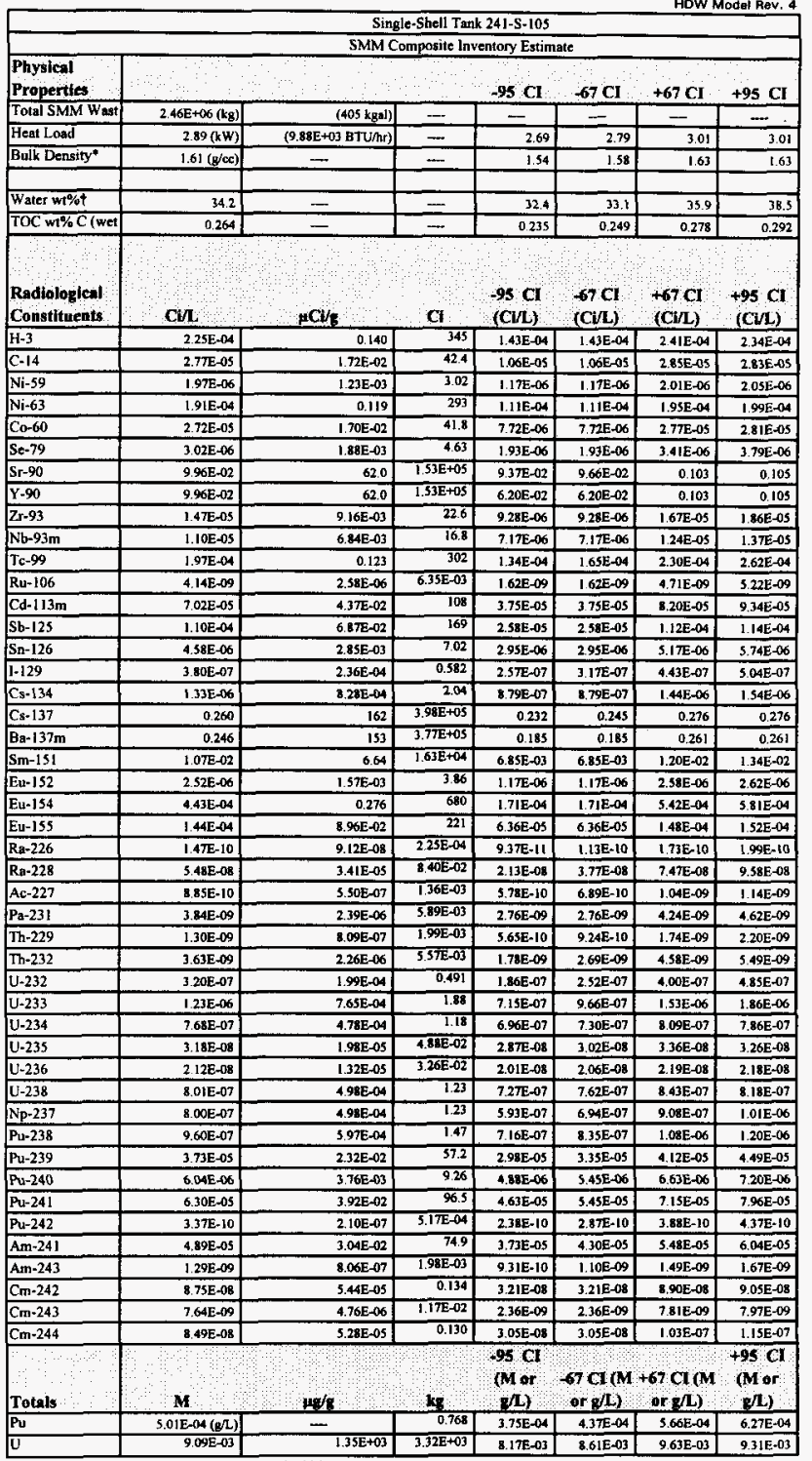

- Density is calculared based on $\mathrm{Na}, \mathrm{OH}_{-}$, and $\mathrm{AlO} 2$ -

tWater wt\% derived from the difference of density and totsil dissolved species. 
HDW Model Rev. 4

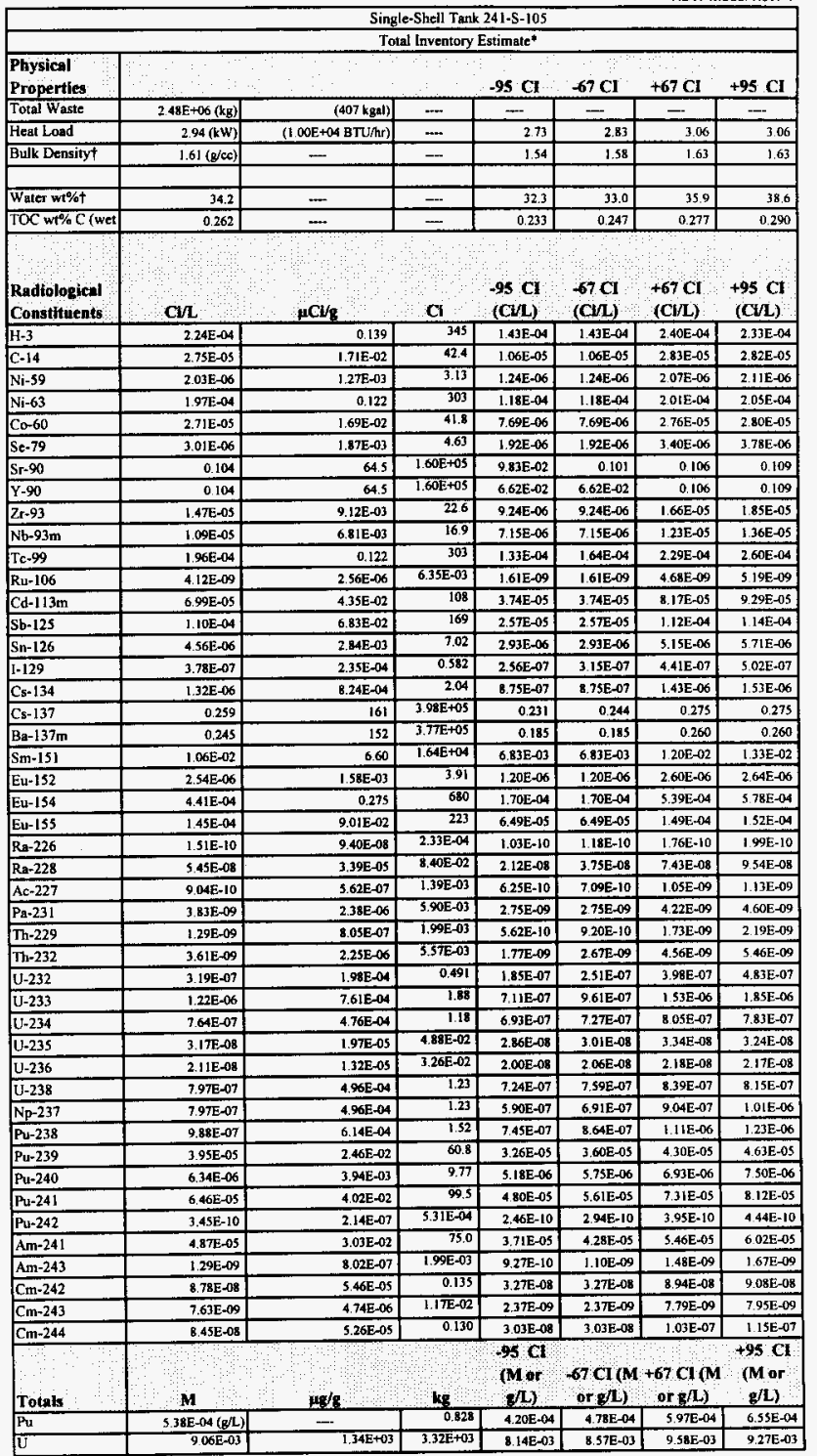

-Unknowns in tank solids inventory are assigned by Tank Layering Model (TLM)

+Volume average for density, mass average Water wt\% and TOC wt\% C. 


\begin{tabular}{|c|c|c|c|c|c|c|c|}
\hline \multirow{4}{*}{$\begin{array}{l}\text { Physical } \\
\text { Properties }\end{array}$} & \multicolumn{7}{|c|}{ Single-Shell Tank 24I-S-106 } \\
\hline & \multicolumn{7}{|c|}{ TLM Solids Composite Inventory Estimate* } \\
\hline & & & & & & & \\
\hline & & $\therefore$ & & $-95 \mathrm{CI}$ & $-67 \mathrm{CI}$ & $+67 \mathrm{CI}$ & $+95 \mathrm{CI}$ \\
\hline Total TLM Wast & $2.15 \mathrm{E}+05(\mathrm{~kg})$ & $(32.0 \mathrm{kgal})$ & $=$ & - & $=$ & $=$ & -- \\
\hline Heat Load & $3.00 \mathrm{E}-03(\mathrm{~kW})$ & (10.3 BTU/hr) & 一 & $2.52 E-03$ & 2.90E-03 & $3.11 \mathrm{E}-03$ & $3.20 \mathrm{E}-03$ \\
\hline Bulk Density & $1.77(g / c)$ & $m$ & $=$ & 1.47 & 1.57 & 1.92 & 2.02 \\
\hline Void Fraction & 0.651 & $\ldots$ & 一 & 0.488 & 0.557 & 0.780 & 0.825 \\
\hline Water $w 1 \%$ & 24.5 & - & - & 4.37 & 12.2 & 45.5 & 55.1 \\
\hline TOC wt\% C (wet) & 0 & 二 & $\cdots$ & 0 & 0 & 0 & $\mathbf{0}$ \\
\hline $\begin{array}{l}\text { Chemical } \\
\text { Constituents }\end{array}$ & mole/L & ppon: & $\mathrm{kg}$ & $\begin{array}{r}95 \mathrm{CI} \\
\text { (mole/L) }\end{array}$ & $\begin{array}{c}-67 \mathrm{CI} \\
\text { (mole } \mathrm{C})\end{array}$ & $\begin{array}{c}+67 \mathrm{Cl} \\
\text { (mole/L) }\end{array}$ & $\begin{array}{l}+95 \mathrm{CI} \\
\text { (molefL) }\end{array}$ \\
\hline $\mathrm{Ne}+$ & 7.86 & $1.02 \mathrm{E}+05$ & 2. $19 E+04$ & 2.23 & 3.91 & 107 & 12.9 \\
\hline $\mathrm{Al} 3+$ & tI.2 & $171 \mathrm{E}+05$ & $3.66 \mathrm{E}+04$ & 5.84 & 7.28 & 14.0 & 16.1 \\
\hline Fej+ (total Fe $)$ & 0.165 & $5.20 \mathrm{E}+03$ & $1.12 \mathrm{E}+03$ & 0.153 & 0.160 & 0.170 & 0.175 \\
\hline $\mathrm{Cr} 3+$ & 2.04E-03 & 59.8 & 12.8 & $171 \mathrm{E}-03$ & 1.97E-03 & $2.10 \mathrm{E}-03$ & $2.17 \mathrm{E}-0.3$ \\
\hline Bi3+ & 0 & 0 & 0 & 0 & 0 & 0 & 0 \\
\hline Last+ & 0 & 0 & 0 & 0 & 0 & 0 & 0 \\
\hline Hg2+ & $4.08 \mathrm{E}-03$ & 462 & 99.1 & $4.02 \mathrm{E}-03$ & 4.06E-03 & 4. $10 \mathrm{E}-03$ & 4. $11 \mathrm{E}-03$ \\
\hline $\mathrm{Zr}$ (as ZrO(OH)2) & 0 & 0 & 0 & 0 & 0 & 0 & 0 \\
\hline $\mathrm{Pb} 2+$ & 0.118 & $1.38 \mathrm{E}+04$ & $2.95 \mathrm{E}+03$ & 0.108 & 0.113 & 0.122 & 0.126 \\
\hline $\mathrm{Ni2+}$ & $1.02 \mathrm{E}-\mathrm{CB}$ & 33.7 & 7.24 & 8.54E -04 & 9.84E-04 & $1.51 \mathrm{E}-03$ & $3.79 \mathrm{E}-03$ \\
\hline $5 r^{2}+$ & 0 & 0 & 0 & 0 & 0 & 0 & 0 \\
\hline $\mathrm{Mn} 4 \mathrm{t}$ & 0 & 0 & 0 & 0 & 0 & 0 & 0 \\
\hline $\mathrm{Ca} 2+$ & 0.121 & $2.73 \mathrm{E}+03$ & 586 & $6,72 \mathrm{E}-02$ & $9.87 E-02$ & 0.141 & 0.161 \\
\hline $\mathrm{K}+$ & $1.54 \mathrm{E}-03$ & 33.9 & 7.27 & $1.29 \mathrm{E}-03$ & $1.48 \mathrm{E}-03$ & 1.59E-03 & 1.64E-03 \\
\hline OH- & 41.7 & $4.00 \mathrm{E}+05$ & $8.59 E+04$ & 20.0 & 25.9 & 53.0 & 61.2 \\
\hline NO3. & 0.572 & $2.00 \mathrm{E}+04$ & $4.30 \mathrm{E}+03$ & 0.485 & 0.554 & 0.591 & 0.610 \\
\hline $\mathrm{NO2-}$ & $\begin{array}{l}0.958 \\
0.29\end{array}$ & $2.49 \mathrm{E}+04$ & 5.34E+03 & 0.799 & 0.925 & 0.990 & 1.02 \\
\hline $\cos 2=$ & 0.121 & 4.09E+03 & 878 & $6.72 \mathrm{E}-02$ & $9.87 E-02$ & 0.141 & 0.161 \\
\hline PO43- & 0 & 0 & 0 & 0 & 0 & 0 & 0 \\
\hline SO42- & 8.39E-03 & 455 & 97.6 & 1.04E-03 & 8. $10 \mathrm{E}-03$ & $8.67 \mathrm{E}-03$ & 8.94E-03 \\
\hline Si (as SiOJ2-) & $2.01 \mathrm{E}-02$ & 319 & 68.4 & 1.69E- 02 & $1,94 \mathrm{E}-02$ & $8.18 \mathrm{E}-02$ & 0.175 \\
\hline F. & 0 & 0 & 0 & 0 & 0 & 0 & 0 \\
\hline $\mathrm{Cl}-$ & $7.07 \mathrm{E}-03$ & 141 & 30,3 & $5.93 \mathrm{E}-03$ & 6.83E-03 & $7.30 \mathrm{E}-03$ & $7.53 \mathrm{E}-03$ \\
\hline C6HSO73- & 0 & 0 & 0 & 0 & 0 & 0 & 0 \\
\hline EDTA4. & 0 & 0 & 0 & 0 & 0 & 0 & 0 \\
\hline HEDTA3- & 0 & 0 & 0 & 0 & 0 & 0 & 0 \\
\hline Slycolate- & 0 & 0 & 0 & 0 & 0 & 0 & 0 \\
\hline acetate- & 0 & 0 & 0 & 0 & 0 & 0 & 0 \\
\hline oxbiatiez:- & 0 & 0 & 0 & 0 & 0 & 0 & 0 \\
\hline DBP & 0 & 0 & 0 & 0 & 0 & 0 & 0 \\
\hline butanol & 0 & 0 & 0 & 0 & 0 & 0 & 0 \\
\hline NH3 & 2.05E-04 & 1.97 & 0,422 & $1.61 \mathrm{E}-04$ & $1.80 \mathrm{E}-04$ & 2.38E-04 & 2.49E-04 \\
\hline Fe(CN)64- & 0 & 0 & 0 & 0 & 0 & 0 & 0 \\
\hline
\end{tabular}

*Unknowns in tank solids inventory are esigned by Tank Lyering Model (TLM). 
HNF-SD-WM-ER-323, Rev. 1

HDW Model fiev. 4

\begin{tabular}{|c|c|c|c|c|c|c|c|}
\hline & \multicolumn{7}{|c|}{ Singie-Shell Tank 241-S-106 } \\
\hline \multicolumn{8}{|c|}{ SMM Composite Inventory Estimate } \\
\hline $\begin{array}{l}\text { Physical } \\
\text { Properties }\end{array}$ & & & & $-95 \mathrm{Cl}$ & $-67 \mathrm{CI}$ & $+67 \mathrm{Cl}$ & $+95 \mathrm{CI}$ \\
\hline Total SMM Wast & $2.80 \mathrm{E}+06(\mathrm{~kg})$ & $(447 \mathrm{kgal})$ & $\ldots$ & $\rightarrow$ & $=$ & $=$ & - \\
\hline Heat Load & $3.65(\mathrm{~kW})$ & $(1.25 \mathrm{E}+04 \mathrm{BTU} / \mathrm{hr})$ & 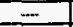 & 3.47 & 3.56 & 3.76 & 3.85 \\
\hline Bulk Density & $1.66(\mathrm{~g} / \mathrm{cc})$ & - & $\ldots$ & 1.60 & 1.63 & 1.63 & 1.69 \\
\hline Water $w t \%$ & 31.0 & $\ldots$ & - & 28.8 & 29.8 & 32.3 & 34.4 \\
\hline TOC wt\% C (wet & $0 . \overline{342}$ & $=$ & $\cdots$ & 0.306 & 0.324 & 0.360 & 0.377 \\
\hline $\begin{array}{l}\text { Chemienl } \\
\text { Constifuents }\end{array}$ & mole/L & ppm & $\mathrm{kg}$ & $\begin{array}{l}-95 \mathrm{CI} \\
\text { (male/L) }\end{array}$ & $\begin{array}{r}-67 \mathrm{CI} \\
\text { (mole/L) }\end{array}$ & $\begin{array}{l}+67 \mathrm{Cl} \\
\text { (mole/L) }\end{array}$ & $\begin{array}{r}+95 \mathrm{CI} \\
(\mathrm{mole} / \mathrm{L})\end{array}$ \\
\hline $\mathrm{Na}+$ & 15.2 & $2.1 \mathrm{E}+05$ & $5.90 \mathrm{E}+05$ & 13.9 & 14.6 & 15.6 & 16.1 \\
\hline Al3t & 2.08 & $3.38 \mathrm{BE}+04$ & $9,47 E+04$ & 1.85 & 1.95 & 2.21 & 2.15 \\
\hline Fe3 + (lothl Fe) & $9.22 \mathrm{E}, 03$ & $3 \mathrm{ii}$ & 871 & $7.46 \mathrm{E}-03$ & $8.32 \mathrm{E}-03$ & $1.01 \mathrm{E}-02$ & 1. 10E-02 \\
\hline $\mathrm{Cr}+\mathrm{t}$ & $0 . \overline{207}$ & $6.50 \mathrm{E}+03$ & $1.82 \mathrm{E}+04$ & 0.160 & 0.185 & 0.215 & 0.223 \\
\hline$B i 3+$ & $6.53 \mathrm{E} .04$ & 82.7 & 232 & $5.92 \mathrm{E}-04$ & $6.23 \mathrm{E}-04$ & $6.87 \mathrm{E} 404$ & 7.18E-04 \\
\hline La3t+ & $3.27 \mathrm{E}-08$ & 2.75E-03 & $7,69 \mathrm{E}-03$ & $2.40 \mathrm{E}-08$ & $2.83 \mathrm{E}-08$ & $3.72 \mathrm{E}-08$ & 4,15E-08 \\
\hline $\mathrm{Hg} 2+$ & $5.82 \mathrm{E}-06$ & 0705 & 1.97 & $5,49 \mathrm{E}-06$ & S.68E-06 & $5.89 \mathrm{E}-06$ & $5.95 \mathrm{E}-06$ \\
\hline $\mathrm{ZT}_{\mathrm{T}}$ (as ZrO(OH)2) & $1.32 \mathrm{E}-04$ & 7.30 & 20.4 & $1.21 \mathrm{E}-04$ & $1.25 \mathrm{E}-04$ & $13 \pi-04$ & $1.44 \mathrm{E}-04$ \\
\hline $\mathrm{Pb} 2+$ & $7.06 \mathrm{E}-04$ & 88.3 & 247 & 6.08E-04 & $6.56 \mathrm{E}-04$ & $7,55 \mathrm{E} 04$ & $8.03 E-04$ \\
\hline $\mathrm{Ni2+}$ & 5.89E-03 & 209 & 586 & $5,72 \mathrm{E}-03$ & $5.80 \mathrm{E}-03$ & $5.99 \mathrm{E}-03$ & $6.07 \mathrm{E}-03$ \\
\hline $\mathbf{S r 2 +}$ & 0 & 0 & 0 & 0 & 0 & 0 & 0 \\
\hline$\overline{\mathrm{Mn} 4+}$ & $3.04 \mathrm{E}-03$ & 101 & 283 & $2.20 \mathrm{E}-03$ & $2.61 \mathrm{E}-03$ & $348 \mathrm{E}-03$ & 3.89E-03 \\
\hline $\mathrm{Ca}+$ & $3.00 \overline{\mathrm{e}}-02$ & 727 & $2.04 \mathrm{E}+03$ & $265 \mathrm{E}-02$ & $2.82 \mathrm{E}-02$ & $3.18 \mathrm{E}-02$ & $3.35 \mathrm{E}-02$ \\
\hline $\mathrm{K}+$ & $6.36 \mathrm{E}-02$ & $1.50 \mathrm{E}+03$ & $4.21 E+03$ & $5.80 \mathrm{E}-02$ & $6.07 \mathrm{E}-02$ & $0.68 \mathrm{E}-02$ & $0.91 \mathrm{E}-02$ \\
\hline $\mathrm{OH}-$ & 12.7 & $1.31 \mathrm{E}+05$ & $3.66 \mathrm{E}+05$ & 11.3 & 120 & 13.5 & 13.3 \\
\hline NO3. & 4.93 & $1.85 \mathrm{E}+05$ & $5.17 \mathrm{E}+05$ & 4.56 & 4.74 & $5,0 \mathrm{~B}$ & 5.10 \\
\hline NO2- & 2.90 & B.06E+04 & $2.26 \mathrm{E}+05$ & 2.54 & 2.71 & 3.09 & 327 \\
\hline CO32- & 0.291 & $1.05 \mathrm{E}+04$ & $2.95 \mathrm{E}+04$ & 0.261 & 0.275 & 0.306 & 0.314 \\
\hline PO43- & $5.70 \mathrm{E}-02$ & $3.27 \mathrm{E}+03$ & $9.15 E+03$ & $5.16 \mathrm{E}-02$ & $3.34 \mathrm{E}-02$ & $5.83 E-02$ & $596 \mathrm{E}-02$ \\
\hline SO42. & 0.185 & $1,076+04$ & $3.00 \mathrm{E}+04$ & 0.138 & 0.160 & 0.210 & 0.232 \\
\hline Si (As SiO32-) & $7.16 \mathrm{E}-02$ & TIE+03 & $3.40 \pm+03$ & $5.94 E-02$ & $6.54 \mathrm{E}-02$ & $7.77 \mathrm{E}-02$ & $83 \% \mathrm{E}-02$ \\
\hline F- & $3.13 \bar{E}-02$ & 359 & $1.00 E+03$ & $2.58 \mathrm{E}-02$ & $281 \mathrm{E}-02$ & $3.39 \mathrm{E}-02$ & $3.61 E-02$ \\
\hline Cl- & 0.256 & $5.49 \mathrm{E}+03$ & $1.54 \mathrm{E}+04$ & 0.230 & 0.243 & 0.271 & 0.274 \\
\hline C6 65073 & $2.34 \mathrm{E}-02$ & $2,67 E+03$ & $7.48 \mathrm{E}+03$ & $2.17 E-02$ & 2.25E-02 & $2.43 \mathrm{E}-02$ & $2.5 \mathrm{IE}-02$ \\
\hline EDTA4- & $2.10 E-03$ & 366 & $1.02 \mathrm{E}+03$ & $1.06 \mathrm{E}-03$ & $1.57 \mathrm{E}-03$ & $2.64 \mathrm{E}-03$ & $3.16 \mathrm{E}-03$ \\
\hline FEDTA3 & $3.45 \mathrm{E}-03$ & 571 & $1.60 E+03$ & $1.37 \mathrm{E}-03$ & $2.39 \mathrm{E}-03$ & 4.52E-03 & $5.56 \mathrm{E}-03$ \\
\hline glycolate. & $4.48 E-02$ & $2.03 \mathrm{E}+03$ & $5.69 E+03$ & $2.44 \mathrm{E}-02$ & $3.44 \mathrm{E}-02$ & $5.53 \mathrm{E}-02$ & $6.53 \mathrm{E}-02$ \\
\hline ncetate- & $2.43 \mathrm{E}-03$ & 86.6 & 243 & $1,9 \pi \mathrm{E}-03$ & $2.20 \mathrm{E}-03$ & $2.66 \mathrm{E}-03$ & $2.89 \mathrm{E}-03$ \\
\hline oxaliatez- & $429 \mathrm{E}-0 \mathrm{~B}$ & $2.28 \mathrm{E}-03$ & $6.38 \mathrm{E}-03$ & $3.81 \mathrm{E}-08$ & 4.04E-08 & $4.53 \mathrm{E}-0 \mathrm{~B}$ & 4. $76 \mathrm{E}-08$ \\
\hline$\overline{D B P}$ & 1.5TE-02 & $1.92 \mathrm{E}+03$ & $3.37 \bar{E}+03$ & $1.21 \mathrm{E}-02$ & $1.36 \mathrm{E}-02$ & $1.66 \mathrm{E}-02$ & $1.80 \mathrm{E}-02$ \\
\hline butanol & $1.51 \mathrm{E}-02$ & 676 & $1.89 \mathrm{E}+03$ & $1.21 \mathrm{E}-02$ & $1.36 \mathrm{E}-92$ & $1.66 \mathrm{E}-02$ & $1.80 \mathrm{E}-02$ \\
\hline NH3 & $7.44 \mathrm{E}-02$ & 764 & $2.14 E+03$ & $6.07 \mathrm{E}-02$ & $6.61 \mathrm{E}-02$ & 8.49E-02 & $9.72 \mathrm{E}-02$ \\
\hline Fe(CN)64- & 0 & 0 & 0 & 0 & 0 & 0 & 0 \\
\hline
\end{tabular}

* Density is calculated based on $\mathrm{Na}, \mathrm{OH}-$ and $\mathrm{AlO}$.

†Water wt\% derived from the difference of density and total dissolved species. 
HOW Model Rev. 4

\begin{tabular}{|c|c|c|c|c|c|c|c|}
\hline \multicolumn{8}{|c|}{ Singie-Shell Tank 241-S-106 } \\
\hline & \multicolumn{7}{|c|}{ Total Inventory Estimate* } \\
\hline $\begin{array}{l}\text { Physical } \\
\text { Properties }\end{array}$ & & & $\because$ & $.95 \mathrm{CI}$ & $-67 \mathrm{CI}$ & $+67 \mathrm{CI}$ & $+93 \mathrm{CI}$ \\
\hline Total Waste & $3.02 \mathrm{E}+06(\mathrm{~kg})$ & (479 kgat) & -- & $\ldots$ & - & $\rightarrow$ & $\cdots$ \\
\hline Heat Load & $3.66(\mathrm{~kW})$ & (1.25E+04 BTU/hr) & $=$ & 3.47 & 3.56 & 3.76 & 3.85 \\
\hline Bullk Density† & $1.66(\mathrm{~g} / \mathrm{c})$ & - & -- & 161 & 1.64 & 1.68 & 1.70 \\
\hline Water wt\% $†$ & 306 & $\ldots$ & - & 28.5 & 29.3 & 32.0 & 33.6 \\
\hline TOC w1\% C (wet & 0.317 & 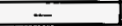 & - & 0.284 & 0.300 & 0.334 & 0.350 \\
\hline $\begin{array}{l}\text { Chemical } \\
\text { Constituents }\end{array}$ & moler. & ppm & $\mathbf{k s}$ & $\begin{array}{l}-95 \mathrm{Cl} \\
\text { (moled) }\end{array}$ & $\begin{array}{c}-67 \mathrm{Cl} \\
\text { (moleft) }\end{array}$ & $\begin{array}{c}+67 \mathrm{Cl} \\
\text { (moledi) }\end{array}$ & $\begin{array}{l}+95 \mathrm{CI} \\
\text { (molen) }\end{array}$ \\
\hline $\mathrm{Na}+$ & 14.7 & $2.03 \mathrm{E}+05$ & $6.12 \mathrm{E}+03$ & 13.5 & 14.2 & ISI & 15.5 \\
\hline $\mathrm{Al3}+$ & 2.69 & $4.36 \mathrm{E}+04$ & 1.31E+05 & 2.31 & 243 & 2,86 & 2.99 \\
\hline Fe? $+($ (totel Fe) & $1.96 \mathrm{E}-02$ & 659 & $1.99 \mathrm{E}+03$ & $1.80 \mathrm{E}-02$ & $1.88 E-02$ & 2.05E-02 & $2,13 E-02$ \\
\hline $\mathrm{Cr} 3+$ & 0.193 & $6.04 E+03$ & $1.82 \mathrm{E}+04$ & 0.150 & 0.173 & 0.201 & 0.208 \\
\hline $\mathrm{Bi3+}$ & 6.11E-04 & 76.8 & 232 & $5.52 \mathrm{E}-04$ & $5 B 1 E-04$ & $6.41 \mathrm{E}-04$ & $6.70 \mathrm{E}-04$ \\
\hline $1.3+$ & $3.05 E-05$ & $2.55 \mathrm{E}-03$ & $7.69 \bar{E}-03$ & $2.24 \mathrm{E}-08$ & 2.64E-08 & $3.47 \mathrm{E} .08$ & $3.87 E-08$ \\
\hline $\mathrm{Hg}_{2+}$ & 2.78E-04 & 33.5 & 101 & 2.74E-04 & $276 \mathrm{E}-\mathrm{B4}$ & $2.79 \mathrm{E}_{0} 04$ & 2.80E-04 \\
\hline $\mathrm{Zr}$ (as $\mathrm{ZrO}(\mathrm{OH}) 2)$ & $1.24 \mathrm{E}-\mathrm{OA}$ & 6.78 & 20.4 & $1.13 E-04$ & $1.17 \mathrm{E}-04$ & $1.28 \mathrm{E}-04$ & 1.34E-04 \\
\hline $\mathrm{Pb} 2+$ & $8.52 \mathrm{E}-03$ & $1.06 \mathrm{E}+03$ & $3.20 \mathrm{E}+03$ & $7.88 E-03$ & $8.26 \mathrm{E}-03$ & $8.75 \mathrm{E}-03$ & $898 \mathrm{E}-03$ \\
\hline $\mathrm{Ni2+}$ & $5.57 \mathrm{E}-03$ & 157 & 593 & $5.40 \mathrm{E}-03$ & 5.48E-03 & 5.65E-03 & $5.78 \mathrm{E}-03$ \\
\hline $\mathrm{sr2+}$ & 0 & 0 & 0 & 0 & 0 & 0 & 0 \\
\hline Mn4+ & $2.84 \mathrm{E} .03$ & 93.8 & 283 & 205E-03 & $2.44 \mathrm{E}-03$ & 3.24E- -03 & $3.63 \mathrm{E}-03$ \\
\hline $\mathrm{Ca2+}$ & $3.61 \mathrm{E}-02$ & 869 & $2.62 \mathrm{E}+03$ & $3.25 \mathrm{E}-02$ & $344 \mathrm{E}-02$ & 3.77E-02 & $3.93 \mathrm{E}-02$ \\
\hline $\mathbf{K +}$ & $5.93 \mathrm{E}-02$ & $1.40 \mathrm{E}+03$ & $4.22 E+03$ & $5.42 E-02$ & $5.67 \mathrm{E}-02$ & $0.25 \mathrm{E}-02$ & $6.46 E-02$ \\
\hline OH- & 14.7 & $1.50 \mathrm{E}+05$ & $+52 \mathbf{E}+05$ & 13.2 & 13.7 & 15.4 & 15.9 \\
\hline NO3. & 464 & $1.73 \mathrm{E}+0 \mathrm{~s}$ & $5.22 \mathrm{E}+05$ & 430 & 4.46 & 4.78 & 4.80 \\
\hline NO2- & 2.77 & $7.67 \mathrm{E}+04$ & 2.31E+05 & 2.43 & 2.59 & 2.95 & 3.11 \\
\hline CO32- & 0.279 & T.C1E+04 & $3,04 E+04$ & 0.252 & 0.265 & 0.294 & 0.301 \\
\hline PO43- & $3.325-02$ & $3.04 E+03$ & 9.15E+03 & $4.81 \mathrm{E}-02$ & 4,995-02 & $5.44 \mathrm{E}-02$ & $5.96 \mathrm{E}-02$ \\
\hline SO42- & 0.123 & $9.98 \mathrm{E}+03$ & $3.01 \mathrm{E}+04$ & 0.129 & 0.150 & 0.196 & 0.217 \\
\hline Si (as SiÖ2-) & $6.8 \mid E-02$ & $1.15 E^{+003}$ & $3.47 \mathrm{E}+03$ & $5.68 \mathrm{E}-02$ & $6.24 \mathrm{E}-02$ & $7.39 \mathrm{~F}-02$ & $7.94 \mathrm{E}=02$ \\
\hline F- & $2.92 E-02$ & 333 & $1.00 \mathrm{E}+0 \mathrm{~J}$ & $2.40 \mathrm{E}-02$ & $2.62 \mathrm{E}-02$ & $3.17 \mathrm{E}-02$ & 3.37E-02 \\
\hline $\mathrm{Cl}$ & 0.240 & $5.11 \mathrm{E}+03$ & $1.54 E+04$ & 0.216 & 0.227 & 0.254 & 0.256 \\
\hline C6H5O73. & $2.18 \mathrm{E}-02$ & $248 \mathrm{E}+03$ & $748 \mathrm{E}+03$ & $2.02 \mathrm{E}-02$ & $2.10 \mathrm{E}-02$ & $2.26 \mathrm{E}-02$ & $2.34 \mathrm{E}-02$ \\
\hline EDTA4- & $1.96 \mathrm{E}-613$ & 340 & $1.02 \mathrm{E}+03$ & $9.93 \mathrm{E}-04$ & $1.47 \mathrm{E}-03$ & $2.46 \mathrm{E}-03$ & $2.95 \mathrm{E}-03$ \\
\hline HEDTA3- & $3.22 \mathrm{E}-03$ & 531 & $1.60 \mathrm{E}+03$ & $1.28 \mathrm{E}-03$ & $2.23 \mathrm{E}-03$ & $4.22 \mathrm{E}-03$ & 5.19E-03 \\
\hline Blyoolate- & $4.18 \mathrm{E}-02$ & $1.89 E+03$ & $5.69 \mathrm{E}+03$ & $2.28 \mathrm{E}-02$ & $3.21 E-02$ & $5.16 \mathrm{E}-02$ & $6.09 \mathrm{E}-02$ \\
\hline acetate- & $2.27 \mathrm{E}-03$ & 80.5 & 243 & 1.84E-03 & $2.05 E-03$ & $2.495-03$ & 2.70E-03 \\
\hline axalate2- & $4.00 E-08$ & 2.12E-03 & 6,38E-0? & $3.56 \mathrm{E}-0 \mathrm{0}$ & 3.77E- $0 B$ & $4.23 \mathrm{E}-0.8$ & 4. $44 \mathrm{E} \leadsto \mathrm{B}$ \\
\hline DEP & $1.41 \mathrm{E}-02$ & $1.78 \mathrm{E}+03$ & $5.37 \mathrm{E}+03$ & $1.13 \mathrm{E}-02$ & $1.2 \pi \mathrm{E}-02$ & $1.55 \mathrm{E}-02$ & $1.68 \mathrm{E}-02$ \\
\hline bulanol & 1.41E-02 & 628 & $1.89 E+03$ & $1.13 \mathrm{E}-02$ & $1.27 \mathrm{E}-02$ & $1.55 \mathrm{E}-02$ & $1.68 \mathrm{E}-02$ \\
\hline NH3 & $6.94 \mathrm{E}-02$ & 710 & $2.14 \mathrm{E}^{+03}$ & $5.67 \mathrm{E}-02$ & $6.17 \mathrm{E}-02$ & $7.92 \mathrm{E}-02$ & $9.075-02$ \\
\hline Fe(CN)64- & 8 & 0 & 0 & 0 & 0 & 0 & 0 \\
\hline
\end{tabular}

"Unknowns in tank solids inventory are assigned by Tank Layering Model (TLM).

+Water wt\% derived from the difference of density and total dissolved species. 
HNF-SD-WM-ER-323, Rev. 1

HOW Model Rev, 4

\begin{tabular}{|c|c|c|c|c|c|c|c|}
\hline \multirow[b]{3}{*}{$\begin{array}{l}\text { Physical } \\
\text { Properties }\end{array}$} & \multicolumn{7}{|c|}{ Single-Shell Tank 241-S-106 } \\
\hline & \multicolumn{7}{|c|}{ TLM Solids Composite Inventory Estimate* } \\
\hline & & & & $-95 \mathrm{CI}$ & $-67 \mathrm{CI}$ & $+67 \mathrm{CI}$ & $+95 \mathrm{CI}$ \\
\hline Total TLM Wast & $2.15 E+05(k \mathrm{~B})$ & $(32.0 \mathrm{kgat})$ & $\cdots$ & 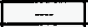 & - & 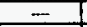 & - \\
\hline Heat Load & $3.00 \mathrm{E}-03(\mathrm{~kW})$ & (10.38TU/hr) & -.. & $2.52 \mathrm{E}-03$ & $2.90 \mathrm{E}-03$ & $3.1 \mathrm{EE}-03$ & $3.20 \mathrm{E}-03$ \\
\hline Bulk Density & $1.77(\mathrm{~g} / \mathrm{cc})$ & $\cdots$ & - & 1.47 & 1.57 & 1.92 & 2.02 \\
\hline Void Fraction & 0.631 & $\cdots$ & $\cdots$ & 0.488 & 0.557 & 0.780 & 0.825 \\
\hline Water wt\% & 24.5 & 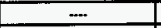 & $\ldots$ & 4.37 & 12.2 & 45.5 & 55.1 \\
\hline $\mathrm{TOC}$ wt\% C (wet) & 0 & $=$ & $\ldots$ & 0 & 0 & 0 & 0 \\
\hline $\begin{array}{l}\text { Radiological } \\
\text { Constituents }\end{array}$ & CUL & $\mu C V_{/ S}$ & C1 & $\begin{array}{l}-95 \mathrm{CI} \\
(\mathrm{CUL})\end{array}$ & $\begin{array}{l}67 \mathrm{CI} \\
(\mathrm{CiL})\end{array}$ & $\begin{array}{l}+67 \mathrm{Cl} \\
(\mathrm{CV} / \mathrm{L})\end{array}$ & $\begin{array}{l}+95 \mathrm{CI} \\
(\mathrm{CU} / \mathrm{L})\end{array}$ \\
\hline $\mathrm{H}-3$ & $1.01 E-06$ & $5.71 E-04$ & 0.123 & $6,33 \mathrm{E}-07$ & $8.98 \mathrm{E}-07$ & $1.13 \mathrm{E}-06$ & $1.24 E-06$ \\
\hline C-14 & $1.09 \mathrm{E}-07$ & $6.16 \mathrm{E}-05$ & 132E-02 & $9.15 \mathrm{E}-0 \mathrm{~g}$ & $1.05 \mathrm{E}-07$ & $1.13 \mathrm{E}-07$ & 1.16E-07 \\
\hline $\mathrm{Ni}-59$ & $3.09 \mathrm{E}-08$ & $1.75 \mathrm{E}-05$ & $3.75 E-03$ & $2.59 \mathrm{E}-08$ & $2.99 \mathrm{E}-08$ & $4.60 \mathrm{E}-0 \mathrm{8}$ & $1.15 \mathrm{E}-07$ \\
\hline $\mathrm{Ni}-63$ & $2.90 \mathrm{E}-06$ & $1.64 \mathrm{E}-03$ & 0.351 & $2.43 E-06$ & $2.80 \mathrm{E}-06$ & $4.31 \mathrm{E}-06$ & $108 \mathrm{E}-05$ \\
\hline $\operatorname{Co}-60$ & $4.71 \mathrm{E}-08$ & $2.66 \mathrm{E}-05$ & 5.71E-03 & $3.96 \mathrm{E}-0 \mathrm{~B}$ & $4.56 \mathrm{E}-08$ & 4.87E-08 & $5.02 \mathrm{E}-08$ \\
\hline Se-79 & $2,37 \mathrm{E}-0.8$ & $1.34 \mathrm{E}-05$ & 2.87E-03 & $1,99 \mathrm{E}-0 \mathrm{~g}$ & $2.29 \mathrm{E}-08$ & $2.45 \mathrm{E}-08$ & $2.53 \mathrm{E}-08$ \\
\hline Sr-90 & $2.05 \mathrm{E}-03$ & 1.16 & 248 & $1.72 \mathrm{E}-03$ & $1.98 \mathrm{E}-03$ & 2.12E-03 & $2.18 \mathrm{E}-03$ \\
\hline$Y-90$ & $2.05 \mathrm{E}-03$ & 1.16 & 248 & $1.72 E-03$ & $198 E-03$ & 2. 12E-03 & 2. $1 \mathrm{BE}-0.3$ \\
\hline $2 x-93$ & 1.12E-07 & 6.34E-05 & $1.36 \mathrm{E}-02$ & $9.42 \mathrm{E}-08$ & 1.09E-07 & 1.16E-07 & $1.20 \mathrm{E}-07$ \\
\hline $\mathrm{Nb}-93 \mathrm{~m}$ & $9.13 \mathrm{E}-08$ & 5.15E-OS & $1.11 \mathrm{E}-02$ & $7.66 \mathrm{E}-08$ & B. $32 \mathrm{E}-08$ & $9.43 \mathrm{E}-08$ & $973 \mathrm{E} .08$ \\
\hline Tc-99 & 7.84E-07 & $4.42 \mathrm{E}-04$ & $9.49 \mathrm{E}-02$ & $6.5 \pi-07$ & $7.57 \mathrm{E}-07$ & 8. $10 \mathrm{E}-07$ & 8.35E-07 \\
\hline $\mathrm{Ru}-106$ & $2.76 \mathrm{E}-13$ & 1.56E-10 & 3.34E-08 & $2.31 \mathrm{E}-13$ & $2.66 \mathrm{E}-13$ & $2.85 \mathrm{E} \cdot 13$ & $2.94 \mathrm{E}-13$ \\
\hline $\mathrm{Cd}=113 \mathrm{~m}$ & $3.51 \mathrm{E}-07$ & $1.98 \mathrm{E}-04$ & 4.25E-02 & $2.94 \mathrm{E}-07$ & 3.39E-07 & $3.62 \mathrm{E}-07$ & $3.74 E-07$ \\
\hline Sb-125 & $7.89 \mathrm{E}-08$ & $446 \mathrm{E}-05$ & $9.56 \mathrm{E}-03$ & $6.62 \mathrm{E}-08$ & 7.63E-08 & $8.16 \mathrm{E}-08$ & S AIE-08 \\
\hline $5 n-126$ & $3.61 \mathrm{E}-08$ & 2.04E-05 & $4.38 E-03$ & $3.03 \mathrm{E}-08$ & 3.49E-08 & 3.73E-08 & 3.85E-08 \\
\hline $1-129$ & $1,50 \mathrm{E}-09$ & $\begin{array}{l}8.47 \mathrm{E}-07 \\
\end{array}$ & $1.82 \mathrm{E}-04$ & $1.26 \mathrm{E}-09$ & 1.45E-09 & $1.55 \mathrm{E}-09$ & $1.60 \mathrm{E}-09$ \\
\hline $\mathrm{Cs}=134$ & $1.65 \mathrm{E}-09$ & $9.34 \mathrm{E}-07$ & $2.00 \mathrm{E}-04$ & $1.39 \mathrm{E}-09$ & $1.60 \mathrm{E}-09$ & $1.71 \mathbf{E}-09$ & $1.76 \mathrm{E}-09$ \\
\hline Cs- 137 & $2.35 \mathrm{E}-03$ & 1.33 & 285 & 1.97E-03 & $2.27 \mathrm{E}-03$ & 2.43E-03 & $2.50 \mathrm{E}-03$ \\
\hline $\mathrm{Ba}-137 \mathrm{~m}$ & $222 \mathrm{E}-03$ & 1.26 & 269 & $1.87 \mathrm{E}-03$ & 2.15E-03 & $230 \mathrm{E}-03$ & 2.37E-03 \\
\hline Sm-151 & 8.47E-05 & $4.78 \mathrm{E}-02$ & 10.3 & $7.10 \mathrm{E}-05$ & $8.18 E-05$ & $8.75 \mathrm{E}-05$ & $9.02 \mathrm{E}-0 \mathrm{~S}$ \\
\hline Eu-152 & $1.75 \mathrm{E}-07$ & $9.89 \mathrm{E}-05$ & $2.12 \mathrm{E}-02$ & $1.74 \mathrm{E}-07$ & $1.75 \mathrm{E}-07$ & $1.76 \mathrm{E}-07$ & 1.76E-07 \\
\hline Eu-154 & 1.15E-06 & $6.48 \mathrm{E}-04$ & 0.139 & $9.63 \mathrm{E}-07$ & 1.11E-06 & $1.19 \mathrm{E}-06$ & $1.22 \mathrm{E}-06$ \\
\hline Eu-155 & $8.33 \mathrm{E}-06$ & $4.71 \mathrm{E}-03$ & 1.01 & $3.27 \mathrm{E}-06$ & 8. 32E- 06 & $835 \mathrm{E}-06$ & B.36E-06 \\
\hline $\mathrm{Rn}-226$ & $2.83 \mathrm{E}-12$ & $1.60 \mathrm{E}-09$ & $3.42 \mathrm{E}-07$ & $2.37 \mathrm{E}-12$ & $2.73 \mathrm{E}-12$ & 2.92E-12 & 3.01 E-12 \\
\hline $\mathrm{Ra}-228$ & $2.92 \mathrm{E}=16$ & $1.65 \mathrm{E}-13$ & $3.54 \mathrm{E}-11$ & $2.90 \mathrm{E}-16$ & $2.91 \mathrm{E}-16$ & $292 \mathrm{E}-16$ & $2.93 \mathrm{E}-16$ \\
\hline$A c=227$ & $1,45 \mathrm{E}-11$ & $8.19 \mathrm{E}-09$ & $1.76 \mathrm{E}-06$ & $1.22 \mathrm{E}-11$ & $1.40 \mathrm{E}-11$ & $1.50 \mathrm{E}-11$ & $1.55 \mathrm{E}+11$ \\
\hline$P_{a-231}$ & $3.44 \mathrm{E} \cdot 11$ & $1.94 \mathrm{E}-08$ & 4.17E-06 & $2.89 \mathrm{E}-11$ & $3.32 \mathrm{E}-11$ & $3.55 \mathrm{E}-11$ & $3.67 \mathrm{E}-11$ \\
\hline Th-229 & 4.13E-14 & $2.33 \mathrm{E}-11$ & $5,00 \mathrm{E}-09$ & $4.09 \mathrm{E}-14$ & 4.12E-14 & 4.13E-14 & 4.14E-14 \\
\hline Th-232 & $4.04 \mathrm{E} \cdot 17$ & 2.28E- 14 & $4.89 \mathrm{E}-12$ & $3,39 \mathrm{E}-17$ & $3.90 \mathrm{E}-17$ & 4.17E-17 & $4.30 \mathrm{E}-17$ \\
\hline U.232 & $6.47 \mathrm{E}-10$ & $3.65 E-07$ & $7,83 \mathrm{E}-05$ & $5.62 \mathrm{E}-10$ & $6.12 \mathrm{E}-10$ & $6.72 \mathrm{E}-10$ & $6.90 \mathrm{E}-10$ \\
\hline U-233 & $2.39 \mathrm{E}-11$ & $1.35 \mathrm{E}-08$ & 2.90E-06 & $2.08 \mathrm{E}-11$ & $2.26 \mathrm{E}-11$ & $2.49 \mathrm{E}-11$ & $2.55 \mathrm{E}-11$ \\
\hline U-234 & $1.48 E-05$ & $8.36 \mathrm{E}-03$ & 1.79 & 1.29E-05 & 1,40E-05 & 1.54E-05 & 1.58E-05 \\
\hline U-235 & $6.29 \mathrm{E}-07$ & $3.55 \mathrm{E}-04$ & $3.62 \mathrm{E}-02$ & $5.46 \mathrm{E}-07$ & 5.95E-07 & $6.53 \mathrm{E}-07$ & $6.71 \mathrm{E}-07$ \\
\hline U-236 & $3.26 \mathrm{E}-07$ & $1.84 \mathrm{E}-04$ & $3.95 \mathrm{E}-02$ & $283 \mathrm{E}=07$ & 3.08E-07 & 3.39E-07 & $3.48 \mathrm{E}-07$ \\
\hline U-238 & $1.44 \mathrm{E}-05$ & 8. 14E-03 & 1.75 & $1.25 \mathrm{E}-05$ & $1.36 \mathrm{E}-05$ & $1.50 \mathrm{E}-05$ & 1.54E-05 \\
\hline $\mathrm{Np}-237$ & $5,48 \mathrm{E}-09$ & $3.09 \mathrm{E}-6 \mathrm{6}$ & $0.64 E-04$ & $460 \mathrm{E}-09$ & $5.30 \mathrm{E}-09$ & 5.6TE-09 & $5.84 \mathrm{E}-09$ \\
\hline Pu-238 & $2.51 \mathrm{E}-05$ & $1.42 \mathrm{E}-02$ & 3.04 & $2.40 \mathrm{E}-05$ & 2.45E -05 & $2.5 \pi-05$ & 2.62E-05 \\
\hline Pu.239 & 1.61E-03 & 0.908 & 195 & $1.54 \mathrm{E}-03$ & 1.57E-03 & 1.64E-03 & $1.68 \mathrm{E}-03$ \\
\hline Pu-240 & $2.27 \mathrm{E}-04$ & 0.128 & 27.5 & $2.17 \mathrm{E}-04$ & $2.22 \mathrm{E}-04$ & $2.32 \mathrm{E}-04$ & $2.37 \mathrm{E}-04$ \\
\hline $\mathrm{Pu}-241$ & $1.39 \mathrm{E}-03$ & 0.782 & 168 & $1.32 \mathrm{E}-03$ & 1.35E-03 & $1,42 \mathrm{E}-03$ & $1.45 \mathrm{E}-03$ \\
\hline$P u-242$ & $5.88 \mathrm{E}-09$ & $3.32 \mathrm{E}-06$ & $7.12 \mathrm{E}-04$ & $5.62 \mathrm{E}-09$ & 5.75E-00 & 6.01E-09 & 6. $14 \mathrm{E}-09$ \\
\hline $\mathrm{Am}-241$ & $4.09 \mathrm{E}-07$ & $2.31 \mathrm{E}-04$ & 4.95E 02 & $3,43 \mathrm{E}-07$ & $3.95 \mathrm{E}-07$ & $4.22 \mathrm{E}-07$ & $4.36 \mathrm{E}-07$ \\
\hline Am-243 & $3.73 \mathrm{E}-12$ & 2.10E-09 & $451 \mathrm{E}-07$ & $3.13 \mathrm{E}-12$ & $3.60 \mathrm{E}-12$ & $3.85 \mathrm{E}-12$ & $3.97 \mathrm{E}-12$ \\
\hline $\mathrm{Cm}-242$ & $3.65 \mathrm{E}-09$ & $2.06 \mathrm{E}-06$ & $4.42 E-04$ & $3.62 \mathrm{E}-09$ & 3.65E-09 & $3.66 \mathrm{E}-09$ & $3.6 \% \mathrm{E}-09$ \\
\hline $\mathrm{Cm}-243$ & $8.34 \mathrm{E}-11$ & $471 \mathrm{E}-08$ & 1.01E-05 & $8.27 \varepsilon-11$ & $832 \mathrm{E}-11$ & 8.35E-11 & $8.36 \mathrm{E}-11$ \\
\hline $\mathrm{Cm}-244$ & $1.30 \mathrm{E}-10$ & $7.33 \mathrm{E}-08$ & 1.57E-05 & 1.09E-10 & $1.25 \mathrm{E}-10$ & $1.34 \mathrm{E}-10$ & $138 \mathrm{E} \cdot 10$ \\
\hline Totals & $\mathbf{M}$ & $\mathrm{H} / \mathrm{s}$ & $\mathrm{kg}$ & $\begin{array}{l}-96 \mathrm{Cr} \\
\text { (Mor } \\
\text { g/L) }\end{array}$ & $\begin{array}{l}67 \mathrm{CL}(\mathrm{L} \\
0 \mathrm{or} / \mathrm{L})\end{array}$ & $\begin{array}{l}+67 \mathrm{CM} \\
\text { or } / \mathrm{L}) \\
\end{array}$ & $\begin{array}{r}+95 \mathrm{CI} \\
(\mathrm{M} \text { or } \\
\mathrm{g} / \mathrm{L})\end{array}$ \\
\hline $\mathrm{Pu}$ & $2.69 \mathrm{E}-02(\mathrm{~g} / \mathrm{L})$ & - & 3.26 & $2.5 \pi-02$ & 2.63E-02 & $2.75 \mathrm{E}-02$ & 2.81E-02 \\
\hline $\mathrm{U}$ & 0.181 & $2.44 E+04$ & $5.23 \mathrm{E}+03$ & 0.158 & 0.172 & 0.188 & 0.194 \\
\hline
\end{tabular}

"Unknowns in tank solids inventory are assigned by Tank Layering Model (TLM). 
HDW Model Rev, 4

\begin{tabular}{|c|c|c|c|c|c|c|c|}
\hline & & Sing & e-Shell Tan & $241-5-106$ & & & \\
\hline & & SMMC & mposite $\operatorname{lnv}$ & entory Estim & & & \\
\hline Physical : & & & & & & & \\
\hline Propertics & & & & $95 \mathrm{Cl}$ & $-67 \mathrm{Cl}$ & $+67 \mathrm{CI}$ & $+95 \mathrm{CI}$ \\
\hline Total SMM Wast & $2.80 \mathrm{E}+06(\mathrm{~kg})$ & $(447 \mathrm{kgal})$ & - & $\ldots$ & - & - & -- \\
\hline Heat Load & $3.65(\mathrm{~kW})$ & $(1.25 \mathrm{E}+04 \mathrm{BTU} / \mathrm{hr})$ & - & 3.47 & 3.56 & 3.76 & 3.85 \\
\hline Bulk Density & $1.66(\mathrm{~g} / \mathrm{cc})$ & - & - & 1.60 & 1.63 & 1.68 & 1.69 \\
\hline & & & & & & & \\
\hline Water w1\%† & 310 & $=$ & - & 28.8 & 29.8 & 32.3 & 34.4 \\
\hline TOC wt $\% \mathrm{C}$ (wet & 0.342 & $=$ & - & 0.306 & 0.324 & 0.360 & 0.377 \\
\hline $\begin{array}{l}\text { Radlological } \\
\text { Constituents }\end{array}$ & CVL & $\mu C \mathrm{~g}$ & Cl & $\begin{array}{l}95 \mathrm{Cl} \\
\text { (CUL) }\end{array}$ & $\begin{array}{l}-67 \mathrm{Cl} \\
(\mathrm{Cl} / \mathrm{L})\end{array}$ & $\begin{array}{l}+67 \mathrm{Cl} \\
(\mathrm{Ci} / \mathrm{L}) \\
\end{array}$ & $\begin{array}{r}+95 \mathrm{Ct} \\
(\mathrm{C} / \mathrm{L})\end{array}$ \\
\hline $\mathrm{H}-3$ & $2.82 E-04$ & 0.171 & 478 & $1.74 E-04$ & $1.74 \mathrm{E}-04$ & $2.95 \mathrm{SE}-04$ & $2.90 \mathrm{E}-04$ \\
\hline$C-14$ & 3.45E-05 & $2.096-02$ & 38.4 & 1.19E-05 & $1.19 E-05$ & $3.52 \mathrm{E}-05$ & $3.51 \mathrm{E}-05$ \\
\hline Ni-59 & $2.26 \mathrm{E}-06$ & $1,36 \mathrm{E}-03$ & 3.82 & $1.20 \mathrm{E}-06$ & $1.20 \mathrm{E}-06$ & $2.31 \mathrm{E}-06$ & $2.36 \mathrm{E}-\mathrm{-06}$ \\
\hline $\mathrm{Ni}-63$ & $2.20 \mathrm{E}-04$ & \begin{tabular}{l|l|}
0.133 \\
\end{tabular} & 372 & $1.15 \mathrm{SE}-04$ & $1.15 \mathrm{E}-04$ & $2.25 \mathrm{E}-04$ & 2.30E-04 \\
\hline Co-60 & $354 \mathrm{E}-05$ & $2.14 \mathrm{E}-02$ & 39.9 & $9.52 \mathrm{E}-06$ & $9.52 \mathrm{E}-106$ & $3.5 \mathrm{gE}-05$ & $3.61 \mathrm{E}-05$ \\
\hline Se-79 & $3.57 \mathrm{E}-06$ & $2.16 \mathrm{E}-03$ & 6.04 & $2.13 \mathrm{E}-06$ & 2.13E-06 & 4.09E-06 & 4.99E-06 \\
\hline Sr-90 & 0.112 & 67,6 & $1.80 \tilde{E}+05$ & 0.105 & 0.108 & 0.116 & 0.119 \\
\hline Y.90 & 0.112 & 67.6 & $1.89 \mathrm{EE}+05$ & $6.21 E-02$ & $6.21 \mathrm{E}-02$ & 0.116 & 0.119 \\
\hline $2 r-93$ & $1.75 \mathrm{E}-05$ & 1.06E-02 & 29.6 & $1.03 E-05$ & $1.03 \mathrm{E}-05$ & $2.01 \mathrm{E}-05$ & $2.26 \mathrm{E}-05$ \\
\hline $\mathrm{Nb}-93 \mathrm{~m}$ & $1.29 \mathrm{E}-05$ & $7.77 \mathrm{E}-03$ & 21.8 & $7.81 E-06$ & $781 \mathrm{E}-06$ & $1.4 \pi E-05$ & 1.64E-05 \\
\hline Tc-99 & $2.47 \mathrm{E}-04$ & 0.149 & 418 & $1.63 \mathrm{E}-04$ & $2.04 \mathrm{E}-04$ & $2.90 E-04$ & 3.32E-04 \\
\hline$R u-106$ & $5, \mathrm{BOE}-09$ & $3.50 \mathrm{E}-06$ & 9.81E-03 & $2.46 \mathrm{E}-09$ & $2.46 \mathrm{E}-09$ & $6.35 E-09$ & $7.23 \mathrm{E}-09$ \\
\hline $\mathrm{Cd}-113 \mathrm{~m}$ & 8.82E-05 & $3.33 \mathrm{E}-02$ & 149 & $4.49 \mathrm{E}-05$ & $449 \mathrm{E}-05$ & 1.04E-04 & 1. 19E-04 \\
\hline Sb-125 & $1.46 \mathrm{E}-04$ & $8.80 \mathrm{E}-02$ & 246 & $3.37 \mathrm{E}-05$ & 3.37E-05 & $1.47 \mathrm{E}-04$ & $1.49 \mathrm{E}-04$ \\
\hline Sn-126 & $3.41 \mathrm{E}-06$ & $3.2 \pi \mathrm{E}-03$ & 9.15 & $3.25 \mathrm{E}-\infty 6$ & $3.25 E-06$ & $6.19 \mathrm{E}-06$ & $694 E-06$ \\
\hline $\mathrm{I}-129$ & $4.76 \mathrm{E}-07$ & $2.87 E-04$ & 0.805 & $3.13 \mathrm{E}-07$ & $3.92 \mathrm{E}-07$ & $5,60 \mathrm{E}-07$ & $6.41 E-07$ \\
\hline$C_{s-134}$ & $1.72 E-06$ & 1.04E-03 & 2.92 & $9.10 \mathrm{E}-07$ & $9.10 \mathrm{E}-07$ & $\begin{array}{ll}1.82 E-06 \\
\end{array}$ & $191 \mathrm{E}-06$ \\
\hline Cs-137 & 0.300 & 181 & $5.078+05$ & 0.276 & 0.288 & 0.313 & 0.325 \\
\hline$B a-137 m$ & 0.284 & 171 & $4.80 \mathrm{E}+03$ & 0.175 & 0.175 & \begin{tabular}{l|l}
0.296 \\
\end{tabular} & 0298 \\
\hline $5 m-15 t$ & $1.26 \mathrm{E}-02$ & 7.61 & $2.13 E+04$ & $7.55 \mathrm{E}-03$ & 7.5SE-03 & $1,44 E-02$ & $1.62 \mathrm{E}-02$ \\
\hline$E u-152$ & $3.14 \mathrm{E}-06$ & 1.90E-03 & 5.32 & $1.36 \mathrm{E}-06$ & $1.36 \mathrm{E}-06$ & $3.20 \mathrm{E}-06$ & $3.25 \mathrm{E}-06$ \\
\hline Eu-154 & $5.78 E-04$ & 0.349 & 978 & $2.18 E-04$ & $2.18 \mathrm{E}-04$ & $7.08 \mathrm{E}-04$ & $7.60 \mathrm{E}-04$ \\
\hline Eu-155 & $1.80 \mathrm{E}-04$ & \begin{tabular}{l|l|}
0.109 \\
\end{tabular} & 305 & $7.38 E-05$ & $7.38 E-05$ & $1.84 \mathrm{E}-04$ & $18 \%-04$ \\
\hline RA-226 & $1.57 \mathrm{E}-10$ & $9.51 \mathrm{E}-0 \mathrm{~B}$ & $2.66 \mathrm{E}-0 \mathrm{~A}$ & $1.13 \mathrm{E}-10$ & $1.13 \mathrm{E}-10$ & $1.80 \mathrm{E}-10$ & $201 \mathrm{E}-10$ \\
\hline Ra-228 & $5.17 E-08$ & $3.12 \mathrm{E}-05$ & $8.74 E-02$ & $2.06 \mathrm{E}-03$ & $3.58 \mathrm{E}-08$ & $7.01 E_{0} 08$ & B.97E-08 \\
\hline$A c=227$ & $9.48 \mathrm{E} \cdot 10$ & S.73E-07 & $1.60 \mathrm{E}-03$ & $6.89 \mathrm{E}-10$ & $6.89 \mathrm{E}-10$ & $1.08 E-09$ & $1.16 \mathrm{E}-09$ \\
\hline $\mathrm{Pa}-231$ & $4.28 \mathrm{E}-09$ & $2.58 \mathrm{E}-06$ & $7.24 E-03$ & $2.84 \mathrm{E}-09$ & $2.84 \mathrm{E}-99$ & \begin{tabular}{|l|l|}
$4.80 E-09$ \\
\end{tabular} & $5.30 \mathrm{E}-09$ \\
\hline Th. 229 & $1.24 E-09$ & $7.47 \mathrm{E}-07$ & $2.09 \mathrm{E}-03$ & 5.5SE-10 & $8.88 \mathrm{E}-10$ & $1.64 E-09$ & $2.07 \mathrm{E}-09$ \\
\hline Th-232 & \begin{tabular}{ll|}
$3.56 \mathrm{E}-199$ \\
\end{tabular} & $2.15 \mathrm{E}-06$ & $6.03 \mathrm{E}-03$ & $1.85 \mathrm{E}-09$ & $2.69 \mathrm{E}-09$ & 4. 44E-09 & $5.28 \mathrm{E}-09$ \\
\hline $\mathrm{U}-232$ & 3.23E-07 & $1.95 \mathrm{E}-04$ & 0.547 & 1.99E-07 & $2.59 \mathrm{E}-07$ & 3.97E-07 & 4.75E-07 \\
\hline $\mathrm{U}-233$ & $1.24 \mathrm{E}-06$ & 7.49E-S4 & 2.10 & $9.63 \mathrm{E}-07$ & $9.96 \mathrm{E}-07$ & $1.52 \mathrm{E}-06$ & $1.82 \mathrm{E}-06$ \\
\hline $\mathrm{U}-234$ & $8.07 E-07$ & $4.88 \mathrm{E}-04$ & 1.37 & $7.47 E-07$ & $7.76 \mathrm{E}-07$ & 8.41E-07 & $8.31 \mathrm{E}-07$ \\
\hline 0.235 & \begin{tabular}{|l|l|}
$3.33 \mathrm{E}-08$ \\
\end{tabular} & $2.01 \mathrm{E}-0 \mathrm{~S}$ & $5.63 \mathrm{E}-02$ & 3.07E-08 & $3.19 \mathrm{E}-0 \mathrm{~B}$ & $3.48 E-08$ & $3.43 \mathrm{E}-08$ \\
\hline U-236 & \begin{tabular}{ll|}
$2.39 \mathrm{E}-08$ \\
\end{tabular} & 1.44E-05 & 404E-02 & 2.29E-08 & $2.34 E-08$ & $2.44 E-08$ & $2.46 \mathrm{E}-0 \mathrm{~B}$ \\
\hline U.238 & \begin{tabular}{l|l|}
$8.30 \mathrm{E}-07$ \\
\end{tabular} & $5.01 \mathrm{E}-\mathrm{MA}$ & 1.40 & 7.69E-07 & \begin{tabular}{ll|}
$7.98 E-07$ \\
\end{tabular} & $8.65 \mathrm{E}-07$ & $8.53 \mathrm{E}-07$ \\
\hline Np-237 & $9.66 \mathrm{E}-07$ & 5.84E-04 & 1.64 & $0.918-07$ & $8.26 \mathrm{E}-07$ & $1.11 \mathrm{E}-06$ & $1.25 \mathrm{E}-06$ \\
\hline Pu-238 & $1.18 \mathrm{E}-06$ & $7.13 \mathrm{E}-04$ & 2.00 & $8.586-07$ & $1.02 E-06$ & $1.33 \mathrm{E}-06$ & $1.50 \mathrm{E}-06$ \\
\hline Pu-239 & $4.26 E_{-05}$ & $2.57 \mathrm{E}-02$ & 72.0 & $3.43 E-05$ & 3.84E-05 & $4.68 \mathrm{E}-05$ & $5.08 \mathrm{E}-05$ \\
\hline $\mathbf{P}_{12}=240$ & $7.06 \mathrm{E}-06$ & $4.27 \mathrm{E}-03$ & 12.0 & $5.52 E-06$ & $6.28 \mathrm{E}-06$ & 7.85E-06 & $8.60 \mathrm{E}-06$ \\
\hline Pu-24I & 7.81E-05 & $4.72 \mathrm{E}-02$ & 132 & 5.61E-05 & $6.09 \mathrm{E}-05$ & 8.94E-05 & $1.00 \mathrm{E}-04$ \\
\hline $\mathrm{Pu}_{\mathrm{u}-242}$ & $4.26 \mathrm{E} \cdot 10$ & $2.57 \mathrm{E}-97$ & $7.20 \mathrm{E}-04$ & 2.94E-10 & 3.39E-10 & $4.93 \mathrm{E}-10$ & $3.57 \mathrm{E}-10$ \\
\hline $\mathrm{Am}-241$ & 5.79E-05 & $3.50 \mathrm{E}-02$ & 97.9 & 4.25E-05 & S.01E-05 & $0.57 \mathrm{TE}-0 \mathrm{~S}$ & $7.32 \mathrm{E}-05$ \\
\hline$A m-243$ & $1.73 \mathrm{E}-09$ & $1.06 \mathrm{E}-96$ & 2.96E-03 & $1.27 \mathrm{E}-09$ & 1.50F-09 & $2.01 \mathrm{E}-09$ & 2.25E-09 \\
\hline $\mathrm{Cm}-242$ & $1.09 \mathrm{E}-07$ & \begin{tabular}{ll|}
$6.57 \mathrm{E}-05$ \\
\end{tabular} & 0.184 & $3.55 \mathrm{E}-08$ & 3.55E-08 & $\begin{array}{l}1.12 E-07 \\
\end{array}$ & $1.14 \mathrm{E}-07$ \\
\hline $\mathrm{Cm}=243$ & $9.83 \mathrm{E}-09$ & $3.94 \mathrm{E}-06$ & $1.66 \mathrm{E}-02$ & 2.BAE-09 & $2.84 \mathrm{E}-09$ & $1.01 \mathrm{E}-0 \mathrm{8}$ & $1.04 \mathrm{E}-08$ \\
\hline Cm-244 & 1.10E-07 & 6.05E-05 & 0.186 & $3,80 \mathrm{E}-08$ & 3.80E-08 & $\begin{array}{l}1.35 \mathrm{E}-07 \\
\end{array}$ & $1.50 \mathrm{E}-07$ \\
\hline Totals & $\mathbf{M}$ & $\mu / g$ & kg & $\begin{array}{l}95 \mathrm{CI} \\
(\mathrm{M} \text { or } \\
8 \mathrm{C})\end{array}$ & $\begin{array}{l}67 \mathrm{CI}(\mathrm{M} \\
\text { or } / \mathrm{L})\end{array}$ & $\begin{array}{l}+67 \text { CI (M } \\
\text { or } /(\mathrm{L})\end{array}$ & $\begin{array}{c}+95 \mathrm{CI} \\
(\mathrm{Mor} \\
\mathrm{g} / \mathrm{L})\end{array}$ \\
\hline Pu & $5.47 \mathrm{E}-04 \mathrm{~g}(\mathrm{~g} / \mathrm{L})$ & - & 0.926 & $3.91 \mathrm{E}-04$ & $4.68 \mathrm{E}-04$ & $6.27 \mathrm{E}-04$ & $7.03 E-04$ \\
\hline $\mathrm{U}$ & $9.47 \mathrm{E}-03$ & $1.36 \mathrm{E}+03$ & $3.81 E+03$ & $8.70 \mathrm{E}-03$ & 9.06E-03 & $9.91 E-03$ & $9.7 \mathrm{SE}-03$ \\
\hline
\end{tabular}

"Density is calculated based on $\mathrm{Na}$, OH-, and $\mathrm{AlO} 2$ -

tWater wt\% derived from the difference of density and total dissolved species. 


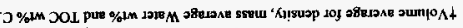

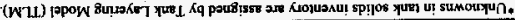

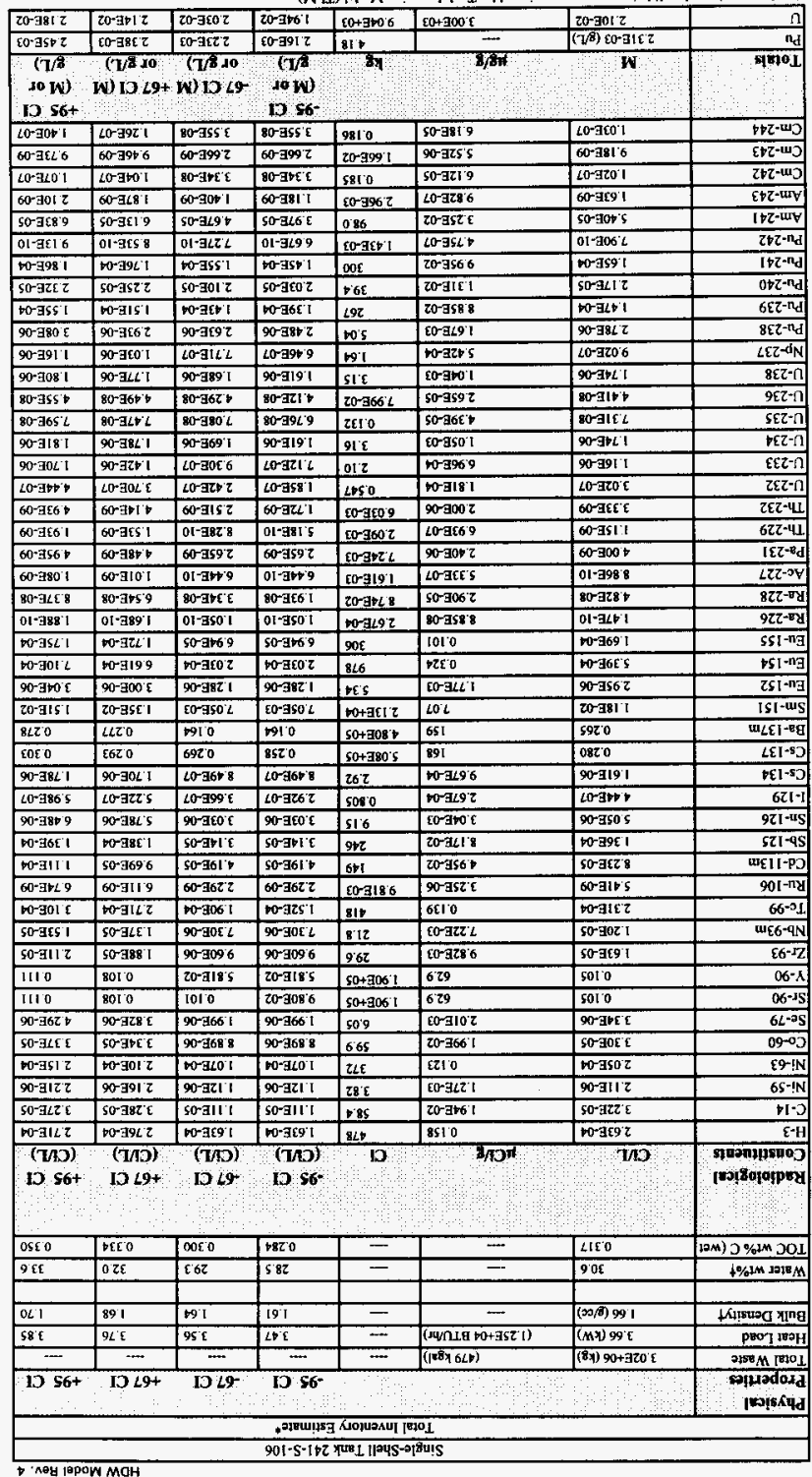


HNF-SD-WM-ER-323, Rev. 1

HDW Model Rev. 4

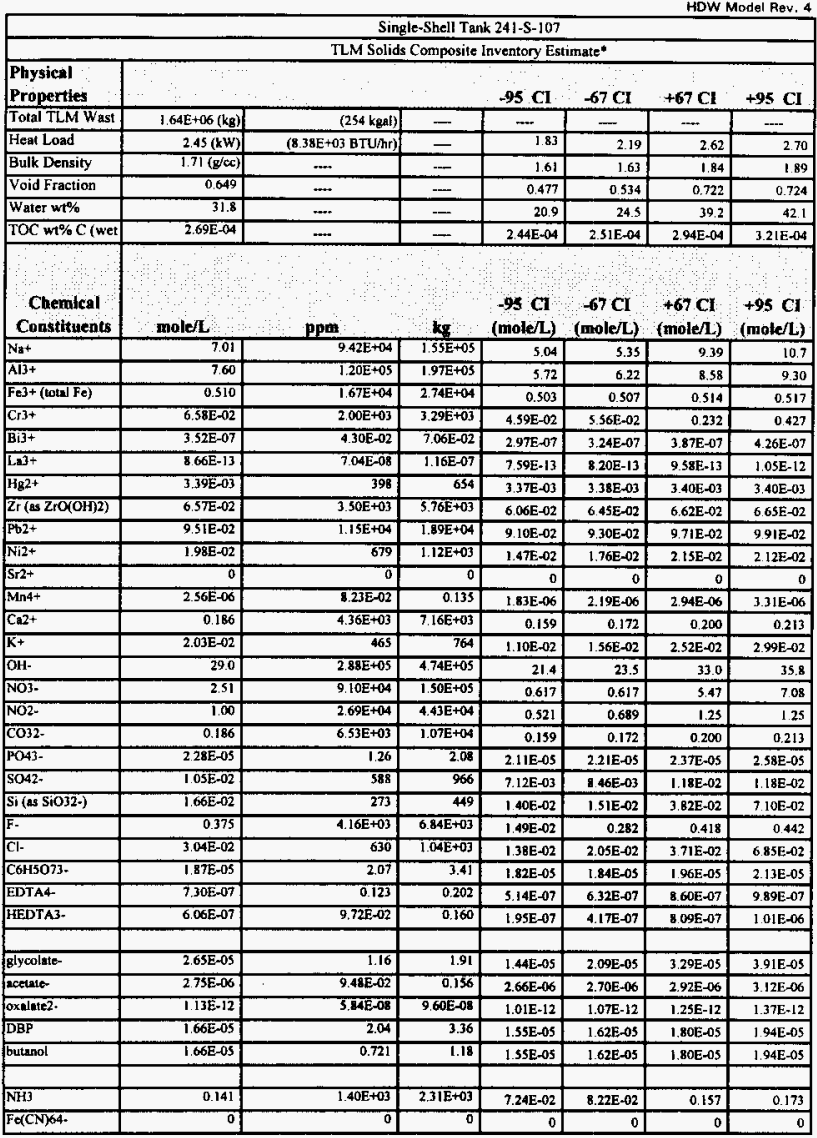

*Unkunowns in tank solids invertory are assigned by Tank Layering Model (TLM) 
HNF-SD-WM-ER-323, Rev. 1

HDW Model Rev. 4

\begin{tabular}{|c|c|c|c|c|c|c|c|}
\hline \multirow[b]{3}{*}{$\begin{array}{l}\text { Physical } \\
\text { Properties }\end{array}$} & \multicolumn{7}{|c|}{ Single-Shell Tank 241-S-107 } \\
\hline & \multicolumn{7}{|c|}{ SMM Composite Inventory Estimate } \\
\hline & & & & $.95 \mathrm{CI}$ & $-67 \mathrm{CI}$ & $+67 . \mathrm{Cl}$ & $+95 \mathrm{CI}$ \\
\hline Total SMM Wast & $6.46 \mathrm{E}+05(\mathrm{~kg})$ & (122 kgal) & - & --- & - & $\cdots$ & $\ldots$ \\
\hline Heat Load & $0.732(\mathrm{kw})$ & $(2.50 \mathrm{E}+03 \mathrm{BTU} / \mathrm{hr})$ & $\rightarrow$ & 0.670 & 0.710 & 0.755 & 077 \\
\hline Bulk Density* & $1.40(\mathrm{~g} / \mathrm{cc})$ & $m$ & - & 1.37 & 1.38 & 1.41 & 1.42 \\
\hline Water wt\% & 49.0 & $m$ & - & 46.8 & 47.8 & 50.4 & 51.9 \\
\hline TOC wt \% C (wet & 0.898 & 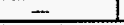 & - & 0.468 & 0.679 & 1.12 & 1.33 \\
\hline $\begin{array}{l}\text { Chemical } \\
\text { Constituents }\end{array}$ & molelL & ppin: & ig & $\begin{array}{l}95 \text { CI } \\
\text { (molen) }\end{array}$ & $\begin{array}{l}-67 \mathrm{CL} \\
(\mathrm{molen})\end{array}$ & $\begin{array}{l}+67 \mathrm{CI} \\
\text { (mole/h) }\end{array}$ & $\begin{array}{r}+95 \mathrm{Cl} \\
(\mathrm{mote} / \mathrm{L})\end{array}$ \\
\hline $\mathrm{Na}+$ & 9,39 & $1.54 \mathrm{E}+05$ & $9.97 \mathrm{E}+04$ & 8.60 & 9.00 & 9.73 & 10.0 \\
\hline $\mathrm{Al} \mathbf{3}+$ & 2.05 & $2.03 \mathrm{E}+04$ & $1.31 E+04$ & 0.975 & 1.01 & 1.09 & 1.13 \\
\hline Fe3+ (total Fe) & $7.79 \mathrm{E} \cdot 03$ & 311 & 201 & $6.49 \mathrm{E}-03$ & 7.13E-03 & $8.46 \mathrm{E}-03$ & $9.09 \mathrm{E}-03$ \\
\hline $\mathrm{C}+3+$ & $9.29 \mathrm{E}-02$ & $3.45 \mathrm{E}+03$ & $2.23 \mathrm{E}+03$ & $7.83 \mathrm{E}-02$ & $8.61 E-02$ & 9.5SE- 02 & $9.81 E-02$ \\
\hline Bi3+ & $8.42 \mathrm{E}-0 \mathrm{~d}$ & 126 & 81.2 & $7.67 \mathrm{E}-04$ & $8.04 E-04$ & 8.79E-04 & $9.16 \mathrm{E}-04$ \\
\hline $\mathrm{La3+}$ & $2.73 \mathrm{E}-05$ & 2.72 & 1.75 & $1.98 \mathrm{E}-05$ & 2.35E-05 & 3.12E-0s & 3.49E-05 \\
\hline $\mathrm{Hg} 2+$ & $5.80 \mathrm{E}-06$ & 0.832 & 0.537 & $5,41 \mathrm{E}-06$ & 5.63E-06 & $5.89 \mathrm{E}-06$ & $5.97 \mathrm{E}-06$ \\
\hline $\mathrm{ZT}$ (as $\mathrm{ZrO}(\mathrm{OH}) 2)$ & $1.55 E-04$ & 10.1 & 6.52 & $1.41 \mathrm{E}-04$ & $1.46 \mathrm{E}-04$ & $1.60 \mathrm{E}-04$ & $1.68 \mathrm{E}-04$ \\
\hline $\mathrm{Pb2+}$ & 6.44E-04 & 93.4 & 61.6 & $5.20 \mathrm{E}-04$ & $5.81 \mathrm{E}-04$ & $7.07 \mathrm{E}-04$ & $7.68 \mathrm{E}-04$ \\
\hline Ni2+ & $4.33 \mathrm{E}-03$ & 182 & 117 & 4.14E-03 & $4,24 \mathrm{E}-03$ & 4.38E-03 & 4.43E- -03 \\
\hline $\mathrm{Sr} 2+$ & 0 & 0 & 0 & 0 & 0 & 0 & 0 \\
\hline Mndt & 2.64E-03 & 104 & 67.0 & $2.02 E-03$ & $232 E-03$ & 2.96E-03 & 3.27E-03 \\
\hline $\mathrm{Ca} 2+$ & $2.33 \mathrm{E}-02$ & 668 & 431 & $2.14 \mathrm{E}-02$ & $2.23 \mathrm{E}-02$ & $2.43 \mathrm{E}-02$ & $2.52 \mathrm{E}-02$ \\
\hline $\mathrm{K}+$ & $4.35 \mathrm{E}-02$ & $1.22 \mathrm{E}+03$ & 786 & $3.97 \mathrm{E}-02$ & $4.15 \mathrm{E}-02$ & $4.56 \mathrm{E}-02$ & $4.76 \mathrm{E}-02$ \\
\hline $\mathrm{OH}-$ & 6.38 & $7.76 \mathrm{E}+04$ & 5.01E+04 & 5.93 & 6,14 & 6.63 & 6.78 \\
\hline NO3- & 3.21 & $1.42 \mathrm{E}+05$ & $9.19 \mathrm{E}+04$ & 2.97 & 3.11 & 3.26 & 3.31 \\
\hline NO2- & 1.58 & $5.21 \mathrm{E}+04$ & $3.37 \mathrm{E}+04$ & 1,32 & 1.44 & 1.72 & 1.86 \\
\hline CO32. & 0.319 & $1.37 \mathrm{E}+04$ & $8.86 \mathrm{E}+03$ & 0.286 & 0,303 & 0.338 & 0.348 \\
\hline PO43- & $602 E-02$ & $4.09 \mathrm{E}+03$ & $264 E+03$ & 5.23E- 02 & $5.51 \mathrm{E}-02$ & 6.16E-02 & $6.30 \mathrm{E}-02$ \\
\hline 5042. & 0.171 & $1.17 \mathrm{E}+04$ & $7.59 E+03$ & 0.137 & 0.153 & 0.190 & 0.206 \\
\hline Si (as Sí032-) & $5,57 \mathrm{E}-02$ & $1.12 \mathrm{E}+03$ & 723 & $4,68 \mathrm{E}-02$ & $5.12 \mathrm{E}-02$ & $6.03 \mathrm{E}-02$ & $6.47 E-02$ \\
\hline F- & 4.13E-02 & 362 & 363 & $3.49 \mathrm{E}-02$ & $3.76 \mathrm{E}-02$ & $4.45 \mathrm{E}-02$ & 4.70E-02 \\
\hline C. & 0.162 & $4.12 \mathrm{E}+03$ & $2.66 \mathrm{E}+03$ & 0147 & 0.154 & 0.170 & 0,175 \\
\hline C6 $65073-$ & $1.90 \mathrm{E}-02$ & $2.57 \mathrm{E}+03$ & $1.66 \mathrm{E}+03$ & 1.726-02 & $1.81 \mathrm{E}-02$ & $2.00 \mathrm{E}-02$ & $2.09 \mathrm{E}-02$ \\
\hline EDTA4: & $2.04 \mathrm{E}-02$ & $4.20 \mathrm{E}+03$ & $2.71 E+03$ & $5.54 \mathrm{E}-03$ & $1.28 \mathrm{E}-02$ & $2.80 \mathrm{E}-02$ & $3.55 \mathrm{E}-02$ \\
\hline HEDTA3. & $3.94 \mathrm{E}-02$ & $7.72 \mathrm{E}+03$ & $4.99 \mathrm{E}+03$ & $9.66 \mathrm{E}-03$ & $2.42 \mathrm{E}-02$ & S. 47E-02 & $6.96 \mathrm{E}-02$ \\
\hline Elycolate- & $8.79 \mathrm{E}-02$ & $4.72 \mathrm{E}+03$ & $3.05 \mathrm{E}+03$ & $5: 82 E-02$ & $7.27 \mathrm{E}-02$ & 0.103 & 0.118 \\
\hline acetnte- & 4.52E-03 & 191 & 123 & $3.71 \mathrm{E}-03$ & $4.10 \mathrm{E}-03$ & 4.93E-03 & $5.33 \mathrm{E}-03$ \\
\hline Oxalate 2- & 3.58E-05 & 2.25 & 1.46 & 3,17E-05 & $3.37 \mathrm{E}-0 \mathrm{~s}$ & 3.79E- 05 & $399 \mathrm{E}-0 \mathrm{~S}$ \\
\hline DBP & $1.24 \mathrm{E}-02$ & $1.87 \mathrm{E}+03$ & $1.21 \mathrm{E}+03$ & $1.02 \mathrm{E}-02$ & $1.13 \mathrm{E}-02$ & $1.35 \mathrm{E}-02$ & $1.46 \mathrm{E}-02$ \\
\hline butanol & $1.24 \mathrm{E}-02$ & 660 & 426 & $1.02 \mathrm{E}-02$ & $1.13 \mathrm{E}-02$ & $1.33 \mathrm{E}-02$ & $1.46 E-02$ \\
\hline $\mathrm{NH3}$ & $5.52 \mathrm{E}-02$ & 671 & 433 & 4.51E-02 & $4.91 E-02$ & $6.30 \mathrm{E}-02$ & $7.20 \mathrm{E}-02$ \\
\hline Fa(CN)64- & $\overline{0}$ & 0.1 & 0 & 0 & 0 & 0 & 0 \\
\hline
\end{tabular}

*Density is calculated based on $\mathrm{Na}, \mathrm{OH}$-, and $\mathrm{AlO}_{2}$ -

tWater wi\% derived from the difference of density and total dissolved species. 
HDW Madel Rev, 4

\begin{tabular}{|c|c|c|c|c|c|c|c|}
\hline & \multicolumn{7}{|c|}{ Single-Shell Tank 24I-S-107 } \\
\hline & \multicolumn{7}{|c|}{ Total Inventory Estimate* } \\
\hline $\begin{array}{l}\text { Physical } \\
\text { Properties }\end{array}$ & & & & $95 \mathrm{CI}$ & $-67 \mathrm{CI}$ & $+67 \mathrm{CI}$ & $+95 \mathrm{CI}$ \\
\hline Total Waste & $2.29 \mathrm{E}+06(\mathrm{~kg})$ & (376 kgal) & $\ldots$ & - & $\cdots$ & ـ & - \\
\hline Heat Lond & $3.19(\mathrm{~kW})$ & $(1.09 \mathrm{E}+04 \mathrm{ETU} / \mathrm{hr})$ & -- & 2.55 & 2.92 & 3.36 & 3.43 \\
\hline Bulk Densityt & $1.61(g / c)$ & - & - & 1.54 & 1.55 & 1.70 & 1.73 \\
\hline Water wt\%t & 36.7 & - & - & 28.4 & 30.9 & 420 & 44.2 \\
\hline TOC wi\% C (wet & 0.253 & $\ldots$ & $\ldots$ & 0.131 & 0.191 & 0,316 & 0.377 \\
\hline $\begin{array}{l}\text { Chemical } \\
\text { Constituents }\end{array}$ & mole/L & ppin & kg: & $\begin{array}{l}95 \mathrm{Cl} \\
(\mathrm{molel})\end{array}$ & $\begin{array}{c}-67 \mathrm{Cl} \\
\text { (molelL) }\end{array}$ & $\begin{array}{c}+67 \mathrm{Cl} \\
(\mathrm{mole} / \mathrm{L})\end{array}$ & $\begin{array}{l}\text { +9s Cl: } \\
\text { (niole/L) }\end{array}$ \\
\hline $\mathrm{Na}+$ & 7.78 & $1.11 \mathrm{E}+05$ & $2.55 \mathrm{E}+05$ & 6.41 & 6.61 & 9.43 & 10.2 \\
\hline Al3+ & 5,47 & $9.18 \mathrm{E}+04$ & $2.10 \mathrm{E}+05$ & 4.20 & 4.55 & 6.14 & 6.62 \\
\hline Fej+(total Fe) & 0.347 & $1.21 \mathrm{E}+04$ & $2.76 \mathrm{E}+04$ & 0.343 & 0.345 & 0.350 & 0.352 \\
\hline$\overline{C r} 3+$ & $7,46 \mathrm{E}-02$ & $2.41 E+03$ & $5.52 \mathrm{E}+03$ & 6.14E-02 & $6.79 \mathrm{E}-02$ & 0.185 & 0.314 \\
\hline Bi3+ & $2.73 \mathrm{E}-04$ & 35.5 & 81.3 & 2.49E-04 & $2.61 \mathrm{E}-04$ & 2.86E-04 & $297 \mathrm{E}-04$ \\
\hline Labt & $8.87 \mathrm{E}-06$ & 0.766 & 1.75 & $6.43 \mathrm{E}-06$ & $7.62 \mathrm{E}-06$ & $1.01 \mathrm{E}-05$ & $1.13 \mathrm{E}-05$ \\
\hline $\mathrm{Hg}_{2+}+$ & $2.29 \mathrm{E}-03$ & 286 & 655 & $2.28 \mathrm{E}-03$ & $2.29 \mathrm{E}-03$ & $2.30 \mathrm{E}-03$ & $2.30 \mathrm{E}-03$ \\
\hline $\operatorname{Zr}(\mathrm{Ar} \mathrm{ZrO}(\mathrm{OH}) 2)$ & 4.44E-02 & $2.52 \mathrm{E}+03$ & $5.77 \mathrm{E}+03$ & 4.10E-02 & $4.36 \mathrm{E}-02$ & $4,48 \mathrm{E}-02$ & $4.50 \mathrm{E}-02$ \\
\hline $\mathrm{Pb2+}$ & $6.44 \mathrm{E}-02$ & $8.30 \mathrm{E}+03$ & $1.90 \mathrm{E}+04$ & $6.17 \mathrm{E}-02$ & $6.30 \mathrm{E}-02$ & $6.58 \mathrm{E}-02$ & $6.71 \mathrm{E}-02$ \\
\hline $\mathrm{Ni2+}$ & TABE-02 & 539 & $1.23 \mathrm{E}+03$ & $1.13 E-02$ & $1,33 \mathrm{E}-02$ & $1.59 \mathrm{E}-02$ & $1.57 \mathrm{E}-02$ \\
\hline $\mathrm{s} 2+$ & 0 & 0 & 0 & 0 & 0 & 0 & 0 \\
\hline $\mathrm{Mn} A+$ & 8.59E 04 & 29.3 & 67.2 & $6.56 \mathrm{E}-04$ & 7.5SE-04 & $9.63 \mathrm{E}-04$ & $1.06 \mathrm{E}-03$ \\
\hline $\mathrm{Ca2t}$ & 0.133 & $3,32 \mathrm{E}+03$ & $7.59 \mathrm{E}+03$ & 0.115 & 0.124 & 0.142 & 0.151 \\
\hline $\mathrm{K}^{+}$ & $2.79 \mathrm{E}-02$ & 677 & I.55E+03 & $2.08 \mathrm{E}-02$ & $2.43 \mathrm{E}-02$ & $3.15 \mathrm{E}-02$ & $3.50 \mathrm{E}-02$ \\
\hline OH- & 21.7 & $2.29 \mathrm{E}+05$ & $5.24 \mathrm{E}+05$ & 16.5 & 17,9 & 24,3 & 26.3 \\
\hline $\mathrm{NO} 3-$ & 2.74 & 1.05E+03 & $2.41 E+05$ & 1.44 & 1.46 & 4.73 & 5.79 \\
\hline NO2- & 1.19 & $3405+04$ & $7.80 \mathrm{E}+04$ & 0.850 & 0.988 & 1.37 & 137 \\
\hline $\cos 2$ & 0.229 & 8.56E+03 & 1.96E+04 & 0.212 & 0.220 & 0.239 & 0.247 \\
\hline PO43- & $1.96 \mathrm{E}-02$ & $1.16 E+03$ & $2.64 E+0.3$ & $1.70 \mathrm{E}-02$ & $1.79 \mathrm{E}-02$ & $2.00 \mathrm{E}-02$ & $2.05 E-02$ \\
\hline SO42- & $6.26 \mathrm{E}-02$ & $3.74 E+03$ & $8.55 \mathrm{E}+03$ & $5.14 \mathrm{E}-02$ & $5.67 \mathrm{E}-02$ & $6.80 \mathrm{E}-02$ & $7.39 \mathrm{E}-02$ \\
\hline Si (as Si032-) & $2.93 \mathrm{E}-02$ & 512 & $1.17 \mathrm{E}+03$ & 2.64E-02 & $2.7 \mathrm{TE}-02$ & $4.38 \mathrm{E}-02$ & $6.57 \mathrm{E}-02$ \\
\hline $\bar{F}-$ & 0.266 & 3.15E+03 & $7.21 \mathrm{E}+03$ & $2.34 \mathrm{E}-02$ & 0.204 & 0.296 & 0.312 \\
\hline Cl- & $7.33 \mathrm{E}-02$ & $1.6 \mathrm{IE}+03$ & $3.69 \mathrm{E}+03$ & $6.23 E-02$ & $6,77 \mathrm{E}-02$ & 7,77t:-02 & $985 E-02$ \\
\hline C6H5O73- & $6.19 \mathrm{E}-03$ & 728 & $1.67 \mathrm{TE}+03$ & $5.59 \mathrm{E}-03$ & $5.88 \mathrm{E}-03$ & $6.50 \mathrm{E}-03$ & $6.80 \mathrm{E}-03$ \\
\hline EDTA4- & $6.62 \mathrm{E}-03$ & $1,19 E+03$ & $2.7] E+03$ & $1.80 \mathrm{E}-03$ & 4.15E-03 & $9.10 \mathrm{E}-03$ & $1.15 \mathrm{E}-02$ \\
\hline HEDTA3- & $1.28 \mathrm{E}-02$ & $2.18 E+03$ & $4.99 E+03$ & $3.14 \mathrm{E}-03$ & $7.85 \mathrm{E}-03$ & $1.77 \mathrm{~T}-02$ & $2.26 \mathrm{E}-02$ \\
\hline glycolate- & $2.85 \mathrm{E}-02$ & $1.33 \mathrm{E}+03$ & $3.05 E+03$ & $1.89 \mathrm{E}-02$ & $2.36 \mathrm{E}-02$ & $3.35 \mathrm{E}-02$ & $3.83 \mathrm{E}-02$ \\
\hline Acetute- & $1.47 \mathrm{E}-03$ & 53,9 & 123 & $1.21 E-03$ & $1.33 \mathrm{E}-03$ & $1,60 \mathrm{E}-03$ & $1.73 \mathrm{E}-03$ \\
\hline oxalate2- & $1.16 \mathrm{E}-05$ & 0.636 & 1.46 & 1.03E-05 & 1.09E-0S & $1.23 \mathrm{E}-05$ & $1.29 \mathrm{E}-05$ \\
\hline DBP & 4.05E-03 & 529 & J.21E+03 & $3.34 \mathrm{E}-03$ & 3.69E- 03 & $441 \mathrm{E}-03$ & $4.75 \mathrm{E}-0.03$ \\
\hline butanol & $4,05 E-03$ & 187 & 427 & $3.34 \mathrm{E}-03$ & $3.69 \mathrm{E}-03$ & 4.41E-03 & 4.75E-03 \\
\hline $\mathrm{NH3}$ & 0.113 & $1.20 \mathrm{E}+03$ & $2.74 E+03$ & $6.62 \mathrm{E}-02$ & $7,3 \mathrm{JE}-02$ & 0.125 & 0.137 \\
\hline $\mathrm{Fe}_{(\mathrm{CN}) 64-}$ & 0 & 0 & 0 & 0 & 0 & 0 & 0 \\
\hline
\end{tabular}

"Unknowns in tank solids inventory are assigned by Tank Layering Model (TLM)

tWater wt\% derived from the difference of density and total dissoived species. 


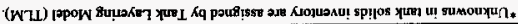

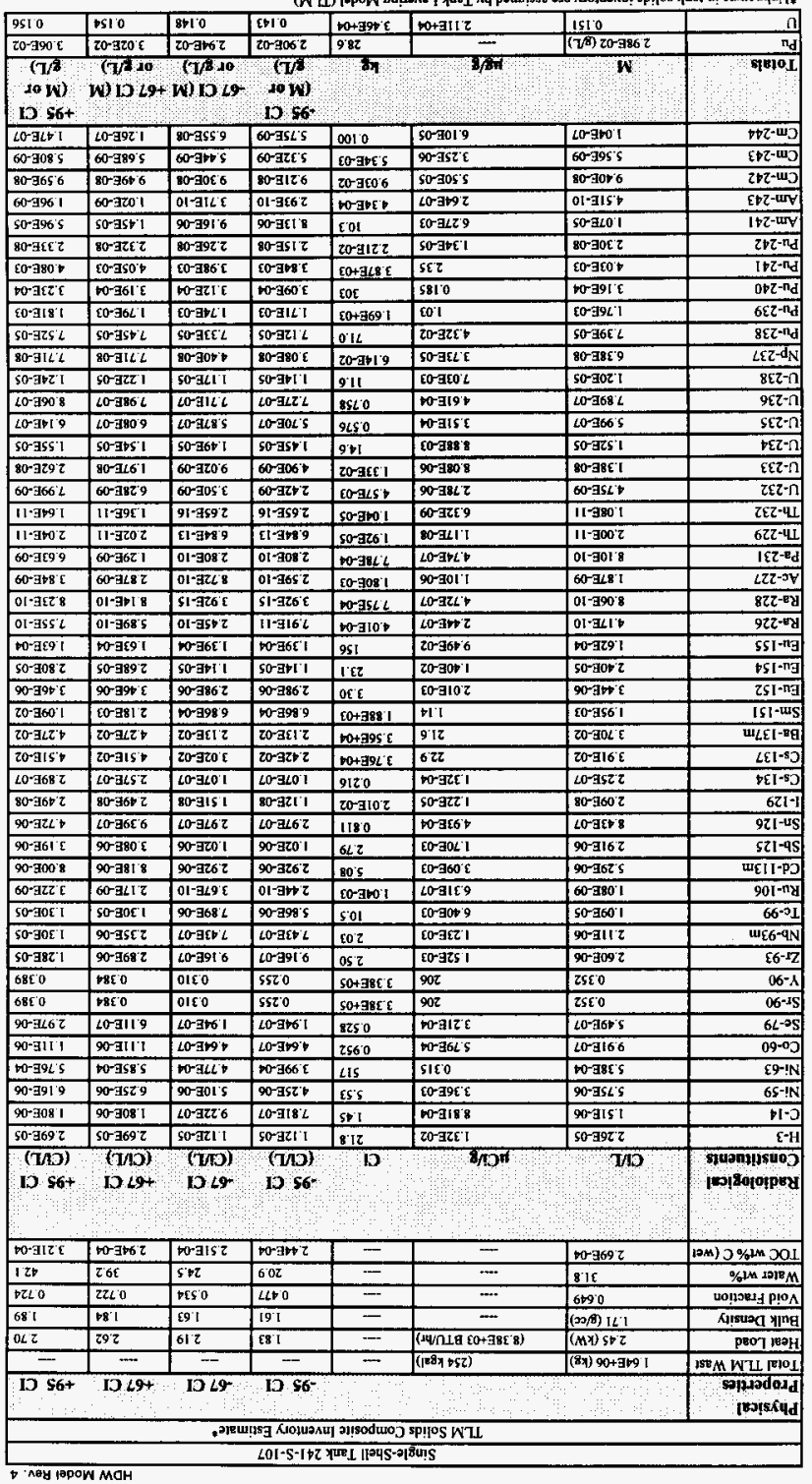




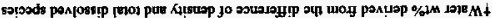

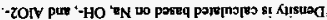

\begin{tabular}{|c|c|c|c|c|c|c|c|}
\hline $20 \div 3999$ & ton-365'9 & COOAZE9 & 20-30r'9 & 602 & $60+701$ I & $\varepsilon 0-35+9$ & ก \\
\hline b0-3SLSS & 50-381's & $60-310^{\circ}$ & to-atrf & चाट०0 & $=$ & $\left(7^{4 I)}+0-909 t\right.$ & nd \\
\hline 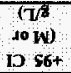 & 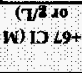 & $\begin{array}{l}\text { (T/S } \\
\text { W) } 1 \mathrm{~L} L \mathrm{l}\end{array}$ & $\begin{array}{l}\text { (T/ } \\
\text { To: } \\
156\end{array}$ & 4 & 97. & $\mathbf{W}$ & 51010I \\
\hline Lotatti & $\angle 0-90 E^{\prime} 1$ & $80-3685$ & $80-36015$ & $20-781 \div 5$ & 50 - 320 ' & Lo-azl t & $76 z-130$ \\
\hline $80-705^{\prime} 1$ & $80-3951$ & $60-2102$ & $60-910 \mathrm{~L}$ & so-azg's & $90-31 C^{\prime} 8$ & 50-12z I & 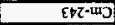 \\
\hline L0-359'1 & $20-a 8+1$ & $80-318 \mathrm{C}$ & $80-3182$ & $20-31199$ & $50-39+6$ & Lo-Az\& ? & $\tau b \tau-w 0$ \\
\hline $60-3602$ & $60-9881$ & $60-3+11$ & $60-3971$ & $00-365 t$ & $90-9811$ & $60-3191$ & ERZ-tuY \\
\hline $50-709 \% 5$ & soganos & SO- $368^{\prime} \varepsilon$ & So-gef & 902 & $20-961 \mathrm{c}$ & $50-49 t+2$ & itz-ury \\
\hline $00-356-5$ & $0[-756 t$ & $01-396 \mathrm{E}$ & 0l-38t ? & tor3902 & LO-361 E & ol-gst & $z \nabla z-n_{d}$ \\
\hline $50-3 \times C 6$ & $50-3968$ & so-alz' & 50-784 9 & $\forall \angle L E$ & 70-70s's & s0-301'8 & $I \in z-n_{d}$ \\
\hline 90.9866 & $90-3 t+2$ & $90-3<29$ & 900:ILS & gI' & $60-900^{\prime} \mathrm{t}$ & 90 -3589 & $00 z^{-n} d$ \\
\hline $50-9196$ & S0:G1Et & $50-969-\mathrm{E}$ & $50^{-765 \mathrm{C}}$ & 581 & 20-a9e'z & $50-900+$ & $6 \varepsilon z-n_{d}$ \\
\hline $90-3 z p \mid$ & 90-30E: I & $90 \mathrm{H} 90 \mathrm{I}$ & LOCGDe 6 & $9+50$ & 6003968 & $90038[1$ & $8 \varepsilon z+n d$ \\
\hline $40-38+8$ & $\angle 0-3 \angle t L L$ & $\angle 0-36 e^{\prime} 5$ & Lo-30t' & $\angle 620$ & 60-309't & Lot9569 & $\angle E Z^{-}-\mathrm{d} N$ \\
\hline $40-30<9$ & $40-\sin 9$ & LO-GEt 9 & LOAIEC 9 & 2000 & nralet & Lor:3559 & $8 \mathrm{E2}-\Omega$ \\
\hline $80-\exists \subset 8^{\prime} 1$ & $80-908$ 't & $80-95<1$ & $80-9161$ & cor-7618 & so-glzl & $80-7 \pm 21$ & $9 \varepsilon z-\Omega$ \\
\hline $80.39 \mathrm{c} 2$ & Botate $t$ & $80^{\circ} 572 z$ & $80-3006$ & $20-350^{\circ} 1$ & 50'A59 I & $80-38 t 2$ & SEZ- \\
\hline$\angle 0^{0}-3185$ & $\angle 0$-IbL'S & L0-7655 & Lo-at's & $09 \tau_{0}^{\circ}$ & 60-g50't & LOAGE'S & $D \varepsilon Z-\Omega$ \\
\hline $90-3562$ & $90=\pi 1 v \tau$ & $90 \because \leqslant \angle P !$ & $90-3901$ & $288^{\prime} 0$ & corget I & श्र0.916 I & $\operatorname{EEZ} \cap n$ \\
\hline$\angle O=\exists O L L$ & $20-90 C^{9}$ & corsts & LOGGSLZ & O\&Z0 & t0-G95' & L0-786 & $2 \varepsilon \tau-\Omega$ \\
\hline $60 \div 3156$ & 6007908 & oogeG & 600GItP $\varepsilon$ & $50-700^{\circ} \mathrm{C}$ & $90^{\circ}$ - $59{ }^{\prime}$ & $60-3059$ & tEZt-LL \\
\hline $60-95 \angle \mathrm{E}$ & 6079862 & $60-3 \times 91$ & $60-7501$ & EO-G50'I & 90-a29' I & $60-39 z^{\prime} z$ & $622-411$ \\
\hline $60-\exists 56 E$ & $60-395 \mathrm{C}$ & 60-3112 & $60-$ gilz & $20-3 \angle P \cdot t$ & $90-a \angle z z$ & $60-3 \angle 1 \varepsilon$ & I£Z-вd \\
\hline $01-3028$ & 01-GIS'L & $01-7266$ & $01-9264$ & wo-791 $\varepsilon$ & Lo-96"'t & $01-3689$ & $\angle z z-5 \gamma$ \\
\hline $40-3+91$ & L0-36Z I & $80-7089$ & $80-1960+$ & $20-a 5 b^{\prime} b$ & $50-7689$ & $80-3596$ & $8 z z-x y$ \\
\hline $01-3 z \varepsilon^{\prime}$ & 0I-GIz' 1 & $11-785 \mathrm{C}$ & $11-985 L$ & SO-AZO'S & $80-38 C L$ & $01-960 \mathrm{I}$ & $9 z z-10 y$ \\
\hline 60.3852 & PO'AEE' $\tau$ & $100 \mathrm{~J} 6 \mathrm{ZI}$ & pra6z I & $0 \%$ & $6+10$ & 70-980z & $551^{-\pi g}$ \\
\hline $60-7619$ & t01-308 5 & Do- 3812 & to-aBI' $z$ & $t z z$ & 9060 & bo-atoto & ts $1^{-n g}$ \\
\hline 90.362 & $90-368 \mathrm{C}$ & $90-3912$ & 90-9912 & 191 & corg6t $z$ & $90-38+\mathrm{E}$ & $\left.z S\right|^{-n}$ \\
\hline $20-9611$ & $20-3901$ & fo-455: & 50-ass's & $80+38 z+$ & 699 & E0-aㄴ? 6 & ISI-tuS \\
\hline$\angle 120$ & 2020 & $891^{\circ} 0$ & 5910 & $20+211$ i 6 & $|+|$ & $\angle 600$ & 때LE $1^{-\mathrm{Eg}}$ \\
\hline 6220 & 6120 & $86 l^{\prime} 0$ & 8810 & $100+3596$ & 601 & $60 \tau 0$ & $\angle E I-8 D$ \\
\hline $90.368 \mathrm{E}$ & 90-318? ? & 90-gz।'z & 90-385 ! & $5 x_{1}$ & E0-9.96। & $9030<2$ & $t \varepsilon I-8 j$ \\
\hline $20-9996$ & 209906 & Lo-Gidz & 40-stbzt & 65I'0 & 60-390'z & Lo-atr' $\varepsilon$ & $62[-]$ \\
\hline 90-3115 & $90-395+$ & $90-78 E 2$ & 90-ABE: $z$ & DE! & co-ass' z & $90-386 \mathrm{E}$ & 921 -4S \\
\hline 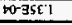 & b0-30r 1 & so-gst' & 50-ast't & 825 & $20-3568$ & po-3St'I & $521-95$ \\
\hline $50-3+16$ & $50 \mathrm{ALO} 8$ & $50-369 \mathrm{E}$ & S0-769 $\mathrm{C}$ & $81 \varepsilon$ & $20-926 t$ & $50-3889$ & Шहा।-PO \\
\hline $60-3199$ & $60-9119$ & $60^{-360^{\prime} \mathrm{g}}$ & $60-360 \mathrm{f}$ & for $3<52$ & $90-7 \angle 6 \mathrm{E}$ & 60-39s 5 & $901^{-n y}$ \\
\hline $100.31+2$ & roalliz & $60-3 \angle+1$ & $50-3911$ & 528 & $8 z 10$ & no-76L' & $66-21$ \\
\hline $50-70 \tau 1$ & $50-3101$ & $90-369$ 's & 90-ang's & $25 b$ & 10-30<' 9 & $90-39\{6$ & wE6-qN \\
\hline $50.9 \angle 91$ & $50-96+1$ & $90-319 \mathrm{~L}$ & $90-319 L$ & $\angle 65$ & f0-7Sz 6 & 50-962' I & $\varepsilon 6^{-1} Z$ \\
\hline $20-3956$ & to-70e' 6 & $20-79 \varepsilon^{\circ} 5$ & 20-G9ts & $60+\mathrm{d} L 1$ & 969 & $20-7506$ & $06-\lambda$ \\
\hline $20-7556$ & $20-3066$ & $20-39 L^{\circ} 8$ & $20-9 n+8$ & $60+3111$ & $9+9$ & $20-9806$ & $06-5$ \\
\hline $90-36 \varepsilon \varepsilon$ & $90-3201 \mathrm{C}$ & $90-415 \mathrm{I}$ & $90-3 / S^{\prime} 1$ & $z 21$ & 10-78: '1 & 90-3E9 $\mathrm{Z}$ & $6 L^{-2} S$ \\
\hline SO-JEOE & so-3 562 & $90-3676$ & $90-76+6$ & $\tau \varepsilon 1$ & $20-\exists+0 ' z$ & S0-798' $\mathrm{Z}$ & $09-03$ \\
\hline $60.3<91$ & 100-BEg'। & $50^{-361 / 8}$ & $50-3618$ & 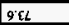 & 1110 & po-365 I & $\varepsilon 9-! N$ \\
\hline $90-30 L 1$ & 90-399 1 & Lo-31t8 & $\angle 0-\mathrm{AIt} 8$ & ISLO & ร0-391' I & 90 - 129 & $65-1 \mathrm{~N}$ \\
\hline $90-9852$ & $50-\exists 5 S^{\prime} z$ & $90-35 \% 8$ & $90-35+8$ & $9+1$ & $20-3081$ & 50-3Is 2 & $+1-0$ \\
\hline H0-3S6 I & to-7!B I & $50-3568$ & 50-7\$क6' & $Z^{\prime} 8 L$ & 1210 & t0-969't & $\varepsilon-H$ \\
\hline $\begin{array}{l}(\mathrm{MO}) \\
\text { 10 } 56+\end{array}$ & $\begin{array}{l}(\mathrm{T} / \mathrm{J}) \\
10 / 9+\end{array}$ & $\begin{array}{l}\text { (พา) } \\
1949\end{array}$ & $\begin{array}{l}(7 / 4) \\
1056\end{array}$ & 10 & 8/אי & $\mathrm{TaP}$ & 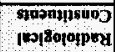 \\
\hline 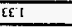 & $2 ! 1$ & $6 \angle 90$ & 8960 & - & $=$ & 8680 & $\mathrm{JPM}) \mathrm{\%} \% \mathrm{M} \mathrm{OOL}$ \\
\hline 615 & 105 & $8 L t$ & 890 & $=$ & $=$ & 065 & t\%2M Jอ1в $M$ \\
\hline tot & $1+1$ & 851 & $L E !$ & 一 & - & $(\infty, A)$ ot 1 & . Sppsued y[ng \\
\hline$\angle L L O$ & SSCO & $01 \angle 0$ & $0 \angle 9^{\prime} 0$ & - & (I4)LG $\left.\varepsilon 0+\exists 0 \mathrm{~S}^{\prime} \mathrm{z}\right)$ & $(M Y) \geq \varepsilon c^{\prime} 0$ & peo] JeOH \\
\hline- & 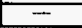 & -- & $\infty$ & - & $(10 \times 1 \times z 21)$ & $(5 \times) 50+39+9$ & ISEM NWNS IEIOL \\
\hline \multirow[t]{3}{*}{$596+$} & IO 494 & ID. 29 & 1756 & $\therefore$ & & & $\begin{array}{l}\text { sprododd } \\
\text { foojtsild }\end{array}$ \\
\hline & \multicolumn{6}{|c|}{ 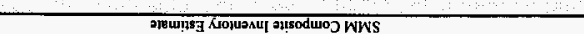 } & \\
\hline & & & $c 01-5 \cdot 1+2$ & I I & & & \\
\hline
\end{tabular}


HNF-SD-WM-ER-323, Rev. 1

HDW Model Rev. 4

\begin{tabular}{|c|c|c|c|c|c|c|c|}
\hline \multicolumn{8}{|c|}{ Single-Sheil Tank 241-S.107 } \\
\hline & \multicolumn{7}{|c|}{ Total Inventory Estimate* } \\
\hline $\begin{array}{l}\text { Physical } \\
\text { Properties }\end{array}$ & & & & $-95 \mathrm{CI}$ & $-67 \mathrm{CI}$ & $+67 \mathrm{CI}$ & $+95 \mathrm{CI}$ \\
\hline Total Waste & $2.29 \mathrm{E}+06(\mathrm{~kg})$ & $(376 \mathrm{kgal})$ & - & - & - & - & $\ldots$ \\
\hline Heat Load & $3.19(\mathrm{~kW})$ & $(1.09 \mathrm{E}+04 \mathrm{BTU} / \mathrm{hr})$ & - & 2.55 & 2.92 & 3.36 & 3.43 \\
\hline Bulk Density't & $1.61(\mathrm{~g} / \mathrm{cc})$ & $\cdots$ & $=$ & 1.54 & 1.55 & 1.70 & 1.73 \\
\hline Water wt\% & 36.7 & $m$ & - & 28.4 & 309 & 42.0 & 442 \\
\hline TOC wt\% C (wet & \begin{tabular}{l|l|}
0.253 \\
\end{tabular} & $\ldots$ & $=$ & 0.131 & 0.191 & 0.316 & 0,377 \\
\hline $\begin{array}{l}\text { Rediological } \\
\text { Constituents }\end{array}$ & CUL & HCH/s & $\mathrm{CH}$ & $\begin{array}{l}-95 \mathrm{Cr} \\
\text { (C) }\end{array}$ & $\begin{array}{l}-67 \mathrm{CI} \\
\text { (CUL) }\end{array}$ & $\begin{array}{r}+67 \mathrm{CI} \\
(\mathrm{Cl} / \mathrm{L})\end{array}$ & $\begin{array}{r}+95 \mathrm{Cl} \\
(\mathrm{CV} / \mathrm{C})\end{array}$ \\
\hline $\mathrm{H}-3$ & $7.02 E-05$ & $4.36 \mathrm{E}-02$ & 999 & $4.43 \mathrm{E}-05$ & $443 \mathrm{E}-05$ & $7.40 \mathrm{E}-05$ & $7,87 E-05$ \\
\hline C-14 & $9.17 \mathrm{E}-06$ & $5,70 \mathrm{E}-03$ & 13.1 & $3.76 \mathrm{E}-06$ & $3.76 \mathrm{E}-06$ & $9.37 \mathrm{E}-06$ & $9.40 \mathrm{E}-06$ \\
\hline $\mathrm{Ni}-59$ & $4.41 \mathrm{E}-06$ & $2.74 \mathrm{E}-03$ & 6.28 & $3.40 \mathrm{E}-06$ & $3.97 \mathrm{E}-06$ & $4.75 \mathrm{E}-06$ & $4.68 \mathrm{E}-06$ \\
\hline $\mathrm{Ni}-63$ & 4.15E-04 & 0.258 & 59] & $3.21 \mathrm{E}-04$ & $3.74 E-04$ & $4.47 \mathrm{E}-04$ & 4.40E-04 \\
\hline $\operatorname{Co}-60$ & 9.94E-06 & $6.18 \mathrm{E}-03$ & 142 & $3.75 \mathrm{E}-06$ & $3,75 E-06$ & $1.02 \mathrm{E}-05$ & 1 . 05E-05 \\
\hline Se-79 & $1.23 \mathrm{E}-06$ & $7.62 \mathrm{E}-04$ & 1.74 & $8.80 \mathrm{E}-07$ & 80E-07 & 1.35E-06 & $2.83 \mathrm{E}-06$ \\
\hline Sr-90 & 0.267 & 166 & $3.80 E+05$ & 0.202 & 0.238 & $0.2 \mathrm{Bg}$ & 0.292 \\
\hline$Y-90$ & 0.267 & 166 & $3,80 E+05$ & 0.202 & 0.239 & 0.289 & 0.292 \\
\hline$Z r-93$ & $5.95 \mathrm{E}-06$ & $3.70 \mathrm{E}-03$ & 8.47 & $4.23 \mathrm{E}-06$ & $4.23 E-06$ & $6.58 \mathrm{E}-06$ & $1.27 \mathrm{E}-05$ \\
\hline $\mathrm{Nb}-93 \mathrm{~m}$ & 4.46E-06 & $2.77 \mathrm{E}-03$ & 6.35 & $3.25 E-06$ & $3.25 \mathrm{E}-06$ & $4.90 \mathrm{E}-06$ & $1.17 \mathrm{E}-05$ \\
\hline Tc.99 & $6.53 \mathrm{E}-05$ & 4 OGE-02 & 930 & $4.52 E-05$ & $550 \mathrm{E}-05$ & $7.57 \mathrm{E}-45$ & 8. 58E-05 \\
\hline $\mathrm{Ru}-106$ & $2.53 \mathrm{E}-09$ & $1.57 \mathrm{E}-06$ & $3.60 \mathrm{E}-03$ & $1.73 \mathrm{E}-09$ & $1.73 \mathrm{E}-09$ & $3.26 \mathrm{E}-09$ & $3.96 \mathrm{E}-09$ \\
\hline $\mathrm{Cd}-113 \mathrm{~m}$ & 2.39E-05 & $1.61 \mathrm{E}-02$ & 36.9 & $1.55 \mathrm{E}-0 \mathrm{~S}$ & $1.55 \mathrm{E}-05$ & $2.96 \mathrm{E}-0.5$ & 3.32E-05 \\
\hline Sb-125 & $4.26 \mathrm{E}-05$ & $2.65 \mathrm{E}-02$ & 80.6 & $1.57 \mathrm{E}-0 \mathrm{~s}$ & $1.57 \mathrm{E}-05$ & $4.41 \mathrm{E}-05$ & 4. 56E-05 \\
\hline $5 \mathrm{n}-126$ & I.86E-06 & $1.16 \mathrm{E}-03$ & 2.65 & $1.34 \mathrm{E}-06$ & 1.34E-06 & $2.05 \mathrm{E}-06$ & $443 \mathrm{E}-06$ \\
\hline $\mathrm{I}-129$ & $1.26 \mathrm{E}-07$ & $7.83 \mathrm{E}-05$ & 0.179 & $8.69 \mathrm{E}-08$ & $1.06 \mathrm{E}-07$ & $1.46 \mathrm{E}-07$ & 1.65E-0? \\
\hline $\mathrm{Cs}-134$ & $1.03 \mathrm{E}-06$ & 6.39E- -94 & 1.46 & $6.63 \mathrm{E}-07$ & $8.42 \mathrm{E}-07$ & $1.22 \mathrm{E}-06$ & $1.40 \mathrm{E}-06$ \\
\hline Cs.137 & $9.41 \mathrm{E}-02$ & 58.5 & $1.34 E+05$ & $8.42 E-02$ & $891 \mathrm{E}-02$ & $9.81 \mathrm{E}-02$ & 0.101 \\
\hline $\mathrm{Ba}+137 \mathrm{~m}$ & $8.90 \mathrm{E}-02$ & 55.3 & $1.27 \mathrm{E}+05$ & $6.72 \mathrm{E}-02$ & $672 \mathrm{E}-02$ & $9.28 \mathrm{E}-02$ & $9.54 \mathrm{E}-02$ \\
\hline $\mathrm{Sm}-151$ & $4.33 \mathrm{E}-03$ & 2.69 & $6.16 E+03$ & $3.12 \mathrm{E}-03$ & $3.12 \mathrm{E}-03$ & $4.77 \mathrm{E}-03$ & $1.02 \mathrm{E}-02$ \\
\hline Eu-152 & $3.45 \mathrm{E}-06$ & $2.15 E-03$ & \begin{tabular}{l|l|}
4.91 \\
\end{tabular} & $3.02 \mathrm{E}-06$ & $3.02 \mathrm{E}-96$ & $3.58 \mathrm{E}-06$ & $371 \mathrm{E}-06$ \\
\hline Eu-154 & $1.73 \mathrm{E}-04$ & 0.108 & 247 & $8.70 \mathrm{E}-05$ & 8.70E-95 & $2.05 \mathrm{E}-04$ & $2.17 \mathrm{E}-04$ \\
\hline Eu-155 & $1,7 \pi \mathrm{E}-04$ & 0.110 & 252 & $1.32 \mathrm{E}-04$ & $1.52 \mathrm{E}-04$ & $1.85 \mathrm{E}-04$ & $1.93 \mathrm{E}-04$ \\
\hline $\mathrm{Ra}-226$ & $3.17 E \cdot 10$ & $1.97 \mathrm{E}-07$ & 4 4.5IE-04 & $9.31 \mathrm{E}-11$ & $2.03 \mathrm{E}-10$ & $4.31 \mathrm{E}-10$ & $5.41 \mathrm{E}-10$ \\
\hline $\mathrm{Ra}-228$ & $3.18 \mathrm{E}-08$ & 1.98E-05 & $4.53 \mathrm{E}-02$ & $1,3 \mathrm{BE}-08$ & $2.26 \mathrm{E}-0 \mathrm{~g}$ & $4.25 \mathrm{E}-08$ & 5.38E-08 \\
\hline$A_{c}=227$ & 1.49E-09 & 9.25E-07 & 2.12E-03 & $4.18 \mathrm{BE}-10$ & 8.24E-10 & 2.15E-09 & 2.79E-09 \\
\hline $\mathrm{Pa}-231$ & $1.58 \mathrm{E}-09$ & 9.80E-07 & $2.24 \mathrm{E}-03$ & $1.15 \mathrm{E}-09$ & $1.15 E-09$ & I.89E-09 & 5.43E-09 \\
\hline Th-229 & $7.48 \mathrm{E}-10$ & $465 \mathrm{E}-0 ?$ & $1.06 \mathrm{E}-03$ & $3.53 \mathrm{E}-10$ & $5.46 \mathrm{E}-10$ & $9.82 E-10$ & $1.23 \mathrm{E}-09$ \\
\hline Th-232 & $2.12 \mathrm{E}-09$ & 1.32E-06 & 3.01E-03 & $1.12 E-09$ & $1.61 \mathrm{E}-09$ & $2.62 \mathrm{E}-09$ & 3.11E-09 \\
\hline U-232 & $1.65 \mathrm{E}-07$ & $1.02 \mathrm{E}-04$ & 0.235 & $9.27 \mathrm{E}-0 \mathrm{~B}$ & 1.28E-07 & $2.08 \mathrm{E}-07$ & $2.53 \mathrm{E}-07$ \\
\hline$U-233$ & $6.29 \mathrm{E}-07$ & $3.91 \mathrm{E}-04$ & 0.895 & $3.53 \mathrm{E}-07$ & $4.88 \mathrm{E}-07$ & $7.93 \mathrm{E}-07$ & 9.67 E-07 \\
\hline U-234 & $1.04 \mathrm{E}-05$ & 6.49E-03 & 14,9 & $9.98 \mathrm{E}-06$ & 1.02E-05 & $1.06 E_{-0} 05$ & 1.07E-05 \\
\hline U-235 & 4.12E-07 & 2.56E-04 & 0.587 & $3.93 \mathrm{E}-07$ & 4.04E-07 & $4.18 \mathrm{E}-07$ & $4.22 \mathrm{E}-07$ \\
\hline $\mathrm{U}-236$ & $5.39 \mathrm{E}-07$ & 3.35E-04 & 0.767 & $497 \mathrm{E} \cdot 07$ & $5.27 \mathrm{E}-07$ & $5.44 E-07$ & $5.50 \mathrm{E}-07$ \\
\hline $\mathrm{U}-238$ & $8.33 \mathrm{E}-06$ & S.1BE-03 & 11.9 & $7.885-06$ & 8.14E-06 & $846 \mathrm{E}-06$ & $8.56 \mathrm{E}-06$ \\
\hline $\mathrm{Np-237}$ & $2.52 \mathrm{E}-07$ & $1.56 \mathrm{E}-04$ & 0.358 & 1.86E-07 & $2.18 E-07$ & $2.86 \mathrm{E}-07$ & $3.19 \mathrm{E}-07$ \\
\hline Pu-238 & S.03E- 05 & $3.13 \mathrm{E}-02$ & 71.6 & $4.85 \mathrm{E}-05$ & $4.99 \mathrm{E}-0.5$ & S.07E-05 & 5.11E-05 \\
\hline Pu-239 & $1.20 \mathrm{E}-03$ & 0.748 & $1.71 E+03$ & $1.17 \mathrm{E}-03$ & 1.19E-03 & 1.22E-03 & $1.24 \mathrm{E}-03$ \\
\hline Pu-240 & 2. $15 \mathrm{E}-04$ & 0.134 & 307 & 2.11E-04 & $2.13 \mathrm{E}-04$ & $2.18 E-04$ & $2.20 \mathrm{E}-04$ \\
\hline Pu-241 & $2.75 \mathrm{E}-03$ & 1.71 & $3.91 \mathrm{E}+03$ & $262 \mathrm{E}-03$ & 2.72E-03 & 2.76E-03 & $2.78 \mathrm{E}-03$ \\
\hline $\mathrm{Pu}-242$ & $1.57 \mathrm{E}-08$ & $9.73 \mathrm{E}-06$ & $2.23 \mathrm{E}-02$ & $1.47 \mathrm{E}-0.8$ & $1.54 \mathrm{E}-0 \mathrm{~g}$ & $1.58 \mathrm{E}-08$ & 1.59E-08 \\
\hline Am-241 & 2.17E-05 & 1. $35 \mathrm{E}-02$ & 30.9 & $1.81 \mathrm{E}-05$ & $1.99 \mathrm{E}-05$ & $243 \mathrm{E}-05$ & $5.4] \mathrm{E}-05$ \\
\hline Am-243 & $8.38 \mathrm{E}-10$ & S.21E-07 & $1.19 \mathrm{E}-03$ & $7.14 \mathrm{E}-10$ & $7.71 \mathrm{E}-10$ & $1.22 \mathrm{E}-09$ & $1.84 \mathrm{E}-09$ \\
\hline $\mathrm{Cm}-242$ & $1.06 \mathrm{E}-07$ & $6.61 \mathrm{E}-05$ & 0.151 & $8.88 \mathrm{E}-0 \mathrm{8}$ & 8.88E-08 & 1.11E-07 & $1.16 \mathrm{E}-07$ \\
\hline $\mathrm{Cm}-243$ & 7.70E-09 & $4.79 \mathrm{E}-96$ & $1.10 \mathrm{E}-02$ & $6.03 E-09$ & $6.03 \mathrm{E}-09$ & 8.16E-09 & $8.61 E-09$ \\
\hline Cm-244 & 1.07E-07 & 6.64E-A5 & 0.132 & 4.01E-08 & $8.06 \mathrm{E}-08$ & 1.21E-07 & $1,35 \mathrm{E}-07$ \\
\hline Totels & $\mathbf{M}$ & $\mathrm{ng} / \mathrm{g}$ & $k e$ & $\begin{array}{l}95 \mathrm{Cl} \\
(\mathrm{Mor} \\
(\mathrm{L})\end{array}$ & $\begin{array}{c}67 \mathrm{CI}(\mathrm{M} \\
\text { or } \mathrm{gL})\end{array}$ & $\begin{array}{c}+67 \mathrm{CI}(\mathrm{M} \\
\text { or } \mathrm{g} / \mathrm{L})\end{array}$ & $\begin{array}{r}+95 \mathrm{Cl} \\
(\mathrm{M} \mathrm{or} \\
\mathrm{g} / \mathrm{L})\end{array}$ \\
\hline $\mathrm{Pu}$ & $2.03 \mathrm{E}-02(\mathrm{~g} / \mathrm{L})$ & - & 28.8 & $1.97 \mathrm{E}-02$ & $2.00 \mathrm{E}-\mathrm{O} 2$ & $205 E-02$ & $208 \mathrm{E}-02$ \\
\hline $\bar{u}$ & 0.104 & $1.54 \mathrm{E}+04$ & $3.54 E+04$ & $9.87 E-02$ & 0.102 & 0.106 & 0.107 \\
\hline
\end{tabular}

-Unknowns in tank solids inventory are assigned by Tank Layering Model (TLM)

+ Volume average for density, mass average Water $w \%$ and $\mathrm{TOC} w \mathrm{w} \% \mathrm{C}$ 
HNF-SD-WM-ER-323, Rev. 1

HDW Model Rev. 4

\begin{tabular}{|c|c|c|c|c|c|c|c|}
\hline \multirow[b]{3}{*}{$\begin{array}{l}\text { Physical } \\
\text { Properties }\end{array}$} & \multicolumn{7}{|c|}{ Single-Shell Tank 241-S-108 } \\
\hline & \multicolumn{7}{|c|}{ TLM Solids Composite Inventory Estimate* } \\
\hline & & & & $.95 \mathrm{Cl}$ & $-67 \mathrm{CI}$ & $+67 \mathrm{Cl}$ & $+95 \mathrm{CI}$ \\
\hline Total TLM Wast & $3.32 \mathrm{E}+04(\mathrm{~kg})$ & $(5.00 \mathrm{kgal})$ & $m$ & - & $\cdots$ & - & $=$ \\
\hline Heat Load & $0.123(\mathbf{k W})$ & (421 BTU/hr) & - & 894E-02 & 0.109 & 0.132 & 0.136 \\
\hline Bulk Density & $1.76(\mathrm{~g} / \mathrm{cc})$ & $\ldots$ & - & 1.51 & 1.53 & 2.11 & 2.24 \\
\hline Void Fraction & 0.536 & 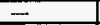 & - & $6.00 \mathrm{E}-02$ & 0.217 & 0.737 & 0.743 \\
\hline Water wt\% & 27.0 & - & $m$ & 2.64 & 10.0 & 42.6 & 44.2 \\
\hline TOC wt\% C (wet & 0 & - & - & 0 & 0 & 0 & 0 \\
\hline $\begin{array}{l}\text { Chemical } \\
\text { Constituents }\end{array}$ & mole/L & ppin & $\mathrm{kg}$ & $\begin{array}{l}95 \mathrm{Cl} \\
\text { (mole/L) }\end{array}$ & $\begin{array}{c}-67 \mathrm{CI} \\
\text { (moleril) }\end{array}$ & $\begin{array}{l}+67 \mathrm{CI} \\
\text { (mole/L) }\end{array}$ & $\begin{array}{r}+95 \mathrm{CI} \\
\text { (thole/L) }\end{array}$ \\
\hline $\mathrm{Nat}$ & 8.13 & $106 \mathrm{E}+05$ & $3.53 \mathrm{E}+03$ & 3.05 & 3.56 & 14.7 & 18.2 \\
\hline $\mathrm{A} 13+$ & 6.99 & $1.07 E+05$ & $3.56 \mathrm{E}+03$ & 6.58 & 6.74 & 715 & 7.15 \\
\hline$F_{e}^{3}+($ total Fe $)$ & 1.01 & $3.22 \mathrm{E}+04$ & $1.07 \mathrm{E}+03$ & 0.994 & 1.00 & 1.02 & 1.03 \\
\hline $\mathrm{Cr} 3+$ & $6.19 E-02$ & $1.83 E+03$ & 60.8 & $7.08 \mathrm{E}-03$ & $3.39 E-02$ & 0.522 & 1.06 \\
\hline Bij+ & 0 & 0 & 0 & 0 & 0 & 0 & 0 \\
\hline Lajt & 0 & 0 & 0 & 0 & 0 & 0 & 0 \\
\hline $\mathrm{Hg}^{2+}$ & 0 & 0 & 0 & 0 & 0 & 0 & 0 \\
\hline $\mathrm{Zs}(\mathrm{aj} \mathrm{ZrO(OH)2)}$ & 0 & 0 & 0 & 0 & 0 & 0 & 0 \\
\hline Pb2t & 0 & 0 & 0 & 0 & 0 & 0 & 0 \\
\hline $\mathrm{Ni2+}$ & $5.07 \mathrm{E}-02$ & $1.69 \mathrm{E}+03$ & 36.3 & $3.66 \mathrm{E}-92$ & 4.45E-02 & $5.54 \mathrm{E}-02$ & 5.4SE-02 \\
\hline 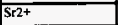 & 0 & 0 & 0 & 0 & 0 & 0 & 0 \\
\hline Min4t & 0 & 0 & 0 & 0 & 0 & 0 & 0 \\
\hline $\mathrm{Cn} 2+$ & 0.220 & $5,02 \mathrm{E}+03$ & 167 & 0.146 & 0.182 & 0.258 & 0.294 \\
\hline$\overline{K+}$ & $1.12 \mathrm{E}-02$ & 250 & 8.30 & $1.28 \mathrm{E}-03$ & $5.25 \mathrm{E}-03$ & $1,52 \mathrm{E}-02$ & $1.52 \mathrm{E}-02$ \\
\hline OH- & 25.6 & $2.48 \mathrm{E}+05$ & $8.23 E+03$ & 23.1 & 24.3 & 26.9 & 28.4 \\
\hline NO3- & 5.29 & $1.87 \mathrm{E}+05$ & $6.20 \mathrm{E}+03$ & $6.03 \mathrm{E}-02$ & 6.03E-02 & 13.5 & 179 \\
\hline NO2- & 146 & $3.82 \mathrm{E}+04$ & $1.27 \mathrm{E}+03$ & 0.132 & 0.595 & 2.15 & 2.15 \\
\hline CO32: & 0.220 & $7.51 E+03$ & 249 & 0.146 & 0.182 & 0.258 & 0.294 \\
\hline PO43- & 0 & 0 & 0 & 0 & 0 & 0 & 0 \\
\hline SO42- & 1.04E-02 & 569 & 18.9 & $1.19 \mathrm{E}-03$ & $4.87 \mathrm{E}-03$ & $1.41 E-02$ & $1.41 \mathrm{E}-02$ \\
\hline $5 i$ (as $\left.\mathrm{SiO}_{2}-\right)$ & 8.06E-03 & 129 & 4.28 & $9.21 \mathrm{E}-04$ & $3.77 \mathrm{E}-03$ & $109 \mathrm{E} .02$ & $109 \mathrm{E}-02$ \\
\hline F- & 0 & 0 & 0 & 0 & 0 & 0 & 0 \\
\hline $\mathrm{Cl}$ & $5.16 \mathrm{E}-02$ & $1.04 \mathrm{E}+03$ & 34.6 & $5.91 \mathrm{E}-03$ & $2.42 \mathrm{E}-02$ & $7.00 \mathrm{E}-02$ & 0.157 \\
\hline C6H5O73- & 0 & 0 & 0 & 0 & 0 & 0 & 0 \\
\hline EDTA4- & 0 & 0 & 0 & 0 & 0 & 0 & 0 \\
\hline HEDTA3. & 0 & 0 & 0 & 0 & 0 & 0 & 0 \\
\hline glycolate- & 0 & 0 & 0 & 0 & 0 & 0 & 0 \\
\hline acctale- & 0 & 0 & 0 & 0 & 0 & 0 & 0 \\
\hline oxalinte2- & 0 & 0 & 0 & 0 & 0 & 0 & 0 \\
\hline DBP & 0 & 0 & 0 & 0 & 0 & 0 & 0 \\
\hline butanol & 0 & 0 & 0 & 0 & 0 & 0 & 0 \\
\hline $\mathrm{NH3}$ & 0.256 & $2,47 E+03$ & 82.1 & 6.55E- 02 & $9.25 \mathrm{E}-02$ & 0.270 & 0.284 \\
\hline Fe(CN)64 & 0 & 0 & 0 & 0 & 0 & 0 & 0 \\
\hline
\end{tabular}

"Unknowns in unk solids inventoty are assigned by Tunk Layering Model (TLM). 
HDW Model Rev. 4

\begin{tabular}{|c|c|c|c|c|c|c|c|}
\hline \multirow{4}{*}{$\begin{array}{l}\text { Physical } \\
\text { Properties }\end{array}$} & \multicolumn{7}{|c|}{ Single-Shell Tank 241-S-108 } \\
\hline & \multicolumn{7}{|c|}{ SMM Composite lnventory Estimate } \\
\hline & & & & & & & \\
\hline & & & & $-95 \mathrm{CI}$ & $-67 \mathrm{CI}$ & $+67 \mathrm{Cl}$ & $+95 \mathrm{CI}$ \\
\hline Total SMM Wast & $3.14 \mathrm{E}+06(\mathrm{~kg})$ & $(497 \mathrm{kgBl})$ & 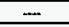 & $\ldots$ & $\ldots$ & + & $=$ \\
\hline Heat Load & $3.98(\mathrm{~kW})$ & (1.36E+04 BTU/hr) & $\cdots$ & 373 & 3.85 & 4.12 & 4.14 \\
\hline Bulk Density* & $1.67(\mathrm{~g} / \mathrm{cc})$ & - & - & 1.60 & 1.64 & 1,70 & 1.70 \\
\hline Water wt\% & 30.0 & $\ldots$ & - & 27.8 & 28.7 & 31.5 & 34.0 \\
\hline TOC wt\% C (wet & 0.321 & - & $=$ & 0.286 & 0.303 & 0.338 & 0.355 \\
\hline $\begin{array}{l}\text { Chenical } \\
\text { Constiftuents } \\
\end{array}$ & moler & ppio & $\mathrm{kg}$ & $\begin{array}{l}-95 \mathrm{CI} \\
\text { (mole/t) }\end{array}$ & $\begin{array}{l}-67 \mathrm{CI} \\
\text { (molen })\end{array}$ & $\begin{array}{c}+67 \mathrm{CI} \\
\text { (mole/L) }\end{array}$ & $\begin{array}{l}+95 \mathrm{CI} \\
\text { (mole/L) }\end{array}$ \\
\hline $\mathrm{Nat}$ & 15.4 & $2.13 \mathrm{E}+05$ & $6.68 E+05$ & 13,9 & 14.8 & 15.9 & 16.3 \\
\hline A]3+ & 2.15 & $3.47 \mathrm{E}+04$ & $1.09 \mathrm{E}+05$ & 186 & 2.00 & 2.31 & 2.22 \\
\hline $\mathrm{Fe} 3+($ total $\mathrm{Fe}$ ) & $9.59 \mathrm{E}-03$ & 320 & $1.01 \mathrm{E}+03$ & $7.88 \mathrm{E}-03$ & $8.72 \mathrm{E}-03$ & $1.05 \mathrm{E}-02$ & $1.13 \mathrm{E}-02$ \\
\hline $\mathrm{Cr} 3+$ & 0.221 & $687 \mathrm{E}+03$ & $2.16 \mathrm{E}+04$ & 0.163 & 0.194 & 0.226 & 0.231 \\
\hline $\mathrm{Bi3+}$ & $8,02 E-04$ & 100 & 315 & $7.21 \mathrm{E}-04$ & $7.61 \mathrm{E}-04$ & $8.43 \mathrm{E}-04$ & $8.82 \mathrm{E}-04$ \\
\hline Lnjt & 1.17E-09 & 9.74E-05 & 306E-04 & 8.91E-10 & $1.03 \mathrm{E}-09$ & $1.32 \mathrm{E}-09$ & $1.45 \mathrm{E}-09$ \\
\hline $\mathrm{Hg} 2^{+}$ & $6.71 \mathrm{E}-06$ & 0.806 & 2.53 & $6.29 \mathrm{E}-06$ & $6.53 \mathrm{E}-06$ & $6.80 \mathrm{E}-06$ & $6.89 \mathrm{E}-06$ \\
\hline $\mathrm{Zr}$ (as $\mathrm{ZrO}(\mathrm{OH}) 2$ ) & 1.66E-04 & 9.06 & 28.5 & $1.51 \mathrm{E}-04$ & $1.56 \mathrm{E}-0.94$ & $1.72 E-04$ & $1.0 \mathrm{E}-04$ \\
\hline $\mathrm{Pb2+}$ & $7,92 \mathrm{E}-04$ & 98.3 & 309 & $6.64 E-09$ & $7.2 \pi \mathrm{E}-104$ & $8.58 E-04$ & 9.21E-04 \\
\hline $\mathrm{Ni} 2+$ & $6.26 \mathrm{E}-03$ & 220 & 691 & 6.04E-03 & $6.15 E-03$ & $6.37 \mathrm{E}-03$ & $6.48 \mathrm{E}-03$ \\
\hline $5 \sqrt{2+}$ & 0 & 0 & 0 & 0 & 0 & 0 & 0 \\
\hline Mn4t & 3.01E-03 & 98.8 & 311 & 2.18E-03 & $2.59 \mathrm{E}-03$ & $3.42 \mathrm{E}-03$ & $383 E-03$ \\
\hline $\mathrm{C}_{2+}$ & $3.20 \mathrm{E}-02$ & 767 & $2.41 \mathrm{E}+03$ & $2.77 \mathrm{E}-02$ & $2.98 \mathrm{E}-02$ & 3.41E-02 & $3.62 \mathrm{E}-02$ \\
\hline $\mathrm{K}+$ & $6.48 \mathrm{E}-02$ & $1.52 E+03$ & $4.77 \mathrm{E}+03$ & $5.79 \mathrm{E}-02$ & 6. $11 \mathrm{E}-02$ & $6.88 \mathrm{E}-02$ & $7.01 \mathrm{E}-02$ \\
\hline OH- & 13.0 & $1.32 k+05$ & $4.16 \mathrm{E}+0.5$ & 11.2 & 12.1 & 14.0 & 13.5 \\
\hline NO3- & 5.17 & $1.92 \mathrm{E}+05$ & $6.03 \mathrm{E}+05$ & 4.72 & 4.94 & 5.35 & 5.35 \\
\hline NO2- & 2.92 & $8.03 \mathrm{E}+04$ & $2.53 \mathrm{E}+05$ & 2.55 & 2.73 & 3.10 & 3.27 \\
\hline CO32- & 0.284 & $1.02 \mathrm{E}+04$ & $3.21 E+04$ & 0.256 & 0.268 & 0,304 & 0.307 \\
\hline PO43- & $6.33 \mathrm{E}-02$ & $3.60 \mathrm{E}+03$ & $1.13 \mathrm{E}+04$ & 5.64E-02 & $5.88 \mathrm{E}-02$ & $6.48 \mathrm{E}-02$ & $6.62 \mathrm{E}-02$ \\
\hline SO42- & 0.188 & $1.08 E+04$ & $3.40 \mathrm{E}+04$ & 0.143 & 0.165 & 0.213 & 0.234 \\
\hline Si (as SiO32-) & $7.18 \mathrm{E}-02$ & $121 E+03$ & $3.79 E+03$ & $6.00 \mathrm{E}-02$ & $6,58 \mathrm{E}-02$ & $7.78 \mathrm{E}-02$ & $8.35 \mathrm{E}-02$ \\
\hline F- & $3.83 \mathrm{E}-02$ & 436 & $1.37 \mathrm{E}+03$ & $3.13 \mathrm{E}-02$ & 3.43E-02 & $418 \mathrm{E}-02$ & $4.45 \mathrm{E} .02$ \\
\hline $\mathrm{Cl}-$ & 0.261 & $5.54 \mathrm{E}+03$ & $1.74 E+04$ & 0.229 & 0.244 & 0.279 & 0.278 \\
\hline C6H5O73- & $2.20 \mathrm{E}-02$ & $2.48 \mathrm{E}+03$ & $7.81 E+03$ & $2.03 \mathrm{E}-02$ & $2.11 \mathrm{E}-02$ & $2.28 \mathrm{E}-02$ & $2.36 \mathrm{E}-02$ \\
\hline EDTA4: & 2.37E-03 & 409 & $1.29 \mathrm{E}+03$ & 1.1SE-03 & 1.75E- 03 & $3.00 \mathrm{E}-03$ & 3.61E-03 \\
\hline HEDTA3- & $3.90 \mathrm{E}-03$ & 640 & $2.01 E+03$ & $1.46 \mathrm{E}-03$ & $2.65 \mathrm{E}-03$ & S.15E-03 & $6.38 \mathrm{E}-03$ \\
\hline glycolate- & $3.44 E-02$ & $1.55 E+03$ & $4.86 \mathrm{E}+03$ & $1.996-02$ & $2.70 \mathrm{E}-02$ & $4.19 \mathrm{E}-02$ & $4.90 \mathrm{E}-02$ \\
\hline Rcetate- & $2.73 \mathrm{E}-03$ & 96.3 & 303 & 2.21E-03 & $2.46 \mathrm{E}-03$ & 2.99E-03 & 3.24E-03 \\
\hline oxalste2- & $1.54 \mathrm{E}-09$ & $8.09 \mathrm{E}-05$ & 2.54E-04 & $1.38 \mathrm{E}-09$ & 1.46E.-09 & $1.61 \mathrm{E}-09$ & 1.69E-09 \\
\hline DBP & $1.48 \mathrm{E}-02$ & $187 \mathrm{E}+03$ & $5.87 \mathrm{E}+03$ & $1.19 \mathrm{E}-02$ & $1.34 \mathrm{E}-02$ & $1.63 \mathrm{E}-02$ & $1.77 \mathrm{E}-02$ \\
\hline butenol & $1.48 \mathrm{E}-02$ & 658 & $2.07 \mathrm{E}+03$ & $1.19 \mathrm{E}-02$ & $1.34 \mathrm{E}-02$ & $1,63 \mathrm{E}-02$ & $1.77 \mathrm{E}-02$ \\
\hline NH3 & $7.75 \mathrm{E}-02$ & 739 & $2.48 \mathrm{E}+03$ & $6.43 E-02$ & $6.95 \mathrm{E}-02$ & 8. 78E-02 & $9.97 \mathrm{E}-02$ \\
\hline Fo(CN)64- & 0 & 0 & 0 & 0 & 0 & 0 & 0 \\
\hline
\end{tabular}

-Density is calculated based on $\mathrm{Na}, \mathrm{OH}-$, and $\mathrm{AlO2}$.

tWater wt\% derived from the difference of density and total dissolved species. 
HDW Model Rev. 4

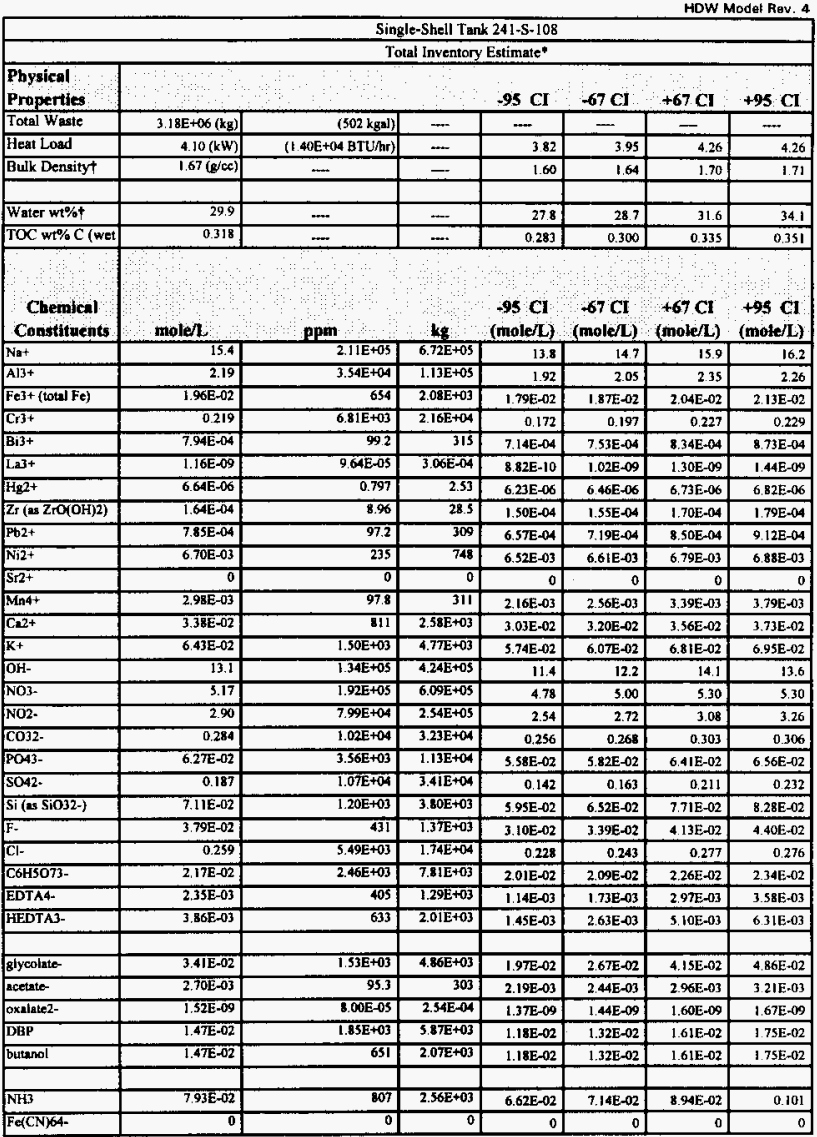

-Unknowns in tank solids inventory are assigned by Tank Layering Model (TLM)

+Water wt\% derived from the difference of density and total dissolved species 


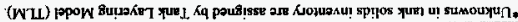

\begin{tabular}{|c|c|c|c|c|c|c|c|}
\hline E0: $\exists 102$ & $60-3 \angle 0 \tau$ & ro:G9I 2 & poraSLI & 689 & $\angle 0 z$ & $50-3551$ & $n$ \\
\hline $20-9101$ & E0-3xo' 6 & $\hat{E} 0-39 \mathcal{L}^{\prime} \boldsymbol{9}$ & $50-3 / 965$ & $6+10$ & 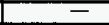 & $\left.(1 /)^{A}\right)\left(00-968^{\prime} \mathrm{L}\right.$ & $n_{d}$ \\
\hline$\left(\mathbb{1}^{3}\right.$ & (1200 & $6 \mathrm{i}^{8} 10$ & tid & 87 & yon: & $\mathbf{W}$ & stmos \\
\hline $10 \mathrm{~W})$ & Wo10 $69+$ & W) 1 < & to $\mathbf{x}$ ) & & & & \\
\hline $1956+$ & & & 1356 & & & & \\
\hline $6003 \angle 9 \varepsilon$ & $60-3 \angle 95$ & $60-3 L 271$ & $01-360^{\circ}[5$ & s0-zIITs & 907-3BS: & $60-30 L Z z$ & $t \tau^{2}-\mathrm{ur}$ \\
\hline $60-92 / \%$ & $60-72 L \bar{L}$ & $60-9 \operatorname{ltg} \mathrm{f}$ & $60-919^{\prime} \varepsilon$ & $50-3869$ & $90-9012$ & $60-369 \mathrm{C}$ & \{tz-шई \\
\hline $20-3291$ & $\angle 0-329$ & $40-3657$ & $\angle 0-38 S I$ & $\varepsilon 0-2 \not 0, \varepsilon$ & $50-3916$ & कoralgl & $2 t \tau-140$ \\
\hline $60-3 b E^{\prime} 1$ & $01-360^{\circ} \mathrm{I}$ & $11-3 b 5 \cdot f$ & $21-3998$ & 90-ack! I & 90-9IC 6 & $11+7 \angle 5: C$ & $\varepsilon b z-\omega \mathrm{V}$ \\
\hline por3ED I & so-301' & $90-96 L \mathrm{E}$ & $\angle 0-3926$ & ร5ा० & 50-319\% & $900960 \%$ & $\operatorname{lnt}$ - बाiv \\
\hline 6or-abct & $60-\mathrm{ABO}^{2} \mathrm{z}$ & $600-9951$ & 60-31Et & ร0:350 \& & $90-9601$ & $60-3281$ & $2 t z-\pi_{d}$ \\
\hline $00-360 ' s$ & Do:-3SS" & toragot $\mathrm{c}$ & po:-358 2 & $15 t$ & $92 z^{\prime} 0$ & $D 0 \cdot \mathrm{A} \angle 6^{\prime} \mathrm{E}$ & $I t z-\pi_{d}$ \\
\hline $50-3998$ & $50-3 \varepsilon L L$ & $50-9 R 25$ & $500958 \mathrm{~b}$ & $8 Z I$ & Z0-3Ss $\varepsilon$ & $50-79 L^{\prime} 9$ & $0+2-\pi d$ \\
\hline poraso's & porsop's & norapor & porJ6E' & 26.8 & 6920 & $p 0 \theta \theta \varepsilon^{\prime}$ & $6 \varepsilon z^{-n_{d}}$ \\
\hline $90-38+8$ & $90-7 \angle 5 L$ & $90-399 \mathrm{~s}$ & 90-3SL" & szio & $E 0-3 L L E$ & $90-3199$ & $8 \mathfrak{E Z ^ { - 1 } \mathrm { nd }}$ \\
\hline$\angle 0,96 E 1$ & LO-365 I & $80-3187$ & $80-3811$ & $\angle 0-9166 !$ & $50-3985$ & $\angle 0-3501$ & $\angle E \tau^{-d} \mathbb{N}$ \\
\hline \begin{tabular}{|l|l|}
$0-969{ }^{\prime}$ & \\
\end{tabular} & $\angle 0-359^{\prime} \mid$ & $80-969 \%$ & $80-36 \mathrm{El}$ & E0-300 z & $50-3269$ & $\angle 0-3 z z^{\prime} 1$ & $8 \varepsilon 2-1$ \\
\hline $60-\operatorname{d95} 7$ & $60-3952$ & $01-3288$ & 013912 & 50-795: & $90-3601$ & $60^{-9}-988 \mathrm{I}$ & $9 \varepsilon z-n$ \\
\hline $60-3569$ & $600-3569$ & $60-9002$ & 01.9985 & $507 \exists 696$ & $90-3267$ & 60 -3EI'S & $552-n$ \\
\hline $20-329^{\circ} 1$ & $10-3291$ & $800-3855$ & $80-99 \varepsilon^{\prime} t$ & g0-asszz & $50-38<9$ & $\angle 0-3611$ & $p \varepsilon z=\pi$ \\
\hline$\overline{\epsilon 1-3 \mid L^{\prime} z}$ & $E 1-a \mid L z$ & $71-96 C^{6}$ & b1-98zZ & $60-3 \angle L \varepsilon$ & 01-3bl! & $\varepsilon 1-300 \%$ & $\varepsilon £ z-\Omega$ \\
\hline$z 1-301 / 2$ & 213012 & $z 1-35 r z$ & $81 \cdot 366 \mathrm{~s}$ & $80-3006$ & $60-986 \mathrm{Z}$ & $21 \cdot 9+2 \mathrm{~S}$ & $2 \mathrm{Ez}-\Omega$ \\
\hline $91-3108$ & $91-9108$ & $91-\exists \angle L Z$ & $\angle L-79 L ' 9$ & II-GtI! & ह1-AOS $\varepsilon$ & $91-916 \mathrm{~s}$ & $z \varepsilon z-4 \mid 1$ \\
\hline$z 1 \cdot 38<1$ & $21 \cdot 984: \mathrm{t}$ & zI- ISLI $_{1}$ & $21 \cdot 36 L 1$ & so-aber & $60-3101$ & $Z 1=0 \angle L t$ & $6 z 2-41$ \\
\hline $80-9894$ & $60-9002$ & OT-G96' & $11-3128$ & SO-G9E'I & $40-960 \%$ & T1-a81' & $I \varepsilon Z-B_{d}$ \\
\hline $80 \div 3201$ & $60-3252$ & $60-9007$ & $01 \cdot 990 \mathrm{E}$ & $50-3006$ & $90-\mathrm{al} L z$ & $60-39 L 5$ & $\angle Z Z-2 Y$ \\
\hline $51-3 b c^{\prime} 6$ & $51-9 b E 6$ & st-assí 6 & 51-Z1006 & $01-9 \leqslant L I$ & zT-38z's & $51-997^{\prime} 6$ & $8 z \tau-x y$ \\
\hline $60-310 z$ & $60-3951$ & $0 \mathrm{I}-\mathrm{a}+09$ & $01-38+1$ & sorapoz & LOSGSI 9 & $60-380 \mathrm{I}$ & $9 z z-\nabla y$ \\
\hline to-acf' & bo-acE & poragr' & Do-3it? & $s z 9$ & 8810 & DOR $30 E$ E & कSI-ng \\
\hline $50=166 z$ & $50-356 \tau$ & 50-310 I & $90-3 \angle 6 z$ & 8000 & $20-3 e z ;$ & 50-39!'z & $\left.\operatorname{ts} I^{-n}\right]$ \\
\hline $90-890 \mathrm{C}$ & $90-390^{\circ} \mathrm{L}$ & $90-\mathrm{G} 169$ & $90-358^{\prime} 9$ & $z \varepsilon 10$ & $50-386 \varepsilon$ & $90 \cdot 300^{\circ} \mathrm{L}$ & $z s i^{-n g}$ \\
\hline $20-9+92$ & co-36ez & HOAEOO & no-396i & rze & 9260 & $80-91<1$ & ISI-UIS \\
\hline $20-9189$ & $20-7 / 89$ & $20-3906$ & $20-30011$ & $10+7001$ & $z^{\prime \prime o \varepsilon}$ & $20-30 E 5$ & $\mathrm{WIE}_{\mathrm{LE}}-\mathrm{E} \mathrm{G}$ \\
\hline 20-392L & $20-39 z L$ & Z0-act' $\varepsilon$ & 200-38t! & $60+3901$ & 6.18 & $20-9095$ & $\left\langle\left[\mathrm{~T}^{-5} \mathrm{~s}\right.\right.$ \\
\hline BorgzS' & $80-92 s^{\prime} \varepsilon$ & $80-3 z s^{\prime} \mid$ & $60-36 \Gamma^{2} \mathrm{C}$ & no-apl's & 50-35s 1 & $80-3 z \angle z$ & $\operatorname{te}[-8 \mathrm{~s}]$ \\
\hline 80-3ELt & 80-3el't & $80-a 261$ & $60-3 B+\varepsilon$ & +0 - $99<5$ & $50-3 \mp \angle 1$ & $80-300$ & $6 \tau \mathrm{L}^{-1}$ \\
\hline 50-3p [1] & 90-3001 & $\angle 0-39+\varepsilon$ & $80-35+8$ & $20-300 \div 1$ & po:312t & LOF $36 E L$ & $97 I^{-1 U S}$ \\
\hline $90-38 L^{\prime} 1$ & $90-38 L^{\circ} \mathrm{I}$ & $\angle 0-a+19$ & $\angle 0-9051$ & $20-39+2$ & pog $\angle L L$ & 90-बाहा & $s z I^{-q S}$ \\
\hline $90-\overline{38} 6$ & $90-38+6$ & $90-\mathrm{BLC} \mathrm{E}$ & L0, 9008 & zह1'0 & $60-386 \mathrm{E}$ & $90-3669$ & $u_{\varepsilon} \|[-P J$ \\
\hline $2 t^{-}-\pi 8 E^{\prime} \varepsilon$ & Z1-38EE & $21-9 L I 1$ & $\varepsilon 1$ - $398 z$ & $80-3 z c^{\prime}$ & $60-32 \cdot 1$ & $z \mathrm{I}-\mathrm{gos} z$ & $901^{-n y}$ \\
\hline s0-asiz & Sorasiz & $90=360$ & $90-3181$ & $000^{\circ} 0$ & $50-\mathrm{azO}^{2} 6$ & s0-38s I & $66^{-5} \mathrm{I}$ \\
\hline 50-בIZ'E & $90-315 z$ & $\angle 0-3898$ & $10-3 z 1 z$ & $20-315 \varepsilon$ & $50-390^{\circ} 1$ & $90-358$ & us6-9N \\
\hline $50-3 £ 0^{\circ} \mathcal{E}$ & $90 \% 380 \varepsilon$ & $90-3901$ & $\angle 0-365 z$ & $20-36 z^{b}$ & $50-3621$ & $90-3 \angle z z$ & $\varepsilon 6-\sqrt{Z}$ \\
\hline 601 & 201 & $218^{\circ} 0$ & 2990 & $60+39<1$ & 625 & 6260 & $06-\lambda$ \\
\hline 501 & 201 & 2180 & 2990 & $n+39<1$ & 625 & 8260 & $06-15$ \\
\hline $90 \cdot 35 I L$ & Lto-3ts'g & $\angle 0$-aszz & $20-30 \mathrm{~s} s$ & $80-360^{6} 6$ & po-anc $z$ & $\angle 0-318$ & $6 L-2 S$ \\
\hline $90^{\circ}$ 3IZ' t & $90-31 z^{\prime}$ & $60.381 \%$ & $\angle 0 \cdot \mathrm{azO} I$ & $20-3691$ & to- $660 \mathrm{~s}$ & $\angle 0 \div 3668$ & $09^{-0} 3$ \\
\hline $50-96 \div \mathrm{I}$ & $00-32 s^{\prime}$ & EQ-gZZ'T & $\varepsilon 0-\mathrm{a} 00^{\prime} 1$ & 292 & $06<\%$ & ह0-36\& ! & $\varepsilon 9-! N$ \\
\hline $50-3091$ & $50-3291$ & SO-GIEI & SO-ZZZO I & $182^{\prime} 0$ & $60-39+8$ & 50-96t ! & $6 \xi-! N$ \\
\hline $90-\bar{Z} \angle 0 \mathrm{E}$ & $90-3 \angle 0 \varepsilon$ & $90-39011$ & L0-365 $z$ & $20-3 \mathrm{k} z+b$ & $60-96 z^{\prime} 1$ & 9r-39z $z$ & $+[-\partial$ \\
\hline $50 \cdot 301+5$ & $50-301 \%$ & sorg $\angle P I$ & $90-\exists 50 \varepsilon$ & Ess: & $20-7 \angle 91$ & $50-926, z$ & $\varepsilon+H$ \\
\hline (บท) & $(\mathrm{T} / \mathrm{T})$ & (1/T) & $(7 / m)$ & 5 & 8mor & in & 9uगmipsua \\
\hline $1956+$ & $10<9+$ & $10<9$ & 1256 & & & & jojąapop:y \\
\hline 0 & 0 & 0 & 0 & $=$ & - & 0 & 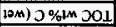 \\
\hline 200 & $9 \tau b$ & 001 & 592 & - & - & $0<2$ & $\% 1 \mathrm{M}$ IDI甘 $M$ \\
\hline$E+\angle 0$ & $\angle E L O$ & $\angle I 20$ & z0-9009 & $=$ & $=$ & $9 E s 0$ & tool25edA P!O \\
\hline$p z z$ & $11 z$ & ES 1 & ISI & $\cdots$ & - & $(00 / 1) 9(1$ & 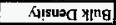 \\
\hline 9510 & zह10 & $601 \%$ & $20-366.8$ & $\cdots$ & 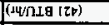 & $(M y) \in Z 10$ & P8O7 189H \\
\hline$=$ & $\infty$ & - & - & $\cdots$ & $(108 \times 100 \%$ & 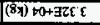 & ${ }_{158} M$ WTI |E) I \\
\hline \multicolumn{7}{|c|}{ D $96+\quad$ IO $69+\quad$ ID $69 \quad \therefore$ D $56 \%$} & 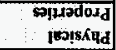 \\
\hline \multicolumn{8}{|c|}{ 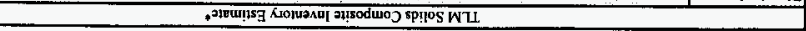 } \\
\hline & & & $801 \cdot 5=[t$ & $\overline{E \perp\|\|_{1 S}}$ & IS & & \\
\hline
\end{tabular}


HNF-SD-WM-ER-323, Rev. 1

HDW Model Rev. 4

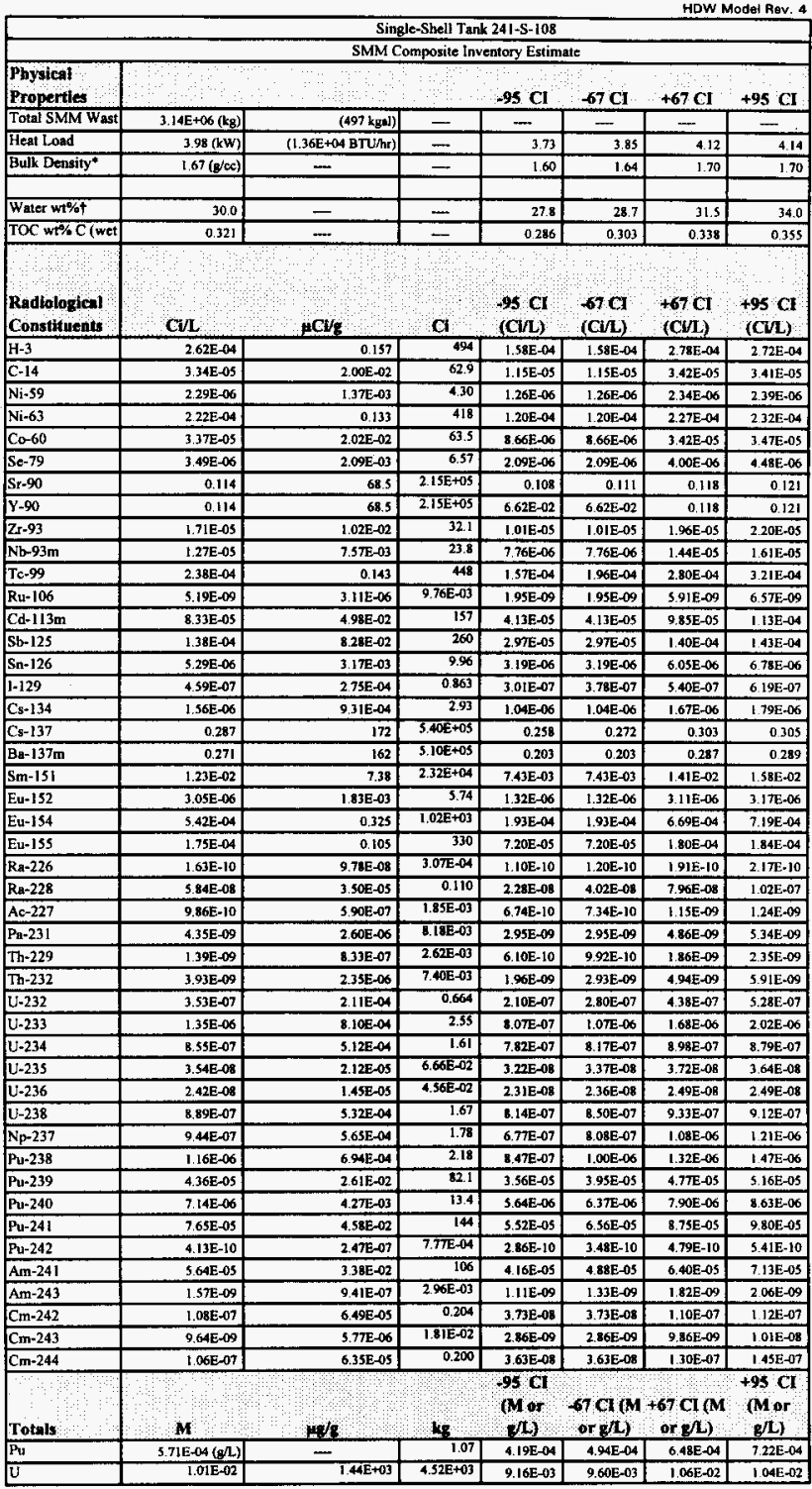

-Density is calculated based on $\mathrm{Na}$, OH-, and $\mathrm{AlO2}$

+Water wt\% derived from the difference of density and total dissolved species. 
HNF-SD-WM-ER-323, Rev. 1

HDW Model Rev. 4

\begin{tabular}{|c|c|c|c|c|c|c|c|}
\hline \multirow[b]{3}{*}{$\begin{array}{l}\text { Physical } \\
\text { Properties }\end{array}$} & \multicolumn{7}{|c|}{ Single-Shell Tank 241-S-108 } \\
\hline & \multicolumn{7}{|c|}{ Total Inventory Estimate" } \\
\hline & & & & $-95 \mathrm{CI}$ & $-67 \mathrm{CI}$ & $+67 \mathrm{CJ}$ & $+95 \mathrm{CI}$ \\
\hline Total Waste & $3.18 \mathrm{E}+06(\mathrm{~kg})$ & $(502 \mathrm{kgB}:)$ & (-) & - & $=$ & $=$ & $\cdots$ \\
\hline Heat Load & $4.10(\mathrm{~kW})$ & $(1.40 \mathrm{E}+04 \mathrm{BTU} / \mathrm{hr})$ & $\ldots$ & 3.82 & 3.95 & 426 & 4.26 \\
\hline Bulk Densityt & $1.67(g / c c)$ & $\cdots$ & - & 1,60 & 1.64 & 1.70 & 1.71 \\
\hline Water wt $\%+$ & 200 & . & & & & & \\
\hline TOC wt\% C (wet) & 29.9 & - & - & 27.8 & 28.7 & 31.0 & 34.1 \\
\hline & 0.3181 & $=$ & $=$ & 0.283 & $0.300 \mid$ & 0.335 & 0351 \\
\hline $\begin{array}{l}\text { Radiological } \\
\text { Constituents }\end{array}$ & CUL & $\mathrm{HC} / \mathrm{s}$ & $\mathrm{Cl}$ & $\begin{array}{l}-95 \mathrm{CI} \\
(\mathrm{CH} / \mathrm{L})\end{array}$ & $\begin{array}{l}67 \mathrm{CI} \\
(\mathrm{CVL})\end{array}$ & $\begin{array}{r}+67 \mathrm{CI} \\
(\mathrm{Cu} / \mathrm{L})\end{array}$ & $\begin{array}{l}+95 \mathrm{CI} \\
(\mathrm{CH} / \mathrm{L})\end{array}$ \\
\hline $\mathrm{H}-3$ & $2.60 \mathrm{E}-04$ & 0.156 & 494 & $1.56 \mathrm{E}-04$ & $1.56 \mathrm{E}-04$ & $2.76 \mathrm{E}-04$ & $2.70 \mathrm{E}-04$ \\
\hline $\mathrm{C}-14$ & $3.31 \mathrm{E}-05$ & $1.98 \mathrm{E}-02$ & 62.9 & $1.14 \mathrm{E}-05$ & $1.14 \mathrm{E}-05$ & $3.39 \mathrm{E}-05$ & 3.38E-05 \\
\hline $\mathrm{Ni}-59$ & $241 \mathrm{E}-06$ & 1.44E 03 & 4.58 & $1.39 \mathrm{E}-06$ & $1.39 \mathrm{E}-\infty 6$ & $2.46 \mathrm{E}-06$ & $251 \mathrm{E}-06$ \\
\hline $\mathrm{Ni}-63$ & $2.34 \mathrm{E}-04$ & 0.140 & 444 & $1.33 \mathrm{E}-04$ & $1.33 \mathrm{E}-04$ & 2.39E-04 & $2+4 E-04$ \\
\hline $\operatorname{Co}-60$ & $3.34 \mathrm{E}-05$ & $2.00 \mathrm{E}-02$ & 63.5 & $8.58 \mathrm{E}-06$ & $8.58 E-06$ & $3.39 \mathrm{E}-05$ & 3.44E-05 \\
\hline Se-79 & $3.46 \mathrm{E}-06$ & $2.07 \mathrm{E}-03$ & 6.58 & $2.08 \mathrm{E}-06$ & $2.08 \mathrm{E}-06$ & $3.96 \mathrm{E}-06$ & 4 44E-06 \\
\hline Sr-90 & 0.123 & 73,3 & $2,33 \mathrm{E}+05$ & 0.116 & 0.119 & 0.126 & 0.129 \\
\hline$Y-90$ & 0.123 & 73.3 & 2.33E+05 & $7.47 E-02$ & $747 E-02$ & 0.126 & 0.129 \\
\hline $\mathrm{Zr} \cdot 93$ & 1.69E-05 & $1.0 \mathrm{EE}-02$ & 32.1 & $9,99 \mathrm{E}-06$ & $9,99 \mathrm{E}-06$ & $1.94 \mathrm{E}-05$ & 2.18E-09 \\
\hline $\mathrm{Nb}-93 \mathrm{~m}$ & $1.25 E-05$ & 7.5iE-03 & 23.8 & $7.70 \mathrm{E}-06$ & $7.70 \mathrm{E}-06$ & 1.43E-05 & $1.60 \mathrm{E}-05$ \\
\hline Te. 99 & $2.36 \mathrm{E}-04$ & 0.141 & 448 & $1.55 \mathrm{E}-04$ & 1.95E-04 & $2.78 E-04$ & $3.18 \mathrm{E}-04$ \\
\hline Ru-106 & $5.14 E-09$ & $3.07 \mathrm{E}-06$ & $9.76 E-03$ & $193 \mathrm{E}-09$ & $1.93 \mathrm{E}-09$ & 5.86E-09 & $6.51 \mathrm{E}-09$ \\
\hline Cd-113m & $8.25 E-05$ & $4.94 \mathrm{E}-02$ & 157 & $4.10 \mathrm{E}-05$ & $4,10 \mathrm{E}-05$ & $9.76 \mathrm{E}-05$ & 1.12E-04 \\
\hline $\mathrm{Sb}-125$ & 1.37E-04 & $8.19 \mathrm{E}-02$ & 260 & 2.94E-05 & $2.94 E_{0}-05$ & $1.39 \mathrm{E}-04$ & $141 \mathrm{E}-04$ \\
\hline $\mathrm{Sn}-126$ & 5.25E-06 & $3.14 \mathrm{E}-03$ & 9.97 & $3.17 \mathrm{E}-06$ & $3.17 \mathrm{E}-06$ & $6.00 \mathrm{E}-06$ & $6.72 \mathrm{E}-06$ \\
\hline 1.129 & 4.55E-07 & $2.72 \mathrm{E}-04$ & 0.864 & $2.98 \mathrm{E}-07$ & 3.74E- -17 & $5.35 \mathrm{E}-07$ & 6 6 13E-07 \\
\hline Cs-134 & 1.54E-06 & $9.21 \mathrm{E}-04$ & 2.93 & $1.03 \mathrm{E}-06$ & $1.03 \mathrm{E}-06$ & $1,66 \mathrm{E}-06$ & 1.77E-06 \\
\hline Cs-137 & 0.285 & 170 & 5.41E+0S & 0.256 & 0.270 & 0.301 & 0.303 \\
\hline $\mathrm{Ba}-137 \mathrm{~m}$ & 0.269 & 161 & $5.11 \mathrm{E}+0.5$ & $0.20 !$ & 0.201 & 0.284 & 0.286 \\
\hline$S_{m-151}$ & $1.22 \mathrm{E}-02$ & 7.31 & $2.32 \mathrm{E}+04$ & $7.37 \mathrm{E}-03$ & $7.37 \mathrm{E}-03$ & $1.40 \mathrm{E}-02$ & $1.57 \mathrm{E} .02$ \\
\hline Eu-152 & $3.09 \mathrm{E}-06$ & $1.85 \mathrm{E}-03$ & 5.87 & $1.37 \mathrm{E}-06$ & $1.37 E-06$ & $3.15 \mathrm{E}-06$ & $3.20 \mathrm{E}-06$ \\
\hline Eu-154 & $5.37 \mathrm{E}-04$ & 0.321 & $1.02 E+03$ & 1.91E-04 & 1,91E-04 & $6.62 \mathrm{E}-04$ & $7.12 \mathrm{E}-04$ \\
\hline Eu-155 & $1.77 \mathrm{E}-04$ & 0.105 & 336 & $7.46 \mathrm{E}-05$ & $7.46 \mathrm{E}-05$ & 1.81E-04 & $185 \mathrm{E}-04$ \\
\hline $\mathrm{Ra}-226$ & $1.73 \mathrm{E}-10$ & $1.03 E-07$ & $3.28 \mathrm{E}-04$ & $1.29 \mathrm{E}-10$ & $1.30 \mathrm{E}-10$ & $1.95 \mathrm{E}-10$ & $2.17 \mathrm{E}-10$ \\
\hline $\mathrm{Ra} \cdot 228$ & $5.79 \mathrm{E}-08$ & $3.46 \mathrm{E}-05$ & 0.110 & $2.26 \mathrm{E}-08$ & $3.98 \mathrm{E}-08$ & 7.88E-08 & $1.01 \mathrm{E}-07$ \\
\hline Ac-227 & $1.02 \mathrm{E}-09$ & $6.12 \mathrm{E}-07$ & $1.94 \mathrm{E}-0 \mathrm{3}$ & $7.68 \mathrm{E}-10$ & $7.74 \mathrm{E}-10$ & 1.15E-09 & $1.23 \mathrm{E}-09$ \\
\hline Pa-23I & $431 \mathrm{E}-49$ & $2.58 \mathrm{E}-06$ & B.19E 03 & $2.93 \mathrm{E}-09$ & 2.93E-09 & 4.81E-09 & $5.30 \mathrm{E}-09$ \\
\hline Th-229 & 1.38E-09 & 8.24E-07 & $2.62 \mathrm{E}-03$ & $6.04 \mathrm{E}-10$ & $9.83 \mathrm{E}-10$ & $1.84 \mathrm{E}-09$ & $2.33 \mathrm{E}-09$ \\
\hline Th-232 & $3.90 \mathrm{E}-09$ & 2.33E-06 & 7 (40E-03 & $1.94 \mathrm{E}-09$ & $2.90 \mathrm{E}-09$ & $4.89 \mathrm{E}-09$ & $585 \mathrm{E}-09$ \\
\hline $\mathrm{U} .232$ & $3.49 \mathrm{E}-07$ & $2.09 \mathrm{E}-04$ & 0.664 & $2.08 E-07$ & $2.77 \mathrm{E}-07$ & 4.33E-07 & $5.22 \mathrm{E}-07$ \\
\hline $\mathrm{U}-233$ & $134 \mathrm{E}-06$ & $8.02 \mathrm{E}-04$ & 2.55 & $7.99 \mathrm{E}-07$ & $1.06 \mathrm{E}-06$ & $1.66 \mathrm{E}-06$ & $200 \mathrm{E}-06$ \\
\hline U-234 & 8.48E-07 & 5.07E-04 & 1.61 & $7.75 \mathrm{E}-07$ & 8.10E-07 & 8.89E-07 & $8.71 \mathrm{E}-07$ \\
\hline U-235 & $3.51 \mathrm{E}-08$ & $2.10 \mathrm{E}-05$ & $6.67 \mathrm{E}-02$ & 3. 20E-08 & $3.34 \mathrm{E}-08$ & 3.69E-08 & $3.60 \mathrm{E}-08$ \\
\hline $\mathrm{U}-236$ & $2,40 \mathrm{E}-03$ & $1.44 \mathrm{E}-05$ & $4.56 \mathrm{E}-02$ & $2.29 \mathrm{E}-08$ & $2.34 \mathrm{E}-08$ & 2.47E-08 & $2.47 \mathrm{E}-0 \mathrm{~B}$ \\
\hline U-238 & $8.82 \mathrm{E}-07$ & $5.27 \mathrm{E}-04$ & 1.68 & 8.08E-07 & B.43E-07 & $9.24 \mathrm{E}-07$ & 9.04E-07 \\
\hline $\mathrm{Np}-237$ & $9.36 \mathrm{E}-07$ & $5,60 \mathrm{E}-04$ & 1.78 & $6.72 \mathrm{E}-07$ & B.01E-07 & $1.07 \mathrm{E}-06$ & $120 \mathrm{E}-06$ \\
\hline Pu-238 & $1.21 E-06$ & $7.26 \mathrm{E}-04$ & 2.31 & 9.04E-07 & $1.06 \mathrm{E}-06$ & $1.37 \mathrm{E}-06$ & $1.53 \mathrm{E}-06$ \\
\hline Pu-239 & $4.79 \mathrm{E}-05$ & $2.86 \mathrm{E}-02$ & 91.0 & $3.99 \mathrm{E}-05$ & $4.38 \mathrm{E}-05$ & 5.19E-0S & $5.58 \mathrm{E}-05$ \\
\hline Pu-240 & $774 \mathrm{E}-06$ & $4.63 \mathrm{E}-63$ & 14.7 & $6.26 \mathrm{E}-06$ & $6.98 \mathrm{E}-06$ & $3.49 \mathrm{E}-06$ & $9.21 \mathrm{E}-06$ \\
\hline Pu-241 & $7.97 \mathrm{E}-05$ & $4.77 \mathrm{E}-02$ & 151 & $5.86 \mathrm{E}-05$ & $6.89 \mathrm{E}-05$ & 9.05E-05 & 1.01E-04 \\
\hline Pu-242 & $4.27 \mathrm{E}-10$ & $2.56 \mathrm{E}-07$ & $8.12 \mathrm{E}-04$ & 3.01E-10 & $3.63 \mathrm{E}-10$ & $4.92 \mathrm{E}+10$ & 5.54E-10 \\
\hline Am-24! & $5.60 \mathrm{E}-05$ & $3.35 \mathrm{E}-02$ & 106 & $4,12 \mathrm{E}-05$ & 4.84E-05 & 6.35E-05 & $7.07 \mathrm{E}-05$ \\
\hline Am-243 & $1.56 \mathrm{E}-09$ & $9.32 \mathrm{E}-07$ & $296 \mathrm{E}-03$ & $1.10 \mathrm{E}-09$ & $1.32 \mathrm{E}-09$ & $1.80 \mathrm{E}-09$ & 2.04E-09 \\
\hline $\mathrm{Cm}-242$ & $1.09 \mathrm{E}-07$ & $6.51 \mathrm{E}-05$ & 0.207 & 3.85E-08 & $385 E-08$ & I.IIE-07 & $1.13 \mathrm{E}-07$ \\
\hline $\mathrm{Cm}-243$ & $9.58 \mathrm{E}-09$ & $5.73 \mathrm{E}-06$ & 182E-02 & 2.87E-09 & $2.87 E-09$ & $9.80 \mathrm{E}-09$ & 1.00E-08 \\
\hline $\mathrm{Cm}-244$ & $1.05 \mathrm{E}-07$ & $6.29 \mathrm{E}-05$ & 0.200 & $3.59 \mathrm{E}-0 \mathrm{~s}$ & $3.59 \mathrm{E}-08$ & $1.29 \mathrm{E}-07$ & 1.44E-07 \\
\hline Totals & $\mathbf{M}$ & $\mu / \mathrm{s}$ & ks: & $\begin{array}{l}-95 \mathrm{CI} \\
(\mathrm{M} \text { or } \\
\text { dL) }\end{array}$ & $\begin{array}{l}67 \mathrm{Cl}(\mathrm{M} \\
\text { or } / \mathrm{L})\end{array}$ & $\begin{array}{l}+67 \mathrm{CI}(\mathrm{M} \\
\text { or } \mathrm{k} / \mathrm{L})\end{array}$ & $\begin{array}{c}+95 \mathrm{Cl} \\
(\mathrm{M} \mathrm{or} \\
\mathrm{g} / \mathrm{L})\end{array}$ \\
\hline $\mathrm{Pu}$ & $644 \mathrm{E}-04(\mathrm{~g} / \mathrm{L})$ & $\ldots$ & 1.22 & 4.94E-04 & $5.68 \mathrm{E}-04$ & $7.21 \mathrm{E}-0.04$ & $7.94 \mathrm{E}-04$ \\
\hline U & $1.00 \mathrm{E}-02$ & $1.43 \mathrm{E}+03$ & $4.53 \mathrm{E}+03$ & $9.08 \mathrm{E}-03$ & $953 \mathrm{E}-03$ & \begin{tabular}{ll|}
$1.05 E-02$ \\
\end{tabular} & $1.03 \mathrm{E}-0$ ? \\
\hline
\end{tabular}

- Unknowns in tank solids inventory are assigned by Tank Layering Model (TLM)

†Volume average for density, mass average Water wt $\%$ and TOC wt\% C. 
HDW Model Pey, 4

\begin{tabular}{|c|c|c|c|c|c|c|c|}
\hline \multicolumn{8}{|c|}{ Single-Shell Tank 241-S.109 } \\
\hline & \multicolumn{7}{|c|}{ TLM Solids Composite lnventory Estimate* } \\
\hline $\begin{array}{l}\text { Physical } \\
\text { Properties }\end{array}$ & & 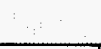 & & $-95 \mathrm{CI}$ & $-67 \mathrm{CI}$ & $+67 \mathrm{CI}$ & $+95 . \mathrm{CI}$ \\
\hline Total TLM Wast & $871 \mathrm{E}+04(\mathrm{~kg})$ & $(13,0 \mathrm{kgal})$ & - & $\cdots$ & -- & $\cdots$ & - \\
\hline Heat Load & $1.22 \mathrm{E}-03(\mathrm{~kW})$ & (4.17 BTU/hr) & - & $1.02 \mathrm{E}-03$ & $1.18 \mathrm{E}-03$ & $1.26 \mathrm{E}-03$ & $130 \mathrm{E}-03$ \\
\hline Bulk Density & $1.77(\mathrm{~g} / \mathrm{cc})$ & 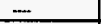 & - & 1.47 & 1.57 & 1.92 & 2.02 \\
\hline Void Fraction & 0.651 & - & $\rightarrow$ & 0.488 & 0.557 & 0.780 & 0825 \\
\hline Water wt\% & 24.5 & - & $=$ & 4.37 & 12.2 & 45.5 & 55.1 \\
\hline TOC wt\% C (wet & 0 & - & $=$ & 0 & 0 & 0 & 0 \\
\hline $\begin{array}{c}\text { Chemical } \\
\text { Constituents }\end{array}$ & mole/L & ppm & $\mathrm{kg}$ & $\begin{array}{l}\text { 9s Cl } \\
\text { (mole/L) }\end{array}$ & $\begin{array}{l}-67 \mathrm{CI} \\
\text { (mole } / \mathrm{L})\end{array}$ & $\begin{array}{l}+67 \mathrm{Cl} \\
(\mathrm{mole} / \mathrm{L})\end{array}$ & $\begin{array}{l}+95 \mathrm{Cl} \\
(\mathrm{mole} / \mathrm{L})\end{array}$ \\
\hline Nat & 7.86 & $1.02 \mathrm{E}+05$ & $8.89 \mathrm{E}+03$ & 2.23 & 3.91 & 107 & 12.9 \\
\hline $\mathrm{Al3+}$ & $\pi 12$ & $1.71 E+05$ & $1.49 \mathrm{E}+04$ & 5.84 & 7.28 & 140 & 16.1 \\
\hline$F 3+(\operatorname{total} \mid F t)$ & 0.165 & $5.20 \mathrm{E}+03$ & 453 & 0.153 & 0.160 & 0.170 & 0.175 \\
\hline $\mathrm{Cr}^{3}+$ & 2.04E-03 & 59.8 & 5.21 & $1.71 \mathrm{E}-03$ & $1.97 \mathrm{E}-03$ & $2.10 \mathrm{E}-03$ & $217 \mathrm{E}-03$ \\
\hline Bi3+ & 0 & 0 & 0 & 0 & 0 & 0 & 0 \\
\hline Laj+ & 0 & 0 & 0 & 0 & 0 & 0 & 0 \\
\hline $\mathrm{Hg} 2+$ & $4.09 \mathrm{E}-03$ & 462 & 40.3 & $4.02 \mathrm{E}-03$ & 4.06E-03 & $4,10 \mathrm{E}-03$ & 4.1IE-03 \\
\hline $\mathrm{Zr}(\operatorname{ar} \mathrm{ZnOOH}) 2)$ & 0 & 0 & 0 & 0 & 0 & 0 & 0 \\
\hline Pb2+ & 0.118 & $1.38 \mathrm{E}+04$ & $1.20 \mathrm{E}+03$ & 0.108 & 0.113 & 0.122 & 0.126 \\
\hline $\mathrm{Ni2+}$ & 102E-03 & 33.7 & 2.94 & $8.54 E-04$ & 9.84E-0A & $1.51 \mathrm{E}-03$ & $3.79 \mathrm{E}-03$ \\
\hline $5 \sqrt{2+}$ & 0 & 0 & 0 & 0 & 0 & 0 & 0 \\
\hline Mndt & 0 & 0 & 0 & 0 & 0 & 0 & 0 \\
\hline $\mathrm{Cn} 2+$ & 0.121 & $2.73 \mathrm{E}+03$ & 238 & $6.72 \mathrm{E}-02$ & $9.87 \mathrm{E}-02$ & 0.141 & 0.161 \\
\hline $\mathrm{K}+$ & $1.54 \mathrm{E}-03$ & 33.9 & 2.95 & $1.29 \mathrm{E}-03$ & $1.48 \mathrm{E}-03$ & 1.59E-03 & 1.64E-03 \\
\hline $\mathrm{OH}-$ & 41.7 & 4.00E+05 & $349 \mathrm{E}+04$ & 20.0 & 25.9 & 53.0 & 61.2 \\
\hline NO3- & 0.572 & $2.00 \mathrm{E}+04$ & $1.75 \mathrm{E}+03$ & 0.485 & 0.554 & 0.591 & 0,610 \\
\hline NO2* & 0.958 & 2.49E+04 & 2. $17 \mathrm{E}+03$ & 0.799 & 0.925 & 0.990 & 1.02 \\
\hline $\cos 2-$ & 0.121 & $4,09 E+03$ & 356 & $6.72 \mathrm{E}-02$ & $9.87 \mathrm{E}-02$ & 0.141 & 0.161 \\
\hline PO43. & 0 & 0 & 0 & 0 & 0 & 0 & 0 \\
\hline $5042-$ & 8.39E-03 & 455 & 39.6 & $7.04 \mathrm{E}-03$ & 8.10E-03 & $8.67 \mathrm{E}-03$ & $8.94 E-03$ \\
\hline $\mathrm{Si}$ (as SiO32-) & 2.01E 02 & 319 & 27.8 & $1.69 \mathrm{E}-02$ & $1.94 \mathrm{E}-02$ & 8. $18 \mathrm{E}-02$ & 0.175 \\
\hline F- & 0 & 0 & 0 & 0 & 0 & 0 & 0 \\
\hline $\mathrm{Cl}-$ & 7.07E- 03 & 141 & 12.3 & $5.93 \mathrm{E}-03$ & $6.83 \mathrm{E}-03$ & $7.30 \mathrm{E}-03$ & $7.53 \mathrm{E}-03$ \\
\hline C6H5073- & 0 & 0 & 0 & 0 & 0 & 0 & 0 \\
\hline EDTA4- & 0 & 0 & 0 & 0 & 0 & 0 & 0 \\
\hline HEDTA3- & 0 & 0 & 0 & 0 & 0 & 0 & 0 \\
\hline glycolate- & 0 & 0 & 0 & $\mathbf{0}$ & 0 & 0 & 0 \\
\hline acetale- & 0 & 0 & 0 & 0 & 0 & 0 & 0 \\
\hline axalute2" & 0 & 0 & 0 & 0 & 0 & 0 & 0 \\
\hline DBP & 0 & 0 & 0 & 0 & 0 & 0 & 0 \\
\hline butanol & 0 & 0 & 0 & 0 & 0 & 0 & 0 \\
\hline NH3 & $205 E-04$ & 1.97 & 0.171 & $1.6 \mathrm{IE}-04$ & 1.80E-04 & $2.38 \mathrm{E}-04$ & $2.49 \mathrm{E}-04$ \\
\hline FE(CN)64- & 0 & 0 & 0 & 0 & 0 & 0 & 0 \\
\hline
\end{tabular}

-Unknowns in tank solids inventory ate usigned by Tenk Layeriag Model (TLM). 
HDW Model Pev, 4

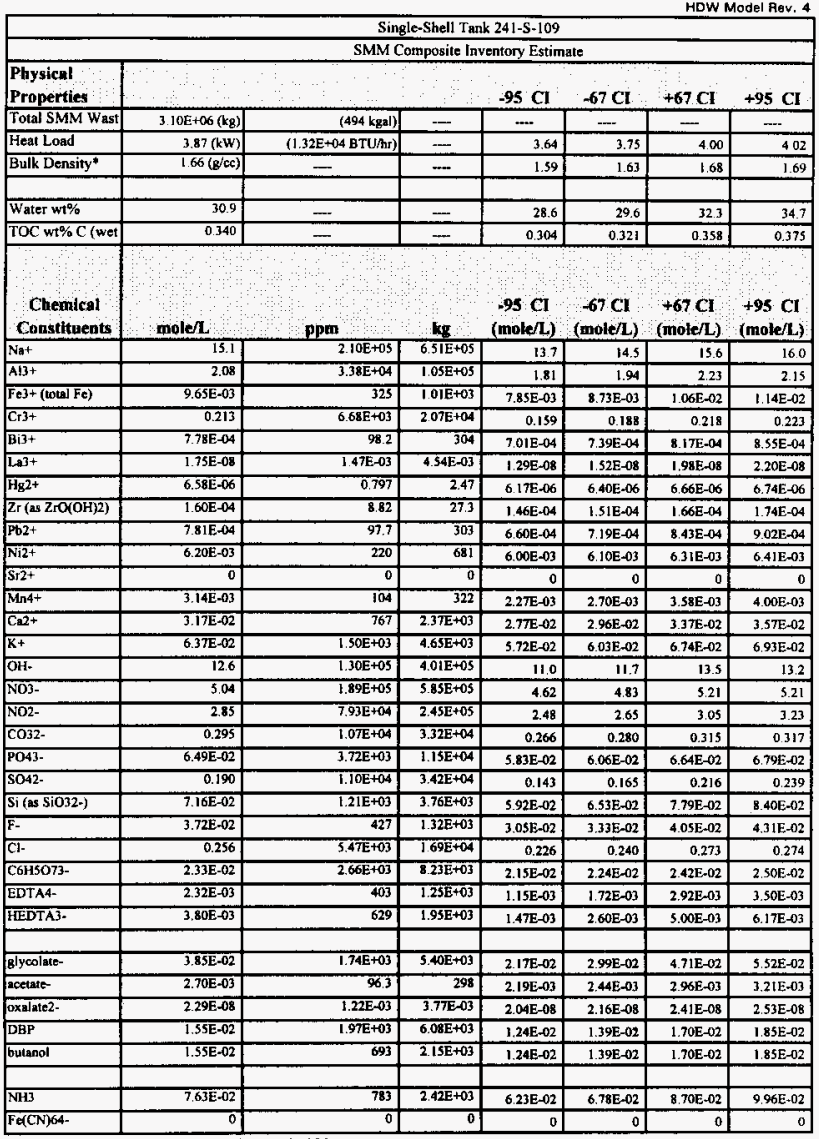

*Density is calculated based on $\mathrm{Na}, \mathrm{OH}-$, and $\mathrm{AlO} 2$ -

+Water wt\% derived from the difference of density and total dissolved species. 


\begin{tabular}{|c|c|c|c|c|c|c|c|}
\hline \multirow{4}{*}{$\begin{array}{l}\text { Physical } \\
\text { Properties }\end{array}$} & \multicolumn{7}{|c|}{ Single-Shell Tank 24 I-S-109 } \\
\hline & \multicolumn{7}{|c|}{ Total Inventory Estimate* } \\
\hline & & & & & & & \\
\hline & & & & $.95 \mathrm{CI}$ & $-67 \mathrm{CI}$ & $+67 \mathrm{Cl}$ & $+95 \mathrm{CI}$ \\
\hline Total Waste & $318 \mathrm{18E}+06(\mathrm{~kg})$ & (507 kgal), & $=$ & $=$ & $=$ & - & $\ldots$ \\
\hline Heat Load & $3.87(\mathrm{~kW})$ & (1.32E+04 BTU/hr) & - & 3.64 & 3.75 & 401 & 4.03 \\
\hline Bulk Densityt & $1.66(\mathrm{~g} / \mathrm{ccc})$ & - & - & 160 & 1.63 & 1.68 & 1.69 \\
\hline & & & & & & & \\
\hline Water wt\%t & 30.7 & $=$ & $=$ & 28.4 & 29.4 & 32.1 & 34.4 \\
\hline TOC wt\% C (wet & 0.331 & - & - & 0.295 & 0.313 & 0.348 & 0.365 \\
\hline $\begin{array}{c}\text { Chemical } \\
\text { Constituents }\end{array}$ & molen & ppm & $\mathrm{kg}$ & $\begin{array}{r}-9 \mathrm{CI} \\
(\text { mote/l) }\end{array}$ & $\begin{array}{r}67 \mathrm{CI} \\
\text { (mole/L) }\end{array}$ & $\begin{array}{l}+67 \mathrm{Cl} \\
\text { (mole/L) }\end{array}$ & $\begin{array}{r}+9 s \mathrm{CI} \\
\text { (mole } / \mathrm{L})\end{array}$ \\
\hline $\mathrm{Na+}$ & 14.9 & $2.07 \mathrm{E}+05$ & $6.60 \mathrm{E}+05$ & 13.5 & 14.3 & 15.4 & 15.8 \\
\hline $\mathrm{Al3}+$ & 2.31 & $3.76 \mathrm{E}+04$ & $1.20 \mathrm{E}+05$ & 2.05 & 2.18 & 2.46 & 2.41 \\
\hline Fes+(tots Fe) & $1.36 \mathrm{E}-02$ & 459 & $1.46 \mathrm{E}+03$ & $1.19 \mathrm{E}-02$ & $1.27 \mathrm{E}-02$ & $145 E-02$ & 1.54E-02 \\
\hline $\mathrm{Cr}^{3+}$ & 0.207 & 6.49E+03 & $2.07 \mathrm{E}+04$ & 0.155 & 0.183 & 0.212 & 0.217 \\
\hline $\mathrm{Bi} \mathbf{i 3 +}$ & $7.58 \mathrm{E}-04$ & 95.5 & 304 & $6.83 \mathrm{E}-04$ & $7.20 \mathrm{E}-04$ & 7.96- -04 & $8.33 \mathrm{E}-04$ \\
\hline Laj+ & $1.70 \mathrm{E}-08$ & $1.43 \mathrm{E}-03$ & 4.54E-03 & $1.26 \mathrm{E}-08$ & $1.48 \mathrm{E}-08$ & $1.93 \mathrm{E}-08$ & 2.15E-08 \\
\hline $\mathrm{Hg}_{2+}$ & T.11E-04 & 13.4 & 42.7 & $1.09 \mathrm{E}-04$ & $1.10 \mathrm{E}-04$ & $1.1 \mathrm{EE}-04$ & $1.12 \mathrm{E}-04$ \\
\hline Zr (as Zró(OH)2) & 1.56E-04 & 8.57 & 27.3 & 1.42E-04 & $1.47 \mathrm{E}-04$ & 1.62E-04 & 1.69E-04 \\
\hline $\mathrm{Pb} 2+$ & $3.78 \mathrm{E}-03$ & 472 & $1.50 E+03$ & $3.53 \mathrm{E}-03$ & $368 \mathrm{E}-03$ & $3.85 E-03$ & $3.90 \mathrm{E}-03$ \\
\hline $\mathrm{Ni2+}$ & $6.07 \mathrm{E}-03$ & 215 & 684 & 5.8TE-03 & 5.97E-03 & $6.17 \mathrm{E}-03$ & $6.27 \mathrm{E}-03$ \\
\hline $5 n+2+$ & 0 & 0 & 0 & 0 & 0 & 0 & 0 \\
\hline Min4t & 3.06E-03 & 101 & 322 & $2.21 \mathrm{E}-03$ & $2.63 \mathrm{E}-03$ & $3.49 \mathrm{E}-03$ & $3.90 \mathrm{E}-03$ \\
\hline $\mathrm{Cln}_{2}+$ & $3.40 \mathrm{E}-02$ & 820 & $2.61 E+03$ & $3.00 E-02$ & $3.20 \mathrm{E}-02$ & $3.59 \mathrm{E}-02$ & 3. $78 \mathrm{E}-02$ \\
\hline $\mathrm{Kt}$ & $6.2 \mathrm{IE}-02$ & $1.46 \mathrm{E}+03$ & $4.66 \mathrm{E}+03$ & $5.58 \mathrm{E}-02$ & 5.88E-02 & $6.57 \mathrm{E}-02$ & $6.73 \mathrm{E}-02$ \\
\hline $\mathrm{OH}-$ & 13.4 & $1.37 \mathrm{E}+05$ & $4.36 \mathrm{E}+05$ & 11.8 & 12.5 & 14.2 & 13.9 \\
\hline NO3- & 4.93 & $1.84 E+05$ & $5.86 \mathrm{E}+05$ & 4.52 & 4.72 & 5.10 & 5.10 \\
\hline $\mathrm{NO2-}$ & 2.80 & $7.78 \mathrm{E}+04$ & 2.48E+03 & 2.44 & 2.61 & 2.99 & 3.17 \\
\hline CO32: & 0.291 & $1.05 \mathrm{k}+04$ & $3.35 \mathrm{E}+04$ & 0.262 & 0.276 & 0.310 & $0,3: 2$ \\
\hline PO43- & $6.32 \mathrm{E}-02$ & $3.62 \mathrm{E}+03$ & $1.15 \mathrm{E}+04$ & $5.68 E-02$ & 5 . 90E-02 & $6.47 \mathrm{E}-02$ & 6.61E-02 \\
\hline SO42- & 0.186 & $1.08 \mathrm{E}+04$ & $3.42 \mathrm{E}+04$ & 0.139 & 0.161 & 0.211 & 0.233 \\
\hline Si (as SiOB2-) & $7.03 \mathrm{E}-02$ & $1.19 E+03$ & $3.79 \mathrm{E}+03$ & $5.82 \mathrm{E}-02$ & $6.41 \mathrm{~L}-02$ & $7.64 \mathrm{E}-02$ & $8.24 E-02$ \\
\hline F- & $3.63 \mathrm{E}-02$ & 415 & $1.32 \mathrm{E}+03$ & $2.97 \mathrm{E}-02$ & $3.25 \mathrm{E}-02$ & $3.95 \mathrm{E}-02$ & $4.20 \mathrm{E}-02$ \\
\hline Cl- & 0.249 & $5.32 \mathrm{E}+03$ & 1.69E+04 & 0.220 & 0.234 & 0.206 & 0.267 \\
\hline C6HSO73- & $2.27 \mathrm{E}-02$ & $2.59 \mathrm{E}+03$ & $8.23 \overline{\mathrm{E}+03}$ & $2.10 \mathrm{E}-02$ & 2.18E-02 & $2.36 \mathrm{E}-02$ & $2.44 \mathrm{E}-02$ \\
\hline EDTA4- & $2.26 \mathrm{E}-03$ & 392 & $1.25 \mathrm{E}+03$ & $1.12 \mathrm{E}-03$ & $1.68 \mathrm{E}-03$ & $2.84 \mathrm{E}-03$ & $3.41 \mathrm{LE}-03$ \\
\hline FEDTA3. & $3.70 \mathrm{E}-03$ & 611 & $1.95 \bar{E}+03$ & $1.43 \mathrm{E}-03$ & $2.54 \mathrm{E}-03$ & $4.87 \mathrm{E}-03$ & $6.01 \mathrm{E}-03$ \\
\hline & & & & & & & \\
\hline g glycolate- & 3.75E-02 & $1.70 E+03$ & $5.40 \mathrm{E}+03$ & $2.12 E-02$ & $2.92 \mathrm{E}-02$ & $4.58 \mathrm{E}-02$ & $5.38 E-02$ \\
\hline acetste- & $2.63 \mathrm{E}-0.3$ & 93.6 & 298 & $2.13 E-03$ & $2.38 E-03$ & $2.89 \mathrm{E}-03$ & 3.13E-03 \\
\hline oxalinte2- & $2.23 \mathrm{E}-0 \mathrm{~B}$ & $1.18 E-03$ & $3.77 \mathrm{E}-03$ & $1.99 \mathrm{E}-08$ & $2.11 \mathrm{E}-08$ & $2.35 \mathrm{E}-08$ & 2.47E- 08 \\
\hline DEP & $1.31 \mathrm{E}-02$ & $1.91 \mathrm{E}+03$ & $6.08 \mathrm{E}+03$ & $1,21 \mathrm{E}-02$ & $1.36 \mathrm{E}-02$ & $1.66 \mathrm{E}-02$ & $1.80 E-02$ \\
\hline \begin{tabular}{|l} 
batanol \\
\end{tabular} & $1.51 \mathrm{E}-02$ & 674 & $2.15 E+03$ & $1.21 \mathrm{E}-02$ & $1.36 \mathrm{E}-02$ & $1.66 \mathrm{E}-02$ & $1.80 \mathrm{E}-02$ \\
\hline & & & & & & & \\
\hline $\mathrm{NH3}$ & $7.43 \mathrm{E}-02$ & 762 & $2.42 \mathrm{E}+03$ & $6.07 \mathrm{E}-02$ & $6.61 \mathrm{E}-02$ & $8.48 \mathrm{E}-02$ & $9.70 \mathrm{E}-02$ \\
\hline $\mathrm{FG}(\mathrm{CN}) 64-$ & 0 & 0 & 0 & 0 & 0 & 0 & 0 \\
\hline
\end{tabular}

"Unknowns in tank solids inventory are assigned by Tank Layering Model (TLM).

+ Water wt\% derived from the difference of density and total dissolved species. 
HNF-SD-WM-ER-323, Rev. 1

HDW Model Rev, 4

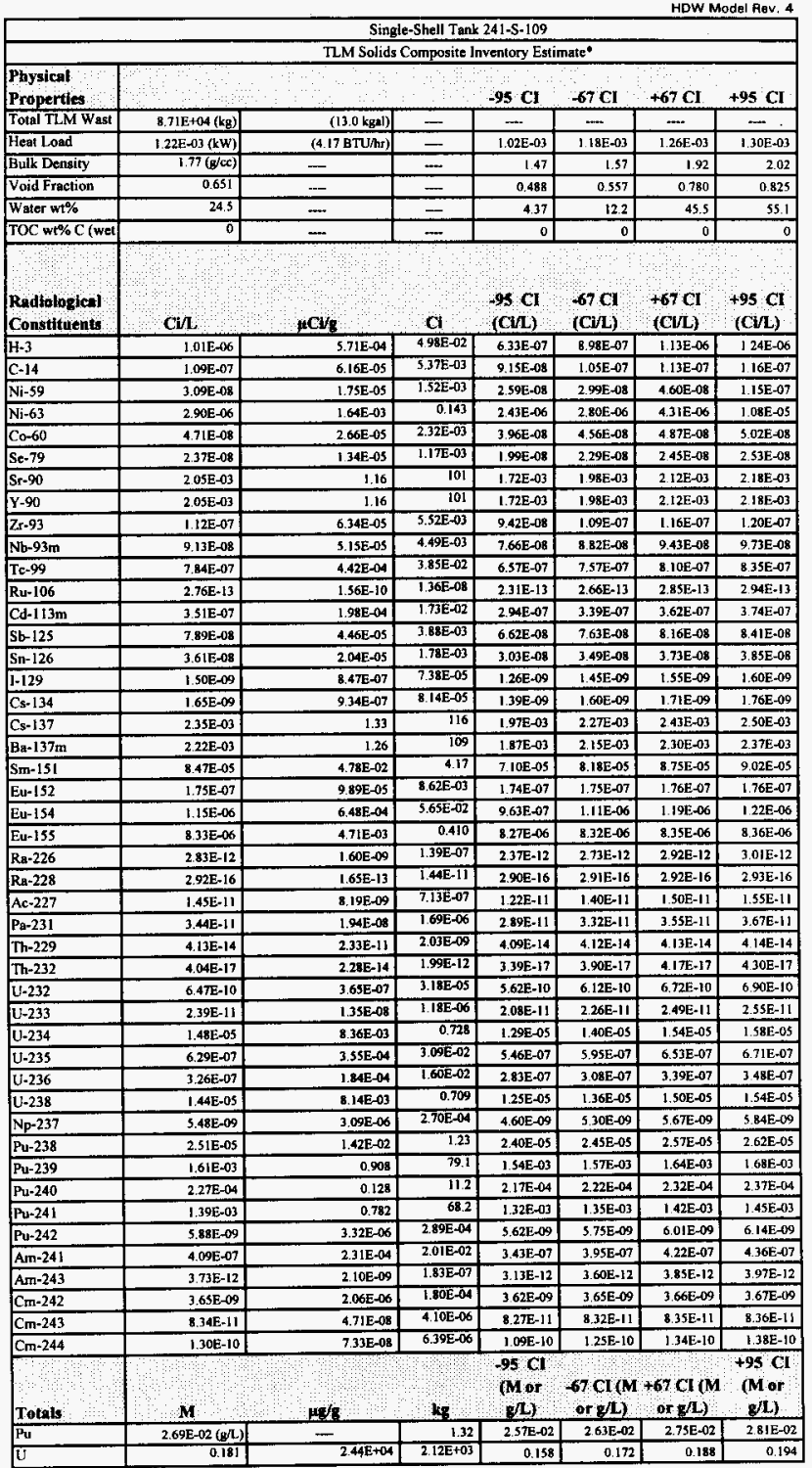

"Unknowns in tank solids inventory are assigned by Tark Layering Model (TLM) 
HNF-SD-WM-ER-323, Rev. 1

HDW Model Rev. 4

\begin{tabular}{|c|c|c|c|c|c|c|c|}
\hline \multirow{4}{*}{$\begin{array}{l}\text { Physical } \\
\text { Propertics }\end{array}$} & \multicolumn{7}{|c|}{ Single-Shell Tank 241-S-109 } \\
\hline & \multicolumn{7}{|c|}{ SMM Composite Inventory Estimate } \\
\hline & & & & & & 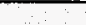 & \\
\hline & & & & $-95 \mathrm{CI}$ & $-67 \mathrm{CI}$ & $+67 \mathrm{CI}$ & $+95 \mathrm{CI}$ \\
\hline Total SMM Wast & $3.10 \mathrm{E}+06(\mathrm{~kg})$ & (494 kgel) & - & $\cdots$ & - & $\cdots$ & - \\
\hline Heat Load & $3.87(\mathrm{~kW})$ & $(1.32 \mathrm{E}+04 \mathrm{BTU} / \mathrm{hr})$ & - & 3.64 & 3,75 & 4.00 & 4.02 \\
\hline Bulk Density* & $1.66(g / c c)$ & $=$ & 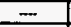 & 1.59 & 1.63 & 1.68 & 1.69 \\
\hline & & & & & & & \\
\hline Water wtot & 30.9 & - & $\omega$ & 28.6 & 29.6 & 32.3 & 347 \\
\hline $\mathrm{TOC}$ wt $\% \mathrm{C}$ (wet & 0.340 & $\cdots$ & - & 0.304 & 0.321 & 0.358 & 0375 \\
\hline $\begin{array}{l}\text { Rodtological } \\
\text { Constltueots }\end{array}$ & $\mathrm{CvL}$ & HEV & $\mathrm{Cl}$ & $\begin{array}{l}-95 \mathrm{CT} \\
\text { (CUL) }\end{array}$ & $\begin{array}{l}-67 \mathrm{CI} \\
\text { (C)L) }\end{array}$ & $\begin{array}{l}+67 \mathrm{CI} \\
(\mathrm{Cl} / \mathrm{L}) \\
\end{array}$ & $\begin{array}{l}+95 \mathrm{CI} \\
(\mathrm{CM})\end{array}$ \\
\hline $\mathrm{H}-3$ & $2.62 E-04$ & 0.158 & 490 & $1.52 \mathrm{E}-04$ & $1.52 \mathrm{E}-0 \mathrm{~s}$ & $2.77 \mathrm{E}-04$ & $2.71 E-04$ \\
\hline C-14 & 3.41E-0S & $2.06 E-02$ & 63.8 & $1.10 \mathrm{E}-0 \mathrm{~S}$ & $1.10 \mathrm{E}-0 \mathrm{~s}$ & $3.48 \mathrm{E}-05$ & $348 \mathrm{E}-05$ \\
\hline Ni-59 & 2.29E-D6 & $1.38 \mathrm{E}-03$ & 4.28 & $1.20 \mathrm{E}-06$ & $1.20 \mathrm{E} \cdot 06$ & $2.34 E-06$ & $2.40 \mathrm{E}-06$ \\
\hline $\mathrm{Ni}+63$ & $2.23 \mathrm{E}-04$ & 0.134 & 416 & 1.15E-04 & 1.1SE-04 & $2.28 E-04$ & $2.33 \mathrm{E}-04$ \\
\hline Co-60 & 3.49E-05 & $2.10 \mathrm{E}-02$ & 65.2 & $8.45 \mathrm{E}-06$ & B.45E-06 & 3.53E-05 & 3.58E-05 \\
\hline Se-79 & $3.50 \mathrm{E}-06$ & $2.11 \mathrm{E}-03$ & 6.54 & $2.02 \mathrm{E}-06$ & $2.02 \mathrm{E}-06$ & $4.03 \mathrm{E}-06$ & $4.54 \mathrm{E}-06$ \\
\hline S $\mathrm{r}-90$ & 0114 & 69.0 & $2.14 \mathrm{E}+05$ & 0.107 & 0.111 & 0.118 & 0.122 \\
\hline$Y-90$ & \begin{tabular}{l|l|}
0.114 \\
\end{tabular} & 69,0 & 2.14E+05 & $6.35 \mathrm{E}-02$ & $6,35 \mathrm{E}-02$ & 0.118 & 0.122 \\
\hline $\mathrm{Zr}-93$ & $171 \mathrm{E}-05$ & $1.03 \mathrm{E}-02$ & 31.9 & $972 \mathrm{E}-06$ & $9.72 \mathrm{E}-06$ & $1.97 \mathrm{E}-05$ & $2.23 \mathrm{E}-05$ \\
\hline $\mathrm{Nb}-93 \mathrm{~m}$ & $1.26 \mathrm{E}-05$ & $7.63 \mathrm{E}-03$ & 236 & 7.48E-06 & $7.48 \mathrm{E}-06$ & 1.45E-05 & $1.63 \mathrm{E}-05$ \\
\hline Tc-99 & $243 E-04$ & 0.147 & 454 & 1.57E-M4 & $1.99 \mathrm{E}-04$ & $2.87 E-04$ & $3,30 \mathrm{E}-04$ \\
\hline$R \mu-106$ & $5.43 \mathrm{E}-09$ & $3.28 E-06$ & $1.02 \mathrm{E}-02$ & $2.02 \mathrm{E}-09$ & $2.02 \mathrm{E}-09$ & $6.19 \mathrm{E}-09$ & $6.89 \mathrm{E}-09$ \\
\hline $\mathrm{Cd}-113 \mathrm{~m}$ & B. $42 E-05$ & 5.08E-02 & 157 & 4.00E-05 & $4.00 E-05$ & $1.00 \mathrm{E}-04$ & $1.16 \mathrm{E}-04$ \\
\hline Sb-125 & 1.44E-04 & $8.68 \mathrm{E}-02$ & 269 & 2.95E-05 & 2.95E-05 & $1.46 \mathrm{E}-04$ & $148 \mathrm{E}-04$ \\
\hline$S n=126$ & $5.29 \mathrm{E}-06$ & $3.20 E-03$ & 9.90 & $3.09 \mathrm{E}-06$ & $3.09 \mathrm{E}-06$ & $6.09 \mathrm{E}-06$ & $6.86 \mathrm{E}-06$ \\
\hline$[-129$ & $4.68 \mathrm{E}-07$ & $2.83 \mathrm{E}-04$ & 0.875 & $3.02 \mathrm{E}-07$ & $3.83 E-07$ & 5.54E-07 & $6.36 \mathrm{E}-07$ \\
\hline$C=134$ & 1.53E-N6 & $9.25 \mathrm{E}-04$ & 2.86 & $1.03 E-06$ & $1.03 \mathrm{E}-06$ & 1.64E-06 & $1.75 \mathrm{E} .06$ \\
\hline $\mathrm{Cs}-137$ & 0.277 & 167 & 5.19E+05 & 0.251 & 0.263 & 0.293 & 0.295 \\
\hline $\mathrm{Ba}=137 \mathrm{~m}$ & 0.262 & 158 & 4. $9 \mathrm{lE}+05$ & 0.196 & 0.190 & 0.277 & 0.279 \\
\hline $5 \mathrm{~m}-151$ & $1.23 \mathrm{E}-02$ & 7.45 & $2.31 \mathrm{E}+04$ & 7.18E-03 & $7.18 E-03$ & 1.42E-02 & $1.60 \mathrm{E}-02$ \\
\hline$E_{u}-152$ & $3.12 \mathrm{E}-06$ & $1.88 \mathrm{E}-03$ & 5.83 & $1.296-06$ & 1.29E-06 & $3.18 E-06$ & $3.23 \mathrm{E}-05$ \\
\hline Eu-154 & $5.57 \mathrm{E}-04$ & 0.336 & $1.04 E+03$ & 1.89E- 04 & $1.89 \mathrm{E}-04$ & $6.90 \mathrm{E}-04$ & $7.43 \mathrm{E}-04$ \\
\hline Eu-155 & $1.79 \mathrm{E}-04$ & 0.108 & 336 & $7.09 \mathrm{E}-05$ & $7.09 \mathrm{E}-05$ & $1.84 \mathrm{E}-04$ & $1,88 \mathrm{E}-04$ \\
\hline $\mathrm{Ra}-226$ & $161 \mathrm{E}-10$ & $9.72 \mathrm{E}-08$ & $3.01 E-04$ & $1.11 \mathrm{E}-10$ & 1.15E-10 & $186 \mathrm{E}-10$ & $2.11 \mathrm{E}-10$ \\
\hline Ra-228 & $5,94 \mathrm{E}-08$ & $3.59 \mathrm{E}-05$ & 0.111 & $2.33 \mathrm{E}-08$ & $4.09 \mathrm{E}-08$ & $8.08 E-08$ & $1.04 \mathrm{E}-07$ \\
\hline Ac-227 & $9.72 \mathrm{E}-10$ & $5.87 \mathrm{E}-07$ & $1.82 \mathrm{E}-03$ & $6.81 \mathrm{E}-10$ & $7076-10$ & $1.12 E-09$ & $1.21 \mathrm{E}-09$ \\
\hline $\mathrm{Pa}-231$ & $4.31 \mathrm{E}-09$ & 2.51E-06 & 8.07E-03 & 2.84E-49 & 2.84E-09 & $4.85 \mathrm{E}-09$ & $5.36 \mathrm{E}-09$ \\
\hline$T_{1}=229$ & $1.42 \mathrm{E}-0 \mathrm{~S}$ & 8.55E- 07 & $2.65 \mathrm{E}-03$ & 6.24E-10 & L.01E-09 & $188 E-09$ & $2.38 \mathrm{E}-09$ \\
\hline Th-232 & $402 \mathrm{E}-09$ & $2.43 \mathrm{E}-06$ & $7.51 \mathrm{E}-03$ & $2.02 \mathrm{E}-09$ & $3.00 \mathrm{E}-69$ & $5.03 E-09$ & $6.01 \mathrm{E}-09$ \\
\hline $\mathrm{U}-2.32$ & $361 E-07$ & $2.18 \mathrm{E}-\mathrm{OA}$ & 0.676 & 2.17E-07 & $2.87 \mathrm{E}-07$ & $4.47 \mathrm{E}-07$ & $5.38 \mathrm{E}-07$ \\
\hline U-233 & 1.39E-06 & 8.37E-04 & 2.59 & 8.33E-07 & 1. 10E-06 & 1.71E-06 & $2.06 \mathrm{E}-46$ \\
\hline U.234 & 842E-07 & S.08E-04 & 1.57 & 7.73E-07 & S.0SE-07 & A.81E-07 & $8.66 \mathrm{E}-07$ \\
\hline U-235 & $348 E-08$ & 2.10E-05 & $6,50 E_{-02}$ & $3.18 \mathrm{E}-08$ & $3.32 \mathrm{E}-\mathrm{A} \mathrm{s}$ & $3.65 E-08$ & $3.58 \mathrm{E}-03$ \\
\hline U-236 & $2,41 \mathrm{E}-0 \mathrm{~B}$ & 1.46E-05 & $4.51 \mathrm{E}-02$ & $2.30 \mathrm{E}-08$ & 2.35E- 08 & $2.47 \mathrm{E}-08$ & $2.49 \mathrm{E}-08$ \\
\hline $\mathrm{U}-238$ & $8.7 \pi-07$ & 5.30E-04 & 164 & 8.07E-07 & $8.40 \mathrm{E}-07$ & $9.17 \mathrm{E}-07$ & $9.01 \mathrm{E}-07$ \\
\hline $\mathrm{Np}-237$ & 9.52E-07) & 5.75E-04 & 1.78 & $6.71 \mathrm{E}-07$ & $8.08 \mathrm{E}-07$ & 1. $10 \mathrm{E}-06$ & $1.24 \mathrm{E}-06$ \\
\hline Pu-238 & 1.19E-06 & $7.19 \mathrm{E}-04$ & 2.23 & 8.61E-07 & $1.02 \mathrm{E}-06$ & $1.36 \mathrm{E}-06$ & 1.52E-06 \\
\hline Pu-239 & $4.40 \mathrm{E}-05$ & $2.66 \mathrm{E}-02$ & 82.3 & $3,55 \mathrm{E}-05$ & $3.97 \mathrm{E}-05$ & 4.83E-05 & $3.24 \mathrm{E}-05$ \\
\hline Pu-240 & $7.24 \mathrm{E}-06$ & 4.37E-03 & 135 & $5.66 \mathrm{E}-06$ & 6.44E-06 & $8.04 \mathrm{E}-06$ & B.81E-06 \\
\hline$P u-241$ & $7.89 \mathrm{E}-05$ & $4,76 \mathrm{E}-02$ & 147 & $564 \mathrm{E}-05$ & $6.74 \mathrm{E}-05$ & $904 \mathrm{E}-05$ & 1.01E-04 \\
\hline $\mathrm{Pu}-242$ & $4.28 \mathrm{E}-10$ & $2.58 \mathrm{E}-07$ & 7.99E-04 & $2.93 \mathrm{E}-10$ & $3.59 \mathrm{E}-10$ & $4.96 \mathrm{E}-10$ & $5.62 \mathrm{E}-10$ \\
\hline Am-241 & $5.66 \mathrm{E}-05$ & 3.42E-02 & 100 & 400E-05 & $4.86 E-05$ & 6.46E-05 & $7.22 \mathrm{E}-05$ \\
\hline Am-243 & $1.63 \mathrm{E}-09$ & 9.85E-07 & 3.05E-03 & 1.14E-09 & $1.37 \mathrm{E}-09$ & $1.89 \mathrm{E}-09$ & $2.14 \mathrm{E}-09$ \\
\hline $\mathrm{Cm}-242$ & $1.12 \mathrm{E}-07$ & 6.78E-05 & 0.210 & $3.74 \mathrm{E}-08$ & $3.74 E-08$ & 1.15E-0? & $1.17 \mathrm{E}-07$ \\
\hline $\mathrm{Cm}-243$ & $1.01 \mathrm{E}-08$ & 6,09E-06 & $1.89 \mathrm{E}-02$ & 2.94E-09 & $2.94 \mathrm{E}-09$ & $1.03 \mathrm{E}-0 \mathrm{~s}$ & 1.06E-08 \\
\hline $\mathrm{Cm}-244$ & $1.11 \mathrm{E}-07$ & $6.71 E-05$ & 0.208 & $3.76 \mathrm{E}-08$ & $3.76 \mathrm{E}-09$ & $1.36 \mathrm{E}-07$ & 1.52E-07 \\
\hline & & & & $\begin{array}{l}95 \mathrm{Ct} \\
\text { M or }\end{array}$ & $67 \mathrm{Cl}(\mathrm{M}$ & $\begin{array}{r}+6 \mathrm{Cl}(\mathrm{M} \\
\end{array}$ & $\begin{array}{c}+95 \mathrm{CI} \\
\text { (Mor }\end{array}$ \\
\hline Totills: & $\mathbf{M}$ & Hes: & $\mathrm{ks}$ & $2 / \mathrm{L})$ & or gili) & or $/ \mathrm{L})$ & $g(\mathrm{~L})$ \\
\hline $\mathrm{Pu}$ & $5.69 \mathrm{E}-04(\mathrm{~g} / \mathrm{L})$ & $=$ & 1.06 & 4.09E-04 & 4.88E-04 & $6.50 \mathrm{E}-04$ & 7.28E-04 \\
\hline $\mathrm{U}$ & 992E-03 & $1.43 \mathrm{E}+03$ & $4.41 \mathrm{E}+03$ & $9.04 \mathrm{E}-03$ & $9.45 \mathrm{E}-03$ & $1.04 E-02$ & $1.02 \mathrm{E}-02$ \\
\hline
\end{tabular}

- Density is calculated based on $\mathrm{Na}, \mathrm{OH}$-, and $\mathrm{AlO} 2$.

$\uparrow$ Water wt $\%$ derived from the difference of density and total dissolved species. 
HNF-SD-WM-ER-323, Rev. 1

HDW Madel Rev. 4

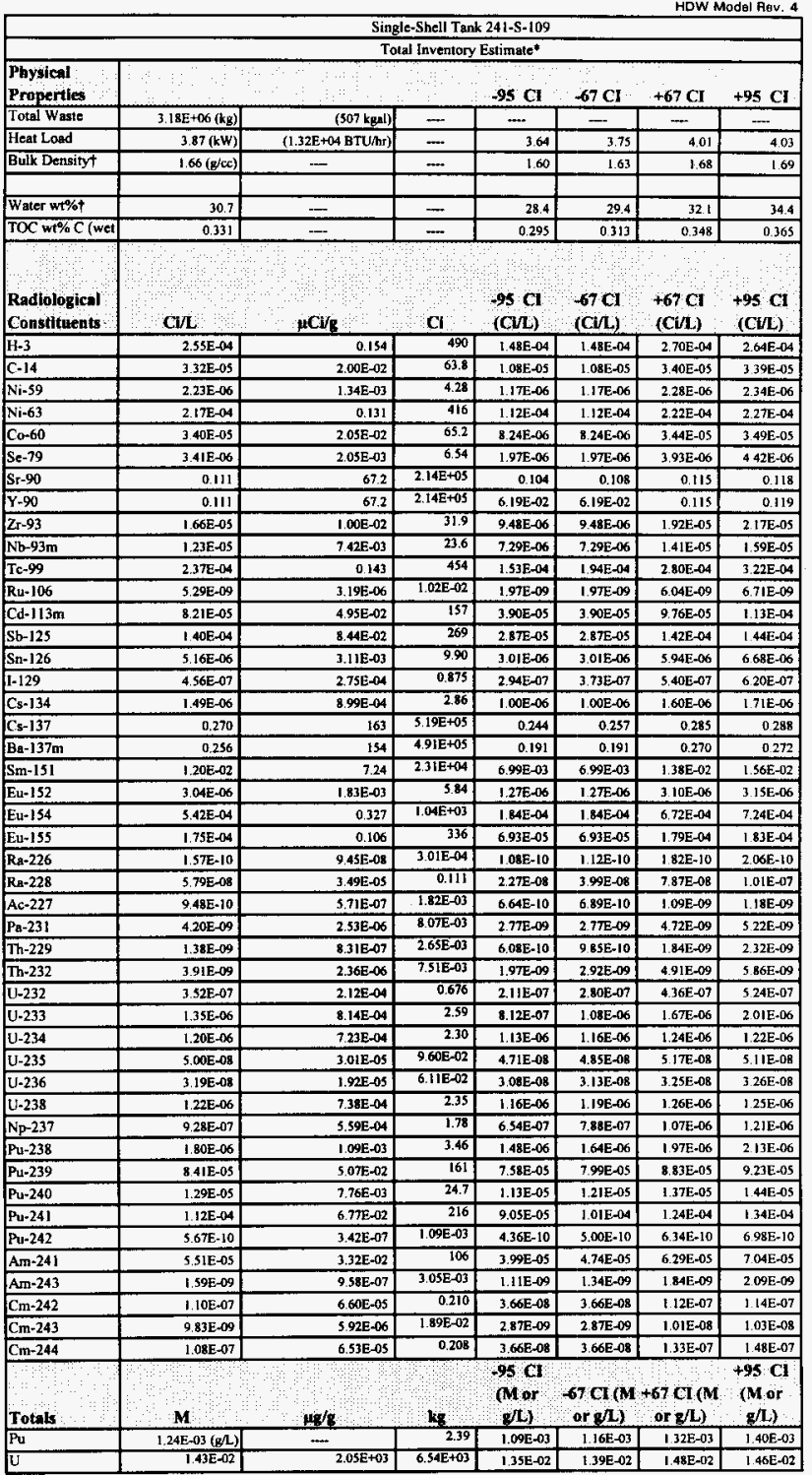

"Unknowns in tank solids inventory are assigned by Tonk Layering Model (TLM)

+ Volume average for density. mass average Water wt\% and TOC wi $\% \mathrm{C}$ 
HNF-SD-WM-ER-323, Rev. 1

HDW Model Rev. 4

\begin{tabular}{|c|c|c|c|c|c|c|c|}
\hline \multicolumn{8}{|c|}{ Single-Shell Tank 241-S-110 } \\
\hline & \multicolumn{7}{|c|}{ TLM Solids Composite Inventory Estimate* } \\
\hline $\begin{array}{l}\text { Physical : } \\
\text { Properties }\end{array}$ & $\because$ & & . & $-95: C I$ & $-67 \mathrm{CI}$ & $+67 \mathrm{CI}$ & $+95 \mathrm{CI}$ \\
\hline Total TLM Wast & $7.53 \mathrm{E}+05(\mathrm{~kg})$ & $(113 \mathrm{kgal})$ & $\ldots$ & - & - & - & 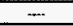 \\
\hline Heat Load & $2.20(\mathbf{k W})$ & $(7.50 \mathrm{E}+03$ BTU/hs $)$ & $\ldots$ & 1.59 & 1.95 & 2.36 & 2.43 \\
\hline Bulk Density & $1.76(\mathrm{~g} / \mathrm{cc})$ & - & $m$ & 1.56 & 1.58 & 2.04 & 2.14 \\
\hline Void Fraction & 0.560 & $\ldots$ & 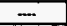 & 0.185 & 0.309 & 0.719 & 0.723 \\
\hline Water wt\% & 26.5 & $\ldots$ & - & 6.48 & 12.7 & 38.3 & 39.4 \\
\hline TOC wi\% C (wet & 0 & - & - & 0 & 0 & 0 & 0 \\
\hline $\begin{array}{l}\text { Chemical } \\
\text { Constituents }\end{array}$ & molel & ppin & $\mathrm{kg}$ & $\begin{array}{l}-95 \text { CI } \\
\text { (molerL) }\end{array}$ & $\begin{array}{c}-67 \mathrm{CI} \\
(\mathrm{mole} / \mathrm{L})\end{array}$ & $\begin{array}{l}+67 \mathrm{CH} \\
(\mathrm{mole} / \mathrm{L})\end{array}$ & $\begin{array}{c}+95 \mathrm{CI} \\
\text { (molen) }\end{array}$ \\
\hline $\mathrm{Nat}$ & B.07 & $1.05 E+05$ & $7.93 \mathrm{E}+04$ & 4.07 & 4.47 & 13.2 & 16.0 \\
\hline $\mathrm{A} / 3+$ & 7.88 & $1.21 \mathrm{E}+05$ & $910 E+04$ & 6.74 & 7.05 & B.48 & 8.91 \\
\hline $\mathrm{Fe} 3+(10451 \mathrm{Fe})$ & 0.833 & $2.64 E+04$ & $1.99 \mathrm{E}+04$ & 0.818 & 0.825 & 0.841 & 0.848 \\
\hline $\mathrm{Cr}^{3+}$ & $4.92 \mathrm{E}-02$ & 1.45E+03 & 1.09E+03 & $6.01 \mathrm{E}-03$ & 2.71E-02 & 0.411 & 0.835 \\
\hline $\mathrm{Bi3+}$ & 0 & 0 & 0 & 0 & 0 & 0 & 0 \\
\hline L83+ & 0 & 0 & 0 & 0 & 0 & 0 & 0 \\
\hline $\mathrm{Hg} 2+$ & $8.66 \mathrm{E}-04$ & 98.8 & 74.3 & 8.54E-04 & $8.61 \mathrm{E}-04$ & $8.70 \mathrm{E}-04$ & $8.73 E-04$ \\
\hline $\mathrm{Zr}($ as $\mathrm{ZrO}(\mathrm{OH}) 2)$ & 0 & 0 & 0 & 0 & 0 & 0 & 0 \\
\hline $\mathrm{Pb2+}$ & $250 \mathrm{E}-02$ & $2.94 E+03$ & $2.21 \mathrm{E}+03$ & $2.30 \mathrm{E}-02$ & 2.41E-02 & $2595-02$ & 2.67E-02 \\
\hline $\mathrm{Ni2+}$ & 4.01E-02 & $1.34 E+03$ & $1.01 \mathrm{E}+03$ & $2.90 \mathrm{E}-02$ & $3.53 \mathrm{E}-02$ & 4.38E-02 & 4.32E-02 \\
\hline $5+2+$ & $\overline{0}$ & 0 & 0 & 0 & 0 & 0 & 0 \\
\hline Mn4+ & 0 & 0 & 0 & 0 & 0 & 0 & 0 \\
\hline $\mathrm{Cn} 2 \mathrm{t}$ & 0.199 & $4.53 \mathrm{E}+03$ & $3.41 \mathrm{E}+03$ & 0.140 & 0.169 & 0.229 & 0.258 \\
\hline $\mathrm{K}+$ & $9.17 \mathrm{E}-03$ & 204 & 153 & $1.34 \mathrm{E}-03$ & $4.47 \mathrm{E}-03$ & $1.23 \mathrm{E}-02$ & $1.23 \mathrm{E}-02$ \\
\hline $\mathrm{OH}-$ & 29.0 & $2.80 \mathrm{E}+05$ & 2.11E+05 & 24.4 & 25.7 & 31.4 & 33.2 \\
\hline $\mathrm{NO}_{-}-$ & 4.29 & $1.51 E+05$ & $1.14 E+05$ & 0.169 & 0.169 & 10.7 & 14.2 \\
\hline NO2. & 1.35 & $3.53 \mathrm{E}+04$ & $2,66 \mathrm{E}+04$ & 0.307 & 0.672 & 1.90 & 1.90 \\
\hline cose- & 0.199 & $6.78 \mathrm{E}+03$ & $5.10 E+03$ & 0.140 & 0.169 & 0.229 & 0.258 \\
\hline PO43. & 0 & 0 & 0 & 0 & 0 & 0 & 0 \\
\hline $5042-$ & $9.98 \mathrm{E}-03$ & 545 & 410 & $2.72 \mathrm{E}-03$ & $5,62 \mathrm{E}-03$ & $1.29 \mathrm{E}-02$ & 1.29E-02 \\
\hline Si (es Sio32-) & 1.06E-02 & 169 & 127 & 4.99E- 03 & $7.24 \mathrm{E}-03$ & $2.37 \mathrm{E}-02$ & 4. 35E- 02 \\
\hline$F$ - & 0 & 0 & 0 & 0 & 0 & 0 & 0 \\
\hline $\mathrm{Cl}-$ & .22E-02 & 849 & 639 & 6. $15 \mathrm{E}-03$ & 2.05E-02 & $5.66 \mathrm{E}-02$ & 0.125 \\
\hline C6H5O73- & 0 & 0 & 0 & 0 & 0 & 0 & 0 \\
\hline EDTA4- & 0 & 0 & 0 & 0 & 0 & 0 & 0 \\
\hline HEDTA3- & 0 & 0 & 0 & 0 & 0 & 0 & 0 \\
\hline glycointe. & $\overline{0}$ & 0 & 0 & 0 & 0 & 0 & 0 \\
\hline acetate- & 0 & 0 & 0 & 0 & 0 & 0 & 0 \\
\hline oxalate2- & 0 & 0 & 0 & 0 & 0 & 0 & 0 \\
\hline DAP & 0 & 0 & 0 & 0 & 0 & 0 & 0 \\
\hline butanol & 0 & 0 & $\overline{0}$ & 0 & 0 & 0 & 0 \\
\hline $\mathrm{NH3}$ & 0.201 & $1.95 \mathrm{E}+03$ & $1.46 E+03$ & S.17E-02 & $7.29 \mathrm{E}-02$ & 0.213 & 0.224 \\
\hline Fo(CN) 64 - & 0 & 0 & 0 & 0 & 0 & 0 & 0 \\
\hline
\end{tabular}

"Unknowns in tank solids inventory are ateigrod by Tank: Leyering Model (TLM). 
HNF-SD-WM-ER-323, Rev. 1

HDW Model Rev. 4

\begin{tabular}{|c|c|c|c|c|c|c|c|}
\hline \multirow[b]{3}{*}{$\begin{array}{l}\text { Physical } \\
\text { Properties }\end{array}$} & \multicolumn{7}{|c|}{ Single-Shell Tank 241-S- 110} \\
\hline & \multicolumn{7}{|c|}{ SMM Composite Inventory Estimate } \\
\hline & & & & $-95 \mathrm{CI}$ & $-67 \mathrm{CI}$ & $+67 \mathrm{Cl}$ & $+95 \mathrm{CI}$ \\
\hline Total SMM Wast & $1.6 \mathrm{E}+05(\mathrm{~kg})$ & $(277 \mathrm{kgal})$ & - & $\cdots$ & $=$ & - & -+- \\
\hline Heat Load & $2.09(\mathrm{~kW})$ & (3.14E+03 BTU/hr) & $=$ & 1.96 & 2.03 & 2.14 & 2.18 \\
\hline Bulk Density" & $1.59(\mathrm{~g} / \mathrm{cc})$ & - & - & 1.54 & 1.57 & 1.62 & 1.63 \\
\hline & & & & & & & \\
\hline Water wt $\%$ & 346 & $=$ & $\ldots$ & 318 & 33.0 & 36.2 & 38.1 \\
\hline TOC wt\% C (wet & 0.569 & - & - & 0.441 & 0.503 & 0.634 & 6.698 \\
\hline $\begin{array}{c}\text { Chemical } \\
\text { Constituents }\end{array}$ & molefl & ppm : & kg & $\begin{array}{l}95 \mathrm{CI} \\
\text { (mole/L) }\end{array}$ & $\begin{array}{r}-67 \mathrm{CI} \\
\text { (mole } / \mathrm{L})\end{array}$ & $\begin{array}{c}+67 \mathrm{CI} \\
(\mathrm{mole} / \mathrm{L})\end{array}$ & $\begin{array}{c}+95 \mathrm{CI} \\
(\mathrm{mole} / \mathrm{L})\end{array}$ \\
\hline $\mathrm{Nat}$ & 13.9 & $2.00 E+05$ & 3.34E+05 & 12.6 & 132 & 14.4 & 14.9 \\
\hline $\mathrm{Al3}+$ & 1.76 & $2.98 \mathrm{E}+04$ & $4.98 \mathrm{E}+04$ & 1.61 & 1.68 & 1.85 & 1.86 \\
\hline Fe]+(total Fe) & $982 \mathrm{E}-03$ & 344 & 575 & $7.75 \mathrm{E}-03$ & B. $77 \mathrm{E} \omega 03$ & 1.09E-02 & 1. $19 \mathrm{E}-02$ \\
\hline $\mathrm{Cr}^{3}+$ & 0.163 & $5.33 \mathrm{E}+03$ & $8.91 E+03$ & 0.134 & 0.150 & 0.170 & 0.176 \\
\hline $\mathrm{Bi3+}$ & $8.12 \mathrm{E}-04$ & 107 & 178 & 7.49E- 04 & $7.80 \mathrm{E}-04$ & 8.45E-04 & $8.76 \mathrm{E}-04$ \\
\hline $\mathrm{La3+}$ & $7.38 \mathrm{E}-06$ & 0.643 & 1.08 & $5.35 \mathrm{E}-06$ & $6.34 \mathrm{E}-06$ & $8.43 E-06$ & 9.44E-06 \\
\hline Hy $2+$ & $7.06 E-166$ & 0889 & 1.49 & $6.73 \mathrm{E} \cdot 06$ & $6.92 \mathrm{E}-06$ & $7.17 \mathrm{E}-06$ & $7.28 \mathrm{E}-06$ \\
\hline 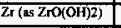 & $1.42 \mathrm{E}-04$ & 8.13 & 13.6 & $1.30 \mathrm{E}-04$ & $1.35 \mathrm{E}-04$ & $1.47 \mathrm{E}-04$ & $1.54 \mathrm{E}-04$ \\
\hline $\mathrm{Pb2+}$ & $8.62 \mathrm{E}-04$ & 112 & 187 & $7.03 \mathrm{E}-04$ & $7.81 \mathrm{E}-04$ & $9.44 \mathrm{E}-04$ & $1.02 E-03$ \\
\hline $\mathrm{Ni2+}$ & $5.77 \mathrm{E}-03$ & 212 & 355 & $5.53 \mathrm{E}-03$ & $5.65 \mathrm{E}-03$ & $5.85 \mathrm{E}-03$ & $5.92 \mathrm{E}-03$ \\
\hline $5 \sqrt{2+}$ & 0 & of & 0 & 0 & 0 & 0 & 0 \\
\hline Mn4+ & $3.58 \mathrm{E}-03$ & 123 & 206 & $2.59 \mathrm{E}-03$ & $3.07 E-03$ & 4.09E-03 & 4.58E-03 \\
\hline Cat+ & $2,98 \mathrm{E}-02$ & 750 & $1.25 \mathrm{E}+03$ & $2.68 \mathrm{E}-02$ & $2.83 E-02$ & $3,13 E \cdot 02$ & $3.28 \mathrm{E}-02$ \\
\hline $\mathrm{K}+$ & $6.00 \mathrm{E}-02$ & $1.47 \mathrm{E}+03$ & $2.46 \mathrm{E}+03$ & $5,39 \mathrm{E}-02$ & $5.68 \mathrm{E}-02$ & $6.33 \mathrm{E}-02$ & $6.64 \mathrm{E}-02$ \\
\hline OH- & 10.8 & $1.15 \mathrm{E}+05$ & $1.92 \mathrm{E}+05$ & 9.84 & 10.3 & 11,3 & 11.4 \\
\hline NO3- & 4.56 & $1.77 \mathrm{E}+05$ & $2.97 \mathrm{E}+05$ & 4.30 & 4.44 & 466 & 4.69 \\
\hline NO2: & 2.55 & $7.36 \mathrm{E}+04$ & $1.23 \mathrm{E}+0.5$ & 2.13 & 2.32 & 2.77 & 2.98 \\
\hline $\mathrm{CO} 32$ & 0.365 & 1.38E+04 & $2.30 \mathrm{E}+04$ & 0.328 & 0.347 & 0.384 & 0.397 \\
\hline PO43- & $6.36 \mathrm{E}-02$ & $3,79 \mathrm{E}+03$ & $6.33 \mathrm{E}+03$ & $5.69 E-02$ & $5.93 \mathrm{E}-02$ & $6.48 E-02$ & 6.59E-02 \\
\hline $\mathrm{SO} 42-$ & 0.209 & $1.26 \mathrm{E}+04$ & 2. $10 \mathrm{E}+04$ & 0.154 & 0.180 & 0.239 & 0.265 \\
\hline Si (ns \$i032-) & $7.24 \mathrm{E}-02$ & $1.28 \mathrm{E}+03$ & $2.13 \mathrm{E}+03$ & $5.82 \mathrm{E}-02$ & $6.52 \mathrm{E}-02$ & $7.97 \mathrm{E}-02$ & $8.67 \mathrm{E}-02$ \\
\hline $\mathrm{F}-$ & 3.95E-02 & 471 & 786 & $3.39 \mathrm{E}-02$ & $3.63 \mathrm{E}-02$ & $4.22 \mathrm{E}-02$ & $443 \mathrm{E}-22$ \\
\hline $\mathrm{Cl}$ - & 0.235 & $5.22 \mathrm{E}+03$ & $8.72 \mathrm{E}+03$ & 0.210 & 0.223 & 0.246 & 0.255 \\
\hline C6H5O73- & $2.82 \mathrm{E}-02$ & $334 \mathrm{E}+03$ & $5.59 \mathrm{E}+03$ & $2.61 \mathrm{E}-02$ & $2.71 \mathrm{E}-02$ & $2.93 \mathrm{E}-02$ & 3.03E- 02 \\
\hline EDTA4- & $7.79 E-03$ & 1.41E+03 & $2.35 E+03$ & $2.75 \mathrm{E}-03$ & $5,21 \mathrm{E}-03$ & $1.04 \mathrm{E}-02$ & 1.29E-02 \\
\hline HEDTA3- & 1.40E-02 & $241 \mathrm{E}+03$ & $4.03 \mathrm{E}+03$ & $396 \mathrm{E}-03$ & $8.89 E-03$ & $1.92 \mathrm{E}-02$ & $2.43 \mathrm{E} .02$ \\
\hline glycolate- & 6.95E-02 & $327 \mathrm{E}+03$ & $5.47 \mathrm{E}+03$ & $4.18 \mathrm{E}-02$ & $5.53 \mathrm{E}-02$ & $8.38 \mathrm{E}-02$ & $9.73 \mathrm{E}-\mathrm{O} 2$ \\
\hline acelnte- & 4.96E-03 & 184 & 307 & $4.03 \mathrm{E}-03$ & $4.48 \mathrm{E}-03$ & S.43E-03 & $5.89 \mathrm{E}-03$ \\
\hline oxalate2- & $9,67 \mathrm{E}-06$ & 0.339 & 0.893 & 8.57E-06 & $9.11 \mathrm{E}-06$ & $1.02 \mathrm{E}-05$ & 1.08E-05 \\
\hline DBP & $1.83 \mathrm{E}-02$ & $2.41 \mathrm{E}+03$ & $4.02 \mathrm{E}+03$ & $1.48 \mathrm{E}-02$ & $1.65 \mathrm{E}-02$ & $2.00 \mathrm{E}-02$ & $2.17 \mathrm{E}-02$ \\
\hline butanol & $1.83 \mathrm{E}-02$ & 849 & $1.42 \mathrm{E}+03$ & $1.48 \mathrm{E}-02$ & $1.65 \mathrm{E}-02$ & $200 \mathrm{E}-02$ & $2.17 \mathrm{E}-02$ \\
\hline $\mathrm{NH3}$ & $7.24 \mathrm{E}-02$ & 772 & $1.29 \mathrm{E}+03$ & $5.63 \mathrm{E}-02$ & $6.27 \mathrm{E}-02$ & $8.48 E-02$ & $9.92 \mathrm{E}-02$ \\
\hline $\mathrm{Fe}(\mathrm{CN}) 64$ & 0 & 0 & 0 & 0 & 0 & 0 & 0 \\
\hline
\end{tabular}

"Density is calculated based on $\mathrm{Na}, \mathrm{OH}-$, and AlO2-

†Water wt\% derived from the difference of density and total dissolved species. 


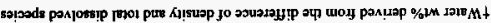

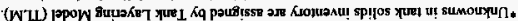

\begin{tabular}{|c|c|c|c|c|c|c|c|}
\hline 0 & 0 & 0 & 0 & 0 & 0 & 0 & $-\mathrm{tg}(\mathrm{N}) \mathrm{NOA}_{\mathrm{H}}$ \\
\hline 5210 & 6110 & $20-3012$ & zo-atris 9 & $50+\mathrm{g} 5 i z$ & $50+3 \neq 1]$ & 0110 & $\mathrm{EHN}$ \\
\hline & & & & & & & \\
\hline 2000051 & $z 0-3 z v^{\prime} 1$ & $20-\exists 4[1$ & 20 -aso' I & $20+\pi z \div \div$ & 585 & $20=\mathrm{gor} 1$ & pournng \\
\hline $20-9451$ & zo-azb' I & $20-7<11 \mathrm{~T}$ & 20-750 1 & $50+\mathrm{H}^{2} \mathrm{O}^{\prime} \mathrm{b}$ & $60+799 \div$ & to-Gor 1 & गGO \\
\hline 90-359L & 90:alL2 & $90-3<+9$ & $90-1609$ & 6680 & $89{ }^{\circ} 0$ & $90-31489$ & - zanejexo \\
\hline fo- $181 \%$ & c0-998'C & 60-381 E & $50-398 z$ & LOE & $\angle Z I$ & 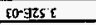 & जintose \\
\hline 20.3169 & 20.756s & 20-9E6 E & $20-3<6$ & $60+3<t \leq 5$ & $10+392 ?$ & zo-ato6: & 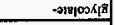 \\
\hline $20-3 z L 1$ & 20 - & co-g1E' & c0-al18 7 & $50+950)$ & $50+2991$ & $20-9<6.6$ & $\neg Y \perp O H H$ \\
\hline $50-3<l^{\prime} 6$ & EO-ALEL & 50-90L'C & CO-GS6'I & co+ass $z$ & 1160 & ro-afs $s$ & +VIOG \\
\hline 203512 & Z0- $980 \mathrm{Z}$ & $20-3561$ & $20-7981$ & $60+765 ' 5$ & E0+3icz & $20-2002$ & $-[\cos H 9 \partial$ \\
\hline 1020 & $\angle 810$ & $0 \angle I^{\prime} 0$ & 2910 & $60+79 C^{\prime} 6$ & $80+398 \mathrm{E}$ & 6010 & 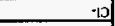 \\
\hline $20-35 I^{\prime} \varepsilon$ & $20-300 \mathrm{f}$ & $20 \mathrm{gss} 2$ & $\tau 0-$ att $z$ & $98 L$ & $+z \varepsilon$ & $20-308 z$ & -3 \\
\hline $20-39 p^{\prime} 9$ & $20-7<6,5$ & $20-966$ & $20-a b+b$ & $60+39 \tau z$ & $\tau \Sigma 6$ & zo-as's & $(-z \operatorname{to} 19 \pi) \mid \mathrm{s}$ \\
\hline 1610 & $z<10$ & IEI O & 2110 & $00+3 s 1 \mathrm{Z}$ & $60+3589$ & 1510 & $-2 \operatorname{cros}$ \\
\hline $20-9896$ & $20-709^{\prime}+$ & zo-alz't & $20-360 t$ & co+jec 9 & $\mathrm{c} 0+\mathrm{a} 19 \mathrm{z}$ & $20-\mathrm{AzS}^{\circ} \mathrm{b}$ & Etrod \\
\hline 6EE0 & OEE $O$ & toe 0 & $16 \tau 0$ & $00+g 18 z$ & $10+99 L^{\prime} \mid$ & $\angle I E 0$ & $-z[00$ \\
\hline 152 & of 2 & 002 & Es't & SOO+J0s I & $+00+3419$ & $0 z z$ & $-\mathrm{ZON}$ \\
\hline 612 & 169 & $\angle Z \varepsilon$ & $81 \mathrm{E}$ & so+9olt & $50+3691$ & set & -EON \\
\hline 221 & $L^{\prime} 91$ & I'SI & $L t I$ & SO+A5ot & $50+3991$ & I'9! & $-\$ 10$ \\
\hline 203600 & $20^{\circ} 39 L$ & $20.900^{\circ}$ & $20-360 \%$ & $60+919 z$ & $50+780 \cdot 1$ & 20-3ist & +8 \\
\hline $20-9266$ & $20-7998$ & $20-3602 \mathrm{~L}$ & $20-7<2 \% 9$ & $50+3990$ & {$[0+\mathrm{a} 25 \mathrm{C}$} & to-38s'L & $+2 t$ \\
\hline E0-J\ZE & $50-316 t$ & to-38t 2 & $60-3081$ & 902 & 158 & co-atsz & $+{ }^{+} \mathrm{uW}$ \\
\hline 0 & 0 & 0 & 0 & 0 & 0 & 0 & $+2+5$ \\
\hline $20-359 \mathrm{I}$ & $20-3891$ & Z0.JED 1 & $20^{\circ} \mathrm{asz} !$ & s0+z9\&! ! & zos & $20-9151$ & $+2 ! N$ \\
\hline $60-3158$ & $60-760 \%$ & $50-9192$ & $50-792 L$ & $20+30+2$ & 166 & $60-3581$ & $+20 \mathrm{~d}$ \\
\hline to- $360^{\circ} 1$ & $50 \cdot 3601$ & $50-9956$ & $50-9926$ & $9 \times 1$ & 195 & bo-310.1 & $(\mathrm{Z}(\mathrm{HO}) \mathrm{OZ} \mathrm{Sx}) \mathrm{IZ}$ \\
\hline $60-9852$ & $+0-z<s \tau$ & 60-ass'z & to-32s: & 852 & EIE & $00-395 \tau$ & $+z^{0} \mathrm{H}$ \\
\hline $90-3069$ & $90-3665$ & $90-3156$ & $90-308 \varepsilon$ & 801 & +00 & 90-ast5 & 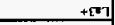 \\
\hline $50-3229$ & t0-700'9 & to-ats s & to-azes & $8 \angle 1$ & res & po-3LLS & $+\pi ! 9$ \\
\hline$\angle E E O$ & $\operatorname{sizo}$ & szlo & 9110 & $10+3001$ & E0+gโI't & oci: 0 & $+[5]$ \\
\hline zsto & osto & $962 \%$ & $\$ 20$ & $10+350 \tau$ & $\varepsilon_{0}+3 \varsigma_{p} \mathrm{~s}$ & $8+20$ & 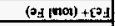 \\
\hline $28 \mathrm{E}$ & $0<\varepsilon$ & $0 \varepsilon \varepsilon$ & $02 \mathrm{C}$ & $50+9\left|p^{\prime}\right|$ & $60+318 \mathrm{~s}$ & ES: & $+\varepsilon \mid Y$ \\
\hline$\varepsilon+1$ & $6 \mathrm{El}$ & 601 & 601 & so+aEit & $50+3 \mid<1$ & 221 & $+N$ \\
\hline $\begin{array}{l}\text { (1/2001) } \\
\text { ID S6t }\end{array}$ & $\begin{array}{l}\text { (1/204) } \\
1569+\end{array}$ & 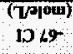 & $\begin{array}{l}\text { (1/p104) } \\
1096\end{array}$ & 87 & $\ln d \mathrm{~d}$ & T/potit & 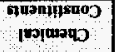 \\
\hline $28+0$ & 8ETO & $\angle D E 0$ & Doro & 二 & - & 2650 & $29 \mathrm{~m}) \mathrm{\partial} \% \mathrm{~m} \mathrm{jOL}$ \\
\hline $9 \angle \varepsilon$ & E9E & $\angle 92$ & $\tau s z$ & $=$ & $=$ & $12 \varepsilon$ & $+\%$ L SOIE $M$ \\
\hline$S L I$ & al & 851 & 951 & - & $=$ & $(x, \pi)$ to 1 & thutsued x[ng \\
\hline $\cos t$ & $6+t$ & $00^{\prime} b$ & $19 \mathrm{E}$ & - & $(44 / 21800+39+1)$ & $(M Y) 6 \tau^{\circ}$ & p8o7 jeaH \\
\hline- & -- & - & - & $=$ & $(1+8 \times 1068)$ & $(z) 90+\exists z+z$ & 25se $M$ [B]O \\
\hline 1D. $56+$ & $1369 t$ & ID $69^{\circ}$ & 1.9 $56^{\circ}$ & & & & 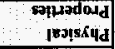 \\
\hline \multicolumn{8}{|c|}{ 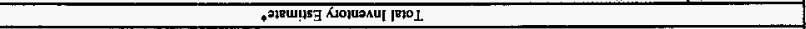 } \\
\hline & & & $011-S^{-1}$ & $1 \|$ IF & suts & & \\
\hline
\end{tabular}


HNF-SD-WM-ER-323, Rev. 1

HDW Model Rev. 4

\begin{tabular}{|c|c|c|c|c|c|c|c|}
\hline \multirow[b]{3}{*}{$\begin{array}{l}\text { Physical } \\
\text { Properties: }\end{array}$} & \multicolumn{7}{|c|}{ Single-Shell Tank $241-\mathrm{S}-110$} \\
\hline & \multicolumn{7}{|c|}{ TLM Solids Composite Inventory Estinute } \\
\hline & $\vdots$ & & & $-93 \mathrm{CI}$ & $-67 \mathrm{CI}$ & $+67 \mathrm{CI}$ & $+95 \mathrm{CI}$ \\
\hline Total TLM Wast & $7.53 \mathrm{E}+05\left(\mathrm{k}_{\mathrm{B}}\right)$ & $(113 \mathrm{kgal})$ & $\ldots$ & - & $\cdots$ & - & + \\
\hline Heat Load & $2.20(\mathrm{~kW})$ & $(7.50 \mathrm{E}+03 \mathrm{BTU} / \mathrm{hr})$ & $\ldots$ & 1.59 & 1.95 & 2.36 & 243 \\
\hline Bulk Density & $1.76(\mathrm{~g} / \mathrm{cet})$ & - & $m$ & 1.56 & 1.58 & 2.04 & 2.14 \\
\hline Void Fraction & 0560 & --- & +- & 0.185 & 0.309 & 0,719 & 0.723 \\
\hline Water wt\% & 26.5 & $\Longrightarrow$ & $=$ & 6.48 & 12.7 & 38.3 & 39.4 \\
\hline TOC wt\% C (wet & 0 & - & $\ldots$ & 0 & 0 & 0 & 0 \\
\hline $\begin{array}{l}\text { Radiological } \\
\text { Constituents } \\
\end{array}$ & $\mathrm{CH} / \mathrm{L}$ & $\mu \mathrm{Cyg}$ & $\mathrm{Ci}$ & $\begin{array}{l}-95 \mathrm{CI} \\
(\mathrm{CWL})\end{array}$ & $\begin{array}{r}67 \mathrm{CI} \\
(\mathrm{Cl} / \mathrm{L})\end{array}$ & $\begin{array}{l}+67 \mathrm{CI} \\
(\mathrm{CU} /)\end{array}$ & $\begin{array}{l}+95 \mathrm{Ct} \\
(\mathrm{C} / \mathrm{L})\end{array}$ \\
\hline $\mathrm{H}-3$ & $2.32 \mathrm{E}-05$ & $1.32 \mathrm{E}-02$ & 994 & $2.62 \mathrm{E}-06$ & $1.18 \mathrm{E}-05$ & $3.25 E-05$ & $3.25 \mathrm{E}-05$ \\
\hline C.14 & $1.81 \mathrm{E}-06$ & $1.03 \mathrm{E}-03$ & 0.772 & $2.27 \mathrm{E}-07$ & $8.58 \mathrm{E}-07$ & $2.44 E-06$ & $2.44 E-06$ \\
\hline $\mathrm{Ni}-59$ & 1.17E-05 & 6.65E-03 & 5.01 & $8.45 \mathrm{E}-06$ & 1.03E-05 & 1.28E-05 & $126 \mathrm{E}-05$ \\
\hline $\mathrm{Ni}=63$ & $1.09 \mathrm{E}-03$ & 0.621 & 468 & $7.89 \mathrm{E}-04$ & $9.61 E-04$ & $1.19 \mathrm{E}-03$ & $118 \mathrm{E}-03$ \\
\hline $\mathrm{Co}-60$ & $7.14 \mathrm{E}-07$ & $4.06 \mathrm{E}-04$ & 0.305 & 9.05E-08 & $3.39 \mathrm{E}-07$ & $9.64 \mathrm{E}-07$ & $9.64 \mathrm{E}-07$ \\
\hline Se-79 & 3. $84 \mathrm{E}-07$ & $2.18 \mathrm{E}-04$ & 0.164 & 4.83E-08 & $1.82 \mathrm{E}-07$ & 5.18E-07 & 5.64E-06 \\
\hline$\$ r-90$ & 0.732 & 416 & $3.13 \mathrm{E}+05$ & 0.522 & 0.640 & 0.802 & 0.814 \\
\hline Y.90 & 0.732 & 416 & $3.13 \mathrm{E}+05$ & 0.522 & 0.640 & 0.802 & 0814 \\
\hline $2 r-93$ & $1.81 \mathrm{E}-06$ & $1.03 \mathrm{E}-03$ & 0.774 & $2.28 \mathrm{E}-07$ & $8.60 \mathrm{E}-07$ & $2.45 \mathrm{E}-06$ & $2.39 \mathrm{E}-0.5$ \\
\hline $\mathrm{Nb}-93 \mathrm{~m}$ & $1.48 \mathrm{E}-06$ & 8.41E-04 & 0.633 & $1.86 \mathrm{E}-07$ & $7.03 \mathrm{E}-07$ & $2.00 \mathrm{E}-06$ & $2.53 \mathrm{E}-05$ \\
\hline Te-99 & $1.26 E_{0} 05$ & 7.19E-03 & 5,41 & $1.59 \mathrm{E}-06$ & $6.01 \mathrm{E}-06$ & 1.71E-05 & $1.71 E-05$ \\
\hline $\mathrm{Ru}-106$ & $2.02 \mathrm{E}-12$ & $1.15 \mathrm{E}-09$ & $8.66 E-07$ & $2.83 \mathrm{E}-13$ & $9.79 \mathrm{E}-13$ & $2.72 \mathrm{E} \cdot 12$ & $272 \mathrm{E}-12$ \\
\hline $\mathrm{Cd}-113 \mathrm{~m}$ & $5.58 \mathrm{E}-06$ & $3.17 \mathrm{E}-03$ & 2.39 & $7.04 \mathrm{E}-47$ & 2.65E- 06 & $7.54 \mathrm{E}-0.06$ & $7.54 \mathrm{E}-06$ \\
\hline $\mathrm{Sb}-125$ & $1.05 \mathrm{E}-06$ & $5.97 \mathrm{E}-04$ & 0.449 & $1.35 \mathrm{E}-07$ & $5.01 \mathrm{E}-07$ & $1.42 \mathrm{E}-06$ & $1.42 \mathrm{E}-06$ \\
\hline $\mathrm{Sn}=126$ & $5,89 \mathrm{E}-07$ & $3.35 \mathrm{E}-04$ & 0.252 & 7.42E-08 & $2.80 \mathrm{E}-07$ & $7.96 \mathrm{E}-07$ & $9.03 \mathrm{E}-06$ \\
\hline $1-129$ & $2.43 \mathrm{E}-08$ & $1,38 E-0 S$ & 1.04E-02 & $3.06 \mathrm{E}-09$ & 1. $15 \mathrm{E}-08$ & $3.28 \mathrm{E}-08$ & $3.28 \mathrm{E}-08$ \\
\hline Cs-134 & $2.18 \mathrm{E}-08$ & $1.24 \mathrm{E}-0 \mathrm{~s}$ & $9.31 \mathrm{E}-03$ & $6.01 \mathrm{E}-09$ & 1.23E-08 & $2.81 \mathrm{E}-08$ & 2.8IE-08 \\
\hline Cs-137 & $4.46 \mathrm{E}-02$ & 25.4 & $1.91 \mathrm{E}+04$ & $1.22 \mathrm{E}-02$ & $251 \mathrm{E}-02$ & $5.77 \mathrm{E}-02$ & $5.77 \mathrm{E}-02$ \\
\hline $\mathrm{Ba}-137 \mathrm{~m}$ & $4.22 \mathrm{E}-02$ & 24.0 & $1.81 \mathrm{E}+04$ & 1.15E -02 & $238 E-02$ & $5,45 \mathrm{E}-02$ & $5.45 \mathrm{E}-02$ \\
\hline Sm-151 & $1.37 \mathrm{E}-03$ & 0778 & 585 & $1.72 \mathrm{E}-04$ & $6.50 \mathrm{E}-04$ & $1.85 E-03$ & $2.08 \mathrm{E}-02$ \\
\hline Ev-152 & $5.55 \mathrm{E}-06$ & 3.15E-03 & 2,37 & $5.43 E+06$ & $5.48 \mathrm{E}-06$ & $5.60 \mathrm{E}-06$ & $5.60 \mathrm{E}-06$ \\
\hline Eu-154 & $1.72 \mathrm{E}-05$ & $9.80 \mathrm{E}-03$ & 7.38 & $2.19 \mathrm{E}-06$ & 8.20E-06 & $2.33 \mathrm{E}-05$ & $2.33 \mathrm{E}-05$ \\
\hline Eu- 155 & $2.62 \mathrm{E}-04$ & 0.149 & 112 & $2.56 \mathrm{E}-04$ & $2.59 \mathrm{E}-04$ & $2.64 \mathrm{E}-(24)$ & $2.64 \mathrm{E}-04$ \\
\hline $\mathrm{Ra}=226$ & $852 \mathrm{E}-10$ & 4.84E-07 & $3.64 \mathrm{E}-04$ & $1,17 \mathrm{E}-10$ & 4.77E-10 & $1.23 \mathrm{E}-09$ & $1.59 \mathrm{E}-09$ \\
\hline Ra-228 & $7.36 \mathrm{E}-15$ & 4.18E-12 & $3.15 \mathrm{E}-09$ & $7.20 \mathrm{E}-15$ & $7.27 \mathrm{E}-15$ & $7,42 \mathrm{E}-15$ & $7.42 \mathrm{E}=15$ \\
\hline Ac-227 & $3.75 \mathrm{E}-09$ & 2.13E-06 & $1.61 \mathrm{E}-03$ & $2.44 \mathrm{E}-10$ & $1.58 \mathrm{E}-09$ & $5.93 E-09$ & $8.02 E-09$ \\
\hline $\mathrm{Pa}-231$ & $5.73 E-10$ & $3.25 \mathrm{E}-07$ & $2.45 E-04$ & $7.20 \mathrm{E}-11$ & $2.72 \mathrm{E}-10$ & $1.62 \mathrm{E}-09$ & 1.32E-08 \\
\hline Th-229 & $140 \mathrm{E}-12$ & $7,96 \mathrm{E}-10$ & 5.99E-07 & $1.37 \mathrm{E}-12$ & $1.38 E-12$ & $1.41 \mathrm{E}-12$ & $1.41 \mathrm{E}-12$ \\
\hline Th-232 & $4.74 \mathrm{E}-16$ & $2.69 \mathrm{E}-13$ & $2.03 \mathrm{E}-10$ & $6.18 \mathrm{E}-17$ & $2.26 \mathrm{E}-16$ & $6.39 E-16$ & $6.39 \mathrm{E}-16$ \\
\hline $\mathrm{v}-232$ & $1.41 \mathrm{E}-10$ & 8.04E-08 & 6.05E-05 & $1.23 \mathrm{E}-10$ & $1.34 E-10$ & 1.47E-10 & $1.51 \mathrm{E}-10$ \\
\hline $\mathrm{U}-233$ & $5.24 \mathrm{E}-12$ & $2.98 \mathrm{E}-09$ & $2.24 \mathrm{E}-06$ & $4.57 \mathrm{E}-12$ & $4.96 \mathrm{E}-12$ & $5.44 \mathrm{E}-12$ & $5.58 \mathrm{E} \cdot 12$ \\
\hline $\mathrm{j}-234$ & $3.24 E-06$ & 1.84E-03 & 1.38 & $2.83 \mathrm{E}-06$ & $3.07 \mathrm{E}-06$ & $3.36 \mathrm{E}-06$ & $3.45 E+06$ \\
\hline U.235 & $1.38 \mathrm{E}-0 ?$ & $7.82 \mathrm{E}-05$ & 5.88E-02 & $1.20 \mathrm{E}-07$ & $1.30 \mathrm{E}-07$ & 1.43E-07 & 1.46E-07 \\
\hline U-236 & 2.07E-08 & $4.02 \mathrm{E}-05$ & $302 \mathrm{E}=02$ & $6.16 \mathrm{E}-08$ & $0.69 \mathrm{E}-08$ & $7,34 \mathrm{E}-08$ & $753 \mathrm{E}-08$ \\
\hline $\mathrm{U}-238$ & $3.16 \mathrm{E}-06$ & $1.79 \mathrm{E}-03$ & 1.35 & $2.75 \mathrm{E}-06$ & $299 \mathrm{E}-06$ & 3.28E-06 & 3.36E-06 \\
\hline $\mathrm{Np}-237$ & $8.22 \mathrm{E}-08$ & $4.67 \mathrm{E}-0.5$ & 1.51E-02 & $1.04 E-08$ & $3.91 \mathrm{E}-08$ & $1.11 \mathrm{E}-07$ & $111 \mathrm{E}-07$ \\
\hline Pu-238 & 1.05E-05 & $5,99 \mathrm{E}-03$ & 4.51 & 9.07E-06 & $979 \mathrm{E}-06$ & 1.13E-05 & $1.20 \mathrm{E}-05$ \\
\hline $\mathrm{Pu}-239$ & $7.13 \mathrm{E}-04$ & 0405 & 305 & $6.08 \mathrm{E}-04$ & $6.60 \mathrm{E}-04$ & $7,67 \mathrm{E}-04$ & 8.18E-04 \\
\hline $\mathrm{Pu}-240$ & 1.01E-04 & $5,766-02$ & 43.4 & 8.64E-05 & $937 \mathrm{E}-05$ & $1.09 \mathrm{E}-04$ & $1.16 \mathrm{E}-04$ \\
\hline Pu-241 & $6.07 E-04$ & 0.345 & 260 & 5.19E-04 & $5.62 \mathrm{E}-0.4$ & $6.52 \mathrm{E}-04$ & $6.96 \mathrm{E}-04$ \\
\hline Pu-242 & $2.68 \mathrm{E}-09$ & $1.53 \mathrm{E}-06$ & 1.15E-03 & $2.28 \mathrm{E}-09$ & $2.48 \mathrm{E}-99$ & 2.89E-09 & $3.09 \mathrm{E}-09$ \\
\hline Am-241 & $6.46 \mathrm{E}-06$ & $3.67 \mathrm{E}-03$ & 2.76 & $8.16 \mathrm{E}-07$ & $3.07 \mathrm{E}-06$ & $8.73 \mathrm{E}-06$ & $1.13 \mathrm{E}_{-} 04$ \\
\hline$A m-243$ & 6.04E-11 & $3.43 \mathrm{E}-08$ & 2.58E-05 & $7.61 \mathrm{E}-12$ & $2.87 E-11$ & $8.16 \mathrm{E}-11$ & 1.06E-09 \\
\hline $\mathrm{Cm}-242$ & $1.28 \mathrm{E}-07$ & $7.25 \mathrm{E}-05$ & $545 E-02$ & $1.25 \mathrm{E}-07$ & 1.26E-07 & $1.29 \mathrm{E}-07$ & $1.29 \mathrm{E}-07$ \\
\hline $\mathrm{Cm}-243$ & $2.92 \mathrm{E}-09$ & $1.66 \mathrm{E}-06$ & i.25E-03 & 2.86E-09 & $2.89 \mathrm{E}-09$ & $2.95 \mathrm{E}-09$ & $2.95 \mathrm{E}-09$ \\
\hline $\mathrm{Cm}-244$ & $2.16 \mathrm{E}-09$ & $1.23 \mathrm{E}-06$ & $9.23 \mathrm{E}-104$ & $2.71 \mathrm{E}-10$ & $1,02 \mathrm{E}-09$ & $2.92 \mathrm{E}-09$ & $2.92 \mathrm{E}-09$ \\
\hline Totals & $\mathbf{M}$ & $\mu / g$ & ks: & $\begin{array}{c}95 \mathrm{Cl} \\
(\mathrm{Mor} \\
\text { o/L) }\end{array}$ & $\begin{array}{r}-67 \mathrm{COM} \\
\text { orgh) }\end{array}$ & $\begin{array}{r}+67 \mathrm{Cl}(\mathrm{M} \\
0 \times \mathrm{g} / \mathrm{L}) \\
\end{array}$ & $\begin{array}{c}+95 \mathrm{Cl} \\
(\mathrm{M} \mathrm{or} \\
\mathrm{g} / \mathrm{L})\end{array}$ \\
\hline $\mathrm{Pu}$ & 1.19E-02 $(\mathrm{g} / \mathrm{h})$ & - & 5.10 & $1.02 E-02$ & $1.10 \mathrm{E}-02$ & 1.28E-02 & $1.37 \mathrm{E}-02$ \\
\hline $\mathbf{U}$ & $3.97 \mathrm{E}-02$ & $5.37 \mathrm{E}+03$ & $4.04 E+03$ & $3.47 \mathrm{E}-02$ & $3,76 \mathrm{E}-02$ & $4.12 \mathrm{E}-02$ & $4.23 \mathrm{E}-02$ \\
\hline
\end{tabular}

*Unknowns in tank solids inventory are assigned by Tank Layering Model (TL.M). 
HNF-SD-WM-ER-323, Rev. 1

HDW Model Rev, 4

\begin{tabular}{|c|c|c|c|c|c|c|c|}
\hline \multirow[b]{3}{*}{$\begin{array}{l}\text { Physical } \\
\text { Properties }\end{array}$} & \multicolumn{7}{|c|}{ Single-Shell Tank $24 \mid-S-110$} \\
\hline & \multicolumn{7}{|c|}{ SMM Composite Inventory Estimate } \\
\hline & & & & $-95 \mathrm{CI}$ & $-67 \mathrm{CI}$ & $+67 \mathrm{CI}$ & $+95 \mathrm{CI}$ \\
\hline Total SMM Wast & $1.67 \mathrm{E}+06(\mathrm{~kg})$ & (277 kgat) & - & - & - & - & - \\
\hline Heat Load & $2.09(\mathrm{kw})$ & $(7.14 \mathrm{E}+03 \mathrm{BTU} / \mathrm{hr})$ & $\ldots$ & 1.96 & 2.03 & 2.14 & 2.18 \\
\hline Bulk Density" & $1.59(\mathrm{~g} / \mathrm{cc})$ & $=$ & - & 1.54 & 1.57 & 1.62 & 1.63 \\
\hline Water wt\% & 34.6 & 二 & - & 31.8 & 33.0 & 36.2 & 38.1 \\
\hline TOC wt\%C (wet & 0.569 & $\cdots$ & $=$ & 0.441 & 0.503 & 0.634 & 0.698 \\
\hline $\begin{array}{l}\text { Radtologicat } \\
\text { Constlituents }\end{array}$ & CILL & $\mathrm{HC} / \mathrm{g}$ & $\mathbf{c i}$ & $\begin{array}{l}-95 \mathrm{Cl} \\
\text { (C) }\end{array}$ & $\begin{array}{l}-67 \mathrm{Cl} \\
(\mathrm{Cl} / \mathrm{L})\end{array}$ & $\begin{array}{l}+67 \mathrm{CI} \\
(\mathrm{Cl} / \mathrm{L})\end{array}$ & $\begin{array}{c}+95 \mathrm{CI} \\
\text { (CUL) }\end{array}$ \\
\hline $\mathrm{H} \cdot 3$ & $2.65 \mathrm{E}-94$ & 0.166 & 278 & $1.38 \mathrm{E}-04$ & 1,38E-04 & $2.73 \mathrm{E}-04$ & $2.75 E-04$ \\
\hline C- 14 & $3.67 \mathrm{E}-05$ & $2.306-02$ & 38.5 & $1.02 \mathrm{E}-05$ & 1.02E-0S & $3.71 \mathrm{E}-05$ & $3.74 \mathrm{E}-05$ \\
\hline $\mathrm{Ni}-59$ & $2.28 \mathrm{E}-06$ & $1.43 E-03$ & 2.39 & $1.03 \mathrm{E}-06$ & $1,03 \mathrm{E}-06$ & 2.34E-06 & $2.40 \mathrm{E}-06$ \\
\hline $\mathrm{Ni}-63$ & $2.23 \mathrm{E}-04$ & 0.140 & 234 & $9.95 \mathrm{E}-05$ & 9.95E-05 & 2.29E-A4 & $2.35 \mathrm{E}-04$ \\
\hline $\operatorname{Cos} 60$ & 3.98E-05 & $249 \mathrm{E}-02$ & \$1.7 & 9.37E-06 & 9.37E-06 & $4.03 \mathrm{E}-05$ & 4.07E-05 \\
\hline Se-79 & $3.55 \mathrm{E}-06$ & $2.23 \mathrm{E}-03$ & 3.72 & $1.86 \mathrm{E}-06$ & $1.86 \mathrm{E}-06$ & 4. $16 E-06$ & 4.75E-06 \\
\hline ST-90 & 0.115 & 71.9 & $1.20 \mathrm{E}+05$ & 0.106 & 0.110 & 0.119 & 0.123 \\
\hline$Y-90$ & 0.115 & 72.0 & $1.20 \mathrm{E}+05$ & $5.62 \mathrm{E}-02$ & $5.62 \mathrm{E}-02$ & 0.119 & 0.123 \\
\hline 2.93 & 1.74E-05 & 1.09E-02 & 18.3 & $8.94 \mathrm{E}-06$ & 8. $94 \mathrm{E}-06$ & $2.05 \mathrm{E}-05$ & 2.34E-05 \\
\hline Nb-93m & $1.27 \mathrm{E}-05$ & $7.95 \mathrm{E}-03$ & 13.3 & 6.74E- 06 & $6.74 \mathrm{E}-06$ & $1.48 \mathrm{E}-05$ & 1.69E.05 \\
\hline Tc-99 & $2.62 \mathrm{E}-04$ & 0.164 & 274 & $1.63 \mathrm{E}-04$ & 2.11E-04 & 3.12E-04 & $3.62 \mathrm{E}-04$ \\
\hline Ru- ]06 & $6.78 \mathrm{E}-09$ & 4.25E-06 & $7.10 \mathrm{E}-03$ & $2.85 \mathrm{E}-09$ & 2.85E-09 & $7.65 \mathrm{E}-09$ & 8.45E-09 \\
\hline $\mathrm{Cd}-113 \mathrm{~m}$ & $9.11 \mathrm{E}-05$ & $3.71 \mathrm{E}=02$ & 95.5 & $4.03 \mathrm{E}-0 \mathrm{~s}$ & 4.03E-05 & 1.09E-04 & $1.27 \mathrm{E}-04$ \\
\hline $\mathrm{Sb}-125$ & $1.69 \mathrm{E}-04$ & 0.106 & 177 & 3.73E-0s & $3.73 E-05$ & $1.71 \mathrm{E}-04$ & I.73E-04 \\
\hline Sn-126 & $5.37 \mathrm{E}-06$ & 3.37E-OS & 5.63 & 283E-06 & $2.83 \mathrm{E}-06$ & $6.29 \mathrm{E}-06$ & 7.17E-A6 \\
\hline 1.129 & 3.04E-07 & $3.16 \mathrm{E}-04$ & 0.528 & $3.13 \mathrm{E}-07$ & $4.06 \mathrm{E}-07$ & $6.03 \mathrm{E}-07$ & $6.98 \mathrm{E}-07$ \\
\hline $\mathrm{Cs}-134$ & $2.03 E-06$ & 1.27E-03 & 2.12 & $1.42 \mathrm{E}-06$ & 1.42E-06 & 2.22E-06 & $2.41 \mathrm{E}-06$ \\
\hline $\begin{array}{l}\text { Cs-137 } \\
\end{array}$ & 0.261 & 164 & $2.73 E+05$ & 0.241 & 0.249 & 0.270 & 0.279 \\
\hline$B a-137 m$ & 0.247 & 155 & $2.595+05$ & 0.166 & 0.166 & 0.256 & 0.264 \\
\hline Sm-151 & $1.25 \mathrm{E}-02$ & 7.84 & $1.31 \mathrm{E}+04$ & 6.57E- 03 & $6.57 \mathrm{E}-03$ & $1.46 \mathrm{E}-02$ & $1.67 E-02$ \\
\hline$E u-152$ & $3.69 \mathrm{E}-06$ & $2.31 \mathrm{E}-03$ & 3.87 & $1.59 \mathrm{E}-06$ & $1.59 \mathrm{E}-06$ & 3.83E-06 & $3.97 \mathrm{E}-06$ \\
\hline Eu-154 & 6.36E-04 & 0.399 & 667 & 2. 12E-04 & $2.12 E-04$ & $7.89 \mathrm{E}-04$ & $8.50 E-04$ \\
\hline Eu- 155 & $2.16 \mathrm{E}-04$ & 0.135 & 226 & $9.05 \mathrm{E}-05$ & $9.05 \mathrm{E}-05$ & $2.24 \mathrm{E}-04$ & $2.32 \mathrm{E}-04$ \\
\hline Ra-226 & 1.49E-10 & 9.33E-09 & $1.56 \mathrm{E}-04$ & $9.62 \mathrm{E}-11$ & $9.62 E-11$ & $1.68 \mathrm{E}-10$ & $1.86 \mathrm{E}-10$ \\
\hline Ra-228 & 1.18E-07 & 7.38E-05 & 0.123 & $4.88 E-08$ & $8.25 \mathrm{E}-08$ & 1.59E-07 & $2.02 E-07$ \\
\hline$A c-227$ & $9.33 \mathrm{E}-10$ & $5.85 \mathrm{E}-0 ?$ & 978E-04 & $6.28 \mathrm{E}-10$ & $6.28 \mathrm{E}-10$ & 1.04E-09 & $1.15 E-09$ \\
\hline $\mathrm{Pa} a-231$ & $4.32 \mathrm{E}-09$ & $2.71 \mathrm{E}-06$ & $4.53 \mathrm{E}-03$ & $2.63 \mathrm{E}-09$ & $2.63 \mathrm{E}-09$ & 4.94E-09 & $5.53 \mathrm{E}-09$ \\
\hline \begin{tabular}{|l|} 
Th-229 \\
\end{tabular} & $2.78 \mathrm{E}-09$ & $1.74 \mathrm{E}-06$ & 2.91E-03 & $1,26 \mathrm{E}-09$ & $2,00 \mathrm{E}-09$ & $3.67 \mathrm{TE}-09$ & $4.63 \mathrm{E}-09$ \\
\hline Th-232 & $7.86 E-09$ & $4.93 E-06$ & $8.25 \mathrm{E}-03$ & 4,05E-09 & $3.92 \mathrm{E}-09$ & 9.81E-09 & 1. $17 E-0 B$ \\
\hline U-232 & $6.26 \mathrm{E}-07$ & $3.92 \mathrm{E}-04$ & 0.656 & 3.49E-07 & 4.84E-07 & $7.99 \mathrm{E}-07$ & $9.64 \mathrm{E}-07$ \\
\hline $\mathrm{U}-233$ & $2.40 \mathrm{E}-06$ & $1.51 E-03$ & 2.52 & 1.34E-06 & $1.86 \mathrm{E}-06$ & $3.03 \mathrm{E}-06$ & $3.69 \mathrm{E}-06$ \\
\hline $\mathrm{U}-234$ & 8.08E-07 & 5.07E-04 & 0.847 & $7.70 \mathrm{E}-07$ & 7.88E-07 & 8.30E-07 & $8.36 \mathrm{E}-07$ \\
\hline $\mathrm{U}-235$ & $3.29 \mathrm{E}-08$ & $2.06 \mathrm{E}-05$ & $3.45 E-02$ & 3.12E-08 & $3.20 \mathrm{E}-08$ & $3.38 E-08$ & 3.41E-08 \\
\hline $\mathrm{U}-236$ & $2.49 \mathrm{~F}-0 \mathrm{~s}$ & $1.56 \mathrm{E}-05$ & $2.61 E-02$ & 2. $40 \mathrm{E}-0 \mathrm{~s}$ & 2.44E-08 & $2.53 \mathrm{E}-08$ & $2.57 \mathrm{E}-08$ \\
\hline U-238 & 8.99E-07 & $5.64 E-04$ & 0.943 & 8.60E-07 & 8.79E- 07 & $9.22 \mathrm{E}-07$ & $9.26 \mathrm{E}-07$ \\
\hline $\mathrm{Np}-237$ & $9.73 \mathrm{E}-07$ & $6.10 \mathrm{E}-04$ & 1.02 & $6.49 \mathrm{E}-07$ & $8.07 \mathrm{E}-07$ & 1.14E-06 & $1.30 \mathrm{E}-06$ \\
\hline Pu-238 & $1,36 \mathrm{E}-00$ & $8.525-04$ & 1.42 & $9.78 \mathrm{E}-07$ & 1 16E- 06 & $1.55 \mathrm{E}-06$ & $1.74 \mathrm{E}-06$ \\
\hline Pu-239 & 4.64E-05 & $2.91 \mathrm{E}-02$ & 48.6 & $3.66 \mathrm{E}-05$ & 4. 14E-05 & $5.13 \mathrm{E}-05$ & 5.61E-05 \\
\hline Pu-240 & $7.86 \mathrm{E}-06$ & $4.93 \mathrm{E}-03$ & 8.24 & $6.05 \mathrm{E}-06$ & $694 \mathrm{E}-06$ & 8.79E-06 & $9.67 \mathrm{E}-06$ \\
\hline Pu-241 & $9.18 \mathrm{E}-05$ & $5.76 \mathrm{E}-02$ & 96.2 & 6.59E-05 & $7.85 \mathrm{E}-05$ & $1.05 \mathrm{E}-04$ & $1.18 \mathrm{E}-04$ \\
\hline Pu-242 & $5.03 \mathrm{E}-10$ & $3.15 E-07$ & $5.27 \mathrm{E}-04$ & $3.48 \mathrm{E}-10$ & $424 \mathrm{E}-10$ & $5.82 \mathrm{E}-10$ & $6.58 \mathrm{E}-10$ \\
\hline$A m-24 !$ & $5.83 \mathrm{E}-05$ & $3.66 \mathrm{E}-02$ & 61.2 & $4,03 \mathrm{E}-05$ & $4.92 \mathrm{E}-05$ & $6.76 \mathrm{E}-05$ & 7.64E-05 \\
\hline Am-243 & $1.98 \mathrm{E}-09$ & $1.24 E-06$ & 2.07E-03 & $1.41 \mathrm{E}-09$ & $1.68 E-09$ & $2.28 \mathrm{E}-09$ & 2.57E-09 \\
\hline $\mathrm{Cm}-242$ & $1.37 \mathrm{E}-07$ & $860 \mathrm{E}-05$ & 0.144 & $5.09 \mathrm{E}-08$ & $5,09 \mathrm{E}-0 \mathrm{3}$ & $1.42 \mathrm{E}-07$ & $1.48 E-0 ?$ \\
\hline $\mathrm{Cm}-243$ & $1.27 \mathrm{E}-08$ & $7.98 E-06$ & $1.33 E-02$ & 4.50E-99 & 4.50E-09 & $132 \mathrm{E}-08$ & $1.37 \mathrm{E}-08$ \\
\hline $\mathrm{Cm}-244$ & $1.33 \mathrm{E}-07$ & 8.32E-05 & 0.139 & $4,79 E-08$ & $4.79 E-08$ & $1.61 \mathrm{E}-07$ & $180 \mathrm{E}-07$ \\
\hline Totals & $\mathbf{M}$ & $\mu \gamma / \mathrm{s}$ & $k=$ & $\begin{array}{l}95 \mathrm{Ct} \\
(\mathrm{M} \phi r \\
\mathrm{g} \mathrm{L})\end{array}$ & $\begin{array}{l}67 \mathrm{CI}(\mathrm{M} \\
6 \mathrm{or})\end{array}$ & $\begin{array}{r}67 \mathrm{CI}(\mathrm{M} \\
\text { or } \boldsymbol{L} \text { ( })\end{array}$ & $\begin{array}{l}+95 \mathrm{CI} \\
(\mathrm{M} \text { or } \\
\mathrm{g} / \mathrm{L})\end{array}$ \\
\hline Pu & $5.77 \mathrm{E}-04(\mathrm{~g} / \mathrm{L})$ & - & 0.605 & $3.93 \mathrm{E}-04$ & 4.84E-04 & $6.71 \mathrm{E}-04$ & $7.60 \mathrm{E}-04$ \\
\hline$U$ & $9.33 E-03$ & $1.39 E+03$ & $2.33 E+03$ & $.83 \mathrm{E}-03$ & $9.07 \mathrm{E}-03$ & $9.61 \mathrm{E}-03$ & $9.66 \mathrm{E}-03$ \\
\hline
\end{tabular}

"Density is calculated based on $\mathrm{Na}, \mathrm{OH}$-, and AlO2-

$\uparrow$ Water wt\% derived from the difference of density and total diseolved species. 


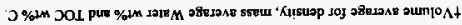

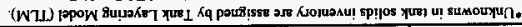

\begin{tabular}{|c|c|c|c|c|c|c|c|}
\hline $20-3681$ & $20-3981$ & zo-3SL' & z0-3L9'1 & $80+7<<9$ & $\varepsilon 0+\pi \varepsilon g Z$ & 20-118 I & ก \\
\hline E0-AEE t & Eo-3ol'b & รO-GEg' & E0-70† $\mathrm{E}$ & 125 & - & $\left(\mathrm{L}^{8)} \mathrm{c} 0-\exists(\mathrm{C} \varepsilon\right.$ & $\mathrm{nd}_{\mathrm{d}}$ \\
\hline $\begin{array}{l}(\mathrm{h} / \mathrm{s} \\
10 \mathrm{w}) \\
\mathrm{s} 96 \mathrm{t}\end{array}$ & $\begin{array}{r}(T / 810 \\
\text { WV } 1029+\end{array}$ & $\begin{array}{l}\text { (T/4 } \\
\text { W) } 10<9\end{array}$ & $\begin{array}{l}(7 \mathrm{f} \\
10 \mathrm{w}) \\
196^{\circ}\end{array}$ & 5 & कामन & $\mathbf{W}$ & 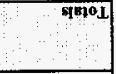 \\
\hline Lo- $\$ 821$ & $10^{\circ} \mathrm{ISI} \mathrm{I}$ & $80-3 C t C$ & $80-\exists L b^{\prime} \varepsilon$ & $0 \mathrm{bl} 0$ & 50-78L's & $80-38+6$ & $t \nabla \tau-\omega)$ \\
\hline 80-7S0 1 & $80^{-} \mathrm{azO} 0^{\circ} \mathrm{I}$ & $60^{-950 \%}$ & $60-7500^{\circ}$ & $20-39+1$ & $90 \cdot 3209$ & $60-3886$ & $c t \tau-\omega\rangle$ \\
\hline$\angle 0-3 z+1$ & $\angle 0^{\circ} \exists 8 E^{\prime} I$ & $80-$ IIILL & $80^{\circ} \mathrm{A} / \mathrm{E}^{\prime} \mathrm{L}$ & 8610 & $50-981 \cdot 9$ & $\angle 0-3 \nabla \varepsilon^{\prime} \mid$ & $z \downarrow z-w \partial$ \\
\hline $60-3581$ & $60-\exists € 9^{\prime} \mid$ & $60-3121$ & 60-ב 201 & $\varepsilon 0-\mathrm{zO} / \mathrm{z}$ & $40-3998$ & $60 \cdot 3 \tau b^{\prime} 1$ & $\varepsilon+\tau-\mathrm{ur} \nabla$ \\
\hline $50-321<$ & $50-366^{\prime} t$ & $50-989 \mathrm{\varepsilon}$ & $50-550$ ' $\varepsilon$ & 659 & $20-3+92$ & 50-JโE $b$ & 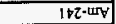 \\
\hline $60 \cdot 3 t z i$ & $60-36511$ & $60-380 \mathrm{I}$ & 60.3201 & 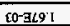 & L0:316'9 & 60- 3 CI I & $z+z^{-n_{n}}$ \\
\hline $100-31002$ & ro-aes? & $00-36 z 2$ & toraglz & 9SE & $\angle 510$ & $60^{\circ}-3162$ & $1+z-n d$ \\
\hline $50-368 \varepsilon$ & SOF $\mathrm{AOLE}$ & $90^{\circ} 3626$ & $50-301 \mathrm{E}$ & 915 & $20-3 \mathrm{El} z$ & $50-305^{\prime} f$ & $0+2-n d$ \\
\hline $50-3<92$ & wo-gtos $z$ & b0-39z' & to-gzlz & DSE & 9610 & t0-300 2 & $6 \mathrm{EZ}^{2}+\mathrm{nd}$ \\
\hline $90-30 t t$ & $90-3 z 2 t$ & $90-328 \varepsilon$ & $90-359 \varepsilon$ & $86\}$ & co-astz & $90-3200$ & $8 \mathrm{EZ}^{-1 \mathrm{n}}$ \\
\hline$\angle 0^{-} B \angle+6$ & $\angle 0$ - & $\angle 0-716 \sigma^{\circ}$ & L0-958't & 501 & to-asct & L0-GSIL & $\angle \varepsilon \tau^{-d} \mathrm{~N}$ \\
\hline $90-3191$ & $90-3651$ & $90-315 \mathrm{I}$ & $90-3 b+1$ & $6 z 2$ & bo-39t'6 & 90-35s'1 & $8 \varepsilon z-\cap$ \\
\hline 80-356 $\mathrm{f}$ & $80-968 \mathrm{C}$ & 80-A|L' & 80-ass' C & $20-3695$ & so-gze'z & $80-328 \mathrm{C}$ & $9 \varepsilon z-\cap$ \\
\hline $80-3859$ & $80-3 c t 9$ & 80-311'9 & $40 \mathrm{al} / \mathrm{s}$ & 20-בfE 6 & 50-as8' & so-aze' 9 & sहz- \\
\hline $90-3 \angle 51$ & 90 - 55 । 1 & $90-39 b^{\prime} 1$ & 90-G6C1 & $8 \pi z$ & 10-9Iz' 6 & 90-ais I & $+\varepsilon z-\Omega$ \\
\hline $90-329 \tau$ & 90:351 & gorazc I & L0-355 6 & $2 s z$ & to-atol & $90-\mathrm{A} 0 \mathrm{C}^{\prime} 1$ & $\varepsilon \varepsilon z-\Omega$ \\
\hline 10-958.9 & $20-919 \mathrm{~s}$ & Lo-ato' $\mathrm{C}$ & $\angle 0-981 z$ & 9590 & toralcz & $40-\mathrm{ath}$ & $\tau \in \tau-\cap$ \\
\hline 60 - $300^{8}$ & $6007 \angle 69$ & $60-30 z^{\prime}$ & $60-988 \mathrm{z}$ & co-352: & $90-30{ }^{\prime} \varepsilon$ & $60-9655$ & $z \varepsilon q-41$ \\
\hline $60-362 \mathrm{E}$ & $60-9192$ & $60-9 z v^{\circ} 1$ & $01-71668$ & E0.3162 & $90-9021$ & $60^{\circ} \mathrm{A} / 6 \mathrm{I}$ & $622-411$ \\
\hline $60-3959$ & $60749 \mathrm{C}$ & $60^{\circ} \mathrm{gtoZ}$ & $60-310 \%$ & $60-38 L^{\circ} b$ & $90-3<0^{\circ} 1$ & $60-3 n t ?$ & IEz-Ed \\
\hline $60-3 \angle A Z z$ & 60-3Rร 2 & $60-3811$ & $0 \mathrm{t}^{-9828}$ & $c 0=38 \% z$ & $90-\mathrm{GLO} \mathrm{I}$ & 60-asL' I & $\angle \tau z-3 V$ \\
\hline$\angle 0-30 b \mid 1$ & LO-GEI I & 80.3985 & soralstic & $\operatorname{szt} 0$ & $50^{-} \mathrm{B} 60^{\circ} \mathrm{S}$ & $80-39 T^{\prime} 8$ & $8 z z^{-7 y}$ \\
\hline $01-35$ t 5 & or-aist & $01-3+52$ & $01-365 !$ & tor 302 's & LOASIZ & ol-azs : & $\left.9 \pi z^{-8}\right)$ \\
\hline to-glbz & bo-ascz & to-90b'1 & to-30t 1 & gfe & 6010 & D0-36z: $z$ & s51-ng \\
\hline to-7609 & to- $759 \%$ & t0-395'I & no-395 ! & $0<9$ & 8LZ 0 & Do-aLS V & $+51^{-n} 3$ \\
\hline $90 \mathrm{~A} \tau \tau_{\mathrm{D}} \mathrm{t}$ & $90 \mathrm{mECt}$ & $90 \mathrm{~B} D t 2$ & $90-3 \neq L z$ & $t 29$ & co-gss $\tau$ & $90-$ IEZ $b$ & $251-\mathrm{ng}$ \\
\hline $20-7+6$ I & $20-7801$ & 90-790's & C0-794's & $00+3<\{1$ & 595 & c0-9LZ 6 & ISI-4IIS \\
\hline 0020 & 5610 & OEI 0 & octo & SO+FLLZ & $\nabla 11$ & 8810 & $\omega_{L \varepsilon 1+B G}$ \\
\hline 1120 & 5020 & 6810 & 080 & SO+1E6? & $I \tau I$ & 8610 & $\angle E[-\mathrm{s})$ \\
\hline $90-3 z<1$ & $90-3851$ & $90-3101$ & $90-3101$ & E) & wo-308 8 & $90-96 t$ & $t \varepsilon 1^{-s}$ \\
\hline$\angle 0^{-}$-HEO'S & LO-GSEV & $\angle 0^{\circ} \mathrm{GS6} z$ & $10-36 z z$ & 6550 & to-gzz'z & LO-GS9 C & $62 I-1$ \\
\hline $90: 302 ' 9$ & 9003690 & $90-3817$ & $90-38 ! \tau$ & 885 & $20-350 Z$ & $90-786$ ¿ & $92 \mathrm{I}^{-\mathrm{uS}}$ \\
\hline เo-GEZ I & torgz $z$ & $50-9892$ & $50-9892$ & $\angle L$ & 20-aze 2 & 100702 I & $52 I-4 S$ \\
\hline $50-3616$ & So-3b6 2 & $50-320 \mathrm{E}$ & $500 \mathrm{S20 \textrm {E }}$ & 626 & $20-3500$ & So-559 9 & uxE II-pD \\
\hline $60-9009$ & $60-3+6 t 5$ & 60-720 2 & $60-\mathrm{GzO} Z$ & EOTEOL 2 & 90-956 Z & 60-718t & 901-ny \\
\hline 100-3192 & $\not 0-\exists 97 z$ & to-AES I & to-9611 & $08 z$ & 5110 & $50-7681$ & $66^{-5} \mathrm{~L}$ \\
\hline SO-ZLSI & $50-30[1$ & 90-acz's & $90-3 z z 5$ & $6 \mathrm{CI}$ & c0-3bcs & 90 aft 6 & ш\{6-qN \\
\hline 50-3 481 & $50-315$ I & 90-3889 & $90-3889$ & 0.61 & 50.358 4 & $50 \mathrm{ng} 21$ & $\varepsilon 6 \cdot I Z$ \\
\hline$\$ 150$ & +160 & $2 s z^{\circ} 0$ & E€飞o & SO+JEE: & $6<1$ & EGt 0 & $06-\lambda$ \\
\hline SIE0 & $P 150$ & $\angle 920$ & $\varepsilon £ Z 0$ & 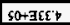 & 641 & $\varepsilon 620$ & 06.15 \\
\hline $90-710 . t$ & $90-7<0 t$ & $90-3501$ & 90-7tt I & $68 \varepsilon$ & $60-3091$ & 90-359? & $6 L=7 S$ \\
\hline $50-31022$ & 5073882 & $90.798^{\circ} 9$ & $90^{\circ} 398^{\prime} 9$ & $0 z$ & $20-3 E 21$ & $50-3+8 z$ & $09-00$ \\
\hline $60-7 \angle 6)$ & $50-\exists 50 \mathrm{~s}$ & bo-gLBC & $00-3 / 8 \mathrm{E}$ & $10 L$ & 6820 & 10-35LV & $\varepsilon 9-! N$ \\
\hline 90-apZs & $90 \mathrm{GECS}$ & $90-321$ ० & $90: 400^{\circ}$ & $0+1$ & Eo-asot & $900 \mathrm{alos}$ & $65 \cdot ! N$ \\
\hline $50-\exists L Z z$ & s0.989: & 90-9E $L L$ & 90-TELL $L$ & 565 & $20-9291$ & $50-7992$ & $+1-3$ \\
\hline $00-32012$ & 00.9861 & tor $350 \mathrm{I}$ & Do-gSO I & 882 & 6110 & DONGSo' I & $\varepsilon=H$ \\
\hline $\begin{array}{l}(7 / 75) \\
1956+\end{array}$ & $\begin{array}{l}(\mathrm{T} / \mathrm{D}) \\
\mathrm{I} 2 / 9+\end{array}$ & $\begin{array}{l}(1 / 0) \\
10<9\end{array}$ & $\begin{array}{l}\text { (T/D) } \\
1296\end{array}$ & & $9 / \mathcal{D}^{7}$ & $\mathbf{T} 15$ & 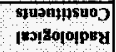 \\
\hline 2850 & $8 \varepsilon+0$ & $L t \varepsilon 0$ & Doco 0 & $\cdots$ & $\cdots$ & 2650 & Lam) ว \%1M $\mathrm{OOI}$ \\
\hline $92 \varepsilon$ & Eq & $\angle 92$ & $\tau \varsigma z$ & $=$ & -- & $1 \tau \varepsilon$ & $+\% 4 \mathrm{H}$ Iэ) M \\
\hline SL't & $\Sigma L I$ & 851 & 951 & -- & $\cdots$ & $(30, \pi) \forall 91$ & +M!!suad YIng \\
\hline 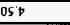 & $60 t 5$ & $00^{\prime}$ & $19 \%$ & $\cdots$ & 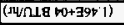 & $(M x)^{\prime} z^{\prime} t$ & PBOT IEכH \\
\hline- & -- & 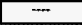 & $\cdots$ & $\cdots$ & (1, & $(8 x) 90+3 z D z$ & 2)รEM /BIOL \\
\hline \multicolumn{7}{|c|}{$1596+1069+$ ID $69^{\circ}$ IO $56^{\circ}$} & 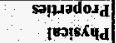 \\
\hline \multicolumn{8}{|c|}{ 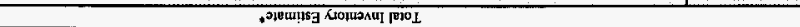 } \\
\hline \multicolumn{8}{|c|}{ 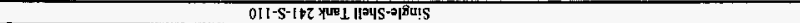 } \\
\hline
\end{tabular}


HDW Model Rev. 4

\begin{tabular}{|c|c|c|c|c|c|c|c|}
\hline \multicolumn{8}{|c|}{ Single-Shell Tank 241-S-111 } \\
\hline & \multicolumn{7}{|c|}{ TLM Solids Composite Inventory Estimate* } \\
\hline $\begin{array}{l}\text { Physical } \\
\text { Properties }\end{array}$ & & $\therefore$ & & $-95 \mathrm{Cl}$ & $-67 \mathrm{CI}$ & $+67 \mathrm{CI}$ & $+95 \mathrm{CI}$ \\
\hline Total TLM Wast & $5.19 \mathrm{E}+05(\mathrm{~kg})$ & $(78.0 \mathrm{kgal})$ & 二 & $=$ & $\cdots$ & $\ldots$ & - \\
\hline Heat Load & $1.63(\mathrm{~kW})$ & $(5.56 \mathrm{E}+03 \mathrm{BTU} / \mathrm{hr})$ & - & 1.18 & 1.44 & 1.75 & I.BO \\
\hline Bulk Density & $1.76(\mathrm{~g} / \mathrm{c})$ & - & - & 1.55 & 1.57 & 2.06 & 2.17 \\
\hline Void Fraction & 0.554 & $\ldots$ & -- & 0.151 & 0.284 & 0.724 & 0728 \\
\hline Water wt\% & 26.6 & $\cdots$ & - & 5.39 & 12.0 & 39.4 & 40.7 \\
\hline TOC wt\% C (wet & 0 & $\ldots$ & $\rightarrow$ & 0 & 0 & 0 & 0 \\
\hline $\begin{array}{l}\text { Chemienl } \\
\text { Contintituents }\end{array}$ & moleț & ppai & Le. & $\begin{array}{l}\text { 9s CI } \\
\text { (mole/L) }\end{array}$ & $\begin{array}{r}67 \mathrm{Cl} \\
\text { (mole/L) }\end{array}$ & $\begin{array}{l}+67 \mathrm{CI} \\
\text { (mole/l) }\end{array}$ & $\begin{array}{r}+95 \mathrm{CI} \\
\text { (molefi) }\end{array}$ \\
\hline $\mathrm{Nat}$ & 8.09 & $1.06 \mathrm{E}+05$ & $5,49 \mathrm{E}+04$ & 3.79 & 4.22 & 13.6 & 16.6 \\
\hline $\mathbf{A l 3 +}$ & 7.64 & $1.17 \mathrm{E}+05$ & $6.08 \mathrm{E}+04$ & 6.81 & 7.03 & 8.07 & 8.38 \\
\hline Fe $3+($ lotal Fe) & 0.883 & $2.80 \mathrm{E}+04$ & 1. $46 E+04$ & 0.867 & 0.874 & $0.89 !$ & 0.899 \\
\hline $\mathrm{Cr}+$ & $5.27 \mathrm{E}-02$ & $1.56 \mathrm{E}+03$ & 809 & 6.30E-03 & $2.90 \mathrm{E}-02$ & \begin{tabular}{l|l}
0442 \\
\end{tabular} & 0.896 \\
\hline Bib+ & 0 & 0 & 0 & 0 & 0 & 0 & 0 \\
\hline Les+ & 0 & 0 & 0 & 0 & 0 & 0 & 0 \\
\hline $\mathrm{Hg} 2+$ & $6.28 \mathrm{E}-04$ & 71.6 & 37.2 & 6.18E-04 & $6.24 E-04$ & 6.30E-04 & $6.32 E-04$ \\
\hline $\mathrm{Z}_{\mathrm{r}}(\Delta \mathrm{ZrO}(\mathrm{OH}) 2)$ & 0 & 0 & 0 & 0 & 0 & 0 & 0 \\
\hline $\mathrm{Pb2+}$ & $1.81 \mathrm{E}-02$ & $2.13 \mathrm{E}+03$ & $1.11 \mathrm{E}+03$ & $1,66 E-02$ & $1.74 \mathrm{E}-02$ & $1.87 \mathrm{E}-02$ & $1,94 \mathrm{E}-02$ \\
\hline $\mathrm{Ni2+}$ & $430 \mathrm{E}-02$ & $1.44 \bar{E}+03$ & 746 & 3.11E-02 & $3.78 E-02$ & $4.70 \mathrm{E}-02$ & $4.63 \mathrm{E}-02$ \\
\hline $\mathrm{Sr}+$ & 0 & 0 & 0 & 0 & 0 & 0 & 0 \\
\hline Mndt & 0 & 0 & 0 & 0 & 0 & 0 & 0 \\
\hline $\mathrm{Cs} 2+$ & 0.205 & $4.66 \mathrm{E}+03$ & $2.42 \mathrm{E}+03$ & 0.142 & 0.173 & 0.237 & 0.268 \\
\hline K+ & $9.735-63$ & 216 & il2 & $1,32 \mathrm{E}-03$ & 4.68E -03 & [. 3LE-07 & 1.3 IE- 02 \\
\hline क्म- & \begin{tabular}{|l|}
28.1 \\
\end{tabular} & $2.71 E+05$ & 1.41E+05 & 24.7 & 25.7 & \begin{tabular}{|l|l|}
29,8 \\
\end{tabular} & 31.1 \\
\hline NO3. & 4.56 & $1.61 \mathrm{E}+0 \mathrm{~s}$ & $835 E+04$ & 0.139 & 0.139 & 11.5 & 15.2 \\
\hline NO2- & 1.38 & $3.61 \mathrm{E}+04$ & $1.88 \mathrm{E}+04$ & 0.259 & 0,650 & 1.97 & 1.97 \\
\hline Co32: & 0.205 & $6.98 \mathrm{E}+03$ & $3.62 \mathrm{E}+03$ & 0.142 & 0.173 & 0.237 & 0.268 \\
\hline PO43- & 0 & 0 & 0 & 0 & 0 & 0 & 0. \\
\hline SO42- & $1.01 \mathrm{E}-02$ & 551 & 286 & $2.30 \mathrm{E}-03$ & $5.41 E-03$ & $1.32 \mathrm{E}-02$ & $1,32 \mathrm{E}-02$ \\
\hline Si (as SiO32-) & 9.91E-03 & 158 & 82.2 & $3.8 \pi-03$ & $0.28 \mathrm{E}-03$ & 1.94E-02 & $3.38 \mathrm{E}-02$ \\
\hline F- & 0 & 0 & 0 & 0 & 0 & 0 & 0 \\
\hline $\mathrm{Cl}+$ & $448 \mathrm{E}-02$ & 902 & 469 & $6.08 \mathrm{E}-03$ & $2.15 \mathrm{E}-02$ & $6.03 \mathrm{E}-02$ & 0.134 \\
\hline C6H5073- & 0 & 0 & 0 & 0 & 0 & 0 & 0 \\
\hline EDTA4- & 0 & 0 & 0 & 0 & 0 & 0 & 0 \\
\hline HEDTA3- & 0 & 0 & 0 & 0 & 0 & 0 & 0 \\
\hline Blycolute- & 0 & 0 & 0 & 0 & 0 & 0 & 0 \\
\hline acetste- & 0 & 0 & 0 & 0 & 0 & 0 & 0 \\
\hline oxeinter- & 0 & 0 & 0 & 0 & 0 & 0 & 0 \\
\hline DBP & 0 & 0 & 0 & 0 & 0 & 0 & 0 \\
\hline Dutanal & 0 & 0 & 0 & 0 & 0 & 0 & of \\
\hline $\mathrm{NH}$ & 0216 & $209 \mathrm{E}+03$ & $1.09 E+03$ & $5.55 E-02$ & $7.83 \mathrm{E}-02$ & 0.229 & 0.240 \\
\hline $\mathrm{Fe}(\mathrm{CN}) 64-$ & 0 & 0 & 0 & 0 & 0 & 0 & 0 \\
\hline
\end{tabular}

Unknowns in tank solids imventory are assigned by Tank Layering Model (TLM). 
HDW Model Rev. 4

\begin{tabular}{|c|c|c|c|c|c|c|c|}
\hline \multirow[b]{3}{*}{$\begin{array}{l}\text { Physical } \\
\text { Properties }\end{array}$} & \multicolumn{7}{|c|}{ Single-Shell Tank 241-S-111 } \\
\hline & \multicolumn{7}{|c|}{ SMM Composite Inventory Estimate } \\
\hline & $\cdots$ & & & $-95 \mathrm{CI}$ & $-67 C I$ & $+67 \mathrm{CI}$ & $+95 \mathrm{CI}$ \\
\hline Total SMM Wast & $3.01 \mathrm{E}+06(\mathrm{~kg})$ & $(460 \mathrm{kgal})$ & $\ldots$ & $\cdots$ & $\ldots$ & $\ldots$ & $\ldots$ \\
\hline Heat Load & $4.23(\mathrm{~kW})$ & (1. 44E+04 BTU/hr) & -- & 3.99 & 4.12 & 4.33 & 4.43 \\
\hline Bulk Density" & $1.73(g / \infty c)$ & - & -- & 1.67 & 1.70 & 175 & 1.77 \\
\hline Water wt\% & 26.4 & -- & - & 23.9 & 25.0 & 27.8 & 29.5 \\
\hline TOC wi $\%$ C (wet & 0.503 & $\ldots$ & - & 0.407 & 0.454 & 0.551 & 0.599 \\
\hline $\begin{array}{l}\text { Chemical } \\
\text { Constituents }\end{array}$ & male/L & ppin & ks & $\begin{array}{c}-95 \mathrm{CI} \\
(\text { mole } / \mathrm{L})\end{array}$ & $\begin{array}{l}-67 \mathrm{CI} \\
(\text { nole/L) }\end{array}$ & $\begin{array}{r}+67 \mathrm{Cl} \\
\text { (mole } / \mathrm{L})\end{array}$ & $\begin{array}{r}+9 \mathrm{~s} \mathrm{cI} \\
(\mathrm{mole} / \mathrm{L})\end{array}$ \\
\hline $\mathrm{Na}+$ & 16.9 & $2.25 \mathrm{E}+05$ & $6.76 E+05$ & 15.6 & 16.2 & 17.5 & 180 \\
\hline $\mathrm{Al} \mathbf{3}+$ & 2.23 & $3.48 E+04$ & $1.05 E+05$ & 2.00 & 2.11 & 2.36 & 2.31 \\
\hline Fej+ (total Fe) & $1.11 \mathrm{E}-02$ & 357 & $1.07 E+03$ & $8.90 \mathrm{E}-03$ & $9.95 \mathrm{E}-03$ & $1.22 \mathrm{E}-02$ & 1.32E-02 \\
\hline $\mathrm{Cr}^{3+}$ & 0.217 & $6.52 \mathrm{E}+03$ & $1.96 \mathrm{E}+04$ & 0.171 & 0.196 & 0.225 & 0.233 \\
\hline $\mathrm{Bi} 3+$ & 9.01E-04 & 109 & 328 & $8.21 \mathrm{E}-04$ & $8.60 \mathrm{~F}-04$ & $9.42 \mathrm{E}-04$ & $9.81 \mathrm{E}-04$ \\
\hline La3+ & $1.61 \mathrm{E}-05$ & 1.29 & 3.88 & $1.16 \mathrm{E}-05$ & $1.38 \mathrm{E}-05$ & $1.83 \mathrm{E}-05$ & 2.05E-05 \\
\hline $\mathrm{Hg} 2+$ & $7.45 \mathrm{E}-06$ & 0.865 & 2.60 & $7.03 \mathrm{E}-06$ & $7.27 \mathrm{E}-06$ & $7.54 \mathrm{E}-06$ & $7.62 \mathrm{E}-06$ \\
\hline $\mathrm{Zr}$ (as Zro(OH)2) & $1.71 \mathrm{E}-04$ & 9.02 & 27.1 & $1.56 \mathrm{E}-04$ & $1.61 \mathrm{E}-04$ & $1.77 \mathrm{E}-04$ & $1.85 \mathrm{E}-04$ \\
\hline $\mathrm{Pb}_{2+}$ & $8.91 \mathrm{E}-04$ & 107 & 321 & $7.64 \mathrm{E}-04$ & 8.26E-04 & $956 \mathrm{E}-04$ & $1.02 \mathrm{E}-03$ \\
\hline Ni2+ & $6.83 \mathrm{E}-03$ & 232 & 698 & 6.64E-03 & $6.73 \mathrm{E}-03$ & $6.92 \mathrm{E}-03$ & $2.00 \mathrm{E}-03$ \\
\hline $\mathrm{Sr} 2+$ & 0 & 0 & 0 & 0 & 0 & 0 & $\theta$ \\
\hline Mn4t+ & $3.80 \mathrm{E}-03$ & 121 & 363 & $2.76 \mathrm{E}-03$ & 3.27E-03 & 4.32E-03 & 4.83E-03 \\
\hline $\mathrm{Ca} 2+$ & $3.50 \mathrm{E}-02$ & 813 & $2.45 \mathrm{E}+03$ & $3.16 \mathrm{E}-02$ & $3,33 \mathrm{E}-02$ & $3.68 \mathrm{E}-02$ & 3. $84 \mathrm{E}-02$ \\
\hline $\mathrm{K}+$ & $7.28 \mathrm{E}-02$ & $1.63 \mathrm{E}+03$ & $496 E+03$ & $6.65 \mathrm{E}-02$ & $6.95 \mathrm{E}-02$ & $7.63 \mathrm{E}-02$ & $7.96 \mathrm{E}-02$ \\
\hline OH- & 13.6 & 1.34E+05 & $4.04 E+05$ & 12.2 & 12.9 & 14.4 & 143 \\
\hline NO3- & 5.54 & $1.59 E+05$ & $5.98 \mathrm{E}+05$ & 5.18 & 5.36 & 5.69 & 5.71 \\
\hline NO2- & 3.16 & $8.43 \mathrm{E}+04$ & $2.53 \mathrm{E}+05$ & 2.72 & 2.93 & 3.39 & 3.61 \\
\hline $\mathrm{CO} 32$ & 0,378 & T.3TE+04 & $3.95 \mathrm{E}+04$ & 0.341 & 0.359 & 0.397 & 0.408 \\
\hline PO43. & $7.39 \mathrm{E}-02$ & $4.07 E+03$ & $122 \mathrm{E}+04$ & $6.64 E-02$ & $6.91 \mathrm{E}-02$ & $7.55 \mathrm{E}-02$ & $7.70 \mathrm{E}-02$ \\
\hline SO42- & 0.229 & $1.27 \mathrm{E}+04$ & $3.82 \mathrm{E}+04$ & 0.172 & 0.199 & 0.259 & 0.286 \\
\hline Si (as $\mathrm{SiO} 32-)$ & $8.35 E-02$ & $1.36 \mathrm{E}+03$ & $4.08 E+03$ & $6.87 \mathrm{E}-02$ & $7.59 \mathrm{E}-02$ & $9.10 \mathrm{E}-02$ & $9.83 \mathrm{E}-02$ \\
\hline F. & 4,35E-02 & 479 & $1.44 \mathrm{E}+03$ & $366 \mathrm{E}-02$ & 3.95E-02 & $470 \mathrm{E}-02$ & $4.97 \mathrm{E}-02$ \\
\hline $\mathrm{Cl}$ & 0.286 & $5.87 E+03$ & $1.72 \mathrm{E}+04$ & 0.261 & 0.273 & 0.301 & 0,308 \\
\hline C6H5O73- & 2.91E-02 & $3.19 \mathrm{E}+03$ & $9.59 \mathrm{E}+03$ & $2.70 \mathrm{E}-02$ & $2.81 E-02$ & $3.02 \mathrm{E}-02$ & 3.12E-02 \\
\hline EDTA4- & 6.48E- 03 & $1.08 E+03$ & $3.25 E+03$ & $2.42 \mathrm{E}-03$ & 4.41E-03 & 8.5TE-03 & $1.06 \mathrm{E}-02$ \\
\hline HEDTA3- & $1.15 \mathrm{E}-02$ & $1.83 \mathrm{E}+03$ & $5.50 \mathrm{E}+03$ & $3.40 \mathrm{E}-03$ & $7.36 \mathrm{E}-03$ & 1.57E-02 & $1.98 \mathrm{E}-02$ \\
\hline glycolate & $6.60 \mathrm{E}-02$ & $2.87 E+03$ & $6.62 \mathrm{E}+03$ & $3.90 \mathrm{E}-02$ & $5.22 \mathrm{E}-02$ & $7.99 \mathrm{E}-02$ & 9.31E-02 \\
\hline weetate- & 4655.03 & 159 & 477 & 3.81E-03 & $4.22 \mathrm{E}-03$ & $5,07 \mathrm{E}-03$ & 5.4BE- 03 \\
\hline oxalate2- & 2.10E-05 & 1.07 & 3.22 & 1.86E-05 & 1.98E-05 & 2.23E- 05 & 2.34E-0S \\
\hline DBP & $1.89 \mathrm{E}-02$ & $2.30 E+03$ & $692 \mathrm{E}+03$ & $1.53 \mathrm{E}-02$ & $1.71 \mathrm{E}-02$ & $2.07 \mathrm{E}-02$ & $2.25 \mathrm{E}-02$ \\
\hline butanol & $1.89 \mathrm{E}-02$ & 812 & $2.44 \mathrm{E}+03$ & $1.53 \mathrm{E}-02$ & $1.71 \mathrm{E}-02$ & 2.07E- 02 & $2.25 \mathrm{E}-02$ \\
\hline $\mathrm{NH3}$ & $8,63 \mathrm{E}-02$ & 850 & $2.55 E+03$ & $6.96 \mathrm{E}-02$ & $7.62 \mathrm{E}-02$ & $9.92 \mathrm{E}-02$ & 0.114 \\
\hline $\mathrm{Fe}(\mathrm{CN}) 64$ & 0 & 0 & 0 & 0 & 0 & 0 & 0 \\
\hline
\end{tabular}

*Density is calculated based on $\mathrm{Na}, \mathrm{OH}$-, and $\mathrm{AlO2}$.

tWater wt\% derived from the difference of density and total dissolved species. 
How Model Rev. 4

\begin{tabular}{|c|c|c|c|c|c|c|c|}
\hline \multirow[b]{3}{*}{$\begin{array}{l}\text { Physical } \\
\text { Praperties }\end{array}$} & \multicolumn{7}{|c|}{ Single-Shell Tank 24!-S-111 } \\
\hline & \multicolumn{7}{|c|}{ Total Inventory Estimate* } \\
\hline & & : & & $-95 \cdot \mathrm{CI}$ & $-67 \mathrm{CI}$ & $+67 \mathrm{CI}$ & $495 \mathrm{CI}$ \\
\hline Total Waste & $3.53 \mathrm{E}+06(\mathrm{~kg})$ & (538 kgal) & $=$ & $\cdots$ & 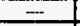 & - & - \\
\hline Heat Load & $5.85(\mathrm{~kW})$ & $(2.00 \mathrm{E}+04 \mathrm{BTU} / \mathrm{hr})$ & -- & 5.22 & 5.57 & 6.08 & 6.06 \\
\hline Bulk Density' & $1.73(\mathrm{~g} / \mathrm{cc})$ & - & - & 1.65 & 1.68 & 1.79 & 1.78 \\
\hline Water wet $\% t$ & 26.4 & - & $\ldots$ & 23.7 & 23.3 & 29.1 & 30.8 \\
\hline TOC wt\% C (wet & 0.429 & - & - & 0.347 & 0.387 & 0.470 & 0.511 \\
\hline $\begin{array}{r}\text { Chemical } \\
\text { Constituents }\end{array}$ & molef L & ppa & kg & $\begin{array}{l}-95 \mathrm{CI} \\
(\mathrm{mole} / \mathrm{L})\end{array}$ & $\begin{array}{r}67 \text { Ct } \\
\text { (molent) }\end{array}$ & $\begin{array}{l}+67 \mathrm{Cl} \\
\text { (mole/L) }\end{array}$ & $\begin{array}{l}+9 s \text { CI } \\
\text { (mole/L) }\end{array}$ \\
\hline $\mathrm{Na}+$ & 15.6 & $2.07 \mathrm{E}+05$ & $7.31 \mathrm{E}+05$ & 13.9 & 14.6 & 16.8 & 16.5 \\
\hline $\mathrm{Al} \mid 3+$ & 3.01 & 4.69E+04 & $1.65 E+05$ & 2.82 & 2.92 & 3.09 & 3.10 \\
\hline Fe3+(total Fe) & 0.137 & $4,43 \mathrm{E}+03$ & $1.56 \mathrm{E}+04$ & 0.135 & 0.136 & 0.138 & 0.139 \\
\hline $\mathrm{Ct}^{3+}$ & 0.193 & $5.79 \mathrm{E}+03$ & $2.04 E+04$ & 0.167 & 0.181 & 0.231 & 0.276 \\
\hline Bi3t & $7.70 \mathrm{E}-04$ & 93.0 & 328 & $7.02 \mathrm{E}-04$ & $7.36 \mathrm{E}-0 \mathrm{~d}$ & 8.05E-04 & B. $39 \mathrm{E}-04$ \\
\hline Lot & $1.37 \mathrm{E}-05$ & 1.10 & 3.88 & 9.95E-06 & $1.18 \mathrm{E}-05$ & 1.57E-05 & $1.75 \mathrm{SE}-0 \mathrm{~S}$ \\
\hline $\mathrm{Hg} 2+$ & $9.73 \mathrm{E}-05$ & 11.3 & 39.8 & $9.60 \mathrm{E}-05$ & 9.68E-05 & $9.77 E-05$ & $980 E-05$ \\
\hline $\mathrm{Zr}(\mathrm{E} \mathrm{ZrO}(\mathrm{OH}) 2)$ & $1.46 \mathrm{E}-04$ & 769 & 27.1 & 1.33E-04 & $1.38 \mathrm{E}-04$ & I.51E-04 & $1.58 \mathrm{E}-04$ \\
\hline $\mathrm{Pb2+}$ & 3.38E-03 & 405 & $1.43 E+03$ & 3.17E-03 & $330 \mathrm{E}-03$ & 3.45E-03 & $349 \mathrm{E}-03$ \\
\hline Ni2t & $1.21 \mathrm{E}-02$ & 410 & 1.44E+03 & $1.03 \mathrm{E}-02$ & $1.13 \mathrm{E}-02$ & $1.27 \mathrm{E}-02$ & $1.24 \mathrm{E}-02$ \\
\hline $5 \mathrm{sr} 2+$ & 0 & 0 & 0 & 0 & 0 & 0 & 0 \\
\hline Mn4+ & $3.25 \mathrm{E}-03$ & 103 & 363 & $2.36 \mathrm{E}-03$ & 2.79E-03 & $3.70 \mathrm{E}-03$ & 4.13E-03 \\
\hline $\mathrm{Ca} 2+$ & $5.96 \mathrm{E}-02$ & $1.38 \mathrm{E}+03$ & $4.87 \mathrm{E}+03$ & $5.10 \mathrm{E}-02$ & $5.59 \mathrm{E}-02$ & $6.28 \mathrm{E}-02$ & $6.59 \mathrm{E}-012$ \\
\hline$\overline{\mathrm{K}+}$ & $6.37 \mathrm{E}-02$ & $1.44 E+03$ & $5.07 \mathrm{E}+03$ & $5.83 \mathrm{E}-02$ & $6.08 E-02$ & $6.66 \mathrm{E}-02$ & $6.94 E-02$ \\
\hline OH- & 15.7 & $1.54 E+05$ & $5.45 \mathrm{E}+05$ & 14,5 & 15.2 & 16.3 & 16.3 \\
\hline No3- & 5.40 & $1.93 \mathrm{E}+05$ & $6.82 E+05$ & 4.54 & 4.71 & 6.34 & 6.64 \\
\hline $\mathrm{NO2}$ & 2.91 & $7.72 \mathrm{E}+04$ & $2.72 E+05$ & 2.53 & 2.70 & 3.10 & 3.29 \\
\hline Co32- & 0.353 & $1.22 E+04$ & $4.31 E+04$ & 0.322 & 0.336 & 0.369 & 0.379 \\
\hline PO43- & $6.32 \mathrm{E}-02$ & $3.47 \mathrm{E}+03$ & $1.22 E+04$ & $5,68 \mathrm{E}-02$ & $5.90 \mathrm{E}-02$ & $6.45 \mathrm{E}-02$ & $6.58 \mathrm{E}-02$ \\
\hline $5042=$ & 0.197 & $1.09 \mathrm{E}+04$ & $3.83 E+04$ & 0.148 & 0.171 & 0.223 & 0.246 \\
\hline $\mathrm{Si}$ (as SiO32-) & $7.28 \mathrm{E}-02$ & 1.18E+03 & $4.16 \mathrm{E}+03$ & $6.02 \mathrm{E}-02$ & $6.04 E-02$ & $7.93 \mathrm{E}-02$ & $855 \mathrm{E}-02$ \\
\hline F. & $3.72 \mathrm{E}-02$ & 409 & $1.44 \mathrm{E}+03$ & 3.13E-02 & $3.38 \mathrm{E}-02$ & $4,02 \mathrm{E}-02$ & 4.25E- 02 \\
\hline $\mathrm{Cl}-$ & 0.251 & $3.14 \mathrm{E}+03$ & $181 \mathrm{E}+04$ & 0.230 & 0.240 & 0.261 & 0270 \\
\hline C6H5O73- & $2.49 \mathrm{E}-02$ & $2.72 \mathrm{E}+03$ & $9.59 \mathrm{E}+03$ & $2.31 \mathrm{E}-02$ & $2.40 \mathrm{E}-02$ & $2.53 \mathrm{E}-\mathrm{O} 2$ & 2.67E-02 \\
\hline EDTA4- & $5.54 \mathrm{E}-03$ & 922 & $3.23 \mathrm{E}+03$ & $2.07 \mathrm{E}-03$ & 3.7TE-03 & $7.33 \mathrm{E}-03$ & $9,07 \mathrm{E}-03$ \\
\hline HEDTAT- & 9.85E-03 & $1.56 \mathrm{E}+03$ & $5.50 \mathrm{E}+03$ & $2.90 \mathrm{E}-03$ & $6.30 \mathrm{E}-03$ & $1.34 \mathrm{E}-02$ & $1.69 \mathrm{E}-02$ \\
\hline glycolate- & 3.64E-02 & $2.45 E+03$ & $8.62 \mathrm{E}+03$ & $3.33 \mathrm{E}-02$ & $4.46 \mathrm{E}-02$ & $6.83 \mathrm{E}-02$ & $7.96 E-02$ \\
\hline icetate- & $3.97 \mathrm{E}-03$ & 135 & 477 & $3.26 \mathrm{E}-03$ & 3.61E-03 & $4.34 \mathrm{E}-03$ & $4.69 \mathrm{E}-03$ \\
\hline oxalute?- & $180 \mathrm{E}-0 \mathrm{~S}$ & 0.914 & 3.22 & $1.59 \mathrm{E}-05$ & $1.69 \mathrm{E}-05$ & $1.90 \mathrm{E}-05$ & 2.00E-05 \\
\hline DBP & $162 \mathrm{E}-02$ & $1.96 \mathrm{E}+03$ & $6.92 \mathrm{E}+03$ & $1.31 \mathrm{E}-02$ & $1.46 \mathrm{E}-02$ & $1.77 \mathrm{E}-02$ & $1.92 \mathrm{E}-02$ \\
\hline butanol & $1.62 \mathrm{E}-02$ & 692 & 244E+03 & $1.31 \mathrm{E}-02$ & $1.46 \mathrm{E}-02$ & $1.77 \mathrm{E}-02$ & $1.92 \mathrm{E}-02$ \\
\hline NH3 & 0.105 & $1.03 \mathrm{E}+03$ & $3.64 \mathrm{E}+03$ & $7.64 \mathrm{E}-02$ & B. $24 E-02$ & 0.116 & 0.129 \\
\hline Fo(CN) $64-$ & 0 & 0 & 0 & 0 & 0 & 0 & 0 \\
\hline
\end{tabular}

"Unknowns in tank solids inventory are assigned by Tank Layering Model (TLM)

tWater wt\% derived from the difference of density and total dissolved species. 


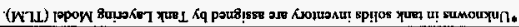

\begin{tabular}{|c|c|c|c|c|c|c|c|}
\hline z0०ةा1 & Z0-360E & $20-a u z z$ & 20-35sz & $80+350 z$ & $\varepsilon 0+\pi S_{6}^{\prime} \varepsilon$ & zo-azst & 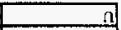 \\
\hline $20-3\left\lfloor z^{\prime} 1\right.$ & $20-381 \%$ & $20-9586$ & E0"7668 & $6 \Gamma^{\prime} \varepsilon$ & - & $\left(\tau^{A}\right) z 0-980^{\prime} \tau$ & $\mathrm{n}_{\mathrm{d}}$ \\
\hline$(\pi / a$ & $(7 / 410$ & $(1 / 8)$ & $\left(\mathrm{T}^{2}\right.$ & का & 8मी & $\mathbf{w}$ & 57 g9oL \\
\hline $10 \mathrm{WU}$ & (1) $10<9+$ & W) 10,9 & row) & & & & \\
\hline $19 \$ 6+$ & & & 1956 & & & & \\
\hline 60-azic & $60-3 z I^{\prime} \varepsilon$ & $600-3601$ & $01-928$ & no-318'9 & $90-\mathrm{AIC]}$ & $60-31 \varepsilon z$ & $p t \tau$-uे \\
\hline $60-991 \mathrm{C}$ & $60-\left.39\right|^{\prime} \varepsilon$ & $60-960 ' \varepsilon$ & $60-9 L 0 t$ & to- 3 šz 6 & $90-88<\div$ & $60-3 E I^{\prime} \varepsilon$ & $\varepsilon b z-4)$ \\
\hline $20-585 \mathrm{I}$ & $40^{-3} 38 c^{\prime} 1$ & LO-aSE I & $\angle 0^{\circ} \operatorname{sen} E^{\prime} !$ & $20-31002$ & $50-3826$ & $\angle 0-\mathrm{a} \angle \varepsilon]$ & $z b c-u v$ \\
\hline $600 \mathrm{gClT}$ & $71-9+2 \cdot 8$ & $11-3901 \varepsilon$ & $21-306 \mathrm{~L}$ & s0-316! & $80-3 \angle 95$ & $11-39 b^{\prime} 9$ & $\varepsilon b z-u v \mid$ \\
\hline b0-al2'1 & 90-35e 6 & $90-Z L Z \bar{f}$ & L0-39+' 8 & $\operatorname{ta} z$ & E0-3E6 & $90-3169$ & $I p \tau^{-\omega}$ - \\
\hline $60 \mathrm{r} 3882$ & $60-3 \angle 9 z$ & $60-3 z z z$ & $60-310 z$ & $10-3 a a_{2}$ & $90-365=1$ & $60-a s+z$ & $\overline{z t z-n_{d}}$ \\
\hline por-10t'9 & $00-3865$ & 60.310's & Do-ats' & z9I & zico & p0-36t's & {$\left[+z^{-n} d\right.$} \\
\hline p0-3907 & $p 0-3001$ & $50-38 \varepsilon_{8}$ & $50-365 \mathrm{~L}$ & $z<z$ & Z0-aE'S & $50-31 Z^{6}$ & $\overline{0 t z-n_{d}}$ \\
\hline $00-365^{\circ} \mathrm{L}$ & $60-3501$ & $00-3685$ & Do-ates & $16 I$ & 8980 & t0-3<F9 & $6 \varepsilon z^{-n} \mathrm{~d}$ \\
\hline $50-3017$ & 50.3501 & $90-3598$ & $90-388 \mathrm{~L}$ & $6 L Z$ & E0:785'S & $90-39+6$ & $8 \varepsilon z^{-n d}$ \\
\hline $60-9617$ & $\angle 0-361^{\prime} 1$ & $80-391 t$ & 80-780 I & $20-965 ' z$ & $50-300 \mathrm{~s}$ & $80-36<-8$ & $\angle \varepsilon Z^{-d} \mathbf{N}$ \\
\hline $90-\exists<+z$ & $90-317 z$ & $90-30 z z$ & 90-360z & 5890 & 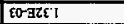 & $90-a z \varepsilon z$ & $9 \varepsilon z-0$ \\
\hline 80-9IS'S & $90-3<55$ & $80-306^{\prime} t$ & 90-315t & zo-aূs'1 & so-g16 2 & so-all's & $9 \mathcal{E Z *}$ \\
\hline$\angle 0-3801$ & $\angle 0-350^{\prime} 1$ & $80-5856$ & 80-ab8 8 & 20-ت96z & so-GSL's & $\angle 0: G 10 \mathrm{I}$ & इ£z-n \\
\hline 90-ges $z$ & $90-\exists L+Z$ & 90-astz & $90-1800^{\circ} t$ & $20<0$ & LO-g5C'1 & 90-38EZ $Z$ & $p \varepsilon z-n$ \\
\hline 21 -30I b & $21 \cdot 966 \mathrm{E}$ & $2[-\bar{G} 59 \mathrm{\varepsilon}$ & ZI-GLEE & $90-3+11$ & $60-9612$ & $21-\exists \mathbf{S 8} \varepsilon$ & 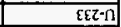 \\
\hline $01-3 I 1]$ & $01-380 \mathrm{t}$ & II-ES8.6 & $11-9806$ & $50-3 \angle 0^{\circ} \varepsilon$ & $90-3165$ & DI-ANo I & $\overline{z \varepsilon z-n}$ \\
\hline $91-3+89$ & 91-3699 & $91-30+z$ & $\angle I-3 \mid \subset C 9$ & $01-36 \mathrm{~b} !$ & ह1- $988 \mathrm{Z}$ & $91-3905$ & $2 \varepsilon \tau-4 \mathrm{I}$ \\
\hline$z[-3 \mid 5)$ & $\tau 1-3151$ & $2\left[-\exists 8 t^{\prime}\right]$ & $z 1-3(p)$ & Lo-aerb & $01-9058$ & 2113051 & $6 z \tau-4 \mathrm{~L}$ \\
\hline $80-3201$ & $60-3 \in \angle T$ & $01-306 z$ & 11-GEt 2 & to-algi & $\angle 0-9 b^{2} \varepsilon$ & or- 76 ! 9 & IEZ- \\
\hline $60-919 \cdot 8$ & $60-3159$ & $60-369 \%$ & $01-3197$ & $50-3611$ & $90-96 z z$ & $600-960 t$ & $\angle Z Z-O Y$ \\
\hline $51-3562$ & SI-ZS6L & $51-9862$ & S1-grLL & Go-afE' $\tau$ & $71-789 t$ & SI'IRB $L$ & $87 z-$ - \\
\hline 60r $\exists 0 \angle t T$ & $60-37 \varepsilon 1$ & ol-3t's & ol-ast't & Do- $90<z$ & L0-302's & 019Asio 6 & 92z-घ्च \\
\hline ro-3csz & H0-בE8 Z & 100: $1 \angle L 2$ & 100-asLz & 0.28 & 0910 & $00-318 t$ & $5 s 1^{-n g}$ \\
\hline $50-36 b^{2} z$ & $50-96+z$ & $90-3 c l>8$ & 90-actz & its & 20-550\% & sorater $\mathrm{t}$ & t51-0 \\
\hline $900-300 \%$ & $90-3009$ & 90-7L85 & 90-328's & 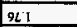 & $50-98 \varepsilon \varepsilon^{\prime} \mathcal{E}$ & $90-9565$ & $z s 1^{-\mathrm{ng}}$ \\
\hline 20 - $\sec z$ & $80-386: t$ & Do-3269 & pora6li & $2 E t$ & IEB 0 & E0-39\$1 & ISI-WS \\
\hline 20 -368's & $20-3685$ & $2 \theta-3 \sin z$ & $20-32 z 1$ & 60+בEC! & LSz & zo-gzst & $\mathrm{w}_{\angle E I 1-\mathrm{g}}$ \\
\hline $20-3819$ & $20-3819$ & $20-3892$ & $20-36 z^{\prime} 1$ & $60+$ gibi & 242 & $20-38<+$ & LEI-S] \\
\hline $80-910 \mathrm{E}$ & $80-310 \varepsilon$ & Borale' I & 60 -abre9 & $60-3 \angle 899$ & so-zzE! & $80-\overline{\varepsilon \varepsilon \tau}$ & $\forall \in[\cdot 5]$ \\
\hline Bo-Jzst & $90-\mathrm{a} s \mathrm{\varepsilon}$ & $80-3 \varepsilon \tau^{\prime} \mid$ & 60-381 द & $80-3 \angle g^{\prime} L$ & sorgas I & $90-909 z$ & $62[-1$ \\
\hline $90-3696$ & Loages 8 & $40-3862$ & $80-30 L 2$ & 9810 & 6or 9655 & LO-AIE 9 & $921-4 \mathrm{~s}$ \\
\hline $90-9 z s^{\prime} 1$ & 90-32s'1 & Lo-azES & $\angle 0-9651$ & Ifro & po-98E9 & $90-921$ & Sटl-qs \\
\hline $90-9808$ & 90-a80 & 90-3z8z & LOAIEL $L$ & $\mathscr{\ell 1}$ & $50-300-\varepsilon$ & 90-IL6's & Шहा II-PJ) \\
\hline $21-3162 z$ & 21 -316 $z$ & $2 \mathrm{zl}-\mathrm{atot}$ & ร1-a+8' z & $\angle 0-39 \varepsilon 9$ & 60-3IZZI & z1-ast z & $90 \mathrm{I}-\pi \mathrm{y}$ \\
\hline 50-318! & s0-3E8'1 & $90-3069$ & $90-3591$ & $66 \mathrm{\varepsilon}$ & $50-369^{\circ} \mathrm{C}$ & 50-ase'1 & $66^{-3} \mathrm{~L}$ \\
\hline so-91Lt & $90-3+12$ & LO-36tL & $\angle 0-3061$ & $\angle 9 \div 0$ & por 3006 & $90-985: 1$ & $w_{\varepsilon 6-q N}$ \\
\hline so: $\angle L S Z$ & $90-1 z 9 z$ & L0-39I' 6 & $\angle 0-a L E Z$ & $z<s_{0}$ & E0-30厂 I & $90-3761$ & $\varepsilon 6^{-1} \mathrm{Z}$ \\
\hline 6280 & $198^{\circ} 0$ & $889^{\circ} 0$ & 1950 & $50+425 z$ & LH & $98 L^{\circ} 0$ & 06-시 \\
\hline$D 280$ & 1980 & 8890 & 1950 & so+AzEz & Cth & $986^{\circ} 0$ & $06^{-1 S}$ \\
\hline $90-7909$ & Lo-ass's & $20-31061$ & $80-320 ' S$ & 1210 & ho-3ะEz & LO-300't & $6 L^{-0}$ \\
\hline gorate l & 90-350 I & $\angle 0-319 \varepsilon$ & $80-3 \angle 56$ & $\operatorname{szz} 0$ & po-atcip & $\angle 0-\exists E 9^{\circ} L$ & $09-0$ \\
\hline E0-agt l & g0-asz'! & 50-3 801 & $500-38+8$ & $\angle \mathrm{PE}$ & 8990 & E0-ZLIT & E9-!N \\
\hline So:ISE I & SO- $I \angle E I$ & $50-9111$ & $90-780^{\circ} 6$ & ILE & $50-351 / 2$ & 50:-392'1 & $65-1 \mathrm{~N}$ \\
\hline $90-319 z$ & 90-3197 & LO-JEI 6 & $\angle 0 \div \exists 9 E Z$ & $0<50$ & E0-301: I & 90-3E6 I & $p 1 \cdot 0$ \\
\hline $50-36 t c$ & s0- $36 t^{\prime} \varepsilon$ & 50-392 I & $90-3 b c z$ & $S E L$ & zo-azbi & $50-36 \circ z$ & $\mathrm{E}-\mathrm{H}$ \\
\hline $\begin{array}{l}(1 / \%) \\
10 \$ 6+\end{array}$ & $(T / /)$ & (1/) & $(1 n)$ & 10 & 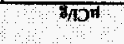 & $T A$ & guanjoxtop \\
\hline 0 & 0 & 0 & 0 & $=$ & - & 0 & IaM) $2 \%^{1 M} \mathrm{DOl}$ \\
\hline$\angle O F$ & $\nabla 6 \varepsilon$ & $02 \mathrm{ZI}$ & $6 \varepsilon \mathrm{S}$ & - & + & $9.9 z$ & \%AM IDIPM \\
\hline 8260 & 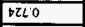 & 1920 & Isio & $\cdots$ & $\cdots$ & DS5 50 & 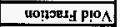 \\
\hline $4 \sqrt{4 ! z}$ & $90 \mathrm{z}$ & $\angle S I$ & s5 1 & $\cdots$ & $=$ & $(x, \theta) 9<1$ & Alisusa ying \\
\hline 081 & SLI I & $\$+1$ & 811 & -- & ( & (Mx) \&9: I & pвo7 280 \\
\hline- & $=$ & $=$ & - & - & $(1884080)$ & $8 \times 1) \leq 0+261$ 's & 758M WרI [B10L \\
\hline \multirow[t]{3}{*}{ ID $56^{+}$} & $15.69+$ & 1929 & ID. $56^{\circ}$ & & & & $\begin{array}{l}\text { oppuadod } \\
\text { fropsiyd }\end{array}$ \\
\hline & \multicolumn{6}{|c|}{ 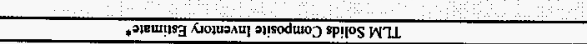 } & \\
\hline & & & III-s-11 & 1 II & & & \\
\hline
\end{tabular}


HNF-SD-WM-ER-323, Rev. 1

HOW Model Rev. 4

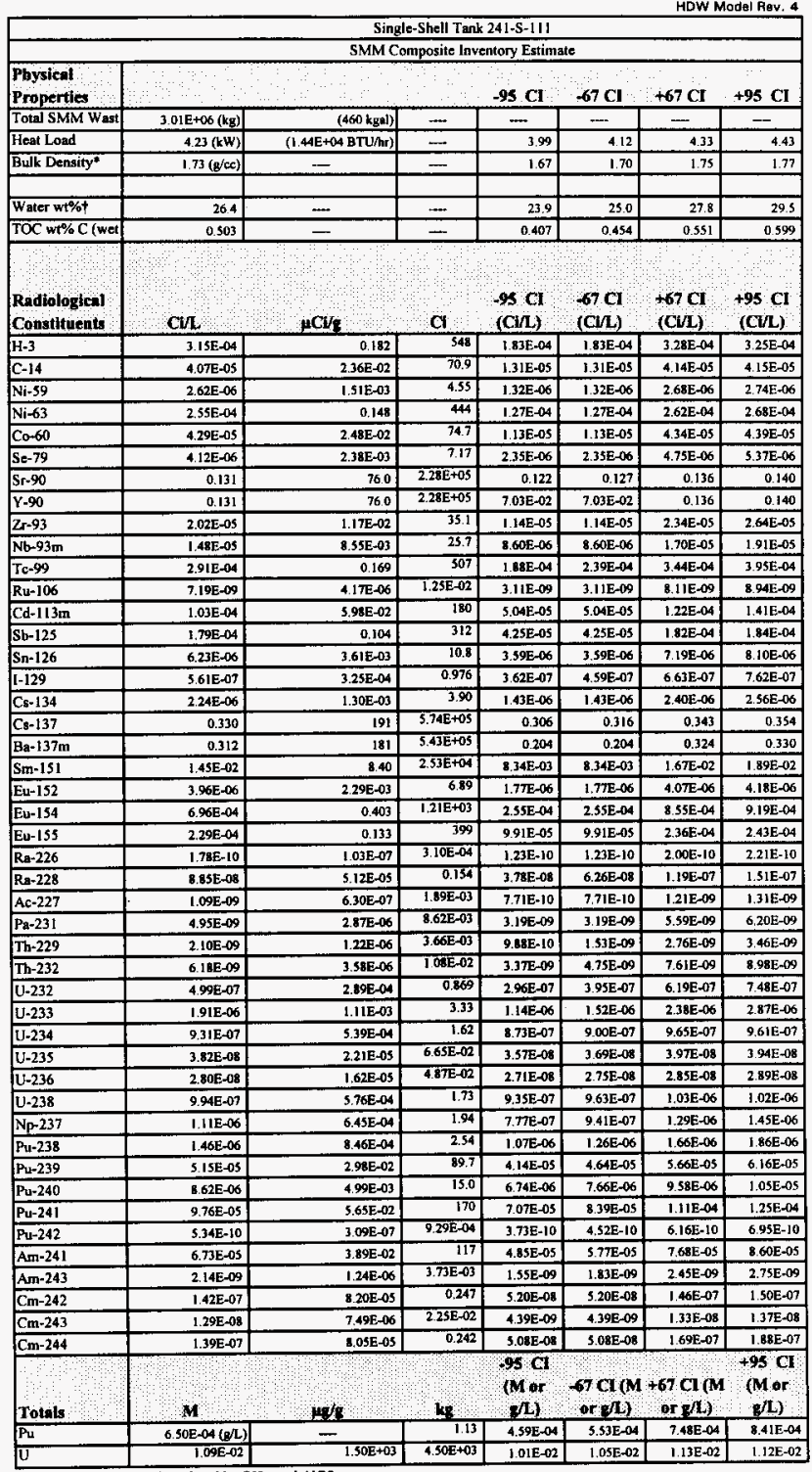

-Density is calculated based on $\mathrm{Na}_{2} \mathrm{OH}$-, and $\mathrm{ANO} 2$ -

+ Water $\mathbf{w} 1 \%$ derived from the difference of density and total diseolved apecies 


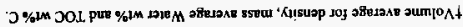

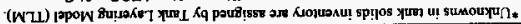

\begin{tabular}{|c|c|c|c|c|c|c|c|}
\hline $20-\pi 8 \mathrm{C}$ & $20-385: 1$ & $20-\mathrm{AzEI}$ & $20-3021$ & $80+35 S 9$ & $60+7981$ & $20-\operatorname{ssc} 1$ & $\Omega$ \\
\hline 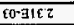 & EO-azz & EO-GEOZ & $\varepsilon 0-9 b 61$ & ELt & $\cdots$ & $\left(7^{8}\right)$ co-azI $z$ & $\mathrm{nd}_{\mathrm{d}}$ \\
\hline $7 / 8$ & $(7 / 8 \times 0$ & $6 / / 80$ & $(\mathrm{~T} / \mathrm{C}$ & 8 & म/क्त: & $\overline{\mathbf{N}}$ & 9ख10, \\
\hline $10 \mathrm{~W}$ & \multirow{2}{*}{\multicolumn{2}{|c|}{ W) $1 \mathrm{~L}<9+$ W) $10<9$}} & $10 W$ & & & & \\
\hline $1056+$ & & & 1356 & & & & \\
\hline $20-319$ & Lo-ast 1 & $80-\exists 8 C t$ & 80-98E & ⿷t?20 & 50-78:9 & $\angle 0^{-9617}$ & $t+2-\omega_{2}$ \\
\hline 80-azz'1 & $80-36$ [1 & 60-alz't & $60-912 t$ & zo-gp\&z & 90-359 9 & $80-3511$ & $\varepsilon t \tau-u \leq 0$ \\
\hline $20-98+1$ & Lo-asti 1 & $80-36 t-9$ & $800-3579$ & $\angle 8 z 0$ & $50-3 b 18$ & coraltil & $z+z-\omega_{0}$ \\
\hline $60-996 z$ & 60-311z & $60-9851$ & $60-3+\varepsilon t$ & co-aSL $\angle$ L & $90-990^{\circ} 1$ & $60-3+81$ & $\varepsilon p \tau-1 \omega \gamma$ \\
\hline $50 \mathrm{as} \times \mathrm{L}$ & $50-3<99$ & So-360s & 50-asz"b & $6 \mathrm{II}$ & $20-3 B 5 \div \varepsilon$ & 50-358'5 & $102-4 \square$ \\
\hline $01-96+6$ & $01 \cdot 918 \cdot 8$ & DI-alt $L$ & 01न & $60-3591$ & L0-389" & $01-3118$ & $z+2-n_{\mathrm{nd}}$ \\
\hline t0-398 I & nor $3 S L 1$ & po-3Is! & to-30t' I & $z \varepsilon f$ & $20-3266$ & torse9 1 & $I t z^{-n_{\mathrm{d}}}$ \\
\hline $50-\operatorname{aft} \tau$ & $50-351 Z z$ & $50-966$ I & 50-316: & $\tau z$ & $20-3021$ & $50-\exists \angle 0 Z$ & $0 b z-n_{d}$ \\
\hline $00-3641$ & AOrJED I & po-aze I & noralet & 182 & $20-9 \% 6$ & to- $38 \varepsilon \mathrm{I}$ & $6 \varepsilon z-n d$ \\
\hline $90-3967$ & $90-36 l z$ & so-astz & 90-38zz & DES & E0raIs I & $90-1797$ & $8 \varepsilon z-1 \mathrm{nd}$ \\
\hline $90-397 \mathrm{I}$ & 90-ב्वान। & LO-ZZLII 8 & $\angle O Z Z \angle C O$ & $96: 1$ & no-3 $3<5$ & $\angle 0^{-a+y^{\prime} 6}$ & $L E Z-d_{N}$ \\
\hline $90-31 Z^{\prime} I$ & $90-\Xi|\tau|$ & 90-99!I & $90-3+1$ ' & $20 z$ & 10-7589 & 90:3611 & $8 \varepsilon z-\Omega$ \\
\hline $80: 322 \mathrm{c}$ & $80-381$ ' & 807al'E & $80^{-} \exists 50^{\circ} \varepsilon$ & $20-30+9$ & $50-9781$ & $80-3 b I ' \varepsilon$ & $9 \varepsilon z-1$ \\
\hline $80-3+8$ & $80-3 z 8 \%$ & 80-afr'p & $80-a z 5, t$ & $20-3196$ & So-3EL 2 & 80-3द्? & $5 \varepsilon z-0$ \\
\hline $900301 ' 1$ & $90-391 \mathrm{~T}$ & $90-3211$ & $90-360^{\circ} \mathrm{I}$ & $z \varepsilon z$ & $60-365 ; 9$ & $90-\mathrm{a}+1 \mathrm{I}$ & $+\varepsilon z-n$ \\
\hline $90-3 s+\tau$ & $90-\mathrm{AEOZ}$ & $90-30 \mathrm{E} 1$ & $\angle 0-3 Z \angle 6$ & Eร'E & porasto 0 & $90-2+9$ & $\varepsilon \mathfrak{\varepsilon \tau - ?}$ \\
\hline LO:36E' 9 & LorgoEs & LOFEE & $\angle 0^{-3 E S C} Z$ & 6980 & $10-39+2$ & $\angle 0-G \angle z+$ & $z \varepsilon z-\cap 1$ \\
\hline $60-7899^{\circ} \mathrm{L}$ & 60-7IS 9 & $60-3907$ & $60-3682$ & $20-3801$ & $90-950 \mathrm{E}$ & 60:-78z's & $z \varepsilon z-4 L$ \\
\hline $60 \cdot 3966^{2} \mathrm{C}$ & $600391 \tau$ & 60-3IET & $01-35+2$ & $50-3995$ & $90-9001$ & $60-9081$ & $6 z 2-4.1$ \\
\hline $600-9<9 ' 5$ & $60-3 \angle 81$ & $60-328 z$ & $60-3287$ & E0-3!8? & $90-1052$ & $60-a z k t$ & {$\left[\varepsilon \tau-{ }^{-8} \mathrm{~d}\right.$} \\
\hline $60=3 \angle 61$ & $60-35<1$ & $60-3 n t \mathrm{t}$ & $60-9 \mathrm{pl} 1$ & $60-380^{\circ} \varepsilon$ & $\angle 0-3 S \angle$ & 60-9ts'1 & $\angle z z-\partial Y$ \\
\hline$\angle 0-36 z^{\prime} 1$ & $\angle 0-910^{\prime} 1$ & Bo-35ES & 80-3Ez $\mathrm{E}$ & $\operatorname{ost} 1^{\circ}$ & 50-3LE & $80-3 \angle 5 L$ & $8 \pi z-87$ \\
\hline $01-3 \leqslant 9 \varepsilon$ & Ot-ast' $\varepsilon$ & $01-38 \varepsilon \tau$ & 101-3102 & b0-708'S & L0-359'1 & ot-ass z & $9 z z-$-в \\
\hline to-3gtz & $m-s z+z$ & po-3szI & rofass I & 28 & $\angle E T O$ & po-alsz & 5sI-ng \\
\hline t00388C & potabe 2 & toralz' & po-3iz? & $50+\mathrm{gzz}$ ! & SDE 0 & D0-386 s & $+51^{-n]}$ \\
\hline $90-3 c t b$ & 90-abet & $90-38 c 2$ & $90-385 \mathrm{Z}$ & 098 & $\{0-35 b z$ & $90-3 b z t$ & $2 s !-\mathrm{ng}$ \\
\hline $20-369 \%$ & $20-355+1$ & EO-apEL $L$ & $\varepsilon 0-g \nabla C L$ & $10+\mathrm{g} L s z$ & $6 \tau L$ & $20-992$ & IS [-wS \\
\hline $6: 820$ & 1820 & 1810 & 1810 & so+zgs's & 851 & ELÉO & 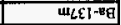 \\
\hline $01 E^{2} 0$ & $66 \tau 0$ & $\angle t^{2} 0$ & 6920 & SO+FB8'S & 491 & 6870 & $\angle E I^{-\infty}$ \\
\hline 90-361z & $90-390 z$ & $90-\mathrm{g} C Z \mathrm{I}$ & 90-3EZ! & $16 \varepsilon$ & E0-AIII & $90-3261$ & $T E[-B D$ \\
\hline Loras99 & L0-91L'S & $\angle 0-996^{\circ} \mathrm{E}$ & $\angle 0-\overline{a t} \varepsilon$ & 786 & $50-36 \angle z$ & LO-AEs' & $62[-1$ \\
\hline $90-a 20 ' L$ & 90-91729 & 90-79/ $\varepsilon$ & 90-391'c & 0.11 & โ0-9EI $\varepsilon$ & $90-3205$ & $92 \mathrm{I}^{-11 \mathrm{~S}}$ \\
\hline po-aLST & porass I & so-asse & $50-759 \cdot \varepsilon$ & EIE & $20-9988$ & pro-ges i & SZI-4S \\
\hline bo-alz' & $00-390^{\circ} 1$ & $50-900 \%$ & so-30t'b & 281 & $20-3515$ & $50-3268$ & $\mathrm{u}_{\varepsilon \mid \mathrm{E} I I} \cdot \mathrm{pD}$ \\
\hline $60-3096$ & $60-31699$ & $60-3992$ & $60-3992$ & zo-gst t & 90-G5s' $\varepsilon$ & $60-$ as 19 & $901-n y$ \\
\hline to-a0b' $\varepsilon$ & p0-996;z & $00-3902$ & 10-369'1 & IIS & $\operatorname{sti0}$ & rogalsz & $66^{-0} \mathrm{~L}$ \\
\hline 5003991 & so-ast! & $90-385 \mathrm{~L}$ & $90-38 S L$ & $29 \tau$ & EO-GEV L & $50-36 z_{1}$ & шह6-qN \\
\hline 50-76z't & S0-9150 $z$ & so- 3001 & $50-3001$ & $\angle S E$ & z0-9101 & so- $35 \angle 1$ & $\varepsilon \sigma^{-5} Z$ \\
\hline $5 \varepsilon 20$ & $\angle E Z O$ & 0010 & $P 610$ & $50+909^{\circ} t$ & IEI & $9 z z 0$ & $06-1$ \\
\hline 5EZO & $\angle E 20$ & 2120 & $E 6 t^{\prime} 0$ & $50+300 p$ & [E] & $9 z 20$ & $06-15$ \\
\hline $90-359$ 'b & $90-\mathrm{szl}$ ' म & $90-3 \angle 0 \mathrm{z}$ & 90-3LOZ $z$ & $62 L$ & $\varepsilon 0-z \in 0 \tau$ & $90-385^{\prime} \varepsilon$ & $6 L^{-0 S}$ \\
\hline $50-\exists \angle C E$ & $50-32 L \varepsilon$ & $90-3 \angle 26$ & $90-3 \angle 66$ & $6+2$ & zo-azlz & $50.989 \mathrm{E}$ & $09^{-0} \mathrm{O}$ \\
\hline por-366 $\mathrm{\varepsilon}$ & $00-3600$ & $00-3662$ & po-3GLt & 162 & $D 2 z 0$ & DO'A8z'E & $\{9-! N$ \\
\hline 90-ZZLI' $\mathrm{t}$ & $90-\operatorname{a\varepsilon z} b$ & $90-3562$ & $90-3562$ & $\angle Z 8$ & co-abe $z$ & $90-390^{\circ}$ & $65 \cdot ! N$ \\
\hline SOFBLSE & SOF.ASS E & sorast' & sorasti & Sil & zorag0z & 50.915 & $t[-5$ \\
\hline $\operatorname{ton} 5282$ & $00-328 z$ & tor-909-1 & to-909! & 955 & 8510 & Do-TEL $Z$ & $\varepsilon-\mathrm{H}$ \\
\hline $\begin{array}{c}\text { (T/10) } \\
1056+\end{array}$ & (प//O) & (1/0) & $(140)$ & $\mathbf{D}$ & $8 \sqrt{n 0^{7}}$ & $7 / 10$ & 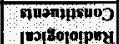 \\
\hline $11 \mathrm{~S}^{\circ} 0$ & Toctro & $\angle 8 E 0$ & LE\&O & $\cdots$ & $\cdots$ & $6 z 50$ & $19 m) \supset \%^{1 m} \supset O L$ \\
\hline $80 E$ & 162 & $\overline{\varepsilon t z}$ & $\overline{L E z}$ & $\cdots$ & $\cdots$ & b.9z & t\%1n vejeM \\
\hline & & & & & & & \\
\hline $8 L 1$ & 661 & 891 & $59 \%$ & -- & - & $(\varpi / B) \varepsilon L 1$ & 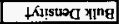 \\
\hline 909 & $80 \%$ & $\angle S 5$ & $\pi z ' s$ & $=$ & $(14 / \cap \Omega 19+0+300 z)$ & $($ (M) $58 \mathrm{~s}$ & 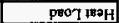 \\
\hline$=$ & $=$ & -- & - & $=$ & $\left(1+\mathbb{Z}^{3} \times 855\right)$ & $(8 \pi) 90+3 \mathrm{cs} \mathrm{c}$ & PISEM $\mathrm{M}$ (8)०1 \\
\hline $1056+$ & $1069+$ & $10 \angle 9^{\circ}$ & $15.56^{\circ}$ & & & & 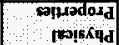 \\
\hline & & & . ग164!1 & 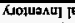 & & & \\
\hline & & & III-S-I & 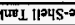 & Du!S & & \\
\hline
\end{tabular}


HDW Model Rev. 4

\begin{tabular}{|c|c|c|c|c|c|c|c|}
\hline & \multicolumn{7}{|c|}{ Single-Shelf Tank 241-S.112 } \\
\hline & \multicolumn{7}{|c|}{ TLM Solids Composite Inventory Estimate* } \\
\hline $\begin{array}{l}\text { Physical } \\
\text { Properties }\end{array}$ & & & & $.95 \mathrm{CI}$ & $-67 \mathrm{CI}$ & $+67 \mathrm{Cl}$ & $+95 \mathrm{Cl}$ \\
\hline Total TLM Wast & $400 \mathrm{E}+04(\mathrm{~kg})$ & $(6.00 \mathrm{kgal})$ & - & - & -- & $\ldots$ & - \\
\hline Heat Load & $7.43 \mathrm{E}-02(\mathrm{~kW})$ & (254 BTU/hr) & - & $5.39 \mathrm{E}-02$ & $6.58 \mathrm{E}-02$ & $7.97 E-02$ & $8.22 \mathrm{E}-02$ \\
\hline Bulk Density & $1.76(\mathrm{~g} / \mathrm{cc})$ & - & - & 1.61 & 1.65 & 1.94 & 2.01 \\
\hline Void Fraction & 0.593 & - & 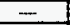 & 0.355 & 0.434 & 0.694 & 0.697 \\
\hline Water wt\% & 25.8 & - & - & 12.3 & 16.7 & 35.7 & 398 \\
\hline TOC wr\% C (wet & 0 & - & - & 0 & 0 & 0 & 0 \\
\hline $\begin{array}{c}\text { Chemical } \\
\text { Constifuents }\end{array}$ & male/L & ppa. & $\mathrm{kg}$ : & $\begin{array}{l}-95 \mathrm{CI} \\
(\mathrm{mole} / \mathrm{L})\end{array}$ & $\begin{array}{c}-67 \mathrm{Cl} \\
\text { (mole/L) }\end{array}$ & $\begin{array}{r}-67 \mathrm{CI} \\
\text { (mole/L) }\end{array}$ & $\begin{array}{l}\text {-9s CI } \\
\text { (mole/L) }\end{array}$ \\
\hline $\mathrm{Nat}$ & 7.99 & $1.04 E+05$ & $4.17 \mathrm{E}+03$ & 5.18 & 5.71 & 11.3 & 13.0 \\
\hline $\mathrm{A} / 3+$ & 9.10 & $1.39 \mathrm{E}+05$ & $5.57 \mathrm{E}+03$ & 6.41 & 7.13 & 10.5 & 11.5 \\
\hline $\mathrm{Fe} 3+($ total Fe $)$ & 0.589 & $1.87 \mathrm{E}+04$ & 747 & 0.580 & 0.584 & 0.594 & 0.599 \\
\hline $\mathrm{Cr} 3+$ & 3.20E-02 & 942 & 37.7 & $4.56 \mathrm{E}-03$ & $1.80 \mathrm{E}-02$ & 0.262 & 0.531 \\
\hline $\mathrm{Bi3+}$ & 0 & 0 & 0 & 0 & 0 & 0 & 0 \\
\hline Le3+ & 0 & 0 & 0 & 0 & 0 & 0 & 0 \\
\hline $\mathrm{Hg} 2+$ & $2.04 E-03$ & 232 & 9.29 & $2.01 \mathrm{E}-03$ & $2.03 \mathrm{E}-03$ & $2.05 E-03$ & $205 E-03$ \\
\hline $\mathrm{Zr}(\mathrm{s} \mathrm{ZrO}(\mathrm{OH}) 2)$ & 0 & 0 & 0 & 0 & 0 & 0 & 0 \\
\hline $\mathrm{Pb} 2+$ & $5.88 \mathrm{E}-02$ & $6.91 E+03$ & 277 & $5.40 \mathrm{E}-02$ & $5.67 \mathrm{E}-02$ & $6.09 \mathrm{E}-02$ & $6.29 \mathrm{E}-02$ \\
\hline Ni2t+ & $2.59 \mathrm{E}-02$ & 861 & 34.5 & $1.88 \mathrm{E}-02$ & $2.28 \mathrm{E}-02$ & $2.82 E-02$ & $2.78 \mathrm{E}-02$ \\
\hline $5+2+$ & 0 & 0 & 0 & 0 & 0 & 0 & 0 \\
\hline Mn4t & 0 & 0 & 0 & 0 & 0 & 0 & 0 \\
\hline $\mathrm{Ca}^{2+}$ & 0.170 & $3,87 \mathrm{E}+03$ & 155 & 0.133 & 0.151 & 0.189 & 0.208 \\
\hline$\overline{K+}$ & $6.38 \mathrm{E}-03$ & 141 & 5.67 & 1,41E-03 & $3,40 \mathrm{E}-03$ & 8.38E-03 & $8,38 E-03$ \\
\hline $\mathrm{OH}$ & 33.7 & $3.24 E+05$ & $1.30 \mathrm{E}+04$ & 22.8 & 25.8 & 39.3 & 43.4 \\
\hline NO3- & 2.93 & $1.03 \mathrm{E}+05$ & $4.12 \mathrm{E}+03$ & 0.316 & 0.316 & 7.02 & 9.24 \\
\hline NO2- & 1.21 & $3.15 \mathrm{E}+04$ & $1.26 \mathrm{E}+03$ & 0.545 & 0.776 & 1.55 & 1.55 \\
\hline $\cos 2-$ & 0.170 & $5.79 \mathrm{E}+03$ & 232 & 0.133 & 0.151 & $0.1 B 9$ & 0.208 \\
\hline PO43. & 0 & 0 & 0 & 0 & 0 & 0 & 0 \\
\hline SO42- & $9.40 \mathrm{E}-03$ & 512 & 205 & 4.79E-03 & 6.63E-03 & $1.12 \mathrm{E}-02$ & 1.12E-02 \\
\hline $\mathrm{Si}(\mathrm{m} \mathrm{STO} 32-)$ & $1.41 \mathrm{E}-02$ & 224 & 8.97 & $1.05 \mathrm{E}-02$ & $1.19 \mathrm{E}-02$ & 4.49E- 02 & $9.16 \mathrm{E}-02$ \\
\hline F- & 0 & 0 & 0 & 0 & 0 & 0 & 0 \\
\hline Cl- & $2.94 \mathrm{E}-02$ & 590 & 23.6 & $6,49 \mathrm{E}-03$ & $1.56 \mathrm{E}-02$ & $3.85 \mathrm{E}-02$ & 8. $20 \mathrm{E}-02$ \\
\hline C6H5073- & 0 & 0 & 0 & 0 & 0 & 0 & 0 \\
\hline EDTA4. & 0 & 0 & 0 & 0 & 0 & 0 & 0 \\
\hline HEDTA3- & 0 & 0 & 0 & 0 & 0 & 0 & 0 \\
\hline Elycolate- & 0 & 0 & 0 & 0 & 0 & 0 & 0 \\
\hline aceture- & 0 & 0 & 0 & 0 & 0 & 0 & 0 \\
\hline oxalmie2- & 0 & 0 & 0 & 0 & 0 & 0 & 0 \\
\hline$\overline{\mathrm{DBP}}$ & 0 & 0 & 0 & 0 & 0 & 0 & 0 \\
\hline butanol & 0 & 0 & 0 & 0 & 0 & 0 & 0 \\
\hline NH3 & 0.128 & $1.23 \mathrm{E}+03$ & 49.4 & $3.29 \mathrm{E}-02$ & 4.6AE- 02 & 0.135 & 0.142 \\
\hline Fe(CN) 64- & 0 & 0 & 0 & 0 & 0 & 0 & 0 \\
\hline
\end{tabular}

"Unknowns in tank solids inventory tre sasigned by Tunk Layering Model (TLM). 
HNF-SD-WM-ER-323, Rev. 1

HDW Madel Rev. 4

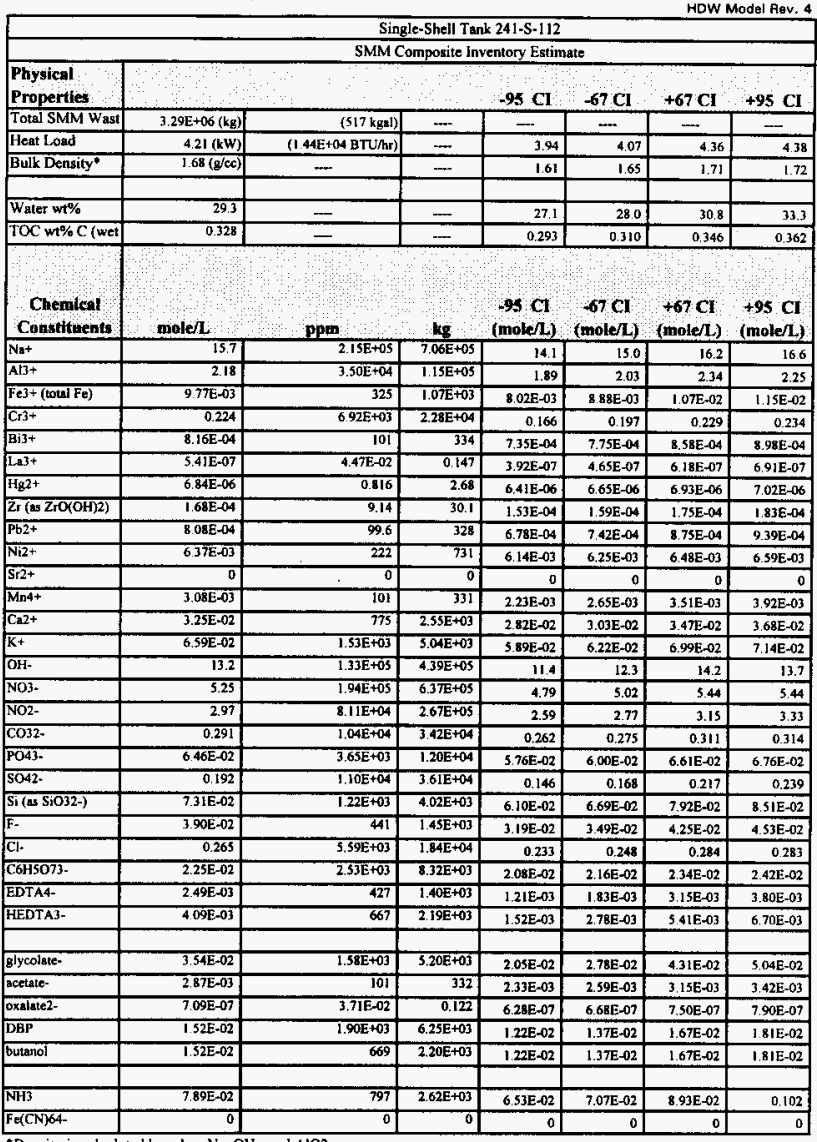

*Density is calculated based an Na, OH-, and $\mathrm{AlO2}$ -

+Water wt\% derived from the difference of density and total dissolved species. 
HDW Model Rov. 4

\begin{tabular}{|c|c|c|c|c|c|c|c|}
\hline \multirow[b]{3}{*}{$\begin{array}{l}\text { Physical } \\
\text { Properties }\end{array}$} & \multicolumn{7}{|c|}{ Single-Shell Tank 241-S-112 } \\
\hline & \multicolumn{7}{|c|}{ Total Inventory Estimate* } \\
\hline & & & & $-95 \mathrm{CI}$ & $-67 \mathrm{CI}$ & $+67 \mathrm{CI}$ & $+95 \mathrm{CI}$ \\
\hline Total Wuste & $3.33 E+06(\mathrm{~kg})$ & $(523 \mathrm{kgal})$ & $=$ & - & $=$ & $\cdots$ & - \\
\hline Heat Load & $4.28(\mathrm{~kW})$ & $(1.46 \mathrm{E}+04 \mathrm{BTL} / \mathrm{hr})$ & - & 4.00 & 4.13 & 444 & 4.45 \\
\hline Bulk Densityt & $1.68\left(g^{\prime} \propto c\right)$ & - & - & 1.61 & 1.65 & 1.71 & 1.72 \\
\hline Water wt\%t & 29.2 & $m$ & - & 27.1 & 28.0 & 30.8 & 33.3 \\
\hline TOC wt\% C (wet & 0.324 & - & - & 0.289 & 0.306 & 0.341 & 0.358 \\
\hline $\begin{array}{c}\text { Chemical } \\
\text { Constituents }\end{array}$ & molerL & ppen: & k8 & $\begin{array}{l}-95 \mathrm{CI} \\
\text { (inble/L) }\end{array}$ & $\begin{array}{l}-67 \mathrm{CI} \\
\text { (nnole/L) }\end{array}$ & $\begin{array}{l}+67 \mathrm{CI} \\
\text { (mole/L) }\end{array}$ & $\begin{array}{l}+95 \mathrm{Cl} \\
(\mathrm{mole} / \mathrm{L})\end{array}$ \\
\hline $\mathrm{Na+}$ & 15.6 & 2.13E+0s & $7.11 \mathrm{E}+05$ & 14.0 & 14.9 & 16.1 & 16.5 \\
\hline $\mathrm{Al3+}$ & 2.26 & $3.62 \mathrm{E}+04$ & $1.21 \mathrm{E}+05$ & 1.98 & 2.11 & 2.42 & 2.33 \\
\hline $\mathrm{Fe} 3+(\operatorname{total} \mathrm{Fe})$ & 1.6AE-02 & 545 & $1.82 \mathrm{E}+03$ & $1.47 \mathrm{E}-02$ & $1.55 \mathrm{E}-02$ & $1,73 \mathrm{E}-02$ & $1.82 \mathrm{E}-02$ \\
\hline $\mathrm{Cr} 3+$ & 0.222 & $6.85 \mathrm{E}+03$ & $2.28 \mathrm{E}+04$ & 0.170 & 0.198 & 0.228 & 0.232 \\
\hline$\overline{\mathrm{Bi3}+}$ & $8.07 \mathrm{E}-04$ & 100 & 334 & $7.27 \mathrm{E}-04$ & $7.66 \mathrm{E}-04$ & $8.48 \mathrm{E}-04$ & 8.88E-04 \\
\hline $\mathrm{La3+}$ & 5.35E-07 & $4.42 \mathrm{E}-02$ & 0.147 & $3.88 \mathrm{E}-07$ & 4.60E-0? & $6.11 E \cdot 07$ & 6.83E-07 \\
\hline Hg2t & 3.01E-05 & 3.59 & 12.0 & $2.97 \mathrm{E}-05$ & J.00E-05 & $3.02 \mathrm{E}-05$ & $3.03 \mathrm{E}-05$ \\
\hline Zr (us Zró(OH)2) & $1.66 \mathrm{E}-04$ & 9.03 & 30.1 & $1.52 \mathrm{E}-04$ & $1.57 \mathrm{E}-04$ & $1.73 \mathrm{E}-04$ & $1.81 \mathrm{E}-04$ \\
\hline $\mathrm{Pb2+}$ & 1.47E-03 & 18] & 604 & $1.3 \mathrm{gE}-03$ & $1.43 \mathrm{E}-03$ & $1.52 \mathrm{E}-03$ & 1.57E-03 \\
\hline $\mathrm{Ni2+}$ & 6.59E-03 & 230 & 766 & $6.39 \mathrm{E}-03$ & $6.49 \mathrm{E}-03$ & $6.69 \mathrm{E}-03$ & 6.79E-03 \\
\hline $\mathbf{S r 2 +}$ & 0 & 0 & 0 & 0 & 0 & 0 & 0 \\
\hline $\mathrm{MnA}^{+}$ & $3.04 E-03$ & 99.3 & 331 & $2.21 \mathrm{E}-03$ & $2.62 \mathrm{E}-03$ & $3.47 \mathrm{E}-03$ & 3.87E-03 \\
\hline $\mathrm{C}_{2+}$ & $3.41 E-02$ & 812 & $2.71 \mathrm{E}+03$ & $3.02 \mathrm{E}-02$ & $3.21 \mathrm{E}-02$ & $3.61 \mathrm{E}-02$ & $380 \mathrm{E}-02$ \\
\hline $\mathbf{K}+$ & $6.52 \mathrm{E}-02$ & $1.52 \mathrm{E}+03$ & $5.05 \mathrm{E}+03$ & $5.83 \mathrm{E}-02$ & $0.16 \mathrm{E}-02$ & $0.92 \mathrm{E}-02$ & $7.06 \mathrm{E}-02$ \\
\hline OH- & 13.4 & $1.362+05$ & $4.52 \mathrm{E}+05$ & 11.7 & 12.5 & 14.4 & 140 \\
\hline No3. & 5.23 & $1.93 \mathrm{E}+03$ & $6.41 E+05$ & 4.85 & 5.03 & 5.38 & 5.38 \\
\hline NO2- & 2.95 & $8.05 E+04$ & $2.68 \mathrm{E}+05$ & 2.57 & 2.75 & 3.13 & 3.31 \\
\hline Co32- & 0.289 & $1.03 \mathrm{E}+04$ & $3.44 \mathrm{E}+04$ & 0.261 & 0.274 & 0.309 & 0.312 \\
\hline PO43. & $6.38 \mathrm{E}-02$ & $3.60 \mathrm{E}+0.3$ & $1.20 \mathrm{E}+04$ & $3.69 \mathrm{E}-02$ & $393 \mathrm{E}-02$ & $6.54 \mathrm{E}-02$ & $668 \mathrm{E}-02$ \\
\hline SO42. & 0.190 & $1.09 \mathrm{E}+0.4$ & 3.62E+04 & 0.144 & 0.166 & 0.215 & 0.237 \\
\hline $\mathrm{Si}(\mathrm{as} \mathrm{SiO} 32-)$ & $7.24 E-02$ & $1.21 \mathrm{E}+03$ & $4.03 \mathrm{E}+03$ & 6.05E-02 & 6.63E-02 & $7.85 \mathrm{E}-102$ & B. 43E-02 \\
\hline $\mathrm{F}$ & $3.86 \mathrm{E}-02$ & 436 & 145E+03 & $3.16 \mathrm{E}-02$ & 3.45E-02 & $4.20 \mathrm{E}-02$ & $4.48 \mathrm{E}-02$ \\
\hline $\mathrm{Cl} \cdot$ & 0.263 & $5.53 \mathrm{E}+03$ & $184 E+04$ & 0.231 & 0.246 & 0.281 & 0.280 \\
\hline C6HSO73- & $2.22 \mathrm{E}-02$ & $2.50 E+03$ & 832E+03 & 2.05E-02 & $2.14 E-02$ & $2.31 \mathrm{E}-02$ & $2.39 \mathrm{E}-02$ \\
\hline EDTA4- & $2.46 \mathrm{E}-03$ & 422 & $1.40 \mathrm{E}+03$ & $1.19 \mathrm{E}-03$ & $1.81 \mathrm{E}-03$ & $3.12 \mathrm{E}-103$ & 3.75E-03 \\
\hline FEDTA3- & 4.04E-03 & 659 & $2.19 \mathrm{E}+03$ & $1.50 \mathrm{E}-03$ & $274 \mathrm{E}-03$ & $5.35 \mathrm{E}-03$ & $6.63 \mathrm{E}-03$ \\
\hline glycolate- & $3.50 \mathrm{E}-02$ & $1.56 \mathrm{E}+0 \mathrm{63}$ & $3.20 \mathrm{E}+03$ & $2.02 \mathrm{E}-02$ & $2.75 \mathrm{E}-02$ & $4.26 \mathrm{E}-02$ & 4.98E-02 \\
\hline ecetate- & 284E-03 & 99.5 & 332 & $2,30 \mathrm{E}-03$ & 2.56E-03 & $3,11 \mathrm{E}-03$ & $3.38 E-03$ \\
\hline oxcalate? & $7.01 \mathrm{E}-07$ & $3.67 \mathrm{E}-12$ & 0.122 & $6.21 \mathrm{E}-07$ & $6.60 \mathrm{E}-07$ & $7.42 \mathrm{E}-07$ & $7,81 \mathrm{E}-07$ \\
\hline$\overline{\text { DBP }}$ & $1.50 \mathrm{E}-02$ & 1.88E+03 & $6.25 E+03$ & $1.21 \mathrm{E}-02$ & $1.35 \mathrm{E}-02$ & $165 \mathrm{E}-02$ & $1.79 \mathrm{E}-02$ \\
\hline butanol & $1,50 \mathrm{E}-02$ & 661 & $2.20 \mathrm{E}+03$ & $1.21 \mathrm{E}-02$ & 1.35E-02 & $1.65 \mathrm{E}-02$ & $1.79 \mathrm{E}-02$ \\
\hline NH3 & $7.94 \mathrm{E}-02$ & 802 & $267 \mathrm{E}+03$ & $6.60 \mathrm{E}-02$ & 1.13E-02 & 8.98E-02 & 0.102 \\
\hline $\mathrm{Fe}(\mathrm{CN}) 64$ & 0 & 0 & 0 & 0 & 0 & 0 & 0 \\
\hline
\end{tabular}

- Unknowns in tank solids inventory are assigned by Tank Layesing Model (TLM).

+ Water wt\% derived from the difference of density and total diswolved apecies. 
HNF-SD-WM-ER-323, Rev. 1

HOW Model Rẹv, 4

\begin{tabular}{|c|c|c|c|c|c|c|c|}
\hline \multirow{4}{*}{$\begin{array}{l}\text { Physical } \\
\text { Properties }\end{array}$} & \multicolumn{7}{|c|}{ Single-Shell Tank 241-S-112 } \\
\hline & \multicolumn{7}{|c|}{ TLM Solids Composite Inventory Estimaie* } \\
\hline & & & & & & & \\
\hline & & & & $-95 \mathrm{CI}$ & $-67 \mathrm{CI}$ & $+67 \mathrm{CI}$ & $+95 \mathrm{CI}$ \\
\hline Total ILM Wast & $4.00 \mathrm{E}+04(\mathrm{~kg})$ & $(6.00 \mathrm{kgal})$ & $m$ & - & $\ldots$ & - & $=$ \\
\hline Heat Load & $7.43 \mathrm{E}-02(\mathrm{~kW})$ & (254 BTU/hr) & $m$ & $5.39 \mathrm{E}-02$ & $6,58 \mathrm{E}-02$ & $7.97 \mathrm{E}-02$ & $8.22 \mathrm{E}-02$ \\
\hline Bulk Density & $1.76(\mathrm{~g} / \propto \mathrm{c})$ & - & $=$ & 1.61 & 1.65 & 1.94 & 2.01 \\
\hline Void Fraction & 0.593 & - & 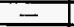 & 0.355 & 0.434 & 0.694 & 0.697 \\
\hline Water w\% & 25.8 & - & - & 12.3 & 167 & 35.7 & 39.8 \\
\hline TOC wr\% C (wet & 0 & - & $=$ & 0 & 0 & 0 & 0 \\
\hline $\begin{array}{l}\text { Radiological } \\
\text { Constlituents }\end{array}$ & CllL & $\mu \mathrm{cu}$ & $\mathbf{a}$ & $\begin{array}{l}95 \mathrm{Cl} \\
\text { (C) }\end{array}$ & $\begin{array}{l}67 \mathrm{Cl} \\
(\mathrm{CVL})\end{array}$ & $\begin{array}{l}+67 \mathrm{CI} \\
(\mathrm{Cl} / \mathrm{L})\end{array}$ & $\begin{array}{r}+95 \mathrm{CI} \\
(\mathrm{Cl} / \mathrm{L}) \\
\end{array}$ \\
\hline $\mathrm{H}-3$ & J.51E-05 & 8.58E-03 & 0.344 & $2.03 \mathrm{E}-06$ & $7.86 \mathrm{E}-06$ & $2.10 \mathrm{E}-05$ & $2.10 \mathrm{E}-05$ \\
\hline C-14 & 1.19E- -06 & $6.73 \mathrm{E}-04$ & $2.69 \mathrm{E}-02$ & $1.84 E-07$ & $5.85 \mathrm{E}-07$ & $1.59 \mathrm{E}-06$ & 1.59E-06 \\
\hline $\mathrm{Ni}-59$ & $7.45 \mathrm{E}-06$ & $4.22 \mathrm{E}-03$ & $\begin{array}{l}0.169 \\
\end{array}$ & $5.38 \mathrm{E}-06$ & 6.55E-06 & 8.14E-A6 & $8.01 E-06$ \\
\hline $\mathrm{Ni}-63$ & $6.95 \mathrm{E}-04$ & 0.394 & 15.8 & $5,02 E-04$ & $6.11 E-04$ & $7.60 \mathrm{E}-04$ & $7.48 E-04$ \\
\hline Co-60 & 4.71E-07 & $2.67 \mathrm{E}-04$ & $107 \mathrm{E}=02$ & 7.47E-AB & $2.33 \mathrm{E}-07$ & $6,30 E-07$ & $6.30 \mathrm{E}-07$ \\
\hline Se-79 & $2.52 \mathrm{E}-07$ & $1.43 \mathrm{E}-04$ & 5.73E-03 & $3.94 \mathrm{E}-08$ & 1.24E-07 & $3.38 \mathrm{~B}-07$ & 3.59E- 06 \\
\hline Sr-90 & 0.465 & 264 & $1.06 E+04$ & 0,332 & 0.407 & 0,510 & 0.517 \\
\hline$Y-90$ & 0.465 & 264 & $1.06 \mathrm{E}+04$ & 0,332 & 0.407 & 0.510 & 0.518 \\
\hline $\mathrm{Zr}-93$ & $1.19 \mathrm{E}-06$ & $6.75 \mathrm{E}-04$ & $2.70 \mathrm{E}-02$ & $1.86 \mathrm{E}-07$ & $587 \mathrm{E}-07$ & $1.59 \mathrm{E}-06$ & $1,52 \mathrm{E}-0.5$ \\
\hline Nb-93m & $9.73 \mathrm{E}-07$ & 5.52E-04 & $2.21 \mathrm{E}-02$ & $1.52 \mathrm{E}-07$ & $4.80 \mathrm{E}-07$ & $1.30 \mathrm{E}-06$ & $1.6 \mathrm{EE}-05$ \\
\hline Tc-99 & $8.32 \mathrm{E}-06$ & $4.72 \mathrm{E}-03$ & 0.189 & $1.30 \mathrm{E}-06$ & $4.10 \mathrm{E}-06$ & $1.11 E-05$ & $1.11 \mathrm{E}-0.0$ \\
\hline Ru-106 & $1.39 \mathrm{E}-12$ & $7.86 \mathrm{E}-10$ & $\begin{array}{l}3.15 \mathrm{E}-0.8 \\
\end{array}$ & $2.81 \mathrm{E}-13$ & $7.22 \mathrm{E}-13$ & $1.83 \mathrm{E}-12$ & 1.83E-12 \\
\hline Cd-113m & $3.67 \mathrm{E}-06$ & $2.08 \mathrm{E}-03$ & 8.34E-02 & 5.75E-07 & $1.81 \mathrm{E}-06$ & 4.92E-06 & $4.92 \mathrm{E}-06$ \\
\hline $\mathrm{sb}-125$ & $6.96 \mathrm{E}-07$ & $3.94 \mathrm{E}-04$ & 1.58E-02 & 1.1SE-07 & $3.47 \mathrm{E}-07$ & 9.29E-07 & $9.29 \mathrm{E}-07$ \\
\hline $5 n-126$ & $3.87 \mathrm{E}-07$ & 2.20E- -4 & $8.80 \mathrm{E}-03$ & $6,03 \mathrm{E}-08$ & $1.91 \mathrm{E} \sim 07$ & $5.19 \mathrm{E}-07$ & $5.74 E-06$ \\
\hline$[-129$ & $1.60 \mathrm{E}-08$ & 9.06E-06 & $363 \mathrm{E}-04$ & $2.49 \mathrm{E}-09$ & $7.88 \mathrm{E}-09$ & $2.14 \mathrm{E}-08$ & $214 E-08$ \\
\hline Cs. 134 & 1.44F-08 & B.18E- 06 & $3.27 \mathrm{E}-04$ & $4.42 \mathrm{E}-09$ & $9.42 \mathrm{E}-09$ & 1.84E-03 & 1.84E-08 \\
\hline $\mathrm{C}_{5}-137$ & $2.92 \mathrm{E}-02$ & 16.6 & 663 & 8.58E-03 & 1.68E-02 & $3.75 \mathrm{E}-02$ & 3.75E- 02 \\
\hline$B A-137 m$ & $276 \mathrm{E}-02$ & 15.7 & 627 & 8.12E-03 & $1.59 \mathrm{E}-02$ & $3.54 E-02$ & $3.54 \mathrm{E} .02$ \\
\hline Sm-15! & $900 \mathrm{E}-04$ & 0.510 & 20.4 & $1,40 \mathrm{E}-04$ & 4.44E-04 & 1.21E-03 & $1.32 \mathrm{E} \cdot 02$ \\
\hline Eu- 152 & $3.59 E-06$ & 2.03E-03 & $8,14 \mathrm{E}-02$ & 3.51E-06 & 3.54E-06 & 3.62E-06 & $3.62 \mathrm{E}-06$ \\
\hline Eu-194 & 1.14E-05 & $6.45 \mathrm{E}-03$ & 0.258 & $1.81 \mathrm{E}-06$ & $5.63 \mathrm{E}-06$ & $1.52 \mathrm{E}-0 \mathrm{~S}$ & 1.52E-05 \\
\hline Eu-155 & $1.69 \mathrm{E}-04$ & $9.602-02$ & 3.85 & 1.66E-04 & $1.67 \mathrm{E}-04$ & $1.71 \mathrm{E}-04$ & $1.71 \mathrm{E}-04$ \\
\hline Ra-226 & $5.42 \mathrm{E}-10$ & 3.07E-07 & $1.23 \mathrm{E} D \mathrm{OS}$ & $7.52 \mathrm{E}-11$ & $3.04 \mathrm{E}-10$ & $7.80 \mathrm{E}-10$ & 1.01E-09 \\
\hline $\mathrm{Ra}-228$ & 4.78E-15 & $2.71 \mathrm{E}-12$ & $1.09 \mathrm{E}-10$ & $4.68 \mathrm{E}-15$ & $4.72 E-15$ & $4.82 \mathrm{E}-15$ & $4.82 \mathrm{E}-15$ \\
\hline Ac-227. & 2.39E-09 & 1.35E-06 & $5.42 \mathrm{E}-05$ & $1.60 \mathrm{E}-10$ & $1.01 \mathrm{E}-09$ & $177 \mathrm{E}-09$ & 5.10E-09 \\
\hline $\mathrm{Pa}-231$ & $3.76 \mathrm{E}-10$ & 2.13E-07 & 8.54E-06 & 5.63E-11 & $1,85 \mathrm{E}-10$ & $1.04 \mathrm{E}-09$ & $8.41 \mathrm{E}-09$ \\
\hline Th-229 & $9.05 \mathrm{E}-13$ & $5.13 \mathrm{E}-10$ & 2.05E- 08 & $8.86 \mathrm{E}-13$ & B.93E.13 & $9.12 \mathrm{E}-13$ & $9.12 \mathrm{E}-13$ \\
\hline Th-232 & $3.16 \mathrm{E}-16$ & $1.79 \mathrm{E}-13$ & $7.17 \mathrm{E}-12$ & $5,40 \mathrm{E}-17$ & 1.58E-16 & $4.21 E-16$ & 4.21E-16 \\
\hline $\mathrm{U}-232$ & $3.26 \mathrm{E}-10$ & 1.85E-07 & $7,40 \mathrm{E}-06$ & $2.83 \mathrm{E}-10$ & $3.08 \mathrm{E}-10$ & $3.38 \mathrm{E}-10$ & $3.47 \mathrm{E} \cdot 10$ \\
\hline $\mathrm{U}-233$ & $1.21 \mathrm{E}-11$ & $6.84 \mathrm{E}-09$ & $2.74 E-07$ & 1.05E-11 & 1.14E-11 & $1.256-11$ & $1.29 \mathrm{E}-11$ \\
\hline $\mathrm{U}-234$ & $7.46 \mathrm{E}-106$ & 4.23E-03 & 0.169 & 6.49E- $\$ 6$ & $7,06 E-06$ & $7.75 \mathrm{E}-06$ & $7.95 \mathrm{E}-06$ \\
\hline $\mathrm{U}-235$ & $3.17 \mathrm{E}-07$ & $1.80 \mathrm{E}-04$ & 7.19E-03 & 2.76E-07 & $3.00 \mathrm{E}-07$ & $3.29 \mathrm{E}-07$ & $3.38 \mathrm{E}-07$ \\
\hline U-236 & $1.64 E_{-107}$ & $9.29 \mathrm{E}-05$ & $3.72 \mathrm{E}-03$ & 1.42E-0? & $1.55 \mathrm{E}-07$ & $1.70 \mathrm{E}-07$ & $1.75 \mathrm{E}-07$ \\
\hline $\mathrm{U}-238$ & $7.27 \mathrm{E}-06$ & 4.12E-03 & 0.165 & $6.32 \mathrm{E}-06$ & 6.87E-06 & $7.54 \mathrm{E}-06$ & $7.75 \mathrm{E}-06$ \\
\hline Np-237 & $5.42 \mathrm{E}-08$ & 3.07E-05 & $1.2 \mathrm{JE}-03$ & 8.62E-09 & $2.68 \mathrm{E}-08$ & $7.25 \mathrm{E}-08$ & 7.25E-08 \\
\hline$P u-238$ & 1.59E-05 & s.99E-03 & 0.360 & 1.49E-05 & 1.54E-05 & $1.63 \mathrm{E}-05$ & $1.68 \mathrm{E}-05$ \\
\hline Pu-239 & $1.04 \mathrm{E}-03$ & 0.590 & 23.6 & $9.73 \mathrm{E}-04$ & $1.01 \mathrm{E}-03$ & $1.07 \mathrm{E}-03$ & $1.11 \mathrm{E}-03$ \\
\hline Pu-240 & $1.47 \mathrm{E}-04$ & 3.34E-02 & 3.34 & $1.38 \mathrm{E}-04$ & $1.42 \mathrm{E}-04$ & 1.52E-04 & $1.57 \mathrm{E}-04$ \\
\hline $\mathrm{Pu}-241$ & 8.91E-104 & 0.505 & 20.2 & 8. 35E-04 & B.63E-04 & $9.20 \mathrm{E}-04$ & $9.47 \mathrm{E}-04$ \\
\hline Pu-242 & $3.85 \mathrm{E}-09$ & 2.18E-06 & 8.74E.05 & 3.59E-09 & $3.72 \mathrm{E}-09$ & $3,98 \mathrm{E}-09$ & $4.11 \mathrm{E}-09$ \\
\hline Am-241 & $4.25 \mathrm{E}-06$ & 2.41E-03 & $9.65 \mathrm{E}-02$ & $6.67 \mathrm{E}-07$ & $2.10 \mathrm{E}-06$ & $569 \mathrm{E}-06$ & $7.18 \mathrm{E}-05$ \\
\hline Am-243 & 3.97E-11 & 2.25E-08 & 9.02E-07 & 6. 19E-12 & $1.96 \mathrm{E}-11$ & $5.32 \mathrm{E}-11$ & $6.71 \mathrm{E}-10$ \\
\hline $\mathrm{Cm}=242$ & 8.23E-08 & 4.67E-05 & $1.87 \mathrm{E}-03$ & $8.06 \mathrm{E}-08$ & B. 13E-08 & $830 \mathrm{E}-08$ & $8.30 \mathrm{E}-08$ \\
\hline $\mathrm{Cm}-243$ & $1.89 \mathrm{E}-09$ & $1.07 \mathrm{E}-06$ & $4.28 \mathrm{E}-05$ & $1.85 \mathrm{E}-09$ & $1,86 \mathrm{E}-09$ & $1.90 \mathrm{E}-09$ & $1.90 \mathrm{E}-09$ \\
\hline $\mathrm{C}_{\mathrm{m}=244}$ & $1.42 \mathrm{E}-09$ & 8.04E-07 & $3.22 \mathrm{E}-05$ & $2.20 \mathrm{E}-10$ & $6.98 \mathrm{E}-10$ & $190 \mathrm{E}=09$ & $1.90 \mathrm{E}-09$ \\
\hline & & & & $+95 \mathrm{CI}$ & 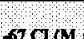 & a: & $+95 \mathrm{CI}$ \\
\hline Totals & $\mathbf{M}$ & $\mathrm{Hg} / \mathrm{s}$ & k. & $\mathrm{p} / \mathrm{L})$ & $0+\phi / 4)$ & or $\pi(\mathrm{J})$ & g/L) \\
\hline $\mathrm{Pu}$ & $1.74 \mathrm{E}-02(\mathrm{~g} / \mathrm{L})$ & - & 0.395 & $1.63 \mathrm{E}-02$ & $1.68 \mathrm{E}-02$ & $1.80 \mathrm{E}-02$ & $1.85 \mathrm{E}-02$ \\
\hline $\mathrm{U}$ & $9.14 E-02$ & $1.23 \mathrm{E}+04$ & 494 & $7.95 \mathrm{E}-02$ & $8.65 \mathrm{E}-02$ & $9.50 \mathrm{E}-02$ & $9.75 \mathrm{E}-02$ \\
\hline
\end{tabular}

-Unknowns in tank solids inventory are assigned by Tank Layering Model (TLM). 
HNF-SD-WM-ER-323, Rev. 1

HDW Model Aev. 4

\begin{tabular}{|c|c|c|c|c|c|c|c|}
\hline \multirow{3}{*}{\begin{tabular}{|l} 
\\
Physical \\
Properties
\end{tabular}} & \multicolumn{7}{|c|}{ Single-Shell Tank 241-S-112 } \\
\hline & \multicolumn{7}{|c|}{ SMM Composite Inventory Estimate } \\
\hline & & & $\because$ & $95 \mathrm{CI}$ & $-67 \mathrm{Cl}$ & $+67 \mathrm{CI}$ & $+95 \mathrm{CI}$ \\
\hline Total SMM Wast & $329 \mathrm{E}+06(\mathrm{~kg})$ & $(517 \mathrm{kgal})$ & - & - & $\ldots$ & - & $\cdots$ \\
\hline Heat Load & $421(\mathrm{~kW})$ & $(1.44 \mathrm{E}+(04 \mathrm{BTU} / \mathrm{hr})$ & - & 3.94 & 4.07 & 436 & 4.38 \\
\hline Bulk Density* & $1.68(\mathrm{~g} / \mathrm{cc})$ & $\cdots$ & - & 1.61 & 1.65 & 1.71 & 1.72 \\
\hline & & & & & & & \\
\hline Weter wt\% & 29.3 & - & - & 27.1 & 28.0 & 30.8 & 33.3 \\
\hline TOC wi $\% \mathrm{C}$, wet & 0.328 & - & $=$ & 0.293 & 0.310 & 0.346 & 0.362 \\
\hline $\begin{array}{l}\text { Radiological } \\
\text { Constifuents }\end{array}$ & $\mathrm{CV}$ & $\mathrm{ACV}$ & $\mathrm{Ci}$ & $\begin{array}{l}-95 \mathrm{Cl} \\
\text { (CiL) }\end{array}$ & $\begin{array}{l}-67 \mathrm{CI} \\
(\mathrm{CM})\end{array}$ & $\begin{array}{l}+67 \mathrm{CI} \\
(\mathrm{CL} / \mathrm{L})\end{array}$ & $\begin{array}{r}+95 \mathrm{CI} \\
(\mathrm{CLL})\end{array}$ \\
\hline $\mathrm{H}-3$ & $2.67 \mathrm{E}-04$ & 0.159 & 523 & $1.60 E-04$ & $1.601-04$ & $2.84 E-04$ & $2.77 \mathrm{E}-04$ \\
\hline C-14 & 3.41E-0S & 2.03E-02 & 66.8 & 1.17E-05 & $1.17 E-05$ & 3.49E-05 & $3.48 \mathrm{E}-05$ \\
\hline Ni-59 & 2.33E-06 & 1,38E-03 & 4.56 & $1.27 E-06$ & $1.2 \pi \mathrm{E}-06$ & $2.38 \mathrm{E}-05$ & $2.43 \mathrm{E}-06$ \\
\hline Ni-63 & 2.26E 04 & 0.134 & 443 & $1.22 \mathrm{E}-04$ & $122 \mathrm{E}-04$ & $2.31 \mathrm{E}-04$ & $237 \mathrm{E}-94$ \\
\hline Co-60 & $3.45 \mathrm{E}-05$ & $2.05 \mathrm{E}-02$ & 67.5 & $8.81 \mathrm{E}-00$ & $8.81 E-06$ & 3.50E-05 & 3.5SE-05 \\
\hline Se-79 & 3.56E-06 & $2.12 \mathrm{E}-03$ & 6.96 & 2.13E-06 & $2.13 E-06$ & 4.08E- -06 & $437 \mathrm{E}-06$ \\
\hline Sr -90 & 0.117 & 69.3 & $2.28 \mathrm{E}+05$ & 0.110 & 0.113 & 0.120 & 0.124 \\
\hline$Y-90$ & 0.117 & 69.3 & $2.28 \mathrm{E}+05$ & $6.71 \mathrm{E}-02$ & $6.71 \mathrm{E}-02$ & 0.120 & 0.124 \\
\hline $2 r-93$ & $1.74 \mathrm{E}-05$ & $1.03 \mathrm{E}-02$ & 34.0 & $1.02 \mathrm{E}-0 \mathrm{5}$ & $1.02 E-05$ & $2.00 \mathrm{E}-05$ & $2.25 E-05$ \\
\hline $\mathbf{N b}-93 \mathrm{~m}$ & $1.29 \mathrm{E}-05$ & $7,66 \mathrm{E}-03$ & 25.2 & $7.87 \mathrm{E}-06$ & $787 \mathrm{E}-06$ & 1.47E- -05 & $164 \mathrm{E}-05$ \\
\hline Tc-99 & $2.43 \mathrm{E}-04$ & 0.145 & 476 & 1.60E-04 & $2.00 \mathrm{E}-04$ & 2.86E-04 & 3.28E-04 \\
\hline Ru-106 & S. IIE-09 & $3,16 \mathrm{E}-06$ & $1.04 \mathrm{E}-02$ & $1.99 \mathrm{E}-09$ & 1.99E- 09 & 6.05E-49 & $6.73 \mathrm{E}-09$ \\
\hline $\mathrm{Cd}-113 \mathrm{~m}$ & 8.50E-05 & $5.05 E-02$ & 166 & 4.20E-05 & $4.20 \mathrm{E}-0.5$ & 1.01E-04 & 1. 15E-04 \\
\hline Sb- 125 & $1.41 \mathrm{E}-04$ & 8.41E.02 & 277 & 3.03E-05 & $3.03 \mathrm{E}-0 \mathrm{~S}$ & $1.44 \mathrm{E}-04$ & $146 \mathrm{E}-0.4$ \\
\hline $5 n-126$ & $5.39 \mathrm{E}-06$ & $3.21 \mathrm{E}-03$ & 10.5 & $3.24 \mathrm{E}-06$ & $3.24 \mathrm{E}-06$ & 6.17E-06 & $6.91 \mathrm{E}-06$ \\
\hline I-129 & 468E-07 & $2.79 \mathrm{E}-04$ & 0.917 & $3.06 \mathrm{E}-07$ & $3.86 \mathrm{E}-07$ & $5.52 \mathrm{E}-07$ & $632 \mathrm{E}-07$ \\
\hline Cs-134 & 1.59E-06 & 9.44E-04 & 3.11 & 1.06E-06 & 1.06E-06 & 1.71E-06 & $182 \mathrm{E}-06$ \\
\hline Cs 137 & 0.291 & 173 & $5.70 \mathrm{E}+05$ & 0.262 & 0.276 & 0.308 & 0.310 \\
\hline $\mathrm{Ba}-137 \mathrm{~m}$ & 0.276 & 164 & $5.39 \mathrm{E}+05$ & 0.206 & 0.206 & 0.291 & 0.293 \\
\hline $5 m-151$ & $1.26 \mathrm{E}-02$ & 7.46 & $2.46 \mathrm{E}+04$ & 7.54E-03 & 7.54E-03 & $1.44 E-02$ & $1.61 \mathrm{E}-02$ \\
\hline Eu- 152 & 3.12E-06 & 1.85E-03 & 6.10 & 1.34E-06 & 1.34E- 06 & $3.18 E-06$ & $3.24 \mathrm{E}-16$ \\
\hline Eu-154 & $5.54 \mathrm{E}-04$ & 0.329 & I.08E+03 & $1.96 \mathrm{E}-04$ & $1.96 \mathrm{E}-04$ & $6.84 \mathrm{E}-04$ & $7.35 \mathrm{E}-04$ \\
\hline Eu- 155 & $1.79 \mathrm{E}-04$ & 0.100 & 350 & 7.34E-05 & $7.34 \mathrm{E}-0 \mathrm{~s}$ & $1.84 \mathrm{E}-04$ & $1.88 \mathrm{E}-04$ \\
\hline $\operatorname{Ra}-226$ & $1.66 \mathrm{E}-10$ & 9.88E-08 & $3.25 E-04$ & $1.12 \mathrm{E}-10$ & $1.22 \mathrm{E}-10$ & $1.94 \mathrm{E}-10$ & $2.21 \mathrm{E}-10$ \\
\hline Rн-228 & $6.02 E-08$ & 3.5BE-0S & 0.118 & 2. 35E-08 & $4.14 \mathrm{E}-08$ & $8.19 \mathrm{E}-08$ & $1.05 \mathrm{E}-07$ \\
\hline Ac-227 & $1.00 \mathrm{E}-09$ & $5.96 \mathrm{E}-07$ & 1.96E-03 & $6.87 \mathrm{E}-10$ & $7.45 \mathrm{E}-10$ & $1.16 \mathrm{E}-09$ & $1.26 \mathrm{E}-09$ \\
\hline $\mathrm{Pa}-231$ & $4.43 \mathrm{E}-09$ & $2.63 E-06$ & $8.67 \mathrm{E}-03$ & $3.00 \mathrm{E}-09$ & $3.00 \mathrm{E}-09$ & $4.95 \mathrm{E}-09$ & $5.45 \mathrm{E}-199$ \\
\hline Th-229 & $1.43 \mathrm{E}-09$ & $8.52 \mathrm{E}-07$ & 2.90E-03 & $6.29 \mathrm{E}-10$ & $1.02 \mathrm{E}-09$ & $1.91 E-09$ & $242 \mathrm{E}-09$ \\
\hline Th-232 & 4.05E-09 & $2.41 \mathrm{E}-06$ & $7.93 \mathrm{E}-03$ & 2.03E-09 & $3.02 E-09$ & $5.09 \mathrm{E}-09$ & $6.08 \mathrm{E}-09$ \\
\hline U-232 & 3.63E-07 & $2.16 \mathrm{E}-04$ & 0710 & 2.16E-07 & $2.83 \mathrm{E}-07$ & 4.50E-07 & $5,42 \mathrm{E}-07$ \\
\hline $\mathrm{U}-233$ & 1.39E-06 & $8.21 \mathrm{E}-04$ & 2.72 & 1. 29E-07 & $1.10 \mathrm{E}-06$ & $1.72 \mathrm{E}-06$ & $2.08 E-06$ \\
\hline U-234 & $8.70 \mathrm{E}-07$ & $5.18 \mathrm{E}-04$ & 1.70 & 7.96E-07 & $8.31 \mathrm{E}-07$ & $9.13 \mathrm{E}-07$ & 8.95E-07 \\
\hline $\mathrm{U}-235$ & $3.60 E-08$ & 2.14E-05 & $7.052-02$ & 3.24E-08 & $343 \mathrm{E}-08$ & 3.78E-08 & $3.70 \mathrm{E}-0 \mathrm{~B}$ \\
\hline $\mathrm{U}-236$ & $2.47 \mathrm{E}-08$ & $1.47 \mathrm{E}-05$ & 4.83E-02 & 2.35E-08 & $2.41 \mathrm{E}-08$ & 2.54E-08 & 2.54E-08 \\
\hline $\mathrm{U}-238$ & $9.06 \mathrm{E}-07$ & $5.39 \mathrm{E}-04$ & 1.77 & $8.31 E-07$ & $8.665-07$ & $9.50 \mathrm{E}-07$ & $929 \mathrm{E}-07$ \\
\hline Np-237 & $9.63 \mathrm{E}-07$ & 5.72E-04 & 188 & $6.09 \mathrm{E}-07$ & $8.23 \mathrm{E}-07$ & $1.10 \mathrm{E}-06$ & $1.24 \mathrm{E}-06$ \\
\hline Pu-238 & $1.19 \mathrm{E}-06$ & $7.05 \mathrm{E}-04$ & 2.32 & 8.64E-07 & $1.02 \mathrm{E}, 06$ & 1.35E.06 & $1.51 \mathrm{E}-06$ \\
\hline $\mathrm{Pu}=239$ & 4.45E-05 & 2.65E-02 & 87.1 & $3.63 \mathrm{E}-05$ & $4.03 \mathrm{E}-05$ & $4.87 \mathrm{E}-05$ & $5.27 \mathrm{E}-05$ \\
\hline Pu-240 & $7.28 \mathrm{E}-06$ & $4.33 \mathrm{E}-03$ & 14.3 & 5.75E- 06 & $6.50 \mathrm{E}-\mathrm{A6}$ & $8.06 E-06$ & $881 \mathrm{E}-06$ \\
\hline Pu-241 & 7.82E-05 & 4.65E-02 & 153 & 5.64E-05 & 6.71E-05 & 8.94E-05 & $1.00 \mathrm{E}-04$ \\
\hline Pu-242 & $4.23 \mathrm{E}-10$ & $2.51 \mathrm{E}-07$ & 8.27E-04 & $2.92 \mathrm{E}-10$ & $3.56 \mathrm{E}-10$ & $489 \mathrm{E}-10$ & $5.54 \mathrm{E}-10$ \\
\hline$A \mathrm{~m}-241$ & $5.75 E-05$ & 3.42E-02 & 112 & $4.23 \mathrm{E}-05$ & $497 \mathrm{E}-05$ & $6.53 \mathrm{E}-05$ & $7.27 \mathrm{E}-\mathrm{OS}$ \\
\hline Am-243 & $1.61 E-09$ & 9.55E-07 & 3.14E-03 & 1.13E- 09 & $1.36 \mathrm{E}-09$ & $1.86 \mathrm{E}-69$ & 2.11E-09 \\
\hline $\mathrm{Cm}-242$ & 1.11E-07 & 6.59E-05 & 0.217 & $3.20 \mathrm{E}-08$ & 3,80E-08 & 1.13E-07 & 1.15E-07 \\
\hline $\mathrm{Cm}-243$ & $9.18 \mathrm{E}-09$ & $587 \mathrm{E}-06$ & $1.93 \mathrm{E}-02$ & $2.93 \mathrm{E}-09$ & 2.93E-09 & $1.01 E-08$ & $1.03 \mathrm{E}-08$ \\
\hline Cm-244 & $1.09 \mathrm{E}-07$ & $0.46 \mathrm{E}-0 \mathrm{~s}$ & 0.213 & $3.70 \mathrm{E}-0 \mathrm{~B}$ & $3.70 \mathrm{E}-08$ & $1.33 \mathrm{E}-07$ & $1.48 \mathrm{E}-07$ \\
\hline Totals & $\mathbf{M}$ & Holg & ks & $\begin{array}{l}05 \mathrm{CI} \\
(\mathrm{Mar} \\
\text { old) }\end{array}$ & $\begin{array}{l}67 \mathrm{CH}(\mathrm{M} \\
\text { ord/L) }\end{array}$ & $\begin{array}{c}+67 \mathrm{Cl}(\mathrm{M} \\
\text { or } / \mathrm{L})\end{array}$ & $\begin{array}{r}+95 \mathrm{CL} \\
(\mathrm{M} \mathrm{ar} \\
\text { gL) }\end{array}$ \\
\hline $\mathrm{Pu}$ & $5.82 E-04(\mathrm{~g} / \mathrm{L})$ & - & 1.14 & $4,26 \mathrm{E}-04$ & $5.03 E-04$ & $6.61 \mathrm{E}-04$ & $7.37 \mathrm{E}-04$ \\
\hline $\mathrm{U}$ & $1.03 \mathrm{E}-02$ & $1.45 \mathrm{E}+03$ & 4.79E+03 & $9.32 \mathrm{E}-03$ & 9.7JE-03 & $1.08 \mathrm{E}-02$ & $1.06 \mathrm{E}-02$ \\
\hline
\end{tabular}

-Density is calculated based on $\mathrm{Na}, \mathrm{OH}$, and $\mathrm{AlO} 2$

+ Water wi\% derived from the difference of density and total dissolved species. 
HNF-SD-WM-ER-323, Rev. 1

HDW Model Pev. 4

\begin{tabular}{|c|c|c|c|c|c|c|c|}
\hline \multirow{3}{*}{\begin{tabular}{|l|} 
\\
\\
Physical \\
Properties:
\end{tabular}} & \multicolumn{7}{|c|}{ Single-Shell Tark 241-S-112 } \\
\hline & \multicolumn{7}{|c|}{ Total Inventory Estimate* } \\
\hline & & & & $.95 \mathrm{CI}$ & $-67 \mathrm{CI}$ & $+67 \mathrm{CI}$ & $+95 \mathrm{Cl}$ \\
\hline Total Waste & $3.33 \mathrm{E}+06(\mathrm{kB})$ & (523 kgai) & $\ldots$ & - & -- & $\because$ & - \\
\hline Heat Load & $4.28(\mathrm{~kW})$ & (1.46E+04 BTU/hr) & - & 4.00 & 4.13 & 4.44 & 4.45 \\
\hline Buik Density ${ }^{\dagger}$ & $1.68(\mathrm{~g} / \mathrm{cc})$ & - & $\cdots$ & 1.61 & 165 & 1.71 & 1.72 \\
\hline Water wi\%t & 29.2 & san. & -- & 27.1 & $2 B .0$ & 308 & 33.3 \\
\hline TOC wt $\%$ C (wet & 0.324 & - & - & 0.289 & 0.306 & 0.341 & 0.358 \\
\hline $\begin{array}{l}\text { Radialogical } \\
\text { Constituents: }\end{array}$ & $C / L$ & $\mu \mathrm{Ci} / \mathrm{s}$ & $\mathrm{Cl}$ & $\begin{array}{l}-95 \mathrm{CI} \\
(\mathrm{CUL})\end{array}$ & $\begin{array}{l}-67 \mathrm{CI} \\
(\mathrm{Ci} / \mathrm{L})\end{array}$ & $\begin{array}{l}+67 \mathrm{CI} \\
(\mathrm{CH} / \mathrm{L})\end{array}$ & $\begin{array}{l}+95 \mathrm{Ct} \\
(\mathrm{Ci} / \mathrm{L})\end{array}$ \\
\hline $\mathrm{H}-3$ & $2.64 \mathrm{E}-04$ & 0.157 & 523 & $1.58 \mathrm{E}-04$ & $1.58 \mathrm{E}-04$ & $2.80 \mathrm{E}-04$ & $2.74 \mathrm{E}-04$ \\
\hline C-14 & $3.37 \mathrm{E}-05$ & $2.00 E-02$ & 66.8 & $1.16 \mathrm{E}-05$ & $1.16 \mathrm{E}-05$ & 3.45E-05 & $3.44 \mathrm{E}-05$ \\
\hline $\mathrm{Ni}-59$ & $239 \mathrm{E}-06$ & $1.42 \mathrm{E}-03$ & 4.73 & $1.34 \mathrm{E}-06$ & $1,34 \mathrm{E}+06$ & 2.44E-06 & $2.49 \mathrm{E}-06$ \\
\hline $\mathrm{Ni}-63$ & 2.32E-04 & 0.138 & 458 & $1.28 \mathrm{E}-04$ & $1.28 \mathrm{E}-04$ & $2.37 \mathrm{E}-04$ & $2.42 \mathrm{E}-04$ \\
\hline $\mathrm{Co}-60$ & $341 \mathrm{E}-05$ & $2.03 \mathrm{E}-02$ & 67.5 & $871 \mathrm{E}-06$ & 8.71E-06 & 3.46E-05 & $3.51 \mathrm{E}-05$ \\
\hline Se-79 & $3.52 \mathrm{E}-06$ & $2.09 \mathrm{E}-03$ & 6.97 & $2.10 \mathrm{E}-06$ & $2.10 \mathrm{E}-\infty 6$ & $4.03 \mathrm{E}-06$ & $4.52 \mathrm{E}-06$ \\
\hline Sr-90 & 0.121 & 71.7 & $2.39 \mathrm{E}+05$ & 0.114 & 0.117 & 0.124 & 0.128 \\
\hline$Y-90$ & $0.12 i$ & 71.7 & $2.39 \mathrm{E}+05$ & $7.17 \mathrm{E}-02$ & $7.17 \mathrm{E}-02$ & 0.124 & 0.128 \\
\hline $\mathrm{Zr}-93$ & $172 \mathrm{E}-05$ & $1.02 \mathrm{E}-02$ & 34,0 & 1.01E-05 & 1.01E-05 & $1.98 \mathrm{E}-0 \mathrm{~S}$ & 2.22E-05 \\
\hline $\mathrm{Nb}-93 \mathrm{~m}$ & $1.28 E-0 S$ & $7.58 \mathrm{E}-03$ & 25,2 & $7.30 \mathrm{E}-06$ & $7.80 \mathrm{E}-06$ & $1.45 \mathrm{E}-05$ & $1.63 \mathrm{E}-05$ \\
\hline Tc-99 & 2.41E-04 & 0.143 & 476 & $1.58 E-04$ & 1.98E-04 & $2.83 E-04$ & $3.24 \mathrm{E}-04$ \\
\hline Ru-106 & $5.25 E-09$ & $3.12 \mathrm{E}-06$ & $1.04 \mathrm{E}-02$ & $1.97 \mathrm{E}-09$ & $1.97 \mathrm{E}-09$ & $5.98 \mathrm{E}-09$ & 6.65E. 09 \\
\hline $\mathrm{Cd}-113 \mathrm{~m}$ & 8. $40 \mathrm{E}-0.5$ & 4.99E- 02 & 166 & 4. 15E-05 & $4.15 \mathrm{E}-05$ & $9.94 \mathrm{E}-0 \mathrm{~S}$ & J.14E-04 \\
\hline $5 b-125$ & $1.40 \mathrm{E}-04$ & $8.31 E-02$ & 277 & $3.00 \mathrm{E}-05$ & $3.00 \mathrm{E}-05$ & 1.42E-04 & $1.44 \mathrm{E}-04$ \\
\hline $5 n-126$ & $5.33 \mathrm{E}-06$ & $3.17 E-03$ & 10.6 & 3.21E-06 & 3.21E-06 & $6.10 E-06$ & $6.84 \mathrm{E}-06$ \\
\hline $1-129$ & $4.63 \mathrm{E}-07$ & 2.75E-04 & 0917 & 3.03E-07 & $3.81 \mathrm{E}-07$ & $5.46 \mathrm{E}-07$ & $6.25 \mathrm{E}-07$ \\
\hline Cs-134 & 1.57E-06 & 9.33E-04 & 3.11 & $1.05 \mathrm{E}-06$ & 1.05E-06 & $1.69 \mathrm{E}-06$ & $1.80 \mathrm{E}-06$ \\
\hline Cs-137 & 0.288 & 171 & $5.71 E+05$ & 0.260 & 0.273 & 0,305 & 0.307 \\
\hline $\mathrm{Ba}-137 \mathrm{~m}$ & 0.273 & 162 & $5.40 \mathrm{E}+05$ & 0.204 & 0.204 & 0.288 & 0.290 \\
\hline $\mathrm{Sm}_{m}+151$ & 1.24E-02 & 7.38 & $2.46 \mathrm{E}+04$ & $7.46 \mathrm{E}-03$ & $7,46 \mathrm{E}-03$ & $1.42 \mathrm{E}-02$ & $1.59 \mathrm{E}-02$ \\
\hline Eu-152 & $3.12 \mathrm{E}-06$ & $1.86 \mathrm{E}-03$ & 6.18 & 1.37E-06 & $1.37 \mathrm{E}-06$ & $3.18 \mathrm{E}-06$ & $3.24 \mathrm{E}-06$ \\
\hline Eu-154 & $5.48 \mathrm{E}-04$ & 0.326 & $1.08 E+03$ & $1.94 \mathrm{E}-04$ & $1.94 \mathrm{E}-04$ & $6.76 E-04$ & $7.27 \mathrm{E}-04$ \\
\hline Eu-155 & 1.79E-04 & 0.106 & 354 & 7.45E-05 & $7.45 \mathrm{E}-05$ & $1.83 \mathrm{E}-0.4$ & $188 \mathrm{E}-04$ \\
\hline Ra-226 & $1.70 \mathrm{E} \cdot 10$ & $1.01 \mathrm{E}-07$ & $3.38 \mathrm{E}-04$ & $1.22 \mathrm{E}-10$ & $1,27 E-10$ & $1.95 \mathrm{E}-10$ & $2.19 \mathrm{E}-10$ \\
\hline $\mathrm{Ra}-228$ & $5.95 \mathrm{E} \cdot 08$ & $3.53 \mathrm{E}-05$ & 0.118 & $2,33 \mathrm{E}-08$ & $4.10 \mathrm{E}-08$ & $8.09 \mathrm{E}-08$ & $104 \mathrm{E}-07$ \\
\hline$A c-227$ & $1.02 E-09$ & 6.05E-07 & $202 E-03$ & $7.38 \mathrm{E}-10$ & $7.64 \mathrm{E}-10$ & $1.16 \mathrm{E}-09$ & $125 \mathrm{E}-09$ \\
\hline $\mathrm{Pa}-231$ & $4.38 \mathrm{E}-09$ & $260 \mathrm{E}-06$ & 8.67E-03 & $2,97 \mathrm{E}-09$ & $2.97 \mathrm{E}-09$ & 4.89E- 09 & $539 \mathrm{E}-09$ \\
\hline Th-229 & $1.42 \mathrm{E}-09$ & 8.42E-07 & $2.80 \mathrm{E}-03$ & $6.22 \mathrm{E}-10$ & $1.01 \mathrm{E}-09$ & $1.89 \mathrm{E}-09$ & $2.39 \mathrm{E}-09$ \\
\hline Th-232 & 4.01E- 09 & $2.38 E-06$ & $7.93 \mathrm{E}-03$ & $2.00 \mathrm{E}-09$ & $2.98 \mathrm{E}-09$ & $5.03 \mathrm{E}-09$ & $601 \mathrm{E}-09$ \\
\hline $\mathrm{U}-232$ & $3.58 E-07$ & $2.13 \mathrm{E}-04$ & 0.710 & 2.13E-07 & 2.84E-07 & $4,44 E,-07$ & $5.36 \mathrm{E}-07$ \\
\hline $\mathrm{U}-233$ & $1,38 \mathrm{E}-06$ & $8.17 \mathrm{E}-0.4$ & 2.72 & $8.20 \mathrm{E}-07$ & $109 \mathrm{E}-06$ & $1.70 \mathrm{E}-06$ & 2.06E- 06 \\
\hline U-234 & 9.46E-07 & $5.62 \mathrm{E}-04$ & 1.87 & $8.73 \mathrm{E}-07$ & 9.07E-07 & $9.88 \mathrm{E}-07$ & 9.70E-07 \\
\hline U-235 & $3,92 \mathrm{E}-08$ & $2.33 \mathrm{E}-05$ & $7.76 \mathrm{E}-02$ & $3.61 \mathrm{E}-08$ & 3.76E- -08 & $4.10 \mathrm{E}-0 \mathrm{~B}$ & $402 \mathrm{E}-08$ \\
\hline U-236 & $2.63 \mathrm{E} .08$ & $1.56 \mathrm{E}-0 \mathrm{~S}$ & $5.20 \mathrm{E}-02$ & 2.51E-08 & 2.57E-08 & $2.69 \mathrm{E}-08$ & 2.70E-08 \\
\hline U-238 & $9.79 \mathrm{E}-07$ & 5.82E-04 & 1.94 & $9.04 \mathrm{E}-07$ & $940 \mathrm{E}-07$ & $1.02 \mathrm{E}-06$ & $1.00 \mathrm{E}-06$ \\
\hline $\mathrm{Np}-237$ & $9.52 \mathrm{E}-07$ & $5.66 \mathrm{E}-04$ & 1.89 & $6.82 \mathrm{E}-07$ & 8. 14E-07 & $1.09 \mathrm{E}-\infty 6$ & $1.23 \mathrm{E}-06$ \\
\hline $\mathrm{Pu}-238$ & $1.35 \mathrm{E}-06$ & $8.04 \mathrm{E}-04$ & 2.68 & $1.04 E-06$ & 1.19E-06 & 1.52E-06 & $1.67 E-06$ \\
\hline Pu-239 & 559E-05 & 3.32E-02 & 111 & $4.78 \mathrm{E}-05$ & $5.18 \mathrm{E}-05$ & 6.00E-05 & $6.40 \mathrm{E}-05$ \\
\hline Pu-240 & $8.89 \mathrm{E}-06$ & $5.28 \mathrm{E}-03$ & 17.6 & $7.57 \mathrm{E}-06$ & 8.12E-06 & 9.66E-06 & $1.04 \mathrm{E}-05$ \\
\hline Pu-241 & $876 \mathrm{E}-05$ & $5.20 \mathrm{E}-02$ & 173 & $6.59 \mathrm{E}-05$ & $7.65 \mathrm{E}-05$ & $9.86 \mathrm{E}-05$ & $1.09 \mathrm{E}-04$ \\
\hline Pu-242 & $4.62 \mathrm{E}-10$ & $2.74 \mathrm{E}-07$ & $9.14 \mathrm{E}-04$ & $3.33 \mathrm{E}-10$ & $3.96 \mathrm{E}-10$ & $5.28 \mathrm{E}-10$ & $5.91 E-10$ \\
\hline$A \mathrm{~m}-241$ & $569 \mathrm{E}-05$ & 3.38E-02 & 113 & 4. $18 \mathrm{E}-05$ & $4.92 \mathrm{E}-05$ & $646 \mathrm{E}-05$ & $7.19 E-05$ \\
\hline Am-243 & $1.59 \mathrm{E}-09$ & $9.44 E-07$ & 3.15E-03 & 1.12E-09 & 1.34E-09 & 1.84E-09 & $2.08 E-09$ \\
\hline $\mathrm{Cm}-242$ & 1.11E-07 & $6.57 \mathrm{E}-05$ & 0.219 & $3.86 \mathrm{E}-08$ & $3,86 \mathrm{E}-08$ & $1.13 \mathrm{E}-07$ & 1.15E-07 \\
\hline $\mathrm{Cm}-243$ & $9.78 \mathrm{E}-09$ & $5.81 \mathrm{E}-06$ & $1.94 \mathrm{E}-02$ & $2.92 \mathrm{E}-09$ & $2.92 \mathrm{E}-09$ & 1.00E-08 & 1.02E-08 \\
\hline $\mathrm{Cm}-244$ & $1.07 E+07$ & $6.38 \mathrm{E}-05$ & 0.213 & $3.66 \mathrm{E}-08$ & $3.66 \mathrm{E}-08$ & $1.31 \mathrm{E}-07$ & $1.47 \mathrm{E}-07$ \\
\hline Totak: & $\mathbf{M}:$ & $\mathrm{ng} / \mathrm{g}$ & kg & $\begin{array}{c}95 \mathrm{Cl} \\
\text { (Mor } \\
\mathrm{g} / \mathrm{L})\end{array}$ & $\begin{array}{c}-67 \mathrm{Cl}(\mathrm{M} \\
\text { or } \mathrm{g} \mathrm{L})\end{array}$ & $\begin{array}{c}+67 \mathrm{Cl}(\mathrm{M} \\
\text { or } \mathrm{g} / \mathrm{L})\end{array}$ & $\begin{array}{c}+95 \text { CI } \\
\text { (M ar } \\
\text { g/L) }\end{array}$ \\
\hline $\mathrm{Pu}$ & $7.75 E-04(\mathrm{~g} / \mathrm{L})$ & - & 1.53 & $6.21 \mathrm{E}-04$ & 6.96E-04 & 8.53E-0.4 & $9.28 \mathrm{E}-04$ \\
\hline U & \begin{tabular}{ll|}
$1.12 \mathrm{E}-02$ \\
\end{tabular} & 1.59E+03 & $5.28 \mathrm{E}+03$ & $1.03 \mathrm{E}-02$ & $1.07 \mathrm{E}-02$ & $1.17 \mathrm{E}-02$ & 1.15E-02 \\
\hline
\end{tabular}

"Unknowns in tank solids inventory are assigned by Tank Layering Model (TLM)

†Volume average for density, mass average Water wi $\%$ and TOC wit C. 
TH!n,$A$ INTENTIONALLY .. I ELANK 
HNF-SD-WM-ER-323, Rev. 1

\begin{tabular}{|c|c|c|c|c|c|}
\hline \multicolumn{6}{|c|}{ DISTRIBUTION SHEET } \\
\hline \multirow{2}{*}{$\begin{array}{l}\text { To } \\
\text { Distribution }\end{array}$} & \multirow{2}{*}{\multicolumn{3}{|c|}{$\begin{array}{l}\text { From } \\
\text { C. H. Brevick }\end{array}$}} & \multicolumn{2}{|l|}{ Page 1 of 1} \\
\hline & & & & \multicolumn{2}{|c|}{$\begin{array}{l}\text { Date } \\
\text { February } 13,1997\end{array}$} \\
\hline \multicolumn{4}{|l|}{ Project Title/Work Order } & \multicolumn{2}{|l|}{ EDT No. } \\
\hline \multicolumn{4}{|c|}{$\begin{array}{l}\text { Supporting Document for the Southwest Quadrant Historical Tank } \\
\text { Content Estimate Report for S-Tank Farm/E18675 }\end{array}$} & \multicolumn{2}{|c|}{$\begin{array}{l}\text { ECN No. } \\
624864\end{array}$} \\
\hline Name & MSIN & $\begin{array}{c}\text { Text } \\
\text { With All } \\
\text { Attach. }\end{array}$ & Text Only & $\begin{array}{l}\text { Attach./ } \\
\text { Appendix } \\
\text { Only }\end{array}$ & $\begin{array}{l}\text { EDT/ECN } \\
\text { Only }\end{array}$ \\
\hline
\end{tabular}

Department of Energy Richland Operations

J. F. Thompson

S7-54 $\quad X$

Fluor Daniel Northwest Inc.

C. H. Brevick

J. W. Funk

L. A. Gadd is

E. D. Johnson

Engr. Publications

S3-10 $\quad X$

S3-09 $X$

S3-04 $\quad X$

S3-09 $\quad X$

Lockheed Mart in Hanford Company

T. M. Brown

J. W. Cammann

M. L. Dexter

J. W. Hunt

G. Jansen

E6-63

B. C. Simpson

A. E. Young

R2-12 $\quad x$

R2-12 $\quad x$

R1-51 $\quad X$

R2-12 $\quad X$

H6-34 $\quad X$

R2-12 $\quad X$

R2-12 $X$

Lockheed Martin Services Inc.

Central Files

Document Control

A3-88 $X$

R1-29 X

Pacific Northwest National Laboratory

T. L. Traub

K. D. Wiemers

$\mathrm{H} 2-53 \quad \mathrm{X}$

$\mathrm{K} 6-51 \quad \mathrm{X}$

P. D. Whitney

$\mathrm{K} 5-12 \quad \mathrm{X}$

OFFSITE

Los Alamos National Laboratory

S. F Agnew

Los Alamos National Laboratory

$X$

CST-14, MS-J586

Bikini Atoll Rd, SM30

Los Alamos, NM 87545

\section{Los Alamos Technical Associates}

T. T. Tran

$X$

903 Bradley Boulevard

Richland, Washington 99352 
THIS PAGE INTENTIONALLY LEFT BLANK 